\title{
DYNAMIC OBJECTS: UNRAVELLING VEGETATION PATTERNS IN A HIGHLY DYNAMIC FLUVIAL ENVIRONMENT
}

\author{
E.A. Addink, H. Douma, Y.T. Duindam, M.G. Kleinhans \\ Department of Physical Geography, Faculty of Geosciences, Utrecht University \\ e.a.addink@uu.nl
}

KEY WORDS: riparian vegetation dynamics, Allier, aerial photographs

\section{EXTENDED ABSTRACT:}

Multitemporal analysis with GEOBIA is complicated by the changing geometry of image objects, even when the scene objects are static. The analysis gets even more complicated when the scene objects are dynamic and geometric changes stem from the scene and image processing. In this study we applied GEOBIA to a time series of photos representing a highly dynamic landscape, i.e. a natural cause of geometric dynamics.

In floodplains of meandering rivers erosion and sedimentation are dominant processes that remove and create banks. Consequently, banks and the vegetation on top have different (maximum) ages at short distances. This vegetation affects the hydrodynamics during high discharge and hence erosion and sedimentation patterns. Vice versa the hydrodynamics affect vegetation through shifting channels, flooding, drought, and seed dispersal.

First attempts to model this interaction produce realistic vegetation patterns (Van Oorschot et al., 2016). However, no ground truth on dynamic vegetation patterns is available. In this project we analyzed emerging vegetation patterns in aerial photographs in five time steps spanning 30 years. The study site was a 2-km stretch of the river Allier near Chatel-de-Neuvre in France. Land cover is characterized by open areas with no or low vegetation dotted by isolated shrubs and trees, next to patches of riparian forest (Geerling et al., 2006). The river shows strong discharge fluctuations, leading to significant channel migration and at least yearly flooding of the entire floodplain.

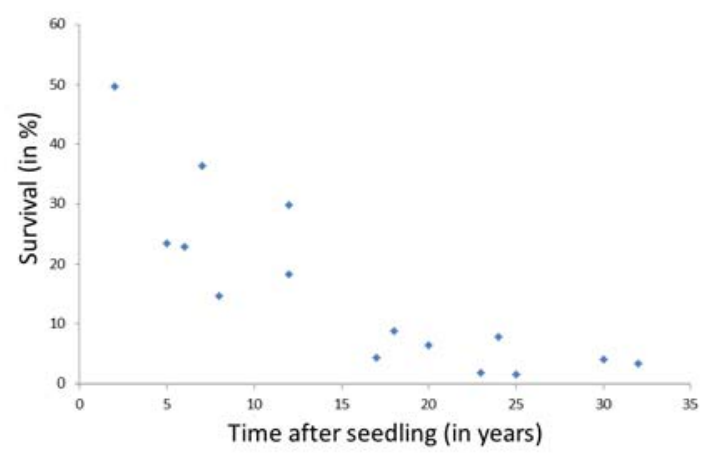

Figure 1. Survival of isolated trees and shrubs
We focused on isolated shrubs and trees as these are the intermediate step from vegetated plains to riparian forests and facilitate the development of new shrubs/trees. We distinguished four categories: small and large objects with a bare or vegetated surface background. We monitored their status in relation to their size, shape and position relative to the river.

First results show that vegetation along the Allier is highly dynamic, caused by a frequent setback to the pioneer stage. Only $20-25 \%$ of the shrubs/trees survive a period of $10+$ years (figure 1). The vast majority of trees/shrubs disappear within 10 years, indicating that $75-80 \%$ of the trees/shrubs are only a few years old. The evolution of forest patches is the exception rather than the rule, even though forest patches are omnipresent. Apparently the trees are highly resistant against flooding and drought once they reach the forest stage.

We will include our findings in a framework for dynamicobject-based image analysis to support studies of changing landscapes.

\section{REFERENCES}

Geerling, G. W., A. M. J. Ragas, R. S. E. W. Leuven, J. H. Van Den Berg, M. Breedveld, D. Liefhebber, and A. J. M. Smits, 2006. Succession and rejuvenation in floodplains along the river Allier (France). Hydrobiologia 565:71-86.

Van Oorschot, M., M. Kleinhans, G. Geerling, and H. Middelkoop, 2016. Distinct patterns of interaction between vegetation and morphodynamics. Earth Surface Processes and Landforms 41:791-808. 


\title{
AN OBJECT-BASED BURNT AREA DETECTION METHOD BASED ON LANDSAT IMAGES - A STEP FORWARD FOR AUTOMATIC GLOBAL HIGH-RESOLUTION MAPPING
}

\author{
E. Woźniak ${ }^{\mathrm{a}}$, S. Aleksandrowicz ${ }^{\mathrm{a}, *}$ \\ ${ }^{a}$ Space Research Centre, Polish Academy of Sciences, Bartycka 18A, 00-716 Warsaw, Poland (ewozniak@cbk.waw.pl, \\ saleksandrowicz@cbk.waw.pl*)
}

KEY WORDS: forest fires, automatic classification, HR mapping

\begin{abstract}
:
This study presents an algorithm for automatically mapping burnt areas using high-resolution images. It is applied to the Landsat 4 , 5, 7 and 8 Land Surface Reflectance product; specifically, images acquired before and after (or during) the same fire season. It is also possible to extend the timeframe and use reference images acquired in the preceding year. This approach was adopted as cloudiness can make the acquisition of long time series impossible. A second advantage is that it avoids huge data transfers. The algorithm combines traditional, pixel-based image processing (calculation of spectral indexes and image differentiation) with object-based procedures (segmentation, reclassification, neighbourhood analysis) and consists of four steps. First, spectral indices (the Normalized Difference Vegetation Index and Normalised Burnt Ratio), and differences between image layers are calculated. The second is a multi-resolution segmentation, which uses the Normalised Burnt Ratio and near infrared layers. At this phase, masking of clouds, water and deserts takes place using atmospherically-corrected Landsat images. This is followed by the classification of 'core' burnt areas based on automatically-adjusted thresholds. The characteristics of the whole image (excluding clouds, deserts and water bodies) are analysed to develop functions that establish these thresholds. The fourth step consists of neighbourhood analysis. This focuses on objects that have not been classified as burnt areas, but whose spatial and spectral distances suggest that they may be part of them. The algorithm was tested in various areas (e.g. Spain, Greece, Siberia, California, Australia and Zambia). Comparisons with manual interpretation show that the fully-automated classification is very accurate (80-100\%). The algorithm can be also applied to MODIS and Sentinel-2 data. It was developed within the framework of the Advanced Forest Fire Fighting (AF3) project, and the results have been used for damage and risk assessment.
\end{abstract}

\section{INTRODUCTION}

\subsection{Literature overview}

The reconstruction, understanding and characterization of the fire history of a terrain is important for improving fire management practices, risk prediction and damage assessment. It also extends our knowledge of the environmental and socioeconomic impacts of fire, climate change, the carbon cycle, biodiversity, ecosystem functioning, and interactions between land use and vegetation. Burnt area mapping defines at least three very important aspects of the fire regime: spatial pattern, size distribution and frequency.

Satellite remote sensing has proven to be an excellent source of information about forest fires. Burnt area studies have been carried out successfully all over the world: in tropical environments (Malingreau et al. 1985, Libonati et al. 2010), in savannas and grassland environments (Silva et al. 2005, Goodwin \& Collett 2014, Hardtke et al. 2015), in Mediterranean zones (Fernandez et al. 1997, Garcia \& Chuvieco 2004, Quintano et al. 2011), and in boreal forests (Laboda et al. 2007, Chen et al. 2016).

Several spectral indices and classification methods have been developed and tested to map burnt areas; these include the Normalised Difference Vegetation Index (NDVI) (Rouse et al. 1973, Chuvieco et al. 2002), the Global Environmental Monitoring Index (Pinty \& Verstraete 1992), the Normalised Burnt Ratio (NBR; the normalised difference of Landsat TM Bands 4 and 7), multi-temporal variations (Key \& Benson 1999, Miller \& Thode 2007, Veraverbeke et al. 2001) and the Burnt Area Index (Martin 1998). Similarly, a wide range of methods are used for fire monitoring: manual digitalisation of burned areas (Silva et al. 2005), near-real-time active fire detection (Giglio et al. 2006), spectral indices (Martin et al. 2006, Laboda et al. 2007), image thresholding (Libonati et al. 2010, Maier 2010, Quintano et al. 2011), fire radiative power estimation (Maier et al. 2013, Williamson et al. 2013), time series analysis (Goodwin \& Collett 2014, Hardtke et al. 2015), object-based approaches (Katagis et al. 2014), and Synthetic Aperture Radar and optical data fusion (Stroppiana et al. 2015).

\subsection{Purpose of the study}

Regional- and global-scale studies of burnt areas have been performed on low resolution $(5 \mathrm{~km})$ National Oceanic and Atmospheric Administration (NOAA) Advanced Very High Resolution Radiometer (AVHRR), or medium-resolution (1 km) Moderate Resolution Imaging Spectroradiometer (MODIS) data (Barbosa et al. 1999, Justice et al. 2002, Roy et al. 2005, Giglio et al. 2006). Historically, high costs and manual or semi-automated mapping methods have limited the use of Landsat images (30 m spatial resolution) to local studies (Russell-Smith et al. 1997, Edwards et al. 2001, Recondo et al. 2002, Felderhof \& Gillieson 2006). However, the Landsat archive was made freely available in 2008, and surface reflectance data is available from 1984 to the present day. Similarly, Sentinel-2 data is freely available. On the other hand, limitations related to image classification methods remain, which highlights the need for the development of automatic classification algorithms for high-resolution data.

Changes in vegetation cover are often unrelated to fire, and can be caused by other factors such as phenological changes, harvesting or soil moisture. In addition, burnt areas are inhomogeneous and their spectral signature is closely related to fire intensity, fuel type, the meteorological conditions of 
combustion, etc. These factors make the development of automated approaches difficult, and require balancing omission and commission errors (Goodwin \& Collett 2014). Furthermore, it may not be possible to transfer methods to other locations or timeframes without recalibration.

Bastarrika et al. (2011) proposed an automated two-phase approach to mapping burnt areas (core burnt pixel identification and burnt region growth) using a series of single date Landsat TM/ETM+ imagery. The algorithm was calibrated and applied to Portugal and California (kappa coefficient $=0.85$ ). A similar approach was applied in the Mediterranean region (Stroppiana et al. 2012), while a trend analysis formed the basis for automatic burnt area mapping in Australia (Goodwin et al. 2014).

Following these studies, this paper describes an algorithm for the automatic mapping of burnt areas. It combines a classical approach, based on the difference between pre- and post-fire images, with an object-based thresholding approach. Full automatization is the result of a recalibration procedure, specifically functions are developed for various parameters. that adjust thresholds between unburnt and burnt areas basing on the difference between pre- and post-fire images. As the method works on two images, it does not require long time series (unlike trajectory analysis methods). This is an important benefit given the burden of data transfer for global mapping, and cloud cover that can prevent the creation of long time series.

\section{METHOD}

\subsection{Data}

The algorithm uses atmospherically-corrected pairs of multispectral optical images (the Landsat 4, 5, 7, and 8 Surface Reflectance product). This product is supplied with cloud and water masks, which are also used. Acquisition dates are key to the algorithm's performance. The first (reference) image should be acquired before the fire season. The second image should be acquired during, or shortly after, it. Alternatively, a reference image acquired one year before the fire season image can be used with no loss of accuracy. Finally, the algorithm requires a thematic layer, namely, the slope layer calculated from Shuttle Radar Topography Mission elevation data.

The algorithm was tested on long time series for two regions in Greece: the surroundings of Athens (path 184 row 34) and the island of Cephalonia (path 185 row 33) and other scenes worldwide (California, Israel, central Siberia, northern and southern Spain, and Australia).

\subsection{Proposed workflow}

Figure 1 presents an overview of the method. It consists of four main steps: (1) the calculation of spectral indices and band differences; (2) the segmentation and exclusion of water bodies, desert and cloud; (3) the detection of 'core' burnt areas; and (4) region growing. All steps were implemented in eCognition software.

\subsection{Raster arithmetic}

The first step consists of calculating a set of additional layers based on pre- and post-fire scenes. The NBR (Key \& Benson 2002) is calculated, along with the NDVI (Rouse at al. 1974).

$$
\mathrm{NBR}=\frac{\mathrm{NIR}-\mathrm{SWIR}}{\mathrm{NIR}+\mathrm{SWIR}}
$$

The differences in near infrared (NIR), short wave infrared (SWIR1, SWIR2) spectral bands and NBR for pre- and post-fire images are calculated. These differences are expressed as relative values and calculated as follows:

$$
N B R_{D I F F}=100-\frac{N B R_{T 2} * 100}{N B R_{T 1}}
$$

where $_{T 1}$ is the pre-fire image, and $T_{2}$ is the post-fire image.

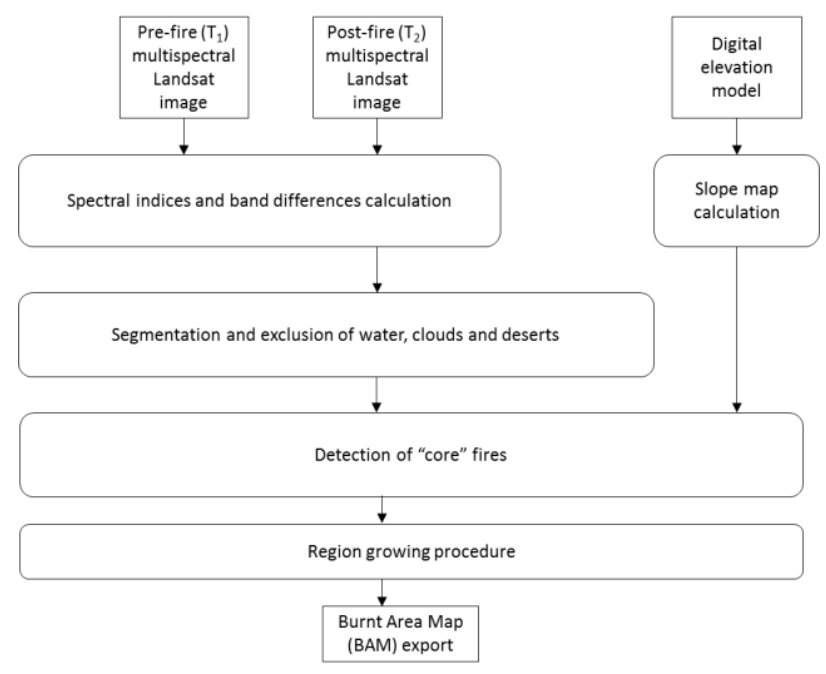

Figure 1. Burnt Area Mapping workflow

\subsection{Segmentation}

Segmentation starts with masking water, cloud and desert, which are excluded from further analysis. The aim is to reduce computation time and avoid any interference with the automatic thresholding process. The first step is based solely on Landsat thematic layers, and segments are classified into an 'exclude from analysis' group. For example, desert areas are masked based on a comparison of the NDVI index for the two images (which should be low in both cases). The second step concerns multiresolution segmentation. Segmentation layers $\mathrm{NBR}_{\mathrm{T} 1}$, $\mathrm{NBR}_{\mathrm{T} 2-\mathrm{T} 1}$, and $\mathrm{NIR}_{\mathrm{T} 1-\mathrm{T} 2}$ are weighted. The scale parameter is fixed and remains unchanged when moving from one scene to another.

\subsection{Core burnt areas classification}

The classification of core burnt areas starts with a coarse classification of the $\mathrm{NBR}_{\mathrm{T} 2}$ layer (Figure 3a). Objects with a low NBR are considered as potential burnt areas. Specifically, objects with $\mu_{o}<\left(\mu_{2}-\sigma_{2}\right)$ (where $\mu_{o}$ is the mean NBR, $\mu_{2}$ is the mean NBR for the scene, and $\sigma_{2}$ is the standard deviation for the scene) are classified as _temp. In the next step, thresholds between unburnt and burnt areas are calculated as a function of the difference between pre- and post-fire images.

$$
\text { threshold }=\mathrm{f}\left(\text { image }_{\text {difference }}\right)
$$

Threshold functions were obtained for all parameters from a reference dataset of pairs of images in different regions. The burnt areas were mapped manually using this dataset. Statistics for unburnt and burnt segments were extracted from the difference image for all parameters and used to calculate thresholds. Thresholds were calculated on the base of the normal distribution (Woźniak et al. 2016). The polynomial regressions between thresholds and difference image were found. An example of a thresholding function is given in Figure 2. Functions for $\mathrm{SWIR} 1_{\mathrm{DIFF}}, \mathrm{SWIR} 2_{\mathrm{DIFF}}, \mathrm{G}_{\mathrm{T} 2}, \mathrm{R}_{\mathrm{T} 2}$ are established in the same way. Areas previously excluded from the analysis (clouds, water, desert) are not taken into account. 


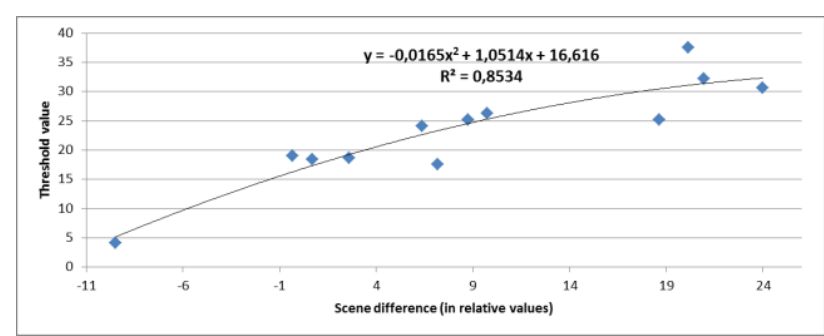

Figure 2. Relationship between scene difference and threshold for burnt and unburnt areas discrimination

However, although the results are good, the approach produces two types of misclassification. The first is the misclassification of crops that are harvested between data acquisitions as burnt areas. The second relates to partial omissions due to the inhomogeneous nature of burnt areas. The first problem is easily addressed using a slope map, as crop areas are usually found on plains Region growing provides a solution for the latter problem.

\subsection{Region growing}

Region growing (Figure 3c) starts with merging objects classified as core burnt areas. Neighbouring objects are classified based on their spectral distance from the core, calculated as the standard deviation of all areas classified as burnt in a scene. If a neighbouring object is classified as a burnt area it is merged into the core and the process is repeated.
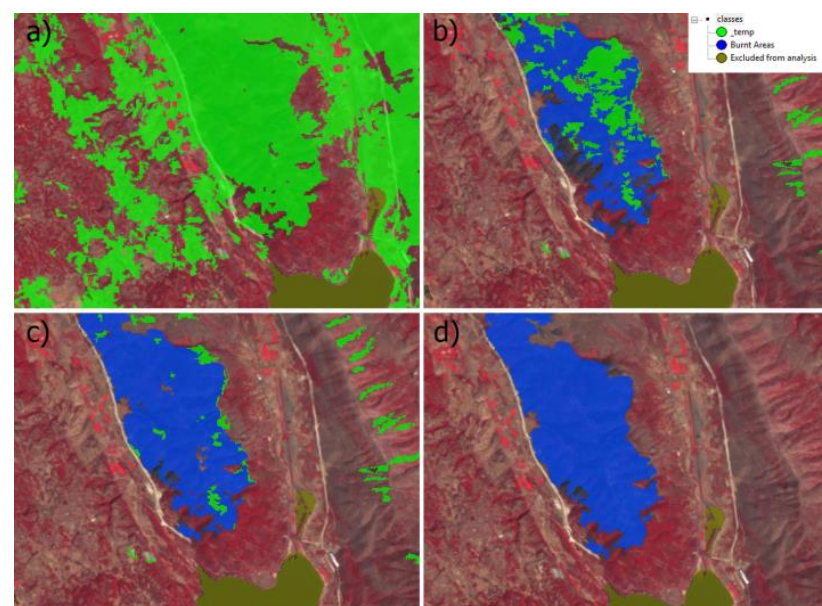

Figure 3. a) Initial NBR classification, b) classification of core burnt areas, c) region growing, $d$ ) post-processing

\subsection{Post-processing}

Post-processing is the final step of the classification (Figure 3d). Here, objects are merged and the minimum mapping unit (1ha) is applied. An enclosure analysis corrects burnt areas obscured by clouds.

\section{RESULTS}

The method was tested for all Landsat satellites in various geographical areas. For the island of Cephalonia and the surroundings of Athens all available Landsat scenes (for years in which at least two acquisitions were available) were classified. Robustness was tested on scenes from Siberia, northern and southern Spain, California, Israel and Australia. Burnt area mapping was validated using stratified random sampling. Stratification was based on the area of detected classes. One point indicated 100 pixels of burnt area and 1, 000 or 10, 000 pixels classified as unburnt depending on the percentage of the burnt area on the image scene. The results were compared with a manual classification of points. Mean overall accuracy for the tested scenes was $97.66 \%$ (mean kappa 0.86 ). Lowest values were obtained for scene from Athens from 1987 (respectively 85.79 and 0.25). Highest values were obtained also for scene from Athens from 1999 (respectively 99.94 and 0.95). Table 1 presents an example of a detailed confusion matrix for scene from Siberia.

\begin{tabular}{|c|c|c|c|c|c|}
\hline & \multicolumn{2}{|c|}{ Classification } & \multirow[b]{2}{*}{ Sum } & \\
\hline & & Burnt & Unburnt & & \\
\hline \multirow{2}{*}{ 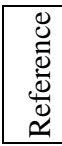 } & Burnt & 14083 & 1069 & 15152 & 92,94 \\
\hline & Unburnt & 1746 & 20892 & 22638 & 92,29 \\
\hline & Sum & 15829 & 21961 & 34975 & \\
\hline & & 88,97 & 95,13 & $\begin{array}{l}\text { Overall } \\
\text { accuracy }\end{array}$ & 92,55 \\
\hline
\end{tabular}

Table 1. Confusion matrix for scene from Siberia.

\section{CONCLUSIONS}

The burnt area mapping method presented here was tested in various areas (e.g. Spain, Greece, Siberia, California, Australia and Zambia). Threshold functions and the region growing procedure proved easily transferable, and accuracy remained satisfactory with no loss in automation.

The approach can also be applied to smaller-scale mapping. Following tuning of threshold functions and segmentation parameters, it was applied to MODIS Land Surface Reflectance (1000 $\mathrm{m}$ resolution) images. The results are more accurate than the fire estimates given in the MODIS QA layer.

Finally, similarities between Landsat and Sentinel-2 data mean that the algorithm can be fully automated when applied to the latter images, which offers an opportunity to fill in gaps in Landsat acquisitions.

\section{ACKNOWLEDGEMENTS}

The algorithm was developed within the framework of the Advanced Forest Fire Fighting (AF3) project (FP7-SEC-2013-1, grant agreement $\mathrm{N}^{\circ} 607276$ )

\section{REFERENCES}

Barbosa, P.M., Gregoire, J.M., \& Cardoso Pereira, J.M. 1999. An Algorithm for Extracting Burned Areas from Time Series of AVHRR GAC Data Applied at a Continental Scale. Remote Sensing of Environment, 69(3), 253-263.

Bastarrika, A., Chuvieco, E., \& Martín, M. P. 2011. Mapping burned areas from Landsat TM/ETM+ data with a two-phase algorithm: Balancing omission and commission errors. Remote Sensing of Environment, 115(4), 1003-1012.

Chen, W., Moriya, K., Sakai, T., Koyama L., \& Cao, C.X. 2016. Mapping a burned forest area from Landsat TM data by multiple methods. Geomatics, Natural Hazards and Risk, 7(1), 384-402.

Chuvieco, E., Martín, M. P., \& Palacios, A. 2002. Assessment of different spectral indices in the red-near-infrared spectral 
domain for burned land discrimination. International Journal of Remote Sensing, 23, 5103-5110.

Edwards, A.C., Hauser, P., Anderson, M., McCartney, J., Armstrong, M., Thackway, R., et al. 2001. A tale of two parks: Contemporary fire regimes of Litchfield and Nitmiluk National Parks, monsoonal northern Australia. International Journal of Wildland Fire, 10, 79-89.

Felderhof, L., \& Gillieson, D. 2006. Comparison of fire patterns and fire frequency in two tropical savanna bioregions. Austral Ecology, 31, 736-746.

Fernandez, A., Illera, P., \& Casanova, J.L. 1997. Automatic mapping of surfaces affected by forest fires in Spain using AVHRR NDVI composite image data. Remote Sensing of Environment 60, 153-162.

Garcia, M., \& Chuvieco, E. 2004. Assessment of the potential of SAC-C/MMRS imagery for mapping burned areas in Spain. Remote Sensing of Environment, 92, 414-423.

Giglio, L., van der Werf, G., Randerson, J., Collatz, G., \& Kasibhatla, P. 2006. Global estimation of burned area using MODIS active fire observations. Atmospheric Chemistry and Physics, 6, 957-974.

Goodwin N.R., \& Collett, L.J. 2014. Development of an automated method for mapping fire history captured in Landsat TM and ETM+ time series across Queensland, Australia. Remote Sensing of Environment, 148, 206-221

Hardtkea,L.A., Blancoa, P.D., del Vallea, H.F., Metternichtb, G.I., \& Sionec, W.F. 2015. Semi-automated mapping of burned areas in semi-arid ecosystems using MODIS time-series imagery. International Journal of Applied Earth Observation and Geoinformation, 38, 25-35.

Justice, C. O., Giglio, L., Korontzi, S., Owens, J., Morisette, J. T., Roy, D., et al. 2002. The MODIS fire products. Remote Sensing of Environment, 83, 244-262.

Katagis, T., Gitas, I.Z., \& Mitri, G.H. 2014. An Object-Based Approach for Fire History Reconstruction by Using Three Generations of Landsat Sensors. Remote Sensing, 6(6), 5480 5496

Key, C. H., \& Benson, N. C. 1999. Measuring and remote sensing of burn severity. In L. F. Neuenschwander, \& K. C. Ryan (Eds.), Proceedings. Joint Fire Science Conference and Workshop, Vol. II, Boise, ID, 15-17 June 1999: University of Idaho and International Association of Wildland Fire (284 pp.).

Loboda, T., O’Neal, \& K., Csiszar, I. 2007. Regionally adaptable dNBR-based algorithm for burned area mapping from MODIS data. Remote Sensing of Environment, 109, 429-442.

Libonati, R., DaCamara, C., Pereira, J., \& Peres, L. 2010. Retrieving middle-infrared reflectance for burned area mapping in tropical environments using MODIS. Remote Sensing Environment, 114, 831-843.

Maier, S. 2010. Changes in surface reflectance from wildfires on the Australian continent measured by MODIS. International Journal of Remote Sensing, 31, 3161-3176.

Maier, S., Russell-Smith, J., Edwards, A., \& Yates, C. 2013. Sensitivity of the MODIS fire detection algorithm (MOD14) in the savanna region of the Northern Territory, Australia. ISPRS Journal of Photogrammetry and Remote Sensing, 76, 11-16.

Martín, M. P. 1998. Cartografia e inventario de incendios forestales en la Península Ibérica a partir de imágenes NOAAAVHRR. Departamento de Geografía. Alcalá de Henares: Universidad de Alcalá.

Martín, M., Gómez, I., \& Chuvieco, E. 2006. Burnt area index (BAIM) for burned area discrimination at regional scale using MODIS data. For. Ecol. Manage. 234s, s221.

Malingreau, J. P., Stephens, G., \& Fellows, L. 1985. Remote sensing of forest fires: Kalimantan and North Borneo in 198283. Ambio, 14, 314-321.

Miller, J.D., \& Thode, A. E. 2007. Quantifying burn severity in a heterogeneous landscape with a relative version of the delta Normalized Burn Ratio (dNBR). Remote Sensing of Environment, 109 (1), 66-80.

Pinty, B., \& Verstraete, M. M. 1992. GEMI: a non-linear index to monitor global vegetation from satellites. Vegetatio, 101, 15-20.

Quintano, C., Fernández-Manso, A., Stein, A., \& Bijker, W. 2011. Estimation of area burned by forest fires in Mediterranean countries: a remote sensing data mining perspective. For. Ecol. Manage, 262, 1597-1607.

Recondo, C., Woźniak, E., \& Perez-Morandaira, C. 2002. Cartografia de zonas quemadas en Asturias durante el periodo 1991-2001 a partir de imaenes Landsat TM, Revista de Teledeteccion, 18, 47-55.

Rouse, J.W., Haas, R.H., Schell, J.A. \& Deering, D.W. 1973. Monitoring vegetation systems in the great plains with ERTS. In, 3rd ERTS Symposium, 309-317 (NASA)

Roy, D., Jin, Y., Lewis, P., \& Justice, C. 2005. Prototyping a global algorithm for systematic fire-affected area mapping using MODIS time series data. Remote Sensing of Environment, 97, $137-162$.

Russell-Smith, J., Ryan, P. G., \& Durieu, R. 1997. A Landsat MSS-derived fire history of Kakadu National Park. Journal of Applied Ecology, 34, 748-766.

Silva, J., Sá, A., \& Pereira, J. 2005. Comparison of burned area estimates derived from SPOT-VEGETATION and Landsat ETM+ data in Africa: influence of spatial pattern and vegetation type. Remote Sensing of Environment, 96, 188-201.

Stroppiana, D., Bordogna, G., Carrara, P., Boschetti, M., Boschetti, L., \& Brivio, P. A. 2012. A method for extracting burned areas from Landsat TM/ETM+ images by soft aggregation of multiple spectral indices and a region growing algorithm. ISPRS Journal of Photogrammetry and Remote Sensing, 69, 88-102.

Stroppiana, D., Azar, R., Calò, F., Pepe, A., Imperatore, P., Boschetti, M., Silva, J.M.N., Brivio, P.A., \& Lanari, R. 2015. Integration of Optical and SAR Data for Burned Area Mapping in Mediterranean Regions. Remote Sensing, 7, 1320-1345

Veraverbeke, S., Lhermitte, S., Verstraeten, W.W., \& Goossens, R. 2001. A time-integrated MODIS burn severity assessment using the multi-temporal differenced normalized burn ratio 
(dNBRMT). International Journal of Applied Earth Observation and Geoinformation, 13, 52-58.

Williamson, G., Price, O., Henderson, S., \& Bowman, D. 2013. Satellite-based comparison of fire intensity and smoke plumes from prescribed fires and wildfires in south-eastern Australia. International Journal of Wildland Fire, 22, 121-129.

Woźniak, E., Kofman, W., Wajer, P., Lewiński, S., \& Nowakowski, A. 2016. The influence of filtration and decomposition window size on the threshold value and accuracy of land-cover classification of polarimetric SAR images. International Journal of Remote Sensing, 37(1), 212-228. 


\title{
APPLYING GEOBIA METHOD TO ANALYZE CLIMATE CHANGES ASSOCIATED TO ENERGY GENERATION - ANALYSIS ABOUT OIL EXPLORATION ONSHORE AT POTIGUAR BASIN
}

\author{
A. M. Alves ${ }^{a}$, V. E. Amaro ${ }^{\text {b }}$ \\ ${ }^{a}$ State University of Rio Grande do Norte, Student of the Graduate Program in Science and \\ Petroleum Engineering - agassiel@gmail.com \\ ${ }^{\mathrm{b}}$ Federal University of Rio Grande do Norte, GEOPRO Laboratory, Department of \\ Geology - amaro@ufrn.br
}

KEY WORDS: Geobia, Oil Explorations, Albedo, Reflectance, Climate Changes.

\begin{abstract}
:
This paper deals with the discussion of the analysis of remote sensing data on onshore oil exploration occurring in the Potiguar Basin, in the state boundaries of Rio Grande do Norte - Brazil, from the perspective of changes in albedo index, surface reflectance and surface temperature associated with to those aspects. The albedo is considered a significant variable in understanding the energy balance that makes up an important element of the analysis of global climate change. Through the use of remote sensing tools and GIS, based on surface reflectivity algorithms and surface temperature from GEObia (GEOgraphic-Object-Based Image Analysis) from the sequence - Pan-sharpened composite, Radiance with atmospheric correction, Reflectance with atmospheric correction, and Surface temperature with emissivity correction, used in scenes produced by OLI sensors (Operational Land Imager) and TIRS (Thermal Infrared Sensor) Landsat- 8 satellite, it was possible to determine the variations in surface reflectance and surface temperature, and compare them to index albedo therein the oilfield based on the method $6 \mathrm{~S}+$ Liang (2000). The research indicates, with the determination of processed values, which areas of oil exploration to the analyzed time, present significant change rates, with a tendency to increase in reflectance and temperature associated with albedo changes, as well as other areas of renewable energy exploration.
\end{abstract}

\section{INTRODUCTION}

The present research is based on the initial analysis of albedo as a relevant factor to energy balance, in earth surface related to 101 onshore production fields, that exist in the limits of Rio Grande do Norte State - Brazil.

Potiguar Basin has a total area of around $49.000 \mathrm{~km}^{2}$, of which $27.000 \mathrm{~km}^{2}$ are offshore and 22.000 onshore, divided between $\mathrm{CE}$ and $\mathrm{RN}$ states. Currently, this basin has been considered the biggest field of exploration in Brazil, in RN state it has an estimated area of $16.903,9 \mathrm{~km}^{2}$, it represents $34,5 \%$ of the total area. (Brazil - AnP, 2014)

Based on the need of observing about the oscillation wether conditions and related systems, index albedo control is necessary because it is a very significant reflectance index of solar radiation. Index albedo focus of interpretation is done in a kind of superficial scale of atmosphere and albedo index of surface.

The Potiguar Basin is located at a semiarid climate region with a severe semiarid subclassification in the center of this climate region, except in the area between coast zone and savanna, the weather is dry and hot, with temperatures over $25^{\circ} \mathrm{C}$ and rainfall annual measures lower than $600 \mathrm{~mm}$. The natural desertification process is present in this region and it has been changed by anthropic action (ANDRADE, 1973, p. 153).

Oil exploration in Potiguar Basin has origin in 50's, in twentieth century ant it had greatly grown, by the time new fields were discovered along the way, and new technologies in exploration were in the same sense, it turned possible oil extraction and natural gas extraction onshore in all 7.071 oil wells in the State (AnP, 2014).

Beside that and according to the Report of Energy Reserch Company - Brasil, EPE (2013) another forms of energy are explored in the State of RN, in a significant way, such as wood, eletric, (thermo and wind power) and biomass of sugar cane.

The albedo index is a concept that is associated to reflectance of solar radiation through the atmosphere and surface, where around $30 \%$ of radiation is reflected back to space, it is done mainly through short waves. It is considered an important sign about global climate changes.

In what is related to local and regional weather conditions, the superficial albedo index is a meaningful variable in the analysis when comparing to other ones (temperature, humidity, precipitation, evaporation / evapotranspiration). The main modulatory of atmospheric and superficial albedo is the cloud cover associated to atmospheric aerosol composition and the amount of solar rays, according to area location in analysis. (VEISSID, PEREIRA, 2000; FADIGAS, 2006; GOLDENBERG, 2007).

On the field of study's reality, the superficial albedo index changes that originated from exploration of biomasses (deforestation), desertification process, besides changes in the atmospheric composition due to the introduction of aerosols and gases from biomass burning, all of those factors are an important tool to the atmosphere energetic balance. (ECK et al., 1998; GALDINO, 2003)

Considering natural aspects related to savanna biome, that form almost total area of Potiguar Basin onshore, in the semiarid, albedo index represent an important variable to be observed, to evaluate a real climate evolution, taking into account natural structure and other relation in the anthrop actions, that occurs in that area.

Intending a comparative analysis, it was made an investigation based on $6 \mathrm{~S}$ method associated with correction rates for albedo calculation defined by Liang (2000), and data superficial reflectance established by applying the method GEOBIA (GEOGRAPHIC-Object-Based Image Analysis, 2014), both by ArGis 10 software. The results point towards a 
wider and detailed description of surface reflectance index through GEOBIA method, considering that all spectrum bands available in Landsat 8 were used in the analysis.

\section{2 . APPLIED METHODOLOGY}

Using remote sensing techniques and SIG, the arising data that were necessary to observe superficial albedo levels at Potiguar Basin were based on images from Landsat 8 satellite that came from USGS, through OLI sensors (Operational Land Imager) sensors and TIRS (Thermal Infrared Sensor) (USGS, 2013).

Five scenes were used to cover all the area we analyzed 216/063(159), 216/064(191), 215/063 (152), 215/064(152) e 214/064(161). The dates were defined taking into account time proximity to measurement period of aphelion of July, 2013, and we chose the scenes where less cover cloud $(<7 \%)$, we follow the tendency work, quoted by Roy, et. all (2014).

In the absence of 214/064 images of the scene with the cloud cover within the set margin of error, and there is no oil exploration field in this area is less than $5 \%$, the eastern end of the study area, the data in this scenario We were not used into account in the statistical analysis.

The outdoor work we had done allowed us to identify geo-reference points to have information spatial exact points for granted, they were associated to WGS- 84 projection system, and it had also granted the biggest precision of about $7,64 \mathrm{~m}$ to images in analysis with a kind of resolution of $14,96 \times 14,96 \mathrm{~m}$ per pixel. We used data from DBOPE (Data bank of Oil Production and Exploration), (BDEP in Brazil), associated to those images.

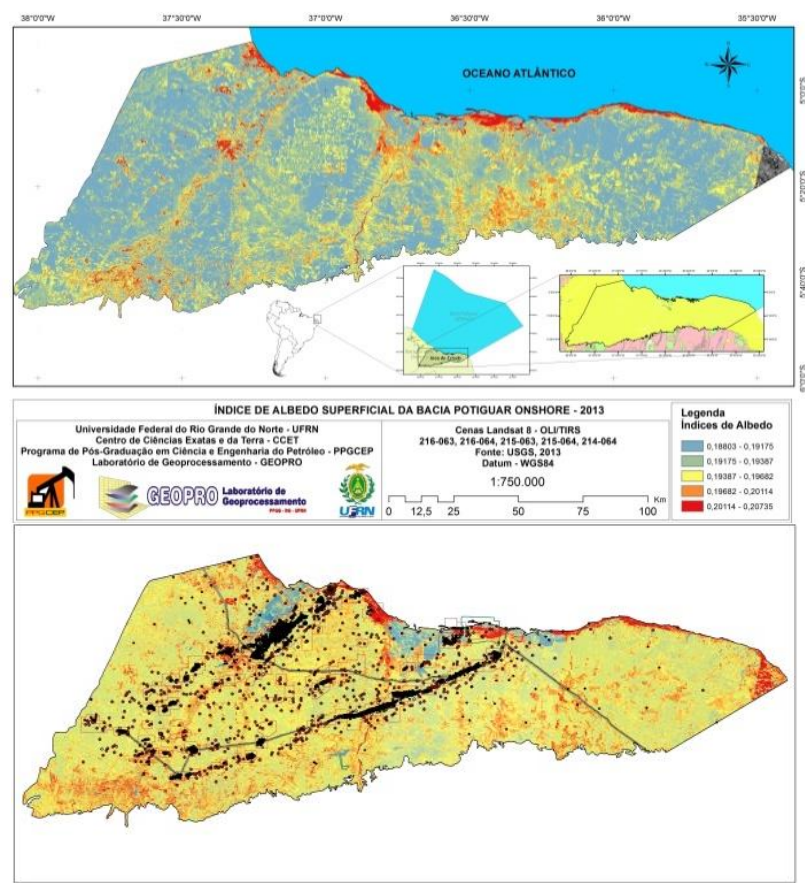

Figure 1. Image of area under analysis. Field location, wells and gas pipelines at Potiguar Basin - PB (BDEP, 2013).

Taking into account the composition of combined scenes, this is: RGB-I (R-6, G-234, B-53, I-7), it was possible to detach different structures of vegetation, explored areas, exposed soil, soil variation, hydrographic reserves and so many other forms of human occupation (Figure 2)
These information easy the reading comprehension of oil exploration area, as well as the comprehension of natural processes of high levels of reflectance.
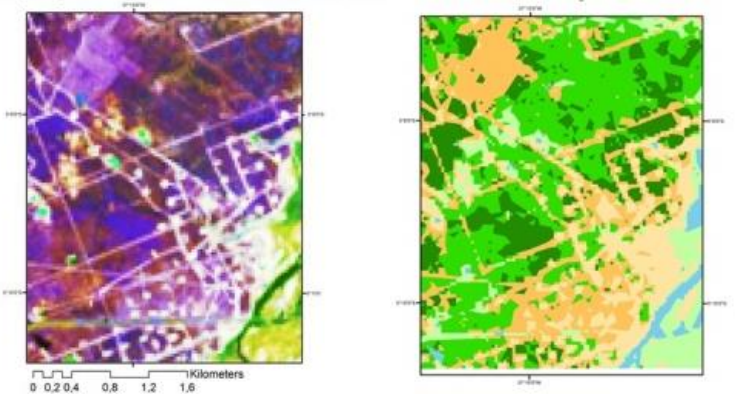

Figure 2. Composition of Images Landsat 8 (RGB-I) (left); and Classification NS from Landsat 8 Scene - 216-063(152/2013) (right).

We had still used information from high levels resolution images as complementary resources, images from satellites Rapideye e Topodata (MMA, 2014), we wanted to check how images were classified. Taking into account these basic information, we applied systems correction of atmosphere and reflectance analysis: the first one the $6 \mathrm{~S}$ (Vermont, et all, 2006) associated to another one proposed by Liang (2000) to albedo. We also used Geobia tool (GEOgraphic-Object-Based Image Analysis, 2014) through the software ArcGis 10 to analyze the medium of surface temperature and reflectance.

Through of Geobia Method, it was possible to do a panoramic reading of all reflectance degree that comes from Potiguar Basin surface, in a regional perspective. In the analysis, the variation through comparative analyzes among preserved areas and another activities developed there.

The method is based on the calculation of reflectance with atmosphere correction:

$\mathrm{R}_{\text {earth }}=\mathrm{R}_{\text {sat }}-\left(\mathrm{R}_{\text {scatter }}-0.01\right)$

Where:

$\mathrm{R}_{\text {earth }}$ : Reflectance of earth`s surface

$\mathrm{R}_{\text {sat }}$ : Reflectance of ToA

$R_{\text {sat }}=\frac{(D N-0.0002)-1}{\cos S Z}$

Where:

DN = Digital Number;

$\cos \mathrm{SZ}=$ cosine of $90^{\circ}$ - degree of solar elevation;

$\mathrm{R}_{\text {scatter }}$ : Reflectance scattered in the atmosphere

$R_{\text {scatter }}=\frac{\left(D N_{\min }-0.0002\right)-1}{\cos S Z}$

Where:

$\mathrm{DN}_{\text {min }}=$ minimum value of $\mathrm{DN}$;

$\cos \mathrm{SZ}=$ cosine of $90^{\circ}-$ degree of solar elevation;

Considering the use of correction system 6S (ANTUNES et al, 2014, p. 03; VERMOTE, 2006) we applied band corrections $2,3,4,5,6$ and 7, it was divided into three following levels: 2, 3, 4 (visible), 5 (infra red low), 6 e 7 (infra red medium).

It is important to point out that the observation in this method is based on the following variables: solar angle, zenithal angle, sun-earth distance, aerossol cover and atmosphere model. 
The atmosphere correction was individually done, by bands, and it had as consequences a high level of interpretation though three steps: calibration and radiance, atmosphere correction (ToA), and finally surface albedo measurement through of method Liang (2000).

Calibration and radiance:

$L_{\lambda}=1+\frac{L_{\max }-L_{\min }}{Q_{\text {cal } \max }-Q_{\text {calmin }}}\left(Q_{\text {calmax }}-Q_{\text {calmin }}\right)+L_{\text {min }}$

Where:

$L_{\lambda}:$ Radiance Value

$\mathrm{L}_{\max }$ : Maximun value radiance (W/Ester-radiano/ $/ \mathrm{m}^{-2} / \mu \mathrm{m}$ )

$L_{\text {min }}$ : Minimun Value Radiance (W/Ester-radiano $/ \mathrm{m}^{-2} / \mu \mathrm{m}$ )

$Q_{\text {calmax }}$ : Maximum value of grey levels used in quantifying data

$Q_{\text {calmin }}$ : Minimum value of grey levels used in quantifying data.

Atmosphere Correction

$\rho_{a p}=\frac{\pi x L_{\lambda x d^{2}}}{E_{\text {sol } x \cos \theta_{s}}}$

Where:

$\pi$ : solid angles in steradian.

$L_{\lambda}$ : Radiance Value;

$\mathrm{d}$ : distance sun-earth in astronomic units (AU);

$\mathrm{E}_{\mathrm{sol}}=$ Solar irradiance on one of the bands in $\mathrm{W} / \mathrm{m}^{-2} / \mu \mathrm{m}$;

After atmosphere correction, OLI sensor bands analyzed were underwent to the calculation of superficial albedo, it was based on method adaptation proposed by Liang (2000, p. 227) to the sensor ETM+ to OLI sensor, but it was done observing short wave adaptation and visible band.

Superficial Albedo:

$\alpha^{O L I}=\left(0.356 \alpha_{2}+0.317 \alpha_{3}+0.130 \alpha_{4}+0.373 \alpha_{5}+\right.$ $\left.0.085 \alpha_{6}+0.072 \alpha_{7}\right)-0.0018[6]$

Where:

$\alpha^{O L I}=$ Sensor Operational Land Image - Landsat 8, adapted from a relation among the bands sensor ETM+; 0,356 $\alpha_{2}$ : Specific value of band calibration.

After composition, classification and ToA albedo analysis, ToA and superficial we did a comparative analysis through the global albedo table proposed by Otterman (1977), where we verify the relationship between natural conditions that induce natural albedo in that area and changes caused by oil fields under our analysis.

\begin{tabular}{lr}
\multicolumn{1}{c}{ Kind of surface } & Albedo \\
\hline $\begin{array}{l}\text { Stable snow cover in high latitudes }\left(>60^{\circ} \text { of }\right. \\
\text { latitude ) }\end{array}$ & 0,80 \\
\hline Stable snow cover in medium latitudes & 0,70 \\
\hline Unstable snow cover in the Autumn & 0,50 \\
\hline Forest covered by stable snow & 0,45 \\
\hline $\begin{array}{l}\text { Forest covered by unstable snow in the } \\
\text { Autumn }\end{array}$ & 0,30 \\
\hline Desert & $\mathbf{0 , 2 8}$ \\
\hline $\begin{array}{l}\text { Forest with falling leaves during a dry } \\
\text { period, cerrado and semi desert. }\end{array}$ & $\mathbf{0 , 2 4}$ \\
\hline
\end{tabular}

\begin{tabular}{lc}
\hline $\begin{array}{l}\text { Forest falling leaves during a rainy } \\
\text { period, thick and semi desert. }\end{array}$ & $\mathbf{0 , 1 8}$ \\
\hline $\begin{array}{l}\text { Estep or decidua forest (temperature over } \\
10^{\circ} \text { in the Spring before snow covering). }\end{array}$ & 0,18 \\
\hline Tundra without snow covering. & 0,18 \\
\hline Acicular Forest (temperatures over $10^{\circ}$ ). & 0,14 \\
\hline Steep and forest during transition period. & 0,13 \\
\hline
\end{tabular}

Tabel 1. Medium albedo values, according to Otterman, 1976.

\section{RESULTS AND DISCUSSION}

If we consider the first results obtained through the application of GeoBia and $6 \mathrm{~S}+$ Liang (2000) techniques, it is possible to observe the following considerations:

The area under analysis has a level of reflectance based on superficial albedo associated to what is estimated by Otterman (1977), in the item "forest falling leaves during a dry period, thick and semi desert" with albedo estimated at 0,18 .

Considering that albedo index in desert zones at $(0,28)$, the area under analysis is in an inferior level, so if we consider data band VIS/IR-P/IR-M $(0,1571)$ and nearer to those areas in relation to VIS/IR-P $(0,2066)$. This fact can be associated to natural conditions level and its chemical-physical properties after the period of higher rain period in the region (jan-jun).

By the establishment of controlled points: preserved vegetation, cover clouds, shadows of clouds, saline deposit and urban areas, it was possible to analyze in a comparative way albedo index related to these areas but with different changes tendencies.

At preserved caatinga region there is a tendency of lower rates of albedo, they are shown in a variation from 0,10 to 0,15. According to Otterman (1977), the medium of albedo related to forests in semiarid tropical areas is approximately about 0,14 (Figure 4).

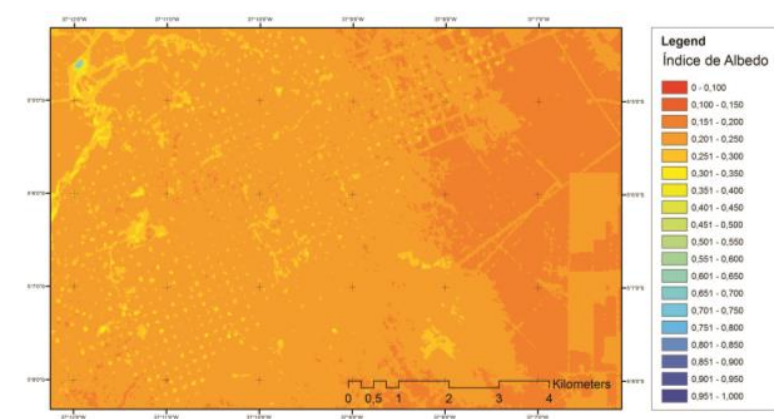

Figure 3: Superficial reflectance values, through Geobia Method from part of the CAM field.

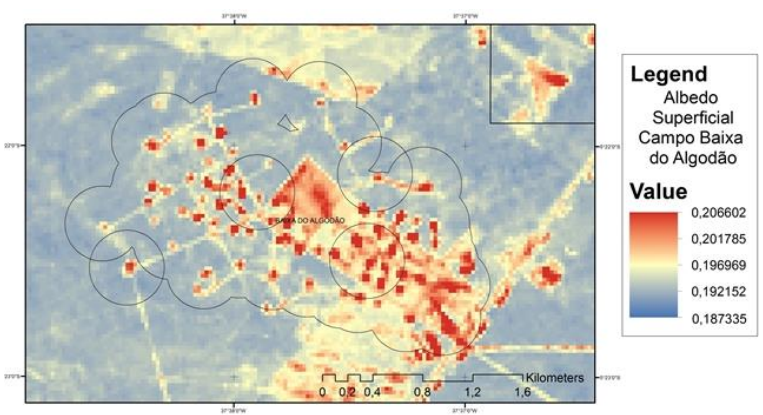

Figure 4. Estimated superficial albedo by the method 6S \& Liang (2000), at BAL field. 
After the selection of fields whose influence presents regional potential of climatic alteration $\left(>3000 \mathrm{~m}^{2}\right.$ - based in used scale 1:750.000), it was done a classification of data in relation to total area of the field and the area of direct influence of exploration (wells, gas pipelines, preprocessing and processing).

These data indicate that many fields presents altered index by presenting parallel activities in its area (agriculture, biomass exploration, urban areas and visible salt exploration in blue area of the figure 03) that alter the perspective in analysis by field, because of this was adopted the selection with $300 \mathrm{~m}$ area just around of the activities of direct exploration of oil and natural gas.

\begin{tabular}{cccccc}
\hline $\mathbf{N}^{\mathbf{o}}$ & Code & Min_EP & Max_EP & Mean_EP & St_Dev_EP \\
\hline 1 & ARG & 0,053504 & 0,645346 & 0,258199 & 0,069645 \\
\hline 2 & BAL & 0,043490 & 0,385675 & 0,122982 & 0,031405 \\
\hline 3 & BEM & 0,104291 & 0,377356 & 0,161016 & 0,049158 \\
\hline 4 & BVS & 0,098585 & 0,356945 & 0,154126 & 0,050452 \\
\hline 5 & BR & 0,070176 & 0,441374 & 0,176264 & 0,051777 \\
\hline 6 & CAM & 0,010811 & 0,538074 & 0,162068 & 0,064278 \\
\hline 7 & ET & 0,002076 & 0,580894 & 0,190535 & 0,061223 \\
\hline 8 & FMQ & 0,079314 & 0,313120 & 0,112323 & 0,024831 \\
\hline 9 & FP & 0,111341 & 0,672718 & 0,253326 & 0,067567 \\
\hline 10 & GMR & 0,160705 & 0,655301 & 0,215433 & 0,044648 \\
\hline 11 & LV & 0,029691 & 0,407038 & 0,143798 & 0,043619 \\
\hline 12 & LOR & 0,061638 & 0,441435 & 0,131587 & 0,039384 \\
\hline 13 & MAG & 0,157249 & 0,661390 & 0,224118 & 0,049708 \\
\hline 14 & MOR & 0,104310 & 0,325440 & 0,125611 & 0,028852 \\
\hline 15 & PJ & 0,082404 & 0,323103 & 0,119520 & 0,024016 \\
\hline 16 & PML & 0,100714 & 0,609326 & 0,193112 & 0,075072 \\
\hline 17 & RE & 0,106152 & 0,491453 & 0,145318 & 0,037128 \\
\hline 18 & REP & 0,100501 & 0,349091 & 0,142265 & 0,042173 \\
\hline 19 & RFQ & 0,043400 & 0,354268 & 0,121550 & 0,028443 \\
\hline 20 & SE & 0,097460 & 0,428327 & 0,158731 & 0,044776 \\
\hline 21 & UPN & 0,080534 & 0,366530 & 0,156513 & 0,033263 \\
\hline 22 & SCR & 0,017223 & 0,701261 & 0,195982 & 0,105308 \\
\hline 23 & SER & 0,059948 & 0,607719 & 0,368373 & 0,143655 \\
\hline 24 & MA & 0,029696 & 0,640282 & 0,302815 & 0,198782 \\
\hline 25 & PITI & 0,018971 & 0,617492 & 0,256322 & 0,110869 \\
\hline & & & & & \\
\hline 1 & & & \\
\hline
\end{tabular}

Table 2. Field General Reflectance Index with regional influence (>3000 $\left.\mathrm{m}^{2}\right)$.

The observation of rates band by band provided the integration of bands of visible electromagnetic spectrum, near infra-red and mid infra-red, what demonstrate that bands of short frequency presents one indicative significant tendency of values of albedo in the area under analysis, providing a quantification more precise of analyzed areas.

Through the quantification with the use of statistic free software ( $\mathrm{R}$ - The R Foudation for Statical Computing - 2015) was possible to analyze the general table of PB (Potiguar Basin), with covering of about $97 \%$ of total area, it was exempt only one part of extreme east where there is no any oil exploration and whose scenario presents clouds covering above average of other scenarios.

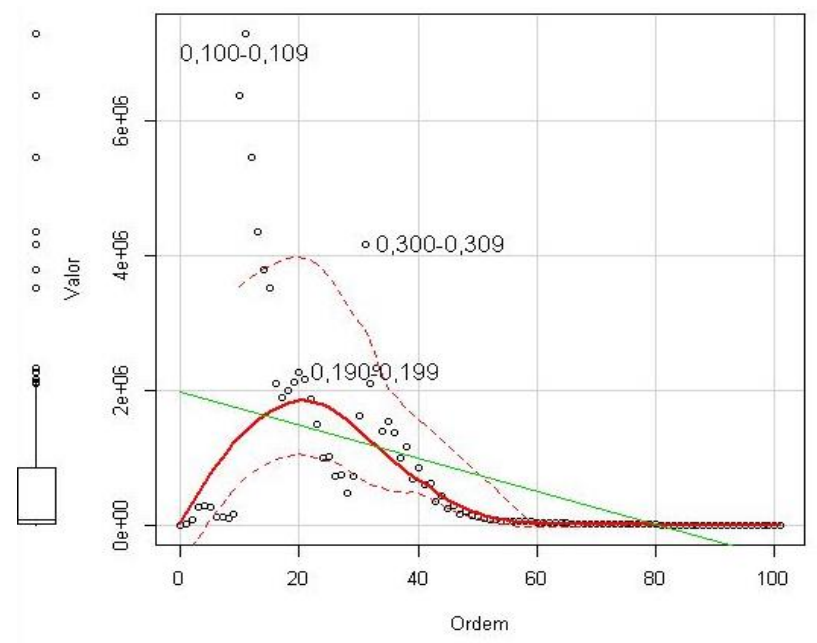

Figure 5. Scatterplot general reflectance data determination of Bacia Potiguar.

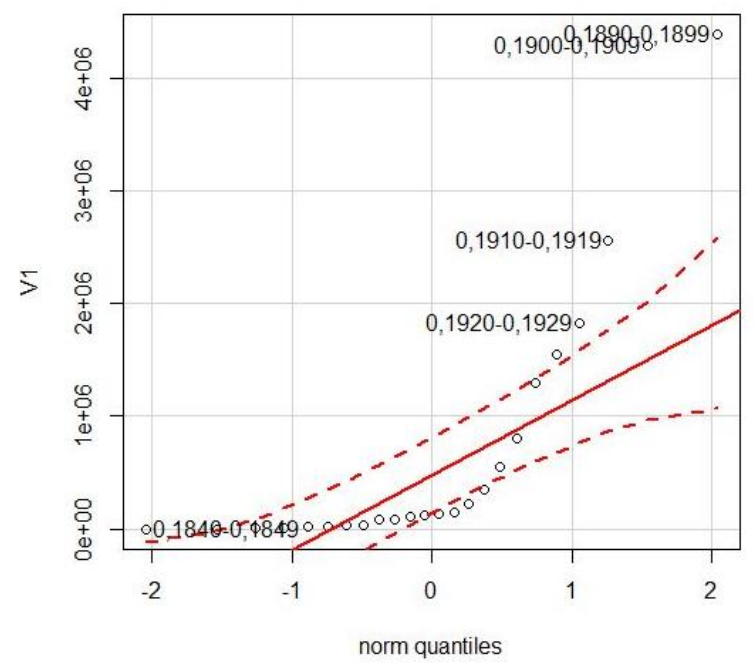

Figure 6. Norm Quantiles of superficial index albedo analysis, highligting the values 0,1900-0,1909 and 0,1890-0,1899 at Potiguar Basin.

We identify 11 oil exploration fields with index that is considered a high level, above average $(>0,16)$, those values are above average of preserved vegetation areas and they're of lower levels in the areas of high index of albedo and natural temperatures.

Those data are not really concluded in their statistic treatment, but it searches to show a kind of general profile, and at the same time it tries to identify oil fields of higher changes influence, that are under observation. A significant order of reflectance in a band from 0,1 to 0,3 fits in data that can be found in the oil exploration area, but the areas highly depends on geosystem where they are located. So, it is necessary a selection and classification of those sub-divisions.

We also applied the analysis of superficial temperature, it is an important aspect in climate analysis, because this way it was possible to see the relationship among rates of albedo, values of superficial reflectance in a wide spectrum and the own changes of temperatures. 


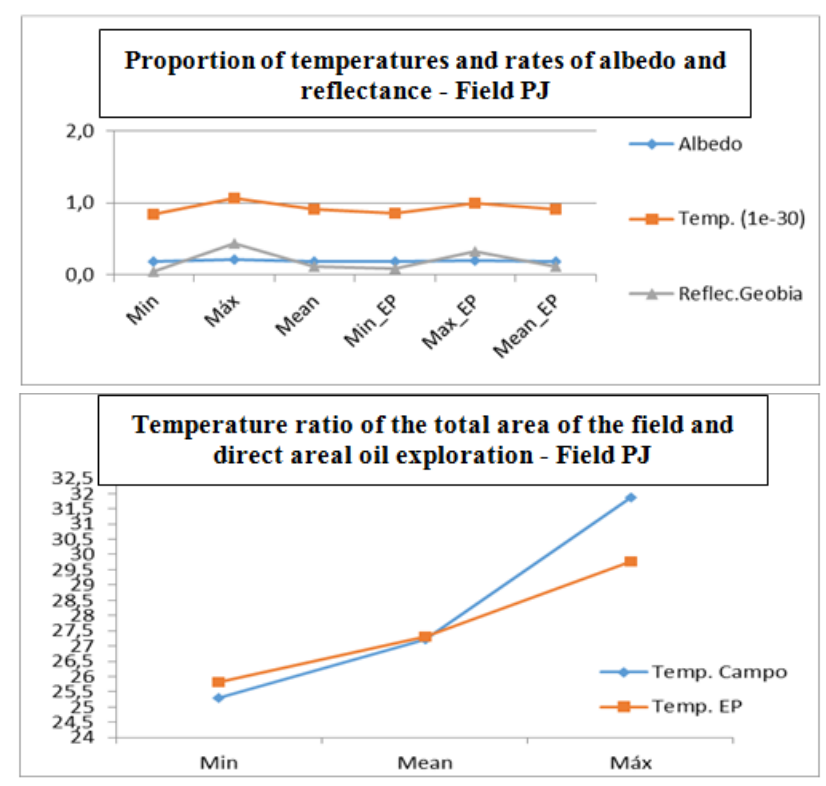

Figure 7. Graphics proportion between temperatures, albedo and relfectance - PJ Field; Relation between the total area of the field and the direct area of exploration - PJ Field.

As we can observe at the figure 7 , variation of temperature are more related to variation on reflectance in general, maximum temperature variation are not direct associated to oil exploration areas, i.e. direct to areas of oil wells or gas pipeline used as reference.

\section{CONCLUSIONS}

The results show that the oil area under analysis presents variations on the superficial albedo index of low significance, with the analysis in course, related to the associated changes on the anthropic activities on the region (farming, urban areas, and the exploration of other energy resources).

On overall perspective in the use of energy exploration activities, considered renewable and non-renewable energy provided changes in the superficial aspects of albedo and superficial temperature jointly in the area under analysis in this research.

Potiguar Basin has natural areas with high levels of reflectance and albedo, located in areas of dune fields, crystalline outcrops and soils with low rate of absorption associated with dryland vegetation due to semi aridity of the region, taking into account it has low potential for solar radiation absorption. Great dune fields on the coast naturally presents high levels of albedo index and temperature without direct anthropic influence. Rocky sandstone outcrops also presents positive variations in its natural aspect.

When we analyze temperature alteration in a comparative way we can see that they are proportionally related to reflectance alterations by Geobia method than to albedo index, however, this relation happens in a more local-regional scope. Meanwhile, the albedo refers to a more regional-global scope.

Those variations are important to understand the final frame of identification in the more affected areas, and those that need a special attention in order to avoid future irreversible degradation scenes.

The relations among the geosystemic structures that compose the areas under study should be considered in order to observe the causes of the alterations on the superficial albedo indexes in exploration areas.

\section{ACKNOWLEGEMENTS}

We are thankful to all support by Professors of PPGCEP/UFRN as well as Academic Community from GEOPRO/UFRN, especially to $\mathrm{PhD}$ Professor Venerando Eustáquio Amaro.

\section{REFERENCES}

ANDRADE, M. C., 1973. The semiard coastal region of northeastern Brasil. In: AMIRAN. D. H. K.; WILSON. A. W. Coastal Deserts: the natural and human environments. The University of Arizona Press, Tucson..

ANTUNES, M. A. H.; FREIRE, R. M. B.; BOTELHO, A. S.; TONIOLLI, L. H. Correções atmosféricas de imagens de satélites utilizando o modelo 6S, 2014. http://www.research gate.net/publication/255635015_correes_atmosfricas_de_image ns_de_satlites_utilizando_o_modelo_6s.

BRASIL, MME. Ministério das Minas e Energia, EPE. Empresa de Pesquisa Energética., 2013. Balanço Energético Nacional 2013: ano base 2012. Rio de Janeiro.

BRASIL. ANP. BDEP, 2014. Banco de Dados de Exploração e Produção. http://app.anp.gov.br/webmaps

ECK, T.F., BRENT, H. N., SHUTSKER, I. \&SETZER, A., 1998. Measurements of irradiance attenuation and estimation of aerosol single scattering albedo for biomass burning aerosols in Amazonia. Journal Geophys. Res. 103 (D24): p.865878. 1998.

FADIGAS, E. A. F. A. ; REIS, L. B. ; CARVALHO, C. E., 2005. Energia, Recursos Naturais e a Prática do Desenvolvimento Sustentável. Manole, São Paulo.

GALDINO, C. A. B., 2003. Passivo ambiental e o setor de exploração de petróleo em terra: abordagem teórica e percepção institucional dos impactos ambientais gerados pela atividade. Dissertação do Programa de Engenharia de Produção da Universidade Federal do Rio Grande do Norte. Natal.

GOLDENBERG, J. ; LUCON, O., 2007 Energia, Meio Ambiente e Desenvolvimento. Edusp, São Paulo.

IGAC - Instituto Geografico Agustin Codazzi. Descripción y correccion de produtos Landsat 8: Landsat Data Continuity Mission. Versión 1.0. Bogotá: Colômbia, 2013.

OTTERMAN, J. Anthropogenic impact on the albedo of the earth., 1977. In: Climatic Change 1. Dordrecht, pp. 137155.

LIANG, S., 2000. Narrowband to broad band conversions of land surface albedo - I algoritms. Remote Sensing of Environment, nov. pp. 213-238

MMA - MINISTÉRIO DO MEIO AMBIENTE. 2014. Geocatálogo. http://www.geocatalogomma.com.br/ 
ROY, D. P., WULDER, M. A., LOVELAND, T. R., ET ALL "Landsat-8: Science and product vision for terrestrial global change research" 2014. Papers in Natural resources. PAPER 459. http://digitalcommons.unl.edu/natrespapers/459.

USGS. Geological Survey., 2013. Satellit Scenes of Landsat-8. http://earthexplorer.usgs.gov/.

UNIVERSITY OF CALGARY., 2014. GEOgraphic-ObjectBased Image Analysis. www.ucalgary.ca/f3gisci/GEOBIA

VEISSID, N.; PEREIRA, E. B., 2000. Estimativa do albedo planetário empregando dados do experimento célula solar do satélite brasileiro SCD2. Brazilian Journal of Geophysics, Vol. 18(1), p. 26.

VERMOTE E, TANRÉ D., J. L. DEUZÉ, M. HERMAN, J. J. MORCRETTE, KOTCHENOVA S. Y., 2006. Second Simulation Of The Satellite Signal In The Solar Spectrum, 6s: An Overview. 6S User Guide Version 3. 


\title{
AN OBJECT-BASED IMAGE INTERPRETATION APPLICATION ON CLOUD COMPUTING INFRASTRUCTURE
}

\author{
R. R. Antunes ${ }^{\text {a }}$, P. N. Happ ${ }^{\text {c }}$, E. S. Bias ${ }^{\text {a }}$, R. S. Brites ${ }^{\text {a }}$, G. A. O. P. Costa ${ }^{\text {b }}$ R. Q. Feitosa ${ }^{\text {b, c }}$ \\ a University of Brasilia, Brazil - edbias@gmail.com, rodrigorantunes@ hotmail.com, brites.ricardo@gmail.com \\ ${ }^{\mathrm{b}}$ Rio de Janeiro State University, Brazil - gilson.costa@ime.uerj.br \\ ${ }^{c}$ Pontifical Catholic University of Rio de Janeiro, Brazil - patrick@ele.puc-rio.br
}

KEY WORDS: Remote Sensing, Object-Based Image Analysis, Distributed Processing, Cloud Computing

\begin{abstract}
:
The rapid increase in the number and in the spatial resolution of aerial and orbital Earth observation systems is generating a huge amount of remote sensing data that need to be readily transformed into useful information for policy and decision makers. A possible approach to tackle the demand for image interpretation tools that can deal efficiently with very large volumes of data is to employ data analysis methods based on distributed computing. This paper presents an object-based, remote sensing image interpretation application executed over cloud-computing infrastructure. The application is implemented with InterCloud, a novel image interpretation platform designed to run on computer grids (physical clusters or cloud-computing infrastructure). The application described in this paper is a land cover/land use classification of a pansharpened GeoEye-1 image, with 19k by $23 \mathrm{k}$ pixels. The image covers an area of the municipality of Goianésia, in Goiás State, Brazil. The site contains sparse urban areas intermixed with rural areas and natural patches of the Brazilian Cerrado biome. Eleven classes of objects, including urban, rural and Cerrado reminiscent targets were considered. In addition to the accuracies of the classification result, in this work we evaluate the scalability capability of InterCloud by performing different runs of the application with different configurations of the cloud infrastructure, in which we vary the number of computing nodes.
\end{abstract}

\section{INTRODUCTION}

The rapid increase in the number and in the spatial, spectral and temporal resolutions of aerial and orbital Earth observation systems is generating a huge amount of remote sensing data (Zhang et al., 2015). As examples, Sentinel-1 from ESA produces around 1.5 GB per day (Grabak, 2014) and EOSDIS project from NASA generates about 16 TB per day (NASA EARTHDATA, 2015). Other space agencies around the world produce a comparable amount of remote sensing image data daily. Moreover, companies like Digital Globe, Planet Labs and Skybox Imaging are about to sum up one hundred satellites orbiting the globe.

This scenario unveils great challenges for the remote sensing community related mainly to the capacity of dealing with very large datasets (Yan Ma et al, 2015; Lee et al., 2015). In fact, the techniques and automatic tools currently available for image interpretation are not ready to handle the massive volumes of remote sensing data being currently produced.

An approach to tackle the demand for image interpretation tools that can deal efficiently with very large datasets is to employ distributed computing based techniques. In this context, cloud computing is a trend (Hashem et al., 2015) as it delivers a powerful infrastructure to perform large-scale computing, usually available in a pay-as-you-go model, which alleviates users from the need to acquire and maintain complex computing hardware.

\footnotetext{
* Corresponding author
}

In this work, we present an object-based, remote sensing image interpretation application executed on cloud-computing infrastructure. The application is implemented with InterCloud, a novel image interpretation platform designed to run on computer grids (physical clusters or cloud-computing infrastructure). InterCloud can exploit the scalability provided by commercial cloud-computing infrastructure services, enabling the interpretation of very large remote sensing datasets in an efficient way.

The land cover/land use classification application uses different modules of InterCloud: distributed segmentation, distributed feature extraction; and the distributed classification. The application was executed using a commercial cloud-computing infrastructure service.

The remainder of this paper is organized as follows. The InterCloud distributed architecture and its different modules are briefly described in Section 3. The interpretation application and the experiments conducted with InterCloud are described in Section 3. Section 4 presents conclusions and gives some directions for future related research.

\section{INTERCLOUD}

InterCloud is a novel, open-source, image interpretation platform, which supports the creation of interpretation models and the execution of such models on cloud computing infrastructure. InterCloud can be regarded as a redesign of InterIMAGE (Costa et al., 2010) conceived to process arbitrarily large datasets in a distributed fashion. 
Although the architecture of InterCloud have already been described before (Ferreira et al., 2014), it is important to recall some of its main aspects. The architecture is composed by three abstraction layers: the project definition layer, the image interpretation layer, and the distribution layer.

The project definition layer supports the definition of all the information required for the execution of the interpretation application. This layer supports the definition of image interpretation models that describes the characteristics or properties of the classes of interest, and the models' processing chains.

The interpretation layer contains the algorithms and methods available to be used on the platform. It is structured on a highlevel programming language that hides the complexity of dealing directly with the distributed programming model. A user with conventional programming skills can embed new algorithms into this layer, so that an end user can select them in the project definition layer. It is worth mentioning that the interface between project and interpretation layers is defined by a translation of the first into processing and data flow instructions in the latter.

The distribution layer is associated to the distributed execution of an interpretation model. Only experts in distributed systems are able to interact with this layer. The interface between the interpretation and the distribution layers is defined by a translation of the first into distributed programming code available on the latter.

Another important aspect of InterCloud is its modular structure: the platform integrates different, independent modules. The modules are based on the same architecture and share the InterCloud core engine. This engine is responsible for the common methods, and the control of execution on the distributed environment.

The three modules used in this work, relate to the three main processing steps in image interpretation: image segmentation, feature extraction and classification (Figure 1).

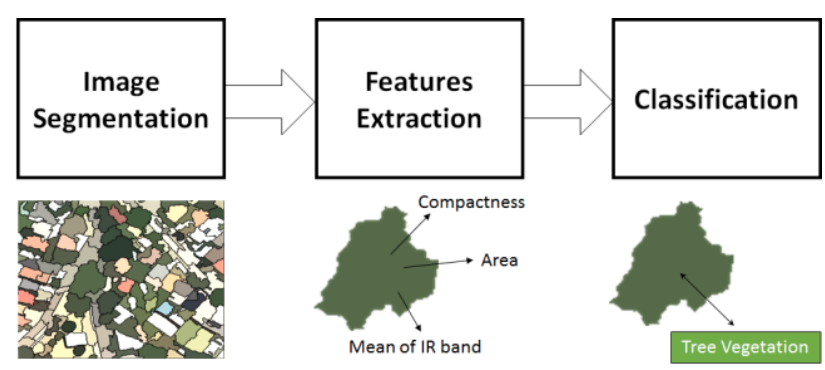

Figure 1. Image interpretation scheme

\subsection{Image Segmentation}

Image segmentation plays a key role in image interpretation since it delineates the segments that should represent image objects to be further classified. This task usually involves a high computational cost, especially when dealing with very large images.

Considering the increasing of earth observation data volume, sequential and parallel solutions fail to deliver the expected performance and scalability for this task. Therefore, distributed processing is an alternative to enable image segmentation of very large remote sensing imagery. This is the main premise in the design of InterCloud Image Segmentation module.

The Image Segmentation module contain different algorithms. In this work, a distributed region growing segmentation method was used (Happ et al., 2015). First, the image is split into tiles that are spatially indexed. Next, an independent region growing segmentation is performed on each tile, in a distributed fashion. Then, the internal segments, i.e. those that do not touch the tile's borders, are preserved. The other segments, i.e. those that touch borders, are grouped for a distributed post-processing step in order to continue the growing process.

The input of this module is a raster image, which is divided into spatially indexed tiles. The tiles are stored on an auxiliary file repository to be retrieved when necessary. The segmentation output is a vector containing the generated segments. This vector is also stored on the same auxiliary file repository.

\subsection{Feature Extraction}

The Feature Extraction module is responsible for distributed computing of segments' properties. It is capable of computing spectral, textural, morphological features, as well as topological relationships among segments.

The user is responsible for selecting which features should be calculated. Then, based on the vector data coming from the segmentation, all the features are computed on a distributed way. The module uses a spatial indexing mechanism in order to group segments on the distributed environment and to retrieve the image tiles when spectral information is required.

The processing output is vector data containing the computed feature values. These values will be latter used in the classification process.

\subsection{Classification}

The classification or recognition step considers segments' properties to associate them to object classes. For this task, complex knowledge-based descriptions can be used, as well as unsupervised and supervised classification methods. Although InterCloud can handle all those alternatives, in this work we employed the latter.

Supervised classification methods are based on samples: the assignment of a segment to a class depends on some sort of similarity of the segment to the samples of that class. Thus, it is important to collect samples that can clearly discriminate one class from the others. In InterCloud, the user must select some segments as samples and label then accordingly in order to create the training dataset. The remaining segments will be considered as the classification dataset.

The distributed supervised classification method is based on (Ayma et al., 2014). The classification dataset is split into disjoined subsets to be distributed, while the training set is stored on an auxiliary storage system. When the execution starts, the training dataset is retrieved to build the classification model for each processing element. Then, the model is used to classify each subset of the classification dataset in the corresponding processing elements. Finally, all the parts of the 
classification dataset are coupled to get the (complete) classification outcome.

Currently InterCloud's classification module contains different machine learning algorithms, like Naïve Bayes Classifier, Decision Trees, Random Forest and Support Vector Machines. The input of this module is a list of segments with corresponding feature values. The samples, with their feature values and class associations are stored on an auxiliary file repository to be retrieved when necessary. The output is a list of classified segments.

\section{EXPERIMENTAL ANALYSIS}

The experimental analysis is based on an object-based application with the InterCloud platform. It is an automatic land cover/land use classification of a remote sensing image involving the distributed segmentation, feature extraction and classification modules. The application was executed using a commercial cloud-computing infrastructure service.

In addition to the accuracies of the classification, we evaluate the scalability capability of InterCloud by performing different runs of the application with different configurations of the cloud infrastructure, in which we vary the number of computing nodes.

We note that this is an ongoing research, and what we show here are preliminary results.

\subsection{Cloud Environment}

The execution environment is a combination of commercial cloud services provided by Amazon Web Services (AWS). The auxiliary storage repository is provided by the Amazon Simple Storage Service (S3). The Amazon Elastic MapReduce (EMR) service was used to dynamically built and manage cluster of Amazon Elastic Compute Cloud (EC2) instances.

The interpretation application was executed using two, four and eight m3.xlarge nodes to run InterCloud. These are 64-bit computers, with $15 \mathrm{~GB}$ of RAM, 4 disks of $420 \mathrm{~GB}$ and an Intel Xeon E5-2670 v2 processor containing 4 cores, operating at 2.5 GHz.

\subsection{Image}

We used a pansharpened GeoEye-1 image with 19404 by 21360 pixels, $0.5 \mathrm{~m}$ resolution and four spectral bands (Figure 2). The image has $3 \mathrm{~GB}$ and covers an area of the municipality of Goianésia, in Goiás State, Brazil. The municipality has a population of 65,767 ; and is located $168 \mathrm{~km}$ from the state capital, Goiânia, and $265 \mathrm{~km}$ from the Brazilian capital, Brasilia. The site contains sparse urban areas intermixed with rural areas and natural patches of the Brazilian Cerrado biome.

\subsection{Interpretation Model}

In the classification we considered 18 different land cover/land use classes of objects from rural and urban tarrgets: Asbestos, Asphalt, Bare Soil A, Bare Soil B, Beige Ceramic, Concrete Floor A, Concrete Floor B, Crops, Dark Ceramic, Gray Ceramic, Light Ceramic, Metallic A, Metallic B, Metallic C, Ploughed Soil A, Ploughed Soil B and Swimming Pool. InterCloud was configured to work with image tiles with $512 \times$ 512 pixels totalizing 1677 tiles.

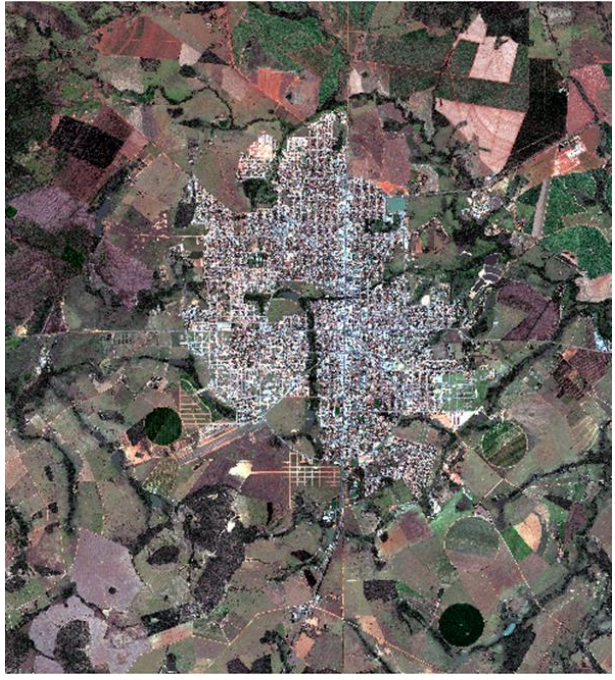

Figure 2. GeoEye-1 image from Goianésia.

The first operation of the interpretation model is the distributed segmentation. A distributed version of the algorithm proposed in (Baatz and Shäpe, 2000) was used in the segmentation. There was no systematic parameter tuning for the particular application. The algorithm's parameter values were determined empirically, and the following configuration was used: scale = 70 , color weight $=0.5$, compactness weight $=0.5$, bands weight $=1,1,1,1$. Figure 3 illustrates part of the segmentation outcome where different colors represent results from different processing elements.

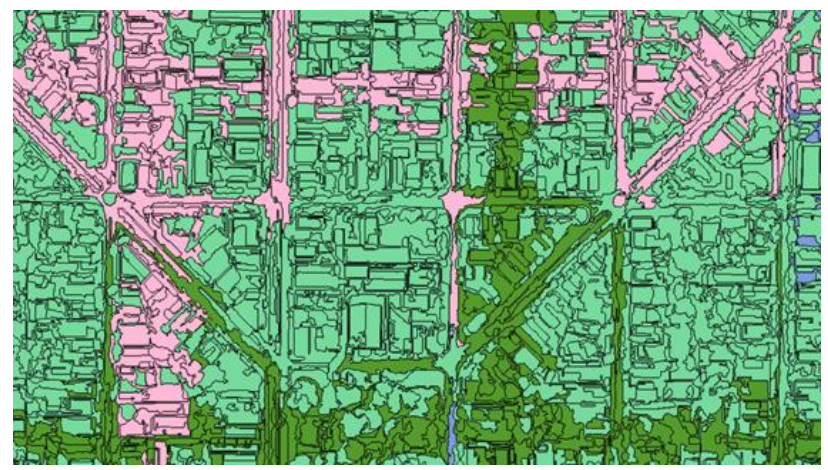

Figure 4. Part of the distributed segmentation outcome.

The next operation is the computation of feature values for each generated segment. Twenty-three features were considered in the classification: nineteen are spectral-based (Brightness, Mean value of bands 1, 2, 3 and 4, Maximum pixel value of bands 1, 2, 3 and 4, Minimum pixel value of bands 1,2, 3 and 4, Ratio of bands 1, 2, 3 and 4, Division of mean value of band 4 by mean value of band 1, and Division of mean value of band 4 by mean value of band 3) and four are based on the segments' geometry (Area, Angle, Compactness, Squareness, and Roundness).

The last operation is the classification step. We collected sample segments for each object class, totalizing 919 samples. Then we created two sets of samples, one for training (with 172 samples) and the other for accuracy assessment (with 747 samples). Then, the distributed Decision Tree (C4.5) algorithm was executed. 


\subsection{Interpretation Results}

The main objective of the experiments reported here was to test the capacity of InterCloud to handle the whole interpretation execution. It is important to note that the same application could simply not be processed using other available interpretation tools like InterImage and eCognition, unless the input image was partitioned into smaller subsets.

Figure 5 shows the processing times obtained from the interpretation execution on different number of computer nodes. Execution times of each step are shown: segmentation, feature extraction and classification. Image segmentation is clearly the most time consuming task, while the classification is the fastest one.

The graph shows that the processing time decreases as the number of processing elements increases and demonstrates the scalability potential of the platform. The exception was the classification procedure, since it was so fast that there were no notable benefits in including additional processing power. Considering 4 nodes, we achieved a speedup of 1.57 and 1.92 for segmentation and feature extraction, respectively. For 8 nodes, speedups of 1.98 and 3.91 were obtained for the same tasks. The feature extraction shows better speedups due to its high parallelizable capability. The image segmentation suffers from dependencies among neighbouring segments, which hinders parallelization.

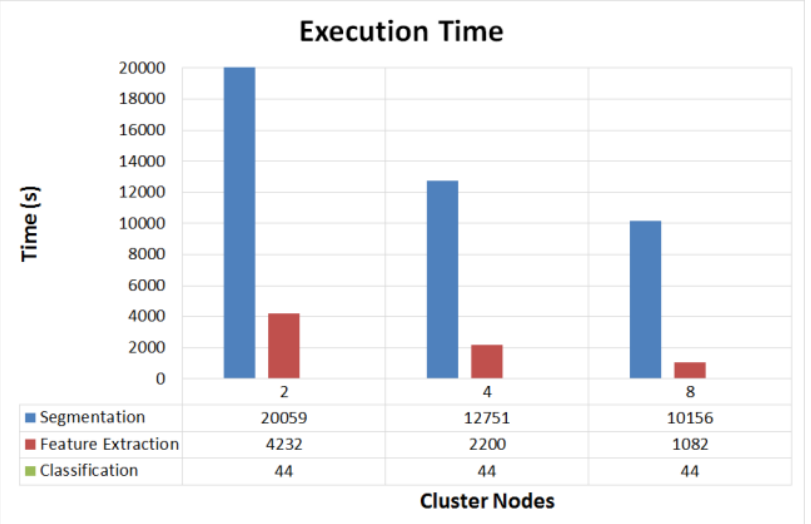

Figure 5. Execution times for each step of interpretation model.

The classification obtained a global accuracy of $53 \%$ and a 0,50 Kappa value. Classification quality was not good basically because of the large number of classes, and because no special tuning of the segmentation parameter values was made.

In order to improve these results, different segmentation runs could be performed for different group of classes. Additionally, some classes could be better classified using a structural, knowledge-based model, since we have better knowledge of their intrinsic characteristics.

\section{CONCLUSION}

This work presents an interpretation application of a large remote sensing image executed over a cloud-computing environment. This application involves image segmentation, feature extraction and supervised classification. We use the InterCloud platform and its modules for this task.
InterCloud is an open-source interpretation tool designed to work with computer grids. The experiments show that its distributed concept allows the execution of large images that cannot be processed with the common available tools. The results also demonstrate its scalability potential showing that the computational performance can be increased when more computer nodes are used.

We note that this is an ongoing research, and what we showed in this paper are preliminary results.

For future work, a better tailored image interpretation model will be designed. We intend to perform different segmentations for distinct groups of classes, and optimize parameter values for those groups. We will also exploit different classification methods and approaches.

\section{ACKNOWLEDGEMENTS}

The authors acknowledge the funding provided by FAPERJ (Carlos Chagas Filho Foundation for Research Support in Rio de Janeiro), CNPq (National Council for Scientific and Technological Development) and CAPES (Coordination for the Improvement of Higher Education).

\section{REFERENCES}

Ayma, V. A., Ferreira, R. S., Happ, P. N., Oliveira, D. A. B., Costa, G. A. O. P., Feitosa, R. Q., Plaza, A., Gamba., P., 2015. On the architecture of a big data classification tool based on a map reduce approach for hyperspectral image analysis, 2015 IEEE International Geoscience and Remote Sensing Symposium (IGARSS), Milan, 2015, pp. 1508-1511.

Baatz M., Schäpe, A., 2011. Multiresolution Segmentation: an optimization approach for high quality multi-scale image segmentation, Angewandte Geographische Informationsverarbeitung XII, Heidelberg.

Costa, G., Feitosa, R., Fonseca, L., Oliveira, D., Ferreira, R., Castejon, E., 2010. Knowledge-based interpretation of remote sensing data with the interimage system: major characteristics and recent developments. In: Addink, E., Van Coillie, F. (Eds.), GEOBIA. ISPRS Working Groups, Gent, Belgium, 2010.

Ferreira, R. S., Oliveira, D. A. B., Happ, P. N., Costa, G. A. O. P., Feitosa, R. Q., Bentes, C., 2014. InterIMAGE 2: The Architecture of an Open Source, High Performance Framework for Automatic, Knowledge-Based Image Interpretation, In: International Geographic Object-Based Image Analysis Conference, Thessaloniki, 2014.

Grabak, P., 2014. Sentinel-1 Mission Status. 15th Meeting of the International Ice Charting Working Group (IICWG).

Happ, P. N., Ferreira, R. S., Costa, G. A. O. P., Feitosa, R. Q., Bentes, C., Gamba, P., 2015. Towards distributed region growing image segmentation based on MapReduce, 2015 IEEE International Geoscience and Remote Sensing Symposium (IGARSS), Milan, 2015, pp. 4352-4355.

Hashem, I. A. T., Yaqoob, I., Anuar, N. B., Mokhtar, S., Gani, A., Khan, S. U., 2015. The rise of 'big data' on cloud computing: Review and open research issues, Information Systems, Volume 47, January 2015, Pages 98-115. 
Lee, J., Kang, M., 2015. Geospatial Big Data: Challenges and Opportunities, Big Data Research, vol. 2, pp. 74-81, June 2015.

Ma, Y., Wu, H., Wang, L., Huang, B., Ranjan, R., Zomaya, A., Jie, W., 2015. Remote sensing big data computing: Challenges and opportunities, Future Generation Computer Systems, vol. 51, pp. 47-60, October 2015.

NASA EARTHDATA., 2015, EOSDIS Annual Metrics Reports [Online]. https://earthdata.nasa.gov/about/systemperformance/eosdis-annual-metrics-reports

Zhang, L., Du, Q., and Datcu, M., 2015. Special section guest editorial: Management and analytics of remotely sensed dig bata, Journal of Applied Remote Sensing, vol. 9, no. 1, pp. 1-2, July 2015. 


\title{
INTEGRATION OF OPEN-SOURCE TOOLS FOR OBJECT-BASED MONITORING OF URBAN TARGETS
}

\author{
R. R. Antunes ${ }^{a}$, E. S. Bias ${ }^{a}$, R. S. Brites ${ }^{a}$ G. A. O. P. Costa ${ }^{b}$ \\ ${ }^{\text {a }}$ Federal University of Brasilia, Brazil - rodrigorantunes@hotmail.com, edbias@gmail.com, \\ brites.ricardo@gmail.com \\ b Rio de Janeiro State University, Brazil - gilson.costa@ime.uerj.br
}

KEY WORDS: Object Based Image Analysis, Data Mining, InterIMAGE, Orange Canvas.

\begin{abstract}
:
The constant increase in population in conjunction with unplanned and irregular urban growth, typical problems in developing countries, can promote a rapid increase in population density and related public infrastructure demand that may be hard to bear with the available economic resources. Efficient monitoring of urban development is thus a key instrument for planners and public policy makers that have to cope with this scenario. This work aims at developing a tool to aid monitoring urban growth from very-high resolution remote sensing images, focussing on the integration of available open-source software and the application of OBIA methods. Specifically, we created a method for detection of urban, land use/land cover classes based on the integration of the InterIMAGE and the Orange Canvas software packages. The image interpretation model for the particular application was constructed with the aid of dataflow building blocks (widgets) for data analysis, structured in the visual programming environment of Orange Canvas. The Classification Tree and the Classification Tree Graph widgets were used to design a decision tree that was later translated in InterIMAGE Decision Rules. The study was conducted over an image from the GeoEye-1 sensor, covering a central area of the city of Goianésia, in the Midwestern region of Brazil. Ten land use/land cover classes were the target of the supervised classification. The results obtained in the experiments confirm that the integration of the two open-source packages can provide for accurate remote sensing image analysis, while facilitating data exploration and the construction of automatic image interpretation models.
\end{abstract}

\section{INTRODUCTION}

A great challenge is posed to urban planners by the fact that intra-urban occupation has been advancing lately, notably in developing countries. Because of this, automatic tools capable of discovering and mapping possible irregularities are on great demand by the public administration. Lang (2008) endorses this argument by describing a growing demand for updated geospatial information combined with quick extraction techniques, to allow for an improved decision-making process and, consequently, an improved management.

Object-based analysis (OBIA) has stood out as an option for the monitoring of urban areas. The diversity of objects that can be identified by the current sensors can be better interpreted by this method due to the possibility of introducing an analyst's knowledge and exploiting multiple features in the classification process. According to Blaschke, Burnett, and Pekkarinen (2004), many geo-information aspects cannot be obtained solely through pixel information, but they could be obtained in a context of proximity to objects of interest. Blaschke (2013) states that OBIA (Object-Based Analysis) allows additional information to be included so as to direct modeling and classification processes. This additional information can be statistics computed considering the pixels that belong to an object, such as: average reflectance; standard deviation of reflectance values; maximum and minimum pixel values, textural and morphological measures of the objects, and topological relationships to other objects.

Integration of data mining software to the InterIMAGE open source image classification system has shown good results so far, according to studies such as: (Bias et al., 2014; Antunes et al., 2014; Passo et al., 2014; Nascimento et al., 2013). By using open-source data mining software packages, it is possible to employ the many available algorithms in object-based classification, and also to extend, adapt or combine existing ones.

Many current object-based studies have used the open-source WEKA data mining software package (Witten and Frank, 2005). In this context, it is interesting to check whether integration between InterIMAGE and other data mining software packages is viable, specially taking into account accuracy of results and efficiency of the overall processes. This study aims at analyzing and assessing the potential of such integration, as well as the quality of the results obtained through object-based urban area classification using InterIMAGE in conjunction with the Orange Canvas open-source data mining software.

\section{MATERIALS AND METHODS}

The study area is the municipality of Goianésia, located in the Midwestern region of Brazil, on the Central Plateau, in the north central region of Goiás State. A pansharpened image from the GeoEye-1 Sensor, acquired in 2013 was used in this study (Fig. 1).

The following open-source software packages were used in the study: QuantumGIS, version 2.8 (QGIS, 2016); Orange Canvas, version 2.7 (ORANGE, 2016); and InterIMAGE, version 1.43 (INTERIMAGE, 2010).

Orange Canvas is a software written in the Python language that offer a broad set of data mining and machine learning algorithms, developed by the Bioinformatics Laboratory of the Faculty of Computer and Information Science at University of Ljubljana, Slovenia, in collaboration with the open source community (Demsar et al., 2013). 
InterIMAGE has been developed by the Computer Vision Lab of the Faculty of Electric Engineering at Pontifical Catholic University of Rio de Janeiro (PUC-Rio) and by the Image Processing and Remote Sensing divisions at the Brazilian National Institute for Space Research (INPE).

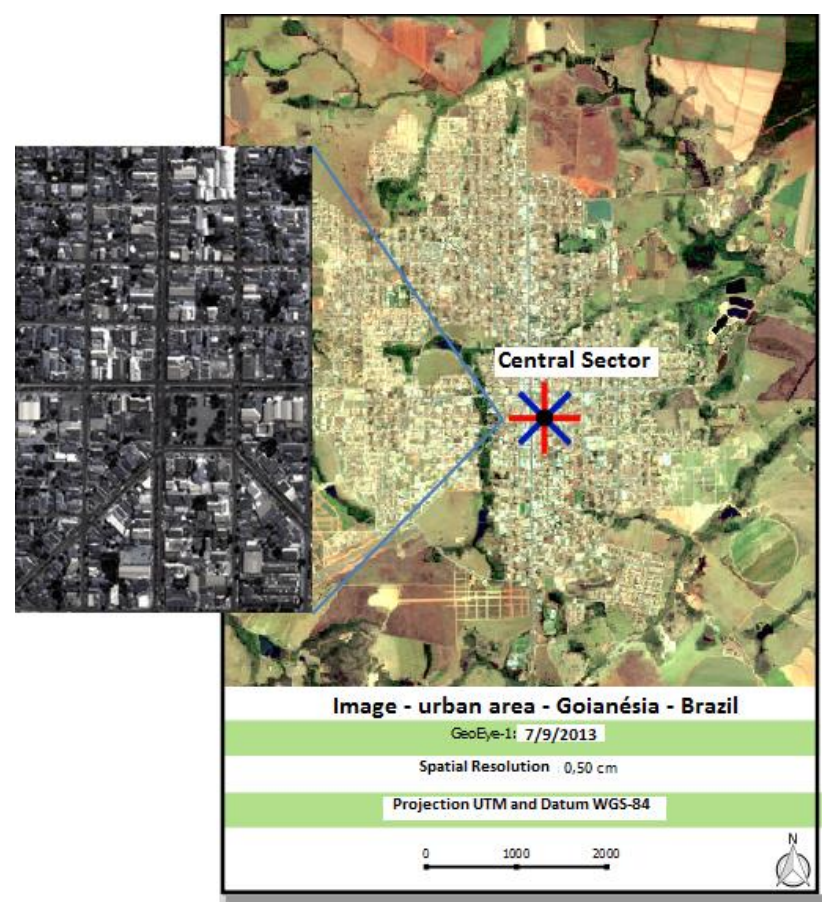

Figure 1. GeoEye-1 image covering the municipality of Goianésia used in this work.

In this work 10 thematic classes were the subject of the classification process, namely: vegetation, swimming pools, shade, urban roads, exposed soil, concrete floor, metal roofing, asbestos roofing, clear ceramics roofing and dark ceramics roofing.

Figure 2 shows the 5 stages of the methodology employed in this study.

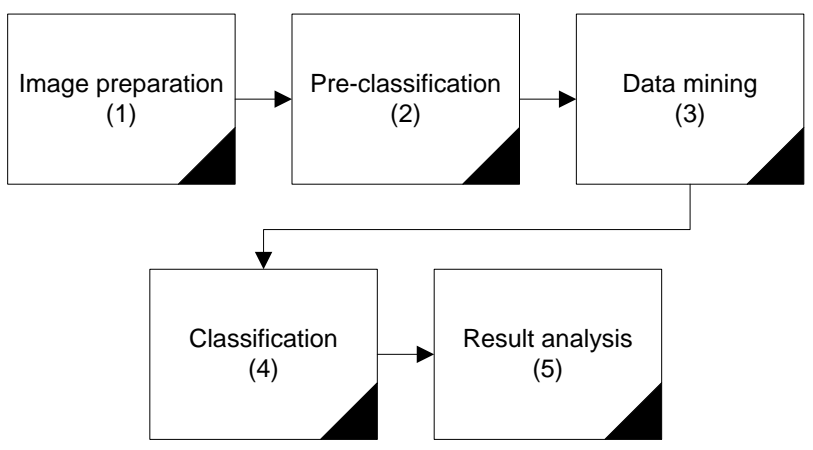

Figure 2. Stages of the methodology.

Stage 1 has to do with image preparation. In this stage, the spectral bands of the GeoEye-1 image were pansharped using the Gram-Schmidt method, and cropped to $977 \times 1531$ pixels with the QuantumGIS sofware.

In Stage 2 the InterIMAGE software package was used for segmentation, feature extraction and sample selection. For some of the classes of interest independent segmentations were produced, using segmentation procedure proposed in (BAATZ \& SHÄPE 2000). The classes and segmentation parameter values used are shown in Table 1 . The segmentation of the other classes: vegetation, shade and roads; were produced through image thresholding based on NDVI and brightness values.

Segment were exported according to the following attributes: shape, spectral statistics, and texture.

\begin{tabular}{|c|c|c|c|c|c|}
\hline Classes & Input Band & $\begin{array}{c}\text { Relative Band } \\
\text { Weight }\end{array}$ & Compacity & Color & Scale \\
\hline Metal Roofing & $0,1,2,3$ & $1,1,1,1$ & 0.8 & 0.4 & 90 \\
\hline $\begin{array}{c}\text { Asbestos } \\
\text { Roofing }\end{array}$ & $0,1,2,3$ & $1,1,1,1$ & 0.7 & 0.5 & 80 \\
\hline $\begin{array}{c}\text { Clear Ceramics } \\
\text { Roofing }\end{array}$ & $0,1,2,3$, & $1,1,1,1$ & 0.5 & 0.5 & 80 \\
\hline $\begin{array}{c}\text { Dark Ceramics } \\
\text { Roofing }\end{array}$ & $0,1,2,3$, & $1,1,1,1$ & 0.5 & 0.5 & 70 \\
\hline $\begin{array}{c}\text { Swimming } \\
\text { Pools }\end{array}$ & $0,1,2,3$ & $1,1,1,1$ & 0.8 & 0.4 & 60 \\
\hline Exposed Soil & $0,1,2,3$ & $1,1,1,1$ & 0.5 & 0.5 & 60 \\
\hline Concrete floor & $0,1,2,3$ & $1,1,1,1$ & 0.5 & 0.5 & 60 \\
\hline
\end{tabular}

Table 1. Segmentation parameter values.

Textural (entropy), spectral statistics (mean, minimum and maximum pixel values, brightness, band ratios) and morphological (primary angle, squareness, circularity, compacity) features were computed for each image segment.

In Stage 3, the Orange Canvas software package was used to explore the classification feature space aiming at defining decision rules for the posterior classification with InterIMAGE, at Stage 4.

Finally, in Stage 5, the classification accuracy was assessed, in terms of Global and TAU accuracy metrics.

\section{DATA MINING AND CLASSIFICATION PROCEDURES}

Orange Canvas provides a visual programming environment, which is based on flowchart building blocks called widgets, to support data analysis.

Widgets are grouped into classes according to their function. Widget flowcharts can be built for input, exploration and visualization of data, such as the flowchart shown in Figure 3.

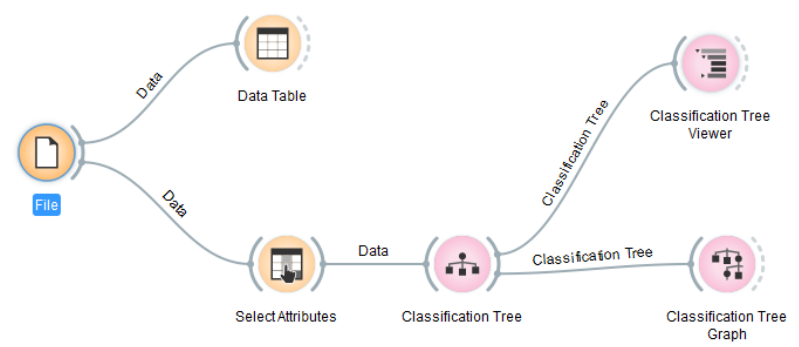

Figure 3. Widgets flowchart. 
The Widget classes in Orange Canvas are:

- Widget File: reads the input data file (data table with data instances).

- Widget Data Table: receives one or more sets of data as input and displays them in spreadsheet form.

- Widget Select Attributes: is used to manually compose a data domain. Through this Widget the user can select the attributes that are actually be going to be used in the data mining process.

- Widget Classification Tree: provides a graphical interface for the C4.5 decision tree learning algorithm, that allows the user to adjust the parameter values of the algorithm and execute it, thus creating a classification tree.

- Widget Classification Tree Viewer: displays the classification tree generated through the Classification Tree Widget in a hierarchical, textual format.

- Widget Classification Tree Graph: displays the classification tree as a graph and allows the user to edit/adjust the classification tree.

Figure 5 shows the decision tree generated with Orange Canvas for this application, as displayed in the Classification Tree Graph Widget. This tree has 32 nodes, 16 leafs, and 16 rules. For the purposes of this study, we consider it a good decision tree, since rules have been attributed to all the specified classes.

According to Tan, Steinback, and Kumar (2005), in a decision tree, a class is attributed to each leaf node. Non-terminal nodes, which include the root node and other internal nodes, contain attribute-test condition that divide entries that have different characteristics. For example, the root node, shown on Figure 5(b), uses the asbestos class and the div4_3 attribute (division of band 4 by band 3) to initiate the separation of classes, continued in descendant nodes, such as Figure 5(c). Figure 5(d) shows a leaf node, associated to the asbestos class.

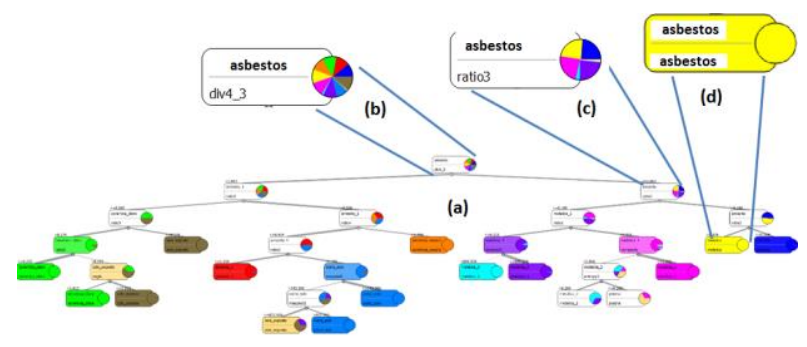

Figure 5. Decision tree generated by the Orange Canvas data mining software. (b) Root node of the tree, represented by the asbestos class. (c) Example of subsequent node of the tree. (d) Example of leaf node.

In this study, the criterion used to select attributes and classes was information gain, which is a measurement of the impurity of data.
Orange Canvas can display the rules associated to the different classes, in textual and graphic form, as shown in Figure 7, for the dark ceramics roofing class.

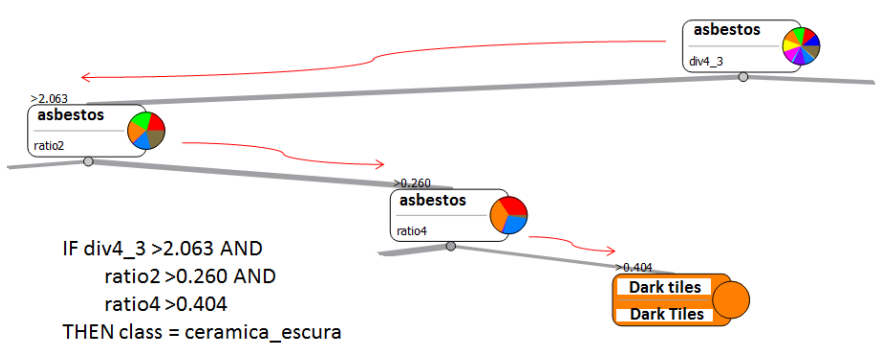

Figure 7. Example of decision rules generated by Orange Canvas.

The rules created with Orange Canvas were subsequently structured in a semantic network in the InterIMAGE software (Figure 8).

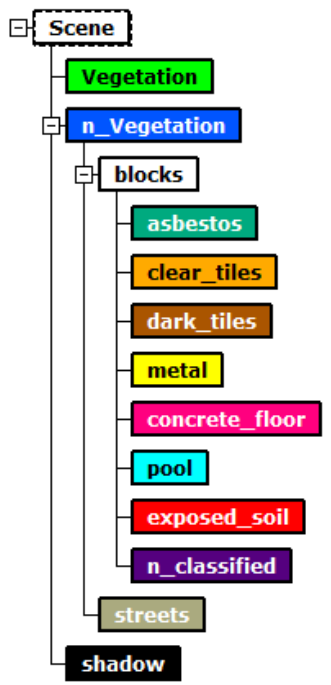

Figure 8. Semantic network in InterIMAGE

The decision rules originated from Orange Canvas were inserted as TopDown decision rules in the InterIMAGE semantic network (Figure 9). For the vegetation, shade, and urban roads classes, the TA_NDVI_Segmenter, TA_Arithmetic, and TA_ShapeFile_Import operators were used.

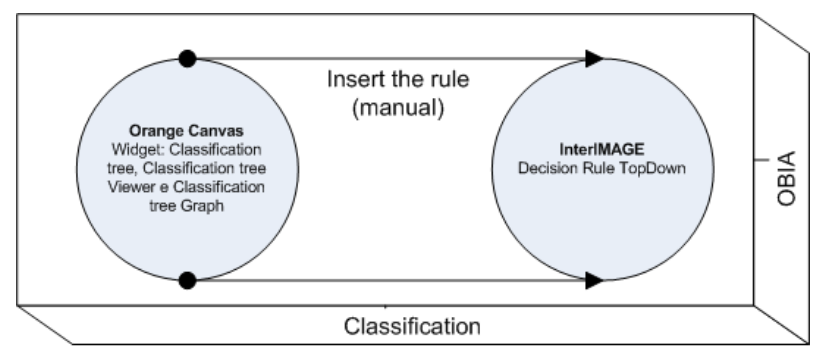

Figure 9. Integration of the Orange Canvas data mining software and InterIMAGE classification software. 


\section{RESULTS AND DISCUSSION}

After classification, a thematic map was generated, classification accuracy was assessed. Global accuracy and the TAU index were computed.

The number of samples was defined using Equation 1, which determined 664 samples (CONGALTON; GREEN, 1999).

$$
N=\frac{B \prod_{i}\left(1-\prod_{i}\right)}{b^{2}}
$$

where $\quad N=$ number of samples

$B=$ obtained from the chi-squared distribution table with (1-k) degrees of freedom

$\Pi_{i}=$ class with the biggest proportion on the map

$k=$ number of classes

$b=$ admissible error

To compose the confusion matrix, classification assessment of random points was carried out using the QuantumGIS software.

The confusion matrix presented $563(85 \%)$ true references and $101(15 \%)$ false references. The result of the classification can be seen on Figure 10, in the form of a thematic map.

Global accuracy was $85 \%$ and the TAU index of agreement was $83 \%$.

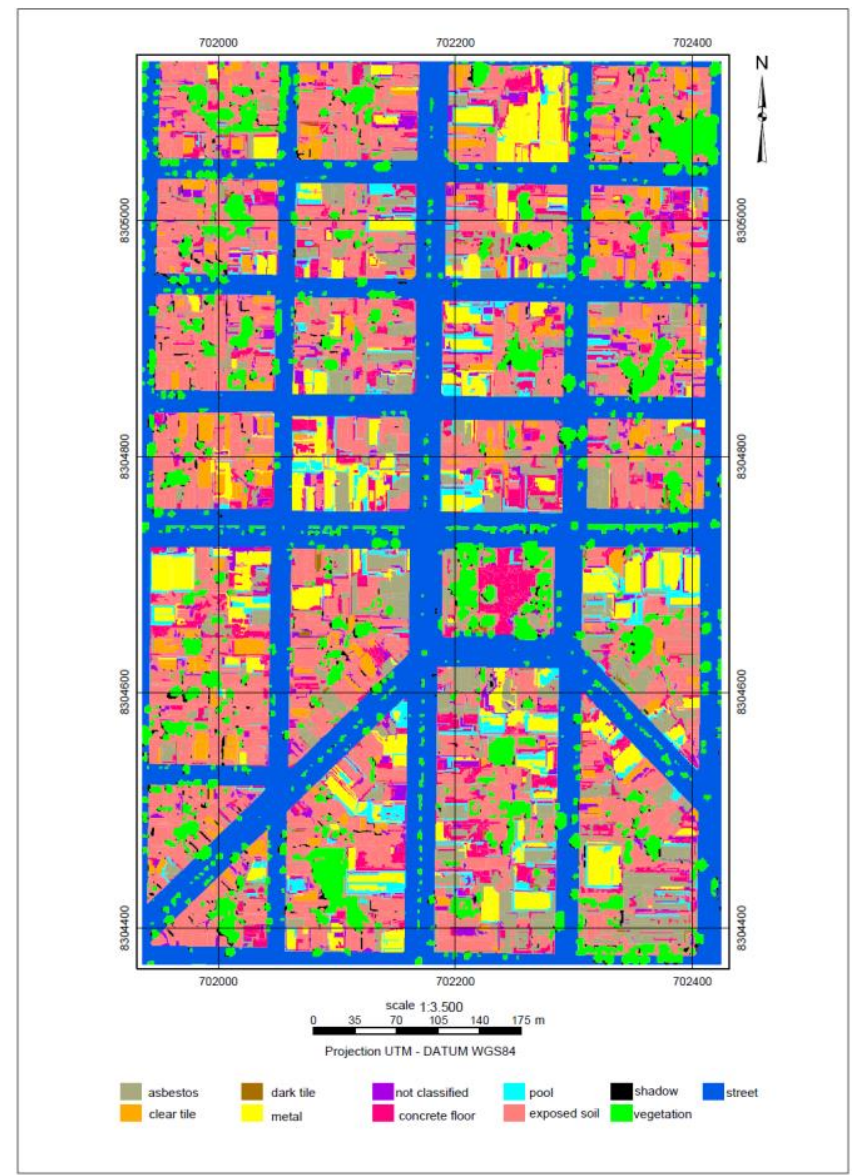

Figure 10. Thematic map of the classification.

The biggest confusion in the classification was between ceramics roofing (dark and clear) and the exposed soil class.
There were difficulties in separating those classes because they have similar spectral characteristics.

\section{CONCLUSION}

The results obtained allowed us to assess the integration of the Orange Canvas data mining software and the InterIMAGE classification software, aiming at analyzing urban occupation. This analysis was performed using and object-based analysis. The following aspects of the methodology and the particular application caried ou in this work can be stressed:

a) The extendablility capacity of the open-source systems InterIMAGE and Orange Canvas are important features for implementing object-based applications.

b) The way Orange Canvas structures classification tree provides for a better organization, exploration and visualization of results.

c) Orange Canvas only provides the C4.5 decision-tree induction algorithm by default. Other algorithms may be implemented on the system, as long as the analyst is capable of programming in the Python language.

d) The $85 \%$ and $83 \%$ obtained respectively for global accuracy and TAU agreement indexes, can be considered very good results. Antunes et al. (2015) classified the same dataset used in this work with the J48 algorithm in the WEKA data mining software, and obtained a global acuuracy of $85 \%$ and TAU agreement of $82 \%$

\section{REFERENCES}

Antunes, R.R., Bias, E.S., Brites, R.S., Costa, G.A.O.P., 2015. Desenvolvimento de técnica para monitoramento do cadastro urbano baseado na classificação orientada a objetos. Estudo de caso: Município de Goianésia, Goiás. Revista Brasileira de Cartografia 67(2), pp. 357-372.

Baatz, M., Schäpe, A., 2000. Multiresolution segmentation: an optimization approach for high quality multi-scale image segmentation. In: XII Angewandte Geographische Informations verarbeitung, AGIT Symposium. Proceedings... Karlsruhe, Germany: Herbert Wichmann Verlag, Salzburg - Austria, pp. 12-23.

Bias, E.S., Antunes, R.R., Pereira, E., Costa, G.A.O.P, Brites, R.S., Rithter, M., 2014. Application of Imagery Analysis Based on Objects as a Tool for Monitoring the Urban Cadastre in Small Municipalities. International Geographic Object-Based Image Analysis Conference, Thessaloniki, 2014.

Blaschke, T., Pekkarinen, A., Burnett, C., 2004. Image Segmentation Methods for Object-based Analysis and Classification. Remote Sensing Image Analysis: Including the Spatial Domain, 211-236. Kluwer Academic Publishers. 2004.

Blaschke, T., 2013. Object Based Image Analysis: A new paradigm remote sensing?. ASPRS, Anual Conference. Baltimore, Maryland.

Passo, D.P., Bias, E.S., Brites, R.S., Costa, G.A.O.P., 2014. Comparison of the accuracy of classifications generated by InterIMAGE and by InterIMAGE integrated with data mining. 
International Geographic Object-Based Image Analysis Conference, Thessaloniki, 2014.

Congalton, R.G., Green, K., 1999. Assessing the accuracy of remotely sensed data: principles and practices. Boca RatonUSA: Lewis Publisher.

Demsar, J., Curk, T., Erjayec, A. 2013. Orange: Data Mining Toolbox in Python. Journal of Machine Learning Research, 14, pp. 2349-2353.

Orange Data Mining - Documentation, 2015. Available at http://orange.biolab.si/ Acessed 21 March 2016.

QGIS BRASIL. Comunidade de usuários QGIS Brasil. 2015. Available at http://qgisbrasil.org/. Acessed 11 August 2015.

INTERIMAGE - Manual do Usuário, 2010. Available at http://www.lvc.ele.puc-rio.br/projects/interimage/ptbr/documentacao/ Acessed 20 September 2014.

Lang. S. Object-based image analysis for remote sensing applications: modeling reality - dealing with complexity. In: Blaschke, T., Lang, S., Hay G. Object-Based Image Analysis. Berlin, German. Springer, 2008.

Nascimento, A.F. et al., 2013. Classificação da Cobertura da Terra, Utilizando os Programas Livres: InterIMAGE, WEKA e QuantumGIS. XVI Simpósio Brasileiro de Sensoriamento Remoto - SBSR, Foz do Iguaçu, Brazil.

Tan, P.N., Steinbach, M., Kumar, V., 2005. Introduction to Data Mining. Addison-Wesley Longman Publishing, Boston, MA, USA.

Witten, I., Frank, E., 2005. Data Mining: Practical Machine Learning Tools and Techniques. 2. ed. San Francisco, CA: Morgam Kaufmann Publishers. 


\title{
CHARACTERIZATION OF THE LAND-COVER AND LAND-USE BY SHAPE DESCRITORS IN TWO AREAS IN PONTA GROSSA, PR, BR
}

\author{
S. R. Ribeiro ${ }^{1 *}$, T. M. Hamulak \\ ${ }^{1}$ Department of Geography, State University of Ponta Grossa, Brazil \\ *corresponding email author: selmar.aranha@gmail.com
}

KEY WORDS: GEOBIA, remote sensing data, Geographic Information Systems - GIS.

\begin{abstract}
:
This study aims to identify the spatial characteristics of the land use and occupation regarding shape (geometry) that these uses are presented in two (2) Landscape units of Hydrographic Basin Pitangui by satellite Geographic Object-Based Image Analysis (GEOBIA). The two (2) croppings of landscape units come from the IRS satellite - P6 (Indian Remote-Sensing Satellite) ResourceSat LISS III sensor. The preprocessings were performed, geometry and atmosphere correction, and the image became segmented in order to extract from it the spatial characteristics and identify the relationship between descriptors of the segments, which were generated from GEOBIA and the use, and occupation of the land. An relevant aspect of this study is that one (1) of landscape units is located in the First Paraná Plateau and the other in the Second Paraná Plateau where there are significant differences concerning geology. The GEOBIA allows segmenting the image by means of regions growth technique and it extracts a relational database considering the spectral, spatial (shape) and texture attributes of the image digital levels. Through this database, we used the descriptors of the spatial attribute (shape) to analyze through graphics and specialization of them with color, to check the relationship between these shape descriptors. It is possible to identify the landscape morphology with this analysis, where small areas do not have defined shapes and the larger areas with geometrically defined shapes. It highlights the importance of analyzing the landscape and its relations with the use and pattern recognition and also the highlight of GEOBIA on this issue which enables to corroborate by the descriptors the visual analysis that the interpreter performs allowing more security and less subjectivity.
\end{abstract}

\section{INTRODUÇÃO}

This study aims to identify the morphology of two landscape units by comparing the shape attribute descriptors, arising Geographic Object - Based Image Analysis (GEOBIA) and this comparison identify which are the archetypes of this attribute with the occupation of landscapes in question, taking into consideration their shape descriptors, to be identified so that ground settlement patterns that characterize both areas.

To ensure the accuracy of the data from this study, we used some preprocessing and after these processing; They were performed targeting and GEOBIA, thereby generating segments and relational database sufficient for the conduct of the study.

\subsection{Segmentation and GEOBIA}

In the Geographic Object or Based Image Analysis (GEOBIA), the important processing is the segmentation of the image. It refers to the process of object image formation that according to Woods and Gonzalez () is the first step in Digital Image Processing (PDI). It consists of dividing the image into homogeneous regions by similarity or discontinuity of algorithms

During the segmentation process, segments are generated or objects through the basic properties of intensity values (digital level) Similar according to the rule used. Vectors are generated taking into consideration the level of abstraction of the segments and also to the relational database on the quantity of processed segments containing the descriptors, spatial, spectral and texture, coming from each region. RIBEIRO, 2003; PRICHOA, 2012; ANTUNES 2014).

\subsection{Object-Oriented Analysis}

The same came up with the preprocessing segmentation, the primary objective of facilitating the domain of a particular area of study to reduce subjectivity mainly in digital classification. She developed GEOBIA, and the most popular algorithms are the Fractal Net Evolution Approach (FNEA) and Full Lambda Schedule Algorithm
(FLSA), designed by Crisp et. al. (2002), this last one used in this study.

Studies by Shapiro and Stockman (2001) indicate two main purposes for using the GEOBIA technique in the analysis: transform the image into fragments for later review and make the change to the representation using their spatial attributes, spectral and texture.

The spatial attributes of shape, have geometric characteristics of the segments, which are translated into the area, length, compactness, convexity strength, roundness, shape factor, stretchiness, rectangularity, main direction, major axis, minor axis, the number of holes, relationship area/ holes.

In this work, we used the attributed form, because of the prior visual analysis difference (photo interpretation) on the morphology of the local landscape.

\section{MATERIALS AND METHODS}

To carry out this work the software utilised were: ENVI 4.7 ENVI EX and ArcGIS 10.2, and the first to the GEOBIA and the last for the analysis of spatial attributes of the relational database. We used the IRS image - P6 Resource Sat Liss III sensor, four spectral bands namely: green, red, near infrared and mid-infrared, with a spatial resolution of $23,5 \mathrm{~m}$, for targeting, available for free on the site INPE (Institute National Space Research). The image corresponds to the scene 328 orbit 96 of the city of Ponta Grossa, of the 07 March 2012. And also the Orth image with a spatial resolution of $5 \mathrm{~m}$, the systematic mapping of the state of Paraná, scale 1: 50,000, SG22 XD - III made available by Parana City, to base and Resource Sat Liss III geometry correction.

Figure 1 shows the block diagram of the methodology used in this study. 


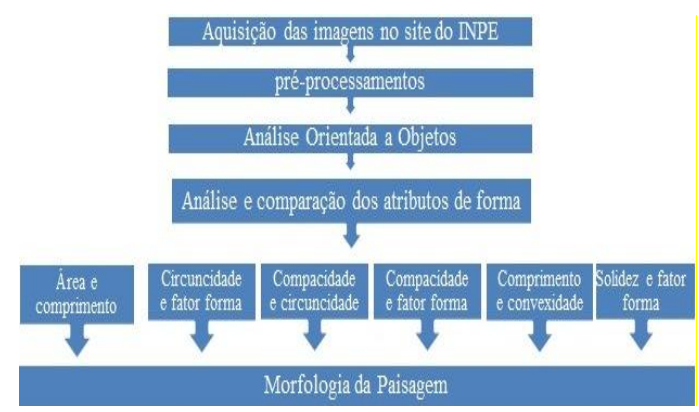

Figure 1. Shows the block diagram with the methodology used in this study.

The preprocessing of the geometric correction, inverse mapping and radiometric correction Dark Object OF Subtraction by key. These refer to the preliminary treatment of the raw data, to calibrate the image radiometry, mitigate the effects of the atmosphere, remove noise and correct geometric distortions arising in the image acquisition process. By GEOBIA is segmented-all spectral bands of images in true color composite false-color R (4) mid-infrared G (3) mid-infrared B (2) red, using the scale factor of 40 and the merger of 50 .

For the recognition of landscape forms, colored compositions were analyzed satellite Resource Satellite, Liss III sensor, and artificial images generated in the GIS of shape descriptors. For visual analysis used the true, false color, as mentioned, since the infrared band, especially next, enhance vegetation.

The artificial images of space descriptors formed by the database of shape descriptors in the GIS wereused in the legend's color scale that distributed the quantitative values and colors for the polygons. The colours are grouped into intervals arranged in the form of classes. The darker color tones highlight the descriptor relationships and the lighter tones generated between the space descriptors the scatter plots. In the representation of artificial images and scatter plots the data were normalized, i.e. the ratio was calculated by the descriptors. Normalization is relevant because of the numerical, difference of descriptors is significant.

\section{RESULTS}

Figure 2 represents the image clippings ResourceSat LISS III, truefalse color- colorful combination R (red) G (near infrared) B (midIR); the first and second unit landscape. Being the first area located at coordinates $603104.697239027 .62 \mathrm{E}$ and $\mathrm{N}$ and the second located at coordinates $583634.727234839 .68 \mathrm{E}$ and N. Located in South America, Brazil, in the state of Paraná in the city of Ponta Grossa.
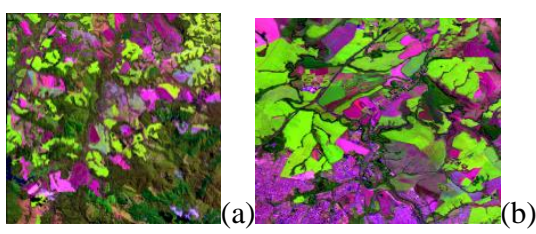

Figure 2. Images that represent the landscape units used in the study first landscape unit (a) located between the coordinates 603,104.69 E 7,239,027.62 $\mathrm{N}$ and second landscape (b) located in coordinates $583634,72 \mathrm{E}$ and $7234839.68 \mathrm{~N}$.

The pre-processing performed in this study were: geometric transformation and the radiometric correction. The geometric correction was obtained employing square error of $6.2 \mathrm{~m}$. The results of GEOBIA with the descriptors are shown in Figures 3 and 4.

Figure 3 (a) and (b) show the segmentation of the first landscape unit (a) and the second landscape unit (b). In Figure 3 (a) it is emphasized that the major joined smaller segments, adding areas to the scale were super-targeted. Note that these areas correspond to areas far as cultivation area or natural vegetation. The second landscape unit, Figure 3 (b), was identified by a significant area of cultivation, and the central point where there were differences between the scale and the merger was to the urban area. This class is unique because of the different forms of the city and high radiance, dividing it into small segments making its analysis difficult. To gain better results the analysis of this class could be separated, however, when interpreted along with other classes will cause confusion, especially because of the image analysis Oriented Geographic Object.

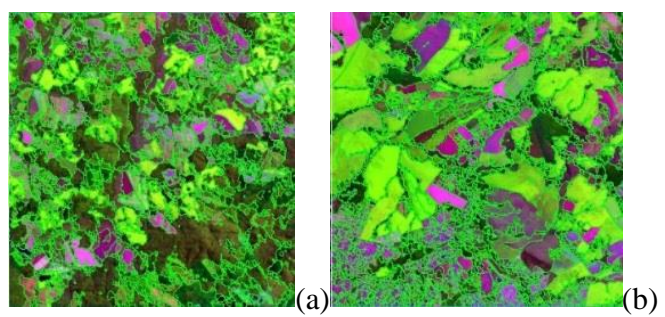

Figure 3 (a) and (b). Image ResourceSat Liss III Color combination representing the true-false color scale of 40 and melt 50 used in the first and second drive landscapes.

\subsection{Separate analysis of descriptors}

With the segmented image could identify differences in the face of the landscape, both in the areas of cultivation, vegetation, as in urban areas. Upon the rising of targeting vectors generated images with shape descriptors in a GIS program. This spatial distribution of shape descriptors allowed the analysis of each of the descriptors and the use and occupation of land in the two landscape units. Figure 5, the first landscape unit, exemplifies this fact, lighter shades and area value in smaller square meters but darker in large quantities and with greater numerical values. These areas are highlighted with arrows.

After these processes comparisons were made between the descriptors of shape attribute arising from AOO, seeking to identify characteristics that have a similar relationship to each other. We carried out the tests consistent in each of the descriptors. However, there were only identified six key combinations representing relations between its characteristics as follows: Area - long, medium circumference-factor, compactness-circumference, compactness form factor, length - convexity, solid - form factor.

\subsubsection{Area - Length}

Figures 4, 5, 6 and 7 show the fields of study, first and second units and the area and length of the descriptors. The analysis of these figures, identifies the brown shade areas are the larger regions comparing it with other areas. These fields range from $4565,06 \mathrm{~m}^{2}$ and $24591,07 \mathrm{~m}^{2}$ (Figure 4), to drive one and the values of $534,687.50 \mathrm{~m}^{2}$ and $2127187,50 \mathrm{~m}^{2}$ to 2 (Figure 6) with small regions shown by shades of yellow, ranging in the legend of $625,00 \mathrm{~m}^{2}$ and $136562,50 \mathrm{~m}^{2}$

In the case of length descriptors in Figures 5 and 7, the highest values are 2444.38 and $24591,07 \mathrm{~m}$ to the first unit in the north-south 
direction, following the Pitangui River. The second unit, 2906.48 to $14,624,64 \mathrm{~m}$ identifies places where there is the presence of large areas of cultivation or soil exposed back to agriculture. The southeast, of figure 7 , there is the urban area, where the regions are small and shown in the yellow color legend.

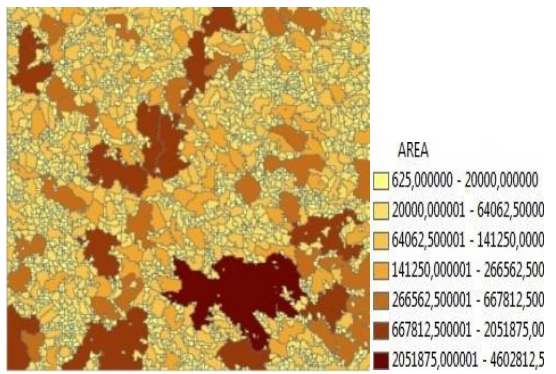

Figure 4 - Image descriptor area of the first unit landscape

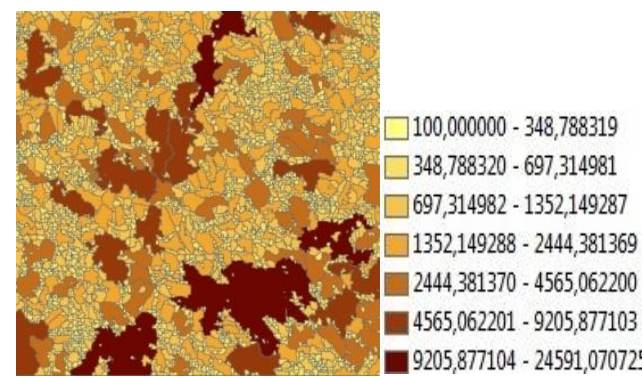

Figure 5 - Descriptor length of the first unit landscape

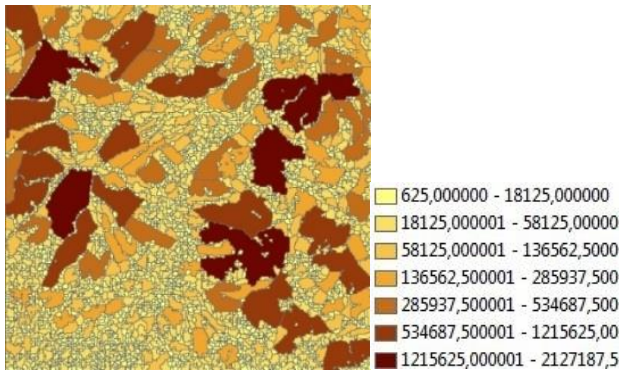

Figure 6 - Descriptor of the second image area landscape unit.

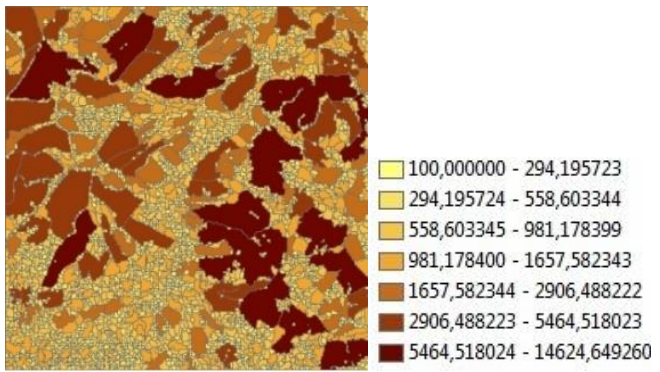

Figure 7. Descriptor of the second image area landscape unit

Figures 8, 9, 10 and 11 show the normalized images (area $\mathrm{x}$ length) and the respective scatter plots of the two landscape units. The Figure 8 and 9 refer to the unit 1 . In the Figure 9 graph, this information is apparent, since the points represented by the medium to dark brown tones are grouped together and grow in both of the $\mathrm{X}$ axis corresponding to the length descriptor. The shaft $\mathrm{Y}$-axis is corresponding to the descriptor area, having only a few points. If scattered in the picture, these correspond to the largest areas.

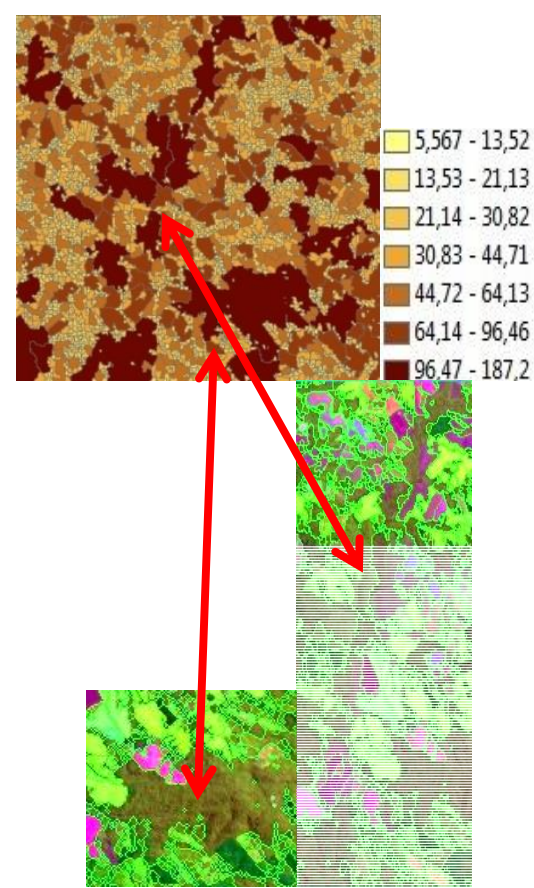

Figure 8 - Standard Image area and length, unit 1

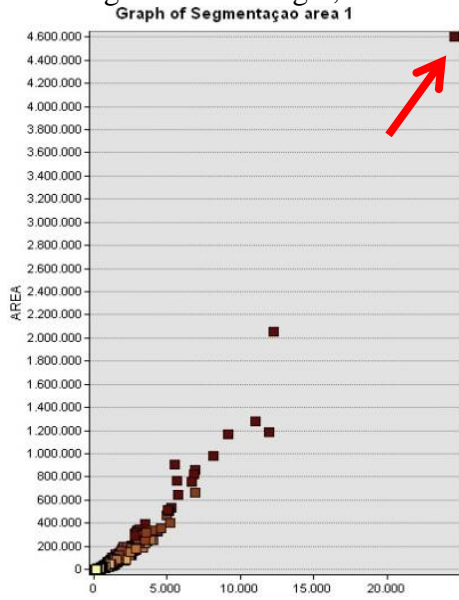

Figure 9 - Graph standard area and length, unit 1.

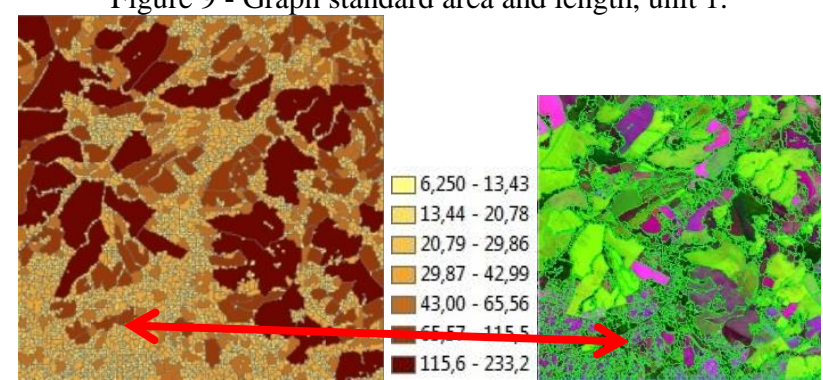

Figure 10 -Image normalized area and length, second landscape unit 


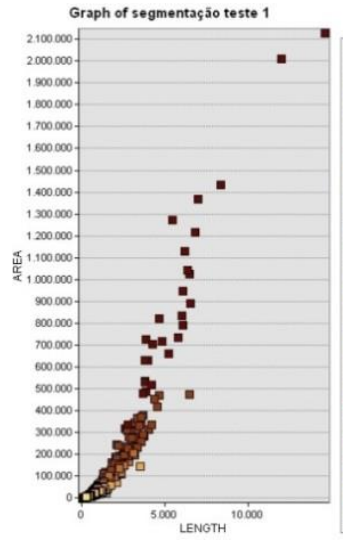

Figure 11 - Graphic standard area and length, the second landscape unit.

A peculiarity found in the first landscape unit, Figure 8 , is a green area (red arrow and inset) with high numerical value area and length area and has a high similarity between descriptors, highlighted in Figure 10 by the graph where the region appears isolated (red arrow).

In Figures 10 and 11 refer to the unit 2. In Figure 11 the normalized image area and length descriptors in brown shades areas are larger and extended. In Figure 11 these scatter graphs show the relation between these descriptors, the increase in size is dependent to the descriptors. The areas of lesser numerical value (red arrow) are in shades of yellow, indicating that they have no similarity in either area or length. Furthermore, they occupy less significant regions to the larger areas. The colored composition in detail also highlights these small areas concerning urban area (red arrow)

When comparing the two landscape units, the first to the second through the area and length of the descriptors, it is identified that the first landscape unit, exhibits small and long areas facing agriculture and vast and extended areas with the presence vegetation. In the second landscape unit, are identified large areas with significant lengths, focused on extensive farming, and small areas with small distances from targeting the urban area of the city of Ponta Grossa.

\subsubsection{Circumcised - Form Factor}

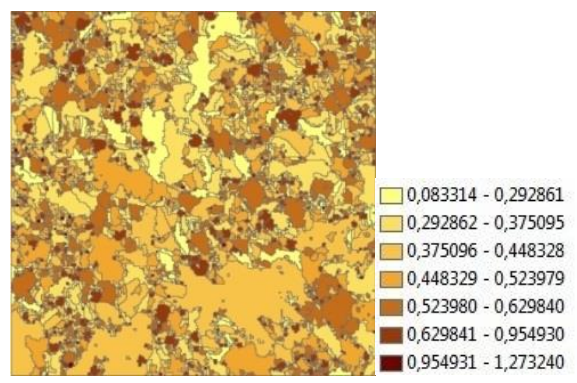

Figure 12. Picture referring to the descriptor circumcised, first landscape unit

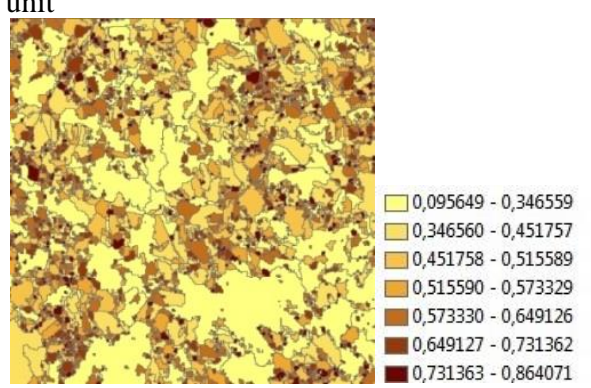

Figure 13 Picture is referring to the descriptor factor first landscape unit form.

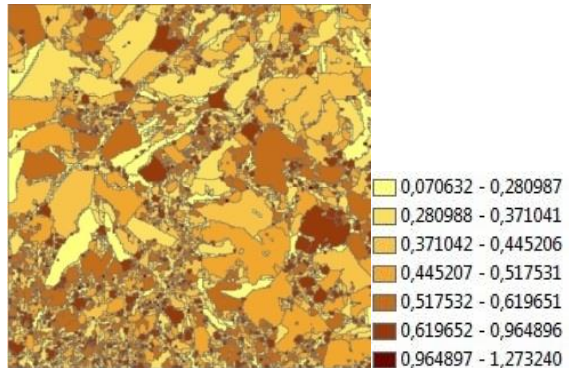

Figura14. Image related to the descriptor roundness second landscape unit.

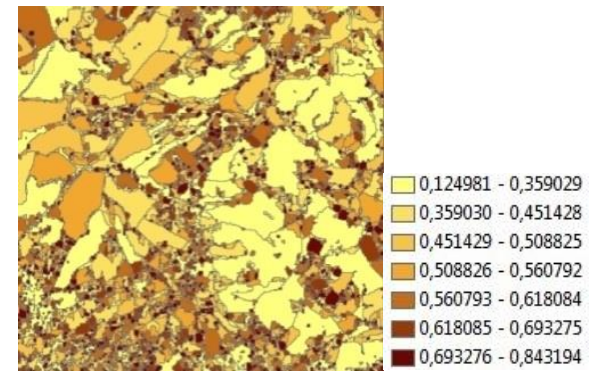

Figure 15. Picture referring to the descriptor formfactor second landscape unit.

Figures 12, 13, 14 and 15 are consistent first and second landscape units, referring to roundness and form factor descriptors. The normalized image roundness between descriptors and form factor are shown in Figures 16 and 18 and; comparative graphs between the two descriptors in Figures 17 and 19.

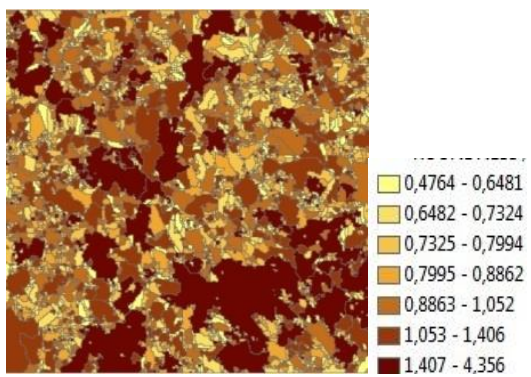

Figure 16. Normalized image among descriptors, unit one, descriptors roundness and formfactor.

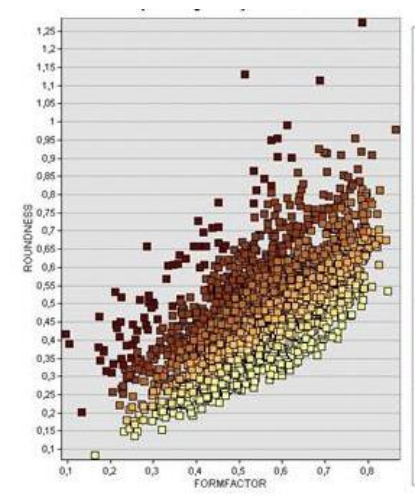

Figure 17 - graph of the normalized image among descriptors, unit one, descriptors Roundness and formfactor. 


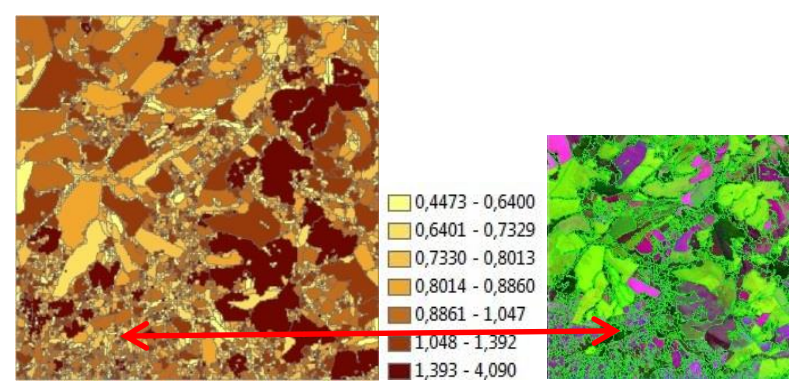

Figure 18. Normalized image among descriptors, unit 2 radius descriptors and form factor.

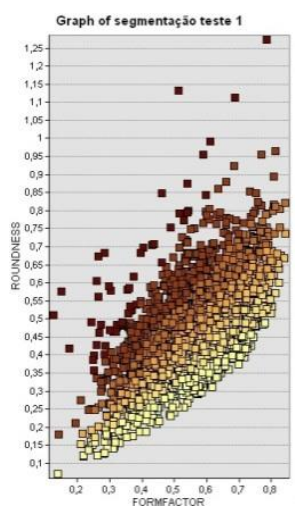

Figure 19. Graph of the normalized image among descriptors, second unit, roundness descriptors and form factor

Given that, the artificial images generated for each of the descriptors and the normalization of the same shows increasing values in the legend where the darkest color match the most significant areas in the descriptor and the lighter ones that have less significance. Notethat in radius image, Figure 12, the greatest radius values are few and scattered. These are highlighted by the color dark and medium brown, ranging in legend between the values of 0.629 and 1.273, as the segments with lower radius cover most of the landscape presenting itself in shades of yellow with values of 0.083314 and 0.375095 .

As for the form factor, figure 13 , it is identified that the greatest figures in shades of dark brown present their values between 0.649127 and 0.864071 and lighter shades of yellow varying between 0.095649 and $0,451,757$.

In figura 18 corresponding to normalized image, identifies that the largest areas present with greater value, highlighted by dark brown color because they are present in more significant amounts in the image.

In the graph, Figure 19, the linear growth between the two descriptors occurs mainly in the areas of smaller, presented in the medium brown color chart. Note the linear growth between the two descriptors as highlighted by the red line.

The large areas on the chart show up in yellow. It is evident that as the descriptor radius, the y- axis, increases the form descriptor factor. And therefore, the $\mathrm{x}$-axis also increases, but differently, causing disorder in and creating a parabola points on the graph

Another feature of these regions is that part of the transition areas between larger areas is that agricultural areas intercalate with areas of vegetation. Because of this, there are some objects identified in small spaces. By this analysis can be stated that in these areas are unclear segmentations caused by existing spectral confusion in the image, i.e., the significant amount of information in one pixel often caused by relief.

In mid-sized regions also the similarity between the descriptors can be identified, as demonstrated in the circled areas. Note that both the image related to radius as in the form factor, are areas similar in brown tones, and in the chart, this similarity is more evident when we note a linear growth between the comparison of these two descriptors, as indicated by the blue arrow.

Figure 14 relating to radius descriptor of the second landscape unit, small areas are identified and presented by dark brown tones, demonstrating that these had a greater scope with the descriptor ranging from 0.619652 and 1.273240 . There are areas where the radius descriptors were less comprehensive presented in yellow tones ranging from 0.070632 and 0.37041 .

Note that in this descriptor; significant regions were the small geared segments or urban area or areas of transition between the large crop fields.

Figure 15 regarding the form descriptor factor in the second landscape unit identified that just as in the radius descriptor the smaller areas were highlighted by shades of dark brown, ranging in legend between 0.618085 and 0.843194 . Larger areas were presented by the shades of yellow, ranging in legend between the values of 0.124981 and 0.451428 .

\subsubsection{Compactness - Form Factor}

Figures 21, 22, 23, and 24 show the first and second landscape unit. These images are descriptors reference to the compactness and form factor. Since the images $25,26,27$ and 28 represent respectively the normalized images and graphics relating to first and second landscape unit. There are 25 and 26 concerning the first landscape unit and 27 to 28 regarding the second landscape unit

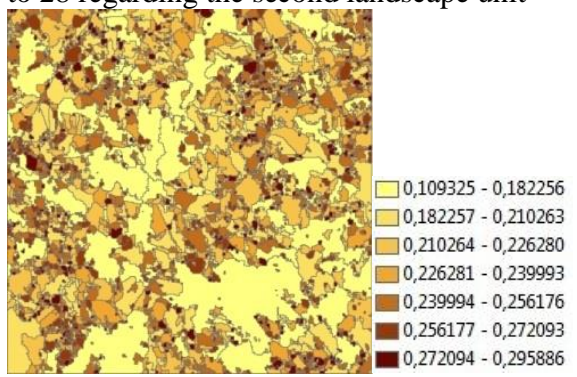

Figure 21. Concerning the first image landscape unit descriptor related to compactness.

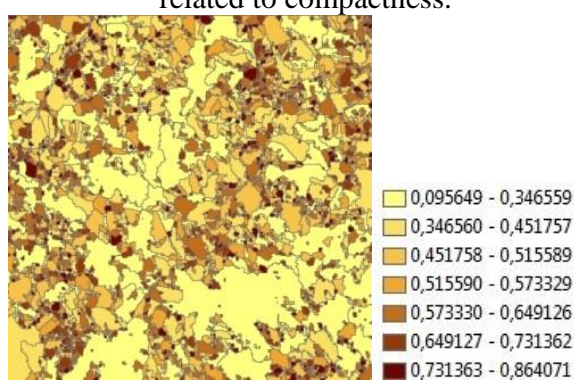

Figure 22. Picture regarding the first landscape unit referring to the descriptor form factor. 


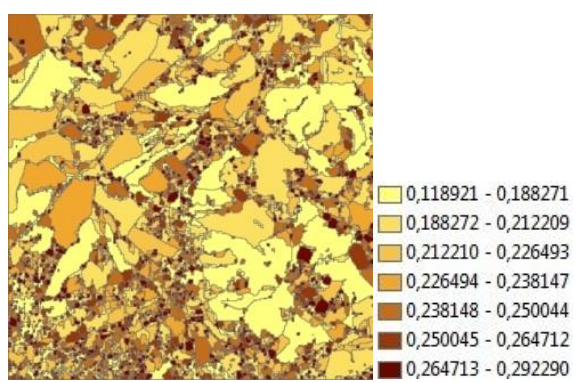

Figure 23. Concerning the second image landscape unit descriptor related to the compactness

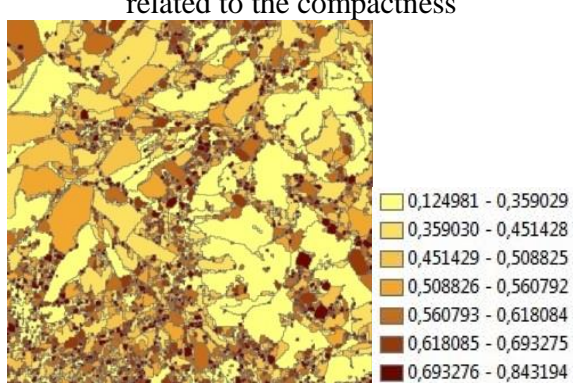

Figure 24. Picture referring the second landscape unit referring to the descriptor form factor.

As we have previously used the compactness and form factor descriptors have the smaller areas with medium brown tones to dark, as they present significant similarity with the descriptors. However in the first landscape unit identifies the presence of large segments of these that appear in shades of yellow, because they have no high compactness and forms defined as the smaller segments, which are presented in a circular fashion.

In the normalized image, Figure 25. It is observed that the smaller areas are shown in shades of yellow and larger areas are presented in dark brown tones. This is because of standardization, that is held direct division of the figures presented the descriptors legends, values referring to smaller areas, highlighted in artificial images from the descriptors by brown tones are now presented in shades of yellow, because the reason for the division is less than the ratio of the larger areas

In this graph in Figure 26, there is linear growth between the two descriptors. It rushes due to the similarity of form factor and compactness descriptors. Are noted by the graph linear growth occurs both in small areas highlighted by shades of dark brown, presented at the beginning of the graph between the values 0.1 and 0.2 for the $\mathrm{x}$-axis refers to the form descriptor factor and $0,11-.19$ descriptor for compactness prescribed on the $y$-axis. As for the areas which are in shades of yellow, they are $\mathrm{x}$-axis between 0,6 and 0,24 and the y-axis values between 0.8 and 0.29 .

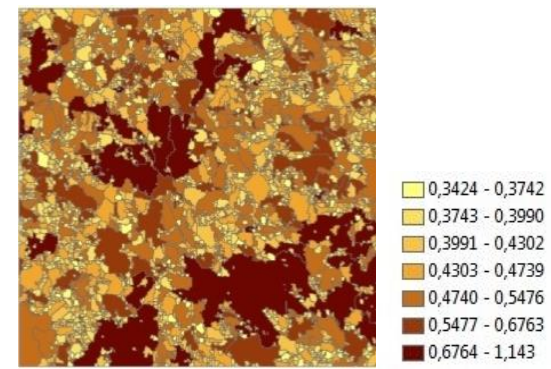

Figure 25. Normalized image regarding the first landscape unit regarding the descriptors compactness and formfactor.

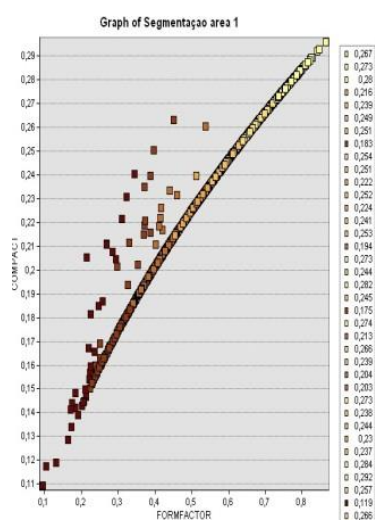

Figure 26. Comparative graph between compactness descriptors and form factor, regarding the first landscape unit.

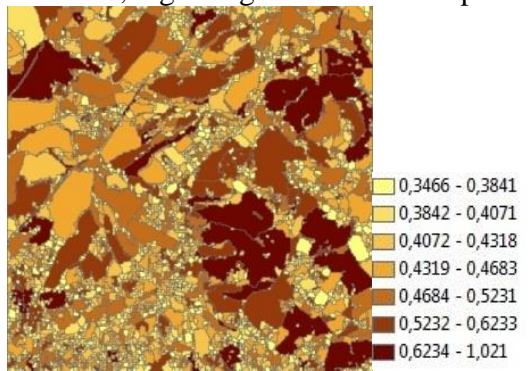

Figure 27. Normalized Image landscape on the second unit between the descriptors and compactness form factor.

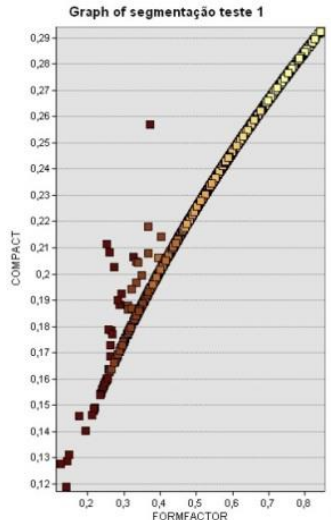

Figure 28.Comparative graph of compactness descriptors and form factor. Second landscape unit.

As previously used the descriptors of compactness and form factor presented in shades of dark brown small areas that have defined shapes and compactness in their spectral response.

It is noted in Figures 25 and 27 refer to normalized image descriptors. Vast areas presented in shades of dark brown to medium brown are because to the fact these are shown to be the defining descriptor form of the image.

In Figures 26 and 28, about the graphics between compactness descriptors and form factor. We noted that shears are directly proportional increasing between the descriptors, i.e., the extension in which the areas became more compact performing as defined.

Note that in the graph based on the $\mathrm{X}$ axis values between 0.0 and 0.2 and $y$-axis values between 0.12 and 0.2 , are the areas in the image for each descriptor are shown in shades of dark brown.

The areas which are in shades of yellow, which refer to larger areas are $\mathrm{x}$-axis values between 0.6 and 0.8 and the $\mathrm{Y}$ axis values between 0 and 0.2429 . 
While comparing these descriptors, it is noted that in both the first area of study and in the second area of study, the descriptors compactness and form factor, are presented in shades of brown. Smaller areas make references to regions where there are little areas of cultivation and vegetation. The case of the first landscape unit, and the second landscape unit such small areas are mainly reference the urban area of Ponta Grossa and the small areas between the vast areas intended for agribusiness.

\section{Conclusion}

The Object-Oriented Analysis made possible to subdivide the landscape units into segments discriminating vectors for each segment database. This study enabled better analysis of the regions and landscape morphology, considering that the descriptors were used in fashion attribute.

When comparing all descriptors, the conclusion is that sound descriptors, form factor, compactness and radius descriptor primarily emphasize small areas because they have rounded shapes. As the descriptors area, length and curvature, these are suitable for large stress regions.

It was identified in the first landscape unit, north-west of Alagados Dam, the largest portions of land are from dense and sparse vegetation considering that these are distributed mainly along the river course, where regions susceptible to flooding. These areas were highlighted particularly by the descriptors area, convexity and length.

In this same landscape unit still identified by the factor, descriptors way radius descriptor, robustness and compactness, smaller areas related to small properties linked to family farming, and even areas with a large amount of spectral confusion regarding the transition areas agriculture regions with regions of vegetation in its two stages of development.

In the second landscape unit used in the present study, located westcentral Alagados Dam, the descriptors area, length and convexity, it was of paramount importance for the identification of large tracts of land geared toward agribusiness.

It is identified through this study that beyond the areas facing agriculture, and urban area of Ponta Grossa there is significant sound descriptors, compactness, radius and form factor. It highlights small areas due to a large amount of information from the reflectance of the urban area. It was subdivided into small areas as the spectral response of object.

Other important points in this landscape unit were to exchange between regions aimed at agriculture, which mainly identifies the presence of exposed soil and vegetation in the process of AOO, subdivided these areas in small arrays, also due to spectral confusion.

Consequently, the landscape morphology in both areas of study proposals is summarized as:

The first area, located north-west of Alagados Dam, is identified mainly by medium regions to mostly small farms focused on family farming and larger areas dominated by vegetation.

The second area, located west central Alagados Dam, identifies large areas related to extensive agriculture, intended mainly for the cultivation of soybeans and wheat, also determines the presence of small amounts of vegetation, which are derived from Riparian forests and small rivers that cross this region, and exchange between the properties.

\section{Referencias}




\title{
PAIRING SEMANTICS AND OBJECT-BASED IMAGE ANALYSIS FOR NATIONAL TERRAIN MAPPING - A FIRST-CASE SCENARIO OF CIRQUES
}

\author{
S. T. Arundel ${ }^{\mathrm{a}}$ \\ ${ }^{a}$ Center of Excellence in Geographic Information Science, U.S. Geological Survey, 1400 Independence Road, Rolla, MO, USA - \\ sarundel@usgs.gov
}

KEY WORDS: Object-based image analysis, national terrain mapping, feature extraction, glacial cirques, semantics

\begin{abstract}
:
As the new National 3D Elevation Program (3DEP) prepares to provide high-resolution lidar coverage for continental United States, Hawaii, and the territories, it is important to consider that terrain information captured in elevation data are pixel-based instead of feature-based. Referencing 3DEP data for semantic access and inferencing requires the transcription of pixels into accessible features. Indeed, accessing and inferencing terrain features renders them more operational for long-term national mapping. Much progress has been made in transcribing pixels into terrain features using Geographic Object-Based Image Analysis (GEOBIA), as compared to traditional, pixel-based image analysis. However, these studies have focused mainly on European terrain, while their applicability and use for United States transcription has not yet been adequately determined. This research evaluates that applicability relative to the mapping of glacial cirques in the Tahoe Basin using an established GEOBIA workflow, run on eCognition. Results suggest that while some parameters of the workflow may require modification, the general workflow steps may apply to other regions.
\end{abstract}

\section{INTRODUCTION}

\subsection{Background}

As the National 3D Elevation Program (3DEP) is preparing to provide high-resolution lidar coverage for the continental United States, Hawaii, and territories, it is important to consider that terrain information captured in elevation data is pixel-based instead of feature-based. Referencing 3DEP data for semantic access and inference requires the transcription of pixels into accessible features. Indeed accessing and inferencing terrain features renders them more operational for long-term national mapping.

'An object view is essential when people communicate about terrain using natural language and when they use landforms as landmarks for navigation and wayfinding' (Mark and Smith, 2004). Much progress has been made in transcribing pixels into terrain features using Geographic Object-Based Image Analysis (GEOBIA), as compared to traditional, pixel-based image analysis. However, these studies have focused mainly on European terrain, while their applicability and use in regions of the United States has not yet been adequately determined. This research evaluates that applicability relative to the mapping of glacial cirques in the Tahoe Basin using an established GEOBIA workflow, run on eCognition. It is important to note that while it is unlikely that the actual threshold values provided by the established model will produce satisfactory results, it is hoped that the general workflow itself can be applied to other regions.

A landform is "a terrain unit created by natural processes in such a way that it may be recognised and described in terms of typical attributes wherever it may occur” (Lobeck, 1939). As the earth's surface is structured into landforms as a result of processes across the geomorphic, geological, hydrological, and ecological realms, these natural objects function as fundamental spatial entities that partition that surface, and define the boundary conditions for processes within these realms (MacMillan and Shary, 2009).

Cirques as landforms are important to study because they are one of the most characteristic forms of glacial landforms in mountain areas (Tricart and Cailleux 1962), and their elevation is an important indicator of paleo equilibrium line altitude (Trenhaile 1976). Identified as bowl-shaped, amphitheater-like depressions associated with glacial erosion, cirques are fairly easy to delineate where they are clearly defined. Evans and Cox (1995) note that larger cirques tend to have better plan and profile closure, and are more likely to develop cols (a saddlelike narrow depression formed by two headward eroding cirques) and tarns (a small lake that collects in a cirque basin when the cirque foot is closed by rock debris. Cirque sizes studied across several regions vary from about $250-1000 \mathrm{~m}$ length by approximately the same values in width, or $\sim 15$ to 25 acres (Evans and Cox, 2015). Hence, cirques smaller than 10 acres were not analyzed in this study.

\subsection{Study Area}

The study area is a small group of mountains in the east central Sierra Nevada range, just west and southwest of Lake Tahoe (Figure 1), covering about $225 \mathrm{~km}^{2}$. Elevation ranges from 1798 m a.s.l. to $3043 \mathrm{~m}$ a.s.l.

The Sierra Nevada is the longest and highest mountain range in the contiguous United States. The backbone of the range is mostly granitic, formed during the Mesozoic, where a chain of volcanoes intruded into the older Palaeozoic rock. By the Late Cretaceous erosion had exposed the deep granite, which was then either uplifted along a north-south fault east of the range, or the eastern basin subsided (Henry, 2009). Soon after the uplift began, the Pleistocene Epoch was marked by the onset of 
global cooling, causing the growth of glaciers in the high Sierras.

The study region experienced extensive and recurring alpine glaciations during the Pleistocene, exhibiting numerous cirques, as well as other typical glacial features, as evidence of glacial erosion. Over 100 glaciers still existed in the range as of 2008 (Basagic, 2008).

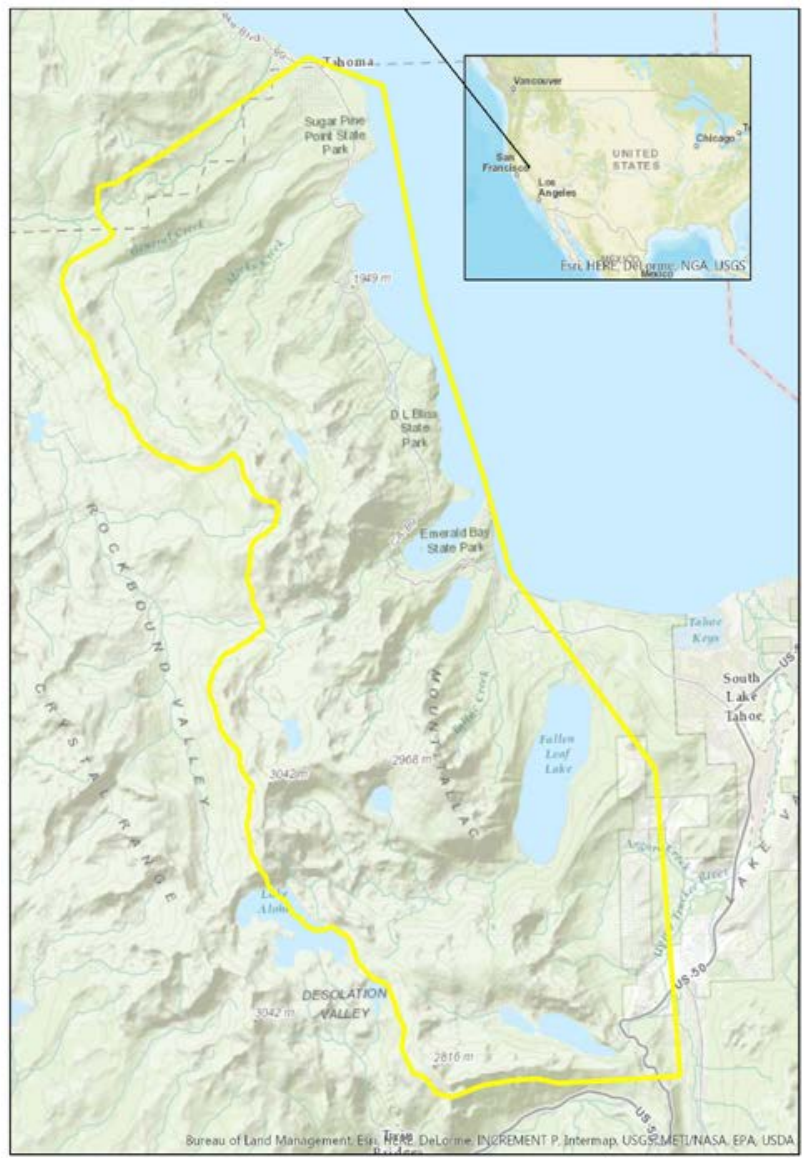

Figure 1.Study area outlined in yellow in the central eastern Sierra Nevadas.

Most of the study area falls within Desolation Wilderness, a federally protected wilderness area in the Eldorado National Forest and Lake Tahoe Basin Management Unit, in El Dorado County, California. Notable topographic features within the region include Mount Tallac, Emerald Bay, Cascade Lake, Fallen Leaf Lake, Echo Lake, Maggies Peaks, Angora Ridge, and Dicks Peak, Jacks Peak, Phipps Peak and Rubicon Peak.

Lidar data were collected for the Tahoe Basin in August of 2010 by Watershed Sciences with an average native pulse density of $>8$ pulses per square meter over terrestrial surfaces. Data from this collection were delivered as a point cloud with an average ground point density of 2.26 points per square meter, and as a DEM with a half meter resolution. The delivery was incorporated into The National Map's $1 / 9,1 / 3$, and 1 arcsecond datasets. The availability of a range of resolutions will allow future comparison of the impact these resolutions have on feature analysis within the same terrain.

\section{METHODS}

Eisank et al. (2010) developed a semantics-based glacial cirque classification workflow for a test area in Austria, in hopes that it would be useful in other regions. The workflow connects the conceptual domain to the software domain using informal semantics, providing a foundation for future work in landform realms even beyond the glacial (Figure 2). The precise methodology used is discussed below.

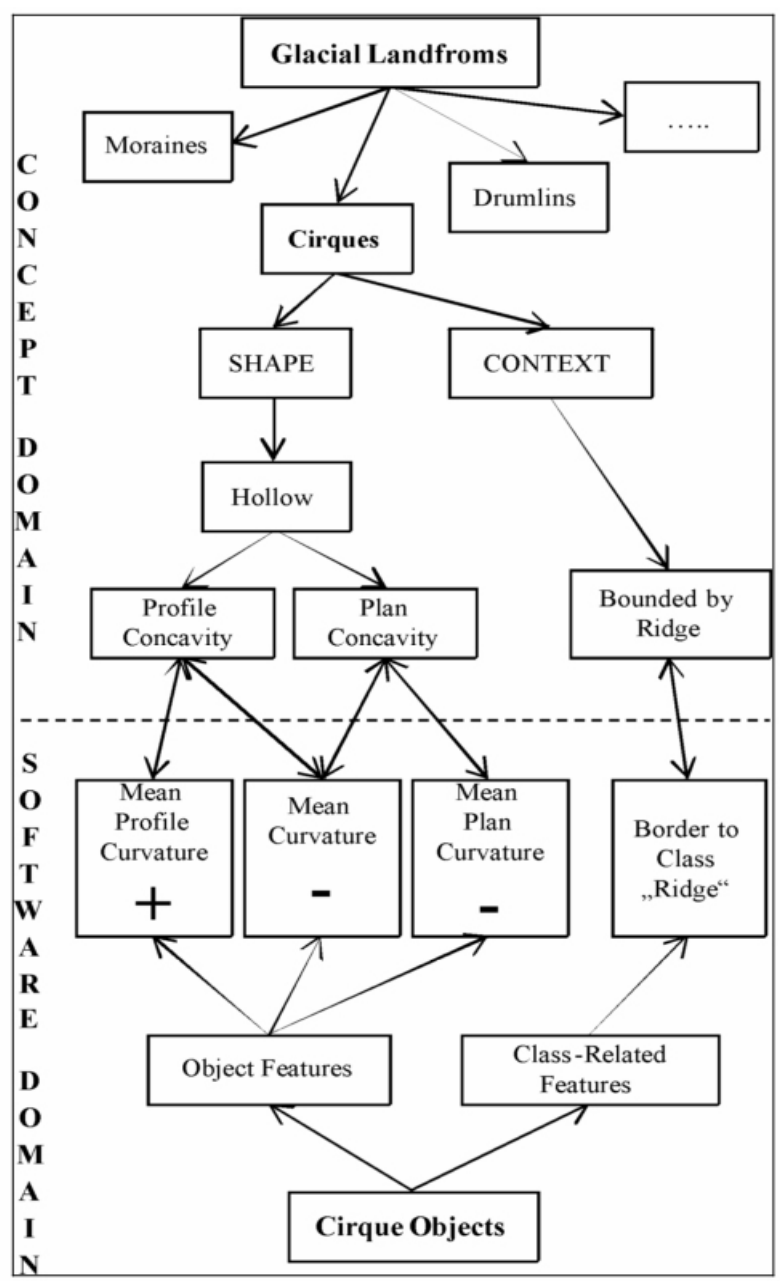

Figure 2. The European semantic model for integrating the cirque concept in OBIA software (from Eisank et al 2010).

\subsection{Reproducing previous work}

An algorithm reproducing as closely as possible the methodology of Eisank et al (2010) was developed to compare to their results, and as a starting point for the Tahoe Basin cirque extraction model. This baseline methodology will hereafter be referred to as the European model.

Three general steps were duplicated from the European model. These steps included classification of ridge-like objects from the mean curvature surface derived from the DEM, masking out ridges from the mean curvature raster to remove them from cirque classification, and classifying cirque objects.

To identify ridge-like objects, mean curvature was calculated from the DEM using ArcGIS 10.2.2, which was smoothed by a 10 x 10 mean filter. Pre-processing steps were completed on a 
larger region to avoid edge-effects. The resulting surface was segmented using the multi-resolution segmentation algorithm in eCognition using the scale parameter of 95 chosen using the Estimation of Surface Parameter tool (ESP) in the European model to best represent ridges in their study area. The ESP tool evaluates scale parameters (SPs) by running stepwise through multi-resolution segmentations of the image, creating imageobjects at iteratively increasing scale (Drăguţ et al., 2010). Upon plotting the local variance (Strahler et al., 1986) against the rate of change (ROC) in image objects at each SP, characteristic scale levels for future segmentation can be assessed.

In the segmentation algorithm, the composition of the homogeneity criterion was based entirely on color, excluding shape (0), and compactness was set at 0.5. Resulting objects with a mean curvature greater than 85 radians $/ 100 \mathrm{~m}$ were classified as ridges. Ridge objects were exported as vectors for use in the masking process.

Masking was performed using ArcGIS by first erasing the ridge polygons from the study area polygon. The resulting vectors were used to clip the raster, using the polygon outlines.

The masked mean curvature surface was subjected to a multiresolution segmentation at scale parameters 43, 11 and 220, values selected in the European model using the ESP tool. Resulting objects with a negative mean curvature that bordered ridge objects were classified as cirques.

\subsection{Improving on previous work}

Based on output from reproducing the European model, adjustments were generated that fell into three basic categories: streamlining of ridge production, improvements to cirque segmentation and enhancements to the cirque classification. Of the three, changes to the ridge extraction model were the least complex.

2.2.1 Ridge extraction: Ridge extraction followed the setup and segmentation steps of the European model exactly, including the use of 95 as the scale parameter for segmentation, as this value produced satisfactory ridge-like objects. However, the classification was altered by greatly decreasing the lower threshold of the curvature values from 85 to 25 but restricting the objects assigned to the class to those objects with a length to width ratio of 1.75 or higher. Then ridge objects were merged and those with a length shorter than $100 \mathrm{~m}$ were removed from the ridge class. The resultant ridge objects were then exported to a polygon vector file for use in the cirque extraction. They were then subtracted from the original mean curvature raster for use in cirque extraction.

2.2.2 Cirque extraction: In order to maximize segmentation for cirque delineation, both the unmasked and masked mean curvature rasters were evaluated for characteristic scale parameters using ESP. The five most characteristic SPs were calculated as those with the highest change in ROC from the previous level, for each image (Table 1). The spikes in the rate of change in the unmasked image were much greater and SPs were easier to detect. ROC fell to almost 0 by SP $=43$ in the masked image, and changed little for the rest of the algorithm run (Figure 3). Because of the difficulty in detecting characteristic SPs for the masked image, the unmasked image was used for subsequent modeling.

\begin{tabular}{|l|l|}
\hline Image layer & Detected scale parameters \\
\hline Filtered mean curvature & $36,66,83,88,211$ \\
\hline $\begin{array}{l}\text { Filtered mean curvature } \\
\text { with excluded ridge areas }\end{array}$ & $18,28,41,68,81$ \\
\hline
\end{tabular}

Table 1. Suitable scale parameters for segmentation of mean curvature surfaces

Objects were first segmented using an SP of 1 as a baseline segmentation that provided input into segmentation at the first identified SP (36). The output objects were then classified as ridges where they matched those output from the ridge extraction. Unclassified objects were classified as cirques where they have a negative mean curvature (concave), but also bordered a ridge object with at least $2 \%$ of its border. Cirque objects were then merged and those smaller than 10 acres $\left(\sim 40,000 \mathrm{~m}^{2}\right)$ were removed from the cirque class.

The output from the first characteristic SP segmentation provided input to the next (66) and so on, until cirques were classified on the five object levels. Cirque objects were exported as a separate shapefile for each level.

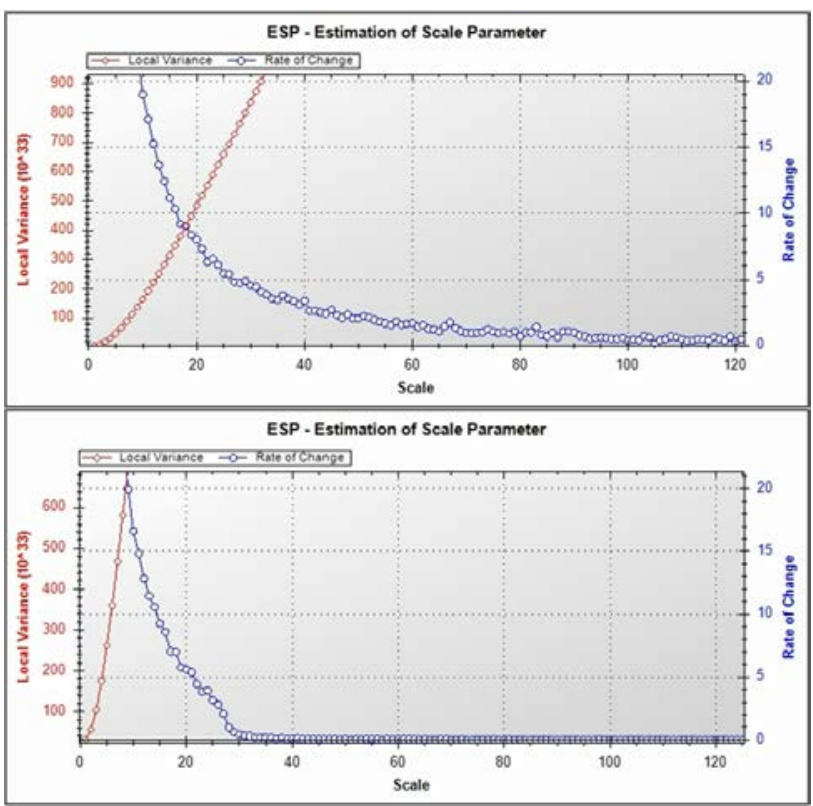

Figure 3. Output from the ESP tool run on the unmasked (top) and masked (bottom) mean curvature, used to estimate characteristic scale parameters for segmentation. Local Variance $\left(10^{\wedge} 33\right)$ is displayed on the left-hand y-axis and Rate of Change is displayed on the right-hand $\mathrm{y}$-axis. The $\mathrm{x}$-axis displays scale parameter.

\section{RESULTS}

\subsection{Reproduction}

Replication of the European model's segmentation parameters resulted in objects that were quite satisfactory for classifying ridges within the study area. Ridge-like objects are clearly outlined. However, classification of objects into the Ridge class fell short; missing ridgelines are clearly identifiable (Figure 5). 


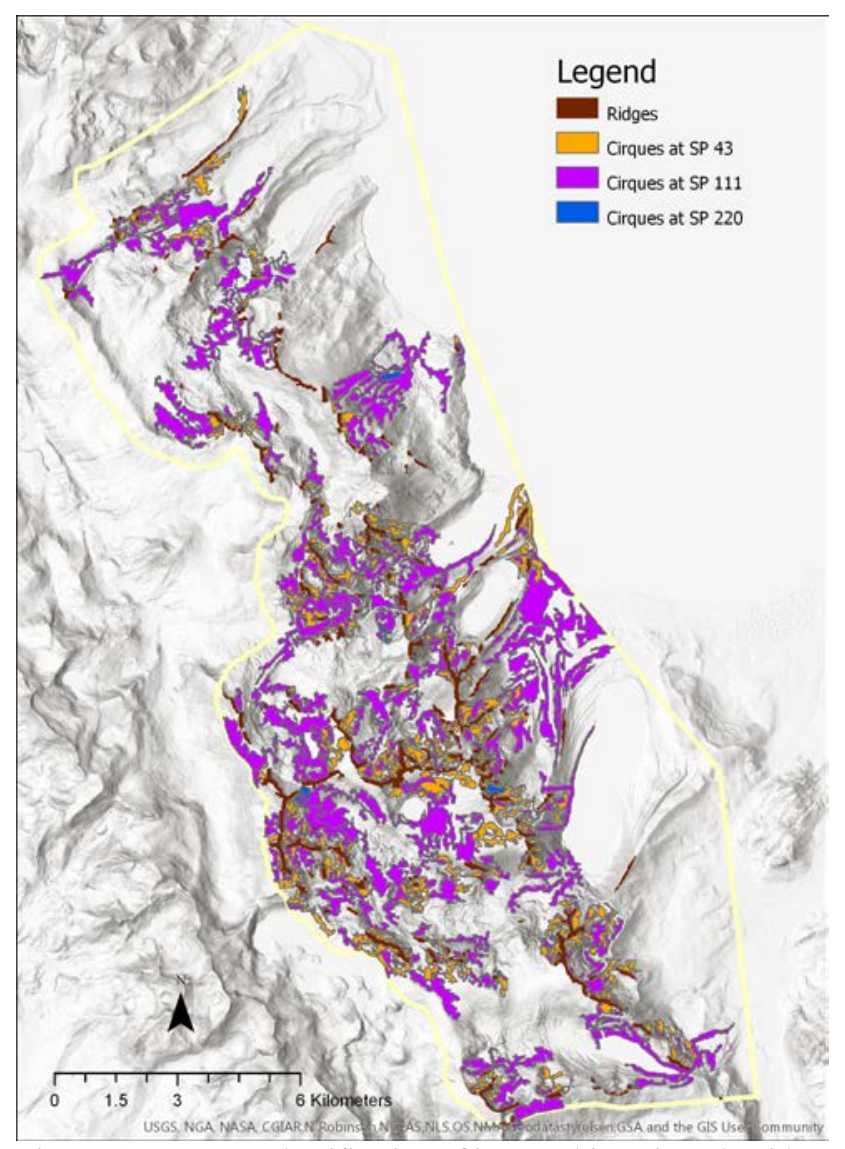

Figure 5. European classification of image objects into the ridge class and three levels of cirque objects based on increasing scale parameters.

\subsection{Improvements}

With improvements to the workflow and threshold values, ridges and cirques were more suitably delineated (Figure 6). Ridges clearly follow visual ridges more closely, and gaps in coverage by the European model have been closed in many places. Cirques are more contained and closer to their signature arm-chair shape.

While the three image segmentations seem to offer adequate objects to represent cirques, the classification still proves inadequate. For example, on Mt Tallac in the southern part of the study area, several older, larger cirques have been re-eroded by smaller glaciers, resulting in the overlay of smaller cirques (Figure 7). The classification was successful at capturing the smaller cirques but not their older, larger parent cirques.

The use of derived profile and plan curvature was not incorporated into the European model, although it was considered in the conceptual-to-software domain semantics. The inclusion of these layers in the cirque segmentation and classification, following the European workflow (Figure 2), may improve their representation.

\section{DISCUSSION}

The expectation that actual threshold values would require alteration for the entire model was proven incorrect by the original ridge segmentation. This is a promising result. These results also validate the concept that the general cirque workflow can apply beyond the region for which it was originally developed, contradicting the widely-held concern that GEOBIA produces methods that are rarely transferable (Arvor et al., 2013). With just minor changes in the algorithm parameters, the workflow was adequately successful. The results support the use of natural language semantics in producing the object extraction workflow.

A next step in the research is to understand how plan and profile curvature can improve such a workflow. Slope calculations may also positively impact the model. Future work will also compare the extraction at the different available DEM scales, as well as from the lidar point cloud.

Ideally, results should be quantitatively compared to reference datasets. However, cirque mapping is a tedious process (which is one of the justifications for automated extraction). A future plan to compare these results, and improve the delineation, includes expert digitization of the study area cirques by glacial geomorphologists.

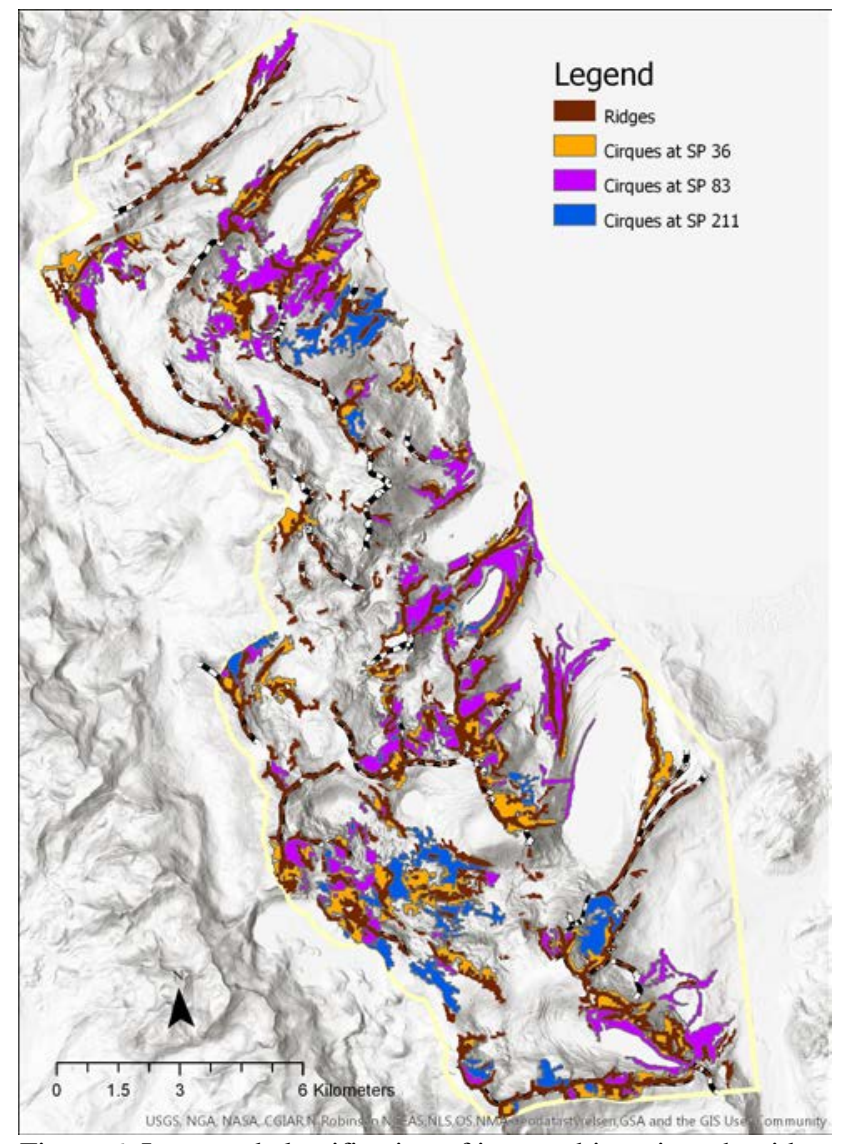

Figure 6. Improved classification of image objects into the ridge class and three levels of cirque objects based on increasing scale parameters.

\section{CONCLUSION}

An existing, semantically-driven GEOBIA workflow for extracting glacial cirques was tested for its transferability to another geographical region. While some changes were made to the workflow, the general steps were successful in delineating cirques in the southwestern Tahoe Basin of California. Future work includes quantitative comparison to expert-driven cirque mapping, and inclusion of slope, plan and profile curvatures in the segmentation and classification model. 


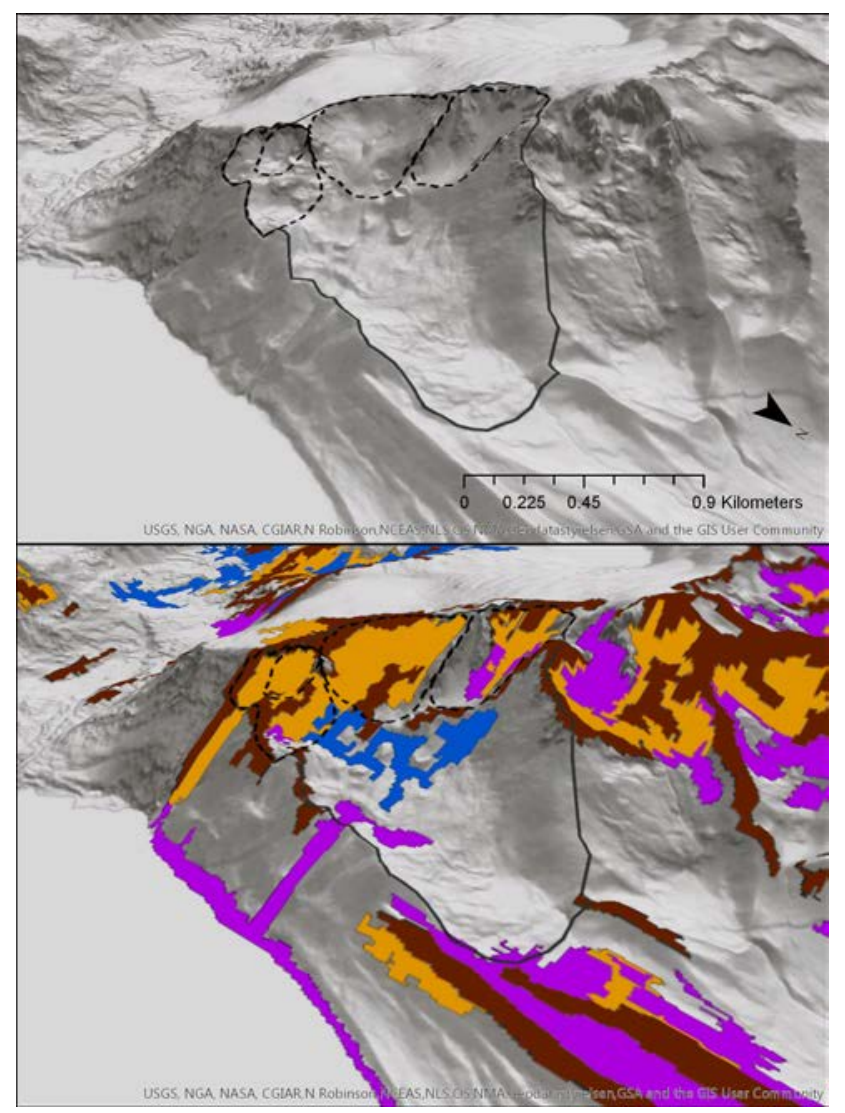

Figure 7. Example of nested cirques delineated by hand in $\mathrm{Mt}$ Tallac (top), along with cirque objects created by the improved model.

\section{ACKNOWLEDGEMENTS (OPTIONAL)}

This research was funded by the National Geospatial Program as part of the U.S. Geological Survey.

\section{REFERENCES}

Arvor, D., Durieux, L., Andrés, S., Laporte, M.A., 2013. Advances in Geographic Object-Based Image Analysis with ontologies: A review of main contributions and limitations from a remote sensing perspective. ISPRS J. Photogramm. Remote Sens. 82, 125-137. doi:10.1016/j.isprsjprs.2013.05.003

Basagic, H.J.I., 2008. Quantifying twentieth century glacier change in the Sierra Nevada, California. Portland State University.

Drăguț, L., Tiede, D., Levick, S.R., 2010. ESP: a tool to estimate scale parameter for multiresolution image segmentation of remotely sensed data. Int. J. Geogr. Inf. Sci. 24, 859-871. doi:10.1080/13658810903174803

Eisank, C., Drăguţ, L., Gotz, J., Blaschke, T., 2010. Developing a semantic model of glacial landforms for object-based terrain classification-the example of glacial cirques. Proc. Int. ....

Evans, I.S., Cox, N.J., 2015. Size and shape of glacial cirques: comparative data in specific geomorphometry. Geomorphol. Geosci. 63, 79-82.
Evans, I.S., Cox, N.J., 1995. The form of glacial cirques in the English Lake District, Cumbria. Zeitschrift fur Geomorphol. 39, 175-202.

Henry, C.D., 2009. Uplift of the Sierra Nevada , California. Sierra 575-576. doi:10.1130/G20197.1.Unruh

Lobeck, A.K., 1939. Geomorphology. McGraw-Hill Book Company, New York. doi:10.1002/sce.3730240525

MacMillan, R.A., Shary, P.A., 2009. Landforms and Landform Elements in Geomorphometry, in: Hengl, T., Reuter, H.I. (Eds.), Geomorphometry-Concepts, Software, Applications. Elsevier, Amsterdam, pp. 227-254.

Mark, D.M., Smith, B., 2004. A science of topography: From qualitative ontology to digital representations, in: Bishop, M P and Shroder Jr, J.F. (Ed.), Geographic Information Science and Mountain Geomorphology. Springer-Praxis, Chichester, England, pp. 75-97.

Strahler, A.H., Woodcock, C.E., Smith, J.A., 1986. On the nature of models in remote sensing. Remote Sens. Environ. 20, 121-139.

Trenhaile, A.S., 1976. CIRQUE MORPHOMETRY IN THE CANADIAN CORDILLERA*. Ann. Assoc. Am. Geogr. 66, 451-462. doi:10.1111/j.1467-8306.1976.tb01101.x

Tricart, J., Cailleux, A., 1962. Le modèle glaciaire et nival. Inf. Geogr. 28, 221. 


\title{
QUANTIFYING BUSHFIRE MAPPING UNCERTAINTY USING SINGLE AND MULTI- SCALE APPROACH: A CASE STUDY FROM TASMANIA, AUSTRALIA
}

\author{
J. Aryal ${ }^{\mathrm{a} *}, \mathrm{R}$. Louvet ${ }^{\mathrm{a}, \mathrm{b}}$ \\ a Discipline of Geography and Spatial Sciences, School of Land and Food, University of Tasmania, Hobart, 7001, Australia \\ jagannath.aryal@utas.edu.au; \\ b UMR ESPACE 7300 CNRS, Universit'e d'Avignon, France \\ Romain.Louvet@utas.edu.au
}

KEYWORDS: Bushfires, Tasmania, Earth Observation, MODIS, Landsat, Sentinel-2, Uncertainty

\begin{abstract}
:
More than 72,000 hectares of western Tasmania were burnt in 2016 due to bushfires. Bushfires in Tasmania has high social, economical, and environmental impacts. The remote delineation of these bushfires has paramount importance for decision-making authorities to help people in emergencies and planning. Considering the fact that delineation uncertainty from Earth Observation [EO] data is inevitable, this study uses MODIS, Landsat and Sentinel-2 imageries covering the 2016 burnt areas from Tasmania. We test the hypothesis that the difference in Normalised Difference Vegetation Index (NDVI) before and after the fire event can detect the accurate delineation of burnt areas and hence the changes. MODIS, Landsat and Sentinel-2 products before and after fire are used independently in delineating and mapping bushfire boundaries. We map in three thematic classes burnt, damaged and both. Delineated boundaries are examined for uncertainty and error maps are produced. The uncertainty examination and validation are performed using ground truth data obtained from local fire authorities. Developed error metrics are used to obtain statistical measures like sensitivity, specificity, positive predictive value, negative predictive value, kappa coefficient and overall accuracy. Our results show that there is minimal difference in overall accuracy from both the sensors MODIS: [0.94 vs 0.92] and Sentinel [0.94 vs 0.93 ] for the classes burnt $\&$ damaged $v s$ only burnt.
\end{abstract}

Furthermore, we propose a conceptual framework for bushfire mapping uncertainty in a multiple-scale environment incorporating sensitive thematic parameters that could affect initiation of fire and blaze direction. The parameters considered in our framework are: vegetation type [landcover], vegetation density [vegetation indices], drought [soil moisture, air moisture, precipitation], temperature [air temperature, soil temperature], topography [elevation, slope, aspect, ruggedness, topography position index], and wind [speed, direction]).

\section{INTRODUCTION}

\subsection{Bushfires in Australia}

Bushfires have been part of the Australian environment since before human settlement of the continent (ABS, 2016). Bushfires in Australia are increasing (Dutta et al, 2016). Bushfires are complex natural disasters that bring catastrophic consequences to the socio-economic and ecological environment of a country. Due to its unique continental position, Australian states and territories experience different sizes of bushfires. In the past, Tasmania suffered their worst bushfires on 'Black Tuesday' $7^{\text {th }}$ February 1967 when approximately 264,000 ha were burnt, 1,700 houses destroyed and 61 people killed (ABS, 2016). Recently, in January 2016, north and north-west Tasmania (Fig.1) experienced bushfires. The burnt areas were located within the World Heritage Area (WHA) and attracted wide attention. The bushfires devastated areas which are home to unique and iconic Tasmanian alpine flora including pencil pines, king billy pines, and cushion plants. Some of these vegetation communities were more than 1,000 years old. Fire ecologists and experts declared that these killed vegetation communities wouldn't grow back and this incident may be sign of a system collapse due to drier summer caused by climate change (Radionz, 2016). Considering the significant heritage importance of the affected areas and the lost vegetation communities, it is essential to know the accurate extent of the affected areas. In this study, the bushfire affected areas that are extremely important to nature conservation and heritage are chosen for remote delineation using Earth Observation [EO] data. The main motivation of this research work is to quantify the uncertainty associated with the delineation of bushfire affected areas. The uncertainty is observed with the calculation of various accuracy measures based on ground truth data. These measures include: sensitivity, specificity, false positive rate, false negative rate, positive predictive value, negative predictive value, kappa coefficient and overall accuracy. These measures are calculated for multisensor data. In this study, we used the change of Normalised Difference Vegetation Index (NDVI) combined with k-means clustering to map the removal of vegetation caused by bushfires. The difference in the NDVI before and after the fire event can provide delineation of burnt areas and these can be improved then by clustering. Mapping burnt areas based on NDVI have been widely tested (van Leeuwen et al., 2010). Other indices such as the Normalised Burn Ratio (NBR) (Veraverbeke et al., 2011) and the Burned Area Index (BAI) (Chuvieco et al., 2002) are also used in mapping burnt areas. In order to test the

\footnotetext{
* Corresponding author
} 
proposed method we analysed the multi-sensor products. Landsat 8 image before the fire (27 December 2015) is analysed and the results are compared with the analysed Sentinetal-2 image after fire (14 ${ }^{\text {th }}$ March 2016). Sentinel-2 was used because Landsat 8 cloud-free images were not available. The Sentinel-2 Multispectral Instrument (MSI) acquires 13 spectral bands ranging from Visible and Near-Infrared (VNIR) to Shortwave Infrared (SWIR) wavelengths along a $290-\mathrm{km}$ orbital swath. The MSI sensor data are complementary to data acquired by the U.S. Geological Survey (USGS) Landsat 8 Operational Land Imager (OLI) and Landsat 7 Enhanced Thematic Mapper Plus (ETM+). Being complimentary to Landsat $8 \mathrm{OLI}$, Sentinel-2 is used for comparison. Independently, we analysed MODIS burned area products from $27^{\text {th }}$ December 2015 and $14^{\text {th }}$ March 2016. We discuss in section 2 the specifications on data, details on study area and methodological approach. Section 3 presents results and discussions, and in section 4 we present conclusions and future directions.

\section{DATA, STUDY AREA AND METHODOLOGY}

\subsection{Earth Observation [EO] and Ground Truth Data}

The EO data used for the analysis are Landsat 8 OLI, Sentinel2 , and MODIS.

\subsubsection{Before fire}

Landsat 8 OLI is used for before the fire analysis. The specifications of the product are as follows:

Product: Landsat 8 Operational Land Imager, 9 bands, date: 27/12/2015 (361/2015), spatial resolution: $30 \mathrm{~m}$, temporal resolution: 16 days.

Similarly, MODIS terra is used for before the fire analysis. The specifications of the product are as below:

Product: MODIS Terra, bands: 1 to 7 , date: 27/12/2015 (361/2015), spatial resolution: $500 \mathrm{~m}$, temporal resolution: daily.

\subsubsection{After fire}

For after fire event analysis, Sentinel-2 is used and compared with Landsat 8 OLI and, MODIS is analysed and compared with MODIS product. The specifications are as follows:

Product: Sentinel-2, 13 bands, date 14/03/2016 (74/2016), spatial resolution: 10,20 , and $60 \mathrm{~m}$, temporal resolution: 10 days.

Near infrared band (band 8) and red band (band 4) with $10 \mathrm{~m}$ spatial resolution are used in computing NDVI from Sentinel-2 product.

Product: MODIS Terra, bands 1 to 7 , date 14/03/2016 (105/2016), spatial resolution: $500 \mathrm{~m}$, temporal resolution: daily.

\subsubsection{Ground Truth Data}

The fire ground truth data is brought together from several sources / agencies (e.g, Tasmania Fire Service; Parks and
Wildlife; Forestry Tasmania; Forico). The 'official' fire history is released in late July of each year. It is available on LISTmap (The state mapping authority of Tasmania) where we can see the source and method of data capture for each polygon. General information from previous year's metadata can be seen at the link below (The LIST, 2016):

https://data.thelist.tas.gov.au/datagn//srv/eng/main.home?uuid= b94d4388-995d-416a-9844-a39de2798bed

Generally, larger fires have had their boundary mapped by viewing from a helicopter during the fires providing us realtime ground truth data. The ground truth in this study is realtime data captured from helicopter. The ground truth data (Figure 1) are used in validating the produced maps based on normalised difference vegetation index, difference and unsupervised classification.

\subsection{Study Area}

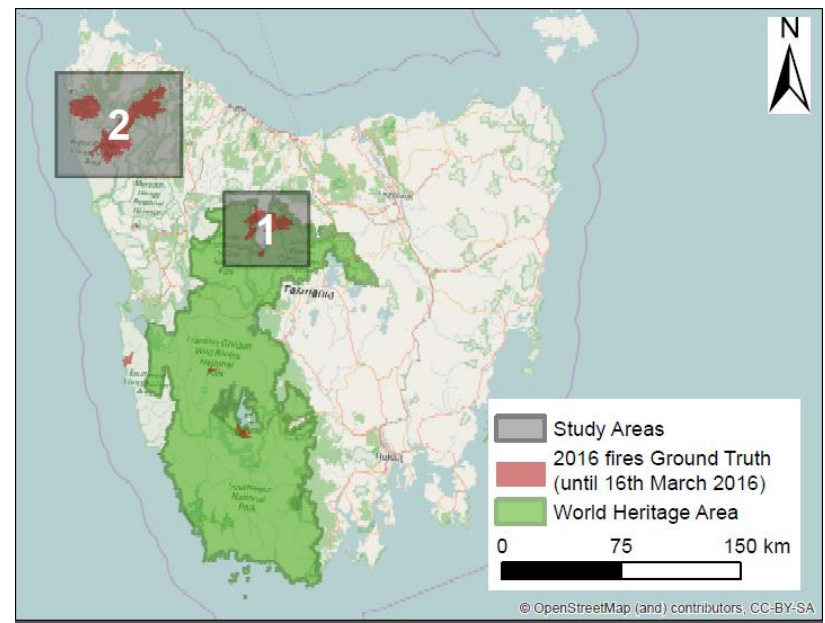

Figure 1. Two bushfire affected areas from north and north-west Tasmania, Australia

The study area 1 is the part of the World Heritage Area. As depicted in Figure 1, two study areas are considered: 'study area 1 ' and 'study area 2'. The real-time picture of fire (Figure 2) shows the surrounding environment of the study area including vegetation communities.

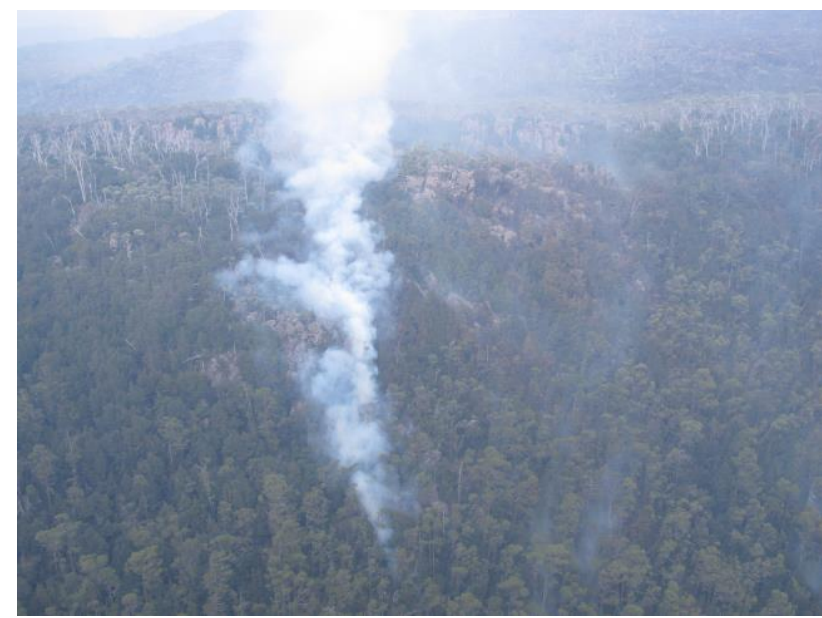

Figure 2. Real-time picture of a fire event including vegetation communities, Tasmania, Australia 


\subsection{Methodology: Single-scale approach}

\subsubsection{True / infrared colour composite}

All EO data are visualised by forming two different colour composites namely true colour composite and infrared colour composite. This visualisation help to better understand the burnt areas after and before the fire, i.e. presence / absence of vegetation. True colour composites for Landsat 8 (Bands 4, 3, 2), Sentinel-2 (Bands 4, 3, 2) and MODIS (Bands 1, 4, 3) and infrared colour composite for Landsat 8 (Bands 5, 4, 3), Sentinel-2 (Bands 8, 4, 3) and MODIS (Bands 2, 1, 4) are observed in ascertaining the burnt area and vegetation area. This manual visualisation provides confirmation for NDVI calculation.

\subsubsection{NDVI Difference Map}

NDVI is calculated using the following equations for the respective $\mathrm{EO}$ data.

$$
\begin{aligned}
& \text { NDVI for Landsat } 8=\frac{\text { band } 5 \text {-band } 4}{\text { band } 5+\text { band } 4} \\
& \text { NDVI for Sentinel }-2=\frac{\text { band } 8-\text { band } 4}{\text { band } 8+\text { band } 4} \\
& \text { NDVI for MODIS }=\frac{\text { band } 2-\text { band } 1}{\text { band } 2+\text { band } 1}
\end{aligned}
$$

Where for Landsat 8, Sentinel-2 and MODIS, the near-infra red bands are respectively band 5 , band 8 , and band 2 ; and the red bands are respectively band 4 , band 4 and band 1 . The calculated NDVI is used to produce change detection map based on difference of NDVI. The difference is computed by subtracting NDVI value after the fire event from NDVI value before the fire event. Since burnt areas are spaces where there is no more vegetation, fire events should correspond to a positive NDVI before the fire and a negative NDVI after the fire. Therefore, NDVI difference values inferior to 0 is selected as a first step to delineate bushfires. Mathematical morphology is then used to correct these extents. A mask layer is created and used with the other bands in EO data after the fire in order to refine the bushfires delineation based on clustering.

2.3.3 Spectral signature extraction, unsupervised classification, and validation using ground truth

For Sentinel-2 and MODIS after fire data, the unsupervised classification is made using $\mathrm{k}$-means clustering based on observed spectral signatures (eg; Figure 3) and false colour composite visualisation, reclassification is made in three categories namely: "burnt", "unburnt", and an intermediary class we called "damaged" which should represent slightly burnt areas. The classified map is validated using ground truth data and error maps are produced along with statistical measures.

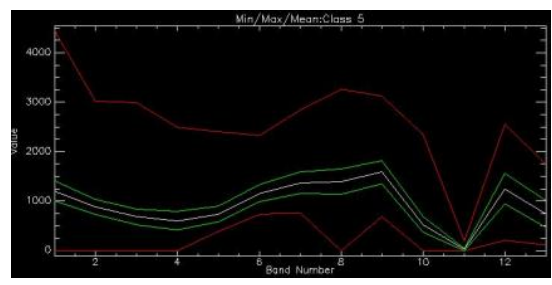

Figure 3: Spectral signature for the class "damaged", flatter than burnt.
2.4 Methodology: Multiple-scale approach and proposed conceptual framework

The single-scale approach provides information on one level. However, a phenomenon like bushfire is the outcome of many variables not only vegetation. With this in mind, we wish to extend the developed method in this work for multiple scales. We propose a framework that can integrate thematic parameters contributing for fire in hierarchies (Aryal and Josselin, 2014; Blaschke et al 2014). The framework integrates parameters like vegetation type [landcover], vegetation density [vegetation indices], drought [soil moisture, air moisture, precipitation], temperature [air temperature, soil temperature], topography [elevation, slope, aspect, ruggedness, topography position index], and wind [speed, direction]) in delineating the likely fire from Earth Observation data. This conceptual framework is not presented in this paper.

\section{RESULTS AND DISCUSSIONS}

3.1 Visualisation of ground truth and MODIS product overlay for study area 1 and 2

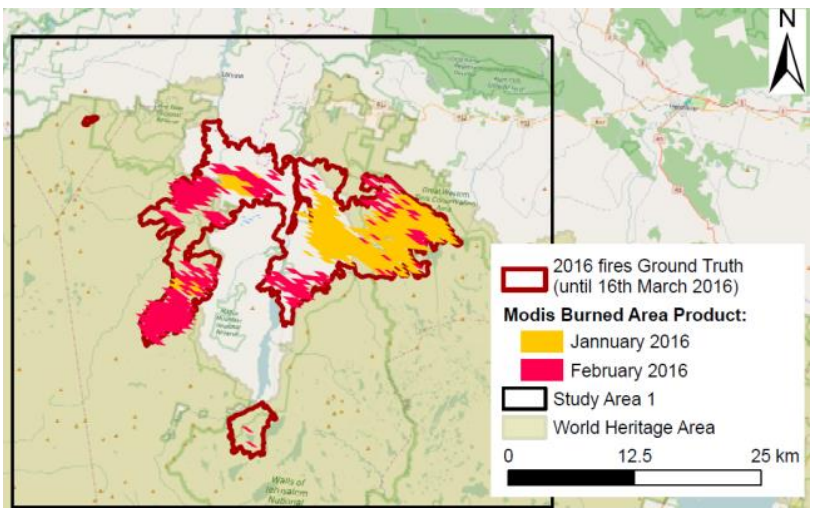

Figure 4: Visualisation of an overlay of ground truth data and MODIS product for study area 1

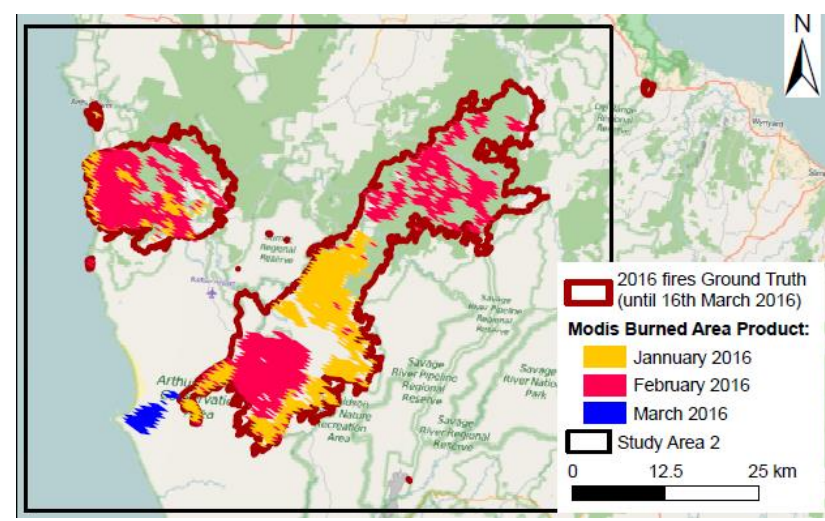

Figure 5: Visualisation of an overlay of ground truth data and MODIS product after fire event for study area 2

The above visualisations (Figure 4 and 5) show alignment of fire ground truth data in many places with MODIS product for both the study area. However, uncertainty is further visualised 
with the aid of colour composite, difference maps and error maps.

3.2 Visualisation of colour composite, NDVI difference map, and error maps for study area 1 and 2
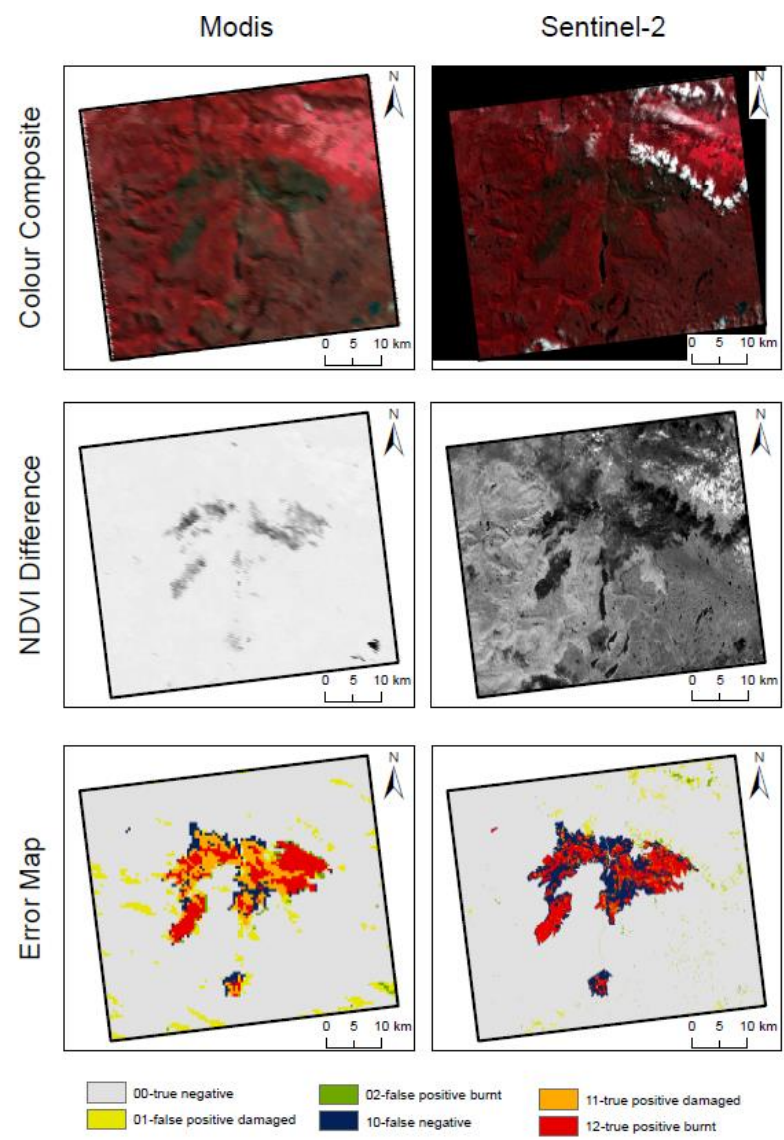

Figure 6. Colour composite, NDVI difference map and error map for study area 1

For both study areas 1 and 2 uncertainty of delineation is visualised (Figures 6 and 7). Statistical measures for associated uncertainties are computed using the "confusionMatrix()" function from the caret package (Classification And REgression Training) in $\mathrm{R}$, an example for burnt class is provided in Table 1. This shows that there is minimal difference between the sensors in extracting these bushfires extent when considering only the "burnt" class, but an increase in precision for MODIS when adding the intermediary class "damaged" with more true positives (Table 2). The difference between the classifications according to the sensor are statistically significant based on the McNemar's test ( $\mathrm{p}$-value $<2.2 \mathrm{e}-16$ ) even the accuracies are close.

Table 1: Statistical measures for MODIS and Sentinel-2 in extracting burnt area based only on burnt class.

\begin{tabular}{|l|l|l|l|l|}
\hline & Accuracy & Sensitivity & Specificity & Kappa \\
\hline MODIS & 0.92 & 0.56 & 0.99 & 0.65 \\
\hline Sentinel-2 & 0.93 & 0.56 & 0.99 & 0.67 \\
\hline
\end{tabular}

Table 2: Statistical measures for MODIS and Sentinel-2 in extracting burnt area using burnt and damaged classes.

\begin{tabular}{|l|l|l|l|l|}
\hline & Accuracy & Sensitivity & Specificity & Kappa \\
\hline MODIS & 0.94 & 0.89 & 0.94 & 0.78 \\
\hline Sentinel-2 & 0.94 & 0.64 & 0.98 & 0.72 \\
\hline
\end{tabular}

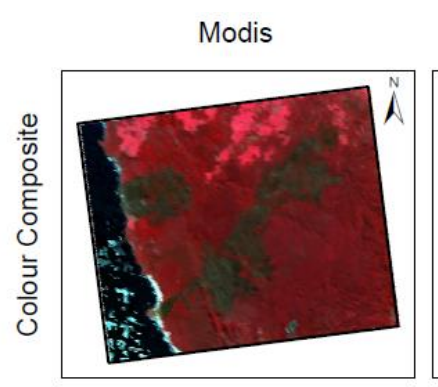

Sentinel-2
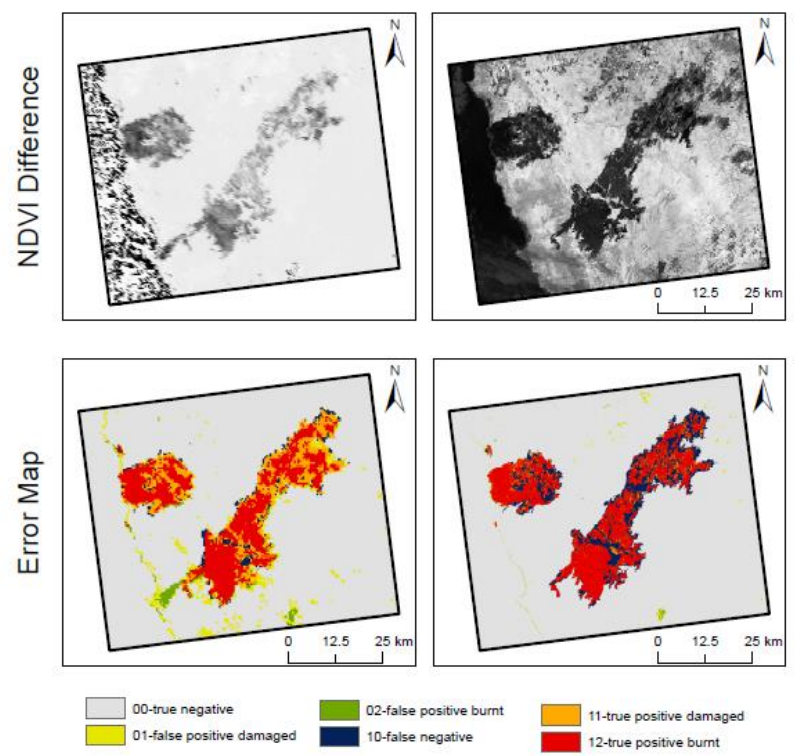

Figure 7. Colour composite, NDVI difference map and error map for study area 2

\subsection{Discussions}

The largest error is false negative. When looking at the colour composites, it seems that those false negatives are indeed unburnt vegetation (red in false colour) surrounded by burnt area. This could be due to the ground truth data having a convex shape and considering unburnt vegetation within that shape as burnt. On the other hand, false positive are mostly isolated pixel. Therefore this could be improved with more mathematical morphology (closing).

In terms of real-world features, clouds, shadows, and lakes, were included in the mask layers based on the NDVI differences of Landsat 8 and Sentinel-2. This was later corrected by using k-means clustering algorithm. The "damaged" class improves the true positives more for MODIS than for Sentinel-2, and add most of the false negatives for both: a sensitivity of 0.89 vs 0.55 and positive predictive value of 0.74 vs 0.9 with MODIS, while in the case of Sentinel-2 the sensitivity of 0.64 vs 0.56 and positive predictive value of 0.90 vs 0.97 .

The overall accuracy doesn't really change neither according to the sensor nor using both damaged and burnt classes. For example, in the case of MODIS 0.94 vs 0.92 overall accuracies; 0.78 vs 0.65 Kappa values while in the case of Sentinel-2 it is 0.94 vs 0.93 overall accuracies and 0.72 vs 0.67 Kappa values (Table 1 and 2). 


\section{CONCLUSIONS AND FUTURE RESEARCH}

This study shows that there is little difference in overall accuracies from both EO data MODIS and Sentinel-2 in mapping the fire event in single analysis scale. The proposed framework for multiple-scale will be implemented and compared in the future extension of this work.

\section{ACKNOWLEDGEMENTS}

We would like to thank the University of Tasmania for a grant to conduct this research project. This study is a part of the project funded to Jagannath Aryal. A travel support from the University of Tasmania and the School of Land \& Food are highly acknowledged. The authors wish to thank Department of Primary Industries, Parks, Water and Environment (DPIPWE) of the Tasmanian Government for providing the ground truth data.

\section{REFERENCES}

Aryal, J., Josselin, D. (2014). Environmental object recognition in a natural image: An experimental approach using geographic object-based image analysis (GEOBIA). International Journal of Agricultural and Environmental Information Systems, 5 (1), pp. 1-18. DOI: 10.4018/ijaeis.2014010101

Australian Bureau of Statistics [ABS] (2016). http://www.abs.gov.au/ausstats/abs@.nsf/0/ccb3f2e90ba779d3c a256dea00053977

Blaschke, T., Hay, G.J., Kelly, M., Lang, S., Hofmann, P., Addink, E., Queiroz Feitosa, R., van der Meer, F., van der Werff, H., van Coillie, F. and Tiede, D. (2014). Geographic Object-Based Image Analysis - Towards a new paradigm, ISPRS Journal of Photogrammetry and Remote Sensing, 87, pp180-191, doi $=\{10.1016 / j$.isprsjprs.2013.09.014

Chuvieco, E., Martín, M. P, Palacios, A. (2002). Assessment of different spectral indices in the red-near-infrared spectral domain for burned land discrimination. International Journal of Remote Sensing 23(23), 5103-5110.

Dutta, R, Das, A, and Aryal, J. (2016). Big data integration shows Australian bush-fire frequency is increasing significantly, 3, 2, The Royal Society Open Science,

http://rsos.royalsocietypublishing.org/content/3/2/150241, DOI: $10.1098 /$ rsos. 150241

The List, Tasmanian Government (2016) https://data.thelist.tas.gov.au/datagn/srv/eng/main.home?uuid=b 94d4388-995d-416a-9844-a39de2798bed

Radionz (2016). Tasmanian bushfires: the ecological impact. Interview of Professor David Bowman of the University of Tasmania http://www.radionz.co.nz/audio/player/201787346

van Leeuwen, W., Casady, G., Neary, D., Bautista, S., Alloza, J., Carmel, J., Wittenberg, L., Malkinson, D., Orr, B. (2010). Monitoring post-wildfire vegetation response with remotely sensed time series data in Spain, USA and Israel. International Journal of Wildland Fire 19 (1), 75-93.
Veraverbeke, S., Harris, S., Hook, S. (2011). Evaluating spectral indices for burned area discrimination using MODIS/ASTER (MASTER) airborne simulator data. Remote Sensing of Environment. 115(10), 2702-2709. 


\title{
IDENTIFYING SUITABLE SEGMENTATION PARAMETERS FOR AN OBJECT-BASED IMAGE CLASSIFICATION
}

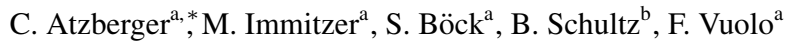 \\ ${ }^{\text {a }}$ Institute of Surveying, Remote Sensing and Land Information (IVFL), University of Natural Resources and Life Sciences, \\ Vienna (BOKU), Peter-Jordan-Straße 82, A-1190 Wien - \\ (clement.atzberger, markus.immitzer, sebastian.boeck, francesco.vuolo)@boku.ac.at \\ ${ }^{\mathrm{b}}$ Instituto Nacional de Pesquisas Espaciais (INPE), Divisão de Sensoriamento Remoto (DSR) - schultz@dsr.inpe.br
}

KEY WORDS: Unsupervised Segmentation Evaluation, Parameter Optimization, Global Score

\begin{abstract}
:
Only well-chosen segmentation parameters ensure optimum results of object-based image classifications. Manually defining suitable parameter sets can be a time-consuming approach, not necessarily leading to optimum results. Moreover, the manual approach is obviously subjective. An automated evaluation approach is needed to reduce human intervention and to provide objective criteria for ranking different parameter sets.

In this work, we test three different ways to find the optimum segmentation.

(i) We used a supervised approach integrating the segmentation and classification tasks. The segmentation is optimized directly with respect to the overall accuracy of the subsequent classification.

(ii) Using the global score value considering within- and between segment heterogeneity, we run an unsupervised segmentation optimization to find the best segmentation parameters.

(iii) Using manually delineated objects we calculate discrepancy measurements like euclidean distance between automatic generated objects and the manual delineated objects in a supervised segmentation evaluation.

For all approaches, we present fully autonomous workflows for supervised and unsupervised object-based classification, combining image segmentation, segmentation evaluation and image classification. Starting from a fixed set of randomly selected and manually delineated training samples, suitable segmentation parameters are automatically identified. Finally, we compare the results of the three different approaches.
\end{abstract}

\footnotetext{
${ }^{*}$ Corresponding author
} 


\title{
ON THE USABILITY OF DEEP NETWORKS FOR OBJECT-BASED IMAGE ANALYSIS
}

\author{
Nicolas Audebert ${ }^{\mathrm{a}, \mathrm{b}}$, Bertrand Le Saux $^{\mathrm{a}}$, Sébastien Lefèvre ${ }^{\mathrm{b}}$ \\ ${ }^{a}$ ONERA, The French Aerospace Lab, F-91761 Palaiseau, France (nicolas.audebert,bertrand.le_saux)@onera.fr \\ ${ }^{\mathrm{b}}$ Univ. Bretagne-Sud, UMR 6074, IRISA, F-56000 Vannes, France - sebastien.lefevre@ irisa.fr
}

KEY WORDS: deep learning, vehicle detection, semantic segmentation, object classification

\begin{abstract}
:
As computer vision before, remote sensing has been radically changed by the introduction of Convolution Neural Networks. Land cover use, object detection and scene understanding in aerial images rely more and more on deep learning to achieve new state-of-theart results. Recent architectures such as Fully Convolutional Networks (Long et al., 2015) can even produce pixel level annotations for semantic mapping. In this work, we show how to use such deep networks to detect, segment and classify different varieties of wheeled vehicles in aerial images from the ISPRS Potsdam dataset. This allows us to tackle object detection and classification on a complex dataset made up of visually similar classes, and to demonstrate the relevance of such a subclass modeling approach. Especially, we want to show that deep learning is also suitable for object-oriented analysis of Earth Observation data. First, we train a FCN variant on the ISPRS Potsdam dataset and show how the learnt semantic maps can be used to extract precise segmentation of vehicles, which allow us studying the repartition of vehicles in the city. Second, we train a CNN to perform vehicle classification on the VEDAI (Razakarivony and Jurie, 2016) dataset, and transfer its knowledge to classify candidate segmented vehicles on the Potsdam dataset.
\end{abstract}

\section{INTRODUCTION}

Deep learning for computer vision grows more popular every year, especially thanks to Convolutional Neural Networks (CNN) that are able to learn powerful and expressive descriptors from images for a large range of tasks: classification, segmentation, detection ... This ubiquity of $\mathrm{CNN}$ in computer vision is now starting to affect remote sensing as well, as they can tackle many tasks such as land use classification or object detection in aerial images. Moreover, new architectures have appeared, derived from Fully Convolutional Networks (Long et al., 2015), able to output dense pixel-wise annotations and thus able to achieve fine-grained classification. Such architectures have quickly become state-of-theart for popular datasets such as PASCAL VOC2012 (Everingham et al., 2014) and Microsoft COCO (Lin et al., 2014). In an Earth Observation context, these FCN models are now especially appealing, as dense prediction allows us performing semantic mapping without requiring any pre-processing tricks. Therefore, using FCN for Earth Observation means we can shift from superpixel segmentation and region-based classification (Lagrange et al., 2015 Audebert et al., 2016. Nogueira et al., 2016) to fully supervised semantic segmentation (Marmanis et al., 2016).

FCN models have been successfully applied for remote sensing data analysis, notably land cover mapping on urban areas (Marmanis et al., 2016. Paisitkriangkrai et al., 2015). For example, FCN-based models are now the state-of-the-art on the ISPRS Vaihingen Semantic Labeling dataset (Rottensteiner et al., 2012. Cramer, 2010). Therefore, even though remote sensing images do not share the same structure as natural images, traditional computer vision deep networks are able to successfully extract semantics from them, which was already known for deep CNN-based classifiers (Penatti et al., 2015). This encourages us to investigate further: can we use deep networks to tackle an especially hard remote sensing task, namely object segmentation ? Therefore, this work focuses on using deep convolutional models for segmentation and classification of vehicles using optical remote sensing data.

To tackle this problem, we design a two-step pipeline for segmentation and classification of vehicles in aerial images. First, we use the SegNet architecture (Badrinarayanan et al., 2015) for semantic segmentation on the ISPRS Potsdam dataset. This allows us generating a pixel-level mask on which we can extract connected components to detect the vehicle instances. Then, using a CNN trained on vehicle classification using the VEDAI dataset (Razakarivony and Jurie, 2016), we classify each instance to infer the vehicle type and to eliminate false positives. We then show how to exploit this information to provide new pieces of data about vehicle types and vehicle repartition in the scene.

Our work is closesly related to (Marmanis et al., 2016, Paisitkriangkrai et al., 2015) who also use Fully Convolutional Network for urban area mapping. However we focus only on small objects, namely vehicles, although we use the full semantic mapping as an intermediate product in our pipeline. Moreover, regarding (Paisitkriangkrai et al., 2015), our work rely solely on the deeply learnt representation of the optical data, as we do not include any expert features in the process. On the vehicle detection task, (Chen et al., 2014) tried to detect vehicle in satellite images using deep CNN, but only regressed the bounding boxes. On the contrary, our pipeline is able to infer the precise object segmentation, which could be regressed into a bounding box if needed. In addition, we also classify the vehicles into several subcategories using a classifier trained on a more generic dataset, thus performing transfer learning.

\section{PROPOSED METHOD}

\subsection{Semantic segmentation}

Many deep network architectures are available for semantic segmentation, including the original FCN (Long et al., 2015) or many variants such as DeepLab (Liang-Chieh et al., 2015). We choose to use the SegNet architecture (Badrinarayanan et al., 2015), since it is well-balanced between accuracy and computational cost. SegNet's symmetrical structure and its use of pooling/unpooling layers is very effective for precise relocalisation of features according to its authors. Indeed, preliminary tests underlined that SegNet performs very well, including for small object localization. Results with other architectures such as FCN reported either no 


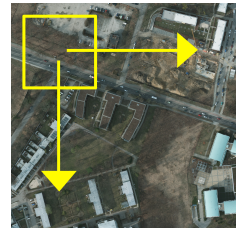

Sliding window on RGB data

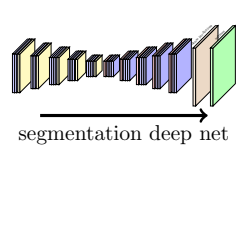

\footnotetext{
Semantic map
}

Figure 1. Illustration of our vehicle segmentation + classification pipeline

improvement or an unsignificant improvement. However, note that any deep network trained for semantic segmentation can be used in our pipeline.

SegNet is based on the convolutional layers of the VGG-16 model (Simonyan and Zisserman, 2014). VGG-16 was designed for the ILSRVC competition and trained on the ImageNet dataset (Russakovsky et al., 2015). Each block of the encoder is comprised of 2 or 3 convolutional layers followed by batch normalization (Ioffe and Szegedy, 2015) and rectified linear units. A maxpooling layer reduces the dimensions between each block. In the decoder, symetrical operations are applied in the reverse order. The unpooling operation replaces the pooling in the decoder: it relocates the value of the activations into the mask of the maximum values ("argmax") computed at the pooling stage. Such an upsampling results in a sparse activation map that is densified by the consecutive decoding convolutions. This allows us upsampling the feature activations up to the original input size, so that after the decoder, the final output feature maps have the same dimensions as the input, which is necessary for pixel-level inference.

We initialize the weights of the encoder using VGG-16's weights trained on ImageNet. By doing so, we leverage the expressiveness of the filters learned by VGG-16 when training on this very diverse dataset. This initialization allows us improving the final segmentation accuracy compared to a random initialization and also makes the model converge faster.

We train SegNet on semantic segmentation on the ISPRS Potsdam dataset. This translates to pixel-wise classification, i.e each pixel is classified as belonging to one class from the ISPRS benchmark: "impervious surface", "building", "tree", "low vegetation", "car" or "clutter". From this semantic map, we can then extract the vehicle mask which labels all the pixels inferred to belong to the "car" class.

\subsection{CNN classification}

After generating the full semantic map from one tile, we can extract the vehicle mask. We perform connected components extraction to find all candidate vehicles in the predicted map. To separate vehicles that are very close to each other in the RGB image and that might belong to the same connected component, we use a morphological opening to open the gap and separate the objects. In addition, to smooth the predictions and eliminate small artifacts and imperfections from SegNet's predicted map, we removed connected components that are too small to be a vehicle (surface $<32 \mathrm{px}$ ). For each vehicle (any remaining connected component), we extract a rectangular patch around its bounding box, including $16 \mathrm{px}$ of spatial context on all sides. This patch is then fed to a CNN classifier trained to infer the vehicle's precise class. The full pipeline is illustrated in Figure 1

We compare $3 \mathrm{CNN}$ for vehicle classification: LeNet (Lecun et al., 1998), AlexNet (Krizhevsky et al., 2012) and VGG-16 (Simonyan and Zisserman, 2014). We use the VEDAI dataset to train these models, so that the Potsdam vehicles will be entirely unseen data. This is to show how knowledge learnt by deep networks on remote sensing data can be transferred efficiently from one dataset to another, under the assumption that provided resolutions $(12.5 \mathrm{~cm})$ and data sources (RGB) are the same. The classifiers learn to discriminate between the following 11 classes of vehicles from VEDAI, with a very high variability: "car", "camping car", "tractor", "truck”, "bike”, "van”, "bus", "ship", "plane”, "pick up" and "other vehicles".

LeNet is a model introduced by (Lecun et al., 1998) designed for character recognition in greyscale image. Our version takes in input a $224 \times 224$ color image. It is a comparatively small model with few parameters $(\simeq 461 \mathrm{~K}$ parameters $)$ that can be trained very quickly.

AlexNet is a very popular model introduced in (Krizhevsky et al., 2012) that won the ILSVRC challenge in 2012. It takes in input a $227 \times 227$ color image. As there are three final fully connected layers, AlexNet is a relatively big network with $61 \mathrm{M}$ parameters. We fine-tune the last layer of the reference ImageNet trained implementation of AlexNet.

Finally, VGG-16 is another popular model designed by (Simonyar and Zisserman, 2014), on which is based the SegNet segmentation network introduced in Section 2.1 It outperformed AlexNet on the ImageNet benchmark in 2014. It takes in input a $224 \times 224$ color image. VGG-16 is the biggest of our three models with $138 \mathrm{M}$ parameters. Once again, we fine-tune only the last layer of the reference weights of VGG-16 trained on ImageNet. It is the biggest and slowest of the three models tested in this work.

\section{EXPERIMENTS}

\subsection{Experimental setup}

3.1.1 VEDAI This VEDAI dataset Razakarivony and Jurie, 2016 ) is comprised of 1268 RGB tiles (1024×1024px) and the associated IR image at $12.5 \mathrm{~cm}$ spatial resolution. For each tile, annotations are provided detailing the position of the vehicles and the associated bounding box (defined by its 4 corners). We crossvalidated the results on three of the suggested splits of the dataset for the training and testing sets. The training set for the CNN is built by extracting square patches around each vehicle bounding box, padded with 16 px of spatial context on each side. We use data augmentation to increase the number of samples by including rotated $\left(\frac{\pi}{2}, \pi\right.$ and $\left.\frac{3 \pi}{2}\right)$ and mirrored versions of the patches containing vehicles.

We train each model during 50 epochs $(\simeq 1000000$ iterations) with a batch size of respectively 128 for LeNet and AlexNet and 32 for VGG-16, and a learning rate of 0.001 , divided by 10 after 30 epochs. Training takes about 25 minutes for LeNet, 60 minutes for AlexNet and 10 hours for VGG-16 on NVIDIA K20c GPU. 
Table 1. Average CNN accuracies on VEDAI (3 train/test splits)

\begin{tabular}{ccccccccccc}
\hline Model/Class & Car & Truck & Ship & Tractor & Camping car & Van & Pick up & Plane & Other & Global \\
\hline LeNet & $74.3 \%$ & $54.4 \%$ & $31.0 \%$ & $61.1 \%$ & $85.9 \%$ & $38.3 \%$ & $67.7 \%$ & $13.0 \%$ & $47.5 \%$ & $66.3 \pm 1.7 \%$ \\
AlexNet & $\mathbf{9 1 . 0 \%}$ & $84.8 \%$ & $81.4 \%$ & $83.3 \%$ & $98.0 \%$ & $\mathbf{7 1 . 1 \%}$ & $85.2 \%$ & $91.4 \%$ & $\mathbf{7 7 . 8 \%}$ & $87.5 \pm 1.5 \%$ \\
VGG-16 & $90.2 \%$ & $\mathbf{8 6 . 9 \%}$ & $\mathbf{8 6 . 9 \%}$ & $\mathbf{8 6 . 5 \%}$ & $\mathbf{9 9 . 6 \%}$ & $\mathbf{7 1 . 1 \%}$ & $\mathbf{9 1 . 4 \%}$ & $\mathbf{1 0 0 . 0 \%}$ & $\mathbf{7 7 . 2 \%}$ & $\mathbf{8 9 . 7} \pm 1.5 \%$ \\
\hline
\end{tabular}
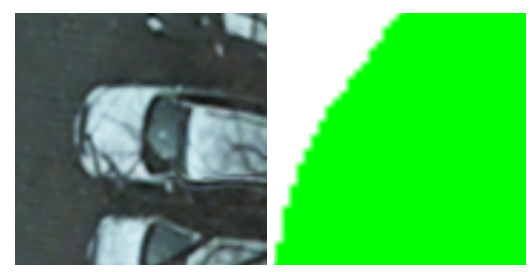

(a) RGB data (b) Ground truth

Figure 2. Car partially occluded by a tree on the Potsdam dataset and misclassification according to the ground truth

3.1.2 ISPRS Potsdam This ISPRS Potsdam Semantic Labeling dataset (Rottensteiner et al., 2012) is comprised of 38 RGB tiles $(6000 \times 6000 \mathrm{px})$ and the associated IR and DSM images at $5 \mathrm{~cm}$ spatial resolution. A comprehensive ground truth is provided for 24 tiles, which are the tiles we will work with. We split randomly the dataset in train/validation/test with 70/10/20 proportions. For a fair comparison of the two datasets (ISPRS Potsdam and VEDAI) in the same framework, we only use the RGB data and downsample the resolution from $5 \mathrm{~cm} /$ pixel to $12.5 \mathrm{~cm} / \mathrm{pixel}$. However, note that SegNet would perform even better for small object segmentation on the high resolution tile.

We build our segmentation training set by sliding a window of $128 \times 128 \mathrm{px}$ over each high resolution tile, including an overlap of $75 \%$ (32 px stride). For this experiment, we use all the classes from the ground truth. This means that we not only train the model to predict the vehicle mask, but also to assign a label to each pixel according to the ISPRS classes, except "clutter": "impervious surface", "building", "tree", "low vegetation" and "car". The model is trained by Stochastic Gradient Descent for 10 epochs ( $\simeq 100000$ iterations) with a batch size of 10 and a learning rate of 0.1 , divided by 10 after 3 and 8 epochs. This takes around 24 hours with a NVIDIA K20c GPU.

At testing time, we process the tiles by sliding a window of $128 \times$ $128 \mathrm{px}$ with an overlap of 50\%. For overlapping pixels, we average the multiple predictions on each pixel. Processing one tile takes around 70 seconds on a NVIDIA K20c GPU. The predicted semantic map contains all classes from the ISPRS benchmark. We extract only the mask for the "car" class as we are mainly interested in how SegNet behaves for small objects, especially vehicles. We report results before and after removing objects smaller than a predefined threshold.

To compute vehicle classification metrics, we build manually an enhanced ground truth by subdividing the "car" class into several subcategories: "cars", "vans", "trucks", and "pick ups". We discard other vehicles that are present in the RGB data (especially construction vehicles) but not in the original ground truth.

\subsection{Results}

3.2.1 VEDAI As detailed in Table 1 both VGG-16 and AlexNet are able to discriminate between the different classes of the VEDAI dataset, with respectively $89.7 \%$ and $87.5 \%$ global accuracy. LeNet is lagging behind and performs significantly worse than its deeper counterpart, with an accuracy of only $66.3 \%$. This result is not

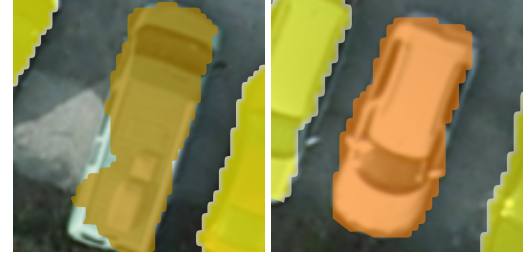

(a) Van misclassi- (b) Car misclassified as truck

fied as van

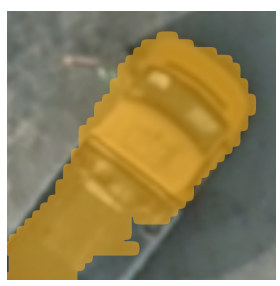

(c) Pick up misclassified as van
Figure 3. Successful segmentation but misclassified vehicles in the Potsdam dataset

surprising, as LeNet is a very small network in comparison to both AlexNet and VGG-16 and therefore has a dramatically lower expressive power. The LeNet model performs adequately on "easy" classes, such as cars and camping cars, that have low intra-class variability and high inter-class variability. However, it does not work well on vans, which are difficult to distinguish from cars from bird's view. Moreover, classes with high intra-class variability (especially trucks and planes) are often misclassified by LeNet.

Indeed, we argue that as VGG-16 and AlexNet were pre-trained on ImageNet and fine-tuned on VEDAI, these models were already initialized with very powerful and expressive visual descriptors learnt on a highly diverse image dataset. Therefore, both networks benefited from a pre-training phase on a comprehensive dataset of natural images requiring complex filters able to analyze shapes, textures and colors, which helped training on VEDAI, even though ImageNet does not contain any remote sensing image. This fine-grained recognition based on textures and shape allow the CNN to discrminate between classes with a low inter-class variability such as cars and vans, and perform well on classes with high intra-class variability such as planes or trucks. This supports evidence that transfer learning and fine-tuning of ImageNet-trained deep convolutional neural networks is an effective way to process aerial images as shown in previous works (Penatti et al., 2015).

\subsubsection{ISPRS Potsdam}

Vehicle segmentation The overall accuracy of the SegNet semantic mapping on the downsampled ISPRS dataset is $89.5 \%$, excluding clutter and eroding borders by $3 \mathrm{px}$. Compared to the state-of-the-art result of $90.3 \%$ This result is quite good considering that we use a downscaled version of the dat $2^{2}$ When considering only the "car" class (which comprises all kinds of terrestrial motorized vehicles), the F1 score at pixel level is $88.4 \%$ (95.5\% with the eroded borders). We also report a pixel-wise precision of $87.8 \%$ and a recall of $89.0 \%$. This indicates that the vehicle mask generated from SegNet's semantic map should be very accurate and can be used for further object-based analysis. html

1 http://www2 isprs .org/potsdam-2d-semantic-labeling.

${ }^{2}$ However, we only report our result on a validation set and not on the full testing set, as full semantic mapping of the ISPRS Potsdam dataset is out of the scope of this work. 


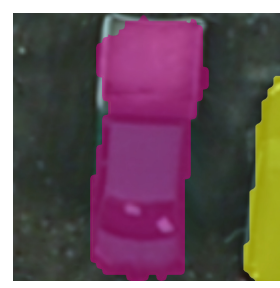

(a) Pick up

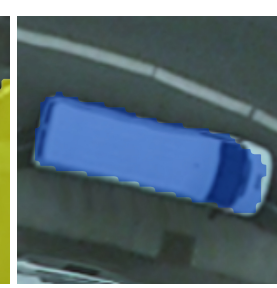

(b) Van

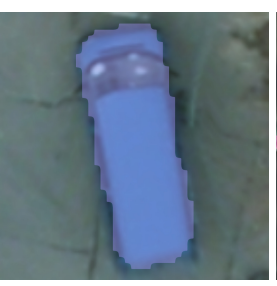

(c) Van

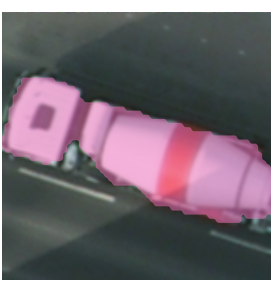

(d) Truck

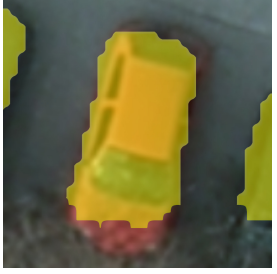

(e) Car

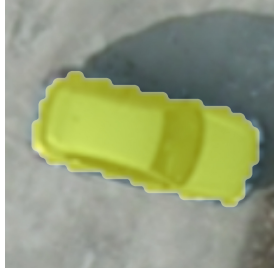

(f) Car

Figure 4. Successful segmentation and classification of vehicles in the Potsdam dataset

Table 2. Vehicle counts in testing tiles of ISPRS Potsdam

\begin{tabular}{cccccc}
\hline Tile \# & 2_11 & 7_12 & 3_11 & 5_12 & 7_10 \\
\hline Cars in ground truth & 110 & 351 & 168 & 428 & 253 \\
Predicted cars & 115 & 342 & 182 & 435 & 257 \\
\hline
\end{tabular}

This is supported by qualitative inspection of the semantic maps on car-heavy areas, such as the one presented in Figure 5

The mean Intersection over Union (IoU) reaches 75.6\%. However, there is a high variance in the IoU depending on the specific surroundings of each car. Indeed, because of occlusions due to trees, the ground truth sometimes is labelled as tree even if the car is visible in the RGB image. This causes SegNet to misclassify (according to the ground truth) most of the pixels, resulting in a low IoU despite the visual quality of the segmentation. This phenomenon is illustrated by Figure 2 However, as SegNet manages to still infer the car's contours, this should not impede too severely later vehicle extraction and classification.

Counting cars We extract the vehicle mask from the semantic map of Potsdam generated by SegNet. Each connected component is expected to be a vehicle instance. We count the number of predicted instances in each tile of the testing set. Detailed results are reported in Table 2 and show that our model can be used to estimate precisely the number of vehicles in the original tile with an average relative error of less than $4 \%$.

We also show that we can use this information to generate heat maps of the vehicle repartition in the tile (cf. Figure 6. Such a visualization provides an indicator of the vehicle density in the urban area, and exacerbates high traffic roads and "hot spots" corresponding to parking lots around points of interest (e.g. hospitals or shopping malls). This can be useful for many urban planning applications, such as parking lot sizing, traffic estimation, etc. In temporal data, this could be used to monitor traffic flows, but also study the impact of the road blocks, jams, etc.

Classification Using our AlexNet-based CNN classifier, we are able to classify the candidate vehicle instances generated by SegNet on the Potsdam dataset. We compare the predicted results to our enhanced vehicle ground truth. On our testing set, we classify correctly $75.0 \%$ of the instances. We are able to improve even further this result by switching to the VGG-16 based classifier which brings accuracy to $77.3 \%$ (cf. Table 3 for detailed results). It should be noted that the dataset is predominantly comprised of cars, and that trucks and pick ups are less represented, which decreases their influence on the global accuracy score. Figure 4 illustrates some vehicle instances where our deep network-based segmentation and classification pipeline was successful, while Figure 3 shows some examples of correct segmentation but subsequent misclassification.

The fact that the average accuracy of the models on Potsdam are lower than results reported on VEDAI suggests that our networks
Table 3. Classification results on the enhanced Potsdam vehicle ground truth

\begin{tabular}{cccccc}
\hline Class & Car & Van & Truck & Pick up & Global \\
\hline AlexNet & $81 \%$ & $42 \%$ & $\mathbf{5 0 \%}$ & $\mathbf{5 0 \%}$ & $75 \%$ \\
VGG & $\mathbf{8 3 \%}$ & $\mathbf{5 5 \%}$ & $\mathbf{5 0 \%}$ & $33 \%$ & $\mathbf{7 7 \%}$ \\
\hline
\end{tabular}

suffered from overfitting. One hypothesis that we make is that VEDAI and Potsdam are images taken from two similar but subtly different environments. Indeed, Potsdam is a urban european city whereas VEDAI images have been shot over Utah, on a more rural american environment. Therefore, vehicle brands and different land covers around the cars might influence the classifiers. More comprehensive regularization (e.g. dropout (Srivastava et al., 2014) during fine-tuning and/or training on a more diverse dataset would help alleviate this phenomenon.

\section{CONCLUSION}

In this work, we presented a two-step framework to detect, segment and classify vehicles from aerial RGB images using deep learning. More precisely, we showed that deep network designed for semantic segmentation such as SegNet are useful for scene understanding of remote sensing data and can be used to segment even small objects, such as cars and trucks. We reported results on the ISPRS Potsdam dataset showing very promising results on wheeled vehicles, achieving a F1 score of $77.3 \%$ on this specific class.

In addition, we presented a simple deep learning based method to improve further this analysis by classifying the different types of vehicle present in the scene. We trained several deep $\mathrm{CNN}$ on the VEDAI dataset and transferred their knowledge to the Potsdam dataset. Our best model, fine-tuned from VGG-16, was able to classify successfully more than $77 \%$ of the vehicles in our testing set.

Finally, this work meant to provide useful pointers for applying deep learning to scene understanding in Earth Observation with an object-oriented approach. We showed that not only deep networks are the state-of-the-art for semantic mapping, but that they can also be used to extract useful information at an object level, with a direct application on vehicle detection and classification.

We showed that it is possible to enumerate and extract individual object instances from the semantic map in order to analyze the vehicle distribution in the images, leading to the localization of points of interest such as high traffic roads and parking lots. This has many applications in traffic monitoring and urban planning, such as analyzing parking lots occupancy, finding pollutants vehicles in unauthorized zones (combined with a classifier), etc. 


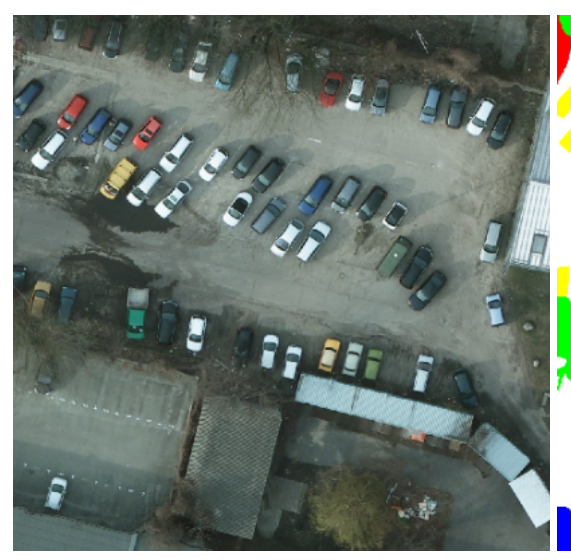

(a) RGB data

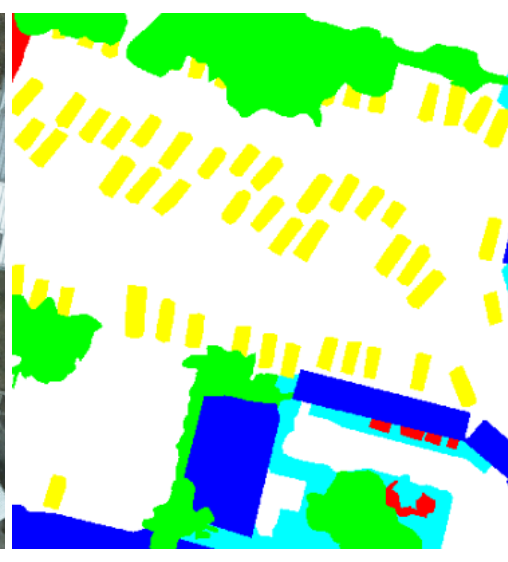

(b) Ground truth

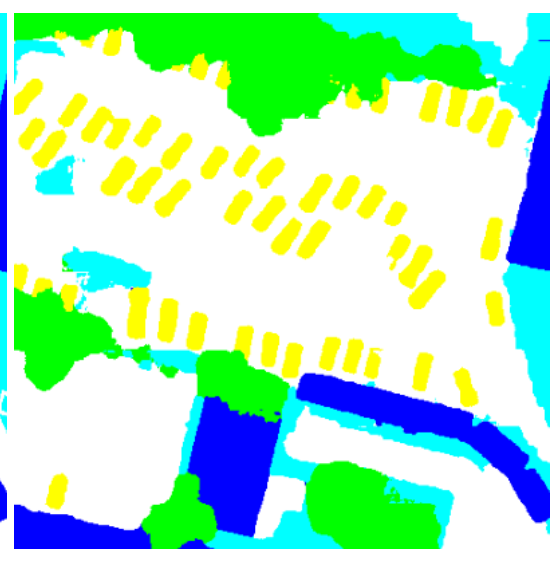

(c) SegNet prediction

Figure 5. SegNet results on a parking lot (extracted from the ISPRS dataset, best viewed in color)

\section{ACKNOWLEDGEMENTS}

The Potsdam data set was provided by the German Society for Photogrammetry, Remote Sensing and Geoinformation (DGPF): http://www.ifp.uni-stuttgart.de/dgpf/DKEP-Allg.html The Vehicle Detection in Aerial Imagery (VEDAI) dataset was provided by S. Razakarivony and F. Jurie.

$\mathrm{N}$. Audebert' work is funded by ONERA-TOTAL research project Naomi.

The authors would like to thank A. Boulch and A. Chan Hon Tong for fruitful discussions on object detection and classification.

\section{REFERENCES}

Audebert, N., Le Saux, B. and Lefèvre, S., 2016. How Useful is Region-based Classification of Remote Sensing Images in a Deep Learning Framework? In: IEEE International Geosciences and Remote Sensing Symposium (IGARSS), Beijing, China.

Badrinarayanan, V., Kendall, A. and Cipolla, R., 2015. SegNet: A Deep Convolutional Encoder-Decoder Architecture for Image Segmentation. arXiv preprint arXiv:1511.00561.

Chen, X., Xiang, S., Liu, C. L. and Pan, C. H., 2014. Vehicle Detection in Satellite Images by Hybrid Deep Convolutional Neural Networks. IEEE Geoscience and Remote Sensing Letters 11(10), pp. 1797-1801.

Cramer, M., 2010. The DGPF test on digital aerial camera evaluation - overview and test design. Photogrammetrie - Fernerkundung-Geoinformation 2, pp. 73-82.

Everingham, M., Eslami, S. M. A., Gool, L. V., Williams, C. K. I., Winn, J. and Zisserman, A., 2014. The Pascal Visual Object Classes Challenge: A Retrospective. International Journal of Computer Vision 111(1), pp. 98-136.

Ioffe, S. and Szegedy, C., 2015. Batch Normalization: Accelerating Deep Network Training by Reducing Internal Covariate Shift. In: Proceedings of The 32nd International Conference on Machine Learning, pp. 448-456.

Krizhevsky, A., Sutskever, I. and Hinton, G. E., 2012. ImageNet Classification with Deep Convolutional Neural Networks. In: F. Pereira, C. J. C. Burges, L. Bottou and K. Q. Weinberger (eds), Advances in Neural Information Processing Systems 25, Curran Associates, Inc., pp. 1097-1105.

Lagrange, A., Le Saux, B., Beaupere, A., Boulch, A., ChanHon-Tong, A., Herbin, S., Randrianarivo, H. and Ferecatu, M.,
2015. Benchmarking classification of earth-observation data: From learning explicit features to convolutional networks. In: Geoscience and Remote Sensing Symposium (IGARSS), 2015 IEEE International, pp. 4173-4176.

Lecun, Y., Bottou, L., Bengio, Y. and Haffner, P., 1998. Gradientbased learning applied to document recognition. Proceedings of the IEEE 86(11), pp. 2278-2324.

Liang-Chieh, C., Papandreou, G., Kokkinos, I., Murphy, K. and Yuille, A., 2015. Semantic Image Segmentation with Deep Convolutional Nets and Fully Connected CRFs. In: Proceedings of the International Conference on Learning Representations.

Lin, T.-Y., Maire, M., Belongie, S., Hays, J., Perona, P., Ramanan, D., Dollár, P. and Zitnick, C. L., 2014. Microsoft COCO: Common Objects in Context. In: D. Fleet, T. Pajdla, B. Schiele and T. Tuytelaars (eds), Computer Vision - ECCV 2014, Lecture Notes in Computer Science, Springer International Publishing, pp. 740-755. DOI: 10.1007/978-3-319-10602-1_48.

Long, J., Shelhamer, E. and Darrell, T., 2015. Fully Convolutional Networks for Semantic Segmentation. In: Proceedings of the IEEE Conference on Computer Vision and Pattern Recognition, pp. 3431-3440.

Marmanis, D., Wegner, J. D., Galliani, S., Schindler, K., Datcu, M. and Stilla, U., 2016. Semantic Segmentation of Aerial Images with an Ensemble of CNNs. ISPRS Annals of Photogrammetry, Remote Sensing and Spatial Information Sciences 3, pp. 473480 .

Nogueira, K., Penatti, O. A. B. and Dos Santos, J. A., 2016. Towards Better Exploiting Convolutional Neural Networks for Remote Sensing Scene Classification. arXiv:1602.01517 [cs]. arXiv: 1602.01517 .

Paisitkriangkrai, S., Sherrah, J., Janney, P. and Hengel, A. V.D., 2015. Effective semantic pixel labelling with convolutional networks and Conditional Random Fields. In: Proceedings of the IEEE Conference on Computer Vision and Pattern Recognition Workshops, pp. 36-43.

Penatti, O., Nogueira, K. and Dos Santos, J., 2015. Do deep features generalize from everyday objects to remote sensing and aerial scenes domains? In: Proceedings of the IEEE Conference on Computer Vision and Pattern Recognition Workshops, pp. 4451 .

Razakarivony, S. and Jurie, F., 2016. Vehicle Detection in Aerial Imagery: A small target detection benchmark. Journal of Visual Communication and Image Representation 34, pp. 187-203. 


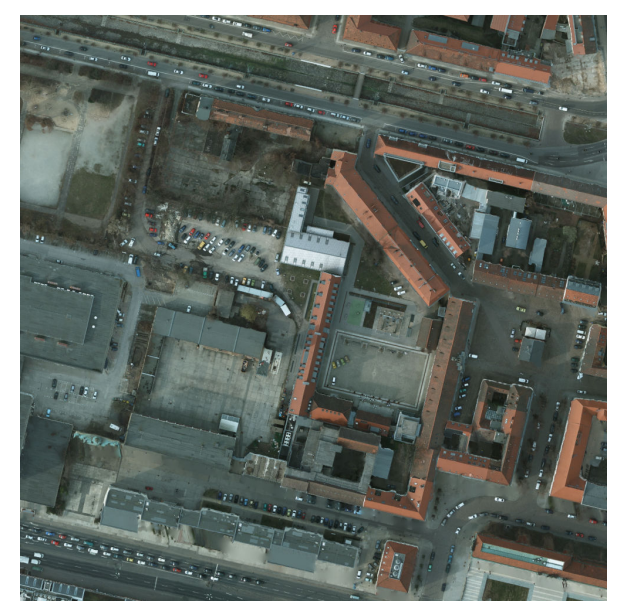

(a) RGB data

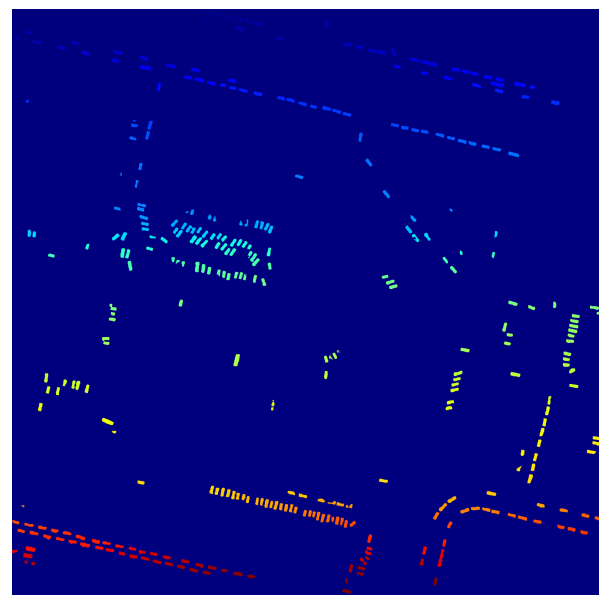

(c) Predicted vehicles

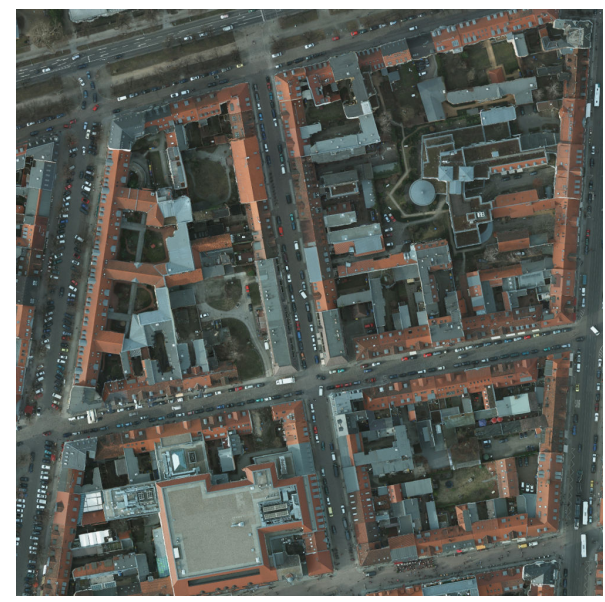

(e) RGB data

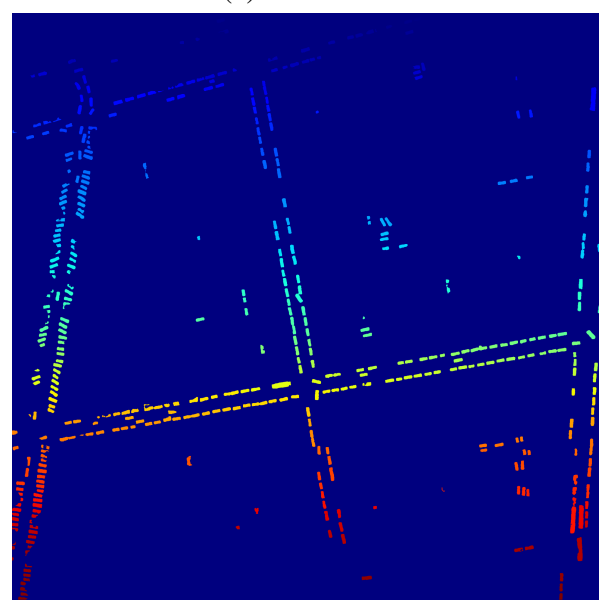

(g) Predicted vehicles

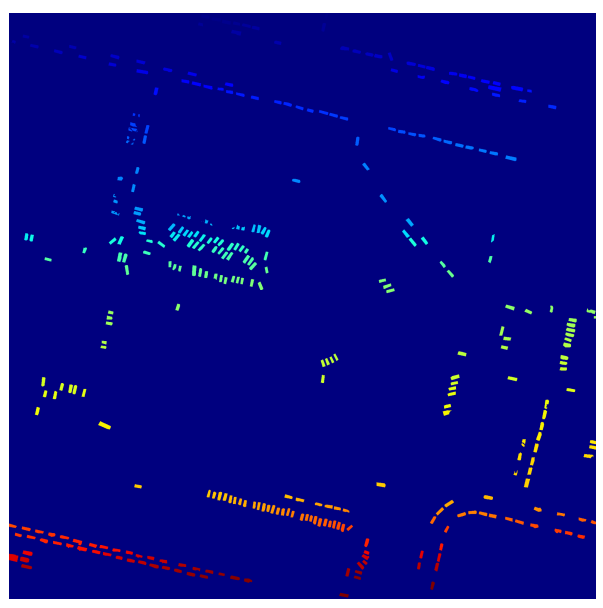

(b) Vehicle ground truth

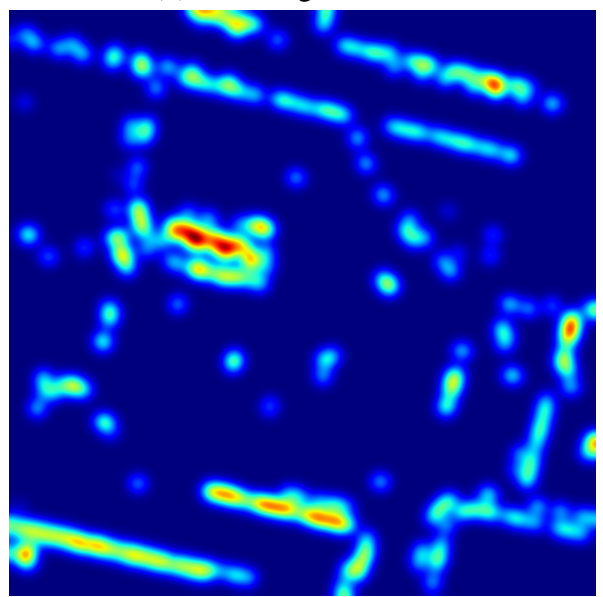

(d) Vehicle occupancy heat map

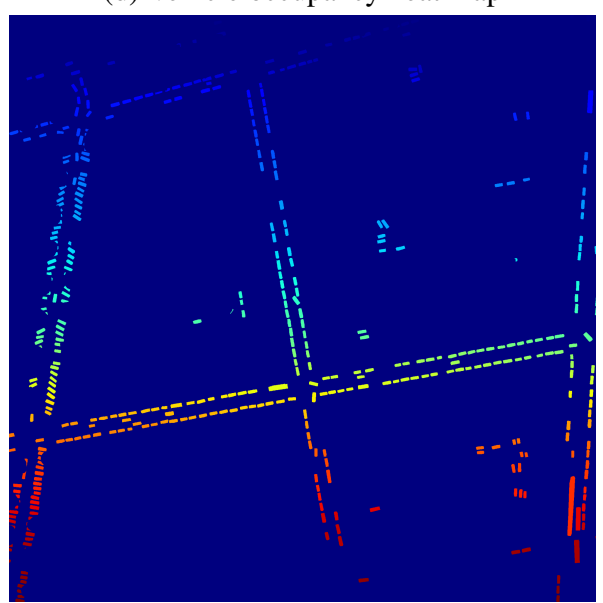

(f) Vehicle ground truth

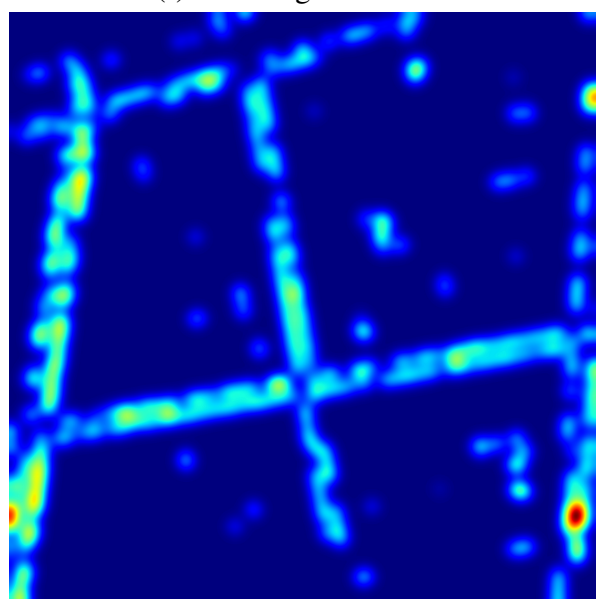

(h) Vehicle occupancy heat map

Figure 6. Visualizing vehicles in the ISPRS Potsdam dataset (best viewed in color) 
Rottensteiner, F., Sohn, G., Jung, J., Gerke, M., Baillard, C., Benitez, S. and Breitkopf, U., 2012. The ISPRS benchmark on urban object classification and $3 \mathrm{~d}$ building reconstruction. ISPRS Ann. Photogramm. Remote Sens. Spat. Inf. Sci 1, pp. 3.

Russakovsky, O., Deng, J., Su, H., Krause, J., Satheesh, S., Ma, S., Huang, Z., Karpathy, A., Khosla, A., Bernstein, M., Berg, A. C. and Fei-Fei, L., 2015. ImageNet Large Scale Visual Recognition Challenge. International Journal of Computer Vision 115(3), pp. 211-252.

Simonyan, K. and Zisserman, A., 2014. Very Deep Convolutional Networks for Large-Scale Image Recognition. arXiv:1409.1556 [cs]. arXiv: 1409.1556.

Srivastava, N., Hinton, G., Krizhevsky, A., Sutskever, I. and Salakhutdinov, R., 2014. Dropout: A simple way to prevent neural networks from overfitting. J. Mach. Learn. Res. 15(1), pp. 1929-1958. 


\title{
AUTOMATED NEAR REAL-TIME EARTH OBSERVATION LEVEL 2 PRODUCT GENERATION FOR SEMANTIC QUERYING
}

\author{
A. Baraldi ${ }^{\mathrm{a}, *}$, Dirk Tiede ${ }^{\mathrm{b}}$, Martin Sudmanns ${ }^{\mathrm{b}}$, Mariana Belgiu ${ }^{\mathrm{b}}$, and Stefan Lang ${ }^{\mathrm{b}}$ \\ ${ }^{a}$ Department of Agricultural and Food Sciences, University of Naples Federico II, Italy - andrea6311@gmail.com \\ b Paris-Lodron Universität Salzburg, IFFB Geoinformatik, Schillerstraße 30, A-5020 Salzburg - (dirk.tiede, martin.sudmanns, \\ mariana.belgiu, stefan.lang)@sbg.ac.at
}

\begin{abstract}
KEY WORDS: Algebra for spatiotemporal objects and events, Cognitive science, Earth observation Level 2 product, Semantic content-based image retrieval, Semantic network, Vision
\end{abstract}

\begin{abstract}
:
Existing Earth observation (EO) content-based image retrieval (CBIR) systems support human-machine interaction through queries by metadata text information, image-wide summary statistics or either image, object or multi-object examples. No semantic CBIR (SCBIR) system in operating mode has ever been developed by the remote sensing (RS) community. At the same time, no EO dataderived Level 2 prototype product has ever been generated systematically at the ground segment, in contrast with the visionary goal of the Global Earth Observation System of Systems (GEOSS) implementation plan for years 2005-2015. Typical EO Level 2 products include: (i) a multi-spectral (MS) image corrected for atmospheric, adjacency and topographic effects, and (ii) a scene classification map (SCM), encompassing cloud and cloud-shadow quality layers. This work presents an original hybrid (combined deductive and inductive) feedback EO image understanding for semantic querying (EO-IU4SQ) system as a proof-of-concept where systematic multi-source EO big data transformation into Level 2 products is accomplished as a pre-condition for SCBIR, in agreement with the object-based image analysis (OBIA) paradigm and the Quality Assurance Framework for Earth Observation (QA4EO) guidelines. In the hybrid EO-IU4SQ system, statistical model-based/bottom-up/inductive/machine learning-from-data algorithms and physical model-based/top-down/deductive/human-to-machine knowledge transfer approaches are combined with feedback loops to take advantage of the complementary features of each and overcome their shortcomings.
\end{abstract}

\section{INTRODUCTION}

Existing Earth observation (EO) content-based image retrieval (CBIR) systems support human-machine interaction through queries by metadata text information (e.g., EO image acquisition time, geographic area of interest, etc.), summary statistics (e.g., image-wide cloud cover quality index), or by either image, object or multi-object examples (Smeulders et al., 2000), see Figure 1. To our best knowledge, no semantic CBIR (SCBIR) system, capable of processing semantic queries such as "retrieve all EO images in the image base where there is a lake adjacent to a highway", has ever been developed by the remote sensing (RS) community in operating mode (Dumitru et al., 2015). To be considered in operating mode an EO SCBIR system is required to score "high" in a set of community-agreed outcome and process $(\mathrm{OP})$ quantitative quality indicators $\left(\mathrm{Q}^{2} \mathrm{Is}\right)$ including degree of automation, accuracy, efficiency, scalability to changes in sensor specification and user requirements, robustness to changes in input parameters, robustness to changes in input data, timeliness from data acquisition to product generation, and costs in manpower and computer power (Baraldi and Boschetti, 2012), in agreement with the Quality Assurance Framework for Earth Observation (QA4EO) Calibration/Validation (Cal/Val) guidelines (Group on Earth Observation, 2010). According to these definitions the Earth Observation Image Librarian (EOLib), considered the state-ofthe-art in SCBIR system prototypes (Dumitru et al., 2015), is affected by several structural limitations in operating mode. EOLib adopts an inductive supervised data learning algorithm, specifically, a support vector machine (SVM), to accomplish 1D image classification, where the vector data sequence consists of image convolutional values generated by 2D spatial filters (Dumitru et al., 2015). Due to the inherent ill-posedness of machine learning-from-data algorithms (Cherkassky and Mulier, 1998), EOLib is semi-automatic and site-specific; it requires user interaction, first, to collect supervised training samples and, second, to define the SVM's free-parameters based on heuristics. In addition, any 1D image classifier is an orderless encoder invariant to permutations, where spatial topological information in the image domain is lost. This is in contrast with an unequivocal fact: human chromatic and achromatic visions are nearly as affective in scene-from-image representation. It means that spatial information, either topological (e.g., adjacency, inclusion, etc.) or non-topological (e.g., metric distance, angle measure, etc.), dominates color information in both the 4D scene domain and the (2D) image domain (Matsuyama and Hwang, 1990). Unlike traditional 1D image interpretation approaches, such as EOLib's, topology preserving mapping of convolutional local filters into 2D retinotopic maps explains the increasing popularity of deep convolutional neural networks in computer vision applications (Cimpoi et al, 2014).

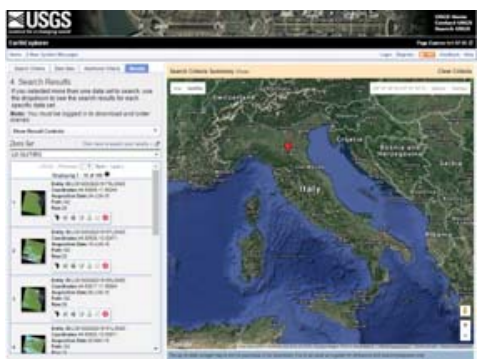

Figure 1. Existing EO CBIR systems support image retrieval by metadata text information, summary statistics or image example. No SCBIR capability is supported in operating mode.

Our conjecture is that existing EO CBIR systems support no semantic querying because they lack EO image understanding 
capabilities, where spatial information dominates color information (Matsuyama and Hwang, 1990). This conjecture has two corollaries. First, it implies that a pre-condition of SCBIR is the computational solution of the multi-disciplinary cognitive problem of image interpretation (vision), i.e., the complexity of the SCBIR problem is not inferior to the complexity of vision. Vision is an inherently ill-posed problem (Matsuyama and Hwang, 1990) in the Hadamard sense, i.e., it is NP-hard. To infer stable symbolic percepts in the 4D spatiotemporal scene domain from ever-varying sub-symbolic sensations (quantitative sensory data) in the (2D) image domain, vision is affected by a semantic information gap and by a 4D-to-2D data dimensionality reduction problem.

The second corollary of our conjecture about an ongoing lack of SCBIR solutions is that existing EO image understanding systems (EO-IUSs) score "low" in operating mode, i.e., they fall short in transforming multi-source EO big data into comprehensive, timely and operational information products. If this thesis holds true, then existing EO-IUSs do not comply with the visionary goal of the Group on Earth Observation (GEO), formulated in the Global Earth Observation System of Systems (GEOSS) implementation plan for years 2005-2015 and in the QA4EO Cal/Val guidelines (Group on Earth Observation, 2010). The thesis that existing EO-IUSs score "low" in operating mode is supported by the fact that no EO data-derived Level 2 prototype product has ever been generated systematically at the ground segment (European Space Agency, 2015). By definition an EO Level 2 product includes an EO image corrected for atmospheric, adjacency and topographic effects, and a general-purpose user- and applicationindependent scene classification map (SCM).

To contribute toward filling an ongoing information gap from EO big data to EO value-adding products and services, an innovative EO Image Understanding for Semantic Querying (EO-IU4SQ) system is presented as a proof-of-concept, where automated multi-source EO big data spatiotemporal analytics is accomplished as a pre-condition for SCBIR, see Figure 2. In particular the EO-IU4SQ system consists of two hybrid (combined deductive and inductive) inference subsystems provided with feedback loops: an EO-IU subsystem is followed by an EO-SQ subsystem. The former transforms automatically (i.e., without user interaction) and in near real-time any multisource EO multi-spectral (MS) image, provided with a radiometric calibration metadata file in agreement with the QA4EO Cal/Val requirements, into an EO Level 2 product consisting of: (a) an enhanced EO image corrected for atmospheric and topographic effects, and (b) an SCM whose legend includes quality layers, such as cloud and cloud-shadow, in addition to the general-purpose user- and applicationindependent 8-class land cover (LC) taxonomy adopted by the initial Dichotomous Phase (DP) of the two-phase Food and Agriculture Organization of the United Nations (FAO) - Land Cover Classification System (LCCS) (Di Gregorio and Jansen, 2000). The 8-class LCCS-DP taxonomy consists of the three "nested" dichotomous layers: (i) vegetation/non-vegetation, (ii) terrestrial/aquatic and (iii) managed/natural. The three DP layers deliver as output the following eight LCCS-DP classes. (1) Cultivated and Managed Terrestrial (non-aquatic) Nonvegetated Areas. (2)Natural and Semi-Natural Terrestrial Vegetation. (3) Cultivated Aquatic or Regularly Flooded Areas. (4) Natural and Semi-Natural Aquatic or Regularly Flooded Vegetation. (5) Artificial Surfaces and Associated Areas. (6) Bare Areas. (7) Artificial Waterbodies, Snow and Ice. (8) Natural Waterbodies, Snow and Ice. The general-purpose 8class LCCS-DP legend is preliminary to the LCCS Modular
Hierarchical Phase (MHP), consisting of a hierarchical battery of application- and user-specific one-class LC classifiers.

Unlike alternative hierarchical LC class taxonomies whose Level 1 is multi-class, such as the CORINE Land Cover (CLC) and the EOLib's LC map legend (Dumitru et al., 2015), the two-phase LCCS-DP and -MHP taxonomies are "nested" endto-end, starting from the first-level LCCS-DP layer vegetation/non-vegetation, whose quality assurance becomes paramount according to an intuitive garbage in garbage out (GIGO) quality principle. To date EO-IUSs typically detect vegetation by inspecting a vegetation spectral index and this spectral index analysis, either single-date or multi-temporal, is typically conducted pixel-based. Conceptually a spectral index is a scalar value equivalent to the slope of one tangent to a multi-spectral (MS) signature; no MS signature characterized by a spectral shape and intensity can be parameterized by one or more angular coefficient in one point without an information loss in both shape and intensity. In practice, an n-channel MS signature is projected onto a scalar variable, equivalent to a panchromatic image affected by an nD-to-1D data dimensionality reduction. In addition, as a special case of 1D image analysis pixel-based image analysis completely ignores both non-topological and topological spatial information in the image domain. The conclusion is that existing EO-IUSs are likely to fall short at level 1 of the 3-level LCCS-DP taxonomy.

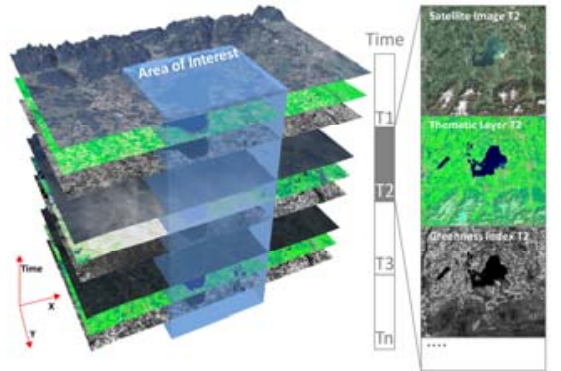

Figure 2. The EO-IU4SQ system for spatiotemporal analytics of multi-source EO big data. Each EO image is automatically provided with numeric/quantitative and categorical/qualitative information layers.

To accomplish multi-source EO big data transformation into Level 2 products the EO-IU4SQ system complies with the object-based image analysis (OBIA) paradigm (Blaschke et al., 2014), where spatial information, either topological or nontopological, dominates color information. In addition the two EO-IU and EO-SQ subsystems adopt a hybrid inference approach provided with feedback loops, where statistical/ bottom-up/inductive/ machine learning-from-data algorithms and physical/ top-down/ deductive/ human-to-machine knowledge transfer approaches are combined to take advantage of the complementary features of each and overcome their shortcomings (Baraldi and Boschetti, 2012). These properties make the EO-IU4SQ system architecture alternative to the design adopted by the majority of EO-IUSs systems, including EOLib's, consisting of a feedforward inductive inference system architecture.

For the sake of brevity, in the rest of this paper the proposed EO-IU4SQ system is described at the two levels of understanding of architecture (design) and knowledge/information representation, whereas the two levels of system understanding of algorithms and implementation are merely outlined, see Figure 3. According to Marr, the linchpin of success of any information processing system is system 
design and knowledge/information representation, rather than algorithms and implementation (Marr, 1982).

\section{EO-IU4SQ SYSTEM DESIGN AND INFORMATION REPRESENTATION}

\subsection{System design}

The EO-IU4SQ system architecture encompasses a hybrid feedback EO-IU subsystem preliminary to a hybrid feedback EO-SQ subsystem, see Figure 3 and Figure 4. These two subsystems share: (i) a fact base of EO data and data-derived products, (ii) a knowledge base available in addition to facts, and (iii) an inference engine, which applies the knowledge base to the fact base to infer new information, such as EO Level 2 products.

\subsection{Knowledge/information representation}

The EO-IU4SQ system's knowledge base consists of four types of knowledge, see Figure 3.

(1) 4D spatiotemporal scene-domain knowledge in user-speak, known as world model. In the 4D scene domain, spatial information typically dominates color information (Matsuyama and Hwang, 1990). Represented as a semantic network in a graphic user interface (GUI) (Growe, 1999), the world model can be thought of as an entity-relationship (ER) conceptual model where classes of real-world 4D objects, represented as nodes, are provided with attributes, either spatiotemporal or not, including appearance properties, while inter-class relationships, represented as arcs, are either spatiotemporal or not, such as part-of and subset-of (Sonka et al., 1994; Laurini and Thompson, 1992). In EO data applications, the world model consist of land cover (LC) classes belonging to a discrete and finite LC class taxonomy to be community-agreed upon in advance, such as the FAO-LCCS taxonomy (Di Gregorio and L. Jansen, 2000). To date grammars (syntactic models) can rarely be inferred from supervised (labeled) or unsupervised (unlabeled) data by statistical approaches. Hence, grammars typically require significant human interaction, e.g., to identify non-spatial inter-class relationships including part-of and subset-of (Sonka et al., 1994). In addition, "no amount of syntax will ever produce semantics" (Mayo, 2003). Hence, the world model is expected to be passed on to the EO-IU4SQ system by human domain-experts. This is a top-down scenedomain knowledge transfer, from human-to-machine. Typically investigated by artificial intelligence (Laurini and Thompson, 1992), top-down knowledge transfer is the dual problem of (complementary not alternative to) inductive machine learningfrom-data, which is currently dominating the RS literature.

(2) Image-domain knowledge in techno-speak, accounting for quantitative visual features such as color, texture (perceptual spatial grouping of texture elements), local shape and interobject spatial relationships, either topological such as inclusion, adjacency, etc., or non-topological, such as metric distances and inter-angle measures (Smeulders et al., 2000; Matsuyama and Hwang, 1990; Sonka et al., 1994). In the (2D) image domain, spatial information, i.e., local shape, texture and inter-object spatial relationships, typically dominates color information.

(3) Knowledge about the sensor transfer functions (translation rules) capable of mapping the scene-domain knowledge in userspeak (e.g., in meter units) onto the image-domain knowledge in techno-speak (e.g., in pixel units).

(4) Standard rule base consisting of physical laws, methods, processes, first-principle models, if-then rules, etc., to be employed by the EO-IU4SQ inference engine to generate new information about the observed 4D scene domain from the existing fact base of EO images and products.

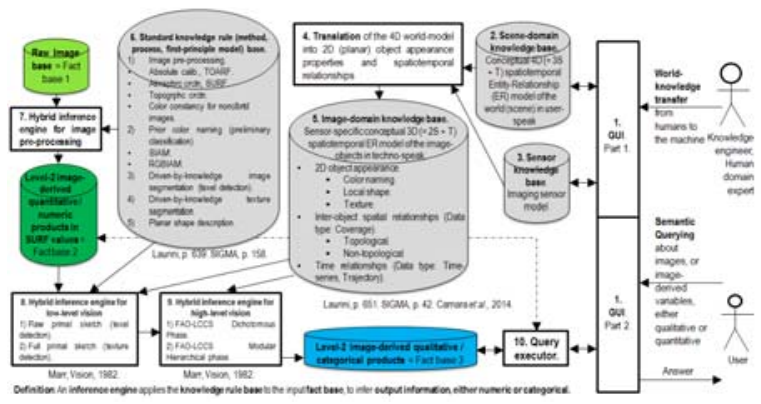

Figure 3. EO-IU4SQ system architecture.

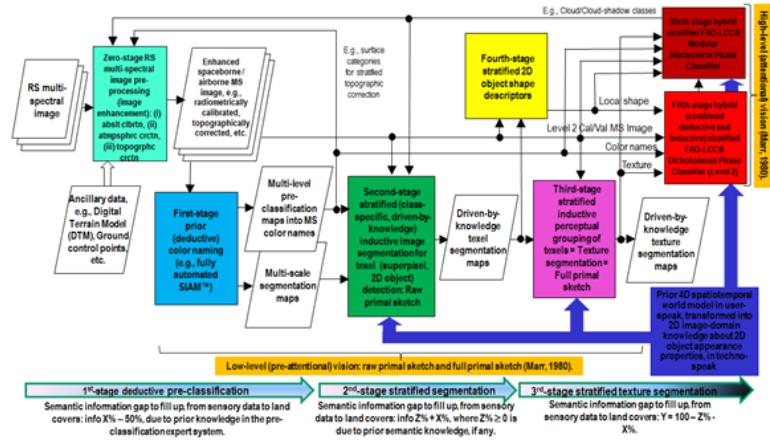

Figure 4. Hybrid feedback EO-IU subsystem based on a convergence-of-evidence approach to low- and high-level EO image understanding (classification). Visual evidence stems from color, local shape, texture and inter-object spatial relationships. Low-level EO image classification is general-purpose, user- and application independent, such as an EO Level 2 SCM product provided with an 8-class LCCS-DP map legend. High-level EO image classification is user- and application-specific, such as an EO SCM product provided with an LCCS-MHP legend.

\subsection{Standard rule base}

Well-known visual features to be investigated by standard computer vision algorithms are color, local shape, texture and inter-object spatial relationships (Matsuyama and Hwang, 1990; Smeulders et al., 2000; Sonka et al., 1994). Visual attributes in the image domain should never be confused with materials (surface types) in the 4D scene domain. For example, MS color names, just like spectral endmembers in hyper-spectral images, cannot always be inverted to unique LC class names (Baraldi and Boschetti, 2012). According to a convergence-of-evidence computer vision approach it is the combination of visual attributes, with special emphasis on spatial information, eligible for identifying a target material in the scene domain. To support a convergence-of-evidence vision approach, the EO-IU4SQ's standard rule base encompasses algorithms for EO data enhancement (pre-processing) and pre-attentional (low-level) vision. In agreement with the Marr terminology (Marr, 1982) pre-attentive vision consists of two phases: a raw primal sketch for zero-crossing (ZX) segment and texel (texture element) detection followed by a full primal sketch for texture segmentation (perceptual spatial grouping of texels). These standard image pre-processing and low-level vision algorithms are summarized below.

(1) EO image pre-processing (EO data enhancement). 
(i) Absolute radiometric Cal, in compliance with the QA4EO Cal requirements. A battery of sensor-specific EO image radiometric calibrators, e.g., AVHRR, SPOT-1 to SPOT-7, Pleaiades, Landsat-4 to Landsat-8, MeteoSat, RapidEye, WorldView, QuickBird, Ikonos, etc., is required to transform digital numbers into a radiometric unit of measure, specifically, top-of-atmosphere reflectance (TOARF). Sensory data Cal is a well-known "prerequisite for physical model-based analysis of airborne and satellite sensor measurements in the optical domain" (Schaepman-Strub et al., 2006). In other words, EO data $\mathrm{Cal}$ is a necessary not sufficient condition for both physical model-based and hybrid inference-based data understanding systems, including the hybrid EO-IU4SQ system. Whereas physical variables can be investigated by physical, statistical and hybrid inference systems, uncalibrated sensory data can be investigated by statistical data models exclusively. Although they do not require physical variables as input, statistical models can benefit from physical variables, since physical units of measure harmonize data acquired across time, space and sensors. Irrespective of this unquestionable benefit, in the RS literature, where statistical models dominate physical ones, EO data Cal is largely oversighted, in disagreement with the QA4EO Cal requirements.

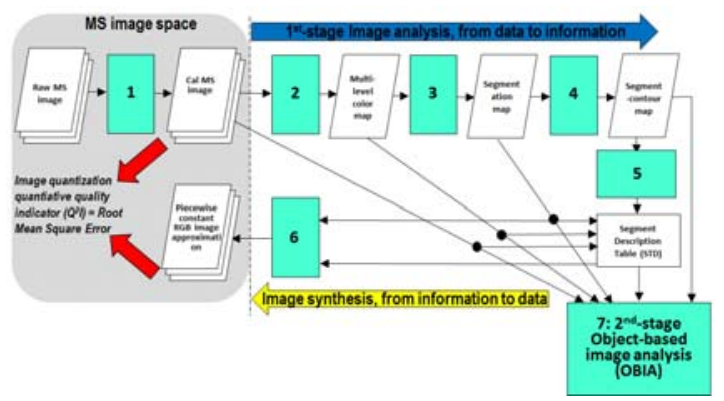

Figure 5. The SIAM software toolbox for prior knowledgebased vector quantization, VQ. Phase 1-of-2 = Encoding phase/Image analysis - Stage 1: MS data calibration into TOARF or SURF values. Stage 2: Prior knowledge-based SIAM color space partitioning. Stage 3: Well-posed twopass connected-component multi-level image labeling. Stage 4: Well-posed segment-contour extraction. Stage 5: Well-posed Segment Description Table (STD) allocation and initialization. Phase 2-of-2 = Decoding phase/Image synthesis - Stage 6: Piecewise constant MS image approximation ('Object-mean view') and per-pixel VQ error estimation. Stage 7: Object-based image analysis (OBIA).

(ii) "Stratified" ("layered", class-conditional) approach to atmospheric correction of TOARF into surface reflectance (SURF) values. Atmospheric correction is a typically ill-posed physical model-based inversion problem. To become better conditioned for numerical treatment, an atmospheric correction algorithm can be run on class-conditional EO data layers (Vermote and Saleous, 2007), such as MS color names detected by a static decision tree for MS reflectance space polyhedralization (Baraldi and Boschetti, 2012).

(iii) "Stratified" topographic correction (TOC). TOC is a typical chicken-and-egg dilemma. To become better posed for automated numerical solution, an inherently ill-conditioned TOC algorithm is run on informative EO image strata. These image masks comprise MS color names, automatically detected by a deductive (prior knowledge-based) MS reflectance space polyhedralization such as the Satellite Image Automatic
Mapper (SIAM) (Baraldi and Boschetti, 2012), see Figure 5, overlapped with illumination-specific information layers inferred from ancillary data sources, specifically, a digital surface model (DSM) and the image metadata sun and sensor positions. Illumination-specific image masks are threefold: horizontal areas where no TOC is required, slopes facing the sun and slopes facing away from the sun. In the latter two illumination-specific layers, a stratified TOC is applied per MS color name (Baraldi at al., 2010).

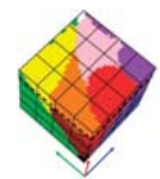

Figure 6. Unlike a MS space polyhedralization impossible to visualize when the number of channels is superior to three, an RGB data cube polyhedralization is intuitive to display. For example, a "universal" inventory of eleven basic color (BC) names in 20 human languages (black, white, gray, red, orange, yellow, green, blue, purple, pink and brown) can be mapped onto the RGB cube based on perceptual evidence (Griffin, 2006).

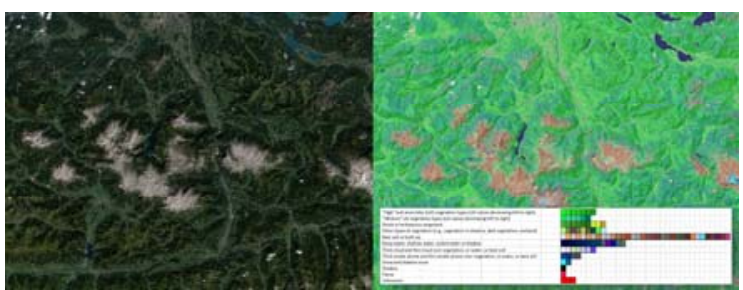

Figure 7. Left: Sentinel-2A (S2A) image of Austria, acquired on 2015-08-18, $10 \mathrm{~m}$ resolution, radiometrically calibrated into TOARF values and depicted in true colors: $\mathrm{R}=$ visible red channel, $\mathrm{G}=$ visible red, $\mathrm{B}=$ visible blue. No histogram stretching for visualization purposes. Right: Automatic SIAM mapping of the S2A image onto a legend of MS color names (spectral categories), depicted as pseudo-colors (green as vegetation, blue as water or shadow, etc.).

(iv) Color constancy in non-calibrated images. In human vision, color constancy ensures that the perceived color of objects remains relatively constant under varying illumination conditions, so that they appear identical to a "canonical" (reference) image, subject to a "canonical" (known) light source of controlled quality, such as a white light source (Gijsenij et al., 2010). In short, solution of the color constancy problem is the recovery of an illuminant-independent representation of the reflectance values in a scene. In practice color constancy supports image harmonization and interoperability when no radiometric calibration parameter is available. A novel selforganizing statistical algorithm for image color constancy (patent pending), inspired by human vision, is typically applied to MS images provided with no radiometric calibration metadata file, such as those acquired by consumer-level RGB color cameras, either true- or false-color, mounted onboard Unmanned Aerial Vehicles (UAVs) (Vo et al., 2016).

(2) Pre-attentive vision: Raw and full primal sketch.

(i) Raw primal sketch: Prior knowledge-based MS reflectance space polyhedralization into color names. When a numeric/quantitative color variable is mapped onto a categorical/nominal color variable, color attributes of target LC classes can be called by name, in line with symbolic human 
reasoning. Proposed to the RS community in recent years (Baraldi and Boschetti, 2012), the SIAM software product is an expert system (prior knowledge-based decision tree) for deductive/top-down vector quantization (VQ) and VQ quality assessment in a MS color space radiometrically calibrated into TOARF or SURF values, see Figure 5. Since it relies exclusively on a priori knowledge available in addition to data, any expert system, such as SIAM, is fully automated, i.e., it requires neither user-defined parameters nor training data to run. In the VQ encoding phase, SIAM partitions the MS reflectance space into static (non-adaptive to data) hyperpolyhedra, not necessarily convex or connected, equivalent to a dictionary (codebook) of MS color names (codewords). Each MS pixel is mapped onto one MS polyhedron associated with a color name. Unfortunately, when the MS space dimensionality is greater than three, a prior dictionary of mutually exclusive and totally exhaustive hyperpolyhedra is difficult to think of and impossible to visualize, see Figure 6. Next, for image analysis purposes a 2D multi-level VQ map is automatically generated, see Figure 7, together with its segmentation map, see Figure 8. In the VQ decoding phase, equivalent to image synthesis, a VQ quality assessment is accomplished in compliance with the QA4EO Val requirements, via a segmentwise-constant MS image reconstruction, see Figure 5. In VQ problems, a typical community-agreed $\mathrm{Q}^{2} \mathrm{I}$ is the per-vector encoding-decoding Euclidean distance (Cherkassky and Mulier, 1998). According to the existing literature (Baraldi et al., 2016), SIAM provides a very substantial contribution to the detection of the first two-ofthree levels of the LCCS-DP taxonomy, namely, vegetation/non-vegetation and terrestrial/aquatic, based on context-insensitive spectral properties exclusively. To improve LC classification accuracy, reliability and informativeness, preliminary spectral-based classification by SIAM must be combined with additional evidence stemming from spatial information according to a convergence-of-visual-evidence approach, refer to Figure 4.

(ii) Raw primal sketch: Prior knowledge-based RGB cube polyhedralization into color names, see Figure 6 . The RGB Image Automatic Mapper (RGBIAM, patent pending) is a novel expert system capable of partitioning an RGB data cube, either true- or false-color, into a pre-defined dictionary of RGB color names. The RGBIAM software design is the same as SIAM's, see Figure 5. When a color space dimensionality is equal to three, a prior dictionary of mutually exclusive and totally exhaustive polyhedra, either convex or not, either connected or not, is intuitive to think of and easy to visualize, see Figure 6. The novel RGBIAM's static decision tree requires as input an RGB image subject to color constancy for data harmonization when no radiometric Cal parameter is available, in compliance with the QA4EO Cal guidelines.

(iii) Raw primal sketch: Prior knowledge-based MS reflectance space polyhedralization into color names through time. Any post-classification LC change/no-change (LCC) detection overall accuracy, OA-LCC $\mathrm{LC}_{1,2} \in[0,1]$, is such that $\mathrm{OA}-\mathrm{LCC}_{1,2} \leq$ $\left(\mathrm{OA}-\mathrm{LC}_{1} \times \mathrm{OA}-\mathrm{LC}_{2}\right)$, where OA-LC $\mathrm{L}_{1}$ and $\mathrm{OA}-\mathrm{LC}_{2}$ are the two single-date OAs. For example, if OA-LC $\mathrm{L}_{1}=0.90, \mathrm{OA}^{-\mathrm{LC}_{2}}=$ 0.90 , then OA-LCC ${ }_{1,2} \leq 0.81$. It means that, in general, postclassification LC change/no-change detection-through-time is recommended if and only if single-date thematic map accuracies score "high". When two SIAM maps of the same geographic area of interest are available at the same spatial resolution at times $\mathrm{T} 1$ and $\mathrm{T} 2>\mathrm{T} 1$, post-classification through time is straightforward according to a two-way square contingency table (association matrix, cross-tabulation matrix) to be community-agreed upon, whose two input categorical variables at times T1 and T2 are the same SIAM legend.

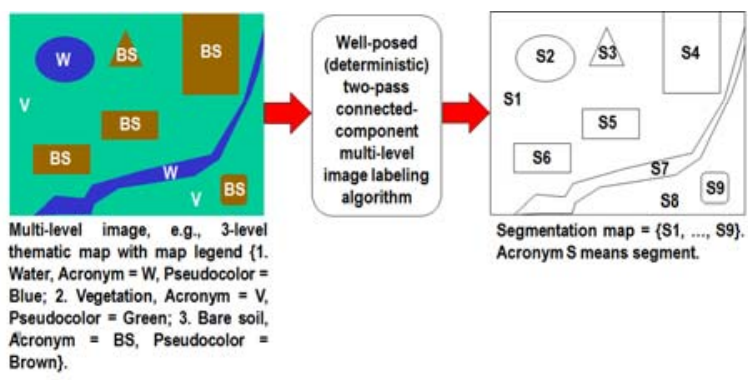

Figure 8. One segmentation map is deterministically generated from one multi-level image, such as a thematic map, but the vice versa does not hold, i.e., many multilevel images can generate the same segmentation map. In this example, nine image-objects/segments $\mathrm{S} 1$ to $\mathrm{S} 9$ can be detected in the thematic map. Each segment consists of a connected set of pixels sharing the same thematic map label. Each stratum/layer/level consists of one or more segments, e.g., stratum Vegetation (V) consists of the two disjoint segments S1 and S8. In any multi-level image domain, three labeled spatial primitives co-exist and are provided with parent-child relationships: pixel with level label, segment with level label and stratum with level label. This overcomes the ill-fated dichotomy between traditional pixel-based versus segment-based image analysis (OBIA) in the numeric image domain.

(3) Raw primal sketch: Two-pass connected-component multilevel image labelling, see Figure 8. Any SCM whose legend is a discrete and finite dictionary of LC class labels is a multi-level image (Sonka et al., 1994). As such, it can be partitioned into planar spatial units (image-objects), either (0D) pixel, (1D) line, (2D) polygon or multi-part image-object (Open Geospatial Consortium, 2015), where each image-object is a connected set of pixels featuring the same LC class label, by a well-posed (deterministic) two-pass connected component multi-level image labeling algorithm (Sonka et al., 1994). Each SCMderived planar object in the image domain is provided with both a segment identifier and an LC class label in the SCM's taxonomy, in compliance with the increasingly popular objectbased image analysis (OBIA) paradigm (Blaschke et al., 2014).

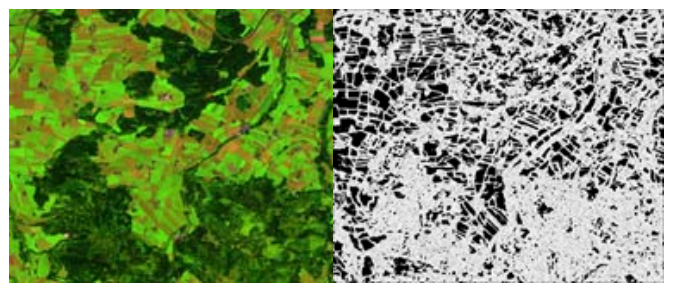

Figure 9. Left: Sentinel-2A image of Austria, radiometrically calibrated into TOARF values, depicted in false colors $(\mathrm{R}=$ MIR, G = NIR, B = Visible Blue), $10 \mathrm{~m}$ resolution. Right: Automated image segmentation into ZX segments. Segment contours automatically depicted with 8-adjacency cross-aura values in range $\{0,8\}$ (Baraldi and Boschetti, 2012).

(4) Raw primal sketch: Consistent with the Mach band illusion (Pessoa, 1996), novel physical model-based automated ZX contour-pixel detection and ZX segment detection. If we 
require that a computer vision system should be able to predict perceptual effects, such as the well-known Mach bands illusion where bright and dark bands are seen at ramp edges, then the number of published vision models becomes surprisingly small (Pessoa, 1996). The novel ZX contour-pixel detector consists of a multi-scale multi-orientation quasicomplete even-symmetric spatial wavelet transform, eligible for image analysis, coding and synthesis (Baraldi and Parmiggiani, 1996). In Marr's words an intermediate information primitive in the image domain, called ZX segment, was defined as "a piece of the contour whose intensity slope (rate at which the convolution changes across the segment) and local orientation are roughly uniform" (Marr, 1982, p. 60). Hence, it is at the level of detection of ZX segments that $0 \mathrm{D}$ ZX pixels turn into sub-symbolic discrete 2D image-objects (polygons). To automatically extract 2D ZX segments from $0 \mathrm{D}$ ZX pixels, an original physical model-based algorithm, featuring no free-parameter to be user-defined, was implemented in operating mode, see Figure 9.

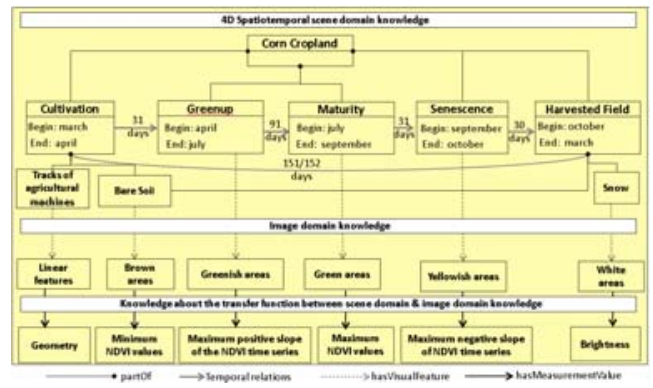

Figure 10. Semantic network of a real-world object with a cyclic behavior, such as a corn cropland in the northern hemisphere.

(5) Full primal sketch: "stratified" texture segmentation. A numeric/quantitative texture variable (perceptual spatial grouping of texels) is mapped onto a categorical/nominal texture variable, suitable for human symbolic reasoning. A multi-scale texture profile of the spatial distribution of ZX contour-pixels is accomplished in line with (Nagao and Matsuyama, 1980). For each spatial scale, move an $\mathrm{N} \times \mathrm{N}$ window over the picture of binary $\mathrm{ZX}$ contour-pixels. If the window contains more than $\operatorname{sqrt}(2) \times \mathrm{N}$ binary $\mathrm{ZX}$ contourpixels, mark the central point of the window as "high" texture.

(6) Planar shape description. Given an image segmentation map consisting of planar objects, see Figure 8, each planar object can be described in geometric (shape) and size terms by a minimally dependent and maximally informative (mDMI) set of planar shape and size indexes, such as area, characteristic spatial scale, scale-invariant roundness, convexity, rectangularity, elongatedness, straightness of boundaries and simple connectivity (Soares et al., 2014). The implemented mDMI set of planar shape indexes is alternative to existing libraries of planar shape descriptors available in commercial EO image processing software toolboxes, provided with no quality assessment in terms of feature independence and informativeness.

\subsection{GUI}

The EO-SQ subsystem's GUI supports the following humanmachine interactions.

(1) Scene-domain knowledge transfer from human-to-machine. The world model can be graphically represented as a semantic network with LC classes as nodes and inter-class relationships, including events, as arcs between nodes (Growe, 1999). Observations of the 4D real world are discrete n-tuples (space $\mathrm{x}$, $\mathrm{y}$ and $\mathrm{z}$, time $\mathrm{t}$; "theme", plus other numeric or categorical attributes, e.g., weight, size, etc.), where the 4-tuple (x, y, z, t) is the location in space and time of the observation, while attribute "theme" identifies the real-world phenomenon or object being observed (Reis Ferreira et al., 2014). Hence, "theme" may account for semantics involved with the observed object or phenomenon. All possible combinations of attributes (space, time, theme), can be modeled as three data types, called time series, coverage, and trajectory, where one attribute is measured, the second is fixed and the third is controlled. According to Reis Ferreira et al. (2014), a time series represents the measured variations of a theme over a controlled time in a fixed location; a trajectory measures locations of a fixed theme over a controlled time and a coverage measures attribute theme within a controlled spatial extent at a fixed time. A world model is an ontology of real-world geospatiotemporal objects/continuants and events/occurrents derived from the three spatiotemporal data types time series, coverage and trajectory. A real-world object/continuant (e.g., a car) is an identifiable (discrete) entity, i.e., it is provided with a unique "identity", which remains constant during its lifetime, while its attributes, whether spatiotemporal or not, including semantics or "theme", can change during its lifetime. An object is present as a whole unit at each moment of its existence. In EO applications, a real-world object can be: (i) a periodic object whose identity is fixed while its attributes change with a cyclic behavior, i.e., the object's identity comprises a given sequence of different states in a fixed time periodicity, see Figure 10, or (ii) persistent/non-periodic objects, e.g. forest area. An event is an individual episode with a definite beginning and an end. It only exists as a whole across the interval over which it occurs, either instant or durative. An event does not change over time. While an event can involve one or more objects, the same object can be involved in any number of events. Events can be: (i) instant, or (ii) durative, including short-term transition events and slowly transient events.

(2) Graphic selection of existing semantic queries/decision rules or generation of new semantic queries/decision rules, in compliance with the world model. There are two types of queries. (i) To accomplish SCBIR operations, where the fact base is investigated for EO image retrieval purposes. e.g., retrieve EO images that are cloud-free across the selected geographic area of interest (AOI). (ii) To infer new information layers from the fact base, e.g., detect flooded areas as a postclassification time-series analysis of single-date thematic maps.

\subsection{Array fact base}

To accomplish efficient geospatial data querying and analysis through space and time within a user-defined AOI and a target time interval, the EO-IU4SQ system prototypical implementation stores its fact base, consisting of multi-sensor multi-temporal EO images, e.g., Landsat-5/7/8 and Sentinel-2A images, each one provided with its information products, either numeric or categorical, in an array database implemented as the Rasdaman (Baumann et. al, 2015). An array database instantiates multiple spatiotemporal data cubes in compliance with the Open Geospatial Consortium (OGC) standards (OGC, 2015), to guarantee inter-system harmonization and compatibility. In a spatiotemporal data cube the third dimension is time, defined in eXtensible Markup Language (XML) as a 1D temporal coordinate system accessible Unified Resource Identifiers (URIs). Time overlays the 2D spatial coordinate 
system, specified by European Petroleum Survey Group (EPSG) codes defined in XML using URIs. Similar to standard relational databases investigated by the Structured Query Language (SQL), an array database consisting of data cubes can be queried by a declarative query language. In an array database storage-related characteristics, such as indexing, tiling and horizontal scaling, can be investigated and optimized independently of application logic In addition, the data cube model has been proven to be scalable and reliable in operational applications (Baumann et. al, 2015). Based on these considerations it was selected as storage backend in the EOIU4SQ system prototypical implementation.

Once users successfully create and store semantic queries in the web-based query interface, these queries are published as OGC compliant Web Processing Services (WPSs) and become available within the client-server architecture, to be executed by any WPS client remotely on a single server or server cluster, see Figure 11. This client-server architecture, providing EO data processing capabilities together with the "ready-toanalyze" data, guarantees fast response to queries.

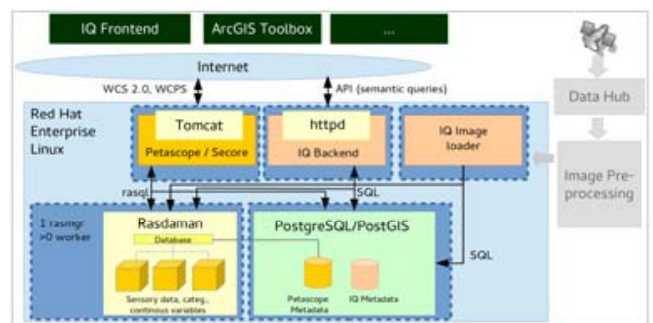

Figure 11. Web-based Image Querying (IQ) system. IQ can be intended as a synonym of EO-IU4SQ (Sudmanns et. al, 2016).

\subsection{Hybrid inference engine}

The EO-IU4SQ's hybrid inference engine combines the rule base and the fact base to infer new information products. It consists of four inference subsystems.

(i) Transformation of space domain knowledge into image domain knowledge through the sensor transfer functions. It provides: (a) an estimate of the EO imaging sensor's physical properties, including spatial, spectral and temporal resolutions, considered necessary to intercept the target LC phenomenon. (b) An estimate of the $2 \mathrm{D}$ visual attributes, such as categorical color names, numeric spectral index values, planar shape descriptors, texture values and spatial relationships, mapped from each target LC class described in the 4D world model, see Figure 10 .

(ii) Automated EO image pre-processing (enhancement), e.g., absolute radiometric Cal, stratified atmospheric correction, stratified TOC, etc., refer to Section 2.3.

(iii) Low-level (pre-attentional) EO image processing, either raw primal sketch, e.g., texels detected as ZX segments, or full primal sketch, e.g., texture segmentation as perceptual grouping of texels (Marr, 1982; Sonka et al., 1994), refer to Section 2.3.

(iv) High-level (attentional) EO image interpretation (classification). It is split into two: a preliminary Level 2 cloud/cloud-shadow detection (Baraldi and Tiede, 2015) plus application-independent general-purpose 8-class LCCS-DP classification, see Figure 12, followed by an LCCS-MHP classification phase, consisting of a hierarchical battery of application- and user-specific one-class LC classifiers. In the two-phase LCCS classification, each final LC classifier adopts a nested (hierarchical, stratified) convergence-of-visual-evidence
OBIA approach, where independent sources of visual evidence are pixel-based color properties in addition to object-based spatial properties, specifically, local shape, texture and spatial relationships, either topological or non-topological (Matsuyama and Hwang, 1990).

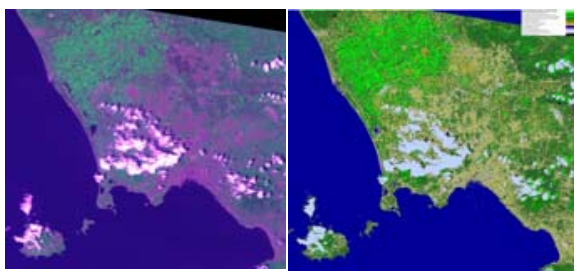

Figure 12. Left: 4-band (B, G, R, NIR) ALOS AVNIR-2 image of Campania, Italy, radiometrically calibrated into TOARF values and depicted in false colors $(R=M I R, G=$ NIR, B = Visible Blue), $10 \mathrm{~m}$ resolution. No histogram stretching for visualization purposes. Right: Level $2 \mathrm{SCM}$ product, whose legend comprises the 8-class LCCS-DP taxonomy, plus Cloud/Cloud-shadow quality layers.

\section{RESULTS}

In the EO-IU4SQ system prototype implemented as a proof-ofconcept, a semantic spatiotemporal query is formulated by a user through the EO-SQ subsystem's GUI to the fact base of EO images provided with derived products by the EO-IU subsystem. In this GUI the user selects (1) the AOI, (2) the target time period and creates or selects via graphical elements the decision-rule pipeline for semantic querying the fact base. Once it is executed, the query results are shown in the image domain and/or as summary statistics (3). The query output product can be downloaded as a geoTiff or re-used for further queries. In Figure 13 the GUI shows an AOI overlapping with the Hohe Tauern National Park (Austria), where a glacier extent invariant within a target time interval was inferred in near realtime from the fact base to exclude seasonal snow.

\section{DISCUSSION}

The QA4EO Val guidelines require each step in an EO data processing pipeline to be provided with community-agreed OP$\mathrm{Q}^{2}$ Is, featuring a degree of uncertainty in measurement, to assess the propagation of errors in comparison with reference standards. According to the GEO, in Stage 3 Val "spatial and temporal consistency of the product with similar products are evaluated by independent means over multiple locations and time periods representing global conditions. In Stage 4 validation, results for Stage 3 are systematically updated when new product versions are released and as the time-series expands" (Group on Earth Observation, 2010).

To comply with the QA4EO Stage 4 Val requirements, the EOIU and EO-SQ subsystems must be provided with OP-Q ${ }^{2}$ Is estimated by independent means on large-scale EO image timeseries. This is a huge validation process still ongoing (Baraldi et al., 2016). Although it has not been subject to a Stage 4 Val process yet, the proposed EO-IU4SQ system implementation works as a proof-of-concept, where automated Level 2 product generation is accomplished as a pre-condition for semantic querying.

In future works, within the proposed EO-IU4SQ system architecture (Figure 3) any information processing block prototype will be considered eligible for permanent optimization, modification or replacement to improve the EOIU or EO-SQ subsystem capabilities in operating mode. 
Furthermore, the semantic network formalism required to graphically represent the world model will be augmented (Growe, 1999) and integrated with an algebra capable of describing spatiotemporal data types and operations in a language-independent and formal way (Reis Ferreira et al., 2014).

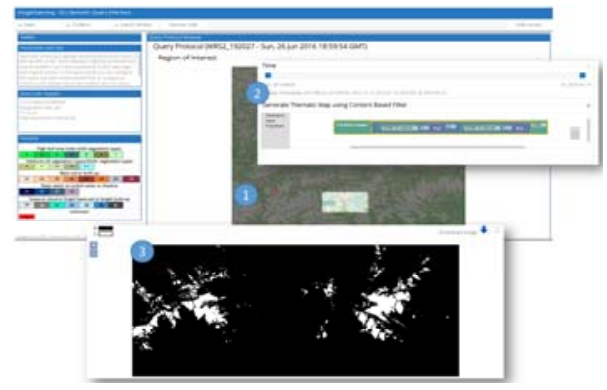

Figure 13. Semantic querying to infer new information layers from the fact base. In this example a glacier extent invariant within a time interval was inferred in near realtime from the fact base to exclude seasonal snow.

\section{ACKNOWLEDGEMENTS}

The test implementation of the EO-IU4SQ system, called Image Querying (IQ) system, was awarded 1st place in the Copernicus Masters 2015 - T-Systems Big Data Challenge. This study was supported by the Austrian Research Promotion Agency (FFG), in the frame of project AutoSentinel2/3, ID 848009.

\section{REFERENCES}

Baraldi, A., Boschetti, L., 2012. Operational automatic remote sensing image understanding systems: Beyond geographic object-based and object-oriented image analysis - Part 2, Remote Sens., 4, pp. 2768-2817.

Baraldi, A., Tiede, D., 2015. AutoCloud+, ESA Invitation to tender - Next Generation EO-based Information Services, $\mathrm{ESA} / \mathrm{AO} / 1-8373 / 15 / \mathrm{I}-\mathrm{NB}$.

Baraldi, A., Gironda, M., Simonetti, D., 2010. Operational three-stage stratified topographic correction of spaceborne multi-spectral imagery, IEEE Trans. Geosci. Remote Sensing, 48(1), pp. 112-146.

Baraldi, A., Humber, M., Tiede, D., Lang, S., 2016. Stage 4 validation of the Satellite Image Automatic Mapper software process and Level 2 products, Int. J. of RS, submitted.

Baraldi, A., Parmiggiani, F., 1996. Combined detection of intensity and chromatic contours in color images, Optical Eng., 35(5), pp. 1413-1439.

Baumann, P., Mazzetti, P., Ungar, J., Barbera, R., Barboni, D., Beccati, A., Bigagli, L., 2015. Big data analytics for earth sciences, Int. J. Digital Earth, pp. 1-27.

Blaschke, T., Hay, G., Kelly, M., Lang, S., Hofmann, P., Addink, E., Queiroz Feitosa, R., van der Meer, F., van der Werff, H, van Coillie, F., Tiede, D., 2014. Geographic objectbased image analysis - towards a new paradigm," ISPRS J. Photogram. Remote Sens., 87, pp. 180-191.

Cherkassky, V., Mulier, F., 1998. Learning from Data: Concepts, Theory, and Methods. New York, NY: Wiley.

Cimpoi M., Maji S., Kokkinos I., Vedaldi A., 2014. Deep filter banks for texture recognition, description, and segmentation, CoRR, abs/1411.6836.

Di Gregorio, A., Jansen, L, 2000. Land Cover Classification
System (LCCS), FAO Corporate Document Repository.

Dumitru, C., Cui, S., Schwarz, G., Datcu, M., 2015. Information content of very-high-resolution SAR images: Semantics, geospatial context, and ontologies," IEEE J. Selected Topics Applied Earth Obs. Remote Sens., 8(4), pp. 1635-1650.

European Space Agency, 2015. Sentinel-2 User Handbook, Standard Document, Issue 1 Rev 2.

Reis Ferreira, K., Camara, G., Vieira Monteiro, A., 2014. An algebra for spatiotemporal data: From observations to events, Trans. in GIS, 1(2), pp. 253-269.

Gijsenij, A., Gevers, T., van de Weijer, J., 2010. Computational color constancy: Survey and experiments, IEEE Trans. Image Proc., 20(9), pp. 2475-2489.

Griffin, L., 2006. Optimality of the basic color categories for classification, J. R. Soc. Interface, 3, pp. 71-85.

Group on Earth Observation/Committee on Earth Observation Satellites (GEO/CEOS), 2010. A Quality Assurance Framework for Earth Observation, version 4.0.

Growe, S., 1999. Knowledge-based interpretation of multisensor and multitemporal remote sensing images, Int. Archives of Photogram. Remote Sens., 32, Part 7-4-3 W6.

Laurini, R., Thompson, D. 1992. Fundamentals of Spatial Information Systems. London, UK: Academic Press.

Marr, D., 1982. Vision. New York, NY: Freeman and C.

Matsuyama, T., Hwang, V., 1990. SIGMA - A Knowledgebased Aerial Image Understanding System. New York, NY: Plenum Press.

Mayo, M., 2003. Symbol grounding and its implications for artificial intelligence, $26^{\text {th }}$ Australian Computer Science Conference, Adelaide, Australia, 16.

Nagao, M., Matsuyama, T., 1980. A Structural Analysis of Complex Aerial Photographs. Plenum Press, New York.

Open Geospatial Consortium (OGC) Inc., 2015. OpenGIS ${ }^{\circledR}$ Implementation Standard for Geographic information - Part 1: Common architecture.

Pessoa, L., 1996. Mach Bands: How many models are possible? Vision Res., 36(19), pp. 3205-3227.

Smeulders, A., Worring, M., Santini, S., Gupta, A., Jain, R., 2000. Content-based image retrieval at the end of the early years, IEEE Trans. Pattern Anal. Machine Intell., 22(12), pp. 1349-1380.

Soares, J., Baraldi, A., Jacobs, D., 2014. Segment-based simpleconnectivity measure design and implementation, Tech. Rep., University of Maryland, College Park.

Sonka, M., Hlavac, V., Boyle, R., 1994. Image Processing, Analysis and Machine Vision, London, U.K.: Chapman \& Hall.

Vermote, E., Saleous, N., 2007. LEDAPS surface reflectance product description - Version 2.0, University of Maryland, College Park, Dept Geography, Code 614.5.

Sudmanns, M., Tiede, D., Augsten, N., Baraldi, A., Belgiu, M., Lang, S., 2016. Array-Datenbanken für semantische inhaltsbasierte Suche und Analyse in Satellitenbildarchiven. Dreiländertagung der SGPF, DFPF und OVG. Bern, Switzerland.

Vo, A., Truong-Hong, L., Laefer, D., Tiede, D., d'OleireOltmanns, S., Baraldi, A., Shimoni, M., 2016. Outcome of the 2015 IEEE GRSS Data Fusion Contest. Part-B: 3D contest," IEEE J. Selected Topics Applied Earth Obs. Remote Sens., X, pp. YYY-ZZZ. 


\title{
3D SEMANTIC LABELING OF ALS POINT CLOUDS BY EXPLOITING MULTI-SCALE, MULTI-TYPE NEIGHBORHOODS FOR FEATURE EXTRACTION
}

\author{
R. Blomley ${ }^{\mathrm{a}}$, B. Jutzi ${ }^{\mathrm{a}}$, M. Weinmann ${ }^{\mathrm{a}, \mathrm{b}}$ \\ ${ }^{\text {a }}$ Institute of Photogrammetry and Remote Sensing, Karlsruhe Institute of Technology (KIT), \\ Englerstr. 7, 76131 Karlsruhe, Germany - (rosmarie.blomley, boris.jutzi, martin.weinmann)@kit.edu \\ ${ }^{\mathrm{b}}$ Université Paris-Est, IGN, LaSTIG, MATIS, \\ 73 avenue de Paris, 94160 Saint-Mandé, France - martin.weinmann@ign.fr
}

KEY WORDS: ALS, LiDAR, Point Cloud, Feature Extraction, Multi-Scale, Classification

\begin{abstract}
:
The semantic labeling of 3D point clouds acquired via airborne laser scanning typically relies on the use of geometric features. In this paper, we present a framework considering complementary types of geometric features extracted from multi-scale, multi-type neighborhoods to describe (i) the local 3D structure for neighborhoods of different scale and type and (ii) how the local 3D structure behaves across different scales and across different neighborhood types. The derived features are provided as input for several classifiers with different learning principles in order to show the potential and limitations of the proposed geometric features with respect to the classification task. To allow a comparison of the performance of our framework to the performance of existing and future approaches, we evaluate our framework on the publicly available dataset provided for the ISPRS benchmark on 3D semantic labeling.
\end{abstract}

\section{INTRODUCTION}

The classification of 3D point clouds has become a topic of great interest in photogrammetry, remote sensing, and computer vision. In this regard, particular attention has been paid to those $3 \mathrm{D}$ point clouds acquired via airborne laser scanning (ALS) as e.g. shown in Figure 1 since these allow an automated analysis of large areas in terms of assigning a (semantic) class label to each point of the considered 3D point cloud (Chehata et al., 2009; Shapovalov et al., 2010; Mallet et al., 2011; Niemeyer et al., 2014; Blomley et al., 2016). However, it still remains challenging to classify ALS point clouds due to the relatively low point density, the irregular point distribution and the complexity of observed scenes. The latter becomes even more significant if one faces many object classes of interest (e.g. buildings, ground, cars, fences/hedges, trees, shrubs or low vegetation). To compare the performance of different approaches for classifying such ALS point clouds, the ISPRS benchmark on 3D semantic labeling has recently been launched with a given ALS dataset.

In this paper, we present a framework for the classification of 3D point clouds obtained via airborne laser scanning. This framework focuses on classifying each 3D point by considering a respective feature vector which, in turn, has been derived by concatenating complementary types of geometric features (metrical features and distribution features) which are extracted from multiple local neighborhoods of different scale and type. Using such multi-scale, multi-type neighborhoods as the basis for feature extraction not only allows for a description of the local 3D structure at each considered 3D point, but also for a description of how the local 3D structure changes across different scales and across different neighborhood types which, in turn, may significantly alleviate the classification task. In a detailed evaluation, we demonstrate the performance of our framework on the given ISPRS benchmark dataset. Thereby, we consider classification results derived using several classifiers which rely on different learning principles. Based on the derived results, we finally discuss both advantages and limitations of our framework in detail.

This paper represents an extension of our previous work (Blomley et al., 2016). Concerning the framework, the main contribu- tion consists in the use of multiple classifiers with different learning principles to obtain an impression about their relative performance. Concerning the experiments, the main contribution consists in the use of the recently released ISPRS benchmark dataset to allow a comparison of the performance of our framework to the performance of other existing and future approaches. Concerning the discussion, the main contribution consists in a focus on the overall performance of the presented framework relying on a multi-scale, multi-type neighborhood instead of a focus on the relative performance of different single-scale, multi-scale and multi-scale, multi-type neighborhoods or a focus on the relative performance of different feature sets.

After briefly discussing related work in Section 2, we present our proposed framework in detail in Section 3. To demonstrate the performance of this framework, we consider a commonly used ALS benchmark dataset and provide the results obtained with our framework in Section 4. Subsequently, the derived results are discussed in Section 5. Finally, in Section 6, we provide concluding remarks and suggestions for future work.

\section{RELATED WORK}

In the context of 3D point cloud classification, the main steps typically address the recovery of a suitable local neighborhood for each $3 \mathrm{D}$ point (Section 2.1), the extraction of geometric features based on information preserved in the local neighborhood (Section 2.2) and the classification of each 3D point based on the derived features (Section 2.3). In the following subsections, we outline related work with respect to these three steps.

\subsection{Neighborhood Recovery}

To describe the local 3D structure at a considered 3D point $\mathbf{X}$, specific characteristics within a local neighborhood are typically considered. Accordingly, an appropriate neighborhood definition is required which may be based on different constraints:

- Single-scale neighborhoods are used to describe the local 3D structure at a specific scale. The most commonly applied 


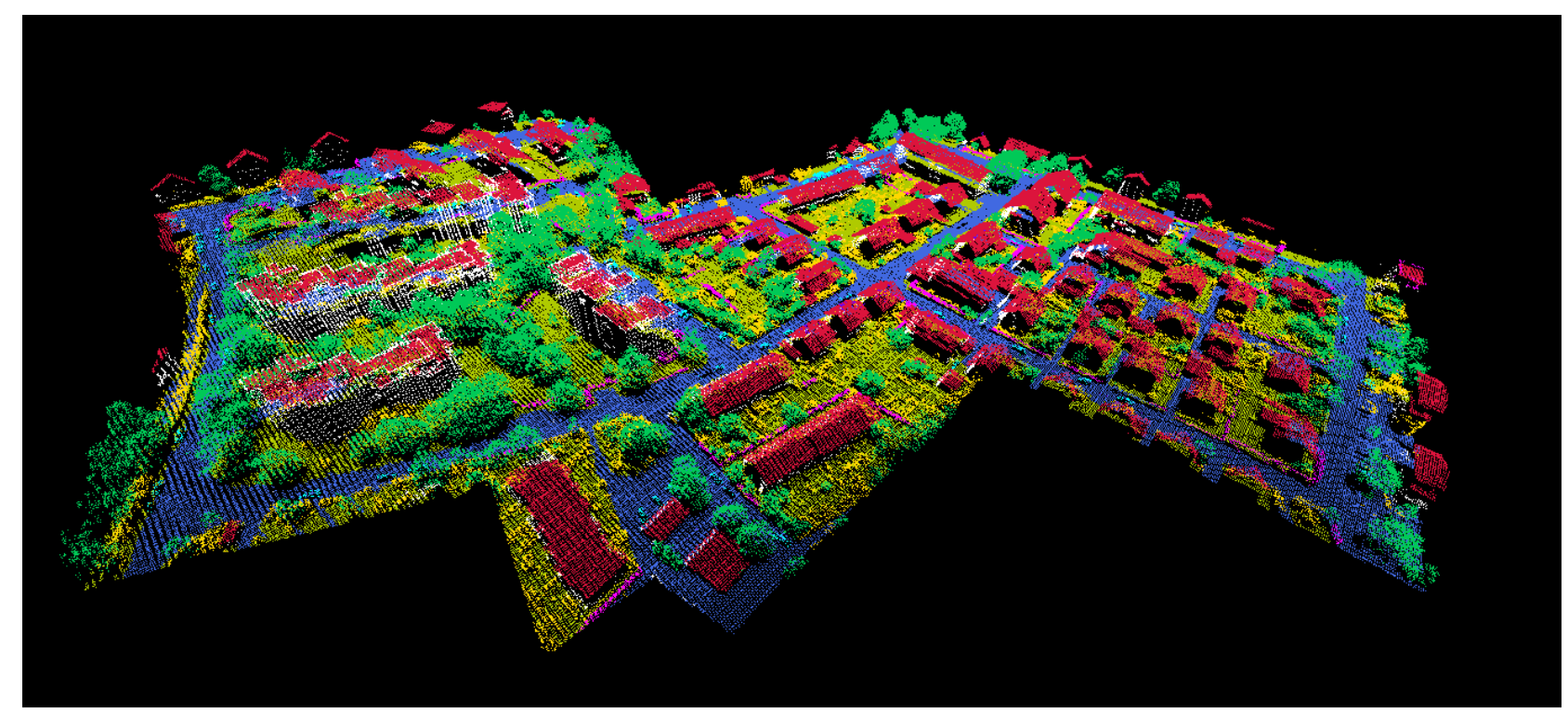

Figure 1. Exemplary 3D point cloud acquired via airborne laser scanning: the color encoding shows the assigned semantic class labels (Powerline: violet; Low Vegetation: yellowish green; Impervious Surfaces: royalblue; Car: cyan; Fence / Hedge: pink; Roof: crimson; Façade: white; Shrub: gold; Tree: emerald-green).

single-scale neighborhoods are represented by $(i)$ a spherical neighborhood formed by all 3D points within a sphere around $\mathbf{X}$, which is parameterized with a fixed radius (Lee and Schenk, 2002), (ii) a cylindrical neighborhood formed by all 3D points within a cylinder whose axis passes through $\mathbf{X}$ and whose radius is fixed (Filin and Pfeifer, 2005), or (iii) a neighborhood formed by the $k \in \mathbb{N}$ nearest neighbors of $\mathbf{X}$ (Linsen and Prautzsch, 2001). While the involved scale parameter is still typically selected based on heuristic or empiric knowledge about the scene and/or the data, there are also few approaches to automatically select the scale parameter in a generic way (Pauly et al., 2003; Mitra and Nguyen, 2003; Demantké et al., 2011; Weinmann et al., 2015a).

- Multi-scale neighborhoods not only allow to describe the local 3D structure at specific scales, but also to describe how the local 3D geometry behaves across scales. Respective approaches have been presented with a combination of cylindrical neighborhoods with different radii (Niemeyer et al., 2014; Schmidt et al., 2014) or a combination of spherical neighborhoods with different radii (Brodu and Lague, 2012). Furthermore, it has been proposed to extract features from different entities such as voxels, blocks and pillars $(\mathrm{Hu}$ et al., 2013) or points, planar segments and mean shift segments (Xu et al., 2014). In all these cases, however, scale parameters are selected based on heuristic or empiric knowledge about the scene and/or the data.

\subsection{Feature Extraction}

Based on the selected neighborhood definition, the spatial arrangement of $3 \mathrm{D}$ points within the neighborhood may be considered to derive a suitable description for a $3 \mathrm{D}$ point $\mathbf{X}$ to be classified. This geometric description is typically represented in the form of a feature vector. To define the single entries of such a feature vector, features belonging to very different feature types may be used:

- The first feature type comprises parametric features which represent the estimated parameters when fitting geometric primitives such as planes, cylinders or spheres to the given data (Vosselman et al., 2004).

- The second feature type comprises metrical features which address a description of local context by evaluating certain geometric measures. The latter typically involve shape measures represented by a single value which specifies one single property based on characteristics within the local neighborhood (West et al., 2004; Jutzi and Gross, 2009; Mallet et al., 2011; Weinmann et al., 2013; Guo et al., 2015). Such features are to some degree interpretable as they describe fundamental geometric properties of the local neighborhood.

- The third feature type comprises sampled features such as distribution features which address a description of local context by sampling the distribution of a certain metric e.g. in the form of histograms (Osada et al., 2002; Rusu et al., 2008; Tombari et al., 2010; Blomley et al., 2014).

Among these feature types, especially metrical features and distribution features are widely but separately used for a variety of applications.

\subsection{Classification}

Once a feature vector has been derived for a 3D point to be classified, the feature vector is provided as input for a classifier which should allow to uniquely assign a respective (semantic) class label. Thereby, the straightforward solution consists in applying standard classifiers such as a Support Vector Machine classifier, a Random Forest classifier, a Bayesian Discriminant classifier, etc. for classifying 3D points based on the derived feature vectors (Lodha et al., 2006; Lodha et al., 2007; Mallet et al., 2011; Guo et al., 2011; Khoshelham and Oude Elberink, 2012; Weinmann et al., 2015a). Respective classifiers are available in numerous software tools and easy-to-use, but the achieved labeling typically reveals a noisy appearance since no spatial correlation between class labels of neighboring 3D points is taken into account.

To account for the fact that the class labels of neighboring 3D points might be correlated, contextual classification approaches 


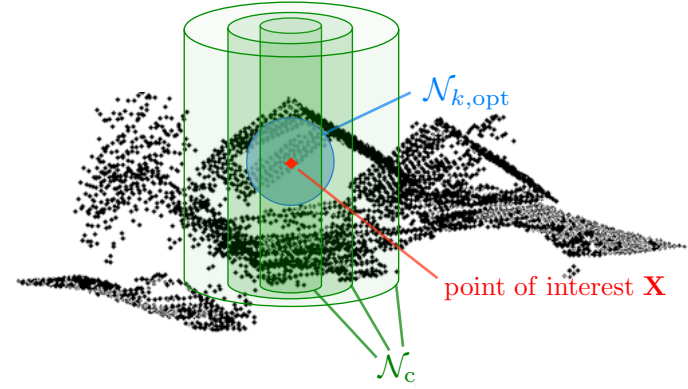

Figure 2. Neighborhood definitions used in this work as the basis for extracting features for a considered 3D point $\mathbf{X}$ : cylindrical neighborhoods $\mathcal{N}_{\mathrm{c}}$ with different radii (green) and spherical neighborhood $\mathcal{N}_{k, \text { opt }}$ formed by an optimal number $k_{\text {opt }}$ of nearest neighbors (blue).

may be applied where, in addition to the feature vector of the considered $3 \mathrm{D}$ point $\mathbf{X}$, the spatial relationship to other neighboring $3 \mathrm{D}$ points is considered in order to assign the class label. An approach for a contextual classification of ALS point cloud data has for instance been presented by applying a Conditional Random Field on the basis of cylindrical neighborhoods (Niemeyer et al., 2014), and the respective classification results represent the baseline for the ISPRS benchmark on 3D semantic labeling.

\section{METHODOLOGY}

For each 3D point $\mathbf{X}$ to be classified, the proposed framework successively applies three main steps which consist in the recovery of a local neighborhood (Section 3.1), the extraction of geometric features based on those 3D points within the local neighborhood (Section 3.2) and the supervised classification of the derived feature vector (Section 3.3).

\subsection{Recovery of Local Neighborhoods}

To obtain an appropriate local neighborhood as the basis for feature extraction, we focus on the use of a multi-scale, multi-type neighborhood (Blomley et al., 2016) as e.g. given in Figure 2. The multi-scale, multi-type neighborhood used in the scope of this work comprises four cylindrical neighborhoods $\mathcal{N}_{\mathrm{c}}$ with radii of $1 \mathrm{~m}, 2 \mathrm{~m}, 3 \mathrm{~m}$ and $5 \mathrm{~m}$ (Niemeyer et al., 2014; Schmidt et al., 2014), and a spherical neighborhood $\mathcal{N}_{k, \text { opt }}$ formed by the $k$ closest neighbors, whereby the scale parameter $k$ is derived via eigenentropy-based scale selection (Weinmann et al., 2015a; Weinmann, 2016) and may hence be individual for each 3D point $\mathbf{X}$.

\subsection{Extraction of Geometric Features}

The derived multi-scale, multi-type neighborhood serves as the basis for extracting features. In the following, we distinguish between two feature types. Shape measures describe features that comprise a single value each, whereby the value specifies one (mathematical) property of the whole set of 3D points within the evaluated local neighborhood (Section 3.2.1). As a consequence, such features are typically interpretable. In contrast, shape distributions are characterized by the fact that each feature is represented by a collection of values e.g. in the form of histograms (Section 3.2.2), where single values are hardly interpretable. Finally, all features are concatenated to a respective feature vector and a subsequent normalization is carried out so that each feature may contribute approximately the same, independent of its unit and its range of values. For more details on the applied normalization, we refer to (Blomley et al., 2016).
3.2.1 Shape Measures: We follow (Weinmann et al., 2015a) and define a variety of geometric 3D properties as features:

- Height: $H=Z$

- Local point density: $D=\frac{\# \text { 3D points within the local neighborhood }}{\text { volume of the local neighborhood }}$

- Verticality: $V=1-\mathbf{n}_{Z}$ where $\mathbf{n}$ is the normal vector

- Maximum height difference $\Delta H$ within the neighborhood

- Standard deviation of height values $\sigma_{H}$ within the neighborhood

For the spherical neighborhood $\mathcal{N}_{k, \text { opt }}$ whose scale parameter has been determined via eigenentropy-based scale selection, we additionally consider the radius $R$ of the considered local neighborhood.

Furthermore, we extract covariance features (West et al., 2004; Pauly et al., 2003) which are derived from the normalized eigenvalues $\lambda_{i}(i \in\{1,2,3\})$ of the $3 \mathrm{D}$ structure tensor, where $\lambda_{1} \geq$ $\lambda_{2} \geq \lambda_{3} \geq 0$ and $\lambda_{1}+\lambda_{2}+\lambda_{3}=1$ :

- Linearity: $L_{\lambda}=\frac{\lambda_{1}-\lambda_{2}}{\lambda_{1}}$

- Planarity: $P_{\lambda}=\frac{\lambda_{2}-\lambda_{3}}{\lambda_{1}}$

- Sphericity: $S_{\lambda}=\frac{\lambda_{3}}{\lambda_{1}}$

- Omnivariance: $O_{\lambda}=\sqrt[3]{\lambda_{1} \lambda_{2} \lambda_{3}}$

- Anisotropy: $A_{\lambda}=\frac{\lambda_{1}-\lambda_{3}}{\lambda_{1}}$

- Eigenentropy: $E_{\lambda}=-\lambda_{1} \ln \left(\lambda_{1}\right)-\lambda_{2} \ln \left(\lambda_{2}\right)-\lambda_{3} \ln \left(\lambda_{3}\right)$

- Sum of eigenvalues: $\Sigma_{\lambda}=\lambda_{1}+\lambda_{2}+\lambda_{3}$

- Local surface variation: $C_{\lambda}=\frac{\lambda_{3}}{\lambda_{1}+\lambda_{2}+\lambda_{3}}$

3.2.2 Shape Distributions: Originally, this type of sampled features has been proposed to describe the shape of a complete object (Osada et al., 2002). The used adaptation of shape distributions to describe geometric properties of the local neighborhood around a considered 3D point $\mathbf{X}$ has been presented in (Blomley et al., 2014).

Generally, shape distributions are histograms of shape values, which may be derived from random point samples by applying (distance or angular) metrics as shown in Figure 3:

- A3: angle between any three random points

- D1: distance of one random point from the centroid of all points within the neighborhood

- D2: distance between two random points

- D3: square root of the area spanned by a triangle between three random points

- D4: cubic root of the volume spanned by a tetrahedron between four random points

Since the histogram counts of randomly sampled shape values within each local neighborhood constitute the feature values, appropriate histogram binning thresholds and a suitable number of random pulls are crucial prerequisites. Following (Blomley et al., 2014), we select 10 histogram bins, i.e. 10 feature values will be produced from each metric, and we perform 255 pulls from the local neighborhood. The binning thresholds of the histogram are estimated by applying the adaptive histogram binning procedure presented in the aforementioned reference. 


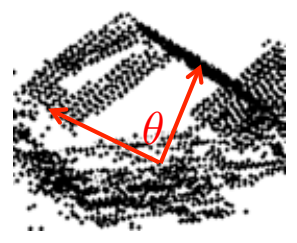

A3

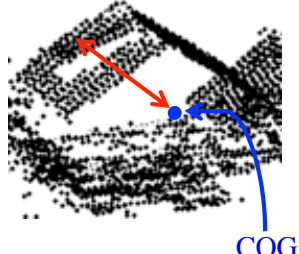

D1

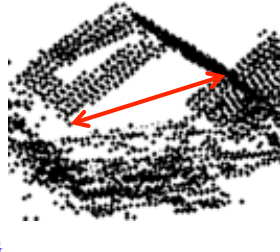

D2

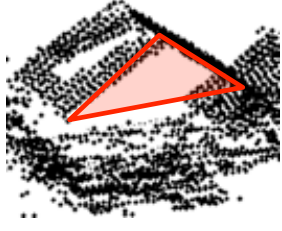

D3

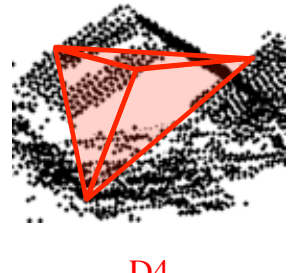

D4

Figure 3. Visualization of the considered shape distribution metrics: angle between any three random points (A3), distance of one random point from the centroid of all points within the neighborhood (D1), distance between two random points (D2), square root of the area spanned by a triangle between three random points (D3), and cubic root of the volume spanned by a tetrahedron between four random points (D4).

\subsection{Supervised Classification of Feature Vectors}

For each considered 3D point $\mathbf{X}$, all features are concatenated to a respective feature vector and provided as input for classification. To obtain general conclusions about the suitability of using multi-scale, multi-type neighborhoods as the basis for feature extraction, we involve several classifiers relying on different learning principles.

3.3.1 Nearest Neighbor Classifier: This classifier relies on the principle of instance-based learning, where each feature vector in the test set is directly compared to the feature vectors in the training set and the class label of the most similar training example is assigned. As similarity metric, we use the Euclidean distance.

3.3.2 Linear Discriminant Analysis Classifier: This classifier relies on the principle of probabilistic learning, where the aim is to derive an explicit underlying probabilistic model and infer the most probable class label for each observed feature vector. For this purpose, a multivariate Gaussian distribution is fitted to the given training data, i.e. the parameters of a Gaussian distribution are estimated for each class by parameter fitting. Thereby, the same covariance matrix is assumed for each class and only the means may vary.

3.3.3 Quadratic Discriminant Analysis Classifier: This classifier is very similar to the Linear Discriminant Analysis classifier. The difference consists in the fact that not only the means but also the covariance matrices may vary for different classes.

3.3.4 Random Forest Classifier: This classifier relies on the principle of ensemble learning, where the aim is to strategically combine a set of weak learners to form a single strong learner. More specifically, the combination of decision trees as weak learners is realized in a rather intuitive way via bagging (Breiman, 1996) which focuses on training a weak learner of the same type for different subsets of the training data which are randomly drawn with replacement. Accordingly, the weak learners are all randomly different from each other and, hence, taking the majority vote across the hypotheses of all weak learners results in a generalized and robust hypothesis of a single strong learner (Breiman, 2001).

\section{EXPERIMENTAL RESULTS}

In the following, we briefly describe the involved dataset (Section 4.1) and the conducted experiments (Section 4.2), before presenting the results achieved with our framework (Section 4.3).

\begin{tabular}{lrr}
\hline Class & Training Set & Test Set \\
\hline Powerline & 546 & N/A \\
Low Vegetation & 180,850 & N/A \\
Impervious Surfaces & 193,723 & N/A \\
Car & 4,614 & N/A \\
Fence / Hedge & 12,070 & N/A \\
Roof & 152,045 & N/A \\
Façade & 27,250 & N/A \\
Shrub & 47,605 & N/A \\
Tree & 135,173 & N/A \\
\hline$\sum$ & 753,876 & 411,722 \\
\hline
\end{tabular}

Table 1. Number of 3D points per class. Note that the reference labels are only provided for the training set and not available for the test set.

\subsection{Dataset}

For evaluating the performance of our framework, we use the Vaihingen dataset (Cramer, 2010) - an airborne laser scanning dataset acquired with a Leica ALS50 system over Vaihingen, a small village in Germany. The acquired data corresponds to areas with small multi-story buildings and many detached buildings surrounded by trees. The Vaihingen dataset has been presented in the scope of the ISPRS Test Project on Urban Classification and 3D Building Reconstruction (Rottensteiner et al., 2012), and it meanwhile serves as benchmark dataset for the ISPRS benchmarks on 2D and 3D semantic labeling. More details about this dataset are provided on the ISPRS webpages ${ }^{1}$, where the dataset is also available upon request.

In the scope of the ISPRS benchmark on 3D semantic labeling, nine semantic classes have been defined for the Vaihingen dataset, and these classes are given by Powerline, Low Vegetation, Impervious Surfaces, Car, Fence / Hedge, Roof, Façade, Shrub and Tree. The point-wise reference labels have been determined based on (Niemeyer et al., 2014). The Vaihingen dataset is split into a training set and a test set (see Table 1). The training set is visualized in Figure 1 and contains the spatial $X Y Z$-coordinates, reflectance information, the number of returns and the reference labels. For the test set, only the spatial $X Y Z$-coordinates, reflectance information and the number of returns are provided.

\subsection{Experiments}

The experiments focus on the use of the presented multi-scale, multi-type neighborhood (Section 3.1) for extracting metrical features given by shape measures and distribution features given by shape distributions (Section 3.2) which, in turn, are concatenated to a feature vector serving as input for a respective classifier (Section 3.3).

\footnotetext{
${ }^{1}$ see http://www2.isprs.org/commissions/comm3/wg4/3dsemantic-labeling.html (Accessed: 11 May 2016)
} 


\begin{tabular}{lrrrr}
\hline Metric & NN & LDA & QDA & RF \\
\hline OA & 45.07 & 50.19 & 38.11 & 41.52 \\
$\kappa$ & 32.08 & 38.30 & 27.66 & 30.28 \\
MCR & 38.14 & 49.09 & 38.65 & 41.30 \\
\hline
\end{tabular}

Table 2. Derived classification results for the NN classifier, the LDA classifier, the QDA classifier and the RF classifier: overall accuracy (OA), Cohen's kappa coefficient $(\kappa)$ and mean class recall (MCR).

For the training phase, we take into account that an unbalanced distribution of training examples per class might have a detrimental effect on the training process (Chen et al., 2004; Criminisi and Shotton, 2013). Accordingly, we introduce a class re-balancing by randomly sampling the same number of training examples per class to obtain a reduced training set. For our experiments, a reduced training set comprising 10,000 training examples per class has proven to yield results of reasonable quality. Note that this results in a duplication of training examples for those classes represented by less than 10,000 training examples. Additionally, it has to be considered that the training process might also rely on different parameters. Whereas the Nearest Neighbor (NN) classifier, the Linear Discriminant Analysis (LDA) classifier and the Quadratic Discriminant Analysis (QDA) classifier do not require parameter tuning, the Random Forest (RF) classifier involves several parameters (such as the number $N_{T}$ of decision trees to be used for classification, the minimum allowable number $n_{\min }$ of training points for a tree node to be split, the number $n_{a}$ of active variables to be used for the test in each tree node, etc.) which have to be selected by the user, e.g. by combining a grid search on a suitable subspace with cross-validation.

For the test phase, we use the trained classifiers to assign class labels to those $3 \mathrm{D}$ points of the test set. The spatial $X Y Z$ coordinates and the estimated labels have been provided to the organizers of the ISPRS benchmark on 3D semantic labeling who performed the evaluation. As evaluation metrics, we consider the overall accuracy (OA), Cohen's kappa coefficient $(\kappa)$ and the mean class recall (MCR). Furthermore, we take into account the class-wise evaluation metrics of recall, precision and $F_{1}$-score.

\subsection{Results}

The classification results obtained with the four considered classifiers are visualized in Figures 4 and 5, and they clearly reveal a different behavior. This also becomes visible when looking at the derived values for different evaluation metrics relying on the whole dataset (see Table 2) or at the derived values for the class-wise evaluation metrics (see Tables 3, 4 and 5). The classification metrics of overall accuracy (OA), Cohen's kappa coefficient $(\kappa)$ and mean class recall (MCR) indicate that the Linear Discriminant Analysis (LDA) classifier achieves the best performance $(\mathrm{OA}=50.19 \%, \kappa=38.30 \%$ and $\mathrm{MCR}=49.09 \%)$ for our application, and a look on the respective processing times required for training and testing (see Table 6) reveals that using a LDA classifier is also favorable in this regard. Note that the RF classifier is the only one of these classifiers which relies on a parameter tuning, i.e. several parameters have to be determined via a heuristic grid search (here: $N_{T}=2,000, n_{\min }=1, n_{a}=3$ ).

\section{DISCUSSION}

Based on the derived results, we may easily get an impression about the pros and cons of the proposed framework for the classification of ALS point clouds (Section 5.1). To also account for other strategies for a semantic labeling of $3 \mathrm{D}$ point clouds, we subsequently provide a more general discussion on the pros and cons of point-wise and segment-wise approaches (Section 5.2).

\begin{tabular}{lrrrr}
\hline Class & NN & LDA & QDA & RF \\
\hline Powerline & 50.33 & 89.33 & 40.33 & 74.33 \\
Low Vegetation & 16.81 & 12.37 & 2.29 & 4.45 \\
Impervious Surfaces & 40.21 & 47.63 & 69.37 & 54.32 \\
Car & 11.43 & 28.88 & 35.03 & 22.11 \\
Fence / Hedge & 18.43 & 20.44 & 45.45 & 21.75 \\
Roof & 69.88 & 80.70 & 35.82 & 56.06 \\
Façade & 34.90 & 51.26 & 50.94 & 50.53 \\
Shrub & 31.14 & 38.39 & 10.19 & 21.60 \\
Tree & 70.12 & 72.76 & 58.41 & 66.59 \\
\hline
\end{tabular}

Table 3. Class-wise recall values (in \%) corresponding to the classification results provided in Table 2.

\begin{tabular}{lrrrr}
\hline Class & NN & LDA & QDA & RF \\
\hline Powerline & 7.61 & 3.03 & 1.46 & 0.87 \\
Low Vegetation & 40.70 & 53.25 & 44.43 & 50.58 \\
Impervious Surfaces & 74.25 & 84.98 & 65.51 & 78.18 \\
Car & 12.65 & 31.36 & 6.12 & 13.12 \\
Fence / Hedge & 8.26 & 13.18 & 5.35 & 7.75 \\
Roof & 47.88 & 48.63 & 59.92 & 47.94 \\
Façade & 17.47 & 36.75 & 16.07 & 19.33 \\
Shrub & 23.61 & 28.25 & 20.91 & 33.62 \\
Tree & 49.03 & 57.46 & 37.29 & 44.44 \\
\hline
\end{tabular}

Table 4. Class-wise precision values (in \%) corresponding to the classification results provided in Table 2 .

\begin{tabular}{lrrrr}
\hline Class & NN & LDA & QDA & RF \\
\hline Powerline & 13.21 & 5.85 & 2.81 & 1.73 \\
Low Vegetation & 23.79 & 20.07 & 4.35 & 8.19 \\
Impervious Surfaces & 52.17 & 61.05 & 67.39 & 64.10 \\
Car & 12.01 & 30.07 & 10.42 & 16.47 \\
Fence / Hedge & 11.41 & 16.03 & 9.58 & 11.43 \\
Roof & 56.82 & 60.69 & 44.84 & 51.68 \\
Façade & 23.29 & 42.81 & 24.43 & 27.96 \\
Shrub & 26.86 & 32.55 & 13.70 & 26.30 \\
Tree & 57.71 & 64.21 & 45.52 & 53.30 \\
\hline
\end{tabular}

Table 5. Class-wise $F_{1}$-scores (in $\%$ ) corresponding to the classification results provided in Table 2 .

\subsection{The Proposed Framework}

The main goal of the proposed framework consists in the use of geometric features extracted from multi-scale, multi-type neighborhoods to allow a semantic reasoning on point-level. The use of contextual information or additional non-geometric features is not in the scope of this paper, since we intend to obtain insights on the neighborhoods and the features, both representing important prerequisites for achieving adequate classification results.

Our results with relatively low numbers for different evaluation metrics indicate that the Vaihingen dataset with a labeling with respect to nine semantic classes represents a rather challenging dataset when focusing on a 3D semantic labeling. To a certain degree, this might be due to the fact that some classes might not be representatively covered in the training data - e.g. the class Powerline with only 546 given training examples and the class Car with 4,614 given training examples (see Table 1) - which, in turn, yields poor classification results for these classes.

Furthermore, the derived results indicate that only considering geometric features might not be sufficient for obtaining adequate classification results for all considered classes, since some of these classes might have a quite similar geometric behavior, e.g. the classes Low Vegetation, Fence / Hedge and Shrub. This indeed becomes visible in Figures 4 and 5 where particularly misclassifications among these three classes may be observed for different 

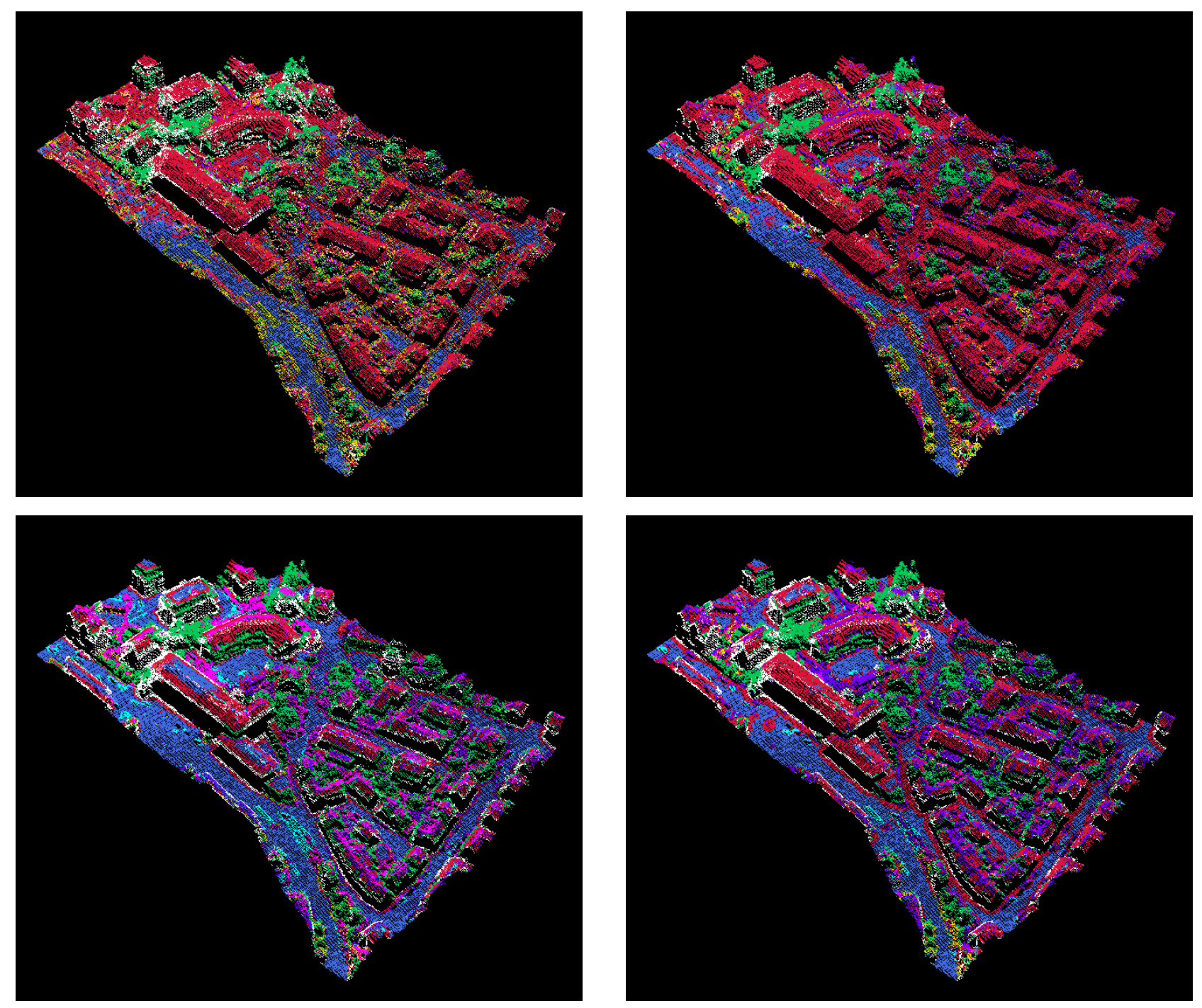

Figure 4. Derived classification results for the NN classifier (top left), the LDA classifier (top right), the QDA classifier (bottom left) and the RF classifier (bottom right) when using the same color encoding as in Figure 1 (Powerline: violet; Low Vegetation: yellowish green; Impervious Surfaces: royalblue; Car: cyan; Fence / Hedge: pink; Roof: crimson; Façade: white; Shrub: gold; Tree: emerald-green).

\begin{tabular}{lrr}
\hline Classifier & $t_{\text {train }}$ & $t_{\text {test }}$ \\
\hline NN & - & $3.30 \mathrm{~h}$ \\
LDA & $18.41 \mathrm{~s}$ & $32.62 \mathrm{~s}$ \\
QDA & $12.17 \mathrm{~s}$ & $97.87 \mathrm{~s}$ \\
RF & $27.36 \mathrm{~s}$ & $153.73 \mathrm{~s}$
\end{tabular}

Table 6 . Required processing times $t_{\text {train }}$ for the training process and $t_{\text {test }}$ for the classification of the test set when using the $\mathrm{NN}$ classifier, the LDA classifier, the QDA classifier and the RF classifier. Note that there is no training process for the NN classifier, since the induction is delayed to the test phase and quite timeconsuming due to the comparison of each feature vector of the test set with each feature vector in the training set.

classifiers. Yet, also the extracted geometric features may not be optimal as some of the neighborhoods used as the basis for feature extraction are relatively large, e.g. the cylindrical neighborhoods with radii of $3 \mathrm{~m}$ and $5 \mathrm{~m}$ which have also been used in (Niemeyer et al., 2014; Schmidt et al., 2014). This, in turn, results in misclassifications at particularly those locations where the cylindrical neighborhood includes 3D points associated to the classes Roof and Impervious Surfaces.

A closer look on the classification results provided in Figures 4 and 5 also reveals seam effects where borders between roofs and façades or between façades and ground are largely categorized into the class Façade, particularly for the QDA classifier and the RF classifier. Furthermore, the QDA classifier provides the best recognition of Impervious Surfaces, while the classification results are rather poor for the classes Low Vegetation, Fence / Hedge and Shrub. In contrast, the LDA classifier provides a good recognition for the classes Roof and Tree, while problems in the separation between Impervious Surfaces and Roof become visible.

\subsection{Point-Wise vs. Segment-Wise Approaches}

In the scope of this paper, we focus on a point-wise classification of $3 \mathrm{D}$ point clouds, since a consideration on point-level results in simplicity and efficiency. The same features are extracted for all $3 \mathrm{D}$ points based on their local neighborhood, and the classifiers are rather intuitive and easy-to-use. Furthermore, the whole process of feature extraction and classification can be highly parallelized. As a result, we efficiently obtain a labeling of reasonable accuracy. Due to the use of standard classifiers for classifying each 3D point based on the respective feature vector, we may expect a slightly noisy appearance of the classified 3D point cloud - which indeed becomes visible in Figures 4 and 5 - since these classifiers do not account for a spatial correlation between class 

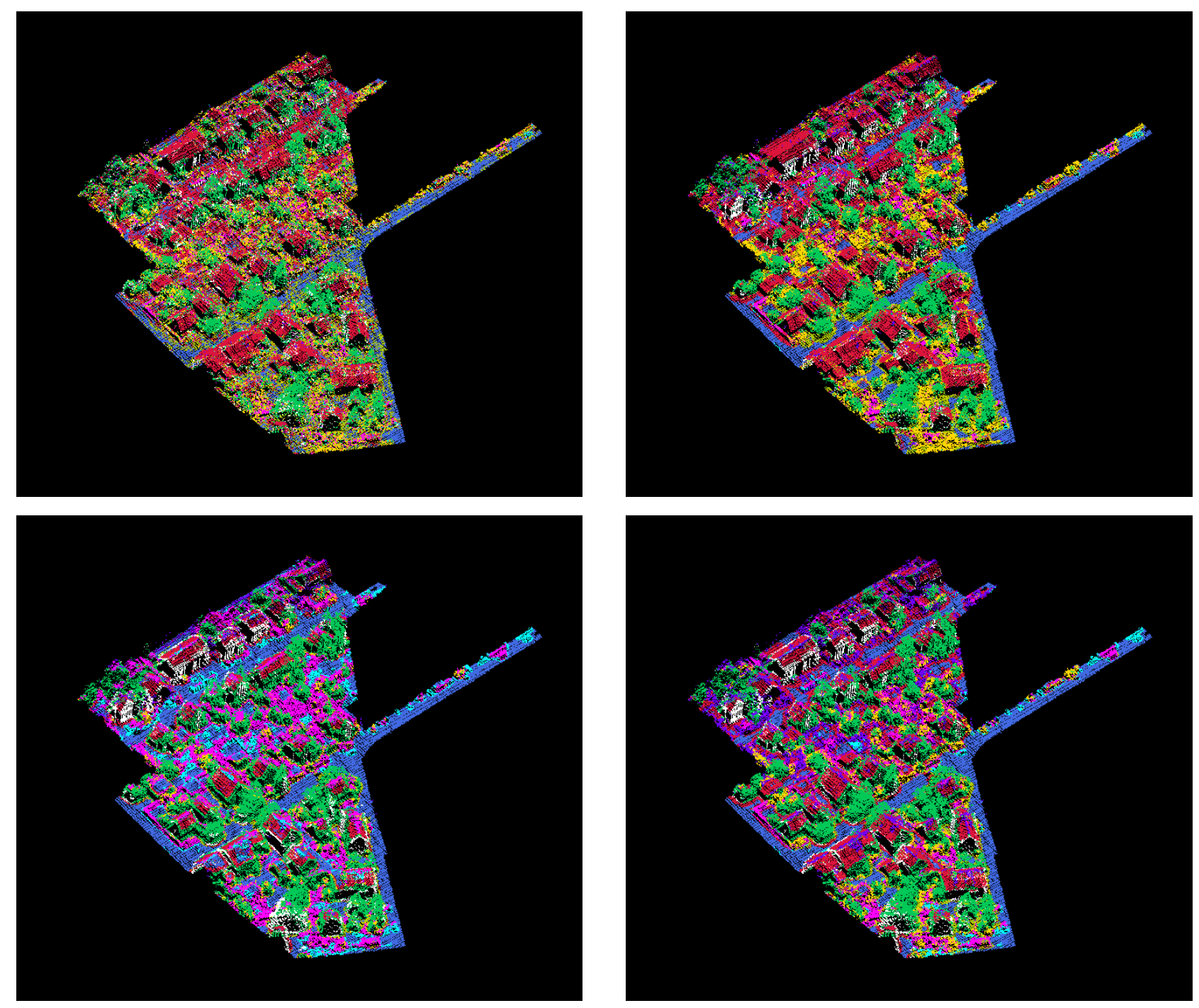

Figure 5. Derived classification results for the NN classifier (top left), the LDA classifier (top right), the QDA classifier (bottom left) and the RF classifier (bottom right) when using the same color encoding as in Figure 1 (Powerline: violet; Low Vegetation: yellowish green; Impervious Surfaces: royalblue; Car: cyan; Fence / Hedge: pink; Roof: crimson; Façade: white; Shrub: gold; Tree: emerald-green).

labels of neighboring 3D points. Such a spatial correlation could for instance be considered by applying approaches for contextual classification such as Conditional Random Fields which are e.g. used in (Niemeyer et al., 2014; Weinmann et al., 2015b).

In contrast, a consideration of 3D point clouds on a segmentlevel certainly relies on the use of an appropriate segmentation approach in order to provide a meaningful partitioning of a $3 \mathrm{D}$ point cloud into smaller, connected subsets which correspond to objects of interest or to parts of these (Melzer, 2007; Vosselman, 2013). Due to the complexity of real-world scenes and the objects therein, however, it often remains non-trivial to obtain a reasonable partitioning without either (i) using the result of an initial point-wise classification or (ii) integrating prior knowledge in the form of object models for those objects expected in the scene. Furthermore, it has to be taken into account that a fully generic segmentation typically results in a high computational effort.

\section{CONCLUSIONS}

In this paper, we have presented a framework for semantically labeling 3D point clouds acquired via airborne laser scanning. Our framework relies on the use of complementary types of geometric features extracted from multi-scale, multi-type neighborhoods as input for a standard classifier. Involving several classifiers with different learning principles, the results derived for a challenging dataset with nine classes indicate that geometric features allow to detect the classes Impervious Surfaces, Roof and Tree - which are characteristic for an urban environment - with an acceptable accuracy. However, there are also classes with a rather similar geometric behavior, e.g. the classes Low Vegetation, Fence / Hedge and Shrub which are not appropriately assigned in the derived classification results. One reason for this has been identified in the fact that some of the neighborhoods used as the basis for feature extraction are relatively large (e.g. the cylindrical neighborhoods with radii of $3 \mathrm{~m}$ and $5 \mathrm{~m}$ ) and thus cause many misclassifications due to a "smoothing" of details.

In future work, we intend to investigate potential sources for improving the classification results. This may include an extension of the presented framework by deriving further features (e.g. from reflectance information, the number of returns, etc.) and/or by considering contextual information inherent in the data. Furthermore, we plan to investigate steps from a semantic labeling of 3D point clouds on point-level to a semantic labeling on objectlevel in order to classify objects of interest and thus allow an object-based scene analysis. 


\section{ACKNOWLEDGEMENTS}

This work was partially supported by the Carl-Zeiss foundation [Nachwuchsförderprogramm 2014].

The Vaihingen dataset was provided by the German Society for Photogrammetry, Remote Sensing and Geoinformation (DGPF) (Cramer, 2010): http://www.ifp.uni-stuttgart.de/dgpf/ DKEP-Allg.html.

\section{REFERENCES}

Blomley, R., Jutzi, B. and Weinmann, M., 2016. Classification of airborne laser scanning data using geometric multi-scale features and different neighbourhood types. In: ISPRS Annals of the Photogrammetry, Remote Sensing and Spatial Information Sciences, Prague, Czech Republic, Vol. III-3, pp. 169-176.

Blomley, R., Weinmann, M., Leitloff, J. and Jutzi, B., 2014. Shape distribution features for point cloud analysis - A geometric histogram approach on multiple scales. In: ISPRS Annals of the Photogrammetry, Remote Sensing and Spatial Information Sciences, Zürich, Switzerland, Vol. II-3, pp. 9-16.

Breiman, L., 1996. Bagging predictors. Machine Learning 24(2), pp. 123-140.

Breiman, L., 2001. Random forests. Machine Learning 45(1), pp. 5-32. Brodu, N. and Lague, D., 2012. 3D terrestrial lidar data classification of complex natural scenes using a multi-scale dimensionality criterion: applications in geomorphology. ISPRS Journal of Photogrammetry and Remote Sensing 68, pp. 121-134.

Chehata, N., Guo, L. and Mallet, C., 2009. Airborne lidar feature selection for urban classification using random forests. In: The International Archives of the Photogrammetry, Remote Sensing and Spatial Information Sciences, Paris, France, Vol. XXXVIII-3/W8, pp. 207-212.

Chen, C., Liaw, A. and Breiman, L., 2004. Using random forest to learn imbalanced data. Technical Report, University of California, Berkeley, USA.

Cramer, M., 2010. The DGPF-test on digital airborne camera evaluation - Overview and test design. PFG Photogrammetrie - Fernerkundung Geoinformation 2 / 2010, pp. 73-82.

Criminisi, A. and Shotton, J., 2013. Decision forests for computer vision and medical image analysis. Advances in Computer Vision and Pattern Recognition, Springer, London, UK.

Demantké, J., Mallet, C., David, N. and Vallet, B., 2011. Dimensionality based scale selection in 3D lidar point clouds. In: The International Archives of the Photogrammetry, Remote Sensing and Spatial Information Sciences, Calgary, Canada, Vol. XXXVIII-5/W12, pp. 97-102.

Filin, S. and Pfeifer, N., 2005. Neighborhood systems for airborne laser data. Photogrammetric Engineering \& Remote Sensing 71(6), pp. 743 755 .

Guo, B., Huang, X., Zhang, F. and Sohn, G., 2015. Classification of airborne laser scanning data using JointBoost. ISPRS Journal of Photogrammetry and Remote Sensing 100, pp. 71-83.

Guo, L., Chehata, N., Mallet, C. and Boukir, S., 2011. Relevance of airborne lidar and multispectral image data for urban scene classification using random forests. ISPRS Journal of Photogrammetry and Remote Sensing 66(1), pp. 56-66.

Hu, H., Munoz, D., Bagnell, J. A. and Hebert, M., 2013. Efficient 3-D scene analysis from streaming data. In: Proceedings of the IEEE International Conference on Robotics and Automation, Karlsruhe, Germany, pp. 2297-2304.

Jutzi, B. and Gross, H., 2009. Nearest neighbour classification on laser point clouds to gain object structures from buildings. In: The International Archives of the Photogrammetry, Remote Sensing and Spatial Information Sciences, Hannover, Germany, Vol. XXXVIII-1-4-7/W5.

Khoshelham, K. and Oude Elberink, S. J., 2012. Role of dimensionality reduction in segment-based classification of damaged building roofs in airborne laser scanning data. In: Proceedings of the International Conference on Geographic Object Based Image Analysis, Rio de Janeiro, Brazil, pp. 372-377.

Lee, I. and Schenk, T., 2002. Perceptual organization of 3D surface points. In: The International Archives of the Photogrammetry, Remote Sensing and Spatial Information Sciences, Graz, Austria, Vol. XXXIV3A, pp. 193-198.
Linsen, L. and Prautzsch, H., 2001. Local versus global triangulations. In: Proceedings of Eurographics, Manchester, UK, pp. 257-263.

Lodha, S. K., Fitzpatrick, D. M. and Helmbold, D. P., 2007. Aerial lidar data classification using AdaBoost. In: Proceedings of the International Conference on 3-D Digital Imaging and Modeling, Montreal, Canada, pp. 435-442.

Lodha, S. K., Kreps, E. J., Helmbold, D. P. and Fitzpatrick, D., 2006. Aerial lidar data classification using support vector machines (SVM). In: Proceedings of the International Symposium on 3D Data Processing, Visualization, and Transmission, Chapel Hill, USA, pp. 567-574.

Mallet, C., Bretar, F., Roux, M., Soergel, U. and Heipke, C., 2011. Relevance assessment of full-waveform lidar data for urban area classification. ISPRS Journal of Photogrammetry and Remote Sensing 66(6), pp. S71S84.

Melzer, T., 2007. Non-parametric segmentation of ALS point clouds using mean shift. Journal of Applied Geodesy 1(3), pp. 159-170.

Mitra, N. J. and Nguyen, A., 2003. Estimating surface normals in noisy point cloud data. In: Proceedings of the Annual Symposium on Computational Geometry, San Diego, USA, pp. 322-328.

Niemeyer, J., Rottensteiner, F. and Soergel, U., 2014. Contextual classification of lidar data and building object detection in urban areas. ISPRS Journal of Photogrammetry and Remote Sensing 87, pp. 152-165.

Osada, R., Funkhouser, T., Chazelle, B. and Dobkin, D., 2002. Shape distributions. ACM Transactions on Graphics 21(4), pp. 807-832.

Pauly, M., Keiser, R. and Gross, M., 2003. Multi-scale feature extraction on point-sampled surfaces. Computer Graphics Forum 22(3), pp. 81-89. Rottensteiner, F., Sohn, G., Jung, J., Gerke, M., Baillard, C., Benitez, S. and Breitkopf, U., 2012. The ISPRS benchmark on urban object classification and 3D building reconstruction. In: ISPRS Annals of the Photogrammetry, Remote Sensing and Spatial Information Sciences, Melbourne, Australia, Vol. I-3, pp. 293-298.

Rusu, R. B., Marton, Z. C., Blodow, N. and Beetz, M., 2008. Persistent point feature histograms for 3D point clouds. In: Proceedings of the International Conference on Intelligent Autonomous Systems, BadenBaden, Germany, pp. 119-128.

Schmidt, A., Niemeyer, J., Rottensteiner, F. and Soergel, U., 2014. Contextual classification of full waveform lidar data in the Wadden Sea. IEEE Geoscience and Remote Sensing Letters 11(9), pp. 1614-1618.

Shapovalov, R., Velizhev, A. and Barinova, O., 2010. Non-associative Markov networks for 3D point cloud classification. In: The International Archives of the Photogrammetry, Remote Sensing and Spatial Information Sciences, Saint-Mandé, France, Vol. XXXVIII-3A, pp. 103-108.

Tombari, F., Salti, S. and Di Stefano, L., 2010. Unique signatures of histograms for local surface description. In: Proceedings of the European Conference on Computer Vision, Heraklion, Greece, Vol. III, pp. 356369.

Vosselman, G., 2013. Point cloud segmentation for urban scene classification. In: The International Archives of the Photogrammetry, Remote Sensing and Spatial Information Sciences, Antalya, Turkey, Vol. XL-7/W2, pp. 257-262.

Vosselman, G., Gorte, B. G. H., Sithole, G. and Rabbani, T., 2004. Recognising structure in laser scanner point clouds. In: The International Archives of the Photogrammetry, Remote Sensing and Spatial Information Sciences, Freiburg, Germany, Vol. XXXVI-8/W2, pp. 33-38.

Weinmann, M., 2016. Reconstruction and analysis of 3D scenes - From irregularly distributed $3 D$ points to object classes. Springer, Cham, Switzerland.

Weinmann, M., Jutzi, B. and Mallet, C., 2013. Feature relevance assessment for the semantic interpretation of 3D point cloud data. In: ISPRS Annals of the Photogrammetry, Remote Sensing and Spatial Information Sciences, Antalya, Turkey, Vol. II-5/W2, pp. 313-318.

Weinmann, M., Jutzi, B., Hinz, S. and Mallet, C., 2015a. Semantic point cloud interpretation based on optimal neighborhoods, relevant features and efficient classifiers. ISPRS Journal of Photogrammetry and Remote Sensing 105, pp. 286-304.

Weinmann, M., Schmidt, A., Mallet, C., Hinz, S., Rottensteiner, F. and Jutzi, B., 2015b. Contextual classification of point cloud data by exploiting individual 3D neighborhoods. In: ISPRS Annals of the Photogrammetry, Remote Sensing and Spatial Information Sciences, Munich, Germany, Vol. II-3/W4, pp. 271-278.

West, K. F., Webb, B. N., Lersch, J. R., Pothier, S., Triscari, J. M. and Iverson, A. E., 2004. Context-driven automated target detection in 3-D data. Proceedings of SPIE 5426, pp. 133-143.

Xu, S., Vosselman, G. and Oude Elberink, S., 2014. Multiple-entity based classification of airborne laser scanning data in urban areas. ISPRS Journal of Photogrammetry and Remote Sensing 88, pp. 1-15. 


\title{
MANGROVE CLASSIFCATION USING SUPPORT VECTOR MACHINES AND RANDOM FOREST ALGORITHM: A COMPARATIVE STUDY
}

\author{
F. Campomanes ${ }^{\text {a }}$, A. V. Pada ${ }^{\text {a }}$ J. Silapan ${ }^{\text {b }}$ \\ ${ }^{\text {a } U n i v e r s i t y ~ o f ~ t h e ~ P h l i p p i n e s ~ C e b u ~ P h i l-L i D A R ~ 2, ~ G o r o r d o ~ A v e n u e, ~ L a h u g, ~ C e b u ~ C i t y ~-~(e n z o . c a m p o m a n e s v, ~ a v s p a d a) @ g m a i l . c o m ~}$ \\ ${ }^{\mathrm{b}}$ University of the Phlippines Cebu, Gorordo Avenue, Lahug, Cebu City - jsilapan@hotmail.com
}

KEY WORDS: Mangroves, Support Vector Machine, Random Forest, LiDAR, OBIA

\begin{abstract}
:
Mangrove forest ecosystems fulfil a number of important functions like supporting the conservation of biological diversity by providing habitats, nurseries, and nutrients for animal species. In the Philippines, mangrove forests are declining due to the growth of aquaculture production. Mangrove forests are slowly being replaced by fishponds. An accurate inventory of what are left of these natural resources is important to know how we can conserve and manage them. This study aims to compare the performance of support vector machines (SVM) with random forest (RF) algorithm in automatically classifying mangrove forests using LiDAR data and orthophotographs in an object based approach. The site is a $36 \mathrm{sq} . \mathrm{km}$. coastal area in Manapla, Negros Occidental, Philippines. Various derivatives were created from the LiDAR data like the pit-free canopy height model (CHM) and intensity. The CHM was used in contrast split segmentation to distinguish between ground and non-ground objects. Only the non-ground objects were segmented further knowing that majority of mangroves are tall. Inventory of short mangroves is not yet included in this study. The non-ground objects were further segmented using multiresolution segmentation with the CHM and RGB bands of the orthophoto using a scale of 15 . The non-ground class was further separated into four classes namely: mangrove, built-up, other trees, and sugarcane. 120 training points and 30 validation points per class were collected by visual inspection using the orthophoto as reference. Several features of the training objects were computed from both the LiDAR and orthophoto derivatives and used for classification. SVM with radial basis function was used to classify the rest of the image and resulted in an overall accuracy of $95.83 \%$. For mangroves, its precision and recall reached $83.33 \%$ and $100 \%$, respectively. In the SVM classification, mangroves were confused with other trees and sugarcane. Another machine learning algorithm, random forest (RF) was used to classify the same area to compare their performance and accuracy. Using the same features, the RF classification achieved an overall accuracy of $99.1667 \%$. For mangroves, the RF classification obtained $100 \%$ and $96.70 \%$ for its precision and recall, respectively. The RF classifier confused other trees with mangroves which caused the error. The accuracies from both machine learning algorithms show that the RF classifier performed better than the SVM classifier and further implies the potential of using RF in classifying mangroves in other areas.
\end{abstract}

\section{INTRODUCTION}

Mangrove forests are very important coastal resources because of the many benefits it gives us especially in our socioeconomic development. It protects our coast from UV-B radiation, "green house" effects, as well as floods and sea level rise (Kathiresan, 2012). They are called the "rainforests of the seas" as they are the backbone of coastal and offshore food webs (Carter et al., 2015). Mapping of these mangroves is a vital input for mangrove management plans to be able to conserve these very important coastal resources.

There have been many approaches to mapping mangroves. Light detection and ranging (LiDAR) and optical remote sensing combined with the support vector machine (SVM) algorithm has been used to map and create a mangrove inventory (David, 2015; Pada, 2016). Other remotely sensed data like Landsat and ALOS-PALSAR have also been used to map mangroves with the random forest (RF) classification (Jhonnerie, 2015).

Comparisons of RF and SVM classification have been done before. RF was found to perform better than SVM in classifying electronic tongue data (Liu, 2013) and RF was also found to be steady and effective compared to SVM in predicting $\beta$-hairpin motifs in proteins (Jia, 2013). However, RF was outperformed by SVM in microarray-based cancer classification (Statnikov, 2008). For mangrove mapping using LiDAR and optical images, RF has not yet been used. Furthermore, a comparison of $\mathrm{SVM}$ and RF in mangrove mapping has not yet been done.

\section{OBJECTIVES}

This study aims to compare the performance of SVM and RF in classifying mangroves using LiDAR and optical imagery with an object based approach. It also aims to provide an alternative or maybe even better method to the currently popular method of using LiDAR and SVM approach.

\section{DATA AND METHODS}

\subsection{Study Area}

The study site that was selected for this study was an area in Manapla, Negros Occidental, Philippines where there are a lot of mangroves in the area based on local data and optical imagery. 


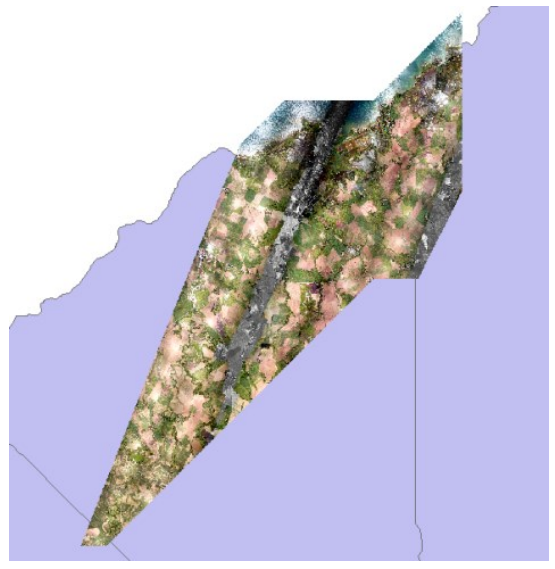

Figure 1. Orthophoto of the study site taken in June 2014. Intensity (from the LiDAR) is shown in areas where there is no orthophoto.

\subsection{Data Used}

LiDAR and orthophoto data was collected in June 2014 with a point density of 2 points/sq. meter at 1 meter resolution (UP TCAGP, 2015). Table 1 shows the details of the LiDAR and orthophoto data acquisition.

\begin{tabular}{|c|c|c|}
\hline Data Source & Resolution & Date Acquired \\
\hline LiDAR & $1 \mathrm{~m}$ & June 2014 \\
\hline Orthophoto & $0.5 \mathrm{~m}$ & June 2014 \\
\hline
\end{tabular}

Table 1. Summary of data sources, spatial resolution, and acquisition date.

\subsection{Training and Validation Points}

Training and validation points were collected through visual interpretation with the optical imagery and other derivatives as reference. Only four (4) classes were collected from the nonground objects namely builtup $(\mathrm{Bu})$, tall sugarcane $(\mathrm{SC} 3)$, other trees (OTr), and Mangroves (Mgr). A total of approximately 150 points per class were collected. By rule of thumb, $20 \%$ of the 150 points, which is 30 points, were used for validation and the rest for training. Table 3 shows the breakdown of points per class.

\begin{tabular}{|c|c|c|}
\hline & Class & Number of Points \\
\hline \multirow{4}{*}{ Training } & Builtup & 120 \\
\cline { 2 - 3 } & Tall Sugarcane & 116 \\
\cline { 2 - 3 } & Other Trees & 120 \\
\cline { 2 - 3 } & Mangroves & 119 \\
\hline \multirow{4}{*}{ Validation } & Builtup & 30 \\
\cline { 2 - 3 } & Tall Sugarcane & 30 \\
\cline { 2 - 3 } & Other Trees & 30 \\
\cline { 2 - 3 } & Mangroves & 30 \\
\hline
\end{tabular}

Table 2. Breakdown of number of points per class.

The training points were then converted to training samples which were used in the classification phase of the methodology.

\subsection{Image Segmentation}

The whole process flow for segmentation was implemented using eCognition Developer (Trimble, 2014). The CHM was used in the contrast split segmentation in determining the ground and non-ground objects as most mangroves are known to be tall. Only the non-ground objects were further segmented using the multiresolution segmentation algorithm (Trimble, 2014 ) with a scale of 15 , shape of 0.3 , compactness of 0.8 , and putting more weight on the canny edge of the CHM and normalized difference vegetation index (NDVI).

\subsection{Features used}

Several features from the LiDAR data and optical imagery were utilized for both SVM and RF algorithms. Among these are the red, green, and red bands from the orthophoto, digital terrain model (DTM), normalized digital surface model (nDSM), canopy height model (CHM), green-red vegetation index (GRVI). Table 2 shows all the 38 features used.

\begin{tabular}{|c|c|}
\hline Statistical Method & Feature \\
\hline Mean & $\begin{array}{c}\text { Red, Green, Blue, nDSM, } \\
\text { CHM, intensity, GRVI, DTM, } \\
\text { number of returns, }\end{array}$ \\
\hline Standard Deviation & $\begin{array}{c}\text { Red, Green, Blue, nDSM, } \\
\text { intensity, number of returns }\end{array}$ \\
\hline GLCM Homogeneity & $*$ \\
\hline GLCM Entropy & $*$ \\
\hline GLCM Ang. 2 ${ }^{\text {nd }}$ moment & $*$ \\
\hline GLCM Standard Deviation & $*$ \\
\hline GLDV Entropy & nDSM, intensity \\
\hline
\end{tabular}

Table 3. Summary of features used. Features with "*” signifies "Red, Green, nDSM, GRVI, intensity".

\subsection{Support Vector Machine Classification}

SVM classification was done in eCognition with the use of the training samples using the mentioned features. Radial basis function was the desired kernel to be used due to the complexity of the training data and C parameter of the SVM was set to 1000 in order to lower bias and penalize misclassification heavily.

\subsection{Random Forest Classification}

The segmented training samples from eCognition were exported as a CSV with all 38 features and used as input in the random forest classification that was implemented in Weka 3.8 (Hall, 2009). Preprocessing was done to the training and test (remaining nonground objects) sets to convert them from CSV to ARFF which is the file format used by Weka. Using the training samples, a classification model was trained using RF with 100 iterations. The trained model was then used to classify the validation samples as well as the remaining test samples.

\subsection{Accuracy Assessment}

The same validation samples were used for both RF and SVM classification results. Accuracy assessment for the SVM result was done in eCognition by generating an error matrix based on samples. Accuracy assessment was done in Weka by using the trained RF model to classify the validation samples. 30 samples per class were classified for a total of 120 points for 4 classes. Precision, recall, and F-measure values were also calculated.

\section{RESULTS AND DISCUSSION}

\subsection{SVM Classification}

SVM was able to completely classify the rest of the image as implemented in eCognition. However, there was obvious misclassification of mangroves in the areas with missing orthophoto. This can be attributed to the fact that the RGB 
values of these areas are zero. Objects classified as mangroves were also still found in areas far from the coast and rivers. Also, for areas with fishponds and water, there is misclassification of builtup. This can be because of the triangulation that happens in the CHM or DSM in areas with water.

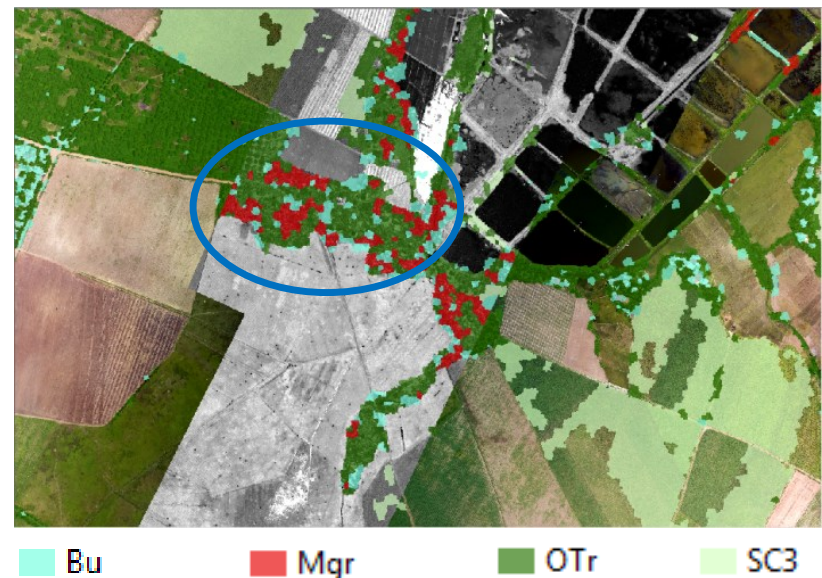

Figure 2. SVM classification result for subset area A. Builtup areas are misclassified as mangroves (encircled in blue).

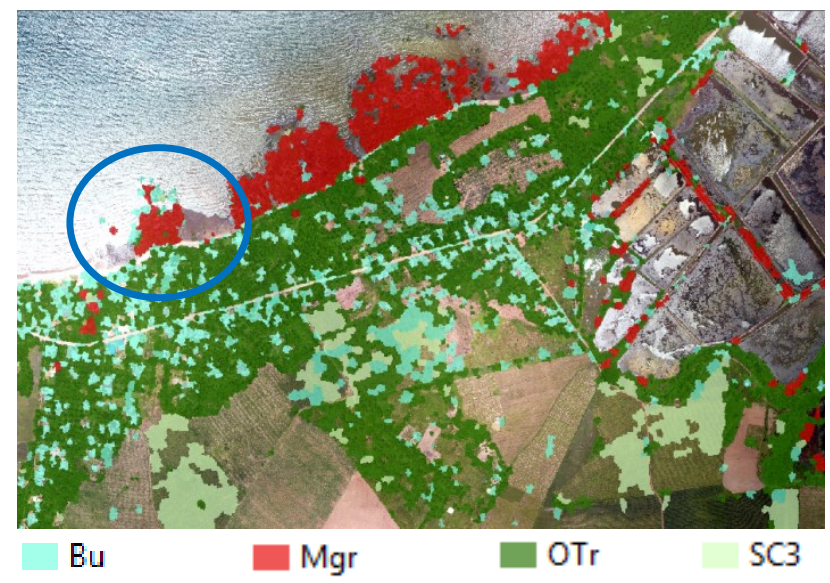

Figure 3. SVM classification result for subset area B.

Mangroves are misclassified as builtup in the upper left area (encircled in blue).

Aside from the mentioned misclassifications, the SVM classifier was generally able to classify mangroves correctly. Of the 30 validation samples for mangroves, 25 were classified correctly, 4 were classified as "Other Trees" or "OTr", and 1 was classified as "Tall Sugarcane" or "SC3". For the 3 remaining classes, all validation samples were classified correctly. 115 samples were correctly classified and 5 were misclassified. With this, an overall accuracy of $95.83 \%$ was obtained.

\begin{tabular}{|c|c|c|c|c|}
\hline Bu & Mgr & OTr & SC3 & \\
\hline 30 & & & & Bu \\
\hline & 25 & & & Mgr \\
\hline & 4 & 30 & & OTr \\
\hline & 1 & & 30 & SC3 \\
\hline
\end{tabular}

Table 4. Confusion matrix for SVM classification result.

\begin{tabular}{|c|c|c|c|}
\hline Class & Precision & Recall & F-Measure \\
\hline $\mathrm{Bu}$ & 1.00 & 1.00 & 1.00 \\
\hline $\mathrm{Mgr}$ & 1.00 & 0.83 & 0.90 \\
\hline $\mathrm{OTr}$ & 0.88 & 1.00 & 0.94 \\
\hline $\mathrm{SC} 3$ & 0.97 & 1.00 & 0.98 \\
\hline Mean & $\mathbf{0 . 9 6 2 5}$ & $\mathbf{0 . 9 5 7 5}$ & $\mathbf{0 . 9 6}$ \\
\hline
\end{tabular}

Table 5. Precision-Recall and F-Measure matrix for SVM classification result.

For the mangrove class, a precision of 1 was obtained while 0.83 was obtained for recall. An F-Measure value of 0.9 was calculated for mangroves. Taking into account all classes, generally higher values were obtained for precision, recall and F-measure at $0.9625,0.9575$, and 0.96 , respectively. Recall and F-measure values obtained for the mangrove class are quite low considering that the primary goal of the study is to correctly classify mangroves.

\subsection{RF Classification}

The trained RF model implemented in Weka was able to classify all objects. Similar to the SVM result, areas with water and fishponds have confusion between builtup and mangroves because of the triangulation of the CHM or DSM in the area.

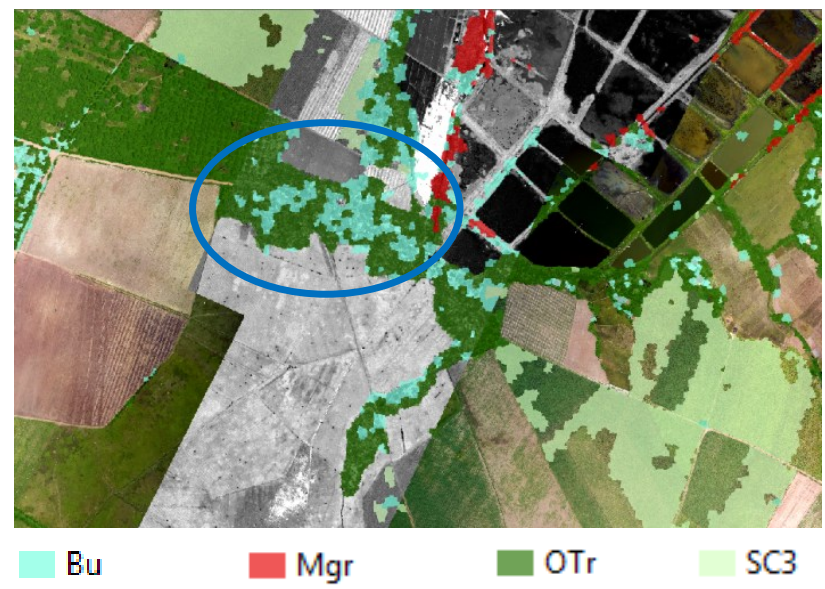

Figure 4. RF classification result for subset area A. Builtup areas are classified correctly, with no misclassification of mangroves.

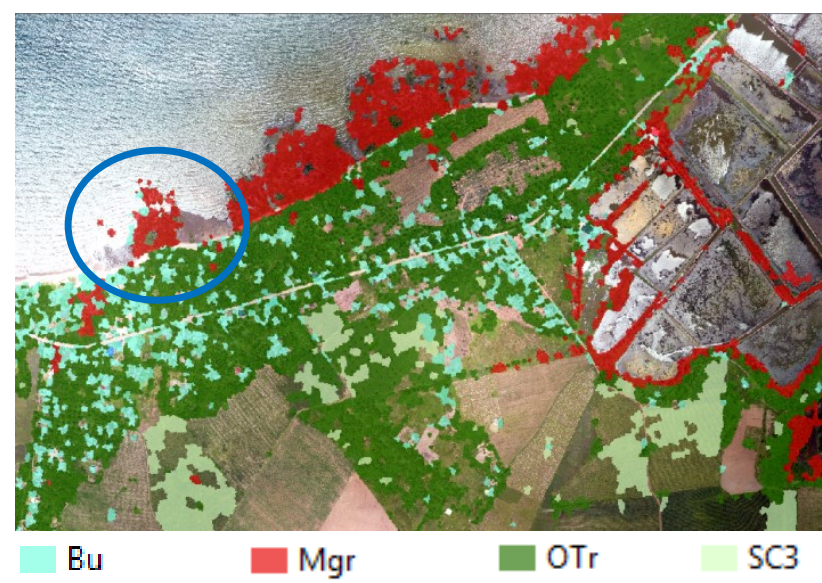

Figure 5 . RF classification result for subset area B. Less misclassification of mangroves as builtup is evident in the area encircled in blue compared to the SVM result.

In figure 4, builtup areas are correctly classified and there is no more confusion with mangroves in the area. Even with the lack 
of RGB information, the classifier was still able to correctly classify the objects. Figure 5 shows little to no misclassification of mangroves as builtup in the upper left portion of the image (encircled in blue). Also, the RF classification result had no errors with regards to mangroves in the non-coastal areas.

Of the 30 validation samples for mangroves, 29 were classified correctly and 1 was classified as "Other trees" or "OTr". Overall, 119 of 120 samples were classified correctly. An overall accuracy of $99.17 \%$ was obtained. Table 6 shows the confusion matrix for the RF classification result.

\begin{tabular}{|c|c|c|c|c|}
\hline Bu & Mgr & OTr & SC3 & \\
\hline 30 & & & & Bu \\
\hline & 29 & & & Mgr \\
\hline & 1 & 30 & & OTr \\
\hline & & & 30 & SC3 \\
\hline
\end{tabular}

Table 6. Confusion matrix for RF classification result.

\begin{tabular}{|c|c|c|c|}
\hline Class & Precision & Recall & F-Measure \\
\hline $\mathrm{Bu}$ & 1.00 & 1.00 & 1.00 \\
\hline $\mathrm{Mgr}$ & 1.00 & 0.97 & 0.98 \\
\hline $\mathrm{OTr}$ & 0.97 & 1.00 & 0.98 \\
\hline $\mathrm{SC} 3$ & 1.00 & 1.00 & 1.00 \\
\hline Mean & $\mathbf{0 . 9 9 2}$ & $\mathbf{0 . 9 9 2}$ & $\mathbf{0 . 9 9 2}$ \\
\hline
\end{tabular}

Table 7. Precision-Recall and F-Measure matrix for RF classification result.

The precision and recall values for the mangrove class are 1 and 0.97, respectively. An F-Measure of 0.98 was also calculated for mangroves. Overall, the RF classifier achieved high accuracies at 0.992 for precision, recall, and F-measure.

\subsection{Comparison of SVM and RF}

Table 8 shows the precision, recall and F-measure values per class and for both SVM and RF.

\begin{tabular}{|c|c|c|c|c|c|c|}
\hline & \multicolumn{2}{|c|}{ Precision } & \multicolumn{2}{c|}{ Recall } & \multicolumn{2}{c|}{ F-Measure } \\
\hline Class & SVM & RF & SVM & RF & SVM & RF \\
\hline $\mathrm{Bu}$ & 1.00 & 1.00 & 1.00 & 1.00 & 1.00 & 1.00 \\
\hline $\mathrm{Mgr}$ & 1.00 & 1.00 & 0.83 & 0.97 & 0.90 & 0.98 \\
\hline $\mathrm{OTr}$ & 0.88 & 0.97 & 1.00 & 1.00 & 0.94 & 0.98 \\
\hline $\mathrm{SC} 3$ & 0.97 & 1.00 & 1.00 & 1.00 & 0.98 & 1.00 \\
\hline Mean & $\mathbf{0 . 9 6 2 5}$ & $\mathbf{0 . 9 9 2}$ & $\mathbf{0 . 9 5 7 5}$ & $\mathbf{0 . 9 9 2}$ & $\mathbf{0 . 9 6}$ & $\mathbf{0 . 9 9 2}$ \\
\hline
\end{tabular}

Table 8. Comparison of accuracies obtained by SVM and RF classifiers.

After comparing the performance of the SVM and RF classifiers based on their obtained accuracies, it was found that RF performed better in the mangrove class as well as the other classes. The RF classifier got higher values in precision, recall, f-measure, and also achieved a higher overall accuracy of $99.17 \%$ (119 of 120) compared to the $95.83 \%$ (115 of 120) that the SVM classifier obtained. This could be because of the characteristic of SVM to have difficulty in training a good model if there are a lot of training samples. For mangrove mapping, there is a need for a lot of training samples to be able to differentiate classes especially mangroves from other trees. $\mathrm{RF}$, on the other hand, because of how they are constructed, are expected to handle a large number of training data.

\section{CONCLUSION AND RECOMMENDATIONS}

Based on the results, it can be said that mangroves can be classified using a trained RF model in an object based approach and should be tested in a different study site to check its applicability for different data. Furthermore, based on the data and performance of both classifiers, it can be said that both the SVM and RF classifiers performed well in classifying mangroves but it should be noted that RF performed better than SVM in areas with no orthophoto. Also, RF performed better in terms of accuracy as it obtained higher values in precision, recall, F-measure, and overall accuracy.

\section{ACKNOWLEDGEMENTS}

The authors would like to acknowledge the Department of Science and Technology (DOST) of the Philippines as the funding agency and the Department of Science and Technology - Philippine Council for Industry, Energy and Emerging Technology Research and Development (DOST-PCIEERD) as the monitoring agency.

\section{REFERENCES}

Carter, H., Schmidt, S., Hirons, A., 2015. An International Assessment of Mangrove Management: Incorporation in Integrated Coastal Zone Management, Diversity 7, pp. 74-104.

David, L. C., Ballado, A., 2015, Mapping Mangrove Forest from LiDAR Data using Object-Based Image Analysis and Support Vector Machine: The Case of Calatagan, Batangas, Humanoid, Nanotechnology, Information Technology, Communication and Control, Environment and Management, pp. 1-5.

Hall M., Frank, E., Holmes, G., Pfahringer, B., Reutemann, P., Witten, I., 2009. The WEKA Data Mining Software: An Update, SIGKDD Explorations, Vol. 11, Issue 1, pp. 10-18.

Jhonnerie, R., Siregar, V., Nababan, B., Prasetyo, L. B., Wouthuyzen, S., 2015. Random Forest Classification for Mangrove Land Cover Mapping Using Landsat 5 TM and ALOS PALSAR Imageries. Procedia Environmental Sciences 24, pp. 215-221.

Jia, S., Hu, X., Sun, L., 2013, The Comparison between Random Forest and Support Vector Machine Algorithm for Predicting $\beta$-Hairpin Motifs in Proteins. Engineering 5, pp. 391395.

Kathiresan K., 2012, Importance of Mangrove Ecosystem. International Journal of Marine Science, Vol. 2, No.10 pp. 7089.

Pada, A. V., Silapan, J., Cabanlit, M. A., Campomanes F., Garcia, J. J., 2016, Mangrove Forest Cover Extraction of the Coastal Areas of Negros Occidental, Western Visayas, Philippines using LiDAR Data, Int. Arch. Photogramm. Remote Sens. Spatial Inf. Sci. XLI-B1, pp. 73-79.

Statnikov, A., Wang. L., Aliferis, C., 2008, A Comprehensive Comparison of Random Forests and Support Vector Machines for microarray-based cancer classification. BMC Bioinformatics.

Trimble, 2014. eCognition Developer Reference Book, pp. 3239.

UP TCAGP, 2015, DREAM Lidar Data Acquisition and Processing for Lucena River Floodplain, Disaster Risk And Exposure Assessment for Mitigation Program, DOST Grants-inAid Program, 55 pp. 


\title{
ASSESSING DOWNSCALING LIMITS OF SPATIAL RESOLUTION FOR AWIFS AND LANDSAT 8 DATA AS COMPARED TO LISS IV
}

\author{
Chauhan A. ${ }^{\mathrm{a}}$, Denis. M. D ${ }^{\mathrm{b}^{*}}$, Kumar. $\mathrm{M}^{\mathrm{c}}$ \\ ${ }^{\mathrm{a}}$ Researcher akankshachauhan6392@gmail.com, ${ }^{\mathrm{b}}$ Professor (derrickmdenis@gmail.com), ${ }^{\mathrm{c} A s s i s t a n t ~ P r o f e s s o r, ~}$ \\ mukesh_fo@yahoo.co.in, Dept. of Soil, Water, Land Engineering and Management, Vaugh School of Agricultural \\ Engineering and Technology, Sam Higginbottom Institute of Agriculture Technology and Sciences, Allahabad, \\ Uttar Pradesh, India, 211007
}

KEY WORDS: Downscaling, AWiFS, LANDSAT8, LISS IV, Kappa coefficient

\begin{abstract}
Downscaling spatial information has a certain limit. Change in resolution of input image from its original resolution either loses or gains information. To understand this limit accuracy of the downscaled information has to be assessed. This research assesses the downscaled information of AWiFS and LANDSAT 8 as compared and validated by LISS IV. A total of 200 sample points were collected using Systematic Random Sampling. The results showed that the overall accuracy for the supervised classification was $95 \%$ for LISS IV where Kappa statistics was 0.92. As the resolution LANDSAT 8 was reduced from $10 \%$ to $50 \%$ the overall accuracy varied from $84 \%$ to $73.5 \%$ with 0.79 to 0.65 Kappa coefficient. For AWiFS at the same reducing resolutions, the overall accuracy varied from $80 \%$ to $67 \%$ with 0.72 to 0.56 kappa coefficients. The results showed that the downscaling limits for AWiFS and LANDSAT8 are only $10 \%$ of their actual resolutions. Any downscaling beyond this limit will result in precious information being lost.
\end{abstract}

\section{INTRODUCTION}

Downscaling refers to an increase in spatial resolution(Atkinson, 2012). Its affect can be observed in image classification (Althausen, 2002; Barnsley, 1999; Estes \& Loveland., 1999; Lefsky \& Cohen, 2003) The parameters for considering a particular resolution as input depends upon the user's needs, funds available and its availability as open source. In general, at a local scale, high spatial resolution data such as IKONOS and QuickBird data are helpful but expensive. At a regional scale, medium spatial resolution data such as those from Landsat TM and Terra Advanced Space borne Thermal Emission and Reflection Radiometer (ASTER) are the most frequently used and are available as open source. At a continental or global scale, coarse spatial resolution data such as Advanced Very High Resolution Radiometer (AVHRR), Moderate Resolution Imaging Spectroradiometer (MODIS), and System Pour l'Observation de la Terre (SPOT) vegetation data are preferable. Atmospheric condition such as clouds and vapor are often an obstacle for capturing highquality optical sensor data.

An image consists of an array of pixels or grid cells and many layers or bands. Each pixel has a Digital Number (DN) representing the intensity of the received signal reflected or emitted by a given area of the earth surface. The size of the area belonging to a pixel is called the spatial resolution. Further bands are created by the sensor that collects energy in specific wavelengths of the electro-magnetic spectrum.

*Corresponding author derrickmdenis@gmail.com
The classification of remotely sensed data has long attracted the attention of the remote sensing community. Scientists and practitioners have made great efforts in developing advanced classification approaches and techniques for improving classification accuracy (Aplin, Atkinson, \& Curran, 1999; Blaschke, 2010; Franklin, 2002; G.M.Foody, 1996; Gallego, 2004; Ghimire, Rogan, \& Miller, 2010; Gong.P \& Howarth, 1992; Kontoes, G.Wilkingson, A.Burril, S.Goffredo, \& Megier, 1993; Lu \& Weng, 2007; Pal \& Mather, 2003; San Miguel-Ayanz \& Biging, 1997; Stuckens, Coppin, \& Bauer, 2000) Classification of remotely sensed data generally produces discrete information categories. It is implicitly assumed that spectral class memberships are precisely defined, so that the attribution of a pixel to a land-cover category is always feasible (Curran \& Williamson, 1985). Implicit in the traditional classification process is the concept that each feature vector should be mapped into one of the classes of interest.

In reality some classes have very close spectral reflectance values and therefore they overlap in the feature space. Overlap can also indicate mistakes in the definition of the training sites. This produces spectral confusion. Hence it has to go through several iterations of training site adjustment, signature development, and signature evaluation before achieving satisfactory signatures. Scatter Plot is useful for evaluating the quality of one's signatures.

Today, validation or accuracy assessment is an integral component of most mapping projects in- corporating remotely sensed data. Other spatial information may not be so stringently evaluated, but at least requires meta-data that documents how the information was generated. In the 1980s, the use of the error matrix became a common tool for representing the accuracy of individual map categories. By 
the 1990s, most maps derived from remotely sensed imagery were required to meet some minimum accuracy standard. Accuracy assessment or validation is a key component of any project employing spatial data. This assessment is important to know how well you are doing and to learn from your mistakes, the ability to quantitatively compare methods and the ability to use the information resulting from spatial data analysis in some decision-making process.

A common method for accuracy assessment involves the use of an error matrix, for which the literature has provided the meanings of and calculation methods for overall accuracy, producer's accuracy, user's accuracy, and kappa coefficient (C.P.Smits, Dellepiane, \& Schowengerdt, 1999; G. R.

\section{MATERIALS AND METHOD}

\section{Datasets used}

Topo sheet of Allahabad district (63G15) was used as a raw material of 1:50,000 scales along with three separate datasets i.e. LISS IV, LANDSAT 8 and AWiFS. Each scene was geo referenced and suitable image enhancements are applied to facilitate the delineation and interpretation of different thematic information.The satellite data, Resourcesat-2 (LISS IV) of January 2013, LANDSAT 8 (OLI) of February 2014 and IRS P6 (AWiFS) of February 2010 for Allahabad were used in this study. These image encompasses of large areas of farmlands, residents, wetlands, some of water bodies and more. These datasets consists of different pixels and covers the study area. The advantage of these datasets is availability of the referenced image produced from field survey, which is used for accuracy purpose. Data collection is first step in any assessment procedure, and may be the single most important factor in accuracy assessment. Since an assessment will meaningless if the reference data cannot be trusted. After collecting all the data from various sources we were mosaic all scenes of all satellite data and the study area has been extracted from mosaiced scenes by using the boundary of as area of interest (AOI) tool. All together seven classes were identified and classified for both supervised and unsupervised classification. These classes are shown inTable 1.

\begin{tabular}{|l|l|l|}
\hline $\begin{array}{l}\text { Sr. } \\
\text { No. }\end{array}$ & Class & Associate Class \\
\hline 1. & Agriculture land & Cultivated land, Harvested land \\
\hline 2. & Agriculture crop & $\begin{array}{l}\text { Agricultural land, Grassland, } \\
\text { Turf and grass. }\end{array}$ \\
\hline 3. & $\begin{array}{l}\text { River/Water } \\
\text { Bodies }\end{array}$ & $\begin{array}{l}\text { Streams, Lakes, Reservoirs, } \\
\text { Ponds and canals. }\end{array}$ \\
\hline 4. & Barren Land & $\begin{array}{l}\text { Exposed soil, Bare exposed rock } \\
\text { and Transitional areas. }\end{array}$ \\
\hline 5. & Settlement & $\begin{array}{l}\text { Residential, Industrial, Industrial } \\
\text { and commercial complexes, Mixed } \\
\text { urban or built-up land and Road. }\end{array}$ \\
\hline 6. & Forest/Trees & $\begin{array}{l}\text { Evergreen forest land, Shrub } \\
\text { and bushes }\end{array}$ \\
\hline 7. & Sand & Bank of river \\
\hline
\end{tabular}

Table 1 Classes identified in the study area.

Preprocessing of satellite images prior to image classification was done. There after resampling was done to do the accuracy study. This was followed by supervised and unsupervised classification. The classification was done in five classes and given in Table 6. Scatter plots were obtained.
Congalton, 1991; R. G. Congalton \& Green, 2008; Foody, 2002) Since multiple sources of sensor data are now readily available, image analysts have more choices to select suitable remotely sensed data for a specific study. Considering these facts three varying resolution remotely sensed data i.e. Linear Imaging Self Scanner (LISS IV) having $5.8 \mathrm{~m}$ spatial resolution, LANDSAT $8,30 \mathrm{~m}$ spectral resolution and Advanced Wide Field Sensor (AWiFS), $56 \mathrm{~m}$ spectral resolution data were considered for accuracy assessment using error matrix ,producer's accuracy ,user's accuracy, overall accuracy and kappa coefficient to assess downscaling limits of spatial resolution for AWiFS, IANDSAT 8 as compared to LISS IV

\section{Accuracy Assessment}

Accuracy assessments are performed on the thematic information. . The percentage of overall accuracy was calculated using following formula:

Overall accuracy $=$ Total number of correct samples X 100 \% Total number of samples.

Besides the overall accuracy, classification accuracy of individual classes was calculated in a similar manner. The two approaches are user's accuracy and producer's accuracy. The producer's accuracy is derived by dividing the number of correct pixels in one class divided by the total number of pixels as derived from reference data. In this study, the producer's accuracy measures how well a certain area has been classified. It includes the error of omission which refers to the proportion of observed features on the ground that is not classified in the map. Meanwhile, user's accuracy is computed by dividing the number of correctly classified pixels in each category by the total number of pixels that were classified in that category.

Kappa coefficient $(\mathrm{K})$ is another measurement used in this study. It is calculated by multiplying the total number of pixels in all the ground verification classes $(\mathrm{N})$ by the sum of the confusion matrix diagonals ( Xkk ), subtracting the sum of the ground verification pixels in a class time the sum of the classified pixels in that class summed over all classes $(\Sigma \mathrm{Xk} \Sigma$ $\mathrm{Yk} \Sigma$ ), where $\mathrm{Xk} \Sigma$ is row total and $\mathrm{Yk} \Sigma$ is column total, and dividing by the total number of pixels squared minus the sum of the ground verification pixels in that class times the sum of the classified pixels in that class summed over all classes. The value of Kappa lies between 0 and 1, where 0 represents agreement due to chance only. 1 represents complete agreement between the two data sets. Negative values can occur but they are spurious. It is usually expressed as a percentage (\%). Kappa statistic can be a more sophisticated measurement to classifier agreement and thus gives better interclass discrimination than overall accuracy. The calculation of Kappa statistic $k \square$ is as follows: 


$$
\widehat{k}=\frac{N \sum_{i=1}^{r}\left(x_{i i}\right)-\sum_{i=1}^{r}\left(x_{i+} \times x_{+i}\right)}{N^{2}-\sum_{i=1}^{r}\left(x_{i+} \times x_{+i}\right)}
$$

Where,

$\mathrm{xij}=$ number of counts in the ijth cell of the confusion matrix

$\mathrm{N}=$ total number of counts in the confusion matrix

$\mathrm{xi}+=$ marginal total of row $\mathrm{i}$

$\mathrm{x}+\mathrm{i}=$ marginal total of column $\mathrm{i}$

\section{RESULT AND DISCUSSION}

Radiometric Normalization of LISS IV data is done to significantly reduce the mean absolute differences between reference scene pixels and corresponding pixels from subject scenes that may be used to fill cloud gaps, as expected. The selection of suitable band combination is essential for LISS IV satellite images for visual interpretation. This enhancement technique was carried out in order to produce a better image classification for digital classification. The images of Allahabad area appear much better after performed enhancement technique. Brightness and contrast level panels were used to adjust the image view to an appropriate level. The image after enhancement clearly showed the separation of the land cover features. Band combinations of 4-3-2 (RGB) for LISS IV was selected as the best combination and later used for digital classification. It has been seen that before histogram matching mean and standard deviation of reference scene is far from corrected scene. But after the histogram matching mean and standard deviation of corrected scene comes very close to mean and standard of reference scene. The statistics of LISS IV scenes before and after radiometric normalization are given in Table 2, Table 3 and Table 4.

Table 2: Statistics of LISS IV scenes before and after radiometric normalization for scene 1 and scene 3

\begin{tabular}{|l|c|c|c|}
\hline $\begin{array}{l}\text { Before Histogram } \\
\text { match }\end{array}$ & Band2 & Band3 & Band4 \\
\hline Std. dev.(scene1) & 38.65 & 36.48 & 56.28 \\
\hline Std. dev.(scene3) & 37.97 & 35.14 & 76.32 \\
\hline Mean (scene1) & 72.37 & 66.08 & 102.47 \\
\hline Mean (scene3) & 69.81 & 58.30 & 130.31 \\
\hline $\begin{array}{l}\text { After Histogram } \\
\text { match }\end{array}$ & & & \\
\hline Std. dev.(scene1) & 37.70 & 34.54 & 75.75 \\
\hline Mean (scene1) & 69.35 & 57.04 & 128.61 \\
\hline
\end{tabular}

Table 3: Statistics of LISS IV scenes before and after radiometric normalization for scene 2 and scene 3

\begin{tabular}{|l|c|c|c|}
\hline $\begin{array}{l}\text { Before Histogram } \\
\text { match }\end{array}$ & Band2 & Band3 & Band4 \\
\hline Std. dev.(scene2) & 44.95 & 39.62 & 54.62 \\
\hline Std. dev.(scene3) & 37.97 & 35.14 & 76.32 \\
\hline
\end{tabular}

\begin{tabular}{|l|c|c|c|}
\hline Mean (scene2) & 85.32 & 74.34 & 101.11 \\
\hline Mean (scene3) & 69.81 & 58.29 & 130.31 \\
\hline $\begin{array}{l}\text { After Histogram } \\
\text { match }\end{array}$ & & & \\
\hline Std. dev.(scene2) & 37.62 & 34.63 & 75.70 \\
\hline Mean (scene2) & 69.19 & 57.25 & 128.88 \\
\hline
\end{tabular}

Table 4: Statistics of LISS IV scenes before and after radiometric normalization for scene 4 and scene 3

\begin{tabular}{|l|c|c|c|}
\hline $\begin{array}{l}\text { Before Histogram } \\
\text { match }\end{array}$ & Band2 & Band3 & Band4 \\
\hline \multicolumn{1}{|c|}{ Std. dev.(scene4) } & 37.61 & 33.31 & 76.71 \\
\hline Std. dev.(scene3) & 37.97 & 35.14 & 76.32 \\
\hline Mean (scene4) & 67.42 & 54.22 & 132.42 \\
\hline Mean (scene3) & 69.81 & 58.29 & 130.31 \\
\hline $\begin{array}{l}\text { After Histogram } \\
\text { match }\end{array}$ & & & \\
\hline Std. dev.(scene4) & 37.60 & 34.70 & 75.69 \\
\hline Mean (scene4) & 69.09 & 57.44 & 128.80 \\
\hline
\end{tabular}

The histogram of the difference image with an ample amount of pixels in the tails clearly indicates changes.

Supervised Classification of LISS IV image was done after performing all corrections in the image. The image was classified with classes viz; river/water bodies, urban, agriculture land, agriculture crop, forest/trees, sand and barren land.. Area Statistics of land use land cover classes of supervised classification of LISS IV at $5.6 \mathrm{~m}$ are given in Table 5.

Table 5 Area Statistics of land use land cover classes of supervised classification of LISS IV

\begin{tabular}{|l|c|c|}
\hline \multicolumn{1}{|c|}{ Class } & $\begin{array}{l}\text { LISS IV @ } \\
\text { 5.6 mtr res }\end{array}$ & $\begin{array}{l}\text { LISS } \\
\text { IV@ 5.6 } \\
\text { mtr res }\end{array}$ \\
\hline Particular & Area(ha) & Area\% \\
\hline Barren land & 14848 & 2.924 \\
\hline River / Water bodies & 10384 & 2.045 \\
\hline Settlement & 4742.6 & 0.935 \\
\hline Agriculture land & 235724 & 46.423 \\
\hline Sand & 9980.61 & 1.966 \\
\hline Agriculture crop & 133385 & 26.268 \\
\hline Forest / Trees & 98706.6 & 19.439 \\
\hline Total & 507770.81 & $100 \%$ \\
\hline
\end{tabular}




\section{Scatter Plot of Supervised Image of LISS IV}

To compare the supervised classification results for LISS IV with scatter plot is presented, we have found for convince to perform them on the same number of Landcover classes (i.e.7 classes).The method has been implemented on the samples of multispectral images So when we draw a scatter plot for supervised classification it shows different classes as per the classification and the drawn plot shows how much the classification is correct. The closeness of different pixels is the sign of good classification and due to same reflectance of various pixels it may sometimes be overlaps and if scatter plot shows in scatter form and the pixels looks far away from one another it shows the low image quality or imperfect classification. Figure 1,2,3 shows the results of the SCATTER module, which represents the overall layout of reflectance values in the image. The signature plot shows the approximate range reflectance values for each class signature. The most problematic classes are the two overlapping ovals in the bottom of the plot. These represent build-up (the large green oval) and roads (the small blue oval), which are difficult to separate because of reasons mentioned above.

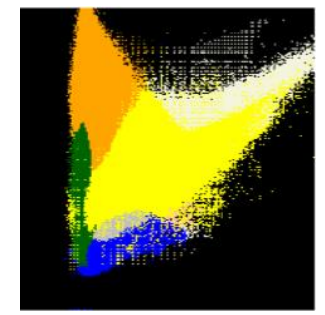

Figure 1 Scatter plot for Supervised classification of LISS IV for band 2 and 3

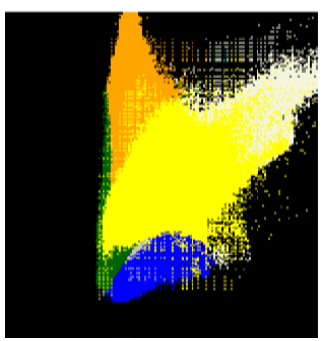

Figure 2 Scatter plot for Supervised classification of LISS IV for band 1 and 3

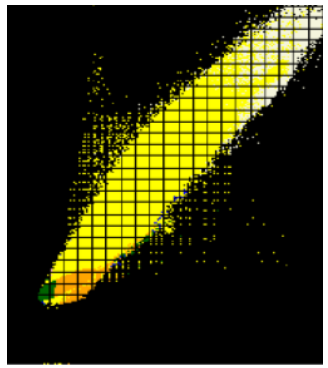

Figure 3 Scatter plot for Supervised classification of LISS IV for band 1 and 2

\section{Accuracy Assessment}

In this study, the number of reference points used for the accuracy assessment of classification were 200, most of which were taken from the field visit, and the remaining from the topographic maps. It can be shown in the table 4.20 that the overall classification accuracy for all three satellite images for both classification. Extraction of agriculture crops may be extracted more accurately. Very small indistinctive scattered urbanized areas couldn't be detected in unsupervised classification, and they were omitted from table of classification because of their insignificant effects. The accuracy of the land cover classification from supervised and unsupervised techniques were evaluated and presented as an error or confusion in the form of matrix table.

Evaluating the accuracy of the classification was done here by applying the accuracy assessment methods. A set of reference pixels is usually used where points on the classified image for which actual data are (or will be) known. The relationship between these two compared information is commonly summarized in an error matrix in appendix. The number of rows and columns in the error matrix should be equal to the number of categories whose classification accuracy is being assessed (R.M.Lillisand \& R.M.Kiefer, 2000)

The accuracy assessment generated from the supervised classification technique showed an overall classification accuracy was $95 \%$ with Kappa Statistic of 0.92 for LISS IV, which indicates a good agreement between thematic maps generated from image and the reference data. This amount of agreement is generally considered a good statistical return.

For instance, in unsupervised classification technique, firstly we were performed unsupervised classification and then we find out the accuracy for LANDSAT 8 and AWiFS at their different resolutions which were reduced at $10 \%$ from their original resolution.

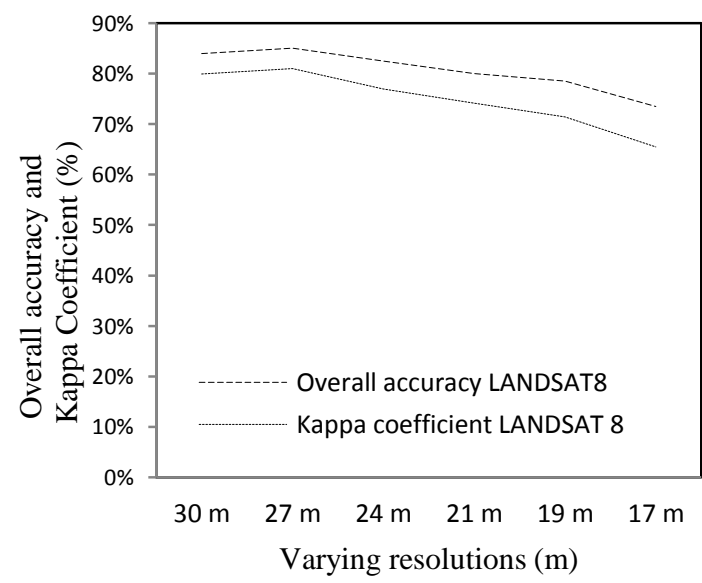

Figure 4 Change in Overall accuracy and Kappa Coefficient with respect to decrease in resolution for LANDSAT8 


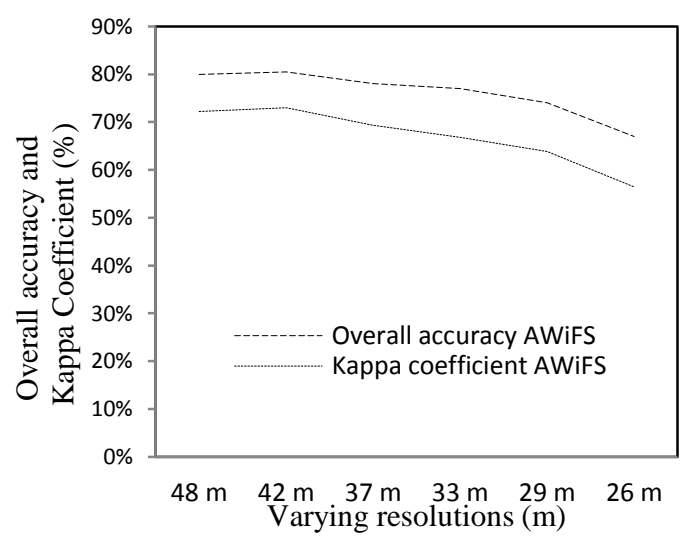

Figure 5 Change in Overall accuracy and Kappa Coefficient with respect to decrease in resolution for AWiFS

Likewise LANDSAT 8 with 30 m resolution, and a reduced resolution at $10 \%$ at $30,28,24,22,19,17 \mathrm{~m}$ respectively. At these resolutions the overall accuracy was $84 \%, 85 \%$, $82.5 \%, 80 \%, 78.5 \%, 73.5 \%$ with $0.79,0.80,0.76,0.74$, $0.71,0.65$ Kappa statistic respectively. Accordingly, AWiFS with $48 \mathrm{~m}$ resolution and a reduced resolution $10 \%$ the over all accuracy was $80 \%, 80.5 \%, 78 \%, 77 \%, 74 \%$, $67 \%$ with $0.72,0.72,0.69,0.66,0.63,0.37$ Kappa statistic respectively.

Table 6 presents the comparison of overall accuracy and Kappa statistic between the supervised and unsupervised classification of LISS IV, LANDSAT 8 and AWiFS at their original resolutions. It shows that the accuracy was increase at the reduction of $10 \%$ resolution, but after that the accuracy was get increased. The statistical approach of the accuracy assessment consists of different multivariate statistical analysis. A used measure is KAPPA (Cohen, 1960). KAPPA is designed to compare results from different regions or different classifications.

Table 6 Comparison of accuracy for supervised and unsupervised classification

\begin{tabular}{|c|c|c|}
\hline Method & $\begin{array}{c}\text { Overall } \\
\text { accuracy }\end{array}$ & Kappa \\
\hline Supervised (LISS IV) & $95 \%$ & 0.9256 \\
\hline $\begin{array}{c}\text { Unsupervised } \\
\text { (LANDSAT 8) }\end{array}$ & $84 \%$ & 0.7990 \\
\hline Unsupervised (AWiFS) & $80 \%$ & 0.7219 \\
\hline
\end{tabular}

REFERENCES

Althausen, J. D. (2002). What remote sensing system should be used to collect the data? In Manual of Geospatial Science and Technology, ed. J. D. Bossler, J. R. Jensen, R. B. McMaster and C. Rizos, 276. New York: Taylor \& Francis.

Aplin, P., Atkinson, P. M., \& Curran, P. J. (1999). Per-field classification of land use using the forthcoming very fine spatial resolution satellite sensors: problems and potential solutions. Advances in Remote Sensing and GIS Analysis
In Table 6 the Kappa statistic illustrated that kappa accuracy and coefficient was better by using supervised compare to unsupervised, where kappa coefficient is slightly higher for supervised with 0.92 and unsupervised with 0.79 and 0.72 .

The figures 4 and 5 shows the graphs between overall accuracy and resolutions for LANDSAT 8 and AWiFS shows the increase and decrease in the accuracy at different resolutions.

\section{Discussion}

This discussion summaries the extent of change in spatial resolution that can be accepted to produce classification maps with high accuracies. Overall the supervised classifications are the most appropriate to create land use land cover map of the study area. As the pixel merge or disaggregate, their spatial characteristic also change. Such as sand and settlement.

Here KAPPA (Cohen, 1960) is used very successfully to validate the results. It shows that that accuracy of a resolution is increased by $10 \%$ reduction. Any further increase will result in decreasing accuracy. Hence downscaling limits of spatial resolution for AWIFS and LANDSAT 8 as compared to LISS IV is up to $10 \%$ of their original resolutions, any resampling beyond this will lead to precious information being lost.

\section{CONCLUSION}

This paper has reviewed the factors and techniques to be considered when understanding the limits of resampling of remotely sensed data. Here in this study again it is established that the supervised classification is more appropriate technique to classify an image or satellite data. Unsupervised classification gives lesser results due to merging of pixel reflectance. The techniques and considerations discussed here need to be applied over all spatial data. Techniques developed for other spatial data need to be tested for use with remotely sensed data. Scatter plot justify the result of supervised classification. Hence we concluded that we can reduce the resolution of any LANDSAT8 and AWiFS at only $10 \%$ from their original resolution with acceptable accuracy.

Acknowledgement : The auther acknowledges the funding and support received from the Sam Higginbottom Institute of Agriculture Science and Technology, Deemed University towards presentation of this research paper.

Atkinson, P. M. (2012). Downscaling in remote sensing. Int. J. Appl. Earth Observ. Geoinf. http://dx.doi.org/10.1016/j.jag.2012.04.012.

Barnsley, M. J. (1999). Digital remote sensing data and their characteristics. I. In Geographical Information Systems: Principles, Techniques, Applications, and Management, , 2nd New York: John Wiley \& Sons. 
Blaschke, T. (2010). Object based image analysis for remote sensing journal Photogrammetric Remote Sensing, 65(2).

C.P.Smits, Dellepiane, S. G., \& Schowengerdt, R. A. (1999). Quality assessment of image classification algorithms for landcover mapping: A review and a proposal for a cost-based approach. Int J Remote Sensing, 20, 1461.

Cohen, J. (1960). A coefficient of agreement for nominal scales, Educ. Psychol. Measurement 20(1):37-46., 20(1), 3746. Congalton, G. R. (1991). A review of assessing the accuracy of classifications of remotely sensed Data. Remote Sensing of Environment, 37(1), 3546.

Congalton, R. G., \& Green, K. (2008). Assessing the Accuracy of Remotely Sensed Data: Principles and Practice, 2nd ed, 183. Boca Raton, FL: CRC Press/Taylor \& Francis Group.

Curran, P. J., \& Williamson, H. D. (1985). The accuracy of ground data used in remote sensing investigations. International Journal of Remote Sensing, 6, 17371651.

Estes, J. E., \& Loveland., T. R. (1999). Characteristics, sources, and management of remotely-sensed data. In Geographical Information Systems: Principles, Techniques, Applications, and Management, 2nd ed., ed. P. Longley, M. Goodchild, D. J. Maguire, and D. W. Rhind, 667. New York: John Wiley \& Sons.

Foody, M. G. (2002). Status of land cover classification accuracy assessment. Remote Sensing of Environment, 80(185). Franklin, S. E. (2002). Evidential reasoning with Landsat TM, DEM and GIS data for land cover classification in support of grizzly bear habitat mapping. International Journal of Remote Sensing, 23, 4633.

G.M.Foody. (1996). Foody, G. M. 1996. Approaches for the production and evaluation of fuzzy land cover classification from remotely-sensed data. Int J Remote Sensing 17:1317. International Journal of Remote Sensing 17(17), 1317.

Gallego, F. J. (2004). Remote sensing and land cover area estimation. International Journal of Remote Sensing, 25, 3019.
Ghimire, B., Rogan, J., \& Miller, J. (2010). Contextual land cover classification: incorporating spatial dependence in land cover classification models using random forests and the Getis statistic. Remote Sensing Lett 1(45).

Gong.P, \& Howarth, P. J. (1992). Frequency-based contextual classification and gray-level vector reduction for land-use identification. Photogramm Eng Remote Sens 58(423).

Kontoes, C., G.Wilkingson, A.Burril, S.Goffredo, \& Megier, J. (1993). An experimental System from the Integration of GIS Data in Knowledge-base Image Analysis for Remote Sensing of Agriculture. International Journal of Geographical Information Systems, 7(3), 247262.

Lefsky, M. A., \& Cohen, W. B. (2003). Selection of remotely sensed data. In Remote Sensing of Forest Environments: Concepts and Case Studies, ed. M. A. Wulder and S. E. Franklin, 13. Boston, MA: Kluwer Academic.

Lu, D., \& Weng, Q. (2007). A survey of image classification methods and techniques for improving classification performance. International Journal of Remote Sensing, 28, 823.

Pal, M., \& Mather, P. M. (2003). An assessment of the effectiveness of decision tree methods for land cover classification. Remote Sensing of Environment, 86(554).

R.M.Lillisand, \& R.M.Kiefer. (2000). Remote Sensing and Image Interpretation. 4th Edition, New York: John Wiely and Sons $724 \mathrm{p}$.

San Miguel-Ayanz, J., \& Biging, G. S. (1997). Comparison of single-stage and multi-stage classification approaches for cover type mapping with TM and SPOT data. Remote Sensing of Environment 5992.

Stuckens, J., Coppin, P. R., \& Bauer, M. E. (2000). Integrating contextual information with per-pixel classification for improved land cover classification. Remote Sensing of Environment, 71, 282. 


\title{
AN OBJECT-BASED META KNOWLEDGE MODEL FOR A DISTRIBUTED IMAGE INTERPRETATION SYSTEM
}

\author{
G. A. O. P. Costa ${ }^{\text {a }}$, P. Hofmann ${ }^{\text {b }}$, P. N. Happ ${ }^{\text {c }}$, R. Q. Feitosa ${ }^{\text {a, c }}$ \\ a Rio de Janeiro State University, Brazil - gilson.costa@ime.uerj.br \\ ${ }^{\mathrm{b}}$ University of Salzburg, Austria - peter.hofmann@sbg.ac.at \\ c Pontifical Catholic University of Rio de Janeiro, Brazil - \{patrick, raul $\} @$ ele.puc-rio.br
}

KEY WORDS: Remote Sensing, Object-Based Image Analysis, Knowledge-Based Systems, Ontology

\begin{abstract}
:
This paper introduces the interpretation meta knowledge model devised for the InterCloud platform. InterCloud is a remote sensing image interpretation platform designed to run on computer clusters or on cloud computing infrastructure. The system is capable of distributing data processing tasks, such as segmentation, feature extraction and classification procedures over the processing elements of a computer grid in a transparent way to the user. Moreover, InterCloud can exploit the potential scalability offered by commercial cloud computing infrastructure services, enabling the interpretation of very large remote sensing datasets in an efficient way. The proposed meta model comprises two types of knowledge: declarative and procedural. The former describes the characteristics of the classes of objects expected to be found in the scene to be interpreted, and the relationships among those classes. The latter describes the functions and procedures that should be applied over the data in order to achieve the desired interpretation. In the proposed knowledge model, the user expresses declarative knowledge through the definition of an ontology, so-called descriptive ontology, which conveys the formal naming and definition of the properties and interrelationships of the object classes in a particular application. Procedural knowledge is expressed by the so-called task ontology, which is represented by a directed graph, in which the nodes represent operations over the input images or over the segments generated by segmentation operations. Besides segmentation, crisp or fuzzy classification operations can be defined by the user. The graph edges define the data flow between operations, which are triggered by the control process as soon as their inputs are produced by the preceding operations. In this paper we illustrate the main components of the meta knowledge model through a theoretical application.
\end{abstract}

\section{INTRODUCTION}

To this date, many knowledge-based or cognitive systems (Sagerer et al., 1990; Liedtke et al., 1997; Bückner et al., 2001; Costa et al., 2010; Trimble, 2014) have been proposed for remote-sensing image interpretation. The main focus of those systems is the modelling of the classes of objects expected to be found in a scene through the explicit representation of prior knowledge about their spectral, morphological or topological characteristics.

There are several advantages of embodying image understanding knowledge into explicit structures (Crevier and Lapage, 1997). First, knowledge can be easily added to a knowledge base, without modifying pre-existing rules. When laid out explicitly, knowledge can be more easily validated, since contradictions and omissions become apparent. Knowledge structures also favour interactive problem solution, providing a way to explore alternative means of extracting information from images. Last but not least, explicit represented knowledge provides for easier collaboration, for knowledge interchange among those tackling similar problems.

Ontologies represent a way to explicitly specify domain conceptualizations (Gruber, 1993). In the remote sensing domain, ontologies have been used to describe the classes of objects of interest for particular applications, mostly intending to support feature extraction methods (Arvor et al., 2013). For example, Forestier et al. (2012) designed ontologies that represent urban features (e.g. house, garden, roads) and groups of urban features (e.g. urban blocks) in QuickBird imagery.
Forestier et al. (2013) also presented a knowledge-based framework for remote sensing image interpretation of coastal areas. Kohli et al. (2012) developed an ontology dedicated to identifying informal settlements from remotely sensed imagery. Belgiu et al. (2014) developed ontologies of the objects to be identified in remote sensing data by means of machine learning techniques.

In this work, we introduce the meta knowledge model devised for the InterCloud platform. InterCloud is a remote sensing image interpretation platform designed to run on computer clusters. InterCloud can exploit the potential scalability offered by commercial cloud computing infrastructure services, enabling the interpretation of very large remote sensing datasets in an efficient way.

The proposed meta knowledge model comprises two types of knowledge: declarative and procedural. The former describes the characteristics of the classes of objects expected to be found in the scene to be interpreted, and the relationships among those classes. The latter describes the functions and procedures that should be applied over the data in order to produce the desired interpretation.

In the proposed meta model, the user expresses declarative knowledge through the definition of an ontology, so-called descriptive ontology, which conveys the formal naming and definition of the properties and interrelationships of the object classes in a particular application. Such properties can include crisp or fuzzy descriptions of the related features values, which are used by the distributed interpretation control engine in the 
direct selection of image objects or in assigning class membership values to the objects.

Procedural knowledge is expressed by the so-called task ontology, which is represented by a directed graph, in which the nodes represent operations over the input images or over the segments generated by segmentation operations. Besides segmentation, crisp or fuzzy classification operations can be defined by the user. The graph edges define the data flow between operations, which are triggered by the control process as soon as their inputs are produced by the preceding operations.

\section{INTERCLOUD OVERVIEW}

InterCloud is a remote sensing image interpretation platform designed to run on computer clusters or on cloud computing infrastructure. The system employs the MapReduce programing model (Dean et al., 2004) to distribute data processing tasks, such as segmentation (Happ et al., 2015), feature extraction and classification procedures (Ayma et al., 2015) over the processing elements of a computer grid in a transparent way to the user. Moreover, InterCloud can exploit the potential scalability offered by commercial cloud computing infrastructure services, enabling the interpretation of very large remote sensing datasets in an efficient way. InterCloud can be regarded as a redesign of InterIMAGE (Costa et al., 2010) conceived to process arbitrarily large datasets in a distributed fashion.

Although the architecture of InterCloud has been described before (Ferreira et al., 2014), we recall some of its main aspects. The architecture is composed by three abstraction layers: the project definition layer, the image interpretation layer, and the distribution layer.

The project definition layer supports the definition of all required information for the execution of an interpretation application. Such information can be described through semantic networks and dataflow graphs that represent processing chains. The first enables the description of the classes of objects expected to be found by the interpretation process, and the latter enables the description of the chain of operations that should be carried in the interpretation process, and that will be distributed over the processing elements in a transparent way to the end user.

The interpretation layer comprises the algorithms and methods available in the platform. It is structured on a high-level programming language that hides the complexity of dealing directly with the distributed programming model. A user with conventional programming skills can embed new methods into this layer, so that the end user can select them in the project definition layer. Finally, the distribution layer is responsible for the distributed execution of the interpretation model.

In the current implementation of InterCloud, the project definition layer lacks a graphical user interface (GUI), so that end user interaction is restricted by the fact that the user needs to describe the interpretation model through a traditional programming language (i.e., Java). Additionally, InterCloud lacks conceptually structured knowledge representation formalisms to guide the user in the definition of interpretation models, and that provide high level abstractions for the definition of such models, so that application domain experts can define complex models without the support of programmers.

This work intends to fill this gap, by proposing knowledge representation abstractions based on the concept of ontologies. In the following we describe those formalisms, which are currently being considered in the implementation of InterCloud's GUI.

\section{META KNOWLEDGE MODEL}

In this work we propose an ontology-based meta knowledge model for remote sensing image interpretation. In this section we define the basic components of the meta model and present some examples to illustrate such components in a simple, theoretical application.

Firstly, what we understand by knowledge are facts and heuristics. Facts constitute the body of information available and heuristics are rules of good judgment, of plausible reasoning, that characterize expert-level decision making.

In the case of object-based image interpretation, facts are related to image objects, which represent the occurrence of instances of object classes on the scene represented by an image. Image objects that belongs to a specific class share common characteristics, which are based on particular properties, which are related to features that can be measured or quantified. Such features may be associated to (i) statistics computed over spectral intensity values of the pixels that belong to the image objects, to (ii) to the shape of image objects, to (iii) topological relationships among objects of specific classes, and to (iv) temporal relationships among objects that occur in images acquired at different points in time. In any case, the description of the characteristics shared by objects of a specific class and the relationships among objects from different classes must be formally described in order to be processed by an automated procedure. We call such kind of knowledge descriptive knowledge.

In this context, heuristics are associated to the way descriptive knowledge is used or processed, so as to find the location of image objects of the classes of interest, on a particular scene. This is associated to a different kind of knowledge, which has to do with the order of processing of tasks, which aim at measuring the properties of image objects and determining the association of the objects to specific classes. We call such kind of knowledge procedural knowledge.

In our meta knowledge model, descriptive knowledge is explicitly represented through a so-called descriptive ontology, and procedural knowledge is represented through a task ontology.

\subsection{Descriptive Ontology}

In the proposed meta model, descriptive ontologies are represented by a graphical knowledge representation formalism, which is based on semantic networks (Sagerer and Niemann, 1997).

The nodes of the semantic network may represent either object classes or lingual descriptors. Lingual descriptors represent generic characteristics of the objects of a class. Lingual descriptors are defined loosely, in linguistic terms, which are qualitative and imprecise by nature. Such imprecision means 
that lingual descriptors cannot be actually computed by an automated process, so they need to be associated to features that can be quantitatively measured. Therefore, each lingual descriptor must be associated to what we call a descriptor definition.

A descriptor definition is composed by a set of measurable features, which are associated to corresponding fuzzy sets. Possibility values derived from the fuzzy sets can be combined by fuzzy operators, so that the computation of a descriptor will produce a single possibility value.

As many lingual descriptors may be associated to an object class, lingual descriptors may also be combined through fuzzy operators. In this way, the possibility values associated to each lingual descriptor may be combined so as to produce a single membership value for an image object, which can be later used by a particular process identified in the task ontology, e.g., defuzzification or spatial conflict resolution.

Figure 1 illustrates a simple descriptive ontology. The edges of the semantic network have different semantic meanings. When the edges connect class nodes, they represent structural relationships between classes, such as is- $a$ and part-of. When the edges connect lingual descriptors they represent operators that combine possibility values, i.e., $t$-norms or $t$-conorms.

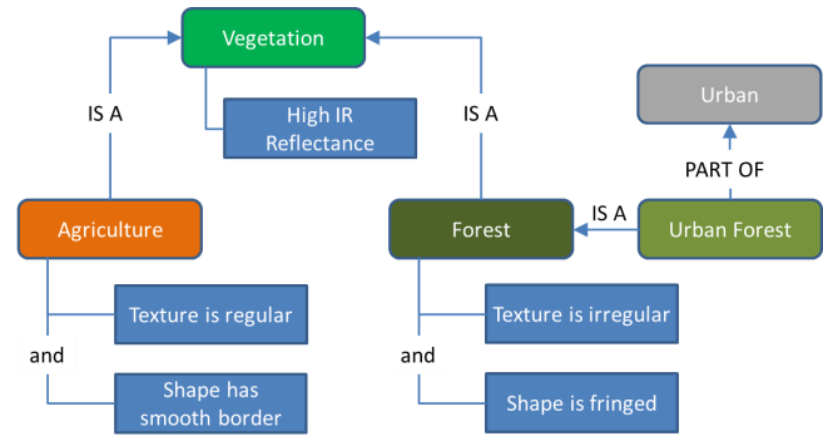

Figure 1. Descriptive ontology example.

Figure 2 shows an example of a lingual descriptor definition. The edges connect feature nodes to the descriptor, they also represent $t$-norms or $t$-conorms.

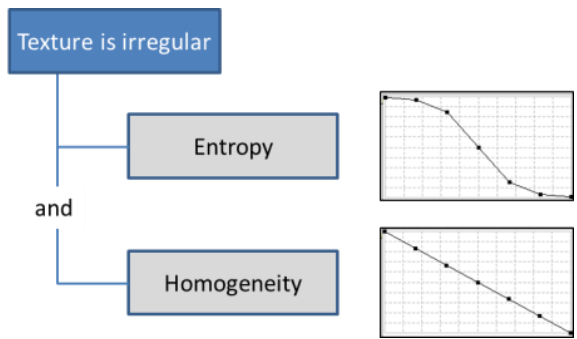

Figure 2. Lingual descriptor definition.

\subsection{Task Ontology}

The task ontology can be understood as a dataflow description. It represents a processing chain that will somehow use the definitions of the descriptive ontology in the interpretation process, in order to find occurrences of instances (image objects) of the classes of interest.

Task ontologies are represented by a direct graph, whose nodes represent either processes, data sets (of raster images or vector data), or object classes; and whose edges indicate the processing flow.

Note that, as more than one edge may come out of one node, the edges can be numbered, so that the control process will know the exact order of processing of the connected nodes. Figure 3 shows a task ontology associated to the descriptive ontology illustrated in Figure 1.

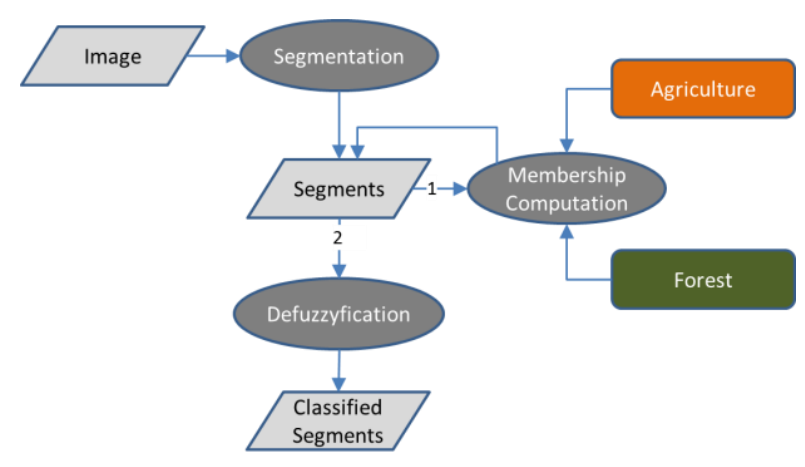

Figure 3. Task ontology example.

It is interesting to observe that for the same descriptive ontology, many different task ontologies may be defined. In Figure 4 we show a different task ontology, associated to the descriptive ontology illustrated in Figure 1. In this task ontology different image segmentations (from different algorithms or from a same algorithm with different parameter values) are performed for the different classes, and a spatial conflict resolution procedure is carried out on the resulting objects, based on their membership values.

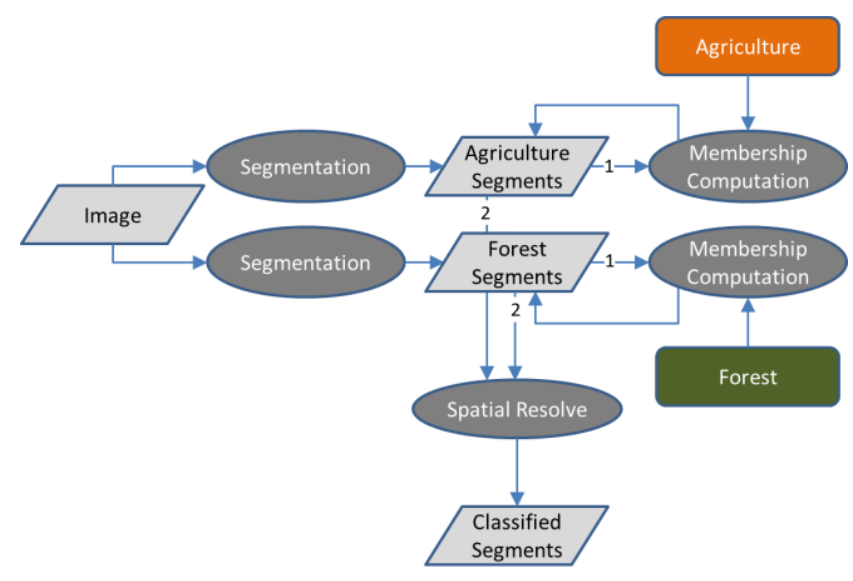

Figure 4. Alternative task ontology.

Figure 5 shows yet another example of a task ontology. This time classification is carried out with a machine learning, supervised technique. In this case, the classification feature space may be defined directly from the features that compose the definition of the lingual descriptors associated to the object classes, or the user may define other features by configuring the parameters of the machine learning procedure. 


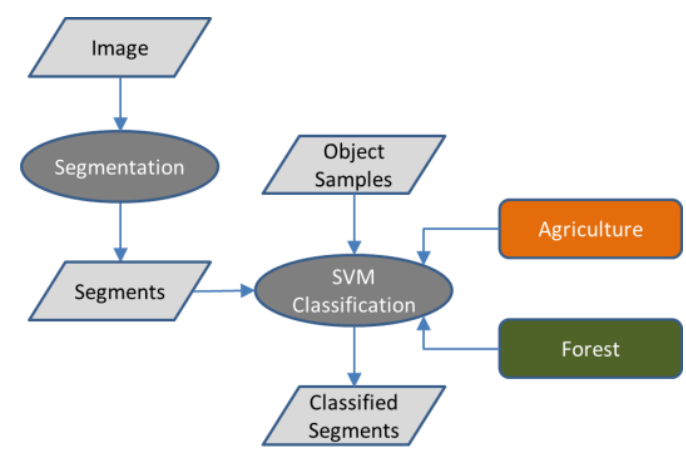

Figure 5. Task ontology with machine learning process.

\section{CONCLUSIONS AND FUTURE WORK}

In this work we presented a meta knowledge model for InterCloud, a distributed object-based image interpretation system. The meta model is able to represent descriptive knowledge and procedural knowledge, through descriptive ontologies and task ontologies, respectively.

In the descriptive ontologies, the characteristics of object classes are defined though lingual descriptors, which are later specified in terms of descriptor definitions, which associate the linguistic terms to concrete, measurable features computed on the image segments.

We believe that lingual descriptors represent an abstraction level that can facilitate knowledge interchange among users tackling similar problems, thus providing a bridge for transferability of image interpretation models.

The task ontologies enable the definition of complex processing chains, and provide a sensible way to control the image interpretation process.

We are currently in the process of detailing the meta model and the corresponding graphical user interface, which we plan to make available in a future version of InterCloud.

\section{ACKNOWLEDGEMENTS}

The authors acknowledge the funding provided by FAPERJ (Carlos Chagas Filho Foundation for Research Support in Rio de Janeiro), $\mathrm{CNPq}$ (National Council for Scientific and Technological Development) and CAPES (Coordination for the Improvement of Higher Education).

\section{REFERENCES}

Arvor, D., Durieux L., Andrés S., Laporte M.-A., 2013. Advances in Geographic Object-Based Image Analysis with Ontologies: a review of main contributions and limitations from a remote sensing perspective. ISPRS Journal of Photogrammetry and Remote Sensing, 82, 125-137.

Ayma, V.A., Ferreira, R.S., Happ, P.N., Oliveira, D.A.B., Costa, G. A. O. P., Feitosa, R. Q., Plaza, A., Gamba, P., 2015. On the architecture of a big data classification tool based on a map reduce approach for hyperspectral image analysis. 2015 IEEE International Geoscience and Remote Sensing Symposium (IGARSS), Milan, 2015, pp. 1508-1511.
Belgiu, M., Tomljenovic, I., Lampoltshammer, T., Blaschke, T., Höfle, B., 2014. Ontology-Based Classification of Building Types Detected from Airborne Laser Scanning Data. Remote Sensing, 6, pp. 1347-1366.

Bückner, J., Pahl, M., Stahlhut, O., Liedtke, C.-E., 2001. GEOAIDA - A Knowledge Based Automatic Image Data Analyzer for Remote Sensing Data. In: ICSC Congress On Computational Intelligence Methods and Applications 2001 CIMA 2001, 2001, Bangor, Wales, Great Britain. Proceedings of the Congress on Computational Intelligence Methods and Applications 2001 - CIMA 2001. International Computing Sciences Conventions - University of Wales in Bangor, U.K.

Costa, G.A.O.P., Feitosa, R.Q., Fonseca, L.M., Oliveira, D.A.B., Ferreira, R.S., Castejon, E., 2010. Knowledge-based interpretation of remote sensing data with the interimage system: major characteristics and recent developments. In: Addink, E., Van Coillie, F. (Eds.), GEOBIA. ISPRS Working Groups, Gent, Belgium, 2010.

Crevier, D., Lepage, R., 1997. Knowledge-Based Image Understanding Systems: A Survey. Computer Vision and Image Understanding, 67 (2), pp. 61-185.

Dean J., Ghemawat, S., 2004. MapReduce: Simplified Data Processing on Large Clusters. OSDI'04: Sixth Symposium on Operating System Design and Implementation, San Francisco, CA, 2004.

Ferreira, R.S., Oliveira, D.A.B., Happ, P., Costa, G.A.O.P, Feitosa, R.Q., Bentes, C., 2014. InterIMAGE 2: The Architecture of an Open Source, High Performance Framework for Automatic, Knowledge-Based Image Interpretation. International Geographic Object-Based Image Analysis Conference, Thessaloniki, 2014.

Forestier, G., Puissant, A., Wemmert, C., Gançarski, P., 2012. Knowledge-based region labeling for remote sensing image interpretation. Computers, Environment and Urban Systems, 36, pp. $470-480$.

Forestier, G., Wemmert, C. \& Puissant, A., 2013. Coastal image interpretation using background knowledge and semantics. Computers \& Geosciences, 54.

Gruber, T. R., 1993. A translation approach to portable ontology specifications. Journal of Knowledge Acquisition for Knowledge-Based Systems, 5, pp. 199-220.

Happ, P.N., Ferreira, R.S., Costa, G.A.O.P., Feitosa, R.Q., Bentes, C., Gamba, P., 2015. Towards distributed region growing image segmentation based on MapReduce. 2015 IEEE International Geoscience and Remote Sensing Symposium (IGARSS), Milan, 2015, pp. 4352-4355.

Kohli, D., Sliuzas, R., Kerle, N., Stein, A., 2012. An ontology of slums for image-based classification. Computers, Environment and Urban Systems, 36, pp. 154-163.

Liedtke, C.-E., Bückner, J., Grau, O., Growe, S., Tönjes, R., 1999. Aida: A System for the Knowledge Based Interpretation of Remote Sensing Data. In: Third International Airborne Remote Sensing Conference and Exhibition, 1999, Copenhagen, Denmark, Proceedings of the Third International 
Airborne Remote Sensing Conference and Exhibition. Environmental Research Institute of Michigan.

Niemann, H., Sagerer, G., Schröder, S., Kummert, F., 1990.

ERNEST: A Semantic Network System for Pattern Understanding. IEEE Transactions on Pattern Analysis and Machine Inteligence, 12 (9).

Sagerer, G., Niemann, H., 1997. Semantic Networks for Understanding Scenes: Advances in Computer Vision and Machine Intelligence. 1.ed. New York: Plenum Publishing Corporation.

Trimble, 2014. eCognition Developer 8.7.2. Reference Book, München. 


\title{
USING PURE AND MIXED OBJECTS IN THE TRAINING OF OBJECT-BASED IMAGE CLASSIFICATIONS
}

\author{
H. Costa ${ }^{\text {a* }}$, G. M. Foody ${ }^{\text {a }}$ D. S. Boyd ${ }^{\text {a }}$ \\ ${ }^{a}$ School of Geography, University of Nottingham, Nottingham NG7 2RD, UK - (lgxhag, giles.foody, \\ doreen.boyd)@nottingham.ac.uk
}

KEY WORDS: Artificial neural networks, OBIA, Over-segmentation, Sample size, Under-segmentation, WorldView.

\begin{abstract}
:
Training of object-based land cover classifications is often performed with objects generated via image segmentation. The objects are commonly assumed to be thematically pure or excluded from training if a mixture of classes is associated with them. However, excluding mixed objects has several consequences such as reducing the size of the training data sets. In this study, it is hypothesized that mixed objects may be used in the training stage of a classification to increase the accuracy with which land cover may be mapped from remotely sensed data, with outputs evaluated in relation to a conventional analysis using only pure objects in training. WorldView-2 data covering the University Park campus of the University of Nottingham were submitted to a series of segmentation analyses in which a range of under- to over-segmentation outputs were intentionally produced. Training objects representing four classes (bare soil, impervious surfaces, vegetation, and water) were selected from the segmentation outputs, resulting in training samples of varying size and proportion of mixed objects. A single-layer artificial neural network equivalent to multinomial logistic regression and able to use both pure and mixed training units was adopted as the classifier. A visual inspection of the results shows that using mixed training objects produced land cover maps of higher quality. Furthermore, the overall and class-specific accuracy of the classifications was systematically higher when mixed training was used (e.g. up to $48 \%$ in overall accuracy). The advantage of using mixed objects in training was beneficial even when the size of the mixed training samples was equivalent to that of the pure training samples.
\end{abstract}

\section{INTRODUCTION}

In object-based image analysis it is common for some of the objects generated via image segmentation to be of mixed thematic composition as a result of under-segmentation error (Clinton et al. 2010). This violates the commonly made assumption of object purity that is implicit in a conventional classification analysis, and thus can be a problem throughout the analysis. For instance, mixed objects can result in degraded training statistics and act to reduce mapping accuracy (Wang et al. 2004; Gao et al. 2011; Hirata and Takahashi 2011). Therefore, mixed objects are often excluded from the training stage so that they are not used in the derivation of training statistics (Dean and Smith 2003; Smith 2010; Dronova et al. 2011; Cai and Liu 2013).

Excluding mixed objects has, however, several consequences. Using only pure objects has the drawback of representing only homogenous areas in training whereas afterwards the classifier may be confronted with some mixed objects. Thus pure objects may be not totally representative of the characteristics of the objects that a classifier has to classify. Furthermore, excluding mixed objects has the consequence that the size of the training samples will inevitably be reduced.

Deviation from the object purity assumption can, however, sometimes be made throughout a classification analysis (e.g. Foody 1999a). Specifically, impure units can be accounted for in training (Foody 1997; Zhang and Foody 2001; Eastman and Laney 2002; Matthew 2012), class allocation (Wang 1990; Foody 1996; Dronova et al. 2011), and testing stages of a supervised image classification (Foody 1995; Binaghi et al.
1999; Stehman et al. 2007). However, little research has been undertaken on the use of mixed units in training object-based image classifications.

This paper sets out to test the hypothesis that mixed objects may be used in the training stage of a classification to increase the accuracy with which land cover may be mapped from remotely sensed data, with outputs evaluated in relation to a conventional analysis using only pure objects in training.

\section{MATERIALS}

\subsection{Study area}

A square area of $2502 \mathrm{~m}$ per $2502 \mathrm{~m}$ over the University Park campus of the University of Nottingham in the United Kingdom was used to undertake the analyses (Figure 1). The central coordinates of the study area are $52^{\circ} 566^{\prime} 28.212^{\prime \prime} \mathrm{N}$ and $1^{\circ} 11^{\prime} 44.3217^{\prime \prime W}$. A total of four land cover classes were defined: bare soil, impervious surface, vegetation, and water.

\subsection{Spectral data}

A WorldView-2 (WV2) image of acquired on 26 May 2012 was used (Figure 1). This image is comprised of eight spectral wavebands (coastal, blue, green, yellow, red, red edge, NIR1, and NIR2) with a spatial and radiometric resolution of $2 \mathrm{~m}$ and 11 bit respectively.

\footnotetext{
* Corresponding author
} 


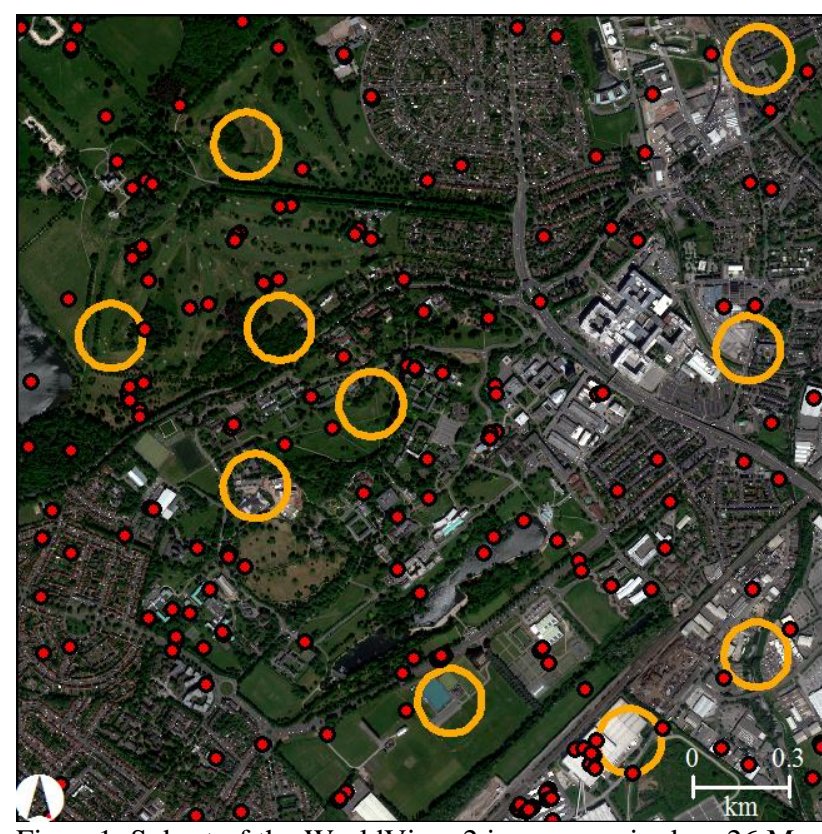

Figure1. Subset of the WorldView-2 image acquired on 26 May 2012 over the study area (University Park campus of the University of Nottingham and its environs). The circular areas of $100 \mathrm{~m}$ radius outlined orange are clusters randomly located used for selecting training objects. The red points locate the pixels of the random testing sample used to assess the accuracy of the classification produced using segmentation scale $=50$ and pure training.

\subsection{Reference data}

Reference data were produced to assist the production of training and testing samples. Visual interpretation of the WV2 image (including the spectral wavebands and a panchromatic band of $0.5 \mathrm{~m}$ spatial resolution) was the basis for populating training and testing samples with land cover class labels. Imagery available through Google Earth was also inspected.

The training samples were produced via cluster sampling (Whiteside et al., 2014) in which the primary sampling unit, the clusters, was defined to be ten circular areas of $100 \mathrm{~m}$ radius randomly located (Figure 1). Thus, a total of $\sim 5 \%$ of the study area was allocated to training purposes. The land cover in the clusters was delimited by visual interpretation as explained above. The secondary sampling unit was the objects generated via image segmentation that intersected the clusters (see 3.2).

Testing samples were produced to assess the accuracy with which WV2 data was classified based on each of the segmentation outputs produced. Therefore, a total of eight samples were produced. They comprised 50 randomly selected pixels per mapped class (Figure 1 shows an example). Although the classifications were object-based, the pixel is a legitimate and practical option for accuracy assessment (Stehman and Wickham, 2011). Land cover in the testing pixels was determined by visual interpretation. Although it may be beneficial to address the potential thematic mixed nature of the pixels, only the dominant class was considered for simplicity.

\section{METHODS}

A series of analyses were undertaken to explore the potential of using mixed objects in training object-based classifications. The methods used included segmentation of the WV2 image, production of training samples, classification of the segmentation outputs, and assessment of the classification accuracy.

\subsection{Image segmentation}

The eight spectral bands of the WV2 image were segmented to generate objects using the multiresolution algorithm implemented in GeoDMA software (Körting et al., 2013), version 0.2.1, which is based on the popular algorithm of Baatz and Schäpe (2000). This is a region-based algorithm that uses spectral and shape properties of the objects being generated, and the most influential parameter of the algorithm, and hence most often manipulated, is scale.

A series of segmentation analyses were undertaken in which the parameter scale was manipulated in order to produce a range of under- to over-segmented outputs. The value of the scale parameter was set at $30,50,70$, and 90 while the remaining parameters were set at 50. As a result, four segmentation outputs were obtained, ranging from over-segmented results (mostly composed of small and possibly pure objects) to undersegmented results (mostly composed of large and possibly mixed objects). In this study an object was taken to be pure if the dominant class covered more than $90 \%$ of the object's area, similar to Cai and Liu (2013).

\subsection{Training}

Training samples were produced to classify each of the segmentation outputs generated. The training samples were formed by the objects generated with each of the parameter settings that intersected the primary sampling unit defined, the 10 random clusters (section 2.3). Therefore, the set of training objects used varied between the segmentation outputs (ranging from over-segmented to under-segmented training objects) while the same geographical area was used in training each classification.

The eight spectral bands were used to calculate training statistics, which were the mean and standard deviation of the pixel values associated with the training objects. The mean digital number provides a value of central tendency whereas standard deviation provides a value of variability (texture). As a result, 16 bands were used as discriminating variables in classifications.

The training objects were assigned reference class labels accordingly to those visually delimited in the clusters. The proportion of the area that each class occupied in a training object was calculated. The proportions calculated were 0.0 if the class was absent and 1.0 if the object was pure. Intermediate values for at least two classes were calculated when the object was of mixed class composition.

Two training strategies were followed. First, the traditional procedure of using only pure objects at the training stage was tested (i.e. the mixed objects, whose dominant class covered $<90 \%$ of the object, were excluded). Second, all of the training objects were used even if the dominant class covered $<90 \%$ of the area. The fractional coverage of the classes found in the objects was used as a measure of class membership, and objects were allowed multiple and partial membership. 


\subsection{Classification}

A multinomial log-linear classifier was applied via a neural network with no hidden layer (R Core Team, 2014; Venables and Ripley, 2002) to produce land cover maps. This neural network allows the objects of mixed class composition to be used in training in a form similar to that explained in Foody (1997) for per-pixel classification. The mean and standard deviation of the objects across the WV2 spectral bands were used as discriminating variables.

Although the classifier used produces soft classifications, traditional hard land cover maps were obtained by allocating each object the label of the class with which it had the greatest membership. Each segmented output generated was thus used to produce hard land cover maps based on different training strategies: pure and mixed.

\subsection{Accuracy assessment}

The testing samples were used to assess the accuracy of the classifications. Confusion matrices comparing the reference labels of the testing pixels and the classification labels of the corresponding objects were constructed. Overall accuracy and per-class estimates of accuracy (user's and producer's accuracy) were calculated in terms of proportion of area correctly classified as described in Olofsson et al. (2014), including confidence intervals at the $95 \%$ confidence level.

The accuracy of the four segmentation outputs produced was also assessed. In this case, the accuracy assessment essentially aimed at determining whether the training data sets used were over-segmented, under-segmented, or balanced. The magnitude of under- and over-segmentation errors is associated with the presence of mixed objects. An empirical discrepancy method proposed by Möller et al. (2013) and slightly refined by Costa et al. (2015) was used in this study to assess segmentation accuracy. This method essentially compares the objects generated to a reference data set to measure the geometric match between them. The land cover delimited in the clusters by visual interpretation (previously used for producing the training data sets) was reused as reference data. The outcome of the method is a metric, $\mathbf{M}^{\mathrm{g}}$, that measures the strength and type of error. Negative $\mathbf{M}^{\mathrm{g}}$ values indicate that under- segmentation error dominates while positive $\mathrm{M}^{\mathrm{g}}$ values represent the opposite case in which over-segmentation error dominates. So, $\mathbf{M}^{\mathrm{g}} \sim 0$ is deemed to be indicative of optimal segmentation accuracy as the two types of error are balanced (Möller et al., 2013).

\section{RESULTS}

The segmentation outputs were, as expected, notably oversegmented when the scale parameter was small as the objects generated were noticeably smaller than when the scale parameter was set at a large value. Figure 2 shows the level of under/over-segmentation error of the training samples. Negative $\mathrm{M}^{\mathrm{g}}$ values indicate that under- segmentation error dominates while positive $\mathrm{M}^{\mathrm{g}}$ values represent the opposite case in which over-segmentation error dominates. The $\mathrm{M}^{\mathrm{g}}$ value closest to 0 was that of parameter scale 50 .

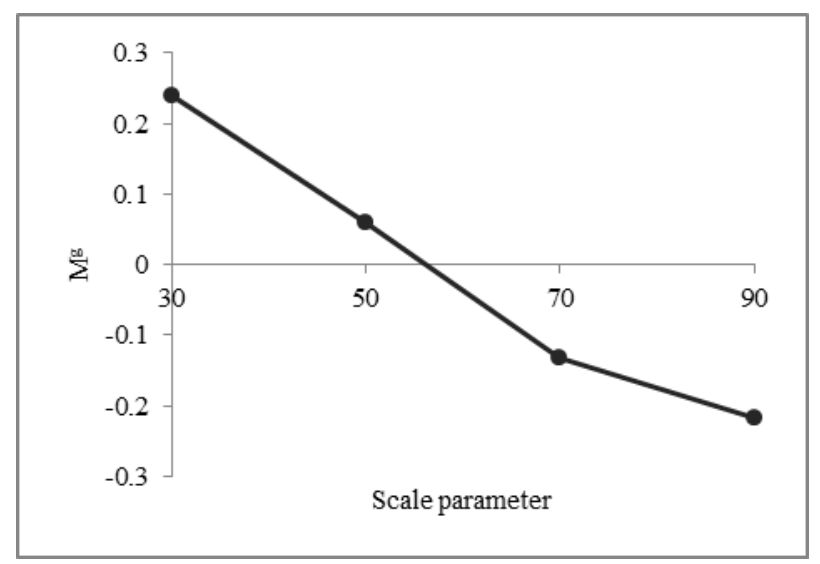

Figure 2. Image segmentation accuracy.

The difference between the classifications produced with pure and mixed training was evident with mixed training affording classification higher accuracy. Figure 3 shows the classifications of the segmentation output produced with scale $=50$. The estimated overall accuracy of the maps is $43.0 \pm 10.4 \%$ (pure training, Figure $3 \mathrm{a}$ ) and $91.5 \pm 5.3 \%$ (mixed training, Figure $3 b$ ) respectively. Figure 4 shows the overall accuracy of all the classifications. Mixed training enabled classification to achieve higher accuracy values than pure training for all of the segmentation settings used.

The per-class estimators of accuracy were also higher when the training stage of the classifications was mixed (Figure 5). For example, the user's accuracy of class vegetation of the map of Figure $3 b$ (produced with mixed training) was larger than that of the map shown if Figure $3 \mathrm{a}$ (produced with pure training), specifically $94.0 \pm 6.3 \%$ and $48.0 \pm 14.0 \%$ respectively.

\section{DISCUSSION}

The common practice of using only pure objects in training may compromise the accuracy of object-based classifications. Excluding mixed objects from training reduces the value of a training data set and thus should not be adopted. One of the advantages of allowing mixed objects to derive training statistics is that the size of the training data sets is larger than when only pure objects are used. Because in this study the mixed training strategy did not exclude mixed objects from training, the size of the mixed training data sets was larger than that of the pure training data sets (Table 1). Therefore, the difference between the accuracy values of the classifications trained with pure and mixed data sets are partly caused by different sizes of the training samples.

\begin{tabular}{|l|c|c|}
\hline Scale & Pure & Mixed \\
\hline 30 & 544 & 767 \\
50 & 278 & 420 \\
70 & 171 & 262 \\
90 & 130 & 207 \\
\hline
\end{tabular}

Table 1. Size (number of objects) of the training samples 

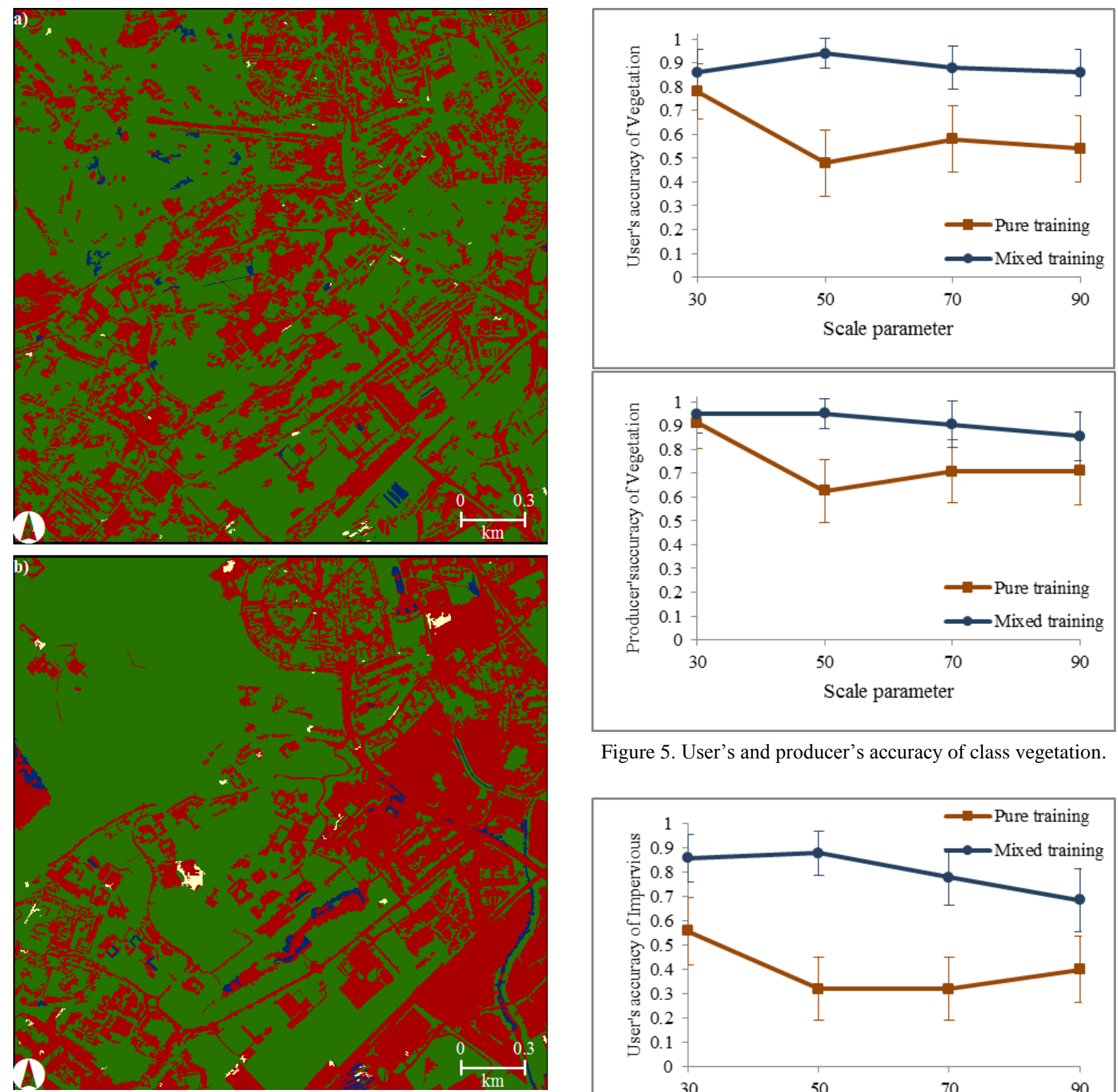

Figure 5. User's and producer's accuracy of class vegetation.

Figure 3. Segmentation output produced using scale $=50$ and classified using a) pure and b) mixed training. Yellow, red, green, and blue represent bare soil, impervious, vegetation, and water, respectively.
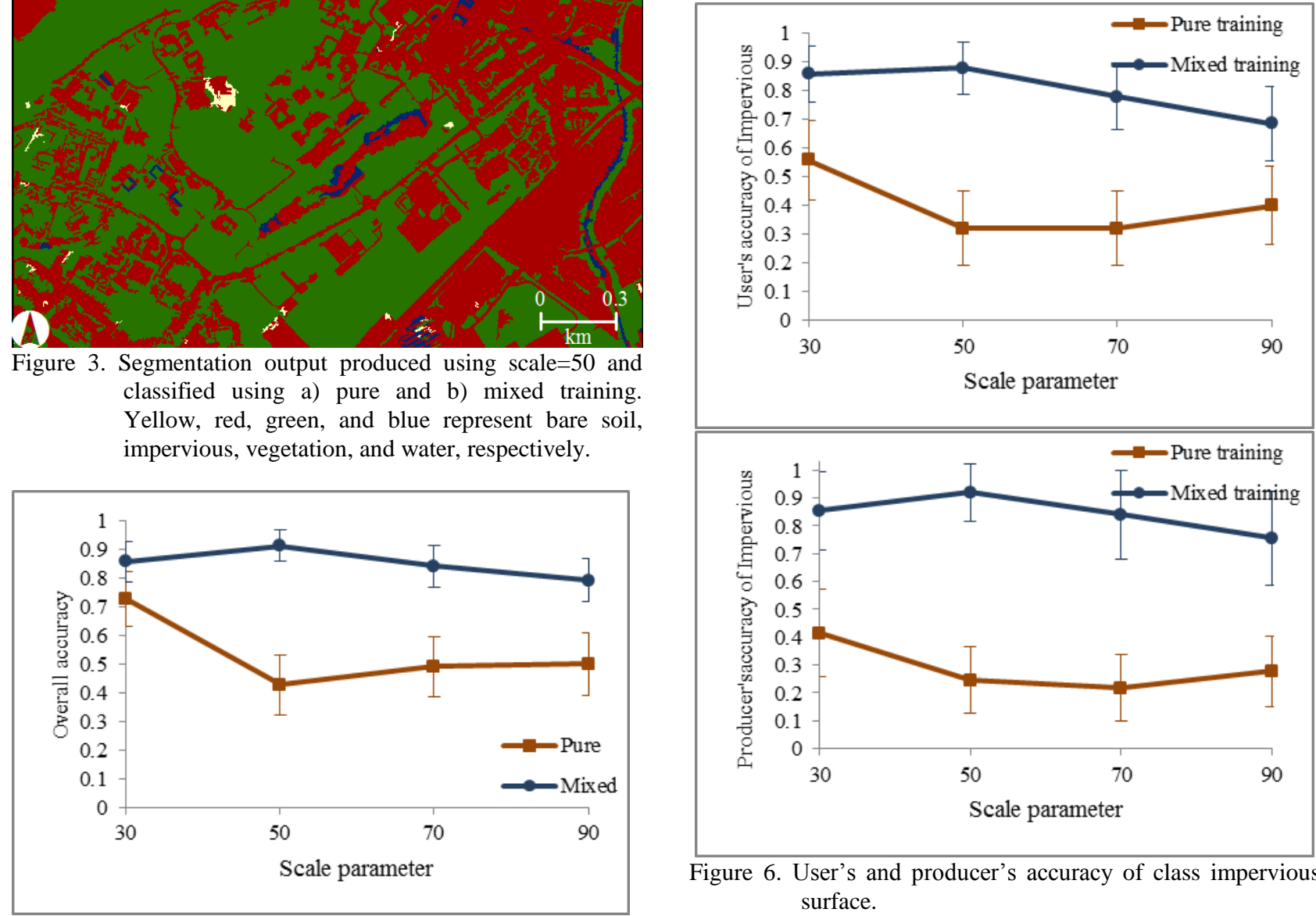

Figure 6. User's and producer's accuracy of class impervious surface.

Figure 4. Accuracy of the classifications 
The size of the training data sets is not, however, the only factor explaining the results. Note that the accuracy of the classification of scale $=30$ that used pure training (544 training objects) was lower than that of classification of scale $=90$ with mixed training (207 training objects). The results, thus, suggest that using pure training data sets is a drawback in that only homogenous areas are represented at the training stage whereas afterwards the classifier may be confronted with some mixed objects. Mixed training, on the contrary, informs on the occurrence of mixed objects.

To highlight the advantage of using mixed training over pure training, an additional analysis was performed without having to deal with different sizes of the training samples. The size of the mixed training data set for the segmentation output produced with scale $=50$ was reduced from 420 to 228 objects, the latter being the size of the corresponding pure training data set (Table 1). This was achieved by excluding randomly selected training objects, with all of the objects, pure and mixed, having the same probability of being excluded. This allowed the size of the training data set to be reduced without changing substantially the inherent ratio of pure to mixed objects. The reduced mixed training data set enabled classification accuracy to reach $84.2 \pm 7.2 \%$, which is lower than the accuracy of the classification that used the entire mixed training data set, but still significantly larger than when using the pure training data set (Figure 4).

\section{CONCLUSIONS}

Image segmentation is a necessary stage for object-based image classification. Commonly segmentation errors produce objects of mixed class composition, which commonly are excluded from the derivation of statistics at the training stage. However, including mixed objects in training is advantageous because the size of a training data set is not reduced and they better represent the characteristics of the objects that a classifier will be confronted with for producing a map via image classification.

\section{ACKNOWLEDGEMENTS}

The WorldView-2 data used was provided by the Earth Observation Technology Cluster, a knowledge transfer initiative funded by the Natural Environment Research Council (grant NE/H003347/1).

\section{REFERENCES}

Baatz, M., Schäpe, A., 2000. Multiresolution Segmentation: an optimization approach for high quality multi-scale image segmentation, in: Strobl, J., Blaschke, T., Griesebner, G. (Eds.), Angewandte Geographische Informationsverarbeitung XII. Beiträge zum AGIT-Symposium Salzburg 2000. Herbert Wichmann Verlag, Heidelberg, Germany, pp. 12-23.

Costa, H., Foody, G.M., Boyd, D.S., 2015. Integrating user needs on misclassification error sensitivity into image segmentation quality assessment. Photogrammetric Engineering and Remote Sensing, 81(6), pp. 451-459.

Foody, G.M., 1997. Fully fuzzy supervised classification of land cover from remotely sensed imagery with an artificial neural network. Neural Computing \& Applications, 5(4), pp. 238-247.
Körting, T.S., Fonseca, L.M.G., Câmara, G., 2013. GeoDMAGeographic Data Mining Analyst. Computers \& Geosciences, 57, pp. 133-145.

Möller, M., Birger, J., Gidudu, A., Gläßer, C., 2013. A framework for the geometric accuracy assessment of classified objects. International Journal of Remote Sensing, 34(24), pp. 8685-8698.

Olofsson, P., Foody, G.M., Herold, M., Stehman, S.V., Woodcock, C.E., Wulder, M.A., 2014. Good practices for estimating area and assessing accuracy of land change. Remote Sensing of Environment, 148, pp. 42-57.

R Core Team, 2014. R: a language and environment for statistical computing. R Foundation for Statistical Computing, Vienna, Austria.

Stehman, S.V., Wickham, J.D., 2011. Pixels, blocks of pixels, and polygons: Choosing a spatial unit for thematic accuracy assessment. Remote Sensing of Environment, 115, pp. 30443055 .

Venables, W.N., Ripley, B.D., 2002. Modern applied statistics with S. Fourth Edition ed. Springer, New York.

Whiteside, T.G., Maier, S.W., Boggs, G.S., 2014. Area-based and location-based validation of classified image objects. International Journal of Applied Earth Observation and Geoinformation, 28, pp. 117-130. 


\title{
SUPPORTED MAPPING WITH MULTI SENSOR IMAGES THROUGH STRATEGY FOCUSED ON CUSTOMIZATION AND INTEGRATION OF GENERALIZED CLASSES BY GEOBIA
}

\author{
C.B.M. Cruz ${ }^{\text {a }}$, P.M.M. Almeida ${ }^{\mathrm{a}, \mathrm{b}}$, R.S. Barros ${ }^{\mathrm{a}}$, R.S. Vicens ${ }^{\mathrm{c}}$, E.M.F.R. Souza ${ }^{\mathrm{a}}$, E.P.A. Caris ${ }^{\mathrm{a}}$, \\ M.C. Fernandes ${ }^{a}$, P.M.L. Menezes ${ }^{\text {a }{ }^{*}}$ \\ ${ }^{a}$ Dept. of Geography, Federal University of Rio de Janeiro - (cmad, almeida.pmm, barros.rafael, manoelcoutofernandes, \\ elizabethmfr, elizabethmfr, pmlmenezes)@gmail.com \\ ${ }^{\mathrm{b}}$ UCB, Castelo Branco University, Dept of Teachers Formation (Geography) \\ ${ }^{\mathrm{c}}$ UFF, Dept. of Geography - rsvicens@gmail.com
}

KEY WORDS: Land use, Land cover, Data Integration

\begin{abstract}
:
Rio de Janeiro presents itself as a land of contrasts, with the second largest metropolitan area in Brazil and a total area of 43,778 $\mathrm{km}^{2}$. Its landscape consists of remnants of the Atlantic Forest and environments with different levels of anthropic impacts. The complexity of its territory combines natural and anthropic covers related to each other at different intensity levels, being a challenge in defining mapping techniques of land use and land cover with greater spatial and temporal detail. Supported by the Secretary of State for Environment, this initiative accepted the challenge of developing a methodology for mapping about $45 \%$ of the state in 1:25,000. This mapping aims to support decision making regarding the land use planning and the monitoring of deforestation actions. Using GEOBIA techniques and images of different resolutions, we structured a methodology for the classification of four macro-classes very different spectrally (Natural Forested Areas, Natural Non-Forested Areas, Anthropic Agropastoral Areas, Anthropic Non-Agropastoral Areas), identifying objects on the ground from 0.5 ha, to meet demands from the Rural Environmental Registry (CAR, in portuguese) and the forest monitoring. The mapping was divided into Working Groups (WG) that customized solutions through Process Trees in eCognition environment. This choice is based on the need to minimize inconsistencies in the interpretive process, improving the level of specialization in short time. Thus, each WG is responsible for the classification of a set of functionally related classes. The integration process of the classes in a single mapping was also supported by GEOBIA. It is believed that the presentation of this strategic view may contribute to challenges of similar mapping, allowing the achievement of cartographic goals in short term.
\end{abstract}

\section{INTRODUCTION}

Institutional mapping aiming at applications related to land use planning is hardly anchored in complex methodological solutions that aim at improving results. When the definition of unusual methods which can be implemented in short time, ensuring quality results, is necessary, it is common for the State and research groups, usually linked to universities, to form partnerships.

This paper presents the experiment for mapping, in the 1:25,000 scale, part of the Atlantic Forest corridor, located in the state of Rio de Janeiro, area of fundamental importance for the maintenance of the water resources, the forest remnants and the biodiversity. The state, with approximately $43,000 \mathrm{~km}^{2}$, presents the main remnants of the Atlantic Forest in Brazil, which occupy about $30 \%$ of its territory. The current mapping covers an area around $20,000 \mathrm{~km}^{2}$, composed by four hydrographic regions of the state, with a minimum mapping area of $0.5 \mathrm{ha}$.

The map intends to offer support to decision-making statewide, related both to issues from the Rural Environmental Registry (CAR, in portuguese) as to actions of forest conservation and recuperation. CAR's goal is to promote identification and integration of environment information of rural properties and possessions, aiming at environmental planning, monitoring, regularization and combating deforestation. As rural properties in the state of Rio de Janeiro are mostly small, the aid of the government through technical support is provided by law. Thus, a system with the necessary bases for the survey of spatial information to define Permanent Preservation Areas (PPA) and Legal Reserves (LR) is indicated. In the specific case of the state of Rio de Janeiro, greater detail scales are desired to meet the demands. Thus, this proposal is justified by the need for compliance with legal requirements related to CAR, in addition to related interests for the forest monitoring with great spatial and temporal detail, in support of the fight against deforestation, among other malpractices.

It is noteworthy that there is no consolidated solution to generate a mapping of this nature, especially if we include the need for short deadlines for implementation. This framework points to the integration of research from different academic groups that, together and supported by experienced professionals from other institutions, participated in this technical solution.

The work is anchored on a Partnership Agreement celebrated together by the company Porto do Açu Operações S.A. and the institution Coordination of Projects, Research and Technology Studies (COPPETEC/UFRJ), having as an intermediate the Secretary of State for Environment (SEA, in portuguese) of Rio de Janeiro. The Laboratory of Remote Sensing and Environment Studies ESPAÇO of UFRJ, with the support from

$1 *$ Corresponding author 
the laboratories of Cartography GEOCART (UFRJ) and of Physical Geography LAGEF (UFF), built the methodological solution of this work.

The mapping area is composed by the Hydrographic Regions (HR) of the Médio Paraíba do Sul, Piabanha, Dois Rios and part of the Baixo Paraíba do Sul and Itabapoana, respectively identified by HR III, IV, VII and IX (figure 1).

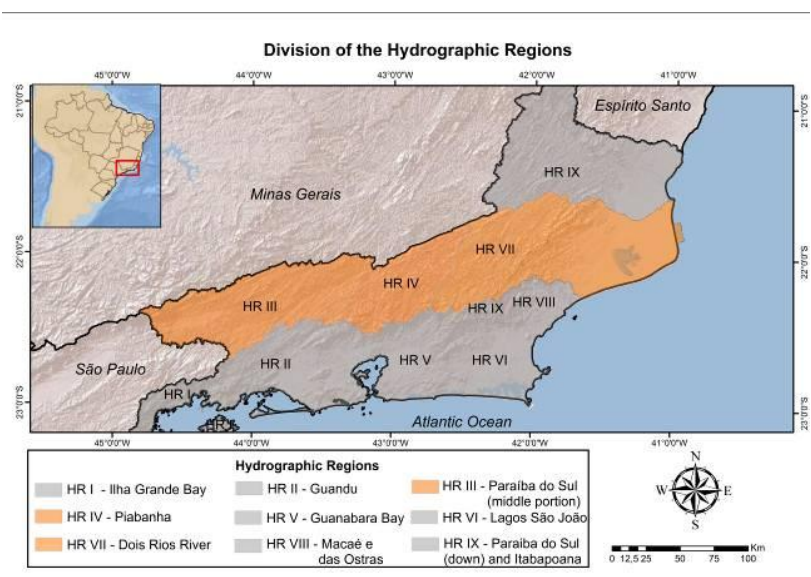

Figure 1: Division of the Hydrographic Regions of the state of

Rio de Janeiro (the mapping area is identified in orange)

The mapping was based on orbital imaging through different sensors and spatial resolutions. The integration of different sensors aimed at meeting the peculiarities of each class, as the temporal dynamics and the best spectral and spatial characterization.

The legend proposed for the final mapping was defined by two hierarchical levels, which were developed in two steps (Table $1)$.

\begin{tabular}{|l|l|}
\hline MACRO-CLASSES & \multicolumn{1}{|c|}{ DETAILING } \\
\hline $\begin{array}{l}\text { NATURAL Sandbar } \\
\text { FORESTED AREAS } \\
\text { (ANF, in portuguese) }\end{array}$ & $\begin{array}{l}\text { Forested Cogtal Sation and Mangrove } \\
\text { Vegetan }\end{array}$ \\
\hline $\begin{array}{l}\text { NATURAL NON- } \\
\text { FORESTED AREAS } \\
\text { (NNF) }\end{array}$ & $\begin{array}{l}\text { Non-Forested Coastal Sandbar } \\
\text { Vegetation }\end{array}$ \\
\hline $\begin{array}{l}\text { ANTHROPIC } \\
\text { AGROPASTORAL } \\
\text { AREAS (AAG) }\end{array}$ & $\begin{array}{l}\text { Silviculture } \\
\text { Areas of Consolidated Use } \\
\text { ANTHROPIC NON- } \\
\text { AGROPASTORAL } \\
\text { AREAS (ANA) }\end{array}$ \\
\hline WATER BODIES & \multicolumn{2}{|}{} \\
\hline
\end{tabular}

Each macro-class corresponds to a grouping of different, but functionally related, covers. This is another complexity for the knowledge modeling in support of the classification by GEOBIA. The classification was performed in hierarchical levels, supported by spectral, temporal, thematic and numeric data, in addition to the specialized knowledge about the spatial context in which each class is inserted.

Working Groups (WG) were defined in order to focus, in a specialized way, on each macro-class. The working teams were leveled so that the used interpretive criteria were homogeneous. The classification method to be adopted considered the most advanced technological processes available:
- Integration of multisensor and multidate data.

- Knowledge modeling, organizing bases and thresholds defined in the bibliography which helps us to build rules for classification.

- Data mining assisting the definition of parameters which characterize the classes, when the previous knowledge is considered not sufficient.

- Classification based on geographic objects, which allows the use of complementary data to the spectrum, increasing chances of achieving more complex legends.

The final product sought consistency in terms of connectivity for the entire state of Rio de Janeiro (link between hydrographic regions) and its structure is compatible to the manipulation through Geographic Information Systems (GIS).

\section{DATA PRE-PROCESSING}

In order to meet the proposed objectives, we used images acquired by four sensor systems with different resolutions: TM/Landsat 5 of 2008, OLI/Landsat 8 of 2014, RapidEye of 2014 and Worldview-2 of 2014, in addition to the Digital Elevation Model (DEM) from the SRTM mission. The year of 2014 was taken as reference for the mapping, being accepted images acquired within one year before or after this reference. In some few areas it was not possible to obtain RapidEye and Worldview-2 images without cloud cover in the proposed period, being necessary to move further away from the year of reference.

The Landsat 5 and 8 images were acquired for free on the USGS website with the spectral bands of visible light, near infrared and short wave infrared, all of them with 30 meters of spatial resolution and orthorectified. These images were used mainly for the detection of changes in order to identify the areas considered consolidated (agropastoral use previous to June of 2008). The consolidated areas need to be identified throughout Brazil on account of CAR, which deals with such areas in a different way.

The RapidEye images are acquired with 6.5 meters of spatial resolution and provided orthorectified with pixels resampled to 5 meters. In Brazil these images were acquired by the Ministry of the Environment (http://geocatalogo.mma.gov.br/) in a license option that allows them to be available to all government agencies with no extra cost, which encourages their use. For containing the near infrared band, in addition to the bands of visible light, these images were useful for generating indexes that allowed the identification of certain classes.

The Worldview-2 images were the main product in this mapping, being acquired from the DGBM (DigitalGlobe Basemap) system, which had five acquired licenses for one year for the development of this project, as well as for the generation of other products for the Secretary of State for Environment. The detailing presented by these images, as well as the relatively lower cost of acquisition by the DGBM was really important in this project. The fact that the license is valid for one year was very interesting, because it allowed new images to be acquired whenever necessary, for example for scenes with excessive presence of clouds or with problems in the geometric correction. Some major disadvantages were also observed, such as the absence of the near infrared band, which is very important in studies related to vegetation, and the problem of the great radiometric diversity observed between different 
scenes, which required a great effort of radiometric equalization. The images available on DGBM correspond only to the bands of visible light, merged with the panchromatic and resampled to 8 bits.

The SRTM DEM was acquired with pixel of 1 arcsecond (approximately 30 meters in Ecuador) and it was very useful for the separation of classes that can be differentiated, among other factors, depending on the altimetry, as was the case of high fields and mangroves.

The methodology predicted that the preliminary effort of preprocessing would be performed by a specific WG, including the preparation of the seed KIT for classification. Thus, all the WG received a standard eCognition project, or seed, to start their classification. This caution was taken, among other things, to facilitate the integration of the classes for the final mapping. This way the images would be distributed in KITs corresponding to pre-defined parts of the official Hydrographic Regions of the state of Rio de Janeiro. The mapping area was divided into 15 parts or KITs, containing, in addition to the images and mentioned DEM, the same segmentation and the mapping of the existing water bodies (water class). The segmentation was generated, after several tests, with a Scale Factor of 30, and weight of 0.5 for shape and 0.1 for compactness. The figure 2 presents the division in subprojects.

All the images were evaluated in terms of geometry and radiometry before being used in the mapping. The Landsat 5 and 8 images were obtained with orthorectification and atmospheric correction directly from the USGS website. Because they have already been the subject of several geometric reviews by our research group, it was decided not to assess the Landsat images in this project (CRUZ and BARROS, 2012). Considering their role of supporting the detection of changes on the cover, it was performed the radiometric normalization of the images of 2008 (Landsat 5) and the current images (Landsat 8). The equalizations were made on the Orthoengine software from PCI Geomatica 2015, while the normalization was made on the R software (CANTY et al, 2004; SCHROEDER et al., 2006).

The RapidEye and Worldview-2 images required more caution in the pre-processing step. The RapidEye images used in this mapping had the support of the official Cartographic Base of Brazil in the 1:25,000 scale for the orthorectification, which contributed for a better geometric correction. The Worldview-2 images are available orthorectified on DGBM. Thus, a geometry review was made comparing the relative displacement between the Worldview-2 and RapidEye images, as well as their absolute position using 20 control points obtained from the $1: 25,000$ cartographic base, identifiable in the images available for the teams. The displacements between the images and the cartographic base were below 15 meters in the tested areas and were acceptable to the $1: 25,000$ scale.

After several tests involving the detailing desired for the mapping in the 1:25,000 scale and the processing capacity and available storage, yet combined to the mapping deadline, it was decided to resample the Worldview-2 images to 2 meters. This way we optimize the processing without compromising the interpretation to the final scale.

Both RapidEye and Worldview-2 images passed by the radiometric equalization on Orthoengine from PCI Geomatica 2015 , in order to minimize the problems caused by the large radiometric variation observed. In figure 3 it is possible to see the variation between the Worldview- 2 images that compose the KIT, named HR3C, before and after the equalization.

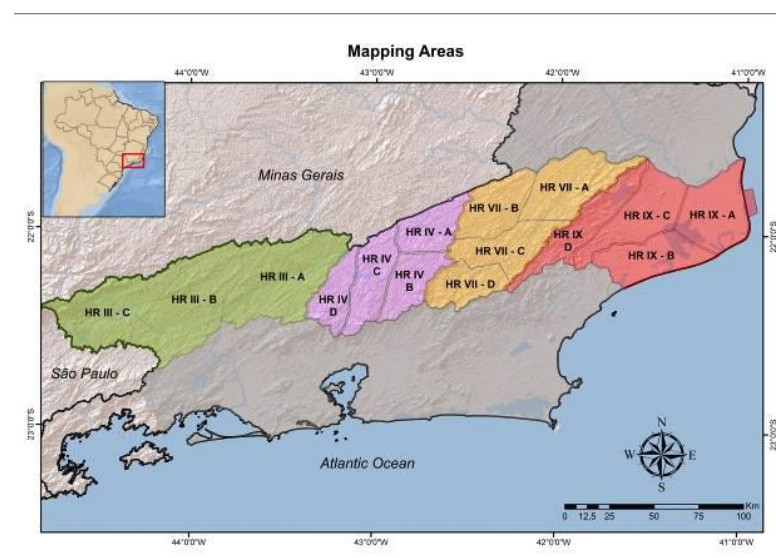

Figure 2: Subprojects for mapping
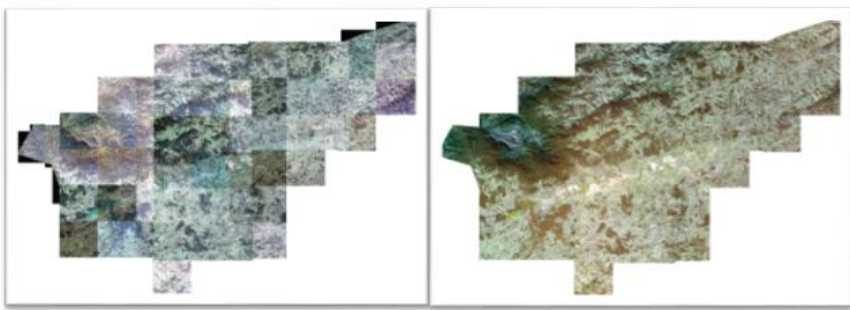

Figure 3: Effect from the Radiometric Equalization between Wordview-2 scenes

After the pre-processing steps, the elaborated KIT for each subproject was delivered with all the mentioned data, as well as the mapping of the Water Bodies class, thus ensuring the further integration of the parts. From these KITs, the teams started the mapping of the macro-class under their responsibility.

\section{KNOWLEDGE MODELING AND CLASSIFICATION}

\subsection{Mapping of the Macro-Classes}

The choice of specialized modeling by WG was fundamental for the conclusion of the mapping in short term, because it allowed the fast leveling of the photo interpreters and the enhancement of the method along the sequential classifications.

The specialized modeling by macro-classes presents benefits, such as: i) lower dependence on previous know-how by the team - once each group is able to focus on the modeling of a single class; ii) mapping speed; iii) continuous verification of commission errors by class/WG; iv) identification of the most complex classes for modeling, candidates for further increase of efforts.

The four macro-classes mapped in this work are detailed in table 2, which presents the set of functional classes that were grouped in each one of them. The subclasses diversity demanded a lot of effort on the modeling and the classification.

\begin{tabular}{|c|c|c|}
\hline CLASS & DEFINITION & DETAILING \\
\hline Natural & It comprises a set of & Dense Forest, Open \\
Forested & forest structures on & Forest; Seasonal \\
Areas & different successional & Forest; Mixed Rain \\
(ANF) & stages of development, & Forest, Coastal \\
& & \\
\hline
\end{tabular}




\begin{tabular}{|c|c|c|}
\hline & $\begin{array}{l}\text { distributed by different } \\
\text { environments and } \\
\text { geographic situations. }\end{array}$ & $\begin{array}{l}\text { Sandbar vegetation } \\
\text { and Mangroves. } \\
\text { Different conservation } \\
\text { states were included. }\end{array}$ \\
\hline $\begin{array}{l}\text { Natural } \\
\text { Non- } \\
\text { Forested } \\
\text { Areas } \\
\text { (NNF) }\end{array}$ & $\begin{array}{l}\text { Natural areas with, } \\
\text { eventually, non- } \\
\text { forested vegetation } \\
\text { (shrubby or } \\
\text { herbaceous), in } \\
\text { addition to other } \\
\text { natural non-forested } \\
\text { indentation. }\end{array}$ & $\begin{array}{l}\text { Relic communities; } \\
\text { outcroppings; active } \\
\text { gullies; coastal } \\
\text { sandbar vegetation, } \\
\text { marsh areas. }\end{array}$ \\
\hline $\begin{array}{l}\text { Anthropic } \\
\text { Agropastor } \\
\text { al Areas } \\
\text { (AAG) }\end{array}$ & $\begin{array}{l}\text { Areas used for } \\
\text { production of food, } \\
\text { fibers and agribusiness } \\
\text { commodities. Both } \\
\text { heterogeneous } \\
\text { agricultural areas and } \\
\text { extensive } \\
\text { pasturelands. }\end{array}$ & $\begin{array}{l}\text { Temporary and } \\
\text { permanent crops; } \\
\text { planted pastures; } \\
\text { silviculture; in } \\
\text { addition to proven } \\
\text { agropastoral areas. } \\
\text { Then the consolidated } \\
\text { areas were separated } \\
\text { (previous to June of } \\
\text { 2008). }\end{array}$ \\
\hline $\begin{array}{l}\text { Anthropic } \\
\text { Non- } \\
\text { Agropastor } \\
\text { al Areas } \\
\text { (ANA) }\end{array}$ & $\begin{array}{l}\text { All types of land use } \\
\text { of non-agropastoral, } \\
\text { non-forested and non- } \\
\text { water nature. }\end{array}$ & $\begin{array}{l}\text { Urban areas (villages, } \\
\text { cities, industrial } \\
\text { complexes); mining } \\
\text { areas; exposed soil } \\
\text { areas, parks. }\end{array}$ \\
\hline
\end{tabular}

Table 2: Detailing of the Macro-classes

Each WG worked independently on the definition of the best way to identify their mapping class. For this, they have considered: (i) the team's previous knowledge on the software and image interpretation; (ii) the experience with GEOBIA; (iii) the spectral characteristics of the targets inserted in the class and; (iv) the scope of the class. These two last aspects, in addition to the complexity of the used data, reflected in the difficulty to achieve a single classification model, replicable for all the 15 projects.

The ANF class presents great extent on the mapping area, mainly on the HR III and IV, which comprise part of the Mountain Region of the state. The modeling of this class was one of the most complex, because its distribution occurs in different lighting conditions due to sharp relief and the incorporation of different vegetation structures, with different compositions and conservation states. The team chose to use, on each project, the tiles division feature available at eCognition, starting with a general classification model, for further tile-totile adjustments. In a general way, the scale factor of the selected segmentation varied between 20 and 50, considering only the WorldView images. The main adopted descriptors were: the first PCA component; HUE and Intensity; in addition to an extra descriptor, resulting of the subtraction of HUE by PCA.

The modeling of the NNF class was performed with three different approaches, proper for the identification of: (i) altitude fields and rocky outcroppings; (ii) other types of outcrops and; (iii) exposed soils. For the altitude fields, the adopted technique was the GEOBIA, through the NDVI average (of the RapidEye image) and the altitude (SRTM). As the outcroppings and exposed soils have a very punctual occurrence, their identification was performed through visual classification.

The AAG class has the largest spatial expression in the area. It presents significant spectral diversity, as it incorporates both "green" and "non-green" regions, and compared to this characteristic the modeling of this class followed the top-down logic that first separates "green" and "non-green" targets, further applying data mining to eliminate areas which do not belong to this macro-class. Within this class, the silviculture areas were treated in a particular way. Their identification used a mixed of techniques, including the use of a previous mapping from 2009 and some textural patterns (AMORIM et al., 2012).

The greatest challenge for mapping the ANA class was its subclasses' heterogeneity. One of the greatest difficulties was the separation between urban and rural areas. In the case of urban areas, different construction intensities were found, besides the presence of exposed soils and small green areas. The relief was another difficulty factor, because many cities were shaded, being hard to delimit. The variation of the angle of incidence of high-resolution sensors was revealed to be problematic in these cases. Thus, a top-down and multiresolution approach was chosen, starting from macro segmentation with scale parameter between 40 and 50, based on the Landsat and RapidEye images. In the modeling, the main adopted descriptors were the Red band average of the RapidEye and Landsat images.

Each WG was responsible for the reintegration of the parts of each Hydrographic Region. This reintegration was performed on the original project with the seed segmentation through the use of mode function. Thus, a single segmentation for the mapping and the association of the predominant class for each object was reestablished.

\subsection{Mapping Integration}

The mapping integration of all WGs left off the individual delivery of each macro-class and the silviculture class, in raster format, with two identified classes: the one in the WG's interest and the area of no interest (or areas not classified by the WG). The spatial resolution of these five raster files was equal to the $2 \mathrm{~m}$ original. Choosing the raster structure is justified by the fact that the classes integration is made through the Combine Raster function on ArcGIS, which was shown to be the most operational way, given the data volume involved.

The Combine Raster results in a combination of all classes against all classes, presenting, at the end, a raster file with the total number of combinations found. In order that the operation occurs satisfactorily, the original files must be organized. The table which accompanies the Combine raster, was done on Excel, where the integration statistics were more easily calculated, allowing the establishment of rules for the next step of the integration, on eCognition. The calculated statistics by HR, were: (i) percentage of classification without confusion (pixels identified by a single WG); (ii) percentage of unclassified areas (pixels not identified by any WG); (iii) percentage of the confusions diversity between WG, being separated by types of confusion. In average, all the HR presented the following percentages: (i) 80 to $90 \%$ of classification without confusion; (ii) 2 to $8 \%$ of unclassified areas and; (iii) 10 to $15 \%$ of confusion areas. The Combine file was then regrouped in a set of 12 to 15 classes, representing the 
observed arrangements. For each situation, the best actions to be taken in support of the final integration modeling on eCognition were discussed in group.

The final project created on eCognition was structured with the images used by the WG, the most detailed segmentation (20 or 40) and the Combine file regrouped in raster format. Figure 4 presents the main steps performed on eCognition, which aimed at solving the identified confusions and omissions.

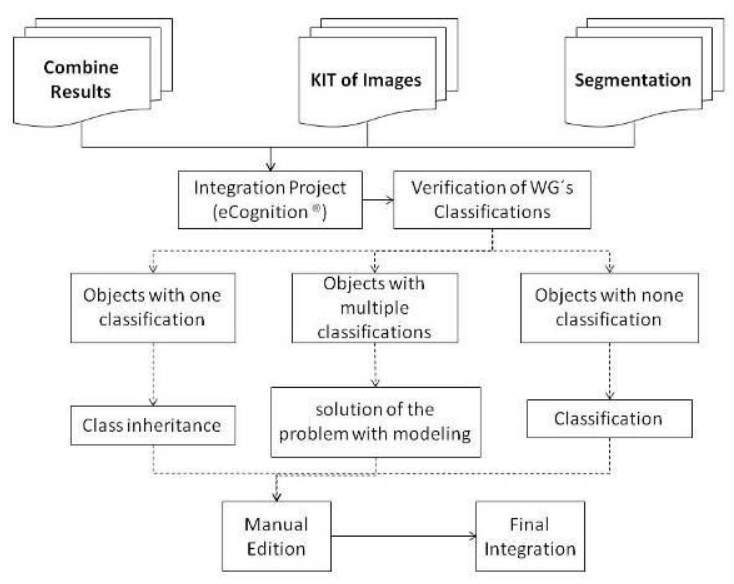

Figure 4: Flowchart with the integration steps on eCognition

First, the mapping areas without confusion were identified, associating the objects of segmentation by mode to the macroclasses. Next, the confusion areas, mapped by more than one WG, were treated. Areas smaller than 0.5 ha were ignored in this step for further treatment with generalization operations. Confusions between the ANF and AAG classes were predominant in all HR, which was expected given that they are the classes with the most spatial expression in the area and for the difficulty to separate the initial secondary vegetation, usually in transition areas. The modeling used, mainly, the NDVI average of the RapidEye image. Smaller confusions, between other classes, were treated in a particular way. The areas not classified by any WG were modeled from spectral characteristics and context.

After the modeling, the obtained results passed by a manual edition, prioritizing more complex cases. The final mapping, exported from eCognition, passed yet by generalization operations on ERDAS (Clump and Eliminate features) to eliminate noise and small unidentified areas. Redefining the topology was one last operation performed by the ArcGIS software.

Each HR, totally integrated, was returned to the WG for a last verification of the decisions taken regarding the unclassified and confusion areas. The final product was taken to the field for validation and the elimination of possible doubts.

Figure 5 presents the final layout of the Hydrographic Region of the Médio Paraíba do Sul, HR III, where it can be perceived the predominance of the ANF (Natural Forested Areas) and AAG (Anthropic Agropastoral Areas) classes.

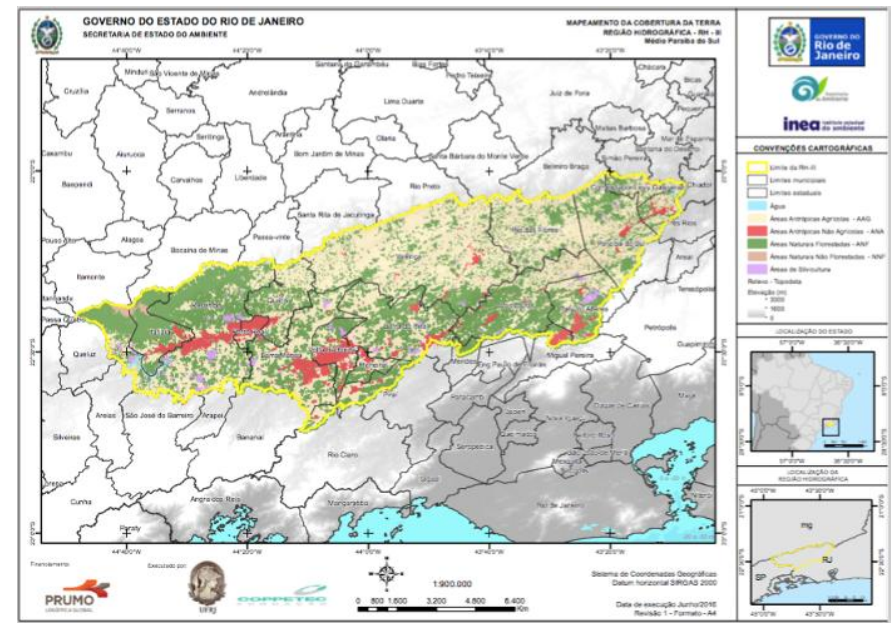

Figure 5: Final layout of the Hydrographic Region of the Médio Paraíba do Sul, HR III. (Legend: • Water Bodies; AAG; $\bullet$ ANF; $\bullet$ NNF; $\bullet$ ANA and $\bullet$ Silviculture

\section{RESULTS AND DISCUSSION}

The use of GEOBIA in cartographic production is still rare in Brazil, in spite of the country having an enormous territorial size (approximately 8.5 million $\mathrm{km}^{2}$ ) and presenting innumerous difficulties for the generation and update of several mappings, as much as in high detail scale, as in thematic.

With experience in other two great mapping initiatives supported by GEOBIA, also aimed at cartographic production in partnership with governmental institutions, the research group from the ESPAÇO laboratory, coordinated the mapping of the Atlantic Forest's biome (CRUZ et al., 2007) in the 1:250,000 scale and the land use and cover of the state of Rio de Janeiro regarding the Ecological and Economic Zoning (CRUZ et al., 2009) in the 1:100,000 scale. Both experiences presented interesting results, in terms of team learning and knowledge improvement for modeling, as well as the investments and deadlines.

The present experiment contributes to solutions that require detailing (1:25,000 scale), accuracy and agility on execution. The total performance time of mapping an area of approximately $20,000 \mathrm{~km}^{2}$, with a minimum area of $0.5 \mathrm{ha}$, was divided into: (i) 4 months of pre-processing; (ii) 6 months of mapping and integration (simultaneous with 2 months of preprocessing); (iii) 2 months for final editions and validation.

The greatest difficulty in this method was the absence of infrared bands on the DGBM (DigitalGlobe Basemap) system, specially the near infrared, demanding the incorporation of another sensor, RapidEye's, and more efforts of processing and data integration; in addition to increasing the data volume to be treated on eCognition and difficulties in modeling. Currently the DGBM system is starting to incorporate images with NIR.

The common cartographic challenge of mapping extensive areas with great detail requires methodological solutions which offer viability and a good relation between cost and benefits. In Brazil, a country whose cartography lacks detail and, mainly, update, it is foreseen that investments would be made.

The project's current step is the validation by obtaining field data. In spite of still not having the accuracy obtained on the final mapping, the integration process of the parts through 
Combine Raster allowed the comprise of greater weakness and certainties of the mapping, directing the following edition step.

Thus, the analysis of the mapping final accuracy is in progress. The field works for collecting points are being structured with mixed teams of members from all the WG, increasing the decision's synergy.

\section{REFERENCES}

AMORIM, H.B.; FRANCELINO, M.R.; SALAMENE, S.; PEDREIRA, L.O.L.; ASSUMPÇÃO FILHO, L.I.; CAPITANO, R.C.; MOURA, T.A. Estimate of the Area Occupied by Reforestation Programs in Rio de Janeiro State. Cerne, Lavras, v. 18, n. 1, p. 27-32, jan./mar. 2012.

CANTY, M.J.; NIELSEN, A.A.; SCHIMIDT, M. Automatic radiometric normalization of multitemporal satellite imagery. Remote Sensing of Environment, 91, 441-451, 2004.

CRUZ, C.B.M.; BARROS, R.S. Contribution of New Sensors to Cartography. In: Cartography, Chapter XX, Editora InTech, 2012, v.01, 20 pg. ISBN 979-953-307-910-2.

CRUZ, C.B.M.; ROSÁRIO, L.S.; ABREU, M.B.; ALMEIDA, P.P.M.; VICENS, R.S.; CRONEMBERGER, F.M. Classificação Orientada a Objetos na Geração do Mapa de Uso e Cobertura da Terra do estado do Rio de Janeiro. XIV Simpósio Brasileiro de Sensoriamento Remoto, INPE. 77897796. Natal, RN. ISBN: 978-85-17-00044-7. INPE, 2009.

CRUZ, C.B.M.; VICENS, R.S.; SEABRA, V.S.; REIS, R.B.; FABER, O.A.; RICHTER, M.; ARNAULT, P.K..; ARAUJO, M. Classificação Orientada a Objetos no Mapeamento dos Remanescentes da Cobertura Vegetal do Bioma Mata Atlântica na escala 1:250.000. XIII Simpósio Brasileiro de Sensoriamento Remoto, INPE. 5691-5699. Florianópolis, SC. ISBN: 978-85-17-00031-7. INPE, 2007.

SCHROEDER, T.A.; COHEN, W.B.; SONG, C.; CANTY, M.J.; YANG, Z. Radiometric correction of multi-temporal Landsat data for characterization of early successional forest patterns in western Oregon. Remote Sensing of Environment, 103, 16-26, 2006.

SOKOLONSKI, H.H. "Manual Técnico de Uso da Terra." Rio de Janeiro: IBGE (1999). 


\title{
IMPROVING THE SPEED OF MULTIRESOLUTION SEGMENTATION USING SLIC SUPERPIXELS
}

\author{
O. Csillik ${ }^{\mathrm{a}, *}$, S. Lang ${ }^{\mathrm{a}}$ \\ ${ }^{a}$ Department of Geoinformatics - Z_GIS, University of Salzburg, 5020, Salzburg, Austria - (ovidiu.csillik, stefan.lang)@ sbg.ac.at
}

KEY WORDS: pixels, superpixels, OBIA, oversegmentation, runtime, eCognition

\begin{abstract}
:
The popular multiresolution segmentation (MRS) algorithm is time and memory consuming when dealing with large images because it uses the pixel-grid for the initial object representation. In this study, we have tested a new workflow for image segmentation of remote sensing data, starting the MRS (using the ESP2 tool) from the superpixel level (using SLIC superpixels) and aiming at dramatically reducing the amount of time and computational resources needed to automatically partition relatively large datasets of very high resolution (VHR) remote sensing images. Tests were done on Quickbird and WorldView-2 data and the results show that the proposed workflow outperforms the traditional approach (MRS starting from pixels). The computational time was reduced in all cases, the biggest improvement being from $5 \mathrm{~h} 35 \mathrm{~min}$ to $13 \mathrm{~min}$, for a WorldView-2 scene with 8 bands and an extent of 12.2 million pixels. This also comes with a slight improvement of the geometric accuracy of the extracted objects. This approach has the potential to enhance the automation of big remote sensing data analysis and processing, especially when time is an important constraint.
\end{abstract}

\section{INTRODUCTION}

The aim of image segmentation is to partition an image into relatively homogeneous objects, non-overlapped and spatially adjacent (Blaschke, 2010). The pixel-grid is used as the underlying representation for many image segmentation algorithms, including the popular multiresolution segmentation (MRS) (Baatz and Schäpe, 2000). However, pixels are not natural entities and are unlikely to match the content of the space represented (Fisher, 1997). It would be more natural and efficient to work with perceptually meaningful entities that are derived from pixels using a low-level grouping process (Ren and Malik, 2003; Neubert and Protzel, 2012). To achieve this, we can partition an image into superpixels, which are the result of perceptual grouping of pixels based on similar characteristics (e.g. color) (Neubert and Protzel, 2012).

In computer vision, using superpixels (Achanta et al., 2012) to speed up later-stage processing are becoming increasingly popular in many applications (Achanta et al., 2012; Neubert and Protzel, 2012; Van den Bergh et al., 2012). In remote sensing few studies have used superpixels (Thompson et al., 2010; Guangyun et al., 2015; Ortiz Toro et al., 2015; Vargas et al., 2015). Even so, they did not tackle the usage of superpixels in order to improve the computational efficiency of segmenting remote sensing data.

We have tested an alternative workflow for image segmentation of remote sensing data, starting the MRS from the superpixels level and aiming at dramatically reducing the amount of time and computational resources needed to partition relatively large datasets of VHR remote sensing data. This approach is compared with the traditional one, starting the MRS from the pixel level, regarding the geometric accuracy of the objects and the computational time.
The following section (Section 2) describes the dataset used, the theoretical background of the superpixels, the workflow of generating objects and the accuracy measures. Section 3 compares the results of the two approaches in terms of accuracy and computational time. Section 4 drafts the major implications of the results, while Section 5 concludes the main findings of our study.

\section{METHODS}

\subsection{Datasets}

Tests were conducted on very high resolution remote sensing data (T1, T2 - Quickbird and T3 - WorldView-2). The spatial resolution ranges from $0.5 \mathrm{~m}$ (T3) to $0.6 \mathrm{~m}$ ( $\mathrm{T} 1$ and $\mathrm{T} 2$ ), respectively, as well as the number of bands, between 4 and 8 . The extent (in pixels) ranges between approx. 4 mil. pixels (T1), 12.2 mil. pixels (T3) and 12.3 mil. pixels (T2). Test area T1 covers a dense residential and services area in the city of Salzburg, Austria. The T2 test area is comprised of dense clustered residential and commercial buildings with large green spaces in between. Test area T3 represents a sensitive riparian habitat with forests, agricultural fields and water bodies. We have used different extents, landscapes and number of bands to assess how the computational time is affected by these factors.

\subsection{Simple Linear Iterative Clustering (SLIC) superpixels}

Simple Linear Iterative Clustering (SLIC) (Achanta et al., 2012; Achanta et al., 2010) have been proven to outperform other state-of-the-art superpixel methods, because of its simplicity, adherence to boundaries, computational speed and more memory efficiency (Achanta et al., 2012). SLIC has only one parameter, which is the desired number of equally sized superpixels to be generated.

\footnotetext{
* Corresponding author
} 
SLIC is an adapted $k$-means clustering, but what it makes it so fast and computationally efficient is that SLIC does not compare each pixel with all pixels in the scene. For a region of approximate size $S \times S$, the distance $D$ (which combines distance of color proximity and spatial proximity) is computed in a region $2 S \times 2 S$ around the superpixel center, reducing dramatically the number of $D$ calculations. Achanta et al. (2012) found out that 10 iterations for superpixels derivation are sufficient for most images and, therefore, we are using this threshold for SLIC superpixels generation.

The same authors proposed a parameter-free SLIC version (SLICO), which generates regular shaped superpixels across the scene, regardless of textured or non-textured regions in the image, while SLIC is influenced by the texture, generating smooth regular-sized superpixels in the smooth regions and highly irregular superpixels in the textured regions (Achanta et al., 2012).

For generating superpixels, we have used a freely available GDAL implementation (GDAL-segment), available on https://github.com/cbalint13/gdal-segment. Superpixels were generated using SLIC and SLICO algorithms (Achanta et al., 2012), as implemented in the tool mentioned, with an initial size of clustering of $10 \times 10$ pixels. We have used this value after trial-and-error, in order to avoid an extreme oversegmentation or too large superpixels (Figure 1).

\subsection{Multiresolution segmentation: pixels vs. superpixels}

One of the biggest issues of MRS is the selection of parameters, of which the most important one is the scale parameter (SP). Drăguţ et al. (2010) developed Estimation of Scale Parameter tool (ESP) to detect optimal scales based on local variance graph, using a single layer. Drăguţ et al. (2014) extended this approach into an automated tool (ESP2) for multiple layers. The ESP2 tool is a fully automated methodology for the selection of scale parameters to extract three distinct scales using MRS, implemented in the eCognition Developer software (Trimble Geospatial).

For reasons of objectivity, we have used the ESP2 tool starting from the pixel level, as usual, and starting from the superpixel level, using a hierarchical bottom-up region merging approach (i.e. starting from an initial level, the next level is generated based on the previous one) to derive only the finest level of objects (Level 1 of the hierarchy approach of ESP2).

\subsection{Segmentation accuracy of the results}

We evaluated the segmentations results by comparing the geometries of resulted objects with 50 manually digitized reference objects for each test area. We have used 5 measures of accuracy: Area fit index (AFI) (Lucieer and Stein, 2002), Oversegmentation (OS), Under-segmentation (US), Root mean square (D) (Clinton et al., 2010) and Quality rate (QR) (Winter, 2000 ), with a minimum percent overlap of $50 \%$. In the case of a perfect match between the geometries of objects, AFI, OS, US and $\mathrm{D}$ would be 0 and QR would be 1 . The measures are implemented into an eCognition tool by Eisank et al. (2014).

Besides geometry comparison, we measured the computational time needed for both approaches. For the ESP2 starting from pixel level we retain only the time needed to run the tool, while for ESP2 starting from superpixel level, we added to the time
ESP2 needs to run the computational time of generating superpixels.
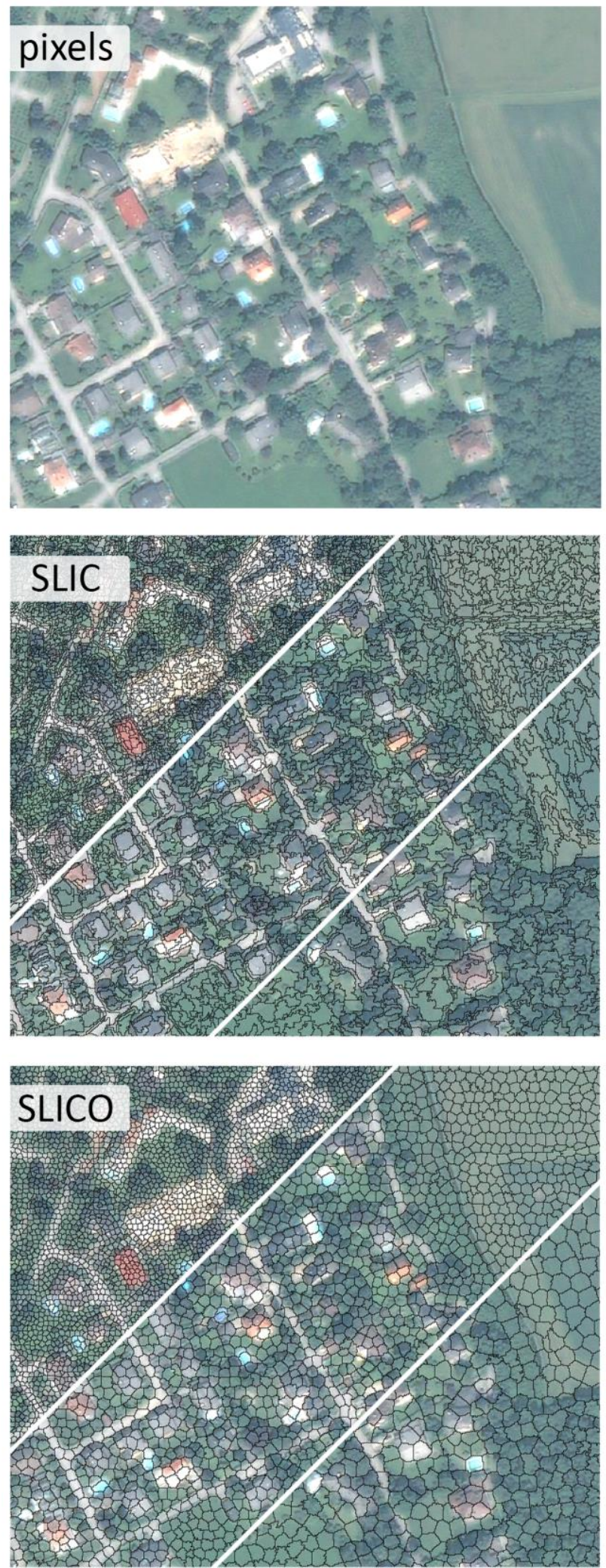

Figure 1. Visual comparison of superpixels produced by SLIC and SLICO methods, on a subset of T2. The average superpixel size in the upper left of the image is $5 \times 5$ pixels, in the middle of the image is $10 \times 10$ pixels and $15 \times 15$ pixels in the lower right. 


\begin{tabular}{|c|c|c|c|c|c|c|c|c|c|c|}
\hline \multirow{2}{*}{ Test } & & \multicolumn{3}{|c|}{ Segmentation results } & \multicolumn{5}{|c|}{ Segmentation accuracy metrics } & \multirow{2}{*}{ Time } \\
\hline & & Number of & SP & Number of objects & AFI & OS & US & $\mathrm{D}$ & QR & \\
\hline \multirow[t]{3}{*}{$\mathrm{T} 1$} & Pixels & $1,403,574$ & 69 & 3,109 & 0.499 & 0.560 & 0.121 & 0.405 & 0.414 & $1 \min 29 \mathrm{~s}$ \\
\hline & SLIC & 13,835 & 81 & 2,017 & 0.388 & 0.463 & 0.122 & 0.338 & 0.499 & $27 \mathrm{~s}$ \\
\hline & SLICO & 13,906 & 61 & 2,757 & 0.447 & 0.515 & 0.122 & 0.374 & 0.454 & $24 \mathrm{~s}$ \\
\hline \multirow[t]{3}{*}{$\mathrm{T} 2$} & Pixels & $12,696,684$ & 172 & 4,670 & 0.174 & 0.229 & 0.067 & 0.169 & 0.729 & $2 \mathrm{~h} 42 \min 40 \mathrm{~s}$ \\
\hline & SLIC & 123,153 & 173 & 4,204 & 0.088 & 0.161 & 0.079 & 0.127 & 0.782 & $13 \min 02 \mathrm{~s}$ \\
\hline & SLICO & 125,842 & 148 & 5,354 & 0.335 & 0.386 & 0.075 & 0.278 & 0.584 & $9 \min 49 \mathrm{~s}$ \\
\hline \multirow[t]{3}{*}{$\mathrm{T} 3$} & Pixels & $12,217,001$ & 220 & 1,632 & 0.100 & 0.148 & 0.052 & 0.111 & 0.813 & $5 \mathrm{~h} 35 \mathrm{~min} 24 \mathrm{~s}$ \\
\hline & SLIC & 131,415 & 212 & 1,702 & 0.062 & 0.124 & 0.066 & 0.099 & 0.823 & $13 \min 03 \mathrm{~s}$ \\
\hline & SLICO & 121,525 & 172 & 2,338 & 0.223 & 0.275 & 0.066 & 0.200 & 0.688 & $10 \mathrm{~min} 46 \mathrm{~s}$ \\
\hline
\end{tabular}

Table 2. Comparison of segmentation accuracy and computational time for the three test areas (T1 to T3), using pixels, SLIC and SLICO superpixels, respectively.

\section{RESULTS}

All the tests were conducted on a computer station with Intel Core i5-4590 CPU $(3.30 \mathrm{GHz})$ processor with $8 \mathrm{~GB}$ RAM, using a 64-bit Windows 7 operating system.

Generating SLIC and SLICO superpixels is a very fast and memory efficient procedure. In the case of SLIC superpixels, the scored runtime was $2 \mathrm{~s}$ for $\mathrm{T} 1,18 \mathrm{~s}$ for $\mathrm{T} 2$ and $26 \mathrm{~s}$ for $\mathrm{T} 3$, while for SLICO was $3 \mathrm{~s}$ for T1, 34s for T2 and 37s for T3. It is obvious that computing SLICO superpixels is a slightly slower procedure and this could be mainly because SLICO generates regular shaped superpixels across the scene, thus having supplementary computational constraints (i.e. compactness) (Figure 1).

The complexity of the three test areas was significantly reduced by aggregating similar pixels into superpixels. Due to this fact, SLIC and SLICO superpixels are making a big difference in segmentation, regarding total computational time. Larger the scene and bigger the number of bands, bigger the time needed for ESP2 to run starting from a pixel-grid (from $1 \mathrm{~min} 29 \mathrm{~s}$ to $5 \mathrm{~h}$ $35 \mathrm{~min} 24 \mathrm{~s}$ ) (Table 2). Runtime of ESP2 starting from SLIC superpixels is higher than that of ESP2 starting from SLICO superpixels, the difference ranging between $3 \mathrm{~s}$ for $\mathrm{T} 1$ and $3 \mathrm{~min}$ $13 \mathrm{~s}$ for $\mathrm{T} 2$. This is mainly because the scale parameter detected for the latter case is smaller and therefore the processing of ESP2 stops earlier. This is explained by the fact that SLICO superpixels have compactness constraints to follow a regular lattice and, as a consequence, it can omit meaningful image boundaries, increasing the internal heterogeneity of the superpixels. SLIC superpixels have reduced the runtime by a magnitude of $229 \%$ for T1, $1146 \%$ for $\mathrm{T} 2$ and $2476 \%$ for T3, respectively.

The scale parameters for pixel-grid and SLIC superpixels are similar. The most evident case is for T3 (220 and 212, respectively), where approximately the same number of objects were extracted in the end (1632 and 1702, respectively).

In all cases, using SLIC superpixels outperformed the other two approaches regarding the geometric accuracy of the final objects (Table 2). Compared to the pixel approach, SLIC has better values of QR for T1 (0.499 compared to 0.414$)$, for T2 $(0.782$ compared to 0.729 ) and for T3 (0.823 compared to 0.813$)$. The only indicator where SLIC had worse values than the pixel approach is the US, the difference ranging from 0.001 for $\mathrm{T} 1$ and 0.014 for $\mathrm{T} 3$, but this could be considered a negligible difference when aiming at considerably improving the runtime. The oversegmentation of the scene using SLICO superpixels negatively impacts the accuracy measures and, therefore, they are better than the pixel approach only in the case of $\mathrm{T} 1$ (QR of 0.454 compared to 0.414 ), where the number of objects is slightly smaller. In test area $\mathrm{T} 1$, even if there is a big difference in number of objects extracted between pixel approach and SLIC superpixels (3109 and 2017, respectively), the US has the same value, while OS decrease by 0.1 . This could mean that SLIC superpixels are better adhering to the boundaries and, therefore, creating more meaningful objects than those generated by starting from a pixel-grid.

\section{DISCUSSION}

Using the adapted workflow, we are able to increase the maximum extents on which ESP2 for MRS runs. Supplementary tests (not shown here) proven that for scenes of tens of millions of pixels ESP2 is successfully fast and memory efficient when starting from superpixels, while in the case of starting from pixels it crashes due to the immense amount of resources needed to compute the statistics at the pixel-level. The size of the superpixels should carefully be chosen, not to worsen the computational efficiency (by generating too small superpixels) or to contain more than one class inside a superpixel (by having too coarse superpixels).

We have used SLIC and SLICO superpixels because they were shown that outperform the other state-of-the-art superpixel algorithm (Achanta et al., 2012). In our study, applying MRS on SLIC superpixels has proven to be the most efficient in terms of geometric accuracy of final objects. One minor drawback of using SLIC and SLICO superpixels in MRS is that we introduce further parameterization in the process, but as implemented in the GDAL-segment tool, the only parameters you have to set is the desired size of the generated superpixels, that means how finer the generated superpixels should be.

Using VHR images with different characteristics gave us an overview of their effects in generating superpixels. For the same size of desired superpixels, the larger the extent of the scene and higher the number of layers, the higher the computational time. But the most important influence over the runtime is the extent of the generated superpixels: smaller superpixels will require longer time than larger superpixels. 


\section{CONCLUSION}

In this paper, we have tested a workflow to efficiently partition an image into objects by using SLIC superpixels as the starting point for MRS. When compared to the traditional approach (starting from pixel-grid), our approach outperformed both in terms of geometric accuracy of the extracted objects and computational time. This approach has the potential to enhance the automation of big remote sensing data analysis and processing, especially when time is an important constraint.

\section{ACKNOWLEDGEMENTS}

This work was supported by the Austrian Science Fund (FWF) through the Doctoral College GIScience (DK W1237-N23). WorldView-2 imagery was provided through the FP7 Project MS.MONINA (Multi-scale Service for Monitoring NATURA 2000 Habitats of European Community Interest), Grant agreement No. 263479 and the INTERREG Project EuLE (EuRegional Spatial Analysis). Quickbird imagery was kindly provided by the Department of Geoinformatics - Z_GIS, Salzburg, Austria.

\section{REFERENCES}

Achanta, R., Shaji, A., Smith, K., Lucchi, A., Fua, P., Süsstrunk, S., 2012. SLIC Superpixels Compared to State-ofthe-Art Superpixel Methods. Pattern Analysis and Machine Intelligence, IEEE Transactions on, 34, pp. 2274-2282.

Achanta, R., Shaji, A., Smith, K., Lucchi, A., Fua, P., Süsstrunk, S., 2010. Slic superpixels. École Polytechnique Fédéral de Lausssanne (EPFL), Technical Report 149300.

Baatz, M., Schäpe, A., 2000. Multiresolution Segmentation-an optimization approach for high quality multi-scale image segmentation, In: Angewandte Geographische Informationsverarbeitung. Strobl, J., Blaschke, T., Griesebner, G. (Eds.), Wichmann-Verlag, Heidelberg, pp. 12-23.

Blaschke, T., 2010. Object based image analysis for remote sensing. ISPRS Journal of Photogrammetry and Remote Sensing, 65, pp. 2-16.

Clinton, N., Holt, A., Scarborough, J., Yan, L.I., Gong, P., 2010. Accuracy assessment measures for object-based image segmentation goodness. Photogrammetric Engineering and Remote Sensing, 76, pp. 289-299.

Drăguţ, L., Csillik, O., Eisank, C., Tiede, D., 2014. Automated parameterisation for multi-scale image segmentation on multiple layers. ISPRS Journal of Photogrammetry and Remote Sensing, 88, pp. 119-127.

Drăguţ, L., Tiede, D., Levick, S., 2010. ESP: a tool to estimate scale parameters for multiresolution image segmentation of remotely sensed data. International Journal of Geographical Information Science, 24, pp. 859-871.

Eisank, C., Smith, M., Hillier, J., 2014. Assessment of multiresolution segmentation for delimiting drumlins in digital elevation models. Geomorphology, 214, pp. 452-464.

Fisher, P., 1997. The pixel: a snare and a delusion. International Journal of Remote Sensing, 18, pp. 679-685.
Guangyun, Z., Xiuping, J., Jiankun, H., 2015. Superpixel-Based Graphical Model for Remote Sensing Image Mapping. Geoscience and Remote Sensing, IEEE Transactions on, 53, pp. 5861-5871.

Lucieer, A., Stein, A., 2002. Existential uncertainty of spatial objects segmented from satellite sensor imagery. Geoscience and Remote Sensing, IEEE Transactions on, 40, pp. 2518-2521.

Neubert, P., Protzel, P., 2012. Superpixel benchmark and comparison, Proc. Forum Bildverarbeitung, pp. 1-12.

Ortiz Toro, C., Gonzalo Martín, C., García Pedrero, Á., Menasalvas Ruiz, E., 2015. Superpixel-Based Roughness Measure for Multispectral Satellite Image Segmentation. Remote Sensing, 7, pp. 14620-14645.

Ren, X., Malik, J., 2003. Learning a classification model for segmentation, In: Computer Vision, 2003. Proceedings. Ninth IEEE International Conference on. IEEE, pp. 10-17.

Thompson, D.R., Mandrake, L., Gilmore, M.S., Castano, R., 2010. Superpixel Endmember Detection. Geoscience and Remote Sensing, IEEE Transactions on, 48, pp. 4023-4033.

Van den Bergh, M., Boix, X., Roig, G., de Capitani, B., Van Gool, L., 2012. Seeds: Superpixels extracted via energy-driven sampling, In: Computer Vision-ECCV 2012. Springer, pp. 1326.

Vargas, J., Falcao, A., dos Santos, J., Esquerdo, J., Coutinho, A., Antunes, J., 2015. Contextual superpixel description for remote sensing image classification, In: Geoscience and Remote Sensing Symposium (IGARSS), 2015 IEEE International, pp. 1132-1135

Winter, S., 2000. Location similarity of regions. ISPRS Journal of Photogrammetry and Remote Sensing, 55, pp. 189-200.

Revised July 2016 


\title{
SUPERPIXELS: THE END OF PIXELS IN OBIA. A COMPARISON OF STATE-OF-THE- ART SUPERPIXEL METHODS FOR REMOTE SENSING DATA
}

\author{
O. Csillik* \\ Department of Geoinformatics - Z_GIS, University of Salzburg, 5020, Salzburg, Austria - ovidiu.csillik@sbg.ac.at
}

KEY WORDS: SLIC, SLICO, SEEDS, LSC, segmentation, computer vision

\begin{abstract}
:
In computer vision, using superpixels or perceptually meaningful atomic regions to speed up later-stage processing are becoming increasingly popular in many applications. Superpixels are used as a pre-processing stage to organize an image into a low-level grouping process through oversegmentation, thus simplifying the computation in later stages. However, in remote sensing domain few studies use superpixels. Even so, there is no comparison between superpixel methods and their suitability for remote sensing images. In this study, we compare four state-of-the-art superpixel methods: Simple Linear Iterative Clustering (SLIC and SLICO), Superpixels Extracted via Energy-Driven Sampling (SEEDS) and Linear Spectral Clustering (LSC). We applied them to very high resolution remote sensing data of different characteristics (extent, spatial resolution and landscape complexity) in order to see how superpixels are affected by these factors. The four algorithms were compared regarding their computational time, ability to adhere to image boundaries and the accuracy of the resulted superpixels. Furthermore, we discuss the individual strengths and weaknesses of each algorithm and draw further applications of superpixels in OBIA.
\end{abstract}

\section{INTRODUCTION}

Nowadays, remote sensing community have to deal with the big volume, variety and velocity of the acquired geospatial data (Chen et al., 2015). Besides many advantages, this also comes with shortcomings, like the limited computational capabilities and a lack of powerful tools to handle the complexity of the data, especially when time is an important constrain in delivering of high quality geospatial information (e.g. Tiede et al., 2011).

What makes the computation so intensive is that many image segmentation algorithms and spatial analysis have as the underlying representation the pixel-grid. In the case of very high resolution (VHR) data the local spatial autocorrelation between the pixels is high, thus an object will be composed by many pixels with the same characteristics (Chen et al., 2012). To overcome this, it would be more natural and efficient to work with superpixels, which are an oversegmentation of the image, a low-level grouping of similar pixels in agreement to some desired homogeneity criterions (e.g. color) (Ren and Malik, 2003; Neubert and Protzel, 2012). Moving to superpixels allows us to measure feature statistics on a naturally adaptive domain rather than on a fixed window (Fulkerson et al., 2009). Furthermore, creating the final objects that matches the reality is a simpler task, by finding the superpixels which are part of the object (Fulkerson et al., 2009).

Using superpixels instead of pixels in the segmentation process have certain advantages: (1) superpixels carry more information than pixels and adhere better to the natural image boundaries (Neubert and Protzel, 2012; Guangyun et al., 2015); (2) superpixels are perceptually meaningful objects, having the scale between the pixel level and the object level (Achanta et al., 2012; Neubert and Protzel, 2012); (3) superpixels are of low computational complexity and can speed-up the subsequent image processing (Ren and Malik, 2003; Li and Chen, 2015); (4) superpixels reduce the susceptibility to noise and outliers and capture image redundancy (Shi and Wang, 2014) and (5) because superpixels are results of an oversegmentation, most structures in the image are conserved (Ren and Malik, 2003).

In computer vision, superpixels are used in many applications. However, in remote sensing superpixels are not used to their full capacity, even if they can improve or speed-up later processing. The main objective of this study is to give a first comparison of four state-of-the-art superpixel methods, applied on remote sensing data.

The following section (Section 2) describes the datasets used, briefly explains the superpixel algorithms and the evaluation methodology. Section 3 compares the results, while Section 4 explains and discusses the main findings and further directions.

\section{METHODS}

\subsection{Datasets}

We have used very high resolution remote sensing data: a Quickbird scene of $0.6 \mathrm{~m}$ spatial resolution and WorldView-2 scene of $0.5 \mathrm{~m}$ spatial resolution, as well as a LiDAR derived DSM, of $1 \mathrm{~m}$ spatial resolution. All three datasets have an extent of 4 million pixels. In the rest of the article, we will refer to the datasets as QB (Quickbird), WV2 (WorldView-2) and DSM (Digital Surface Model). QB and WV2 datasets are located in the city of Salzburg, with residential, industrial and urban green areas, while DSM covers a rural area, with sparse housing and forest patches. In order to run the superpixel algorithms, all three datasets were used in a RGB combination with an 8-bit color depth, for QB and WV2 the red, green and blue bands being used.

\footnotetext{
* Corresponding author
} 


\subsection{Superpixel algorithms}

Many superpixel algorithms exist, each with its advantages and limitations (Neubert and Protzel, 2012; Achanta et al., 2012). For this study, we compared 4 state-of-the-art superpixel algorithms, namely Simple Linear Iterative Clustering (SLIC and SLICO - parameter free) (Achanta et al., 2012), Superpixels Extracted via Energy-Driven Sampling (SEEDS) (Van den Bergh et al., 2012) and Linear Spectral Clustering (LSC) ( $\mathrm{Li}$ and Chen, 2015). In computer vision, the 4 algorithms were found to be very efficient and accurate, outperforming many existing algorithms (Achanta et al., 2012; Van den Bergh et al., 2012; Li and Chen, 2015). For derivation of superpixels, we have used the open-source GDAL implementation, available on https://github.com/cbalint13/gdalsegment. In the following paragraphs we are briefly describing the methods and, for more details, the reader is referred to the sources mentioned for each algorithm.

SLIC is a gradient-ascent based algorithm, which starts from a rough initial clustering of pixels and iteratively refine the clusters until some criterions are met to form the superpixels (Achanta et al., 2012). SLIC is an adapted $k$-means clustering and, by default, the only parameter of the algorithm is $k$, the desired number of approximately equally sized superpixels (Achanta et al., 2012). What makes SLIC fast and computational efficient is that, when clustering the pixels, it does not compare each pixel with all pixels in the scene. For a region of size $S \times S$, a distance $D$ (distance of color proximity and spatial proximity) is computed around the superpixel center, minimizing the number of $D$ calculations, and, therefore, improving the speed over conventional $k$-means clustering, where each pixel must be compared with all cluster centers (Achanta et al., 2012).

The same authors (Achanta et al., 2012) proposed a parameterfree version of SLIC (SLICO), which generates regular shaped superpixels across the scene, regardless of textured or nontextured regions in the image, while SLIC is influenced by the texture, generating smooth regular-sized superpixels in the smooth regions and highly irregular superpixels in the textured regions (Achanta et al., 2012).

SEEDS algorithm is a simple hill-climbing optimization which starts from an initial superpixel partitioning and continuously refines the superpixels by modifying the boundaries (Van den Bergh et al., 2012). The algorithm is based on a robust and fast to evaluate energy function, based on enforcing color similarity between the boundaries and the superpixel color histogram (Van den Bergh et al., 2012).

In LSC, each image pixel is mapped to a point in a ten dimensional feature space where weighted $k$-means is applied for segmentation ( $\mathrm{Li}$ and $\mathrm{Chen}, 2015)$. Non-local information is implicitly preserved due to the equivalence between the weighted $k$-means clustering in this ten dimensional feature space and normalized cuts in the original pixel space ( $\mathrm{Li}$ and Chen, 2015).

For a better overview of the algorithms, for each test area we have derived superpixels starting from initial sizes of $5 \times 5$, $10 \times 10,15 \times 15$ and $20 \times 20$, respectively. A number of 10 iterations for superpixels clustering and refinement was used for each method.

\subsection{Evaluation}

Comparison of the algorithms was done qualitatively and quantitatively. Each superpixel segmentation results were visually inspected in order to draw conclusions about the quality of boundary adherence of each algorithm. Since time is an important issue of an algorithm, we also retained the time needed for each algorithm to derive the superpixels. As a measure of internal homogeneity of the superpixels, we compared the overall standard deviation (SD) computed for the derived superpixels.

\section{RESULTS}

\subsection{Runtime}

The fastest algorithm is SEEDS, while the slowest is LSC (Figure 1). When increasing the size of the superpixels, the differences in time between the algorithms tend to significantly decrease, LSC and SLIC having similar values. This is due to the fact that a small number of superpixels are derived $(20 \times 20$ pixels, approx. 10.000 superpixels) and the computational effort is less significant compared to the derivation of the finest superpixel segmentation $(5 \times 5$ pixels, approx. 160.000 superpixels). LSC and SLICO are slower compared to SLIC and SEEDS and this can be explained by the fact that the former two are having additional compactness constraints, deriving a more regular lattice of superpixels than the latter two (e.g. Figure 2).

Because for all three test areas we have used three bands as input, there are no significant differences between datasets, for the same method.

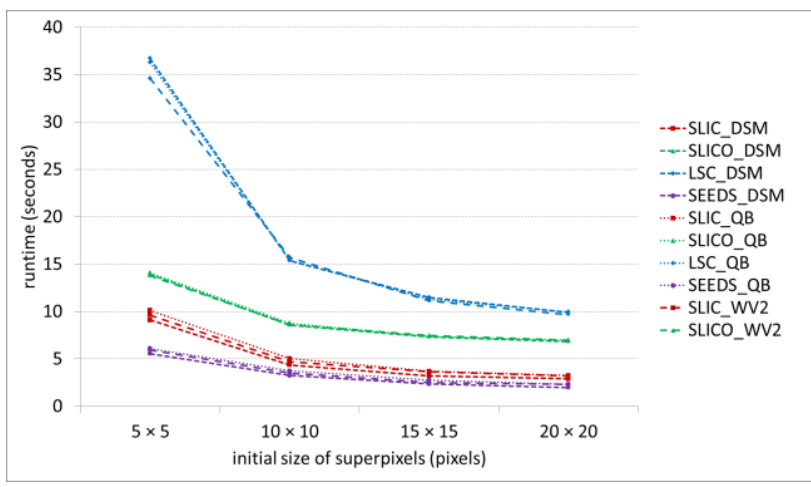

Figure 1. Runtime (in seconds) for each algorithm and for each test area, starting from initial size of the superpixels of $5 \times 5$, $10 \times 10,15 \times 15$ and $20 \times 20$, respectively.

\subsection{Visual evaluation}

In the case of an initial size of superpixels of $5 \times 5$ pixels, the oversegmentation is high and the superpixels boundaries tend to follow the boundaries of natural features within the test areas (Figure 2, 3 and 4). Since the approx. size of superpixels is 25 pixels, there are low chances that a superpixel contains information from more than one class. For DSM test area, due to the fine transition between elevation of low buildings and their surroundings, LSC algorithm can omit some of these boundaries. 
When increasing the size of superpixels ( $10 \times 10$ pixels $)$, patterns starts to be visible in the scenes. Even if a superpixel is now approx. 100 pixels in size, there is a good adherence of the superpixels boundaries to the features in the images and, therefore, low changes that a superpixel contains information of more than one class. In non-textured regions (e.g. industrial buildings in Figure 3), all the algorithms produce superpixels that come closer to a regular lattice.

The initial superpixel size of $15 \times 15$ pixels is appropriate for detection of buildings in DSM test area (Figure 4), for SLIC, LSC and partially SLICO algorithms. In the same case, SEEDS have the tendency to create false superpixels where the there is
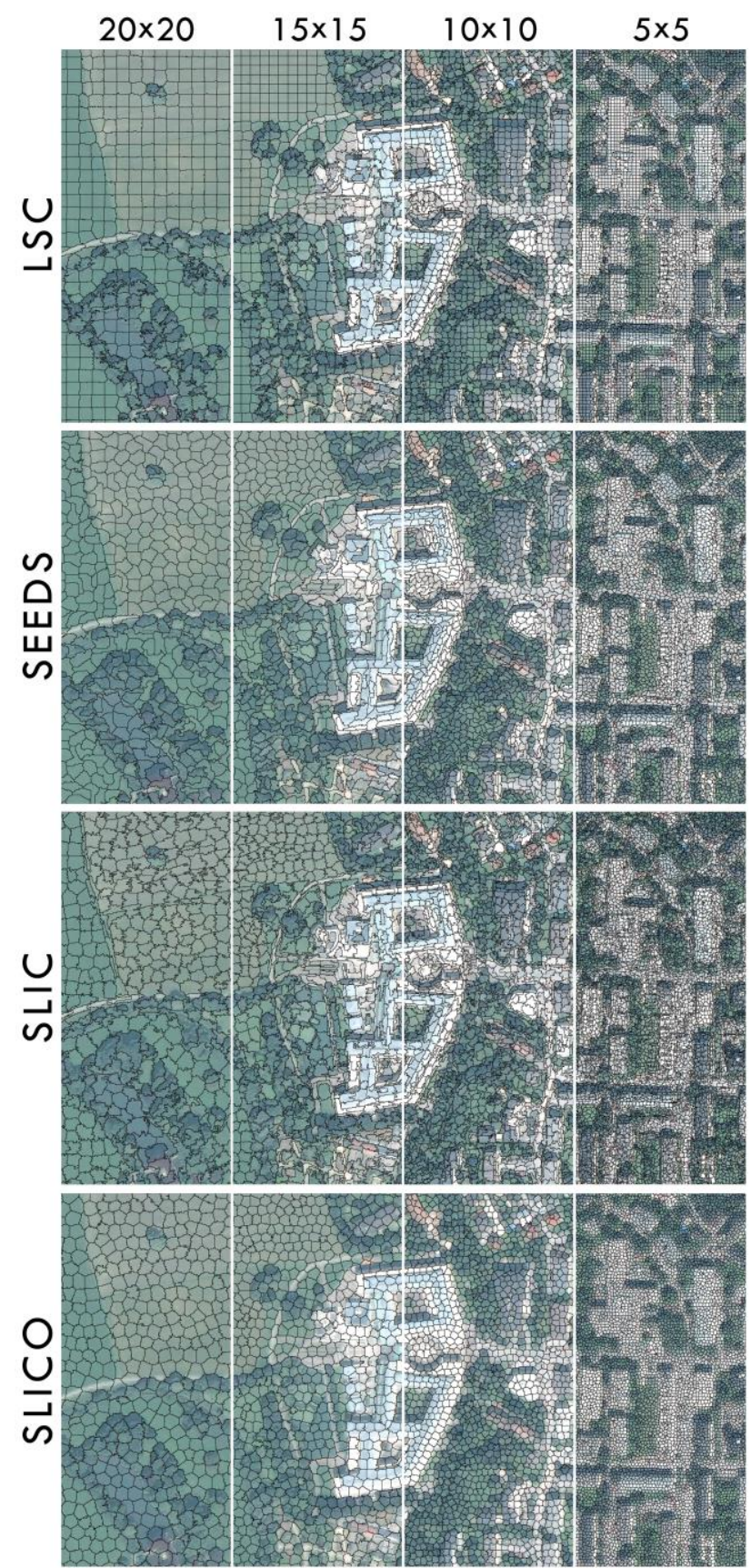

Figure 2. Results for the QB test area representing the LSC, SEEDS, SLIC and SLICO segmentation, starting from initial size of the superpixels of $5 \times 5,10 \times 10,15 \times 15$ and $20 \times 20$, respectively. a smooth transitions of boundaries (Figure 4). At this size of superpixels, individual trees and road segments can accurately be detected by all four algorithms. For $15 \times 15$ pixels size for superpixels there is a higher chance that a superpixel contains information from more than one class.

The last tested size of superpixels, $20 \times 20$ pixels, is reaching the capabilities of some algorithms to follow the correct boundaries, because many objects inside the scene have the size smaller than the size of superpixels. However, objects with similar or larger size are having the boundaries well detected (e.g. road segments, grass fields, rooftop parts, trees and forest patches in Figure 2 and 3). In the case of DSM, only SLIC and partially
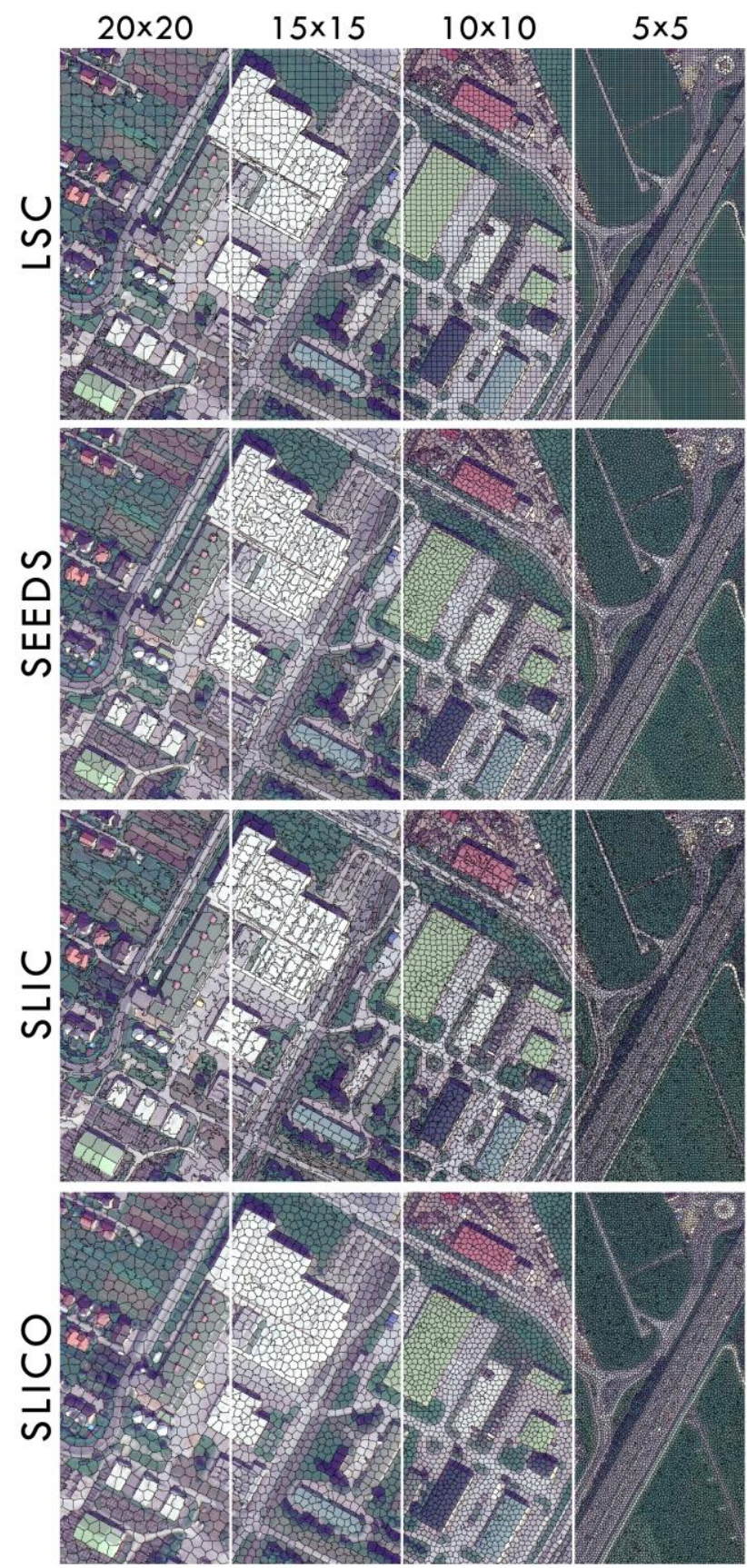

Figure 3. Results for the WV2 test area representing the LSC, SEEDS, SLIC and SLICO segmentation, starting from initial size of the superpixels of $5 \times 5,10 \times 10,15 \times 15$ and $20 \times 20$, respectively. 
SLICO superpixels adhere to the boundaries of buildings, while LSC and SEEDS fails (Figure 4). In the same test area, LSC performs better at delineating the boundaries of the forest patch (Figure 4).
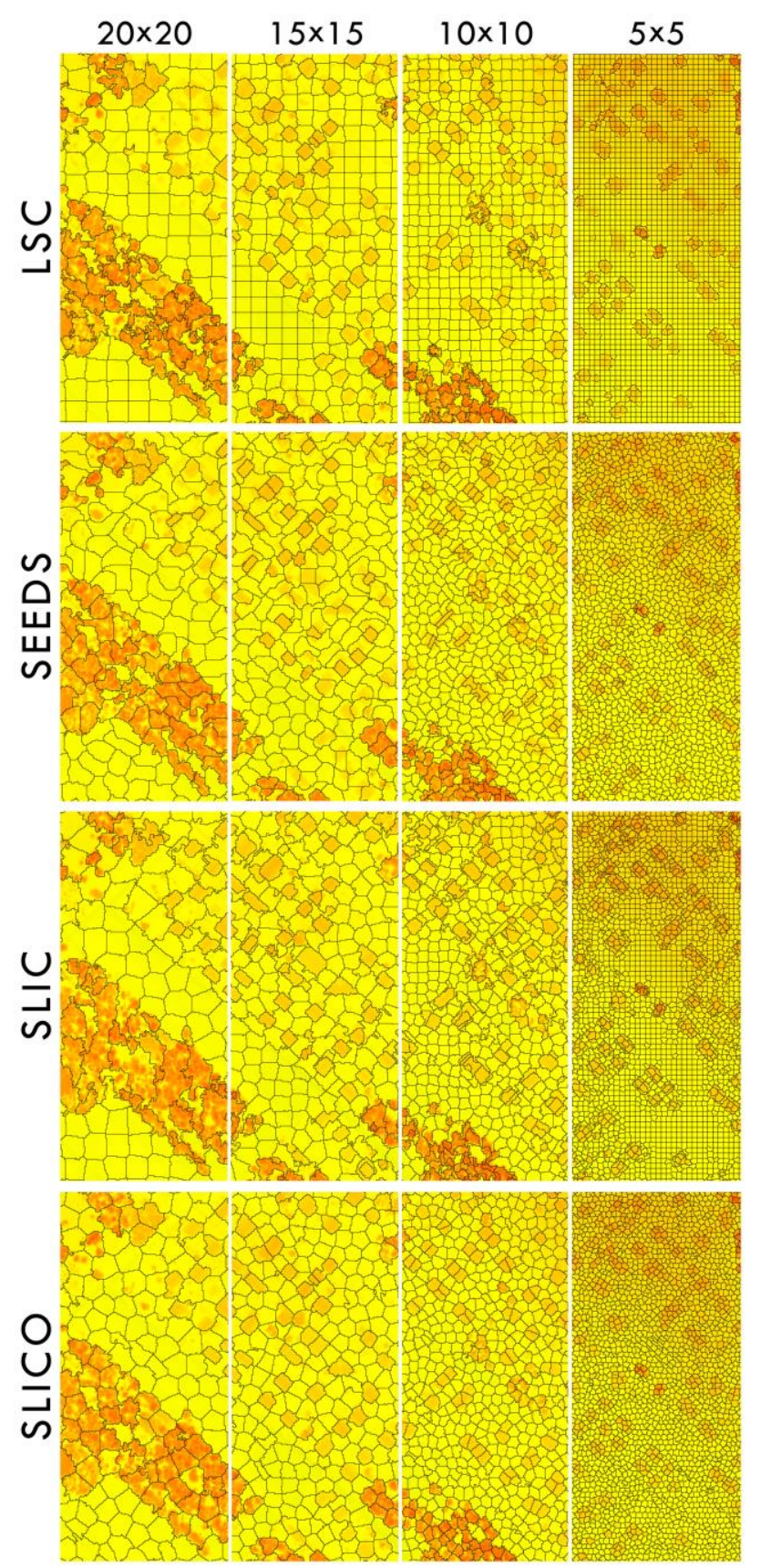

Figure 4. Results for the DSM test area representing the LSC, SEEDS, SLIC and SLICO segmentation, starting from initial size of the superpixels of $5 \times 5,10 \times 10,15 \times 15$ and $20 \times 20$, respectively.

\subsection{Superpixels homogeneity}

In the case of QB test area there are no big differences regarding the SD of the final superpixels (Figure 5). However, small differences occur between the algorithms. At the finest level, SLIC has the most internal homogeneous superpixels while SEEDS is on the opposite side. At the coarser level, LSC, SLIC and SEEDS have similar values of SD of approx. 15, while SLICO superpixels have a SD value of approx. 17.

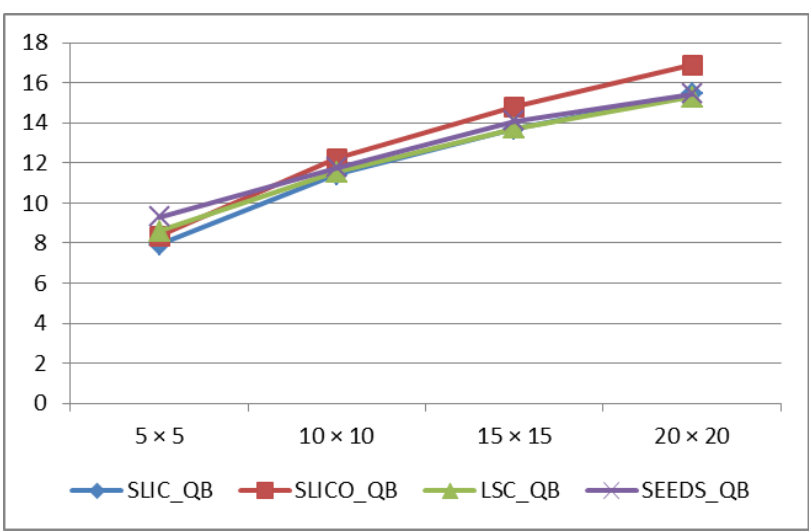

Figure 5. SD values (vertical axis) of the superpixels from QB test area, starting the algorithms from initial size of the superpixels of $5 \times 5,10 \times 10,15 \times 15$ and $20 \times 20$, respectively.

In the case of WV2 the differences regarding the SD of the final superpixels are more obvious than in the previous test area (Figure 6). SLIC algorithm outperform the other at all sizes of the generated superpixels. As in the previous study area, SLICO superpixels have good values for smaller sizes of the superpixels and tend to get worse at the coarser scale, because of the compactness constraints. LSC and SEEDS have similar values for all four sizes of superpixels.

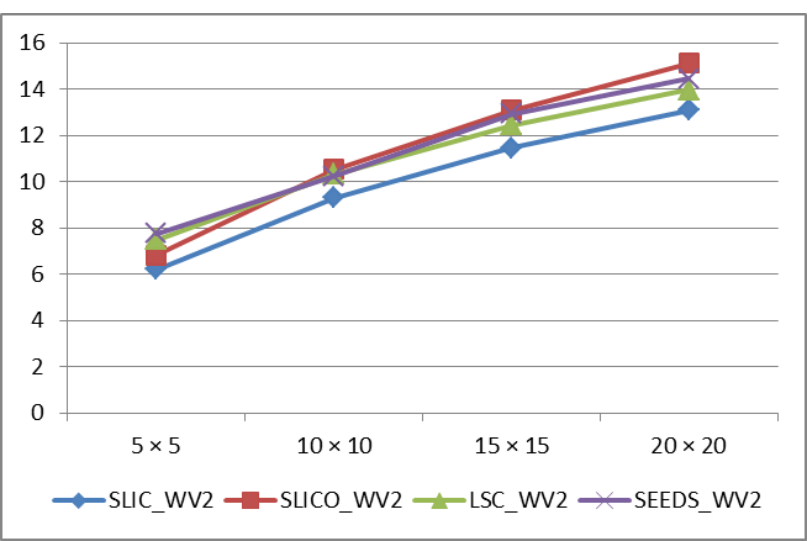

Figure 6. SD values (vertical axis) of the superpixels from WV2 test area, starting the algorithms from initial size of the superpixels of $5 \times 5,10 \times 10,15 \times 15$ and $20 \times 20$, respectively.

In the last test area (DSM), SLIC superpixels are having the best values of SD at all tested sizes (Figure 7). The SD of LSC superpixels is significantly getting worse as the size of the superpixels increases. This can be explained by the fact that LSC mixes the buildings with their surroundings at the size of superpixels of approx. $20 \times 20$ pixels, while the others don't (Figure 4). The SEEDS superpixels have the worse SD for the finest size of superpixels, but tends to reduce the difference to SLIC as the size of the superpixels increases. 


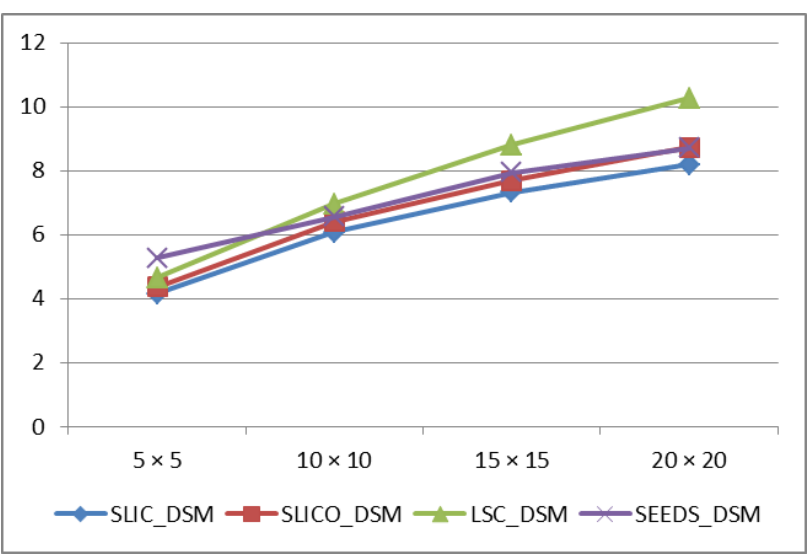

Figure 7. SD values (vertical axis) of the superpixels from DSM test area, starting the algorithms from initial size of the superpixels of $5 \times 5,10 \times 10,15 \times 15$ and $20 \times 20$, respectively.

\section{DISCUSSIONS AND CONCLUSION}

This study offers an initial overview of comparing four state-ofthe-art superpixel methods: SLIC, SLICO, SEEDS and LSC. The fastest one was SEEDS, closely followed up by SLIC. The desired size of initial superpixels influences the speed of the computation. Therefore, the size of the superpixels should be carefully chosen: a smaller size is increasing the runtime, while a larger size is mixing up more than one class inside a superpixel. We suggest that an initial size of $10 \times 10$ pixels for the superpixels is a good compromise between the speed and accuracy of the final superpixels.

In this study, we have used 10 iterations for clustering and refinement of superpixels, while this value was found to be sufficient (Achanta et al., 2012). However, a higher number of iterations can lead to a better adherence of the superpixels to the boundaries of natural features in the scene but affecting the runtime negatively.

Regarding internal homogeneity of resulted superpixels, SLIC overcomes the other algorithms. This is mainly because SLIC generates superpixels that don't have a compactness constraint. Therefore, SLIC superpixels better follow even the most irregular shapes in the image.

Overall, after comparing runtime and homogeneity of the resulted superpixels for all three test areas, we can came to the conclusion that SLIC superpixels are the best choice when taking into account the two aspects: speed and accuracy. We suggest that superpixels should be preferred against the rigid structure of pixels, having in mind all the advantages described in this study. Further studies are needed in order to have a more detailed comparison amongst many other existing superpixel algorithms and their possible usage in OBIA applications.

\section{ACKNOWLEDGEMENTS (OPTIONAL)}

This work was supported by the Austrian Science Fund (FWF) through the Doctoral College GIScience (DK W1237-N23). WorldView-2 imagery was provided through the FP7 Project MS.MONINA (Multi-scale Service for Monitoring NATURA 2000 Habitats of European Community Interest), Grant agreement No. 263479 and the INTERREG Project EuLE (EuRegional Spatial Analysis).

\section{REFERENCES}

Achanta, R., Shaji, A., Smith, K., Lucchi, A., Fua, P., Süsstrunk, S., 2012. SLIC Superpixels Compared to State-ofthe-Art Superpixel Methods. Pattern Analysis and Machine Intelligence, IEEE Transactions on, 34, pp. 2274-2282.

Chen, J., Dowman, I., Li, S., Li, Z., Madden, M., Mills, J., Paparoditis, N., Rottensteiner, F., Sester, M., Toth, C., Trinder, J., 2016. Information from imagery: ISPRS scientific vision and research agenda. ISPRS Journal of Photogrammetry and Remote Sensing, 115, pp. 3-21.

Chen, Y.X., Qin, K., Liu, Y., Gan, S.Z., Zhan, Y., 2012. Feature modelling of high resolution remote sensing images considering spatial autocorrelation. ISPRS - International Archives of the Photogrammetry, Remote Sensing and Spatial Information Sciences, 1, pp. 467-472.

Fulkerson, B., Vedaldi, A., Soatto, S., 2009. Class segmentation and object localization with superpixel neighborhoods, In: ICCV, Vol. 9, pp. 670-677.

Guangyun, Z., Xiuping, J., Jiankun, H., 2015. Superpixel-Based Graphical Model for Remote Sensing Image Mapping. Geoscience and Remote Sensing, IEEE Transactions on, 53, pp. 5861-5871.

Li, Z., Chen, J., 2015. Superpixel segmentation using linear spectral clustering, In: Computer Vision and Pattern Recognition (CVPR), 2015 IEEE Conference on, pp 1356-1363. Neubert, P., Protzel, P., 2012. Superpixel benchmark and comparison, Proc. Forum Bildverarbeitung, pp. 1-12.

Ren, X., Malik, J., 2003. Learning a classification model for segmentation, In: Computer Vision, 2003. Proceedings. Ninth IEEE International Conference on. IEEE, pp. 10-17.

Shi, C., Wang, L., 2014. Incorporating spatial information in spectral unmixing: A review. Remote Sensing of Environment, 149, pp. 70-87.

Tiede, D., Lang, S., Füreder, P., Hölbling, D., Hoffmann, C., Zeil, P., 2011. Automated damage indication for rapid geospatial reporting. Photogrammetric Engineering \& Remote Sensing, 77, pp. 933-942.

Van den Bergh, M., Boix, X., Roig, G., de Capitani, B., Van Gool, L., 2012. Seeds: Superpixels extracted via energy-driven sampling, In: Computer Vision-ECCV 2012. Springer, pp. 1326.

Revised July 2016 


\title{
COMBINING MULTIPLE RESOLUTIONS INTO HIERARCHICAL REPRESENTATIONS FOR KERNEL-BASED IMAGE CLASSIFICATION
}

\author{
Y. Cui ${ }^{\mathrm{a}}$, S. Lefèvre ${ }^{\mathrm{a}}$, L. Chapel $^{\mathrm{a}}$, A. Puissant ${ }^{\mathrm{b}}$

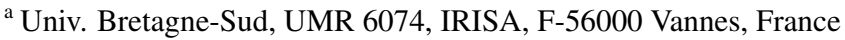 \\ \{yanwei.cui, laetitia.chapel, sebastien.lefevre\}@irisa.fr \\ ${ }^{\mathrm{b}}$ Univ. Strasbourg, UMR 7362 LIVE, F-67000 Strasbourg, France \\ anne.puissant@live-cnrs.unistra.fr
}

KEY WORDS: multi-resolution remote sensing, multi-source fusion, hierarchical representation, structured kernel, image classification.

\begin{abstract}
:
Geographic object-based image analysis (GEOBIA) framework has gained increasing interest recently. Following this popular paradigm, we propose a novel multiscale classification approach operating on a hierarchical image representation built from two images at different resolutions. They capture the same scene with different sensors and are naturally fused together through the hierarchical representation, where coarser levels are built from a Low Spatial Resolution (LSR) or Medium Spatial Resolution (MSR) image while finer levels are generated from a High Spatial Resolution (HSR) or Very High Spatial Resolution (VHSR) image. Such a representation allows one to benefit from the context information thanks to the coarser levels, and subregions spatial arrangement information thanks to the finer levels. Two dedicated structured kernels are then used to perform machine learning directly on the constructed hierarchical representation. This strategy overcomes the limits of conventional GEOBIA classification procedures that can handle only one or very few pre-selected scales. Experiments run on an urban classification task show that the proposed approach can highly improve the classification accuracy w.r.t. conventional approaches working on a single scale.
\end{abstract}

\section{INTRODUCTION}

Geographic object-based image analysis (GEOBIA) framework has gained increasing interest recently, especially in the case of very high resolution remote sensing images (Blaschke et al., 2014). One of the key features for GEOBIA framework is the hierarchical image representation through a tree structure, where objectsof-interest can be revealed through various scales, and where the topological relationship between objects (e.g. A is part of $\mathrm{B}$, or $\mathrm{B}$ consists of A) can be easily modeled. In the classification context, however, most papers in literature address one scale only, as being pointed out in a recent survey paper (Blaschke, 2010).

Features extracted from multiple scales are important for improving the object-based classification accuracy, as the underlying tree structure models the hierarchical relationship among the objects (Blaschke, 2010). Two important topological information across the scales can be extracted from hierarchical representation: context features and objects spatial arrangement features.

Context features correspond to the spatial interactions between one region and its surrounding regions. For instance, trees can be classified as residential area instead of forest zone given surrounding regions being buildings and roads. Such context information can help to disambiguate similar regions during the classification phase (Liu et al., 2008). Through hierarchical representation, context features can model the evolution of one region and describe it at different levels. Integrating such complementary information leads to some classification accuracy improvement (Shackelford and Davis, 2003). Since the spatial position is also implicitly taken into account, it often produces a spatially smoother classification map avoiding "salt and pepper" effect (Bruzzone and Carlin, 2006, Lefèvre et al., 2014).

Objects spatial arrangement features model the decomposition of an object and the interactions among its subparts. For instance, a residential area is much easier to be identified when knowing it is composed of houses and roads. Including such information can highly improve the classification rate when spatial interaction between subparts is considered as a critical feature (Tang et al., 2013, Cui et al., 2015).

Although features extracted from the multiscale representations are considered as discriminative characteristics for classification, dedicated machine learning algorithms still remain largely unexplored for learning directly from such representations. Recently, advanced machine learning algorithms have been introduced in the GEOBIA framework. Methods such as Support Vector Machine (SVM) (Tzotsos and Argialas, 2008), and Random Forests (Stumpf and Kerle, 2011) have been proposed in order to overcome conventional issues of previous GEOBIA classification procedures (Shackelford and Davis, 2003, Benz et al., 2004), e.g. manual thresholding and a subjective selection of suitable features. A few dedicated methods have been introduced for taking into account the multiscale features extracted from hierarchical representation (Bruzzone and Carlin, 2006). However, such algorithms able to fully benefit from the multiscale representations remain largely underdeveloped.

Meanwhile, remote sensing image fusion approaches tend to develop under the GEOBIA framework. These techniques aim to integrate information from different sources, and to produce fused data with more detailed information. For instance, combining high-resolution imagery and LIDAR data allows better accuracy achievements in an urban area classification task (Chen et al., 2009). As the availability of multi-resolution remote sensing data is rapidly increasing, developing methods able to fuse images from multiple sources and multiple resolutions to improve classification accuracy is becoming an important topic in remote sensing (Zhang, 2010, Gomez-Chova et al., 2015).

In this paper, we propose a new approach i) to build a hierarchical image representation from a pair of images with different resolutions (captured with two different sensors) under the GEOBIA 
Context information extracted from coarser levels built on MSR image

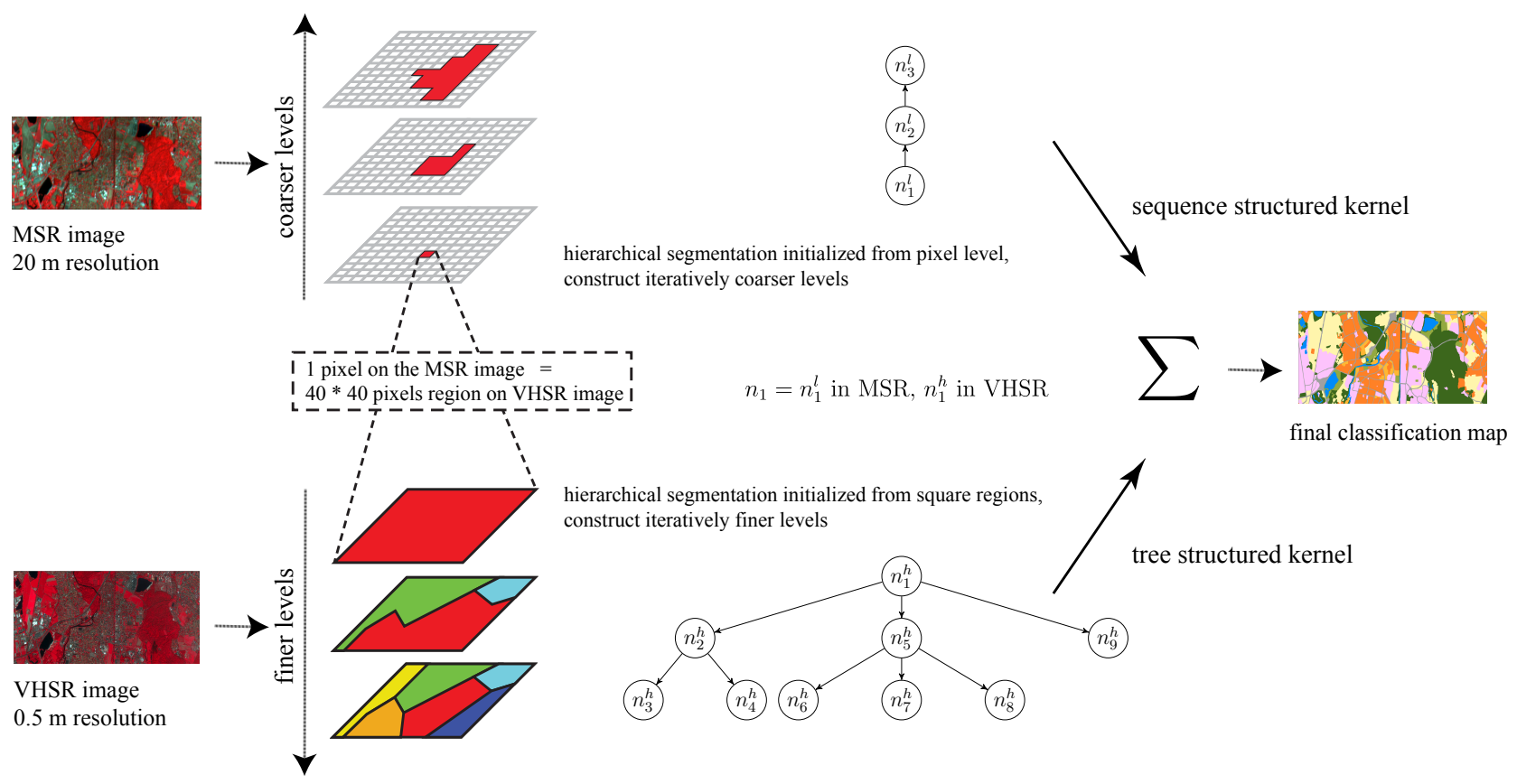

Subregions arrangement information extracted from finer levels built on VHSR image

Figure 1. Illustration of the hierarchical image representation for one data instance $n_{1}$ to be classified. Each data instance corresponds a pixel of the MSR image $n_{1}^{l}$, and a $40 \times 40$ square region on the VHSR image $n_{1}^{h}$. It associates the context information thanks to the coarser levels of the hierarchy built from the MSR image, and the subregion spatial arrangement information thanks to the finer levels constructed on the VHSR image. Both complementary information are taken into consideration thanks to two dedicated structured kernels, then fused together through a composite kernel that provides the classification output.

framework, and ii) to apply dedicated kernel methods to perform supervised classification directly from the constructed tree.

To build a hierarchical image representation, we rely on two images: a Low Spatial Resolution (LSR) or Medium Spatial Resolution (MSR) image on the one side, and a High Spatial Resolution (HSR) or Very High Spatial Resolution (VHSR) image on the other side. Such a hierarchical representation allows one to benefit of the context information on the coarser levels built from LSR/MSR image, and of the subregions spatial arrangement information on the finer levels built from the HSR/VHSR image.

To perform image classification from a hierarchical representation, we propose to combine structured kernels computed on two types of structured data: a sequence structured kernel (Cui et al., 2016) allows learning the context information with ancestor regions at coarse levels on LSR/MSR image, while a tree structured kernel (Cui et al., 2015) on HSR/VHSR image makes possible the modeling of the spatial arrangement between subregions. Both kernels exploit complementary information from the hierarchical representation, therefore they are combined at the end. Evaluations show that exploiting multiscale features through a hierarchical representation with dedicated kernels significantly improves the classification accuracy w.r.t. only one single scale.

The paper is organized as follows. We illustrate our main contributions, which include: i) the construction of a hierarchical image representation using two resolution images at different resolutions with different sensors (Sec. 2), and ii) the kernel to learn directly on the constructed tree (Sec. 3). Then in Sec. 4, we detail the experimental setup and discuss the results. Conclusion and future directions are given at the end of the paper.

\section{HIERARCHICAL REPRESENTATION WITH MULTIPLE RESOLUTION IMAGES}

Hierarchical image representation is capable of revealing objectsof-interest through various scales. To construct such representations, one of the most widely adopted techniques is the bottomup iterative region merging approach e.g. HSeg (Tilton, 1998). Starting from the pixel level or any other initial partition (e.g. in superpixels), it merges the most similar regions into a new region at each iterative step, until finally the whole image becomes one single region. A threshold parameter (e.g. a list of similarity criteria following ascending order) is often provided for users to generate the final representation output, with each level being the segmentation map that fulfills the threshold conditions.

Here we build a hierarchical representation with multiple resolution images through two separate steps: i) use LSR/MSR to construct coarser levels of context information on the one side, and ii) use HSR/VHSR image to generate finer levels of subregions spatial arrangement information on the other side, as illustrated in Fig. 1.

Firstly, we initialize our segmentation at the pixel level on the LSR/ MSR image and construct iteratively the coarser levels. Let $n_{1}$ be a data instance to be classified. Within the LSR/MSR image, it corresponds to a pixel $n_{1}^{l}$ and can be represented as a sequence $S=\left\{n_{1}^{l}, \ldots, n_{P}^{l}\right\}$ that models the evolution of the pixel $n_{1}^{l}$ through the hierarchy. Each node $n_{i}^{l}$ is described by a $D$ dimensional feature $\boldsymbol{x}_{i}^{l}$ that encodes the region characteristics, e.g. spectral information, size, shape, etc.

Secondly, we use the HSR/VHSR image to provide the fine details of the observed scene for each data instance $n_{1}$. Indeed, one 
pixel of the LSR/MSR image $n_{1}^{l}$ always corresponds to a square region of the HSR/VHSR image $n_{1}^{h}$. To do so, we initialize the top level of the multiscale segmentation to be the square regions, then construct the finer levels. Through the hierarchy, the data instance $n_{1}$ can be modeled as a tree $T$ rooted in $n_{1}^{h}$ which encodes subregions and the spatial arrangement among them. The characteristics of region $n_{i}^{h}$ is also described by a $D$-dimensional feature $\boldsymbol{x}_{i}^{h}$.

In the end, each data instance $n_{1}$ can be represented by an ascending sequence $S$ data from the LSR/MSR image, and a descending tree $T$ data generated from the HSR/VHSR image. Learning directly on such representations requires the development of dedicated machine learning algorithms.

\section{STRUCTURED KERNELS FOR LEARNING ON HIERARCHICAL REPRESENTATIONS}

\subsection{Structured kernels}

To learn from hierarchical representations, we use structured kernels computed on the constructed structures: a sequence structured kernel allows learning context information with ancestor regions at coarser levels on the LSR/MSR image, while a tree structured kernel on the HSR/VHSR image makes possible the modeling of spatial arrangement between subregions. The classification map relies on the composition of both structured kernels.

Both tree and sequence kernels can be view as instances of the convolution kernel (Haussler, 1999) that defines a general framework to construct structured kernels. It states that a kernel on a complex structure can be formed by tailoring simple kernels computed on its substructures. Formally, let $G, G^{\prime}$ two structured data and $s, s^{\prime}$ their substructures, then the kernel between $G, G^{\prime}$ can be written as:

$$
K\left(G, G^{\prime}\right)=\sum_{s \in G, s^{\prime} \in G^{\prime}} K\left(s, s^{\prime}\right)
$$

In order to capture the hierarchical nature of multiscale representation trees and encode the parent-child relationships among the nodes, subpath substructure has been defined and successfully applied in (Cui et al., 2015) for tree structured data and in (Cui et al., 2016) for sequence structured data. It can be written as $s=\left(n_{(1)}, n_{(2)}, \cdots n_{(t)}, \cdots n_{(p)}\right), s \in S$, with $(t)$ being the relative position of a node in the subpath, following an ascending order $1 \leq t \leq p$, and $p$ being the subpath length. Fig. 2 gives an example of a sequence and a tree, with enumeration of all their subpaths $s$ respectively.

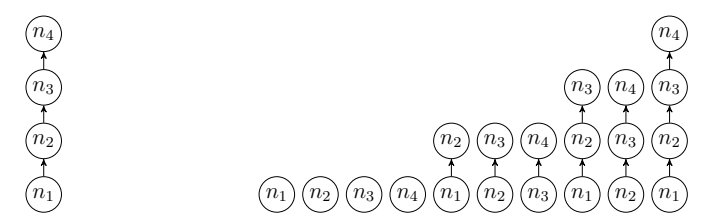

(a) A sequence $S$ and all its subpaths $s$.
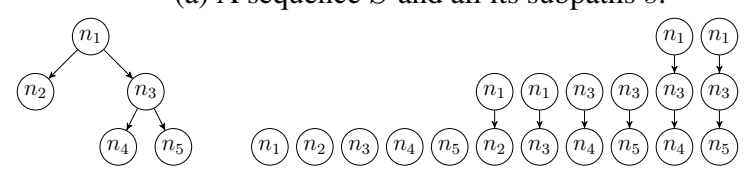

(b) A tree $T$ and all its subpaths $s$.

Figure 2. Examples of structured data and related substructures.
The kernel between two subpaths $s$ and $s^{\prime}$ with equal length $|s|=$ $\left|s^{\prime}\right|=p$ is defined as the product of atomic kernels (e.g. Gaussian kernel as in Eq. (7)) computed on individual nodes $k\left(n_{(t)}, n_{(t)}^{\prime}\right)$ :

$$
K\left(s, s^{\prime}\right)=\prod_{t=1}^{p} k\left(n_{(t)}, n_{(t)}^{\prime}\right) .
$$

\subsection{Kernel computation}

We propose here an unified algorithm for computing the sequence and tree kernels based on subpaths. This efficient algorithm can bring down the overall complexity to quadratic w.r.t. the size of structures $O\left(|G|\left|G^{\prime}\right|\right)$. The basic idea is to iteratively compute the kernel on subpaths $s$ and $s^{\prime}$ of length $p$ using previously computed kernels on the subpaths of length $p-1$. The atomic kernel $k\left(n_{i}, n_{j}^{\prime}\right)$ between each pair of nodes $\left(n_{i} \in G, n_{j}^{\prime} \in G^{\prime}\right)$ thus needs to be computed only once, avoiding redundant computations.

Regarding the sequence kernel, we define a two-dimensional matrix $M$ of size $|S| \times\left|S^{\prime}\right|$, where each element $M_{i, j}$ is computed iteratively as:

$$
M_{i, j}=k\left(n_{i}, n_{j}^{\prime}\right)\left(1+M_{i-1, j-1}\right) .
$$

where $M_{i, 0}=M_{0, j}=0$ by convention.

The overall kernel value is then computed as the sum of all the matrix elements.

$$
K\left(S, S^{\prime}\right)=\sum_{i=1}^{|S|} \sum_{j=1}^{\left|S^{\prime}\right|} M_{i, j}
$$

For the tree kernel, we slightly modify the iteration in Eq. (3) by changing $M_{i-1, j-1}$ to $M_{\text {parent }\left(n_{i}\right) \text {,parent }\left(n_{j}^{\prime}\right)}$, where parent $\left(n_{i}\right)$ refers as parent index of the node $n_{i}$. It can be constructed by presenting the tree as a sequence of nodes with a pre-order depthfirst traversal algorithm (Hopcroft et al., 1983). By convention, the parent index of the root of a tree is 0, see Fig. 3 for an example.

\section{Pre-order traversal tree}

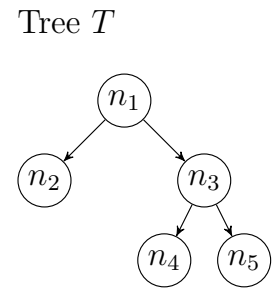

\begin{tabular}{|c|c|c|c|c|c|}
\hline index & 1 & 2 & 3 & 4 & 5 \\
\hline node & $n_{1}$ & $n_{2}$ & $n_{3}$ & $n_{4}$ & $n_{5}$ \\
\hline
\end{tabular}

Parent index

\begin{tabular}{|c|c|c|c|c|c|}
\hline node & $n_{1}$ & $n_{2}$ & $n_{3}$ & $n_{4}$ & $n_{5}$ \\
\hline parent & 0 & 1 & 1 & 3 & 3 \\
\hline
\end{tabular}

Figure 3. A tree $T$ and its associated pre-order depth-first traversal order and parent index table.

The overall complexity for both kernels is bounded by the computation of the two-dimensional matrix $M$, which yields $O\left(|G|\left|G^{\prime}\right|\right)$.

\subsection{Kernel combination}

Kernel values must be independent of the size of the structures and should lie in the $(0,1]$ interval. We thus normalize the kernel value by using the following standard strategy: 


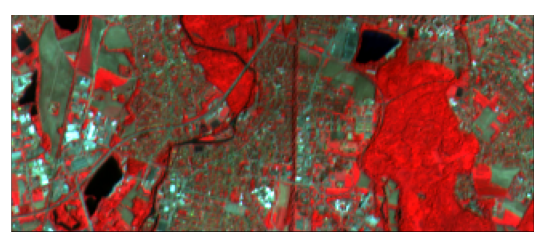

(a) Spot-4 image

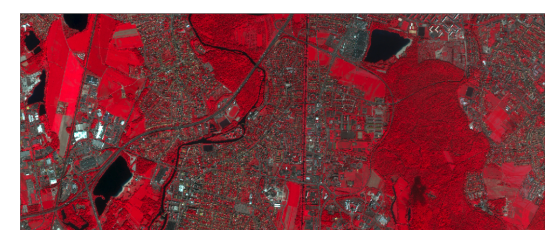

(b) Pleiades image

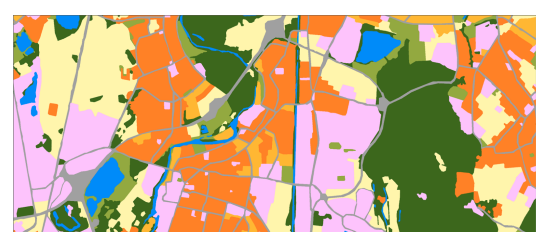

(c) Ground truth image

Figure 4. Urban scene taken over South of Strasbourg, France. From left to right: false color image of Spot-4 (C) CNES 2012) with $20 \mathrm{~m}$ resolution, false color image of Pleiades (C) CNES 2012, distribution Airbus DS / Spot Image) with $50 \mathrm{~cm}$ resolution, and the associated ground truth (C) LIVE UMR 7362, adapted from OCSOL CIGAL 2012) with eight thematic classes.

$$
K^{*}\left(G, G^{\prime}\right)=\frac{K\left(G, G^{\prime}\right)}{\sqrt{K(G, G)} \sqrt{K\left(G^{\prime}, G^{\prime}\right)}}
$$

The final kernel between two data instances $n_{1}, n_{1}^{\prime}$ is computed using a linear combination of the two structured kernels, with a parameter $\rho \in[0,1]$ that controls the importance ratio between the two kernels:

$$
K\left(n_{1}, n_{1}^{\prime}\right)=\rho \times K^{*}\left(S, S^{\prime}\right)+(1-\rho) \times K^{*}\left(T, T^{\prime}\right),
$$

where $n_{1}$ (resp. $n_{1}^{\prime}$ ) is described by $S$ (resp. $S^{\prime}$ ) on the LSR/MSR image and $T\left(\operatorname{resp} T^{\prime}\right)$ on the HSR/VHSR image.

\section{EXPERIMENTS}

\subsection{Study area}

In this paper, we focus on urban land-use classification in the South of Strasbourg city, France. We consider 8 thematic classes of urban patterns as shown in Tab. 1 (class details) and in Fig. 4c (ground truth image), see (Kurtz et al., 2012) for more details. Two images from different sources are used:

- MSR: Spot-4 20 m resolution, 4 bands: Green, Red, NIR, MIR. Image with $326 \times 135$ pixels (Fig. 4a).

- VHSR: Pleiades 0.5 m resolution, 4 bands: Red, Green, Blue, NIR. Image with $13040 \times 5400$ pixels (Fig. 4b).

Table 1. List of classes, their color, and number of pixels in ground truth (on the MSR image, Fig. 4c).

\begin{tabular}{|l|c|c|}
\hline Class & Color & Nb of pixels \\
\hline Water surfaces & Blue & 1653 \\
Forest areas & Dark green & 9315 \\
Urban vegetation & Light green & 1835 \\
Road & Grey & 3498 \\
Industrial blocks & Pink & 8906 \\
Individual housing blocks & Dark orange \\
Collective housing blocks & Light orange & 9579 \\
Agricultural zones & Yellow & 1434 \\
\hline Total & & $\mathbf{4 7 9 0 1 0}$ \\
\hline
\end{tabular}

\subsection{Experimental setup}

We conduct experiments considering a one-against-one SVM classifier, using the Java implementation of LibSVM (Chang and Lin, 2011). The following scenarios are considered:
- Scenario 1: Gaussian kernel at single level on the MSR image $v$ s. sequence kernel taking into account the context information at multiple levels on the MSR image.

- Scenario 2: Gaussian kernel at single level on the VHSR image $v s$. tree kernel taking into account the subregions spatial arrangement information at multiple levels on the VHSR image.

- Scenario 3: Composite kernel combining both the context and the subregions spatial arrangement information extracted from a hierarchical representation using the two resolution images.

To generate the hierarchical image representation, we rely on HSeg, whose parameters have been empirically fixed as follows:

- On the MSR image, we generate, from the bottom level of single pixels, 7 additional levels of hierarchical segmentation by increasing the region dissimilarity criteria $\alpha=$ $\left[2^{-2}, 2^{-1}, \ldots, 2^{4}\right]$. We observe that with such parameters, the number of segmented regions is roughly decreasing by a factor of 2 between each level.

- On the VHSR image, we generate, from the top (root) level of each square region of size $40 \times 40$ pixels (i.e. equivalent to a single MSR pixel), 4 additional levels of hierarchical segmentation by decreasing the region dissimilarity criteria $\alpha=\left[2^{4}, 2^{3}, \ldots, 2^{1}\right]$. Using such parameters, we observe that the number of segmented regions is roughly increasing by a factor of 2 between each level.

Each region in the hierarchical representation is described by a 8dimensional feature vector $\boldsymbol{x}$, which includes the region average of the 4 original multi-spectral bands, Soil Brightness index (BI) and NDVI, as well as Haralick texture measurements computed with gray level co-occurrence matrix homogeneity and standard deviation. These features are considered as standard ones in the urban analysis context (Forestier et al., 2012).

We use Gaussian kernel for the atomic kernel $k(\cdot, \cdot)$ defined for a pair of nodes $n_{i}, n_{j}^{\prime}$ with respective features $\boldsymbol{x}_{i}, \boldsymbol{x}_{j}^{\prime}$ as

$$
k\left(n_{i}, n_{j}^{\prime}\right)=\exp \left(-\gamma\left\|\boldsymbol{x}_{i}-\boldsymbol{x}_{j}^{\prime}\right\|^{2}\right) .
$$

Free parameters are determined by 5-fold cross-validation over potential values: the Gaussian kernel bandwidth $\gamma$ and the SVM regularization parameter $C$. We also cross-validate the parameter $\rho \in[0,1]$ in Eq. (6) for relative contribution of each kernel. The comparison between different approaches is done by using identical randomly chosen 200 samples per class for training and the rest for testing. All reported results are computed over 10 repetitions of each experiment. 
Table 2. Classwise accuracies, overall accuracies (OA), average accuracies (AA) and Kappa indices with standard deviation in parentheses. Methods with single level and multiple levels on hierarchical image representation are compared as follows: scenario 1: Gaussian kernel with single level on MSR image (single MSR) vs. sequence kernel with multiple levels context information on MSR image (context MSR); scenario 2: Gaussian kernel with single level on VHSR image (single VHSR) vs. tree kernel with multiple levels subregions spatial arrangement information on VHSR image (subregions VHSR); scenario 3: composite kernel combining both sequence and tree kernel using both MSR and VHSR images (composite). All results are computed over 10 repetitions with best results being boldfaced. Significant differences between single level Gaussian kernels and structured ones using a Wilcoxon test are underlined.

\begin{tabular}{|l||c|c||c|c||c|}
\hline Class & single MSR & context MSR & single VHSR & subregions VHSR & composite \\
\hline Water surfaces & $84.90(2.5)$ & $84.58(2.2)$ & $92.49(1.3)$ & $91.69(1.4)$ & $90.40(1.6)$ \\
Forest areas & $\underline{80.32}(1.5)$ & $77.96(2.1)$ & $84.78(0.8)$ & $\underline{85.80}(0.9)$ & $86.76(1.1)$ \\
Urban vegetation & $25.99(4.4)$ & $\underline{73.63}(2.1)$ & $36.84(4.9)$ & $38.16(3.4)$ & $73.19(2.1)$ \\
Road & $38.86(3.1)$ & $\underline{43.39}(2.3)$ & $48.85(1.9)$ & $\underline{51.26}(1.7)$ & $54.19(2.3)$ \\
Industrial blocks & $35.96(3.2)$ & $\underline{70.88}(2.4)$ & $23.24(2.5)$ & $\underline{\underline{34.61}(1.9)}$ & $69.01(1.6)$ \\
Individual housing blocks & $57.09(4.4)$ & $\underline{63.91}(3.3)$ & $51.42(3.3)$ & $\underline{58.02}(2.1)$ & $69.62(1.2)$ \\
Collective housing blocks & $24.13(2.8)$ & $\underline{77.89}(3.0)$ & $35.32(3.6)$ & $\underline{\underline{38.82}}(2.8)$ & $79.52(3.1)$ \\
Agricultural zones & $36.93(3.3)$ & $\underline{67.96}(3.0)$ & $67.79(1.8)$ & $\underline{69.39}(1.7)$ & $77.17(1.9)$ \\
\hline \hline OA & $51.52(1.0)$ & $\underline{\underline{68.98}(0.9)}$ & $55.91(0.7)$ & $\underline{60.53}(0.4)$ & $\mathbf{7 4 . 4 7}(0.4)$ \\
AA & $48.02(0.3)$ & $\underline{70.03}(0.5)$ & $55.09(0.3)$ & $\underline{58.47}(0.5)$ & $\mathbf{7 4 . 9 8}(0.3)$ \\
Kappa & $0.426(0.009)$ & $\underline{\underline{0.629}}(0.009)$ & $0.485(0.007)$ & $\underline{0.533}(0.004)$ & $\mathbf{0 . 6 9 3}(0.004)$ \\
\hline
\end{tabular}

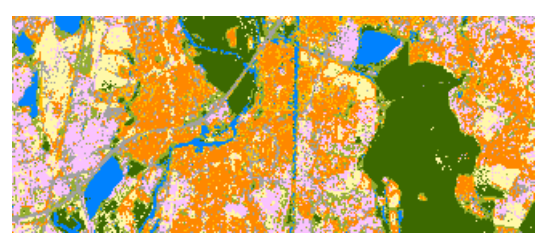

(a) single MSR

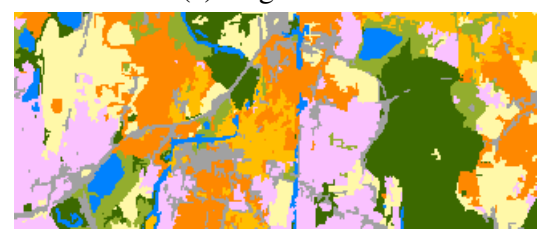

(b) context MSR

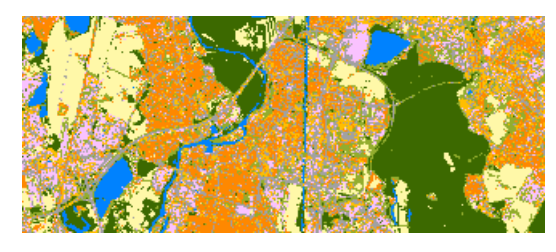

(c) single VHSR

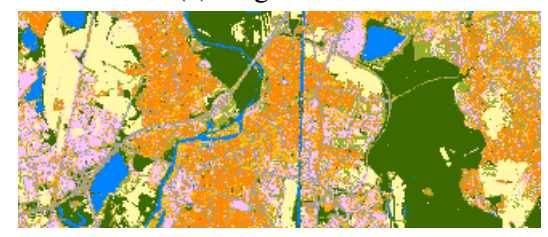

(d) subregions VHSR

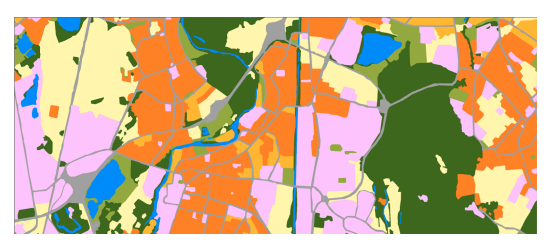

(e) Ground truth image

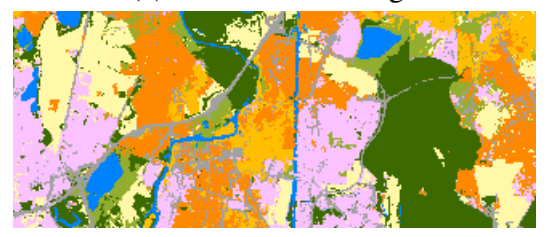

(f) Composite

Figure 5. Classification maps for methods using single level and multiple levels of a hierarchical image representation: scenario 1: single level on Spot-4 image (a) vs. multiple levels context information on Spot-4 image (b); scenario 2: single level on Pleiades image (c) vs. multiple levels subregions spatial arrangement information on Pleiades image (d); scenario 3: combination of context information and subregions spatial arrangement information (f). Ground truth image (e) is also given as reference.

\subsection{Results and discussion}

By taking into account the context information through sequence kernel, the classification results on the MSR image are largely improved comparing to SVM with Gaussian kernel on a single level. We can see in Tab. 2 that per class accuracy is greatly improved for all classes but two. On the VHSR image, the classification accuracy is improved for all classes but two by using subregions spatial arrangement information. Water surface and urban vegetation classification accuracies remain similar since regions are mostly homogeneous. Moreover, the combination of context information and subregions spatial arrangement information yields an additional improvement, mainly focused on the classes road, individual housing blocks and agricultural zones.

As shown in Fig. 5a, the predictions are very noisy with a single level analysis of the MSR image. This is the typical "salt and pepper" problem encountered in remote sensing image classification when the spatial information is not taken into account. Using multiscale information, the spatial dimension is implicitly taken into consideration by the ancestor regions in the hierarchy. Thus a "smoother" prediction map can be obtained (as shown in Fig. 5b). Let us note that we did not use any post-processing technique to produce such classification map, relying only a structured kernel coping with context information. However, we can also observe that small structures such as road networks disappear in certain areas, and enhance wrongly in other ones.

As far as the VHSR image is concerned, the prediction maps are noisy with both single and multiple scales. This is due to the fact that the multiscale features extracted on the VHSR image can no longer serve as context information, and spatial relationships among data instances are no longer taken into account. However, it provides the complementary subregions spatial arrangement information, thus leading to a more precise prediction. This conclusion is easier to be reached through quantitative analysis in Tab. 2, showing that results improve consistently for most of the classes (6 out of 8 ). Classes such as individual housing blocks and industrial blocks are significantly improved, as they can be better characterized by their subregions and the spatial relationships among those regions. Indeed, this shows the advantage of taking into account subregions spatial arrangement information.

The classification map in Fig. 5f shows that the composite kernel manages to combine the advantages from the two complementary information sources. Indeed, we can observe that the prediction seems to achieve a spatial regularization for the large regions, while providing precision for the small structures such as road 
networks. Therefore, it leads to the best classification accuracy.

\section{CONCLUSION}

In this paper, we introduced a novel multiscale approach for combining multiresolution images under the GEOBIA framework. Based on a hierarchical representation generated from images of different resolutions, we propose to use a sequence kernel to take into account the context information built on MSR data, and a tree kernel to capture subregions spatial arrangement information from VHSR data. Both kernels are integrated together through a simple but efficient kernel combination to output final classification results. Evaluations on an urban scene classification problem show that our proposed multiscale approach can significantly improve the classification accuracies w.r.t. methods that use only a single spatial scale and only one image.

This paper demonstrates the need of integrating more dedicated machine learning algorithms to take into consideration the topological relationships between objects under the GEOBIA framework. However, the main issue remains the current quadratic kernel computation complexity. In the future, we plan to investigate efficient algorithms, e.g. random Fourier features (Bo and Sminchisescu, 2009), to further bring down the computation complexity, and make the proposed approach more adaptable for big remote sensing data.

\section{ACKNOWLEDGEMENTS}

The authors acknowledge the support of the French Agence Nationale de la Recherche (ANR) under reference ANR-13-JS020005-01 (Asterix project), and the support of Région Bretagne and Conseil Général du Morbihan (ARIA doctoral project).

\section{REFERENCES}

Benz, U. C., Hofmann, P., Willhauck, G., Lingenfelder, I. and Heynen, M., 2004. Multi-resolution, object-oriented fuzzy analysis of remote sensing data for gis-ready information. ISPRS Journal of Photogrammetry and Remote Sensing 58(3), pp. 239-258.

Blaschke, T., 2010. Object based image analysis for remote sensing. ISPRS Journal of Photogrammetry and Remote Sensing 65(1), pp. 2-16.

Blaschke, T., Hay, G. J., Kelly, M., Lang, S., Hofmann, P., Addink, E., Feitosa, R. Q., van der Meer, F., van der Werff, H., van Coillie, F. and Tiede, D., 2014. Geographic object-based image analysis towards a new paradigm. ISPRS Journal of Photogrammetry and Remote Sensing 87, pp. 180 - 191.

Bo, L. and Sminchisescu, C., 2009. Efficient match kernel between sets of features for visual recognition. In: Advances in Neural Information Processing Systems, pp. 135-143.

Bruzzone, L. and Carlin, L., 2006. A multilevel context-based system for classification of very high spatial resolution images. IEEE Transactions on Geoscience and Remote Sensing 44(9), pp. 2587-2600.

Chang, C.-C. and Lin, C.-J., 2011. Libsvm: a library for support vector machines. ACM Transactions on Intelligent Systems and Technology (TIST) 2(3), pp. 27.

Chen, Y., Su, W., Li, J. and Sun, Z., 2009. Hierarchical object oriented classification using very high resolution imagery and lidar data over urban areas. Advances in Space Research 43(7), pp. 1101-1110.
Cui, Y., Chapel, L. and Lefèvre, S., 2015. A subpath kernel for learning hierarchical image representations. In: Graph-Based Representations in Pattern Recognition, Lecture Notes in Computer Science, Vol. 9069, pp. 34-43.

Cui, Y., Chapel, L. and Lefèvre, S., 2016. Combining multiscale features for classification of hyperspectral images: a sequence based kernel approach. In: 8th IEEE International Workshop on Hyperspectral Image and Signal Processing: Evolution in Remote Sensing (WHISPERS 2016).

Forestier, G., Puissant, A., Wemmert, C. and Gançarski, P., 2012. Knowledge-based region labeling for remote sensing image interpretation. Computers, Environment and Urban Systems 36(5), pp. $470-480$.

Gomez-Chova, L., Tuia, D., Moser, G. and Camps-Valls, G., 2015. Multimodal classification of remote sensing images: a review and future directions. Proceedings of the IEEE 103(9), pp. 1560-1584.

Haussler, D., 1999. Convolution kernels on discrete structures. Technical report, Department of Computer Science, University of California at Santa Cruz.

Hopcroft, J. E., Ullman, J. D. and Aho, A. V., 1983. Data structures and algorithms. Addison-Wesley Boston, MA, USA.

Kurtz, C., Passat, N., Gancarski, P. and Puissant, A., 2012. Extraction of complex patterns from multiresolution remote sensing images: A hierarchical top-down methodology. Pattern Recognition 45(2), pp. 685-706.

Lefèvre, S., Chapel, L. and Merciol, F., 2014. Hyperspectral image classification from multiscale description with constrained connectivity and metric learning. In: 6th International Workshop on Hyperspectral Image and Signal Processing: Evolution in Remote Sensing (WHISPERS 2014).

Liu, Y., Guo, Q. and Kelly, M., 2008. A framework of regionbased spatial relations for non-overlapping features and its application in object based image analysis. ISPRS Journal of Photogrammetry and Remote Sensing 63(4), pp. 461-475.

Shackelford, A. K. and Davis, C. H., 2003. A combined fuzzy pixel-based and object-based approach for classification of highresolution multispectral data over urban areas. IEEE Transactions on Geoscience and Remote Sensing 41(10), pp. 2354-2363.

Stumpf, A. and Kerle, N., 2011. Object-oriented mapping of landslides using random forests. Remote Sensing of Environment 115(10), pp. 2564-2577.

Tang, H., Shen, L., Qi, Y., Chen, Y., Shu, Y., Li, J. and Clausi, D. A., 2013. A multiscale latent dirichlet allocation model for object-oriented clustering of vhr panchromatic satellite images. IEEE Transactions on Geoscience and Remote Sensing 51(3), pp. 1680-1692.

Tilton, J. C., 1998. Image segmentation by region growing and spectral clustering with a natural convergence criterion. In: IEEE International Geoscience and Remote Sensing Symposium (IGARSS), Vol. 4, IEEE, pp. 1766-1768.

Tzotsos, A. and Argialas, D., 2008. Support vector machine classification for object-based image analysis. In: Object-Based Image Analysis, Springer, pp. 663-677.

Zhang, J., 2010. Multi-source remote sensing data fusion: status and trends. International Journal of Image and Data Fusion 1, pp. 5-24. 


\title{
REPLACING THE USE OF TEXTURE AND SEALED AREA IN URBAN FABRIC CLASSIFICATIONS BY INTEGRATING VOLUME AND OBJECT BASED DISTANCE CALCULATIONS
}

\author{
R. de Kok $^{\mathrm{a},{ }^{*}}$, P. Wezyk ${ }^{\mathrm{b}}$, B. Hejmanowska ${ }^{\mathrm{c}}$, J. Książek $^{\mathrm{c}}$ \\ a ProGea.PL, \\ ${ }^{\mathrm{b}}$ Laboratory of Geomatics, Institute of Forest Resource Management, Faculty of Forestry, University of Agriculture in Krakow, \\ ${ }^{c}$ AGH University of Science and Technology, Kraków, Poland, Faculty of Mine Surveying and Environmental Engineering, \\ Department of Geoinformation, Photogrammetry and Remote Sensing of Environment. \\ roeland_de_kok@hotmail.com
}

KEY WORDS: Urban Fabric, Distance to Neighbours, Perception, Thiessen, GEOBIA

\begin{abstract}
:
The classification of urban fabric can be achieved using the information of individual buildings and measure their distance to all image objects in the map. The distance to a variety of building types return various areas, which can be associated with dense and less dense urban fabric. The resulting classification can be used as an alternative or as a control mechanism on normal urban fabric classification. The method can be used to extend existing manuals on urban fabric.
\end{abstract}

\section{REDEFINING URBAN FABRIC}

\subsection{Urban fabric by proxy}

The Urban fabric is described in terms of "dense", "less dense", "continuous" and "discontinuous". These classifications are usually applied in projects such as Corine and Urban Atlas (EU, 2011). The driving factor for the urban fabric classification is the layer of "soil sealing" and the loosely defined "texture" (Bossard et.al. 2000, EU, 2011). The role of both the Red and the Infrared channel for mapping the vegetation is essential. In remote sensing, the degree of imperviousness can be estimated with proxy parameters that quantify the cover of green vegetation, which en gross can be considered inversely correlated with the degree of surface imperviousness in urban or built-up areas (Gangkofner et. Al, 2010) The surface imperviousness can than play a role in classifying dense and less dense urban fabric.

\subsection{Classifying by building type}

This study introduces a method to classify urban fabric without relying on the availability of the Red and the Infrared spectral channels. Although the manual for Urban Atlas explicitly states that building type should not play a role in urban fabric classification (EU, 2011) it is the intuitive and historically developed human perception on the topographical map that allows to distinguish urban zones related to building type (see also Chapter 2.1). For reasons of perception and the strive for simulating the unique human vision with computer vision (Marr, 1982), the core dataset used in this study is based on data that allows the extraction of the size and height of individual buildings. There are different datasets for retrieving such information; Lidar data that goes back till end of 20th century. Alternatively, classic photogrammetric imagery that relies on parallax (see o.a. Avery and Gradon, 1992). This data has the advantage that it goes back till beginning of 19th century.

\subsection{Extracting groups of single buildings}

The level of detail in cadastral maps deviates from satellite based urban mapping. Details on individual buildings in cadastral GIS are standard. Most in the form of the building footprints. The visualization of the cadastral map only hints at the inherent grouping of neighbouring buildings with similarities in footprint (see chapter 2.1 on perception). Between urban mapping for international use, based on satellite data and the cadastral maps for local use, based on surveying, there are huge differences in the budget, production timeline, users and mapping techniques. The aim is to move the information derived from grouped individual buildings up to the level of 25:000 (trans) national scale, making it comparable to the mapping scale of Corine and Urban Atlas.

After demonstrating initial results, the presented study can function as a basis to discuss the relevance of urban maps from historical data. The results can also become an alternative to overcome the problems related to urban change detection methods. The analysis could further be applied in the near future for a full automatic tagging on existing Urban Atlas polygons that might require an update.

\subsection{The primal sketch to group image parts}

Urban fabric is classified by a proxy parameter. The measurement by proxy does not explain the real physically density of urban area. There is few conceptual information in the manuals on this topic and even no information on the ontology of urban fabric. Although, outside of the manuals, the scientific literature is extensive (o.a. Montenergro, 2009). Starting with a classical figure from the Urban Atlas mapping guide (EU, 2006), it is Fig $1 \mathrm{~A}$. This figure can be related to the very early manual of Corine landcover ( Bossard e.a. , 2000). It is one of the rare illustrations related to the concept of urban fabric. Now any explicit information on this image is just dropped in the 2006 Urban Atlas guide, maybe for good reasons.

\footnotetext{
* Corresponding author
} 


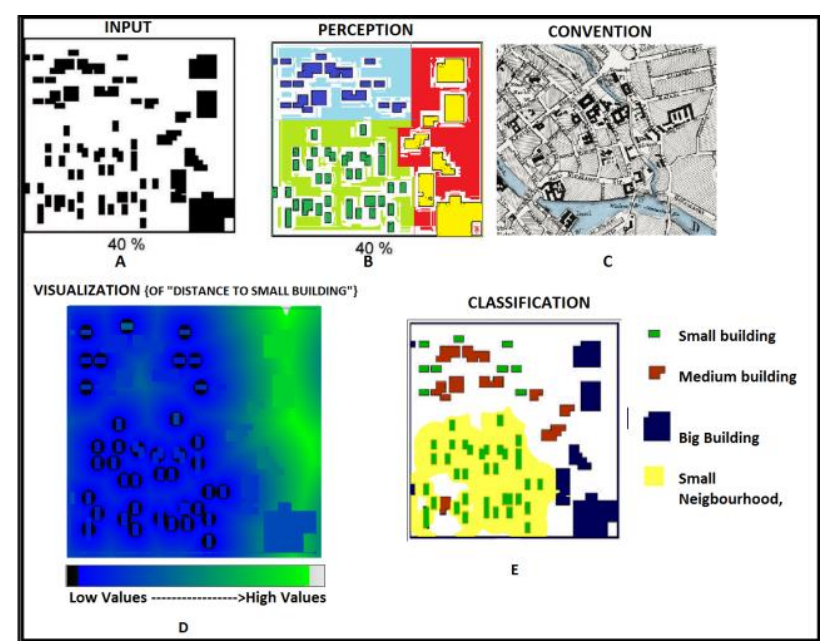

Figure 1A-E. Illustrations on the various concepts of map interpretation. A; Computer Vision Input value 40\%, B; A sketch on Fig 1 A to illustrate the Human Perception, C, Historical Convention on the topographic map around 1850, D; Feature Value Visualization on Fig1A, E; Image Classification.

Although Fig 1 A might seem extremely simple, the perception (Marr, 1982) is quite complicated. First of all, the classical layout of the historical topographic map at large scale depicts urban areas with individual footprints of selected buildings. Fig $1 \mathrm{C}$ is a snapshot of a 19th century map of Alexanderplaz, Berlin. The Fig $1 \mathrm{C}$ is related to cartographic convention on how to display the symbol of a large building with the printing technique of that time. The " $40 \%$ "annotation is a radical break with that convention. No person would ever "see" " $40 \%$ ". This " $40 \%$ " annotation in Fig1A is a computer vision fossil. It only hints at the simplest value at which the image can be processed using the most basic image processing techniques. With a revolution in computer vision, the $40 \%$ must be reduced to only one of the multitude of measurements that makes Fig 1 A accessible to computer processing. So, if a person does not see " $40 \%$ ", than what is the true perception of Fig 1 A ?

Although a more in-depth explanation on perception would surely improve the manuals, a first attempt is achieved using Fig. 1B which is derived from Fig1A in limited accordance with the very extensive theory on perception used by Marr (Marr, 1982). In Fig. 5B a "primal sketch" uses manually added colours to illustrate how a person "sees" the city. Perception in different stages, as described by Marr (1982) involves the areas that are interpreted as belonging to a group or cluster in the image. A naive perception of the urban fabric would separate the Fig $1 \mathrm{~A}$ in different building types and zones. This interpretation process is approached by adding the colours in Fig 1B. In addition to the explanation on the visual interpretation in a modern manual, we are now in urgent need of an extension of the manuals with computer protocols.

\subsection{The process tree}

To illustrate this need for an extension of the manual, the image-object-feature "distance to building" is used. This is an example to explain the need of a process protocol accompanying the illustration of the manual. Except for the initial $40 \%$ in Fig $1 \mathrm{~A}$, more and more complex values can be extracted from the conceptual illustration of Fig. 1A. Fig 1 D shows the distance of all pixels towards the nearest small buildings. The yellow area in Fig 1 E ("Goal1" in the following process tree) is classified with the following process. For the final paper, the protocol will be added here:..

The yellow polygon of Fig 1 E classified by the to be shown protocol only approaches the manually coloured green district of Fig $1 \mathrm{~B}$ and could be associated by proxy as an area of sparse urban fabric with small houses. It is clear that not all small (green) buildings in Fig $1 \mathrm{D}$ are surrounded by a yellow area. The rule set can be expanded and refined for generalization purposes. However, the role of it's essential feature "Distance to Neighbour" remains.

\section{FROM SIMULATION TO REAL DATA}

\subsection{Thiessen area by proxy}

The feature display of Fig $1 \mathrm{D}$ can be applied to real data as well. Fig 2 Shows the study area over Cracow with the castle Wawel along the river Wisla $(20 \times 10 \mathrm{Km})$, where Fig. 3 shows the same feature distance to building in exact area of Fig 2 with the feature as used in Fig 1D.

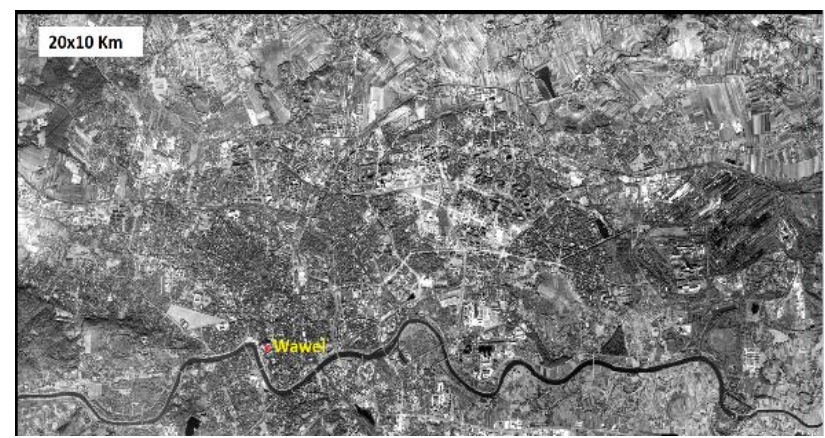

Figure 2.A WVII image with the study area of Cracow and the Wawel castle along the river Wisla

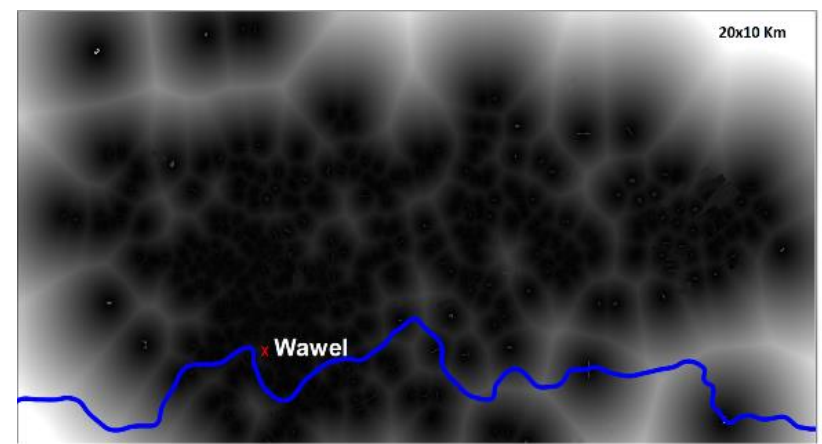

Figure 3 Visualization of the feature Distance to large buildings

Fig 3 displays the attribute "Distance to Building Volume Category $60 "$ in grey values. The Building category 60 is a selected subset of buildings with large volume derived from the 2006 Lidar image of Cracow. The River Wisla as a blue line draped over this to ease visual comparison. Fig 3 shows the distance of all 1,3 million objects towards their nearest object in the class Building 60. If the distance is very small, the value is low and the display is dark. Vice versa, when the distance to any building in category 60 is very large, the value is high and therefore bright. There are 541 large buildings in class 60 and 541 "Bubbles". These bubbles approach the characteristics of Thiessen polygons. 


\subsection{The repetitive patterns of city blocks}

Intuitive and empirical findings on urban mapping support the assumption that similar buildings are encountered as grouped in city blocks. At least for European conditions this is often the case. This hints at the fractal nature of European cities, where repetitive patterns can be observed within city blocks. Buildings within a certain category of „Building Volume” can then be expected to have several neighbours with similar values for „Building Volume”. Although this seems to be logic for planned city blocks, the characteristic of similarity for neighbouring buildings still can be observed among organic/historical growth patterns in European cities. It is now assumed that neighbourhoods with very large values for „Building Volume” cover the area of Dense Urban fabric. Vice versa, the buildings with small values for "Building Volume" are associated with sparse urban fabric. The latter form the use case for this study using the simplest parameters to prove the case. A visualization of all seven different distances to respective seven building categories can be combined in a colour composite (Fig4) to enhance the nested appearance of areas with similarities in distance to the same building category. Now these features must be translated to the protocol of the process tree.

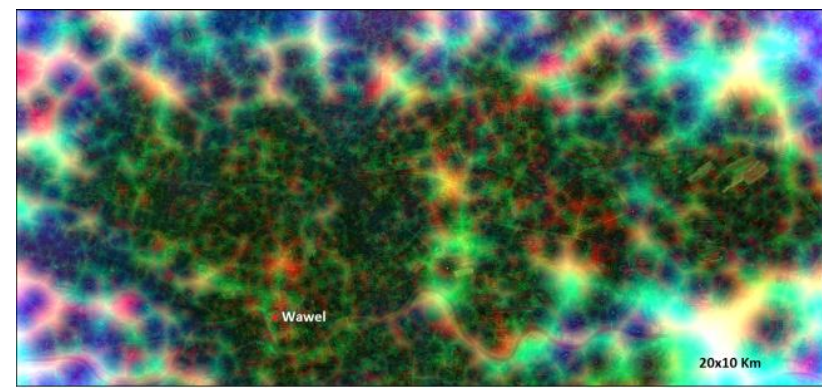

Figure 4 Visualization of the feature Distance to Buildings for 7 building classes. Each of the 1.3 million objects have 7 values for distance to building in 7 categories.

There are classes that can be calibrated better than large volume buildings and become the core classes in this introduction study to explain the central role of the feature distance to building class.

\section{EVALUATION}

\subsection{The Urban Atlas as reference}

The most recent version of urban atlas can now be used to Evaluate the classification in 7 classes.

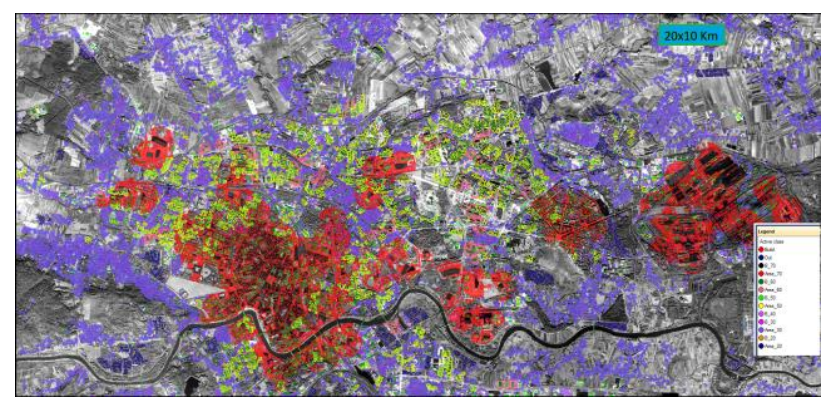

Figure 5 Visualization of the classification
To confront the classification results with a state of the art detailed product. The various classes must be split because not all urban areas are within the urban fabric. Most notably the class of Allotment gardens show a typical deviation from the other urban classes. The in-depth role will be described in the follow up of this extended abstract.

\section{CONCLUSIONS}

The classification of areas grouped by similar distance to building-type offers at the least a signal or flagging procedure to highlight urban atlas polygons that require an update.

\section{REFERENCES}

Avery, T.E., Gradon, L., 1992. Fundamentals of Remote Sensing and Airphoto Interpretation Berlin. 1992. PrenticeHall, Inc.

Bossard,M., Feranec,J., Otahel,J., 2000 CORINE Land Cover Technical Guide Addendum 2000, European Environment Agency Technical Report 40 http://www.eea.europa.eu/publications/tech40add

European Union, (2011). Mapping Guide for a European Urban Atlas. Available at http://www.eea.europa.eu/data-andmaps/data/urban-atlas.

Gangkofner, U., Weichselbaum, J., Kuntz, S., Brodsky, L. Larsson, K., de Pasquale, V., 2010 2010, Update of the European High-resolution Layer of Built-up Areas and Soil Sealing 2006 with Image2009 Data.Remote Sensing for Science, Education, and Natural and Cultural Heritage Rainer Reuter (Editor) EARSeL,

Lillesand, T.M, Kiefer, R.,Chipman J., 2015. Remote Sensing and Image Interpretation (7th edition).. John Wiley \& Sons,

Marr, D. (1982). Vision: A Computational Investigation into the Human Representation and Processing of Visual Information. San Francisco, CA: W. H.Freema

Montenegro, N., Duarte, J.P., 2009, CUMINCAD Computational Ontology of Urban Design: Towards a City Information Model , Computation: The New Realm of Architectural Design, Istanbul Technical University, Turkey, 


\title{
APPLICATION OF GEOBIA TO MAP THE SEAFLOOR
}

\author{
M. Diesing a * \\ ${ }^{a}$ Centre for Environment, Fisheries and Aquaculture Science, Lowestoft, United Kingdom - markus.diesing@ cefas.co.uk
}

KEY WORDS: Marine, Seafloor, Sonar, Multibeam Echosounder, Habitat

\begin{abstract}
:
Geographic Object-Based Image Analysis (GEOBIA) has been successfully employed to map terrestrial environments. However, $71 \%$ of Earth's surface is covered by seawater and standard optical methods suitable for mapping the land surface have limited application in such environments. Application of GEOBIA to marine environments has nevertheless been attempted and can generally be subdivided into three domains: 1 . The intertidal zone and shallow subtidal zone have been mapped with optical data and application of GEOBIA in such environments can be seen as a seaward extension of terrestrial approaches. 2. Photographs of the seafloor give very detailed but spatially limited information. GEOBIA methods have been applied to classify benthic species and habitats and estimate seafloor complexity among others. 3. Due to the rapid attenuation of light in water, the method of choice to map the seafloor employs sound. Modern multi-beam echosounders map the seafloor in high detail. Such sensors measure the topography (water depth) and the strength of the returning signal (backscatter), which can be used to characterise the seafloor substrates and habitats. This contribution will focus on the application of GEOBIA to marine acoustic datasets. A generic workflow for object-based acoustic seafloor mapping will be showcased and the current state of the application of GEOBIA to marine acoustic data will be discussed.
\end{abstract}

\section{INTRODUCTION}

It has been said that the Earth's ocean is the final frontier and that we know more about the surface of Mars than the floor of our oceans. Only $5-10 \%$ of the seafloor is mapped with a resolution comparable to that on land (Wright and Heyman, 2008) due to the fact that optical methods are incapable of penetrating the water column apart from the shallowest marginal parts of the world ocean. Unsurprisingly, there have been limited attempts so far to apply GEOBIA to marine data sets with the aim to map the seafloor.

Marine Object-Based Image Analysis (MOBIA), here defined as the application of GEOBIA to marine data sets with the aim to map the seafloor geomorphology, geology and habitats, is a nascent science discipline, but most advanced where optical remote sensing imagery collected from satellites and remotely operated aircraft systems ('drones') has been utilised to map intertidal and shallow subtidal habitats. Among the most frequently mapped habitats are mangroves (Conchedda et al., 2008; Heumann, 2011; Wang et al., 2004), saltmarsh (Moffett and Gorelick, 2013; Ouyang et al., 2011), seagrass (Lathrop et al., 2006; Lyons et al., 2012; Roelfsema et al., 2014) and coral reefs (Benfield et al., 2007; Knudby et al., 2011; Phinn et al., 2012).

Seafloor photographic images, collected from various platforms, such as drop-frames, benthic sledges, remotely operated vehicles and autonomous underwater vehicles, might be interpreted separately or stitched together to derive georeferenced photo-mosaics. Such image data sets could be analysed with GEOBIA methods to map habitats and derive (semi-)quantitative information (e.g. seafloor complexity); however a significant drawback is the very limited footprint on the order of $1 \mathrm{~m}^{2}$ to several $100 \mathrm{~m}^{2}$ and few examples exist in the peer-review literature (Lacharité et al., 2015).
The preferred method to map the seafloor in sufficient detail utilises sound rather than light, as sound is much less attenuated and travels over significant distances in the water column. Modern multibeam echosounders (MBES) survey the seafloor in high detail and typically measure two parameters: the travel time of the sound from the transducer to the seafloor and back to the receiver, which can be converted to water depths and the intensity of the returned signal, commonly known as backscatter strength. From the depth data, it is possible to construct detailed digital elevation models and derive secondary variables, such as seafloor slope and rugosity. The backscatter strength is influenced by various factors, such as the geometry of the sonar-target system, the physical characteristics and the intrinsic nature of the seafloor (Blondel, 2009), but yields an interpretable acoustic image of the makeup of the seafloor surface. It is mainly this kind of imagery that has proved most useful for mapping seafloor sediments and habitats (Brown et al., 2011). Due to the fact that MBES typically survey with one acoustic frequency those backscatter images are essentially limited to one band.

The resolution of acoustic seafloor imagery is dependent on the beam width and the water depth. Typical shallow water MBES collect data with a spatial resolution of c. $1 \mathrm{~m}$ to $5 \mathrm{~m}$. Features of interest are typically larger than that (H-resolution case) and object-based approaches are appropriate under these circumstances (Blaschke et al., 2014). Since Lucieer's (2008) paper on object-based mapping of benthic marine habitats, uptake of GEOBIA methods has been slow but is currently accelerating with studies published in the peer-review literature (e.g. Diesing et al., 2014; Lucieer and Lamarche, 2011; Montereale Gavazzi et al., 2016) and presented at conferences. This contribution will showcase a generic workflow for categorical seafloor mapping based on acoustic data sets and ground-truth observations.

\footnotetext{
* Corresponding author
} 


\section{GENERIC WORKFLOW}

Because the interpretation of seafloor acoustic imagery is significantly less unequivocal than optical images of the land surface, ground-truth observations are usually required. These might be seafloor still images interpreted with respect to substrate or habitat type or physical samples of the seafloor taken with grab samplers. Collected sediment samples might be analysed for their grain-size distribution and content of benthic species and classified accordingly. The need for samples does mean that the classification process is typically sample-based rather than rule-based. A generic workflow is shown in Figure 1.

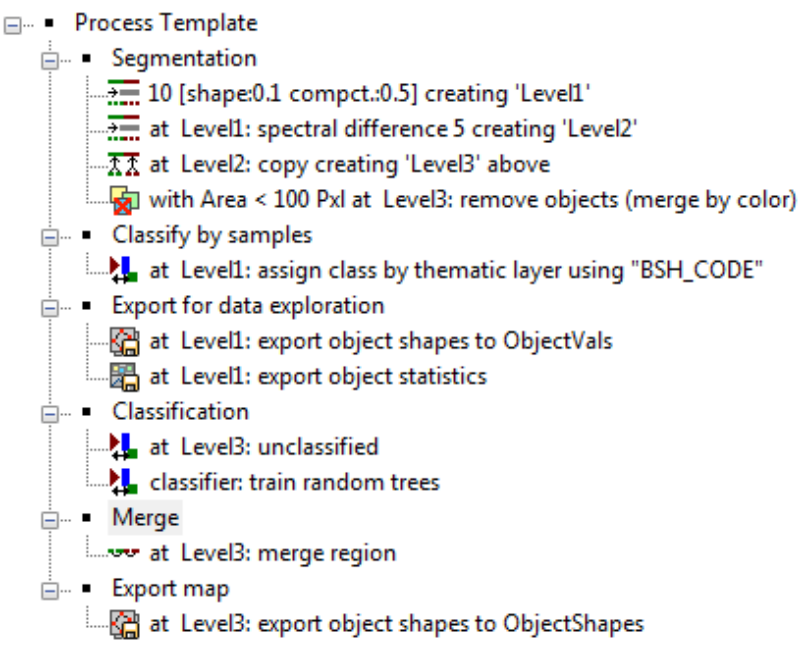

Figure 1. Generic workflow for acoustic seafloor mapping.

\subsection{Segmentation}

Segmentation is typically carried out with the multiresolution segmentation algorithm utilising suitable image layers. The scale parameter might be estimated via the Estimation of Scale Parameter tool (Drăguţ et al., 2010) or via visual assessment of the segmentation results. Further segmentation steps might include merging of objects with the spectral difference algorithm and removal of small objects of a size below a defined minimum mapping unit.

\subsection{Classification by samples}

Ground-truth information brought into the project as a thematic layer (shape file) is utilised to classify image objects that coincide with these samples. Image object features that are deemed potentially useful for further classification are then selected and feature values extracted for every classified object.

\subsection{Feature selection}

We have developed a browser-based tool utilising Shiny, a web application framework for $\mathrm{R}$, to select important and remove correlated features. Feature selection is carried out with the Boruta algorithm (Kursa and Rudnicki, 2010) and correlated features can be identified with a correlation matrix.

\subsection{Data exploration/model building}

Additionally, the tool allows the user to display the data as box plots and density curves for the selection of suitable thresholds for classification. Alternatively, Conditional Inference (CI) analysis (Hothorn et al., 2006) might be carried out. The resulting decision tree (Figure 2) is easily translatable into an eCognition rule-set.

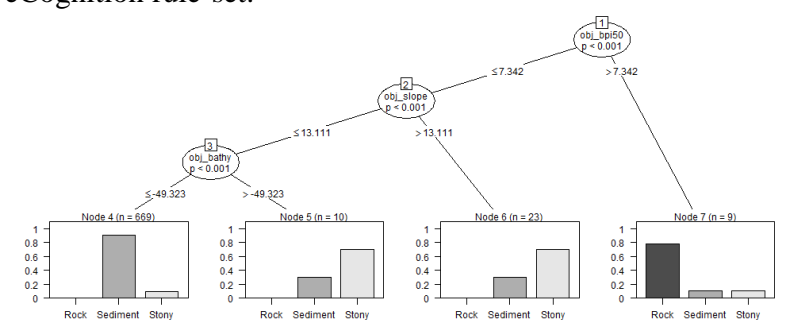

Figure 2. Example of CI decision tree that can be translated into a rule-set.

\subsection{Classification}

Classification in eCognition might be carried out in three different ways, a) by applying simple rules and thresholds as derived by data exploration, b) by translating the CI decision tree into a rule-set or c) by utilising the implemented machine learning algorithms (e.g. Random Forest, Breiman, 2001). Once acceptable results are achieved, image objects are merged by class and the final results exported as a shape file.

\section{DISCUSSION}

Automated and repeatable acoustic seafloor classification is still in its infancy, but progress could be accelerated by learning from terrestrial remote sensing (Diesing et al., 2016). The same is true for the application of GEOBIA to acoustic data. It is encouraging to see the increasing amount of studies presented at conferences such as GeoHab (geohab.org). However, more research is needed to fully assess and exploit the true potential of GEOBIA in relation to acoustic seafloor mapping. Only a limited amount of features available for classification is typically utilised. These are most frequently object statistics and to a lesser extent texture and shape features. There has been little usage of object relationships and the concept of hierarchy in landscapes (Burnett and Blaschke, 2003), represented as segmentations at different levels, has hardly been investigated so far.

A limiting factor in acoustic seafloor mapping is the missing spectral resolution of backscatter data, which typically consists of one band. Hence, it is not possible to derive band ratios or exploit spectral signatures. The potential for improved seafloor classification with 3-band acoustic backscatter data has recently been demonstrated (Hughes-Clarke, 2015; Tamsett et al., 2016) and it is hoped that multi-spectral MBES will become the standard in seafloor mapping.

\section{ACKNOWLEDGEMENTS}

This work was funded by the Cefas Research and Development Fund.

\section{REFERENCES}

Benfield, S.L., Guzman, H.M., Mair, J.M., Young, J. a. T., 2007. Mapping the distribution of coral reefs and associated sublittoral habitats in Pacific Panama: a comparison of optical satellite sensors and classification methodologies. Int. J. Remote Sens. 28, 5047-5070. doi:10.1080/01431160701258062

Blaschke, T., Hay, G.J., Kelly, M., Lang, S., Hofmann, P., Addink, E., Queiroz Feitosa, R., van der Meer, F., van der Werff, H., van Coillie, F., Tiede, D., 2014. Geographic Object- 
Based Image Analysis - Towards a new paradigm. ISPRS J. Photogramm. Remote Sens. 87, 180-191.

Blondel, P., 2009. The handbook of sidescan sonar. Springer, Berlin, Heidelberg, New York.

Breiman, L., 2001. Random Forests. Mach. Learn. 45, 5-32.

Brown, C.J., Smith, S.J., Lawton, P., Anderson, J.T., 2011. Benthic habitat mapping: A review of progress towards improved understanding of the spatial ecology of the seafloor using acoustic techniques. Estuar. Coast. Shelf Sci. 92, 502520. doi:10.1016/j.ecss.2011.02.007

Burnett, C., Blaschke, T., 2003. A multi-scale segmentation/object relationship modelling methodology for landscape analysis. Ecol. Modell. 168, 233-249.

Conchedda, G., Durieux, L., Mayaux, P., 2008. An object-based method for mapping and change analysis in mangrove ecosystems. ISPRS J. Photogramm. Remote Sens. 63, 578-589. doi:10.1016/j.isprsjprs.2008.04.002

Diesing, M., Green, S.L., Stephens, D., Lark, R.M., Stewart, H.A., Dove, D., 2014. Mapping seabed sediments: Comparison of manual, geostatistical, object-based image analysis and machine learning approaches. Cont. Shelf Res. 84, 107-119.

Diesing, M., Mitchell, P., Stephens, D., 2016. Image-based seabed classification: what can we learn from terrestrial remote sensing? ICES J. Mar. Sci. doi:10.1093/icesjms/fsw118

Drăguţ, L., Tiede, D., Levick, S.R., 2010. ESP: a tool to estimate scale parameter for multiresolution image segmentation of remotely sensed data. Int. J. Geogr. Inf. Sci. 24, 859-871.

Heumann, B.W., 2011. An object-based classification of mangroves using a hybrid decision tree-support vector machine approach. Remote Sens. 3, 2440-2460. doi:10.3390/rs3112440

Hothorn, T., Hornik, K., Zeileis, a, 2006. party: A Laboratory for Recursive Part (y) itioning. R Packag. version 0.9-0, URL http//CRAN. R-project. org. doi:10.1.1.151.2872

Hughes-Clarke, J.E., 2015. Multispectral Acoustic Backscatter from Multibeam, Improved Classification Potential, in: United States Hydrographic Conference 2015. National Harbor, MD, USA, pp. 1-18.

Knudby, A., Roelfsema, C., Lyons, M., Phinn, S., Jupiter, S., 2011. Mapping Fish Community Variables by Integrating Field and Satellite Data, Object-Based Image Analysis and Modeling in a Traditional Fijian Fisheries Management Area. Remote Sens. 3, 460-483.

Kursa, M., Rudnicki, W., 2010. Feature selection with the Boruta Package. J. Stat. Softw. 36, 1-11.

Lacharité, M., Metaxas, A., Lawton, P., 2015. Using objectbased image analysis to determine seafloor fine-scale features and complexity. Limnol. Oceanogr. Methods 13, 553-567. doi:10.1002/lom3.10047

Lathrop, R.G., Montesano, P., Haag, S., 2006. A Multi-scale Segmentation Approach to Mapping Seagrass Habitats Using Airborne Digital Camera Imagery. Photogramm. Eng. Remote Sens. 72, 665-675. doi:10.14358/PERS.72.6.665
Lucieer, V., Lamarche, G., 2011. Unsupervised fuzzy classification and object-based image analysis of multibeam data to map deep water substrates, Cook Strait, New Zealand. Cont. Shelf Res. 31, 1236-1247. doi:10.1016/j.csr.2011.04.016

Lucieer, V.L., 2008. Object-oriented classification of sidescan sonar data for mapping benthic marine habitats. Int. J. Remote Sens. 29, 905-921.

Lyons, M.B., Phinn, S.R., Roelfsema, C.M., 2012. Long term land cover and seagrass mapping using Landsat and objectbased image analysis from 1972 to 2010 in the coastal environment of South East Queensland, Australia. ISPRS J. Photogramm. Remote Sens. 71, 34-46. doi:10.1016/j.isprsjprs.2012.05.002

Moffett, K.B., Gorelick, S.M., 2013. Distinguishing wetland vegetation and channel features with object-based image segmentation. Int. J. Remote Sens. 34, 1332-1354. doi:10.1080/01431161.2012.718463

Montereale Gavazzi, G., Madricardo, F., Janowski, L., Kruss, A., Blondel, P., Sigovini, M., Foglini, F., 2016. Evaluation of seabed mapping methods for fine-scale classification of extremely shallow benthic habitats - Application to the Venice Lagoon, Italy. Estuar. Coast. Shelf Sci. 170, 45-60. doi:10.1016/j.ecss.2015.12.014

Ouyang, Z.T., Zhang, M.Q., Xie, X., Shen, Q., Guo, H.Q., Zhao, B., 2011. A comparison of pixel-based and objectoriented approaches to VHR imagery for mapping saltmarsh plants. Ecol. Inform. 6, 136-146. doi:10.1016/j.ecoinf.2011.01.002

Phinn, S.R., Roelfsema, C.M., Mumby, P.J., 2012. Multi-scale, object-based image analysis for mapping geomorphic and ecological zones on coral reefs. Int. J. Remote Sens. 33, 37683797. doi:10.1080/01431161.2011.633122

Roelfsema, C.M., Lyons, M., Kovacs, E.M., Maxwell, P., Saunders, M.I., Samper-Villarreal, J., Phinn, S.R., 2014. Multitemporal mapping of seagrass cover, species and biomass: A semi-automated object based image analysis approach. Remote Sens. Environ. 150, 172-187. doi:10.1016/j.rse.2014.05.001

Tamsett, D., McIlvenny, J., Watts, A., 2016. Colour Sonar: Multi-Frequency Sidescan Sonar Images of the Seabed in the Inner Sound of the Pentland Firth, Scotland. J. Mar. Sci. Eng. 4, 26. doi: $10.3390 /$ jmse 4010026

Wang, L., Sousa, W.P., Gong, P., 2004. Integration of objectbased and pixel-based classification for mapping mangroves with IKONOS imagery. Int. J. Remote Sens. 25, 5655-5668. doi:10.1080/014311602331291215

Wright, D.J., Heyman, W.D., 2008. Introduction to the Special Issue: Marine and Coastal GIS for Geomorphology, Habitat Mapping, and Marine Reserves. Mar. Geod. 31, 223-230.

Revised June 2016 


\title{
OBJECT-BASED VHSR IMAGE CLASSIFICATION USING MULTIBAND COMPACT TEXTURE UNIT DESCRIPTOR
}

\author{
K. Djerriri ${ }^{\mathrm{a},{ }^{*}}{ }^{*}$, A. Safia $^{\mathrm{b}}$, R. S. Cheriguene ${ }^{\mathrm{a}}$, H. S. Rahli ${ }^{\mathrm{a}}$, M. S. Karoui ${ }^{\mathrm{a}}$ \\ ${ }^{\text {a }}$ Centre des Techniques Spatiales, Département Observation de la Terre, Algeria; \\ ${ }^{\mathrm{b}}$ Centre for Research and Applications in Remote Sensing (CARTEL), Department of Geomatics, Sherbrooke University, Canada; \\ kdjerriri@cts.asal.dz
}

KEY WORDS: Multispectral images, Object-based Image Analysis, Multiband Compact Texture Unit

\begin{abstract}
:
In remote sensing, texture is commonly used to support spectral information particularly when spectral signatures of class of interest are similar. It is usually extracted using panchromatic band instead of multispectral bands. This is because panchromatic band has rich texture content due to its fine spatial resolution. Recent space-borne and pansharpening techniques can deliver multispectral images with a submetric resolution which are also good candidates for texture analysis. The difficulty in extracting texture in multispectral images is the fact that existing and widely used methods are limited to analyzing spatial relationship between pixels in a single band at a time. When multispectral images are used texture characterization is usually performed by analyzing spatial relationships in each spectral band independently. This ignores inter-band spatial relationships which can be a source of valuable source of information.

This paper evaluates the capability of a recently proposed method named multiband compact texture unit. This method extracts texture by characterizing simultaneously spatial relationship in the same band and across the different bands. This evaluation is performed in the context of object-based classification paradigm using WorldView-2 image of a forest area. For that image-objects were generated through superpixel segmentation. Classification in the object-feature space is performed suing $\mathrm{K}$ nearest neighbor algorithm. The proposed approach is compared to two groups of methods. The first group includes texture methods that use only spatial relationships in the same band: Gabor features wavelets and Granulometry. The second group includes methods that use intra-band and inter-band spatial relationships: integrative gray-level co-occurrence matrix, opponent Gabor features and opponent local binary patterns.

Experimental results show that texture extracted using both intra-band and inter-band spatial relationship improves the classification accuracy compared to when it is extracted in each spectral band independently. Among the methods of the second group that use both intra-band and inter-band spatial relationships, the multiband compact texture unit method produces the best results.
\end{abstract}

\section{INTRODUCTION}

Texture has been widely used for the analysis of remote sensing data particularly when spectral information cannot deliver sufficient results. It has been used to analyze medium spatial resolution images and it continues to play an increasing role in Very High Spatial Resolution (VHSR) images due to its capability to decrease the effect of high intra-class variability that characterizes VHSR images.

Most of the previous works on texture analysis have focused on extracting texture information using panchromatic bands due to their finer spatial resolution compared to multispectral ones. However, with the improvement of the spatial resolution of VHSR images, there is a growing interest on extracting texture from multispectral bands. Unfortunately, most of the existing texture methods were developed to extract texture from a single band and there is no general agreement on how to deal with images of many spectral bands. A widely adopted solution for extracting texture from multispectral images is to use existing monoband texture methods by analyzing each band independently. However, reducing the texture of multispectral images to a set of independent grayscale texture represents a very simplified solution as it does not take into account the multidimensional nature of image pixels.

Recently the authors in Safia and He (Safia and He, 2015a) introduced the concept of multiband texture as an extension of the concept of grayscale texture: "Multiband texture is defined as the spatial distribution of the tonal variations in the same band plus the spatial distribution of the tonal variations across different bands". The extraction of multiband texture by analyzing both intra- and inter-band spatial interactions simultaneously in many bands results in high calculation complexity. This is the main reason why the few available multiband texture methods are restricted to extracting intra- and inter-band spatial interactions between only two bands, which is an important limitation. Safia and He in (Safia and He, 2015a), proposed a new multiband texture descriptor which can extract multiband texture between a large numbers of bands simultaneously. The new descriptor is based on the Compact Texture Unit (C-TU) model proposed by the authors for monoband texture (Safia and He, 2013a) which has the advantage of having low computational complexity.

The process of texture extraction is time and resources consuming because it is performed locally using a moving window centered at each pixel in the image. The use of VHSR images for texture analysis makes the situation even harder. In recent years, object-based approach in contrast to pixel-based one has gained a great interest in the remote sensing community. When applying object-based method to image classification, images are decomposed to objects and after that classification will be conducted in object-feature space. Imageobjects as the basic classification unit were generated through image segmentation. Many studies reported that object-based approach can be an alternative to classic pixel-based image analysis particularly for VHSR images (Blaschke, 2010a).

This paper presents an evaluation of the multiband compact texture unit descriptor for simultaneous intra-band and interband texture analysis in an object-based classification scheme using WorldView-2 data in a forest environment. Segmentation step is performed using superpixels approach proposed in

* Corresponding author 
(Achanta, Shaji, Smith, Lucchi, Fua and Süsstrunk, 2012). To evaluate the pertinence of multiband texture features, the stateof-art texture features such as Gray-Level Co-Occurrence Matrix (GLCM), Gabor features and Local Binary Patterns (LBP) are also extracted for comparison purpose. Different feature descriptors were compared by means of classification accuracy. A simple K-nearest neighbors (KNN) classifier is employed for the classification in object-feature space.

\section{METHODS}

In this research, object-based image analysis is adopted. It is performed in three steps: superpixel based image segmentation, multiband compact texture unit feature extraction and supervised classification using KNN classifier.

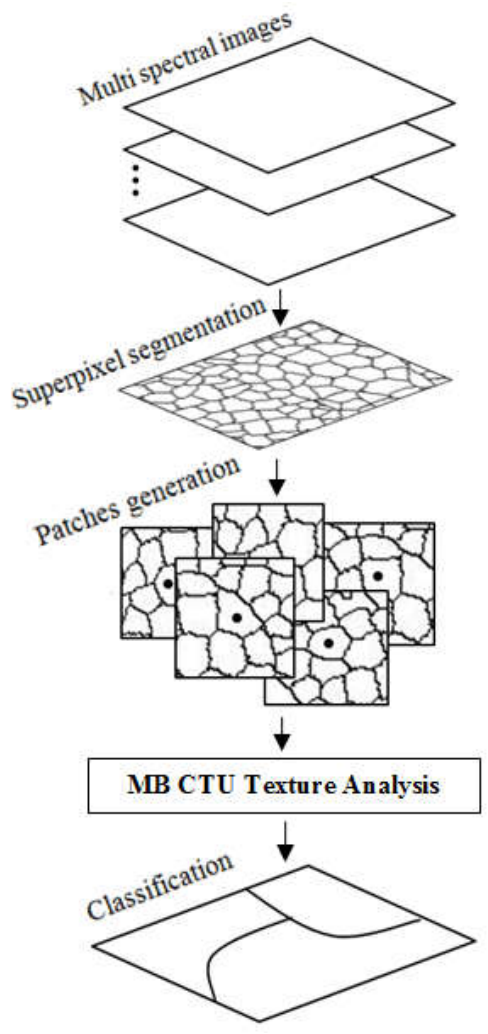

Figure1. Flowchart of the proposed methodology

\subsection{Image Segmentation}

Texture extraction is done generally over fixed size moving window. In this paper texture analysis is performed based on patches derived from superpixels (Achanta, Shaji, Smith, Lucchi, Fua and Süsstrunk, 2012). In order to take into account the limits between the different classes in the original image, we compute superpixels with the SLIC algorithm (Achanta, Shaji, Smith, Lucchi, Fua and Süsstrunk, 2012). Superpixels typically cover the whole image; they are distributed regularly with respect to the nature of the input image. The desirable variation of superpixels size is preferably small and the boundary of superpixels has to match the natural boundary of the different objects present in the image. The parameters of the superpixel extraction are tuned in order to get around 100 200 pixels by superpixel. The patches are 64 by 64 pixels subimages. One patch is generated for each superpixel. Figure1 presents the process of patch creation.

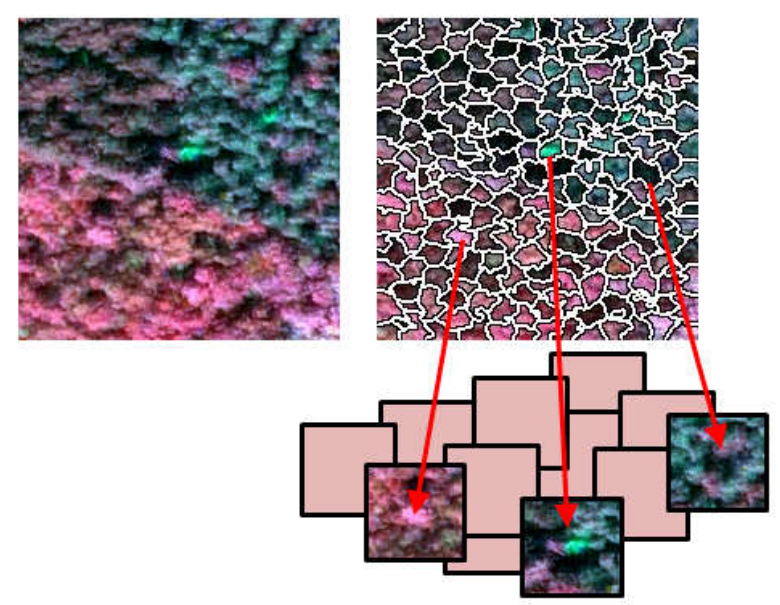

Figure2. Generation of patches from image

\subsection{Compact Texture Unit Analysis}

The compact texture unit was proposed to reduce the computational complexity of the "texture spectrum" texture analysis approach (Wang and He, 1990a), while maintaining equivalent performances. The image is scanned using a moving window.

\subsubsection{Monoband C-TU descriptor}

At each position, $\mathrm{N}$ (spatial resolution of the C-TU model) differences are calculated around the central pixel. The set $\Omega$ of all these differences over the entire image characterizes the local spectral variations contained in the image. The histogram giving the distribution of the elements in the set $\Omega$ is then divided into $\mathrm{M}$ regular subdivisions (spectral resolution of the C-TU model); each of them is assigned a unique code. Thus each local neighborhood is transformed into its equivalent spatial pattern called the Compact Texture Unit (C-TU).

A Compact Texture Unit Number (NC-TU) is assigned to each C-TU using the following sum formula:

$$
N_{C-T U}=\sum_{i=1}^{N} \zeta_{M, N}^{\Omega}\left(v_{i}-v_{0}\right)
$$

Where, $\mathrm{M}$ is the spectral resolution of the C-TU model, $\mathrm{N}$ : the spatial resolution of the C-TU model, $\mathrm{v}_{\mathrm{i}}$ : value of a given neighbourhood pixel, $\mathrm{v}_{0}$ : value of the central pixel, $\Omega$ is the set of the local spatial variation in the image and $\zeta_{\mathrm{MN}}^{\Omega}$ : function that associates $\mathrm{N}$ and $\mathrm{M}$ to a unique code.

The set of codes depends on the parameters $\mathrm{N}$ and $\mathrm{M}$. The authors in Safia and He (2013a) provided these codes for different spatial resolutions $\mathrm{N}$, and different spectral resolutions $\mathrm{M}$. The statistics of the occurrence frequency function of all the NCTU in the image or in a moving window provide a powerful tool for texture characterization. This is similar to the way with which an histogram allows spectral characterization. Figure 3 
shows the results of this transformation starting from a local neighborhood to the generation of the corresponding $\mathrm{N}_{\mathrm{CTU}}$.

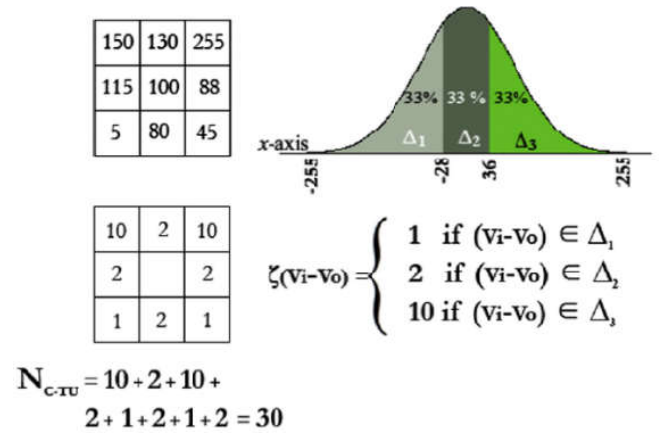

Figure 3. Transformation of a $3 \times 3$ neighborhood into a C-TU number using spectral resolution: $\mathrm{M}=3$ (Safia and $\mathrm{He}, 2013 \mathrm{a}$ ).

\subsubsection{Multiband C-TU descriptor}

The multiband descriptor extends the idea of monoband C-TU model to multispectral images. For that instead of using relative values of pixels in the same spectral band, it uses pixel values from all available spectral bands. To illustrate that, let's consider a 3 by 3 pixels local window in a three band image Figure 4(a). Spectral values in this window can be reorganized in order to form a new multiband neighborhood as follows. The central pixel of this multiband neighborhood can be taken as the value of the central pixel of the local window in the first spectral band. Twenty four neighbors can then be attributed to this new multiband neighborhood with their values taken as the values of the eight neighbors of the local window in the three spectral bands. This 24-pixel multiband neighborhood is shown in Figure 4(b).

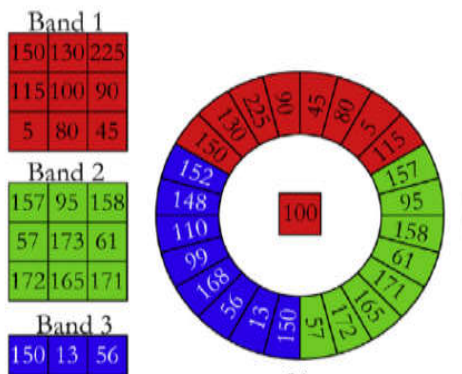

(b)

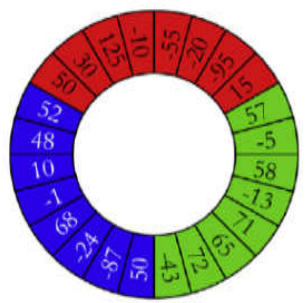

(c)
15811099
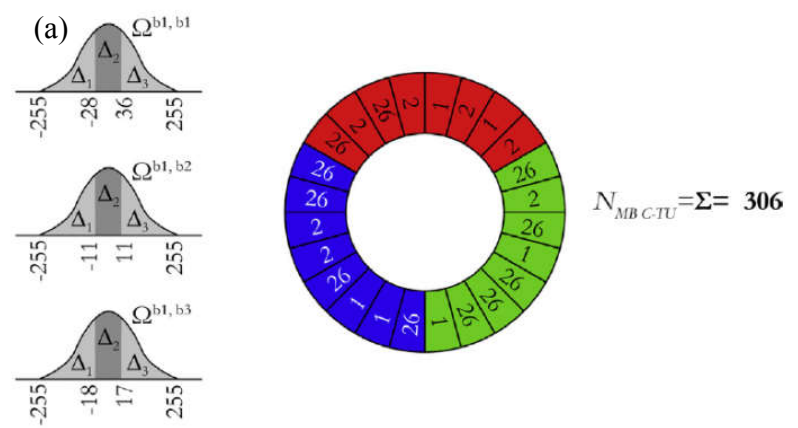

Figure 4. Extraction of the multiband C-TU descriptor for a three-band image using a $3 \times 3$ pixel multispectral neighborhood (Safia and $\mathrm{He}, 2015$ ).
The relative measures of pixel values in this 24-pixel multiband neighborhood, conducted exactly as in the monoband C-TU descriptor allows the characterization of different local spatial relation types. Indeed, when the central pixel from spectral band 1 is compared with the eight pixels from the same band, it is the classic intra-band local spatial relations which are characterized. This defines intra-band texture of spectral band 1 . When the central pixel is compared to the eight pixels from spectral band 2, it is the local inter-band spatial relations which are characterized. This defines inter-band texture of the spectral band 1 as viewed from the perspective of spectral band 2 . Similarly, comparison with pixels neighbors from band 3 leads to inter-band texture of spectral band 1 as viewed from the perspective of spectral band 3. In this way, a multiband neighborhood with pixels values from $p$ spectral bands contains $p$ types of local spectral variations: $p-1$ inter-band local variations plus one intra-band local variations. Result of the intra- and interband relative measures for the three-band case is presented in Figure 4(c).

\section{STUDY AREA AND USED DATA}

The validation of the proposed approach was conducted using pan-sharpened WorldView-2 multispectral image, acquired overthe Eastern Townships of Quebec, Canada, in August 2010. For that a subset of 1000 by 1000 pixels for which a detailed ground truth was available has been selected as test image. The subset represents typical forest landscapes with different pure and mixed forest stands (Figure 5).

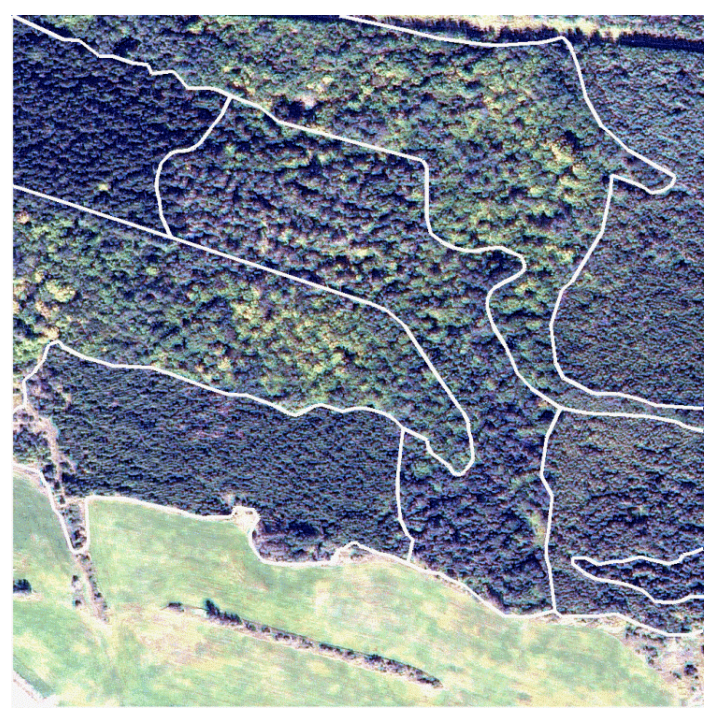

Figure 5. True color composite of the used image.

\section{EXPERIMENTS AND RESULTS}

After SLIC based segmentation, each superpixel was classified using a supervised nearest-neighbor rule (1-NN) with Euclidean distance algorithm (Altman, 1992). Training data were collected for each class in the test image. Continuous ground locations in test site showed important intra class texture variations. During the classification process, the p-bands compact textureunit numbers images were scanned with the generated superpixels. For each superpixel in the segmentation, the histogram of $\mathrm{N}_{\mathrm{MB}}$ C-TU was calculated for a patch of 64 by 64 pixels around its centroid and compared those of the training dataset. The pixels 
of the superpixels were labeled as the class that minimizes the Euclidean distance.

Classification accuracy assessment was conducted to assess the benefit compare of characterizing texture as a set of intra- and inter-band spatial interactions compared to the classic strategies that use only intra-band spatial interactions. To provide an idea about the performance of the multiband C-TU a comparison of existing methods that use inter-band and intra-band spatial relationships is presented. The overall correct classification and Kappa index provided a measure of agreement between the classifications produced and the ground data maps. These ground maps have the same size as the test images to allow a pixel by pixel accuracy assessment based on all pixels in the test images.
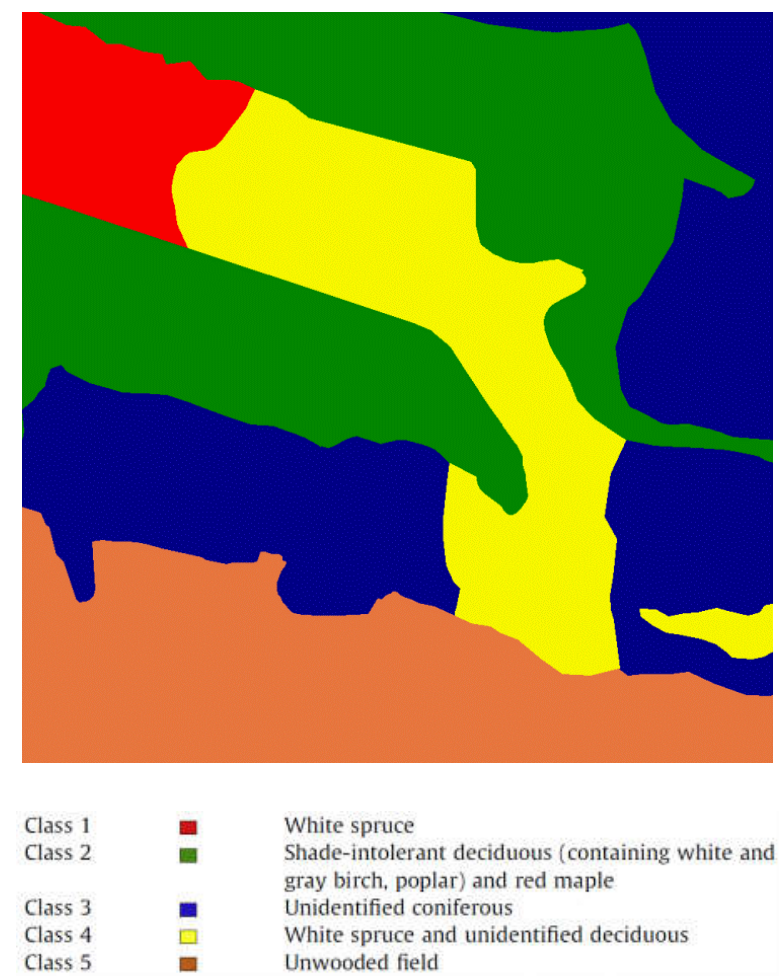

Figure 6 . Ground truth of the study area

The results of the object-based classification using multiband C-TU descriptor have been compared to six other texture features. Three of them use only intra-band characterization techniques: Gabor, wavelets and Granulometry features. The remaining three methods use intra-band and inter-band spatial relationships: integrative co-occurrence matrices, opponent Gabor features and opponent color local binary patterns (Bianconi, Harvey, Southam, Fernández, 2011). The comparison study was conducted using three bands image (nir, red and green spectral bands). Some of the resulting classified images are given in Figure. 7. The accuracy in terms of classification rate and Kappa index are summarized in Table 1.

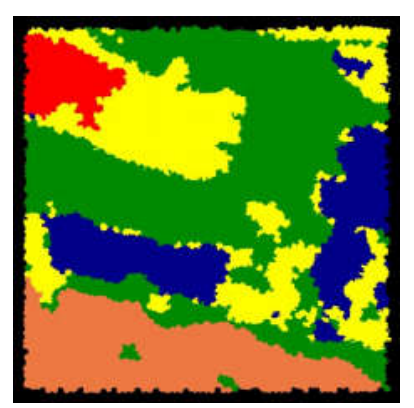

a. GLCM

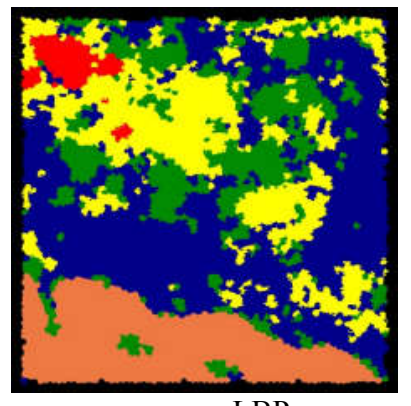

c. LBP

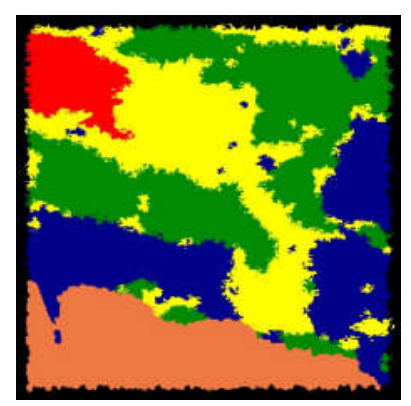

b. $\mathrm{MB} \mathrm{C}-\mathrm{TU}$

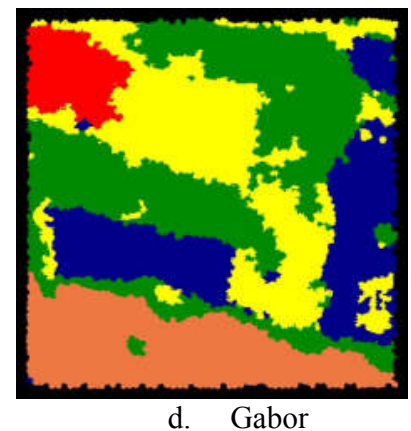

Figure 7 .Classifications of the used image

\begin{tabular}{|c|c|c|c|c|}
\cline { 2 - 5 } \multicolumn{1}{c|}{} & \multicolumn{4}{c|}{ Intra-bands } \\
\cline { 2 - 5 } \multicolumn{1}{c|}{} & Gabor & Wavelet & \multicolumn{2}{c|}{ Granulometry } \\
\hline OA (\%) & 72.83 & 66.94 & \multicolumn{2}{c|}{77.31} \\
\hline Kappa & 0.647 & 0.565 & \multicolumn{2}{c|}{0.704} \\
\hline & \multicolumn{4}{c|}{ Intra \& inter-bands } \\
\hline OA (\%) & 74.27 & $\mathbf{8 1 . 0 0}$ & 60.92 & 77.31 \\
\hline Kappa & 0.662 & $\mathbf{0 . 7 5 3}$ & 0.488 & 0.705 \\
\hline
\end{tabular}

Table 1.Classification rates of the conducted classifications

Table 1 shows that except for OLBP method, using both intraband and inter-band spatial relationships for texture analysis improves the classification rate compared to the classic intraband texture. For example the MB C-TU recorded an improvement of $14.06 \%$ compared the classic intra-band texture extracted using wavelet. The comparison between the three texture methods that use intra-band and inter-band spatial relationships shows that the multiband C-TU recorded the highest performances in terms of overall accuracy and kappa index. The improvement was $20.1 \%, 6.73 \%$ and $3.69 \%$ compared to the opponent LBP, IGLCM and opponent Gabor methods respectively. 


\section{CONCLUSION}

Texture of multispectral images is usually processed by analyzing each spectral band independently. In this work, an object-based classification has been conducted, where the basic processing units were patches of $64 \times 64$ pixels created around the segments from superpixel segmentation. The texture descriptor named multiband $\mathrm{C}-\mathrm{TU}$ was used to describe texture of multispectral images as multiband spatial information, which is the result of intra-band and inter-band spatial interactions. This descriptor is based on the idea of characterizing texture using relative pixel values in a multispectral neighborhood instead of a monoband neighborhood. The multibandC-TU descriptor can be extracted with low computationally complexity. The experimental results were promising and showed that extracting texture of multispectral images as a multiband texture is more efficient than the classic approaches based on only intra-band spatial interactions.

\section{REFERENCES}

Achanta, R., Shaji, A., Smith, K., Lucchi, A., Fua, P., \&Süsstrunk, S. (2012). "SLIC superpixels compared to state-ofthe-art superpixel methods". IEEE transactions on pattern analysis and machine intelligence, 34(11), 2274-2282.

Altman, N. S. (1992). "An introduction to kernel and nearestneighbor nonparametric regression". The American Statistician 46 (3): 175-185.

Bianconi, F., Harvey, R., Southam, P., \&Fernández, A. (2011). "Theoretical and experimental comparison of different approaches for color texture classification" .Journal of Electronic Imaging, 20(4), 043006-043006.

Blaschke, T. (2010). Object based image analysis for remote sensing. ISPRS journal of photogrammetry and remote sensing, 65(1), 2-16.

Safia, A., \& He, D. C. (2013). "Improving the Texture Spectrum Model Using a Compact Texture Unit Descriptor". Journal of Communication and Computer, 2, 234-251.

Safia, A., \& He, D. C. (2015). "Multiband compact texture unit descriptor for intra-band and inter-band texture analysis". ISPRS Journal of Photogrammetry and Remote Sensing, 105, 169-185.

Wang, L., \& He, D. C. (1990). "Texture classification using texture spectrum. Pattern Recognition”, 23(8), 905-910. 


\title{
SMALL SCALE LANDFORM MAPPING BY INTEGRATED OPTICAL (2D) AND TERRAIN (3D) UAV DATA
}

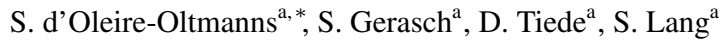 \\ ${ }^{\text {a }}$ Department of Geoinformatics - Z_GIS, University of Salzburg \\ Schillerstr. 5020 Salzburg, Austria - sebastian.doleire-oltmanns@sbg.ac.at
}

KEY WORDS: landform mapping, UAV, monitoring, local scale, ditch

\begin{abstract}
:
The potential of UAV-based data for fine-scaled remote sensing applications has been investigated for several years and is still increasing. The presented study is attached to the field of landform mapping. Mapping and extracting of landforms contributes to different sectors such as agriculture, disaster management and nature conservation. In this study recent experiences and results from working in a nature conservation area are presented. The chosen study area 'Haarmoos' is located southwest of the lake Abtsdorf, Bavaria and is declared under the European wide Natura 2000 network. Optical imagery and terrain data were analyzed in an integrated approach based on object-based image analysis (OBIA). The classification delivered good results. Ditches may be classified well by the presented approach whereas a tradeoff was made between local rule set adaption and applicability to further data sets. The validation of the classification results was based on three steps.
\end{abstract}

\section{INTRODUCTION}

The potential of UAV-based data for fine-scaled remote sensing applications has been investigated for several years and is still increasing. This is due to optical sensors with even higher spatial resolution as well as new type of sensors that may be carried on UAVs. In the field of landform mapping the use of UAVs has recently started to become more attractive for local scale applications and is increasingly used for photogrammetric and remote sensing applications (Colomina and Molina, 2014). Mapping and extracting of landforms contributes to different sectors such as agriculture, disaster management and nature conservation. This study presents recent experiences and results in dealing with landform mapping on small scales in a conservation area based on optical (2D) and derived terrain (3D) data acquired from UAV missions. Object-based image analysis (OBIA) has already been applied in several studies on landform mapping and supports a strategy of integrated data analysis across different scales (Anders et al., 2011, D’Oleire-Oltmanns et al., 2013).

\section{METHODOLOGY}

\subsection{Study area}

The chosen study area Haarmoos is located southwest of the lake Abtsdorf, south of the city of Laufen, Bavaria (see figure 1). The area is a historically drained wetland site, a fen which is nowadays protected by different nature conservation acts: the site is declared under the European wide Natura 2000 network and adheres to federal regulations of bird protection as a unique habitat for certain wet meadow breading birds in Bavaria. As these wet meadows requires a distinct amount of water constantly available, the maintenance and even extension of drainage ditches is considered as potential risk to this sensitive environment. The application of UAV-based remote sensing in combination with e.g. VHR optical satellite data as well as in-situ data offers a valuable approach for the mapping and monitoring of such sensitive and partly inaccessible habitats.

\footnotetext{
${ }^{*}$ Corresponding author
}

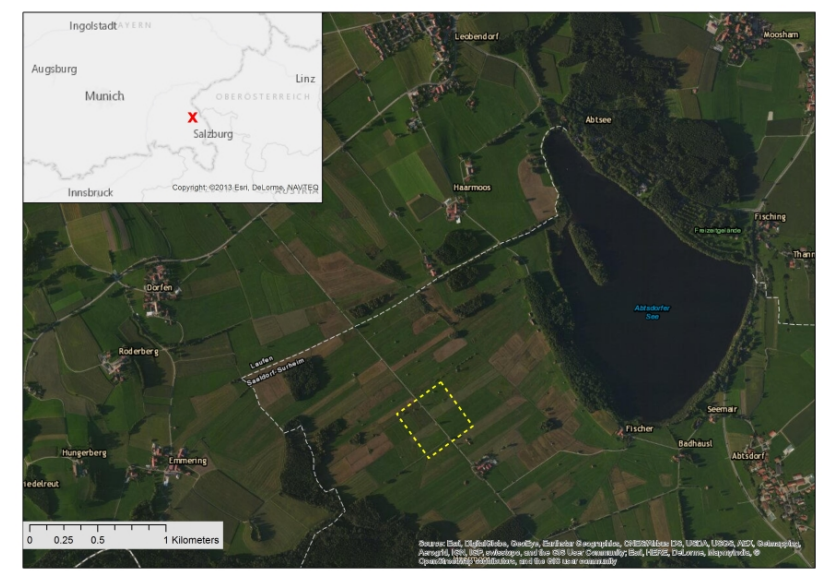

Figure 1. Study area located south-west of the lake Abtsdorf. The dashed yellow line illustrates the extent of the mapped area during the UAV campaign.

In order to provide a more realistic impression of the study area some oblique aerial photographs have been acquired. In figure 2 the road parallel to the main ditch may be identified from left to right in the middle of the photograph. Furthermore the structure of the area characterized by field plots and ditches in alternating order is visible.

\subsection{Data acquisition and photogrammetric processing}

The data acquisition has been conducted with a multicopter that is equipped with a consumer-grade RGB camera (model RICOH GXR). Flight campaigns followed pre-defined flightpaths that met all requirements for future photogrammetric processing of the aerial photographs: in-track and parallel overlap of both $70 \%$ and a flying height of $100 \mathrm{~m}$ above ground ensuring a Ground Sample Distance (GSD) below $5 \mathrm{~cm}$ per pixel.

Distributed Ground Control Points (GCPs) were measured with a dGPS to increase the accuracy during the triangulation of the aerial photographs and the calculation of data products such as 


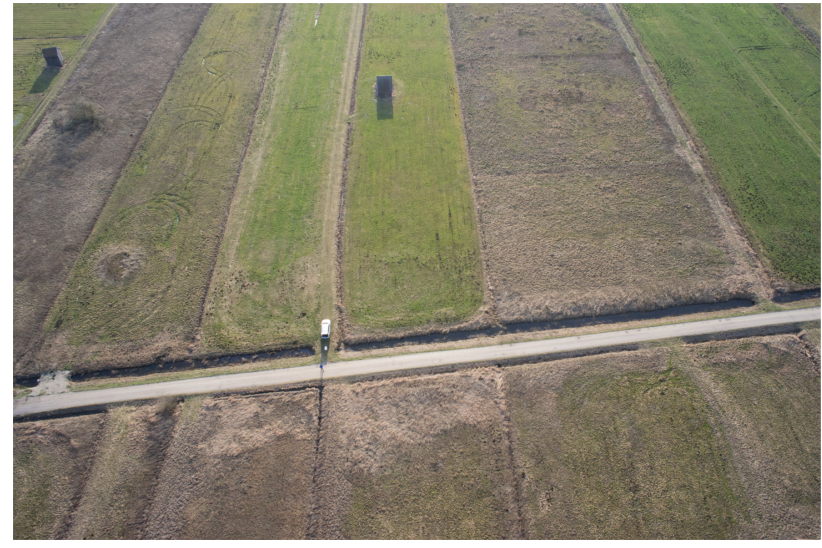

Figure 2. Oblique aerial photograph providing a more detailed view on the study area. The ditches between the field plots may be easily identified. In the center area the UAV pilot and the parked car may be found.

orthomosaics and Digital Surface Models (DSMs). In figure 3 the distribuion of the GCPs is illustrated.

\section{Ground Control Points}

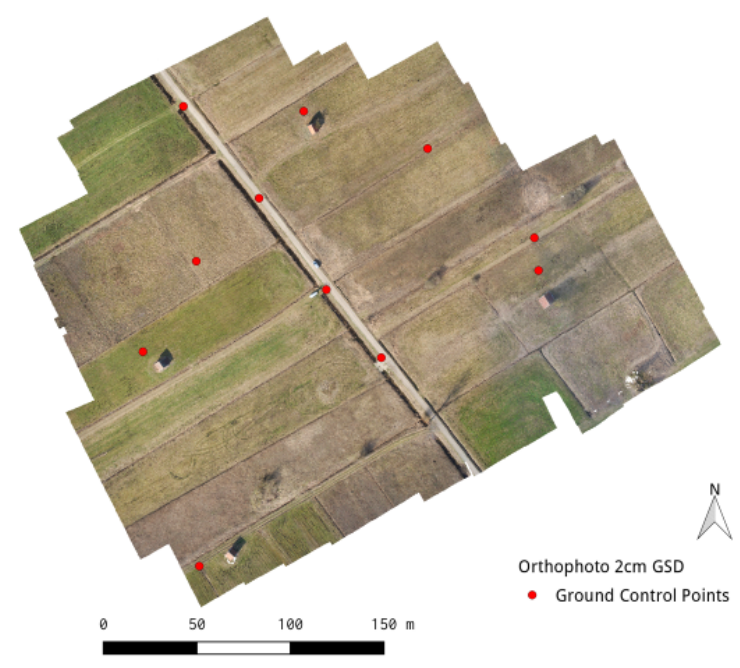

Figure 3. Distribution of GCPs (red points) over the mapped area.

Two flights were conducted to cover the chosen study site. The acquired aerial photographs were processed by using a software environment (Agisoft PhotoScan) that is based on the Structurefrom-Motion (SfM) algorithm. The acquired raw images were triangulated based on the software implicit feature matching routines. Orientation of the image block in WGS84 took place based on the measured reference coordinates. The measured GCP coordinates were assigned to the corresponding image pixel containing the GCP. This allows to reference the image block accurately with a much higher precision than relying only on the accuracy of the onboard GPS values.

After referencing and optimization of the triangulation several data products were calculated: A point-cloud representing the three dimensional surface of the mapped study site was generated. Based on this point cloud a mesh data set was derived that builds the basis for the calculation of the Digital Surface Model
(DSM). The DSM had a resolution of $2 \mathrm{~cm}$ per pixel which represents the mapped study site on a very high level of detail.

\subsection{Classification of ditches and validation}

Preprocessing. The resulting elevation model had an original resolution of $2 \mathrm{~cm}$ per pixel. Since the project's scope lies on the landform as such and not on distinct vegetation features or single trunks, the elevation model was smoothed using a moving window with a size of $15 \times 15$ pixel. This step preserved the general landform needed for the analysis and reduced the amount of influence of single vegetation features. In addition, a slope layer was derived from the elevation model according to (Mitasova and Hofierka, 1993). The size of one image layer was around 2 GB. To speed up the following rule-set development in CNL (Cognition Network Language within the eCognition software, Trimble Geospatial), we decided to choose a subset of the data with a size of approximately $0.25 \mathrm{ha}$, selecting a heterogeneous area in order to develop a rule-set which would also be applicable for the whole dataset (see figure 3).

OBIA. At an initial step, the height model and the slope were inspected visually. For a human expert, it was already possible to differentiate the ditches from other terrain forms (see figure 3 ). Based on these observations the goal was to develop a classification rule-set that mimics the human interpreter best possible. But also provides an easy to use and easy to understand framework for further adaption to other datasets. The following steps were conducted:

1. Initial segmentation based on the Multiresolution Segmentation algorithm by (Baatz and Schäpe, 2000). The aim was to derive homogeneous objects in respect to the two parameters slope and elevation (input layers for the segmentation process, the RGB images were not used). The ESP tool from Dragut et al. (Dragut et al., 2010) was used to automate the process of parameter selection and achieve a result with maximized local variance between the image segments.

2. Resulting objects were classified as ditch objects when the slope threshold (manually defined) exceeded 14 degrees. Falsely classified objects (non ditch) with high slope values were removed from the classification through excluding objects by relative local height values. The form descriptor length to width ratio was used to differentiate between ditch objects and non-elongated object with similar slope values. As a final step, objects with lower slope values surrounded by the objects of the class ditch (ditch bottoms) were also classified as ditch. Only 9 different rules were needed for the ditch extraction routine, which was possible due to the clear distinction of ditches in the data set (elongated structures showing local minimum height values in the centre and high slopes values at the border), keeping therefore the complexity of the rule-set low and allow an easier transferability to other areas.

Validation. Three steps were taken in order to validate the results from the classification approach. In the first step, we compared the classification result to the RGB orthophoto. With the knowledge of the field campaign, we were able to identify the position of the ditches. This first indication was used to further improve and change the algorithm until the presented state. As a second step, the classified ditches were assessed against a manually delineated reference data set. This dataset was created by an expert independently of and before the automatized delineation (see figure 4). 

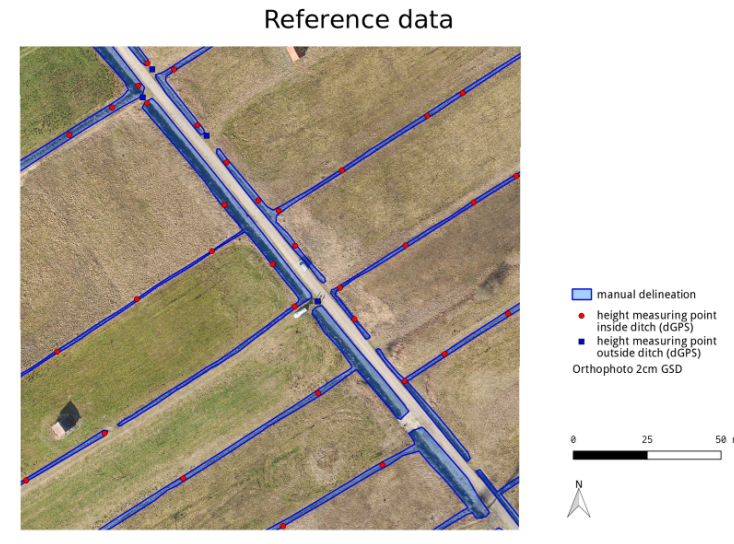

Figure 4. Manually delineated reference data (blue polygons) and the distribution of height measuring points are illustrated. Points are colored according to their location inside (red) or outside(blue) the ditch.

In a third step, the classification was compared with ground truth data that was acquired by a second survey (see figure 4). For this purpose, we captured the height of 40 different locations with a differential GPS. Most heights where located directly in the ditches (red circles) and some additional heights were captured between the ditches (blue rectangles) on the small bridges between the meadow sites. In addition to the evaluation of the $2 \mathrm{D}$ position, the heights of the points were compared to buffered elevation model areas with a radius of $20 \mathrm{~cm}$.

\section{RESULTS AND DISCUSSION}

The first look onto the result reveals a good classification result. The protruding lines forming the ditches are clearly visible in the classification, too. On a closer look, the classification was found to over classify the main ditch. This was also detected when the classification was compared to the expert segmentation of the ditches (see underlying classification result in figure 5).

\section{Rule set based classification result}

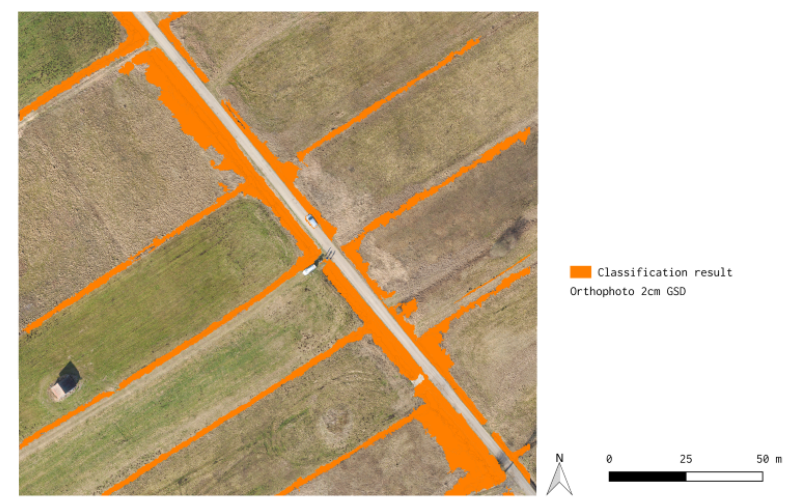

Figure 5. Classification Results (orange). Some overclassification along the main ditch is visible. The overall classification result is showing valid outcomes.

In figure 6 the classification results and the reference data is illustrated for validation purpose. The good classification outcome is illustrated more detailed in figure 7 showing a subset of the overall area that was analyzed.

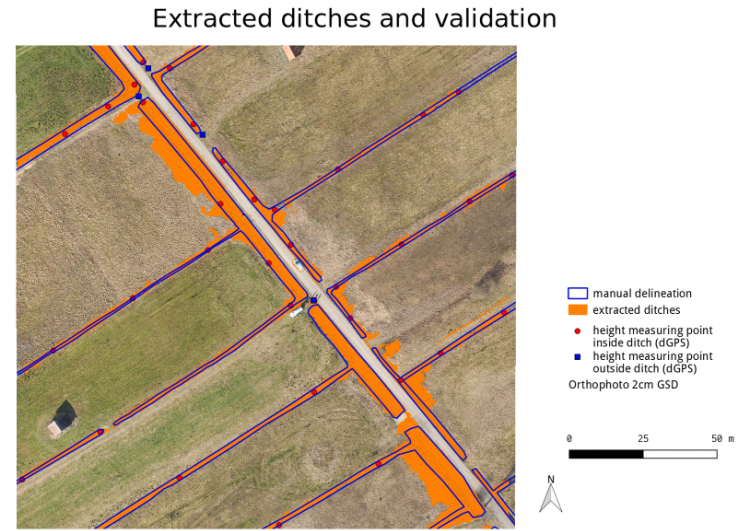

Figure 6. Illustration of the classification outcomes (orange) and the reference data (blue outlines) including the height measuring points.

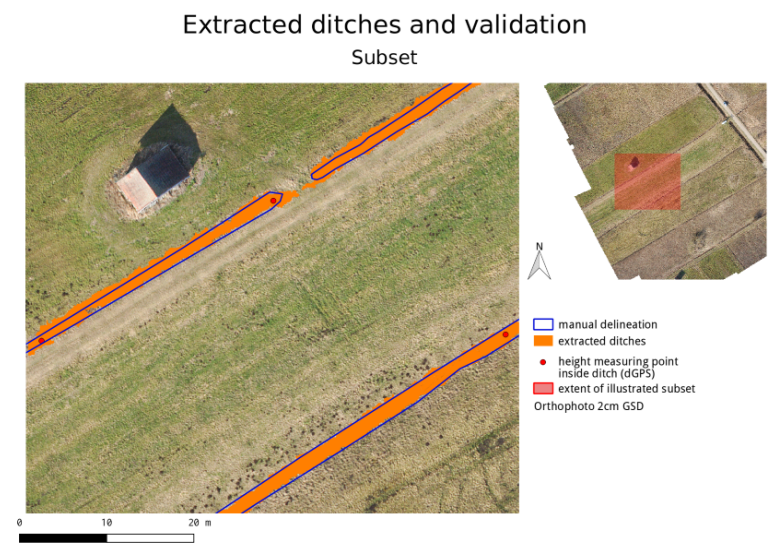

Figure 7. A subset of the classification outcomes (orange) and the reference data (blue outlines). The location of the subset is indicated by the red rectangular in the overview.

In comparison to the ground truth data, all but one point were correctly classified as ditches. The height difference between the points and the elevation model was found to be significant. The mean error is located at $0.5 \mathrm{~m}+/-0.41 \mathrm{~m}$ (see figure 8) This error can be explained by the fact, that the ground of the ditches was measured, but at the time of the UAS flight, some ditches were filled with water partially. Another important factor is the vegetation to be found within the ditches as well as vegetation which grows over the ditches. These impacts the calculation of the elevation model from the SfM data.

Because of the chosen methodology, the high slope value was used as classification criterion mainly. Therefore the rule set tends to classify rough surfaces such as high grass as ditch. This can be observed by an overclassification of the main drainage ditch along the road. Further rules were tested on the subset as well as on the large scale, but were mostly found to train the rule set too well for the given subset. A tradeoff was made between local rule set adaption and applicability to other data sets.

\section{CONCLUSION}

Mapping and extracting of landforms is a continuous topic contributing to different sectors such as agriculture, disaster manage- 


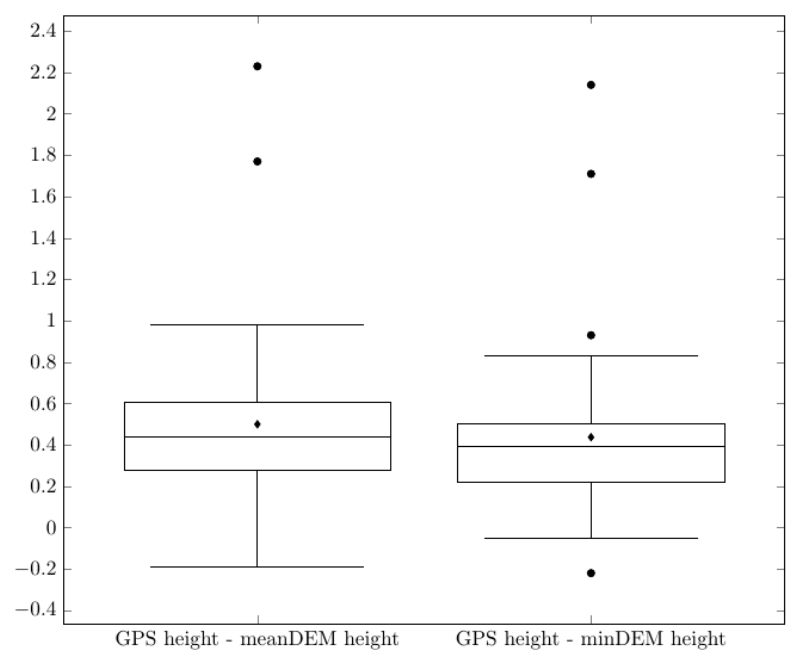

Figure 8. Boxplots illustrating the validation outcomes between GPS height and mean DEM height (left) and GPS height and min DEM height (right).

ment and nature conservation. This study presents recent experiences and results in coping with landform mapping on small scales in a conservation area based on optical (2D) and derived terrain (3D) data acquired from UAV missions. The resulting orthoimage mosaic as well as the elevation model has been analyzed by means of the OBIA concept. The elevation model had an original resolution of $2 \mathrm{~cm}$ per pixel that was smoothed over an area of $30 \mathrm{~cm}$, which preserved the general landform required for the analysis and reduced the amount of influence of single vegetation features. Automated extraction of ditches was conducted based on a clear and simple rule-set, which was possible due to the clear distinction of the ditches in the data sets. A threefold validation approach has been applied for evaluation of the classification results. The height difference between the points and the elevation model was found to be significant. The mean error is located at $0.5 \mathrm{~m}+/-0.41 \mathrm{~m}$ and can be explained by the fact, that the ground of the ditches was measured, but at the time of the UAS flight, some ditches were filled with water partially.

\section{REFERENCES}

Anders, N. S., Seijmonsbergen, A. C. and Bouten, W., 2011. Segmentation optimization and stratified object-based analysis for semi-automated geomorphological mapping. Remote Sensing of Environment 115(12), pp. 2976-2985.

Baatz, M. and Schäpe, A., 2000. Multiresolution segmentation: an optimization approach for high quality multi-scale image segmentation.

Colomina, I. and Molina, P., 2014. Unmanned aerial systems for photogrammetry and remote sensing: A review. ISPRS Journal of Photogrammetry and Remote Sensing 92, pp. 79-97.

D’Oleire-Oltmanns, S., Eisank, C., Dragut, L. and Blaschke, T., 2013. An Object-Based Workflow to Extract Landforms at Multiple Scales From Two Distinct Data Types. IEEE Geoscience and Remote Sensing Letters 10(4), pp. 947-951.

Dragut, L., Tiede, D. and Levick, S. R., 2010. Esp: a tool to estimate scale parameter for multiresolution image segmentation of remotely sensed data. International Journal of Geographical Information Science 24(6), pp. 859-871.
Mitasova, H. and Hofierka, J., 1993. Interpolation by regularized spline with tension. ii: Application to terrain modeling and surface geometry analysis. Mathematical Geology 25(6), pp. 657669. 


\title{
SEMANTIC CLASSIFICATION OF URBAN BUILDINGS COMBINING VHR IMAGES AND GIS DATA
}

\author{
S. Du* , F. Zhanga , X. Zhang ${ }^{\mathrm{a}}$

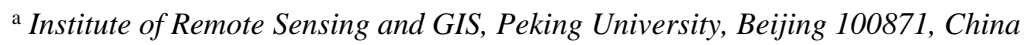

KEY WORDS: Very high resolution (VHR) images, urban buildings, semantic classification, random forest, object-based image analysis (OBIA)

\begin{abstract}
:
While most existing studies have focused on extracting geometric information on buildings, only a few have concentrated on semantic information. The lack of semantic information cannot satisfy many demands on resolving environmental and social issues. This study presents an approach to semantically classify buildings into much finer categories than those of existing studies by learning random forest (RF) classifier from a large number of imbalanced samples with high-dimensional features. First, a two-level segmentation mechanism combining GIS and VHR image produces single image objects at a large scale and intra-object components at a small scale. Second, a semi-supervised method chooses a large number of unbiased samples by considering the spatial proximity and intra-cluster similarity of buildings. Third, two important improvements in RF classifier are made: a voting-distribution ranked rule for reducing the influences of imbalanced samples on classification accuracy and a feature importance measurement for evaluating each feature's contribution to the recognition of each category. Fourth, the semantic classification of urban buildings is practically conducted on two different kinds of cities, and the results demonstrate that the proposed approach is effective and accurate. The seven categories used in the study are finer than those in existing work and more helpful to studying many environmental and social problems.
\end{abstract}

\section{INTRODUCTION}

As main sites of urban activities and important components of cities, urban buildings are vital foundations of urban studies. Semantic classification of buildings intends to label buildings using a set of semantic categories cognized and conceptualized by people, such as low-story shantytowns, middle-story apartments, high-story apartments, administrative buildings, commercial buildings, etc. These categories strongly correlate with urban environment analyses (e.g. ecological and environmental evaluation), urban resource allocation (e.g. resource management, transportation planning, and disaster reduction) and urban social analyses (e.g. population estimation, and market research) (Wu et al., 2005). Existing work has focused on how to extract building contours or accurately distinguish buildings from non-buildings. However, geometric information alone cannot fulfill the demands on urban ecology, resources and social researches (Paul et al., 2001). Therefore, semantic classification of urban buildings is required.

Most existing studies have focused on extracting geometric information on buildings while only a few have concentrated on semantic analysis. In addition, some important issues still remain to be resolved. First, existing work on semantic analyses has distinguished too few categories to satisfy the many demands in environmental or social sciences (Graesser et al., 2012; Kohli et al., 2012; Belgiu et al., 2014; Lu et al., 2014). Second, there have been no appropriate segmentation scales and algorithms to produce single image objects for diverse buildings. This lack of computational methods leads to low classification accuracies as image features strongly depend on segmentation scales. Third, a small number of manually chosen samples and features may be practical for classifying a few categories of buildings. To distinguish more building categories greatly varying in sizes, shapes, structures, and spectrums, however, a large number of samples, high-dimension and heterogeneous features are required. In this situation, the samples are often imbalanced, and the features are often auto-correlated and have distinct importance for distinguishing different categories (Du et al., 2015). Unfortunately, there is still a lack of related work to reduce the influences of imbalanced samples on classification and to evaluate feature importance to classifying each category.

Aimed to resolve the issues raised above, this study presents a two-level segmentation mechanism (i.e. a large-scale layer constrained by GIS data for producing single image objects and a small-scale layer providing intra-object component features) and a semi-supervised method to choose a large number of unbiased samples by considering the spatial proximity and intracluster similarity of buildings. Random forest (RF) classifier is used to semantically classify buildings, for it is capable of handling a large number of samples and high-dimension and heterogeneous features. Moreover, to improve classification accuracy and evaluate feature importance, two improvements in RF classifier are presented: a voting-distribution-ranked rule for reducing the influences of imbalanced samples and a feature importance measurement for each category based on Gini descent and path tracing strategy.

\section{METHODOLOGY}

\subsection{Category system of urban buildings}

Urban buildings made of various materials with assorted styles and appearances in the real physical world are the basis of cognizing semantic category by people and of sensing buildings by remote sensors. In the geoinformatic world, buildings are abstracted into contours in GIS data and into image pixels or

\footnotetext{
* Corresponding author
} 
image objects in VHR images. Accordingly, they are described from the aspects of spectrum, shapes, and textures. In the cognition world, people cognize, understand, and communicate their ideas about buildings through appropriate semantic categories. Therefore, building a semantic category system helps to transform the feature representations in the geoinformatic world to the concepts in the cognition world.

The goal of semantic classification is to build relationships between the concepts of buildings in the cognition world and the features of buildings in the geoinformatic world. Therefore, the semantic category system can be built by discriminating the appearances and functions of urban buildings, including lowstory (LS) shantytowns, medium-story (MS) apartments, highstory (HR) apartments, administrative (AD) buildings, commercial (CM) buildings, industrial (ID) buildings, and auxiliary (AU) buildings.

\subsection{The presented procedure}

To solve the issues raised, such as the complete segmentation of buildings, the choice of training samples, and the classification approach of semantic categories, this study presents an approach to semantic classification of urban buildings.

Four steps are required.

(1) VHR image segmentation constrained by GIS data. Since existing segmentation methods cannot produce single image object for each building, this study adopts a segmentation constrained by GIS data to obtain a single object for each building. The pixels inside each GIS contour are first merged into a single object, and then image features are computed.

(2) Features extraction of buildings. Image features are the bridge connecting building objects to semantic categories. In this study, four types of features are used, including spectrum, texture, geometry, and spatial distribution. The first three choose samples and classify buildings while the last evaluates clustering results and chooses samples.

(3) Samples selection with semi-supervised method. A large number of samples is required to semantic classification. The ISODATA algorithm is first used to cluster buildings according to spectrum, geometry, and texture features. Then, intra-cluster similarity is used to choose corresponding samples of semantic categories in a semisupervised way.

(4) Semantic classification of buildings using improved RF classifier. The three steps above can collect a larger number of samples as well as high-dimension and heterogeneous features for each sample. To train a classifier from these complex features and huge samples, a semantic classification approach using RF classifier is presented. Furthermore, RF is improved to evaluate the contribution of each feature to each category, which reduces the influences of imbalanced samples.

\subsection{Improved RF classifier for semantic classification}

To reasonably exploit samples and their features, the RF classifier is employed to randomly select samples and features to train decision trees and to integrate all trained decision trees to vote for the most popular category (Breiman, 2000). In this study, $\mathrm{RF}$ is improved to evaluate the contribution of each feature to classifying each category and to reduce the influences of imbalanced samples on classification results.

Based on bagging integration learning algorithm, RF classifier trains each decision tree independently (Pal, 2005) and the votes of all decision trees determine the final results. The training steps are as follows: (1) to choose a subset of samples using Bootstrap sampling methods, (2) to choose randomly $\sqrt{M}$ features from $M$ ones for each node, (3) to construct a CART decision tree with the chosen samples by using GINI coefficient as information gain, and (4) to build N CART decision trees until a RF is built.

The classification process of decision trees is exactly same as that of the training process. For each building, each tree independently predicts a category; accordingly, the resulting category is the most popular category.

Traditional RF uses the simple majority voting rule to make decisions and tends to misclassify the minority categories. Therefore, imbalanced samples heavily affect the classification accuracy. Existing work on learning imbalanced data includes pseudo balanced random forest (BRF) and weighted random forest (WRF) (Chen et al., 2004). BRF copies a small number of samples for the minority categories and randomly chooses the same number of samples for the majority categories. WRF weighs the samples of different categories to reduce the differences in samples. However, BRF needs to identify adaptively the number of copied samples, causing it to change the original distribution of samples. Meanwhile, WRF necessitates the specification of weight for each category. However, how to specify weights is still unresolved. Therefore, a new voting rule - the voting-distribution ranked rule - is proposed to replace the simple majority voting rule.

Supposing there are $N$ decision trees and $K$ categories, and each tree has one vote for a sample $(o)$, then the votes of the $N$ trees can be represented as a distribution $\operatorname{Vote}(o)=$ $\left(n_{1}, n_{2}, \ldots, n_{K}\right)$ with $\sum_{i=1}^{K} n_{i}=N$. The simple majority vote rule (Kontschieder et al., 2014) assigns a sample to the most popular category.

The vote distributions of out-of-bag (OOB) samples are significant in discovering confused categories. For example, let $n_{1}$ and $n_{2}$ be the first and second maximum votes; if they are close, unclassified samples tend to be misclassified. Due to the influence of imbalanced samples, it is easier for majority categories than minority categories to obtain more votes. Once the votes of the majority categories are larger than those that they deserved, misclassification will occur. Fortunately, imbalanced samples do not hide the vote distribution over categories, even though they affect the number of votes for each category. Let $p_{i}=n_{i} / N(i=1,2, \ldots, K)$ be the probability of the $i-t h$ category, then the vote distribution of unclassified sample $o$ is defined as $\operatorname{prob}(o)=\left(p_{1}, p_{2}, \ldots, p_{K}\right)$. For each OOB sample, a vote distribution can be obtained; as a result, multiple vote distributions can be obtained for each category since there are many OOB samples for each category. The reliable distributions (ranked in top 5\% in the probabilistic distributions) of each category are averaged into a representative one. Accordingly, for an unclassified sample, its vote distribution is compared with the representative distributions of the $K$ categories. The sample is then assigned to the category with the largest similarity.

\section{EXPREMENTAL RESUTLS}

To verify the feasibility of the presented approach, a series of experiments are conducted, including sample selection, semantic classification of traditional RF, semantic classification of improved RF for handling imbalanced samples, and evaluation of feature importance.

\subsection{Study area and used data}

Since urban buildings vary notably in sizes, shapes, structures and spectrums, the experiments will focus on them to test the presented method. The study area is located at Haidian and 
Xicheng districts in Beijing city (Fig. 1), which belongs to the city expansion area and is full of high-tech companies, culture and education industry, commercial and service industry, and research institutions. In addition, due to rapid urbanization in recent years, the area has a large number of informal settlements and developing zones. Therefore, it is very significant to analyze and address the semantic categories of buildings in this area.

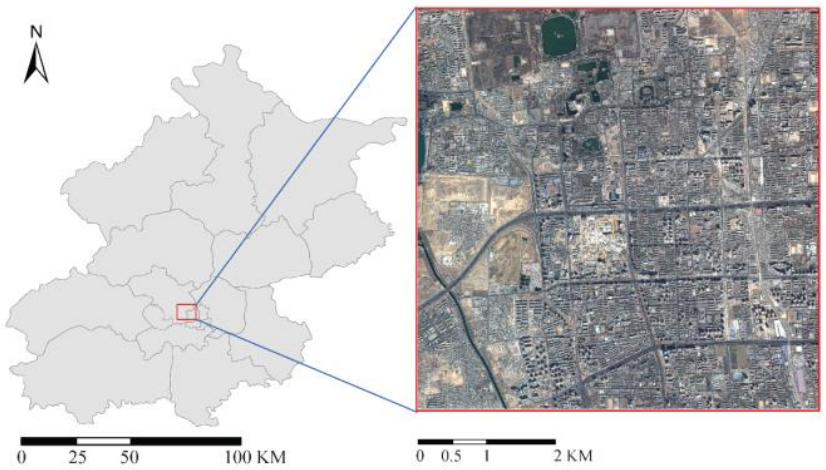

Fig. 1. Study area and Quickbird data.

For semantic classification of buildings, both GIS and Quickbird data are used:

(1) Quickbird image Data. Panchromatic band with resolution of $0.61 \mathrm{~m}$ and four multispectral bands with resolution of $2.44 \mathrm{~m}$ are fused to produce a four band data with resolution of $0.61 \mathrm{~m}$. The spectral, geometric, and texture features are extracted from Quickbird image.

(2) GIS data of buildings. To obtain a single-image object for each building, the contours in GIS data were used. The study area covers $38.7 \mathrm{~km}^{2}$ and contains 8831 buildings. The largest contour, about $96462 \mathrm{~m}^{2}$, represents a LS shantytown or ID building, while the smallest contour is only $70 \mathrm{~m}^{2}$ and refers to an AU building.

Before semantic classification and sample selection, twolevel segmentation was conducted and 307 image features were computed for each image object. In total, 8831 image objects and 15258 sub-objects were obtained. The largest object has 118 subobjects while the smallest object has only one sub-object.

\subsection{Classification results with the simple majority vote rule}

A RF classifier with 200 decision trees is trained using the chosen 2747 samples, and the 6084 unclassified buildings are classified using the trained RF. For each OOB sample, each decision tree will have one vote for classification. The final category is the one with the most votes, and the confusion matrix of accuracy assessment is created based on the predicted and existing categories of OOB samples. The overall accuracy is $71.50 \%$, and overall kappa coefficient 0.59 . The overall accuracy is greatly reduced by the misclassification of 303 high-story apartments and 35 commercial buildings.

\subsection{Classification results with the voting-distribution ranked rule}

The imbalanced samples result from the real distribution of buildings in each category, and they will lead to a certain bias when identifying the categories using the simple majority voting rule. The minority categories will probably be misclassified. Due to the small number of samples, MS apartments - as are CM and AD buildings - are often confused with HR apartments. This confusion makes it difficult to correctly classify these buildings by the simple majority voting rule. The voting-distribution ranked rule (Du et al., 2015) is used to reclassify unclassified buildings (Fig. 2). The overall accuracy increases to $79.54 \%$, and the kappa coefficient remarkably to 0.72 , demonstrating that our new vote rule can improve the classification accuracy: the misclassified MS and HR apartments are greatly reduced.

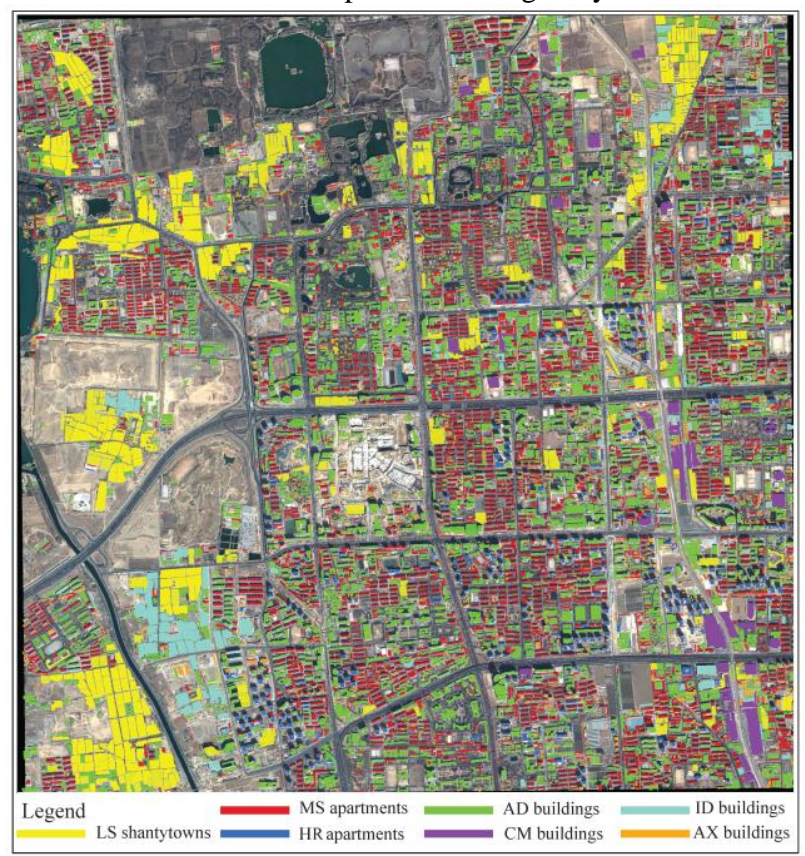

Fig. 2. The classification results with the improved voting rule.

\subsection{Evaluation of feature importance}

The image features used in this study are highly correlated, highdimensional and heterogeneous; therefore, it is necessary to evaluate the contributions of these features to classification. Based on the trained RF, the feature importance analysis method (Gini descent) was used to evaluate the importance of the 307 features.

In Table 1, texture and geometric features have great contributions to most categories, while spectral ones have poor performances due to their confusions. LS shantytowns are much larger than other buildings, and thus geometric features (e.g. area and perimeter) are significant. In addition, AU buildings are usually relatively small and easily distinguished by geometric features. Geometric features also play important roles in classifying MS apartments and ID and CM buildings because of the buildings' unique shapes and areas; however, HR apartments often have complex roofs and structures, showing unique characteristics of texture, thus they can be identified by texture features. Composed of diverse sub-objects, AD buildings vary greatly in materials and styles; thus, they can be classified by combining geometric and texture features. In other words, geometric and texture features are more important to classification than spectral ones. 
Table 1. The feature importance scores to seven categories.

\begin{tabular}{cccc}
\hline \multirow{2}{*}{ Semantic category } & \multicolumn{3}{c}{ Feature contribution rank (\%) } \\
\cline { 2 - 4 } & $\begin{array}{c}\text { spectru } \\
\mathrm{m}\end{array}$ & geometry & texture \\
\hline LS shantytowns & 15.36 & 31.80 & 52.84 \\
MS apartments & 12.30 & 45.14 & 42.56 \\
HR apartments & 25.94 & 15.37 & 58.69 \\
AD buildings & 10.71 & 40.27 & 49.02 \\
CM buildings & 3.39 & 55.00 & 41.62 \\
ID buildings & 15.27 & 38.28 & 46.45 \\
AU buildings & 15.80 & 63.07 & 21.13 \\
\hline
\end{tabular}

\section{CONCLUSION}

This study presents an improved classification approach for semantic classification of urban buildings. Four scientific tasks are resolved. Initially, GIS data were used to constrain the image segmentation for producing a single-image object for each building. Then, at the second level, each image object is further split into sub-objects to measure the internal heterogeneity of buildings. Next, ISODATA algorithm was used to group image objects into clusters by using extracted features, and a large number of unbiased samples were chosen by considering spatial proximity and intra-cluster similarity. The chosen samples reflect the real distributions of buildings in the physical world. Subsequently, the voting-distribution ranked rule can improve RF classifier by reducing classification error caused by imbalanced samples. The classification results of the improved and original RF were compared, and the accuracy increased from $71.50 \%$ to $79.54 \%$.

\section{REFERENCES}

Belgiu, M., Tomljenovic, I., Lampoltshammer, et al., 2014. Ontology-based classification of building types detected from airborne laser scanning data. Remote Sensing, 6, pp. 1347-1366.

Breiman, L., 2000. Randomizing outputs to increase prediction accuracy. Machine Learning, 40(3), pp. 229-242.

Chen, C., Liaw, A., Breiman, L., 2004. Using random forest to learn imbalanced data. Technical Report of Department of Statistics, UC, Berkeley.

Du, S., Zhang, F., Zhang, X., 2015. Semantic classification of urban buildings combining VHR image and vector data: a random forest approach. ISPRS Journal of Photogrammetry and Remote Sensing, 105, pp. 107-109.

Graesser, J., Cheriyadat A., Vatsavai R. R., Chandola, V., Long, J., and Bright E., 2012. Image based characterization of formal and informal neighborhoods in an urban landscape. IEEE Journal of selected topics in applied earth observations and remote sensing, 5(4), pp. 1164-1176.

Kohli, D., Sliuzas, R., Kerle, N., Stein, A., 2012. An ontology of slums for image-based classification. Computers, Environment and Urban Systems, 36, pp. 154-163.
Lu, Z., Im, J., Rhee, J., Hodgson, M., 2014. Building type classification using spatial and landscape attributes derived from LIDAR remote sensing data. Landscape and Urban Planning, 130, pp. 134-148.

Pal, M., 2005. Random forest classifier for remote sensing classification. International Journal of Remote Sensing, 26(1), pp. 217-222.

Paul, S., et al., 2001. Census from heaven: an estimate of the global human population using night-time satellite imagery. International Journal of Remote Sensing, 22(16), pp. 3061-3076.

Wu, S., Qiu, X., and Wang, L., 2005. Population estimation methods in GIS and remote sensing: a review. GIScience \& Remote Sensing, 42(1), pp. 80-96. 


\title{
ASSESSING UNCERTAINTIES ASSOCIATED WITH DIGITAL ELEVATION MODELS FOR OBJECT BASED LANDSLIDE DELINATION
}

\author{
B. Feizizadeh ${ }^{a *}$, T. Blaschke ${ }^{b}$ \\ ${ }^{a}$ Dept. of Remote Sensing and GIS, University of Tabriz, Blvd 29 Bahman, Tabriz, Iran - Feizizadeh@tabrizu.ac.ir \\ ${ }^{\mathrm{b}}$ Dept. of Geoinformatics, University of Salzburg, 5020- Salzburg Austria - Thomas.Blaschke@ @bg.ac.at
}

KEY WORDS: Uncertainty analysis, DEM, Object based landslide mapping, Iran

\begin{abstract}
:
Digital elevation models (DEMs) are representations of topography with inherent errors that constitute uncertainty. DEMs data are often used in object based analyses without quantifying the effects of these errors. The main objective of this research is to establish a semi-automated object-based image analysis (OBIA) methodology for modelling uncertainty associated with DEMs when applied for locating landslides. In order to assess the uncertainty of DEMs, the Monte Carlo Simulation methodology was employed for evaluation of the effects of uncertainty on elevation and derived topographic parameters. The effect of DEM error is investigated, using stochastic conditional simulation to generate multiple equally likely representations of an actual terrain surface. Accordingly, distributional measures including accuracy surfaces, spatial autocorrelation indices, and variograms were also employed to quantify the magnitude and spatial pattern of the uncertainty. The semi-automated object based rule-sets for landslide delineation were developed based on two approaches a): without uncertainty assessing of DEMs and b): under applying uncertainty assessing of DEMs to examine the probable and possible uncertainties in delineating the landslides and measuring the improved accuracy after minimizing these associated uncertainties. The results of two approaches were validated using a landslide inventory database and very accurate GPS dataset. Results indicated very significant improvement in accuracy of results (> $28 \%$ ) when employing DEMs under uncertainty assessment. This research demonstrates how application of this methodology can address DEMs uncertainty, contributing to more responsible use of elevation and derived topographic parameters, and ultimately results obtained from their use.
\end{abstract}

\section{INTRODCTION}

Digital Elevation Models (DEMs) are known as key and fundamental data for variety of application in domain of geoscience. "DEMs represent the topography that drives surface flow and are arguably one of the more important data sources for deriving variables" (Wechsler, 2007, 1481). Due to the decrease in data and computer costs, increase in computing power as well as developing of geoscience technologies, the DEMs data has become increasingly available to spatial data users (Wechsler, 2007). The term of DEMs are often assumed to be accurate representations of the real-world terrain surfaces. However, as the name of "Model" is clearly indicates uncertainty is associated with any spatial modelling. Based on this observation it can be stated that DEMs are only an approximation representation of the true terrain surface (Oksanen, 2006; Venzin, 2013).The inherent uncertainty with DEMs may have significant impact on its accuracy and even some times leads inaccurate results (Feizizadeh and Blaschke 2014). Technically speaking, systematic and random errors have significant impacts on the accuracy of DEMs. These errors can range from elevation data acquisition to geospatial applications (Oksanen, 2006; Venzin, 2013). As already mentioned, DEMs frequently can be produced either based on remote sensing satellite images or topography dataset. However, both of these methods are subject to uncertainties due to measurement errors, interpolations of point data and digitization errors (Temme et al., 2009; Venzin, 2013). Wise (2000) and Reuter et al. (2009) classified uncertainties in DEMs into three category including a) artifact, blunders or gross error which can have huge impact on the derived land surface parameters, b) systematic errors which implies by data collection method and c) random errors or noise which are inherent in any DEMs and are caused by the measurement itself (i.e. single noise).It is critical to assess the inherent uncertainty associated with DEMs. Such an uncertainty analysis leads to reduce the errors, and its relative propagation of these errors on other derived parameters or models extracted from DEMs (Abd Aziz et al., 2012). In this regard, the accuracy of DEMs the Root Mean Square Error of Elevation (RMSE) is a most widely used technique which indicates the difference between the estimated and true value of DEMs products (Wise, 2000). However, according to Wechsler (2007) the RMSE method sometimes does not calculate an accurate assessment of how precisely each grid in a DEM represents topographical features. To solve this issue, number of researchers proposed spatial simulation methods for assessing the uncertainty of elevation estimates in each DEMs grid (Holmes et al., 2000; Carlisle, 2005; Wechsler and Kroll, 2006; Abd Aziz et al., 2012). The spatial simulation process analyses the spatial correlation in data to produce equiprobable estimates (realizations) of each particular grid in the DEMs. These realizations provide a range within which the true estimate lies and can be used to quantify the uncertainty at each DEM grid (Wechsler, 2007). Within this research we aimed to assess the inherent uncertainty associated with DEMs when applied to object based landslide mapping. Our research methodology make use of spatial analysis and statistical approaches such as Monte Carlo Simulation.

\section{STUDY AREA DATASET}

The study area was Ahar Chay- Basin which is sub-area of Urmai lake basin in northern-west of Iran. Geology setting of Ahar Chay- Basin is responsible for natural hazards and in particular to landslides. The landslide inventory database of study area indicated 32 landslide events which clearly acknowledge the potentiality of slopes in Ahar Chay- Basin for to landslides and mass movements (Feizizadeh and Blaschke 2012; Feizizadeh et al., 2012). Weathering, groundwater impacts, unstable slopes

\footnotetext{
* Corresponding author
} 
geological formation such as marlaceous and sedimentation combined with human impact issues such as land use/cover changes make the Ahar Chay- Basin prone to landslide and mass movements.

Within this research the semi-automated object-based approach was employed for delineating landslides. For this goal, DEMs derived from topography map 1:25 $000(10 \mathrm{~m})$ was used to extract morphometric features. In order to measure the improved accuracy under applying uncertainty analysis, the DEMs was employed in two approaches. Within the first approach the row DEMs data was used as base of landslide delineation by applying OBIA techniques. While, in the second approach the uncertainty analysis was performed on DEMs data and the accuracy of DEMs was improved by minimizing the associated uncertainties. Accordingly, the revised DEMs was employed for object based landslide delineation. To delineate landslides in object based manner, the information gathered from different parameter features provided by OBIA and landslide inventory dataset as thematic GIS layers.

\section{METHDOLOGY}

In order to determine the uncertainty associated with DEMs, it is necessary to compute the error at first step. Within this research the error associated with DEMs was computed as following: -Error

$\left(x_{\circ}\right) e\left(x_{\circ}\right)=Z\left(x_{0}\right)-\hat{Z}\left(x_{\circ}\right)$

$\mathrm{e}\left(\mathrm{x}_{\circ}\right)$ is error in location

$\mathrm{Z}\left(\mathrm{x}_{\circ}\right)$ is the accurate number

$\hat{\mathrm{Z}}\left(\mathrm{x}_{0}\right)$ is the calculated amount

- Bias

Bias is fundamental model which can be calculated from average of error based on statistical approaches as following:

$\mathrm{ME}=\frac{1}{\mathrm{n}} \sum_{\mathrm{i}=1}^{\mathrm{n}}\left(\widehat{\mathrm{Z}}\left(\mathrm{x}_{\mathrm{i}}\right)-\mathrm{Z}\left(\mathrm{x}_{\mathrm{o}_{\mathrm{i}}}\right)\right)$

Precision

Precision or true value is also another fundamental model which can be calculated by measuring the error distribution from average as following:

$$
\mathrm{STD}=\sqrt{\frac{\sum_{\mathrm{i}=1}^{\mathrm{n}}\left(\overline{\mathrm{e}}_{\mathrm{i}}-\hat{\mathrm{e}}_{\mathrm{i}}\right)^{2}}{\mathrm{n}-1}}
$$

Accuracy in DEMs is also known as sum of precision and unBias. According to this, the difference between the estimated and true value is often available for final DEMs products and indicated by the RMSE (Wise, 2000):

$$
\text { RMSE }=\sqrt{\frac{\sum_{\mathrm{i}=1}^{\mathrm{n}}\left(\mathrm{z}\left(\mathrm{s}_{\mathrm{i}}\right)-\hat{\mathrm{z}}_{\mathrm{REF}}\left(\mathrm{s}_{\mathrm{i}}\right)\right)^{2}}{\mathrm{n}}}
$$

where $\mathrm{z}\left(\mathrm{s}_{\mathrm{i}}\right)$ refers to the $i^{\text {th }}$ interpolated elevation, $\hat{\mathrm{z}}_{\mathrm{REF}}\left(\mathrm{s}_{\mathrm{i}}\right)$ refers to the $i^{\text {th }}$ known or measured elevation and $\mathrm{n}$ is the number of samples.

However, it is believed that measuring the error associated with DEMs is not easy task. Sometimes small error can have a significant impact on accuracy of DEMs, whereas large errors may cancel out in other cases. Even though DEMs with a high spatial resolution are often interpreted as less uncertain, in order to model this uncertainty our research also make use the advantage of Monte Carlo Simulation which is known as effective approach for uncertainty analysis.

\subsection{MONTE CARLO SIMULATION ON DEM}

Monte Carlo simulation approach is one the most widely used approaches for uncertainty analysis. This methods has been employed by many researchers to evaluate error associated with GIS datasets (Wechsler and Charles, 2006). In order to make of Monte Carlo Simulation for DEMs uncertainty analysis, it is required to recognize DEMs as only one possible realization of the true elevation surface. Within this approach multiple simulations can be used to quantify DEMs uncertainty through evaluation of statistics associated with a distribution of realizations (Venzin, 2013). Based on the Monte Carlo approach, the error propagation problem can be formulated as follows:

$U=g\left(A_{1, \ldots, A_{n}}\right)$

Where $U$ is the output of a GIS operation $g$ on $n$ different inputs $A_{i}$. According to this equation, the objective of uncertainty analysis is to compute the uncertainty in the output $\mathrm{U}$, given the operation $g$ and the uncertain input $A_{i}$. The Monte Carlo method aims to compute $\mathrm{U}$ multiple times with inputs $a_{i}$ which are randomly sampled from their joint probability distribution of $A_{i}$ (Venzin, 2013). According to Temme et al. (2009) the Monte Carlo Simulation consists of the following steps:

I. Repeat $\mathrm{N}$ times:

a) Generate a random realization $a_{i}$

b) For each $a_{i}$, compute and store the output $\mathrm{u}=\left(a_{i}, \ldots, a_{n}\right)$

II. Compute and store sample statistics from the $N$ output $u$

In order to generate a random sample $a_{i}$ of $A_{i}$, a pseudo random number generator is required (Van Niel and Laffan, 2003). Due to the joint probability distribution, higher number of realizations $\mathrm{N}$ leads to obtain more stable results. Brown and Heuvelink (2006) indicates that the accuracy of Monte Carlo approach is proportional to the square root of the number of runs $\mathrm{N}$. This means that any degree of accuracy can be reached with a large enough number of realizations $\mathrm{N}$, but also that the computation time of an uncertainty analysis quadruples if we want to double the accuracy. Each Monte Carlo Simulation run results in one realization. The sum of the realizations can be used to derive several statistical estimators such as the standard deviation describing the uncertainty of the computed product (Venzin, 2013). The minimum amount of $\mathrm{N}$ should be at least 100 and that less than 20 simulations are insufficient. Oksanen (2006) also highlighted that the minimum amount of simulation has to be about 500. Within this research we considered 1000 runs for assessing uncertainty associated with DEMs. Accordingly in order to create this distribution a number $(\mathrm{N})$ of random error fields are generated where each cell represents the possible error at a co-located elevation. Each random field is added to the DEMs generating a new realization of the elevation surface (Wechsler and Charles, 2006).

\subsection{OBJECT BASED LANDLIDE DELEIANTION}

Within this research we follow methodology proposed by Blaschke et al. (2014) for object-based landslide mapping. This methodology makes use of integrated approach of spectral and spatial characteristics for modelling and delineating landslides. As first step of this methodology, the multi-resolution segmentation process was performed to create objects which were analysed with respect to their spatial, spectral, and textural parameters. The segmentation was performed using five scale parameters $(5,7,8,9$ and 10$)$, with the same shape $(0.3)$ and compactness $(0.5)$ factors. Then, the data were parameterized according to the specific requirements for mapping landslides 
and incorporated into a multi-scale optimization routine (Stumpf et al., 2011). In this way we aimed to integrate the spectral, spatial, and morphometric characteristics of landslides. Slope and flow directions were derived from the DEMs. The landslide inventory dataset were used as samples to develop object based rule-set for landslide mapping. An analysis of the landslide inventory map indicated that all of the 32 landslides objects occurred at high elevations $(>1450 \mathrm{~m}$ ) in areas with slopes (> 7 $\%$ ). Based on this dataset, whole objects were then classified into either "potential landslide" or "no landslide". According to the previous works regarding geomorphology of Ahar Chay- Basin, most of the known landslides are categorized as rotational landslides (Feizizadeh and Blaschke 2012, Feizizadeh et al., 2012). The morphological characteristics of these rotational slides exhibit abrupt changes in slope morphology, with concavity in the depletion zone of the landslide and convexity in the accumulation zone (Akbarimehr, 2013). In doing so, to delineate landslides object based techniques including mean, standard deviation, GLCM texture parameters (GLCM Contrast, GLCM Correlation, GLCM Entropy, GLCM Mean, GLCM Standard Devotion) were computed as base of rule-sets. Landslide inventory data were also employed as sampling data for obtaining thresholds.

\section{RESULTS}

Results of obtained landslide objects for both approaches are represented in figure 1. Based on the results of first approach (landside delineation using row DEMs) totally 44 landslide object were identified and the area affected by landslides were measured approximately 822 hectares. While, the identified landslide objects from the second approach by employing revised DEMs was counted 36 with area about 484 hectares.

In order to validate results, the accuracy assessment process was performed by applying known landslide locations within the study area. It is believed that, accuracy assessment is fundamental step in object based landside delineation and the quality of a detected landslide inventory depends on its accuracy (Moine et al., 2009; Blaschke et al., 2014). According to Moine et al. (2009) defining the accuracy of a landslide inventory is not straightforward, and standards do not exist. However, to evaluate the accuracy of our results, the OBIA-derived landslides were compared to an existing landslide inventory map of the study area. This landslide inventory database was used as a reference to implement the accuracy assessment. Results of accuracy assessment indicated the overall accuracy of $68.04 \%$ for landslide map delineated from row DEMs. While this overall accuracy significantly improved to $96.92 \%$ when the revised DEMs was employed as base of landslide delineation rule-sets. Figure 2 represent comparison between known landslides and landslides delineated from both DEMs.

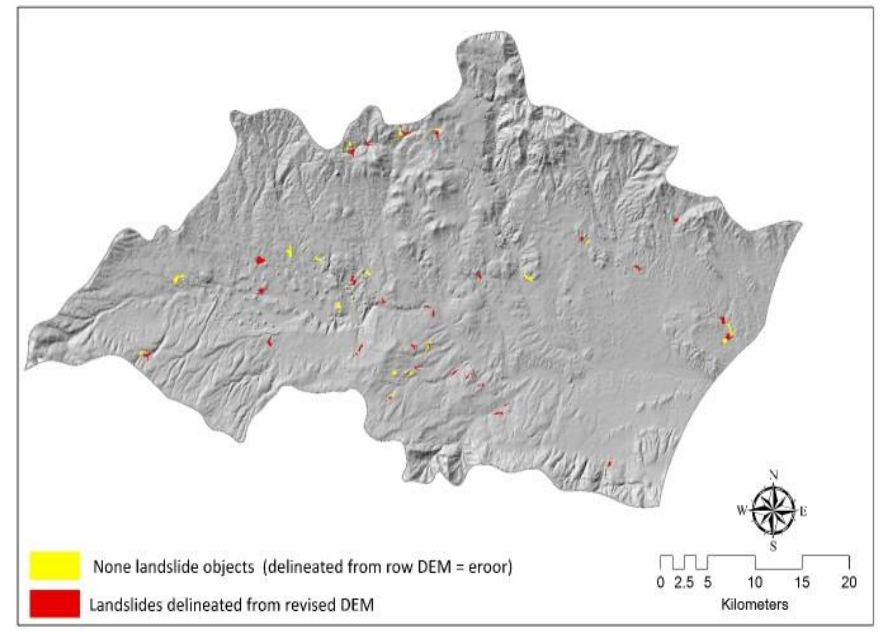

Figure 1. Landslides delineated from both DEMs

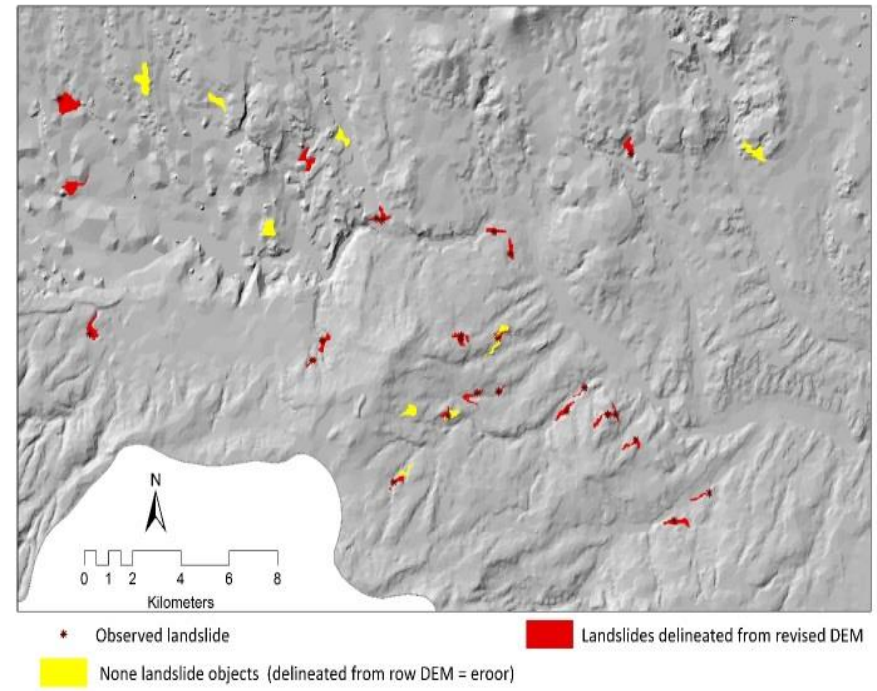

Figure 2. Results of accuracy assessment

\section{REFERENCES}

Akbarimehr, M., Motagh, M., Haghshenas-Haghighi, M., 2013. Slope Stability Assessment of the Sarcheshmeh Landslide, Northeast Iran, Investigated Using InSAR and GPS Observations". Remote Sensing. vol. 5, pp. 3681-3700.

Abd Aziz, A., Steward, B.L., Kaleita,A., Karkee, M., 2012. Assessing the effects of DEM uncertainty on erosion rate estimation in agricultural filed, journal of agriculture and biosystem engraining, 55(3): 785-798.

Blaschke, T., Feizizadeh, B., Holbling, D., 2014.Object-Based Image Analysis and Digital Terrain Analysis for Locating Landslides in the Urmia Lake Basin, Iran, IEEE Journal of Selected Topics in Applied Erath Observation and Remote Sensing, 7( 12), 4806-4817.

Carlisle, B. H., 2005. Modeling the spatial distribution of DEM error. Trans. GIS 9(4): 521-540.

Feizizadeh, B., Blaschke, T., 2014. Uncertainty analysis of GISMulticriteria based landslide susceptibility mapping. International Journal of Geographic Information Science, DOI:10.1080/13658816.2013.869821. 
Feizizadeh, B., Blaschke, T., 2012. GIS-Multicriteria Decision Analysis for landslide susceptibility mapping: comparing three methods for the Urmia lake basin, Iran. Natural Hazards, vol. 65 (3), pp. 2105-2128.

Feizizadeh, B., Blaschke, T., Nazmfar, 2012. GIS-based ordered weighted averaging and Dempster Shafer methods for landslide susceptibility mapping in Urmia lake Basin, Iran". International Journal of Digital Earth, DOI:10.1080/17538947.2012.749950.

Holmes, K. W., Chadwick, O. A., Kyriakidis, P. C., 2000. Error in a USGS 30-meter digital elevation model and its impact on terrain modelling. J. Hydrol. 233(1-4): 154-173.

Lu, P., Stumpf, A., Kerle, N., Casagli, N., 2011. Object-Oriented Change Detection for Landslide Rapid Mapping. IEEE Geoscience and Remote Sensing Letters, vol. 8 (4), pp. 701-705, 2011.

Moine, M., Puissant, A., Malet J. P., 2009. Detection of landslides from aerial and satellite images with a semiautomatic method. Application to the Barcelonnette basin (Alpes-de-Haute-Provence, France). In: J. P. Malet, A. Remaitre and T. Bogaard (Eds.), "Landslide Processes: From Geomorphological Mapping to Dynamic Modelling”, CERG, Strasbourg, France, pp. 63-68, 2009

Oksanen, J., 2006. Digital elevation model error in terrain analysis. PhD thesis, Faculty of Science, University of Helsinki.

Reuter, H.I., Hengl, T., Gessler, P., Soille, P., 2009. Preparation of DEMs for Geomorphometric Analysis. In: Tomislav, H\& Hannes, I.R (eds) Developments in soil science. Chapter 4, pages $87-120$.

Temme, A., Heuvelink, G., Schoorl, J., and Claessens, L., 2009. Geostatistical Simulation and Error Propagation in Geomorphometry. In Hengl, T. and Reuter, H. I., editors, Geomorphometry Concepts, Sof tware, Applications, volume 33 of Developments in Soil Science, chapter 5, pages 121-140.

Venzin., 2013, Analyzing the Impact of High Resolution DEM Uncertainty on Hydrological Models Using a Parallel Computing Approach, Master Thesis, Department of Geography, GIScience, Geocomputation, University of Zurich.

Wechsler, S.P., Charles, N.K., 2006. Quantifying DEM uncertainty and its effect on topographic parameters, Photogrammetric Engineering \& Remote Sensing, 72, No. 9, September 2006, pp. 1081.

Wechsler, S. P., 2007. Uncertainties associated with digital elevation models for hydrologic applications: a review. Hydrology and Earth System Sciences, 11(4):1481-1500.

Wise, S., 2000. Assessing the quality for hydrological applications of digital elevation models derived from contours. Hydrological Processes, 14(11-12):1909-1929. 


\title{
FOREST COVER CHANGE ANALYSIS BY OBJECT BASED METHOD USING SPOT AND RAPIDEYE IMAGES
}

\author{
Y. Gao a*, I. Gonzalez a, R. Gonzalez ${ }^{\text {b }}$, J.G. Lopez-Sanchez a , M.Skutsch a, Jaime Paneque-Gálvez ${ }^{\text {a }}$, Jean François Mas ${ }^{\text {a }}$ \\ ${ }^{a}$ Centro de Investigaciones en Geografía Ambiental, UNAM, Antigua carretera a Pátzcuaro 8701, Morelia, 58190, México - (ygao, \\ mskutsch,jfmas)@ciga.unam.mx; igonzalez@pmip.unam.mx; jairogabo@gmail.com,jpanequegalvez@gmail.com \\ b Institute of Environmental Science and Technology (ICTA), Universitat Autónoma de Barcelona (UAB), Rafael.Gonzalez@uab.cat
}

KEY WORDS: Segmentation, Object-based image classification, SPOT-5, RapidEye, Forest degradation, Deforestation, Dinamica

\begin{abstract}
:
In this paper we present forest cover change analysis by object based method with SPOT-5 (2007) and RapidEye (2013) images for an Ejido in the state of Jalisco, Mexico. We identified three classes in images of each date: 1) forest 2) degraded forest and 3) non-forest. An object based image analysis was applied to first segment the images into objects, and then classify the objects into the above three classes. We compared the results from this object based model with a model based on pixel based method. Classified images from both methods were evaluated with verification data composed of 254 random points and object based methods obtained slightly higher overall accuracy than the pixel based methods. Forest cover changes were analysed by constructing a model in DINAMICA (3.0.6) in which the forest classes of two dates were compared, and the forest cover changes were derived including deforestation, degradation, regeneration, and revegetation. The results show that although the overall accuracies of the classifications show no significance difference by McNemar's test, except the classifications between MLC and MD for 2007, the obtained forest change results show big variance between adopted methods and it is rather difficult to compare them. The future study will apply test data for forest change classes and decide the best change results with the highest accuracies.
\end{abstract}

\section{INTRODUCTION}

\subsection{Background}

Forest covers about $30 \%$ of the land area of the Earth. Changes of forest cover can affect many environmental processes and quantifying forest cover change plays an important role to address issues such as global carbon budget, ecosystem dynamics, etc. Satellite images are important data sources for reliable forest cover mapping and forest cover change quantification. For example, the combination of coarse spatial resolution images, e.g. from MODIS sensor with images of finer spatial resolution such as SPOT, or Landsat can be very useful in identifying and quantifying forest cover change of large area (Gao et al. 2011). One of the relatively simple change detection methods generates change classes by comparison of multitemporal image classification results. The multi-temporal satellite images were first classified into forest cover types, and then compared pixel by pixel to form spatially explicit forest cover change data. This method requires accurate classification results due to that the accuracy of the forest cover change results depends on the accuracy of the classification results.

Image classification assigns the image pixels into land cover categories by certain algorithm. Pixel base classification usually only implements spectral information of the images. It encounters problems when classifying high resolution satellite images with the increased noise-signal ratio. Besides, there are often spatial characteristics in image segments (groups of pixels) that are useful in the classification especially of high spatial resolution satellite images. Object based classification groups similar and adjacent pixels into objects based on both spectral and spatial information and classifies the segmented image by objects. Both pixel based and object based image classification methods have been proved successful, depending on the study area and sensor choices. This paper tested both pixel based and object based methods for forest cover change analysis with the purpose to find out which method could achieve results with higher accuracy. The assumption is that the multi-temporal classifications with high overall accuracies also lead to change map with high overall accuracies.

\section{STUDY AREA, DATA, AND METHODOLOGY}

\subsection{Study area}

The test area, el "ejido" la Laja, is a village with 760 habitants (INEGI, 2010), located in the Mixtlan municipality in the west of Jalisco state, west Sierra Madre region. The dominant climate is humid and semi-humid, with an altitude of 1440 meters above sea level, and an average annual temperature of 19.8 degrees. The main forest types are pine and oak (Gobierno de Jalisco 2010) (Figure 1).

\footnotetext{
* Corresponding author
} 


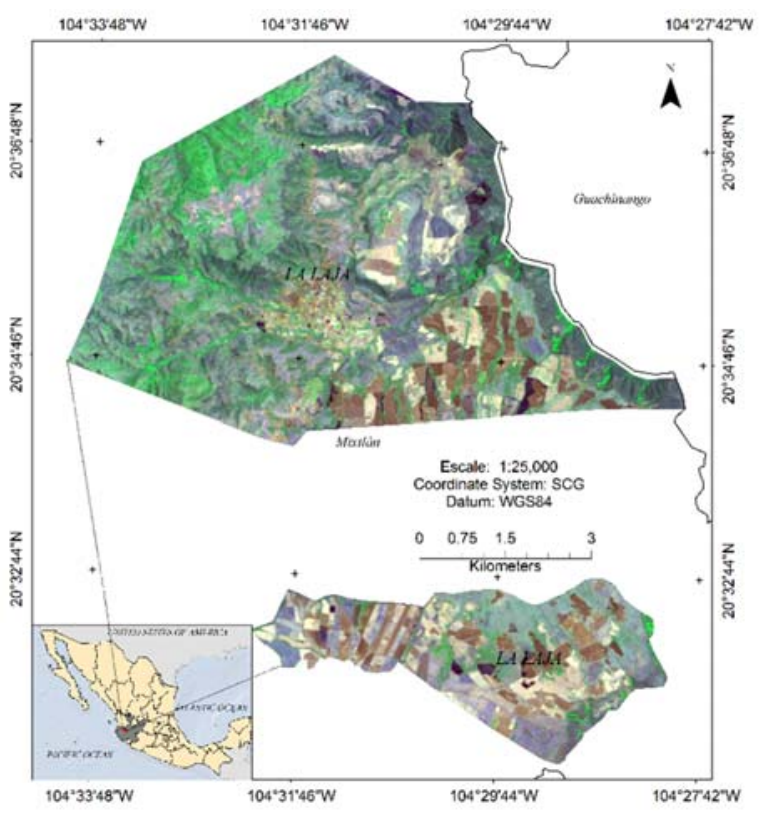

Figure 1. The study area

\subsection{Data}

The data for this analysis include SPOT 5 (for image analysis of 2007) and RapidEye images (for image analysis of 2013). SPOT-5 satellite sensor functioned from May 2002 to March 2015. Its applications include medium-scale mapping (1:25000 and 1:10000), urban and rural planning, and natural disaster management, among others

(http://www.satimagingcorp.com/satellite-sensors/othersatellite-sensors/spot-5/).

RapidEye sensor was launched in August 2008 and offers image data with large-area coverage, frequent revisit intervals, high resolution, and multispectral capabilities. Its capabilities can be applied to agriculture, forestry, cartography, and mining, among others. The specifications of SPOT-5 and RapidEye sensor are summarized in table 1 .

\begin{tabular}{|c|c|c|c|c|}
\hline & \multicolumn{2}{|c|}{ Image bands (nm) } & \multicolumn{2}{|c|}{$\begin{array}{l}\text { Spatial Resolution } \\
(\mathrm{m})\end{array}$} \\
\hline & SPOT & RapidEye & SPOT & RapidEye \\
\hline Pan & $480-710$ & 1 & $\begin{array}{c}5 \\
\text { (nadir) }\end{array}$ & \\
\hline Blue & 1 & $440-510$ & 1 & 5 \\
\hline Green & $500-590$ & $520-590$ & 10 & 5 \\
\hline Red & $610-680$ & $630-685$ & 10 & 5 \\
\hline Red Edge & 1 & $690-730$ & 1 & 5 \\
\hline Near IR & $780-890$ & $760-850$ & 10 & 5 \\
\hline $\begin{array}{c}\text { Shortwave } \\
\text { IR }\end{array}$ & $\begin{array}{c}1580- \\
1750\end{array}$ & 1 & 20 & 1 \\
\hline
\end{tabular}

Table 1. Specifications of SPOT-5 and RapidEye satellite sensor (adapted from http://www.satimagingcorp.com/satellitesensors/other-satellite-sensors/spot-5/)

For carrying out the classifications, the training data were selected for three classes: forest, degraded forest, and non-forest, for images of 2007 and 2013, respectively. The selection is based on the visual inspection of the classified satellite images. For the accuracy assessment of the classifications, two sets of test data with random points for both 2007 and 2013, were created by visual interpretation.

\subsection{Methodology}

2.3.1. Multi-temporal image classifications: Object based classification was carried out in SPRING which requires two parameters for image segmentation: area and similarity. The decision for those parameters was based on a trial and error method by visual inspection of the segmentation results. The segments were classified into thematic classes in SPRING. Pixel based MLC classifies based on the probability function constructed training data, and a pixel will be classified to the class to which it has the highest probability. Pixel based MD classifies a pixel to a land cover category to which it has the shortest distance.

2.3.2. Accuracy assessment: Two sets of test data were created for the study area for both 2007 and 2013 by visual interpretation of random points. Error matrix was built with the classification data and the test data, based on which the overall accuracy, the use's and producer's accuracies were calculated. The overall accuracy calculates the proportion of the assessed area that is classified correctly. The user's accuracy calculates the proportion of pixels classified as a class that truly belong to that class, and the producer's accuracy calculates the proportion of pixels from a class that are classified as that class. User's and producer's accuracies are related to commission and omission errors. Kappa coefficient was not used since it may underestimate classification accuracy by overestimating the chance agreement (Pontius et al. 2011).

2.3.3. The statistic of the McNemar's test: Because the same set of reference data was used in accuracy assessment for both pixel-based and object-based classification, dependency may exist among the resultant confusion matrices. The McNemar's test was applied to test the statistical significance of the difference between those classifications. McNemar's test is based on an assumption that different classification approaches have the same number of correctly and incorrectly classified pixels. It is a non-parametric test and the statistic follows a chisquare distribution of one freedom degree. The formula for the statistic of the McNemar's test is presented in equation 1:

$X^{2}=\frac{\left(f_{12}-f_{21}\right)^{2}}{f_{12}+f_{21}}$

Where $f_{12}$ represents the number of pixels correctly classified by one method but misclassified by the other, while $f_{21}$ for reverse. For this test, an overlay analysis was carried out with the two classifications in comparison and the test data.

2.3.4. Post-classification comparison for change detection: The two-date (2007 \& 2013) change detection was carried out by comparing the classification results of the corresponding dates. A model was constructed in DINAMICA EGO to analyse forest cover change. Deforestation is defined as changes from forest to non-forest, and from degraded forest to non-forest; forest degradation is defined as changes from forest to degraded forest; reforestation is defined as changes from non-forest to forest, and to degraded forest; and revegetation is defined as changes from degraded forest to forest. A change matrix 2007 2013 is presented in table 2.

\begin{tabular}{l|c|c|c|c}
\hline \multicolumn{2}{c|}{} & \multicolumn{3}{|c}{ Forest cover 2013 } \\
\cline { 3 - 5 } & Forest & Degraded forest & Non-forest \\
\hline \multicolumn{2}{c|}{ Forest } & Permanence & Degradation & Deforestation \\
\hline
\end{tabular}




\begin{tabular}{c|c|c|c|c}
\hline $\begin{array}{c}\text { Forest } \\
\text { cover }\end{array}$ & $\begin{array}{c}\text { Degraded } \\
\text { forest }\end{array}$ & Revegetation & Permanence & Deforestation \\
\cline { 2 - 5 } 2007 & $\begin{array}{c}\text { Non- } \\
\text { forest }\end{array}$ & Reforestation & Reforestation & Permanence \\
\hline
\end{tabular}

Table 2. Change matrix $2007-2013$.

\section{RESULTS}

This part presents the results of classification and change detected based on the post-classification analysis.

\subsection{Multi-temporal classifications}

The multi-temporal classifications (2007 and 2013) were carried out with object based, pixel based MLC, and pixel based MD classifiers. Training data were created for three categories: forest, degraded forest, and non-forest for the images of 2007 and 2013, respectively.

\subsection{Accuracy assessment}

The accuracy of the classifications was evaluated by test data comprised of 250 random points using error matrix. The results of the overall accuracy, user's and producer's accuracies for the individual classes are summarized in the table 3.

\begin{tabular}{|c|c|c|c|}
\hline Accuracy (\%) & Forest & $\begin{array}{l}\text { Degraded } \\
\text { forest }\end{array}$ & Non-forest \\
\hline \multicolumn{4}{|c|}{ Object based $2007 / 2013:$ Overall accuracy $=68.5 / 70.2$} \\
\hline User's accuracy & $73.3 / 66.3$ & $53.3 / 63.2$ & $76.4 / 81$ \\
\hline $\begin{array}{l}\text { Producer's } \\
\text { accuracy }\end{array}$ & $76.7 / 88.4$ & $57.1 / 52.7$ & $69.4 / 73.9$ \\
\hline \multicolumn{4}{|c|}{ Pixel based MLC 2007 / 2013: Overall accuracy = 68.1 / 69.8} \\
\hline User's accuracy & $72.7 / 63.6$ & $50 / 67.7$ & $82.5 / 78$ \\
\hline $\begin{array}{l}\text { Producer’s } \\
\text { accuracy }\end{array}$ & $74.4 / 91.3$ & $61.4 / 46.2$ & $67.3 / 77.2$ \\
\hline
\end{tabular}

\begin{tabular}{l|l|l|l}
\hline \multicolumn{4}{c}{ Pixel based MD 2007 / 2013, Overall accuracy = 63.8 / 69 } \\
\hline User's accuracy & 74.4 / 65.5 & $41.7 / 58.9$ & $93 / 85.3$ \\
\hline $\begin{array}{l}\text { Producer's } \\
\text { accuracy }\end{array}$ & 70.9 / 82.6 & 68.6 / 58.2 & $54.1 / 69.6$ \\
\hline
\end{tabular}

Table 3. results of accuracy assessment for 2007 and 2013 for the tested classifications.

\subsection{Results of the statistic of the McNemare's test}

The McNemar's test was applied to evaluate if there is significant difference between the classification results by different methods. As an example, between the classifications by pixelbased MD and by object-based method, the $X^{2}$ value from the McNemar's test was 3.6. With one degree of freedom, this result has a p-value greater than 0.05 , indicating a statistically no significant difference. In a similar way, the McNemar's test was applied to the classifications of 2007, and those of 2013. The results are summarized in the table 4 .

\begin{tabular}{c|c|c|c|c}
\hline \multicolumn{2}{c|}{$\begin{array}{c}\text { Comparisons } \\
(2007 / 2013)\end{array}$} & $X^{2}$ & $P$ & $\begin{array}{c}\text { Significan } \\
\text { ce }\end{array}$ \\
\hline $\begin{array}{c}\text { Object } \\
\text { based }\end{array}$ & MD & $3.6 / 0.21$ & $P<0.1 / P<0.75$ & No / No \\
\hline $\begin{array}{c}\text { Object } \\
\text { based }\end{array}$ & MLC & $0.03 / 0.04$ & $P<0.90 / P<0.90$ & No / No \\
\hline MLC & MD & $4.17 / 0.11$ & $P<0.05 / P<0.75$ & Yes / No \\
\hline
\end{tabular}

Table 4 . The result of the statistic of the McNemar's test.

\subsection{Change matrices by post-classification comparison}

Forest cover change from 2007 - 2013 was analysed by the comparison of the post-classifications with the methods of object based, the pixel based MLC, and the pixel based MD. The results are presented in change matrices in the table 5,6 , and 7.

\begin{tabular}{c|c|c|c|c}
\hline \multicolumn{2}{c|}{ Object based (ha) } & \multicolumn{3}{|c}{ Forest cover 2013 } \\
\cline { 3 - 5 } \multicolumn{2}{c|}{} & Forest & Degraded forest & $\begin{array}{c}\text { Non- } \\
\text { forest }\end{array}$ \\
\hline $\begin{array}{c}\text { Forest } \\
\text { cover }\end{array}$ & Forest & 1534.2 & 265.9 & 111.4 \\
\cline { 2 - 5 } 2007 & Degraded forest & 378.4 & 1115.9 & 379.1 \\
\cline { 2 - 5 } & Non-forest & 115.5 & 242.2 & 1313.1 \\
\hline
\end{tabular}

Table 5. Post-classification comparison by object based method.

\begin{tabular}{l|c|c|c|c}
\hline \multicolumn{2}{c|}{ Pixel based MLC (ha) } & \multicolumn{3}{|c}{ Forest cover 2013 } \\
\cline { 3 - 5 } \multicolumn{2}{c|}{} & Forest & Degraded forest & $\begin{array}{c}\text { Non- } \\
\text { forest }\end{array}$ \\
\hline \multirow{2}{*}{$\begin{array}{c}\text { Forest } \\
\text { cover } \\
2007\end{array}$} & Forest & 1529.5 & 194.1 & 164.3 \\
\cline { 2 - 5 } & Degraded forest & 425.6 & 1116 & 411.5 \\
\hline
\end{tabular}

Table 6. Post-classification comparison by pixel based MLC classifier.

\begin{tabular}{c|c|c|c|c}
\hline \multicolumn{2}{c|}{ Pixel based MD (ha) } & \multicolumn{3}{c}{ Forest cover 2013 } \\
\cline { 3 - 5 } \multicolumn{2}{c|}{} & Forest & Degraded forest & Non-forest \\
\hline \multirow{2}{*}{$\begin{array}{c}\text { Forest } \\
\text { cover }\end{array}$} & Forest & 1298 & 293.5 & 55.3 \\
\cline { 2 - 5 } 2007 & Degraded forest & 353.4 & 1624.3 & 656.1 \\
\cline { 2 - 5 } & Non-forest & 145.2 & 199.7 & 830.3 \\
\hline
\end{tabular}

Table 7. Post-classification comparison by pixel based MD classifier.

\subsection{Forest change $2007-2013$}

Based on the results of the change matrices, quantities of the forest changes, including deforestation (DEF), forest degradation (F-DEG), reforestation (REF), and revegetation (REV) were obtained. The results are summarized in table 8 (ha).

\begin{tabular}{l|l|l|l|l|l}
\hline (ha) & DEF & F-DEG & REF & REV & Total \\
\hline $\begin{array}{l}\text { Object } \\
\text { based }\end{array}$ & 490.5 & 265.9 & 357.7 & 378.4 & 1492.5 \\
\hline MLC & 575.8 & 194.1 & 306.9 & 425.6 & 1502.4 \\
\hline MD & 711.4 & 293.5 & 344.9 & 353.4 & 1703.2 \\
\hline
\end{tabular}

Table 8 . The obtained forest cover changes in quantity (ha) $2007-2013$.

\section{DISCUSSION AND CONCLUSION}

This study performed forest cover change analysis by postclassification comparison method. The classification was carried out by three different methods: object based method, pixel based MLC and MD methods. The objective was to evaluate if object based method could outperform pixel based method.

The classifications did not show big difference in the overall accuracies. By the McNemar's test, there is significant difference only between the pixel based MLC and MD classifications for the images of 2007. As for the accuracies of the individual classes, degraded forest has the lowest user's and producer's accuracies for both 2007 and 2013. At the same time, non-forest has the highest overall accuracies. Degraded forest covers a wider spectral range than that of the forest and non-forest categories, which makes it difficult to be separated from other classes. There is no significant difference in the classifications by the pixel based method and the object based method for the selected study area using SPOT-5 images. Since the change analysis result 
depends on the accuracies of the classification results, this seems to suggest that the change results by those three methods would also be similar. In table 8, the obtained forest change results in total are similar by the post-classifications of object based method and pixel based MLC method. The change results in quantities for the individual categories have the same tendencies: deforestation $>$ revegetation $>$ reforestation $>$ forest degradation. This result makes it difficult to decide which method is more suitable for the forest change analysis. For future study, it is needed to derive test data for change categories and perform accuracy assessment for change maps.

\section{REFERENCES}

INEGI, 2010. Censo Nacional de población y vivienda 2010.

Gobierno de Jalisco, 2010, www.jalisco.gob.mx/es/jalisco/municipios/mixtlan.

Gao, Y., Skutsch, M., Drigo, R., Pacheco, P., Masera, O., 2010. Assessing deforestation from biofuels: Methodological challenges. Applied Geography. 31, 508-518.

Pontius, P.G., \& Millones, M., 2011, Death to Kappa: birth of quantity disagreement and allocation disagreement for accuracy assessment. International Journal of Remote Sensing. 32, 44074429. 


\title{
A WEB-BASED PLATFORM FOR REMOTE SENSING IMAGE ANNOTATION
}

\author{
A. Garcia-Pedrero ${ }^{\mathrm{a}, \mathrm{b}, *}$, C. Gonzalo-Martín ${ }^{\mathrm{a}, \mathrm{b}}$, M. Lillo-Saavedra ${ }^{\mathrm{c}, \mathrm{d}}$, C. Ortiz-Toro ${ }^{\mathrm{a}, \mathrm{b}}$ \\ ${ }^{a}$ Center for Biomedical Technology, Universidad Politécnica de Madrid, Campus de Montegancedo, \\ Pozuelo de Alarcón 28233, Spain - \{am.garcia\}@alumnos.upm.es. \\ ${ }^{\mathrm{b}}$ Escuela Técnica Superior de Ingenieros Informáticos, Universidad Politécnica de Madrid, Campus de Montegancedo, \\ Boadilla del Monte 28660, Spain \\ ${ }^{\mathrm{c}}$ Faculty of Agricultural Engineering, University of Concepción, Chile \\ ${ }^{\mathrm{d}}$ Water Research Center for Agriculture and Mining, CRHIAM, University of Concepción, Chile
}

KEY WORDS: Image annotation, object-based, active learning

\begin{abstract}
:
The generation of labeled data for training automated methods used in the analysis of remote sensing images is a challenging task. Approaches as Active Learning aim to perform accurate classifications in a scenario of a few annotated data. These approaches generally require the interaction between the user and the machine learning method during training phase. However, in the remote sensing area, it is difficult to find a tool that facilitates this interaction. In this work, an interactive web-based platform to perform the training of method for remote sensing image annotation by means of an active learning approach is proposed. The platform integrates open-source GIS technologies and object-based approach in order to facilitate the interaction between the user and the active learning approach. While some usability aspects should be improved, the obtained results show the potential of the proposed platform.
\end{abstract}

\section{INTRODUCTION}

The daily generation of terabytes of Earth-Observation data opens an opportunity to both industry and scientific communities to fully exploit these data in order to provide services to end-users (Ma et al. 2015). However, the analysis of these voluminous data is not possible by manual means, and therefore it is required the use of automatic and semiautomatic tools. In this regard, several machine learning and pattern recognition techniques have been used in remote sensing (RS) applications in order to facilitate the image annotation. During training step, these methods mostly require a large amount of annotated (labeled) samples that provides a good statistical separability of the classes to provide final accurate results (Xia et al. 2015).

Nevertheless, the generation of that labeled (annotated) collection is a challenging task, especially for large area mapping (Olofsson et al. 2014). In this context, Active Learning (AL) approaches aim to perform accurate classifications in a scenario of a few annotated data (Stumpf et al. 2014, Tuia and Munoz-Mari 2013 Crawford et al. 2013, Tuia et al., 2009). In a basic AL scheme, an user, also called oracle, provides annotated information and knowledge about the desired classes, while the classifier provides both its own interpretation of the distribution of the classes and the most relevant observations that are needed in order to solve the discrepancies encountered during training phase (Crawford et al. 2013). As one can imagine, the interaction between the user and the machine learning algorithm during training phase is fundamental.

To the best of our knowledge, there is not a platform that facilitates this interaction for remote sensing image annotation. In this work, we propose an interactive web-based platform to perform AL approach for remote sensing image annotation. The platform integrates open-source GIS technologies to display raster and vector datasets in a web browser, while internally a machine learning method is trained through AL approach. The proposed platform uses image-objects as minimum processing units instead of pixels.

${ }^{*}$ Corresponding author
Image-objects are labeled during AL process according to a set of classes established a-priori by the user. This reduces dramatically the number of instances to process in very-high resolution remote sensing images.

The rest of the paper is organized as follows. Section 2 describes the architecture of the system. In Section 3 the methodology adopted for the development of the proposed platform is described. Section 4 shows the results of a small experiment to test the proposed platform. Finally, main conclusions are given in Section 5

\section{SYSTEM ARCHITECTURE}

The proposed architecture (Figure 1 follows a web-based application scheme consisting of two main layers (server and client layers) and several modules. Each module has a different purpose, and is implemented using technology based on open standards and free software.

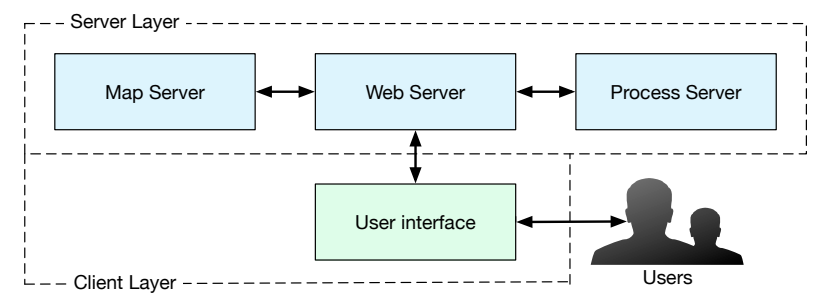

Figure 1. Architecture of the proposed platform.

\subsection{Server Layer}

This layer represents the main component in the system. It consists of three sub-modules: Map Server, Web Server, and Process Server modules.

The Map Server module contains the source imagery (raster) and vector data to be used by the system. A spatial database was implemented to optimize storage and query vector data. PostgreSQL 1

1 https://www. postgresql.org 
with PostGIS ${ }^{2}$ plugin was selected as management system of this database. GeoServer ${ }^{3}$ was chosen for rendering raster and vector data. It can handle data directly from most common spatial databases including PostgreSQL/PostGIS databases, as well as different raster formats such as GeoTIFF.

The Web Server module hosts the source code of the application developed using HTML5, Django, Python, JavaScript and CSS programming languages. It deals with incoming traffic and requests from client browsers. For this purpose, Nginx ${ }^{4}$ web server is used, which allows to serve static content quickly and efficiently, has a low memory usage (ideal for virtual environments), and offers a free-of-charge license. Moreover, Python scripts are used along the server layer for managing the communications between the users and the Web Server module, and between the last and the Process Server module for processing the data (e.g., segmentation, classification). Django is used to provide easy configuration management platform and connect to the database.

The Process Server module is mainly used for processing tasks such as image segmentation, and classification. This module receives the processing requests from Web Server module, which in turn receives from users.

In this work, all these modules are housed in the same computer, therefore they are limited by the capabilities of this equipment. For developing and testing this will not cause any problem, however for production applications that usually requires more capacity, this modules can be easily distributed in different machines using technologies such as Docker ${ }^{5}$

\subsection{Client Layer}

This layer defines the interactions between the users through an internet browser and the proposed system. It consists of a User Interface module, which is a web page implemented using AJAX and Javascript technologies. They are basically codes running on the user's computer, in particular in the web browser, which facilitate user interaction with the platform. In this regard, Openlayers $3^{6}$ an open-source Javascript library, was used to create interactive maps in the web browser with no server-side dependencies. It provides a complete control of map controls, map events, map layers, among others features. These features were exploited to display geospatial data (vector and raster) as a map.

Other libraries used to facilitate the creation of the web page were Jquery 7 , and Bootstran ${ }^{8}$ Jquery eased the implementation of manipulation operations and AJAX requests, while Bootstrap provided the framework to design a responsive front-end.

\section{METHODOLOGY}

As mentioned in Section 1 , the proposed platform aims to facilitate the process of active learning through an interactive process between the user and the classification method. Moreover, to ease and reduce the time of sampling, an object-based approach was implemented. Thus, the user instead of selecting pixels by manually delineating objects, simply selects the previously generated segments. As can be shown in Figure 2 in addition to the user interface (web page), two other main submodules were implemented: image segmentation and active learning. These submodules are explained in detail below.

\footnotetext{
2 http://postgis.net

3 http://geoserver.org

4 https://www.nginx.com

5 https://www.docker.com

6 http://openlayers.org

7 http://jquery.com

$\varepsilon$ http://getbootstrap.com
}

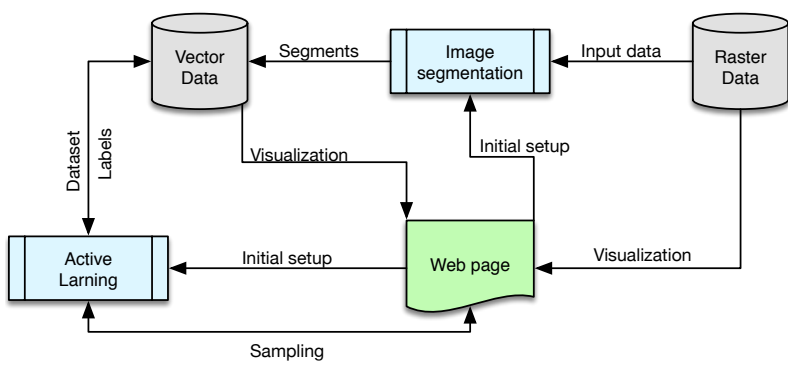

Figure 2. Flowchart of the methodology adopted to implement the platform.

\subsection{Image segmentation}

A superpixel method was selected to segment multispectral imagery. These type of methods produces a controlled over-segmentation of the image; in this regard, the generated superpixels tend to be similar in size and color, as well as belonging to only one object. In this work, a modified version of the SLIC algorithm (Achanta et al. 2012) for segmenting multispectral images (Garcia-Pedrero et al. 2015) was implemented. This method has two configuration parameters: a compactness factor and the superpixel size. The first one balances spectral proximity and space proximity, while the second one, as its name suggests, controls the number of pixels that in average comprises each superpixel.

\subsection{Active Learning}

The user-classifier interaction of AL approach is described as follows: 1) The user provides a small dataset of labeled regions. 2) The classifier learns the class distribution of the labeled data. 3) Random non-labeled regions are submitted to the classifier, which determines the regions with maximum uncertainty that should be labeled by the user. The last process is known as query. 4) Steps 1 to 3 are repeated until the classifier is trained. 5) Finally, the classifier is used to annotate the entire image.

Label Propagation (LP) algorithm (Bengio et al. 2006), implemented in Pedregosa et al. (2011), was selected as the classification method to be trained through an AL approach. LP is a graph-based method which main assumption is that instances occupying the same manifold are very likely to share the same label. Thus this method propagates labels of the labeled data to the unlabeled data instances according to the intrinsic data manifold structures found in the training data. There is currently no feature extraction module. Therefore, the segments are only characterized using the mean value of the pixels that they contain.

\subsection{User Interface}

The user interface, shown in Figure 3 incorporates the following features: (1) It allows the selection of different color composites for visualization. (2) It allows to locate a particular point of the image by geographical coordinates. (3) Tracking location is available. (4) It allows to show and hide the object (segment) boundaries. (5) It allows to label one or more segments at same time (Figure 4 . This operation is only possible if the object boundaries are visible. (6) It allows zoom in and zoom out operations for better viewing.

A configuration screen is also available to set the parameters necessary for the proper functioning of the platform. These parameters include: (1) the different color composites; (2) the parameters of the segmentation and classification algorithms; (3) AL parameters which include the number of labeled objects required to start the process, the number of elements (regions with higher uncertainty) to be manually labeled during a AL request (query), and the number of requests (iterations) necessary to complete the training. 


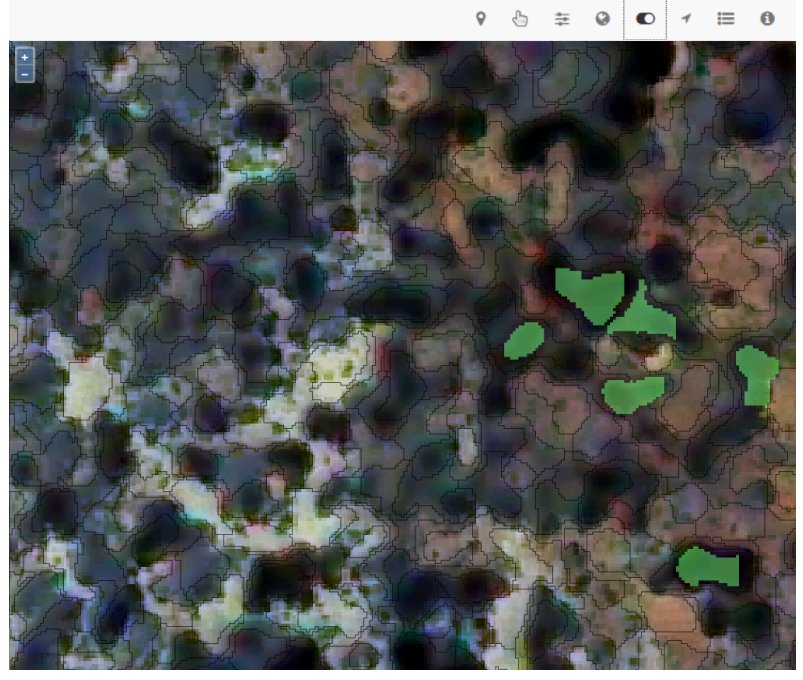

Figure 3. Overview of the proposed platform.

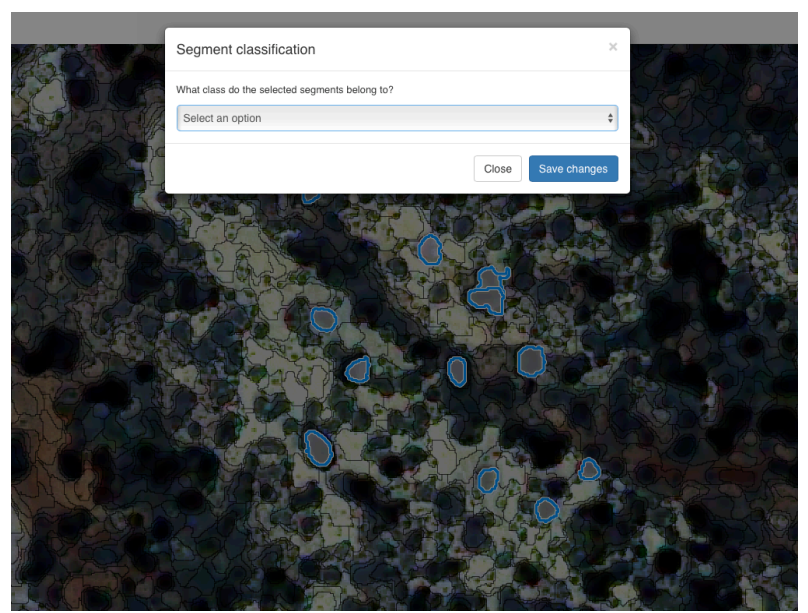

Figure 4. Manual labeling of several segments.

\section{EXPERIMENTAL RESULTS}

To prove the effectiveness of the proposed platform, a small experiment was carried out. A WorldView-2 (WV-2) dataset acquired on May 16th, 2011, was used for the analysis. The scene (show in Figure 3 is mostly characterized by scattered shrubs, bare soil, buildings and roads. Image annotation problem was reduced to the annotation of only two classes: non-shrub and shrub.

The use of the platform is described as follows: (1) An initial set of 50 regions was labeled by the user in order to start the AL approach. (2) The classifier was trained with the labeled elements. (3) Then 100 unlabeled segments were randomly selected by AL approach, the 5 elements most relevant were displayed to the user to label them manually. The segments are labeled one to one. To do this, the view is positioned in one of the elements to label, after this, the next element is displayed. (4) Steps 2 and 3 were repeated until reach a total of 50 iterations, which completed the training of the classifier.

A visual inspection after classifying 100 unlabeled regions points out a good correspondence between the obtained results (shown in Figure 5] and the expected by the user.

At this time, the platform is only available for the internal use of a small user group, however, it will open to a wider group in future releases.

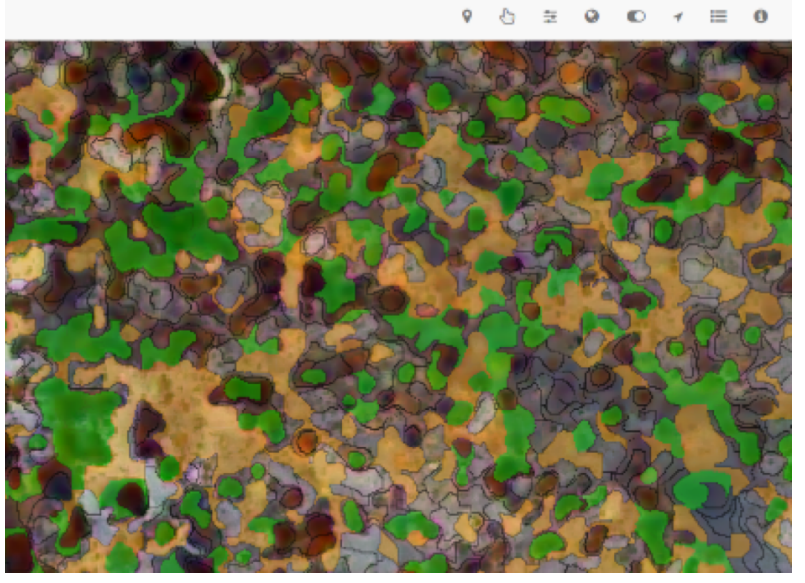

Figure 5. Results of the labeling process by the classifier trained by the AL approach.

\section{CONCLUSIONS}

A web-based platform for the annotation of remote sensing imagery was presented. The proposed platform allowed the interactive training, using an active learning approach, of a machine learning method for automatic image annotation. The use of the platform has facilitated the annotation of images without the need of large training set. The obtained results are promising, however some issues must be solved to improve the user experience. One of the main problems is the lack of an intuitive way to label more than one segment during the query made by the active learning approach. Another one is the lack of a feature extraction module. New releases of the platform will intended to solve these problems.

\section{ACKNOWLEDGEMENTS}

A. Garcia-Pedrero (grant 216146) acknowledges the support for the realization of his doctoral thesis to the Mexican National Council of Science and Technology (CONACyT). This work has been partially funded by FONDEF IT13I20002, and the Centro de Recursos Hídricos para la Agricultura y la Minería CRHIAM (CONICYT -FONDAP-1513001), and by the Universidad Politécnica de Madrid (AL16-PID-17).

\section{References}

Achanta, R., Shaji, A., Smith, K., Lucchi, A., Fua, P. and Susstrunk, S., 2012. SLIC Superpixels Compared to Stateof-the-Art Superpixel Methods. Pattern Analysis and Machine Intelligence, IEEE Transactions on 34(11), pp. 2274-2282.

Bengio, Y., Delalleau, O. and Le Roux, N., 2006. Label propagation and quadratic criterion. Semi-supervised learning.

Crawford, M. M., Tuia, D. and Yang, H. L., 2013. Active learning: Any value for classification of remotely sensed data? Proceedings of the IEEE 101(3), pp. 593-608.

Garcia-Pedrero, A., Gonzalo-Martin, C., Fonseca-Luengo, D. and Lillo-Saavedra, M., 2015. A geobia methodology for fragmented agricultural landscapes. Remote Sensing 7(1), pp. 767787.

Ma, Y., Wu, H., Wang, L., Huang, B., Ranjan, R., Zomaya, A. and Jie, W., 2015. Remote sensing big data computing: challenges and opportunities. Future Generation Computer Systems 51 , pp. 47-60. 
Olofsson, P., Foody, G. M., Herold, M., Stehman, S. V., Woodcock, C. E. and Wulder, M. A., 2014. Good practices for estimating area and assessing accuracy of land change. Remote Sensing of Environment 148, pp. 42-57.

Pedregosa, F., Varoquaux, G., Gramfort, A., Michel, V., Thirion, B., Grisel, O., Blondel, M., Prettenhofer, P., Weiss, R., Dubourg, V., Vanderplas, J., Passos, A., Cournapeau, D., Brucher, M., Perrot, M. and Duchesnay, E., 2011. Scikit-learn: Machine learning in Python. Journal of Machine Learning Research 12, pp. 2825-2830.

Stumpf, A., Lachiche, N., Malet, J.-P., Kerle, N. and Puissant, A., 2014. Active learning in the spatial domain for remote sensing image classification. IEEE Transactions on Geoscience and Remote Sensing 52(5), pp. 2492-2507.

Tuia, D. and Munoz-Mari, J., 2013. Learning user's confidence for active learning. IEEE Transactions on Geoscience and Remote Sensing 51(2), pp. 872-880.

Tuia, D., Ratle, F., Pacifici, F., Kanevski, M. F. and Emery, W. J., 2009. Active learning methods for remote sensing image classification. IEEE Transactions on Geoscience and Remote Sensing 47(7), pp. 2218-2232.

Xia, G.-S., Wang, Z., Xiong, C. and Zhang, L., 2015. Accurate annotation of remote sensing images via active spectral clustering with little expert knowledge. Remote Sensing 7(11), pp. $15014-15045$. 


\title{
VALUE OF FEATURE REDUCTION FOR CROP DIFFERENTIATION USING MULTI- TEMPORAL IMAGERY, MACHINE LEARNING, AND OBJECT-BASED IMAGE ANALYSIS
}

\author{
J. K. Gilbertson ${ }^{\mathrm{a}}$, A. Van Niekerk ${ }^{\mathrm{a}, \mathrm{b} *}$ \\ ${ }^{\text {a }}$ Department of Geography and Environmental Studies, University of Stellenbosch, Stellenbosch, South Africa - 16255135@sun.ac.za \\ b School of Plant Biology, University of Western Australia, 35 Stirling Hwy, Crawley WA 6009, Perth, Australia - avn@ sun.ac.za
}

KEY WORDS: Feature Selection, Machine Learning, Object-Based Image Analysis, Multi-Temporal, Supervised Classification

\begin{abstract}
This study examined the value of automated and manual feature selection, when applied to machine learning and object-based image analysis (OBIA), for the differentiation of crops in a Mediterranean climate. Five Landsat8 images covering the phenological stages of seven major crops types in the study area (Cape Winelands, South Africa) were acquired and processed. A statistical image fusion technique was used to enhance the spatial resolution of the imagery. The pan-sharpened imagery was used to produce a range of spectral features, textural measures, indices and colour transformations, after which it was segmented using the multi-resolution (MRS) algorithm. The entire set of 205 features (41 per image capture date) was then subjected to different feature selection and reduction methods. The feature selection and reduction methods included manual feature removal (i.e. grouping into semantic themes), filter methods (such as classification and regression trees (CART) and random forest (RF)), and statistical principal components analysis (PCA). The experiments were carried out in two scenarios, namely 1) on all input images in combination; and 2) on each individual image date. The feature subsets were used as input to decision trees (DTs), k-nearest neighbour (k-NN), support vector machine (SVM), and random forest (RF) machine learning classifiers. In order to assess the value of each feature reduction method (comprising feature reduction and selection techniques), overall accuracy, kappa coefficient and McNemar's test were employed to assess classification accuracy and compare the results. The results show that feature selection was able to improve the overall crop identification accuracy for the DT, k-NN, and RF classifiers, but was unable to do so for SVM. SVM scored the highest overall accuracy and kappa coefficient, even without applying feature reduction or selection. Based on these results it was concluded that, although feature selection can aid the crop differentiation process, it is not a necessity.
\end{abstract}

\section{INTRODUCTION}

Accurate crop maps are required for the health of an economy's agricultural sector, as they can be used for yield forecasting and keeping agricultural database statistics up to date (Monfreda et al. 2008). The production of crop maps has traditionally been done through field visits, which are costly and biased. Remote sensing has been proposed as a cost-effective solution (Castillejo-Gonzalez \& Lopez-Granados 2009), as it can be linked to climate, soil properties, terrain characteristics, and light-use efficiency and therefore provide farmers with crucial information on crop health and moisture content, resulting in better decisions about irrigation and fertilization (Monfreda et al. 2008; Xin et al. 2015). In recent years, multi-temporal optical data has been the preferred data source for crop type mapping. However, multi-temporal approaches often lead to large datasets and features, which often result in the so-called 'curse of dimensionality'. This phenomenon occurs when an increase in the number of input features leads to a decrease in classification accuracy due to feature space sparseness (Gislason et al. 2006; Rodriguez-Galiano et al. 2012). One way of mitigating this effect is through feature selection (Rodriguez-Galiano et al. 2012) and/or reduction (Zhang et al. 2009).

\section{MAIN BODY}

\subsection{Study Site}

The study was carried out in the Cape Winelands region of South Africa (Figure 1). The $1040 \mathrm{~km}^{2}$ study site, which extends from $33^{\circ} 34^{\prime} 39^{\prime \prime}$ to $33^{\circ} 52^{\prime} 17^{\prime \prime} \mathrm{S}$ and $18^{\circ} 32^{\prime} 24^{\prime \prime}$ to $18^{\circ} 54^{\prime} 43^{\prime \prime} \mathrm{E}$, was selected based on the availability of multitemporal cloud free Landsat8 imagery and the variety of winter and summer crops produced in the region. The study site has a Mediterranean climate with cool wet winters and warm dry summers, an average annual rainfall of $550 \mathrm{~mm}$, a mean annual temperature minima of $11^{\circ} \mathrm{C}$ and a maxima of $22^{\circ} \mathrm{C}$ (Tererai et al. 2015).

\footnotetext{
* Corresponding author
} 


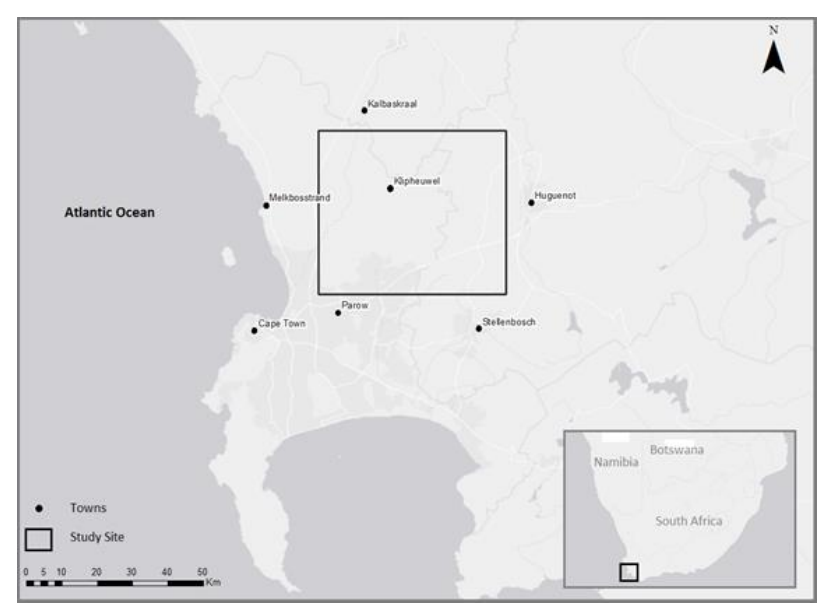

Figure 1. Location of the study area in the Western Cape, South Africa.

The area produces a wide range of crops, the most common of which are canola, grapes (mainly for wine production), lucerne (alfalfa), lupine, olives, managed pasture, and wheat. The phenological and agricultural production stages of these crops are shown in Figure 2. The annuals canola, lupine, pasture grasses and wheat are grown during southern hemisphere winter (April to August), while grapes are harvested during summer months (December to February). Lucerne is cut throughout the year, while olives are harvested during the early winter months.

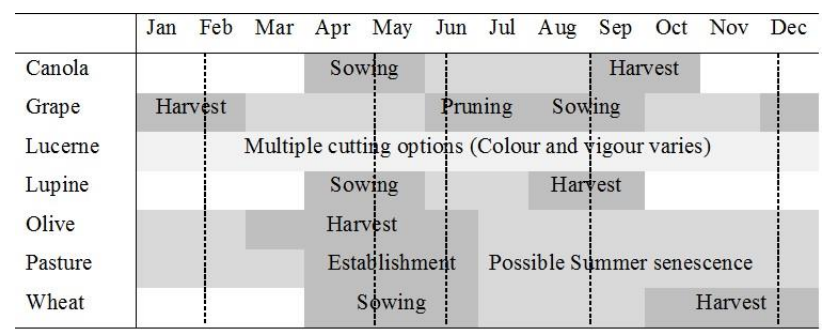

Figure 2. Phenological and agricultural production phases of targeted crops. Dark grey represents important agricultural phases, light grey the growth of the crop, and white represents bare field. The selected imagery dates are indicated with dotted lines.

\subsection{Segmentation}

Multiresolution segmentation (MRS) was chosen as the segmentation algorithm. This function uses scale, shape, compactness, and layer weighting as input parameters. The ESP (Estimation Scale Parameter) tool (developed by Dragut et al. (2010)), which attempts to quantitatively estimate an optimal scale parameter, was applied but recommended an unrealistically large scale factor. A qualitative assessment was consequently carried out by comparing systematic segmentation outputs (with increasing scale parameters) to shapefiles of digitised and verified crop fields. The literature on similar segmentation attempts was also consulted. Based on visual assessment, a favourable delineation of objects was achieved using 0.45, 0.55, and 14 for shape, compactness and scale factor respectively, with a slightly heavier weighting for the green, red, near-infrared, and short wave infrared spectral bands.

\subsection{Image Feature Set Generation}

Several other features (in addition to the Landsat8 spectral bands) were used as input to the classifications. Table 2 outlines the 205 features considered (41 per image capture date). For the spectral features, mean and standard deviation values were calculated for each of the objects. Coastal aerosol (band 1), cirrus (band 8), thermal infrared 1 (band 10), and thermal infrared 2 (band 11) were excluded as they were deemed unsuitable for crop type differentiation. Nine indices commonly used in vegetation studies were generated from the remaining bands. The textural features considered included contrast, correlation, and entropy, as recommended by Clausi (2002). Several image transforms relating to principal component analysis (PCA), colour transformations and tasselled cap were carried out. Coefficients for the latter were obtained from Baig et al. (2014).

Table 1. Features used as input for the DT, NN, SVM, and RT classifiers.

\begin{tabular}{l|ll} 
Type & Subtype & Features \\
\hline $\begin{array}{l}\text { Spectral } \\
\text { Features }\end{array}$ & Mean & $\begin{array}{l}\text { Blue, Green, Red, NIR, SWIR 1, } \\
\text { SWIR 2, Panchromatic } \\
\text { Green, Red, NIR, SWIR 1, SWIR 2 }\end{array}$ \\
\hline Indices & SD & $\begin{array}{l}\text { ARVI, EVI, GCI, GNDVI, Green } \\
\text { Index, NDVI, RGR, SAVI, SRI }\end{array}$ \\
\hline $\begin{array}{l}\text { Textural } \\
\text { features }\end{array}$ & GLCM & $\begin{array}{l}\text { Contrast, Correlation, Entropy, } \\
\text { Homogeneity } \\
\text { Correlation, Entropy, Mean }\end{array}$ \\
\hline $\begin{array}{l}\text { Image } \\
\text { transforms }\end{array}$ & GLDV & PCA \\
& Tasselled & $\begin{array}{l}\text { PC1, PC2, PC3, PC4 } \\
\text { Brightness, Greenness, Wetness, } \\
\text { Transformation 4, Transformation 5, } \\
\text { Transformation 6 } \\
\text { Hue, Saturation, Intensity }\end{array}$
\end{tabular}

\subsection{Feature Selection}

A number of feature selection and reduction methods were used in this study. This included the two filter-based feature selection methods CART and RF, which were carried out using Salford Systems Data Mining and Predictive Software. The feature reduction technique PCA was performed in ArcMap. Manual feature subset selection was also done.

For RF the number of trees to build was set to 1000 for better performance, as computation time and power was not as issue. The number of variables to be considered at each node was based on the Breiman (2001) recommendation of the square root of $\mathrm{K}$ (where $\mathrm{K}$ is the number of predictors). Boot strap sample size was left at AUTO and a balanced class weighting was applied, as recommended by the Salford Systems user's manual.

For CART the search intensity was set to the maximum (400), the splitting method was set to GINI (as done by Yu et al. (2006) and Lewis (2000)), while the V-fold cross-validation was set to 10, as done by Lewis (2000) and recommended by the Salford Systems user's manual. The maximum number of nodes and depth was set to AUTO, the "apply no penalties to any variables" setting was enabled and a threshold level of 15 was set for enabling intelligent categorical split search.

Variable importance, as produced by CART and RF, was used to select a relevant subsets of features. Feature reduction and selection were done iteratively with user defined intervals of 75 , $50,25,20,15,10,5,4,3,2$, and 1 variable(s). The CART variable importance scores tapered off as soon 75 features were used, so no selection iterations larger than 75 were implemented. 
PCA was generated using the original image bands, and the number of components used was based on eigenvalue percentages so as to remove components that accounted for insignificant covariance.

\subsection{Classification Software}

The Supervised Learning and Image Classification Environment (SLICE) software developed by the Centre for Geographical Analysis was used for classification and accuracy assessment. SLICE was developed using the $\mathrm{C}++$ programming language and libraries from OpenCV 2.2 (Bradski \& Pisarevsky 2000) and Libsvm (Chang and Lin 2011). SLICE includes a range of classification algorithms, including decision trees (DTs), knearest neighbour (k-NN), random forests (RF) (called random trees in OpenCV), and support vector machine (SVM). For SVM the radial basis function kernel was chosen for this study, as recommended by $\mathrm{Hsu}$ et al. (2010), and for the k-NN classification k was set to 1 as used by Qian et al. (2015). The geospatial data abstraction library is used to manipulate shapefiles and raster files. A 3:2 sample split ratio was employed for classification and accuracy assessment (the same set of training and testing samples were used for all experiments).

\section{RESULTS AND DISCUSSION}

The results (Table 2) show that feature selection and reduction improved classification accuracies for three out of the four classifiers. In Figures 3 and 4 it is clear that DT, NN, and RF initially benefits from feature selection/reduction, but then accuracy is reduced as the information content diminishes. The two tree classifiers showed smaller improvements with feature selection/reduction compared to NN. This is expected, given that $\mathrm{NN}$ has been shown to be sensitive under conditions of high dimensionality (Myburgh \& Van Niekerk 2014).

Table 2. Overall accuracies and kappa coefficients for all classifications scenarios. All scenarios are also assigned a run

\begin{tabular}{rll|llllllll}
\multicolumn{10}{c}{ number. } \\
\hline Run & Feature & Feature & DT & DT & NN & NN & RF & RF & SVM & SVM \\
No. & Reduction & Count & OA & K & OA & K & OA & K & OA & K \\
\hline 1 & None & 205 & 81,8 & 0,78 & 78,9 & 0,75 & 87,5 & 0,82 & 95,9 & 0,95 \\
2 & CART & 75 & 86,3 & 0,83 & 88,1 & 0,85 & 89,3 & 0,87 & 93,9 & 0,92 \\
3 & CART & 60 & 83,0 & 0,79 & 87,1 & 0,84 & 88,7 & 0,86 & 92,3 & 0,90 \\
4 & CART & 50 & 80,2 & 0,76 & 78,4 & 0,74 & 84,9 & 0,82 & 91,9 & 0,90 \\
5 & CART & 40 & 75,2 & 0,70 & 77,7 & 0,73 & 84,5 & 0,81 & 90,2 & 0,88 \\
6 & CART & 30 & 73,8 & 0,68 & 76,9 & 0,72 & 82,3 & 0,79 & 87,7 & 0,85 \\
7 & CART & 20 & 73,8 & 0,68 & 64,6 & 0,58 & 79,4 & 0,75 & 82,4 & 0,79 \\
8 & CART & 10 & 68,2 & 0,62 & 63,4 & 0,57 & 72,5 & 0,67 & 74,0 & 0,69 \\
9 & CART & 5 & 55,8 & 0,47 & 60,0 & 0,52 & 65,2 & 0,58 & 70,4 & 0,64 \\
10 & RF & 150 & 78,9 & 0,75 & 82,0 & 0,78 & 85,0 & 0,82 & 90,5 & 0,88 \\
11 & RF & 100 & 83,7 & 0,80 & 91,6 & 0,90 & 89,5 & 0,87 & 93,7 & 0,92 \\
12 & RF & 75 & 84,8 & 0,82 & 94,9 & 0,93 & 89,5 & 0,87 & 94,9 & 0,93 \\
13 & RF & 60 & 81,8 & 0,78 & 89,9 & 0,88 & 88,5 & 0,86 & 92,4 & 0,91 \\
14 & RF & 50 & 81,0 & 0,77 & 88,2 & 0,86 & 86,6 & 0,84 & 92,1 & 0,90 \\
15 & RF & 40 & 80,2 & 0,76 & 87,5 & 0,85 & 86,3 & 0,84 & 89,8 & 0,87 \\
16 & RF & 30 & 76,7 & 0,72 & 84,4 & 0,81 & 79,2 & 0,75 & 90,8 & 0,89 \\
17 & RF & 20 & 76,4 & 0,72 & 78,0 & 0,74 & 79,1 & 0,75 & 89,0 & 0,87 \\
18 & RF & 10 & 73,4 & 0,69 & 73,5 & 0,68 & 78,9 & 0,75 & 82,1 & 0,78 \\
19 & RF & 5 & 61,3 & 0,54 & 58,8 & 0,51 & 74,1 & 0,69 & 68,8 & 0,63 \\
20 & Band Means & 35 & 71,3 & 0,66 & 88,7 & 0,86 & 86,2 & 0,83 & 90,5 & 0,88 \\
21 & Band Means \& SD & 60 & 72,6 & 0,67 & 89,8 & 0,87 & 86,0 & 0,83 & 92,9 & 0,91 \\
22 & PCA All Dates & 20 & 67,5 & 0,61 & 83,1 & 0,79 & 84,5 & 0,81 & 89,1 & 0,87 \\
23 & PCA Per Image & 20 & 82,1 & 0,78 & 93,0 & 0,91 & 90,1 & 0,88 & 96,2 & 0,95 \\
24 & TCT & 30 & 78,0 & 0,73 & 94,5 & 0,93 & 84,4 & 0,81 & 92,7 & 0,91 \\
25 & Indices & 45 & 67,2 & 0,61 & 64,6 & 0,58 & 78,6 & 0,74 & 87,8 & 0,85 \\
26 & Transforms & 110 & 85,7 & 0,83 & 84,1 & 0,81 & 92,1 & 0,90 & 95,2 & 0,94 \\
27 & Texture Values & 35 & 49,8 & 0,40 & 56,2 & 0,48 & 55,4 & 0,47 & 58,1 & 0,50 \\
& & & & & & & & & &
\end{tabular}

Rodrigues-Galiano et al. (2012) tested RF classification and feature selection on 972 potential variables under similar conditions to this study (multi-seasonal data, Mediterranean climate, classifiers, feature ranking method). Some of the best results achieved in their study were achieved when using reduced feature sets (of around 117). This finding corresponds to the results obtained in this study which indicate that the best classifications were obtained when 75-205 features were used as input. It seems that, for multi-seasonal differentiation of crop types, a feature set of approximately 100 is ideal.

\section{CONCLUSION}

This paper aimed to determine whether or not feature selection/reduction is of any value when discriminating crops using multi-temporal Landsat8 imagery. The results show that feature selection can, under certain scenarios, prove useful, but is ultimately not beneficial when the SVM classifier is used. The DT, NN, and RF classifiers did, however, improve with variable selection based on CART and RF. Given the effort of feature selection and/or reduction, we recommend using SVM (with the full set of features) for mapping crop types using multi-temporal Landsat8 imagery.

\section{ACKNOWLEDGEMENTS}

This work forms part of a larger project titled "Wide-scale modelling of water use and water availability with earth observation/satellite imagery" which was initiated and funded by the Water Research Commission (WRC) of South Africa (contract number K5/2401//4). More information about this project is available in the 2014/2015 WRC Knowledge Review available at www.wrc.org.za. The authors thank the USGS for providing the Landsat 8 data, the Western Cape Department of Agriculture for supplying the crop data census, and the Centre for Geographic Analysis for use of the classification and accuracy assessment software.

\section{REFERENCES}

Bradski G.R., \& Pisarevsky V., 2000. Intel's computer vision library: applications in calibration, stereo, segmentation, tracking, gesture, face and object recognition, 1(1), pp. 2796.

Breiman, L., 2001. Random Forests. Machine Learning, 45(1), pp. 5-32.

Castillejo-Gonzalez, I., \& Lopez-Granados, F., 2009. Object and pixel-based analysis for mapping crops and their agroenvironmental associated measures using QuickBird imagery. Computers and Electronics in Agriculture, 68(2), pp.207-215.

Chang C.C., \& Lin C.J., 2011. LIBSVM: A library for support vector machines. ACM Transactions on Intelligent Systems and Technology, 2(3), pp. 27.

Clausi D.A. 2002. An analysis of co-occurrence texture statistics as a function of grey level quantization. Canadian Journal of Remote Sensing, 28(1), pp 45-62.

Dragut L., Tiede D., \& Levick S., 2010. ESP: a tool to estimate scale parameter for multiresolution image segmentation of remotely sensed data. International Journal of Geographic Information Science, 24(6), pp. 859-871. 
Gislason P.O., Benediktsson J.A., \& Sveinsson J.R., 2006. Random forests for land cover classification. Pattern Recognition Letters 27(4), pp. 294-300.

Hsu C.W., Chang C.C., \& Lin C.J., 2003. A practical guide to support vector classification. Available at: http://www.csie.ntu.edu.tw/ cjlin/papers/guide/guide.pdf

Lewis R.J., 2000. An Introduction to Classification and Regression Tree (CART) Analysis. In Annual Meeting of the Society for Academic Emergency Medicine in San Francisco, California. 1-14.

Monfreda C., Ramankutty N., \& Foley J. A., 2008. Farming the planet: 2. Geographic distribution of crop areas, yields, physiological types, and net primary production in the year 2000. Global biogeochemical cycles 22(1).

Myburgh G., \& Van Niekerk A., 2014. Effect of feature dimensionality on object-based land cover classification: A comparison of three classifiers. South African Journal of Geomatics 2(1), pp. 13-27.

Qian Y., Zhou W., Yan J., Li W., \& Han L., 2015. Comparing Machine Learning Classifiers for Object-Based Land Cover Classification Using Very High Resolution Imagery. Remote Sensing, 7(1), pp. 153-168.

Rodriguez-Galiano V.F., M. Chica-Olmo, F. Abarca-Hernandez P.M., Atkinson, and Jeganathan C., 2012. Random Forest classification of Mediterranean land cover using multi-seasonal imagery and multi-seasonal texture. Remote Sensing of Environment 121(1), pp. 93-107.

Tererai F., Gaertner M., \& Jacobs S.M., 2015. Resilience of invaded riparian landscapes: The potential role of soil-stored seed banks. Environmental Management 55(1), pp.86-99

Yu Q.P., Gong N., Clinton G., Biging M., Kelly M., \& Schirokauer D., 2006. Object-based Detailed Vegetation Classification with Airborne High Spatial Resolution Remote Sensing Imagery. Photogrammetric Engineering and Remote Sensing 72(7), pp. 799-811.

Xin Q., Broich M., Suyker A.E., Yu L., \& Gong P. 2015. Multiscale evaluation of light use efficiency in MODIS gross primary productivity for croplands in the Midwestern United States. Agricultural and Forest Meteorology 201(1), pp. 111-119.

Zhang, Y., Wu, L., Neggaz, N., Wang, S. and Wei, G., 2009. Remote-sensing image classification based on an improved probabilistic neural network. Sensors, 9(9), pp.7516-7539. 


\title{
DETECTING ATLANTIC FOREST PATCHES APPLYING GEOBIA AND DATA MINING TECHNIQUES
}

\author{
C. D. Girolamo Neto ${ }^{\text {a }}$, A. C. M. Pessôa ${ }^{\text {b }}$ T. S. Körting ${ }^{\text {a }}$, L. M. G. Fonseca ${ }^{\text {a }}$ \\ a Image Processing Division - National Institute for Space Research - INPE - cesare@ dsr.inpe.br; (thales; leila)@dpi.inpe.br \\ ${ }^{\mathrm{b}}$ Remote Sensing Division - National Institute for Space Research - INPE - ana.pessoa@inpe.br \\ Av. dos Astronautas, 1758, São José dos Campos, SP, Brazil.
}

\section{KEY WORDS: Land cover, Classification, Landsat-8, Random Forest, Artificial Neural Networks, Feature selection}

\begin{abstract}
:
Brazilian Atlantic Forest is one of the most devastated tropical forests in the world. Considering that approximately only $12 \%$ of its original extent still exists, studies in this area are highly relevant. In this context, this study maps the land cover of Atlantic Forest within the Protected Area of 'Macaé de Cima', in Rio de Janeiro State, Brazil, combining GEOBIA and data mining techniques on an OLI/Landsat-8 image. The methodology proposed in this work includes the following steps: (a) image pan-sharpening; (b) image segmentation; (c) feature selection; (d) classification and (e) model evaluation. A total of 15 features, including spectral information, vegetation indices and principal components were used to distinguish five patterns, including Water, Natural forest, Urban area, Bare soil/pasture and Rocky mountains. Features were selected considering well-known algorithms, such as Wrapper, the Correlation Feature Selection and GainRatio. Following, Artificial Neural Networks, Decision Trees and Random Forests classification algorithms were applied to the dataset. The best results were achieved by Artificial Neural Networks, when features were selected through the Wrapper algorithm. The global classification accuracy obtained was of $98.3 \%$. All the algorithms presented great recall and precision values for the Natural forest, however the patterns of Urban area and Bare soil/pastures presented higher confusion.
\end{abstract}

\section{INTRODUCTION}

Land use and cover (LUC) analysis can be used to determinate how a specific area is being used, highlighting the anthropogenic interactions with the environment. In order to access patterns of LUC changes, it is vital to use data from remote sensing imagery (Brannstrom et al., 2008). This technology allows the generation of LUC maps, showing areas being occupied by pastures, crops, natural vegetation, river courses and other features. They can also indicate areas of risk or those heavily degraded.

One of the most devastated Brazilian biomes is the Atlantic Forest. The second largest Brazilian forest has only $12 \%$ of its initial extent preserved (Ribeiro et al., 2009). A large part of the occupation of this biome has occurred due to the expansion of urban centers and agricultural areas. Among Rio de Janeiro and São Paulo states, most of Atlantic Forest patches are usually in Protected Areas (PA's). LUC analysis on these territories is even more important when considering the possibility of degrading preserved natural vegetation areas (Figueroa \& Sánchez-Cordero, 2008).

A procedure used to perform the LUC classification is the Geographic Object-Based Image Analysis (GEOBIA), which aims to classify an image based on similar characteristics of its objects. In addition to the spectral properties, GEOBIA can evaluate features associated with the shape, texture, contextual and semantic relationships of objects, increasing the chances of a more reliable classification (Camargo et al., 2009).

Another methodology that has been constantly used on image classification is the Data Mining (DM). DM helps GEOBIA in the process of identifying patterns on objects. In this context, some DM classification algorithms have been used on LUC analysis, for instance: Decision Trees were used for vegetation mapping (Colstoun et al., 2003), temporal analysis of agricultural crops (Körting, 2012) and urban LUC (Pinho et al., 2012). Random Forests were applied to classify LUC on various locations (Smith, 2010; Müller et al., 2015). Artificial Neural Networks were used assessing Natural vegetation LUC (Moreira et al., 2013) and Agricultural areas (Andrade et al., 2013). The choice to use each algorithm requires the analysis of the problem. The results of Decision Trees are easy to visualize (Witten et al., 2011), Random Forests can avoid overfitting, and are also not very sensitive to noisy data (Breiman, 2001). The main advantage of Artificial Neural Networks is to solve complex problems (Haykin, 2009) and may outperform other classifiers on LUC classification (Song et al., 2012).

Considering the importance of LUC information on Atlantic Forest areas and the potential of GEOBIA and DM techniques on image classification, this study aims to map the land use and cover of Atlantic Forest within the PA of 'Macaé de Cima', in Rio de Janeiro State, Brazil, using GEOBIA and DM techniques on an OLI/Landsat-8 image.

\section{METHODOLOGY}

\subsection{Study site and Data}

The study site is the PA of Macaé de Cima - Rio de Janeiro State (Figure 1). It is located between the coordinates of $22^{\circ} 17^{\prime} \mathrm{S}-22^{\circ} 27^{\prime} \mathrm{S}$ and $42^{\circ} 35^{\prime} \mathrm{W}-42^{\circ} 12^{\prime} \mathrm{W}$, on the municipalities of Macaé and Nova Friburgo. It has an area of 350.000 square meters on steep region with rocky mountains and small valleys, with $72 \%$ of Atlantic Forest cover (INEA, 2007).

Data was obtained from the Operational Line Imager (OLI) sensor from Landsat- 8 satellite, path/row 216/76 acquired on $10 / 14 / 2014$. 


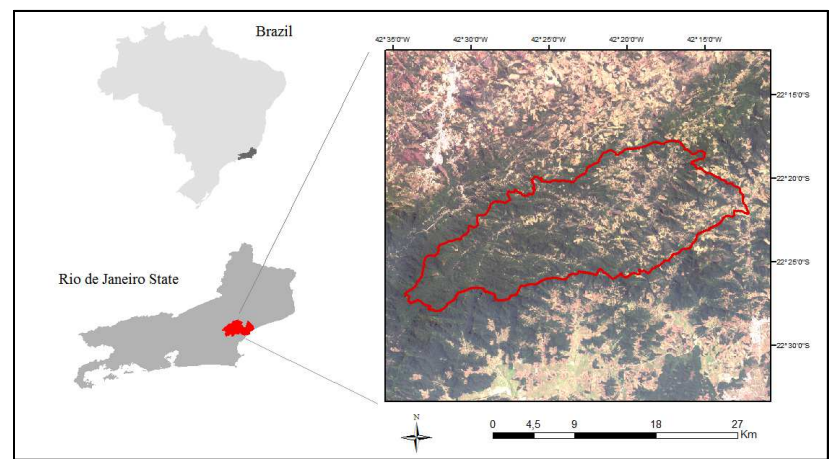

Figure 1. Study site on a true color composition (R4, G3, B2) from the OLI sensor.

\subsection{Image Pan-sharpening}

The original image was pan-sharpened using the Gram-Schmidt method (Laben et al., 2000). Bands 2 to $7(0.450$ to $2.300 \mu \mathrm{m})$ from OLI sensor were used, since this type of pan-sharpening method has generally good results when it is carried out with the same sensor bands (Klonus \& Ehlers, 2009). In addition, this method presented good results when compared to other pan-sharpening methods when applying LUC classification (Bendini et al., 2015).

\subsection{Image Segmentation}

In order to generate the objects for the classification process, the pan-sharpened images were segmented on eCognition software (version 8.64), using the Multi-resolution Segmentation algorithm (eCognition, 2011). Bands 2 to 7 were used in this process and the internal parameters in the algorithm (Scale, Shape and Compactness) were calibrated in order to optimize the segmentation results.

\subsection{Dataset generation and feature selection}

The features obtained from the processed image are on Table 1 . All of them presented mean values from each object (excluding max. diff.).

\begin{tabular}{|l|l|}
\hline Feature & Meaning \\
\hline Band_1 & Surface reflectance from band 1 \\
Band_2 & Surface reflectance from band 2 \\
Band_3 & Surface reflectance from band 3 \\
Band_4 & Surface reflectance from band 4 \\
Band_5 & Surface reflectance from band 5 \\
Band_6 & Surface reflectance from band 6 \\
Band_7 & Surface reflectance from band 7 \\
PCA1 & Principal Component 1 \\
PCA2 & Principal Component 2 \\
PCA3 & Principal Component 3 \\
NDVI & Normalized Difference Vegetation Index \\
NBR & Normalized Burn Ratio \\
B6/B5 & Relation Band6/Band5 \\
Brightness & Average of means from bands 1 to 7 \\
Max. Diff. & Maximum difference between bands \\
Class & Name of the class \\
\hline
\end{tabular}

Table 1. Features of the dataset.

The objects could be classified to distinguish 5 patterns, including Water, Natural forest, Urban area, Bare soil/pasture and Rocky mountains. A visual interpretation of the image was done in order to select approximately 120 samples of each class. A high resolution image, acquired on 30/05/2014 by Google Earth software, was used as a support for the interpretation. The Water class had no more than 80 samples, so it was excluded from the data set, since there was no more Water areas left to be identified in the image.

On the prepared dataset a feature selection process was carried out. This process tends to reduce the computational cost and raise the classification accuracy, by eliminating irrelevant and redundant features of the dataset (Guyon \& Elisseeff, 2003). Tree feature selection methods were used: Wrapper (John \& Kohavi, 1997), Correlation Feature Selection (Hall, 1999) and the GainRatio (Witten et al., 2011).

\subsection{Classification and evaluation}

The classification was performed on the WEKA software, version 3.7.9. (Hall et al., 2009). The algorithms used for classification were Decision Trees (5 and 10 instances per leaf), Artificial Neural Networks (back propagation with one hidden layer with 10 neurons) and Random Forest (100 trees). As a first task, these classifiers were evaluated on a 10 -fold cross validation. Later on, an external data set was used for the evaluation. It contained 30 visually classified samples of each class that were not on the training set. The confusion matrix was used to determine measures such as accuracy, error, precision and recall.

\section{RESULTS AND DISCUSSION}

The GainRatio algorithm ranked the features from the dataset. From the 16 features we decided to reduce the number to 10 , according to the values of information gain ratio. The best merit found on the new dataset was from the NDVI feature, so we decided to test how this feature would influence the classification. We tested the dataset with and without the NDVI feature for the algorithms presented before. The accuracies of the classifiers are presented on Table 2.

\begin{tabular}{|c|c|c|c|c|}
\hline & \multicolumn{2}{|c|}{ With NDVI } & \multicolumn{2}{c|}{ Without NDVI } \\
\hline Algorithm & $\begin{array}{c}\text { Cross } \\
\text { validation }\end{array}$ & Test set & $\begin{array}{c}\text { Cross } \\
\text { validation }\end{array}$ & Test set \\
\hline $\begin{array}{c}\text { Decision Tree } \\
(5)\end{array}$ & 96,6 & 96,7 & 96,6 & 96,7 \\
\hline $\begin{array}{c}\text { Decision Tree } \\
(10)\end{array}$ & 96,8 & 95,8 & 96,8 & 95,8 \\
\hline $\begin{array}{c}\text { Random } \\
\text { Forest }\end{array}$ & 96,6 & 96,7 & 97,0 & 97,5 \\
\hline $\begin{array}{c}\text { Artificial Neural } \\
\text { Networks }\end{array}$ & 97,9 & 97,5 & 98,1 & 97,5 \\
\hline
\end{tabular}

Table 2. Overall accuracy (\%) of GainRatio feature selection with and without NDVI.

The influence of using the NDVI feature was very low. There was no alteration on Decision Trees results and it was noticed a slight improvement on the 10 -fold cross validation without using the NDVI on Artificial Neural Networks and Random Forest. Usually, NDVI tends to apply better results on the classification when it is used with others sensors bands (DeFries $\&$ Townshend, 1994). However, when we analyse the remaining features selected we noticed that band 4 was always selected on all modelling situations. When band 4 is removed instead of the NDVI, the same results are obtained. When both were removed, all results were the same or lower than the previous 
classification, so we assumed that band 4 and NDVI might have correlated information for the classification (since NDVI uses band 4 on its formula). The results of the other algorithms can be found on Table 3 .

\begin{tabular}{|c|c|c|c|c|}
\hline & \multicolumn{2}{|c|}{ CFS } & \multicolumn{2}{c|}{ Wrapper } \\
\hline Algorithm & $\begin{array}{c}\text { Cross } \\
\text { validation }\end{array}$ & Test set & $\begin{array}{c}\text { Cross } \\
\text { validation }\end{array}$ & Test set \\
\hline $\begin{array}{c}\text { Decision Tree } \\
\text { (5) }\end{array}$ & 96,6 & 96,7 & 96,2 & 95,0 \\
\hline $\begin{array}{c}\text { Decision Tree } \\
\text { (10) }\end{array}$ & 96,8 & 95,8 & 96,4 & 95,0 \\
\hline $\begin{array}{c}\text { Random } \\
\text { Forest }\end{array}$ & 97,2 & 96,7 & 97,2 & 96,7 \\
\hline $\begin{array}{c}\text { Artificial Neural } \\
\text { Networks }\end{array}$ & 97,2 & 97,5 & 98,1 & 98,3 \\
\hline
\end{tabular}

Table 3. Overall accuracy (\%) of Correlation Feature Selection and Wrapper for the three algorithms.

The best results were obtained by Artificial Neural Networks with the Wrapper feature selection. The algorithm which presented the second best results was the Random Forest with the GainRatio feature selection. Both scored $98.1 \%$ accuracy on 10 -fold cross validation. We show the confusion matrix of both of these classifiers in order to enhance this discussion (Table 4 and 5). We selected the matrix from the cross-validation because it contained more instances and the errors became more visible, but similar results were spotted on the test set Matrix.

\begin{tabular}{|c|c|c|c|c|c|}
\cline { 2 - 6 } \multicolumn{2}{c|}{} & \multicolumn{4}{c|}{ Classified as } \\
\cline { 2 - 6 } \multicolumn{2}{c|}{} & $\begin{array}{c}\text { Bare soil } \\
\text { /pasture }\end{array}$ & $\begin{array}{c}\text { Natural } \\
\text { forest }\end{array}$ & $\begin{array}{c}\text { Urban } \\
\text { area }\end{array}$ & $\begin{array}{c}\text { Rocky } \\
\text { mountains }\end{array}$ \\
\hline \multirow{2}{*}{$\begin{array}{c}\text { Bare soil } \\
\text { /pasture }\end{array}$} & 119 & 0 & 2 & 0 \\
\cline { 2 - 6 } & $\begin{array}{c}\text { Natural } \\
\text { forest }\end{array}$ & 0 & 121 & 0 & 0 \\
\cline { 2 - 6 } & $\begin{array}{c}\text { Urban } \\
\text { area }\end{array}$ & 2 & 1 & 107 & 3 \\
\cline { 2 - 6 } & $\begin{array}{c}\text { Rocky } \\
\text { mountains }\end{array}$ & 0 & 0 & 1 & 112 \\
\hline
\end{tabular}

Table 4. Confusion matrix for Artificial Neural Networks with Wrapper feature selection.

\begin{tabular}{|c|c|c|c|c|c|}
\hline & \multicolumn{4}{|c|}{ Classified as } \\
\hline & & $\begin{array}{l}\text { Bare soil } \\
\text { /pasture }\end{array}$ & $\begin{array}{c}\text { Natural } \\
\text { forest }\end{array}$ & $\begin{array}{c}\text { Urban } \\
\text { area }\end{array}$ & $\begin{array}{c}\text { Rocky } \\
\text { mountains }\end{array}$ \\
\hline \multirow{4}{*}{ 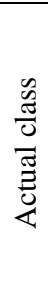 } & $\begin{array}{l}\text { Bare soil } \\
\text { /pasture }\end{array}$ & 117 & 0 & 3 & 1 \\
\hline & $\begin{array}{c}\text { Natural } \\
\text { forest }\end{array}$ & 0 & 120 & 0 & 1 \\
\hline & $\begin{array}{c}\text { Urban } \\
\text { area }\end{array}$ & 6 & 0 & 107 & 1 \\
\hline & $\begin{array}{c}\text { Rocky } \\
\text { mountains }\end{array}$ & 1 & 0 & 1 & 111 \\
\hline
\end{tabular}

Table 5. Confusion matrix for Random Forest with GainRatio feature selection (without NDVI).

Our main objective within this paper is to evaluate the land cover of Atlantic forest. For this purpose, the recall values for this class were $100 \%$ and $99,2 \%$ for the respective classifiers. This result shows that almost all areas of Natural forest were classified correctly. There was almost no confusion of other classes being classified as Natural forest, since the precision values of the models were $99,2 \%$ and $100 \%$. Both of these models worked very well on classifying the forest patches and can be used to map these tiles. The only confusion was a misclassified Forest area with a Rocky mountain area. This error may have occurred because of the presence of vegetation in some mountains, which behave similarly as a vegetation patch (Figure 2).

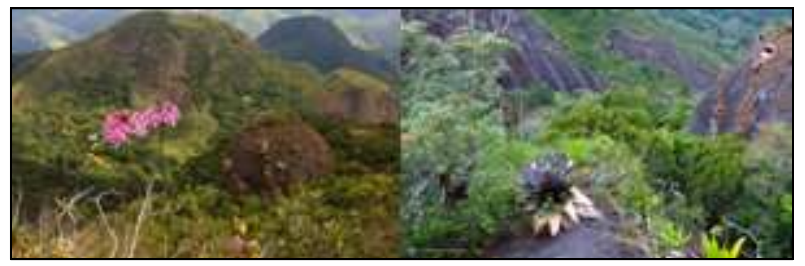

Figure 2. Rocky mountains with some vegetation at the peak of Pedra Riscada - Macaé/RJ.

When considering the other classes, some deficiencies can be spotted. There was a clear confusion between Bare soil/pasture and Urban area. On both classifiers, samples of Bare Soil/pasture were misclassified as Urban area and vice-versa. The Random Forest model was more problematic with this confusion, since the 9 samples were misclassified. The recall values for the Urban area class was considered low on both models $(94,7 \%$ and $93,8 \%)$ when compared to the other classes. The confusion between these classes is a problem noticed before (Kux \& Araujo, 2008; Novack \& Kux, 2010). Considering this is a protected area and it has small agricultural actives, these classes might be encountered together and some objects may contain a mix of both classes.

\section{CONCLUSIONS}

Brazilian Atlantic Forest suffers from devastation and must be protected. Thus, the mapping of its remaining patches is crucial. An automated procedure was adopted involving GEOBIA and DM techniques. Algorithms as Artificial Neural Networks and Random Forest produced encouraging results on identifying these areas (up to $98.3 \%$ accuracy). The only misclassification occurred with some Rocky mountains that have vegetation on it. When considering other investigated patterns, the confusion between Bare soil/pasture and Urban area was more notable. Overall, using GEOBIA with classification algorithms as Artificial Neural Networks or Random Forest is a viable tool for mapping remaining areas of Atlantic Forest on protected areas. However, further adaptations might be required when on sites that have a different class distribution.

\section{REFERENCES}

Andrade, L. N.; Vieira, T. G. C.; Lacerda, W. S.; Volpato, M. M. L. \& Davis Jr, C. A. 2013. Application of artificial neural networks in the classification of coffee areas in Machado, Minas Gerais State. Coffee Science, 8(1), pp. 78-90.

Bendini, H. N.; Girolamo Neto, C. D.; Körting, T. S.; Marujo, R. F. B.; Tranbaquini, K.; Eberhardt, I. D. R.; Sanches, I. D.; Fonseca, L. M. G. 2015. Effects of Image Fusion Methods on Sugarcane Classification with Landsat-8 Imagery. In: XVII Brazilian simposion of remote sensing, pp. 2498-2505.

Brannstrom, C.; Jepson, W.; Filippi, A. M.; Redo, D.; Xu, Z.; \& Ganesh, S. 2008. Land change in the Brazilian Savanna (Cerrado), 1986-2002: comparative analysis and implications for land-use policy. Land Use Policy, 25(4), pp. 579-595. 
Breiman, L. 2001. Random forests. Machine learning, 45(1), pp. 5-32.

Camargo, F. F.; Florenzano, T. G.; Almeida, C. M.; Oliveira, C. G. \& Feitosa, R. Q. 2009. Object-Based Analysis and ASTER/Terra Data for Classifying Relief Units. Bol. Ciênc. Geod., 15(1), pp. 81-102.

Colstoun, E. C. B.; Story, M. H.; Thompson, C.; Commisso, K.; Smith, T. G. \& Irons, J. R. 2003. National Park vegetation mapping using multitemporal Landsat 7 data and a decision tree classifier. Remote Sensing of Environment, 85(3), pp. 316-327.

DeFries, R. S. \& Townshend, J. R. G. 1994. NDVI-derived land cover classifications at a global scale. International Journal of Remote Sensing, 15(17), pp. 3567-3586.

eCognition, 2011. eCognition Developer 8.64.1 users guide. Trimble Germany.

Figueroa, F. \& Sánchez-Cordero, V. 2008. Effectiveness of natural protected areas to prevent land use and land cover change in Mexico. Biodiversity and Conservation, 17(13), pp. 3223-3240.

Guyon, I.; Elisseeff, A. 2003. An introduction to variable and feature selection. Journal of Machine Learning Research. 3, pp. 1157-1182.

Hall, M. A. 1999. Correlation-based feature selection for machine learning. 178p.

Hall, M. A.; Frank, E.; Holmes, G.; Pfahringer, B.; Reutemann, P.; Witten, I. H. 2009. The WEKA Data Mining Software: An Update; SIGKDD Explorations. 11(1) pp. 10-18.

Haykin, S. 2009. Neural Networks and Learning Machines. Prentice-Hall.

INEA - Instituto Estadual do Ambiente/RJ (Rio de Janeiro state environmental institute). 2007. http://www.inea.rj.gov.br/ Portal/Agendas/BIODIVERSIDADEEAREASPROTEGIDAS/ UnidadesdeConservacao/INEA_008619\#/ConselhoConsultivo. (06 may 2016).

John, G. H.; Kohavi, R. 1997. Wrappers for feature subset selection. Artificial Inteligence. 97(1-2), pp. 273-324.

Klonus, S. \& Ehlers, M. 2009. Performance of evaluation methods in image fusion. In: 12th International Conference on Information Fusion, IEEE. pp. 1409-1416.

Körting, T. S. 2012. GeoDMA: a toolbox integrating data mining with object-based and multi-temporal analysis of satellite remotely sensed imagery. $119 \mathrm{p}$.

http://urlib.net/8JMKD3MGP7W/3CCH86S. (03 apr. 2016).

Kux, H. J. H. \& Araujo, E. H. G. 2008. Object-based image analysis using QuickBird satellite image and GIS data, case study Belo Horizonte (Brazil). In: Blaschke, T.; Lang, S.; Hay, G. J. Object-Based Image Analysis-Spatial Concepts for Knowledge-Driven Remote Sensing Applications. pp. 531-553.
Laben, C. A.; Bernard V. \& Brower W. 2000. Process for enhancing the spatial resolution of multispectral imagery using pan-sharpening, US Patent $6,011,875$.

http://www.google.com/patents/US6011875. (18 apr. 2016).

Moreira, G. F.; Fernandes, R. B. A.; Fernandes Filho, E. I.; Vieira, C. A. O. \& Santos, K. A. 2013. Automated

Classification of Use and Land Cover from Landsat Images. Revista Brasileira de Geografia Física, 6(1), pp. 58-65.

Müller, H.; Rufin, P.; Griffiths, P.; Siqueira, A. J. B. \& Hostert, P. 2015. Mining dense Landsat time series for separating cropland and pasture in a heterogeneous Brazilian savanna landscape. Remote Sensing of Environment, 156, pp. 490-499.

Novack, T. \& Kux, H. J. H. 2010. Urban land cover and land use classification of an informal settlement area using the open-source knowledge-based system InterIMAGE. Journal of Spatial Science, 55(1), pp. 23-41.

Pinho, C. M. D.; Fonseca, L. M. G.; Körting, T. S.; Almeida, C. M.; Kux, H. J. H. 2012. Land-cover classification of an intra-urban environment using high-resolution images and object-based image analysis. International Journal of Remote Sensing, 33(19), pp. 5973-5995.

Ribeiro, M. C.; Metzger, J. P.; Martensen, A. C.; Ponzoni, F. J.; Hirota, M. M. 2009. The Brazilian Atlantic Forest: How much is left, and how is the remaining forest distributed? Implications for conservation. Biological Conservation, 142(6), pp. 1141-1153.

Smith, A. (2010). Image segmentation scale parameter optimization and land cover classification using the Random Forest algorithm. Journal of Spatial Science, 55(1), pp. 69-79.

Song, X.; Duan, Z. \& Jiang, X. 2012. Comparison of artificial neural networks and support vector machine classifiers for land cover classification in Northern China using a SPOT-5 HRG image. International Journal of Remote Sensing, 33(10), pp. 3301-3320.

Witten, I. H.; Frank, E.; Hall, M. A. 2011. Data Mining: Practical machine learning tools and techniques. Morgan Kaufmann. 


\title{
DEEP LEARNING FOR SUPERPIXEL-BASED CLASSIFICATION OF REMOTE SENSING IMAGES
}

\author{
C. Gonzalo-Martín ${ }^{\mathrm{a}, \mathrm{b} *}$,A. Garcia-Pedrero ${ }^{\mathrm{a}, \mathrm{b}}$, M. Lillo-Saavedra ${ }^{\mathrm{c}, \mathrm{d}}$, E. Menasalvas ${ }^{\mathrm{a}, \mathrm{b}}$ \\ ${ }^{a}$ Center for Biomedical Technology, Universidad Politécnica de Madrid, Campus de Montegancedo, \\ Pozuelo de Alarcón 28233, Spain - \{consuelo.gonzalo\}@ upm.es \\ ${ }^{\mathrm{b}}$ Escuela Técnica Superior de Ingenieros Informáticos, Universidad Politécnica de Madrid, Campus de Montegancedo, \\ Boadilla del Monte 28660, Spain \\ ${ }^{\mathrm{c}}$ Faculty of Agricultural Engineering, University of Concepción, Chile \\ ${ }^{\mathrm{d}}$ Water Research Center for Agriculture and Mining, CRHIAM, University of Concepción, Chile
}

KEY WORDS: Convolutional Neural Networks, Remote Sensing Image Classification, Superpixels

\begin{abstract}
:
Recently deep learning-based methods have demonstrated excellent performance on different artificial-intelligence tasks. Even though, in the last years, several related works are found in the literature in the remote sensing field, a small percentage of them address the classification problem. These works propose schemes based on image patches to perform pixel-based image classification. Due to the typical remote sensing image size, the main drawback of these schemes is the time required by the window-sliding process implied in them. In this work, we propose a strategy to reduce the time spent on the classification of a new image through the use of superpixel segmentation. Several experiments using CNNs trained with different sizes of patches and superpixels have been performed on the ISPRS semantic labeling benchmark. Obtained results show that while the accuracy of the classification carried out by using superpixels is similar to the results generated by pixel-based approach, the expended time is dramatically decreased by means of reducing the number of elements to label.
\end{abstract}

\section{INTRODUCTION}

Recently deep learning-based methods have demonstrated excellent performance on different artificial-intelligence tasks, including speech recognition (Hinton et al., 2012a), natural language processing (Dahl et al. 2012), and computer vision (Krizhevsky et al. 2012). In the later area, Convolutional Neural Networks (CNNs) play a major role for processing visual-related problems such as image classification (Lee et al., 2009, Sermanet et al. 2013), object detection (Girshick et al. 2014), and face recognition (Taigman et al. 2014).

CNNs are biological-inspired variants of feed-forward neural networks, where each layer is a non-linear feature detector performing local processing of contiguous features within each layer Biem 2014). In this regard, the CNN architecture is able to exploit the strong spatially local correlation present in natural images by enforcing a local connectivity pattern between neurons of adjacent layers. This leads to higher conceptual representation as information moves up to the output layer. In this context, a CNN is able to generate patterns from an image in a hierarchical manner similar to that of the mammalian visual cortex. Empirical studies have demonstrated that these methods often provided better results than traditional machine learning methods (Larochelle et al. 2009 Salakhutdinov and Hinton, 2009).

Two different approaches to exploit the hierarchical analysis capacity of CNN for image analysis can be found in literature: feature extraction and classification. In feature extraction approach, pre-trained CNN models are used to automatically extract image features that later are analyzed by traditional machine learning methods (Sharif Razavian et al. 2014). In classification approach, a CNN is trained from scratch using a large set of images (Krizhevsky et al. 2012), which requires high performance equipment for processing (e.g., graphics processing units).

\footnotetext{
${ }^{*}$ Corresponding author
}

As far as our knowledge the use of CNNs for processing remotely sensed imagery is relatively recent. Particularly, CNNs have been used in remote sensing area for generating thematic maps following a pixel-based approach (Paisitkriangkrai et al. 2015, Zou et al. 2015). In a pixel-based approach, during training phase, training images are broken down into overlapping patches, where each patch is centered on a pixel which provide the class for the whole patch. Once CNN is trained with these patches, it is applied to test images using a window-sliding approach in order to provide a label to each pixel in the images (pixel-based classification). As it is known, window-sliding approach is a highly time-consuming process, especially when images have billions of pixels as very-high resolution remote sensing images.

In this work, we propose a strategy to reduce the time spent to classify a new image through the use superpixel segmentation. Superpixels (SPs) are a form of image segmentation, but the focus lies more on a controlled oversegmentation, not on segmenting meaningful objects. By controlling the size and compactness of the SPs, the image can be divided into several homogeneous regions with determined number of pixels. Thus it is possible to create SPs that can be completely contained by a patch of determined size. In this way, SPs are expected to maintain all the characteristics of a reduced environment inside of the area learned by a CNN. This allows to generate, during testing phase, only patches which centers correspond to the centroids of the SPs. This reduces the number of elements that must be considered in the window-sliding approach during labeling process of a new image, which is a bottleneck of this type of approaches.

Several experiments using CNNs trained with different sizes of windows and SPs have been performed on the ISPRS semantic labeling benchmark. Obtained results show that while the accuracy of the classification carried out by using SPs is similar to the results generated by the conventional window-sliding approach, the expended time is dramatically decreased by means of reducing the number of elements to label. 


\section{DATA AND METHODS}

\subsection{Data description}

In this work, the ISPRS labeling contest dataset ${ }^{1}$ has been used This dataset consists of very-high resolution true ortho-photo (TOP) tiles and their corresponding digital surface models (DSMs) The images includes different urban scenes from a relatively small village with many detached buildings and small multi story buildings (Vaihingen, Germany). In addition, sixteen labeled scenes serving as ground-truth data are part of this dataset. These images have been classified manually into six land cover classes: impervious surfaces, building, low vegetation, tree, car, and clutter/background. This last class includes different types of landcovers that have a small presence in the analyzed scenes.

\subsection{Superpixels}

Superpixel processing is carried out by the SLIC algorithm (Achanta et al. 2010), which is based on the well-known k-means method to group pixels in a conventional color space. SLIC superpixels are generated according to two criteria: spectral similarity (limited to three channels) and spatial proximity. In the SLIC procedure, the generation of SPs is based on the assumption that limiting the search space to a region proportional to the desired SP size reduces considerably the calculation time. In fact, its computational complexity is linear in the number of pixels in the image (Achanta et al. 2012). Moreover, a weighted distance that combines spectral and spatial proximity allows controlling the size and compactness of the SP (Achanta et al. 2012). Therefore, it has two parameters: $k$, the desired number of superpixels, and $c$, the compactness factor. A larger value of $c$ emphasizes the importance of the spatial proximity resulting in more compact SPs.

In this work, a modified version of SLIC (Gonzalo-Martín et al. 2016. Garcia-Pedrero et al. 2015) is used to generate SPs. This version extends the definition of spectral proximity provided by the original SLIC to work with multispectral images of $B$ bands. The first step of the segmentation framework begins with the sampling of $k$ initial cluster centers on a regularly spaced grid of $g$ pixels. The initial centers are defined as:

$$
C_{i}=\left[p^{1}, p^{2}, \ldots, p^{B}, x, y\right]^{T}, \quad i=1,2, \ldots, k
$$

where $p^{b}$ represents the spectral value in band $b-t h$ of pixel $p$ at position $x$ and $y$, and $B$ denotes the number of spectral bands. To produce similar sized superpixels, the grid interval is defined as $g=N / k$, where $N$ is the total number of pixels in the image. $g$ determines the size of the superpixels, the greater value of $g$, the larger the SPs. This allows to adapt superpixels to specific requirements for real-world applications as a determined scale of analysis.

In the next step, each pixel $p$ is associated to the nearest cluster center whose search space overlaps its location. The search region is enclosed to an area of $2 g \times 2 g$ pixels around each superpixel center. Then the cluster centers are updated to be the mean vector of all pixels belonging to the cluster. Both steps are repeated iteratively until a maximum of 10 iterations, since no further significant changes in superpixel quality could be observed (Achanta et al. 2010).

The clustering distance is a weighted relationship between spectral and spatial measures. The first measure ensures superpixel homogeneity, and the second one enforces compactness and regularity

1 http://www2.isprs .org/commissions/comm3/wg4/ 2d-sem-label-vaihingen.html Last access: June 2016. in superpixels shape. In order to work with multispectral images, the spectral square distance between pixels $i$ and $j$ is defined as follows:

$$
d_{c}^{2}=\sum_{b=1}^{B}\left(p_{i}^{b}-p_{j}^{b}\right)^{2}
$$

The spatial square distance is calculated as:

$$
d_{s}^{2}=\left(x_{i}-x_{j}\right)^{2}+\left(y_{i}-y_{j}\right)^{2}
$$

where $x$ and $y$ denote the position of the pixel. Finally, the clustering distance is calculated as:

$$
D=d_{c}+\left(\frac{c}{g}\right) d_{s}
$$

where $c$ controls the compactness of the superpixels. According to Garcia-Pedrero et al. (2015), a 3.9\% of the maximum pixel value in image as $c$ is optimal.

\subsection{Convolutional Neural Network}

The concept of CNN is not new, in fact, it was first proposed in 1980 by (Fukushima, 1980) with the name of NeoCognitron, and later refined by (LeCun et al., 1989). CNNs have some characteristics that distinguish them from traditional feed-forward neural networks. Unlike traditional feed-forward layers, convolutional layers have neurons with limited receptive fields, i.e., they only process a local image region that affects a particular element in the output. Moreover, as their name reflects, the output of this layer is computed as a spatial convolution using a learned filter over its input. The result of this convolution is a set of features of the image. Nowadays, both technological and algorithmic advances have allow the implementation and use of CNNs. Regarding technological advances, it should be mentioned the advent on the market of affordable graphics processing units (GPUs), together the availability of large databases of annotated images. From an algorithmic point of view, two main contributions have been the proposal of the rectified linear unit (ReLu) (Jarrett et al. 2009), which allows a faster training, and the dropout strategy (Hinton et al. 2012b) to reduce overfitting. All these advances were used by Krizhevsky et al. (2012) achieving outstanding results in image processing tasks. This work may be considered as the starting point for the widespread use of CNNs.

$\mathrm{CNN}$ architecture typically comprises several layers of different types (Castelluccio et al. 2015):

Convolutional layers. As their name suggests, they compute the convolution of the input image with the weights of the network. These layers are characterized by few parameters: the size of filters, the filter spatial support, the step between different windows and an optional zero-padding which controls the size of the layer output. The analysis of the image is done at different scale in the different layers. As the layers are deeper, the features extracted from the image are higher-level.

Pooling layers. The mission of these layers is to reduce the size of the input layer through some local non-linear operations. Their most important parameters are the support of the pooling window and the step between different windows.

Normalization layers. Their objective is to improve generalization of the CNN. For that, they use inhibition schemes inspired in the real neurons of the brain. Neurons typically used in these layers are sigmoid type. 
Fully-connected layers. These layers are typically used in the last levels of the network. Since the size of the image is reduced in the previous layers, their full connectivity is not a limitation for using them. These layers have the capacity of abstracting the low-level information generated in previous layers for a final decision.

\subsection{Architecture}

The CNN architecture used in this work is inspired on the approach presented in Paisitkriangkrai et al. (2015). A scheme of this architecture is shown in Figure 1 It is defined by the alternation of three convolutional layers, which compute the convolution between the input of each layer and a set of learned filters; and other additional layers (contrast normalization and max-pooling layers), which apply a non-linear transformation (rectified linear unit - ReLU) and sub-samples the output of the corresponding convolutional layer, respectively. The role of these additional layers is to improve the robustness of the network to distortions and small translations. Moreover, two fully-connected layers are included at the end of the architecture. The output of these layers feeds a $k$-way soft-max layer which produces a probability distribution over 6 class labels. To reduce overfitting in the fullyconnected layers, the dropout method has been used (Hinton et al. 2012a). In Figure 1 the expression $i$ x $j \times k$ under each convolutional layer represents the size of the kernels associated to this layer, where the number of kernels for the three first layers are 32, 64, and 128, respectively. Each of these kernels generates a feature map for feeding the next layer.

\subsection{Superpixel-based labeling approach}

As mentioned above, CNNs have demonstrated a good performance in computer vision tasks such as classification where a single label is assigned to the entire image. However, to perform a pixel-based classification it is necessary to break the image down into overlapping patches. Each patch is centered on a pixel which provide the class for the entire patch. $\mathrm{CNN}$ is trained using a large set of patches randomly selected trying to maintain a good distribution of the classes of interest. During the testing phase a window-sliding approach is commonly followed to provide a label to each pixel of a test image. This approach, shown in Figure 2(a). consists in applying a trained CNN to a patch defined by a window, the output label is then assigned to the center pixel of the window. To produce a thematic map, several windows are generated by centering a window in each pixel of the image. A mirror padding strategy is used to label the pixels around the boundaries of the image. Since all pixels must be processed, the sliding window approach is a time consuming task, especially if we consider that a very-high resolution image usually has billions of pixels.

To alleviate this drawback, an alternative approach based on the use of superpixels during labeling process was explored in this work. In the proposed approach, images are segmented into superpixels using the method described in Section 2.2 the resulting superpixels are then used as minimum processing units during labeling process. Since superpixels are the product of a controlled over-segmentation of the image; they tend to be similar in size and color, as well as belonging to only one object. These properties allowed to generate superpixels completely contained by a window of determined size, maintaining all the characteristics of a reduced environment inside of the area to analyze with a CNN. The proposed method reduces the number of windows to those centered on the pixels corresponding to the centroids of the superpixels, as shown in Figure 2(b), then superpixels are labeled according to the output of the CNN obtaining a thematic map. Thus the time required for labeling a new image is drastically reduced.

\section{EXPERIMENTS AND RESULTS}

Since the objective of this work is to prove the effectiveness of the superpixel-based labeling approach (Section 2.5) regarding a traditional window-sliding approach, a set of experiments using different superpixels and windows sizes (W) have been carried out. Twelve images of the labeled dataset have been used for training, while the remaining four $(15,28,34,37)$ were used for testing purposes. From training images, a total of 10000 patches for each class have been randomly selected, then using a proportion of 70-30 to create training and validation sets, respectively. A total of twelve different CNNs were generated, one for each combination between three window sizes $(32 \times 32,48 \times 48,64 \times 64)$ and four SP sizes $(20,30,40,50)$. Each CNN was trained using a different training and validation sets, obtaining an accuracy of about $80 \%$ in the validation set.

Once CNNs were trained, each of them was used for labeling the four testing images. The labeling process was carried out using both the conventional pixel-based (window-sliding) approach and the superpixel-based approach. F1-score values were calculated in each case to measure the error in the classification, and how these results are affected by the labeling approaches. Table 1 summarizes the obtained results.

Figures 3(a), 3(b), and 3(c) represent the differences in percentage between F1-score after applying the different approaches for labeling testing images, using a window size of 32,48 , and 64, respectively. As can be observed, the differences increase as the SP size does. On the other hand, as can be observed in Figure 3(d) differences between both labeling approaches decrease as the window size increases. However the use of larger windows also increase the calculation time due to the increase in the amount of data to be processed (larger patches).

The results show that the pixel-based approach is always slightly better, however in general terms, the difference between the two labeling approaches is negligible. If we estimate the time required for the labeling process in term of processing data, in our experiments, depending on the SP size, the reduction has been around a $96 \%$ of data, when the labeling process is performed based on superpixels against the same process based on pixels (sliding-window).

Figure 4 shows the comparison between the classification results obtained with the pixel-based approach (Figure 4(a) and the superpixel-based approach (Figure 4(b) for a W=64 and SP size of 50. In agreement with numerical results, the differences between both images are contemptible. However, in order to show the possible discrepancies between both images, their difference is displayed in Figure 4(c) As can be appreciated, these differences are mainly related to the edges between regions of different classes.

All experiments have been done using an NVIDIA GTX970 GPU with 1664 CUDA cores and 4 GB of memory. Codes were developed in python using Caffe framework (Jia et al. 2014). The size of the trained CNN has been determined by the time and memory limitations imposed by the GPUs used. In our experiments, the average time reduction has been from 5.5 hours for the pixel-based labeling against $20 \mathrm{~min}$. for the SP-based one.

\section{CONCLUSIONS}

From the results obtained in the experiments carried out in this work, it is concluded that the proposed methodology based on superpixels to automatically label satellite imagery using CNN, 


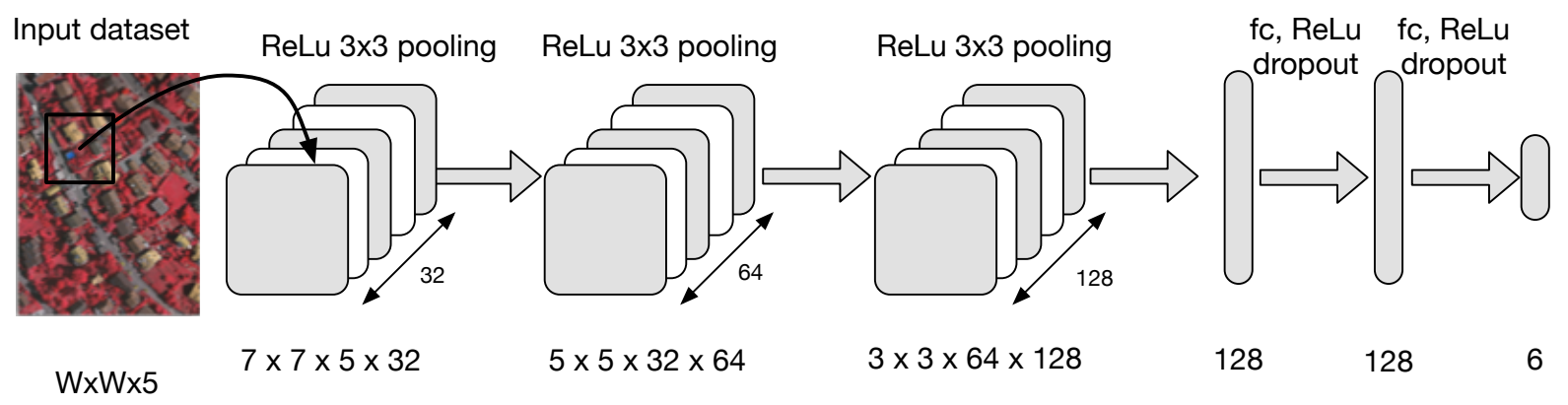

Figure 1. CNN architecture used during the experiments.

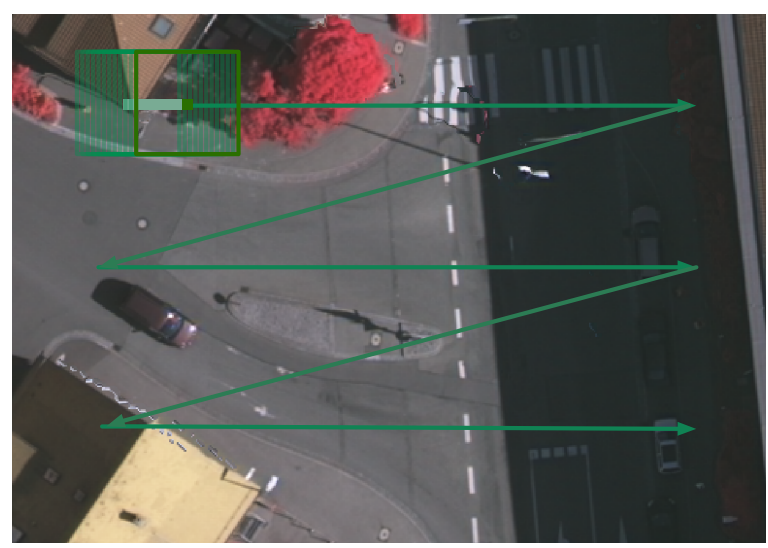

(a) Window-sliding approach

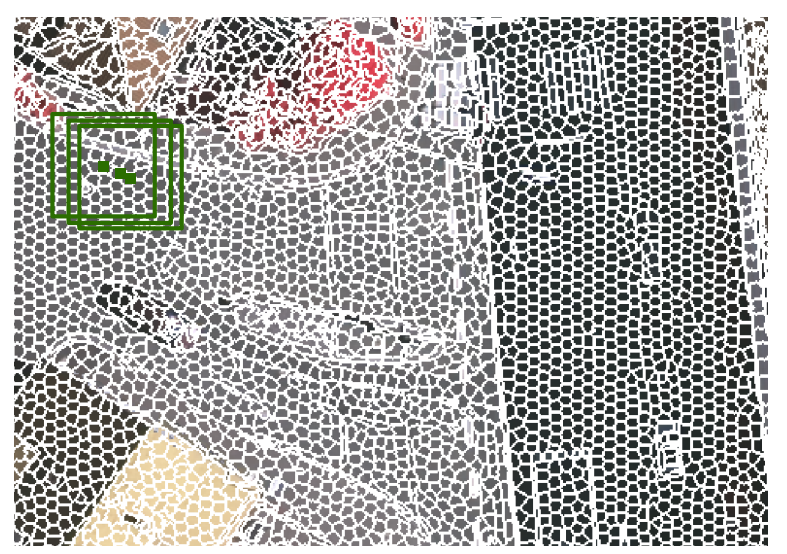

(b) Our approach

Figure 2. Green boxes indicate the windows that serve to feed a CNN during the labeling process in order to generate the final thematic map of the scene under analysis.

Table 1. Classification results (F1-values) obtained by applying the different labeling approaches. The difference between both approaches is shown in percentage.

\begin{tabular}{|c|c|c|c|c|c|c|c|c|c|c|c|c|c|}
\hline \multirow{3}{*}{$\begin{array}{l}\text { W } \\
\text { size }\end{array}$} & \multirow{3}{*}{ Image } & \multicolumn{12}{|c|}{ Superpixel size } \\
\hline & & \multicolumn{3}{|c|}{20} & \multicolumn{3}{|c|}{30} & \multicolumn{3}{|c|}{40} & \multicolumn{3}{|c|}{50} \\
\hline & & Pixel & SP & $\begin{array}{l}\text { Diff. } \\
(\%)\end{array}$ & Pixel & SP & $\begin{array}{l}\text { Diff. } \\
(\%)\end{array}$ & Pixel & SP & $\begin{array}{l}\text { Diff. } \\
(\%)\end{array}$ & Pixel & SP & $\begin{array}{l}\text { Diff. } \\
(\%)\end{array}$ \\
\hline \multirow{4}{*}{32} & 15 & 0.7202 & 0.7187 & 0.1435 & 0.7256 & 0.7233 & 0.2294 & 0.7248 & 0.7219 & 0.2854 & 0.7204 & 0.7166 & 0.3830 \\
\hline & 28 & 0.7312 & 0.7297 & 0.1541 & 0.7346 & 0.7326 & 0.1985 & 0.7306 & 0.7269 & 0.3684 & 0.7384 & 0.7335 & 0.4908 \\
\hline & 34 & 0.7468 & 0.7449 & 0.1858 & 0.7531 & 0.7511 & 0.2041 & 0.7530 & 0.7496 & 0.3386 & 0.7574 & 0.7530 & 0.4344 \\
\hline & 37 & 0.7748 & 0.7732 & 0.1635 & 0.7703 & 0.7678 & 0.2475 & 0.7765 & 0.7732 & 0.3328 & 0.7629 & 0.7585 & 0.4417 \\
\hline \multirow{4}{*}{48} & 15 & 0.7555 & 0.7539 & 0.1643 & 0.7584 & 0.7567 & 0.1770 & 0.7515 & 0.7486 & 0.2865 & 0.7515 & 0.7474 & 0.4093 \\
\hline & 28 & 0.7510 & 0.7493 & 0.1624 & 0.7463 & 0.7435 & 0.2798 & 0.7532 & 0.7497 & 0.3478 & 0.7631 & 0.7587 & 0.4450 \\
\hline & 34 & 0.7591 & 0.7575 & 0.1634 & 0.7618 & 0.7597 & 0.2129 & 0.7692 & 0.7666 & 0.2614 & 0.7703 & 0.7657 & 0.4561 \\
\hline & 37 & 0.7936 & 0.7914 & 0.2107 & 0.7911 & 0.7887 & 0.2335 & 0.7932 & 0.7899 & 0.3301 & 0.7976 & 0.7940 & 0.3638 \\
\hline \multirow{4}{*}{64} & 15 & 0.7401 & 0.7388 & 0.1238 & 0.7617 & 0.7598 & 0.1923 & 0.7540 & 0.7510 & 0.2999 & 0.7548 & 0.7511 & 0.3699 \\
\hline & 28 & 0.7659 & 0.7648 & 0.1135 & 0.7670 & 0.7647 & 0.2264 & 0.7534 & 0.7502 & 0.3148 & 0.7626 & 0.7579 & 0.4706 \\
\hline & 34 & 0.7645 & 0.7626 & 0.1861 & 0.7581 & 0.7561 & 0.1993 & 0.7699 & 0.7666 & 0.3308 & 0.7715 & 0.7673 & 0.4200 \\
\hline & 37 & 0.7986 & 0.7971 & 0.1540 & 0.7971 & 0.7958 & 0.1272 & 0.7978 & 0.7954 & 0.2384 & 0.7979 & 0.7944 & 0.3478 \\
\hline
\end{tabular}

provides a dramatically computer time reduction, estimated on the base of number of data to be processed, with a negligible decrease of the label accuracy. It is hoped that the results can be improved by increasing the deep of the $\mathrm{CNN}$, as well as the size of the windows, which will be feasible with faster GPUs.

\section{ACKNOWLEDGEMENTS}

This work has been funded by the Universidad Politécnica de Madrid (AL16-PID-17). A. Garcia-Pedrero (grant 216146) acknowledges the support for the realization of his doctoral thesis to the Mexican National Council of Science and Technology (CONAСyT).

\section{References}

Achanta, R., Shaji, A., Smith, K., Lucchi, A., Fua, P. and Süsstrunk, S., 2010. Slic superpixels. École Polytechnique Fédéral de Lausssanne (EPFL), Tech. Rep.

Achanta, R., Shaji, A., Smith, K., Lucchi, A., Fua, P. and Susstrunk, S., 2012. SLIC Superpixels Compared to State- 


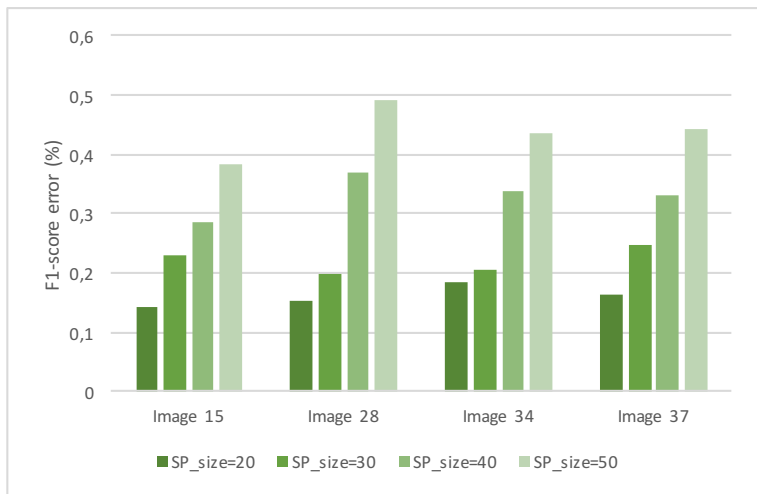

(a) $\mathrm{W}=32$

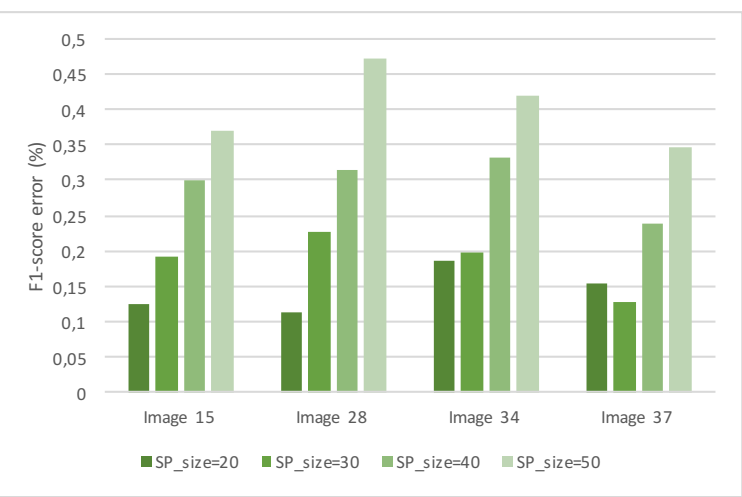

(c) $\mathrm{W}=64$

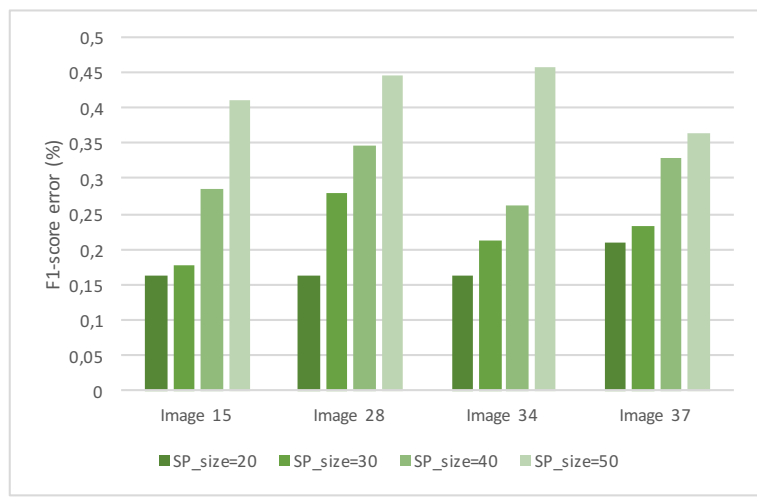

(b) $\mathrm{W}=48$

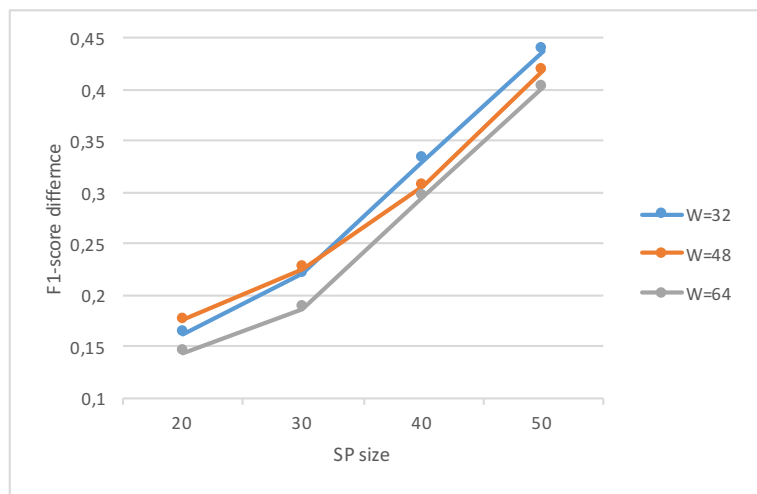

(d) \% Average F1-score error

Figure 3. \% F1-score errors.

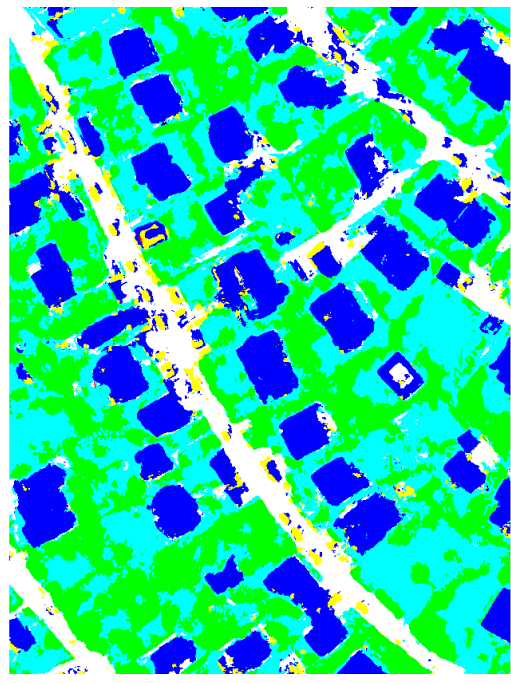

(a) Window-sliding approach

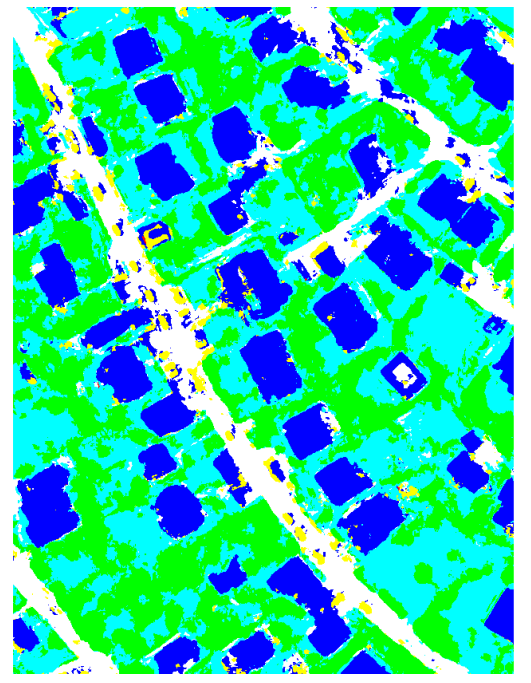

(b) Superpixel approach

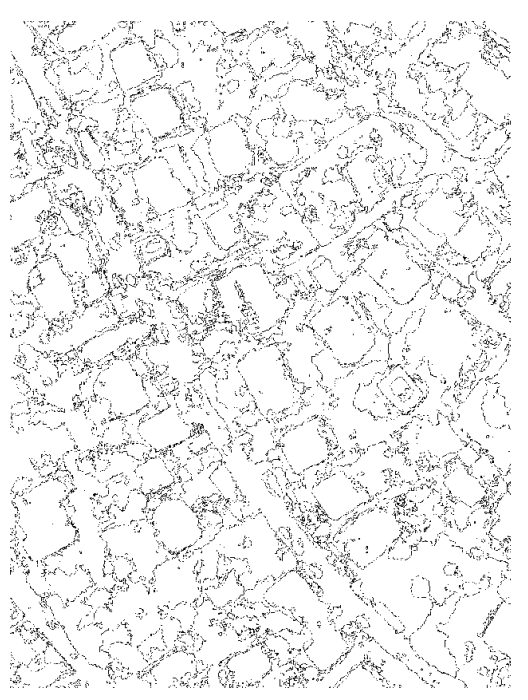

(c) Differences between both approaches

Figure 4. Labeling results.

of-the-Art Superpixel Methods. Pattern Analysis and Machine Intelligence, IEEE Transactions on 34(11), pp. 2274-2282.

Biem, A., 2014. Neural networks: A review. In: C. C. Aggarwal (ed.), Data Classification: Algorithms and Applications, Chapman and Hall/CRC, chapter 8, pp. 206-236.

Castelluccio, M., Poggi, G., Sansone, C. and Verdoliva, L., 2015.
Land use classification in remote sensing images by convolutional neural networks. arXiv preprint arXiv:1508.00092.

Dahl, G. E., Yu, D., Deng, L. and Acero, A., 2012. Context-dependent pre-trained deep neural networks for largevocabulary speech recognition. Audio, Speech, and Language Processing, IEEE Transactions on 20(1), pp. 30-42. 
Fukushima, K., 1980. Neocognitron: A self-organizing neural network model for a mechanism of pattern recognition unaffected by shift in position. Biological cybernetics 36(4), pp. 193-202.

Garcia-Pedrero, A., Gonzalo-Martin, C., Fonseca-Luengo, D. and Lillo-Saavedra, M., 2015. A geobia methodology for fragmented agricultural landscapes. Remote Sensing 7(1), pp. 767 787.

Girshick, R., Donahue, J., Darrell, T. and Malik, J., 2014. Rich feature hierarchies for accurate object detection and semantic segmentation. In: The IEEE Conference on Computer Vision and Pattern Recognition (CVPR).

Gonzalo-Martín, C., Lillo-Saavedra, M., Menasalvas, E., FonsecaLuengo, D., García-Pedrero, A. and Costumero, R., 2016. Local optimal scale in a hierarchical segmentation method for satellite images. Journal of Intelligent Information Systems 46(3), pp. $517-529$.

Hinton, G., Deng, L., Yu, D., Dahl, G. E., r. Mohamed, A., Jaitly, N., Senior, A., Vanhoucke, V., Nguyen, P., Sainath, T. N. and Kingsbury, B., 2012a. Deep neural networks for acoustic modeling in speech recognition: The shared views of four research groups. IEEE Signal Processing Magazine 29(6), pp. 82-97.

Hinton, G. E., Srivastava, N., Krizhevsky, A., Sutskever, I. and Salakhutdinov, R. R., 2012b. Improving neural networks by preventing co-adaptation of feature detectors. arXiv preprint arXiv:1207.0580.

Jarrett, K., Kavukcuoglu, K., Lecun, Y. et al., 2009. What is the best multi-stage architecture for object recognition? In: 2009 IEEE 12th International Conference on Computer Vision, IEEE, pp. 2146-2153.

Jia, Y., Shelhamer, E., Donahue, J., Karayev, S., Long, J., Girshick, R., Guadarrama, S. and Darrell, T., 2014. Caffe: Convolutional architecture for fast feature embedding. arXiv preprint arXiv: 1408.5093.

Krizhevsky, A., Sutskever, I. and Hinton, G. E., 2012. Imagenet classification with deep convolutional neural networks. In: Advances in neural information processing systems, pp. 10971105.

Larochelle, H., Bengio, Y., Louradour, J. and Lamblin, P., 2009. Exploring strategies for training deep neural networks. The Journal of Machine Learning Research 10, pp. 1-40.

LeCun, Y., Boser, B., Denker, J. S., Henderson, D., Howard, R. E., Hubbard, W. and Jackel, L. D., 1989. Backpropagation applied to handwritten zip code recognition. Neural computation 1(4), pp. 541-551.

Lee, H., Grosse, R., Ranganath, R. and Ng, A. Y., 2009. Convolutional deep belief networks for scalable unsupervised learning of hierarchical representations. In: Proceedings of the 26th Annual International Conference on Machine Learning, ACM, pp. 609-616.

Paisitkriangkrai, S., Sherrah, J., Janney, P. and Hengel, A., 2015. Effective semantic pixel labelling with convolutional networks and conditional random fields. In: Proceedings of the IEEE Conference on Computer Vision and Pattern Recognition Workshops, pp. 36-43.

Salakhutdinov, R. and Hinton, G. E., 2009. Deep boltzmann machines. In: International conference on artificial intelligence and statistics, pp. 448-455.

Sermanet, P., Eigen, D., Zhang, X., Mathieu, M., Fergus, R. and LeCun, Y., 2013. Overfeat: Integrated recognition, localization and detection using convolutional networks. CoRR.
Sharif Razavian, A., Azizpour, H., Sullivan, J. and Carlsson, S., 2014. Cnn features off-the-shelf: an astounding baseline for recognition. In: Proceedings of the IEEE Conference on Computer Vision and Pattern Recognition Workshops, pp. 806-813.

Taigman, Y., Yang, M., Ranzato, M. and Wolf, L., 2014. Deepface: Closing the gap to human-level performance in face verification. In: The IEEE Conference on Computer Vision and Pattern Recognition (CVPR).

Zou, Q., Ni, L., Zhang, T. and Wang, Q., 2015. Deep learning based feature selection for remote sensing scene classification. IEEE Geoscience and Remote Sensing Letters 12(11), pp. 23212325 . 


\title{
AUTOMATIC DETECTION OF LANDSLIDES IN OBJECT-BASED ENVIRONMENT USING OPEN SOURCE TOOLS
}

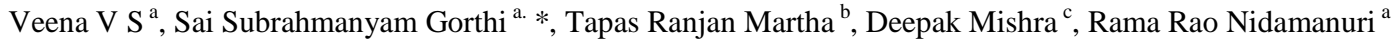 \\ ${ }^{a}$ Dept. of Earth and Space Sciences, Indian Institute of Space Science and Technology, Trivandrum, India \\ ${ }^{\mathrm{b}}$ Geosciences Group, National Remote Sensing Centre, Hyderabad, India \\ ${ }^{c}$ Dept. Of Avionics, Indian Institute of Space Science and Technology, Trivandrum, India \\ email: saisubrahmanyam.gorthi@gmail.com
}

KEY WORDS: Simple Linear Iterative Clustering, Object Based Image Analysis, Green Normalized Difference Vegetation Index, Digital Elevation Model, Principal Component Analysis, Speeded Up Robust Features

\begin{abstract}
:
Automatic detection of landslides from very high resolution satellite images is a prerequisite for rapid damage assessment and supporting disaster management activities. In this study, a novel method using open source tools was developed for extracting landslides from bi-temporal satellite images based on Object Based Image Analysis (OBIA). The methodology employed involves image segmentation followed by elimination of non-landslide candidates using object based change detection techniques and lastly, unsupervised classification. Brightness of a post-landslide image is higher in comparison to its pre-landslide image hence a suitable threshold value for post image brightness was set to demarcate the landslide affected regions from the other land cover types. Further, landslide diagnostic parameters such as difference in Green Normalized Difference Vegetation Index (GNDVI), Digital Elevation Model (DEM, slope, Principal Component Analysis (PCA) and difference in Top of Atmosphere (ToA) values were used to eliminate challenging false candidates such as snow cover, barren land and river sediments. The objects retained after eliminating false candidates are then classified into two classes using k-means clustering algorithm. The local features associated with an image can be computed by finding the key points using a Speeded Up Robust Feature (SURF). Performance of this method was investigated using Resourcesat-2 LISS-IV multispectral $(5 \mathrm{~m})$ bi-temporal satellite image covering parts of Uttarakhand state in India. Results show that the proposed methodology will aid rapid inventorisation of landslides.
\end{abstract}

\footnotetext{
* Corresponding author
} 


\section{INTRODUCTION}

Landslide inventory from remote sensing images is essential. Any effort to reduce the risk of landslide should start with an inventory, a detailed map showing where the landslides have occurred. The possibility of detection of historical landslides provides a reference strategy for setting long term disaster management activities while rapid inventorisation of newly triggered landslide is less for immediate disaster response. Therefore, an automatic technique to detect landslides from very high resolution satellite imaging is crucial in overcoming the limitations that exist in traditional field inspection method and for the efficient utilization of high resolution data availability.

Generally, the regions corresponding to a landslide region are identified based on its spectral characteristics. Detection of landslide with the traditional pixel based approach seems less relevant because the same pixel intensity value may indicate different land cover class like barren land, cloud cover, etc. So it becomes too difficult for finding a criterion for efficient land cover classification as well. In this research work, we develop an OBIA method to automatically detect landslides from multispectral remote sensing imaging which treats image into various image regions rather than traditional pixel based approach. It tries to overcome the limitation of traditional pixel based approach and provide segmentation based object oriented analysis to improve the classification accuracy.

Remote sensing techniques have been widely used from 1990 for landslide research and for this purpose different spatial and spectral resolution images have been employed. Research work conducted so far includes photo-interpretation and inventory of large landslides, determinant factor analysis, stereo-plotting of movements, and automatic detection by textural analysis. The irregular boundaries and surface textures of landslides mean that they often produce characteristic features that can be enhanced in remote sensing imaging through textural analysis. Over the past few decades, many studies have been carried for landslide detection. Interpretations of landslides from aerial photographs have been developed from on-screen images. Li-Wei et.al (2006) used FORMOSAT 2 images for identification of landslides because of its advantage of low cost and frequent revisit. Their technique explores discriminative features, such as texture and topological features. Texture features were extracted using log polar wavelet packet transformation whereas for topological feature slope is derived. Finally a contextual based classifier that combines spectral and spatial information was used for homogeneous object identification. Zhongbin Li et.al (2016) developed a semi-automated approach from bi-temporal satellite images which uses a change detection based threshold analysis to generate initial zero level curve. Method includes generating difference image using change vector analysis, detecting landslide candidates using change detection based threshold method and removing false candidates using some morphological operations. Landslide boundaries are detected using edge based and region based level set evolution. HaiRong Ma et.alb (2016) proposed a method to detect shallow landslides using Worldview2 images. Features like high soil brightness and low vegetation coverage helped them to identify shallow landslides on remote sensing images. To eliminate back scrap, shadow index was used. FORMOSAT-2 multispectral imagery provides poor landslide identification since it considers only spectral features. LiDAR data have provided landslide maps in forested areas. In general, the research work conducted for Landslide Inventory Map methods can be classified into three groups: Pixel based approaches, Object based approaches and hybrid approaches. With respect to change detection algorithm, landslides are land cover changes and can be detected by using pre and post landslide images. Usually change detection method based on pixel approach has the limitation of 'salt and pepper effect'. There comes the significance of grouping homogeneous pixels into an object or into 'super pixels'.

In this study, an algorithm to automatically detect landslide from satellite imaging was developed by incorporating a change detection based threshold analysis and followed by unsupervised classification. For change detection analysis, we considered bi-temporal satellite images. Methodology was developed using open source tools where some contextual features were used at object level to detect landslides. Segmentation based object oriented analysis with certain optimal parameters were used to segment image into homogeneous image objects. Thus segments become the object primitives and they can be used further for image analysis. Simple Linear Iterative Clustering algorithm was used to cluster image objects. It was followed by a change detection technique, which enhanced the change detected regions by comparing pre and post landslide images. One of the most important properties observed in post landslide image is the increase in the brightness level for the areas surrounded by the landslide affected region. Parameters that can be considered for discriminating landslides from change detected regions were considered at object level. They were used with suitable threshold values based on our domain knowledge. From the change detected output, key points were computed. Associated with each key point, key point descriptors were described manually. Further on this array, K-means clustering algorithm was performed to cluster each objects into their respective classes.

\section{METHODOLOGY}

Automatic detection of landslides from remote sensing imagery consists of the following sub-blocks:

1) Image segmentation using Simple Linear Iterative Clustering algorithm (SLIC).

2) Elimination of non-landslide candidates using object based change detection technique.

3) Classification for discriminating landslides.

\subsection{Bi-temporal Satellite Images}

Test input images used for this research work was a Resourcesat-2 LISS-IV multispectral (5m) bi-temporal satellite image covering parts of Uttarakhand state in India. Bi-temporal satellite images are the images captured at two different instant of time of the same place and will be considered as Post and Pre-landslide images throughout this paper.

\subsection{Segmentation of Post Landslide Image Using SLIC}

A necessary prerequisite for Object Oriented Image Analysis is successful image segmentation. To obtain useful information from an image, the segmentation process splits an image into unclassified "object primitives" that forms the basis for the image objects and the rest of the image analysis. Segmentations, and the resulting characteristics of object primitives and eventual image objects, are based on shape, size, color, and pixel topology controlled through parameters set by the user. The values of the parameters define how much influence spectral and spatial characteristics of the image layers will have in defining the shape and size of the image objects. 


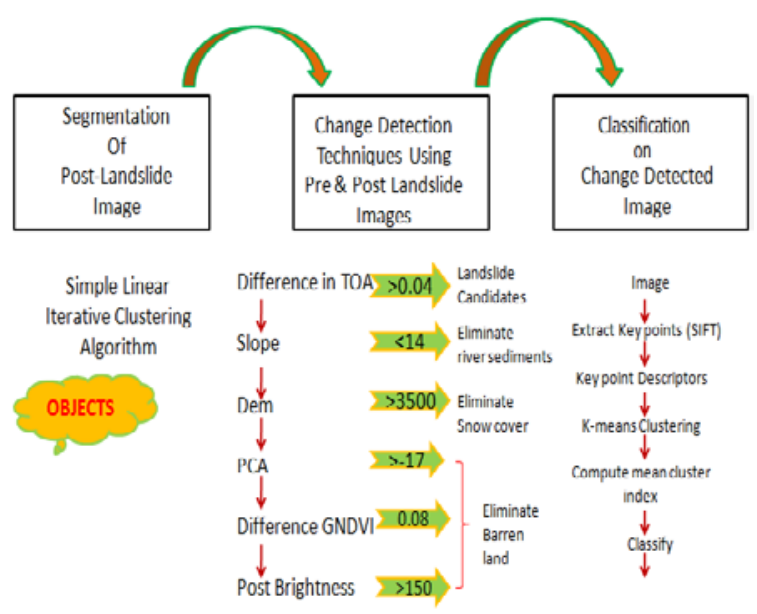

Figure 1. Block diagram of the proposed scheme

The user modifies the settings depending on the objective, as well as image quality, bands available, and image resolution. As a general rule, 'good' image objects should be as large as possible, but small enough to show contours of interest and to serve as building blocks for objects of interest not yet identified. In this research work, we have used SLIC to create image objects which decompose an image into visually homogeneous region based on the spatially localized version of K-means clustering algorithm. It has two parameters like region size and regularizer. The following pseudo code was used for computing SLIC.

1. Image is divided into grid with region size. 2. Centre of each grid is used to initialize corresponding Kmeans.

3. Finally, K mean centers and clusters are refined by Lloyd algorithm. Lloyd's algorithm starts by an initial placement of some number $\mathrm{k}$ of point sites in the input domain. In mesh smoothing applications, these would be the vertices of the mesh to be smoothed; in other applications they may be placed at random, or by intersecting a uniform triangular mesh of the appropriate size with the input domain. It then repeatedly executes the following relaxation step: -The Voronoi diagram of the $\mathrm{k}$ sites is computed. -Each cell of the Voronoi diagram is integrated and the centroid is computed. •Each site is then moved to the centroid of its Voronoi cell. Voronoi diagram construction algorithms can be highly nontrivial, especially for inputs of dimension higher than two, the steps of calculating this diagram and finding the centroids of its cells may be approximated by a suitable discretization in which, for each cell of a fine grid, the closest site is determined, after which the centroid for a site's cell is approximated by averaging the centers of the grid cells assigned to it. During K-mean iteration each pixel can be assigned to only $2 \times 2$ centers corresponding to grid tiles adjacent to pixel. Regularizer, $\lambda$ sets the tradeoff between clustering appearance and spatial regularization.

$$
\lambda=\frac{\text { Regularizer }}{\text { Region Size }}
$$

SLIC starts by dividing the image domain into regular grid $\mathrm{MxN}$ tile where

$$
M=\frac{\text { Image Width }}{\text { Region Size }} ; \quad N=\frac{\text { Image height }}{\text { Region Size }}
$$

Region is initialized with each grid center

$$
\mathbf{x}_{\mathbf{i}}=\operatorname{round}[\mathrm{M}] ; \quad \mathbf{y}_{\mathbf{i}}=\operatorname{round}[\mathrm{N}]
$$

To avoid placing these centers on the top of discontinuities (edges) centers are moved in a 3x3 neighborhood to minimize edge strength

$$
\text { edge }(\mathrm{x}, \mathrm{y})=\left\|I_{(X+1, Y)}-I_{(X-1, Y)}\right\|^{2}+\| I_{(X, Y+1)}-I_{(X, Y-1)}
$$

Regions are obtained by running K-means clustering algorithm started from centers. K means uses a Lloyd algorithm, i.e., to reestimating centers as the average of corresponding feature vectors of the pixel assigned to them. After convergence SLIC eliminates any connected region whose area is less than min [Region Size] pixels. This is done by merging region to neighbours.

\subsection{Proposed Change Detection rule set}

Usually for landslide regions the brightness is more as compared to its corresponding pre-landslide region, a change detection method was proposed to effectively detect change detected regions (Martha et al., 2012). For change detection algorithm, we considered some suitable parameters like difference in Top of Atmosphere (ToA) reflectance among pre and post landslide images, difference in Green Normalised Difference Vegetation Index (GNDVI) among pre and post landslide images, fourth and fifth principal component, slope, DEM to effectively eliminate false candidates from the change detected regions. Here, we explore two methods for change detection: 1) Feature based thresholding, and 2) Change detection based thresholding. The following sub-sections descried these methods in detail.

\subsubsection{Feature Based Threshloding}

In this subroutine, we set appropriate threshold for each feature value to eliminate the non-landslide candidates from the Post image. The thresholds used are discussed below.

2.3.1.1. Difference in ToA: Based on our domain knowledge, we can say that the brightness value for a landslide occurred region is more as compared to its corresponding pre-landslide region. This is due to the exposure of fresh rock surface. So a difference in ToA among pre and post landslide image were considered to effectively detect the change occurred regions. We compute threshold conditions for detecting those regions. There are three ToA bands (NIR, green and red), and the difference of their means gives the difference in ToA of the two images. The equation used for the calculation of difference in brightness is,

$$
\begin{aligned}
\text { Difference in ToA }= & \text { Post image } \\
& \text { image }_{(\text {ToA band1 }+ \text { ToAband } 1+\text { ToAband2 }+ \text { ToAband } 3)}
\end{aligned}
$$

2.3.1.2. Difference in GNDVI: GNDVI uses NIR (Near InfraRed) and green bands of the electromagnetic spectrum. It is same as to the NDVI, but here red band is replaced by green band. Equation for the calculation of GNDVI is,

$$
\frac{\text { TOA }_{\text {NIR }}-\text { TOA }}{\text { Green }}
$$

Significance for choosing GNDVI parameter for landslide detection:

$$
\text { - } \quad \text { It can be used for eliminating barren lands. }
$$


- GNDVI is very sensitive to landslides; decrease in GNDVI value in post image indicates vegetation loss due to landslides.

- It can be used for detecting small scale changes.

2.3.1.3. Principal component analysis: PCA is an image analysis technique used for reducing the dimensionality of a data set. Some of the original bands may be highly correlated and, to save on data storage space and computing time, such bands should be combined into new less correlated eigen images by PCA. Newly triggered landslides were primarily concentrated in the PC4. Because of high brightness, some barren land may be misclassified as landslide candidates. To effectively eliminate such false candidates PCA of stacked pre and post landslide images were used for separation of barren land. Threshold value for removal of barren land is set to -17 .

2.3.1.4. DEM: DEM is always used as a generic term, only representing height information. DEM has been widely used in assessment of landslide susceptibility risk. Some challenging features like snow cover, cloud cover have high brightness values which may be misclassified as landslide candidates. So DEM layer is used to eliminate snow covered areas since snow is confined to high altitude regions. Threshold value to eliminate snow cover was taken to be 3500 , in our work. Objects with value greater than or equal to 3500 are considered as snow cover line.

2.3.1.5. Slope: Slope layer is very useful for the identification of the false positives like river and river sand. River sand has high brightness, small slope and low relief. Most of the landslides occur in very steep slope. So it is easy to separate landslides from false positives using slope angle. At some regions river sediments show same brightness characteristics as that of a landslide region which leads to the misclassification of river sediments into landslide candidates. So slope can be used as the feature to identify landslide candidates since landslide occurs in steep slopes. Objects with slope values less than or equal to 14 is considered as river sediments.

2.3.1.6. Post image brightness: Finally post image brightness is used for removal of barren land from landslide candidates. Post image brightness with values less than or equal to 100 is used for discriminating barren land from remaining candidates.

\subsubsection{Change Detection Based Threshold (CDT)}

It can be used for detecting changes among two registered images acquired from same geographical position at different instant of time. CDT comprises of mainly two steps: 1) Difference Image 2) Threshold. In this study pre and post landslide image is considered for the difference image computation. The magnitude of change vector for a specific pixel is defined as

$$
\rho(X)=\sqrt{\sum_{b=1}^{n}\left(X_{b, t_{1}}-X_{b, t_{2}}\right)^{2}}
$$

where $X_{b, t_{1}}$ and $X_{b, t_{2}}$ are pixel values obtained at time $t_{1}$ and $t_{2}$ respectively where ' $b$ ' is the band and ' $n$ ' is the total number of bands. Greater values of $\rho(X)$ indicates that the pixel $X$ have big change from $t_{1}$ to $t_{2}$. Therefore brighter pixels in $\rho(\mathrm{X})$ indicate landslide candidates. False candidates (non landslides) might be present within the difference image. To overcome this, a threshold is set at object level that distinguishes landslide candidates from non landslide ones within the Difference Image (DI). The magnitude of each pixel was compared at object level with the set threshold value. If it is greater than or equal to a threshold, the object is labelled as landslide candidate; otherwise not. The threshold value is formulated as:

$$
\mathrm{X}_{\mathrm{DI}}\left\{\begin{array}{l}
\text { Landslide, } \rho(\mathrm{X}) \geq \bar{\rho}+\alpha^{*} \sigma \\
\text { Nonlandslide, otherwise }
\end{array}\right.
$$

where $X_{D I}$ value of object $X$ in DI. $\bar{\rho}$ and $\sigma$ are mean and standard deviation of $\rho$ respectively. $\alpha$ is fixed as 1.5 .

\subsection{Classification}

After change detection, the objects that left within the image are used for classification. The method involves feature point detection, feature descriptors, visual word dictionary and a classifier (Cheng et.al, 2013). In feature point detection, the pixels which correspond to edges and corners highlights significant information compared to pixels of homogeneous region. Significant pixels can be computed using different approach like SIFT and SURF. They are usually called as key points which indicate local variation of image intensity in the spatial domain. Associated with each key points, descriptors are computed which might be usually SIFT descriptors. But in this work, we have defined the descriptors; the parameters like slope, DEM, PC4 and mean intensity. We observed that these features are apt for landslide identification. Those parameters were considered at object level. i.e., associated with each key point, those parameters values are calculated at object level for the non-zero segments obtained after change detection. K means clustering algorithm was used to cluster key point descriptors in their feature space into two major clusters. Using mean intensity values, DEM, Slope and PC4 the key point descriptors are clustered and encode each key point descriptors by the index of the cluster into landslide class or non-landslide class (Figure 2).

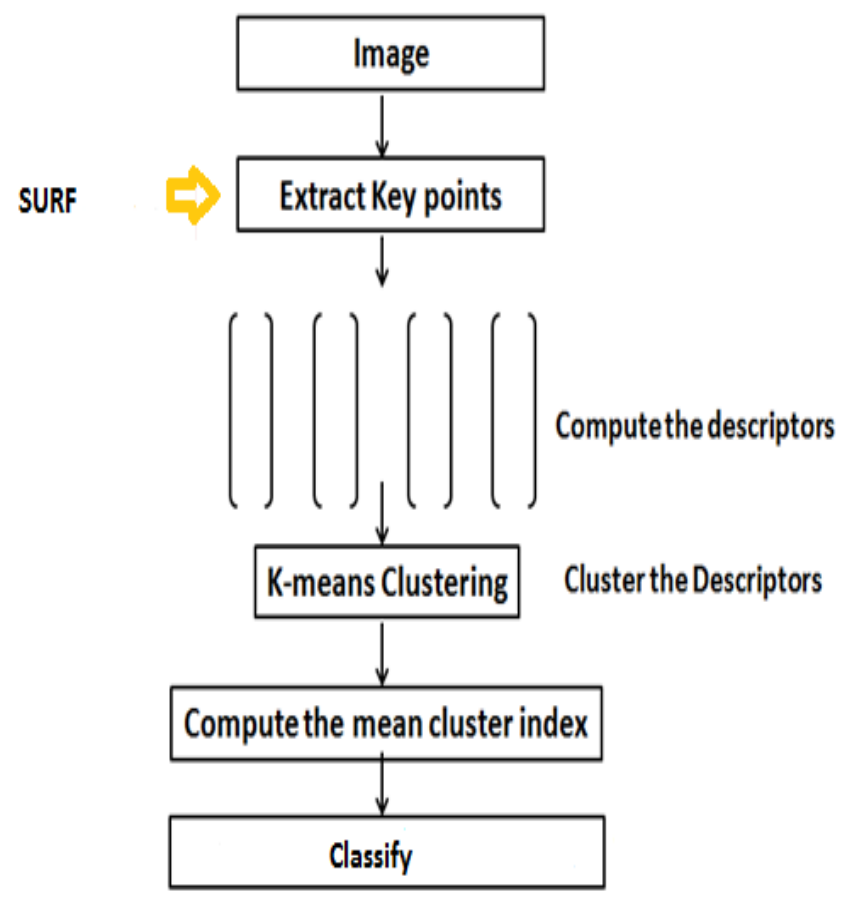

Figure 2. Block diagram for classification scheme 


\section{RESULTS \& DISCUSSION}

For the experimental analysis for landslide detection, bitemporal LISS-IV multispectral images of Chamoli district in Uttarakhand region, which were collected from NRSC, Hyderabad, India, have been used. The ground truth data says that there are 377 landslides in total which includes small, medium and large sizes. In this section, we will discuss our results and inferences drawn out of this exercise.

\subsection{Segmentation}

SLIC segments the post landslide image into objects of different shape and size based on suitable region size. For the current analysis, scale parameter of 30 was used to create objects.

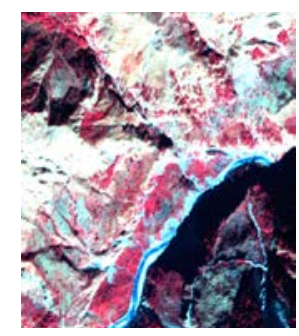

(a) Pre-Segmentation

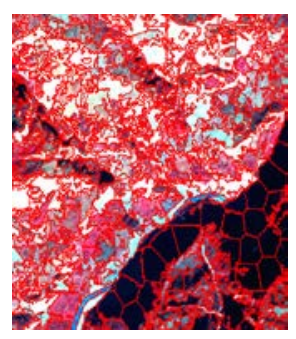

(b) Post-Segmentation
Figure 3. SLIC Segmentation

\subsection{Change Detection}

Two methods of change detection approach were experimented. One was based on proposed change detection rule set (Feature Based Thresholding, Section 2.3.1). Second was based on the difference image concept (Change Detection Based Threshold, Section 2.3.2).

\subsubsection{Results of Proposed Change Detection rule sets}

3.2.1.1. Elimination of false positives based on difference in TOA: The rule set begins with finding those change detected regions by comparing TOA values of pre as well as post images. Landslide objects within the post image have more brightness as compared to corresponding objects in the pre image. Thus, if the threshold value for difference in TOA brightness among the objects was greater than 0.04 , then the object will be considered as landslide candidate. Figure (4) depicts the cropped region within the post landslide image and the black portion indicates the eliminated segment since it doesn't have changes while comparing at object level basis. After taking the difference in TOA for extracting change detected candidates, further elimination of false positives are necessary. To discriminate each one of them and eliminate those false positives, additional parameters and its corresponding threshold conditions were used in this study.

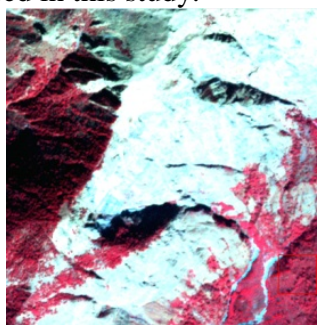

(a) Before ToA thresholding

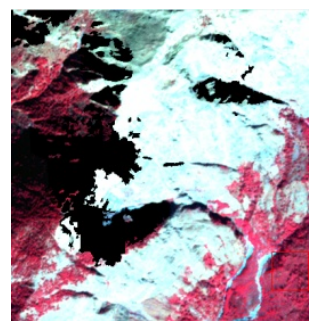

(b) After ToA thresholding
Figure 4. Result after diff. TOA thresholding at object level
3.2.1.2. Elimination of false positives based on slope: It was observed that there exist some river sediments that might have almost the same brightness as that of landslide candidates. Elimination of such regions can be performed well using slope parameter. It was observed that if the mean value of slope within an object is less than or equal to 14 , it indicates river sediments because most of the landslide happens on steep slopes. From the figure (2), it is evident that the river sediments are well eliminated.

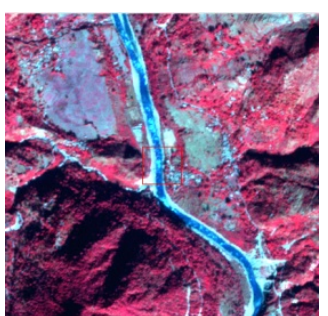

(a) Before Slope thresholding

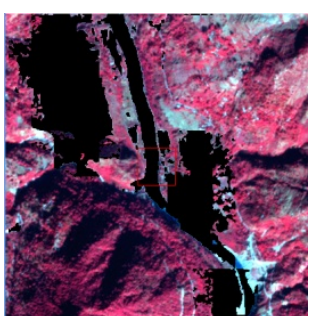

(b) After Slope thresholding
Figure 5.Result after threshold slope at object level

3.2.1.3. Elimination of false positives based on DEM: Once TOA have been calculated, presence of some brightness features like snow, cloud cover may be misclassified as landslides. DEM layer can be used for the elimination of snow covered areas since snow is confined to high altitude regions. For DEM, average value among the object after the segmentation is greater than $3500 \mathrm{~m}$, then the object is confined to be in high altitude and will be considered as a snow cover line. Figure (6) indicates the elimination of snow cover.

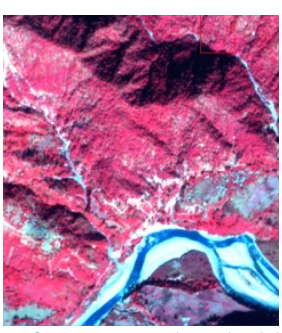

(a) Before DEM thresholding

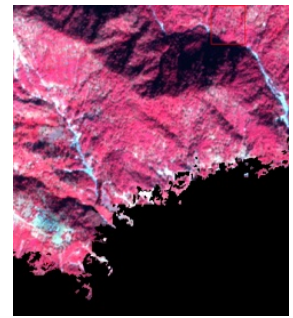

(b) After DEM thresholding
Figure 6. Result after threshold DEM at object level

\subsubsection{Elimination of false positives based on PC4}

Principal Component layers can be used for the elimination of barren land, as is shown in many studies. PC4 and PC5 were used for landslide detection since both are good for detecting landslide triggered regions. Due to the high brightness some barren land may be misclassified as landslide candidates. Computation of PCA was performed by stacking pre and post image layer. Layer stacking was performed on both pre and post landslide images. Many research works suggest that PC4 and PC5 are good features for identifying new landslides. If the average value among the objects within the $4^{\text {th }}$ or $5^{\text {th }}$ principal component was greater than -17 it will be eliminated, hence eliminating the barren land candidates.

\subsubsection{Elimination of false positives based on GNDVI}

GNDVI is a good indicator of landslides because it can be used for the detection of even small scale changes. If the average value of GNDVI among the objects is less than 0.08 , then that condition would eliminate barren lands. Figure (8) indicates 
eliminated barren land by considering difference in GNDVI among the post as well as pre landslide image.

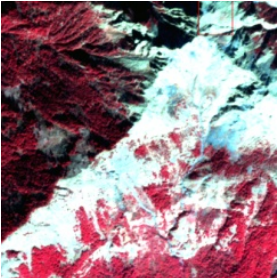

(a) Before PC4 thresholding

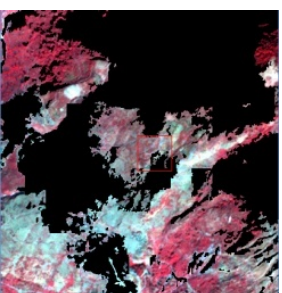

(b) After PC4 thresholding
Figure 7.Result after thresholding PC4
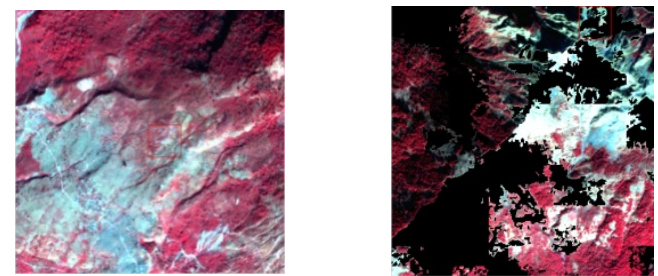

(a) Before GNDVI thresholding (b) After GNDVI thresholding

Figure 8.Result after thresholding diff in GNDVI

3.2.1.6. Elimination of false positives based on post image brightness: Majority of the challenging features is eliminated to a certain extent; but some false positives still exist. Among them barren land can also be eliminated by taking the mean intensity values. Within an image object, if the mean intensity value is less than 110 it indicates that it is barren land, and thus can be eliminated by thresholding.. But it was evident that there still exist some significant regions that need to be further eliminated. Further elimination was done through keypoint detection followed by unsupervised classification, as is discussed in Section 3.3.

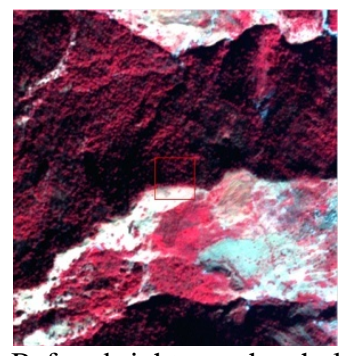

(a) Before brightness threshold

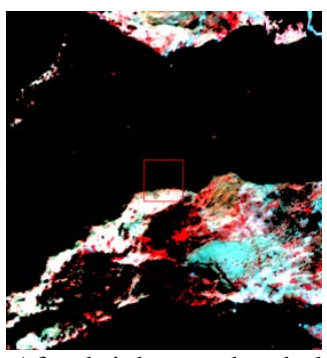

(b) After brightness threshold
Figure 9.Result after threshold brightness values.

\subsubsection{Object level Change Detection based threshold}

Here, a pixel based image analysis technique, which has been converted to object based analysis after segmentation, has been discussed (Hai-Rong, 2016).

3.2.2.1. Elimination of false positives based on difference image: Figure 10 shows the result of difference image threshold at object level obtained by considering 3 bands of pre and post images. Selection of tuning parameter was based on our domain knowledge. From the difference image obtained parameters like mean and standard deviation were computed. Tuning parameter with various trial and error approaches it was opted as 1.5.

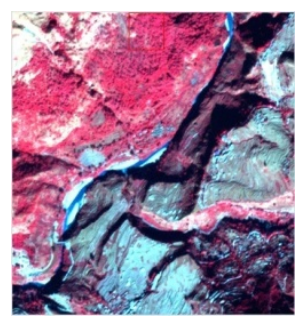

(a) Before CD thresholding

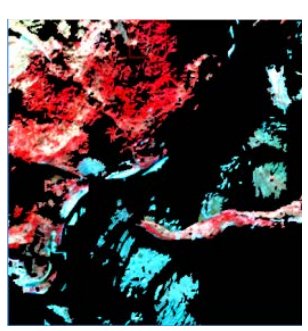

(b) After CD thresholding
Figure 10. Result after CD thresholding at object level

\subsection{Classification}

Change detection was followed by classification. In change detection algorithm, we have considered different parameters for detecting changes, such as detected region which includes the landslide candidates and some false positives as well. Image which obtained after the change detection still includes a large number of false positives. Unsupervised classification using kmeans tries to classify the image into two classes based on the descriptors associated with each of the key points. From the change detected output, the algorithm tries to find the key points by means of SURF algorithm. Figure (11) shows the key points computed over the entire image using SURF detector (Vetrivel, 2016). From the image of dimension 5620 X 4894, about 30,832 corners were detected by applying the threshold value of 10,000 .
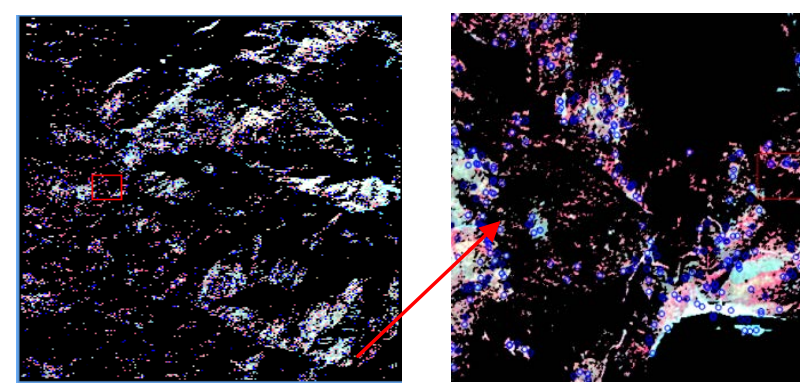

Figure 11. SURF feature detectors

Advantages of using SURF as feature detector is that, key points can be computed over the entire image within a short duration of time. Associated with each key point, key point descriptors are described. In this experiment the descriptors taken are mean intensity value, slope, DEM, PC4 associated with each key point and the mean over its corresponding object. Here, an array was created for all those non-zero objects having key points followed by unsupervised classification (k-means). Two classes were declared and each object was encoded into either of the two class based on their feature vectors.

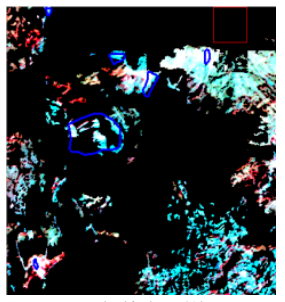

(a) Landslide Class

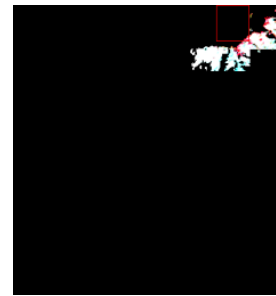

(b) Non Landslide Class
Figure 12. Result obtained after Classification (Blue polygons indicate the overlaid shape files) 


\section{Evaluation Metrics}

In object detection techniques, three measures for evaluating the accuracy of methods are precision, recall and $F$ measures. Precision tells about how accurately the detected objects overlap with the ground truth. Using precision we can measure Fmeasure. Precision measures the fraction of detections that are true positives and Recall measures fraction of positives that are correctly identified. Let as assume Tp, Fp and Fn are the true positive, false positive and false negatives respectively.

Recall

$$
\mathrm{R}=\frac{T_{P}}{T_{P}+F_{n}}
$$

The below tables summarizes the effect of evaluation metric with different key point descriptor and with different non zero segments descriptor followed with parameters like slope, DEM and principal component factors. Table compares the final results performance assessment.

\begin{tabular}{|c|c|c|}
\hline Evaluation Metric & Change Detection & CDT \\
\hline $\mathrm{T}_{\mathrm{p}}$ & 302 & 287 \\
\hline $\mathrm{F}_{\mathrm{n}}$ & 75 & 90 \\
\hline $\mathrm{R}$ & 0.801 & 0.761 \\
\hline
\end{tabular}

Table 1: Summary of the proposed change detection rule set and CVA followed by key point descriptors.

\begin{tabular}{|c|c|c|}
\hline Evaluation Metric & Change Detection & CDT \\
\hline $\mathrm{T}_{\mathrm{p}}$ & 227 & 271 \\
\hline $\mathrm{F}_{\mathrm{n}}$ & 150 & 106 \\
\hline $\mathrm{R}$ & 0.602 & 0.719 \\
\hline
\end{tabular}

Table 2: Summary of the proposed change detection rule set and CVA followed by classification without key point descriptors.

\section{CONCLUSION}

OBIA based extraction of landslides from very high resolution satellite imagery is a promising technology nowadays. This is because of the importance of landslide inventory map in hazard mapping of an area. In this scheme, we have discussed an open source technique to automatically detect landslides of small, medium and of larger types. Based on proposed rule set for change detection and combining the concept of machine learning algorithm false positive elimination within the image have performed to a certain extent. Also, we experimented with a change detection based threshold analysis for false positive elimination based on difference image at object level. But a generalized way to optimize the algorithm still exist which can be further improved at descriptors level. Aforementioned method utilized LISS IV bi-temporal multispectral satellite images of Uttarakhand region. Algorithm shows that for the proposed change detection rule set followed by key point descriptors about $80.1 \%$ recall was obtained. Precision was not calculated due to a large number of false positives in the landslide image. Methodology implies that there exist few more challenging candidates that need to be further eliminated. In future research, we need to solve for such problem by including additional parameters like some texture parameters for enhancing landslide candidates from the current algorithm result.

\section{ACKNOWLEDGEMENTS}

Authors would like to thank Runjhun Chandra, B.Tech (Physical Science) student, IIST, for helping in editing the paper to its final form as per suggestions.

\section{REFERENCES}

[1] Anand Vetrivel, Markus Gerke, Norman Kerle, George vosselman, Identification of structurally damaged areas in airborne oblique images using a Visual-Bag-of-Words approach, Remote sensing 2016

[2] David G Lowe, Distinctive image features from Scale invariant key points, International Journal of Computer Vision, January 2004

[3] Gong Cheng, Junwei Han "A survey on object detection in optical remote sensing images”, ISRPS Journal of Photogrammetry and remote sensing, March 2016, pg.11-28

[4] Gong Cheng, Lei Guo, Tianyun Zhao, Junwei Han, Huihui Li \& Jun Fang "Automatic landslide detection from remote sensing imagery using a scene classification method based on BoVM and Plsa “, International Journal of Remote Sensing, Vol.34,No.1, 10 January 2013,45-59

[5] Hai-Rong Ma, Xinwen Cheng, Lianjn Chen, Haitao Zhang, Hongwei Xiong, Automatic identification of shallow landslides based on Worldview 2 remote sensing images, Journal of applied remote sensing, March 2016, Vol.10, issue 1 [6] Li-Wei Chang,Pi-Feui Hsieh and Ching Weei Lin "Landslide identification based on FORMOSAT-2 multispectral imagery by wavelet based Texture Feature Extraction", IEEE International conference on Geoscience and Remote Sensing Symposium, 2006

[7] Paul L.Rosin, Javier Hervas, Jose I Berredo , Remote sensing image thresholding for landslide motion detection [8] Tapas R. Martha, Norman Kerle, Cees J van Western, Victor Jetten, K. Vinod kumar, Object oriented analysis of mutitemporal panchromatic images for creation of historical landslide inventories, ISRPS Journal of Photogrammetry and remote sensing, January 2012, Vol. 66-issue 1, pg no:105-119 [9] V. Keerthi and A. Senthil Kumar "At-sensor Solar Exoatmospheric Irradiance, Rayleigh Optical Thickness and Spectral parameters of RS-2 Sensors"

[10] Zhongbin Li, Wenzhong Shi, Soe W Myint, Ping Lu, Qunming Wang, Semi automated landslide inventory mapping from bitemporal aerial photographs using change detection and level set method, Remote sensing environment, 2016, pg.215230 


\title{
AN OPEN-SOURCE SEMI-AUTOMATED PROCESSING CHAIN FOR URBAN OBIA CLASSIFICATION
}

\author{
T. Grippa ${ }^{a^{*}}$, M. Lennert ${ }^{a}$, B. Beaumont ${ }^{\text {ab }}$, S. Vanhuysse ${ }^{a}$, N. Stephenne ${ }^{b}$, E. Wolff ${ }^{a}$ \\ ${ }^{a}$ IGEAT, Université Libre De Bruxelles, Belgium - (tgrippa, mlennert, bbeaumon, svnhuyse, ewolff)@ulb.ac.be \\ ${ }^{\mathrm{b}}$ ISSeP, Institut Scientifique de Service Public, Belgium - (b.beaumont, n.stephenne)@issep.be
}

KEY WORDS: Open source, Urban land-cover, OBIA supervised classification, Segmentation, Optimization, GRASS GIS

\begin{abstract}
:
This study presents the development of a semi-automated processing chain for OBIA urban land-cover and land-use classification. Implemented in Python and relying on existing open-source software GRASS GIS and R. The complete tool chain is available in open-access and adaptable to specific user needs. For automation purpose, we developed two GRASS GIS add-ons allowing (1) to optimize segmentation parameters in an unsupervised manner and (2) to classify remote sensing data using several individual machine learning classifiers or their predictions combination through voting-schemes. We tested the performance and transferability of the processing chain using sub-metric multispectral and height data on two very different urban environments: Ouagadougou, Burkina Faso in sub-Saharan Africa and Liège, Belgium in Western Europe. Using a hierarchical classification scheme, the kappa values reached for both cities about 0.78 at the second level ( 9 and 11 classes) and 0.90 at the first level (5 classes).
\end{abstract}

\section{INTRODUCTION}

Land-use/land-cover (LULC) information extraction is one of the main use cases of remote sensing imagery. The advent of sub-meter resolution data brought about the revolution of methods from pixel-based to object-based image analysis (OBIA) involving image segmentation. The latter provides many new opportunities and highly increases the quality of the output, but there remains a number of challenges to address.

First of all, segmentation parameters are often selected after tedious and time-consuming trial-and-error refinement (Zhang et al., 2008; Blaschke, 2010). This method consists in a manual step-by-step segmentation parameters adjustment, relying on subjective visual human interpretation. Despite such efforts, the validity of the selected parameters is usually restricted to the specific scene under study, or even to specific areas within this scene, and they have to be adapted for each dataset. Unsupervised evaluation methods meet the requirements for automation in the OBIA process, as they can be used to automatically adjust segmentation parameters (Zhang et al., 2008).

Second, during the classification step, many authors use rulebased approaches, which can be quite efficient on a specific dataset (e.g. Salehi et al., 2012; O’Neil-Dunne et al., 2013). However, their transferability remains an issue as they also generally rely on manual intervention by the authors, with many choices guided by scene specificities. As an alternative, machine-learning classifiers have proven their efficiency for remote sensing data classification. While identification of the best performing classifier cannot rely on a-priori knowledge, the combination of results of multiple classifiers through ensemble or voting schemes is a solution towards the development of more automated classification processes (Moreno-Seco et al., 2006).

\footnotetext{
Corresponding author
}

Third, much of the work presented on OBIA tool chains is black box. First, specific decisions of authors concerning parameter settings in the manual processes described above are based on their subjective evaluation, which is not always easy to reproduce. Moreover, proprietary software used for the analyses presents issues of lack of peer review options, even if the procedures that are implemented are documented. This concerns the core software and also, in some cases, extensions of that software published closed-source (Drăguţ et al., 2014). In times when reproducibility of research is high on the discussion agenda, the use of free and open-source solutions, including access to the code developed by researchers in their work, becomes paramount.

Linked to the previous point, the question of access to the necessary tools is of great importance, especially for many researchers in poorer countries where the lack of resources reduces the options. Again, free and open-source solutions provide an answer to this issue by creating common pool resources that all researchers can use, but also contribute to. Licensing costs can also be an obstacle to upscaling of processes, especially in times of big data with ever-increasing spatial, spectral and temporal resolutions. Free and open-source software can help researchers surmount this challenge by letting them run their programs on as many different cores or machines as necessary without having to worry about software costs.

In this paper, we present a complete framework for urban LULC mapping from earth observation data, which responds at least partly to the above issues. It was developed in a completely free and open-source environment, using GRASS GIS and R, and immediately reinjected into the wider open-source community. It contains tools for automatic parameter selection for segmentation, statistical characterization of objects and machine-learning techniques combined through a majorityvoting scheme. Care was taken to make the use of this processing chain accessible even to non-programmers. The 
proposed framework was tested on two very different urban environments.

\section{METHODS AND TOOLS}

The entire processing chain relies on existing open-source software. It was implemented in a 'Jupyter notebook' using the GRASS Python scripting library, which allows calling GRASS GIS functions directly from Python. The script is structured into clear steps and documented with references to scientific articles used. The GRASS GIS add-ons implemented in our processing chain are briefly presented below.

\subsection{Segmentation and unsupervised segmentation parameter optimization (USPO) tools}

The segmentation was performed using the i.segment module of GRASS GIS (Eric Momsen, Markus Metz, and GRASS Development Team, 2015). This module implements image segmentation with a region-growing algorithm that requires a standardized 'threshold' parameter below which regions are merged, and a 'minsize' parameter defining the minimum size of regions.

As we looked for automation, we elaborated a new GRASS GIS add-on for unsupervised segmentation parameter optimization (USPO) called i.segment.uspo (Moritz Lennert, and GRASS Development Team, 2016a). This tool is an implementation of methods developed by Espindola et al. (2006) and Johnson et al. (2015). It relies on optimization functions combining measures of weighted variance (as intra-segment homogeneity quality measure) and spatial autocorrelation (as inter-segment heterogeneity quality measure). Two optimization functions are proposed: a simple sum of the normalized criteria values as defined by Espindola et al. (2006), or the F-function as defined by Johnson et al. (2015) which permits to weight the two optimization criteria. On the basis of a range of parameter values provided by the user, the i.segment.uspo tool creates a set of segmentation results, which are then assessed using the optimization function. With this module, it is possible to optimize the segmentation parameter for several subsets of the scene.

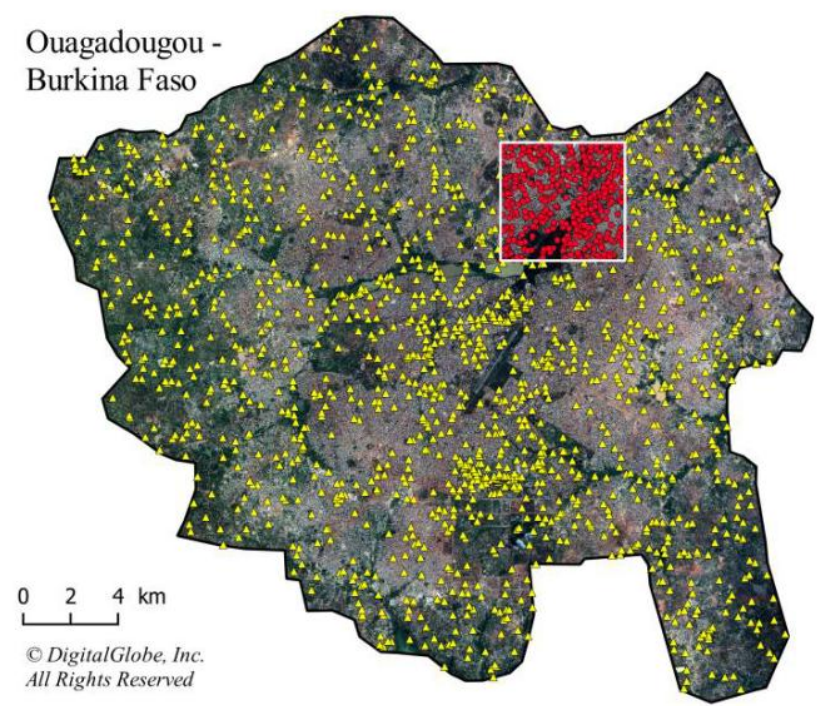

\subsection{Object statistics computation}

Object statistics were computed using the i.segment.stats GRASS GIS add-on (Moritz Lennert, and GRASS Development Team, 2016b) and were used as features in the classification process. This tool allows the computation of both spectral and morphological statistics of objects.

\subsection{Classification by combination of multiple machine learning classifiers}

The classification stage of the processing chain uses the v.class.mIR GRASS GIS add-on (Moritz Lennert, and GRASS Development Team, 2016c). It relies on the utilization of the "Caret" library of R software (R Development Core Team, 2008), and allows the classification of data using Support Vector Machine, Random Forest, Recursive partitioning, and k-Nearest Neighbors classifiers. This add-on automatically tunes classifiers' parameters using repeated cross-validation with 10 iterations of 5-fold cross-validation on the training data set (these default values can be changed). Predictions of individual classifiers are then combined using several types of majority vote. Four voting systems are provided: "Simple Majority Vote" (SMV), "Simple Weighted Vote" (SWV), "Best Worst Weighted Vote" (BWWV) and "Quadratic Best Worst Weighted Vote" (QBWWV) (Moreno-Seco et al., 2006).

\section{CASE STUDIES}

\subsection{Study areas and data}

As a proof of concept, the processing chain was applied to two very distinct urban environments using datasets with different spatial resolutions and consisting of multi-spectral and height data: Ouagadougou (Burkina Faso, in Sub-Saharan Africa) and Liège (Belgium, in Western Europe). More broadly, this work is linked with two research projects dealing with the production (MAUPP project, focusing on African Sub-Saharan cities) and the update (SmartPop project, focusing on the Walloon region in Belgium) of LULC maps. These maps will be used later as input in census population data disaggregation models.

The processing chain was first developed on Ouagadougou,

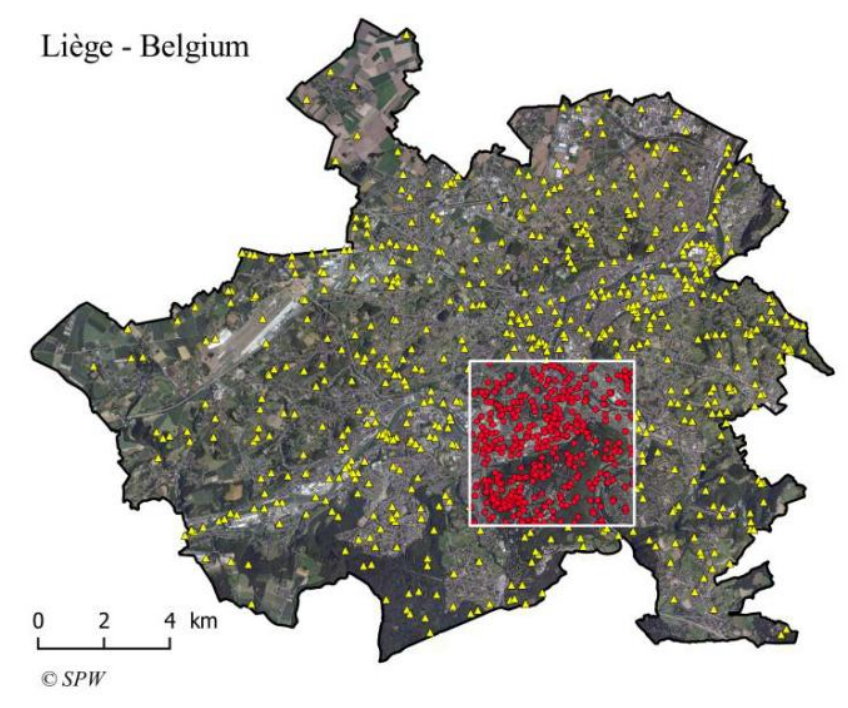

Figure 1. Ouagadougou and Liège case studies. True color composite is used as background. The classification was made on the white-squared subset. Training samples are in yellow while the test samples are in red 
capital of Burkina Faso in Western Africa. Covering more than $615 \mathrm{~km}^{2}$, this city has been facing intensive urban sprawl during the last decades as most of Sub-Saharan cities and is characterized by very different urban patterns, among others planned versus unplanned residential areas. The processing chain was then applied to the Liège area $\left(261 \mathrm{~km}^{2}\right)$, a Western European city located in Belgium which shows strong land artificialization (more than $55 \%$ of the territory). Urban morphologies are more diversified (from isolated houses to $10+$ storey buildings), but urban sprawl is limited and controlled in comparison with Africa.

Data used for Ouagadougou were a pansharpenened stereo WorldView-3 imagery (VNIR product, 0.5 meters) acquired during the wet season (October 2015) and a nDSM produced from WorldView-3 by stereophotogrammetry ( 0.5 meters). For Liège, the data consisted in VNIR aerial orthophotos with a spatial resolution of 0.25 meters acquired on leaf-on (May 2012) and a nDSM extracted from LiDAR data $\left(1-3 \mathrm{pt} / \mathrm{m}^{2}\right)$ that was acquired on leaf-off (winter 2013-2014).

As our processing chain is under development, we focused the classification effort on a $25 \mathrm{~km}^{2}$ subset for both cities (see Figure 1), representative of the diversity of landscapes and urban forms.

\subsection{Legend / Classification scheme}

Because our final goal is to map the land use, the classification scheme is organized in two hierarchical levels (see Table 1).

\begin{tabular}{||l|l|c|c||}
\multicolumn{5}{c|}{ Ouagadougou - Burkina Faso } \\
\hline $\begin{array}{c}\text { Level 1 } \\
\text { LC classes }\end{array}$ & \multicolumn{1}{|c|}{$\begin{array}{c}\text { Level 2 } \\
\text { LULC classes }\end{array}$} & $\begin{array}{c}\text { Training set } \\
\text { size }\end{array}$ & $\begin{array}{c}\text { Test } \\
\text { set size }\end{array}$ \\
\hline \hline \multirow{3}{*}{ Artificial surfaces } & Buildings & 216 & 43 \\
\cline { 2 - 5 } & Swimming pools & 90 & 31 \\
\cline { 2 - 5 } & Asphalt surfaces & 119 & 30 \\
\hline Natural material \\
surfaces & Brown/red bare soil & 130 & 42 \\
\hline \multirow{3}{*}{ Vegetation } & White/grey bare soil & 91 & 30 \\
\hline Water & Trees & 91 & 32 \\
\hline Shadow & Mixed bare soil/vegetation & 99 & 32 \\
\cline { 2 - 5 } & Dry vegetation & 93 & 32 \\
\cline { 2 - 5 } & Other vegetation & 218 & 36 \\
\hline
\end{tabular}

Liège - Belgium

\begin{tabular}{||l|l|c|c||}
\hline \multicolumn{1}{|c|}{$\begin{array}{c}\text { Level 1 } \\
\text { LC classes }\end{array}$} & \multicolumn{1}{|c|}{$\begin{array}{c}\text { Level } 2 \\
\text { LULC classes }\end{array}$} & $\begin{array}{c}\text { Training set } \\
\text { size }\end{array}$ & $\begin{array}{c}\text { Test } \\
\text { set size }\end{array}$ \\
\hline \hline Articial surfaces & Buildings & 62 & 37 \\
\cline { 2 - 5 } & Asphalt surfaces & 86 & 60 \\
\hline $\begin{array}{l}\text { Natural material } \\
\text { surfaces }\end{array}$ & Bare soil & 51 & 42 \\
\hline \multirow{3}{*}{ Vegetation } & Low vegetation $(<1 \mathrm{~m})$ & 55 & 46 \\
\hline \begin{tabular}{l} 
Medium vegetation $(1-7 \mathrm{~m})$ \\
\cline { 2 - 5 }
\end{tabular} & $\begin{array}{l}\text { High vegetation decideous } \\
(>7 \mathrm{~m})\end{array}$ & 49 & 48 \\
\hline $\begin{array}{l}\text { High vegetation coniferous } \\
>7 \mathrm{~m})\end{array}$ & 49 & 36 \\
\hline Water & Inland waters & 72 & 37 \\
\hline Shadow & Shadow & 62 & 39 \\
\hline
\end{tabular}

Table 1. Classification scheme and size of training and test set for Ouagadougou and Liège

The first level contains only land-cover (LC) classes, while the second level is a LULC mix of classes. At both levels, an extra class is dedicated to shadows; their post-processing is out of the scope of this article. The classification was made based on the second-level classes, which were aggregated to match the first-level classes.

\subsection{Sampling scheme}

Sampling was conducted outside the processing chain, by generating random points and labelling them through image interpretation. Although existing geodatabases were used for stratification, a visual interpretation was needed to bypass misregistration or land-cover changes. The training set was generated for the whole area excluding the subset where the classification was produced. An independent test set was generated inside this subset for performance evaluation purposes. We used this approach to avoid potential spatial autocorrelation between the training and test sets (Brenning, 2012).

For Ouagadougou, OpenStreetMap (OSM) datasets were used for stratification as far as possible according to availability, i.e., for the level 2 classes Buildings, Asphalt surfaces, and Inland waters. When OSM datasets consisted of lines, as is the case for asphalt roads and watercourses, buffers were created. Manual sampling was required for Swimming pools and Shadow classes.

For Liège, existing TOP10V (IGN, 2010) and PICC (SPW, 2007) geodatabases were used for the stratification of the majority of level 2 classes. Manual sampling was needed for the class Shadow. Given the production date of the geodatabases used, visual validation of sampling was needed to match the 2013 land-cover status.

In total, 1352 training points and 369 test points were created for Ouagadougou and 549 training points and 388 test points for Liège. The smaller size of the training set for Liège is explained by the reduced number of classes, their higher homogeneity and the intensive use of reference geodatabases. The class-distribution detail is presented in Table 1.

\subsection{Segmentation}

The segmentation and USPO steps were made using multispectral information for each datasets. For Ouagadougou, NDVI was also used as additional layer. The "minsize" parameter was set in order to match the chosen minimum mapping unit. The latter was defined according to the geographical context in order to identify the smallest houses/shelters : $2 \mathrm{~m}^{2}$ for Ouagadougou and $15 \mathrm{~m}^{2}$ for Liège. The intervention of the operator in the segmentation process was limited to set the range of "threshold" parameters to be tested (minimum, maximum and intervals). The optimized threshold was then automatically determined via the i.segment.uspo add-on.

\subsection{Classification feature}

For both case studies, the minimum, maximum, range, standard deviation, sum and median statistics were computed on each multispectral bands, NDVI and nDSM. The latter were completed with morphological attributes of objects (area, perimeter and compactness). 


\section{RESULTS}

Existing open-source solutions were used for developing a semi-automated processing chain for urban OBIA classification. This chain is available at http://maupp.ulb.ac.be/geobia_2016. Anyone interested can use and/or adapt it to match different project-specific needs, by integrating additional steps (e.g., automated image pre-processing, computation of spectral or textural indices, automated sampling based on existing reference geodatasets,...). The transferability and adaptability of the processing chain was confirmed by applying it to two very different urban contexts. Some results are illustrated here after.

The segmentation process using USPO enabled us to obtain very convincing results. As can be seen on Figure 2, the image is segmented into meaningful objects; under-segmentation is nearly absent and over-segmentation is limited.

Accuracy measures for the four individual classifiers and their fusion using four voting systems are presented in Table 2. Individual classifier performance ranking is the same for both cities, with Random Forest (RF) performed best, followed by Support Vector Machine (SVMradial), then Recursive partitioning (Rpart) and finally $\mathrm{K}-\mathrm{Nearest}$ Neighbors classifiers $(\mathrm{kNN})$.

Votes' results are very close to each other and perform similarly as the best individual classifier (RF), excepting for SMV. This appears consistent with Moreno-Seco et al. (2006) who showed that use of voting-systems could either improve, equal or underperform accuracy of individual classifiers.
Using our semi-automated processing chain, we reach kappa values of 0.79 (Ouagadougou) and 0.77 (Liège) at level 2 . When considering the first level, kappa values increase up to 0.90 (Ouagadougou) and 0.89 (Liège). Classification confusions occur between different vegetation classes or between asphalt and bare soil classes for both case studies. In Ouagadougou specific confusion is observed between bare soils classes. This explains the gaps of performance between the two hierarchical classification levels.

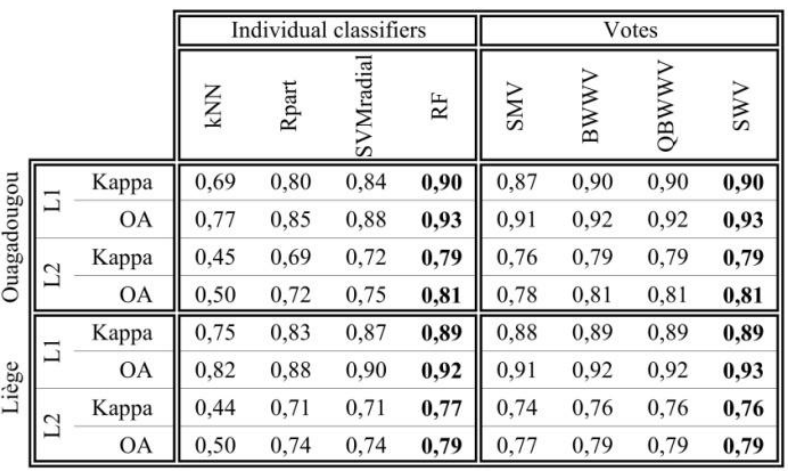

Table 2. Performance evaluation of individual classifiers and four different voting systems (OA: Overall accuracy. L1 and L2: Levels of the classification scheme)

The Building class is of particular importance in the framework of the MAUPP and SmartPop projects since their objective is to disaggregate census population data using LULC maps in order to map the spatial distribution of the population densities inside each city. This class reached per-class kappa values of 0.88
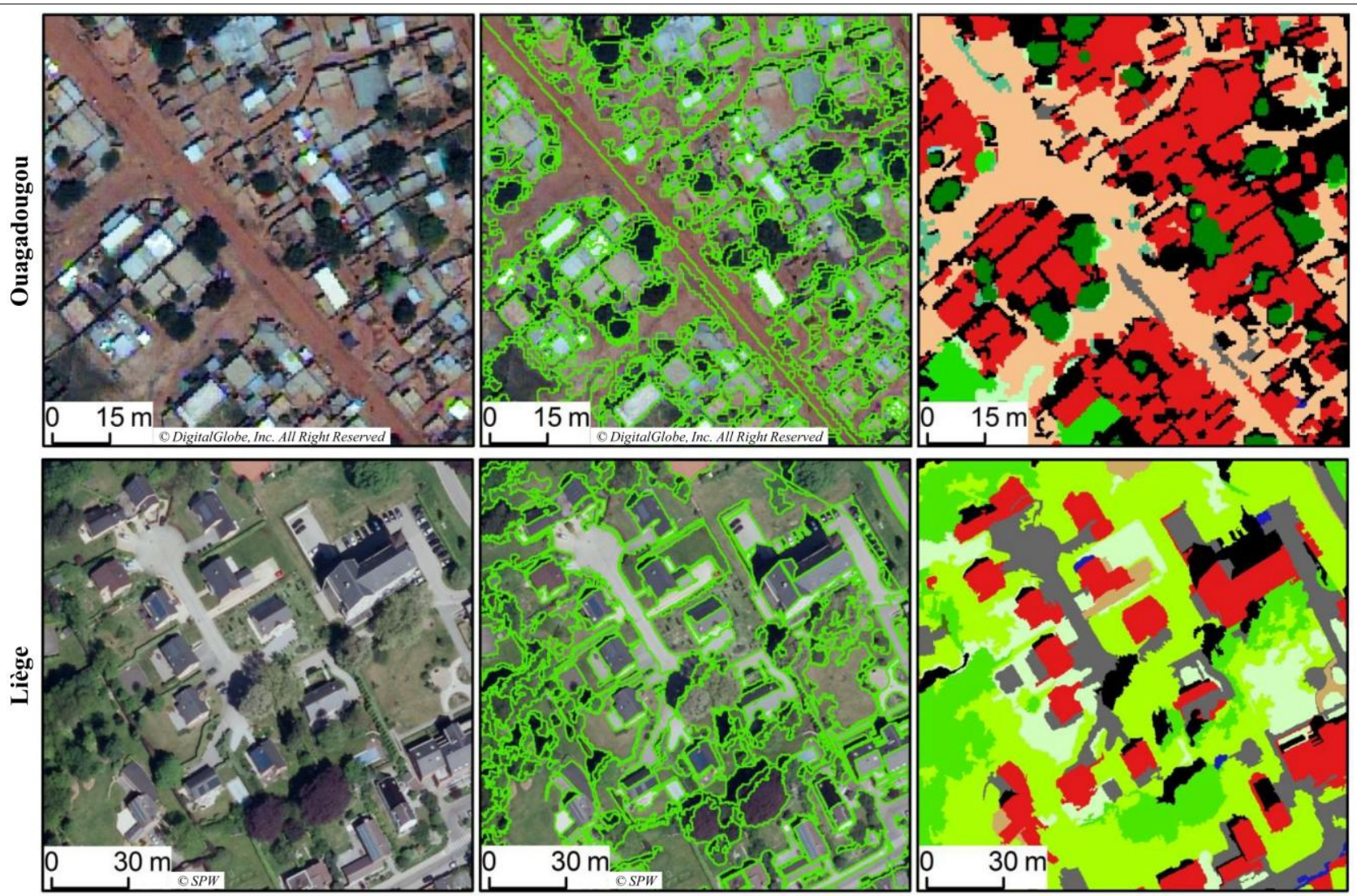

Figure 2. True color composite (left), results of USPO segmentation (center), classification at level 2 with SWV vote (right) on a subset for each case studies 
(Ouagadougou) and 0.97 (Liège).

\section{CONCLUSION AND PERSPECTIVES}

This research is a contribution towards the development of a transparent and open-source fully automated processing chain for urban LULC mapping. This framework is very adaptable and transferable. It proved its ability of being quickly customized in order to match the requirements of different projects, with very different urban morphologies and different datasets. The results achieved on our case studies are very satisfying taking into account the complexity of the urban environments and the detail of the legend.

The generation of training and validation samples still requires strong manual experts' intervention. This remains a challenge to overcome by future research looking for automation, especially when highly accurate reference geodatabases are not available. In that case, alternative data like OpenStreetMap data could be used but their quality is often inconsistent and they should therefore be assessed prior to any automated use.

In order to improve the segmentation and hence the resulting classification, we intend to implement multi-level segmentation and to assess its added value.

Another improvement concerns the features used as input in the classification process. Currently, only relatively simple object statistics are used. Band ratios and several textural indices will be added. They will be automatically computed and submitted to a feature selection procedure.

Regarding the classification, as the potential spatial autocorrelation in the training/validation set can lead to undetected overfitting (Brenning, 2012), we will investigate the possibility of implementing spatial cross-validation instead of the non-spatial one currently used. Moreover, we will explore the possibilities to implement a class-specific weighted vote. Voting-system currently used to combine predictions of the different classifiers are based weights computed with overall accuracy or kappa, but non-best classifiers often outperform the best one's performance regarding to specific classes.

Since the performances of different LULC mapping methods are currently being assessed in the SmartPop project, our open-source semi-automated approach will be compared to a rule-based classification. This second approach integrates existing GIS layers (buildings, roads, rails \& water bodies), updates them and classifies lands outside of these thematic data using rule-based on remotely sensed input data. Constrained segmentation using ancillary vector layers in GRASS GIS will be investigated in the next steps.

In the near future, the processing chain will be tested on different datasets and/or cities. For the MAUPP project, optical and SAR data will be integrated in order to improve the accuracy and the chain will be applied to Ouagadougou (Burkina Faso), Dakar and Saint-Louis (Senegal). For the SmartPop project, Pleiades imagery will be used instead of orthophotos in order to assess the comparative advantage of each dataset. Thereafter, the efficiency of the processing chain will be tested for the automated processing of a very large area (i.e., the Walloon Region in Belgium).

\section{ACKNOWLEDGEMENTS}

This work was funded by the Belgian Federal Science Policy Office (BELSPO) (Research Program for Earth Obervation STEREO III, contract SR/00/304 - as part of the MAUPP project - http://maupp.ulb.ac.be) and by Moerman research program of ISSeP (SmartPop project http://www.issep.be/smartpop).

\section{REFERENCES}

Blaschke, T. (2010). Object based image analysis for remote sensing. ISPRS J. Photogramm. Remote Sens. 65, pp. 2-16.

Brenning, A. (2012). Spatial cross-validation and bootstrap for the assessment of prediction rules in remote sensing: The $\mathrm{R}$ package sperrorest. (IEEE), pp. 5372-5375.

Drăguţ, L., Csillik, O., Eisank, C., and Tiede, D. (2014). Automated parameterisation for multi-scale image segmentation on multiple layers. ISPRS J. Photogramm. Remote Sens. 88, pp. 119-127.

Eric Momsen, Markus Metz, and GRASS Development Team (2015). Module i.segment. Geographic Resources Analysis Support System (GRASS) Software, Version 7.0. Open Source Geospatial https://grass.osgeo.org/grass70/manuals/i.segment.html Foundation.

Espindola, G.M., Camara, G., Reis, I.A., Bins, L.S., and Monteiro, A.M. (2006). Parameter selection for region- growing image segmentation algorithms using spatial autocorrelation. Int. J. Remote Sens. 27, pp. 3035-3040.

Johnson, B.A., Bragais, M., Endo, I., Magcale-Macandog, D.B., and Macandog, P.B.M. (2015). Image Segmentation Parameter Optimization Considering Within- and Between-Segment Heterogeneity at Multiple Scale Levels: Test Case for Mapping Residential Areas Using Landsat Imagery. ISPRS Int. J. GeoInf. 4, pp. 2292-2305.

Moreno-Seco, F., Inesta, J.M., De León, P.J.P., and Micó, L. (2006). Comparison of classifier fusion methods for classification in pattern recognition tasks. In Joint IAPR International Workshops on Statistical Techniques in Pattern Recognition (SPR) and Structural and Syntactic Pattern Recognition (SSPR), (Springer), pp. 705-713.

Moritz Lennert, and GRASS Development Team (2016a). Addon i.segment.uspo. Geographic Resources Analysis Support System (GRASS) Software, Version 7.0. Open Source Geospatial

Foundation.

https://grass.osgeo.org/grass70/manuals/addons/i.segment.uspo. html

Moritz Lennert, and GRASS Development Team (2016b). Addon i.segment.stats. Geographic Resources Analysis Support System (GRASS) Software, Version 7.0. Open Source Geospatial Foundation. https://grass.osgeo.org/grass70/manuals/addons/i.segment.stats. html

Moritz Lennert, and GRASS Development Team (2016c). Addon v.class.mlR. Geographic Resources Analysis Support 
System (GRASS) Software, Version 7.0. Open Source Geospatial Foundation. https://grass.osgeo.org/grass70/manuals/addons/v.class.mlR.htm 1

O'Neil-Dunne, J.P.M., MacFaden, S.W., Royar, A.R., and Pelletier, K.C. (2013). An object-based system for LiDAR data fusion and feature extraction. Geocarto Int. 28, pp. 227-242.

R Development Core Team (2008). R: A language and environment for statistical computing (Vienna, Austria: R Foundation for Statistical Computing).

Salehi, B., Zhang, Y., Zhong, M., and Dey, V. (2012). ObjectBased Classification of Urban Areas Using VHR Imagery and Height Points Ancillary Data. Remote Sens. 4, pp. 2256-2276.

Zhang, H., Fritts, J.E., and Goldman, S.A. (2008). Image segmentation evaluation: A survey of unsupervised methods. Comput. Vis. Image Underst. 110, pp. 260-280. 


\title{
AN OBJECT-BASED SEMANTIC CLASSIFICATION METHOD OF HIGH RESOLUTION SATELLITE IMAGERY USING ONTOLOGY
}

\author{
H. Y. Gu ${ }^{\text {a, * }, \text { H.T. Li }}{ }^{\text {a }}$ L. Yan ${ }^{\text {b }}$ T. Blaschke ${ }^{\text {c }}$ \\ ${ }^{\text {a }}$ Institute of Photogrammetry and Remote Sensing, Chinese Academy of Surveying and Mapping, 28 Lianhuachi Road, P.R. China - \\ (guhy,lhtao)@casm.ac.cn \\ ${ }^{\mathrm{b}}$ School of Geodesy and Geomatics, Wuhan University, Luojiashan, P.R. China - lyan@ @sg.whu.edu.cn \\ ${ }^{c}$ Department of Geoinformatics - Z_GIS, University of Salzburg, Schillerstrasse 30, 5020 Salzburg, Austria -(Mariana.Belgiu, \\ Thomas.Blaschke)@sbg.ac.at
}

KEY WORDS: Geographic Object-based Image Analysis, Ontology, Semantic Network Model, Web Ontology Language, Semantic Web Rule Language, Machine learning, Expert rule, Land-Cover Classification

\begin{abstract}
:
Geographic Object-Based Image Analysis (GEOBIA) techniques have become increasingly popular in recent years and are able to incorporate and develop ontology model within the classification process. They have been claimed to represent a paradigm shift in remote sensing interpretation. Nevertheless, it is lack of formal expression and objective modelling of the whole process of GEOBIA, and lack of the study of semantic classification method using ontology. A major reason is the complexity of the process of GEOBIA. The study has put forward an object-based semantic classification method of high resolution satellite imagery using ontology that aims to fully exploit the advantages of ontology to GEOBIA. A detailed workflow has been introduced that has three steps: ontology modelling, initial classification based on data-driven machine learning method, and semantic classification based on knowledgedriven expert rules method. The whole process of GEOBA is organized organically and expressed explicitly using ontology, and the semantic relations are expressed in the formal language that the computer could operate. Image objects are classified based on ontology model and using machine learning method and expert rules. From the result it is well understood that the method enhances the existing GEOBIA techniques with the help of the ontology, which expresses and organizes the whole process of GEOBIA, and establishes their relations, and provides semantic meaning for GEOBIA. In particular, we found that it provides an ontology model and method for further classifications and large scale applications, and the method using ontology is suitable for automatic classification.
\end{abstract}

\section{INTRODUCTION}

Geographic object-based image analysis (GEOBIA) is devoted to developing automated methods to partition remote sensing (RS) imagery into meaningful image objects, and assessing their characteristics through spatial, spectral, texture and temporal features, thus generating new geographic information in a GISready format (Hay and Castilla,2008). There has been great progress compared to traditional pixel-based image analysis. GEOBIA has the advantages of having a high degree of information utilization, strong anti-interference, a high degree of data integration, extreme precision of classification, and less manual editing (Hay and Castilla,2006; Robertson and King,2011; Duro et al.,2012; Myint et al.,2011). Over the last decade, advances in GEOBIA research have led to numerous workshops, software packages, and peer-reviewed journal papers; five highly successful biennial international GEOBIA conferences; and a growing number of books and university theses (Addink et al.,2012; Blaschke,2010). It has recently been recognized as a new paradigm in remote sensing (Blaschke et al.,2014).

Ontology is originated in the western philosophy and then introduced into the GIS. The concept of domain knowledge is expressed in the form of machine-understandable and is utilised for semantic modelling, semantic interoperability, knowledge sharing and information retrieval service in the field of GIS (Li et al.,2014; Agarwal,2005). Recently, researchers begin to attach importance to the application of ontology in the field of remote sensing, especially in remote sensing image interpretation, which provides a new means for image classification.

Arvor D. et al. (2013) described how to utilise ontology expert knowledge to improve the automation of image processing and analysed the potential applications of GEOBIA, which can provide theoretical support for remote sensing data discovery, multi-source data integration, image interpretation, workflow management and knowledge sharing. Jesús et al. (2013) built a framework for ocean image classification based on ontology; the framework describes how low and high the level content of ocean satellite images can be modeled with ontology. In addition, decision tree classifiers and rule-based expert systems have been presented. Dejrriri et al. (2012) presented GEOBIA and data mining techniques for non-planned city residents based on ontology. Kohli D. et al.(2012) provided a comprehensive framework that includes all potentially relevant indicators that can be used for image-based slum identification. Forestier et al. (2013) built coastal zone ontology to extract coastal zone with background and semantic knowledge. Kyzirakos et al. (2014) provided wildfire monitoring services and combined satellite images and geospatial data with ontology. Belgiu et al. (2014)

\footnotetext{
* Corresponding author
} 
presented an ontology-based classification method for extracting types of buildings where airborne laser scanning data are employed and obtained effective recognition results. Belgiu et al. (2014) provided a formal expression tool to express object-based image analysis technology through ontology.

However, these studies focuses on some aspect that only expert knowledge or specific geographic entity, which is expressed formally using ontology. It is lack of formal expression and objective modelling of the whole process of GEOBIA, and lack of the study of ontology driven semantic classification method, and the whole process of GEOBIA expressed formally using ontology is rare. Therefore, the study puts forward an objectbased semantic classification method of high resolution satellite imagery using ontology that aims to fully exploit the advantages of ontology to GEOBIA.

\section{METHODOLOGY}

The applied workflow of the object-based semantic classification is organized as follows: in the ontology model building step, the models of remote sensing image, object features, land cover class hierarchy and classifiers are built using the procedure described in Section 2.1 (Step 1, Figure 1), and the ontology frame file is built. Subsequently, the remote sensing image is classified using machine learning method and the initial classification result is imported into the ontology frame file (Step 2, Figure 1), which is described in Section 2.2. In the last step, the initial classification result is reclassified to get the final classification result based on the expert rules (Step3, Figure 1), which is described in Section 2.3.

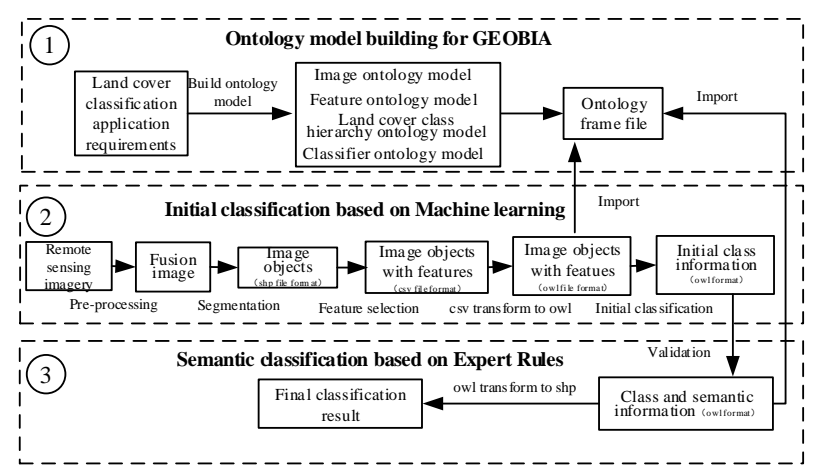

Figure 1. Overview of the methodology followed in this study.

\subsection{Ontology Model for GEOBIA}

Ontology helps in reducing the semantic gap that exists between the image object domain (Arvor et al., 2013). It is important to combine the whole process of GEOBIA into the knowledge formalization using ontology. The ontology model of remote sensing image, object features, land cover class hierarchy and classifiers are built with orientation toward land cover classification requirements. The information of remote sensing image, land cover class hierarchy, object features and machine learning classifiers is expressed in Ontology Web Language (OWL). The expert rules are expressed in Semantic Web Rule Language (SWRL). The FaCT++ reasoner is used to infer the relationship among all the individuals. The knowledge engineering method and the Protégé software developed by Stanford University are chosen to build the ontology model for GEOBIA. Thus the whole process of GEOBIA is expressed and modelled to form the semantic network model.
2.1.1 Ontology Model of the Remote Sensing Image: The ontology construction of the remote sensing image is as follows. 1) A list of important terms and concepts, such as satellite, sensor, image, spatial resolution and spectral resolution, are created. 2) The spectral resolution is defined through the topdown method. It is divided into visible and infrared. Visible is divided into blue, green and red, infrared is divided into near infrared, far infrared and thermal infrared. 3) The slot is defined. The slot includes associated_to, from_band, from_satellite, from_sensor, has_spatial_resolution - and has_spectral_resolution. 4) The slot surface is defined. The range and scope of the slot are defined which is described in table 1 .

Table 1. The range and scope of the slot

\begin{tabular}{|l|l|l|}
\hline Slot & Range & Scope \\
\hline associated_to & Region & Image \\
from_band & Image & - \\
from_satellite & Sensor & Satellite \\
from_sensor & - & Sensor \\
has_spatial_resolution & - & Spatial_resolution \\
has_spectral_resolution & - & Spectral_resolution \\
\hline
\end{tabular}

2.1.2 Ontology Model of the Image Object Features: It makes use of the feature concepts used in the eCognition software to develop a general upper level ontology (Definiens Imaging $\mathrm{GmbH}, 2011)$. The image object features are defined through the top-down method and are divided into six categories: LayerProperty, GeometryProperty, PositionProperty, TextureProperty, ClassProperty, and ThematicProperty. Each class continues to segmentation. For instance, the TextureProperty is divided into ToParentShapeTexture and Haralick. Haralick is divided into GLCMHom, GLCMContrast and GLCMEntropy. It is shown in figure 2.

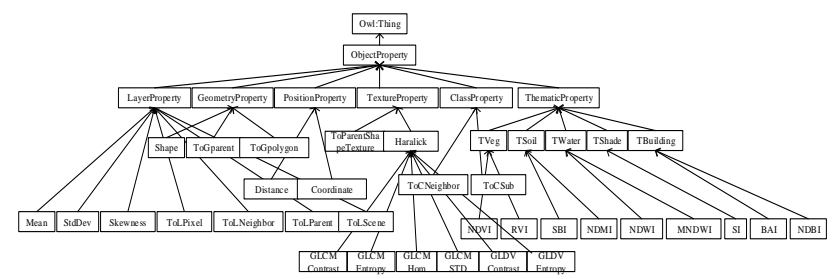

Figure 2. Object features hierarchy in ontology (Every subclass is shown with an "is.a" relationship).

2.1.3 Ontology Model of the Land Cover Class Hierarchy: It makes use the Land Cover Classification System (LCCS) (Di,2005)concepts to develop a general upper level ontology. It includes the various land cover classification scheme. The upper level classes defined in the ontology based on LCCS are shown in Figure 3. Detailed classes can be defined according to the actual situation. 


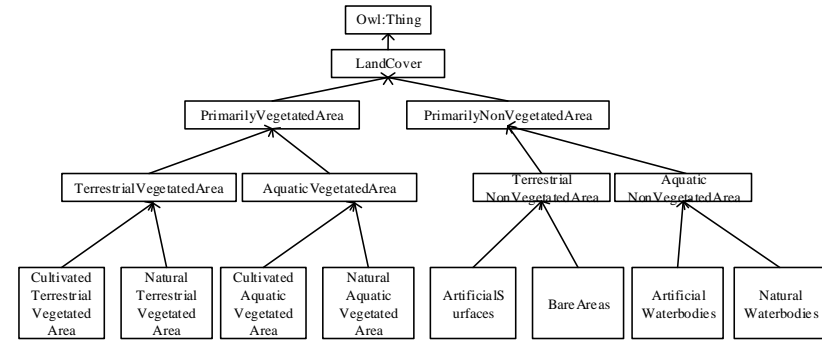

Figure 3. Land cover class hierarchy in ontology(Every subclass is shown with an "is.a" relationship).

2.1.4 Ontology Model of the Classifiers: Ontology is employed to express two typical algorithms, namely, decision tree and expert rules.

(1) Ontology model of the decision tree classifier

1) A list of important terms and concepts of the decision tree classifier is created, such as DecisionTree, Root, Node and Leaf 2) The slot is defined. The slot includes GreaterThan, GreaterThanOrEqual, LessThan and LessThanOrEqual. 3) The individuals of the node of the decision tree are created according to the land cover class hierarchy. The ontology model of the decision tree classifier is shown in figure 4.

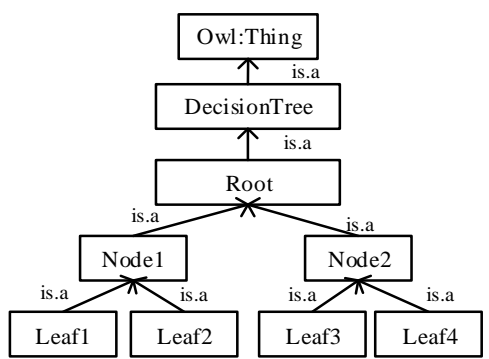

Figure 4. Ontology model of the decision tree classifier

(2) Ontology model of the expert rules

The process of modelling expert rules includes building mark rules and expert rules. Building mark rules is based on a semantic concept, and the process is from low-level features to semantic concepts. Expert rules is obtained based on mark rules and expert knowledge, the process is from advanced features to the identification of land cover. The ontology model of mare rules and expert rules are shown as follows:

(a)Ontology model of the mark rules

The objects are modelled from different semantic aspects according to the common sense knowledge, it is divided into strip and planar from the morphology; regular and irregular from the shape; smooth and rough from the texture; light and dark from the brightness; high, medium and low from the height; adjacent, disjoint and containing from the position relationship. The ontology model of the mark rules is shown in figure 5.

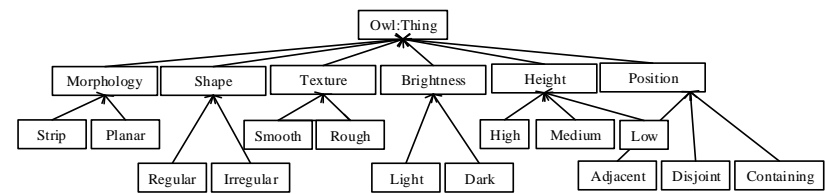

Figure 5. The mark rules in ontology (Every subclass is shown with an "is.a" relationship).

The mark rules are expressed in SWRL, and the semantic relationships between the object features and the classes are built. For example, the Brightness type is expressed in SWRL as follows:

- Mean (?x, ?y), greaterThanOrEqual(?y, 0.38) -> Light (?x);

- Mean (?x, ?y), lessThan (?y, 0.38) -> Dark (?x);

It means, Mean of an object greater than or equal 0.38 denotes Light, whereas that less than 0.38 denotes Dark.

(b)Ontology model of the expert rules

The expert rules for eight types of land cover are acquired from literature. In general, the expert rules are as follows:

- Fieldland = Regular + Planar+ Smooth+ Dark+ Low + Adjacent to Road ;

- Woodland = Irregular+ Planar + High + Rough + Dark + Adjacent to Fieldland;

- Orchardland = Regular + Smooth + Planar + Dark + Adjacent to Fieldland;

- Grassland= Irregular + Planar +Smooth +Dark+ Low + Adjacent to Building;

- Building = Regular + Planar + Rough + High + Light + Adjacent to Road;

- Road $=$ Regular + Strip + Smooth + Light + Low;

- Bareland = Irregular +Planar +Rough + Lght + Low;

- Water =Irregular + Planar + Smooth + Dark + Low+Normal Differential Water Index(NDWI).

The expert rules are expressed in SWRL, and the semantic relationships between the mark rules and the classes are built. For example, the Fieldland is expressed in SWRL as follows:

- Regular (?x), Planar (?x),Smooth(?x),Darklight(?x),Low(?x) $>$ Field (?x);

It means, an image object with Regular, Planar, Smooth, Dark and Low features is a Fieldland. $\mathrm{C}($ ? $\mathrm{X}), \mathrm{X}$ is an individual of $\mathrm{C}$, $\mathrm{P}($ ? $\mathrm{X}$ ? Y) represents attributes, and $\mathrm{x}$ and $\mathrm{y}$ are variables.

The other classifiers such as support vector machine (SVM), random forest could be expressed in SWRL. And the ontology model of the expert rules should be extended and supplemented to realize the semantic understanding of various category of land cover.

2.1.5 Semantic Network Model: The entire semantic network model is formed through the construction of the remote sensing image, land cover class hierarchy, image object features and the classifiers. It is shown in figure 6 .

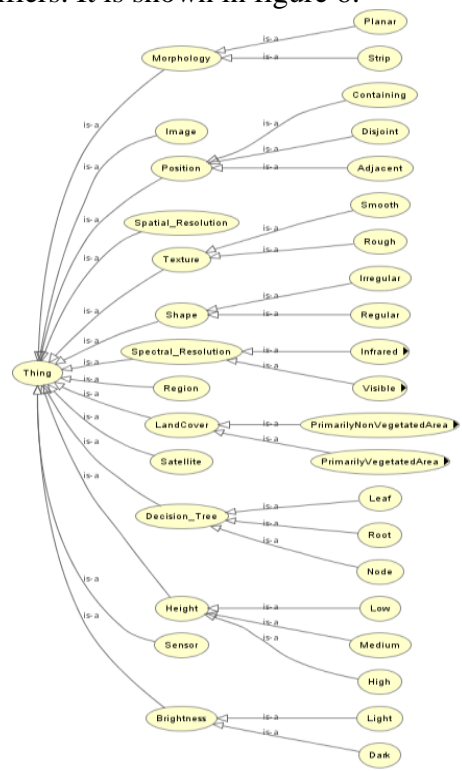

Figure 6. The semantic network model 
The semantic network model is a type of directed network graph that expresses knowledge through the concept and its semantic relations. It has the following advantages. First, the concepts, features and relationships of geographical entities are expressed explicitly, it could reduce the semantic gap between low-level features and high-level semantics. Second, it can be traced back to the parent object, child objects and neighborhood objects through their relationships. Third, it is easy to express semantic relations by computer operable formal language (Tonjes et al., 1999).

\subsection{Initial Classification based on Data-driven Machine Learning}

The process includes pre-processing, segmentation, feature selection, sample collection and initial classification. The software PCI Geomatica developed by the Canadian PCI company is chosen to be the image pre-processing tool, it is good at geometric correction and image fusion. The software FeatureStation developed by the Chinese Academy of Surveying and Mapping is chosen to be the image segmentation and classification tool, it is good at segmentation and decision tree classification. The Protégé plugin developed by Jesús M. A $\mathbf{J}$ is chosen to be the format transformation and semantic classification tool.

2.2.1 Preprocessing: The test site is in Ruili City, Yunnan Province in China. We utilised panchromatic (Pan) ZY-3 data with $2.1 \mathrm{~m}$ resolution and multispectral (MS) ZY-3 data with $5.8 \mathrm{~m}$ resolution (with four bands, including blue, green, red and near-infrared), which were acquired in April 2013. ZY-3 MS imagery is obtained and geometrically corrected to the Universal Transverse Mercator (UTM) projection and then resampled to $2.1 \mathrm{~m}$ to match the Pan image pixel size; it is then fused by using the Pansharp fusion method and the PCI Geomatica software. Figure 7 shows the resulting fused image based on MS bands 4 (near-infrared), 3 (red) and 2 (green). The part of the city selected for the study is characterised by classes identified as fieldland, woodland, grassland, orchardland, bareland, road, building and water.

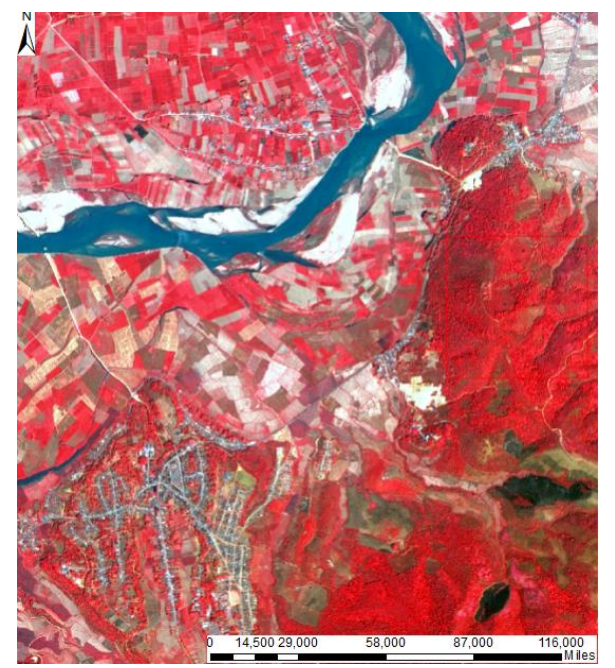

Figure 7. The fusion image of ZY-3(false color)
2.2.2 Image Segmentation: The objective of image segmentation is to keep the heterogeneity within objects as small as possible, at the same time preserving the integrity of the object. The fusion image is segmented using the G-FNEA method which is based on graph theory and fractal net evolution approach (FNEA) within the FeatureStation software. The method could get high efficiency and maintain good feature boundaries (Yang et al. 2015).

There are three parameters in the G-FNEA method: $T$ (scale parameter), $w_{\text {colour }}$ (weight factor for color heterogeneity), and $w_{\text {compt }}$ (weight factor for compactness heterogeneity). A high $T$ value indicates fewer, larger objects than a low $T$ value. The color heterogeneity $w_{\text {colour }}$ describes the spectral information, which is used to indicate the degree of similarity between two adjacent objects. The higher the $w_{\text {colour }}$ value, the greater influence color has on the segmentation process. The $w_{c o m p t}$ value reflects the degree of clustering of the pixels within a region: the lower the value, the more compact the pixels are within the region. It should be noted that the scale parameter is considered to be the most important factor for classification as it controls the relative size of the image objects and has a direct effect on the overall classification accuracy.

There are some methods on automatic determination of appropriate segmentation parameters, such as Estimation of Scale Parameters (ESP)(Dră guţ, L. et al.,2010), Optimised image segmentation (Gao Y. et al.,2011). In the study, the selection of image segmentation parameters is based on an iterative trial-and-error approach that is often utilized in objectbased classification (Myint et al. 2011; Pu et al.2011). It can get good segmentation result with $T=100, \quad w_{\text {colour }}=0.8$, and $w_{\text {compt }}=0.3$.

2.2.3 Feature Selection: The selection of appropriate object features can be based on experience and user knowledge, or can make use of feature-selection algorithms. The Random Forest classifier is capable of handling large numbers of features and a relatively small number of samples (Stumpf, A. et al.,2011). In this study, we make use of experience and user knowledge to guide the initial selection of object features, and thus keep to the following four rules: (1) the most important features of an object are the spectral characteristics, which are independent of test area and segmentation scale, (2) the ratio of bands is closely related to vegetation and non-vegetation, (3) the effect of the shape feature, which is used to reduce the image classification error rate, is small; therefore, it becomes effective when the segmentation scale reaches a certain level, (4) the auxiliary data is dependent on the scale; the smaller the scale, the more important the auxiliary data. Based on the above four rules, twenty-nine features (e.g., ratio, mean, NDWI, Normalized Difference Vegetation Index, homogeneity, and brightness) are selected and stored in Shpfile format, and then converted to OWL format.

2.2.4 Initial Classification: The $\mathrm{C} 4.5$ decision tree method is used for the construction of a decision rule, which includes generation stage and pruning stage (figure 8). 


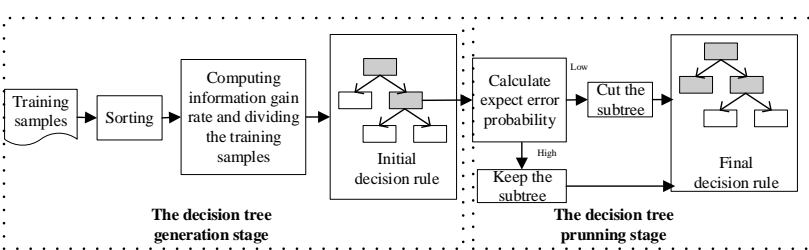

Figure 8. Decision rule based on C4.5 decision tree classifier

Stage 1: The generation of decision tree

1) The training samples are ordered in accordance with the "class, features of sample one, features of sample two, etc". The training and testing samples are selected by visual interpretation of imagery with their selection being controlled by the requirement for precision and representativeness, and by their statistical properties.

2) The training samples are divided. The information gain and information gain rate of all the features of training samples are calculated. The feature is taken as the test attribute, whose information gain rate is the biggest and its information gain is not lower than the mean of all the features, and the feature is taken as a node and leads to a branch. In this circulation way, all the training samples are divided.

3) The generation of decision tree. If all the training samples of the current node belongs to a class, the class is marked as a leaf node and marked for the specify feature; It runs on the same way, at last, it forms a decision tree until all the data of a subset are recorded in the main feature and their feature value are the same, or there is no feature to divide again.

Stage 2: The pruning of decision tree

The possible error probability of sub-node not leaf-node is calculated, the weights of all the nodes are assessed. The subtree is kept if the error rate causes by cutting off the node is high, otherwise, the subtree is cut off. At last, the decision tree with the least expected error rate is the final decision tree which is shown in figure 9. The decision tree is expressed in OWL is shown in figure 10 .

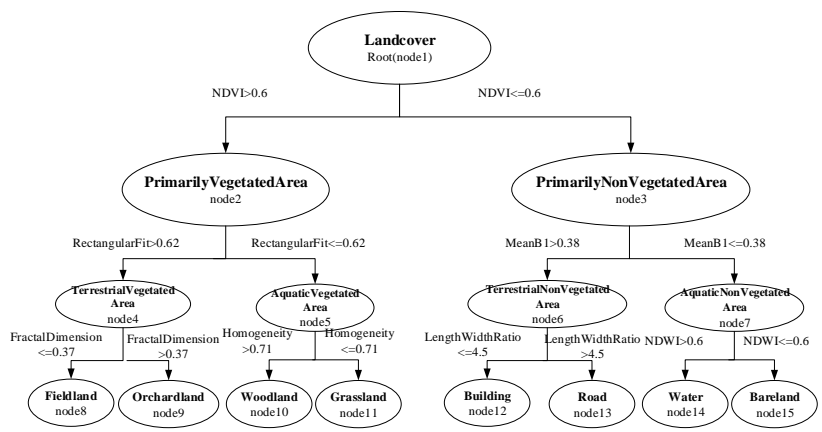

Figure 9. The model of decision tree

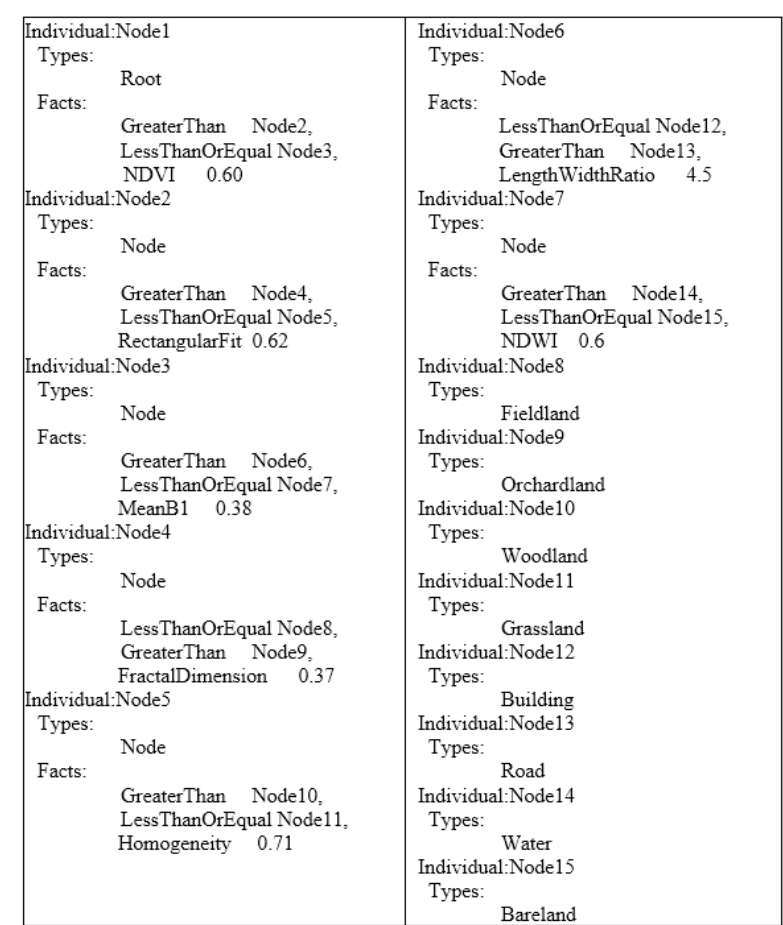

Figure 10. The decision tree is expressed in OWL

The above decision rule is import into the ontology framework, all objects are classified using the decision rule, and the initial classification result is expressed in OWL file format.

\subsection{Semantic Classification based on Knowledge-driven Expert Rules}

On the basis of the initial classification, each object is reclassified by expert rules in SWRL to obtain the semantic information.

2.3.1 Expert Rules Building:The mark rules and expert rules of the eight classes of the test site are expressed in SWRL according to the ontology model of the above mark rules and expert rules.

1) Mark rules are shown as follows:

- RectFit (?x, ?y), greaterThanOrEqual(?y, 0.5) -> Regular (?x);

- RectFit (?x, ?y), lessThan(?y, 0.5) -> Irregular (?x);

- LengthWidthRatio(?x, ?y), greaterThanOrEqual(?y, 1) -> Strip(?x);

- LengthWidthRatio(?x, ?y), lessThan (?y, 1) -> Planar(?x);

- Homo (?x, ?y), greaterThanOrEqual(?y, 0.05) -> Smooth (?x);

- Homo (?x, ?y), lessThan (?y, 0.05) -> Rough(?x);

- Mean (?x, ?y), greaterThanOrEqual(?y, 0.38) -> Light (?x);

- Mean (?x, ?y), lessThan (?y, 0.38) -> Dark (?x);

- MeanDEM(?x, ?y), greaterThanOrEqual(?y, 0.6) -> High(?x);

- MeanDEM (?x, ?y), lessThan(?y, 0.2) -> Low(?x);

- MeanDEM (?x, ?y), greaterThanOrEqual(?y,0.2), lessThan(?y, $0.6)$-> Middle(?x).

It means, RectFit of an object greater than 0.5 denotes Regular shape, whereas that less than 0.5 denotes Irregular shape.

2) Expert rules are shown by the following:

- Regular (?x), Planar (?x),Smooth(?x),Dark (?x),Low(?x) -> Fieldland (?x);

- Irregular (?x), Planar (?x),Rough(?x),Dark (?x),High(?x)-> Woodland (?x); 
- Regular (?x), Planar (?x),Smooth(?x),Dark (?x),Middle(?x) -> Orchardland (?x);

- Irregular (?x), Planar (?x), Smooth (?x),Dark (?x),Middle(?x) -> Grassland (?x);

- Regular (?x), Planar (?x), Rough (?x), Light(?x), High(?x)-> Building(?x);

- Regular (?x), Strip (?x), Smooth (?x), Light(?x),Low(?x) -> Road(?x);

- Irregular (?x), Planar (?x), Rough (?x), Light (?x), Low (?x) $>$ Bareland(?x);

- Irregular (?x), Planar (?x), Smooth (?x), Dark (?x), Low (?x) $>$ Water(?x).

For example, an object with Regular, Planar, Smooth, Dark and Low features is a Fieldland. $\mathrm{C}($ ? $\mathrm{X}), \mathrm{X}$ is an individual of $\mathrm{C}, \mathrm{P}($ ? $\mathrm{X}$ ? $\mathrm{Y}$ ) represents attributes, and $\mathrm{x}$ and $\mathrm{y}$ are variables.

2.3.2 Sematic Classification: The initial classification result is reclassified to get the final classification result based on the expert rules. The exported OWL objects are a way to preserve the semantics of the features the image objects exhibits. The classified objects already exported in OWL format help in retrieving the object features of interest (Figure 11).

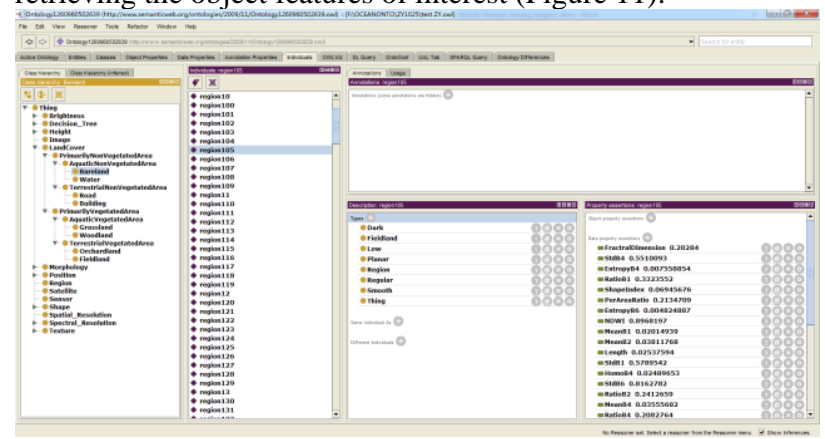

Figure 11. The sematic information of "region 105"

The classification information in OWL format is transformed to Shpfile format which is shown in figure 12.

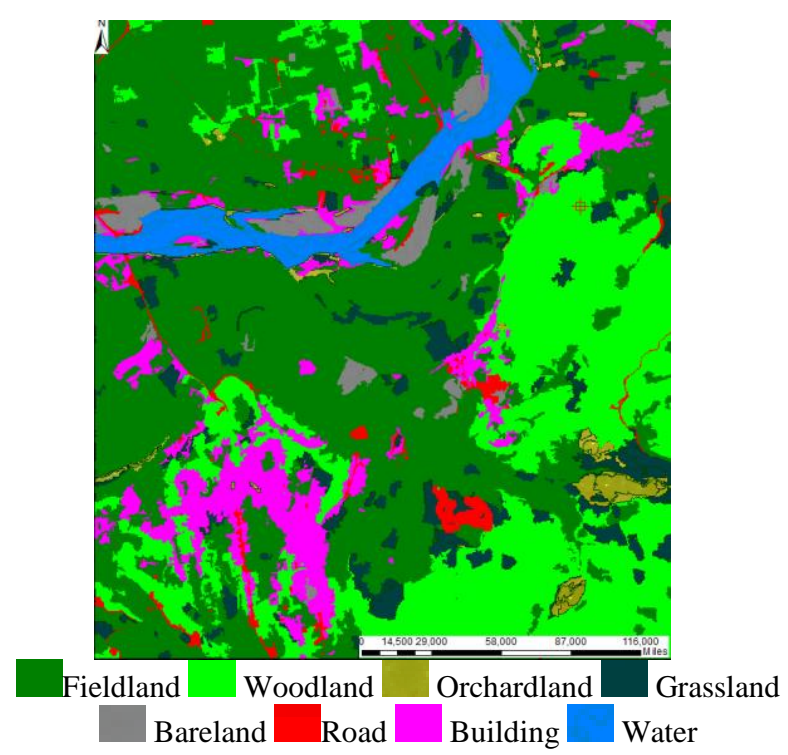

Figure 12. Land cover classification map of ZY-3 in the test site

\section{ANALYSIS AND DISCUSSION}

Accuracy assessment is necessary to validate the result. Error matrix based on samples is selected and used for performing accuracy assessment. The samples are selected depending on the visual interpretation performed over the image. Typical samples are collected from each land-cover type, with their selection being controlled by the requirement for precision and representativeness, and by their statistical properties. The requirement for precision ensures that the samples are accurately selected and that they actually come from the same types of land-cover, the requirement for representativeness ensures that the selected samples are typical of each land-cover type, and the statistical properties ensure that the samples selected are truly representative of the full distribution within each land-cover type. This procedure ensures that similar numbers of samples are used to represent each land-cover type, for both training and testing. Some samples from each landcover type serve as the training samples used to derive the decision tree, while the others are used to test the classification accuracy. In the object-based image analysis, the sample refers to an object. The error matrix of the test is shown in table 2 .

Table 2. The error matrix of the test

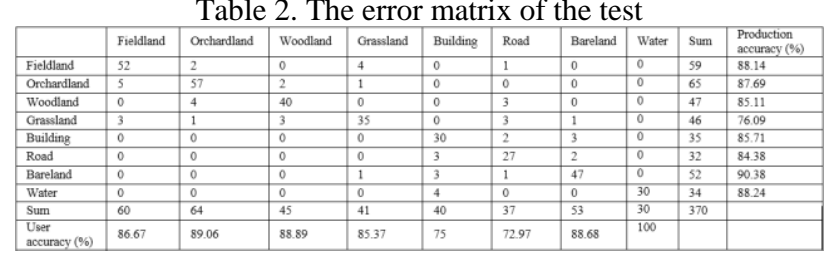

A graphical representation of the classification confusion matrix is shown in Figure 13.

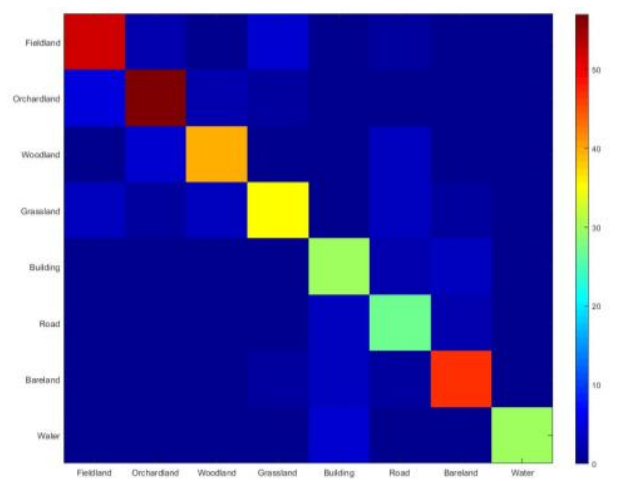

Figure13. Graphic representation of the classification confusion matrix. Rows represent reference class and columns classified data.

The user's and producer's classifications are shown in figure 14.

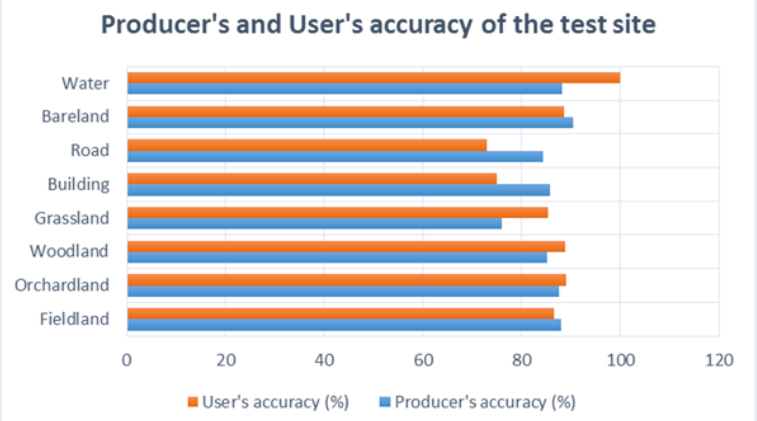

Figure 14. User's and producer's accuracies for land cover classification

The confusion matrix shows that the classification method can distinguish the eight types of land cover, the total accuracy is $85.95 \%$. Road had a poor performance and presented several 
misclassification with and Building and Bareland classes. And Grassland had a poor performance and presented several misclassification with and Fieldland, Woodland and Road classes. Grassland class producer's accuracy is the lowest due to the confusion with classes Fieldland,Woodland and Road. Bareland presented the highest producer's accuracy due to their special spectral and shape comparing with others. The user's accuracy of Road is the lowest due to the misclassification with Building and Bareland classes. Given that the method employs expert rules to restrict, it reduces misclassification and leakage to a certain extent. However, obvious misclassification phenomena of Building and Road exists because the two types of spectral are close. The reason for low classification accuracy for Road is that the road in the study area is located in the Southern suburb of Ruili, which is narrow; thus, the method misinterpretes the spectrum of the Road as the Building spectrum. The shape is further utilised to restrict and high-level information is employed to distinguish.

The uncertainty of the method includes the determination of a segmentation scale, the importance of features, the choice of classifiers, and the determination of parameters. This study focuses on the implementation process of the method, and overall accuracy is used to evaluate the feasibility of the method It should be pointed out that, various elements of GEOBA on the influence of this method is beyond the scope of the study.

From the result it is well understood that the ontology model helps in combining various elements of GEOBIA. It also helps in transferring information from one source to other. The ontology model of image object features uses the structure of eCognition as the upper level knowledge to be further extended. The ontological framework proposed in the study uses the concepts of Land Cover classification System (Di, 2005) knowledge as the upper level knowledge to be further extended. It only builds the decision tree ontology model and expert rule ontology model, they should be extended and supplemented to realize the semantic understanding of various category of land cover. The process of image interpretation in geographic domain is an expert process and many of the parameters need to be tuned depending on the problem domain (Arvor et al., 2013). Thus to improve the GEOBIA, the overall elements of GEOBIA should be modelled using ontology.

\section{CONCLUSIONS AND OUTLOOK}

The study has put forward an object-based semantic classification method of high resolution satellite imagery using ontology that aims to fully exploit the advantages of ontology to GEOBIA. A detailed workflow has been introduced that includes three steps: ontology modelling, initial classification based on decision tree machine learning method, and semantic classification based on expert rules. All kinds of elements for GEOBA were organized organically and expressed explicitly using ontology, and semantic relations were expressed in the OWL and SWRL formal language that the computer could operate. Image objects were classified based on ontology model and using decision tree and expert rules. It could supply objective model and new method for remote sensing image classification, and promote its automation development.

The ontology model of remote sensing imagery, image object feature, land cover class hierarchy and classifiers were built by use of OWL and SWRL, thus the entire semantic network model was built, which lays an ontology model for object classification. These knowledge for model are acquired either from literature (Belgiu,2014) or by using data mining techniques (Maillot, 2004; Belgiu, 2014). Ontology not only proves to be a source of knowledge the domain needs but also fills the semantic gap which exists in performing image classification (Blaschke et al., 2014). Image objects were classified by combining decision tree and experts rules, which not only provide the classification result of the geographical objects, but also master the semantic information of the geographical entities, and realize the reuse of the domain knowledge and the semantic network model. The test has proved the feasibility of the method. The authors are confident that the method has the potential to be applied to the land cover monitoring at regional and global scales.

In addition, the uncertainty of remote sensing information is an important and challenging research field in which many important theoretical and methodological issues need to be addressed. Due to the limitation of our research time and level, this research only discusses the classification that brings uncertainty. However, uncertainty remains in each stage of GEOBIA, an issue which is becoming a matter of concern for more and more researchers, especially for experts in GEOBIA. Nevertheless, it is an emerging method that is still in the process of development and improvement. Further in-depth studies may be required to (a) improve and refine the ontology model, (b) build the ontology model for new classifiers such as deep learning, random forests and random fern, (c) investigate the factors influencing classification, such as the spatial scale, the segmentation method employed, and the choice of samples and object features, and (d) to investigate the automation and 'geointelligence' potential of the ontology-driven object-based semantic classification method.

The method is knowledge-driven and needs to be shared among the experts so as to enhance and share. Thus it is recommended that future researchers and experts utilize the existing ontology to form more domain specific ontology, and to enhance the automation of GEOBIA.

\section{ACKNOWLEDGEMENTS}

This work was supported by the [National Natural Science Foundation of China- Automatic urban geographical conditions information classification by combining high-resolution satellite imagery and multisource space data] under Grant[ 41371406]; and [Central Public-interest Scientific Institution Basal Research Fund] under Grant[7771508].

\section{REFERENCES}

Addink, E.A.; Van Coillie, F.M.B.; De Jong, S.M. 2012. Introduction to the GEOBIA 2010 special issue: from pixels to geographic objects in remote sensing image analysis. Int. J. Appl. Earth Obs. Geoinf., 15, pp.1-6.

Agarwal, P. 2005. Ontological considerations in GIScience. International Journal of Geographical Information Science. 19, pp.501-536.

Arvor D, Durieux L, Andres S, et al. 2013. Advances in Geographic Object-Based Image Analysis with ontologies: Areview of main contributions and limitations from a remote sensing perspective. ISPRS Journal of Photogrammetry and Remote Sensing, 82, pp.125-137. 
Belgiu M, Tomljenovic I, Lampoltshammer T J, et al. 2014. Ontology-based classification of building types detected from airborne laser scanning data. Remote Sensing, 6(2),pp.13471366.

Belgiu M, Hofer B, Hofmann P. 2014. Coupling formalized knowledge bases with object-based image Analysis. Remote Sensing Letters ,5(6), pp.530-538.

Belgiu, M., Dră guţ, L., and Strobl, J., 2014. Quantitative evaluation of variations in rule-based classifications of land cover in urban neighbourhoods using WorldView-2 imagery. ISPRS Journal of Photogrammetry and Remote Sensing, 87, pp.205-215.

Benz, U.C.; Hofmann, P.; Willhauck, G.; Lingenfelder, I.; Heynen, M. 2004. Multi- resolution, object-oriented fuzzy analysis of remote sensing data for GIS-ready information. ISPRS J. Photogramm.58, pp.239- 258.

Blaschke, T. 2010. Object based image analysis for remote sensing. ISPRS J. Photogramm. 65, pp.2-16.

Blaschke, T., G.J. Hay, M. Kelly, S. Lang, P. Hofmann, E. Addink, R. Feitosa, F. van der Meer, H. van der Werff, F. Van Coillie, D. Tiede. 2014. Geographic Object-based Image Analysis: a new paradigm in Remote Sensing and Geographic Information Science. ISPRS International Journal of Photogrammetry and Remote Sensing,87(1), pp.180-191.

Duro, D. C.; Franklin, S. E.; Dubé, M.G. 2012. A comparison of pixel-based and object-based image analysis with selected machine learning algorithms for the classification of agricultural landscapes using SPOT-5 HRG imagery. Remote Sens. Environ. 118 , pp. 259-272.

Dejrriri K, Malki M. 2012. Object-based image analysis and data mining for building ontology of informal urban settlements Proc. SPIE 8537, Image and Signal Processing for Remote Sensing XVIII, 85371I.

Definiens Imaging GmbH. Developer 8 Reference Book; Definiens Imaging GmbH: Munich, Germany, 2011.

Di G., A., 2005. Land cover classification system: classification concepts and user manual: LCCS, Software version 2. ed, Environment and natural resources series. Food and Agriculture Organization of the United Nations, Rome.

Forestier G, Puissant A, Wemmert C, et al. 2012. Knowledgebased region labeling for remote sensing image interpretation. Computers, Environment and Urban Systems,36, pp.470-480.

Gao, Y.; Mas, J. F.; Kerle, N.; Navarrete Pacheco, J. A. 2011. Optimal region growing segmentation and its effect on classification accuracy. International Journal of Remote Sensing, 32(13), pp.3747-3763.

Hay, G.J.; Castilla, G. 2006. Object-based Image Analysis: Strengths, Weaknesses, Opportunities and Threats (SWOT). In Proceedings of 1st International Conference on Object-based Image Analysis (OBIA), Salzburg, Austria, 4-5 July 2006.

Hay, G.J.; Castilla, G. 2008. Geographic Object-Based Image Analysis (GEOBIA): A New Name for a New Discipline. In Object-Based Image Analysis: Spatial Concepts for Knowledgedriven Remote Sensing Applications; Blaschke, T., Lang, S.,
Hay, G.J., Eds.; Springer-Verlag: New York, NY, USA, pp. 8192.

Hofmann, P.; Lettmayer, P.; Blaschke, T.; Belgiu, M.; Stefan Wegenkittl S.; Graf R, Lampoltshammer T. J., Andrejchenko V. 2015.Towards a framework for agent-based image analysis of remote-sensing data, International Journal of Image and Data Fusion, 6(2), pp.115-137.

Jesús M A J, Luis D, José A P F. 2013. A Framework for Ocean Satellite Image Classification Based on Ontologies. IEEE Journal of selected topics in applied earth observations and remote sensing, 6(2), pp.1048-1063.

Kohli, D., Sliuzas, R., Kerle, N., \& Stein, A. (2012). An ontology of slums for image-based classification. Computers Environment and Urban Systems, 36(2), 154-163.

Kyzirakos K, Karpathiotakis M, Garbis G, et al. 2014. Wildfire monitoring using satellite images, ontologies and linked geospatial data. Web Semantics: Science, Services and Agents on the World Wide Web, 24, pp.18-26.

Li, J.L.; He, Z.Y.; Ke, D.L.; Zhu, Q.L. 2014. An Approach for Insight into Geo-ontology Merging based on Description Logics. Geomatics and Information Science of Wuhan University. 39(3), pp.317-321.

Dră guţ, L.; Tiede, D.; Levick, S.R. 2010, ESP: a tool to estimate scale parameter for multiresolution image segmentation of remotely sensed data, International Journal of Geographical Information Science, 24(6),pp:859-871.

Maillot, N., Thonnat, M., and Boucher, A., 2004. Towards ontology-based cognitive vision. Machine Vision and Applications, 16 (1), pp.33-40.

Myint, S.W.; Gober, P.; Brazel, A.; Grossman-Clarke, S.; Weng, Q. 2011. Per-pixel vs. object-based classification of urban landcover extraction using high spatial resolution imagery. Remote Sens. Environ. 115, pp.1145-1161.

OWL Web Ontology Language Reference[EB/OL].http://www.w3.org/TR/owl-ref/.

Robertson, L.D.; King, D.J.; 2011. Comparison of pixel- and object-based classification in land-cover change mapping. Int. J. Remote Sens. 32, pp.1505-1529.

Stumpf, A.; Kerle, N. 2011. Object-oriented mapping of landslides using Random Forests. Remote Sensing of Environment, 115(10), pp. 2564-2577.

SWRL: A Semantic Web Rule Language Combining owl and RuleML[EB/OL].http://www.w3.org/Submission/SWRL/.

Tonjes, R.; Glowe, S.; Buckne,r J.; et al. 1999. Knowledgebased interpretation of RS images using semantic nets. Photogrammetric Engineering\& Remote Sensing. 65,(7), pp.811-821.

Yang Yi, Li Haitao, Han Yanshun, Gu Haiyan. 2015. High resolution remote sensing image segmentation based on graph theory and fractal net evolution approach. 2015 International Workshop on Image and Data Fusion. Kona, Hawaii, USA, 21-23 July 2015. 


\title{
TOWARDS A TYPOLOGY OF LAND-COVER/LAND-USE EVOLUTIONS USING HIGH RESOLUTION SATELLITE IMAGE TIME SERIES: APPLICATION TO THE METROPOLITAN AREA OF STRASBOURG (FRANCE)
}

\author{
F. N. Güttler ${ }^{\mathrm{a}, \mathrm{b}, \mathrm{c}} *$, A. Puissant ${ }^{\mathrm{b}}, \mathrm{P}$. Gançarski $^{\mathrm{c}}$ \\ a INPT, Ecole d'Ingénieurs de Purpan, UMR 1201 DYNAFOR, 31300 Toulouse, France - fabio.nor-guttler@purpan.fr \\ ${ }^{\mathrm{b}}$ Laboratoire Image, Ville, Environnement, UMR 7362 CNRS, University of Strasbourg, 67000 Strasbourg, France - \\ anne.puissant@live-cnrs.unistra.fr \\ ${ }^{c}$ ICube Laboratory, UMR 7357 CNRS, University of Strasbourg, Strasbourg 67000, France - \\ gancarski@unistra.fr
}

KEY WORDS: Temporal GEOBIA, Change Detection, Land-cover Evolution, Urban and Peri-urban Areas, Fuzzy Learning

\begin{abstract}
In this work, we propose an original approach to extract structured information from long-term satellite image time series (SITS) which can be employed to construct a typology of land-cover/land-use (LCLU) evolutions. Through a semi-supervised framework we combine per-pixel and object-based image analysis with data mining techniques. Our experiments are applied to the metropolitan area of Strasbourg (France) using nine high spatial resolution $(20 \mathrm{~m})$ SPOT images acquired between 1992 and 2011. The framework is organized in four main steps: (a) construction of image objects, (b) multi-strategy fuzzy learning approach to predict LCLU class membership at pixel level, (c) generation of structured information at object level and (d) mining structured information across the time-series.
\end{abstract}

\section{INTRODUCTION}

Satellite Image Time Series (SITS) is a relevant source of information for studying evolutions in urban and peri-urban areas. However follow such complex and dynamic areas is challenging. On the one hand, high spatial resolution imagery $(<$ $30 \mathrm{~m}$ ) is required to identify urban spatial organization and the main types of land-cover and land-use (LCLU) at a given date. On the other hand, most of the LCLU evolutions spread over long periods, usually ranging from few months to several years. Three key issues can be associated to the spatiotemporal analysis of SITS: (1) firstly, one should be able to detect where the evolutions took place in a given landscape, (2) then, it is important to determine precisely when those evolutions arise and, (3) finally, it is essential to be able to characterize the different types of evolutions, classifying them into categories with relevant semantic meaning.

As advertised by the scientific community, methods describing multi-temporal behavior are among open challenges in GEOBIA (Blaschke et al., 2014 ; Chen et al., 2012). Chen et al. (2012) organized the techniques used for object-based change detection (OBCD) in four categories: (1) image-object change detection where multi-dates images are segmented separately and the objects are directly compared; (2) class-object change detection where a classification is performed independently for each image before comparison; (3) multitemporal-object change detection where multi-date images are combined and segmented together to produce corresponding change objects and (4) hybrid change detection which combines both object and pixelbased image analysis techniques. Still, most of those change detection techniques cannot deal with long time series (i.e. more than four or five images). Also, they usually do not satisfy the three key issues of the spatiotemporal analysis. In this work, in opposition to the classical approaches, we propose an original approach to extract information related to those three key issues and we tested our method in a long-term SITS containing nine optical images. Our approach is hybrid and combines pixelbased and object-based image processing with data mining techniques in a semi-automatic way. The paper is organized as follows: Section 2 presents the study area and the dataset. Section 3 describes all the steps of the methodology. Experimental results are presented and discussed in section 4. Conclusions are drawn in Section 5.

\section{STUDY AREA AND DATA DESCRIPTION}

\subsection{Study Area}

The study area corresponds to the metropolitan area of Strasbourg, located in the North-East of France (Figure 1). The metropolitan area consists of 28 municipalities and has a territory of about $316 \mathrm{~km}^{2}$. The population is estimated in more than a half million of inhabitants. It is built around a concentric dense city centre inherited from the Middle Ages with surroundings organised in some rings characterized by typical urban fabrics such as discontinuous urban fabric dedicated to housing, leisure activities, commercial and industrial activities, etc. The city is also covered by some urban vegetation and is bordered to the East by an important river (the Rhine). Agricultural areas and patches of forest are present in most of the surrounding municipalities. During the last decades the study area experienced a classical urbanization process of urban sprawl around the inner-city like some European cities in Western Europe.

* Corresponding author 


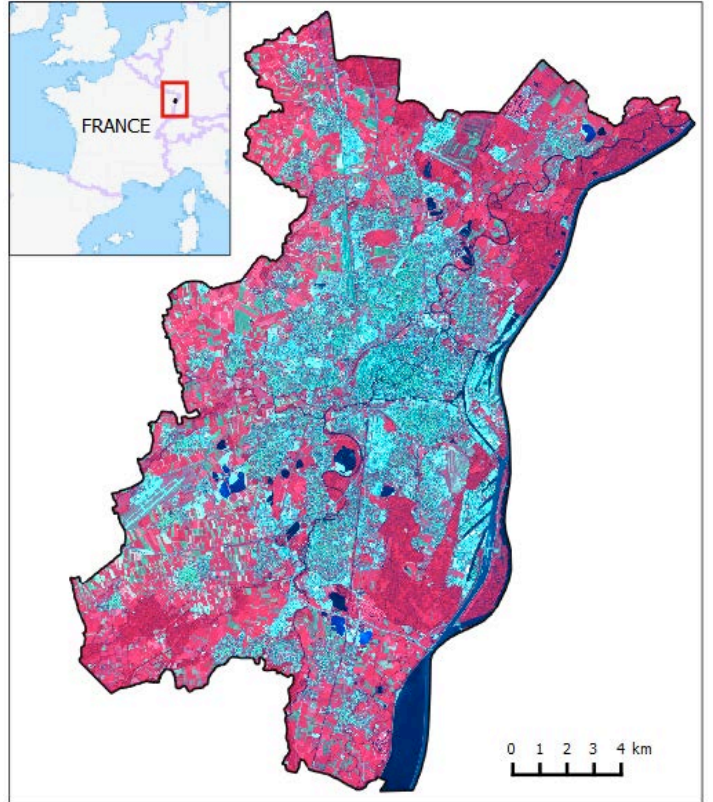

Figure 1. The metropolitan area of Strasbourg (Spot image (C)CNES, 2011)

\subsection{Data and pre-processing}

The main source of data is a collection of nine high spatial resolution SPOT images acquired between 1992 and 2011 (see Table 1).

\begin{tabular}{|r|l|c|l|}
\hline Acquisition date & Satellite & Pixel size $(\mathrm{m})$ & \multicolumn{1}{|c|}{ Spectral bands* } \\
\hline 7 Sept. 1992 & SPOT 1 & $20 \mathrm{~m}$ & G, R, NIR \\
22 July 1996 & SPOT 3 & $20 \mathrm{~m}$ & G, R, NIR \\
17 Aug. 1998 & SPOT 2 & $20 \mathrm{~m}$ & G, R, NIR \\
25 July 1999 & SPOT 4 & $20 \mathrm{~m}$ & G, R, NIR, SWIR \\
8 June 2000 & SPOT 1 & $20 \mathrm{~m}$ & G, R, NIR \\
22 July 2001 & SPOT 4 & $20 \mathrm{~m}$ & G, R, NIR, SWIR \\
5 Sept. 2004 & SPOT 5 & $10 \mathrm{~m}$ & G, R, NIR, SWIR \\
5 July 2010 & SPOT 5 & $10 \mathrm{~m}$ & G, R, NIR, SWIR \\
27 June 2011 & SPOT 5 & $10 \mathrm{~m}$ & G, R, NIR, SWIR \\
\hline * G green, $R=$ red, NIR = near infrared, SWIR = short wave infrared
\end{tabular}

Table 1. Acquisition date and main characteristics (before pre-

processing) of the SPOT images used in this work.

In order to minimize changes related to the seasons (illumination conditions, phenology states, etc.), all the images were acquired during the summer (June to September) and a radiometric normalization process has been applied (Canty et al., 2004). As the original images have dissimilar spatial resolutions, some of them (those from SPOT 5) were resampled to generate a homogeneous SITS with a pixel size of $20 \mathrm{~m}$. For the same reason, we removed the fourth band (short wave infrared) of the images acquired by SPOT 4 and 5. Therefore, only the remaining three bands (green, red, near infrared) were considered for the whole SITS. Finally, a spatial subset was performed to keep only the pixels inside the administrative limit of the Strasbourg metropolitan area as having valid values (see Figure 1).

\section{METHODOLOGY}

The methodological framework is organized in four steps. Step 1 focuses on the construction of two types of image- objects: (a) mono-date objects and (b) time-series objects. In step 2, several spectral and texture features are computed for each timestamp (or date) of the SITS. Step 3 corresponds to a fuzzy supervised learning procedure. It generates LCLU class membership at pixel level, for each timestamp of the SITS. In step 4, structured (thematic, spatial and temporal) information is obtained at object level (mono-date, bi-date and time-series). Finally, some data mining strategies (yet in development) are proposed in order to group similar trajectories and to contribute to the construction of a typology of LCLU evolutions which considers the whole SITS.

\subsection{Construction of image objects}

Mono-date objects: The processing chain starts with multiscale image segmentation. Each image of the time series is segmented independently, but using strictly the same rule-set, which generates separate sets of mono-date objects.

The segmentations were obtained combining the Multiresolution Segmentation Algorithm (MSA) and the Spectral Difference Algorithm, both available in eCognition Developer 8.8.1. MSA is a bottom-up segmentation based on a pairwise region merging technique (Baatz and Schäpe, 2000). To decide whether to merge or not neighboring pixels (or objects) it uses a combination of spectral, shape and scale criteria. The following strategy was employed to tune the MSA algorithm (using two images of the SITS).

Firstly, the ESP2 tool (Drăguţ et al., 2014) was used to obtain an automated estimation of the scale parameter. As a result, the scale values 10 and 31 were selected. Then, with the scale parameter fixed at 10 , we tested and visually compared all the combinations of the shape and compactness criteria (from 0.0 to 1.0 in intervals of 0.1 ). The most appropriated segmentation result was obtained with the following values: shape $=0.4$ and compactness $=0.6$. This segmentation was further enhanced by applying the Spectral Difference Algorithm (SDA value $=4$ ), which merged spectrally similar objects previously separated by the scale parameter constraint. This segmentation result was named "fine level".

Above the fine level, a less detailed segmentation was generated using the second scale value (31) with the MSA. Again, all the combinations of the shape and compactness criteria were tested and compared. The most appropriated segmentation result was obtained with the following values: shape $=0.3$ and compactness $=0.8$. This result was named "medium level".

With the segmentation parameters presented above, a rule-set was created and applied over each timestamp of the SITS.

Time-series objects: Time-series objects (ts-objects) are the result of the topological overlay of all the mono-date objects of the SITS, for a given scale level. More in detail, each ts-object assembles the pixels belonging to the same sequence of monodate objects across time, meaning they possess a similar spectral trajectory.

\subsection{Computing pixel based spectral and texture features}

Before starting the learning step, the optical images were processed and several spectral and textural attributes were computed at pixel level. 
3.2.1 Spectral indices: In total, nine spectral indices were computed using the Orfeo Tool Box (OTB $)^{1}$. Most of them are useful for a better characterization of the vegetation or soil. A detailed description of each index is presented in Table 2 .

\begin{tabular}{|c|c|c|}
\hline $\begin{array}{c}\text { Index } \\
\text { acronym and } \\
\text { type }\end{array}$ & Index name & Formula \\
\hline $\begin{array}{l}\text { NDVI } \\
\text { [Vegetation] }\end{array}$ & $\begin{array}{l}\text { Normalized } \\
\text { difference } \\
\text { vegetation } \\
\text { index }\end{array}$ & $\frac{\text { nir }- \text { red }}{\text { nir }+ \text { red }}$ \\
\hline $\begin{array}{l}\text { TSAVI } \\
\text { [Vegetation] }\end{array}$ & \begin{tabular}{|l|} 
Transformed \\
soil adjusted \\
vegetation \\
index
\end{tabular} & $\frac{0.7(\text { nir }-0.7 \text { red }-0.9)}{0.7 \text { nir }+ \text { red }+0.08\left(1+0.7^{2}\right)}$ \\
\hline $\begin{array}{l}\text { MSAVI } \\
\text { [Vegetation] }\end{array}$ & $\begin{array}{|ll|}\text { Modified } & \text { soil } \\
\text { adjusted } & \\
\text { vegetation } & \\
\text { index } & \\
\end{array}$ & $\begin{array}{l}\quad \frac{(1+L) *(\text { nir }- \text { red })}{n i r+r e d+L} \\
\text { where : } \\
L=1-2 s * N D V I(\text { nir }-0.4 r e d)\end{array}$ \\
\hline $\begin{array}{l}\text { GEMI } \\
\text { [Vegetation] }\end{array}$ & $\begin{array}{l}\text { Global } \\
\text { environment } \\
\text { monitoring } \\
\text { index }\end{array}$ & $\begin{array}{l}\frac{n(1-0.25 n)-\text { red }-0.125}{1-r e d} \\
\text { where: } \\
n=\frac{2\left(n \text { nir }^{2}-r e d^{2}\right)+1.5 \text { nir }+0.5 r e d}{n i r+r e d+0.5}\end{array}$ \\
\hline $\begin{array}{l}\text { NDWI2 } \\
\text { [Water] }\end{array}$ & \begin{tabular}{|l|} 
Normalized \\
difference water \\
index 2
\end{tabular} & $\frac{\text { green }- \text { nir }}{\text { green }+ \text { nir }}$ \\
\hline $\begin{array}{l}\text { RI } \\
\text { [Soil] }\end{array}$ & Redness index & $\frac{\text { red }^{2}}{\text { green }^{3}}$ \\
\hline $\begin{array}{l}\text { CI } \\
{[\text { Soil }]}\end{array}$ & Color index & $\frac{\text { red-green }}{\text { red }+ \text { green }}$ \\
\hline $\begin{array}{l}\text { BI } \\
{[\text { Soil] }}\end{array}$ & $\begin{array}{l}\text { Brightness } \\
\text { index }\end{array}$ & $\sqrt{\frac{\text { green }^{2}+\text { red }^{2}}{2}}$ \\
\hline $\begin{array}{l}\text { BI2 } \\
{[\text { Soil }]}\end{array}$ & $\begin{array}{l}\text { Brightness } \\
\text { index } 2\end{array}$ & $\sqrt{\frac{\text { green }^{2}+r e d^{2}+n i r^{2}}{3}}$ \\
\hline
\end{tabular}

Table 2. Spectral indices computed for each image of the SITS at pixel level.

3.2.2 Texture features: The whole pixel-based Haralick textures features (Haralick et al., 1973) were computed using the functions available at OTB (29 texture features in total). These features are organized in three sets: (a) Simple, (b) Advanced and (c) Higher order. After testing some correlationbased feature subset selection techniques (results not showed here), it was possible to eliminate the 14 most irrelevant and redundant features. The 15 remaining texture features are listed in Table 3. Texture features were calculated using a $5 \times 5$ sliding window (size in pixels).

\footnotetext{
${ }^{1}$ Orfeo Tool Box version 5.4 available at: https://www.orfeotoolbox.org/
}

\begin{tabular}{|l|l|}
\hline Set of texture & Feature name \\
\hline Simple & Correlation \\
Simple & Inverse Difference Moment \\
Simple & Cluster Shade \\
Simple & Cluster Prominence \\
Simple & Haralick's Correlation \\
\hline Advanced & Mean \\
Advanced & Variance \\
Advanced & Sum Entropy \\
Advanced & Difference of Variances \\
Advanced & IC1 (Info Measures of Corr.) \\
Advanced & IC2 (Info Measures of Corr.) \\
\hline Higher order & Grey-Level Non-uniformity \\
Higher order & Run Length Non-uniformity \\
Higher order & Run Percentage \\
Higher order & Low Grey-Level Run Emphasis \\
\hline
\end{tabular}

Table 3. Selected texture features computed for each image of the SITS at pixel level.

\subsection{Computing land-cover / land-use class membership}

3.3.1 Thematic labelling: Taking advantage that ts-objects group pixels sharing a similar spectral trajectory, we used them to guide the thematic labelling. In total, 95 ts-objects (from the medium scale level) were manually selected and labelled into LCLU classes (a label per timestamp). We used a two-level hierarchical nomenclature for this labelling task (see Table 4). The first level has four general LCLU classes; three of them are detailed in the second level that counts nine LCLU classes in total.

\begin{tabular}{|l|l|}
\hline \multicolumn{1}{|c|}{ Level 1 } & \multicolumn{1}{|c|}{ Level 2 } \\
\hline Bare areas & $\begin{array}{l}\text { Bare agriculture } \\
\text { Bare gravel }\end{array}$ \\
\hline Build-up areas & $\begin{array}{l}\text { Build dense } \\
\text { Build industrial-commercial } \\
\text { Build residential }\end{array}$ \\
\hline Vegetation areas & $\begin{array}{l}\text { Woody vegetation } \\
\text { Herbaceous vegetation with high } \\
\text { greenness values } \\
\text { Herbaceous vegetation with low } \\
\text { greenness values }\end{array}$ \\
\hline Water areas & Water \\
\hline
\end{tabular}

Table 4. Land Cover/Land Use (LCLU) classes used in the labelling step.

Together, the 95 labelled ts-objects correspond to a total of 12,224 pixels.

3.3.2 Fuzzy learning strategy: Before starting the learning process, the original SITS was converted into a series of concatenated raster files, each of them containing 27 dimensions (i.e., the 3 former spectral bands +9 spectral indices +15 texture features). The collection of labeled pixels, described in the previous sub-section, was divided in two sets: (a) the training set composed of 8,623 pixels (70 ts-objects) and (b) the validation set with the remaining 3,599 pixels (25 tsobjects).

We designed a multi-strategy fuzzy learning approach that combines three machine learning algorithms: (a) Logistic Regression, (b) Random Forest and (c) Decision Tree Regressor. Coded in Python language, our approach uses the implementations of the above cited algorithms provided by the 
scikit-learn library ${ }^{2}$. More in detail, our approach is based on the "one versus the rest" scheme, meaning that a specific model is constructed for each LCLU class at each timestamp.

The accuracy of the individual models is assessed through 10fold cross-validation using solely the 8,623 pixels of the training set. Root Mean Squared Error (RMSE) was used as the reference metric for model assessment. This experimentation basis was primarily used for tuning the parameters of the three algorithms. Then, the best configuration of parameters per algorithm was selected; i.e. those producing the lowest RMSE considering all the timestamps. Finally, the selected models were deployed over the entire study area.

Model output is expressed as numerical predictions, at pixel level, for each LCLU class (membership values ranging from 0 to 1 ). In order to obtain a single membership value per class, the predictions of the three algorithms were combined by the mean of a weighted average. The weights are inversely proportional to algorithm's RMSE scores obtained for each LCLU class. (Section 4.1 presents results obtained on our dataset.)

At the end of the learning process, the averaged class membership values were exported and converted into a new series of multidimensional raster files, where the number of dimensions is equals to the number of LCLU classes.

After all, we used the validation set (3,599 labelled pixels not used during the learning process) to get an independent assessment of the quality of the results.

\subsection{Generating structured information at object level}

3.4.1 Mono-date objects and LCLU class membership: The fuzzy learning approach applied over the whole study area generates, for each pixel, a vector of LCLU class membership values. For the mono-date objects, the corresponding class membership vectors are computed by averaging pixel vectors. (Section 4.2 and 4.3 present results obtained on our dataset.)

After this operation, for each date, we obtain a set of mono-date objects described by their LCLU class membership. Two additional attributes are computed for each mono-date object. The first is the majority LCLU class (i.e., the label of the class presenting the highest membership value). The second is a confidence estimation of the predicted majority class. Such estimation is based on the membership value of the majority class itself and on its relative importance considering the membership values of the other classes.

3.4.2 Bi-date objects and the types of transition: Besides mono-date and time-series objects, we created also bi-date objects. They correspond to the topological overlay of the mono-date objects from two subsequent timestamps. These objects are computed to better characterize the transitions through the times series. The primary attribute computed for bidate objects are the delta class membership values (i.e., the variation in terms of membership values) for each LCLU class, between two timestamps.

Then, we compute an additional attribute called 'type of transition', which takes into account the magnitude of the delta attributes computed for each LCLU class. Three types of

\footnotetext{
${ }^{2}$ Python machine learning library, version 0.17 .1 , available at: http://scikit-learn.org/
}

transition are considered as function of the observed magnitudes: (a) conservative, (b) semi-conservative and (c) non-conservative. Finally, another attribute, named 'major process', is computed for the bi-date objects presenting nonconservative or semi-conservative types of transition. The attribution of a major process relies on a formal definition of the possible/desired processes that one may be interested in detecting. In the present work, we formalize (as a set of conditions) the following processes: 'urbanization', 'deurbanization', 'retrofit or replacement', 'vegetation increase', 'harvesting', 'poldering' and 'water extension'.

3.4.3 Time-series objects and the connexion of timestamps and transitions: The time-series objects can be viewed as a key element for accessing and connecting information across the time. In that light, all the information coming from mono-date and bi-date objects become connected and can be accessed as temporal sequences through ts-objects. Each sequence consists of a list of transitions and each transition consists of a pair of mono-date objects. (Section 4.4 presents results obtained on our dataset.)

\subsection{Mining structured information}

The temporal sequences are structured in a database allowing multidimensional queries (e.g. one may want to map the spatial footprint of all the vegetation areas converted into residential areas before the year 2000). The objective is to be able to query the database and to generate a map with all the associated temporal and thematic information from such queries.

The next step consists in processing ts-objects sequences using specific tools of sequential pattern mining and temporal clustering. More in detail, sequences presenting similar temporal patterns may be grouped together and their corresponding frequencies should be assessed. This result will provide a synthesis of the temporal patterns based on thematic meaningful transitions. (Section 4.5 presents results obtained on our dataset.)

The paper focuses mainly on the other four steps as the mining step is yet in development.

\section{RESULTS}

In this section we mainly present on the results corresponding to step 3 and 4 of our methodology.

\subsection{Multi-strategy fuzzy learning assessment}

The accuracy assessment of the three learning algorithms (individually) and of the combined model are showed in Table 5 .

As a global result, predictions from the combined model present lower RMSE for most of the LCLU classes. The best predictions were obtained for water and bare areas as well as for herbaceous vegetation (low greenness values). Intermediary quality predictions are observed for the classes build industrialcommercial, woody vegetation and herbaceous vegetation (high greenness values). The less precise predictions are related to the classes build residential and build dense.

\subsection{Class membership for mono-date objects}

At each timestamp of the SITS, mono-date objects are described by LCLU class membership values, ranging from 0 to 1 . Figure 2 shows the spatial distribution of membership values for three LCLU classes (out of nine) in 1992. 


\begin{tabular}{|c|c|c|c|c|c|c|c|c|}
\hline \multirow{2}{*}{ Class label } & \multicolumn{2}{|c|}{ Logistic Regression } & \multicolumn{2}{|c|}{ Random Forest } & \multicolumn{2}{|c|}{ Decision Tree Regressor } & \multicolumn{2}{|c|}{ Combined model } \\
\hline & Mean & Std dev & Mean & Std dev & Mean & Std dev & Mean & Std dev \\
\hline Bare agriculture & 0.113 & 0.066 & 0.108 & 0.075 & 0.121 & 0.068 & 0.102 & 0.069 \\
\hline Bare gravel & 0.100 & 0.033 & 0.089 & 0.027 & 0.113 & 0.030 & 0.089 & 0.026 \\
\hline Build dense & 0.220 & 0.024 & 0.210 & 0.020 & 0.243 & 0.048 & 0.208 & 0.031 \\
\hline Build ind-com. & 0.144 & 0.024 & 0.134 & 0.032 & 0.148 & 0.035 & 0.129 & 0.029 \\
\hline Build residential & 0.267 & 0.027 & 0.269 & 0.027 & 0.294 & 0.032 & 0.267 & 0.027 \\
\hline Woody vegetation & 0.159 & 0.028 & 0.164 & 0.033 & 0.174 & 0.018 & 0.157 & 0.026 \\
\hline Herb. veg. h-green & 0.183 & 0.042 & 0.168 & 0.042 & 0.182 & 0.032 & 0.167 & 0.038 \\
\hline Herb. veg. l-green & 0.114 & 0.033 & 0.080 & 0.038 & 0.100 & 0.038 & 0.087 & 0.035 \\
\hline Water & 0.009 & 0.015 & 0.014 & 0.013 & 0.030 & 0.049 & 0.015 & 0.020 \\
\hline Global RMSE & 0.145 & 0.068 & 0.137 & 0.077 & 0.156 & 0.071 & 0.136 & 0.072 \\
\hline
\end{tabular}

Table 5. Global and per-class Root Mean Squared Error (RMSE) for the three learning algorithms (individually) and for the combined model. RMSE mean and standard deviation calculated using the validation set for the nine timestamps of the SITS.

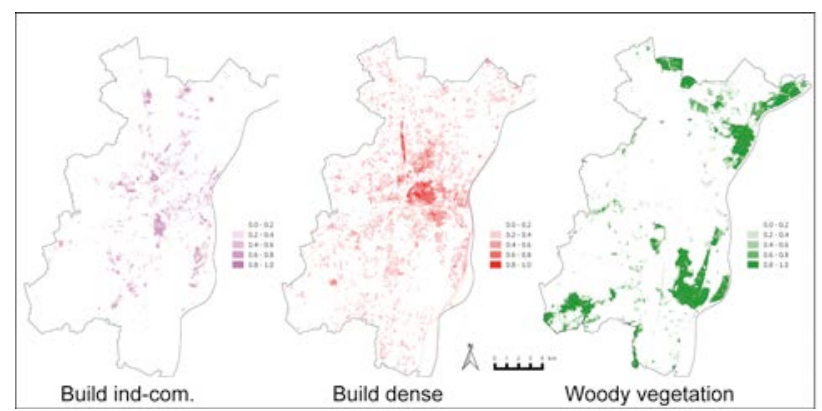

Figure 2. Spatial distribution of membership values for three land-cover/land-use (LCLU) classes: Build industrialcommercial, Build dense, Woody vegetation. Mono-date objects from the first timestamp of the SITS (year 1992).

In order to obtain a less fragmented view of the study area, it is useful to draw the map of the majority LCLU class predicted for each object. Figure 2(A) presents the majority class map obtained for the first image of the series (1992). The second part of the Figure 2(B) represents the confidence of the predictions.
As a general result, higher levels of confidence are observed in woody vegetation and water areas. The lowest levels of confidence are frequently associated to peri-urban areas where the edge separating build-up areas and agricultural areas is not easy to recognise.

\subsection{Class membership temporal trajectories}

Temporal information across the SITS can be obtained from the ts-objects. Indeed, all the attributes computed for mono-date and bi-date objects can be accessed through the ts-objects. For instance, it is possible to compute and analyse class membership trajectories for any ts-object. Figure 3 shows three different class membership trajectories obtained for ts-objects. For a better visual analysis, we plotted the trajectories using the more general level of the LCLU nomenclature (i.e. level 1 containing four general classes).
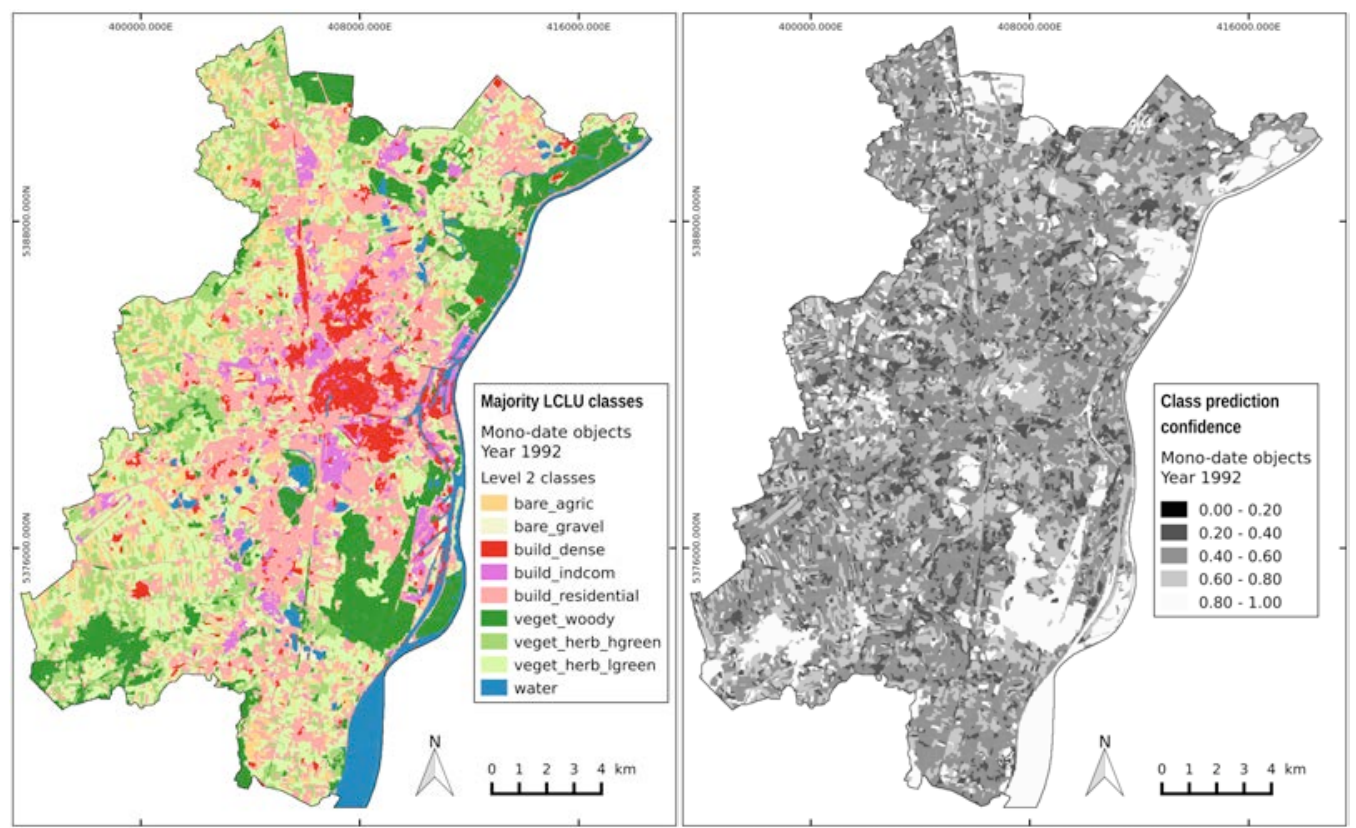

Figure 3. (A) Majority land-cover/land-use (LCLU) classes predicted from the first image of the series. (B) Majority class prediction confidence for the first image of the series. 

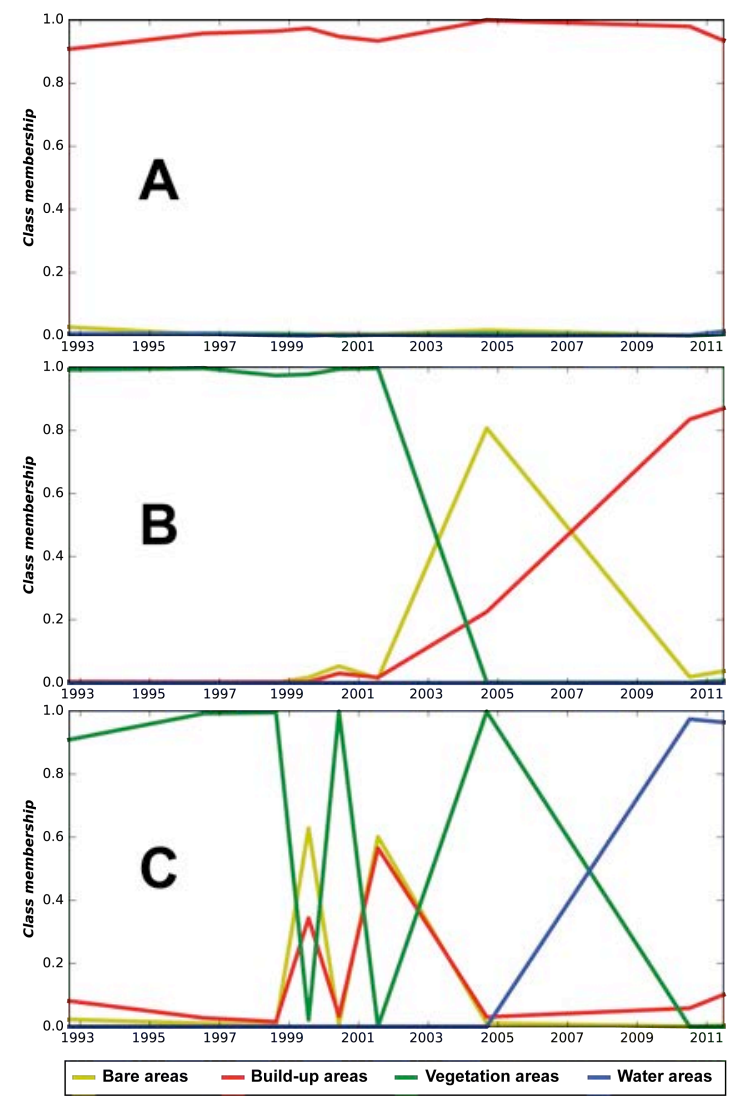

Figure 4. Four class membership trajectories for three tsobjects. (A) Stable area in the city centre of Strasbourg, (B) Vegetated area that was urbanized in the second part of the series (C) Agricultural crop area converted into a gravel extraction pond by the end of the series.

Plot A of Figure 4 shows the membership trajectories of a tsobject located in the historical city centre of Strasbourg. The class membership values are very stable during the entire timeseries, with a clear dominance of the build-up class over the other three classes.

In plot B the temporal behaviour remains stable until the middle of the series, with vegetation membership values around 1 . Between the timestamps of 2001 and 2004, the vegetation membership value falls to 0 while the bare class becomes dominant. However, in the next transition $(2004$ - 2010) the build-up class membership replaces the position of the bare class and this configuration is maintained until the final timestamp (2011). The temporal pattern observed here corresponds to the urbanisation of a meadow area that has been converted into a residential area.

Plot $\mathrm{C}$ presents a more complex temporal pattern. It corresponds to an agricultural crop area located next to a water pond used for gravel extraction. Until 2004, the crop field was observed whether covered by vegetation (when the image was acquired before crop harvesting) or without vegetation (when the image was acquired after crop harvesting). Between 2004 and 2010, the pond has been expanded and the water replaced the former crop field.

\subsection{Transitions and cumulated intensity of change}

Transitions can be analysed as function of the observed magnitudes of change in the class membership values. In order to take into account all the transitions, we computed a global score that considers the types of transition (conservative, semiconservative or non-conservative) for of each bi-date object. This score is a cumulated sum of the non-conservative and semi-conservative transitions, where the first worth 1 and the second 0.5 . Figure 5 shows the spatial distribution of the cumulated intensities of change.

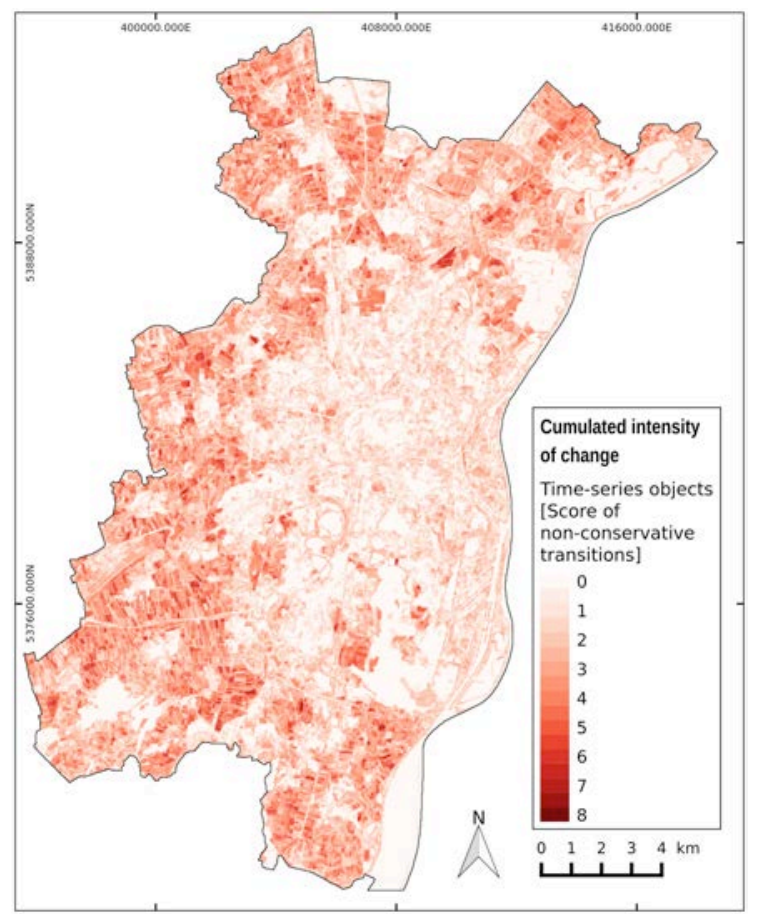

Figure 5. Cumulated intensity of change computed considering all the individual transitions of the SITS.

\subsection{Long-term evolutions}

In order to associate the three key issues to the spatiotemporal analysis of SITS, it is essential to able to characterize the different types of evolutions, grouping them into categories with relevant semantic meaning. First results of long-term evolution are presented in Figure 6. The most relevant is the phenomenon of urban growth from 1992 to 2011.

More precisely, we detect where urbanization took place within the study area. As this type of process was computed for bi-date objects, it is possible to determine between which pair of timestamps the urbanization has been detected. Then, we can calculate the total area becoming urbanized at each transition and compute the cumulated values for the whole SITS (see graph at the bottom of Figure 6). The analyses of other longterm evolutions are in progress, as for instance 'urban retrofit in already build-up areas' and 'expansion of water ponds related to gravel extraction'. 


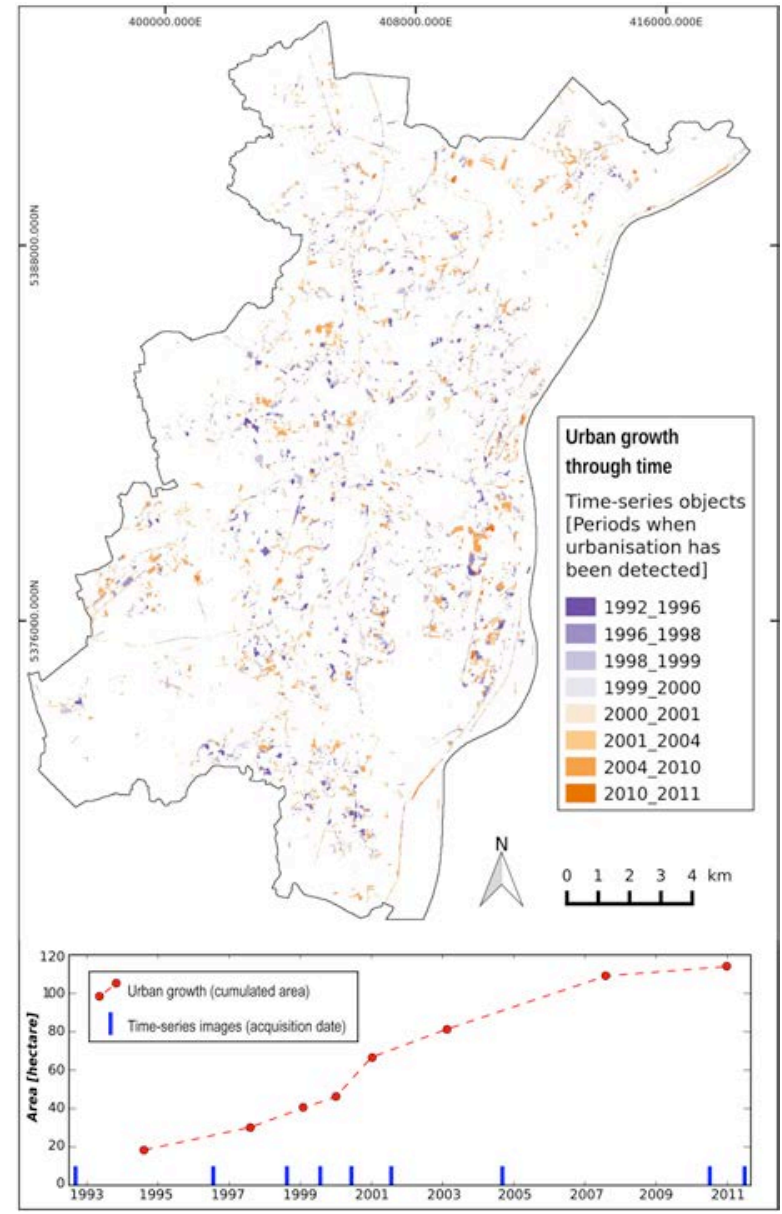

Figure 6. Urban growth from 1992 until 2011. Map presents the different periods when urbanization has been detected.

Graph shows the cumulated curve of urbanized areas throughout the transitions of the SITS.

\section{CONCLUSIONS}

In this paper, we proposed an original and hybrid framework combining per-pixel and object-based image analysis of SITS. Information related to the three key issues of spatiotemporal analysis (where / when / what) has been extracted from a longterm SITS containing nine optical images. First results are relevant and the information extracted is structured in a database allowing multidimensional queries. Further developments are necessary in the mining step (e.g., temporal clustering and sequential pattern mining) in order to propose a typology of LCLU evolutions considering the whole SITS.

\section{ACKNOWLEDGEMENTS}

This work is part of the project COCLICO (ANR- MN-12-01) funded by the French agency of Research (ANR).

\section{REFERENCES}

Baatz, M., Schäpe, A., 2000. Multiresolution segmentation: an optimization approach for high quality multi-scale image segmentation. Angewandte Geographische Informationsverarbeitung, XII(58), pp. 12-23.
Baraldi, A., Boschetti, L., 2012. Operational automatic remote sensing image understanding systems: Beyond geographic object-based and object-oriented image analysis (GEOBIA/GEOOIA). Part 1: Introduction. Remote Sensing, 4(9), pp. 2694-2735.

Blaschke, T., Hay, G.J., Kelly, M., Lang, S., Hofmann, P., Addink, E., Queiroz Feitosa, R., van der Meer, F., van der Werff, H., van Coillie, F., Tiede, D., 2014. Geographic ObjectBased Image Analysis - Towards a new paradigm. ISPRS Journal of Photogrammetry and Remote Sensing 87, pp. 180191.

Canty J., Nielsen A., Schmidt M., 2004. Automatic radiometric normalization of multitemporal satellite, Remote Sensing of Environment, 91, pp. 441-451.

Chen, G., Hay, G.J., Carvalho, L.M.T., Wulder, M.A., 2012. Object-based change detection. In: International Journal of Remote Sensing 33, pp. 4434-4457.

Drăguţ, L., Csillik, O., Eisank, C., \& Tiede, D., 2014. Automated parameterisation for multi-scale image segmentation on multiple layers. ISPRS Journal of Photogrammetry and Remote Sensing, 88, pp. 119-127.

Haralick, R. M., Shanmugam, K., 1973. Textural features for image classification. IEEE Transactions on systems, man, and cybernetics, (6), pp. 610-621. 


\title{
ATHENA: CENTER OF EXCELLENCE IN CYPRUS IN THE FIELD OF REMOTE SENSING FOR CULTURAL HERITAGE IN THE AREAS OF ARCHAEOLOGY AND CULTURAL HERITAGE
}

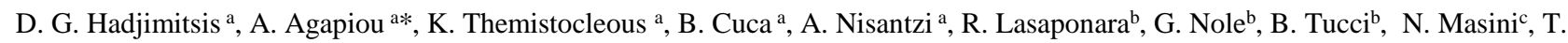 \\ Krauss $^{\mathrm{d}}$, D. Cerra ${ }^{\mathrm{d}}$, U. Gessner ${ }^{\mathrm{d}}$, G. Schreier ${ }^{\mathrm{d}}$,
}

\begin{abstract}
${ }^{a}$ Remote Sensing and Geo-Environment Research Laboratory, Department of Civil Engineering and Geomatics, Cyprus University of Technology, Saripolou str. 2-8, 3036 Limassol, Cyprus; +357 250024 71; d.hadjimitsis@cut.ac.cy; athos.agapiou@cut.ac.cy; vasiliki.lysandrou@cut.ac.cy; k.themistocleous@cut.ac.cy; branka.cuca@cut.ac.cy; argyro.nisantzi@cut.ac.cy;

${ }^{\mathrm{b}}$ National Research Council, Institute of Institute of Methodologies for Environmental Analysis, C.da S. Loya, 85050 Tito Scalo, Italy; rosa.lasaponara@imaa.cnr.it

${ }^{\mathrm{c}}$ National Research Council, Institute of Archaeological and Monumental Heritage, C.da S. Loya, 85050 Tito Scalo, Italy; n.masini@ibam.cnr.it

d DLR - German Aerospace Center, EOC - Earth Observation Center, D-82234 Oberpfaffenhofen, Germany Thomas.Krauss@dlr.de; Daniele.Cerra@dlr.de; Ursula.Gessner@dlr.de; Gunter.Schreier@dlr.de;
\end{abstract}

KEY WORDS: Remote Sensing; Landsat; Cyprus; Fires; Monitoring

\begin{abstract}
:
In periods of economic instability, national considerations are overruling the process of European integration. Cultural Heritage $(\mathrm{CH})$ is an integral element of a European set of values, and respect for heritage is vital for developing a common European identity. The $\mathrm{CH}$ sector has always been facing a number of challenges that have increased with the financial crisis that has hit Europe. To name a few, these include the decrease of public budgets, urbanisation, globalisation, and technological changes. Within this context, $\mathrm{CH}$ professionals are seeking to improve currently used methodologies, in order to better understand, protect and valorise the common European past and common identity.

The use of satellite and other remote sensing (RS) technologies has progressively been established in the field of environmental monitoring. In the domain of $\mathrm{CH}$ and landscape monitoring and in particular with regards to archaeological sites, these technologies have made a significant contribution to research and analysis over the past few decades. The potential use of RS for the understanding, documenting, monitoring and valorization of $\mathrm{CH}$ has long been recognised not only by $\mathrm{RS}$ experts and archaeologists, but also by the public authorities involved in heritage management, that suggested an increasing use of non-invasive technologies (Valletta Convention, 1992).

The ATHENA project aims to strengthen the Cyprus University of Technology's (CUT) Remote Sensing Science and GeoEnvironment Research Laboratory in the field of Remote Sensing Archaeology by creating a unique link between two internationally-leading research institutions: The National Research Council of Italy (CNR) and the German Aerospace Centre (DLR). Through ATHENA, CUT's staff research profile and expertise will be raised, while the S\&T capacity of the linked institutions will come out enhanced.
\end{abstract}

\section{INTRODUCTION}

Cultural Heritage $(\mathrm{CH})$ remains an important sector for several economies within European Union. Several studies have shown that $\mathrm{CH}$ can provide an added value to the real economy of a country. As European Union (2015), highlights that Cultural heritage is now widely appreciated as an essential part of Europe's underlying socioeconomic, cultural and natural capital. This is a significant change in focus as cultural activities have traditionally been regarded as costs to society. The economic benefits of cultural heritage have most commonly been seen in terms of tourism.

According to studies, $\mathrm{CH}$ is also strong connected with tourism sector in Cyprus as well. Though that most tourists visit Cyprus for leisure, Cyprus aims at expanding its "special interest tourism", which includes cultural tourism, health and wellbeing, conference and events tourism, religious tourism, agrotourism, weddings and honeymoons and sports tourism. Cyprus' main advantages as a destination for cultural tourism are its culture and heritage, its clean archaeological sites, easy access to these and valued guide services. For instance, Paphos is a popular coastal town in the southwest of Cyprus. It offers spectacular scenery and some of Cyprus' finest beaches. Paphos offers ancient historical sites, some classified as world heritage sites by Unesco and an attractive harbour. It is expected that Paphos will be subject to intensified cultural activity, as it has been appointed by the EU to be a European Capital of Culture for 2017 (Cyprus Tourism Market Report, 2015). Recent statistics indicates that the archaeological site of Nea Paphos is the most visited monument in the island with more than 200,000 tourists per year.

Though several people recognise the importance of $\mathrm{CH}$ both in terms of sustainable economy as well as in terms of common memory and identity, several actions are needed to be taken so as to protect and safeguard these standing monuments. Nowadays, both natural and anthropogenic hazards are threatening $\mathrm{CH}$ sites and therefore a robust and systematic tool is needed to help stakeholders. In this perspective remote sensing technologies, including space observation and ground 
non-contact techniques can support stakeholders for monitoring and mapping both the monuments and sites as well their threats.

\section{AIMS OF ATHENA PROJECT}

Given the importance of remote sensing technologies for $\mathrm{CH}$, ATHENA, a three-year duration project, aims to strengthen the Cyprus University of Technology's (CUT) in the field of Remote Sensing Archaeology by creating a unique link between two internationally-leading research institutions: The National Research Council of Italy (CNR) and the German Aerospace Center (DLR). The overall objective of the project is to expand the capabilities of the CUT members so as to establish a science centre in the eastern Mediterranean with advance remote sensing capabilities. This objective will be performed through training and other activities such as workshops and summer schools. ATHENA project also allows researchers to focus in new remote sensing technologies and to examine their potential use in archaeological research and prospection.

Such activities include among other the training of CUT personnel in advance remote sensing algorithms and approaches applied in archaeological research, risk estimation and damage assessment such as fire burned area mapping (Lanorte et al (2013). Object oriented analysis is expected to be carried out through different applications of the ATHENA project for classification purposes e.g. monitoring urban expansion in the vicinity of archaeological sites or detection of buried archaeological remains using segmentation techniques.

A recent example of the application of object oriented analysis is the detection of post-fire areas in the region of Troodos, where recent fires occurred in middle of July 2016 have burnt more than 16 square kilometers of forest. In this area, several important historical monuments exist including World Heritage monuments.

As shown in Figure 1, Landsat 8 multispectral image, taken at 24-06-2016 has been able to map the burnt area (see Figure 1, left, NIR-R-G composite). Segmentation analysis of the image was processed so as to create homogenous clusters of pixels in the image (Figure 1, right).
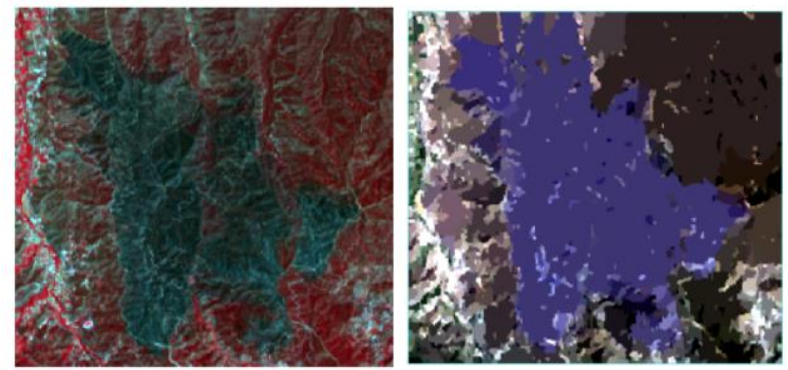

Figure 1: Left: NIR-R-G composite of the Landsat 8 (24-062016) and Right: Segmentation of the multispectral image.

The classification result was able to map the burnt areas using simple rule based approaches. This information was used so as to document the threat of the historical ekllisiastical churches of the sourrounding area, all protected and enlisted in the World Heritage mouments list (know as the ten byzantine chrches of Troodos). The risk map is shown in Figure 2, where burnt areas are indicated with orange colour, villages in red spots and the byzantine churches in green dots. As it is shown three of these ten monuments where in a close vicinity of fire event.

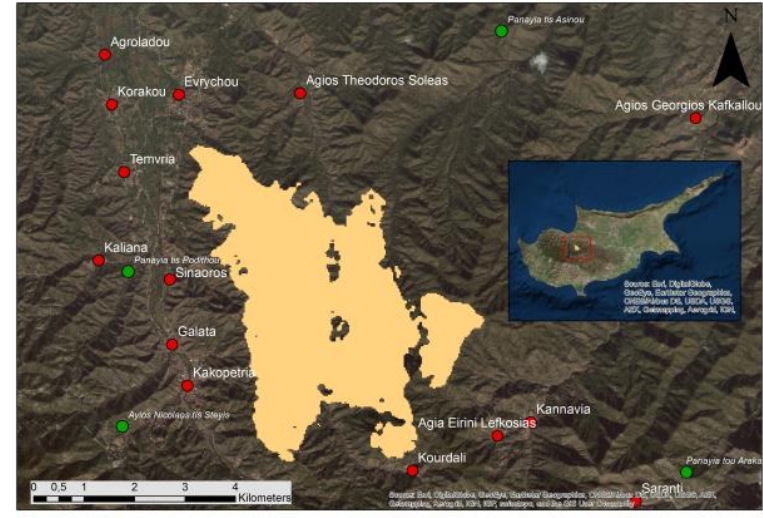

Figure 2: Map indicating the burnt areas (orange color), villages with red dots and the World Heritage Monuments in green dots.

\section{DISCUSSION}

Remote sensing datasets can provide helpful information for stakeholders so as to monitor cultural heritage sites and landscapes. Recent studies have indicated the importance of Cultural Heritage to real economy and tourism. Recent fires occurred in Cyprus have been used as a case study so as to demonstrate the capabilities of such technologies even in case of emergency.

As demonstrated in this case study, protection and monitoring of sites and monuments (either known or still un-known) is feasible using remote sensing data and remote sensing methodologies. Segmentation and rule based classification can be also used in this direction so as to identify in large scale any illegal activities.

The protection of $\mathrm{CH}$ by means of analysing satellite imagery is also in the focus of a EU "strategy for international cultural relations", where specifically the European COPERNICUS program should use its capabilities to monitor sites at risk and to evaluate damage (European Commission, 2016)

In this framework ATHENA project aims to build an advance remote sensing centre in the area of the Eastern Mediterranean and benefit from the new capabilities that new remote sensing sensors provide nowadays.

\section{ACKNOWLEDGEMENTS}

The present communication is under the "ATHENA" project H2020-TWINN2015 of European Commission. This project has received funding from the European Union's Horizon 2020 research and innovation programme under grant agreement No 691936. Thanks are also given to the Remote Sensing and GeoEnvironment Research Laboratory of the Department of Civil Engineering \& Geomatics of the Cyprus University of Technology for the support (http://www.cut.ac.cy).

\section{REFERENCES}

European Commission, 2015. Getting cultural heritage to work for Europe. Report of the Horizon 2020 Expert Group on Cultural Heritage, B-1049 Brussels.

Cyprus Tourism Market Report, 2015. A summary of the significant factors and major drivers of tourism in Cyprus, KPMG Limited. 
Lanorte A, M Danese, R Lasaponara, B Murgante 2013 Multiscale mapping of burn area and severity using multisensor satellite data and spatial autocorrelation analysis International Journal of Applied Earth Observation and Geoinformation 20, $42-51$

European Commission: Towards an EU Strategy for international cultural relations, JOIN(2016) 29 final; Brusels, 8.6.2016 


\title{
INTERSEG: A DISTRIBUTED IMAGE SEGMENTATION TOOL
}

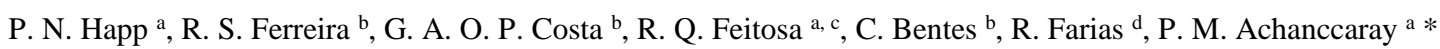 \\ a Pontifical Catholic University of Rio de Janeiro, Brazil - (patrick, raul, pmad9589)@ele.puc-rio.br \\ ${ }^{\mathrm{b}}$ IBM Research Brazil Lab in Rio de Janeiro, Brazil - rosife@br.ibm.com \\ ${ }^{c}$ Rio de Janeiro State University, Brazil - gilson.costa@ime.uerj.br, cris@eng.uerj.br \\ ${ }^{\mathrm{d}}$ Federal University of Rio de Janeiro, Brazil - rfarias@ cos.ufrj.br
}

KEY WORDS: Remote Sensing, Image Segmentation, Object-Based Image Analysis, Distributed Processing, Cloud Computing

\begin{abstract}
:
Motivated by the rapidly increase of remote sensing data over the last years, this paper presents a tool designed for distributed image segmentation. InterSeg is able to handle efficiently very large high-resolution images using scalable distributed segmentation methods over computer clusters. Through a graphic user interface, InterSeg hides the distributed processing complexity, allowing the user to easily perform distributed image segmentation on a cloud-computing environment. Experiments carried out with the tool attest its potential scalability and its capability to exploit distributed processing over many cluster nodes. Moreover, as an opensource solution, the software can be extended with different segmentation algorithms and methods.
\end{abstract}

\section{INTRODUCTION}

Modern advances in Earth Observation technologies are responsible for improvements in spatial, spectral and temporal resolution of remote sensing data. Such improvements, associated with the increasing number of aerial and orbital systems in activity, result in a huge growth in the volume of available remote sensing data. Furthermore, the cost of images provided by private companies has dropped considerably in recent years, and open-access datasets have been widespread, facilitating the access to this kind of information.

Timely analysis of such large datasets represents a great challenge for researchers in a number of application areas, such as agriculture, disaster response, urban planning, and mining operations, among others. These areas usually require automatic methods and tools to interpret remote sensing imagery, allowing the extraction of strategic information to be used by decision makers.

Currently, Object-Based Image Analysis (OBIA) is widely used in the environmental science community (Blaschke et al., 2014), and image segmentation is at the core of OBIA. The procedure is responsible for partitioning an input image in homogeneous pixel agglomerates that will be later classified.

Image segmentation is not a new subject and a wide variety of algorithms have been proposed in the past decades (Haralick and Shapiro, 1985; Pal and Pal, 1993; Dey et al., 2010). In addition, a number of automatic tools for image segmentation are now available. However, dealing efficiently with massive data volumes represents a major obstacle for the current segmentation solutions, which are generally not able to handle very large images.

\footnotetext{
* Corresponding author
}

Distributed computing is a common alternative for the processing of very large datasets, and in this context, cloud computing is a trend, as it can deliver a scalable infrastructure to support different processing needs (Fernández et al., 2014). Moreover, there are currently many cloud computing infrastructure providers that offer a pay-per-use model and deliver great computing power to users that do not need to be concerned with acquiring or maintaining complex hardware.

In this work, we use the distributed computing concept in order to provide a scalable and efficient solution to overcome current limitations for processing very large datasets. We introduce InterSeg, an open-source distributed processing tool designed to run image segmentation on physical or virtual computer clusters. InterSeg is an easy to use, scalable and efficient solution to handle very large remote sensing images.

The remainder of this paper is organized as follows. Some related work on image segmentation is presented in Section 2. An overview of InterSeg is described in Section 3. An example of usage of InterSeg is shown as an experiment in Section 4. Section 5 concludes this working and offers some directions for future work.

\section{RELATED WORK}

Image segmentation aims at identifying regions that represent objects of interest (Russ, 1998). This is an important step in image analysis, since it is responsible for delineating the regions that will be later classified by experts or by automatic classification procedures. Thus, the segmentation quality has a direct influence on the result of the whole image analysis process.

In the past few years, considerable efforts have been made to ease the computational burden of image segmentation of high- 
resolution remote sensing imagery. These efforts usually rely on parallel processing solutions that explore symmetric multiprocessors (Moga et al., 1998), multicore processors (Happ et al., 2010) or even graphic processing units (Backer et al., 2013). In spite of achieving good accelerations, these algorithms still present problems to handle the growing scale of the remote sensing imagery.

Regarding distributed solutions, Tesfamariam (2011) proposes a different approach to edge detection models on a local cluster. Surve and Paddune (2014) and Jin et al. (2014) also present distributed versions based on clustering techniques being the latter focused on cloud computing. Pixel-based analysis techniques are easier to adapt to distributed processing environments, since they represent almost independent tasks at the pixel level. Region growing segmentation, however, has to deal with strong dependencies among adjacent segments, and requires a more complex distribution strategy. In general, the algorithms have to split the image into tiles and process them independently. The key problem is how to handle segments on the borders of the tiles, as there is a high level of dependency among neighboring segments.

InterSeg is based on a generic distributed segmentation strategy that can generate (image tile bordering) segments without artifacts. This strategy is based on image tile division and on a hierarchical stitching of the segments on tile borders (Happ et al., 2015). Additional segmentation algorithms can also be included in the tool as long as they comply with some rules.

\section{INTERSEG OVERVIEW}

InterSeg is an open-source image segmentation tool, which provides distributed processing on computer clusters or on cloud computing infrastructures. It can also be considered as an independent module of InterCloud, a distributed image interpretation platform for handling large remote sensing datasets (Ferreira et al., 2014).

\subsection{InterSeg Architecture}

Similar to InterCloud, the architecture of InterSeg consists of three layers containing a different representation of the segmentation tool in different levels of abstraction (Figure 1). The first layer works as a user interface. It focuses on the enduser and is related to definition of the segmentation inputs and configurations. For this reason, it is known as the project definition layer.

The second layer is associated to the algorithms and methods developed for the tool. It is called segmentation layer and it is organized on a high-level structure in order to hide the distributed programming complexity. A conventional programmer can interact with this layer in order to include new segmentation algorithms and other desired functions. The connection between project and segmentation layers is defined by a translation of the first into processing and data flow instructions present in the latter.

The third layer is the distribution layer. Since it is related to the distributed processing of the image segmentation, only programmers with distributed programming skills are able to interact with this layer. The connection between the segmentation and the distribution layers is defined again by a translation. The high-level instructions are compiled into distributed processing code.

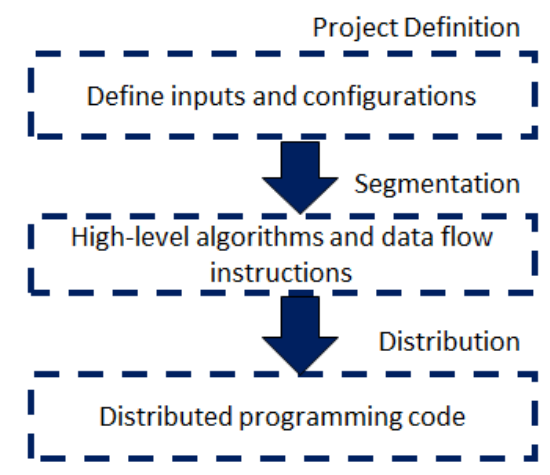

Figure 1. InterSeg's architecture.

There are many alternatives to implement the architecture of InterSeg. In the following, we will present our current implementation.

\subsection{InterSeg Implementation}

The distributed implementation is based on MapReduce (Dean and Ghemawa, 2008); a programming model widely used to process massive data volumes in the cloud (Assunção et al., 2014). More specifically, we use Apache Hadoop, which is MapReduce's most used implementation and offers an opensource, highly scalable and reliable framework for storing and processing large datasets.

In order to manage MapReduce, we use the Pig framework (Gates, 2011). Pig is composed by a high-level language for expressing data-flows (Pig Latin), and by an engine that compiles it into MapReduce jobs. Pig Latin provides an easier way to work with the distributed MapReduce model. It also offers an extension capability, of external libraries and methods, through its User Defined Functions (UDFs).

The image segmentation algorithms are coded in Java and are structured as Pig UDFs in order to be invoked by Pig Latin scripts. Additional algorithms can be embedded following the same structure. A parser translates the project definitions into such scripts, and the definitions are set by the user through a graphical user interface (GUI).

Lastly, the cloud computing services are accessed through an application programming interface (API) provided by the cloud computing infrastructure providers.

\subsection{InterSeg as a tool}

Considering InterSeg as an end-user tool, the focus relies on how to operate it in order to perform a segmentation task. InterSeg and its code are available at http://www.lvc.ele.pucrio.br/wp/?cat=41. It is important to note that the tool is still under development and this is a Beta release, therefore some bugs are expected.

InterSeg has four tabs to define the segmentation project and run it on the cloud. The first is the database description: the user must define a local path or a cloud link for the raster image (Figure 2). If it is possible, InterSeg will load the geographic coordinates and additional information. The user is able to modify them and to set the Coordinate Reference System and the desired tile size. 


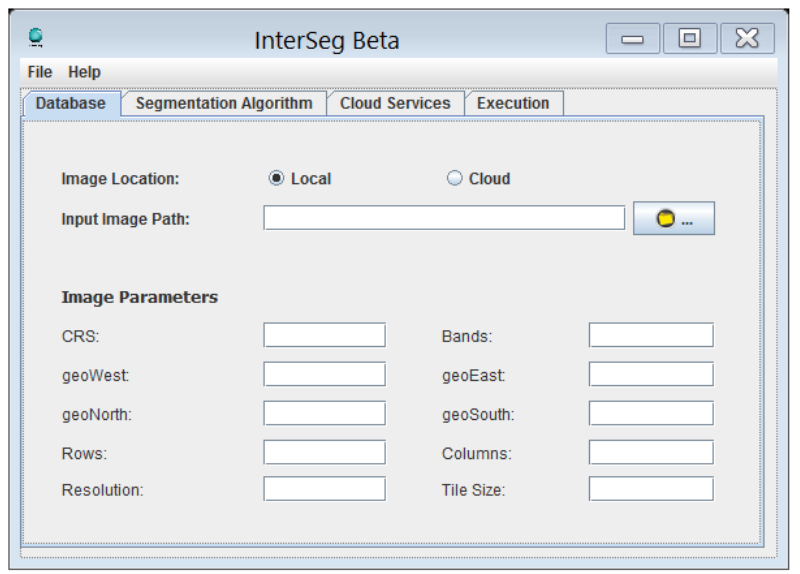

Figure 2. Defining the database

The second tab is related to the segmentation algorithm (Figure 3). The user should select one among the available implementations. At the moment, there are distributed versions of a chessboard, a multiresolution region growing and a thresholding segmentation. The segmentation parameters are automatically loaded after an algorithm is chosen and must be set by the user.

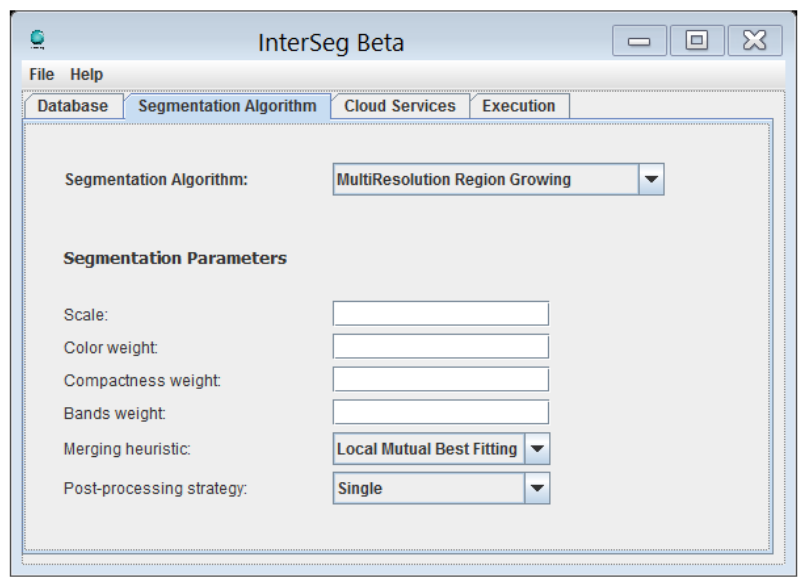

Figure 3. Selecting the segmentation algorithm

The third tab is based on the cloud computing settings (Figure 4). First, the user should choose one from the available cloud infrastructure providers. Then, according to the user selection, the required parameters are automatically loaded to be filled by the user.

The last tab concerns the segmentation execution. The user can select the type of the output such a JSON file or a shapefile. It is also possible to choose the output path and to select a location to download the result. After the whole configuration is done, the start segmentation button initiates the execution.

At the beginning of the process, image tile division is carried out. If the image is in a local path, each generated tile and its respective auxiliary files are uploaded to the defined file storage repository. The Pig script containing the distributed segmentation data-flow is also created and placed in a similar location. Then, the virtual cluster is instantiated at the desired cloud computing service. Next, the distributed segmentation starts and, when it is completed; the outcome is downloaded in the proper format to the desired local path.

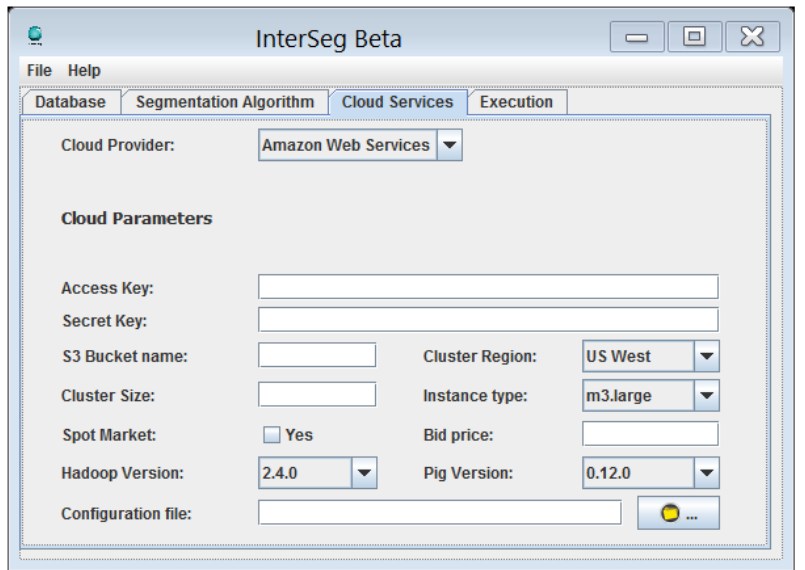

Figure 4. Configuring the cloud computing services

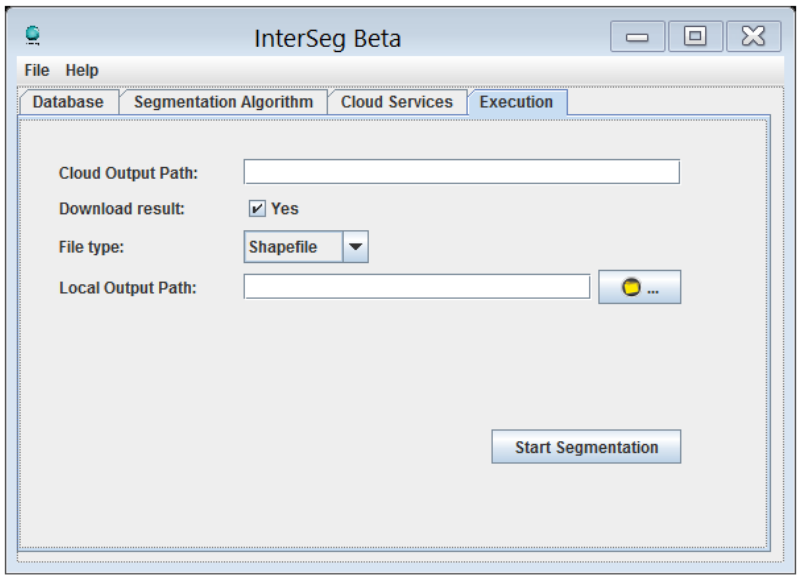

Figure 5. Running the segmentation

\section{EXPERIMENTS AND RESULTS}

To validate InterSeg, we performed an experiment using the tool to segment a Quickbird image on the cloud. The image is a $4000 \times 4000$ pixels subset, called hereafter 4K image (Figure 6), covering some districts of São Paulo city, Brazil. It has four spectral bands (blue, green, red and infrared) and a spatial resolution of 0.61 meters.

The image was replicated in order to assess the performance for large images. We generated images of $8000 \times 8000$ pixels $(8 \mathrm{~K})$ $16000 \times 16000$ pixels $(16 \mathrm{~K})$ and $32000 \times 32000$ pixels $(32 \mathrm{~K})$. The tile size used was $1024 \times 1024$ pixels.

The selected image segmentation algorithm was the distributed version of the multiresolution region growing algorithm proposed in (Baatz and Shäpe, 2001). The parameters were kept the same for every run: scale $=40$; color weight $=0.84$; compactness $=0.8 ;$ bands weights $=1,1,1,1 ;$ merging heuristic = local mutual best fitting; post-processing strategy $=$ single post-processing.

We selected the Amazon Web Services (AWS) as the cloudcomputing provider. AWS provides the Simple Storage Service (S3) as a file repository and the Amazon Elastic MapReduce (EMR) to build a Hadoop cluster using Amazon Elastic Compute Cloud (EC2) instances. We varied the cluster sizes (2, $4,8,16$ and 32 nodes) in order to show the potential scalability of the tool. For every run, instances of type m3.xlarge were used under the spot market. These are machines containing Intel 
Xeon E-5-2670 v2 processors operating at $2.5 \mathrm{GHz}$ with a 64-bit architecture, 15 GB of RAM and 2 disks with 40GB using SSD technology. The Hadoop version was set to 2.4 .0 and the Pig version was 0.12.0.

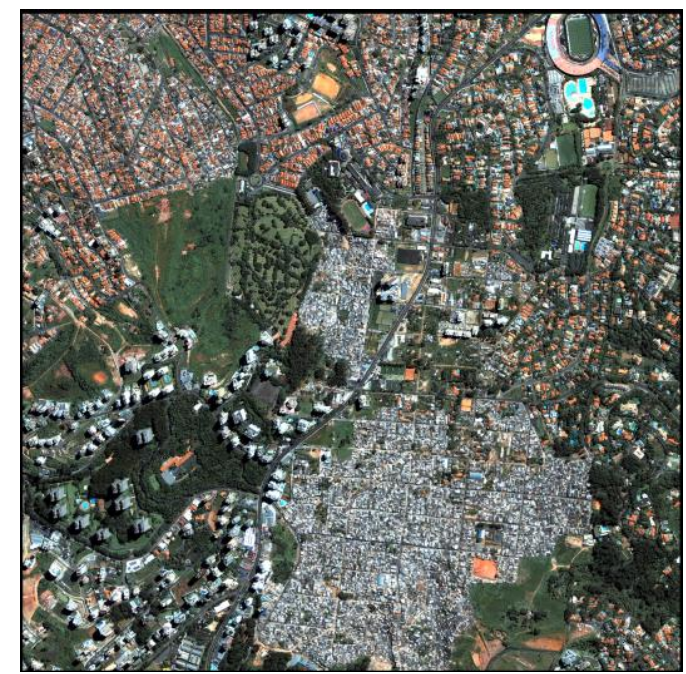

Figure 6. QuickBird image subset called 4K.

All the images were successfully segmented on the cloudcomputing environment. Figure 7 presents the execution times for the $4 \mathrm{~K}, 8 \mathrm{~K}, 16 \mathrm{~K}$ and $32 \mathrm{~K}$ images over $2,4,8,16$ and 32 cluster nodes. It is possible to observe that the execution time decreases, as more nodes are included. This reduction is more significant for larger images, since it can benefit more from the higher number of processing elements available.

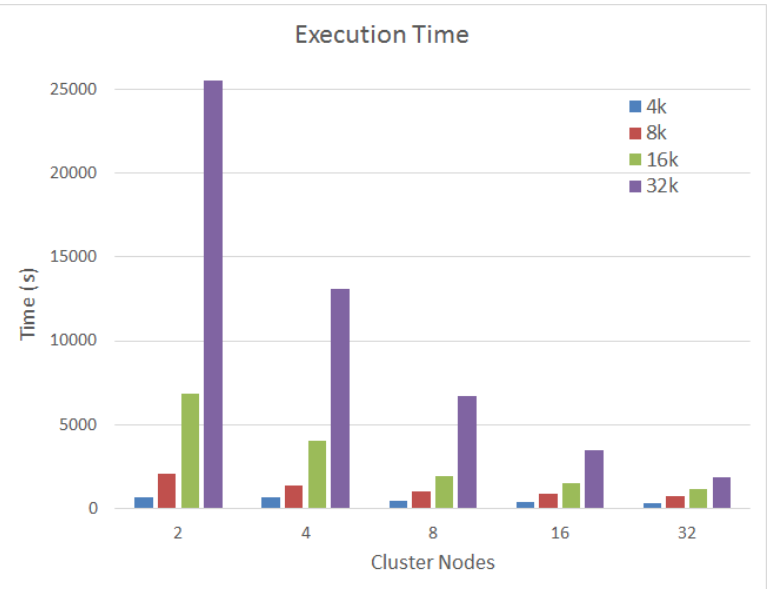

Figure 7. Execution time for every segmentation run

Figure 8 shows the same results, but exposed as speedups relative to the execution over 2 cluster nodes. The plot demonstrates that the speedup tends to increase with the image size. For the largest image, we obtained speedups higher than 13 when using 32 nodes. Small images are not able to exploit the additional processing power, and achieve smaller speedups.

It is worth mentioning that different amounts of cluster nodes produce exactly the same segmentation outcome. Figure 9 shows part of the outcome for $4 \mathrm{k}$ image. The result shows that overall segmentation presents good results with few artifacts. The artifacts are still present due to the simple post-processing strategy used as described in (Happ et al., 2015) Different strategies can produce results without artifacts.

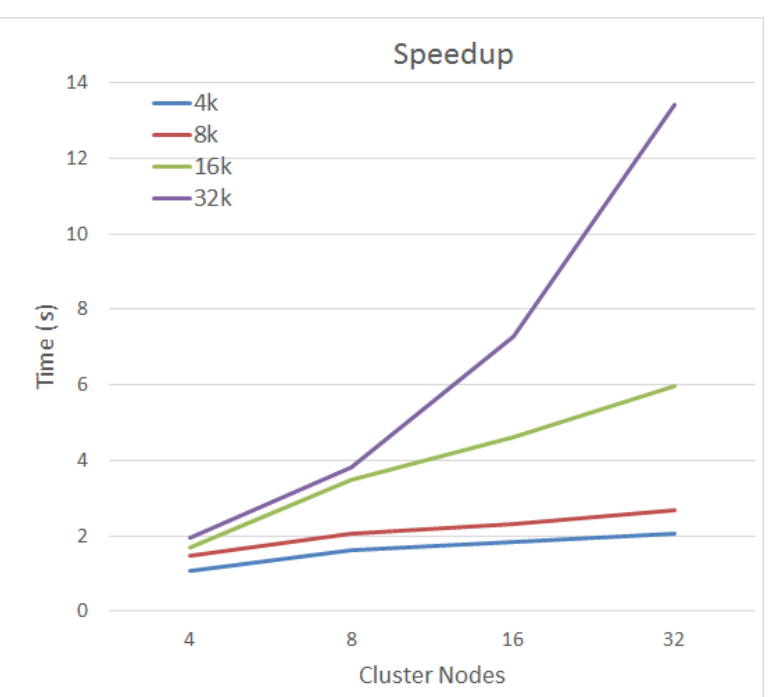

Figure 8. Speedups related to the 2 cluster nodes execution.

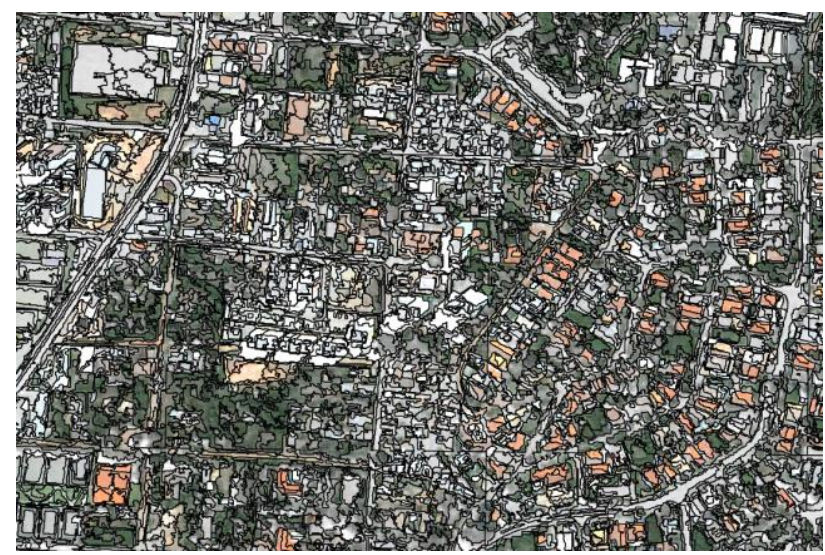

Figure 9. Part of segmentation outcome.

\section{CONCLUSION}

In this work, InterSeg was presented, which is a free tool designed to perform distributed image segmentation over computer clusters. The first Beta release of the software is available at http://www.lvc.ele.puc-rio.br/wp/?cat=41.

Through a graphical user interface, a user is able to choose a segmentation algorithm and process a very large image using a scalable cloud-computing infrastructure. The number of available algorithms, as well as the cloud infrastructure providers, are still limited; however, it is possible to extend the software including new alternatives.

The experiments conducted in this work demonstrated the potential scalability of the software since using an increasing amount of cluster nodes led to higher speedups when the images were large enough.

\section{ACKNOWLEDGEMENTS}

The authors acknowledge the funding provided by FAPERJ (Carlos Chagas Filho Foundation for Research Support in Rio de Janeiro), CNPq (National Council for Scientific and Technological Development) and CAPES (Coordination for the Improvement of Higher Education). 


\section{REFERENCES}

Baatz, M., Schäpe, A., Multiresolution segmentation: an optimization approach for high quality multi-scale image segmentation, In: XII Angewandte Geographische Informationsverarbeitung, Wichmann-Verlag, Heidelberg, 2000.

Backer, M.; Tünnermann, J.; Mertsching, B. Parallel K-Means Image Segmentation Using Sort, Scan and Connected Components on a GPU. In: Facing the Multicore-Challenge III, 108-120. Berlin: Springer, 2013.

Blaschke, T., Hay, G. J., Kelly, M., Lang, S., Hofmann, P., Addink, E., Feitosa, R. Q., Der Meer, F., Der Werff, H., Coillie, F., Tiede, D., Geographic object-based image analysis towards a new paradigm, ISPRS Journal of Photogrammetry and Remote Sensing, vol. 87, pp. 180-191, 2014.

Dean, J., Ghemawa, S., MapReduce: Simplified Data Processing on Large Clusters. Communications of the ACM, v. 51, n. 1, p. 107-113, 2008.

Dey, V., Zhang, V., Zhong, M., A review on image segmentation techniqueswith remote sensing perspective, In: Proc. ISPRS, pp. 5-7, 2010.

Fernández, A., del Río, S., López, V., Bawakid, A., del Jesus, M. J., Benítez, J. M., Herrera, F., Big Data with Cloud Computing: an insight on the computing environment, MapReduce, and programming frameworks, Wiley Interdisciplinary Reviews: Data Mining and Knowledge Discovery, vol. 4, no. 5, pp. 380-409, September/October 2014

Ferreira, R. S., Oliveira, D. A. B., Happ, P. N., Costa, G. A. O. P., Feitosa, R.Q., Bentes, C., InterIMAGE 2: The Architecture of an Open Source, High Performance Framework for Automatic, Knowledge-Based Image Interpretation, In: International Geographic Object-Based Image Analysis Conference, Thessaloniki, 2014.

Gates, A., Programming Pig, O'Reilly, 2011.

Happ, P. N., Ferreira, R. S., Bentes, C., Costa, G. A. O. P., Feitosa, R. Q., Multiresolution Segmentation: a Parallel Approach for High Resolution Image Segmentation in Multicore Architectures, In: 3rd International Conference on Geographic Object-Based Image Analysis, 2010, Ghent, The International Archives of the Photogrammetry, Remote Sensing and Spatial Information Sciences. Enshede: ITC, 2010. v.XXXVII

Happ, P. N., Ferreira, R. S., Costa, G. A. O. P., Feitosa, R. Q., Bentes, C., Gamba, P., Towards distributed region growing image segmentation based on MapReduce, In: 2015 IEEE International Geoscience and Remote Sensing Symposium (IGARSS), Milan, 2015, pp. 4352-4355.

Haralick, R. M., Shapiro, L. G., Image segmentation techniques. Computer Vision Graphics Image Process, 29:100-132, 1985.

Jin, X.; Sieber, R.; Kalacska, M. The challenges of image segmentation in big remotely sensed imagery data. Annals of GIS 20.4 (2014): 233-244, 2014.

M. D. Assunção, Calheiros, R. N., Bianchi, S., Netto, M. A. S., Buyya, R., Big Data computing and clouds: Trends and future directions, Journal of Parallel and Distributed Computing, Aug. 2014.

Moga, A.; Cramariuc, B.; Gabbouj, M., Parallel watershed transformation algorithms for image segmentation, Parallel Computing, 1998.

Pal, N. R., Pal, S. K., A review of image segmentation techniques, Pattern Recognition, 26(9):1277-94, 1993.

Russ, J. C. The image processing handbook - 3rd ed. Materials Science and Engineering Department North Carolina State University Raleigh - North Carolina, 1998.

Surve, A. R.; Paddube, N. S., A Survey on Hadoop Assisted KMeans Clustering of Hefty Volume Images. International Journal on Computer Science and Engineering 6.3 (2014): 113, 2014.

Tesfamariam, E. B. "Distributed processing of large remote sensing images using MapReduce - A case of Edge Detection," 2011. 


\title{
ADAPTIVE MORPHOLOGICAL SEGMENTATION - CONCEPTS AND PYTHON IMPLEMENTATIONS
}

\author{
H. Herold ${ }^{\mathrm{a}, *}$, G. Meinel ${ }^{\mathrm{a}}$ \\ ${ }^{\text {a }}$ Leibniz Institute of Ecological Urban and Regional Development, IOER Dresden, Germany - \\ h.herold@ioer.de
}

KEY WORDS: Mathematical Morphology, Image Analysis, Adaptive Segmentation, Python/NumPy, Implementation

\begin{abstract}
:
Segmentation algorithms based on the set theoretical Mathematical Morphology have been successfully applied in geosciences, remote sensing as well as biomedical image analysis. Morphological operators can be conceptualized as non-linear filters, which allow the modelling of spatial relations of pixels in an image, but also of voxels in volumetric data sets such as acquired by LiDAR. However, the iterative trial-and-error parameterization of algorithms, namely the selection of the morphological operator and structuring element, is one of the biggest issues for the transferability of a developed solution to other datasets. In the light of the GEOBIA topics operationalisation and transferability, the paper gives an overview of the fundamental concepts for automated adaption in morphological image segmentation. There are two fundamental concepts to adaptive morphology, which are highlighted. First, approaches that make use of intrinsic image properties such as the pixel location or gray values for the adaption; and second, supervised approaches that automatically adapt and validate the parameters based on a small set of user-provided samples of the expected segmentation result. Both approaches significantly reduce the efforts of manual parameterization as well as the amount of required problem knowledge and thus contribute to the operationalization and transferability of developed segmentation algorithms or image analysis workflows. Finally, examples of ready-to-use Python-based implementations are given, which solely rely on opensource image analysis frameworks.
\end{abstract}

\footnotetext{
* Corresponding author
} 


\title{
AGENT BASED IMAGE ANALYSIS (ABIA) - PRELIMINARY RESEARCH RESULTS FROM AN IMPLEMENTED FRAMEWORK
}

Peter Hofmann ${ }^{\mathrm{a}, *}$, Vera Andrejchenko ${ }^{\mathrm{a}}$, Paul Lettmayer ${ }^{\mathrm{b}}$, Manuel Schmitzberger ${ }^{\mathrm{b}}$, Michael Gruber ${ }^{\mathrm{b}}$, Izzet Ozan ${ }^{\mathrm{b}}$, Mariana Belgiu $^{\mathrm{a}}$, Roland Graf ${ }^{\mathrm{b}}$, Thomas Josef Lampoltshammer ${ }^{\mathrm{b}}$, Stefan Wegenkittl ${ }^{\mathrm{b}}$, Thomas Blaschke ${ }^{\mathrm{a}}$

anterfaculty Department of Geoinformatics - Z_GIS, Salzburg University, Schillerstr. 30, 5020 Salzburg, Austria. (*peter.hofmann,vera.andrejchenko,mariana.belgiu,thomas.blaschke)@ sbg.ac.at.

bepartment of Information Technology \& Systems Management, Salzburg University of Applied Sciences, Austria. (((mschmitzberger,mgruber,iozan).its-m2014,plettmayer.its-m2013),stefan.wegenkittl,roland.graf)@ fhsalzburg.ac.at,thomas.lampoltshammer@gmail.com

KEY WORDS: Agent Based Image Analysis, Object Based Image Analysis, Software Agents, Autonomous Systems, Adaptive Systems, Multi Agent Systems

\begin{abstract}
:
Object Based Image Analysis (OBIA) has meanwhile been established as a paradigm for analyzing remotely sensed image data. Although the degree of automation for OBIA methods has increased for several applications, especially in the domain of remote sensing, robust and transferable object-based solutions for automated image analysis of sets of images or even large image archives are still rare. One of the reasons for this lack of robustness and transferability is the high complexity of remote sensing image contents: Especially in Very High Resolution (VHR) remote sensing data, under varying imaging conditions or sensor characteristics, the objects' properties can vary unpredictably. Although earlier work has demonstrated that OBIA rule sets bear a high potential of transferability these rule sets need to be adapted manually in order to receive acceptable results, or the classification results need to be adjusted manually in a post-processing step. In order to automate these adaptation and adjustment procedures we investigate the coupling, extension and integration of OBIA with the agent-based paradigm, which is exhaustively investigated in software engineering and robotics. The aims of such integration are a) rule sets which can be adapted autonomously according to varying imaging data, and b) image objects which can adapt and adjust themselves in order to best possibly represent the objects of interest in an image. This paper briefly introduces a framework for Agent Based Image Analysis (ABIA) and presents our first research results.
\end{abstract}

\section{INTRODUCTION}

\subsection{OBIA for VHR image data analysis}

Very high resolution (VHR) remote sensing data and ObjectBased Image Analysis (OBIA) have influenced the remote sensing community since the beginning of the millennium. Although the latter builds on several older concepts and methods, such as image segmentation and knowledge based image interpretation, the particular combination of these concepts allows concepts that are common in Geography and other spatial disciplines, such as multi-scale analysis (Burnett and Blaschke 2003), to be applied. Meanwhile OBIA is an accepted paradigm for image analysis in the remote sensing community (Blaschke et al. 2014), which goes beyond analysing spectral statistical parameters. OBIA allows further object properties such as shape and spatial context to be incorporated (Benz et al. 2004, Blaschke 2010). Nevertheless, due to the scene complexity imaged with VHR data, complex image processing tasks as a kind of 'geo-intelligence' is necessary in order to draw on the full advantage of these data when applying methods of OBIA (Hay and Blaschke 2010).

While OBIA was regarded to be inextricably linked to image segmentation in the first years, since the segments provide the building blocks of object based image analysis (Hay and Castilla, 2008; Lang, 2008), it is meanwhile accepted that an optimum segmentation can only be achieved iteratively and knowledge-based, and that it is strongly bound to the application task and the data used (Baatz et al. 2008). Eventually, segments depict regions that ideally represent the objects of interest in the image of concern. Thus, the additional spatial information contained in objects is of even greater advantage than spectral per-object statistics (Benz et al., 2004; Hay and Castilla, 2008). It has been frequently claimed that this spatial dimension (shape, distances, neighbourhood, topologies, etc.) is crucial to OBIA methods. Authors increasingly recognize that the real potential lies in the intelligence and the chance to formulate user knowledge as 'rule sets' (Hofmann et al. 2011, Belgiu et al. 2014, Lang et al. 2014). However, the creation of robust, object-based solutions for automated image analysis of a set of images or even large image archives still remains extremely challenging (Pinz 2005, Walker and Blaschke 2008, Hofmann et al. 2011, Laliberte and Rango 2011, Kohli 2013). The highly complex content of VHR image data and the hardly predictable variability of the objects' qualities ultimately reduce the robustness and transferability of OBIA rule sets used for image classification. Fully automated, transferable and robust methods of remote sensing image analysis are not in sight yet. Moreover, either the rule sets or the objects' shape or even both need to be manually adapted in order to achieve acceptable results for a variety of images. Against this background, recent developments have paid more attention to the genericity of solutions (Novack et al. 2014) and the reusability of knowledge that has been used to create the complex rule sets (Arvor et al. 2014). However, in order to overcome the limited transferability of OBIA rule sets, we investigate the coupling, extension and integration of OBIA with principles and methods from the agent-based paradigm. In particular, this paper outlines a framework for Agent Based Image Analysis (ABIA), which extends the existing OBIA concepts and methods by those from the agent-based paradigm 
(Hofmann et al. 2015) and demonstrates our current implementations and first results achieved.

\subsection{From object-based to agent-based image analysis}

In OBIA, image analysis usually starts with a more or less arbitrary segmentation of the input data to generate a hierarchical net of image objects. These initially created image objects are then classified, or at least labelled with feature values, intrinsic for each object. Then, an iterative process of selective segmentation improvements and re-classifications begins, and ceases as soon as satisfactory results have been achieved. The criteria to define a current subset to operate on can be spatial, non-spatial, or both (Baatz et al. 2008, Lang 2008). A rule set in this context stores all processing steps, their procedural sequences and sub-sequences in order to re-apply them whenever necessary. Thus, a rule set can be seen as a kind of program written in a so-called Domain Specific Language (DSL), which uses linguistic elements and concepts of the application domain (Hudak 1996). A well-known example for such a DSL in the context of OBIA is the Cognition Network Language (CNL), which is implemented in the software eCognition (Athelogou et al. 2007). CNL provides two principle types of rules, which either calculate values or change the objects' shape and classification rules. While the former is self-explanatory, the latter either selects and processes a subset of objects based on defined criteria, or assigns objects to classes based on defined classification rules. The classification rules can be sample-based or explicit, and can be crisp or fuzzy, or incorporate a-priori probabilities. Additionally, classification rules can follow hierarchical (fuzzy) classification schemes. Fuzzy classification schemes describe each class as a fuzzy set based on fuzzy membership functions and their fuzzy-logic combinations (Benz et al. 2004). Each classification scheme reflects the object classes' remote sensing ontology, that is, their appearance in the image data at hand (Belgiu et al. 2014). Nevertheless, designing a rule set is labour intensive and to a certain degree subjective (Arvor et al. 2014, Belgiu et al. 2014). Its reusability can be limited once the input data changes (Hofmann et al. 2011). Against this background a conceptual framework for Agent Based Image Analysis (ABIA) has been introduced (Hofmann et al 2015), which describes two alternative approaches: (1) the agent-based, autonomous adaptation of OBIA rule sets, and (2) the evolvement of image objects to image object agents. Both approaches intend to allow a flexible reaction of rule sets and image objects on varying image data in order to produce similar classification results. In the present paper we briefly outline the conceptual framework, and present some simple and exemplary implementations thereof, together with some preliminary results. Finally we discuss the results and suggest future work to improve both the concepts and the results.

\section{SOFTWARE AGENTS AND MULTI AGENT SYSTEMS}

\subsection{Software agents}

According to the agent-based paradigm, software agents are flexible and capable of acting autonomously in complex and even unknown environments. Each agent is provided with sensors and effectors in order to interact with its environment and to achieve predefined goals. Thus, they are widely used in robotics and similar application fields. Several software agents can be combined in order to build a Multi Agent System (MAS). In a MAS software agents can interact, communicate, collaborate or negotiate among themselves in order to achieve individual or common goals. In such a system, each agent can have a specific role, which equips it with role-dependent abilities and goals. However, in a MAS, each software agent decides individually and based on its own strategy, when and how to act in a particular situation (Fig. 1).

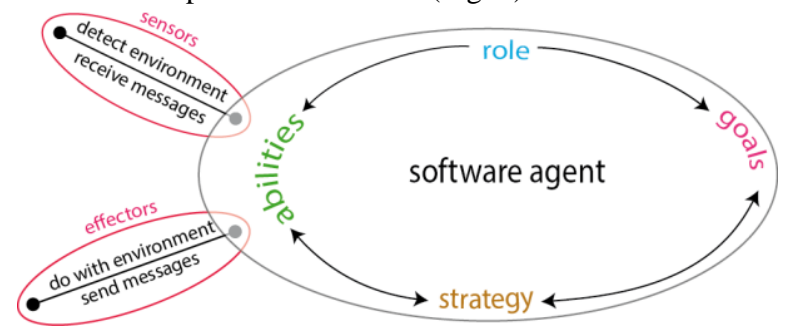

Figure 1. Principle components of individual software agents. Source: Hofmann et al. (2015).

These fundamental abilities have been shown to allow agentbased systems to deal with complex and unpredictable situations, as well as with incomplete information in a much more flexible and robust manner compared to conventional systems (Wooldridge and Jennings 1995, Jennings 2000).

\subsection{Multi agent systems in GIS and image analysis}

A common application of MAS in GIScience is agent based modelling. Agent Based Models (ABMs) simulate the behaviour of complex systems, such as societies or ecosystems. In Geography and related spatial sciences ABMs are used to simulate the evolvement and development of spatial patterns. During the simulation process the assumed behaviour of individuals interacting with their environment is modelled. Typical applications are the simulation of land use change or ecosystems and their spatial patterns (Parker et al. 2003, Macal and North 2005, Brown et al. 2005, Koch 2007, Marceau and Moreno 2008, Yu and Peuquet 2009).

Software agents are also widely applied in robotics. Similar to ABMs, individual software agents can be aggregated to Agent Based Systems (ABS) in order to control or maintain complex processes in industry. In this context ABS are increasingly used for process automation, which requires a high level of robustness and flexibility (Fazel Zarandi and Ahmadpour 2009, Göhner 2013).

However, in image analysis the usage of software agents or MAS is not very common yet. Bovenkamp et al. (2004) and Rodin et al. (2004) both describe a similar approach for object detection in Intra Vascular Ultra Sound (IVUS) images for medical image analysis. Samadzadegan et al. (2009, 2010) report on the application of ABS for building detection in LiDAR data. Mahmoudi et al. (2013) describe an ABS designed to improve OBIA classification results in urban areas. All these approaches have in common the distribution of different tasks of object recognition among respectively defined agents, that is, to parallelize image analysis using software agents. The agents simultaneously operate on an image and share their (intermediate) results. However, all these approaches focus on the potential of software agents to parallelize complex image processing tasks. The improved results are briefly presented by the authors.

\section{CONCEPTUAL FRAMEWORK FOR ABIA}

In contrast to the aforementioned agent-based approaches for image analysis, the conceptual framework for Agent Based Image Analysis (ABIA) outlined by Hofmann et al. (2015) 
focuses on the flexibility of ABS in order to increase the robustness of OBIA methods. Thus, it combines the ability of OBIA for a spatially detailed and semantic rich image analysis with the flexibility and robustness of ABS, aiming at increasing the reusability of OBIA rule sets. Since, in principle, both the rule sets and the image objects can adapt to changing image data, two alternative approaches were described by Hofmann et al. (2015). (1) Similar to ABS in robotics and process control, software agents automatically adapt existing rule sets according to varying image data: Software agents can autonomously change OBIA processing and classification rules. (2) Image objects evolve to image object agents: Each image object can autonomously adapt itself in order to best possibly fulfil its own classification criteria independent of the underlying image data. This approach has similarities to the concept of patch-based cellular automata, as introduced by Wang and Marceau (2013).

In both approaches the ontology of the image content plays a central and regulating role, since it acts as the foundation for the rule set and the object classes defined in the rule set with all their semantic constraints. The class descriptions, that is, the classification rules, can even be directly translated from the ontology (Belgiu et al. 2014). However, the ontology describes the object classes and their semantics independently from any underlying image data, and must not be violated by any agentbased adaptations.

Another aspect that impacts the agents' behaviour is the achieved quality during or after adaptation. Quality by definition reflects the minimum properties of an acceptable classification result (ISO 19157:2013). In remote sensing this refers to correctness and completeness. Both are measured by comparing the classification result with a reference classification that is assumed to be true (Congalton and Green 2009). However, a complete conformity between reference data and classification results is often not achievable (Hofmann and Lohmann, 2007, Albrecht et al. 2010). Thus, evaluating the classification quality should rather be measured according to the user requirements in terms of the required correctness and completeness. Nevertheless, in both ABIA concepts the minimum quality to be achieved has to be defined as a goal.

\subsection{MAS to autonomously adapt existing rule sets}

A MAS designed to change existing rule sets consists at least of one or more Rule Set Adaptation Agents (RSAA). Since the number of potential rule set adaptations can be as numerous as the number of rules and parameters, there is no limit for the number of RSAAs being organized in such a MAS. However, each ABIA-MAS must balance its activities between ontology conformity and quality requirements. That is, only rule set adaptations not violating the ontology and leading to best achievable quality are permitted. In the framework presented by Hofmann et al. (2015), this balancing and evaluation act is performed by one or several dedicated control agents (CAs). Their goals are designed to prohibit adaptations that lead to a contradiction between ontology and rule set, and to achieve a minimum quality, usually at least the quality of the initial classification the rule set was created for. Consequently, the adaptation process is evolutionary, whereby RSAAs and CAs try to find the best adaptations in order to achieve their common goals. During this process, CAs inform RSAAs whether their intended actions are allowed or not, and to what degree their adaptations would be successful in terms of goal achievement. In the outlined MAS, each RSAA is designed to adapt specific elements of a given rule set, which means to adjust parameters, to change classification rules, and to add or remove processing rules, etc. Depending on the type of adaptation, RSAAs can be sub-divided into diverse types of RSAAs. However, each RSAA has individual sensors, effectors, goals and strategies. Additionally, each agent should be provided with a learning mechanism and a cost model allowing it to focus on the most promising adaptation actions (see Fig. 2).

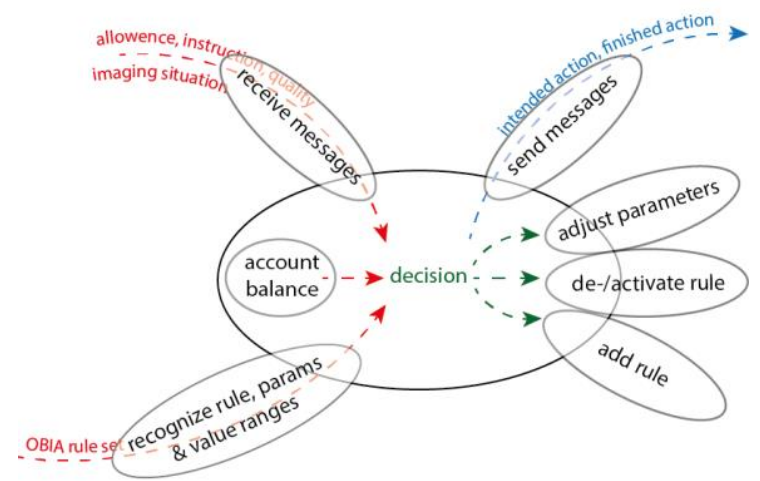

Figure 2. Principle design of a RSAA. Source: Hofmann et al. (2015).

\subsection{Hierarchical net of Image Object Agents}

Alternatively to a MAS consisting of RSAAs and CAs, image objects aka segments can evolve to image object agents (IOAs) and together build a MAS, organized as a (hierarchical) net of IOAs. Similar to the hierarchical net of image objects as outlined in Benz et al. (2004), each IOA is connected with its sub-, super- and neighbour agents. This allows each IOA to request the current status of the connected IOAs, and to provide information about its own status. However, the ABIA concept extends the OBIA approach by adding the ability of each IOA to process itself, and to interact, collaborate and to negotiate with other IOAs. Additionally, IOAs have individual goals or share goals with other IOAs, motivating each IOA to execute or to not execute certain actions. If a cost model is additionally integrated, each IOA aims at reducing costs, too. Implementing learning mechanisms allows each IOA to avoid the repetition of unsuccessful actions, and to focus on the most promising strategies. If an IOA is a member of at least one class, one of its goals will be to fulfil the classification criteria as good as possible, that is, to match the class antetype as described in the ontology and according class definition. Further goals can be to achieve pre-defined quality criteria, as outlined in the section before, or to avoid classification ambiguities and uncertainties. For a MAS built up by IOAs, as depicted here, it can also be sensible to encapsulate global tasks, such as executing the classification, controlling the global quality, prioritising tasks or classes, etc. in one or more CAs.

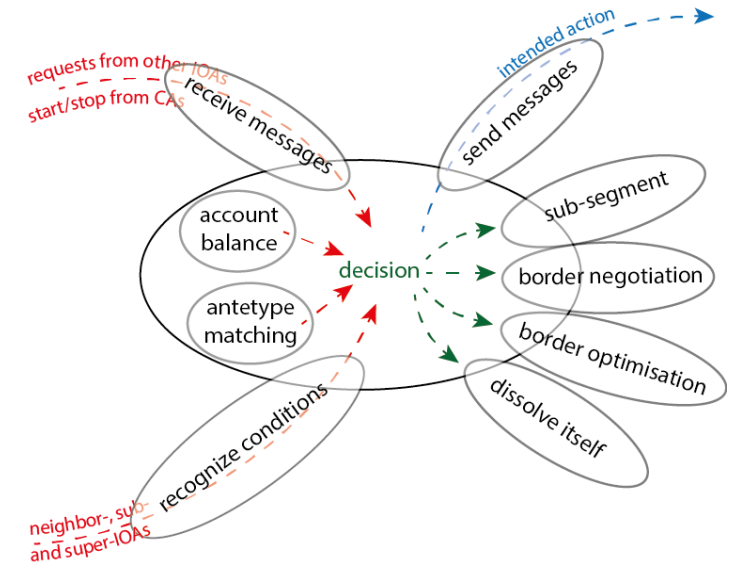


Figure 3. Principle design of an IOA. Source: Hofmann et al. (2015).

\section{CURRENT IMPLEMENTATIONS}

Both of the described approaches of ABIA have been implemented in different environments. The IOA approach as outlined in section 3.2 has been implemented twice: One implementation is based on eCognition and its CNL and has already been realized and documented in Hofmann et al. (2015). The second implementation is introduced here. It integrates existing non-commercial tools for $\mathrm{ABM}$, image processing and GIS with self-coded software. The necessary image processing methods, object property calculations, and fuzzy classification mechanisms, as well as the necessary methods to exchange data between the tools and components (Fig. 4) were implemented by the authors.

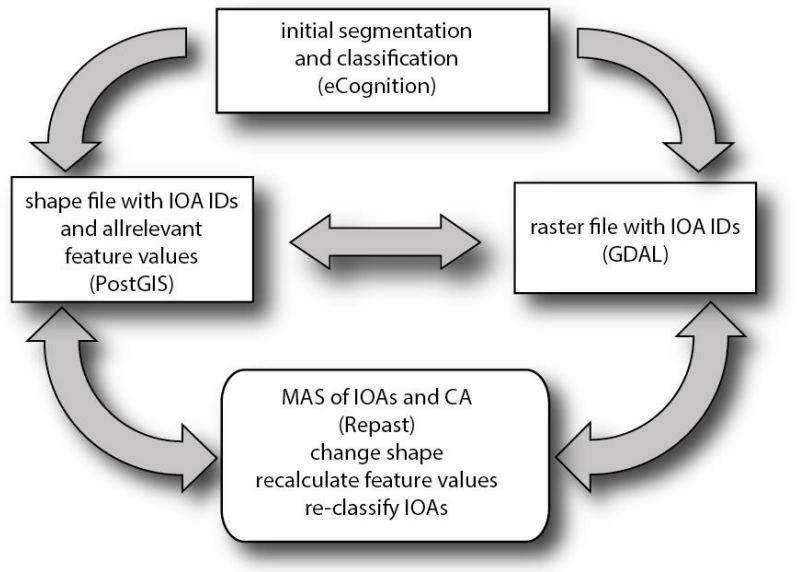

Figure 4. Principle adaptation workflow and components of an ABIA MAS consisting of IOA.

For the realisation of the RSAA approach as outlined in section 3.1, Repast was used for the agent-based processing, and eCognition for the execution of the adapted OBIA rule set. Additionally, a DSL was developed, which allows the specific agents for this approach to be very easily defined (Lettmayer, 2015). Recently, none of the implementations contains a learning method, yet, whereas the integrating implementation of the IOA approach uses a simple cost model.

All approaches were developed based on a simple scenario consisting of a subset of an orthorectified VHR airborne scene from Weilheim, Upper Bavaria, Germany, taken in May 2010. The orthoimage together with a DSM were derived from a stereo pair of the RGB-bands captured by an UltraCam XP (www.ultracamx.com) using software from SimActive (www.simactive.com). The spatial resolution of the orthoimage is $8 \mathrm{~cm}$, and that of the DSM is $35 \mathrm{~cm}$. The radiometric resolution of the optical data is 8bit. Slope and slope of slope (change of slope) were calculated from the DSM per pixel and expressed in degrees. The subset under investigation has a size of 1311 x 869 pixels. The initial segmentation and classification were performed using eCognition, whereby the rule set was designed to delineate roofs and different roof types ("saddled", "flat" and "other"). The CNL rule set consists of an initial multi-resolution segmentation, which uses the R, G, and B band of the image together with the DSM and slope values as input data, and parameters as outlined in Tab. 1.

\begin{tabular}{|c|c|c|c|c|}
\hline Level & Method & scale parameter $^{1)} /$ size $^{2)}$ & shape & compactness \\
\hline 1 & Chessboard & $1^{2)}$ & - & - \\
2 & MRS & $150^{1)}$ & 0.2 & 0.5 \\
\hline
\end{tabular}

Table 1. Segmentation parameters

It then classifies each image object according to a fuzzy class hierarchy describing the different roof types (see Appendix 1), which is based on an appropriate ontology. The initial parameterisation of the segmentation leads to a general oversegmentation of the scene and an incomplete detection of roofs (see Fig. 5).

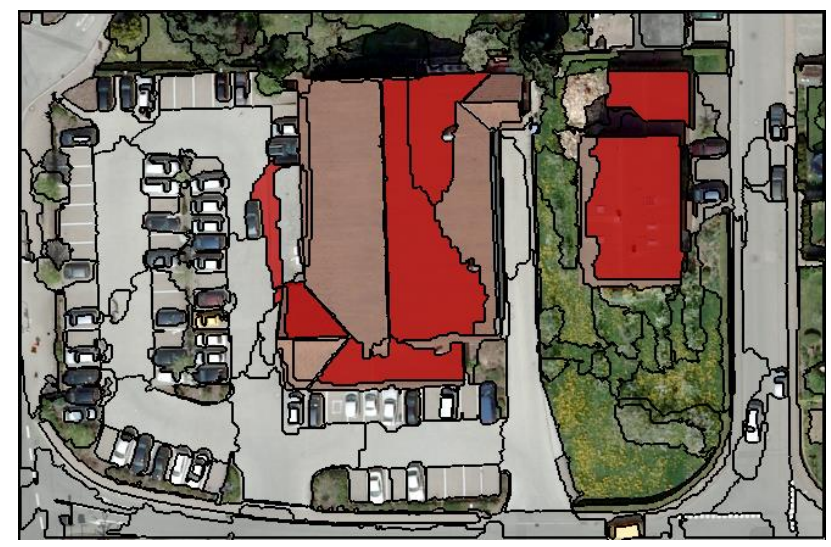

Figure 5. Initial segmentation and classification result.

\subsection{Agent-based adaptation of existing rule set}

As outlined in section 3.1, the MAS for this approach consists of at least one RSAA and one CA. We decided to reduce the technical complexity of the MAS to a minimum in order to demonstrate the principle feasibility,. Consequently, our current configuration of this MAS consists of exactly one RSAA and one CA. The MAS (Fig. 6) tries to adjust the so-called scale parameter of the multi-resolution segmentation (Baatz \& Schäpe, 2000).

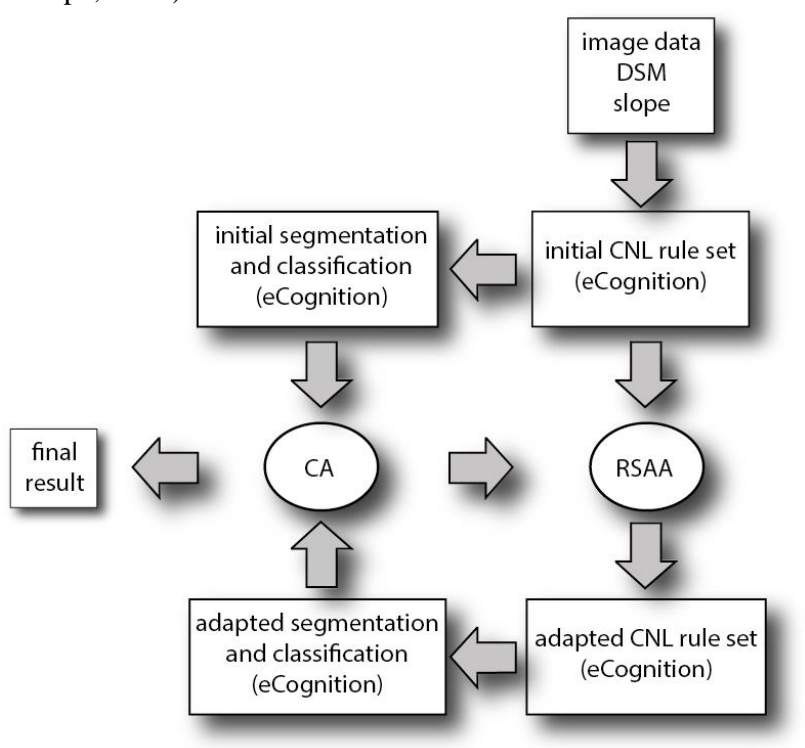

Figure 6. Principle adaptation workflow and components of an ABIA MAS consisting of RSAA.

Goal of the MAS is to adjust the scale parameter in a way that the resulting segments delineate house objects as good as possible. The latter is evaluated through their individual degree of membership to one of the "roof"-type sub-classes: the higher 
the membership degree, the better they delineate a house object. To evaluate the whole scene, the mean best membership value of all "roof"-objects to one of these roof classes is calculated. Unclassified objects remain disregarded. The higher the mean best membership, the more the MAS has achieved its goal. In the present example we accept a classification result that produces at least a mean best membership of 0.7. That is, once this threshold is achieved (within a pre-defined number of iterations aka ticks), the MAS will stop the adaptation process.

RSAA and CA were defined using the so-called Agent Definition Language (ADL), which is a DSL especially designed for agent-based autonomous OBIA rule set adaptation (see Lettmayer, 2015 for details). They are translated in Java code and executed in Repast (Collier, 2003) in order to adapt the XML-coded CNL rule set. In each adaptation tick the adapted rule set is automatically executed with eCognition, and the resulting mean best membership value is evaluated by the CA. In case the goal is not achieved yet, the CA triggers the RSAA to adapt the scale parameter again, and the next adaptation tick starts (Fig. 6).

\subsection{Self-organising net of Image Object Agents}

In principle, IOAs and their behaviour can be implemented in different environments and configurations. The basic idea in the example given is to provide each IOA with the goal to become a best possible member of one of the roof-type classes, and to individually decide on optional actions in order to change its own shape. Hofmann et al. (2015) demonstrate how this can be realised in eCognition using its CNL. However, eCognition does not allow direct access to single pixels of objects, which is essential for autonomous IOA shape adjustment. Additionally, the approach of Hofmann et al. (2015) does not contain a cost model. Thus, we decided to implement the IOA approach by integrating existing GIS and tools: PostGIS (Lijing \& Jing, 2010), and GeoJSON, (Butler et al., 2008), image processing tools, here: GDAL (http://www.gdal.org/), and tools for ABM, here: Repast. Initially segmented and classified objects are processed in this environment. Each IOA can access its own pixels, which allows it to exchange its border pixels with neighbouring IOAs, and vice versa. Currently, only five neighbouring border pixels can be exchanged at once per IOA and tick, due to the still complex exchange mechanism. The latter currently uses a CSV file that stores all exchanging IOAs and their exchangeable pixels. In order to avoid a deterioration of neighbouring IOAs, each IOA prefers those neighbours for pixel exchange that have the lowest membership degree for one of the roof-type classes. The recent cost model decreases an IOA's account by two currency units for each action. If the performed action improved the IOAs class membership, it gets three currency units back, which "rewards" the action's success. Our database stores the evolution of each IOA, namely the shape and all feature values of each IOA at each tick.

\section{RESULTS}

While for the implemented RSAA approach and the IOA approach implemented according to Hofmann et al. (2015) an improvement is observable, this is not the case for the IOA approach using our own integrated environment. The reasons for the latter will be discussed in the next section.

As Fig. 8 indicates, the autonomous adaptation of the scale parameter for the multi-resolution segmentation improves the initial classification results, that is, the mean membership of all house IOAs to one of the roof type classes. While the initial value was at 0.14 , it improved to 0.56 after 100 ticks, which is still below the aspired minimum value of 0.7 (Fig. 7 and 8). The results are similar to those achieved by Hofmann et al. (2015). Nevertheless, parts of the big central building in the lower left corner and the upper right corner were not connected to the main object. Similarly, the garage bordering the smaller building in the central-right part of the image was neither delineated nor classified as a roof-type object (false negatives). Vice versa, the building's shape at its northbound border was wrongly classified as a roof-type object (false positive). The reasons for these misclassifications are discussed in the next section.

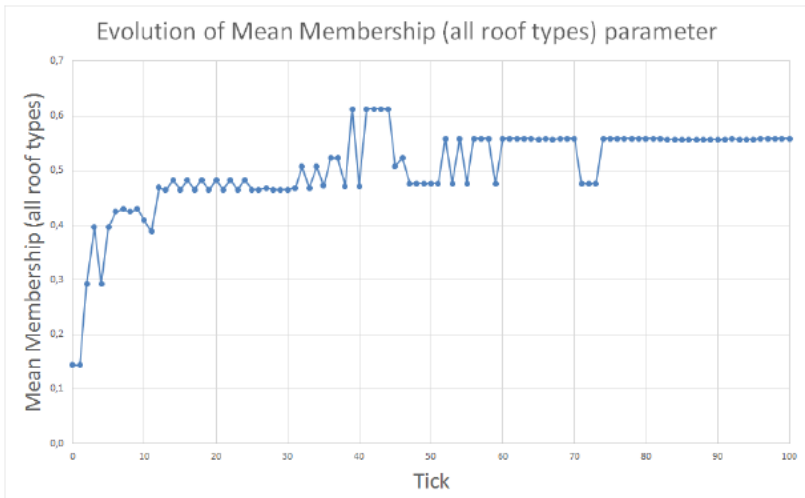

Figure 7. Development of the mean membership of all roof-type IOA for one of the roof-type classes when applying the ABIA MAS consisting of RSAA.

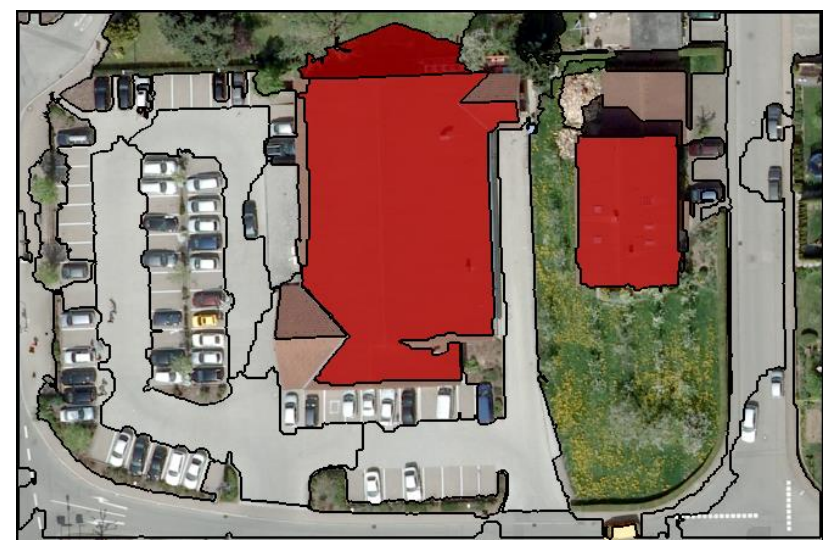

Figure 8. Final segmentation and classification result applying the ABIA MAS consisting of RSAA.

In contrast, for our integrated solution the mean membership of all house IOAs to one of the roof type classes decreases (see Fig. 9).

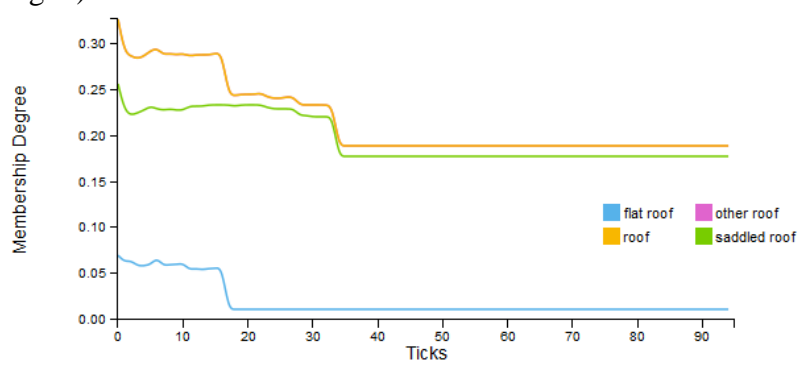

Figure 9. Development of mean membership degrees for rooftype classes applying the integrated IOA approach.

Additionally, even after 100 ticks almost no change of the objects' shape is observable (see Fig 10 in comparison with Fig. $5)$. 


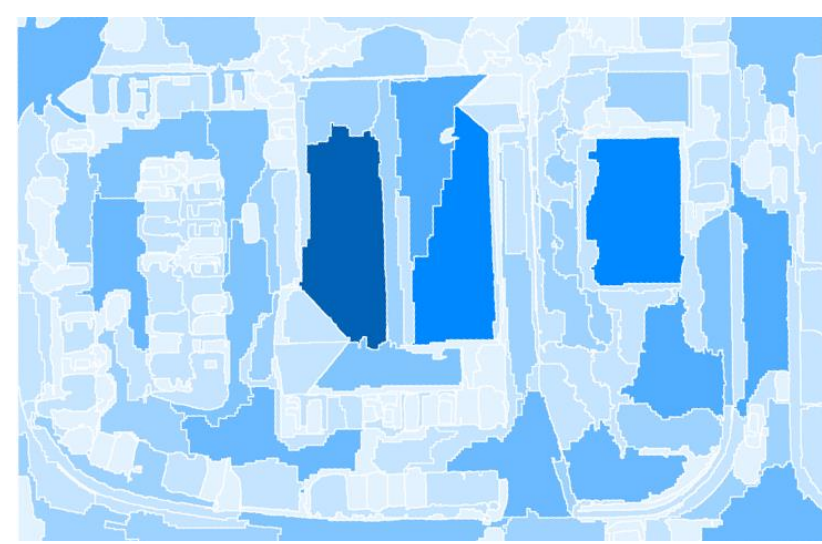

Figure 10. Final segmentation result after applying the ABIA MAS consisting of IOA for 100 ticks.

Nevertheless, we were able to demonstrate that it is possible in principle to realise a MAS of IOAs according to the conceptual framework introduced in Hofmann et al. (2015), and wherein neighbouring IOAs are capable of exchanging border pixels following individual goals, that is, to reduce transaction costs and to improve their own membership to one of the desired classes.

\section{CONCLUSIONS AND DISCUSSION}

We could demonstrate that the implementation of the conceptual framework for ABIA and the different adaptation approaches as described in Hofmann et al. (2015) are feasible in principle. For the example data used we could also show that besides the already demonstrated IOA solution as implemented with CNL in eCognition, rule set adaptation with RSAAs is also capable of improving initial OBIA segmentation and classification results. In this context it turned out that the development and usage of a DSL supports the creation and design of ABIA MASs. That is, the necessary knowledge (here: about JAVA, Repast and eCognition) to create an ABIA MAS can be reduced to a minimum, while the user can focus on the design of necessary software agents. Thus, we plan to further develop and extend the developed ADL in order to make it applicable for the IOA approach also. Nevertheless, the results are not perfect. A potential reason for the false negatives mentioned in section 5 is the already "perfect" shape achieved, when the central parts of the bigger building evolve to a rooftype object. Since the unlinked outer parts are jutties, with locally different slopes and shapes, integrating them with the main part would lead to a reduction of the membership to one of the roof-type classes. The latter would contradict the MAS's goal. For the same reason, the garage roof cannot merge with its bordering shade area or even the bordering building. Thus, leaving the garage unclassified but completely delineating the neighbouring building leads to an overall better result in terms of membership degrees. The detectable false positive area at the upper border of the central building is in fact the shade of this building casted on a neighbouring tree. Since the slope and elevation of the tree is similar to that of buildings, and the casted shade has the building's shape, this segment already fulfils the classification criteria of a roof-type class. Nevertheless, its degree of membership to the class "saddled roof" is only at $\mu=0.15$. Those of the central building and the smaller right-hand building are at $\mu=0.94$ and $\mu=0.99$. However, introducing an explicit distinct class "shade" to the ontology and the class hierarchy could easily solve this problem.

The reasons for the rather disappointing results of our integrated IOA approach are manifold. One is certainly given by the recently missing alternative actions each IOA can execute. Such an alternative action could be, for example, merging with neighbouring IOAs. Especially if the image resolution is as extremely high as in the example given $(0.08 \mathrm{~m})$, merging could certainly accelerate the adaptation process. With this high resolution, exchange of (five neighbouring) border pixels has almost no impact on the IOAs' shape. Thus, the amount of exchangeable pixels (e.g. along the whole border or even inside a to-be-defined buffer) should be increased. Nevertheless, more research with lower resolutions is necessary. Similarly, the presented RSAA approach has no cost model implemented yet, since this is not sensible with only one acting option (adjust the scale parameter) However, if alternative actions were implemented, which is planned, a cost model for RSAAs is also necessary. Last but not least, none of the implemented MAS contains agents with the ability to learn. Having a learning method available for each agent could further support them in their adaptation and optimisation process. Additionally, future research will take into account the possibility of parallelizing ABIA activities.

Besides the presented approach for using IOAs, they could also be used in ABM, as described in section 2.2. In combination with remote sensing data this would offer the opportunity to simulate and validate an $\mathrm{ABM}$ simultaneously based on remote sensing data. In combination with the used ADL, even non-experts could easily perform ABM.

\section{ACKNOWLEDGEMENTS}

The research presented was funded by the FWF basic research project ABIA, grant number FWF P25449. We also thank Thomas Strellen (TS Photogrammetrie \& Geoinformatik, www.photogeo.de) for providing us with the UltraCam XP image data and SimActive (www.simactive.com) for supporting us in the generation of the DSM.

\section{REFERENCES}

Athelogou, M., et al., 2007. Definiens Cognition Network Technology - A Novel Multimodal Image Analysis Technique for Automatic Identification and Quantification of Biological Image Contents. In: Frischknecht, F., Shorte, S.L., (Eds.): Imaging Cellular and Molecular Biological Functions, Springer. Berlin, Heidelberg. pp. 407-421.

Albrecht, F., Lang, S. and Hölbling, D., 2010. Spatial Accuracy Assessment of Object Boundaries for Object-Based Image Analysis. The International Archives of the Photogrammetry, Remote Sensing and Spatial Information Sciences, XXXVIII4/C7.

Arvor, D., Durieux, L., Andrés, S. and Laporte, M.-A., 2013. Advances in Geographic Object-Based Image Analysis with ontologies: A review of main contributions and limitations from a remote sensing perspective. ISPRS Journal of Photogrammetry and Remote Sensing, 82 (0), pp. 125-137.

Baatz, M., Hoffmann, C. and Willhauck, G., 2008. Progressing from object-based to object-oriented image analysis. In: Blaschke, T. Lang, S. and Hay, G. (eds.) Object-Based Image Analysis: Spatial Concepts for Knowledge-Driven Remote Sensing Applications. Berlin, Heidelberg: Springer.

Belgiu, M., Hofer, B. and Hofmann, P., 2014a. Coupling formalized knowledge bases with object-based image analysis. Remote Sensing Letters, 5 (6), pp. 530-538. 
Benz, U. C. et al., 2004. Multi-resolution, object-oriented fuzzy analysis of remote sensing data for GIS-ready information. ISPRS Journal of Photogrammetry and Remote Sensing, 58, pp. 239-258.

Blaschke, T. and Strobl, J., 2001. What's wrong with pixels? Some recent developments interfacing remote sensing and GIS. GIS - Zeitschrift für Geoinformations-systeme, 14 (6), pp. 1217.

Blaschke, T. et al., 2014. Geographic Object-Based Image Analysis - Towards a new paradigm. ISPRS Journal of Photogrammetry and Remote Sensing, 87, pp. 180-191.

Bovenkamp, E. G. P. et al., 2004. Multi-agent segmentation of IVUS images. Pattern Recognition, 37(4), 647-663.

Brown, G. D. et al., 2005. Spatial process and data models: Towards integration of agent-based models and GIS. Journal of Geographical Systems, 7, pp. 25-47.

Burnett, C. and Blaschke, T., 2003. A multi-scale segmentation/object relationship modelling methodology for landscape analysis. Ecological Modelling, 168 (3), pp. 233-249.

Butler, H., Daly, M., Doyle, A., Gillies, S., Schaub, T., Schmidt, C., 2008. The GeoJSON format specification. Rapport technique, 67.

Collier, N., 2003. Repast: An extensible framework for agent simulation. The University of Chicago's Social Science Research, 36, 2003.

Congalton, R. G. and Green K., 2009. Assessing the Accuracy of Remotely Sensed Data-Principles and Practices. Boca Raton, FL CRC Press, Taylor \& Francis Group.

Cracknell A. P., 1998. Synergy in remote sensing - what's in a pixel? International Journal of Remote Sensing, 19 (11), pp. 2025-2047.

Fazel Zarandi, M. H. and Ahmadpour, P., 2009. Fuzzy agentbased expert system for steel making process. Expert Systems with Applications, 36, pp. 9539-9547.

Göhner, P., 2013. Agentensysteme in der Automatisierungstechnik. Berlin, Heidelberg: Springer.

Hay, G. J. and Blaschke, T., 2010. Special issue: geographic object-based image analysis (GEOBIA). Photogrammetric Engineering and Remote Sensing, 76 (2), pp. 121-122.

Hay, G. J. and Castilla, G., 2008. Geographic Object-Based Image Analysis (GEOBIA): A new name for a new discipline. In: Blaschke, T. Lang, S. and Hay, G. (eds). Object-Based Image Analysis: Spatial Concepts for Knowledge-Driven Remote Sensing Applications. Berlin, Heidelberg: Springer.

Hofmann, P. and Lohmann, P., 2007. A strategy for quality assurance of land-cover/land-use interpretation results with faulty or obsolete reference data. The International Archives of the Photogrammetry, Remote Sensing and Spatial Information Sciences, XXXVI (1/W51).

Hofmann, P., Blaschke, T. and Strobl, J., 2011. Quantifying the robustness of fuzzy rule sets in object-based image analysis. International Journal of Remote Sensing, 32 (20), pp. 73597381.
Hofmann, P., et al. 2015. Towards a framework for agent-based image analysis of remote-sensing data. International Journal of Image and Data Fusion, 6 (2), pp. 115-137.

Hudak, P., 1996. Building domain-specific embedded languages. ACM Computing Surveys, 28 (4).

International Organization for Standardization, 2013. ISO 19157:2013. Geographic information - Data quality.

Jennings, N. R., 2000. On agent-based software engineering. Artificial Intelligence, 117, pp. 277-296.

Koch, A., 2007. Geovisualisierung durch Geosimulation. Visuelle Repräsentation humangeographischer Phänomene durch Agentenbasierte Geosimulation. In: Tzschaschel, S., Wild, H., Lentz, S. (eds.) Visualisierung des Raumes. Karten machen - die Macht der Karten. Forum ifl, pp. 191-202.

Kohli, D. et al., 2013. Transferability of Object-Oriented Image Analysis Methods for Slum Identification. Remote Sensing, 5, pp. 4209-4228.

Laliberte, A. S. and Rango, A., 2011. Image Processing and Classification Procedures for Analysis of Sub-decimeter Imagery Acquired with an Unmanned Aircraft over Arid Rangelands. GIScience \& Remote Sensing, 48 (1), pp. 4-23.

Lang, S., 2008. Object-based image analysis for remote sensing applications: modelling reality - dealing with complexity. In: Blaschke, T. Lang, S. and Hay, G. (eds). Object-Based Image Analysis: Spatial Concepts for Knowledge-Driven Remote Sensing Applications. Berlin, Heidelberg: Springer.

Lang, S. et al., 2014. Geons - domain-specific regionalization of space. Cartography and Geographic Information Science, 41 (3), pp. 214-226.

Lettmayer, P., 2015. Domain specific language for agent-based image analysis. Master thesis, University of applied sciences Salzburg.

Lijing Z; Jing Y, 2010. Management methods of spatial data based on PostGIS. In Circuits, Communications and System (PACCS), 2010 Second Pacific-Asia Conference on , Vol.1, No.1, pp.410-413, 1-2 Aug. 2010

Macal C. M. and North, M. J., 2005. Tutorial on agent-based modeling and simulation. In: Kuhl M. E., Steiger N. M., Armstrong F. B. and Joines J. A. (Eds.), 37th Winter Simulation Conference, 2005 Orlando, FL, USA.

Mahmoudi, F. T., Samadzadegan F. and Reinartz, P., 2013. Object oriented image analysis based on multi-agent recognition system. Computers \& Geosciences, 54, pp. 219-230.

Maillot, E. N. and Thonnat, M., 2008. Ontology based complex object recognition. Image and Vision Computing, 26 (1), pp. 102-113.

Motik, B., Patel-Schneider, P. F. and Grau, B. C., 2009. Owl 2 web ontology language direct semantics. W3C recommendation, 27.

Novack, T., Kux, H., Feitosa, R. Q. and Costa, G. A. O. P., 2014. A knowledge-based, transferable approach for blockbased urban land-use classification. International Journal of Remote Sensing, 35(13), pp. 4739-4757. 
Parker, D. C. et al., 2003. Multi-agent systems for the simulation of Land-Use and Land-Cover change: A review. Annals of the Association of American Geographers, 93 (2), pp. 314-337.

Pinz, A., 2005. Object Categorization. Foundations and Trends in Computer Graphics and Vision, 1 (4), pp. 255-353.

Rodin, V. et al., 2004. An immune oriented multi-agent system for biological image processing. Pattern Recognition, 37 (4), pp. 631-645.

Samadzadegan, F., Schenk, T. and Mahmoudi, F. T., 2009. A multi-agent method for automatic building recognition based on the fusion of Lidar range and intensity data. Joint Urban Remote Sensing Event, 2009, 20-22 May 2009, Shanghai, China, 1-6.

Samadzadegan, F., Mahmoudi, F. T. and Schenk, T., 2010. An agent-based method for automatic building recognition from lidar data. Canadian Journal of Remote Sensing, 36 (3), pp.

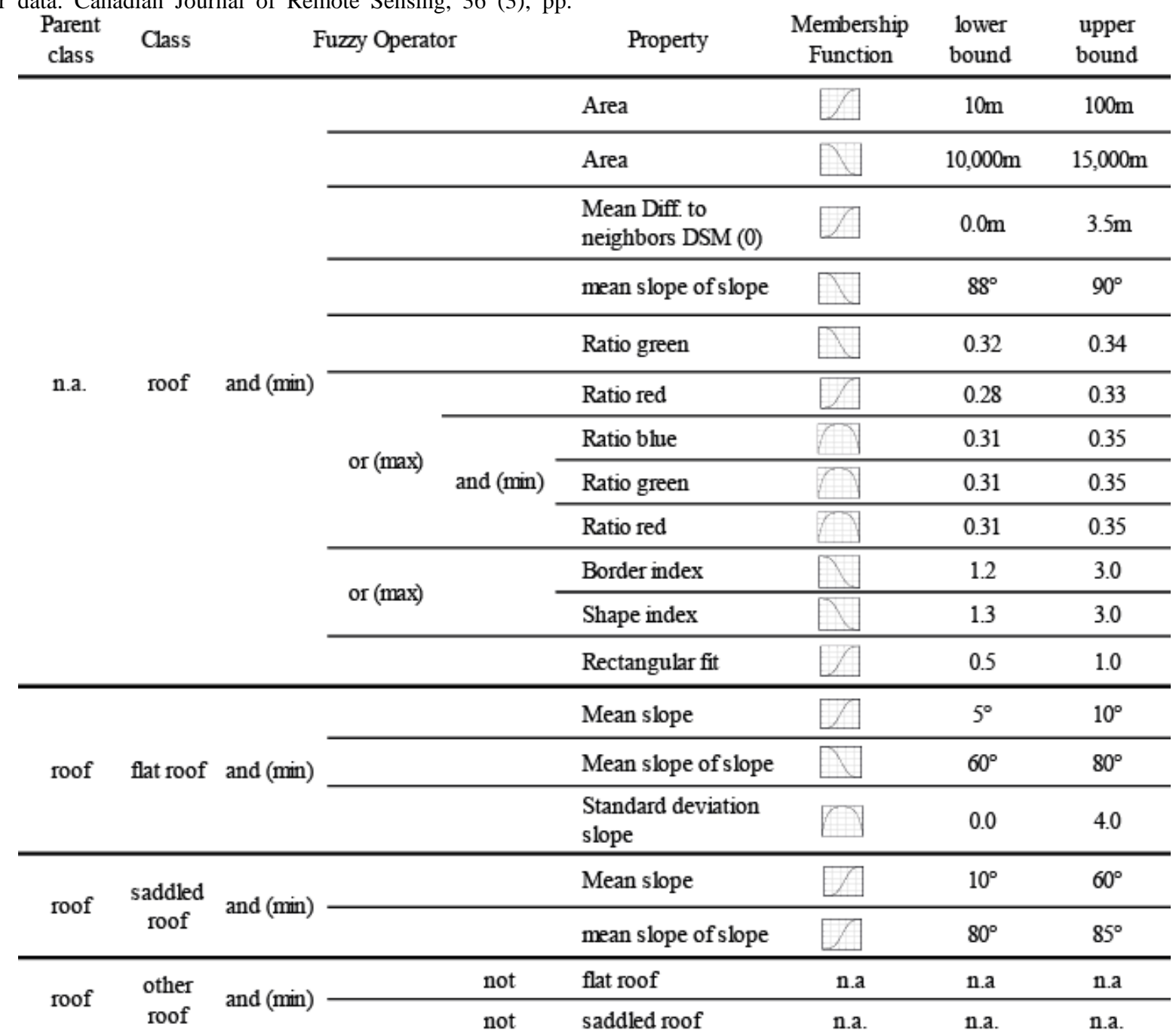

Appendix 1: Classes and class descriptions. Source: Hofmann et al (2015).
211-223.

Walker, J. S. and Blaschke, T., 2008. Object-based landcover classification for the Phoenix metropolitan area: optimization vs. transportability. International Journal of Remote Sensing, 29 (7), pp. 2021-2040.

Wooldridge, M. and Jennings, N. R., 1995. Intelligent agents: Theory and practice. Knowledge Engineering Review, 10 (2), pp. 115-152.

Yu, C. and Peuquet, D. J., 2009. A GeoAgent-based framework for knowledge-oriented representation: Embracing social rules in GIS. International Journal of Geographical Information Science, 23 (7), pp. 923-960.

\section{APPENDIX}

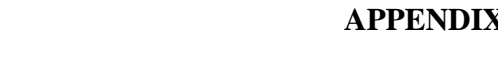




\title{
FROM CLASSIFICTAION RESULTS TO TOPOGRAPHIC MAPS
}

\author{
Joachim Höhle \\ Aalborg University, Department of Development and Planning, Denmark
}

KEY WORDS: Classification, Topographic Mapping, Cartographic Enhancement, Assessment, Accuracy

\begin{abstract}
:
The classification of high resolution multispectral aerial imagery enables very high thematic accuracies when machine learning methods are applied. The use of classification results as topographic maps requires cartographic enhancement and checking of the geometric accuracy. Urban areas are of special interest. The conversion of the classification result into topographic maps of high thematic and geometric quality is subject of this contribution. After reviewing the existing literature on this topic a methodology is presented. It has the goal to achieve high cartographic quality and geometric accuracy for buildings and other topographic objects. The suggested methodology for improving the classification results is described. With the ISPRS data set of the '2D labelling contest' a land cover map of six classes was produced and then enhanced by the proposed method. The classification used a machine learning method applying a variety of attributes including object heights derived from imagery. The cartographic enhancement is carried out with two different levels of quality. The user's accuracy for the classes "impervious surface" and "building" were above $85 \%$ in the 'level 1' map. The geometric accuracy of building corners in the 'level 2' map was assessed by means of reference data derived from a DSM-based orthoimage. The obtained root mean square errors were RMSE_x $=1.2 \mathrm{~m}$ and RMSE_y $=0.7 \mathrm{~m}$. All processing could be carried out with a high level of automation.
\end{abstract}

\section{INTRODUCTION}

Big progress has been achieved in the classification of aerial and satellite images by means of machine learning methods. The classification results, however, are not topographic maps. Generalization of the map content and cartographic quality are absolutely necessary for topographic maps. The geometric accuracy has to meet high demands. Urban areas are of special interest. Important is also that these maps can be produced quickly and updated in short intervals of time. This demand requires automatic processing to a large extent.

In this contribution we focus on automatic 2D mapping of urban areas with detailed content including small objects. The positional accuracy of well-defined objects should be less than one meter. The graphical output must have cartographic quality, which includes simplification of the content and representation of man-made objects by straight and orthogonal lines. Such map data are in need. An overview on the status of the mapping in the world was recently published by (Konecny et al., 2015). The authors have collected data from 244 countries. In the report is stated that in the scale range "1:25000 and bigger" only 30\% of the total world area is produced by the authorities. There exist big differences in the coverage with topographic maps in the mentioned scale range, e.g. 4.7\% (Africa) and 98.1\% (Europe). In some of the areas covered with maps or databases the data may be 10 to 30 years old. These facts demonstrate the need of improvements in the production and maintenance of such data. Private companies, e.g. Google and Microsoft, have created map data for navigation and location based services. They often use volunteers to create these maps. The Open Street Maps (OSM) is one example. The contents and accuracy of such maps differs. Tests indicate that these maps may have a geometric accuracy of $1.6 \mathrm{~m}$ after correction of systematic errors (ElAshmawy, 2016).

The last advancements in the sensor technology and processing methods yield new possibilities to faster produce and update the maps and databases in the important category " $1: 25000$ and bigger". Topographic objects of urban areas need to be mapped in the scale range "1:10000 and bigger". These vector maps may be manually or automatically derived from various source data. The cartographic enhancement and the geometric accuracy are very important issues when topographic maps are the goal. This part will be the main focus point in this work. In the past, research has been carried out in order to find solutions for this task. Some authors proposed methods using lidar data only (Gross and Thoennessen, 2006; Sampath and Shan, 2007). Awrangjep et al., 2010. presented a method, which is based on aerial colour imagery and lidar. This contribution has the goal to automatically produce urban 2D maps of high cartographic quality and of high geometric accuracy using aerial imagery only.

The structure of the paper is the following. Section 2 describes the characteristics of topographic maps and databases. The source data and the classification methods are discussed in Section 3 and 4. The cartographic enhancement of the classification result is dealt with in Section 5. A methodology to improve the cartographic quality is presented in Section 6 . Information on the assessment of the thematic and geometric accuracy is given in Section 7. Examples of cartographic enhancements are part of Section 8. Discussion and conclusion are in Section 9.

\section{CHARACTERISTICS OF LARGE-SCALE TOPOGRAPHIC MAPS AND DATABASES}

Topographic maps of urban areas with many details are produced in scales "1:10000 and bigger". The contents of these maps depend on the purpose of the map. Planning and management are the important applications. The various object types are stored in different layers and can be displayed individually or in combination. The storage in databases allows also an analysis of the map data. The generation and updating of such geographic information systems (GIS) is a major task of mapping organizations today. Details on the assessed accuracy, the time of acquisition, and many other information are stored in metadata. Topographic maps are always georeferenced and may also contain elevations. The separation into a planimetric 
(2D) map and an accompanying digital elevation model (DEM) seems to be a trend in mapping including updating. In the following we discuss 2D maps only.

\subsection{D maps of urban areas}

2D maps of urban areas are sometimes also called technical maps. Such maps should have a high geometric accuracy and a high rate of updating. They are digital vector maps and they are displayed on a computer screen in a range of scales, e.g. 1:1000 to $1: 10000$. Printing of analogue maps may occur on demand only. The production methods are different in the world. Manual digitizing of orthoimages is a fast and cheap method to produce and update digital vector maps today. The level of detail and accuracy of maps produced by this so-called "headson digitizing" depend very much on the resolution of the orthoimages. The smallest object which can be recognized should cover an area of two to three pixels which also must have sufficient contrast to its surroundings. The accuracy of orthoimages should be about two ground sampling distances (GSD). The orthoimages are often used as background information for the map data in vector format. The automatic extraction of points, lines, and areas from orthoimages is subject of this article. This process is called vectorization.

\subsection{Objects of large-scale topographic maps}

Objects of large-scale topographic maps are buildings, car ports, walls, roads, parking lots, paths, bridges, trees, bushes, hedges and many others. In order to represent them on a map they should have a minimum size. This is given by the resolution of the human eye which is about one minute of arc. It means that lines of $0.05 \mathrm{~mm}$ in width can be recognized from a distance of $30 \mathrm{~cm}$. The resolution of the computer screen is given by its pixel size. For example, the pixel size of a $56 \mathrm{~cm}$ screen with $1680 \times 1050$ pixels is $0.28 \mathrm{~mm}$ which corresponds to a resolution of 35 pixels $/ \mathrm{cm}$ or $90 \mathrm{dpi}$. The smallest line which can be displayed on such a screen has a width of $0.28 \mathrm{~mm}$ only. The lines of man-made objects must be straight and orthogonal. The lines should be without gaps and the polygons of area objects have to be closed.

\section{SOURCE DATA OF CLASSIFICATION}

In this contribution the generation of topographic maps is investigated by enhancing the results of classification. The resources are images, auxiliary data and usable features (attributes) of topographic objects.

\subsection{Type of imagery}

The images used for the classification of urban areas should have spectral bands in the visible (red, green, blue) and in the non-visible (near-infra-red) parts of the spectrum. The radiometric resolution should be better than 8 bit corresponding to 256 digital numbers. Furthermore, the images should be metric which means that accuracy values for the camera constant and the position of the principal point have to be determined by a calibration. Modern aerial photogrammetric cameras will meet these demands. The imagery has to be taken with overlap, e.g. $60 \%$, so that heights can be derived. The ground sampling distance (GSD) should have a size that elevations can be determined with sub-meter accuracy. The taken aerial images have to be georeferenced. This means that all images will be connected by means of automatically derived homologous points. This process is called aerotriangulation which requires a few ground control points. Data of sensors for position and attitude, simultaneously recorded with the images, will support this process and enable accurate orientation data of the images. The aerial images can then be transferred into orthoimages. In this transformation, the aerial images are rectified due to their tilts and corrected for differences in terrain elevations. The first task requires the orientation data of the images and the second one needs a digital elevation model. Two elevation models can be used, either the digital surface model (DSM) or the digital terrain model (DTM). The DSM-based orthoimage depicts the buildings in a correct position but their outlines are wriggly lines. In the DTM-based orthoimage, the outlines of buildings are sharp but displaced due to the height above ground. The DTM is derived from the DSM by means of filtering. The size of the orthoimage pixel can differ from the GSD value. Each pixel of the orthoimage may have coordinates of the reference system. The coordinate value is valid either for the centre or for the upper left corner of a pixel. In order to achieve high geometric accuracies, the mentioned parameters have to be known and be correctly used in the processing.

\subsection{Auxiliary data}

In the following procedures in classification and graphic enhancement other data could also be used with advantage. For example, the spatial coordinates of the perspective centres and the heights above ground may be applied to correct the position of buildings when DTM-based orthoimages are used in the classification. Existing maps may be helpful to detect objects. The classification can then be restricted to certain areas, e.g. to roads and parking lots when cars have to be detected. Other data may be digital elevation models (DEMs) that are derived from airborne laser scanning (lidar) or other sensors.

\subsection{Attributes and attribute profiles}

The objects of topographic maps can automatically be detected by means of attributes which characterize the objects. The average height of residential houses (dZ) in suburbs may be known in advance. Other attributes used in classification are spectral signature and normalized difference vegetation index (NDVI). They can be derived from imagery. Also attribute profiles may be used. These are attributes of the standard attributes (dZ, NDVI). The attribute profiles are, for example, the standard deviation of the intensities or of elevations in the neighbourhood of a pixel.

\section{CLASSIFICATION METHODS}

Many classification methods have been developed in the past. Besides the generation of land cover maps with several classes, the extraction of single objects is subject of many studies. The extraction of building boundaries using high resolution images and lidar data is recently published in (Li et al., 2013). Lidar data are used to produce a coarse boundary, which is then refined by means of edges extracted from stereo images. Precise 3D boundaries of buildings are obtained by this methodology. In (Niemeyer et al., 2014) 2D building outlines are generated by means of lidar data using elevations and intensities. This investigation deals with the generation of $2 \mathrm{D}$ land cover maps of six classes using high resolution images only.

The applied method in this investigation is decision tree (DT). The theoretical background of the DT method is given in (Breiman et al., 1984). Experiences with DT classification are published i.a. in (Friedl and Brodley, 1997; Höhle, 2014). 


\section{CARTOGRAPHIC ENHANCEMENT OF THE CLASSIFICATION RESULT}

In order to produce topographic maps from classification results, several steps have to be carried out. Objects which are not part of topographic maps have to be removed and topographic objects have to gain cartographic quality. The thematic and geometric accuracies of the final result have to be accessed.

\subsection{Removal of non-topographic objects}

Topographic maps contain permanent objects only. Cars, boats, people, animals, tents, trampolines, haystacks, and other nonpermanent objects are not part of topographic maps. The classification results may have inhomogeneous areas representing more than one class. Buildings, roads, etc. have to be represented by one colour only. Non-data areas may also be present in the classification result. These areas have to be filled.

\subsection{Cartographic refinements}

Very small objects like garages, oriels, sheds, cellar entrances have to be removed as well. A minimum size has to be applied, e.g., area objects should cover at least $25 \mathrm{~m}^{2}$ in nature. That means, that a simplification of the map content has to take place. The degree of this generalization will vary for different map types, which are characterized by the number of objects and their level of detail.

Man-made objects like buildings, walls, roads, etc. have to be represented in the maps or data bases by straight lines. The outlines of buildings, e.g., consist of orthogonal and parallel lines. Small deviations from linearity, orthogonality and parallelism are easily noticeable by the map user (buyer) and should therefore be corrected. All of these improvements form the cartographic quality. Research on cartographic refinement of classification results is not very much dealt with in literature. In (Li et al., 2012) a methodology is presented. By means of a DSM a building mask is derived. The corner points of buildings can then be detected and line parameters are derived from them. The orientation of the lines is then averaged for a whole district of the city.

\subsection{Degree of automation}

The topographic maps have to be compiled by a high degree of automation. Some manual work may still be necessary. The solutions may be different according to the demands, the available resources, and the skills of the personnel. Topographic maps and data bases of different content and levels of quality have to be considered. Computation times may also be a matter of concern. Efficient algorithms have to be found and applied.

\section{A NEW METHODOLOGY TO IMPROVE CARTOGRAPHIC QUALITY}

The results of the classification may be cartographically enhanced by means of image processing and image analysis techniques. Each class has to be processed for itself. Two approaches are applied in these investigations. The first approach is a simple one where the focus is on a high degree of automation, but the quality of the map is limited. We name the approach 'level 1'. The second approach, called 'level 2', yields a higher cartographic quality for buildings and other man-made objects, but the efforts become higher and some interactions by an operator are needed. The proposed solution is achieved in small steps and will be carried out at the practical tests in Section 8 .

\subsection{Generation of level 1}

For the generation of level 1 quality only a couple of image manipulation are carried out (cf. Figure 1). Details for each step are given in the following.

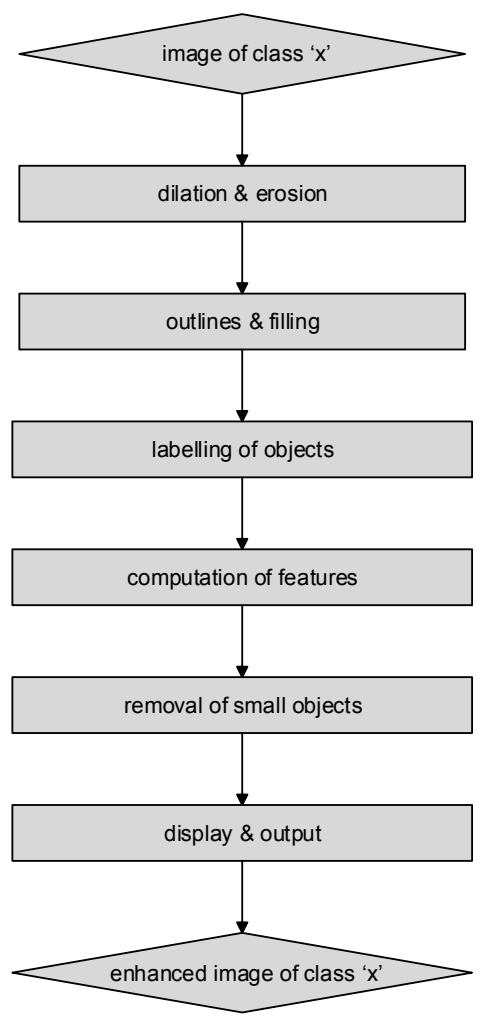

Figure 1. Steps in cartographic enhancement of land cover maps (level 1)

6.1.1 Dilation and erosion

These morphological operations are carried out by filtering. A structuring element (SE) has to be defined beforehand, e.g. a diamond shaped figure covering an area of a few pixels. The manipulations by dilation and erosion first increases the set of pixels and reduces them thereafter. The effect is a smoothing of the boundaries and a removal of some noise.

\subsubsection{Outlines and filling}

The first manipulation thresholds the image by means of a moving rectangular window. Two parameters have to be specified: The size of the window and the offset from the average intensity within the window. The outlines of objects are generated. The second operation generates a filling of the whole object with pixels of the intensity " 1 ". The areas of the objects, e.g. buildings, are then homogeneous.

\subsubsection{Labelling of objects}

The connected sets of pixels with the intensity " 1 " can now be labelled by a digit. The number of objects can then be counted.

6.1.4 Computation of features

Features of objects like position, area, maximum radius, orientation, etc. are derived for each of the objects in the binary image (B). The formula for the area (A) of an object is: 


$$
A=\sum_{i=1}^{n} \sum_{j=1}^{m} B[i, j]
$$

where $\mathrm{i}, \mathrm{j}=$ image coordinates.

The coordinates of the centre of an object $\left(x_{c}, y_{c}\right)$ are calculated by:

$$
\begin{gathered}
x_{c}=\frac{\sum_{i=1}^{n} \sum_{j=1}^{m} j \cdot B[i, j]}{A} \\
y_{c}=\frac{\sum_{i=1}^{n} \sum_{j=1}^{m} i \cdot B[i, j]}{A}
\end{gathered}
$$

The units are pixels. More details about these formulas can be found in (Jain, 1995).

6.1.5 Removal of small objects

Small objects can now be removed using a threshold for the area (A) or the radius of an object. The result is a generalization of the map content.

\subsubsection{Display and output}

The result of three enhancements can quickly be displayed by means of the RGB-channels. Overlaps between classes can then be discovered. In order to have all classes in the map, the images have to be plotted by means of colours. The sequence of plotting should follow the rule that 'hard' objects (buildings, roads, walls) should be plotted at the end. Overlaps with the 'soft' classes (vegetation) are then repressed.

\subsection{Generation of level 2}

The 'level 2' approach uses results of 'level 1'and improves the lines of man-made objects. Each object has to be processed individually. It is extracted from the connected component image using its label. The point cloud of each boundary line has then to be separated from the other point clouds. The parameters of each line can now be calculated by least squares adjustment. The next step is the generation of orthogonal and parallel lines. Corner points are then calculated by intersecting successive lines. The polygons forming buildings, car ports, walls, etc. have also to be closed. The suggested approach is depicted in Figure 2 for the

class "building". It will be explained in more detail in the following.

\subsubsection{Extraction of point clouds belonging to lines}

The boundaries of man-made objects consist of several lines. The boundaries are approximated by straight lines. Parallel and orthogonal lines exist at buildings, walls, car ports, roads, etc.

The first step is the extraction of the point clouds forming the boundary lines. The separation of lines can be done by means of the Hough transform, which uses a voting mechanism. Each point of the point cloud votes for several combinations of parameters. The parameters that receive a majority of votes are the winners (Jain et al., 1995). The lines are modelled by

$$
\rho=x \cdot \cos \theta+y \cdot \sin \theta
$$

where $\rho=$ distance from the origin and $\theta=$ azimuth of the normal vector to the line; $x, y$ are constants in the parameter space $\mathrm{H}(\theta$, $\rho)$.
All points of the point cloud of a building boundary are mapped in the parameter space using combinations of $\theta$ and $\rho$. The cells of the parameter space are used as an accumulator which is incremented by 1 when a point satisfies the equation (3).

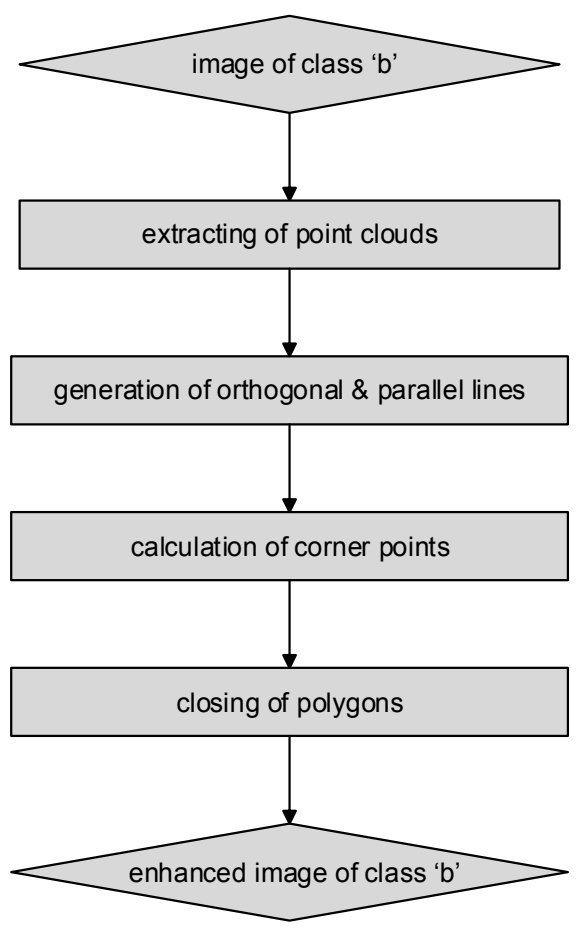

Figure 2. Steps in the cartographic enhancement (level 2) at the example of class "building"

The highest values in the accumulator array $(\mathrm{H})$ correspond to the boundary lines (cf. Figure 3 ). The parameters are analysed in order to decide which point clouds have to be extracted so that all lines of the building can be modelled.

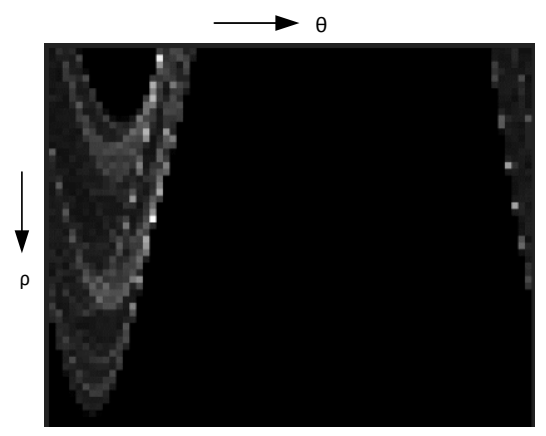

Figure 3. Display of the parameter space $(\mathrm{H})$ of the Hough transform. The pixels with the highest intensities have the parameters $(\theta, \rho)$ of the building's boundary lines.

6.2.2 Generation of orthogonal and parallel lines The extracted point clouds of building outlines are modelled by

$$
a_{i} \cdot x+y+c_{i}=0
$$

and coefficients $\left(a_{i}\right.$ and $c_{i}$ ) are now determined more accurately. We will use a building with four corners as an example (cf. Figure 4). Preliminary coordinates of the corner points $\left(x_{0}, y_{0}\right)$ are obtained by intersection of two consecutive lines $\left(l_{l}\right.$ and $\left.l_{2}\right)$. 


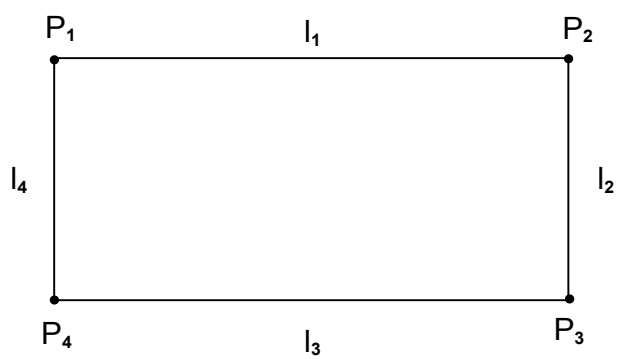

Figure 4. Sketch of building outlines and corner points

$$
\begin{aligned}
& x_{0}=\frac{c_{2}-c_{1}}{a_{1}-a_{2}} \\
& y_{0}=\frac{c_{1} \cdot a_{2}-c_{2} \cdot a_{1}}{a_{1}-a_{2}}
\end{aligned}
$$

The final coordinates of the corner points $\left(\mathrm{P}_{\mathrm{i}}\right)$ are derived in two steps. First, the slope value (a) is found by a weighted average

$$
a=\frac{\sum_{i=1}^{n} w_{i} \cdot a_{i}}{\sum_{i=1}^{n} w_{i}}
$$

where $n$ is the number of lines and $w_{i}$ is a weight. The weight is the number of extracted points for one line which about corresponds to the length of the line. The slope of the lines orthogonal to the main direction of the building are given by

$$
a_{\text {orthogonaF }}-\frac{1}{a}
$$

Such values have to be converted before the averaging. The second step calculates the c-values of equations (5) and (6) by least squares adjustment. These equations are linear and the adjustment is, therefore, very simple. The unknowns (vector $\boldsymbol{x}$ ) are then obtained by least squares adjustment using

$$
A x=b+r
$$

The matrix $(\boldsymbol{A})$ and the vectors $(\boldsymbol{x}, \boldsymbol{b}, \boldsymbol{r})$ have the following designations:

$$
\left(\begin{array}{cccc}
-k_{2} & +k_{2} & 0 & 0 \\
-k_{1} & -k_{3} & 0 & 0 \\
0 & +k_{2} & -k_{2} & 0 \\
0 & -k_{3} & -k_{1} & 0 \\
0 & 0 & -k_{2} & +k_{2} \\
0 & 0 & -k_{1} & -k_{3} \\
-k_{2} & 0 & 0 & +k_{2} \\
-k_{1} & 0 & 0 & -k_{3}
\end{array}\right)\left(\begin{array}{l}
c_{1} \\
c_{2} \\
c_{3} \\
c_{4}
\end{array}\right)=\left(\begin{array}{l}
x_{2} \\
y_{2} \\
x_{3} \\
y_{3} \\
x_{4} \\
y_{4} \\
x_{1} \\
y_{1}
\end{array}\right)+\left(\begin{array}{l}
r_{x 2} \\
r_{y 2} \\
r_{x 3} \\
r_{y 3} \\
r_{x 4} \\
r_{y 4} \\
r_{x 1} \\
r_{y 1}
\end{array}\right)
$$

The matrix elements $k_{1}, k_{2}$, and $k_{3}$ are calculated after

$$
k_{1}=\frac{1}{1+a^{2}}
$$

$$
\begin{aligned}
& k_{2}=\frac{a}{1+a^{2}} \\
& k_{3}=\frac{a^{2}}{1+a^{2}}
\end{aligned}
$$

If there are more than four lines in the building the matrix and the vectors are extended after the same pattern. The unknown $\mathrm{c}_{\mathrm{i}}$ values are found by

$$
\hat{\boldsymbol{x}}=\left(\boldsymbol{A}^{T} \boldsymbol{A}\right)^{-1} \boldsymbol{A}^{T} \boldsymbol{b}
$$

6.2.3 Calculation of corner points

The adjusted coordinates of the corner points are calculated by

$$
\boldsymbol{p}=\hat{\boldsymbol{x}}
$$

Equation 13 can be extended by a weight matrix

$$
\boldsymbol{W}=\operatorname{diag}\left(w_{1}, w_{2}, \ldots w_{n}\right)
$$

and the unknowns $\left(\mathrm{c}_{\mathrm{i}}\right)$ are then derived by

$$
\hat{\boldsymbol{x}}=\left(\boldsymbol{A}^{T} \boldsymbol{W} \boldsymbol{A}\right)^{-1} \boldsymbol{A}^{T} \boldsymbol{W b}
$$

The adjustment by a least squares procedure can also derive accuracy values. The estimated residuals are obtained by

$$
\hat{\boldsymbol{r}}=\boldsymbol{p}-\boldsymbol{b}
$$

from which the variance factor and the covariance matrix for the corner coordinates are derived by

$$
\begin{aligned}
& \hat{\sigma}_{0}^{2}=\frac{\hat{\boldsymbol{r}}^{T} \boldsymbol{W} \hat{\boldsymbol{r}}}{n-u} \\
& \Sigma_{p}=\hat{\sigma}_{0}^{2} \boldsymbol{A}\left(\boldsymbol{A}^{T} \boldsymbol{W} \boldsymbol{A}\right)^{-1} \boldsymbol{A}^{T}
\end{aligned}
$$

The accuracy of the corner coordinates by means of the covariance matrix is an interior accuracy only. For the assessment of the exterior accuracy we need accurate reference values.

6.2.4 Closing of polygons

The polygons have to be closed. It is achieved by repeating the first point in the list to be used in plotting.

\section{ASSESSMENT OF THE THEMATIC AND GEOMETRIC ACCURACY}

The assessment of the accuracies has to be carried out separately for the results of the classification, the enhanced map of level 1, and for the geometric accuracy of the enhanced map of level 2.

\subsection{Assessment of the classification}

The applied accuracy measures are error matrix, and overall user's and producer's accuracy. The formulas and definitions are given in (Congalton and Green, 2009). 


\subsection{Assessment of the enhanced maps}

The assessment of the thematic accuracy by the mentioned measures can also be carried out for the enhanced map of level 1. The assessment of the level 2 results may use an accuracy measure that is based on objects. The number of objects in the scene are then compared with the detected and mapped ones.

\subsection{Assessment of the geometric accuracy}

The assessment of the geometric accuracy is carried out by means of the corner point coordinates. They are well-defined at a DSM-based orthoimage The accuracy measures, Root Mean Square Error (RMSE) and Mean ( $\mu$ ), are calculated for each of the buildings. The Mean is the average displacement of the enhanced map with regard to the reference. The comparison of the two data sets requires that an equal number of corner points exists. In reality this may not always be the case. In (Avbelj et al., 2015) a metric is proposed that evaluates the differences between polygons and line segments. This so-called 'PoLIS' method calculates orthogonal distances of vertices to line segments. We prefer the RMSE/ $\mu$ as measures because of simplicity and because they are used as a standard in topographic mapping.

\section{EXAMPLES OF CARTOGRAPHIC ENHANCEMENTS}

The applied data are part of the ISPRS " $2 \mathrm{D}$ semantic labelling contest" (ISPRS WG III/4, 2014). The selected test site is a city area in Germany where high buildings are close to each other. Trees, bushes and grass planes are situated between the buildings. Many cars are on roads and parking lots.

\subsection{Description of source data}

The original imagery is taken by a photogrammetric camera (Zeiss DMC). The images have four bands (RGB+NIR) and are of very high spatial resolution $(\mathrm{GSD}=0.09 \mathrm{~m})$. The exposure occurred at sunshine which resulted in long shadows beside elevated objects. A digital surface model (DSM), a normalized digital surface model (nDSM), a false-colour orthoimage (cf. Figure 5), and a reference map were derived from the images by the organizers of the test.

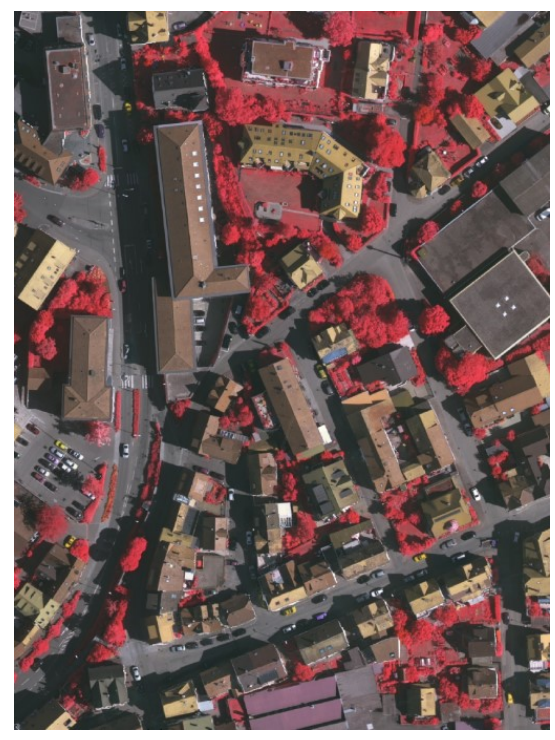

Figure 5. DSM-based ortho image (false-colour)
The reference map is manually produced by means of the DSMbased orthoimage and consists of five major urban land cover classes ("impervious surfaces", "building", "low vegetation", "tree", and "car"). The selected test site covers 4 ha.

\subsection{Classification}

The classification starts with the training of the classifier. The chosen formula in modelling of the classes uses five variables:

$$
r e f \sim n d s m+n d v i+s d \_Z \_5+b 1+s d \_b 1 \_5
$$

where $r e f=$ reference class, $n d s m=$ normalized digital surface model ( $d Z$-value), $n d v i=$ normalized difference vegetation index, $s d Z 5=$ standard deviation of the $Z$-value (elevation), $b 1=$ intensity value of the near-infrared channel (band1) of the 'true' orthoimage, $s d \_b 1 \_5=$ standard deviation of the intensities of band $1(b 1)$.

The ndsm (dZ) attribute is the 'height above ground' and is calculated by

\section{$\mathrm{dZ}=\mathrm{DSM}-\mathrm{DTM}$}

The $n d v i$ is derived from the intensities in the NIR-band and the Red-band according to equation (22).

$$
n d v i=\left(I \_N I R-I \_R\right) /\left(I \_N I R+I \_R\right)
$$

where $I N I R=$ intensity in the NIR-band, $I R=$ intensity in the R-band. The units of $\mathrm{dZ}$ are meters $(\mathrm{m})$ and of $I_{-} N I R$ and $I_{-} R$ digital numbers $(\mathrm{DN})$ in the range $0-255$.

The calculation of the standard deviations of the Z-values $(s d Z 5)$ and of the infra-red band ( $s d b 1$ 5) used the surrounding of $5 \times 5$ pixels of the digital elevation model and of the spectral band 1 respectively. The Z-values are not used as attributes due to the relatively big height differences of $\Delta \mathrm{Z}=38$ $m$ in the area of the test site.

The decision tree (cf. Figure 6) is trained by means of an adjacent map comprising $2995 \times 1783$ pixels (or 4.3 ha) which contains all six classes. The class "clutter/background" consists mainly of water (river) in this area.

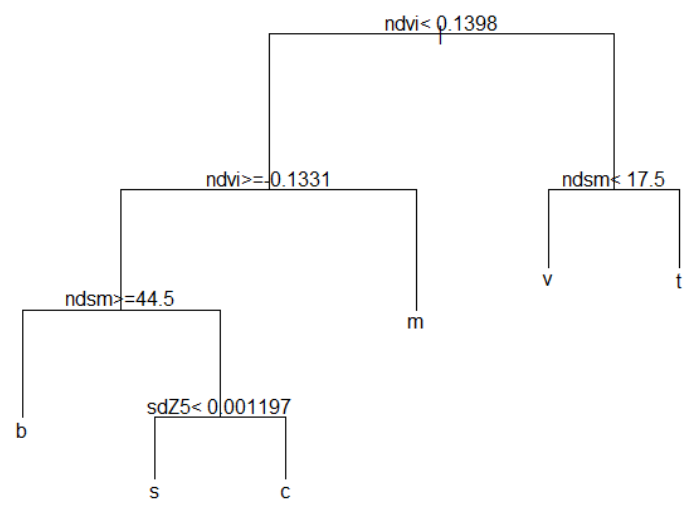

Figure 6. Decision tree derived from an existing land cover map. ( $b=$ "building", $s=$ "impervious surfaces", $\mathrm{c}=$ "car", $\quad \mathrm{m}=$ "clutter/background", $\quad \mathrm{v}=$ ="low vegetation", $\mathrm{t}=$ "tree"; $\mathrm{sdZ} 5=$ standard deviation of elevations in the $5 \times 5$ pixels surroundings [m], ndsm=normalized digital surface model [DN], ndvi=normalized difference vegetation index. 


\subsection{Results}

The derived accuracy measures are contained in Table 1. The user's accuracy reveals that the classes "impervious surfaces" and "building" are above $80 \%$. The classes 'low vegetation' and "trees" are less accurate (59\% and 74\% respectively). The class "car" is $6 \%$ and class "clutter/background" $0 \%$ only. It means that these two classes could not be determined at all.

\begin{tabular}{|c|c|c|}
\hline class & $\begin{array}{c}\text { uacc } \\
{[\%]}\end{array}$ & $\begin{array}{c}\text { pacc } \\
{[\%]}\end{array}$ \\
\hline imp_surf & 84 & 52 \\
\hline building & 88 & 78 \\
\hline low_veg & 59 & 57 \\
\hline tree & 74 & 65 \\
\hline car & 6 & 84 \\
\hline clutter & 0 & 0 \\
\hline
\end{tabular}

Table 1. User's accuracy (uacc) and producer's accuracy (pacc) of the test site

The overall accuracy is calculated with $64.3 \%$ (95\% CI: $64.3 \%$ $64.3 \%$ ). The calculated confidence intervals (CIs) are very narrow due to the big number of checkpoints (4.93 million points). The CI-values for the user's and producer's accuracy are therefore not given.

In order to evaluate the achieved accuracy a comparison with the results of the training area are calculated (cf. Table 2).

\begin{tabular}{|c|c|c|}
\hline class & $\begin{array}{c}\text { uacc } \\
{[\%]}\end{array}$ & $\begin{array}{c}\text { pacc } \\
{[\%]}\end{array}$ \\
\hline imp_surf & 79 & 57 \\
\hline building & 90 & 73 \\
\hline low_veg & 64 & 55 \\
\hline tree & 87 & 87 \\
\hline car & 8 & 65 \\
\hline clutter & 67 & 82 \\
\hline
\end{tabular}

Table 2. User's accuracy (uacc) and producer's accuracy (pacc) of training area.

The user's accuracy for the class "car" is also poor $(8 \%)$, the class "clutter/background", however, $67 \%$. The overall accuracy is $69.9 \%$. It should be mentioned that the class "car" and "clutter/background" are not topographic objects and have, therefore, been removed in the cartographic enhancement.

\subsection{Cartographic enhancement}

The result of the cartographic enhancement (level 1) is depicted in Figure 7. It is carried out after the proposed procedures described in Section 6. For the generation of level 1 quality, the program package "EBImage" was applied (Pau, 2013). The morphological operations used a structuring element of $5 \times 5$ pixels. When generating the outlines of buildings, the selected parameters were $2 \times 2$ pixels (size of the moving window) and 0.01 (thresholding offset from the averaged value). The minimum area of a building to be mapped was assumed to be 25 $\mathrm{m}^{2}$ and for the areas of class "low vegetation" $21 \mathrm{~m}^{2}$. The areas of class "impervious surface" used as threshold a radius of $2 \mathrm{~m}$ and the areas of class "tree" a radius of $4 \mathrm{~m}$. All objects smaller than these thresholds were removed. This generalization produces some areas of no data which have to be filled again. In this way the cartographic quality can be improved.

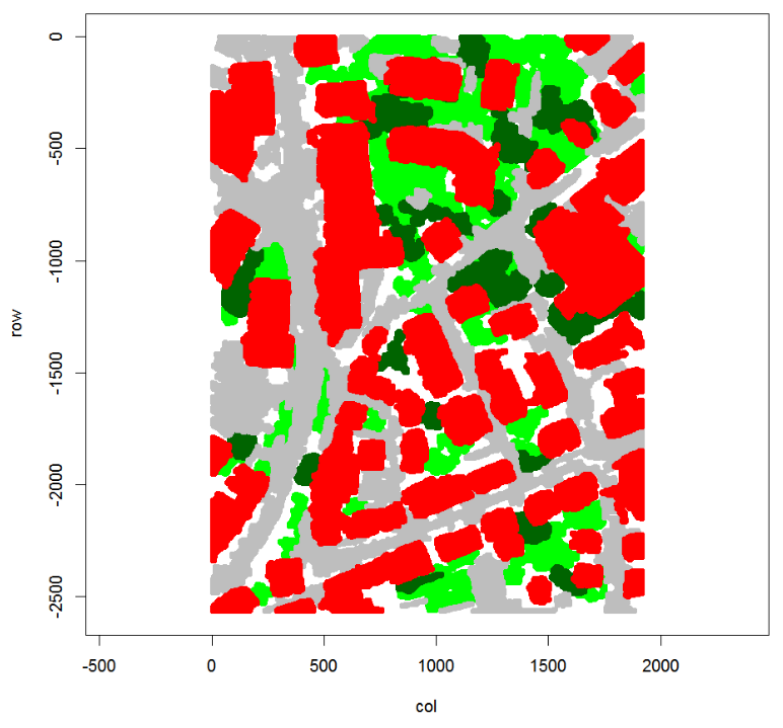

Figure 7. Enhanced land cover map - level 1. (red="building", dark green="tree", green= "low vegetation", gray="impervious surface", white= "no data")

\subsection{Thematic accuracy of the enhanced map}

The thematic accuracy of the topographic objects is contained in Table 3. The use of other attributes and/or thresholds may improve the results. The process of enhancement can be automated as well.

\begin{tabular}{|c|c|c|}
\hline class & $\begin{array}{c}\text { uacc } \\
{[\%]}\end{array}$ & $\begin{array}{c}\text { pacc } \\
{[\%]}\end{array}$ \\
\hline imp_surf & 88 & 52 \\
\hline building & 86 & 82 \\
\hline low_veg & 57 & 58 \\
\hline tree & 28 & 28 \\
\hline
\end{tabular}

Table 3. User's accuracy (uacc) and producer's accuracy (pacc) of enhanced land cover map (level 1)

\subsection{Geometric accuracy}

The coordinate errors calculated from equation (19) are very small $(1.0$ pixel or $0.09 \mathrm{~m})$. This is an interior accuracy only. The root mean square errors, derived from reference values, are absolute errors (cf. Table 4). The averages of all RMSE_x and RMSE y are $1.2 \mathrm{~m}$ and $0.7 \mathrm{~m}$ respectively when the manually derived map (GT) was used as reference. Reference values were also derived by digitizing the corner points of buildings on top of the DSM-based orthoimage. The results are about the same. Altogether 31 corner points have been checked. The averages of the standard deviations $\left(\sigma_{\mathrm{x}}, \sigma_{\mathrm{y}}\right)$ are about the same as the RMSE values. This indicates that the systematic shifts $\left(\mu_{\mathrm{x}}, \mu_{\mathrm{y}}\right)$ of the coordinates with regard to the reference are very small. The results may be improved when a $2 \mathrm{D}$ transformation is applied. Besides the shifts a rotation and scale factors will then be corrected too. Building 41 includes a side the slope of which is close to $90^{\circ}$. In such a case, the line parameters $\theta$ and $\rho$ are calculated. This requires a linearization of equation (3) and iterations when the approximate values of the parameters are not very accurate. Furthermore, the calculation of the corner points will then be based on equation (3) as well. 


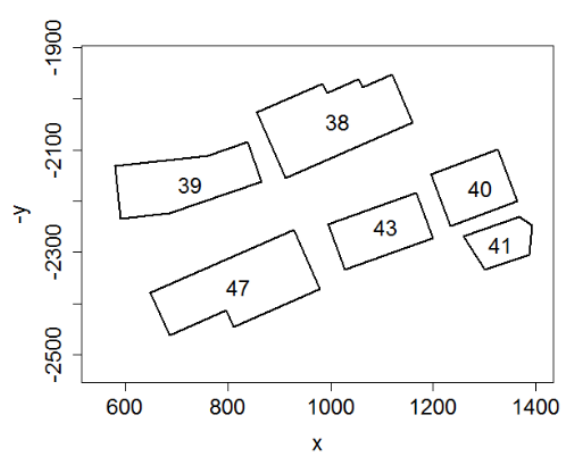

Figure 10. Enhancement of class 'building', level 2

\begin{tabular}{|c|c|c|c|c|c|}
\hline \multirow{2}{*}{$\begin{array}{c}\text { \# of } \\
\text { building }\end{array}$} & \multirow{2}{*}{ of } & \multicolumn{2}{|c|}{$\mathrm{GT}[\mathrm{m}]$} & \multicolumn{2}{c|}{ Ortho [m] } \\
\cline { 3 - 6 } & corners & RMSE $_{\mathrm{x}}$ & RMSE $_{\mathrm{y}}$ & RMSE $_{\mathrm{x}}$ & RMSE $_{\mathrm{y}}$ \\
\hline 38 & 8 & 1.1 & 0.8 & 1.0 & 0.7 \\
\hline 39 & 4 & 1.1 & 0.4 & 1.2 & 0.5 \\
\hline 40 & 4 & 1.2 & 0.5 & 1.1 & 0.4 \\
\hline 41 & 5 & 1.6 & 0.8 & 1.6 & 0.9 \\
\hline 43 & 4 & 0.9 & 0.7 & 1.0 & 0.6 \\
\hline 47 & 6 & 1.3 & 0.8 & 0.9 & 0.6 \\
\hline average & $\mathbf{5 . 2}$ & $\mathbf{1 . 2}$ & $\mathbf{0 . 7}$ & $\mathbf{1 . 1}$ & $\mathbf{0 . 6}$ \\
\hline
\end{tabular}

Table 5. Geometric accuracy of the enhanced map (level 2).

\section{DISCUSSION AND CONCLUSION}

The applied method used pixels of the orthoimages as units. The thematic map used for training contained six classes but was not identical with the area to be classified. The distribution of the areas of the produced land cover map was therefore different from the distribution in the training area. The obtained user's accuracy is pretty good for the classes "building" (88\%) and "impervious surface" (84\%). The detection of cars is poor with the selected approach. The quality of the input data and of the reference data is important for good results and should, therefore, be tested. For example, the accuracy of the elevations (Z) is of lower accuracy at the boundaries of buildings. These areas could have been ignored in the assessment. The obtainable accuracies would then definitely be higher. The cartographic enhancement improves the quality of the land cover map. The thematic accuracy is about the same for the classes "building", "impervious surfaces", and "low vegetation". The class "tree" is much worse due to the threshold for minimum area. The applied classifier (DT) could easily handle the relatively large amount of data. The processing and plotting of all classes in map-like colours required high processing times. The geometric accuracy derived from 31 corners of buildings were RMSE_ $\mathrm{x}=1.2 \mathrm{~m}$ and RMSE_y $=0.7 \mathrm{~m}$. According to the positional accuracy standards for digital geospatial data, e.g. in USA, an accuracy of RMSE_ $x=R M S E \_y=1.0 m$ is required for map scales in the range 1: 2000 to 1:4000 (ASPRS, 2015).

\section{REFERENCES}

ASPRS, 2015. ASPRS Positional Accuracy Standards for Digital Geospatial Data, Photogramm Eng Rem S, 81, (3), pp. A1-A26.

Awrangjep, M., Ravanbakhsb, M., Fraser, C., 2010. Automatic detection of residential buildings using LIDAR data and multispectral imagery, ISPRS J Photogramm 65 (5), 457-467.
Avbelj, J., Müller, R., Bamler, R., 2015.A metric for polygon comparison and building extraction evaluation, IEEE Geosci Remote $S$, vol. 12, no. 1, 5 p.

Breiman, L., Friedman, J., Stone, C.J., Olshen, R.A., 1984. Classification and regression trees. CRC Press.

Congalton, R., G, Green, K., 2008. Assessing the accuracy of remotely sensed data. CRC Press.

El-Ashmawy, K. L. A., 2016. Testing the positional accuracy of OpenStreetMap data for mapping applications, Geodesy and Cartography, vol. 42, issue 1, pp. 25-30.

Friedl, M.A., Brodley, C.E., 1997. Decision tree classification of land cover from remotely sensed data. Remote Sens. Environ. 61, 399-409.

Gross, H., Thoennessen, U., 2006. Extraction of lines from laser point clouds, In: Symposium of ISPRS Commission III: Photogrammetric Computer Vision PCV06. International Archives of Photogrammetry, Remote Sensing and Spatial Information Sciences, pp. 86-91.

Höhle, J., 2014. Generation of 2D land cover maps for urban areas using decision tree classification, ISPRS Annals of the Photogrammetry, Remote Sensing and Spatial Information Sciences, vol. II-7, pp. 15-21.

ISPRS WG III/4, 2014.

http://www2.isprs.org/commissions/comm3/wg4/semanticlabeling.html (12 June 2016).

Jain, R., Kasturi, R., Schunck, B.G., 1995. Machine vision, McGraw-Hill, Inc., ISBN 0-07-113407-7.

Konecny, G.; Breitkopf, U.; Radtke, A.; Lee, K., 2015. The status of topographic mapping in the world - a UNGGIMISPRS project 2012-2015, final report, Leibniz Universität Hannover, 64 pp.

Li, H., Zhong, C., Hu, X., Xiao, L., Huang, X., 2013. New methodologies for precise building boundary extraction from LiDAR data and high resolution image. Sensor Rev, 33/2, pp. 157-165.

Li, Y., Zhu, L., Shimamura, K., Tachibana, K., 2012. A refining method for building object aggregation and footprint modelling using multi-source data. International Archives of the photogrammetry, remote sensing and spatial information sciences, vol. XXXIX-B3, pp.41-46.

Niemeyer, J., Rottensteiner, F., Soergel, U., 2014. Contextual classification of lidar data and building object detection in urban areas. ISPRS J Photogramm 87, pp. 152-165.

Pau, G., Sklyar, O, and W. Huber, 2013. Introduction to EBImage - an image processing and analysis toolkit for R, http://www.bioconductor.org/packages/release/bioc/html/EBIm age.html (13 June 2016)

Sampath, A., Shan, J., 2007. Building boundary tracing and regularization from airborne LiDAR point clouds. Photogramm Eng Remote S 73 (7), pp. 805-812.

\section{ACKNOWLEDGEMENT}

The author thanks the ISPRS WG III/4 for providing test data. 


\title{
AUTOMATIC BUILDING EXTRACTION FROM AIRBORNE LIDAR POINT CLOUD BASED ON MEAN SHIFT SEGMENTATION
}

\author{
Z. Hui ${ }^{\text {a*}}$, Y. Hu ${ }^{\text {a }}$ Y.Y. Ziggah ${ }^{\mathrm{a}}$ \\ a Faculty of Information Engineering, China University of Geosciences, Wuhan 430074 - huizhenyang2008@163.com
}

KEY WORDS: Airborne LiDAR, Building extraction, Mean shift, Segmentation

\begin{abstract}
:
Building extraction is an important part for smart city construction. This paper proposes a novel method for automatic building extraction from airborne LiDAR point cloud. In the present study, filtering was first applied to point cloud, which could help obtain elevated points for generating the DTM. The building-candidate points were then obtained by setting a threshold from the DTM. To distinguish the tree points from building points, three constraints, namely, area constraint, point density constraint and root mean square error constraint were applied to the building-candidate points. By comparing with the reference data generated manually, the evaluation result shows that the proposed method could yield a good performance.
\end{abstract}

\section{INTRODUCTION}

\subsection{Introduction}

The Building extraction from airborne LiDAR point cloud has been a research hotsopt for several years. Many researchers have made many contributions on this area. However, there are still some unsolved problems such as the inability of detecting the false plane generated by tree crowns, low detection accuracy, cumbersome process among others . In this paper, a novel automatic building extraction method has been proposed to resolve the above-mentioned problems.

Building extraction from airborne LiDAR point cloud always involves two main steps, namely, filtering and segmentation. Filtering is a key step which has three main effects, such as downsizing the point cloud, generating DTM and identifying elevated objects. Segmentation plays a role in clustering points with similar characteristics which will help distinguish building points from tree points.

\section{METHODS}

\subsection{Pre-processing}

Due to the multi-path errors and errors in the laser range finder, the obtained point cloud always include outliers, especially low outliers, which will have a significant effect on the following steps. Thus, these outliers should be removed firstly. This paper adopted the method proposed by Hui et al. (2016) to denoise the point cloud.

After the denosing step, the point cloud was organized as grid cells. At this step, only the lowest point in each grid cell was involved in processing. This would help to remove tree points and points on walls. Generally, buildings are composed roofs and vertical walls, so no laser beams could penetrate the building. Thus, there are no points inside the building. In contrast, the laser beams could penetrate brunches or leaves of the tree, capturing not only the tree crown but also surface underneath the tree crown. Therefore, if we select the lowest points in grid cells, most points reflected from tree crown could be removed. Moreover, since laser beams always have incidence angles, there are many points reflected from walls, which will have some effects on the following mean shift segmentation. By selecting the lowest points, these wall points could be removed.

\subsection{Filtering}

This paper adopted the filtering algorithm proposed by Hui et al. (2016) to generate the DTM. For each non-ground points, if its elevation is more than the given threshold $T_{h}$, this point will be labeled as building-candidate points. The threshold was defined as Equation (1).

$$
T_{h}=H_{g}+H_{c}
$$

where $\quad H_{g}=$ the corresponding ground height

$H_{c}=$ a height constant

\subsection{Mean shift segmentation}

The mean shift was first applied to image segmentation (Comaniciu \& Meer, 2002). Here we adopted it for segmenting the building-candidate points. The principle of mean shift is trying to get closer to a local maximum by moving along the mean shift vector as shown in Figure 1.

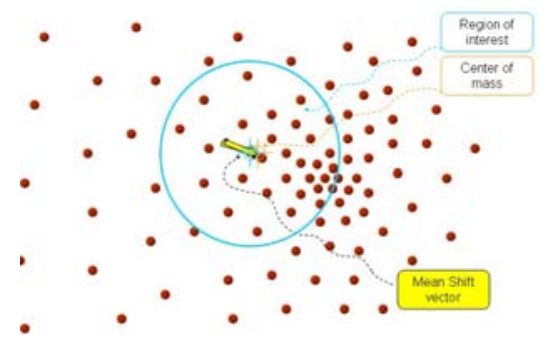

* Corresponding author 
Figure 1. Mean shift sketch map

In this paper, the mean shift vector was defined as Equation (2).

$$
m s(X)=\frac{\sum_{i=1}^{n} X_{i} K^{s}\left(\left\|\frac{X^{s}-X_{i}^{s}}{h^{s}}\right\|^{2}\right) K^{r}\left(\left\|\frac{X^{r}-X_{i}^{r}}{h^{r}}\right\|^{2}\right)}{\sum_{i=1}^{n} K^{s}\left(\left\|\frac{X^{s}-X_{i}^{s}}{h^{s}}\right\|^{2}\right) K^{r}\left(\left\|\frac{X^{r}-X_{i}^{r}}{h^{r}}\right\|^{2}\right)}-
$$

$$
\text { where } \begin{aligned}
n & =\text { the number of points } \\
K^{s} & =\text { horizontal kernel } \\
K^{r} & =\text { vertical kernel } \\
X^{s} & =\text { components of the horizontal vector } \\
X^{r} & =\text { components of the vertical vector }
\end{aligned}
$$

This paper chose uniform as the kernel function considering the rate of convergence and the sensitive of feature. The uniform kernel is defined in Equation (3) as

$$
K(X)= \begin{cases}1 & \text { if }\|X\|<1 \\ 0 & \text { if }\|X\| \geq 1\end{cases}
$$

The mean shift segmentation results are shown in Figure 2(a). It can be found that the building-candidate points were clustered into different segments, including connected components and scattered points. To obtain an accurate building extraction results, three constraints should be applied to the mean shift segments.

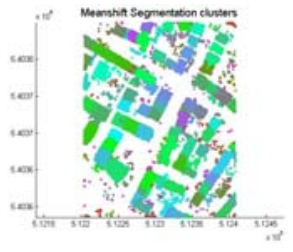

(a)

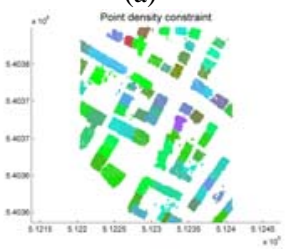

(c)

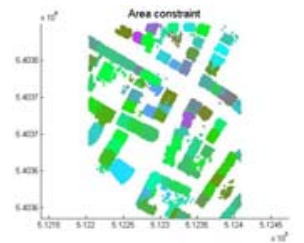

(b)

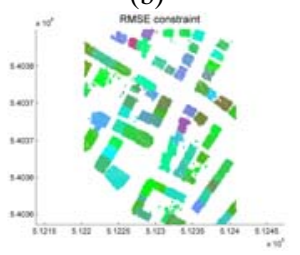

(d)
Figure 2. Result for each step

\subsection{Area, point density and RMSE constraints}

Since buildings are always connected components, their areas are not less than a given threshold. After applying the area constraint, a "clean" result was obtained as shown in Figure 2(b). However, there are still some scattered points, which could be some high trees. To remove these points, point density constraint was applied to the result of last step. By searching the neighbors of each point, if the number of its neighbors is less than a given threshold, this point will be labeled as non-building building points. As mentioned above, some dense tree crowns could generate some false planes. To remove these false planes, root mean square error (RMSE) constraints was applied to the point density constraint result. If RMSE of the segment is more than a given threshold, this segment will be removed. The final result is shown in Figure 2(d).

\section{EVALUATION}

The proposed method was evaluated by comparing the extracted results with the manually corrected results according to the object-based evaluation techniques proposed by Rutzinger et al. (2009). Three indicators defined in Equation (4) were utilized.

$$
\begin{aligned}
& \text { Completeness }=\frac{T P}{T P+F N} \\
& \text { Correctness }=\frac{T P}{T P+F P} \\
& \text { Quality }=\frac{T P}{T P+F P+F N}
\end{aligned}
$$

The evaluated results are shown in Table 1.

\begin{tabular}{|c|c|c|}
\hline Completeness & Correctness & Quality \\
\hline $93 \%$ & $95 \%$ & $91 \%$ \\
\hline
\end{tabular}

Table 1. Margin settings for A4 size paper

\section{CONCLUSION}

On the basis of the results obtained (Figure 2), a conclusion could be drawn that the proposed method can detect buildings effectively and yield a good performance even though there are many dense tree crowns surrounding the buildings.

\section{REFERENCES}

Comaniciu, D., 2002. Mean shift: A robust approach toward feature space analysis. IEEE transactions on Pattern Analysis and Machine Intelligence, 24, pp. 603-619.

Hui, Z., 2016. An Improved Morphological Algorithm for Filtering Airborne LiDAR Point Cloud Based on Multi-Level Kriging Interpolation, Remote Sensing, 8(1), pp. 35.

Rutzinger, M., 2009. A comparisonof evaluation techniques for building extraction fromairborne laser scanning. IEEE Journal of Selected Topics inApplied Earth Observations and Remote Sensing, 2(1), pp. 11-20. 


\title{
AERIAL IMAGE BASED GEOMETRIC REFINEMENT OF BUILDING MODELS DERIVED FROM AIRBORNE LIDAR DATA
}

\author{
M. Jarząbek-Rychard ${ }^{\mathrm{a}, \mathrm{b}} *$, H-G. Maas ${ }^{\mathrm{b}}$ \\ ${ }^{a}$ Institute of Geodesy and Geoinformatics, Wroclaw University of Environmental and Life Science, Poland - \\ malgorzata.jarzabek-rychard@igig.up.wroc.pl \\ ${ }^{\mathrm{b}}$ Institute of Photogrammetry and Remote Sensing, Technische Universität Dresden, Germany - hans-gerd.maas@tu-dresden.de
}

KEY WORDS: building reconstruction, 3D modeling, laser scanning, aerial imagery, edge matching

\begin{abstract}
:
Airborne laser scanning has proven to be a strong basis for the automatic generation of 3D building models. A drawback, however, is often in the point spacing of typical datasets. As a consequence, the precision of roof plane and ridge line parameters is usually significantly better than the precision of gutter lines. To cope with problem the paper presents a novel approach for geometric refinement of building models reconstructed from ALS point clouds using single aerial imagery. The basis idea of our modeling approach it to obtain refined roof corners by direct intersection of 3D roof planes previously extracted from ALS data with viewing planes assigned with the edges detected in high resolution digital photographs. The synergy between LiDAR and optical imagery allows for obtaining building models with high vertical and plannimetric accuracy. In order to evaluate performance of our refinement algorithm, we compare the results of 3D reconstruction executed using only laser scanning data and enhanced by image information. Furthermore, quality assessment of both modeling outputs is performed based on a reference data provided by the ISPRS benchmark for 3D building reconstruction. Integration of linear cues retrieved from single imagery allows for average improvement of planar accuracy of $9 \mathrm{~cm}$ (RMS error for roof plane outlines). The overall quality of final building models calculated on a per-area level reaches nearly $90 \%$.
\end{abstract}

\section{INTRODUCTION}

Accurate and timely updated 3D building models has been considered a critical element of urban scene reconstruction. Virtual models serve as an important information source to support various domains such as urban planning, disaster management, navigation and tourism. Permanently increasing spectrum of applications urgently demands advanced methods for efficient and highly automatized reconstruction algorithm providing up-to-date products. Despite worldwide intensive efforts to improve the modeling process, reconstruction of highly accurate building models still remains as a challenging task (Rottensteiner et al., 2014)

Due to the needs for efficient modeling covering large areas the base information for building extraction mainly comes from airborne data: laser scanning and imagery. For the past two decades numerous research papers concerning building reconstruction were published, which used LiDAR data (e.g. Oude Elberink and Vosselman, 2009; Kim and Shan, 2011; Xiong et al., 2015) or image information (Nex and Remondino, 2012; Bulatov et al., 2014). Airborne laser scanning has proven to be a strong basis for the automatic generation of 3D building models. Vertical accuracy of the reconstructed rooftops is adequate even for highly demanding engineering applications. Unfortunately, building models derived from ALS point clouds are restricted by the ground resolution of datasets. Hence it is difficult to achieve high planimetric accuracy of a reconstructed scene. Compared to laser scanning, optical imagery with its higher spatial resolution usually allows for a more accurate extraction of building edges, accompanied by a higher precision of reconstructed 3D elements. Largely complementary nature of LiDAR and image data forms a basis for an efficient combination of these two techniques. Integration of laser scanning and imagery for 3D reconstruction can be performed in two ways, using parallel or sequential approach (Sohn et al., 2013). In case of the former one, each modeling cues is extracted from two datasets at the same time (Chen et al., 2005; Habib et al., 2011; Demir and Baltasavias, 2012; Zhang et al., 2014). In the sequential fusion approach, building models are generated based on a single information source and later refined by the other data (Perera, 2014; Dal Poz, 2014). The latter method may also depict an opportunity for an effective update of virtual cities already reconstructed from LiDAR. In these terms, existing 3D models can serve as an input for improving their accuracy by newly collected information.

This paper presents a novel approach for sequential refinement of 3D building models using a single aerial image. The core idea of modeling improvement it to obtain refined model edges by intersecting roof planes accurately extracted from 3D point clouds and viewing planes assigned with building edges detected in a high resolution aerial image. Although 3D models reconstructed from LiDAR show deficits in their planimetric accuracy, they serve as a good input information for structural arrangements of roofs and convergence priors. In order to improve the geometric accuracy of roof plane outlines, 3D roof edges are projected into image space and substituted by the best matching linear segments extracted from the image. The chosen lines are projected back to 3D space and intersected with relevant planes previously detected from ALS data. The underlying methodology so far assumes that the input models are topologically correct. The research aim is then to increase the geometric accuracy of reconstructed roofs. In order to evaluate performance of our refinement algorithm, we compare the results of $3 \mathrm{D}$ reconstruction performed only using ALS data

* Corresponding author, 
and enhanced by information retrieved from an aerial image. Furthermore, quality assessment of both modeling outputs is performed based on a comparison to the reference data, according to the validation methods standardized by the International Society for Photogrammetry and Remote Sensing (Rutzinger et al., 2009).

\section{REFINEMENT METHODOLOGY}

\subsection{Projection of a 3D model to the image space}

3D building models previously reconstructed from an ALS point cloud provide structural arrangements of roofs and convergence priors. Initial models subjected to the refinement are reconstructed according to the approach described in Jarząbek-Rychard and Borkowski, 2016) and stored as a list of $x, y, z$ coordinates of roof vertices and their topological relations (connecting edges). The first step of the implemented algorithm is to project an input wireframe model into image space. The projection is performed through the collinearity equations, along with known exterior and interior orientation parameters. First, object space points (3D roof vertices) are transformed into the camera coordinate system. In the second step, the internal camera model and the interior orientation parameters are used to transform camera points into the image space. Finally, projected 2D points are connected according to the topology information provided by input models. As a result (c.f. Fig.1a), initial 3D building boundaries are transformed into planar lines and thus, integrated in the image.

\subsection{Linear feature extraction}

To automatically extract straight line segments from aerial photographs we use the Canny edge detector followed by connected component analyses and Hough transform. The result of Canny operator (presented in Fig.1b) is a binary image with marked edge pixels associated to object boundaries. Straight line segments and related line equations are computed by finding peaks in the Hough space of the binary image. According to the assumption underlying Hough transform, the pixels lying on one line need not all be contiguous. This fact can give misleading results when different objects happen to be aligned by chance. Thus, in order to reduce the searching space for potential pixels belonging to the estimated line, we use connected component labelling (c.f. Fig.1c). The labelling is applied to the boundary image provided by the Canny edge detector. Hough transform is then repeated for each binary image assigned to one connected components (detected straight lines are shown in Fig.1d). Besides building edges, extracted information is also related to other, irrelevant objects such as vegetation, fences, or roof patterns. On the other hand, some desired roof boundaries may not be derived due to shadow areas and occlusions. Hence, all the extracted linear features are treated as sharp boundary information and serve as an input for correspondence matching, performed in the next step.

\subsection{Similarity measure and line matching}

Similarity measures are defined to find correspondences between projected lines of a 3D model and new edges extracted from the image. The aim of line matching is to substitute each building edge initially reconstructed from ALS by the best candidate chosen from the line segments extracted by image processing. At this stage of refinement procedure both edge sets, reference and candidate, follow the same pixel coordinate system. Hence, it is possible to compute mutual geometric relationships and find corresponding pairs of boundary lines. The matching algorithm involves the following criteria to choose the correct image line for each reference edge:

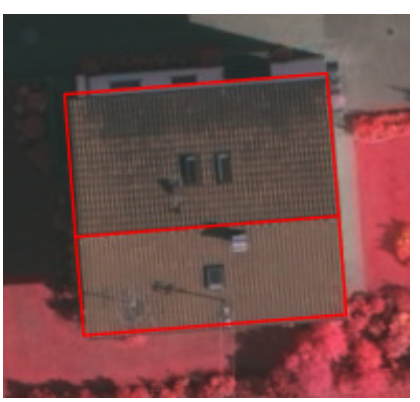

(a)

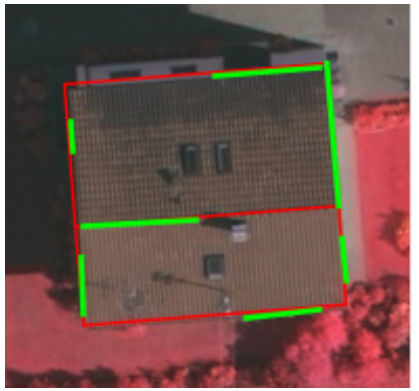

(e)

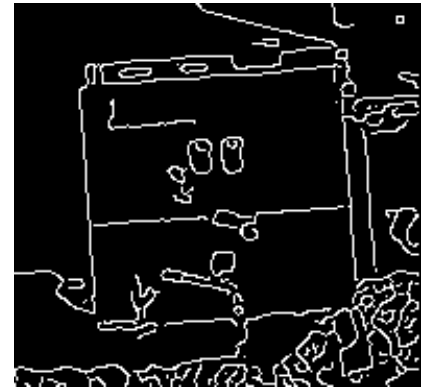

(b)

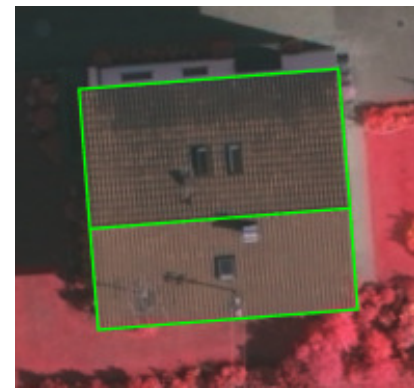

(f)

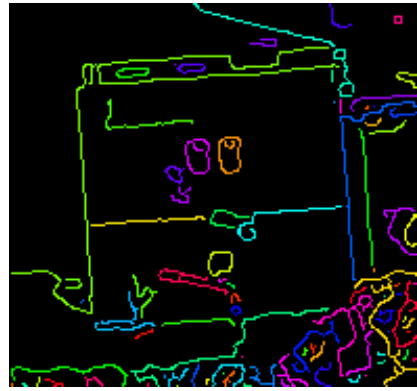

(c)

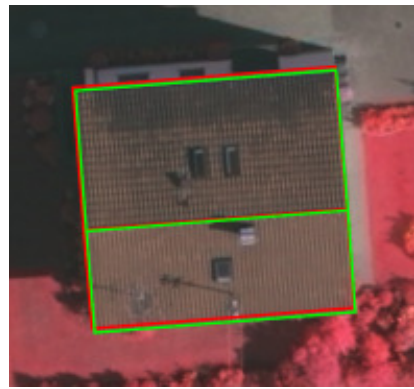

(g)

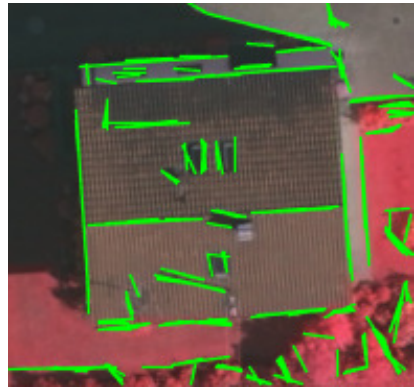

(d)

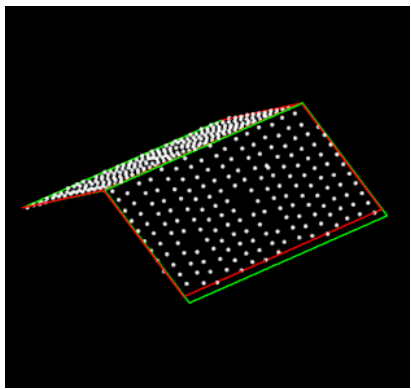

(h)

Figure 1. Refinement process: input wireframe model projected into image space (a), extracted boundary image (b), connected component labelling (c), straight lines detected by Hough transform (d), correspondence matching; if no new line is matched then the input line is preserved (e), refined wireframe model projected into the image (f), comparison of building models before (red) and after (green) refinement - image space (g), comparison of building models before and after refinement - 3D space (h). 
- proximity of both lines defined by the distances from both end-points of a candidate segment to a reference line segment,

- similar orientation defined by the angle between reference and candidate line,

If there is more than one line fulfilling the two requirements above, the closest line is chosen.

Several factors, such as shadows along the desired edge, poor contrast, occlusions, or erroneous references can hinder proper extraction of all relevant building edges. Hence, it is not always possible to find a corresponding line for each reference edge. In this case, the $2 \mathrm{D}$ equation of an initial edge is used in further processing. Figure 1e presents ALS-based building edges and their best matching lines detected from the image. Newly extracted roof edges are rectified and aligned perpendicular or parallel to the main orientation of a building. Because orientation of a ridge line derived from ALS is expected to reach high accuracy, it determines the dominant building direction. For building types that do not include ridge lines (e.g. flat roofs) detected lines are regularly aligned with respect to the longest matched line detected in the image.

\subsection{Reconstruction of a refined 3D model}

A refined 3D building model is generated by the intersection of roof planes extracted from ALS data with viewing planes assigned with newly detected edges. In order to reconstruct viewing planes, viewing ray vectors are generated for both end points of a $2 \mathrm{D}$ line. A viewing plane is then created based on the plane normal derived by the cross product of viewing ray vectors and coordinates of the projection center. Intersection of neighboring viewing planes and $3 \mathrm{D}$ planes of a roof enables to obtain refined 3D coordinates of roof corners. The neighboring planes are identified according to the topology information stored in the input building models. In the final step of building reconstruction $3 \mathrm{D}$ models are subjected to the regularization. As a result, right angles of boundary edges are preserved and 3D lines assigned to ridges and gutters are aligned horizontally. The refined building model projected back to the image space is shown in Fig.1f. Finally, wireframe models constructed before and after refinement are compared in the image space (c.f. Fig.1g) and 3D space (c.f. Fig.1h).

\section{RESULTS AND DISCUSSION}

To verify the performance of the presented approach we used data provided by ISPRS WGIII/4 (Rottensteiner et al., 2014). The dataset (area3 - Vaihingen) presents a purely residential area with 56 detached houses. The ALS point cloud was collected by Leica ALS50 system with a density of 4 points / $\mathrm{m}^{2}$. The aerial images were acquired using an Intergraph / ZI digital mapping camera with a ground sampling distance of 8 $\mathrm{cm}$ and a radiometric resolution of 12 bits. The interior and exterior parameters were determined in the level of one pixel georeferencing accuracy. The refinement method was applied to a set of 3D building models generated on LoD2 (JarząbekRychard and Borkowski, 2016). An overview on the results of our experiment is presented in Fig.1. The both sets of 3D building models, reconstructed from ALS data only and enhanced by image information, are transformed into the image 2D space and compared.

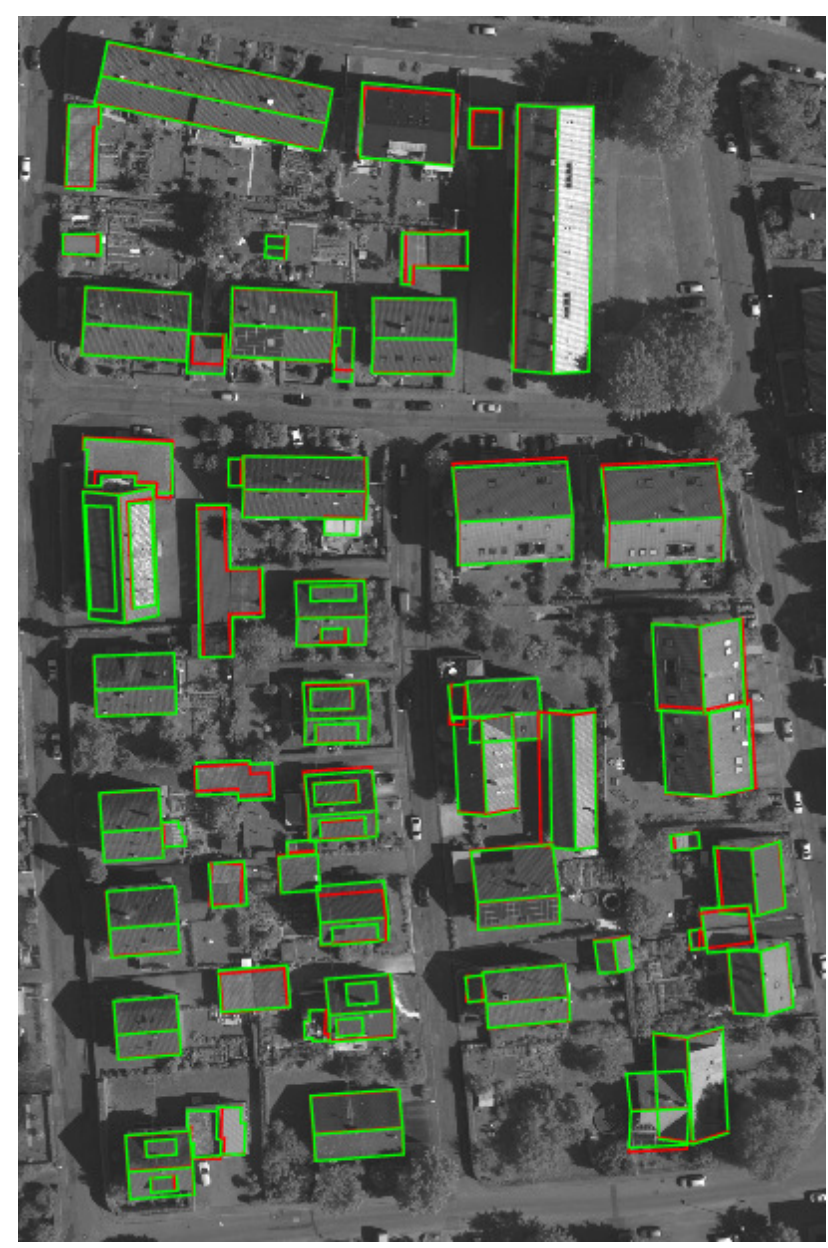

Figure 1. Reconstructed 3D models projected into the image: roof edges generated from ALS point clouds (red) and refined using single aerial imagery (green) (for better visibility in this figure original infrared image is transformed into gray scale).

\begin{tabular}{|l|c|c|}
\hline Edge type & \# edges & mean shift [cm] \\
\hline ridge & 34 & 5,5 \\
gutter & 68 & 17,9 \\
eave & 136 & 13,1 \\
dormer & 56 & 21,1 \\
flat & 94 & 43,3 \\
height jump & 10 & 54,4 \\
adjacent flat & 30 & 12,2 \\
\hline
\end{tabular}

Table 1. Quantitative analysis of the refinement performance: mean planar displacement of building edges.

To get a clear idea of the influence of the applied refinement procedure an average planar displacement of different types of roof edges is calculated. For that reason we computed perpendicular distance between each reference ALS edge line and the end-points of a corresponding line extracted from the image. Table 1 shows quantitative analysis of the resulting changes presented with respect to the edge type. The statistics are illustrated in Fig. 2. 


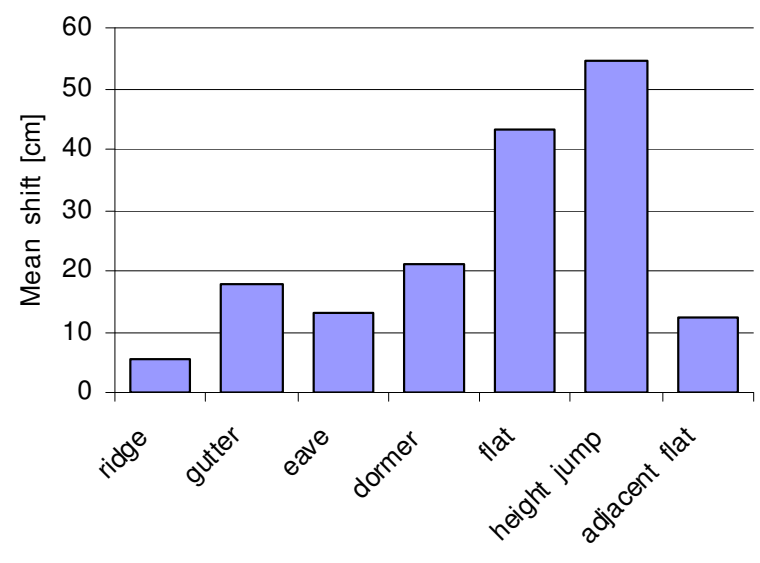

Edge type

Figure 2. Mean planar displacement of building edges presented with respect to the edge type.

The largest displacement $(54 \mathrm{~cm})$ is shown for height jump edges. Such situations result from a limitation of the applied building reconstruction method, in which a gap may arise between a single roof and its adjacent multi roof plane building. The integrated image information allows for a compensation of this effect by shifting the lower height jump edge to the planar position of the upper one. The second biggest change of planar position $(43 \mathrm{~cm})$ is observed for the outlines of flat roofs, which are sensitive to ALS point spacing and directional arrangements of scanning lines. Interestingly, small roofs adjacent to complex buildings, which are also usually flat-type, show much smaller displacement $(12 \mathrm{~cm})$. Such roofs are usually significantly smaller and often affected by shadows. Thus, it is difficult to extract a correct matching line from the image. As expected, the smallest change after applied refinement (about $5 \mathrm{~cm}$ shift) is noted for ridge lines, which are precisely determined from ALS data by an intersection of 3D planes.

\begin{tabular}{|c|c|c|}
\hline Input data & ALS & ALS \& image \\
\hline RMS (reference boundaries) [cm] & 73 & 64 \\
\hline Evaluation on a per-area level: & & \\
\hline True Positives $\left[\mathrm{m}^{2}\right]$ & 7430,5 & 7522,5 \\
\hline False Positives $\left[\mathrm{m}^{2}\right]$ & 276,5 & 262,0 \\
\hline False Negatives $\left[\mathrm{m}^{2}\right]$ & 683,9 & 591,9 \\
\hline True Negatives $\left[\mathrm{m}^{2}\right]$ & 33609,1 & 33623,5 \\
\hline Completeness [\%] & 91,6 & 92,7 \\
\hline Correctness [\%] & 96,4 & 96,6 \\
\hline Quality [\%] & 88,6 & 89,8 \\
\hline
\end{tabular}

Table 2. Qualitative assessment of roof location accuracy performed with respect to the reference data. Integration of linear features extracted from aerial image improves the rate of all performance indicators.

In order to asses the quality of the reconstructed 3D models, both sets of the modeling results (before and after correction) were verified within ISPRS Test Project on Urban Classification and 3D Building Reconstruction. The benchmark allows for the evaluation of the modeling results according to unified criteria against other reconstruction methods. The statistics on qualitative analysis concerning plannimetric accuracy of roof plane outlines are collected in Tab.2. Since all the indicators are improved, it can be stated that the integration of refinement procedure certainly increases the modeling performance. The assumption of the refinement approach preserves initial roof structure of a model. Therefore, only the $3 \mathrm{D}$ position of roof corners is modified. As no new building components can appear in the refined scene, the overall quality
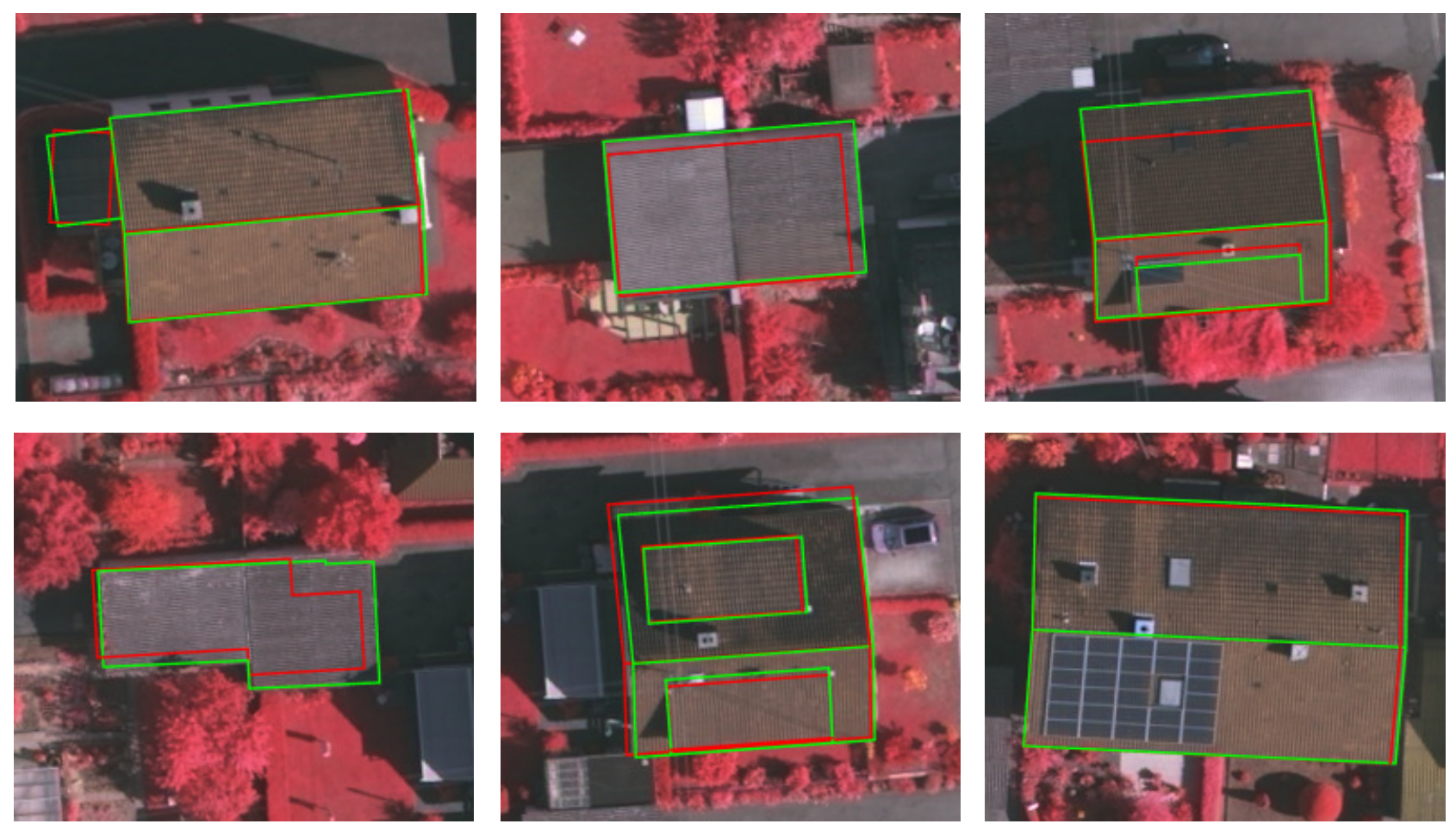

Figure 3. Comparison of building roof edges before (red) and after refinement (green). 
indicators computed per-area level are not expected to highly change. As shown in a table, an average RMS error calculated with respect to the reference boundaries is reduced by $9 \mathrm{~cm}$ (from $73 \mathrm{~cm}$ to $64 \mathrm{~cm}$ ). Positive impact of the refinement procedure on the final results is also revealed in coverage evaluation. The sum of True Positive area increased from $7430 \mathrm{~m}^{2}$ to $7522 \mathrm{~m}^{2}$, while False Positive indicator decreases from $684 \mathrm{~m}^{2}$ to $592 \mathrm{~m}^{2}$. A significant improvement of the refined models geometry is also confirmed by visual comparison of building edges already presented in Fig.1. Especially for instances, where large positional deviations occur (c.f. Fig.3) integration of optical imagery compensates for reconstruction drawbacks and leads to more accurate building models.

\section{CONCLUSIONS}

We have presented a method for sequential refinement of ALSbased building models using single aerial image. The novelty of the proposed reconstruction approach is to extract refined roof corners by direct intersection of 3D roof planes previously detected from laser scanning data with viewing planes assigned to roof edges and extracted from single image. The methodology enables us to benefit from the synergy between LiDAR and optical imagery in the context of high vertical and plannimetric accuracy. Integration of linear cues retrieved from imagery allowed for an average improvement of planar accuracy by $9 \mathrm{~cm}$ (RMS error calculated for roof plane outlines). Visual comparison of the refinement performance indicates that the refinement impact is not distributed regularly. For many instances substantial edge correction is not needed; however in case of large positional deviations the applied refinement clearly compensates reconstruction drawbacks. In this preliminary study, we confirmed that the proposed approach is able to improve the geometric accuracy of 3D building models. The underlying methodology so far assumes that the structural arrangements of model edges reconstructed from ALS data is topologically correct. In the future work we will extend the range of the refinement by a possibility for modification of a model shape and on the simultaneous use of multiple aerial images.

\section{ACKNOWLEDGEMENTS}

This work was supported by National Science Centre, Poland (Project No 2014/13/N/ST10/04892). The Vaihingen data set was provided by the German Society for Photogrammetry, Remote Sensing and Geoinformation (DGPF) http://www.ifp.uni-stuttgart.de/dgpf/DKEP-Allg.html. The authors wish to thank the chair persons of ISPRS Commission III/4 for the evaluation of the results.

\section{REFERENCES}

Bulatov, D., Haufel, G., Meidow, J., Pohl, M., Solbrig, P., Wernerus, P., 2014. Context based automatic reconstruction and texturing of 3D urban terrain for quick response tasks. ISPRS Journal of Photogrammetry and Remote Sensing 93, 157-170.

Chen, L., Teo, T., Rau, J., Liu, J., Hsu, W., 2005. Building reconstruction from LiDAR data and aerial imagery.
Proceedings of the IEEE International Geoscience and Remote Sensing Symposium, vol. 4. pp. 2846 - 2849.

Dal Poz, A. P., 2014. Synergy Between LiDAR and Image Data in Context of Building Extraction, The International Archives of the Photogrammetry, Remote Sensing and Spatial Information Sciences, Vol. XL-1, pp. 89-93.

Demir, N. and Baltsavias, E., 2012. Automated modeling of 3D building roofs using image and LiDAR data. ISPRS Annals of the Photogrammetry, Remote Sensing and Spatial Information Sciences, Vol. I-4, pp.35-40.

Habib, A., Kwak, E., Al-Durgham, M., 2011. Model-based automatic 3D building model generation by integrating LIDAR and aerial images. Archives of Photogrammetry, Cartography and Remote Sensing, Vol. 22, 2011, pp. 187-200

Jarząbek-Rychard M., Borkowski A., 2016. 3D building reconstruction from ALS data using unambiguous decomposition into elementary structures. ISPRS Journal of Photogrammetry and Remote Sensing, vol. 118, pp. 1-12.

Kim, K., Shan, J., 2011. Building roof modeling from airborne laser scanning data based on level set approach. ISPRS Journal of Photogrammetry and Remote Sensing 66, pp.484-497.

Nex, F., Remondino, F. 2012: Automatic roof outlines reconstruction from photogrammetric DSM. Annals of the Photogrammetry, Remote Sensing and Spatial Information Sciences, Vol. I(3), pp. 257-262

Oude Elberink, S., Vosselman, G., 2009. Building reconstruction by target based graph matching on incomplete laser data: analysis and limitations. Sensors 9, pp. 6101-6118.

Perera, G.S.N., 2014. Automated generation of 3D building models from dense point clouds \& aerial photos. PhD Thesis, TU Dresden

Rottensteiner, F., Sohn, G., Gerke, M., Wegner, J.-D., Breitkopf, U., Jung, J., 2014. Results of the ISPRS benchmark on urban object detection and 3D building reconstruction. ISPRS Journal of Photogrammetry and Remote Sensing, Volume 93, 256-271.

Rutzinger, M., Rottensteiner, F., Pfeifer, N., 2009. A comparison of evaluation techniques for building extraction from airborne laser scanning. IEEE Journal of Selected Topics in Applied Earth Observations \& Remote Sens. 2 (1), 11-20.

Sohn, G., Jung, J., Jwa, Y., and Armenakis, C., 2013. Sequential modelling of building rooftops by integrating airborne LiDAR data and optical imagery: preliminary results, ISPRS Annals of the Photogrammetry, Remote Sensing and Spatial Information Sciences, vol. II-3/W1, pp. 27-33.

Xiong, B.; Jancosek, M.; Oude Elberink, S.; Vosselman, G., 2015. Flexible building primitives for 3D building modeling. ISPRS Journal of Photogrammetry and Remote Sensing, Vol. 101, pp. 275-290.

Zhang, W., Wang, H., Chen, Y., Yan, K., Chen, M., 2014. 3D Building Roof Modeling by Optimizing Primitive's Parameters Using Constraints from LiDAR Data and Aerial Imagery. Remote Sensing 6 (9), 8107-8133. 


\title{
Finding complex patterns using template matching
}

\author{
G. Jasvilis ${ }^{\mathrm{a}, *}$, C. Weise ${ }^{\mathrm{b}}, \mathrm{B}$. Zenger-Landolt ${ }^{\mathrm{b}}$ \\ ${ }^{a}$ Trimble, Geospatial Division, Vingriu st.6A, 01141 Vilnius - gediminas_jasvilis@ trimble.com \\ ${ }^{\mathrm{b}}$ Trimble, Geospatial Division, Arnulfstr. 126,80636 München, Germany - (christian_weise, barbara_zenger-landolt)@ trimble.com
}

KEY WORDS: template matching, object-based image analysis, correlation coefficient, omission and commission error

\begin{abstract}
:
Complex patterns that combine pixels of different brightness and colour in a fixed spatial layout pose a challenge for the objectoriented approach to image analysis. This is because the pixels that make up the pattern cannot readily be linked together into an object as they do not share spectral or textural properties. The goal of this study was to determine whether template matching techniques can be used to identify those patterns without a prior segmentation of the image.

We studied three different scenarios that pose specific challenges: the application of template matching is most straightforward in (1) the detection of symbols in scanned maps where different occurrences of a given pattern strongly resemble one another; most notably, symbols always have the same size and orientation. (2) The detection of trees in aerial images of oil palms is more challenging, since the characteristic tree pattern exhibits significant variations from tree to tree. Finally, in the (3) detection of pavement markings in aerial images objects could appear at arbitrary orientations in the image.

Our results show that template matching successfully identifies many target regions. Once target locations are known, objects can be created and the full power of object-based image analysis approaches can be applied to eliminate remaining false positives.
\end{abstract}

\section{INTRODUCTION}

For object-oriented image analysis to exhibit its full strength, meaningful objects have to be created (Baatz, Hoffmann \& Willhauck 2008). This can be challenging for patterns characterized by a specific spatial layout that cannot easily be described with shape or texture features. What is needed is a method that provides information about the likelihood of pattern presence before the segmentation step. In this study, we investigated whether classical template matching techniques (e.g. Nixon \& Aguado 2012, Bradski \& Kaehler 2008) can be used to solve this problem.

There are a variety of different algorithms in common use that differ mostly in how the similarity between a template and an image region is quantified (Bradski \& Kaehler 2008).

One simple method for measuring the similarity $s$ is to use the sum of the squared differences between a template $t$ and an image region $I$ :

$$
s=\sum_{x, y}(t(x, y)-I(x, y))^{2}
$$

Note that for this measure, a simple overall shift in mean intensity in the image can harm the match quality as much as any real change in the shape of the pattern. Therefore, the sum of squared differences may not be a good option for many common use cases, where natural variations in illumination cannot be eliminated.

Another very simple measure for match similarity $s$ is the correlation between a template and an image region $I$.

$$
s=\sum_{x, y} t(x, y) \cdot I(x, y)
$$

Unfortunately, this measure is also affected heavily by shifts in mean illumination. The disadvantage can be eliminated though, when the Pearson's correlation coefficient $r$ is used as similarity measure.

$$
r=\frac{\sum_{x, y}(x-\bar{x})(y-\bar{y})}{\sqrt{\sum_{x, y}(x-\bar{x})^{2}} \cdot \sqrt{\sum_{x, y}(y-\bar{y})^{2}}}
$$

The correlation coefficient can be interpreted as a correlation between a template and an image after both the template and the image have been z-normalized (i.e. rescaled so that the mean is zero and the standard deviation is 1). Illumination and contrast differences are thus eliminated before match quality is evaluated - making the correlation coefficient an ideal measure of match when we want to ensure robustness for variations of pattern brightness and contrast.

Our study shows that template matching based on the correlation coefficient $r$ can successfully identify potential target regions in a number of real life use cases.

\section{USE CASES}

Our study included three different use cases, each highlighting a different challenge.
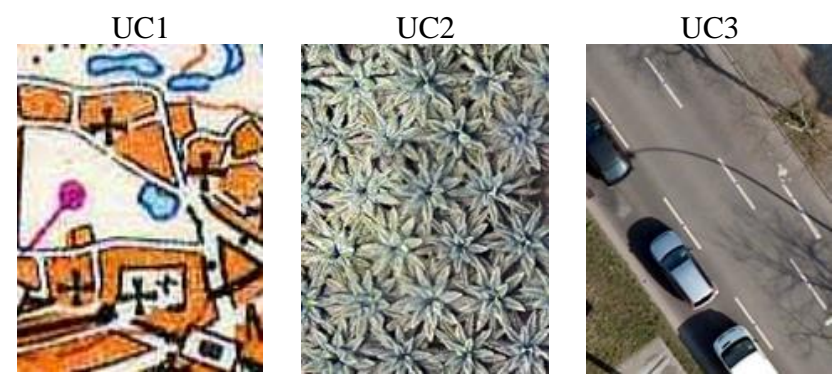

Figure 1. Image thumbnails for the different use cases.

\footnotetext{
* Corresponding author
} 


\subsection{Use case 1: symbols in scanned maps}

Symbols in scanned maps come at a fixed size and a fixed orientation, thus perhaps representing an ideal problem to address with template matching, as we need worry neither about scale nor rotation invariance.

\subsection{Use case 2: trees in aerial images of oil palm plantations}

The detection of palm trees in a plantation is already more complex. The trees have a very characteristic shape, but they do not exactly match one another. There is a significant amount of natural variation or pattern heterogeneity that needs to be taken into account.

\subsection{Use case 3: pavement markings in aerial images}

The detection of specific pavement markings (here the white bars that separate different lanes) pose a new challenge: the markings may appear at an arbitrary number of different orientations in a given image.

\section{METHODS}

\subsection{Template Generation}

Before template matching can be applied to a problem, an adequate template for a given pattern of interest needs to be generated. In this study, templates were generated from manually selected samples. To avoid overlearning, samples were collected in regions outside the designated test region.
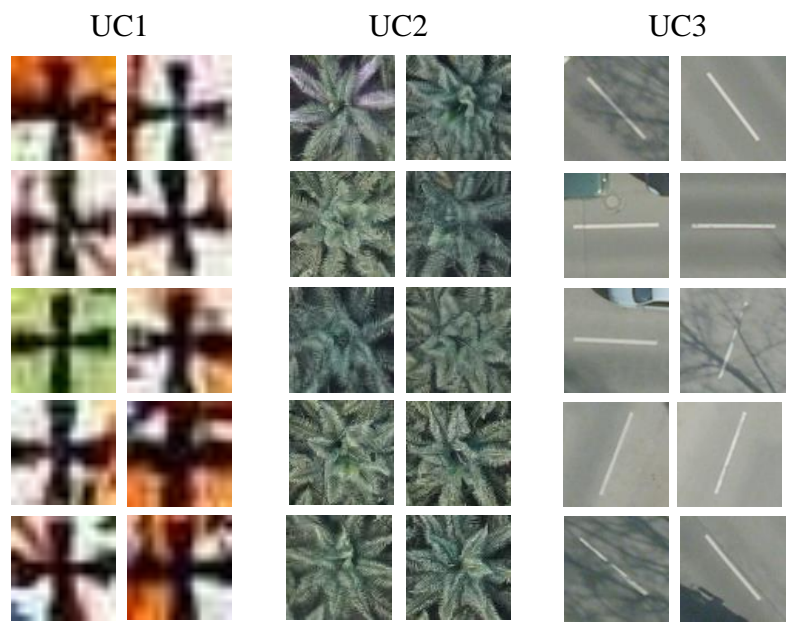

Figure 2. A selection of samples used for template generation

Perhaps the most straightforward way of generating a template is averaging all the samples, and this is the basic approach we used (see e.g. Figure 3/UC1).

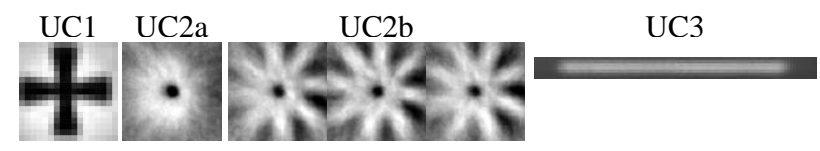

Figure 3: The templates generated for the different use cases.

For the detection of palm trees, the mean template (UC2a) misses characteristic aspects of palm trees and a template group (UC2b) was used instead.
However, when samples show considerable variation (like in the example of palm trees) a simple mean template does not capture the characteristic aspects of the samples (Figure 3/UC2a). To address this, we used a variant of factor analysis to extract more than one template. The variety in the sample space was thus captured not by a single template, but by several distinct templates, which we refer to as template group (Figure 3/UC2b).

Another obvious situation where simple averaging would fail is when samples differ in orientation, as they do in the case of pavement markings. To prevent this, we rotated the sample images so they would all be rotationally aligned before averaging them to obtain a single template image (Figure 3/UC3).

\subsection{Generation of a similarity map}

For reasons already discussed in the introduction section, we used the correlation coefficient $r$ to quantify the quality of the match between a target and an image region.

A similarity map was generated by sliding the template over the image (sliding window), and computing the correlation coefficient $r$ at each location. The result was assigned to the central pixel of the current window. Note that the final similarity map is thus smaller than the original image, with shrinkage depending on the template size.

For the more challenging use cases we applied more than one template to each location. This could be either the different templates of a template group (use case: trees in aerial images of oil palm plantations), or several rotated versions of a single template (use case: pavement markings in aerial images).

\subsection{Target localization}

Once the similarity map is obtained, there still remains the task of localizing the targets. Clearly, a certain similarity threshold must be reached to suggest presence of a target. In addition, we want to avoid finding many "hits" in a very small region. We thus impose two criteria for target presence at a specific location:

a) The similarity map value of the corresponding pixel must exceed a defined threshold $t h r_{r}$. This threshold was set to obtain a small overall error rate while keeping omission and commission errors (see next section) relatively balanced.

b) The match quality of the pixel must represent a local maximum in a neighbourhood corresponding $\left(d_{\min }\right.$ is the radius of this neighbourhood).

\subsection{Accuracy Assessment}

To evaluate the quality of the template matching algorithm we selected for each use case a test image for which we manually created a ground truth. A target counted as correct detection when the distance to a ground truth element was below a defined tolerance. This tolerance was set to a value of about $10 \%$ to $20 \%$ of the template size, depending on the use case. Tolerance was largest for the tree detection (corresponding to $1.5 \mathrm{~m}$ on the ground), as the tree centres cannot be allocated precisely, reflecting an inherent noise in the ground truth.

We also evaluated the number of misses, i.e., locations marked in the ground truth, for which no target was identified in the neighbourhood defined by the tolerance. Finally, we counted also false positives - targets identified by our algorithm that were absent from the ground truth. 
Following standard convention, we used those measures to evaluate the omission and commission errors:

$$
\begin{aligned}
& E r r_{\text {omission }}=\frac{\text { misses }}{\text { misses }+ \text { correct detections }} \\
& E r r_{\text {comission }}=\frac{\text { false positives }}{\text { false positives }+ \text { correct detections }}
\end{aligned}
$$

Those error rates were used to evaluate the power of our template matching algorithms in the different use cases.

\section{RESULTS}

Parameters used for template generation, template matching and target localization are shown in Table 4, together with the results of the accuracy assessment.

\begin{tabular}{|l|c|c|c|}
\hline & UC 1 & UC 2 & UC3 \\
\hline Parameters & 278 & 394 & 60 \\
\# Samples & $17 \times 17$ & $69 \times 69$ & $95 \times 7$ \\
Template size (pxl) & 0.69 & 0.18 & 0.76 \\
th $r_{r}$ & 13 & 50 & 60 \\
$d_{\text {min }}(\mathrm{pxl})$ & 2 & 12 & 10 \\
Tolerance (pxl) & \multicolumn{3}{|l|}{} \\
\hline Results & 436 & 68 \\
\hline Elements in & 127 & & \\
Ground Truth & 107 & 399 & 55 \\
Correct Detection & 17 & 18 & 14 \\
False Positives & 20 & 37 & 13 \\
Misses & 0.137 & 0.043 & 0.203 \\
Commission Error & 0.158 & 0.085 & 0.191 \\
Omission Error
\end{tabular}

Table 4. Parameter settings and accuracy assessment results

Error rates vary between around $6 \%$ for the detection of oil palm trees and around $20 \%$ for the detection of pavement markings. Image thumbnails highlighting the detected targets are shown in Figure 5.
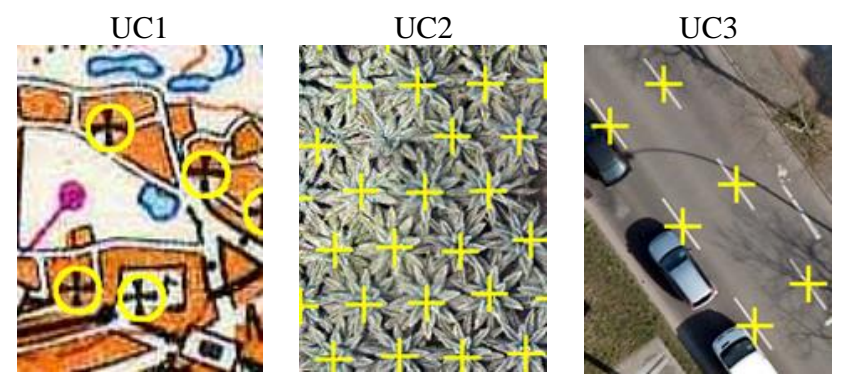

Figure 5: Image thumbnails with identified target locations.

\section{DISCUSSION}

It is difficult to directly compare our results to the literature, as obtained accuracies do not only reflect the power of the algorithms applied. Results are also heavily influenced by the testing method, and of course, the nature and quality of the test images. Nevertheless, it is noteworthy that in spite of the conceptual simplicity of our approach, the detection quality achieved here for tree detection lies well within the range of other published work on the detection of palm trees (Malek,
Bazi, Alajalan, AlHichric and Melgani, 2014; Srestasathiern \& Rakwatin, 2014; Manandhar, Hoegner \& Stilla, 2016).

While we have set the threshold $t h r_{r}$ in this study to achieve a low and balanced error rate, software-supported workflows will typically warrant a different type of threshold adjustment. This is because the omission error is usually associated with a higher cost than a commission error. Consider, for instance, a manual quality assurance step: finding additional targets in an image is by far more time consuming than "clicking away" a selection of false positives.

Clearly, the specific implementations of template matching we used here need not be the final word on the optimal approach. For example, in the symbol detection it appears that not all pixels in the cross template are created equal - some contribute more to the actual pattern than others. Indeed we found that applying a mask which prevents certain pixel locations to enter the correlation computation can lower error rates considerably, from around $15 \%$ to around $10 \%$ in the symbol detection studied here. Furthermore, we observed significant improvement of oil palm tree detection when preprocessing the data layers in a carefully designed manner, before applying template matching.

But even when taking further improvements into account, it appears questionable that template matching will be able to provide an answer to all pattern matching tasks. In our view, template matching simply represents a useful first step. It enables identification of target regions that can then be processed further by classical object-oriented approaches (Baatz, Hoffmann \& Willhauck 2008).

In summary, we have applied template matching to three different use cases of practical relevance. We found that template matching can successfully isolate many targets, in spite of its conceptual simplicity. To leverage this power, template matching capabilities are now made available in eCognition Developer, where the user can choose from a variety of convenient algorithms and tools for generating and applying templates.

\section{REFERENCES}

Baatz, M., Hoffmann, C., and Willhauck, G. (2008). Progressing from object-based to object-oriented image analysis. Object Based Image Analysis, ed. Blaschke, T., Lang, S. and Hay, G. New York.

Bradski, G. and Kaehler, A. (2008). "Histograms and Matching" in Learning OpenCV: Computer Vision with the OpenCV Library (1st ed.), O'Reilly Media, Inc., pp. 193-221

Malek, S., Bazi, Y., Alajlan, N., AlHichri, H. and Melgani, F., 2014. Efficient framework for palm tree detection in uav images. IEEE Journal of Selected Topics in Applied Earth Observations and Remote Sensing, 7(12), pp. 4692-4703.

Manandhar, A., Hoegner, L. and Stilla, U., 2016. Palm tree detection using circular autocorrelation of polar shape matrix. (abstract accepted at ISPRS 2106)

Nixon, M. and Aguado, A. 2012. Template matching. In: Feature Extraction \& Image Processing for Computer Vision (3rd ed.). Academic Press, pp. 222-235

Srestasathiern, P. and Rakwatin, P., 2014. Oil Palm Tree Detection with High Resolution Multi-Spectral Satellite Imagery . Remote Sensing, 6, pp. 9749-9774 


\title{
Object-based Integrated Landscape Change Analysis: synergy of multi-temporal LiDAR and very high resolution orthophotos
}

\author{
M. Kamps ${ }^{\text {a }}$, A.C. Seijmonsbergen ${ }^{\text {a }}$ and W. Bouten ${ }^{\mathrm{a}}$ \\ aIBED, Institute for Biodiversity and Ecosystem Dynamics (IBED) - martijntkamps@ gmail.com - (a.c.seijmonsbergen, \\ w.bouten)@uva.nl
}

KEY WORDS: Data Synergy, OBIA, Landslide, Above Ground Biomass, LiDAR, Orthophotos, Land Cover Change

\begin{abstract}
:
Active landslides have three major effects on a landscape: 1. land cover change, 2. topographical change, and 3. above ground biomass change. Data derived from multi-temporal Light Detection and Ranging technology (LiDAR) is used in combination with multi-temporal orthophotos to quantify changes between 2006 and 2012, caused by a landslide near Doren in Austria. Data synergy is used to optimize accuracies of land cover change, and to improve results of topographical change analysis and aboveground biomass estimations. Topographical change is calculated using differencing of digital terrain models. The above ground biomass is quantified by applying a local-maximum algorithm for tree top detection, in combination with allometric equations. The land cover change classification accuracies were improved from 65\% (using only LiDAR) and 76\% (using only orthophotos) to 90\% (using synergy) for 2006. A similar increase from respectively $64 \%$ and $75 \%$ to $91 \%$ was established for 2012 . The results of the improved land cover classifications were used to optimize the topographical and above ground biomass change calculations. Fine-scale improvements of the classifications included forest edges and shadows, small open spots in the vegetation, and confusion between land cover classes. The enhanced accuracies of the land cover change analysis demonstrate the effectiveness and advantages of using synergy of LiDAR and orthophotos using OBIA. The method has great potential to be transferred to larger areas for use in monitoring, although data size calls for workflows to operate on cloud-based infrastructures that provide sufficient computational power.
\end{abstract}

\section{INTRODUCTION}

The synergy of very high resolution (VHR) imagery such as orthophotos and data derived from Light Detection and Ranging (LiDAR) technology to detect land cover change (LCC), topographical and above ground biomass (AGB) changes through object-based image analysis (OBIA) in an area affected by an active landslides is analyzed. Landslides are common processes that may cause continuous or sudden changes in the topography and the overlying vegetation cover. The growing availability of multi-temporal LiDAR-based elevation data facilitates the combined analysis of fine-scale 3D topographical and forest structure changes occurring in landslide areas.

OBIA has been successfully applied in the segmentation and classification of VHR imagery for mapping LCC change (Machala \& Zejdova, 2014; Zhou et al, 2008) and in many landslide detection studies (Lahousse et al. 2011, Li et al. 2015). In such cases, OBIA generally outperforms pixel-based approaches in LCC studies (Machala \& Zejdova, 2014), although hybrid approaches (Aguirre-Gutierrez et al. 2012, Wang, 2004) may sometimes lead to better accuracies. The synergy between VHR imagery and LiDAR information has been used to analyze 3D-forest structure (Machala \& Zejdova, 2014), to monitor forest canopy height (Wulder \& Seemann, 2003) and to classify land cover (Mücher et al., 2015; Parent et al., 2015). These studies mention LCC classification accuracies of more than eighty percent and emphasize the mutual benefits of the combination of VHR imagery and LiDAR. In addition, embedding elevation data into LCC change detection is also thought to improve the accuracy of segmentation and classification (Desclee et al., 2006). The forest cover in a landslide area can not only be expressed in areal loss, but can also be characterized by changes in AGB, by using tree specific allometric relations derived from multi-temporal LiDAR-based canopy height models (Muukkonen et al., 2005).
LiDAR-based DTM differencing (James et al. 2012) may reveal detailed changes in topography of forested and non-forested parts of the landslide and may aid interpretation of landslide development and its relation to event-based loss of trees. These trends show that the combination of VHR imagery, LiDARbased elevation models and OBIA may result in higher accuracies in change analyses studies. To integrate the various analysis techniques, we present a combined approach using a modular workflow in the commercial eCognition 9.2 software package that automatically analyzes LCC change, and, for the same time frame, quantifies the topographical and the AGB changes of an active landslide near the village of Doren in Vorarlberg, western Austria.

\section{STUDY AREA}

The landslide near Doren is located in the north of Vorarlberg along the river Weißach and is known to be active since 1847 (Fig 1.). Major events occurred in the early 1927, 1935 and 1988 , during which $2-3 \times 10^{6} \mathrm{~m}^{3}$ was mobilized as rock- and soil slides, and debris flows (Jaritz and Marte, 2008). The geology is formed by a tilted sedimentary sequence of alternating Molasse marls, sandstone and conglomerates (Friebe, 2007), that, in combination with a highly fractured rock mass, promotes groundwater flow to potential failure planes, thus reactivating the landslide. The rock underlain area was glacially eroded and covered by $5-10 \mathrm{~m}$ of subglacial till, which was dissected again by the approximately 80 deep valley of the Weißach River. Norway spruce is the dominant tree species on the valley slopes with understory of shrubs and many small, open spots, while meadows (grass), shrubs and some infrastructure are present in the upper, intact low-angle slopes. 


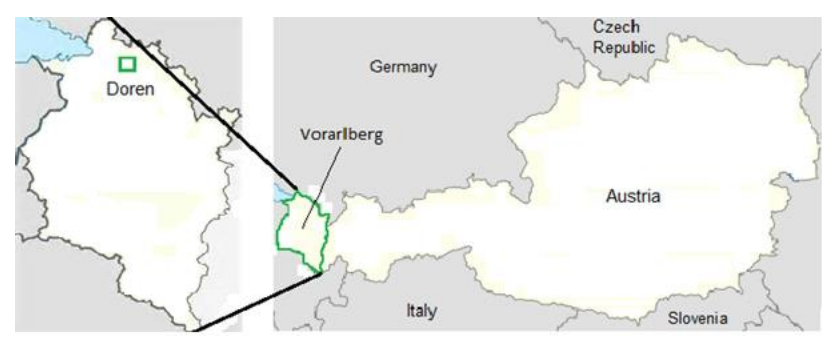

Figure 1. Location of the Doren landslide (coordinates: $47^{\circ} 29^{\prime} 31.9^{\prime \prime} \mathrm{N} 9^{\circ} 52^{\prime} 55.6^{\prime \prime E}$ ) in Vorarlberg, western Austria.

\section{METHODS}

\subsection{Data}

In table 1 metadata of the true colour orthophotos (TCO) and LiDAR data for 2006 and 2012 are presented.

\begin{tabular}{|c|c|c|c|c|}
\hline Year & Data type & Extension & Camera & Accuracy \\
\hline \multirow[t]{2}{*}{2006} & TCO & img & Zeiss RMK TOP $30 / 23$ & Resolution: $0,125 \mathrm{~m} \times 0,125 \mathrm{~m}$ \\
\hline & LIDAR & img & ALTM 2050 & $\begin{array}{l}\text { Point density: } 2 / \mathrm{m} 2 \\
\text { Height accuracy: } 0,1 \mathrm{~m} \\
\text { Location accuracy: } 0,2 \mathrm{~m} \\
\text { Vertical accuracy: }<0,25 \mathrm{~m} \\
\text { Horizontal accuracy: }<0,30 \mathrm{~m}\end{array}$ \\
\hline \multirow[t]{2}{*}{2012} & TCO & .img & Vexcel Ultracam XP RGBI & Resolution: 0,125 $\mathrm{m} \times 0,125 \mathrm{~m}$ \\
\hline & LIDAR & laz & $\begin{array}{l}\text { Harrier 56-009 } \\
\text { LiDAR System }\end{array}$ & $\begin{array}{l}\text { Point density: } 4 \text { to } 8 / \mathrm{m} 2 \\
\text { Height accuracy: } 0,075 \mathrm{~m} \\
\text { Location accuracy: } 0,1 \mathrm{~m} \\
\text { Vertical accuracy: }<0,15 \mathrm{~m} \\
\text { Horizontal accuracy: }<0,25 \mathrm{~m}\end{array}$ \\
\hline
\end{tabular}

Table 1. Metadata of the various datasets

\subsection{Workflow}

Figure 2 shows the workflow used to determine LCC change, topographical change and AGB change. The workflow consists of main procedures $(1-4)$ which have been broken down into several steps (A - D). To compare the added value of using data synergy instead of an individual dataset, three scenarios were calculated, indicated by coloured arrows. The 'synergy' scenario is indicated by a green arrow, while the 'orthophotos only' and the 'LiDAR data only' scenarios are indicated by blue and red arrows respectively.

\subsubsection{Data Pre-processing}

The LAStools extension for ArcGIS was used to interpolate the 2012 point-cloud LiDAR data (Rapidlasso $\mathrm{GmbH}$ ). In eCognition the maximum first return and minimum last return were used to filter out the DTM and DSM respectively.

\subsubsection{Land Cover Change detection}

The following land-cover classes that have been recognized and used in the LCC are: bare soil, grass and shrub land, forest, water, road and buildings. Three scenarios have been processed: one using segmentation and classification synergy of the orthophotos and LiDAR datasets and two scenarios using only the orthophotos and only the LiDAR data. The general procedures and steps of segmentation, classification, accuracy assessment and image differencing is described in four subsections (see also Figure 2).

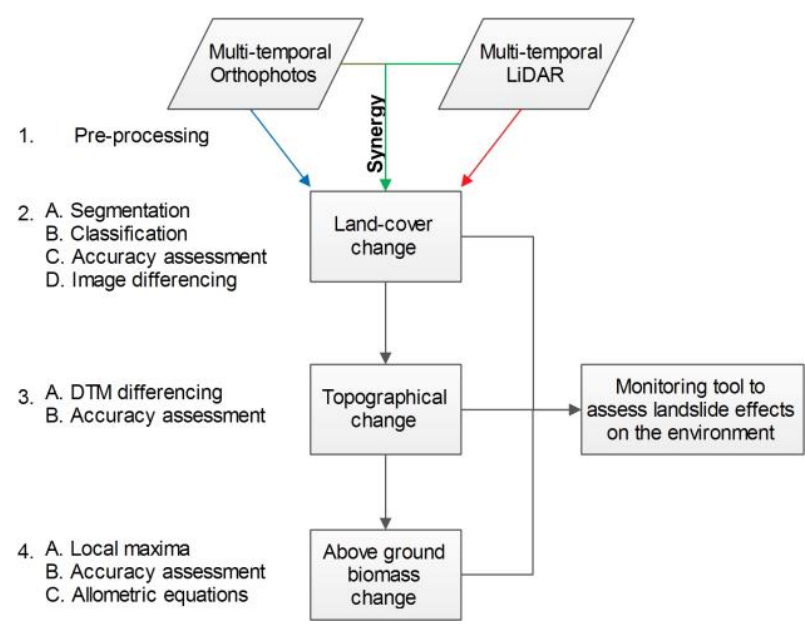

Figure 2. Workflow, in which he 'synergy' scenario is indicated by a green arrow, the 'orthophotos only' and the 'LiDAR data only' scenarios are indicated by blue and red arrows.

Step A. Following successful applications of multiresolution segmentation (MRS) in mountainous environments (AguirreGutierrez et al., 2012; Anders et al, 2013; Eisank et al., 2014) an iterative algorithm was developed producing 125 parameter combinations, similarly to the work of Clinton et al. (2010). The parameter settings used for scale parameter are 10, 20, 30, 40 and 50, for the shape parameter $0.1,0.3,0.5,0.7,0.9$ and for compactness $0.1,0.3,0.5,0.7$ and 0.9 . The accuracy of the segmentation results was determined by comparing the generated objects with the orthophotos based on over-and under segmentation (Esch et al., 2008). Based on the accuracy assessment a MRS using a scale parameter of 50, a shape parameter of 0.3 and compactness of 0.5 , and the spectral information from the orthophotos in combination with the LiDAR-derived canopy height model (CHM) was used.

Step B. The classification consists of two routines: (1) a stratified membership classification based on feature space optimization (FSO) and (2) fuzzy-logic improvements based on context, geometry, spectral and elevation characteristics.

1. For the FSO 200 samples per land-cover class were manually classified based on orthophoto recognition. FSO was then applied to twelve distinct features derived from both the orthophotos and LiDAR.

2. Fuzzy-logic rulesets are used to improve the classification accuracy after membership-based classification. This step is necessary since the overlap of the orthophotos and LiDAR introduces some fine-scale errors, for instance shadowing effects, small open spots in the forest and mismatches between bare soil and the LiDAR model. Fuzzy-logic rulesets that use the spectral characteristics (RGB), elevation information (LiDAR) and contextual information derived from expert knowledge can deal with such errors. These detailed rulesets are specifically used to improve finescale features, optimize classification borders and shadows and minimize errors as a result of dataset overlap.

Step C. Classification accuracy assessment was performed by random selection of 250 objects. A standard confusion error matrix was derived including user and producer accuracies according to the method described by Congalton (1991).

Step D. After image classification post-classification change detection is applied using simple image differencing (Gutierrez 
et al., 2012; Zhou et al., 2008).

\subsubsection{Topographical change}

The topographical change procedure was calculated in two steps (Figure 2).

Step A. The topographical volumetric change was calculated for each land cover class using DTM differencing and multiplying with pixel size.

Step B. Following Wheaton et al. (2009) the propagation error in the DTM differencing process caused uncertainties of 0.29 meters. This represents $0.0066 \mathrm{~m}^{3}$ per pixel volumetric change. Therefore, volumetric changes $<0.01 \mathrm{~m}^{3}$ are disregarded and considered as 'no change areas' to reduce apparent change as a result of data errors (Latypov, 2002).

\subsubsection{Above Ground Biomass change}

The AGB change procedure was calculated in three steps (Figure 2).

Step A. The eCognition local-maxima algorithm was used on the CHM to derive canopy top locations. The local maximum algorithm was applied to areas which were classified as forest with the synergy approach for LCC.

Step B. Accuracy assessment of the canopy tops is performed by manual inspection and comparison of the generated canopy tops with the CHM and orthophotos.

Step C. According to Maier et al. (2006) the Norway Spruce, is the dominant tree species in Vorarlberg. Therefore, the allometric equations for biomass calculation as used by Muukkonen et al. (2005) for Norway Spruce were used:

$$
\mathrm{DBH}=\mathrm{e}^{\left(\left(0.1687+1.2413^{*} \ln ([\mathrm{H}])\right)\right.}
$$

where

$$
\begin{aligned}
& \mathrm{DBH}=\text { diameter at breast height } \\
& \mathrm{H}=\text { tree height }
\end{aligned}
$$

$$
\mathrm{B}=\mathrm{e}^{\left(-4.63873+\left(2.75352 * \ln ([\mathrm{DBH}])-0.08578^{*} \ln ([\mathrm{H}])\right)\right)}
$$

where $\quad \mathrm{B}=$ biomass

The allometric equations are used to first calculate the diameter at breast height $[\mathrm{R} 2=0,880]$ and subsequently the biomass using the height of the canopy tops [R2 $=0,946]$.

\section{RESULTS}

\subsection{Land Cover Change}

Although the segmentation results using the synergy between the datasets could not be quantified, manual inspection reveals that segmentation results improved. Segmentation based solely on the orthophotos shows under segmentation of spectrally similar adjacent land-cover classes, while segmentation based solely on LiDAR-derived data does not distinguish between spectrally different land-cover classes with similar elevation characteristics.

Classification results were substantially improved using the synergy between the datasets. The highest overall accuracy of the classification based on orthophotos only was 76\% (kappa: 0.64 ), for classification based only on LiDAR-derived data $65 \%$ (kappa: 0.46) and for the synergy based classification $91 \%$ (kappa: 0.93). Table 2 shows the confusion matrix for classification based on orthophotos and LiDAR in 2012.

\begin{tabular}{|l|r|r|r|r|r|r|r|}
\hline $\begin{array}{l}\text { Confusion } \\
\text { Matrix }\end{array}$ & Bare soil & Building & Grassland & Road & Trees & Water & \multicolumn{1}{c|}{ Sum } \\
\hline Bare soil & 36 & 0 & 1 & 1 & 1 & 0 & 39 \\
Building & 0 & 5 & 0 & 0 & 0 & 0 & 5 \\
Grassland & 2 & 0 & 63 & 1 & 6 & 0 & 72 \\
Road & 2 & 0 & 1 & 12 & 0 & 0 & 15 \\
Trees & 1 & 0 & 4 & 1 & 117 & 0 & 123 \\
Water & 2 & 0 & 0 & 0 & 0 & 11 & 13 \\
sum & 43 & 5 & 69 & 15 & 124 & 11 & 267 \\
& & & & & & & \\
Producer & 0,84 & 1,00 & 0,91 & 0,80 & 0,94 & 1,00 & \\
User & 0,92 & 1,00 & 0,88 & 0,80 & 0,95 & 0,85 & \\
Overall & 0,91 & & & & & & \\
accuracy & 0,93 & & & & & & \\
Kappa & & & & & & \\
statistic & 0, & & & & & & \\
\hline
\end{tabular}

Table 2. Classification accuracies for 2012

Compared to classifications solely based on membership functions, the fuzzy-logic based stratified classifications improved the accuracy results with $26 \%$ (for 2006) and $18 \%$ (for 2012).

Classification based solely on orthophotos may result in confusion between forest and grassland and classification based solely on LiDAR may cause confusion between grassland and bare soil. Orthophoto-based classification does however separate grassland with bare soil, while LiDAR-based classification separates forests from grassland and bare soil. The spectral and elevation synergy between the datasets therefore results in accurate classification of all land-use classes. Two examples of classification improvements are presented in figure 3.
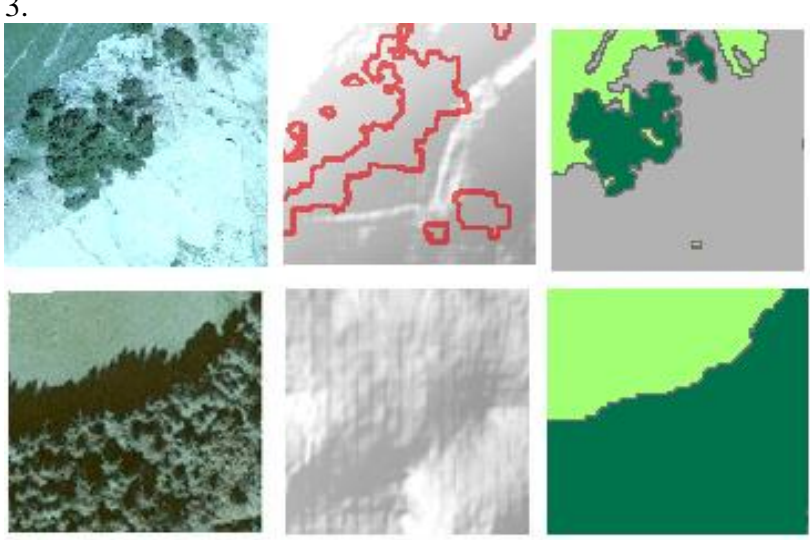

Elevation [m]

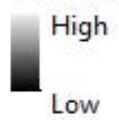

Trees

Grassland

Bare soil

LIDAR error

Figure 3. Two examples of classification improvements using data synergy. The left column shows the orthophoto fragments, the central the LiDAR hillshade and indicated error in red. The right column displays the improved classification result.

In the left column the orthophoto is presented, in the central column the LiDAR dataset and errors indicated in red and in the right column the improved classification result. LiDAR-based errors refer to areas with a CHM higher $>2$ meters, but without vegetation on the orthophotos. These were correctly classified as bare soil using spectral information. The shadow areas in were correctly classified as grassland using LiDAR derived elevation information. 
In table 3 the LCC changes for each land-cover class from 2006 to 2012 are summarized.

\begin{tabular}{|c|c|c|c|c|c|c|c|c|}
\hline Year & & Bare soil & Building & Grass & Road & Trees & Water & Total \\
\hline \multirow[t]{2}{*}{2006} & Sum $[\mathrm{m} 2]$ & |52484,3 & $\begin{array}{l}680,2 \\
\end{array}$ & 86111,4 & N.A. & 241721,6 & 1552,3 & 382549,9 \\
\hline & Percent [\%] & 13,7 & 0,2 & 22,5 & N.A. & 63,2 & 0,4 & \\
\hline \multirow[t]{2}{*}{2012} & Sum [m2] & 41458,0 & 532,7 & 92694,4 & 2364,2 & 244386,0 & 1114,6 & \\
\hline & Percent [\%] & 10,8 & 0,1 & 24,2 & 0,6 & 63,9 & 0,3 & \\
\hline 2006-2012 & Change [\%] & $-2,9$ & $-0,1$ & 1,7 & 0,6 & 0,7 & $-0,1$ & \\
\hline
\end{tabular}

Table 3. Quantification of relative LCC per land-cover class.

Table 4 summarizes the 'from-to' changes between 2006 and 2012. A map of these changes in presented in Figure 4, upper left panel, in relation to the landslide area and surroundings.

\begin{tabular}{|c|c|c|c|c|c|c|}
\hline & GtG & GtB & GtT & GtR & BtB & BtG \\
\hline Sum [m2] & 51405 & 10274,07 & 23078,65 & 1287,33 & 24773,08 & 22204,76 \\
\hline Change [\%] & 13,44 & 2,69 & 6,03 & 0,34 & 6,48 & 5,81 \\
\hline & BtT & BtR & TtT & TtG & TtB & TtR \\
\hline Sum [m2] & 4612,2 & 337,75 & 215888,24 & 19082,68 & 6096,93 & 653,16 \\
\hline Change [\%] & 1,21 & 0,09 & 56,44 & 4,99 & 1,59 & 0,17 \\
\hline
\end{tabular}

Table 4. Quantification of 'from-to' land-cover change. $G=$ grassland, $\mathrm{B}=$ bare soil, $\mathrm{T}=$ Trees, $\mathrm{R}=$ Road. E.g.: $\mathrm{GtB}=$ Grassland to Bare soil

\subsection{Topographical change}

Topographical change is visualized in figure 4, upper right panel. Volumetric changes (in $\mathrm{m}^{3}$ ) are represented in three classes, 1. Removal of material (in red), no change (in yellow) and deposition of material (in green). The resulting patterns reflect the landslide dynamics: red colours dominate in the upper scarp section, and alternate with distinct zones of deposition and no change, which suggests slide-like movement, which is in accordance with observations by Jaritz and Marte (2008). Deposition predominates in the toe area of the slide, without interruptions of vast removal and no change areas, suggesting flow-type accumulations to prevail in the lower section of the landslide. Part of the landslide in the lower eastern edge of Figure seems to be re-activated, probably related to undercutting action of the local Weissach river.

\subsection{Above Ground Biomass change}

The AGB is plotted in $\mathrm{kg}$ for individual tree tops in figure 4, upper right panel. Striking is that small trees (biomass $<250 \mathrm{~kg}$ ) predominantly occur on or close to the lower depositional toe of the landslide, along the edges of forest stands and at canopy openings, probably due to regrowth between 2006 and 2012 . Trees with biomass $>1000 \mathrm{~kg}$ occur mainly in the middle of forest stands and are not affected by the landslide.

\subsection{Combined effects of landslides}

The combined results of the LCC, topographical and biomass change show patterns that align well with the behaviour of large landslides. DEM differencing (figure 4, panel $\mathrm{B}$ and $\mathrm{C}$ ) illustrate that that the landslide is dominated by removal, caused by 'slide-type' movements in the upper scar section, in contrast to the lower section, which indicates 'flow-type' depositional processes. This pattern is reflected in the LCC analysis, because deposition into forested area occurs in the lower toe area, without causing total removal of trees. In total, the forest area slightly increased by $0.86 \%$ compared to 2006 , while biomass decreased $-8.3 \%$. Figure 4, panel A (top-left) illustrates the details and dynamic LCC on the landslide body, in contrast to the relatively stable forested LCC outside the landslide.

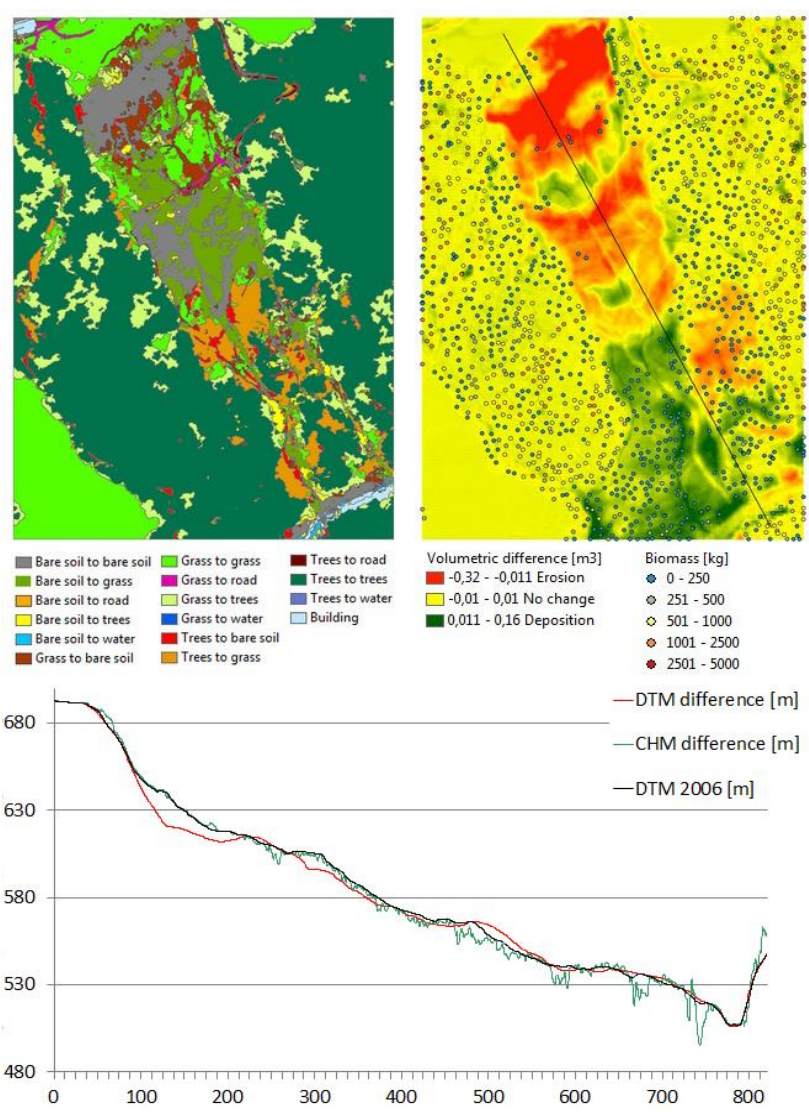

Figure 4. A, upper left panel: 'from-to' classification results of LCC. B, upper right panel: topographical change and AGB for individual tree tops. C, lower panel: topographical cross-section visualizing DTM differences.

\section{DISCUSSION}

Although the quality of the LiDAR data and orthophotos is high, the differences in point densities and the applied filtering and interpolation techniques may cause error propagation in the synergy approach and thus in the resulting LCC. However, elevation differences measured from 50 fixed objects has shown that elevation differences remain below $30 \mathrm{~cm}$. In an active large landslide this is not seen as a major problem.

The synergy of elevation and spectral information to improve segmentation and classification has resulted $18-25 \%$ higher classification accuracies than results based on the single datasets alone. Three distinct errors were detected, related to shadows and LiDAR-based confusion between LC classes. Although synergy solved most of the errors, it remains unsure in a dynamic landslide area, what the effect of time differences between the orthophotos and LiDAR information is.

The orthophotos allow detailed validation of LCC, however, field validation could improve the accuracy assessment of especially AGB; understory, alignment of tree tops on sloping areas and other tree growth specific conditions may hamper exact AGB biomass estimates. In addition, full understanding of the mechanism of landslide dynamics and its combined effects to the landscape, requires some level of field inspection.

The transferability of the workflow to larger areas has great potential, however, VHR datasets require efficient computing 
power, which implies that cloud computing facilities seems a logical future step.

\section{CONCLUSION}

The synergy of orthophotos and LiDAR-based information improved the object-based segmentation and classification results of LCC. Three types of classification errors (shadow effects, small open spots in the forest and LiDAR-based errors) were removed, by using spectral, DTM and CHM information, which increased LCC accuracies by $18 \%$ (for 2016) and 26\% (for 2012). Detailed patterns of stable areas and areas with removal and deposition of material were detected on the landslide, which likely correlate to slide-type and flow-type processes. LCC are most dynamic within the landslide area; major changes occurred from grassland to trees, from bare soil to grassland and from trees to grassland, while the surrounding forested areas remained unchanged.

\section{ACKNOWLEDGEMENTS}

Funding was awarded by the Computational Geo-Ecosystem group of the Institute for Biodiversity and Ecosystem Dynamics (IBED) of the University of Amsterdam. The Land Vorarlberg (www.Voralberg.at) has kindly allowed us to use geographical datasets, orthophotos and LiDAR data, available from the VOGIS repository. The GIS-studio (www.GIS-studio.nl) of the University of Amsterdam is thanked for computational support.

\section{REFERENCES}

Aguirre-Gutiérrez, J., Seijmonsbergen, A. C., \& Duivenvoorden, J. F., 2012. Optimizing land cover classification accuracy for change detection, a combined pixelbased and object-based approach in a mountainous area in Mexico. Applied Geography, 34, 29-37.

Anders, N., Seijmonsbergen, A., and Bouten, W., 2013. Geomorphological change detection using object-based feature extraction from multi-temporal lidar data. Geoscience and Remote Sensing Letters, IEEE, 10(6):1587-1591.

Congalton, R. G., 1991. A review of assessing the accuracy of classifications of remotely sensed data. Remote Sensing of Environment, 37(1):35-46.

Desclee, B., Bogaert, P., and Defourny, P., 2006. Forest change detection by statistical object-based method. Remote Sensing of Environment, 102(1):1-11.

Eisank, C., Smith, M., and Hillier, J., 2014. Assessment of multiresolution segmentation for delimiting drumlins in digital elevation models. Geomorphology, 214:452-464.

Esch, T., Thiel, M., Bock, M., Roth, A., \& Dech, S., 2008. Improvement of image segmentation accuracy based on multiscale optimization procedure. IEEE Geoscience and Remote Sensing Letters, 5(3), 463-467.

Friebe, J. (2007). Geologie der Osterreichischen bundeslander vorarlberg. Geologische Bundesanstalt, Wien, page 154.

James, L. A., Hodgson, M. E., Ghoshal, S., and Latiolais, M. M., 2012. Geomorphic change detection using historic maps and DEM differencing: The temporal dimension of geospatial analysis. Geomorphology, 137(1):181-198.
Jaritz,W., Marte, R., 2008. 75 Jahre Sanierung Großhangbewegung Doren (Vorarlberg) - Ungleiches Ringen zwischen Natur und Technik? Moser/Jaritz Ziviltechniker GmbH. http://mediatum.ub.tum.de/doc/1138083/1138083.pdf

Lahousse, T., Chang, K. T., \& Lin, Y. H., 2011. Landslide mapping with multi-scale object-based image analysis-a case study in the Baichi watershed, Taiwan. Natural Hazards and Earth System Sciences, 11(10), 2715-2726.

Latypov, D., 2002. Estimating relative lidar accuracy information from overlapping flight lines. ISPRS Journal of Photogrammetry and Remote Sensing, 56(4):236-245.

Li, X., Cheng, X., Chen, W., Chen, G., \& Liu, S., 2015. Identification of forested landslides using LiDar data, objectbased image analysis, and machine learning algorithms. Remote Sensing, 7(8), 9705-9726.

Machala, M. and Zejdova, L., 2014. Forest mapping through object-based image analysis of multispectral and lidar aerial data. Eur.J.Remote Sens, 47:117-131.

Maier, B., Tiede, D., \& Dorren, L.K.A., 2006. Assessing mountain forest structure using airborne laser scanning and landscape metrics. Salzburg: Int Archives Photogrammetry Remote sensing Spatial Information Science XXXVI-4/C42.

Mucher, C., Roupioz, L., Kramer, H., Bogers, M., Jongman, R., Lucas, R., Kosmidou, V., Petrou, Z., Manakos, I., and PadoaSchioppa, E., 2015. Synergy of airborne lidar and worldview-2 satellite imagery for land cover and habitat mapping. International Journal of Applied Earth Observation and Geoinformation, 37:48-55.

Muukkonen, P., \& Heiskanen, J., 2005. Estimating biomass for boreal forests using ASTER satellite data combined with standwise forest inventory data. Remote Sensing of Environment, 99(4), 434-447.

Parent, J. R., Volin, J. C., and Civco, D. L., 2015. A fullyautomated approach to land cover mapping with airborne lidar and high resolution multispectral imagery in a forested suburban landscape. ISPRS Journal of Photogrammetry and Remote Sensing, 104:18-29.

Wang, L., Sousa, W. P., \& Gong, P., 2004. Integration of object-based and pixel-based classification for mapping mangroves with IKONOS imagery. International Journal of Remote Sensing, 25(24), 5655-5668.

Wheaton, J. M., Brasington, J., Darby, S. E., and Sear, D. A., 2010. Accounting for uncertainty in dems from repeat topographic surveys: improved sediment budgets. Earth Surface Processes and Landforms, 35(2):136-156.

Wulder, M. A. and Seemann, D., 2003. Forest inventory height update through the integration of lidar data with segmented landsat imagery. Canadian Journal of Remote Sensing, 29(5):536-543.

Zhou, W., Troy, A., and Grove, M., 2008. Object-based land cover classification and change analysis in the Baltimore metropolitan area using multi-temporal high resolution remote sensing data. Sensors, 8(3):1613-1636. 


\title{
Assessment of the interaction of land-cover change on shallow landslide occurrence using an automated object-based approach
}

\author{
M. Kamps ${ }^{a}$, A.C. Seijmonsbergen ${ }^{a}$, M. Rutzinger ${ }^{b c}$ and T. Zieher ${ }^{\text {bc }}$ \\ ${ }^{\text {a} U n i v e r s i t y ~ o f ~ A m s t e r d a m ~-~ I n s t i t u t e ~ f o r ~ B i o d i v e r s i t y ~ a n d ~ E c o s y s t e m ~ D y n a m i c s ~(I B E D) ~-~ m a r t i j n t k a m p s @ g m a i l . c o m ~-~}$ \\ a.c.seijmonsbergen@uva.nl \\ ${ }^{\mathrm{b}}$ University Innsbruck - Institute for Geography \\ ${ }^{c}$ Austrian Academy of Sciences - Institute of Interdisciplinary Mountain Research (IGF) - martin.rutzinger@oeaw.ac.at - \\ thomas.zieher@uibk.ac.at
}

\section{KEY WORDS: Data Synergy, OBIA, Landslide, LiDAR, Orthophotos, Land Cover Change}

\begin{abstract}
Land-cover change could considerably lower landslide triggering rainfall thresholds allowing precipitation events with shorter recurrence intervals to initiate shallow landslides. This research focusses on developing an automated, robust and upscalable workflow to quantitatively assess the effect land-cover change has on initiating rainfall induced shallow landslides in the Laternser Valley. Land-cover is classified using four sets of high resolution orthophotos (198x, 2001, 2006, 2009; 0.25 m spatial resolution) by applying an object-based approach with eCognition software. The correlation between land-cover change and landslide occurrence was assessed by analyzing land-cover change trends in the vicinity ( $<25$ meters) of mapped shallow landslides. The obtained classification accuracy ranges from $76 \%$ for $198 \mathrm{x}$ to $88 \%$ for 2009 . The relative area undergoing land-cover change is $18 \%$ in the whole Laternser valley and $34 \%$ in the vicinity of landslides. Overall land-cover change trends indicate a shift from grassland to forest in the whole Laternser valley. However, in the vicinity of landslides the opposite is observed, namely a shift from forest to grassland and grassland to bare soil. Even though a general vegetation reduction is detected in the vicinity of landslides no correlation between LCC and landslide occurrence could be established yet.
\end{abstract}

\section{INTRODUCTION}

Shallow landslides are geomorphological processes occurring in mountainous regions. Shallow landslide susceptibility depends on several factors such as topography, changing land cover and characteristics of subsurface material. Detailed knowledge of fine-scale past and present land cover change (LCC) is a prerequisite for modelling and analysing landslide susceptibility. Classification of high resolution orthophotos - as a transparent and transferable method - is well suited to efficiently and accurately document LCC and to investigate the relation with known landslide-triggering rainfall events. The aims of this research are:

(1). To develop a workflow for automated object-based LCC detection using high resolution orthophotos in the Laternser valley, Austria.

(2). To analyse the correlation between LCC and the temporal occurrence of rainfall triggered shallow landslides.

\section{STUDY AREA}

The Laternser valley is located in the Eastern Alps in Vorarlberg, the westernmost province of Austria. Vorarlberg is a mountainous province characterized by large topographic differences and heterogeneous land-cover. The Laternser valley is situated between $47.23-47.31^{\circ} \mathrm{N}$ and $9.66-9.84^{\circ} \mathrm{E}$ and comprises of an area of $52.1 \mathrm{~km}^{2}$ (Figure 1). Approximately $68 \%$ of the total area has an inclination between 20 and 50 degrees making it generally susceptible to landslides. The geological setting of Vorarlberg was formed by succession of calcareous, dolomitic and pelitic sediments originating from the Mesozoic era (Friebe, 2007). Vorarlberg has a typical Alpine geomorphology characterized by subglacial till, moraine ridges,

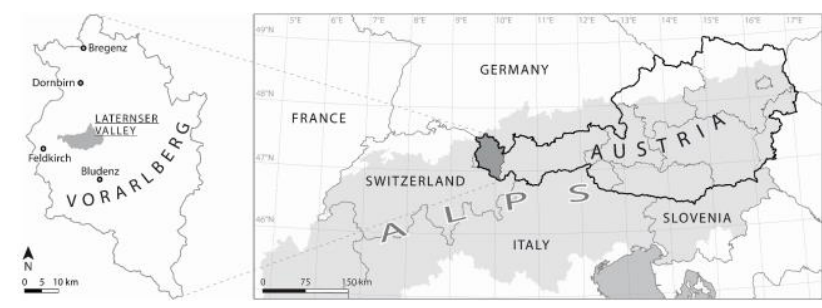

Figure 1. Location of the Laternser Valley in Vorarlberg, western Austria.

ice-marginal landform deposits and cirques (Seijmonsbergen et al., 2014). The Laternser valley is dominated by quaternary, penninic and helvetic nappes (Friebe, 2007) and is drained by a tributary of the Rhine, the river Frutz.

\section{METHODS}

\subsection{Data}

Table 1 shows the meta data of the multi-temporal orthophotos utilized in this research.

\begin{tabular}{|l|l|l|l|l|}
\hline $\begin{array}{l}\text { Orthophoto } \\
\text { series }\end{array}$ & $\begin{array}{l}\text { Photo } \\
\text { scale }\end{array}$ & $\begin{array}{l}\text { GSM } \\
{[\mathrm{m}]}\end{array}$ & $\begin{array}{l}\text { Radiometric } \\
\text { resolution }\end{array}$ & Bands \\
\hline $198 \mathrm{x}$ & $\begin{array}{l}10.000- \\
41.000\end{array}$ & 0.2 & 8 bit & CIR \\
\hline 2001 & $\begin{array}{l}10.000- \\
15.000\end{array}$ & 0.25 & 8 bit & CIR \\
& $10.000-$ & 0.125 & 8 bit & RGB \\
\hline 2006 & 12.000 & & & RGB \\
& $10.000-$ & 0.125 & 8 bit & \\
\hline 2009 & 15.000 & & & \\
\hline
\end{tabular}


Table 1. Meta data of the high resolution orthophoto series. GSD: ground sampling distance, CIR: color infrared imagery, RGB: Red, green, blue; true color imagery. Adopted from Zieher et al., 2016.

A well-documented field- and orthophoto-based inventory of shallow landslides is available for the Laternser Valley in Vorarlberg, developed as part of the project Climate Induced System Status Changes at Slopes and Their Impact on Shallow Landslide Susceptibility (C3S-ISLS). Most of those registered landslides relate to the 1999 and 2005 rainfall events. Figure 2 shows the mapped landslides in three time periods that were analysed in this research. This multi-annual landslide inventory with a 1:400 nominal scale is used as reference dataset for the location of shallow landslides.

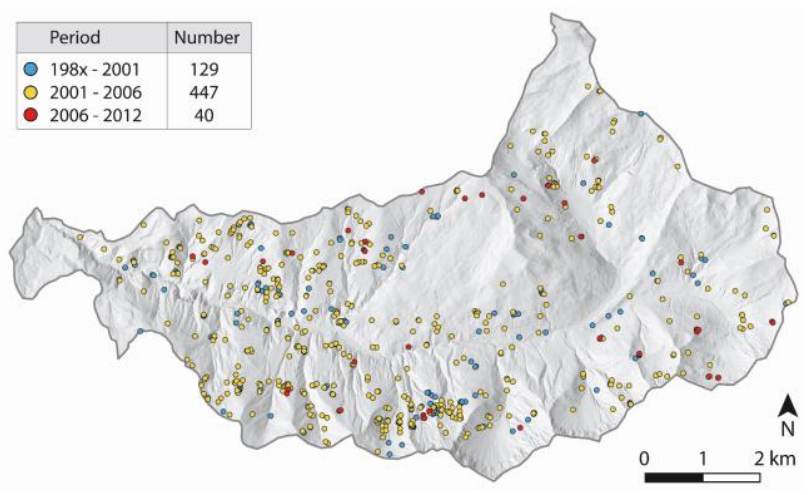

Figure 2. Mapped shallow landslides in the Laternser valley.

\subsection{Workflow}

Figure 3 shows a schematic overview of the general workflow of this research. Four steps are included: pre-processing, analysis, accuracy assessment and the deliverables to work towards the two aims: land-cover change maps and assessing the correlation between LCC and shallow landslide occurrence.

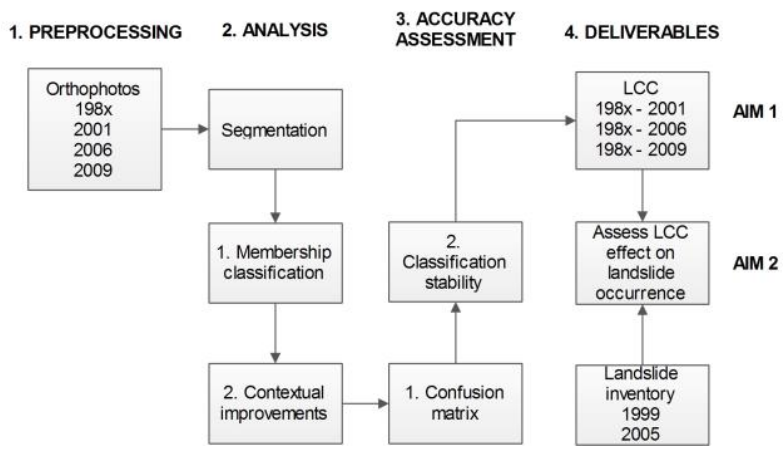

Figure 3. Schematic overview of the workflow.

\subsection{Pre-processing and software}

Pre-processing of the data was performed using ArcGIS 10.3.1 and QGIS 2.4.0. eCognition Developer 9.1.2 software was used to develop the LCC maps. Pre-processing consists of resampling to $0.25 \times 0.25 \mathrm{~m}$ and tiling the orthophotos to tiles of
$2750 \mathrm{~m}$ edge length including an overlap area of $10 \%$ to ensure minimization of object segmentation edge effects.

\subsection{Land Cover Change Analysis}

Our workflow in eCognition Developer for LCC combines segmentation and object-based image analysis of four sets of high resolution orthophotos. Subsequently, post-classification change detection using image differencing was utilized.

Multiscale multiresolution segmentation was utilized to obtain image objects of every land-cover class throughout the whole study area. Scale parameters for multiresolution segmentation were determined with the estimation of scale parameter tool (ESP2) developed by Drăguţ et al. (2014).

The class hierarchy used in this research is: forest, grassland, shadow, bare soil and infrastructure.

To classify the land-cover classes, supervised feature space optimization (FSO) is used to determine the parameter combinations with the highest separation distance (Platt \& Rapoza, 2008). 250 representative (based on expert knowledge) samples of every land-cover class are used by the FSO algorithm on 48 distinct features.

Classification consisted of two steps: (1) stratified membership classification and (2) contextual improvements. The classification workflow was developed and optimized in one tile and subsequently upscaled to the whole of Laternser valley for analysis and validation. A minimum mapping unit of $3.125 \mathrm{~m}^{2}$ (50 pixels) was utilized.

The stratified membership classification was based on the feature thresholds derived from the FSO. The stratified membership classification workflow was designed to be conservative in order to minimize false positives. The contextual improvements were used to classify remaining false negatives.

The contextual improvement rationale was developed to account for landscape homogeneity, variability and overlap in the feature space. The contextual improvements were based on expert interpretation of false positive and false negatives in combination with the original feature space parameters of the objects. Every rule in the workflow only removed a small portion of omission and commission errors.

The land-cover change analysis is performed using postclassification image differencing by automatic comparison of image sub-object hierarchies (Gutierrez et al., 2012; Zhou et al., 2008).

Derivation of LCC trends is utilized for interpretation of LCC in the vicinity $(<25 \mathrm{~m})$ of shallow landslides to quantitatively assess the effect of LCC on rainfall induced landslide occurrence (Begueria, 2006). An automated ArcGIS model is used to calculate the area per land-cover change class using a 25 [m] buffer around the scarpoints derived from the C3S-ISLS project.

\subsection{Accuracy Assessment}

The accuracy assessment consists of two parts: (1) standard confusion matrix, (2) classification stability. 
A stratified sampling scheme was used to obtain 150 samples per period based on the relative occurrence of land-cover classes and the entire range of membership probabilities to obtain a representative sample. Additionally, 150 samples in the vicinity $(<25 \mathrm{~m})$ of landslides were specified to compare the standard overall accuracy with the landslide specific application.

In this study the classification stability was used to differentiate between the segments correctly classified by the initial membership-based classification and the contextual improvements.

\section{RESULTS}

\subsection{Land Cover Change Analysis}

Classification accuracies range from $76 \%$ (198x) to $88 \%$ for the 2009 orthophotos. Kappa statistic falls between $64 \%$ (198x) to $81 \%$ in 2009 . The context based classification ruleset improved $52 \%$ of all accurately classified samples in $198 \mathrm{x}$ and decreases over time to $39 \%$ in 2009 due to better image quality and reduced overlap in the feature space in the more recent years. This research shows the effectiveness of utilizing context to improve classification accuracy after initial membership-based classification in an object-based workflow to deal with overlap in the feature space.

Figure 4 shows the results of the LCC between 198x and 2009 .

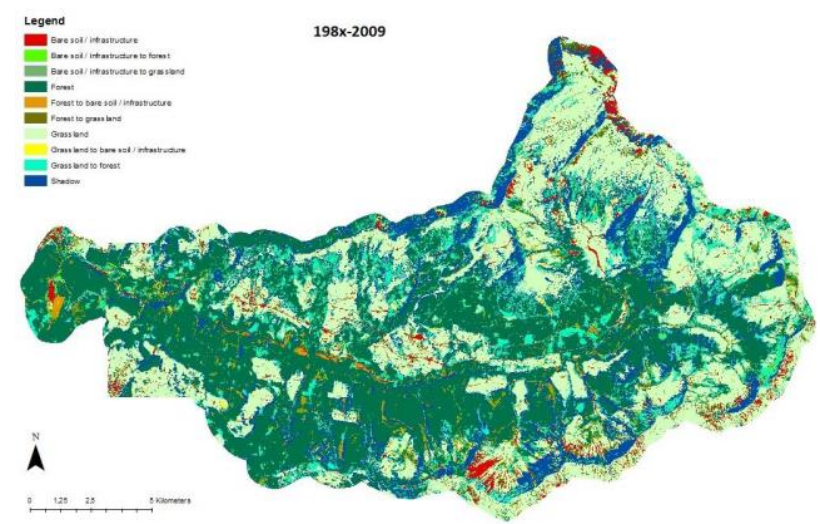

Figure 4. LCC results 198x-2009.

Figure 5 shows the relative area change for every land-cover class from 198x to 2009 in the whole Laternser Valley. A general increase in forest is observed while grassland decreases. Approximately $18 \%$ of the total area undergoes LCC between 198x and 2009. The biggest LCC is the shift from grassland to forest attributing to $10.5 \%$ of the area and $58 \%$ of total LCC. This LCC shift occurs mainly at open areas within the canopy and at the edges of the tree-line.

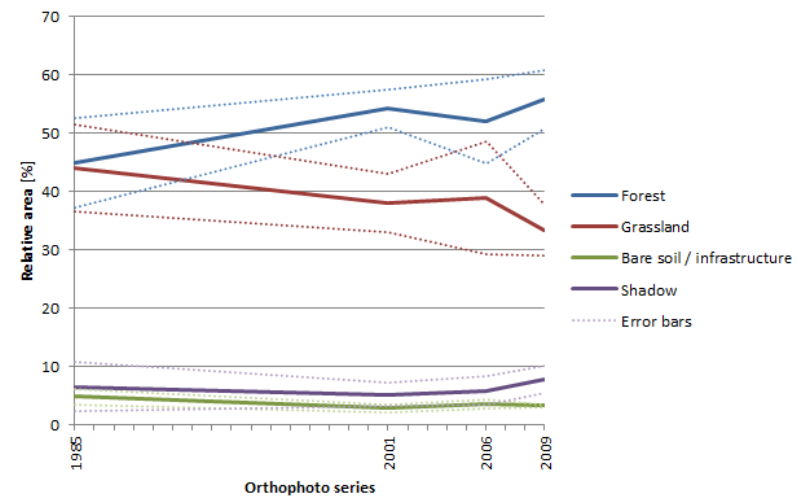

Figure 5. Relative land cover change for the four classes per orthophoto series in the whole Laternser valley. Error bars are derived from the average user/producer accuracy per land-cover class.

\subsection{Change Detection and Landslides}

Land-cover changes that occur in the vicinity $(<25 \mathrm{~m})$ of landslides are shown in figure 6 . Land-cover changes account for $33.9 \%$ (198x-2009) within the $25 \mathrm{~m}$ buffer area. The landcover changes that occur the most are: a shift from grassland to bare soil / infrastructure, and a shift from forest to grassland.

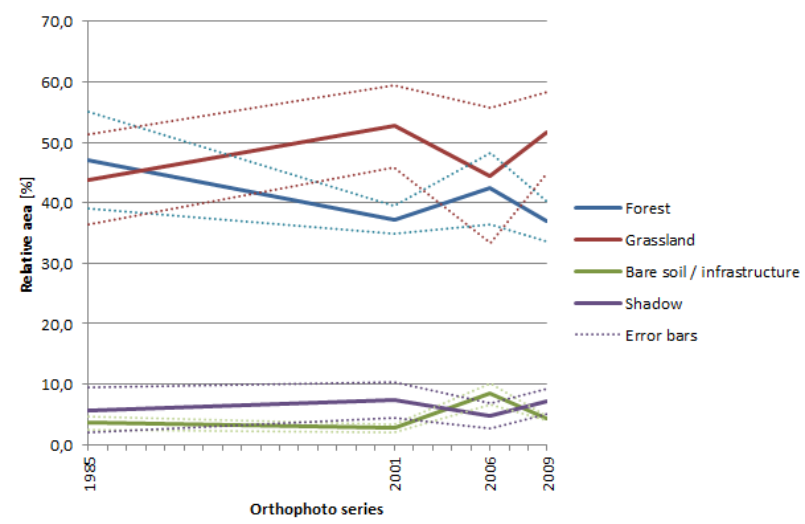

Figure 6. Relative land cover change for the four classes per orthophoto series in the vicinity of landslides. Error bars are derived from the average user/producer accuracy per land-cover class.

\section{CONCLUSION}

In the Laternser valley $18 \%$ of the total area undergoes LCC while in the vicinity of landslides LCC occurs on $34 \%$. Although overall LCC trends indicate a shift from grassland to forest for the Laternser valley, the opposite is observed near shallow landslides. So far, a clear correlation between LCC and landslide occurrence could not be established. The question remains whether the observed land-cover change actually caused the landslides or was the eventual effect of the landslides. Further analysis will incorporate topographic LiDAR data to optimize segmentation and classification and integrate additional explanatory variables for shallow landslide susceptibility such as slope angle, aspect and curvature. Additional ancillary data such as lithology would contribute to strengthening the analysis. 


\section{ACKNOWLEDGEMENTS}

We thank the Federal State of Vorarlberg for providing the necessary data. This work has been conducted within the project C3S-ISLS, which is funded by the Austrian Climate and Energy Fund, 5th ACRP Program.

\section{REFERENCES}

Aguirre-Gutiérrez, J., Seijmonsbergen, A. C., \& Duivenvoorden, J. F. (2012). Optimizing land cover classification accuracy for change detection, a combined pixelbased and object-based approach in a mountainous area in Mexico. Applied Geography, 34, 29-37.

Beguería, S. (2006). Changes in land cover and shallow landslide activity: a case study in the Spanish Pyrenees. Geomorphology, 74(1), 196-206.

Drăguţ, L., Csillik, O., Eisank, C., \& Tiede, D. (2014). Automated parameterisation for multi-scale image segmentation on multiple layers. ISPRS Journal of Photogrammetry and Remote Sensing, 88, 119-127.

Friebe, J. (2007). Geologie der österreichischen bundesländer vorarlberg. Geologische Bundesanstalt, Wien, 154.

Platt, R. V., \& Rapoza, L. (2008). An evaluation of an objectoriented paradigm for land Use/Land cover classification. The Professional Geographer, 60(1), 87-100.

Seijmonsbergen, A. C., De Jong, M. G., de Graaff, L. W., \& Anders, N. S. (2014). Geodiversität von vorarlberg und liechtenstein-geodiversity of vorarlberg and liechtenstein. Haupt Verlag AG.

Zieher, T.; Perzl, F.; Rössel, M.; Rutzinger, M.; Meißl, G.; Markart, G. \& Geitner, C. (2016), 'A multi-annual landslide inventory for the assessment of shallow landslide susceptibility - Two test cases in Vorarlberg, Austria', Geomorphology 259, $40-54$.

Zhou, W., Troy, A., \& Grove, M. (2008). Object-based land cover classification and change analysis in the baltimore metropolitan area using multitemporal high resolution remote sensing data. Sensors, 8(3), 1613-1636. 


\title{
COASTAL CHANGES AND MOVEMENTS IN THE WIDER VLORA (ALBANIA) AREA
}

\author{
U. Kanjir ${ }^{\text {a, * }, ~ N . ~ G r e g o r i c ̌ ~ B o n ~}$ \\ ${ }^{a}$ ZRC SAZU, Institute of Anthropological and Spatial studies, Department of remote sensing, Novi trg 2, 1000 Ljubljana, Slovenia - \\ ursa.kanjir@zrc-sazu.si \\ ${ }^{\mathrm{b}}$ ZRC SAZU, Institute of Anthropological and Spatial studies, Department of anthropology, Novi trg 2, 1000 Ljubljana, Slovenia - \\ natasa.gregoric@zrc-sazu.si
}

KEY WORDS: Change detection, change vector analysis, GEOBIA, urban growth, coastal erosion, migration, Albanian Riviera, ecology, anthropology, multidisciplinary research

\begin{abstract}
:
This paper explores the environmental change monitoring on the Albanian coastline (Vlora area) by analysing optical remote sensing data. Changes along this coastline are driven by coastal erosion and abusive urban construction of mostly tourist complexes and other man-made processes and pressures (e.g. sandpits, poor wastewater management, etc.). The change detection is based on yearly Landsat data of the period between 1984 and 2015. On the basis of mentioned images, each year's Normalized Difference Water Index (NDWI), Normalized Difference Build-up Index (NDBI) and Normalized Difference Vegetation Index (NDVI) are extracted. With the use of change vector analysis technique the combination of these indexes were then compared between each other. The quantitative extent of coastal changes was defined using geographic object-based image analysis (GEOBIA). By obtaining GEOBIA post-classification results we compared both change detection approaches of pairs of one period (2010-2015). The coastline and coastal change maps produced at $30 \mathrm{~m}$ resolution, applying both pixel- and object-based approaches, showed that CVA approach is a preferred technique when analysing Landsat data for coastal change detection. Apart from identifying spatial changes through time, the objective of this study is to show that these landscape changes correspond with the population movements and migrations. This study argues that movements of both people and landscape strongly impact each other and form an intertwining and corresponding relationship.
\end{abstract}

\section{INTRODUCTION}

Over the last decades, ecological environment has changed dramatically due to increasingly intensifying social and economic development. Accurate and timely information about land cover patterns and changes in urban areas is crucial for urban land management decision-making, ecosystem monitoring and urban planning. A large amount of environmental and social data at relatively high spatiotemporal resolutions has become available to monitor and manage natural resources, to assess environmental impact and to observe human patterns. These data are the foundation for better understanding of relationships and interactions between human and natural phenomena.

This study focuses on geomorphologic and demographic changes in wider Vlora area, as it is said that Albania is one of the countries with the highest levels of erosion and migration in Europe (Buchroithner, 2000; Grazhdani and Shumka, 2007; King, 2005; Vullnetari, 2012). This work is ongoing and in continuation of previous research on changes of natural and demographic movements (Kanjir and Gregorič Bon, 2016). It examines land changes detected on Landsat data from 1984 to 2015. Several scholars (Allen and Kupfer, 2000; Baker et al., 2007; Lorena et al., 2002; Xiaolu and Bo, 2011) have conducted studies of change detection using change vector analysis from Landsat data. Authors (Berberoglu and Akin, 2009) undertook a comparison of different change detection techniques in the Mediterranean environment and although different change detection algorithms have their own merits and advantages, change vector analysis resulted in the largest overall accuracy. We first applied change vector analysis on the multi-temporal remote sensing data, where we detected two major types of coastal changes seen in this area: coastal erosion and coastal urbanisation. Furthermore, change vector analysis approach was compared and evaluated with the post-classification change detection results obtained with the geographic object-based image analysis (GEOBIA). Post-classification comparison methods detect land cover change by comparing independently produced classifications of images from different dates (Rogan and Chen, 2004). GEOBIA is a frequently used technique in remote sensing and has demonstrated great potential for classification and change detection of high-spatial resolution multispectral imagery in heterogeneous urban environments (Boldt et al., 2012; Chen et al., 2012; Kanjir et al., 2011; Zhou et al., 2008).

Finally, this paper explores the demographical changes along the southern Albanian coastline using socioeconomic data. It questions if the correlation found between changes seen from optical satellite images and corresponding migration data of Albania from the last three decades could offer insights into the complex environmental and social changes.

\subsection{Changes on the Albanian coastline}

The Albanian coastline has faced abrupt changes in the course of the last decade. The country has over $400 \mathrm{~km}$ of coastline, approximately $150 \mathrm{~km}$ of which at its southern end is highly erosive (Gruda and Dollma, 2013) and of high ecological value. The entire stretch of southern coast, extending from Vlora to Ftelis Bay (Greece), has been a target of intense erosion, about $20-50 \mathrm{~cm}$ per year (Qiriazi and Sala, 2000). The causes of coastal erosion are related to several general and local factors, both human (e.g. removal of inert materials from the riverbeds and coastline, unplanned constructions, hydrological construction on the shores) and naturally driven (e.g. coastal terrigenous material, steep slopes, river deposits, irregular precipitation). Spatial distributions of annual coastal changes are valuable information for coastal monitoring and 
environmental assessment studies. In Albania there is poor periodic control of erosion and hardly any legal regulations are in place for coastal protection.

Apart from erosion being the main reason for land changes in this coastal area, rapid urbanisation has also had a great impact on the land changes, especially after the start of the new millennium when a greater amount of remittances were being sent by migrants, or around 2010 when many of the migrants started to return back home. These newly constructed sites along the coast tend to give priority to capital accumulation before any consideration of social values, and therefore do not follow any urban planning regime.

\subsection{Population movements in Albania}

Albania's population has been subject to migrations throughout the centuries and migrations still play a major role in the lives of many Albanians today. (Gregorič Bon, 2016) has shown how migration is embodied in people's' practices and is reified in the Albanian architecture. The exclusive period when migration flow in the area was abruptly stopped was in 1945 with the rise of the communist regime, when private ownership of cars was forbidden and in-country movements were limited. Nevertheless, due to the development of textile and food industry in Vlora in this period, many people living in rural areas moved to this coastal city. Major migrations out of the country started after 1990 with the collapse of the communist regime, when both authorized and unauthorized migration in Albania acquired epic proportions. Massive migration "exoduses" that have importantly influenced the Albanian politics, economy, society as well as landscape, took place at the beginning of 1990's (King, 2005; Vullnetari, 2012). After the year 2000, the number of migrants stabilised whereas the number of remittances and other material goods sent sporadically to home location increased. Remittances were often the main source of income for particular households and economies in Albania (Wong, 2006) and had influenced the rapid urbanisation of the area. Another important milestone marks the Albanian entrance into the Schengen Zone area in December 2010, which had a stabilizing effect on migration, but at the same time many migrants began to return due to fiscal and economic crisis in Greece and Italy. The years 2010 and 2011 report the largest number of return migration in relation to the total population living in Vlora (INSTAT, 2016), leading to an increase in the process of urbanisation in this area.

\section{DATA AND METHODOLOGY}

\subsection{Study site, data used and time slots}

This research focuses on the wider area of the city of Vlora, located in the southern part of Albania, facing the Adriatic Sea (see Figure 1). Vlora is the municipal seat of the eponymous County, one of the 12 Albanian counties, and it is the second largest port in Albania. Over the last decade, the city's coastal area is being affected by affronting urban construction of mostly touristic complexes (hotels, apartment houses, touristic villages, etc.). This study area therefore represents a complex scene, as the landscape varies in spatial and temporal terms, affected by different factors, both natural and anthropogenic, interacting with each other, and resulting in multiple consequences.

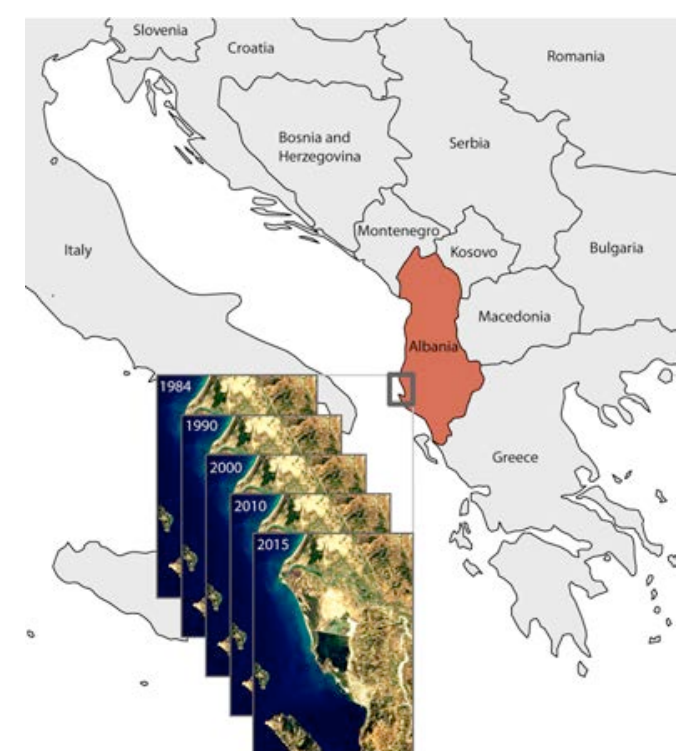

Figure 1. Location of the study area and Landsat data set.

The data set of this study is comprised of five Landsat data collected in 1984, 1990, 2000, 2010 and 2015. Time slots of Landsat data were selected deliberately, as they coincide with the periods of greater political changes in Albania:

- 1984 - 1990: communist regime (1945-90)/forbidden outcountry migrations, limited and controlled in-country migrations.

-1990 - 2000: fall of communism - transition period/massive migrations (mainly to Greece and Italy).

- 2000 - 2010: democracy/stabilized migration, remittances, growing number of returnees, in-country migrations.

- 2010 - 2015: Albania joins the Schengen Area/the number of migrations rises again in 2014.

All five Landsat images used were taken in the same season (late July-August-early September) in order to minimize change detection error introduced by seasonal differences. But for the Landsat 8 image from 2015, all other images are the product of Landsat 5 sensor. All multispectral images have $30 \mathrm{~m}$ of spatial resolution.

To describe population migration patterns for the particular time-periods we have used the INSTAT national statistical data.

\subsection{Image pre-processing}

Images acquired at different times usually have different amounts of haze and dust in the atmosphere. These differences can mask real changes or make similar land covers appear to have changed (Berberoglu and Akin, 2009). To overcome this problem, we applied atmospheric correction using ATCOR software. These corrections are important especially when comparing multi-temporal images (Jianya et al., 2008), e.g. when applying change vector analysis, as this technique is based on pixel-based radiometric comparison.

All images were also geometrically aligned between each other before the analysis. The importance of accurate spatial registration of multi-temporal imagery cannot be overstated, since mis-registration will give rise to false results. If high registration accuracy is not available (sub-pixel), image displacement will lead to inaccuracies in change detection scene.

Images were finally cropped to the desired geospatial extent corresponding to our study interest. 


\subsection{CVA Change detection}

There exist a number of change detection techniques, but none of them is universally optimal, as every method depends upon the application, data and the physical environment. Based on the results of research done by (Berberoglu and Akin, 2009) change vector analysis is the most appropriate model for handling the variability present in the Mediterranean land use/cover. We have used this method to obtain changes of erosion and urbanisation on the Albanian southern coast. The change vector analysis was implemented entirely with the use of open source software R.

Change vector analysis is a simple radiometric technique for land use/land cover change detection. It uses two spectral channels and calculates the magnitude of change and the direction of change between these two images (Malila, 1980). The direction or angle of the change vector is determined by the type of occurring change on the coastline (outline of coastal area) and/or at the coastal area. This procedure involves selecting a threshold to determine the extent of changed areas, and a conclusion on the extent of change is reached depending on whether the change magnitude exceeds a specific threshold.

In order to reduce the amount of redundant information for the detection of coastal erosion and urbanisation in our study area, we have used a combination of different band indexes instead of using satellite spectral bands directly. For the coastal erosion detection, we have used the combination of Normalized Difference Water Index (NDWI) and Normalized Difference Urban Index (UI) - or also called the build-up index (Zha et al., 2003). Furthermore, for the needs of urbanisation growth detection through time CVA we applied Normalized Difference Vegetation Index (NDVI) in combination with UI indexes. For a detailed description of these indexes please see (Kanjir and Gregorič Bon, 2016).

Results of changes in the coastal area of southern Albania detected with the CVA in the selected time periods were later compared with the statistical data of migrations that took place since the date of the first available satellite image from 1984 up until 2015. Before that, the CVA results of this pixel approach were compared with object change detection post-classification analysis, which is described below.

\subsection{GEOBIA classification}

Many Mediterranean land covers exhibit similar spectral characteristics making the separation in feature space by simple per-pixels classifiers difficult, leading to inaccurate classification (Berberoglu and Akin, 2009). We used objectbased approach to conduct the classification separately on the two Landsat images, from 2010 and 2015 respectively. With the GEOBIA approach, objects (segments) are first created and then used for further analysis. Object characteristics such as shape, spatial relations and reflectance statistics can be used for classification and change detection. In our procedure both times Landsat images were segmented using same segmentation parameters. Following the segmentation, we performed an example based feature extraction to classify each object into one of the classes. We were able to classify nine land cover classes on both images: sea, agriculture, urban environment, bare land, greenery, lagoon and shrubs.

Following the classification, we conducted a comparison of both land cover change detection methods for a period between 2010 and 2015 to obtain a change detection difference map.

\subsection{Accuracy assessment of classification}

Accuracy assessment of GEOBIA classification results was done using reference data created from visual interpretation. Stratified random samples were generated and it was determined whether they were correctly classified or not. A total number of 100 points were sampled, for each of the nine classes. Overall accuracies of both classification maps of 2010 and 2015 were $87.3 \%$ and $88.2 \%$ respectively. The final accuracy in GEOBIA strongly depends on the accuracy of the segmentation.

The change detection results derived from GEOBIA image pairs 2010-2015 were compared with CVA results of pairs of the same time period. OBIA change detection of selected period therefore serves as a verification methodology of the results obtained by CVA analysis.

\section{RESULTS}

\subsection{CVA}

3.1.1 Coastal erosion: The results of CVA show strong coastal erosion in the Vjosa river delta. It can be seen from the analysis of Landsat satellite images spanning the period 19842015 that delta erosion has progressively increased. The higher amount of yellow areas on the coast shows higher magnitude (or higher chance) of coastal changes between the selected dates (see Figure 2). Obvious delta shape changes of Vjosa River have probably resulted from the interaction between sediment discharge by the river, anthropogenic removal of coastal sediment, wave energy and tidal effects.
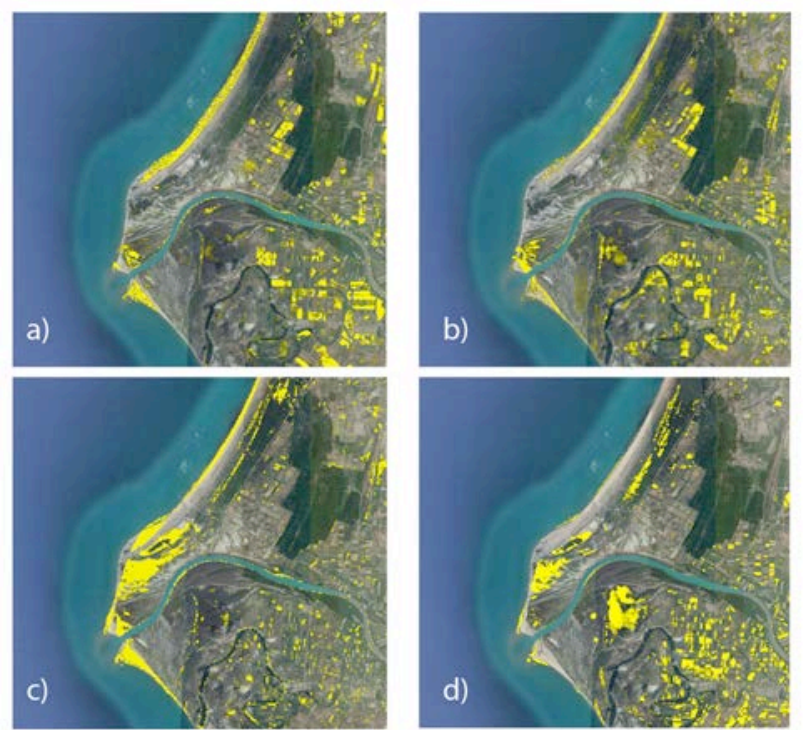

Figure 2. Change vector analysis results of the Vjosa delta area. The Figure presents changes in the periods of 1984-1990 (a), 1990-2000 (b), 2000-2010 (c) and 2010-2015 (d). Background layer on all sub-images is Google Earth image from 2015. 
3.1.2 Urban change: Urbanisation along the Albanian coast has been on a rapid increase since the late 1990s, which is when emigrants' revenue started to grow. This can be clearly seen from the results with the biggest change, taking place between 1990 and 2000 (Figure 3). The extent of urbanisation in and around the city of Vlora appears to be smaller than that brought about by coastal erosion in the delta of Vjosa River. This was expected, due to a lower spatial extent of constructions in the city compared to the greater deposits in Vjosa River.
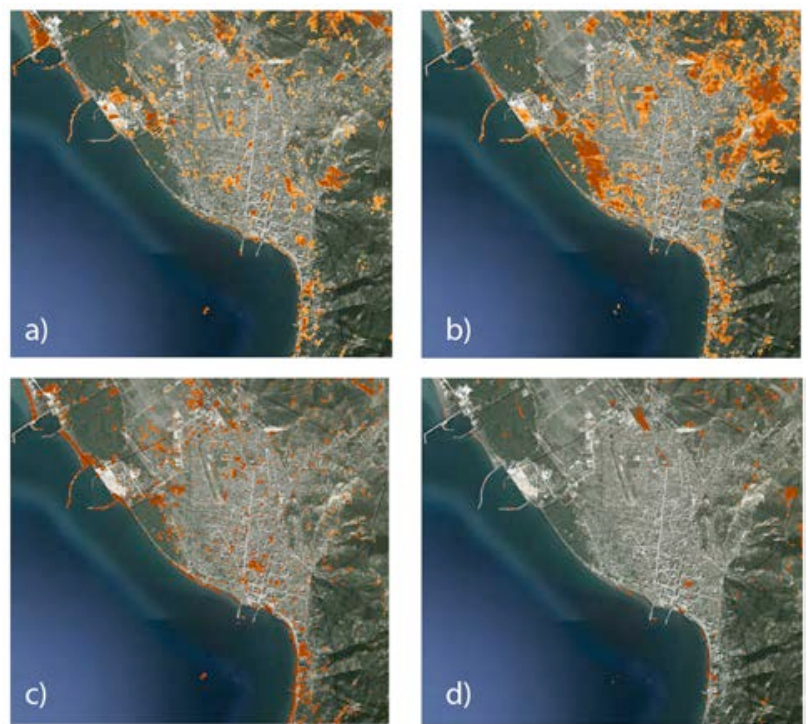

Figure 3. Changes of the urban areas through time in the city of Vlora. The Figure presents changes in the periods of 1984-1990 (a), 1990-2000 (b), 2000-2010 (c) and 2010-2015 (d). Background layer on all sub-images is Google Earth image from 2015.

\subsection{GEOBIA classification}

With the object-based approach we have obtained two classification images (see Figure 4), which were later analysed for change detection, also known as post-classification approach. Similar results as obtained with the CVA technique are detected also with the object-based approach. Major land cover changes can be seen for the most altering areas: at the delta of Vjosa River and around the city of Vlora.
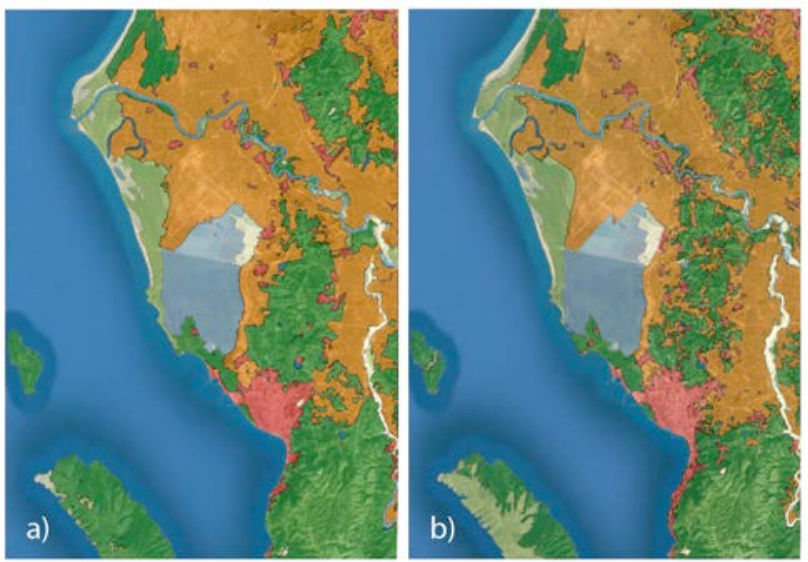

Figure 4. Object-based image analysis results for 2010 (a) and 2015 (b).

On the Figure 5 that shows change detection results from GEOBIA approach (red areas), vegetation changes in the inland can also be noted, but we have not given these changes any weight, as this is not within the scope of our interest. An important decrease in sediment on the coast is highlighted from Landsat data (green arrows), whereas sandy shoreline progradation in the mouth sector of the delta is the sediment brought by the river and by the tidal waves on the areas around the delta (black arrows).

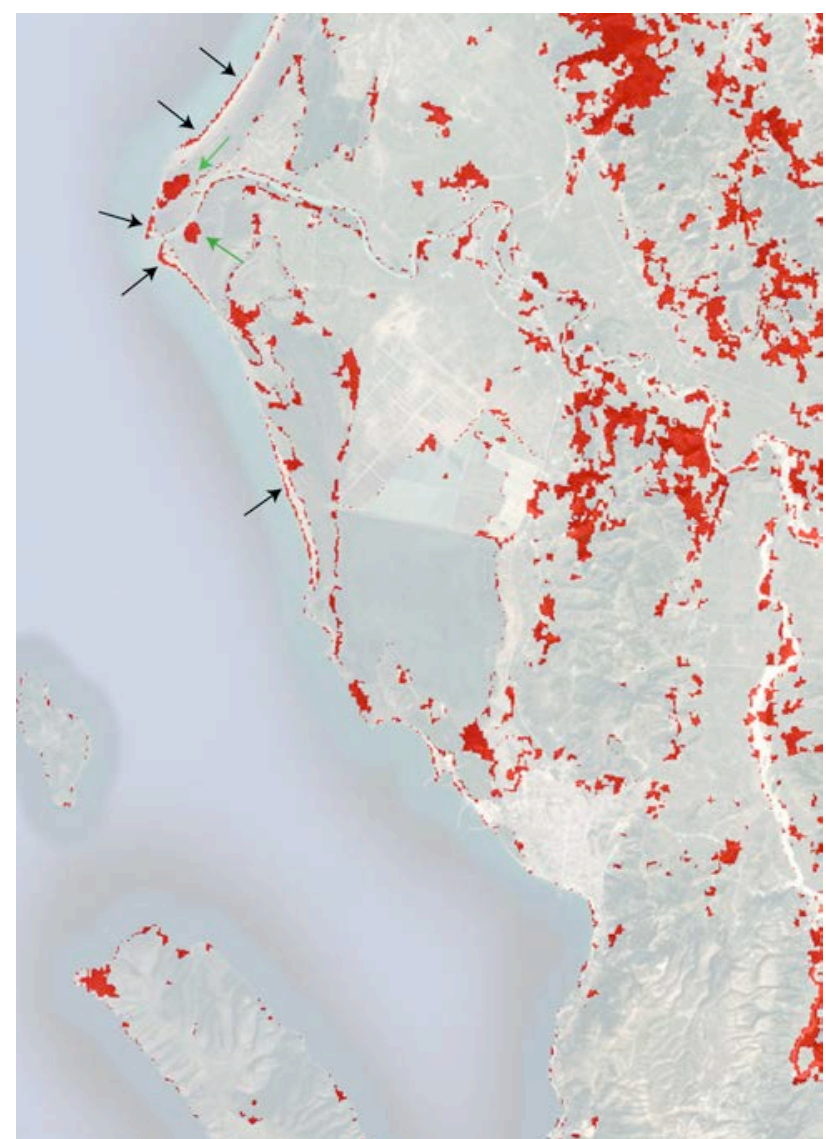

Figure 5. Figure shows change detection results (red areas) that have been detected between two classification maps obtained with the GEOBIA approach. Black arrows indicate progradation areas, whereas green arrows indicate loss of sediment in delta area.

\subsection{Comparison of both change analysis techniques}

Although land changes through time were detected with both change detection techniques, a profound visual examination of an experienced analyst shows that better change detection results are given with the CVA method. In order to test that, we have performed a comparison of change pixels from both techniques on the preselected area at the river delta. CVA approach has detected on the river delta on average $90 \mathrm{~m} \mathrm{(3}$ pixels) of coastal erosion, whereas GEOBIA approach detected $180 \mathrm{~m}$ (6 pixels) of change on average between the years 2010 and 2015 . The resolution of images is $30 \mathrm{~m}$; therefore one sample data (pixel) equals 900 square meters. According to visual estimation of erosion on the same evaluated areas there has been on average around 3 pixels of erosion during the 5 years, which indicates CVA's more exact result. GEOBIA approach performed poorer results due to the obvious exaggeration of object sizes while performing segmentation. CVA approach works on pixel level and is therefore more precise. We expect that the situation would be different when dealing with high-resolution data $(10 \mathrm{~m}$ or higher spatial resolution). 


\subsection{Correlation of land and socioeconomic changes}

While comparing the socio-economic data with the results from the remote sensing change detection analysis by periods, both changes correspond with each other (see Table 1).

\begin{tabular}{|l|l|l|}
\hline Date & Socioeconomic statistics & $\begin{array}{l}\text { Max width of } \\
\text { coastal } \\
\text { (CVA) }\end{array}$ \\
\hline Until 1990 & $\begin{array}{l}\text { Forbidden out country } \\
\text { migrations, limited and } \\
\text { controlled in-country } \\
\text { migrations }\end{array}$ & $<100 \mathrm{~m}$ \\
2000-2010 & $\begin{array}{l}\text { Massive migrations } \\
\text { Stabilized migration, } \\
\text { remittances, growing no. of } \\
\text { returnees, in-country } \\
\text { migration }\end{array}$ & $<400 \mathrm{~m}$ \\
2010-2015 & $\begin{array}{l}\text { Stable, the no. of migrations } \\
\text { rises again in 2014 }\end{array}$ & $<150 \mathrm{~m}$ \\
\hline
\end{tabular}

Table 1. Comparison of the data on migration and CVA results.

First period is marked with almost no migrations and only slight environmental changes. The most extensive migrations took place between 1990 and 2000, where at the same time river mouth mass changes of Vjosa substantially increased in comparison to the first period and the urbanisation growth is the most extensive in this era. The biggest environmental changes in Vjosa River are detected between 2000 and 2010 (up to 400 meters in width) when the migrations are stabilizing but remittances are at their highest, resulting in the most intense urbanisation growth in the Vlora area so far. In the last five years, when only half a decade has been taken into consideration, both migration and environmental changes are still under progress, but up to a much smaller extent than in the previous periods.

\section{CONCLUSION}

In this study we have shown that the environmental changes (coastal erosion and urbanization growth) have greatly influenced the wider Vlora area over the last thirty years. Our results of coastal changes driven by coastal erosion in the studied area confirm the findings of authors (Qiriazi and Sala, 2000), when they mention strong coastal erosion $(20-50$ $\mathrm{cm} /$ year) along the southern Albanian coastline. We argue that the main factor underpinning the erosion is connected with human induced changes in the area (e.g. material displacement). The vulnerability of the Vjosa delta involves a conjunction of various pressing issues attracting more and more scientific and political attention, underpinned by the tensions raised by the planned large hydropower dam projects up the Vjosa River, and the threats such projects pose for the sustainability of the river deltas in general. The future research will focus on the quantification and the extent of changes and their directions as detected from remote sensing data, and subject them to a statistical analysis.

At the same time we have performed a comparison between pixel- and object-based change detection in order to obtain the performance of CVA methodology on Landsat data, which we used as a main change detection methodology on this area. Coastal erosion and urban extension in the city of Vlora showed better performance while being detected with the CVA method comparing to GEOBIA changes, which is in accordance with Berberoglu and Akin (2009) statement. Future research anticipates similar change detection research on high-resolution data (e.g. Sentinel-2 data) to compare techniques and their performance on different remote sensing data.

The detected spatial changes correspond to the social, political, economic and demographic changes of Albania during our period of study. We can therefore state that the changes seen in the environment strongly indicate larger socio-economic, political and demographic changes taking place within the state.

\section{ACKNOWLEDGEMENTS}

This research has been funded by the Slovenian Research Agency (2014-2017).

\section{REFERENCES}

Allen, T.R., Kupfer, J.A., 2000. Application of Spherical Statistics to Change Vector Analysis of Landsat Data: Southern Appalachian Spruce-Fir Forests. Remote Sens. Environ., 74, pp. 482-493.

Baker, C., Lawrence, R.L., Montagne, C., Patten, D., 2007. Change detection of wetland ecosystems using Landsat imagery and change vector analysis. Wetlands, 27, pp. 610-619.

Berberoglu, S., Akin, A., 2009. Assessing different remote sensing techniques to detect land use/cover changes in the eastern Mediterranean. Int. J. Appl. Earth Obs. Geoinformation, 11, pp. 46-53.

Boldt, M., Thiele, A., Schulz, K., 2012. Object-based urban change detection analyzing high resolution optical satellite images. In: Michel, U., Civco, D.L., Ehlers, M., Schulz, K., Nikolakopoulos, K.G., Habib, S., Messinger, D., Maltese, A. (Eds.), Proc. SPIE 8538. Presented at the Earth Resources and Environmental Remote Sensing/GIS Applications III, p. $85380 \mathrm{E}$.

Buchroithner, M.F., 2000. Remote Sensing for Environmental Data in Albania: A Strategy for Integrated Management, Softcover reprint of the original 1st ed. 2000 edition. Springer, Dordrecht, Boston.

Chen, G., Hay, G.J., Carvalho, L.M.T., Wulder, M.A., 2012. Object-based change detection. Int. J. Remote Sens., 33, pp. 4434-4457.

Grazhdani, S., Shumka, S., 2007. New Water Culture of South East European Countries-AQUA 2005An approach to mapping soil erosion by water with application to Albania. Desalination, 213, pp. 263-272.

Gregorič Bon, N., 2016. Rooting Routes: (Non)Movements in Southern Albania. In: Gregorič Bon, N., Repič, J. (Eds.), Moving Places. Relations, Return and Belonging. Berghahn Books, Oxford, New York.

Gruda, G., Dollma, M., 2013. Albania. In: Pranzini, E., Williams, A. (Eds.), Coastal Erosion and Protection in Europe. Routledge, London, New York, pp. 345-354.

INSTAT, 2016. Instituti i Statistikave Republika e Shqiperise [WWW Document]. URL http://databaza.instat.gov.al (accessed 7.8.16). 
Jianya, G., Haigang, S., Guorui, M., Qiming, Z., 2008. A Review of Multi-Temporal Remote Sensing Data Change Detection Algorithms. In: The International Archives of the Photogrammetry, Remote Sensing and Spatial Information Sciences. Presented at the ISPRS Congress Beijing 2008, pp. 757-762.

Kanjir, U., Gregorič Bon, N., 2016. Spatial changes and population movements on the Albanian coastline. In: The International Archives of the Photogrammetry, Remote Sensing and Spatial Information Sciences. Presented at the ISPRS Congress Prague 2016.

Kanjir, U., Veljanovski, T., Oštir, K., 2011. Change detection of urban areas - the Ljubljana, Slovenia case study. In: 2011 Joint Urban Remote Sensing Event. Presented at the 2011 Joint Urban Remote Sensing Event, pp. 425-428.

King, R., 2005. Albania as a laboratory for the study of migration and development. J. South. Eur. Balk., 7, pp. 133155 .

Lorena, R.B., Shimabukuro, Y.E., Brown, I.F., Kux, H.J.J., 2002. A change vector analysis technique to monitor land use/land cover in SW Brazilian amazon: Acre state. Presented at the PECORA 15-Integrating Remote Sensing at the Global, Regional and Local Scale.

Malila, W.A., 1980. Change Vector Analysis: An Approach for Detecting Forest Changes with Landsat. In: Proceedings of the 6th Annual Symposium on Machine Processing of Remotely Sensed Data. Presented at the 6th Annual Symposium on Machine Processing of Remotely Sensed Data, Purdue University, West Lafayette, Indiana, pp. 326-335.

Qiriazi, P., Sala, S., 2000. Environmental Problems of Albania. In: Buchroithner, M.F. (Ed.), Remote Sensing for Environmental Data in Albania: A Strategy for Integrated Management, NATO Science Series. Springer Netherlands, pp. 13-30.

Rogan, J., Chen, D., 2004. Remote sensing technology for mapping and monitoring land-cover and land-use change. Prog. Plan., 61, pp. 301-325.

Vullnetari, J., 2012. Albania on the Move: Links Between Internal and International Migration. Amsterdam University Press.

Wong, M., 2006. The Gendered Politics of Remittances in Ghanaian Transnational Families. Econ. Geogr., 82, pp. 355381.

Xiaolu, S., Bo, C., 2011. Change Detection Using Change Vector Analysis from Landsat TM Images in Wuhan. Procedia Environ. Sci., 11, pp. 238-244.

Zha, Y., Gao, J., Ni, S., 2003. Use of normalized difference built-up index in automatically mapping urban areas from TM imagery. Int. J. Remote Sens., 24, pp. 583-594.

Zhou, W., Troy, A., Grove, M., 2008. Object-based Land Cover Classification and Change Analysis in the Baltimore Metropolitan Area Using Multitemporal High Resolution Remote Sensing Data. Sensors, 8, pp. 1613-1636. 


\title{
ENABLING REPRODUCIBLE OBIA WITH OPEN-SOURCE SOFTWARE IN DOCKER CONTAINERS
}

\author{
C. Knoth ${ }^{\mathrm{a} *}$ D. Nüst ${ }^{\mathrm{a}}$ \\ ${ }^{\text {a } I n s t i t u t e ~ f o r ~ G e o i n f o r m a t i c s, ~ U n i v e r s i t y ~ o f ~ M u ̈ n s t e r, ~ M u ̈ n s t e r, ~ G e r m a n y ~(c h r i s t i a n k n o t h, ~ d a n i e l . n u e s t) @ ~ u n i-m u e n s t e r . d e ~}$
}

KEY WORDS: reproducibility, open-source OBIA, conflict damage assessment, containerisation, Docker

\begin{abstract}
:
While most approaches in Object-Based Image Analysis (OBIA) currently rely on proprietary software, the interest in free and opensource software (FOSS) for OBIA is growing. This interest stems not only from cost savings, but also from benefits concerning reproducibility and collaboration. However, technical challenges hamper practical reproducibility, especially when multiple software packages are involved. In this study, we use the Docker technology to containerise an OBIA workflow in a well-defined FOSS environment. Running the analysis inside a container eliminates the need to recreate the original software environment on the executing computer. We explore the approach using two software stacks (InterIMAGE, and QGIS in combination with Orfeo ToolBox, SAGA and Python libraries) to perform an exemplary analysis detecting destruction of buildings in bi-temporal images of a conflict area. The analysis combines feature extraction techniques with segmentation and object-based analysis to detect changes and to distinguish disappeared buildings from similarly changed non-target structures. The resulting workflow is published as FOSS comprising both the model and a ready to use Docker image including all required software and data. The presented solution advances OBIA in the following aspects: higher transparency of methodology; easier reuse and adaption of workflows; better transferability between operating systems; complete description of software environment; and easy adoption of OBIA workflows by image analysis experts and non-experts.
\end{abstract}

\section{INTRODUCTION}

Openness in conducting research is not a new topic, but there clearly is a recent trend enforcing transparency and availability under the terms Open Science ${ }^{1}$ and Open Access ${ }^{2}$. All stakeholders in the research process contribute rules, incentives or guidelines to foster openness. For example (a) on the funding side, the EU requires open access as part of Horizon $2020^{3}$ and builds the European Open Science Cloud ${ }^{4}$ (b) on the publishing side, journals such as Science (Nosek et al., 2015) and Bioinformatics (Peng, 2009) encourage reproducibility, and (c) researchers themselves argue for reproducibility in "Five selfish reasons to work reproducibly" (Markowetz, 2015) or publish "Ten Simple Rules for Reproducible Computational Research" (Sandve et al., 2013), which essentially argue in favour of a proper scientific workflow simply to be able to reproduce your own results. A core notion of all of these activities is the ideal to publish data, methods, and software along with scholarly publications.

A definition of the term reproducibility is far from trivial. It is even used together with other terms to describe different levels of recreation. The Vienna Principles' definition (Kraker et al., 2016) focuses on traceability, others treat "reproducibility" and "replicability" either as interchangeable (Gentleman and Lang, 2007) or completely different terms (Goodman et al., 2016).

\footnotetext{
*Corresponding author

Note: URLs in this document were last accessed July $1^{\text {st }} 2016$.

${ }^{1}$ https://www.fosteropenscience.eu/foster-taxonomy/open-sciencedefinition

${ }^{2}$ See http://digital-scholarship.org/cwb/WhatIsOA.htm and https://open-access.net/DE-EN/information-on-open-access/historyof-the-open-access-movement

${ }^{3}$ https://ec.europa.eu/programmes/horizon2020/en/h2020section/open-science-open-access

${ }^{4} \mathrm{http}$ ://ec.europa.eu/research/openscience/index.cfm?pg=openscience-cloud
}

For the remainder of this work, we will use "reproduce" and "reproducibility" to say that a third party can run the original analysis using code and data provided by the author of a published work, and that this execution creates the same processing result (following a definition by Peng, 2009).

The referenced guides and rules indirectly define general challenges of reproducibility. Computational sciences, such as GEOBIA, face particular challenges. For example the uniqueness of data (can only be captured once) or processing environments (e.g. supercomputers) can make real replication of results impossible, so that trust in the applied methods must be established instead (Peng, 2011).

To achieve this trust, open sourcing of workflows and the underlying software is crucial. While benefits of free and open source software (FOSS) for business and security have been documented widely ${ }^{5}$, a more important aspect of FOSS in science is the potential for evaluation and scientific collaboration. The licensing models $^{6}$ of FOSS allow to combine individual contributions of small functional parts into a bigger solution for a problem at hand. This modularity at the roots of many open source software projects is propagated by the Unix philosophy (Salus, 1994): Each programme should only provide a specific feature and excel at it. They must allow (technically and legally) maintenance and re-purposing by third parties.

A number of publications at GEOBIA conferences over the last years demonstrate the feasibility of a FOSS approach, for example using ILWIS and MultiSpec (Baldina and Grishchenko, 2014) or R and GRASS GIS (Van De Kerchove et al., 2014). Specific OBIA FOSS projects exist as well, for example InterIMAGE (Costa et al., 2010).

When implementing a complex workflow with FOSS, a large

\footnotetext{
${ }^{5}$ See for example https://opensource.org/advocacy/ case_for_business.php and https://opensource.com/business/13/12/usingopen-source-software

${ }^{6}$ For a quick introduction we recommend http://choosealicense.com/.
} 
number of independent tools are utilised, both visible and invisible to the user. This hampers reproducibility because of compatibility conflicts between different software packages in different versions. In this study we present an approach to mitigate this problem by making the whole analysis including software in specific tested versions available through containerisation.

The implemented workflow detects destruction in a bitemporal image subset of a conflict area and is partly based on previous work (Knoth and Pebesma, 2014). Analysis of conflict damage is a use case where an open approach is specifically useful, because non-profit organisations face budget restrictions and, more importantly, because of the importance of transparency when using complex analysis techniques in politically sensitive environments.

The main contribution of this work is a fully reproducible and open workflow for geographic object-based image analysis (GEOBIA). It is based on mainstream IT containerisation technology and a collection of pieces of FOSS for image analysis and geographic information systems (GIS).

The following sections describe the image analysis workflow (Section 2), how it is implemented with FOSS, and how it is made reproducible using Docker (Section 3). Finally we discuss the solution and its challenges (Section 4) and conclude with a summary and outlook (Section 5).

\section{EXAMPLE ANALYSIS - CONFLICT DAMAGE ASSESSMENT}

\subsection{Data}

The data for our example analysis are two images of a village in Darfur, Sudan. They are available online as part of a blog post by the AAAS Geospatial Technologies Project ${ }^{7}$. The copyright holder DigitalGlobe granted permission to re-publish them as part of this work. The data consists of two preview pictures of remote sensing imagery showing the village Jonjona (located roughly at 13.686, 24.979 (latitude, longitude) west of Al-Fashir) before (December 2004) and after (February 2007) reported attacks in the area (see Figure 1). They were downloaded from the website in .jpg format, manually georeferenced, resampled to a spatial resolution of 0.5 metres (approximating the spatial resolution of current commercial very high resolution satellites), and saved as GeoTIFF files.

\subsection{Analysis workflow}

The general strategy implemented in this study is to segment the pre-conflict layer and analyse the resulting segments regarding their change values using information from the pre- and postconflict temporal layers. The analysis workflow can be divided into three major steps: (i) feature extraction and segmentation, (ii) change analysis, and (iii) extraction of dwelling objects from changed objects. First, a principal component analysis (PCA) is applied to each file in order to compress the highly redundant spectral information of the three RGB bands to one dimension, the first principal component. The first principal components are then used as temporal layers of a bi-temporal data set. The image objects, as basic elements of the object-based analysis, are created using the watershed segmentation algorithm, which is based on the identification of local extrema (OTB Development Team, 2016).

\footnotetext{
${ }^{7}$ American Association for the Advancement of Science, http://www.aaas.org/page/appendix-darfur-sudan-and-chad-imagerycharacteristics
}

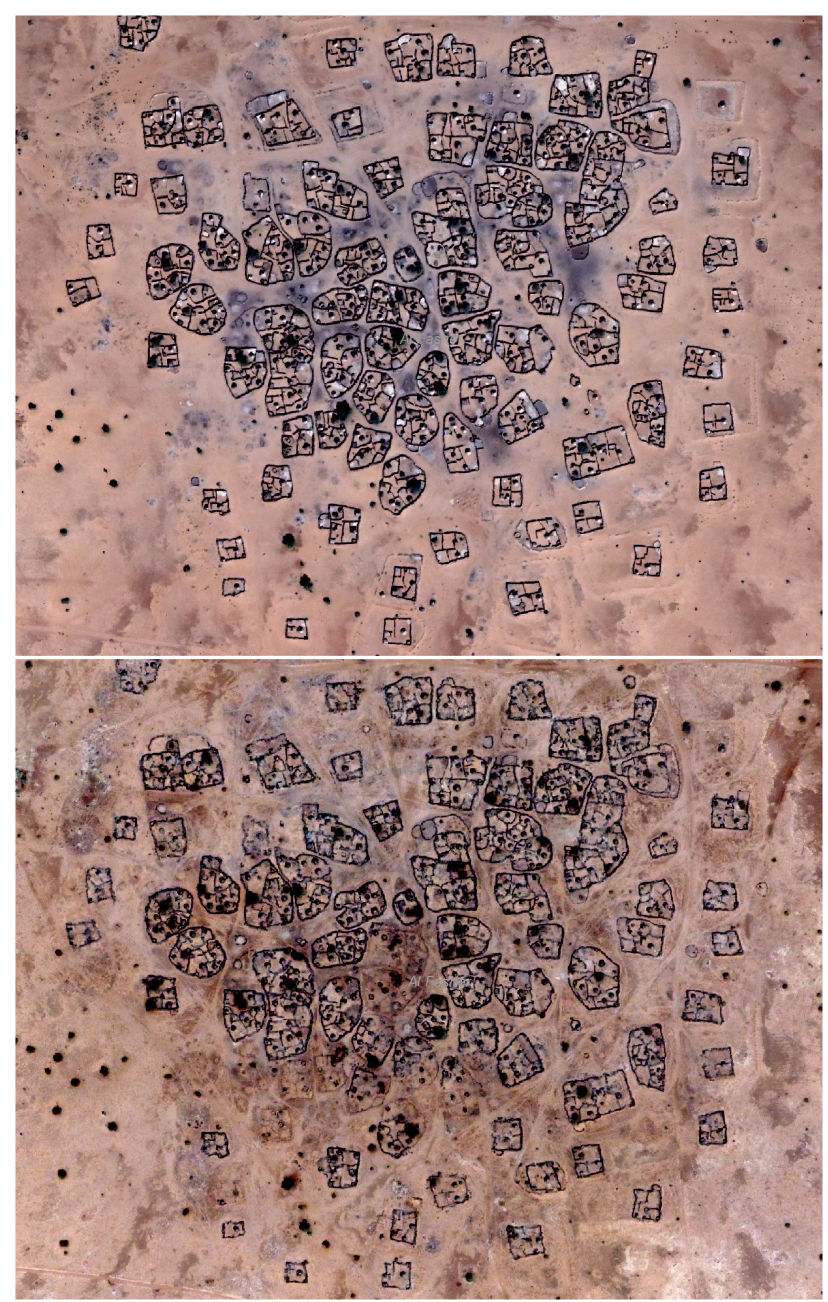

Figure 1. Example image of a village in Darfur before (December 2004, top) and after (February 2007, bottom) a reported attack. (c) 2016 DigitalGlobe

The segmentation is hampered by the specific structural properties of the objects of interest. Most of the dwellings have conical roofs. This results in a heterogeneous spectral response of the differently illuminated parts. Additionally, the buildings are often directly attached to fences or walls, so they sometimes poorly separate from the background. Earlier studies in similar areas have shown, that mathematical morphology can be used to eliminate such interfering features (Sulik and Edwards, 2010, Knoth and Pebesma, 2014). Therefore, a morphological closing operator precedes the segmentation to smooth out small and linear features. The objects resulting from the segmentation are analysed for structural differences between the two points in time. The basis for this change analysis is the difference in mean edge density per object. It is calculated after execution of an edge detection algorithm and used as the change attribute.

We apply two methods to extract the changed objects based on the change attribute: First, a fixed threshold, which can be tuned manually to best distinguish between changed and unchanged objects. Second, a k-means cluster analysis on all image objects, which does not need a predefined threshold. The latter is based on the assumption that when comparing the change of objects, disappeared dwellings differ significantly in the change of edge intensity from unchanged objects. Thus, they can be isolated in the cluster of highest change values using unsupervised clustering. 
Besides the change analysis the objects are further investigated regarding their extent, their shape and their values in the preconflict morphological closing layer in relation to the unfiltered layer, i.e. the impact of the closing operator. This allows to better distinguish between changed dwelling structures and other, similarly changed objects (e.g. fences). The shape of objects is computed using the Shape Index (Lang and Blaschke, 2007). It measures how well an object approximates a circle, i.e. the more the shape differs from a circle, the higher the shape index value.

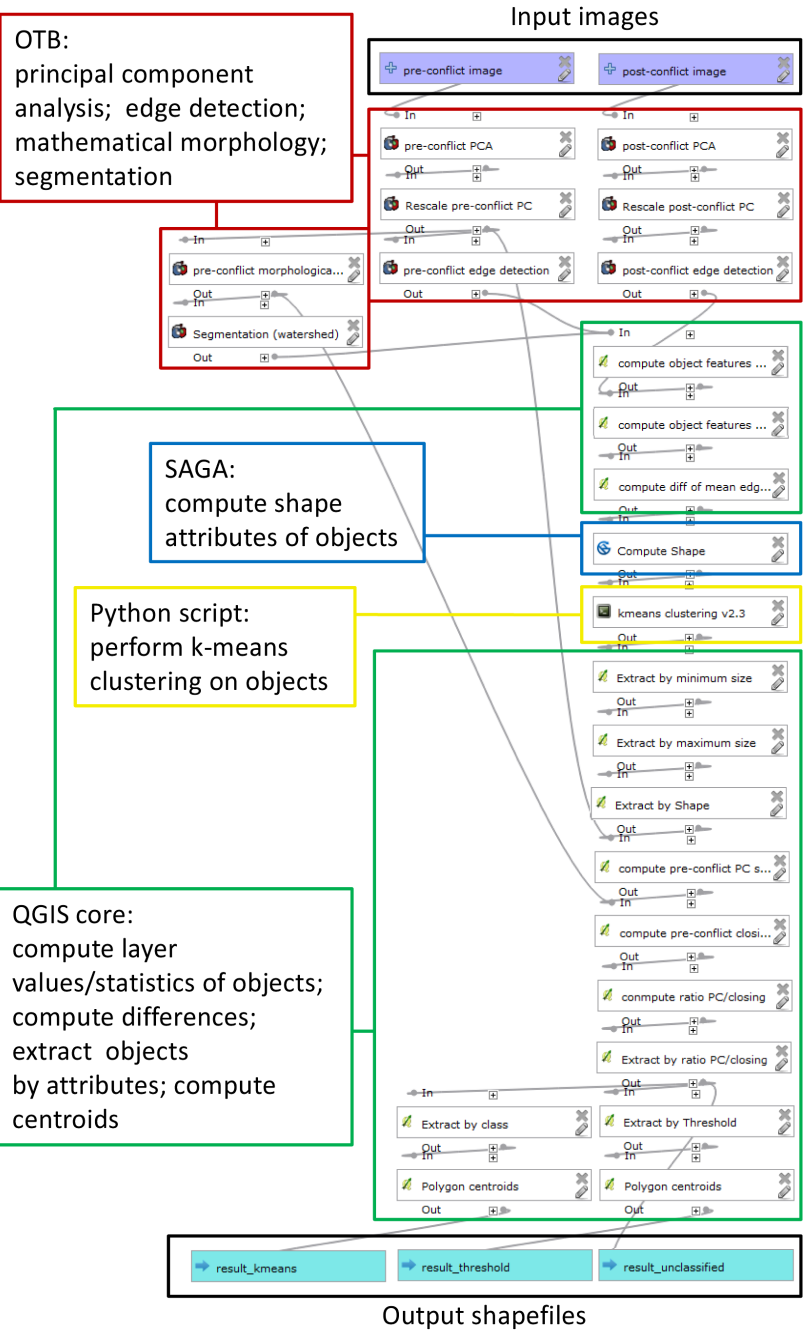

Figure 2. Overview of the analysis workflow in the graphical modeller and the role of the different software packages.

The workflow produces three results (see Figure 2). The first one is a point shapefile showing the centroids of dwelling objects detected as changed (disappeared/destructed) by the predefined threshold. The second output is another point shapefile indicating locations of dwellings belonging to the cluster of highest change as determined by k-means clustering (shown in Figure 3). The third product is a polygon shape file of all segments (changed and unchanged) where only the distinction between dwellings and other objects has been made. The change cluster (resulting from k-means clustering) as well as the computed change feature for each polygon are stored in the corresponding attribute table of this shapefile. This third result can be used to understand the two change detection results and to refine the analysis workflow (e.g. change number of clusters, adapt predefined thresholds etc.).

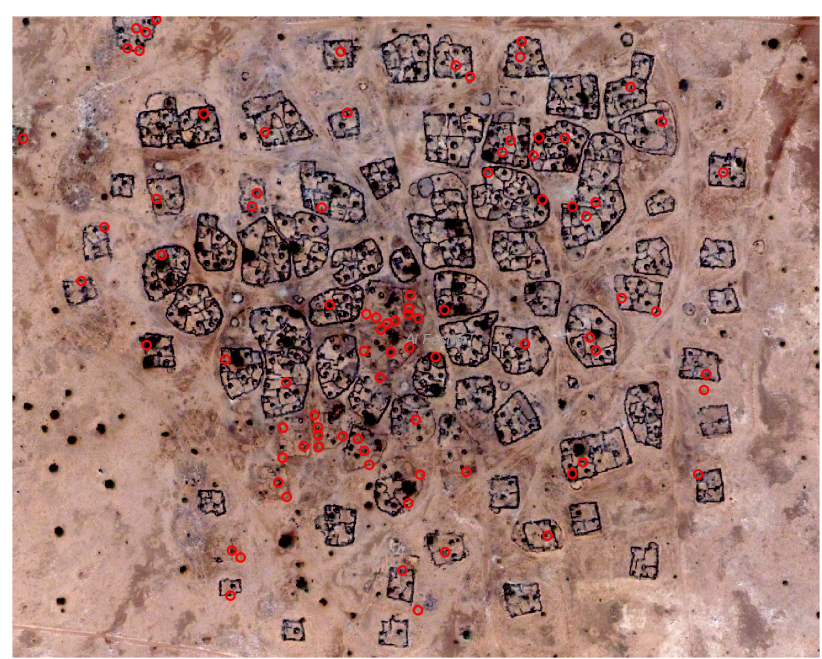

Figure 3. Post-conflict image with result of the destruction detection using k-means clustering of the change values (Image (c) 2016 DigitalGlobe).

\section{PACKAGING OF THE GEOBIA WORKFLOW}

\subsection{Introduction}

This section describes two software stacks for a FOSS-based implementation of the damage assessment workflow. They are evaluated to the extent that the respective implementations allow. The description is limited to the explicitly used pieces of software and leaves out the numerous underlying (system) libraries.

\subsection{QGIS-based automated workflow}

3.2.1 Developing the analysis model One implementation is based on the FOSS GIS QGIS (formerly known as Quantum-GIS, see QGIS Development Team, 2016). The Processing Framework included in QGIS (Graser and Oyala, 2015) provides access to native QGIS algorithms as well as a huge number of geoprocessing capabilities of third-party applications without requiring programming skills. In addition, user-created algorithms written in Python (Rossum, 1995), and subsequently the processing capabilities of any Python library, can be added.

The processing framework provides a graphical modeller for easy integration of the various algorithms into complex analysis models. These models can then be run as a whole on a selected set of input, e.g. layers in the QGIS desktop application.

Figure 2 gives an overview of the analysis steps and the involved software packages in the described workflow (Section 2.2) along with a screenshot of the corresponding modeller view. The applied tools comprise Orfeo Toolbox (OTB, see Inglada and

Christophe, 2009) for image processing and segmentation tasks, native QGIS algorithms e.g. for computing object features based on the image layers, SAGA GIS (Conrad et al., 2015) for calculating shape attributes, and a user script performing a k-means algorithm using SciPy (Jones et al., 2016).

3.2.2 Workspace preparation The user workspace comprises the directories and files shown in Listing 1 . They are stored in a specific directory structure so that the model executor (described in the next section) can identify them correctly. The contents of the workspace are

- a subdirectory data with the two original preview images and the georeferenced data files in TIFF format 
- a Python script file, model.py, calling the actual model using the QGIS Python $\mathrm{API}^{8}$

- analogous to the Python user models and scripts directories, a models and a scripts directory containing respectively

- a .model-file with the user model

- a Python-file with a user algorithm

Listing 1. Workspace directory tree (documentation files not shown).

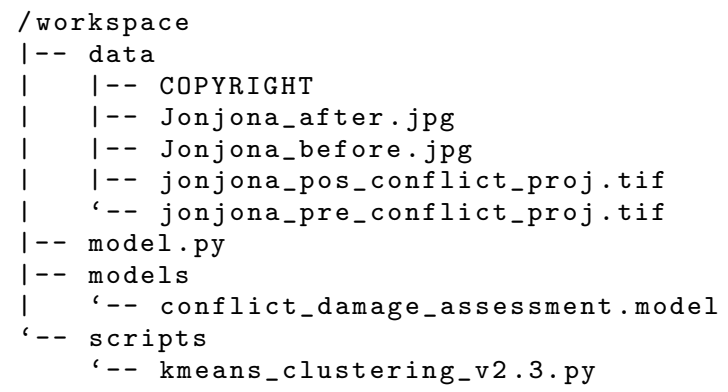

The full workspace is available on GitHub ${ }^{9}$.

3.2.3 Containerisation of workspace and runtime environment After the creation of a QGIS workflow with the QGIS graphical modeller, the next step is packaging all required parts of the analysis. We use a powerful tool for DevOps ${ }^{10}$ called Docker ${ }^{11}$. It provides lightweight virtualisation to package an application and its dependencies, for example in cloud infrastructures.

In this study, we use a Docker image to encapsulate the GEOBIA workflow with a well-defined software environment. The image can be executed anywhere where a Docker host environment is running, including Linux, Windows, and $\mathrm{OSX}^{12}$. The image is build from a human- and machine-readable definition of the complete environment called Dockerfile. It allows a scripted definition of a Docker image, i.e. installation and configuration of contained software and files, and consequently a replication of a runtime environment. When an image is started and running it is called container. A container can be paused, stopped, and restarted, or be removed from the host. While not being intentioned for it, Docker is a means to ensure long term reproducibility of computational research, as demonstrated for example for $\mathrm{R}$ (Boettiger, 2015). A Docker image suffices to capture the data, software, and runtime environment in a well-defined manner and facilitates reproducibility.

In our specific case, the Dockerfile contains commands to install the required software, to copy the workspace into the container at the location /workspace, and to call a Bash ${ }^{13}$ script to execute the actual workflow. The installation commands rely mostly on software packages from the Ubuntu ${ }^{14}$ and UbuntuGISunstable $^{15}$ repositories. The exception is SAGA, which is installed from source in a specific version not available in the repositories to solve compatibility issues with QGIS ${ }^{16}$.

\footnotetext{
${ }^{8}$ based on http://docs.qgis.org/testing/en/docs/ pyqgis_developer_cookbook/intro.html\#using-pyqgis-in-standalonescripts

${ }^{9}$ https://github.com/nuest/docker-qgis-model

${ }^{10}$ http://radar.oreilly.com/2012/06/what-is-devops.html

${ }^{11} \mathrm{http}: / /$ docker.io

12 https://docs.docker.com/engine/installation/

$13 \mathrm{https}$ //www.gnu.org/software/bash/

${ }^{14} \mathrm{http}: / /$ archive.canonical.com/

${ }^{15}$ https://launchpad.net/ ubuntugis/+archive/ubuntu/ubuntugisunstable

${ }^{16}$ See http://hub.qgis.org/issues/13279 for details.
}

The relevant parts of the main Bash script are shown in Listing 2. Omitted lines contain mostly logging commands. The main statements copy the models and script files from the workspace to the required QGIS locations and execute the Python script model.py with a virtual framebuffer using $X_{V F B}{ }^{17}$ because the container does not need a physical display. The copy statements use system environment variables which are shared between the different involved scripts, for example \$QGIS_MODELSCRIPT resolves to the value /workspace/model.py.

Listing 2. Excerpt from the main bash script.

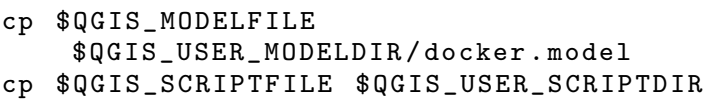

The Python script initiates and configures the QGIS application, for example library paths and logging. Then it creates variables holding the full paths to input and output objects and runs the actual model. Listing 3 shows an excerpt from the file. The runalg function's first argument, "model:docker", loads the model in the file docker.model previously copied to the model directory by the Bash script.

Listing 3. Excerpt from the Python file for model execution: initiate QGIS and run the model.

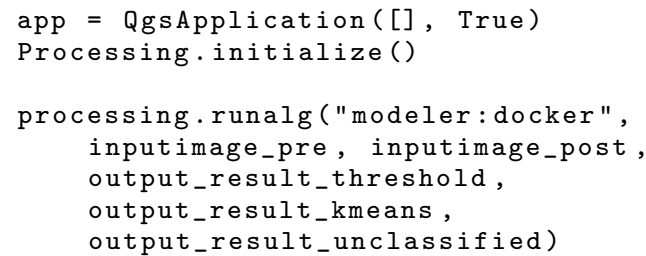

3.2.4 Running the container The container can be executed on any local machine or server if Docker is installed. The image with the analysis is published on Docker Hub ${ }^{18}$. Only the first command shown in Listing 4 is required to run the container, because Docker downloads images automatically from Docker Hub.

Listing 4. Full reproduction commands: run the container from Docker Hub and extract the result.

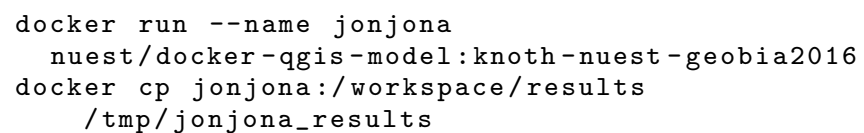

The second command copies the output of the workflow to a directory of the host machine. Its contents are shown in Listing 5. It contains a directory with a timestamp of the current execution, which contains three shapefiles-the actual model output. The shapefiles can now be inspected by the user or be processed further. Figure 3 shows a visualisation of the file result kmeans . shp.

Listing 5. Workspace directory tree after execution (supplementary shapefile files, i.e. .dbf, .prj, .qpj, and .shx, and workspace files (see previous Listing 1 not shown).

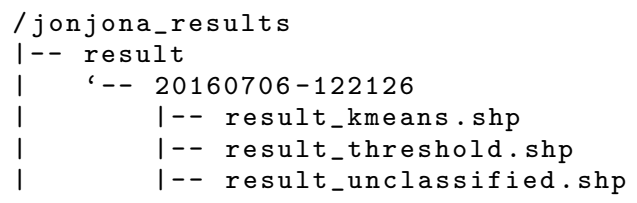

\footnotetext{
${ }^{17}$ https://en.wikipedia.org/wiki/Xvfb

${ }^{18} \mathrm{https} / / /$ hub.docker.com/r/nuest/docker-qgis-model
} 


\subsection{InterIMAGE-based application package}

InterIMAGE is another potential candidate for a FOSS-based OBIA workflow. It provides different segmentation algorithms ${ }^{19}$ including the advanced and widely used multiresolution segmentation (Baatz and Schäpe, 2000) and operators for calculation of attributes like shape, texture or topological characteristics (Costa et al., 2010). A so-called batch mode feature ${ }^{20}$, available since version 1.39, allows the automatic execution of InterIMAGE interpretation projects in the form of semantic networks. The networks store the classes and operators to be executed. Nüst and Knoth (2016) demonstrate running the user interface of the latest available Linux release (1.27) in a Docker container by sharing a local X11 socket.

However, several issues hinder the implementation of the presented use case (see Section 2). The software focuses on image interpretation and not all required algorithms for processing the image layers (e.g. the edge detection) are available in the basic package. More importantly, the latest available download for Linux is outdated ${ }^{21}$. We were not successful in compiling a later version of the source code for Linux as part of this work due to a lack of documentation and community support ${ }^{22}$. Currently only Linux is supported as the operating system inside a container, but support for multi-platform containers (most importantly Windows) is under development ${ }^{23}$. This limitation is not an issue for most FOSS projects because they are usually platform independent, but a straightforward execution of a InterIMAGE interpretation project within a container is not possible.

\section{DISCUSSION}

We successfully demonstrate packaging a complete GEOBIA workflow using FOSS. The created package is transferable between machines (different host operating systems as well as desktop and cloud platforms) and all tools are available free of charge. Our experiments show that because of the numerous involved tools in different versions and potential conflicts between them, containerisation is useful not only for reproducibility by third parties, but also for the original development of an FOSS-based analysis.

However, the approach still has shortcomings when it comes to the overarching goal of reproducible research. First, the presented solution is a one-off effort to containerise a specific workflow. It lacks a strict standardisation beyond the docker run command. Only experienced users can trace the command flow within the container. The currently used scripts are tailored to the actual use case, especially in the case of the file model.py. While most of the file is already generic, namely preparing the QGIS environment, logging, and clocking statements, the actual call to the algorithm is specific to the model. This part of the script should be split up so that only the relevant three to four lines of code must be provided by the workflow author.

Second, the Dockerfile installs automatically the latest available versions of software from the repositories. This proofed to

\footnotetext{
${ }^{19}$ http://wiki.dpi.inpe.br/doku.php?

$\mathrm{id}=$ interimage:operators_documentation

${ }^{20} \mathrm{http} / / /$ wiki.dpi.inpe.br/doku.php?id=interimage:batch_processing

${ }^{21}$ Version 1.27 , see http://www.lvc.ele.pucrio.br/projects/interimage/download

${ }^{22}$ https://groups.google.com/forum/\#!topic/interimage/924t-uZrAMs

${ }^{23}$ See https://blog.docker.com/2016/04/docker-windows-server-tp5/ and https://www.docker.com/microsoft
}

be tricky during development of the use case with incompatibilities or missing features of libraries, resulting in a specific compatible version of one software to be installed manually. Software can be installed in specific versions, but creating these install statements manually is not user-friendly. While the used UbuntuGIS community repository simplifies installation tremendously, the ability to download, build, and install a software package from source within the Dockerfile was crucial to complete the use case at hand.

Third, the approach does not enforce best practices for reproducible research, such as versioning scripts, but could easily accommodate them, for example by putting a git repository inside the workspace directory (Ram, 2013).

Fourth, a general issue is the availability of open data. Especially in GEOBIA, where very high resolution imagery plays an important role in many analyses, the applied images are often not freely available. In these cases it is not possible to publish the data along with the analysis workflow and software.

Finally, the applied FOSS solutions, at the current stage, cannot compete with commercial software packages, such as eCognition, regarding usability and functionality in OBIA. The available functions of FOSS tools already provide a substantial set of algorithms and the analysis is created with a user-friendly interactive modeller in a Desktop environment. But the number of actual OBIA operations in the modeller is limited. They can only partially be re-created, e.g. by combination of other algorithms. However, since FOSS tools are easily extensible, the missing functionality can be contributed as new functions or independent tools. Therefore, we see a high potential for open-source software in OBIA, especially if a growing community of users can be established, who can become active contributors and lead the development of new algorithms.

\section{CONCLUSION AND OUTLOOK}

Docker containers and a combination of established free and open source GIS and image analysis software facilitate reproducible GEOBIA. We build and distribute a container to carry all required software and data in a transparent manner. This is a breakthrough for creating a transferable and executable package of a GEOBIA workflow. The presented analysis goes well beyond simple processing by successfully integrating a large set of tools into a complex multi-step analysis. The shortcomings discussed in the previous section are mostly related to usability. Therefore we see the following potential for future work.

With respect to standardisation, an open standard for packaging GEOBIA software and workflows would allow to follow similar approaches with different software stacks. This opens new possibilities for reviews of scientific work and collaboration between researchers.

The current solution is also mostly useful for users with software development and Linux experience. User interfaces that are delivered to a browser via HTTP can mitigate this limitation and provide a good user experience across platforms.

There is a need for documentation and ready-to-use templates for packaging as well as a user-friendly automation of packaging workflows, for example an "Export to Container"-button in the QGIS workflow modeller. A standardised format would also allow to create new services to store, share, and execute GEOBIA workflow packages in cloud infrastructures. 
With respect to interaction with the container, it would be possible to pass on parameters or external datasets into the container, for example via environment variables or mounting directories as volumes into the container. This way users can manipulate an analysis' parameters or quickly apply a complete workflow to their own data.

Regarding the outputs of the container, the presented solution lacks clear information about the result of the analysis, besides the $\log$ file and the created output files. More research on improving the result interpretation is required to enable machine-based output validation and better result visualisation for users.

But the tools are just one part of the deal: To reach high userfriendliness and adoption of an approach similar to this demonstration, we see a high demand in education of the current and next generation of OBIA users in programming and open source technologies. Although there are commonalities across all scientific disciplines, domain specific requirements demand targeted examples/course material, high-quality specialised FOSS, and bes practises of common use cases. The challenge starts with a definition of reproducibility specifically for (GE)OBIA (Baker, 2016). This important next step needs an open discourse in the GEOBIA community, to which this work intends to contribute.

\section{ACKNOWLEDGEMENTS}

This research has has been conducted in the context of the Gradschool for Geoinformatics ${ }^{24}$. It has partly been supported by the project Opening Reproducible Research ${ }^{25}$ funded by the German Research Foundation (DFG) under project number PE 1632/10-1.

\section{REFERENCES}

Baatz, M. and Schäpe, A., 2000. Multiresolution segmentation an optimization approach for high quality multi-scale image segmentation. In: J. Strobl, T. Blaschke and G. Griesebner (eds), Angewandte Geographische Informations-Verarbeitung XII, Wichmann, Karlsruhe, pp. 12-23.

Baker, M., 2016. Muddled meanings hamper efforts to fix reproducibility crisis. Nature News.

Baldina, E. A. and Grishchenko, M. Y., 2014. Object oriented analysis of multitemporal thermal infrared images. In: Proceedings of the GEOBIA 2014: Geographic Object-Based Image Analysis, Thessaloniki, Greece, pp. 415-418.

Boettiger, C., 2015. An introduction to docker for reproducible research, with examples from the r environment. ACM SIGOPS Operating Systems Review 49(1), pp. 71-79.

Conrad, O., Bechtel, B., Bock, M., Dietrich, H., Fischer, E., Gerlitz, L., Wehberg, J., Wichmann, V. and Böhner, J., 2015. System for Automated Geoscientific Analyses (SAGA) v. 2.1.4. Geosci. Model Dev. 8, pp. 1991-2007.

Costa, G., Feitosa, R., Fonseca, L., Oliveira, D., Ferreira, R. and Castejon, E., 2010. Knowledge-based interpretation of remote sensing data with the InterImage system: Major characteristics and recent developments. In: Proceedings of the GEOBIA 2010: Geographic Object-Based Image Analysis, Ghent, Belgium.

Gentleman, R. and Lang, D. T., 2007. Statistical analyses and reproducible research. Journal of Computational and Graphical Statistics 16(1), pp. 1-23.

\footnotetext{
${ }^{24}$ http://www . uni-muenster.de/Geoinformatics/en/ Studies/study_programs/PhD/

${ }^{25} \mathrm{http}$ ://o2r.info
}

Goodman, S. N., Fanelli, D. and Ioannidis, J. P. A., 2016. What does research reproducibility mean? Science Translational Medicine 8(341), pp. 341ps12-341ps12.

Graser, A. and Oyala, V., 2015. Processing: A Python Framework for the Seamless Integration of Geoprocessing Tools in QGIS. ISPRS International Journal of Geo-Information 4(4), pp. 2219 2245.

Inglada, J. and Christophe, E., 2009. The orfeo toolbox remote sensing image processing software. In: Proceedings of the 2009 IEEE International Geoscience and Remote Sensing Symposium (IGARSS), Cape Town, South Africa, Vol. 4, pp. 733-736.

Jones, E., Oliphant, T., Peterson, P. et al., 2016. SciPy: Open source scientific tools for Python. http://www.scipy.org/ (27 June 2016).

Knoth, C. and Pebesma, E., 2014. Detecting destruction in conflict areas in darfur. In: Proceedings of the GEOBIA 2014: Geographic Object-Based Image Analysis, Thessaloniki, Greece, pp. 165-168.

Kraker, P., Kaier, C., Gutounig, R., Vignoli, M., Dennerlein, S., Aspöck, E., Schmidt, N., Wandl-Vogt, E., Ferus, A., McNeill, G., Steinrisser-Allex, G., Dörler, D., Rieck, K., Heigl, F., imukovi, E. and Enkhbayar, A., 2016. The vienna principles: A vision for scholarly communication in the 21 st century. Zenodo.

Lang, S. and Blaschke, T., 2007. Landschaftsanalyse mit GIS. Ulmer, Stuttgart, pp. 241-243.

Markowetz, F., 2015. Five selfish reasons to work reproducibly. Genome Biology 16, pp. 274.

Nosek, B. A., Alter, G., Banks, G. C., Borsboom, D., Bowman, S. D., Breckler, S. J., Buck, S., Chambers, C. D., Chin, G., Christensen, G., Contestabile, M., Dafoe, A., Eich, E., Freese, J., Glennerster, R., Goroff, D., Green, D. P., Hesse, B., Humphreys, M., Ishiyama, J., Karlan, D., Kraut, A., Lupia, A., Mabry, P., Madon, T., Malhotra, N., Mayo-Wilson, E., McNutt, M., Miguel, E., Paluck, E. L., Simonsohn, U., Soderberg, C., Spellman, B. A., Turitto, J., VandenBos, G., Vazire, S., Wagenmakers, E. J., Wilson, R. and Yarkoni, T., 2015. Promoting an open research culture. Science 348(6242), pp. 1422-1425.

Nüst, D. and Knoth, C., 2016. docker-interimage: Running the latest InterIMAGE linux release in a docker container with user interface. http://zenodo.org/record/55083 (06 July 2016).

OTB Development Team, 2016. The ORFEO Tool Box Software Guide. https://www.orfeotoolbox.org//packages/OTBSoftwareGuide.pdf (27 June 2016).

Peng, R. D., 2009. Reproducible research and biostatistics. Biostatistics 10(3), pp. 405-408.

Peng, R. D., 2011. Reproducible research in computational science. Science 334(6060), pp. 1226-1227.

QGIS Development Team, 2016. QGIS Geographic Information System. http://qgis.osgeo.org (24 June 2016).

Ram, K., 2013. Git can facilitate greater reproducibility and increased transparency in science. Source Code for Biology and Medicine 8, pp. 7.

Rossum, G., 1995. Python reference manual, available at http: //www. python. org/.

Salus, P., 1994. A Quarter-Century of Unix. Addison-Wesley, Boston, chapter 7 of part 2, p. 52. 
Sandve, G. K., Nekrutenko, A., Taylor, J. and Hovig, E., 2013. Ten simple rules for reproducible computational research. PLoS Computational Biology 9(10), pp. e1003285.

Sulik, J. and Edwards, S., 2010. Feature extraction for darfur: geospatial applications in the documentation of human rights abuses. International Journal of Remote Sensing 31(10), pp. 2521-2533.

Van De Kerchove, R., Hanson, E. and Wolff, E., 2014. Comparing pixelbased and objectbased classification methodologies for mapping impervious surfaces in wallonia using orthoimagery and LIDAR data. In: Proceedings of the GEOBIA 2014: Geographic Object-Based Image Analysis, Thessaloniki, Greece, pp. 657661. 


\title{
HOW TO EFFECTIVELY OBTAIN METADATA FROM REMOTE SENSING BIG DATA?
}

\author{
Körting, T. S., Namikawa, L. M., Fonseca, L. M. G., Felgueiras, C. A. \\ National Institute for Space Research - INPE Brazil \\ DPI - Image Processing Division at the OBT - General Earth Observation Coordination \\ Av. dos Astronautas, 1758 São José dos Campos, Brazil \\ \{thales.korting, laercio.namikawa, leila.fonseca, carlos.felgueiras\}@inpe.br
}

KEY WORDS: Big data, Remote sensing, Metadata, Image processing, Vegetation indices, Water indices, Pattern recognition

\begin{abstract}
:
What can be considered big data when dealing with remote sensing imagery? In general terms, big data is defined as data requiring high management capabilities characterized by 3 V's: Volume, Velocity and Variety. In the past, (e.g. 1975), considering the computational and databases resources available, a series of Landsat-1 imagery from the same region could be considered big data. Nowadays, several satellites are available, and they produce massive amounts of data. Certainly, an image data set obtained by a single satellite, for a specific region and along time, fills the 3 V's requirements to be considered big data as well. In order to deal with remote sensing big data, we propose to explore the generation of metadata based on the detection of simple features. Besides the intrinsic geographic information on every remote sensing scene, no additional metadata is usually considered. We propose basic image processing algorithms to detect basic well-known patterns, and include them as tags, such as cloud, shadow, stadium, vegetation, and water, according to what is detectable at each spatial resolution. In this work we show preliminary results using imagery from RapidEye sensor, with 5 meter spatial resolution, composed by two full coverages of Brazil with RapidEye multispectral imagery (around 40k scenes).
\end{abstract}

\section{INTRODUCTION}

Nowadays, several references to the term big data are available, many of them without a proper understanding of what is the real meaning of it. Since 2001, Big data has been defined as data requiring high management capabilities characterized by 3 V's: Volume, Velocity and Variety, as proposed by (Laney, 2001). In (Plunkett et al., 2013), the authors provide examples of what has been considered big data so far. Examples include Web server and application logs, digital video and music, clickstream data, social networks, smartphone location-based services, real-time trading data, blogs and social media. It is clear that most of the given examples are Internet-related, however we can find other examples of big data generation, such as remote sensing.

Remote sensing satellites fill the requirements to be characterized as big data. Since at each day new images are obtained, and previously captured images are also combined as time series, it is possible to confirm the constant growing volume and also the increasing velocity of data gathering. New satellites are being launched and their design life (duration) are frequently overcome. One great example is the Landsat 5 satellite, projected with a 5-year design life, that returned scientifically viable data for 28 years (USGS, 2016). In terms of variety, remote sensing data is expanding the amount of spectral channels (i.e. Landsat 5 and 7 produces images in 8 spectral channels; Landsat 8 , in 11 channels), which means different ways to capture spectral interaction between targets and eletromagnetic radiation. When we focus the analysis in the GEOBIA approach, the variety of data related to the same target increases more, since with the use of spectrally homogeneous regions, we combine the intra-region spectral information, such as average pixel values or texture, with spatial information, such as geometric features, and also relations to the neighborhood. Considering the aforementioned reasons, it is clear that remote sensing is a source of big data.

In this paper, we propose a method to work in a set of images composed by two full coverages of Brazil with RapidEye mul-

\footnotetext{
*Corresponding author
}

tispectral imagery (MMA, 2016), from 2012 and 2014. RapidEye images are generated from a constellation of 5 satellites located at the same orbital plane, and carrying the same sensors (BlackBridge, 2015). Available RapidEye imagery are processed into level 3A, which corresponds to geometric, radiometric and sensor correction, and mosaicked into 25 by $25 \mathrm{~km}$ tiles with a 5 meter pixel size, created from the acquisition sampled at 6.5 meters at the nadir. The multispectral bands are 5: blue $(0.44-0.51 \mu \mathrm{m})$, green $(0.52-0.59 \mu \mathrm{m})$, red $(0.63-0.685 \mu \mathrm{m})$, red edge $(0.69-0.73 \mu \mathrm{m})$, and near infra-red $(0.76-0.85 \mu \mathrm{m})$.

\section{METHODOLOGY}

In this section we describe our proposal to deal with remote sensing big data for metadata generation, which is depicted in the diagram of Figure 1. We also provide references for the used indices and algorithms to detect the presence of target patterns in images.

Our proposal is to integrate a set of simple algorithms for pattern recognition (blocks called Detector for pattern $1 \ldots N$ in Figure 1) without a strong compromise with accuracy, therefore we can consider these algorithms as weak detectors. Our expected level of metadata to be generated is as superficial as tags like cloud, shadow, stadium, vegetation, and water, according to what is detectable in each spatial resolution.

The basic idea in dealing with remote sensing big data plus a stream of incoming images, is to provide a continuous workflow, which means a system that keeps running (see the block New images), allowing the insertion of algorithms for detecting new patterns on-the-fly (block New detectors). With the provided structure, the algorithm can run more than once for the same image, which is useful when parameters are changed or new algorithms are inserted.

\subsection{Pre-processing}

When dealing with remote sensing big data, which should include heterogeneous sources of images, specific parameters for each 


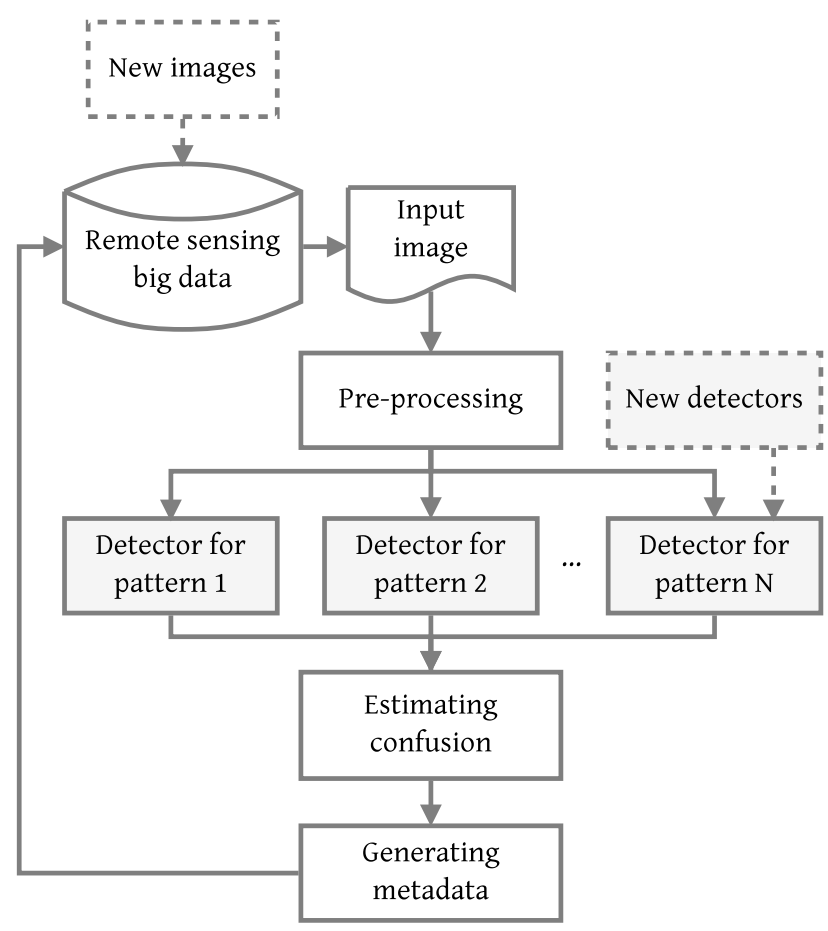

Figure 1. Diagram for metadata discovery in remote sensing big data.

sensor are often unavailable, and therefore algorithms should not rely on them. For this reason, we adopted an uniform normalization of the images, using the well-known min-max method (Equation 1), considering original pixel from image $I$ at row $r$ and column $c(r=1 \ldots H$ and $c=1 \ldots W$, and $H$ and $W$ are the Height and Width of $I$ ). This equation also uses $\min (I)$ and $\max (I)$ as the minimum pixel and maximum pixel values, respectively. As output we create the normalized image $I_{\text {norm }}$ with values in the interval $[0,1]$.

$$
I_{\text {norm }}(r, c)=\frac{I(r, c)-\min (I)}{\max (I)-\min (I)}
$$

This step also includes removal of null pixel values, or no data values, depending on how they are represented by the formats. Objects with these values will not be used by our algorithms. The detectors proposed in our approach are based on well-known remote sensing indices, such as vegetation or water indices. For other patterns without well-known indices, such as stadium, we are developing specific algorithms.

Although most of the classic indices are computed using reflectance information extracted by pixel values and often specific sensor parameters, we assume an approximation of the values by using the normalized images as inputs, since our proposal is not to map exact regions, but presence/absence of patterns.

\subsection{Detectors}

The main idea in our proposal is to create an image $D_{i}$, representing the resemblance of an object $\Gamma$ (pixel/region) to a certain pattern $i$. This resemblance is based on thresholding processed images, represented as target indices, with values $\Phi$. We defined a straightforward rule to be applied on each index, and it is up to the analyst to define the best expected threshold $\zeta$ to consider or not $\Gamma$ in a specific pattern.
In Equation 2, we formalize the pattern detector. Given an object $\Gamma$, its detection index $\Phi$ and a threshold $\zeta$, the outcome of the detector will be one of the following values:

$$
\operatorname{pattern}(\Gamma)= \begin{cases}2, & \text { if } \Phi \geq \zeta \\ 1, & \text { if } \Phi<\zeta \text { and } \Phi \geq 0.95 . \zeta \\ 0, & \text { if } \Phi<0.95 . \zeta\end{cases}
$$

The value 0.95 was defined empirically to distinguish a high from a medium resemblance outcome (values 2 and 1, respectively). When object $\Gamma$ is not detected, the output is value 0 . By applying each detector on the normalized image $I_{\text {norm }}$, the result is an image $D_{i}, i=1 \ldots N$. All detections are then combined to estimate confusion and generate metadata.

Our preliminary results are composed by 5 weak detectors for the patterns cloud, shadow, stadium, vegetation, and water. For detecting the pattern cloud, we defined a threshold in the brightness band, with is basicaly computed by selecting, for each pixel, the maximum value among all bands. For pattern shadow, we applied a threshold in the near infra-red band to detect low values, adapting the proposal for Landsat TM band by (Abreu et al., 2013). The rules for pattern stadium are based on finding parts of images in which a compact block with high resemblance to vegetation contrasts with a surrounding area with low resemblance with vegetation. The average size of each stadium is defined as a relation between the spatial resolution of the image, the number of pixels expected to define the stadium, and it is computed using a basic highpass filtering algorithm. For pattern vegetation, we applied a threshold in the Normalized Difference Vegetation Index (NDVI), as described in (Rouse et al., 1974), that uses bands in the region of red and near infra-red. For pattern water, we applied a threshold in the Normalized Difference Water Index (NDWI), as described in (McFeeters, 1996), that uses bands in the region of green and near infra-red.

\subsection{Estimating confusion}

Independently of the scale of the objects (represented by $\Gamma$ ), in this step we process the detections for each pixel and create an output image $E$ with estimated confusion from the previous step. By summing up individual detection values from $D_{i}, i=1 \ldots N$, it is possible to infer if a pixel was detected as more than one pattern, or even if the object was not classified by any detector. It is important to highlight that in this step we account only for pixels marked with value high resemblance (value 2). Formally, the image $E$ is computed as follows:

$$
E(r, c)=\sum_{i=1}^{N} 1 \Longleftrightarrow D_{i}(r, c)=2
$$

The highest value we obtain in this sum, the more unconfident we are with the resultant detection. In Figure 2 we provide one example of some pattern detections and the estimated confusion.

\subsection{Generating metadata}

Our proposal is to output tags related to the detected patterns for each input image. It is known that certain targets appear in the images with higher probability than others. One example, when analyzing the full coverage of Brazil, is the pattern of vegetation. When compared to, for example, water, there is a strong difference in terms of area of occurrence. 

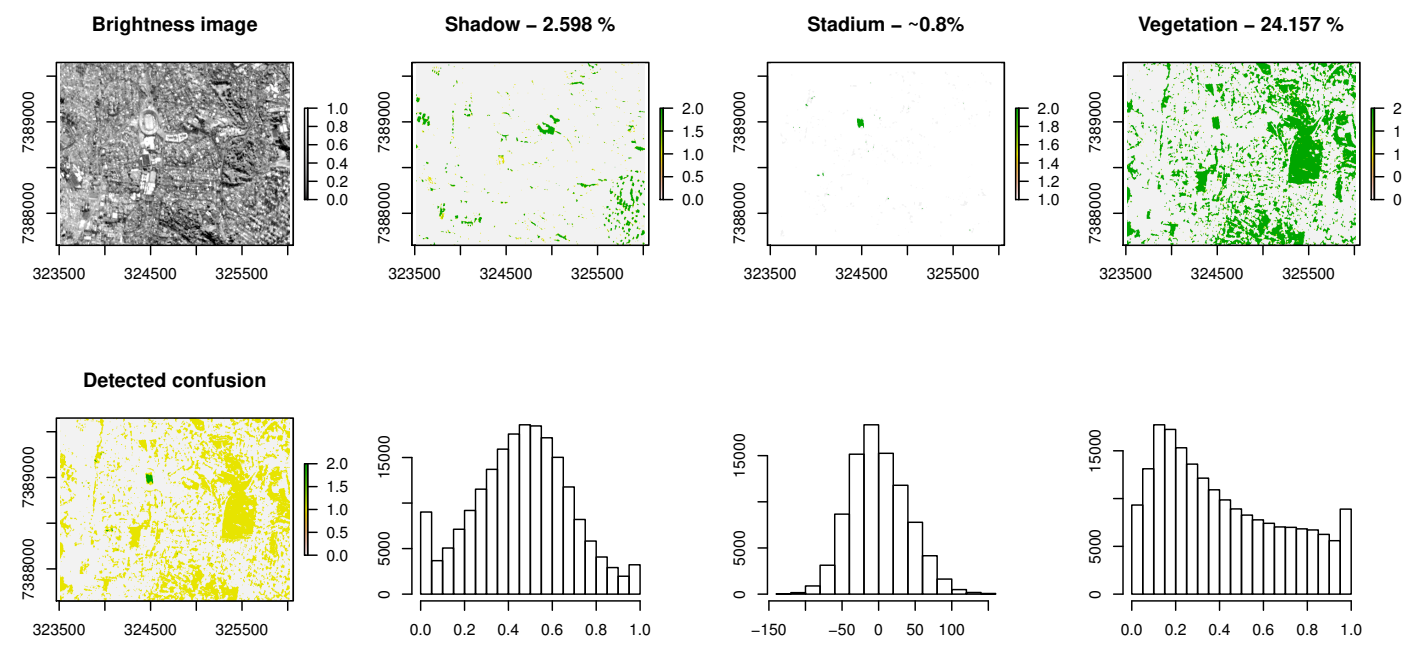

Figure 2. Estimating confusion between 3 pattern detections. Pixels marked as 2 were recognized by 2 pattern detectors. Pixels marked as 1 were detected by a single pattern, and pixels marked with 0 were not detected by any of the pattern detectors.

However, it is up to the analyst to define a minimum estimated proportion of detected targets in one image, as a threshold $\Upsilon_{i}, i=$ $1 \ldots N$, to consider the existence of the pattern $i$ in the image, and therefore creating the tag. As we are dealing with big data, it is mandatory that $\Upsilon_{i}$ is defined as a flexible value, since each remote sensing scene is supposed to include different combinations and proportions of targets. For this purpose, we compute in the following equations the value $P_{i}$, which stands for the estimated amount of pattern $i$ in the input image $\left(\#\right.$ pattern $\left._{i}\right)$, disregard the detected confusion (\#confusion).

$$
\begin{aligned}
\text { \#pattern }_{i} & =\sum_{r=1, c=1}^{H, W} 1 \Longleftrightarrow D_{i}(r, c) \in\{1,2\} \\
\text { \#confusion } & =\sum_{r=1, c=1}^{H, W} 1 \Longleftrightarrow E(r, c)>3 \text { and } D_{i}(r, c)=2 \\
\text { \#total }_{i} & =\text { \#pattern }_{i}+\sum_{r=1, c=1}^{H, W} 1 \Longleftrightarrow D_{i}(r, c)=0 \\
P_{i} & =\frac{\text { \#pattern }_{i}-\text { \#confusion }}{\text { \#total }_{i}}
\end{aligned}
$$

The value 3 for considering a confusion was empirically defined (in Equation $E(r, c)>3$ ), based on the assumption that if more than 3 detectors output a high resemblance for the same pattern, this value should be disconsidered. At the end, the decision for creating or not a tag for pattern $i$ is based on the following rule:

$$
\begin{aligned}
\operatorname{tag} \text { for pattern } i, \text { if } P_{i} & \geq \Upsilon_{i} \\
\text { no-tag,if } P_{i} & <\Upsilon_{i}
\end{aligned}
$$

\section{CONCLUSIONS}

This short paper presented preliminary insights in using remote sensing big data for metadata discovery. To work with big data, the flexibility of the thresholds must be considered, and also a week compromise with positional accuracy should be assumed. The analyst plays an important role on defining thresholds for detecting patterns, and also for creating or not metadata tags for presence of targets.
We are currently running our methodology in a big data set composed by remote sensing images from RapidEye sensor, composed by two full coverages of Brazil with RapidEye multispectral imagery (around 40k scenes). New detectors must be created to extend the metadata generation for more patterns, since we are currently working with 5 patterns, namely cloud, shadow, stadium, vegetation, and water. Although 5 is a small number of patterns, it has allowed us to refine the methodology for estimating confusion and deciding for tag generation. The algorithms were developed in $\mathrm{R}$ language, using packages rgdal, raster, ggplot2 and $s p$.

\section{ACKNOWLEDGEMENTS}

The authors would like to thank FAPESP, under grant 2016/145457, São Paulo Research Foundation (FAPESP).

\section{REFERENCES}

Abreu, E., Fonseca, L., Santos, C. and Ribeiro, V., 2013. Cloud Detection Tool Uma ferramenta para a detecção de nuvens e sombras em imagens de satélite. Anais XVI Simpósio Brasileiro de Sensoriamento Remoto - SBSR (2002), pp. 4234-4241.

BlackBridge, 2015. Satellite Imagery Product Specifications. Technical Report April.

Laney, D., 2001. 3D Data management: Controlling data volume, velocity, and variety.

McFeeters, S., 1996. The Use of the Normalized Difference Water Index (NDWI) in the Delineation of Open Water Features. International Journal of Remote Sensing 17(7), pp. 1425-1432.

MMA, 2016. Geo Catálogo Do Ministério Do Meio Ambiente.

Plunkett, T., Mohiuddin, K., Macdonald, B., Harding, D., Nelson, B., Segleau, D., Sun, H., Mishra, G., Hornick, M., Stackowiak, R. and Laker, K., 2013. Oracle Big Data Handbook. Oracle Press, New York.

Rouse, J., Haas, R., Schell, J. and Deering, D., 1974. Monitoring Vegetation Systems in the Great Plains with ERTS. NASA Special Publication 351, pp. 309.

USGS, 2016. Landsat 8 (L8) Data Users Handbook. 


\title{
MAPPING LAKES ON THE TIBETAN PLATEAU WITH LANDSAT IMAGERY AND OBJECT-BASED IMAGE ANALYSIS
}

\author{
K. Korzeniowska ${ }^{a b}$, O. Korup ${ }^{b}$

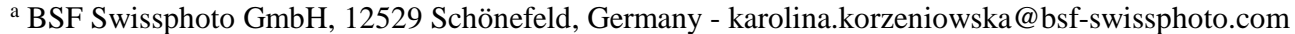 \\ b Geohazards Research Group, University of Potsdam, 14476 Potsdam, Germany - korup@ geo.uni-potsdam.de
}

KEY WORDS: Tibetan Plateau, lakes, LANDSAT, SRTM, MNDWI, OBIA, changes detection

\begin{abstract}
:
The Tibetan Plateau, the world's largest orogenic plateau, hosts thousands of lakes that play a prominent role as water resources, environmental archives, and sources of natural hazards such as glacier lake outburst floods. Previous studies reported that the size of the lakes on the Tibetan Plateau has been changing rapidly in recent years, possibly because of contemporary global warming. Tracking these changes systematically from remote sensing data offers new challenges and opportunities for automated classification methods such as object-based image analysis (OBIA). We present a method for an automatic mapping of lakes combining LANDSAT images, Shuttle Radar Topography Mission (SRTM) digital elevation model (DEM), and OBIA. We tested our method for most of the area of the Tibetan Plateau where lakes formed in tectonic depressions or blocked by glaciers and sediments have different spectral colours, and different physical states of water (frozen and not-frozen). We analysed images obtained in 1995 and 2015. For detecting the lakes we used the Modified Normalized Difference Water Index (MNDWI) combined with OBIA and a slope map derived from the DEM. Our classification of lakes with an area $>10 \mathrm{~km}^{2}$ derived 323 water bodies with a total area of 31,258 $\mathrm{km}^{2}$, which is $2.6 \%$ of the analysed area in 2015 . The same number of lakes had covered only $24,892 \mathrm{~km}^{2}$ in 1995 , so that lakes grew by $\sim 26 \%$ in the past two decades on average. The classification had an overall, producer's and user's accuracies of 0.98 , and a Cohen's kappa of 0.98 , and may be useful step towards quantifying regional-scale hydrological budgets.
\end{abstract}

\section{INTRODUCTION}

The Tibetan Plateau is the world's largest orogenic plateau with a mean elevation exceeding $4000 \mathrm{~m}$ above sea level (a.s.l.), and often named as "the Roof of the World" or "the 3rd Pole of the Earth" (Song et al. 2014), (Wang 2016). The Plateau is surrounded by high mountains ranges such as the Himalayas, the Kunlun Shan, Pamir, and Qilian Shan (Liu and Chen 2000), and hosts glaciers and thousands of lakes that play a prominent role as water resources, environmental archives, and sources of natural hazards such as glacier lake outburst floods.

Previous studies reported that the Tibetan Plateau could be one of the most sensitive places to global warming (Zhang et al. 2011). Symptoms attributed to rising temperatures on the Tibetan Plateau include retreating glaciers (Liu and Chen 2000), (Yang et al. 2008), and the degradation of permafrost (REF). A number of studies reported that the size of the lakes on the Tibetan Plateau has been changing rapidly in recent years, possibly because of a warming climate. Some studies (Yang et al. 2008), (Song et al. 2014) hold that meltwater from the surrounding glaciers significantly changes the size distribution of these lakes. In this context, satellite-based monitoring offers repeatability of images and standardized output of results. Tracking lake changes systematically also offers new challenges and opportunities for automated classification methods such as object-based image analysis (OBIA). Accurate and reliable automatic mapping of landforms or landform elements from remote sensing data decreases subjective operator bias, and allows rapid investigation of large regions. However, the training of such algorithms still requires carefully designed strategies.

We address this issue and present a semi-automatic method for classifying large lakes on the Tibetan Plateau from LANDSAT images and digital elevation models. Our approach is universal to detecting and classifying lakes prone to seasonal ice cover, as is the case on the Tibetan Plateau. We use the modified normalized difference index (MNDWI) to detect water areas, and applied OBIA to extract lake boundaries and distinguish them from rivers and glaciers.

\section{PREVIOUS METHODS FOR DETECTING WATER AREAS}

\subsection{Water detection algorithms}

Methods for extracting water areas from remote sensing data involve analysing objects features such as colour, texture, and shape; thresholding of image bands and band ratio: or spectral water indexes in both supervised and unsupervised classifications (Song et al. 2014). The most common approaches use thresholding of a single band, or a ratio of bands, because they are easy and quick to use (Ryu, Won, and Min 2002), (Song et al. 2014). However, simple thresholding often yields inaccurate classifications that methods based on segmentation or spectral matching hope to improve (Jawak, Kulkarni, and Luis 2015). Frazier et al. (2000) proposed a method applying single-band $(\rho)$ density slicing of LANDSAT images on each individual band separately to determine the most useful ones. They found that the highest accuracy in classifying water areas was based on the $\rho$ short-wave infrared. They also compared the results of band slicing with a maximum likelihood classifier on six bands of LANDSAT for their study area and found that the maximum likelihood approach only slightly outperformed the method of simple $\rho$ density slicing. McFeeters (1996) found that using the green $\rho$ and Near Infrared (NIR) $\rho$ emphasised water areas on top of other objects of the land surface, and proposed the normalized difference water index (NDWI) to delineate water areas: $N D W I=\left(\rho_{\text {Green }}-\rho_{\mathrm{NIR}}\right) /\left(\rho_{\text {Green }}+\rho_{\mathrm{NIR}}\right)$, where the water surfaces have positive values, and other objects have negative values. Rogers and Kearney (2004) suggested using 
the combination of red $\rho$ and SWIR1 $\rho$ as a ratio ( $\rho_{\text {Red }}-$ $\rho$ SWIR 1$) /\left(\rho_{\text {Red }}+\rho_{\text {SWIR } 1)}\right.$ to automatically delineate water boundaries, arguing that only water is more reflective in SWIR1 $\rho$ than red $\rho$. Xu (Xu 2006) found that applying the NDWI misclassified noise of built-up land in urban areas, revealing that the reflectance pattern of built-up areas on green $\rho$ and NIR $\rho$ is similar to these of water. He also found that the built-up areas on SWIR1 $\rho$ have much greater values than on NIR $\rho$, and suggested replacing this band by a modified normalized difference water index $M N D W I=\left(\rho_{\text {Green }}-\right.$ $\rho$ SWIR 1$) /\left(\rho_{\text {Green }}+\rho\right.$ SWIR 1$)$. (Feyisa et al. 2014) proposed nonnormalised multi-band ratios of LANDSAT data to delineate water areas. Their automated water extraction indexes (AWEI) specifically target areas with dark surfaces: $A W E I_{n s h}=4 *(\rho$ Green $\left.-\rho_{\text {SWIR } 1}\right)-\left(0.25 * \rho_{\mathrm{NIR}}+2.75 * \rho_{\mathrm{SWIR} 2}\right)$; in mountainous areas they used $A W E I_{s h}=\rho_{\text {Blue }}+2.5 * \rho_{\text {Green }}-1.5 *\left(\rho_{\text {NIR }}+\rho_{\text {SWIR } 1}\right)-$ $0.25 * \rho$ swIR2 to avoid confusion of deep shadows with water bodies. Another non-normalized water index (WI) proposed for large regions by Fisher, Flood, and Danaher (2016) uses the combination of five LANDSAT bands. The index is applied for surface reflectance data and evaluated as $W I=1.7204+$ $171 \rho_{\text {Green }}+3 \rho_{\text {Red }}-70 \rho_{\text {NIR }}-45 \rho_{\text {SWIR } 1}-71 \rho_{\text {SWIR2 }}$.

\subsection{Lake detection methods}

Habib et al. (2006) suggested three methods for detecting lakes from SPOT images, i.e. the spectral angle mapper (SAM) classification method, the irregular pyramid, and the watershed with markers methods. They concluded that all of these methods had advantages and disadvantages, and to achieve the best results they suggested combining them. Verpoorter, Kutser, and Tranvik (2012) introduced a method for extracting water bodies from LANDSAT GeoCover ${ }^{\mathrm{TM}}$ mosaics (GWEM), delineating lakes $>0.0002 \mathrm{~km}^{2}$ in size. They used thresholding and supervised classification to map water boundaries, and classified lake outlines by texture analysis. In a refinement step they converted lakes boundaries to vector format and evaluated their area, perimeter, and shape for evaluating additional morphometric parameters. In a last step they removed falsely classified shadows. Jiang et al. (2014) proposed an automated method for extracting rivers and lakes (AMERL) from LANDSAT images. They compared their method with the NDWI, MNWDI, and AWEI, and concluded that their method was superior, because it accounts for not only the spectral characteristics of pixels, but also their neighbourhood. Jawak and Luis (2014) suggested a semi-automatic approach for extracting lakes from World View-2 images based on a modified NDWI algorithm, and suggested to modify the NDWI and applying PAN-sharpening of images from $2 \mathrm{~m}$ into $0.5 \mathrm{~m}$ to improve classification.

\section{TEST AREA AND DATA}

\subsection{Test area}

We selected the greater part of the Tibetan Plateau as our test area (Fig. 1). We excluded from the analysis the eastern part of the Tibetan Plateau mainly because of the few high-quality cloud free LANDSAT images. We analysed all lakes on the selected satellite images, also these beyond the Tibetan Plateau margin. We tested our method for semi-automatically extracting lake outlines for most of the Tibetan Plateau where lakes formed in tectonic depressions or behind glaciers and sediments, and have different spectral colours, and different physical states of water (frozen and non-frozen).

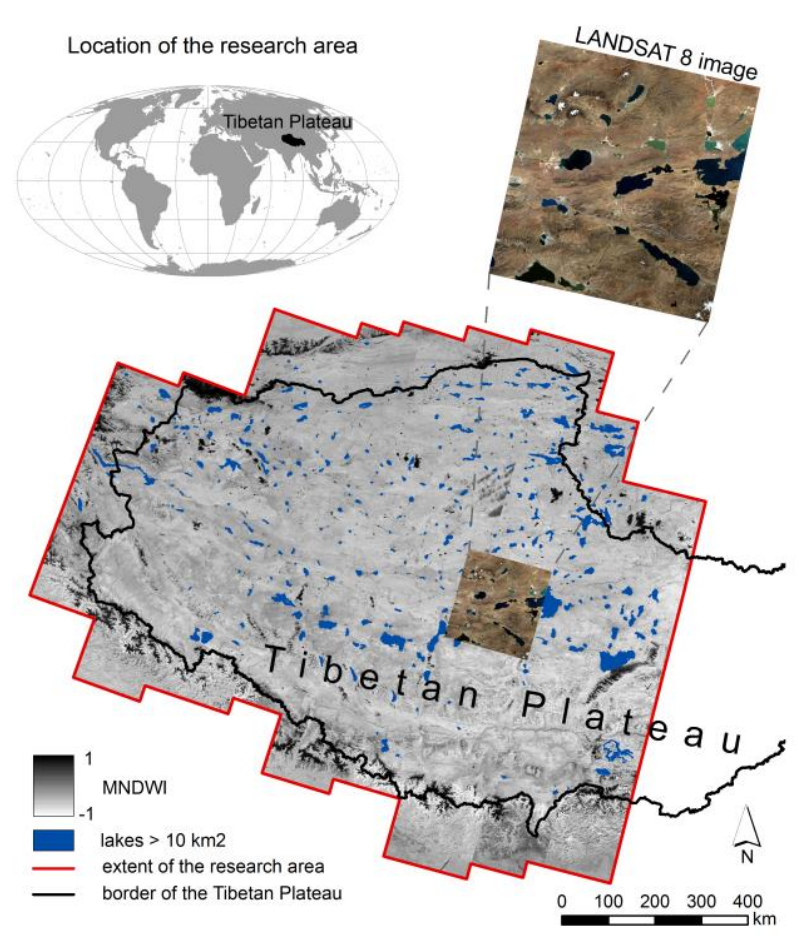

Figure 1. Study area on the Tibetan Plateau and modified normalized difference water index (MNDWI) for major lakes.

\subsection{Data}

We analysed 47 LANDSAT-5 images for 1995, and 47 LANDSAT-8 images for 2015. The size of the study area and the different weather conditions captured on these images required that we analysed different days of the year, i.e. from 25 April to 17 December 1995, and from 8 June to 22 November 2015. Due to the lack of high-quality images for the study area in 1995, we also included 22 images from 1994 and four images from 1996. We selected only images with $<10 \%$ cloud cover, and atmospheric and sun-angle correction provided by the U.S. Geological Survey (http://espa.cr.usgs.gov/). We used Top-OfAtmosphere (TOA) reflectance bands because the cosine effect of different solar zenith angles linked to different time in data acquisition is already removed from these data (Chander et al. 2009). To delineate the lake boundaries we used the SRTM DEM version 4 (Reuter, Nelson, and Jarvis 2007) (http://srtm.csi.cgiar.org/) as a supporting layer in the OBIA analysis. We used the SRTM DEM to generate a local slope map of the Tibetan Plateau. The slope map records the rate of elevation change between neighbouring pixels.

\section{METHODS}

Our workflow uses satellite images taken in all seasons, thus involving seasonally changing water colours and ice cover (Fig. 2 ). Consequently the RGB values of satellite images that are used for classifying water boundaries are not a proper input to delineate the lake shapes. Therefore, we applied an MNDWI algorithm for distinguishing water areas irrespective of the physical state of the water bodies and their visual appearance. In this regard, we tested the modified normalized difference water index $M N D W I$ (Xu 2006). In this algorithm only those pixels representing the water areas should have positive values. However, objects like glaciers, and mountains shadows have also values above the zero, making the classification of water areas in mountainous and ice-covered areas problematic. To 
meet this challenge, we combined the MNDWI and its thresholds with OBIA and SRTM data for a more integrative approach for automatically detecting lakes.
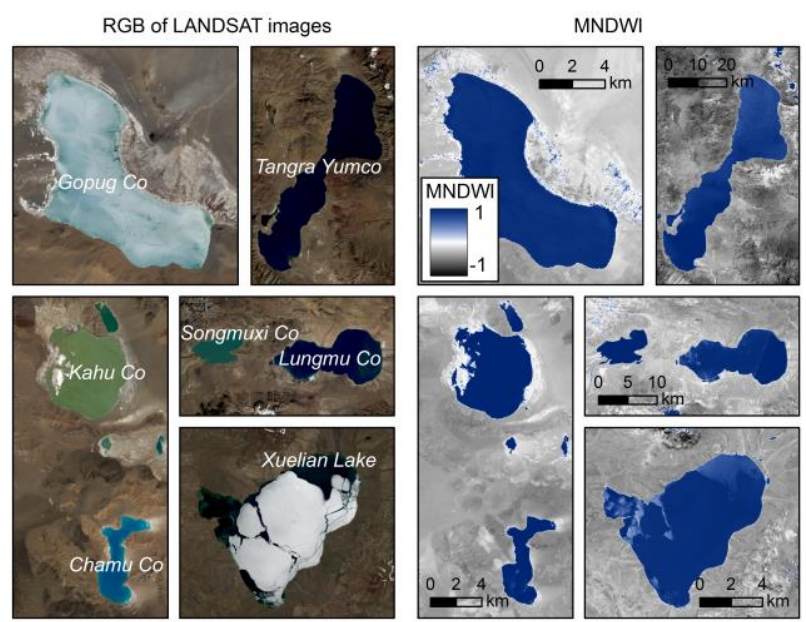

Figure 2. The RGB values of sample lakes on the Tibetan

Plateau and their modified normalized difference water index (MNDWI).

In the first step we generated mosaics using standard method Mosaic to New Raster available in ArcGIS software for bands from all images for 1995 and 2015. Then we evaluated the MNDWI for the whole study area. We also evaluated the slope map $\left[{ }^{\circ}\right.$ ] in a $3 \times 3$ search window from the SRTM DEM data using the Slope function with default options available in ArcGIS software. We then applied a multi-resolution segmentation algorithm (Baatz and Schäpe 2000) to segment the MNDWI values and classify the segments as either 'water' or 'other'. We applied the multi-resolution segmentation only to the MNWDI without the slope map, because the SRTM data date back to March 2000, and might thus influence the segmentation process. Using a manual trial and error approach we selected 100 as the scale of segmentation (Fig. 3). For this scale the segment size was smaller than lakes $>10 \mathrm{~km}^{2}$ in area, and large enough to maintain feasible computing time. We arbitrarily selected 0.1 as the shape, and 0.7 as the compactness parameters, after performing tests with different scales.
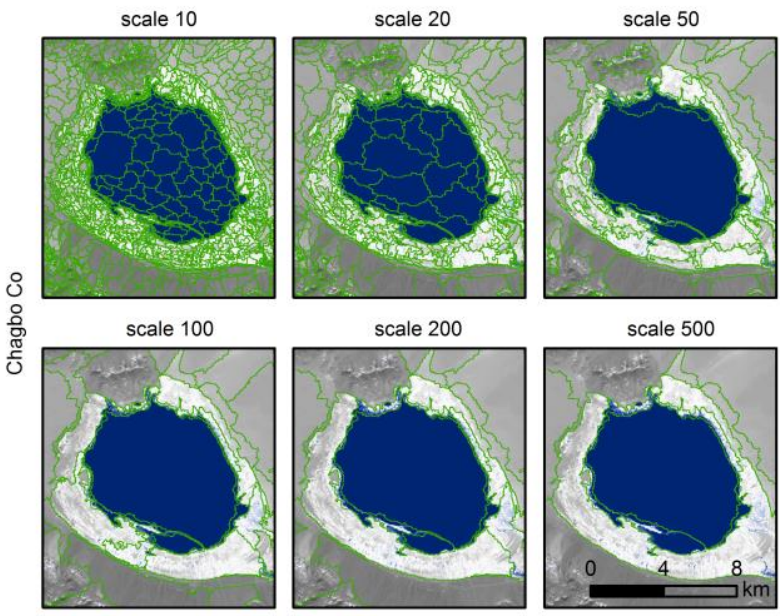

scale 200

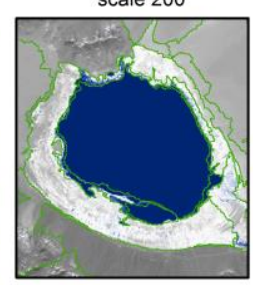

scale 500

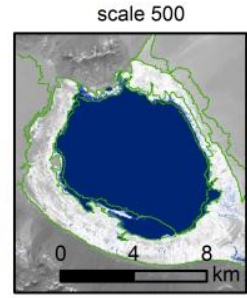

Figure 3. Multiresolution segmentation of Chagbo Co with different scales.

In the next step we used the threshold value of the MNDWI to classify water segments. To differentiate lakes from rivers we used the asymmetry of segments and their relation to the neighbouring segments and also their classification. The asymmetry we computed with the Asymmetry function in the eCognition software, where it is defined as a relative length of the segment to a regular polygon drawn around the segment. We find that many glaciers on the Tibetan Plateau have MNDWI values similar to those of lakes, and that a pure OBIAdriven classification based on MNDWI produced many errors, so that we used the slope map from the SRTM DEM as a supporting layer. We assumed that the glaciers are located on areas with slope $>2^{\circ}$ to distinguish them from lakes. To omit resolution errors, we used only those lakes $>10 \mathrm{~km}^{2}$ and exported them as vector polygons for further analysis. We used the exported lakes polygons to generate a reference data set, and verified visually the correctness of lakes boundaries using natural colour mosaic (RGB) generated from LANDSAT data. In some cases, especially in areas where the lakes are very shallow and visual interpretation of RGB image was difficult, we supported the delineation of lakes boundaries by analysing the MNDWI map. We edited the edges of all lakes in areas where the boundary was incorrectly delineated, and delated all falsely classified polygons not representing lakes.

\section{RESULTS}

Our classification of Tibetan lakes found that in 2015 the total area of lakes $>10 \mathrm{~km}^{2}$ was $31,258 \mathrm{~km}^{2}$, or $2.6 \%$ of the total study area. Twenty years earlier, these same lakes covered only $24,892 \mathrm{~km}^{2}$. The total area of large lakes thus grew by $\sim 26 \%$ over two decades on average.

\subsection{Classification accuracy}

To verify the correctness of our classification we compared the results with manually generated reference data, and computed several standard performance statistics (Sithole and Vosselman 2004), (Congalton 1991), (Cohen 1960). Our OBIA method for extracting lakes with the area $>10 \mathrm{~km}^{2}$ had an overall accuracy of 0.99 (Tab. 1); the producer's and user's accuracy as well as Cohen's kappa for both analysed time slices was $>0.98$.

\begin{tabular}{lcc}
\hline \multicolumn{1}{c}{$\begin{array}{c}\text { Performance } \\
\text { statistics }\end{array}$} & 1995 & 2015 \\
\hline Type I error & 0.0134 & 0.0169 \\
Type II error & 0.0003 & 0.0005 \\
Total error & 0.0006 & 0.0010 \\
Overall accuracy & 0.9994 & 0.9990 \\
Producer's accuracy & 0.9866 & 0.9831 \\
Users accuracy & 0.9850 & 0.9808 \\
Cohen's kappa & 0.9855 & 0.9815 \\
\hline
\end{tabular}

Table 1. Performance statistics for automatic lakes classification

By cross-checking these classification results visually we see that the most of misclassified areas occur on the border of lakes (Fig. 4), especially in areas where the lakes has very complex coastlines or where rivers flows into the lake; it is there that our object-based approach tends to create only single segments. Clouds also favoured misclassification on some images, especially where located directly above the lake or its coasts. The physical state of the lake surface did not influence our automatic OBIA approach (Fig. 4 - Xuelian Lake), and both frozen and not-frozen parts of lake were correctly classified. We also observed that glaciers were distinguished from lakes with high accuracy, and only few glaciers were wrongly classified as lakes. The biggest confusion potential occurs where glacier segments are sharing a border with lake segments. In some 
cases small island lakes were falsely misclassified by adding them into the lake class.

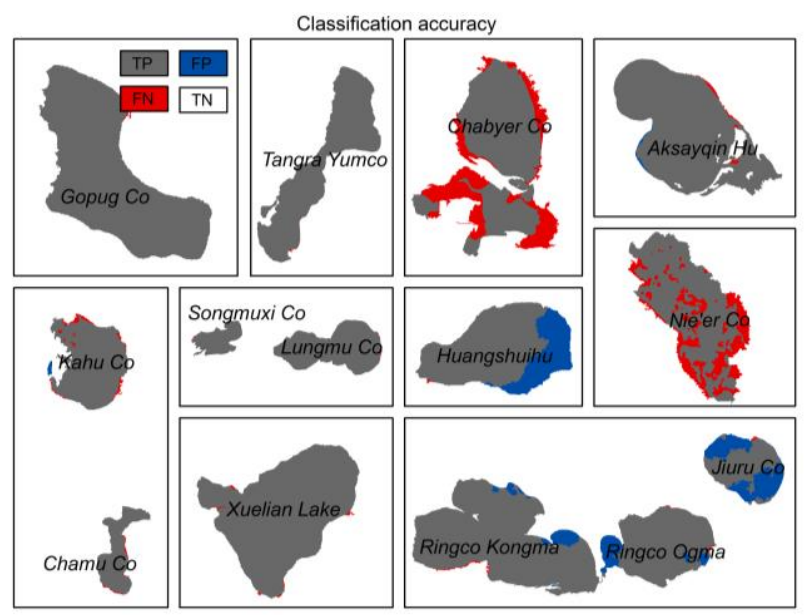

Figure 4. OBIA classification accuracy examples for 2015 data based on confusion matrix; TP is true positive, FP is false positive, $\mathrm{FN}$ is false negative, and $\mathrm{TN}$ is true negative.

A good example of a rather low classification accuracy is Chabyer Co (Fig. 4); there, many pixels were falsely classified as 'lake', because this lake is changing its size rapidly, so that misclassified pixels represent marshy areas where shallow water occurred in the past. Nie'er Co is another example of a lake detected with low accuracy. Although this lake has been correctly detected by the thresholding of the MNDWI, the assumptions which we used in the OBIA approach included small lake islands and surrounding areas as lakes.

\subsection{Lakes changes}

Our analysis shows that in general the total area of lakes on the Tibetan Plateau increased about $0.5 \%$ or some $6,366 \mathrm{~km}^{2}$. Changes in the lakes area are not evenly spread out on the whole area of the Tibetan Plateau (Fig. 5).
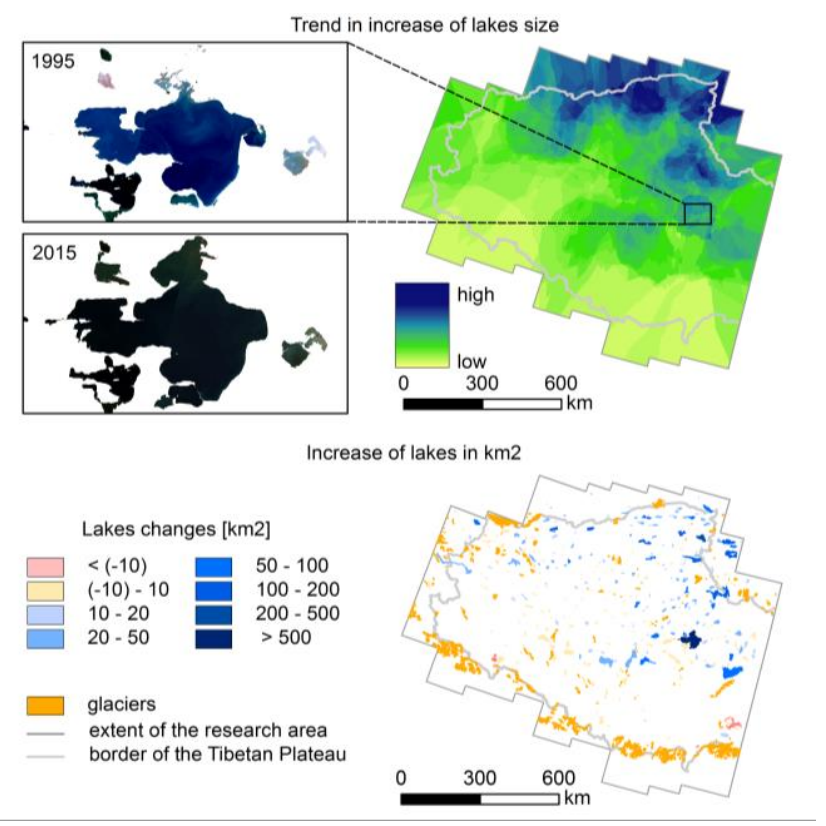

Figure 5 Changes in lake areas on the Tibetan Plateau from 1995 to 2015, compared to glacier area.
Lakes were growing most strikingly in the north-eastern parts, which also hosts the largest number of lakes with individual net increases of $>100 \mathrm{~km}^{2}$. Most lakes with size changes of $<10 \mathrm{~km}^{2}$ in the past two decades are in the southwestern part of the Tibetan Plateau, mainly along the Himalayas and adjacent mountain belts.

\section{DISCUSSION}

Our automatic classification results have a strikingly high accuracy. Most lakes have been correctly detected with only few misclassifications along lake boundaries, especially complex coastline geometries. We found the MNDWI an accurate indicator of all water areas, regardless of whether they sustained ice cover or not. The segmentation algorithm in our workflow helped to reduce the salt-and-pepper noise and delineate more reliably the boundaries of lakes, whereas OBIA allowed finding the segments which were incorrectly classified by the MNDWI thresholding. Spuriously low values for thresholding arose from clouds above the lakes, too low MNDWI values, or mountain shadows. Integrating topography via a slope map enabled us to exclude from the lakes class falsely classified glaciers. We infer that using more accurate DEMs might help to achieve better results, especially if the topographic data were captured closer to the timeframe of interest. We used DEM obtained in 2000 to analyse lakes in 1995 and 2015, meaning that we had 10 to 15 years difference between our elevation data and the satellite images. Any OBIA workflow should be corrected for such possible mismatches, wherever possible.

Our change detection analysis reveals that changes in lake size are not uniform across the Tibetan Plateau, thus supporting similar results from previous works (Song, Huang, and $\mathrm{Ke}$ 2013), (Song et al. 2014), and (Fang et al. 2016). Comparing our findings to a regional glacier map, we see that the most of glaciers are located in the Himalayan Mountains Range where the growth of large lakes is smallest, hence any excess glacial meltwater might mostly feed lakes $<10 \mathrm{~km}^{2}$ in that area. It is equally possible that any growth of lakes in the Himalayas remains shrouded from automated detection because of the locally rough terrain characteristics. Changes to lakes in flatter areas involve commensurate larger increases in area than in mountainous areas. Such topographic boundary conditions may partly explain the large net growth of lake areas in the northeastern Tibetan Plateau, although detailed analyses are clearly desirable to unravel the underlying causes.

\section{CONCLUSIONS}

In this contribution we presented an approach for automatic detection of lakes $>10 \mathrm{~km}^{2}$, and their changes through time, drawing on examples from the Tibetan Plateau. Our method is designed for detecting lakes with different state of the water for large areas, especially those where many glaciers occur. Our approach combined a satellite-derived water index, OBIA, and DEM-derived slope data to automatically extract lakes. Our classification derived 323 lakes with a total area of $31,258 \mathrm{~km}^{2}$, or $2.6 \%$ of the study area in 2015 . The same number of lakes had covered only $24,892 \mathrm{~km}^{2}$ in 1995 , so that lakes grew by $\sim 26 \%$ in the past two decades on average. We conclude that our approach is general and flexible enough to provide easy transferability and its application in areas other than the Tibetan Plateau, which we selected as our testing research area. 


\section{ACKNOWLEDGEMENTS}

This research was funded by the European Union under the Marie Curie Initial Training Network ALErT (Creation of an interactive CAP natural-hazard database), project-number: FP7PEOPLE-2013-ITN-607996. The LANDSAT images used in the study were provided by the U.S. Geological Survey, available from http://espa.cr.usgs.gov/. The SRTM DEM version 4 data were provided by the CGIAR-CSI, available from http://srtm.csi.cgiar.org/.

\section{REFERENCES}

Baatz, Martin, and Arno Schäpe. 2000. "Multiresolution Segmentation: An Optimization Approach for High Quality Multi-Scale Image Segmentation.” Angewandte Geographische Informationsverarbeitung XII. Beiträge zum AGIT-Symposium Salzburg 2000, Karlsruhe, Herbert Wichmann Verlag: 12-23.

Chander, Gyanesh, Brian L Markham, and Dennis L Helder. 2009. "Remote Sensing of Environment Summary of Current Radiometric Calibration Coef Fi Cients for Landsat MSS , TM , ETM + , and EO-1 ALI Sensors." Remote Sensing of Environment 113(5): 893-903.

Cohen, Jacob. 1960. "A Coefficient of Agreement for Nominal Scales.", Educational and Psychological Measurement 20(1): 37-46.

Congalton, Russell G. 1991. "A Review of Assessing the Accuracy of Classifications of Remotely Sensed Data." Remote Sensing of Environment 37(1): 35-46.

Fang, Yue, Weiming Cheng, Yichi Zhang, Nan Wang, Shangmin Zhao, Chenghu Zhuo, Xi Chen, and Anming Bao. 2016. "Changes in Inland Lakes on the Tibetan Plateau over the Past 40 Years." Journal of Geographical Sciences 26(4): 415-38.

Feyisa, Gudina L., Henrik Meilby, Rasmus Fensholt, and Simon R. Proud. 2014. "Automated Water Extraction Index: A New Technique for Surface Water Mapping Using Landsat Imagery." Remote Sensing of Environment 140: 23-35.

Fisher, Adrian, Neil Flood, and Tim Danaher. 2016. "Comparing Landsat Water Index Methods for Automated Water Classification in Eastern Australia." Remote Sensing of Environment 175: 167-82.

Frazier, Paul Shane, and Kenneth John Page. 2000. "Water Body Detection and Delineation with Landsat TM Data." Photogrammetric Engineering \& Remote Sensing 66(12): 1461-67.

Habib, Tarek, Michel Gay, Jocelyn Chanussot, and Pascal Bertolino. 2006. "Segmentation of High Resolution Satellite Images SPOT Applied to Lake Detection." International Geoscience and Remote Sensing Symposium (IGARSS) (April): 3680-83.

Jawak, Shridhar D, Kamana Kulkarni, and Alvarinho J Luis. 2015. "A Review on Extraction of Lakes from Remotely Sensed Optical Satellite Data with a Special Focus on Cryospheric Lakes." J 4(4): 196-213.

Jawak, Shridhar D, and Alvarinho J Luis. 2014. “A Semiautomatic Extraction of Antarctic Lake Features Using Worldview-2 Imagery." (October): 33-46.

Jiang, Hao, Min Feng, Yunqiang Zhu, Ning Lu, Jianxi Huang, and Tong Xiao. 2014. "An Automated Method for Extracting Rivers and Lakes from Landsat Imagery." Remote Sensing 6(6): 5067-89.

Liu, Xiaodong, and Baode Chen. 2000. "Climatic Warming in the Tibetan Plateau During Recent Decades."
International Journal of Climatology 20: 1729-42.

McFeeters, S. K. 1996. "The Use of the Normalized Difference Water Index (NDWI) in the Delineation of Open Water Features." International Journal of Remote Sensing 17(7): 1425-32.

Reuter, H. I., A. Nelson, and A. Jarvis. 2007. 21 International Journal of Geographical Information Science $A n$ Evaluation of Void-Filling Interpolation Methods for SRTM Data.

Rogers, A. S., and M. S. Kearney. 2004. "Reducing Signature Variability in Unmixing Coastal Marsh Thematic Mapper Scenes Using Spectral Indices." International Journal of Remote Sensing 25(12): 2317-35.

Ryu, Joo Hyung, Joong Sun Won, and Kyung Duck Min. 2002. "Waterline Extraction from Landsat TM Data in a Tidal Flat a Case Study in Gomso Bay, Korea." Remote Sensing of Environment 83(3): 442-56.

Sithole, George, and George Vosselman. 2004. "Experimental Comparison of Filter Algorithms for Bare-Earth Extraction from Airborne Laser Scanning Point Clouds." ISPRS Journal of Photogrammetry and Remote Sensing 59(1-2): 85-101.

Song, Chunqiao, Bo Huang, and Linghong Ke. 2013. "Modeling and Analysis of Lake Water Storage Changes on the Tibetan Plateau Using Multi-Mission Satellite Data." Remote Sensing of Environment 135: 25-35.

Song, Chunqiao, Bo Huang, Linghong Ke, and Keith S. Richards. 2014. "Remote Sensing of Alpine Lake Water Environment Changes on the Tibetan Plateau and Surroundings: A Review." ISPRS Journal of Photogrammetry and Remote Sensing 92: 26-37.

Verpoorter, Charles, Tiit Kutser, and Lars J. Tranvik. 2012. "Automated Mapping of Water Bodies Using Landsat Multispectral Data." Limnology and Oceanography: Methods 10: 1037-50.

Wang, Cuizhen. 2016. "A Remote Sensing Perspective of Alpine Grasslands on the Tibetan Plateau: Better or Worse under 'Tibet Warming'?" Remote Sensing Applications: Society and Environment 3: 36-44.

$\mathrm{Xu}$, Hanqiu. 2006. "Modification of Normalised Difference Water Index (NDWI) to Enhance Open Water Features in Remotely Sensed Imagery." International Journal of Remote Sensing 27(14): 3025-33.

Yang, Wei, Tan Dong Yao, Bai Qing Xu, Guang Jian Wu, Ling Long Ma, and Xiao Dong Xin. 2008. "Quick Ice Mass Loss and Abrupt Retreat of the Maritime Glaciers in the Kangri Karpo Mountains, Southeast Tibetan Plateau." Chinese Science Bulletin 53(16): 2547-51.

Zhang, Guoqing et al. 2011. "Monitoring Lake Level Changes on the Tibetan Plateau Using ICESat Altimetry Data (2003-2009)." Remote Sensing of Environment 115(7): 1733-42. 


\title{
TEMPLATE MATCHING TO SUPPORT EARTH OBSERVATION BASED REFUGEE CAMP ANALYSIS IN OBIA WORKFLOWS - CREATION AND EVALUATION OF A DWELLING TEMPLATE LIBRARY FOR IMPROVING DWELLING EXTRACTION WITHIN AN OBJECT-BASED FRAMEWORK
}

\author{
P. Krafft ${ }^{\mathrm{a}}$, D. Tiede ${ }^{\mathrm{a}}$, P. Füreder ${ }^{\mathrm{a}}$ \\ a Department of Geoinformatics - Z_GIS, University of Salzburg, Austria - pascal.krafft@stud.sbg.ac.at, (dirk.tiede, \\ petra.fuereder)@sbg.ac.at
}

\begin{abstract}
KEY WORDS: Object-based image analysis (OBIA), Template matching, Object detection, dwelling extraction, refugee and IDP camps, VHR data
\end{abstract}

\begin{abstract}
:
Accurate and reliable information about the situation in refugee or internally displaced person camps is very important for planning any kind of help like health care, infrastructure or vaccination campaigns. The number and spatial distribution of single dwellings extracted semi-automatically from very high resolution (VHR) satellite imagery as an indicator for population estimations can provide such important information. The accuracy of the extracted dwellings can vary quite a lot depending on various factors. To enhance established single dwelling extraction approaches, we have tested the integration of stratified template matching methods in objectbased image analysis (OBIA) workflows. The created template library aims to be generally applicable in similar conditions. Compared to pre-existing OBIA classifications, the approach could increase in average the producer's accuracy by $12 \%$ and also slightly increase the user's accuracy. These results show that the stratified integration of template matching approaches in OBIA workflows is a possibility to further improve results of semi-automated dwelling extraction, especially in complex situations.
\end{abstract}

\section{INRODUCTION}

The number of refugees, asylum-seekers and internally displaced persons (IDP) again hits an all-time high. By the end of 2015, more than 65 million people were forcibly displaced (UNHCR, 2016) caused by natural disasters, changing environmental conditions and violent conflicts, which constitute the main reasons for displacement. Refugee camps and temporary settlements provide refuge for most of these people (Füreder et al., 2015; UNHCR, 2015). Accurate, reliable and up-to-date information about the situation in refugee and IDP camps like population estimations is very important for planning any kind of help like health care, infrastructure or vaccination campaigns. Camp management is performed typically by humanitarian relief organizations. Often it is impossible to gather such information by field assessments, because of security reasons provoked by conflict situations. Furthermore, there are sometimes incorrect information provided by stakeholders, which try e.g. to increase the number of refugees for various reasons or political interests are leading to reduced numbers of reported IDPs. IDPs have not crossed an international recognized border consequently they stay inside their home countries. Therefore the protection remains legally by their own government making assistance, such as regulated camp management, often very difficult or it is not allowed at all. The number and spatial distribution of single dwellings extracted from very high-resolution (VHR) satellite imagery can provide important information in such situations (Grundy et al., 2012; Spröhnle et al., 2014; Tiede et al., 2013). Nevertheless the accuracy of the extracted dwellings can vary quite a lot depending on various factors, such as the contrast of dwelling types to surroundings, weather situation, but also the dwelling density (Spröhnle et al., 2014). A specific limitation of existing objected-based workflows is related to the initial segmentation of the image. This process creates segments for image areas with usually similar pixel values, which can lead to under-segmentation in the case of similar spectral reflectance of the dwellings and their surroundings (e.g. under dusty conditions). On the other hand dwellings and their cast shadows can help in such situations to still distinguish dwellings from the non-elevated surroundings, but also in this case a proper segmentation of the dwelling and the shadow area is needed. Figure 1 shows an initial image segmentation where the shadow of the left dwelling is not a clearly delineated segment, which makes it hard to use the shadow as a clear identifier for a dwelling in an automated process as it is done by a visual image interpretation in the classification process.

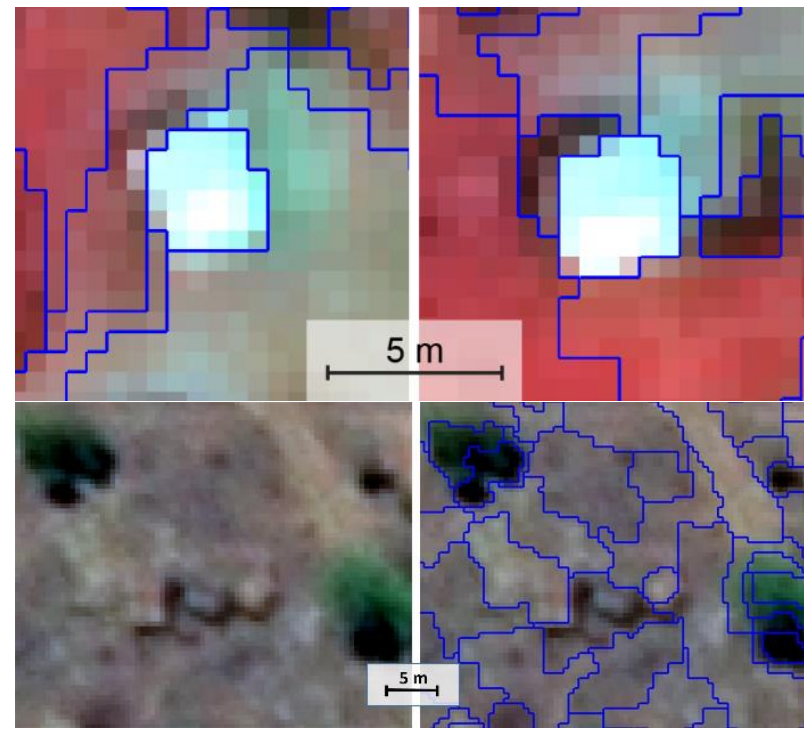

Figure 1. QuickBird 2 subsets of the Yida camp area (acquisition date: 12.10.2012, band combination NIR-R-G) showing an initial image segmentation example of two bright dwellings and the cast shadows (above). Below an example of dark dwellings which show very low contrast to the surrounding, the cast shadow is the only indicator for the presence of dwellings. Segmentation based on homogeneity usually fails in extracting meaningful objects (Yida camp, 03.04.2013, WorldView-2, R-G-B) 
To enhance single object detection in such difficult situations, we have tested the integration of stratified template matching methods in object-based image analysis workflows. In the templates generation process the object to be detected and the surroundings can be taken into account, which is in our case a dwelling and its cast shadow. Thus, matches include information regarding the orientation and can be used in the analyses to reduce false positive alarms.

The concept of template matching can be summarized as the process of comparing patterns with regard to their similarity enabling single objected detection. Although template-matching methods are not new, there is still little research on combining them with OBIA methods in one workflow. The combination of the two approaches has the aim to tackle typical problems of template matching approaches, like e.g. a high number of false positives in complex images by focusing on relevant parts of the image only (e.g. certain parts of refugee camps or certain spectrally relevant objects). The main assumption is, that the incorporation of the shadow effect of dwellings can improve the dwelling detection rate. A visual study of different refugee camps revealed that the same dwelling types often occur (bright, blue, and dark/ brown dwellings) as well as large and small structures with typical geometrical properties (cf. Figure 2). The aim of the established template library is to be generally applicable in similar conditions and to be easily integrated in already existing workflows for object-based dwelling extraction.
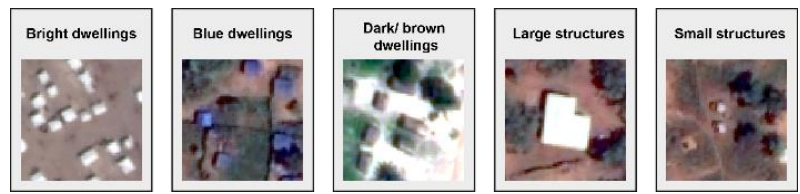

Figure 2. Typical occurring dwelling types detected in refugee and IDP camps in East Africa. Source: Füreder et al., 2015, adapted.

In order to check the accuracy of the approach it was tested on three sites of different complexities regarding dwelling extraction and the results were compared to a visual image interpretation as well as a pre-existing (independent) OBIA classifications.

\section{STUDY AREA AND DATA}

For the creation of the dwelling template library 16 VHR optical images were used. The satellite images cover parts of 10 refugee and IDP camps. These camps are distributed over Eastern Africa. The spatial distribution of the camps is presented in Figure 4. The spatial resolution ranges between $0.5 \mathrm{~m}$ and $0.6 \mathrm{~m}$ ground sample distance (pan-sharpened VHR data)

Existing semi-automated dwelling classifications were provided by the Department of Geoinformatics - Z_GIS, University of Salzburg, within an operational service for Médecins Sans Frontières (MSF) for three images (El Redis, Sudan 12.03.2015, Yida, South Sudan 12.10.2012, Yida 03.04.2013; cf. Table 1) in order to compare the outcome of the dwelling template library with existing and verified results.

\section{METHODOLOGY}

\subsection{Template matching library}

One of the objectives of this work was to create a template library for dwellings of refugee and IDP camps that can easily be integrated in already existing workflows for dwelling extraction, applicable to different kind of dwelling detection. Most of the satellite images are taken when the sun is not at the zenith of the captured region. Therefore, the library is suitable for images where objects with a certain level of height cast a shadow. The samples for the templates are taken from different camps (cf. Figure 4) to capture the variety of dwelling types at different locations, time steps and weather conditions like rainy and dry season or the effects after a sandstorm. Since most of the used images have a ground sample distance (GSD) of $0.5 \mathrm{~m} / 0.6 \mathrm{~m}$, the library is best fitting for this resolution. However, it can also be applied to other image resolutions. The applied workflow for creating a template and integration in the library is presented in Figure 3.
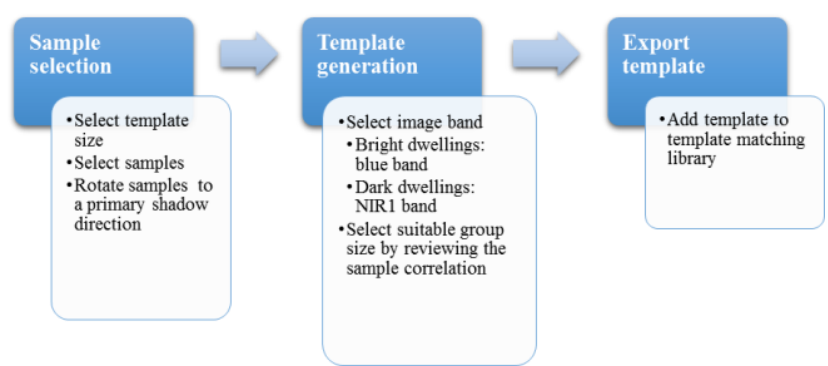

Figure 3. Generalized workflow of template generation applied in this study.

The template library is structured according to the attributes of the templates (colour intensity, dwelling shape, size and shadow direction) that allow to cover several possible combinations of a dwelling type and the cast shadow. Moreover, new dwelling templates can easily be added to the existing library enabling to customize it depending on the specific problem. The naming convention also follows this structure, making it simple for the user to find the right dwelling templates without screening all available template images. The following paragraphs describe the structure of the template library in detail.

\subsubsection{Dwelling shape}

Small objects in satellite image, such as dwellings in refugee and IDP camps look like the image were taken from bird's eye view although the VHR data is usually not collected at nadir. The building lean effect is only visible on tall objects (Baltsavias et al., 2001). Therefore, usually only the roof of a dwelling is visible and in this work the dwelling shapes are mostly referred to twodimensional geometric shapes. Dwellings in refugee camps typically have simple shapes. The predominantly simplified types are quadratic, rectangular cuboids and cones. The template library is therefore structured regarding the following three dwelling types: square, rectangle and round.

\subsubsection{Template size}

The template size is defined by the height and width of a rectangle. Templates should be big enough to cover the dwelling that is centred, the cast shadow and a bit of the surrounding area in order to include the differences of dwelling to surroundings.

\subsubsection{Dwelling brightness}

The templates are calculated in this implementation on one image band only. Hence, they are grayscale, which means that there is no distinction between colours but only between shades of grey. The template library is divided in two intensity levels: Strong intensity - bright dwellings, Weak intensity - dark dwellings. The contrast of bright dwellings compared to surrounding areas was best on the blue band. For dark dwellings, best results were 
achieved on the near infrared (NIR) band. This was detected by empirical testing and comparing the correlation of the samples of a template on the pan-sharpened multi-spectral bands (blue, green, and red and NIR).

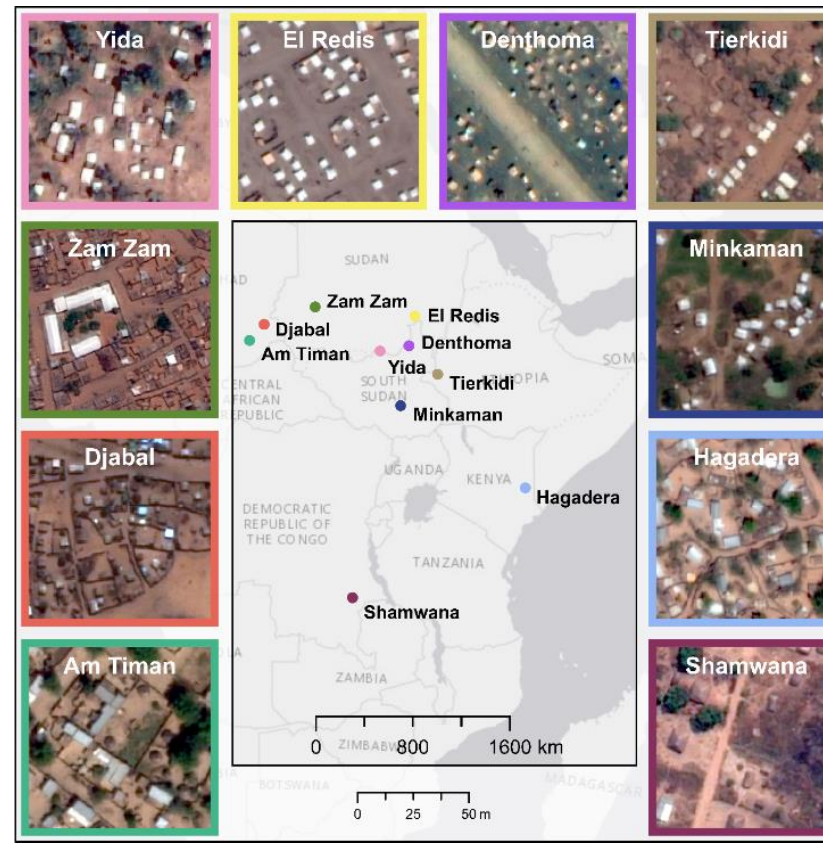

Figure 4. Spatial distribution of the refugee/IDP camps (band combination of images: R-G-B).

\begin{tabular}{|c|c|c|c|c|c|c|c|c|}
\hline & Shape & Square & Square & Rectangle & Rectangle & Rectangle & Rectangle & Round \\
\hline & Color & White & White & White & White & White & White & Grey \\
\hline & $\begin{array}{c}\text { Size } \\
{[\text { [ixel] }}\end{array}$ & $12 \times 12$ & $12 \times 12$ & $36 \times 15$ & $38 \times 15$ & $15 \times 36$ & $36 \times 15$ & $12 \times 12$ \\
\hline 裹 & Name & $\begin{array}{l}\text { Square } \\
1100 \\
\text { White } \\
12 \times 12\end{array}$ & $\begin{array}{l}\text { Square } \\
1001 \\
\text { White } \\
12 \times 12\end{array}$ & $\begin{array}{l}\text { Rectangle } \\
1000 \\
\text { White } \\
36 \times 15\end{array}$ & $\begin{array}{c}\text { Rectangle } \\
\text { 1001 } \\
\text { White } \\
38 \times 15\end{array}$ & $\begin{array}{l}\text { Rectangle } \\
1000 \\
\text { White } \\
15 \times 36\end{array}$ & $\begin{array}{l}\text { Rectangle } \\
1100 \\
\text { White } \\
36 \times 15\end{array}$ & 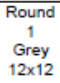 \\
\hline لِّ & $\begin{array}{l}\text { Aerial } \\
\text { view }\end{array}$ & 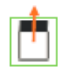 & A & & & & & \\
\hline & $\begin{array}{l}4^{\circ} \\
\text { view }\end{array}$ & 8 & $\square$ & & & & & \\
\hline \multirow{4}{*}{ 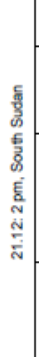 } & $\begin{array}{c}\text { size } \\
\text { [pixel] }\end{array}$ & $12 \times 12$ & $12 \times 12$ & $36 \times 15$ & $38 \times 15$ & $38 \times 15$ & $15 \times 36$ & $12 \times 12$ \\
\hline & Name & $\begin{array}{l}\text { Square } \\
1101 \\
\text { White } \\
12 \times 12 \\
\end{array}$ & $\begin{array}{l}\text { Square } \\
\text { 1000 } \\
\text { White } \\
12 \times 12\end{array}$ & $\begin{array}{c}\text { Rectangle } \\
1100 \\
\text { White } \\
36 \times 15 \\
\end{array}$ & $\begin{array}{c}\text { Rectangle } \\
\text { Wonte } \\
\text { White } \\
30 \times 15 \\
\end{array}$ & $\begin{array}{c}\text { Rectangle } \\
1001 \\
\text { White } \\
30 \times 15\end{array}$ & $\begin{array}{l}\text { Rectangle } \\
1000 \\
\text { White } \\
15 \times 30 \\
\end{array}$ & 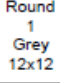 \\
\hline & $\begin{array}{l}\text { Aerial } \\
\text { view }\end{array}$ & & & & & & & \\
\hline & $\begin{array}{l}4^{\circ} \\
\text { view }\end{array}$ & $v$ & & & & & & \\
\hline
\end{tabular}

Figure 5: Structural attributes of the template library such as shadow direction, shape, type and size of dwellings.

Exemplified at predominantly occurring dwelling types on 16 different satellite images in 10 surveyed refugee and IDP camps. The shadows are simulated for the 21.12.2013 at

$12 \mathrm{am}$ and $2 \mathrm{pm}$ for the camp Yida, which is located in South Sudan. The aerial view is north oriented the $45^{\circ}$ view is west south oriented. The orange arrow shows the rotation angle and the green rectangle the (relative) size of the samples for a dwelling template.

\subsubsection{Shadow direction}

The last attribute is the shadow cast by a dwelling. It determines the rotation angle of the samples, which is highly dependent on the shape of the object. Optical VHR satellite images are typically taken at daylight. Hence, the only relevant light source is the sun. Therefore, objects cast usually only simple shadows. As mentioned above, the predominantly simplified dwelling shapes are rectangular cuboids and cones. Latter can only cast a shadow or no shadow. Rectangular cuboids have three possibilities: no shadow, a shadow on one of four sides and shadows on two sides.

The template library is built especially for dwellings showing a cast shadow. Related to the attribute shadow, objects with round layout need only one template, cubic dwellings two templates and dwellings with rectangular layout three templates because the shadow can be on one side plus on the short and the long side as well as on the long and the short side, which cannot be approached by rotating.

Summarized, the library is divided in the following main template classes (Figure 5): (a) dwellings with strong intensity, which means they appear bright on satellite images and dwellings with low intensity (structures occur dark); (b) dwellings of different shapes that can be a square, rectangle or a circle; (c) the direction of the shadow cast by the dwellings, which can be on one side or on two sides.

\subsection{Application of the template library}

The application of the templates from the library is an interactive, but straightforward process, here applied within the eCognition software environment (version 9.1, Trimble Geospatial). In order to choose the right templates from the library it is necessary to take a look at the analysed satellite images and to select the appropriate templates for potentially occurring dwellings. Within the application of the template matching algorithm, the rotation steps of the templates can be adjusted. If, for example, four rotation steps are applied, the rotation angles of the template are $0^{\circ}, 90^{\circ}, 180^{\circ}$ and $270^{\circ}$. A suitable rotation angle is always the shadow orientation of dwellings in the image. A second parameter, which can be customized, is the correlation threshold. If the correlation of the template and the image at a point is higher than the thresholds set in the configuration of the template matching process, a point ("hit") will be created. There are only points created if it is the highest values compared to the neighbouring pixels (local maximum). The resulting point layer consists typically of a lot of false positive alarms, sorting out the false alarms is essential to receive more precise results. This can be done by adjusting the correlation thresholds and filtering queries depending on the rotation angles of the templates.

\subsection{Integration of template matching in an object-based image analysis workflow}

There are three main reasons for combining template matching with objected-based image analysis methods. At first, the application of two or more different templates may result in more than one hit on the same object that means dwellings are double (or more often) counted. Second, the point layers created by applying template matching still consist of too many false positive alarms, which is reflected in a low user's accuracy, which is typical for template matching methods (Cheng and Han, 2016). Third, the templates in the library do not cover every kind 
of possible dwelling. These limitations can be overcome by stratifying the template matching with the help of OBIA methods. Multiple counting of a dwelling can be avoided by segmentation and object resizing using neighbouring information. False alarms such as trees and bushes, that cast also a shadow and therefore look quite similar compared to a dark dwelling on one image band can be sorted out easily by masking out e.g. vegetated area and by stratification of the template matching approach to the camp area only. This tasks can therefore enhance the user's accuracy significantly. Dwellings that are missed by template matching because they look not similar to one of the applied templates, but show a strong spectral distinction from the surrounding can be detected by OBIA methods. Thus, a combination of both methods can improve both, the producer's and user's accuracy. In this research, the developed template library is integrated in an OBIA workflow as outlined below (cf. Figure 5):

1) The area of interest, in this case the camp extent, is derived by an initial rough OBIA classification of dwellings and a dwelling density calculation (Tiede et al., 2013) to stratify the template matching on the camp area only and to safe computation time.

2) The template library is applied, templates and rotation of templates are selected according to a visual inspection of dwelling types and shadow directions.

3) The segmentation of the satellite image is improved by including the correlation layer of the template matching in the segmentation process.

4) Exclusion (masking) of objects that are not relevant for the analysis like vegetation and bare soil (based on spectral information) as well as elongated structures like fences or walls (based on geometrical properties). This steps helps to eliminate false positive alarms created in the template matching process.

5) Classification of the image objects based on mainly two steps: (i) classification of dwellings using the template point layer and (ii) additional classification of dwellings applying OBIA methods (objects which do not match the template matching process, but show characteristics of dwellings regarding spectral information, form and spatial embeddedness).

\section{RESULTS AND DISSCUSION}

\subsection{Results for three test sites}

The template library was applied on three images showing different levels of difficulty regarding dwelling extraction. The used camp sites are El Redis and two different points in time of the camp Yida (cf. Table 1). The provided images cover parts of the Yida camp and the complete El Redis site. In the following, results of three different methods which were applied on the three sets of images are presented. The applied approaches for dwelling detection are: (1) Template library that is based on grayscale image template matching; (2) Template library for dwelling extraction within an object-based framework. As comparison existing and user-validated OBIA classifications for the sites were available.

The output of the template matching implementations is a point file. Applying more than one template creates sometimes several hits on one dwelling. In order to merge these multiple hits to get a meaningful result, the points are buffered with a radius of $1.5 \mathrm{~m}$. Consequently, the diameter is $3 \mathrm{~m}$, which is approximately the size of a small dwelling type.

The complexity regarding dwelling extraction of the image taken from the El Redis camp acquired at 12.03.2015 can be rated as 'low'. It consists mostly of bright dwellings, which can be clearly distinguished from surrounding areas. However, some darker dwellings are harder to distinguish. The structure of the camp is very clear. There is almost no vegetation like trees and bushes within the camp area, which could be wrongly identified as a dwelling. Fences, which can look like a shadow cast by a dwelling, occur rarely. The last two facts reduce false positive alarms created by template matching and misclassification.

\begin{tabular}{|l|l|l|l|l|}
\hline Site & $\begin{array}{l}\text { Acquisition } \\
\text { date }\end{array}$ & Size [pixel] & Sensor & Complexity \\
\hline $\begin{array}{l}\text { El } \\
\text { Redis }\end{array}$ & 12.03 .2015 & $1657 \times 1658$ & WV-2 & Low \\
\hline Yida & 12.10 .2012 & $2698 \times 2337$ & QB & Average \\
\hline Yida & 03.04 .2013 & $3237 \times 2805$ & WV-2 & High \\
\hline
\end{tabular}

Table 1. Sites on which the template library is applied including information about acquisition date, size and complexity regarding dwelling extraction.

Without a differentiation between dwelling types the application of the template library only, results in 2159 detected dwellings. Integrated in an object-based framework the classified dwellings were reduced to 1542 . In comparison to these results the preexisting OBIA classification detects 1426 dwellings. Differentiated between dwelling types the pre-existing OBIA classification detects 1261 bright dwellings, 139 brown dwelling and 26 large structures. The approach of the template library within an OBIA framework extracts 1357 bright dwellings, 168 brown dwellings and 17 large structures (cf. Figure 7). All in all, the new approach combined with OBIA methods detects 116 dwellings more than the pre-existing OBIA classification in the same area.

A reason for the difference is that the pre-existing OBIA classification lacks in creating discrete segments for dwellings that are built near to each other. Thus, a segment can includes more than one object. If the area of this segment is greater than the threshold for large structures, it is classified as a large structure. This could be improved by the new approach by integrating a correlation layer in the segmentation process. In comparison to the image captured from the El Redis camp the complexity increases from 'low' to 'average' in the image from the Yida camp acquired at $12^{\text {th }}$ October 2012. It consists mostly of bright and blue dwellings, typically built with local material and covered by white or blue plastic sheets in the rainy season (UNHCR, 2013). Bright structures can be clearly distinguished from surrounding areas compared to darker structures, which are more difficult to detect. The detection rate of blue dwellings depends on the intensity and similarity of surrounding areas. The camp structure can be described as 'chaotic'. The reason for this is that the development of the camp in its initial phase was not managed by a humanitarian relief organisation (nubareports.org, 2015). Most of the dwellings are located disperse. Between these structures, trees and other vegetation like grassland and rustcoloured earthen patches can be found. This increases the complexity concerning dwelling extraction. Especially if structures are partial hidden by tree crowns or look similar to surrounding areas, the differentiation of dwellings is hampered. The image was taken in the end of the rainy season in October. Therefore, the vegetation appears intense and dwelling roofs are less dusty. Overall, that increases the contrast between dwellings and surrounding areas. Occasionally fences that appear similar to shadows cast by a dwelling, occur. Overall the template library based approach detects in this case 5447 dwellings. The combined method extracted 2913 bright dwellings, 1644 blue dwellings, 90 large structures - in total 4674 dwellings. 


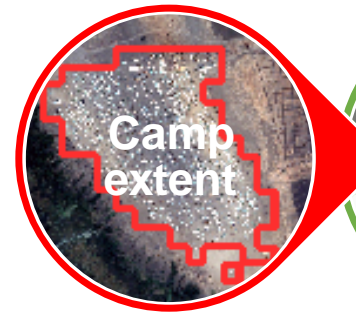

- Rough segmentation on one image band

- Classification of clear dwellings

$\rightarrow$ camp extent

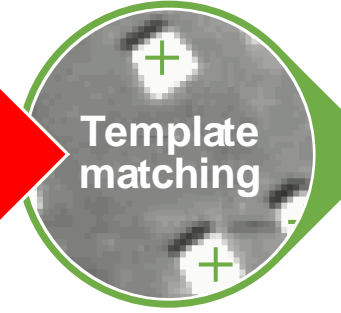

- Application of template library

- Refinement of result using correlation and rotation thresholds

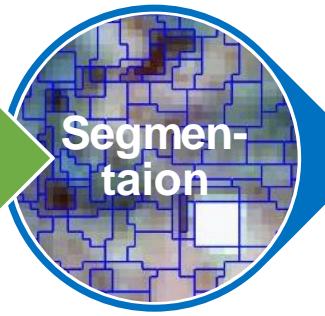

- Segmentation using various image bands, indexes and template correlation layer

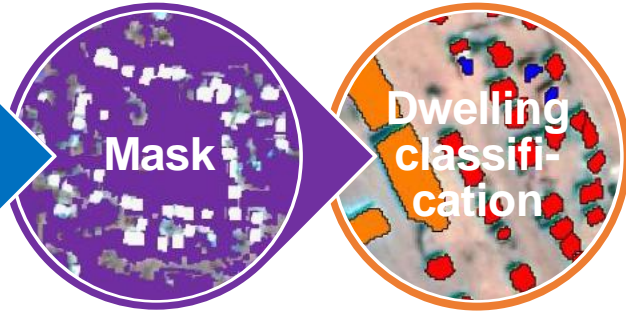

- Creation of a mask, where no dwellings can occur like:

- Vegetation mask

- Ground mask - etc.
- Classification using template points

- Classification using OBIA methods

- Refinement of classes

Figure 5. Overview of the applied combination of OBIA and template matching for dwelling extraction

In comparison, the pre-existing OBIA classification detects 3095 bright dwellings, 1268 blue dwellings and 67 large structures, which is 4430 dwellings in total (cf. Figure 7). The differences in bright and blue dwellings result from slightly different thresholds that distinguish these structures. Overall, the template matching library integrated in an object-based framework approach detects 217 dwellings more than the pre-existing OBIA classification in the same area.

The third image also covers the camp Yida and was taken six months later in early April 2013. The different sizes of the subsets are due to the different GSDs of different sensors (QuickBird with $0.6 \mathrm{~m}$ GSD and WorldView- 2 with a GSD of $0.5 \mathrm{~m}$ ). This scene was chosen in order to compare the effects and impacts of dry versus rainy season. There was only little change in terms of the camp structure, distribution of dwellings and occurrance of fences. However, this time the image is taken at the end of the dry season. The impact of this change is, that the complexity for dwelling extraction increases to 'high' because of several reasons. Dwelling roofs look dusty and are similar to the surroundings, where mainly rust-coloured earth and some trees can be found (cf. Figure 6). All in all, there is less contrast between all image objects that influences the accuracy of the detection of dwellings and classification respectively.
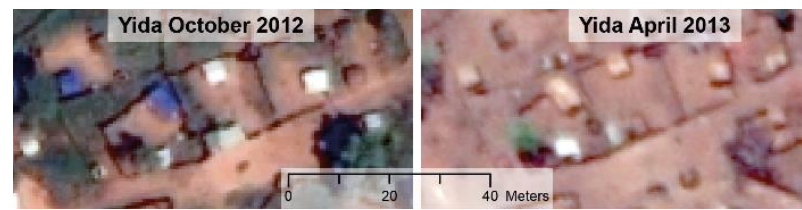

Figure 6. Comparison of the same area of the Yida camp (80m x 40m) between October 2012 (left, QuickBird) and April 2013 (right, WV-2). In the rainy season (left) dwelling show higher contrast to surroundings compared to the dry season (right).

Not differentiated between dwelling types, the template library based approach detects 4789 dwellings. The pre-existing OBIA classification detects 1665 bright, 1839 brown and 264 blue dwellings. Additionally, there are the classes large structure with 538 objects and small structure with 123 objects (in total 4429 dwellings were detected). In comparison, the template library within an object-based framework detected in total 5532 dwellings. Broken down by classes there are 2455 bright, 2277 brown and 289 blue dwellings as well as 330 large structures and 181 small structures (cf. Figure 7). Overall, 1103 additional dwellings are extracted compared to the pre-existing OBIA classification.

It should be emphasized that this time the template matching library detected fewer dwellings than the combined approach. Most likely the applied templates did not cover the whole range of occurring dwellings, especially the dusty conditions caused by dry season result in less clear differentiation between bright and dark structures which is hampering template matching using grey level based templated only.

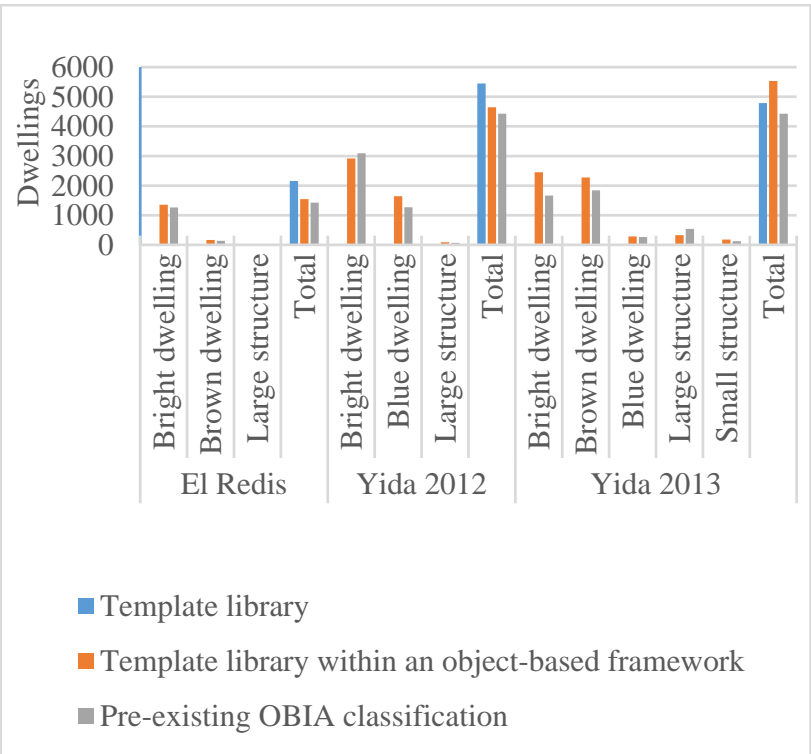

Figure 7. Number of detected dwellings for the three test sites

\subsection{Accuracy assessment}

In general the success of a method coincides with the applicability and accuracy. In order to verify latter, the results from the three test sites were compared in addition with a visual interpretation based on the satellite images. The visual interpretation was performed on randomly selected $200 \mathrm{~m}$ x $200 \mathrm{~m}$ sample areas. In the El Redis camp only two of these sample areas were created because of the small camp size. In the Yida camp five sample areas were created (cf. Figure 8). The sample areas are the same for both years (2012 and 2013), which allows a detailed comparison of the development in these camp parts. The visual interpretation includes a point file for every occurring 
dwelling in the sample areas, including attributes about the dwelling type.

\subsubsection{Accuracy assessment: not differentiated between dwelling types}

For El Redis the visual interpretation revealed 657 dwellings within the randomly selected sample areas. The template library identified 602 dwellings correctly, showing a user's accuracy of approximately $74 \%$ and a producer's accuracy of $92 \%$. Integrated template matching within an object-based framework extracted the same number of correctly extracted dwellings (same producer's accuracy), but the user accuracy increased to approximately $98 \%$ based on the reduction of false positive alarms. In comparison, the pre-existing OBIA classification extracted 542 dwellings correctly with a very high user's accuracy of $96 \%$ but a lower producer's accuracy of $83 \%$ compared to the other approaches (cf. Figure 9).
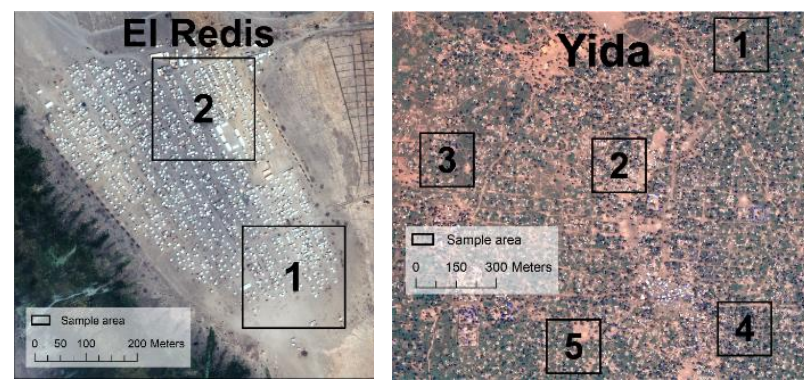

Figure 8. On the left side El Redis camp (2015, band combination R-G-B) and on the right side Yida camp (2012, band combination R-G-B) with square cells (200m x 200m) randomly selected for visual interpretation and accuracy assessment.

For the image of the Yida camp captured in October 2012, the visual interpretation detected 587 dwellings within the sample areas. Applying the template library based approach, 410 dwellings were classified correctly with a user's accuracy of 75\% and a producer's accuracy of $70 \%$. The combination with OBIA methods increased the correctly detected structures to 452 and corresponding the producer's accuracy to $77 \%$. False positive alarms are reduced strongly, which shows the enhanced user's accuracy of $93 \%$. For comparison, the pre-existing OBIA classification extracted 397 dwellings with a high user's accuracy of $90 \%$ and a producer's accuracy of $68 \%$ (cf. Figure 9 ).

For the same area, but based on the image acquired six months later, the visual interpretation revealed 504 dwellings. The template matching only detects 247 dwellings with a user's accuracy of $65 \%$ and a producer's accuracy of $49 \%$. Integrated in an object-based framework the extracted number of dwellings increased to 374 and hence also the producer's accuracy to $74 \%$. Moreover, the user's accuracy rises to $77 \%$. The pre-existing OBIA classification extracted 284 dwellings correct with a user's accuracy of $78 \%$ and a quite low producer's accuracy of $56 \%$ (cf. Figure 9).

\subsubsection{Accuracy assessment: differentiated between dwelling types}

A differentiation between dwelling types was conducted for the application of the template library within an object-based framework and for the pre-existing OBIA classifications only. Applying a template matching implementation frequently results in multiple hits per dwelling. A dark dwelling template can create a positive alarm on structures appearing dark as well as for structures which appear brighter (but with similar contrast) vice versa for a bright dwelling template. Consequently, no precise and accurate distinction of dwelling types can be conducted. Differentiated between dwelling types the template library within an object-based framework detected 598 dwellings correct in the El Redis camp test site. The user's and producer's accuracy decreases less than $1 \%$ compared to the result without the differentiation between dwelling types. Looking at the single classes the producer's accuracy is very high for bright dwellings (95\%) and large structures $(100 \%)$ but only moderate for brown dwellings $(66 \%)$. The overall accuracy reduction of the preexisting OBIA classification (537 extracted structures) is also less than $1 \%$. Important to mention are the high producer's accuracy rates for bright dwellings $(90 \%)$ and large structures $(100 \%)$ while there is a very low value for brown dwellings (33\%), which are much better detected by the integrated template matching approach. The low accuracy values for brown dwellings do not have a lot of influence on the overall accuracy of both classifications because their dwelling share is approximately $15 \%$.

For the image of the Yida camp (October 2012) the template matching combined with OBIA methods extracted 452 dwellings correctly, which is less than without a dwelling distinction. Hence, this reduces the user's and producer's accuracy (user's accuracy: 91\%, producer's accuracy: 75\%). The class-specific user's and producer's accuracy depend again highly on dwelling types (class-specific user's accuracy: $96 \%$ for bright dwellings, $81 \%$ for blue dwellings and $67 \%$ for large structures; classspecific producer's accuracy: $78 \%$ for bright dwellings, $70 \%$ for blue dwellings and $67 \%$ for large structures). As a comparison, the user's accuracy is $88 \%$ and the producer's accuracy is $66 \%$ for the pre-existing OBIA classification. The class-specific user's and producer's accuracy develop in a similar way (class-specific user's accuracy: 93\% for bright dwellings, $80 \%$ for blue dwellings and $33 \%$ for large structures; class-specific producer's accuracy: $77 \%$ for bright dwellings, $48 \%$ for blue dwellings and $67 \%$ for large structures). A particularly conspicuous aspect is that the template matching within an object-based framework detects blue dwellings a lot better than the pre-existing OBIA classification only.

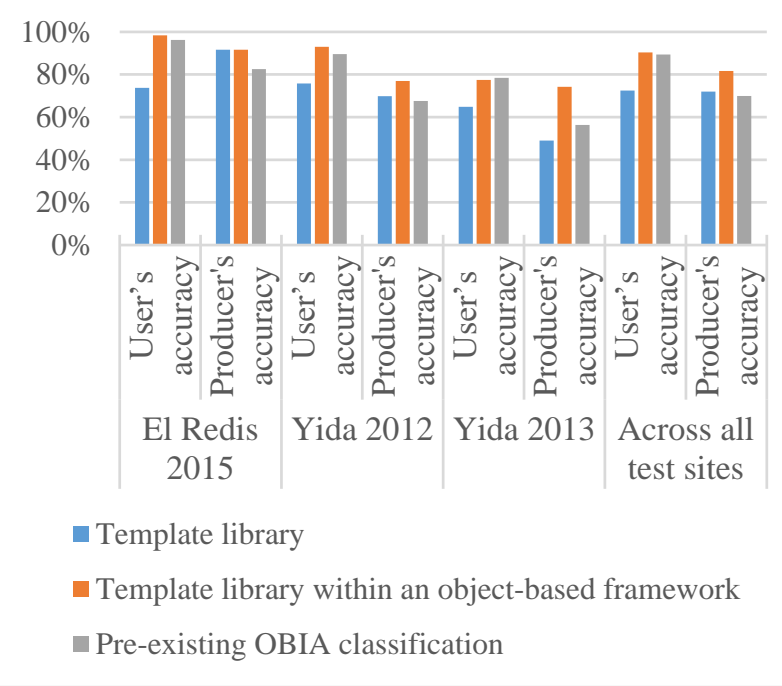

Figure 9. Accuracy assessment for randomly selected sample areas. Dwellings are not differentiated by type.

For the second image taken from the Yida camp, the combined approach detects 346 dwellings correctly (differentiated by type). The producer's accuracy drops to $68 \%$ as well as the user's accuracy to $71 \%$. A detailed look at the class-specific accuracy 
reveals again that darker structures are harder to extract (classspecific user's accuracy: $69 \%$ for bright dwellings, $76 \%$ for brown dwellings, $81 \%$ for blue dwellings, $56 \%$ for large structures and $25 \%$ for small structures; class-specific producer's accuracy: $90 \%$ for bright dwellings, 59\% for brown dwellings, $52 \%$ for blue dwellings, $59 \%$ for large structures and $100 \%$ for small structures). The pre-existing OBIA classification extracted 238 dwellings in total, with a user's accuracy of $65 \%$ and a producer's accuracy of $47 \%$. The class-specific accuracy shows that the detection rate for all classes apart from large structures is low (class-specific user's accuracy: 67\% for bright dwellings, $73 \%$ for brown dwellings, $91 \%$ for blue dwellings, 34\% for large structures and $15 \%$ for small structures; class-specific producer's accuracy: $61 \%$ for bright dwellings, $40 \%$ for brown dwellings, $44 \%$ for blue dwellings, $71 \%$ for large structures and $67 \%$ for small structures) in comparison to the template matching within an object-based framework. Figure 10 presents the accuracy differentiated by the dwelling type summarised across all test sites.

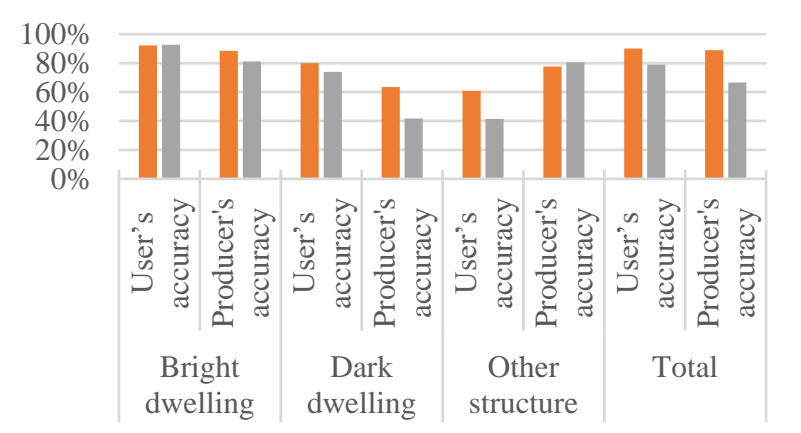

- Template library within an object-based framework

$\square$ Pre-existing OBIA classification

Figure 10. Accuracy assessment for randomly selected sample areas. Dwellings are differentiated by the type.

\section{CONCLUSION AND OUTLOOK}

The main finding of this study is that template matching is suitable for dwelling extraction in refugee and IDP camps using VHR satellite imagery, if it is stratified by OBIA means. Incorporating the shadow effect of dwellings in the templates improved the detection rate. Furthermore, the orientation of a template caused by the shadow can be used as a clear identifier for a dwelling. This is an advantage compared to conventional object-based workflows for dwelling extraction. A visual analysis of several refugee camps in East Africa revealed that there are certain dwelling types which typically occur. Based on this findings a template library for various dwelling types (template samples are taken from ten different camp sites using 16 satellite images) was established. The library is structured according to the dwelling grayscale intensity (strong: bright dwelling, weak: dark dwelling), dwelling shape (square, rectangle, circle), dwelling size (in pixel) and shadow direction (one side, two sides). The bright dwelling templates are created on the blue band and dark dwellings on the NIR band, based on empirical tests. Altogether 18 template classes were created covering typically occurring dwellings. The template matching library aims to be generally applicable in similar conditions. The tests implementations revealed, that if the template library is applied solely, it is sensitive to image areas which look similar to a template such as trees or a white spot on the ground. Consequently, false positive alarms are represented by a low user's accuracy. This drawback could be overcome by a stratified usage in OBIA workflows.

It could be shown, that the combined approach, stratifying the template matching within an initial object-based analysis, increases the average producer's accuracy by $11.7 \%$ and slightly increase the user's accuracy by $0.9 \%$ compared to pre-existing conventional OBIA classifications. This holds also true, if the accuracy assessment is further differentiated into different dwelling types.

These results show, that the integration of template matching in OBIA workflows is a possibility to further improve results of semi-automated dwelling extraction, especially in complex situations. The initial assumptions could therefore be verified: (1) Through the incorporation of the shadow effect of a dwelling in a template library, the extraction rate in difficult (e.g. low contrast, dense dwellings) situations can be improved; (2) it is possible to establish a general template matching library for dwellings to be applied in similar conditions; (3) only the combination of template matching with OBIA methods (stratification) can enhance the accuracy of dwelling extraction compared to template matching solely.

Restrictions of the used template matching implementation are (i) the missing support for multiband images; (ii) templates are not scale invariant and (iii) the rotation angle could not be set directly but only the amount of rotation numbers, which increases the computation time (redundant usage of templates) in the process.

The accuracy of grayscale template matching can further be increased, if a multiband template matching approach is used. First tests in the software Ciratefi (v.1.05) revealed quite promising results, but the combination with OBIA workflows is at the moment limited due to the lack of geo-spatial data support and reduced radiometric depth allowed per image band. For a multiband approach also the templates library needs to be extended, which could hamper the general applicability along with increasing computation time.

\section{ACKNOWLEDGEMENTS}

The research has received funding from the Austrian Research Promotion Agency (FFG) under the Austrian Space Application Programme (ASAP) within the projects EO4HumEn and EO4HumEn+ (EO-based services to support humanitarian operations: monitoring population and natural resources in refugee/IDP camps; contract no: 840081/854041).

\section{REFERENCES}

Baltsavias, E.P., Gruen, A., VanGool, L., 2001. Automatic Extraction of Man-made Objects from Aerial and Satellite Images III, Monte Verit $\{a ̀\}$. Taylor \& Francis.

Cheng, G., Han, J., 2016. A Survey on Object Detection in Optical Remote Sensing Images. ISPRS JOURNAL OF PHOTOGRAMMETRY AND REMOTE SENSING 117, $11-28$.

Füreder, P., Lang, S., Rogenhofer, E., Tiede, D., Papp, A., 2015. Monitoring Displaced People in Crisis Situations Using Multi-temporal VHR Satellite Data During Humanitarian Operations in South Sudan, in: GI_Forum 2015 Geospatial Minds for Society. pp. 391-401.

Grundy, C., Füreder, P., Siddiqui, R., Katsuva Sibongwe, D., Tiede, D., Lang, S., Checci, F., 2012. Validation of satellite imagery methods to estimate population size., in: MSF Scientific Day, 25 May 2012. London, Great Britain.

Lang, S., Tiede, D., Hölbling, D., Füreder, P., Zeil, P., 2010. Earth observation (EO)-based ex post assessment of 
internally displaced person (IDP) camp evolution and population dynamics in Zam Zam, Darfur. International Journal of Remote Sensing 31, 5709-5731.

nubareports.org, 2015. On the move again; 70,000 refugees compelled to leave camp, Nuba Reports. http://nubareports.org/on-the-move-again-70000refugees-compelled-to-leave-camp/ (08.07.2016)

Spröhnle, K., Tiede, D., Schoepfer, E., Füreder, P., Svanberg, A., Rost, T., 2014. Earth Observation-Based Dwelling Detection Approaches in a Highly Complex Refugee Camp Environment - A Comparative Study. Remote Sensing 6, 9277-9297.

Tiede, D., Füreder, P., Lang, S., Hölbling, D., Zeil, P., 2013. Automated Analysis of Satellite Imagery to provide Information Products for Humanitarian Relief Operations in Refugee Camps - from Scientific Development towards Operational Services. PFG Photogrammetrie Fernerkundung - Geoinformation 2013, 185-195.

UNHCR, 2013. UNHCR Statistical Yearbook 2012, 12th edition 172.

UNHCR, 2015. Mid-Year Trends 2015. http://www.unhcr.org/statistics/unhcrstats/56701b969/mi d-year-trends-june-2015.html (08.07.2016)

UNHCR, 2016. Global Trends. Forced displacement in 2015. http://www.unhcr.org/news/latest/2016/6/5763b65a4/glo bal-forced-displacement-hits-record-high.html (08.07.2016) 


\title{
3D object-based feature extraction from 3-stereo DSM in urban context
}

\author{
K. Kulessa \& S. Lang \\ Department of Geoinformatics - Z_GIS, University of Salzburg, Austria - (Kerstin.Kulessa, Stefan.Lang)@sbg.ac.at
}

KEY WORDS: 3D features, triple-stereo, Digital Surface Model, Pléiades, Semi-Global Matching, urban area

\begin{abstract}
:
For urban planning purposes updated information on land-use and urban structures and change dynamics are essential. Satellite Earth observation is a very effective information source, to provide actual representations of the situation 'as is' in regular time steps. The Pléiades constellation offers very high resolution imagery with a triple-stereo acquisition. The intrinsic combination of thematic and height information is an additional asset provided by recent VHR stereo imagery. Digital surface models (DSM) can be derived through stereo matching algorithms, one of which is semi-global matching (SGM). SGM integrates local and global matching methods with a short processing time and accurate surface generation.

We use the generated DSM for extracting 3-D features which enrich object-based class models of composite urban objects. These multi-purpose 3-D object feature datasets can be used for various planning and monitoring purposes, revealing height-related characteristics of urban objects (blocks, neighbourhoods, parks, industrial sites, etc.). In addition object-wise aggregated height information can be used to better assess the adherence of actual building activities to zoning or land development plans, e.g. with respect to maximum or average height in recreational areas etc.

As compared to detailed and potentially more accurate DSMs generated from LiDAR data, a (3-)stereo DSM has the advantage of recording height and spectral information instantaneously, so not to lose any information in high dynamic urban settings due to a time gap. Height and colour information, when integrated by spatial context and multi-scale OBIA methods, enable a wealth of new applications in urban feature characterisation and 3D city model applications.
\end{abstract}

\section{INTRODUCTION}

Urban areas are characterised by continuous change. This is a challenge for urban planning purposes because regularly updated information on the region is required to get an idea on the dynamics of urban structures and the changes in urban landuse. For these purposes it is important to have data with a high spatial resolution because the structure and the dynamics of the city have to be recorded in detail for urban planning, including information on the third dimension. Zhou (2013) used LiDAR heights to improve land-use classification. For urban areas, Laser scanning data, including the height information, is very accurate and has a very high spatial resolution which is also important for cities. A disadvantage of laser scanning data is the high cost and effort for acquisition of the data resulting in very accurate data sets that could not be updated too often due to high costs.

As opposed to this, satellite imagery is nowadays available in high temporal and spatial resolution. This data is already used for a variety of applications in urban areas such as for land-use classification or change detection analysis. Some satellites provide data in stereo or for some time past even tri-stereo acquisition mode. This mode can be used to derive height information from the satellite data with a high resolution which can be used as alternative to laser scanning data (Gehrke et al. 2010). Height information from tri-stereo satellite imagery can be calculated by stereo matching. The height information of this satellite DSM can be also used to improve classifications or even classify objects. This study shows the workflow of how to create a DSM from tri-stereo Pléiades images and explains the
3-D object extraction with eCognition software for statistical analysis in the field of urban planning.

\section{METHODS}

\subsection{Study Area}

This study has been done for the study area of Salzburg in Austria, covering the EuRegio greater region. The extent of the study area has a size of around $165 \mathrm{~km}^{2}$ and is characterised by a variety of topographies. Located north of the Alps, the city of Salzburg is situated in a rather flat basin bounded by the Gaisberg and characterized by some steep hills such as the Kapuzinerberg or Mönchsberg in the flat city area. Furthermore, the region of the study area is shaped by the Salzach River and the Saalach River, which forms the border between Austria and Germany and issues into the Salzach River in the north of the city (see Figure 1). The study area contains two cities: the city of Freilassing on the German side of the Saalach River, which mainly includes single family houses in a city environment of lower building density, and the larger city of Salzburg on the Austrian side. The city of Salzburg has a high building density with multi-story buildings in the inner city and a lower density in the residential areas. 


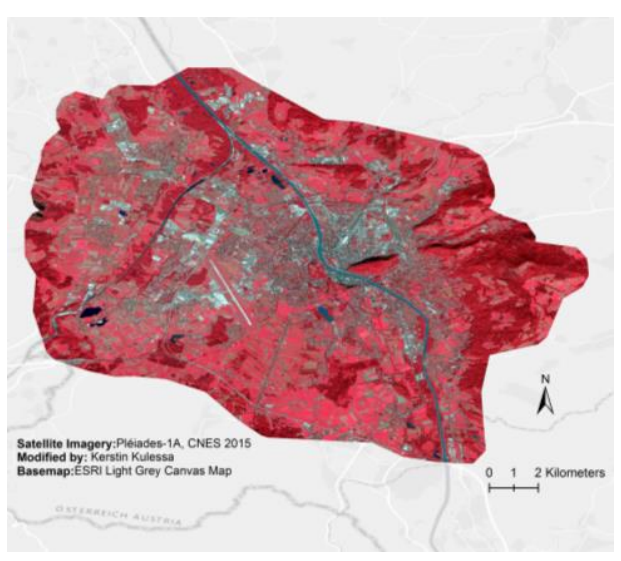

Figure 1. Pléiades false colour image showing the extent of the study area

\subsection{Data}

For this study there has been used Pléiades tri-stereo satellite imagery recorded during one pass. The Pléiades sysrem consists of the twin satellites Pléiades-1A launched in 2011 and Pléiades-1B launched in 2012. The two satellites, equipped with a pushbroom scanner, operate on the same orbit and are located $180^{\circ}$ from each other. This constellation enables a higher frequency of image acquisition which results in a larger temporal resolution. With a viewing angle of $+/-30^{\circ}$, which can be maximised up to $+/-47^{\circ}$ (ASTRIUM, 2012), a daily revisit of nearly every location on the Earth is possible (see Figure 2).

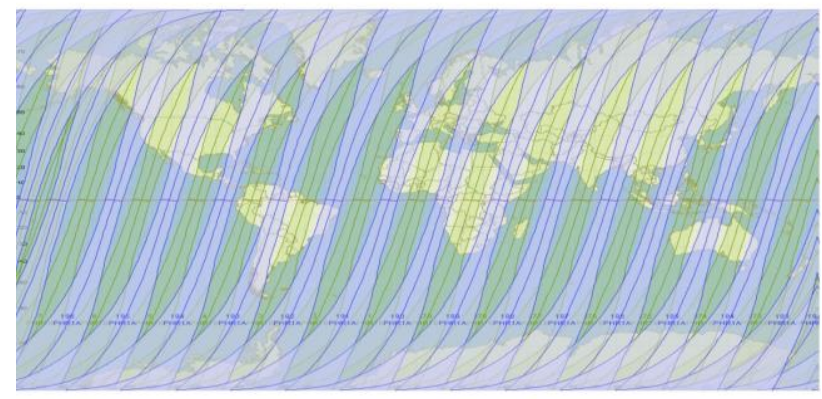

Figure 2. Visibility of Pléiades-1A \& 1B for one day $+/-30^{\circ}$ (ASTRIUM, 2012)

Pléiades satellite record multispectral and panchromatic information. Multispectral images contain the red, green, blue and near infrared band with a resolution of $2.0 \mathrm{~m}$ whereas the panchromatic band has a spatial resolution of $0.5 \mathrm{~m}$. Rated as a very high resolution (VHR) satellite, a large variety of applications is feasible such as hazard mapping, detailed landuse classifications or urban mapping.

Pléiades satellites are able to collect images in tri-stereo acquisition mode, meaning that three images are recorded from the same area within one pass (see Figure 3).

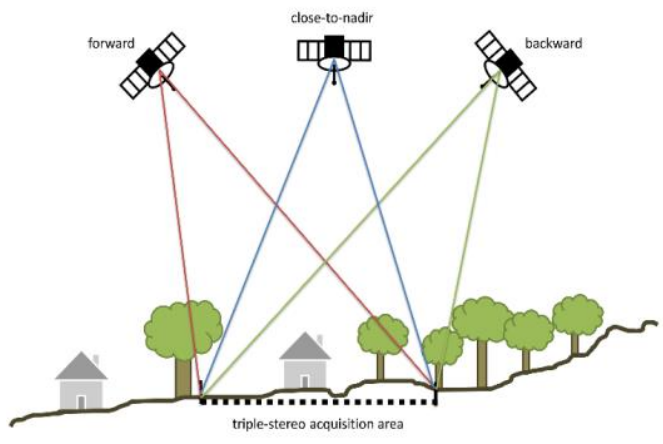

Figure 3. Tri-Stereo acquisition mode

As compared to stereo acquisition, which is the minimum configuration to extract 3D information, tri-stereo data records an image from close-to-nadir acquisition angle. This is of great advantage for urban areas or mountainous regions, because information from areas with urban canyons can be extracted well whereas they cannot be seen with mere stereo images. Moreover, it is of particular importance for urban areas, that the Base over Height $(\mathrm{B} / \mathrm{H})$ ratio is chosen according to the characteristics of the study area before data acquisition. The $\mathrm{B} / \mathrm{H}$ ratio indicates the stereoscopic capacity of a stereo image pair. If a high $\mathrm{B} / \mathrm{H}$ ratio is chosen, areas of urban valleys could not be seen (see Figure 4).
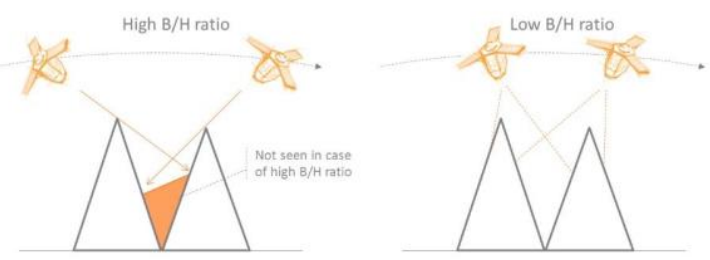

Figure 4. Comparison between a high and low $\mathrm{B} / \mathrm{H}$ ratio (ASTRIUM, 2012)

ASTRIUM (2012) recommends a value between 0.4 and 0.7 for DSM generation and a value smaller than 0.4 for urban area and mountainous regions.

For the study, there has been used tri-stereo satellite imagery acquired on 1 September 2015 for the whole extent of the study area including red, green, blue, NIR and panchromatic band.

\subsection{Stereo Matching}

For the generation of the digital surface model (DSM) there is used a stereo matching algorithm called Semi-Global Matching (SGM). To run the SGM algorithm, it is necessary to have a known epipolar geometry (see Figure 5). 


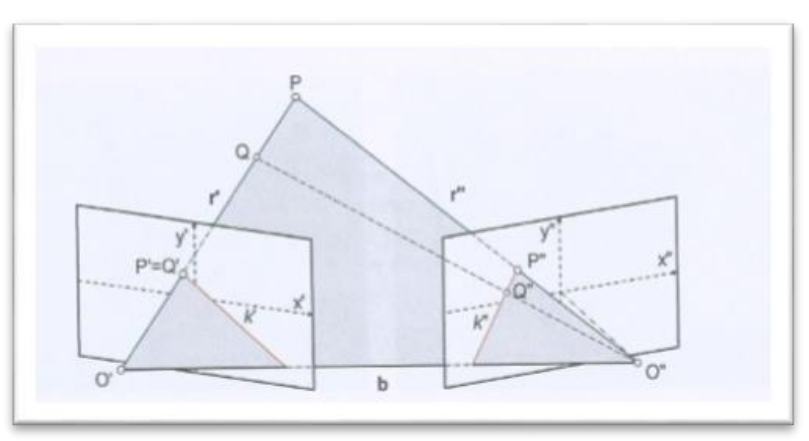

Figure 5. Epipolar Geometry (Luhmann et al., 2014)

The perspective centre $O$ of the camera lies on the same line with the image point $Q$ and the respective point of the recorded object $P$. Having two images, there are also two lines $r$ ' and $r$ '. These two lines form together with the line between the two camera projection centres the epipolar plane which intersects the image planes in a line, called epipolar line. The image point and also the corresponding point lie on this epipolar line. Having a known epipolar geometry reduces the search space because the corresponding point has to be searched only along the epipolar line and not in the whole image. The SGM algorithm as an energy function which should be minimal:

$$
\mathrm{E}(\mathrm{D})=\sum_{P} C\left(p, d_{p}\right)+\sum_{q \epsilon n_{p}} P\left\lfloor\left|d_{p}-d_{q}\right| \geq 1\right]
$$

where $\quad \mathrm{p}=$ pixel $\mathrm{p}$

$\mathrm{d}=$ distance

$\mathrm{N}_{\mathrm{p}}=$ neighbourhood of pixel $\mathrm{p}$

$\mathrm{q}=$ all pixels from the neighbourhood $\mathrm{N}_{\mathrm{p} \text { of }}$ pixel $\mathrm{p}$

Luhmann et al., 2014

This function consists of two parts: the cost function $C$ and the penalty term $P$. The cost function defines the cost needed for matching a pixel with corresponding pixel at a distance $d$ along the epipolar line. The penalty term penalises disparities of all pixels from the neighbourhood of the pixel $p$ which is larger than 1. This results in an exponential growth of computational effort with an increasing size of input data. For this reason, Hirschmueller (2005) did not use the global matching approach but introduced the SGM which decreases the computational effort by using a combination of local and global matching. For SGM eight linear paths through the image get calculated running radially and symmetrically from image boundaries meeting at pixel $p$. Finally, the disparity with the least cost is chosen (Hirschmueller, 2011).

\subsection{DSM generation and object extraction}

This SGM algorithm is meanwhile also implemented to several GIS software such as ERDAS Imagine and usable for satellite imagery. This software has been also used for the DSM generation of this study. After image block creation and triangulation the SGM algorithm has run resulting in three point clouds, one for each image pair. The SGM calculation in ERDAS Imagine can be only calculated for image pairs but not for three images in one step. After the conversion of the point cloud to a raster grid, the DSM derived from Pléiades tri-stereo imagery can be used.

Having height information and spectral information from the same sensor is advantageous because it has been recorded at the same time and the same spectral resolution. This data is then used to extract different objects of the urban areas, such as buildings or vegetation, based on the height value, spectral information and/or shape and neighbourhood relations. The objects have been extracted with an object-based approach in eCognition software. There has been created a ruleset to classify objects according to their shape, spectral values and relation to other objects.

\section{RESULTS}

\subsection{DSM Evaluation}

The quality of the DSM derived from Pléiades tri-stereo satellite imagery is quite good overall. Some outliers occur in the data set due to shadowed areas and high disparity differences. Data gaps, where the algorithm did not find corresponding points, lead to inaccuracies due to interpolation. Comparing the DSM derived from Pléiades with a DSM based on LiDAR data, an overall accuracy of around $1 \mathrm{~m}$ has been achieved, which is a satisfying result, particularly for urban areas. Calculated heights were also compared to height information of the Pléiades DSM with measured GPS points existing all over the study area. This comparison confirmed the accuracy of around $1 \mathrm{~m}$.

\subsection{3-D Objects}

The 3-dimensional objects are created in two steps: first of all the object extraction in eCognition based on spectral information, shape, object relations and/or height information. As a second step the height information has been added to the created objects to get a 3-dimensional image object.

Based this information, statistics are calculated on different levels such as the minimum and maximum height, as well as the volume of objects. True height values can be calculated and not just an estimation of values as e.g. in Shao et al., 2011. In urban areas we could apply different scales for the calculation, for example single buildings or trees, neighbourhoods, complete forests or urban parks. Furthermore, we could use the units of an aerial zoning plan to check for the compliance with height constraints per zone (see Figure 6).

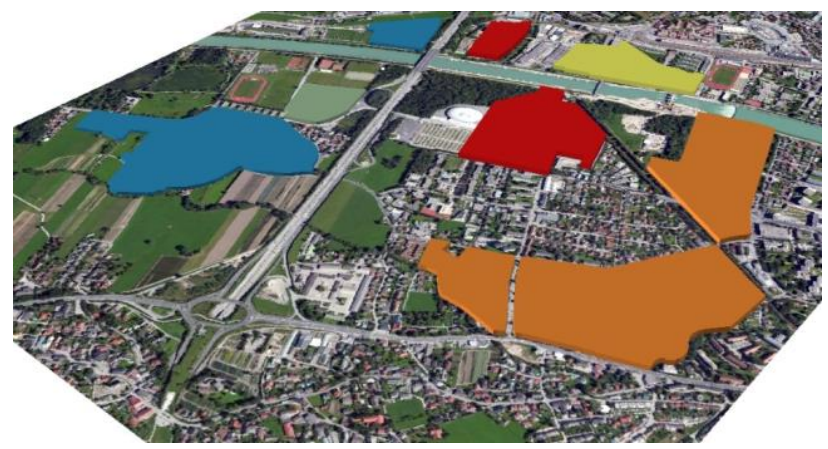

Figure 6. Statistical calculations for units of the aerial zoning plan 
Within the scope of this study, there has been a calculation of statistics which includes the minimum, mean and maximum height of an object and the volume of objects. The following levels of urban objects have been used: single object (single building, tree), building blocks, neighbourhood (neighbouring objects), urban parks and the aerial zoning plan.

Moreover, the height information is not only used to calculate statistics in urban areas but is also helpful in the classification of a scene. A classification is refined by an additional feature: the height of an object. It also works good to use the elevation data for the classification and classify objects according to their heights. Objects of similar heights are then grouped together and form specific areas such as the inner city with a larger building height value in comparison with a lower height value for bushes.

Urban planners can use this information for planning purposes and to monitor dynamics in urban areas. Furthermore, these 3dimensional objects can be used in urban context to monitor changes in the urban areas. Updating the Pléiades data - the spectral information as well as the elevation data - could help to detect changes in the city. To keep comparability it would be advantageous to always use data from the same sensor, such as Pléiades.

Using Pléiades tri-stereo satellite imagery does not have only advantages in this context but could also cause some inaccuracies. In comparison to laser scanning data, Pleíades has a resolution of $0.5 \mathrm{~m}$ in the panchromatic band, whereas LiDAR data reaches much higher resolution nowadays. Furthermore, by taking the calculation of vegetation volumes as an example, the DSM from Pléiades only gives the information on the tree crown which is visible from the perspective of the sensor. Tree trunks could not be seen in this kind of data.

\section{CONCLUSIONS}

Using Pléiades tri-stereo satellite imagery for 3-D object extraction could have different advantages: height and thematic imformation can be extracted simultaneous. This data is good for synergistic use because it comes from the same sensor and there is no time gap in between. If the spatial resolution is sufficient for the respective purpose it could be a good alternative to laser scanning data because it saves costs and the acquisition effort is not that high. Additionally, satellite data can be updated regularly under low costs which make them very useful in urban context, e.g. for urban planning purposes.

For the future, it is important to improve the result of the Pléiades DSM calculated by SGM and develop filtering methods which filter all the outliers and errors from the point cloud. Furthermore, it could be a possibility to use mono satellite imagery from the archive and match multiple mono scenes to get the same result. With this method it is possible to create DSMs from multiple previous mono acquisitions which could be, in comparison with newly ordered tri-stereo data, a good data base for the monitoring of urban dynamics.

\section{REFERENCES}

- ASTRIUM (2012), Pléiades Imagery, User Guide. Technical Reference.

- GEHRKE, S., MORIN, K., DOWNEY, M., BOEHRER, N., \& FUCHS, T. (2010), Semi-global matching: An alternative to LiDAR for DSM generation. In Proceedings of the 2010 Canadian Geomatics Conference and Symposium of Commission I.

- HIRSCHMUELLER, H. (2005), Accurate and efficient stereo processing by semi-global matching and mutual information. In: 2005 IEEE Computer Society Conference on Computer Vision and Pattern Recognition (VCPR`05) (Vol. 2) 807-814.

- HIRSCHMUELLER, H. (2011), Semi-global matching-motivation, developments and applications. Photogrammetric Week, Wichmann/VDE Verlag, Berlin \& Offenbach, 173-182.

- LUHMANN, T.; ROBSON, S.; KYLE, S.; BOEHM, J. (2014), Close-Range Photogrammetry and 3D Imaging. 2nd Edition. Walter de Gruyter GmbH.

- SHAO, Y.; TAFF, G. N.; \& WALSH, S. J. (2011), Shadow detection and building-height estimation using IKONOS data. International journal of remote sensing, 32(22), 6929-6944.

- ZHOU, W. (2013), An object-based approach for urban land cover classification: integrating LiDAR height and intensity data. IEEE Geoscience and Remote Sensning Letters, 10(4), 928-931. 


\title{
RSOBIA - A new OBIA Toolbar and Toolbox in ArcMap 10.x for Segmentation and Classification.
}

\author{
T.P. Le Bas
}

National Oceanography Centre, European Way, Southampton, UK. SO14 3ZH.

tim.lebas@noc.ac.uk

KEY WORDS: OBIA, Segmentation, ArcMap, Classification, Toolbar

\begin{abstract}
:
A new toolbox for ArcMap 10.x will be presented that segments the data layers into a set of polygons. The software is available at htttp://www.codemap.eu/Outputs . Each polygon is defined by a K-means clustering and region growing algorithm, thus finding areas, their edges and boundaries in the imagery. Attached to each polygon are the characteristics of the imagery such as mean and standard deviation of the pixel values, within the polygon. The segmentation of imagery into a jigsaw of polygons also has the advantage that the human interpreter does not need to spend hours digitising the boundaries. The actual segmentation process has been taken from the RSGIS library of analysis and classification routines (Bunting et al., 2014). These routines are freeware but have been modified to be made available in the ESRI ArcGIS software under the Windows operating system. Input to the segmentation process is a multi-layered raster image, for example; satellite imagery, or any set of raster datasets made up from derivatives of topography. The size and number of clusters are set by the user and are dependent on the imagery used.
\end{abstract}

The advantage of having OBIA within the ArcGIS environment is that it can become part of the workflow, either separately or in models. Such integration speeds analysis and allows easier manipulation of data. Meaningful classification of the polygons using their numerical characteristics can be very dependent on the data subject. Many classification systems are available and tailored to the data available. A simple classification tool is provided as a paint function, but it is expected that functionality within ArcMAP is already being used to undertake complex classification rules.

\section{INTRODUCTION}

OBIA (Object Based Image Analysis) is a relatively new technique that mimics human interpretation of imagery. The objectivity and speed of production of results aids the interpreter to choose either the most favourable results from a subjective point of view or the use of statistical parameters and measures for a quantified interpretation.

Software for OBIA is available (e.g. eCognition made by Trimble). The issue with much of these software is the learning curve required to produce useful and recognisable results. If users are willing and able to spend the time learning how to use such software the results can be excellent. An additional issue however is that the data has to be exported from their source software and imported into the OBIA software, followed by any results exported from the OBIA software back into the source software. Often this source software is a GIS system such as ESRI's ArcMap.

A need was therefore seen for OBIA to be integrated into GIS. ESRI's ArcMap is currently used by millions of people daily worldwide and could benefit from access to OBIA. Easy usage and access is essential. A toolbar for push button control was envisaged and RSOBIA (Remote sensing Object Based Image
Analysis) was created. The toolbar is easy to use and requires very little training. For the slightly higher level user a toolbox has also been created, so that the RSOBIA functions can be incorporated into other programming models and allows more complex functions to be created.

\section{SOFTWARE}

The RSOBIA toolbar is added to the ESRI ArcMap version 10.x toolbars. It consists of 3 sections. The first section is designed for use with single band imagery and creates mathematical derivatives of the single band imagery, such as slope maps from topography. The second section has the main segmentation algorithms creating polygonised feature data. The input for segmentation is either multi-band imagery, such as Landsat imagery and RGB pictures, or a combination of single layer grids making a multi-layered dataset. The final section in RSOBIA is for classification and interpretation of the polygonised features.

\subsection{Derivatives}

There are 5 derivatives currently available. The functions offered by the derivatives menu are essentially standard grid manipulation techniques but brought together for ease of use. 


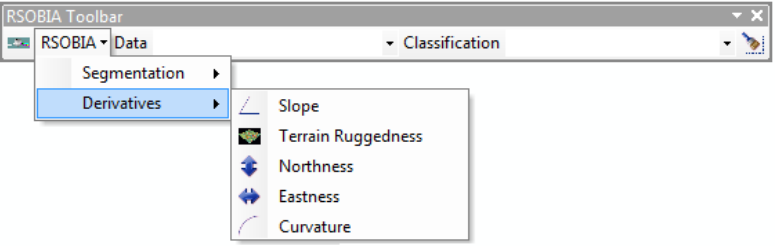

Figure 1: RSOBIA toolbar showing the derivatives sub-menu

\subsubsection{Slope}

This calculates the maximum slope (in any direction) in degrees. Values created are real numbers between 0.0 and 90.0 but a value of -1.0 is used for areas of no dataThis function differs from a shaded relief which is a slope derivative from a particular direction. Slope maps are occasionally used for pseudo-3D display - using the greyscale slope map with the high slope as black, and placed under a colour topography map with partial transparency.

\subsubsection{Terrain Ruggedness}

This is sometimes referred to as Rugosity. Calculates the variation in bathymetry datasets within a neighbourhood. This technique combines the variability of slope and aspect in an area. It is taken from the "Benthic Terrain Modeller" developed by Shaun Wallbridge (Wright et al., 2012).

\subsubsection{Northness}

This is the calculation of the aspect of the slope. Aspect is often calculated is terms of the compass direction 0-360 degrees. For terrain calculations the zero and 360 degree boundary can create issues and so the Northness is calculated being the cosine of the aspect. Values are from 1.0 (fully north facing) to -1 (south facing).

\subsubsection{Eastness}

This is the complement to Northness being the sine of the Aspect. Values are from 1.0 (fully east facing) to -1 (west facing).

\subsubsection{Curvature}

The calculation of the curvature is in the direction of maximum slope of a bathymetry dataset. It is taken from the spatial analyst toolbox. This is useful for highlighting crests and depressions. Values often are between 30.0 (convex crest) and -30.0 (concave depressions).

\subsection{Segmentation}

The segmentation section of the toolbar covers more than just segmentation. There are 5 tools available: layering of single band grids together, segmentation to create polygonised maps, polygon attribute calculations, seeding of polygons with groundtruth point sample attributes and a bespoke wizard tool for multibeam bathymetry and backscatter interpretation.

\subsubsection{Layering}

The layering function is a preparation step for segmentation. This function can combine many single layered files as required and create a multi-layered raster image.

\subsubsection{Segmentation}

Segmentation is the method to aggregate pixels together to create a thematic map. The segmentation process is a licensed tool taking a multi-layered image and creates a set of polygons defined by the statistics associated with the layered image. Clusters of the imagery pixels are created in n-dimensional space and created into classes. Aggregation into geographic regions (polygons) is done according to a minimum polygon size rule, and clustering rules.

There are 3 main parameters required:

i. Number of Clusters: This is the most important parameter and depends on the complexity of the data.

ii. Minimum Object Size: This is the minimum size of any output polygon in terms of pixels. Knowledge of the pixel resolution is crucial.

iii. Layer Weights: During the standard segmentation process each layer is given equal weighting regardless of the differing units used on each layer. The user can provide individual layer weights if, for example, one layer is deemed to provide more important or better imagery.

The segmentation process uses a technique of k-means clustering and uses the iterative removal of outliers (Shepherd et al., 2014). This technique is incorporated in a large software library RSGISLib (Bunting et al., 2014). The library is accessed by the RSOBIA toolbar.
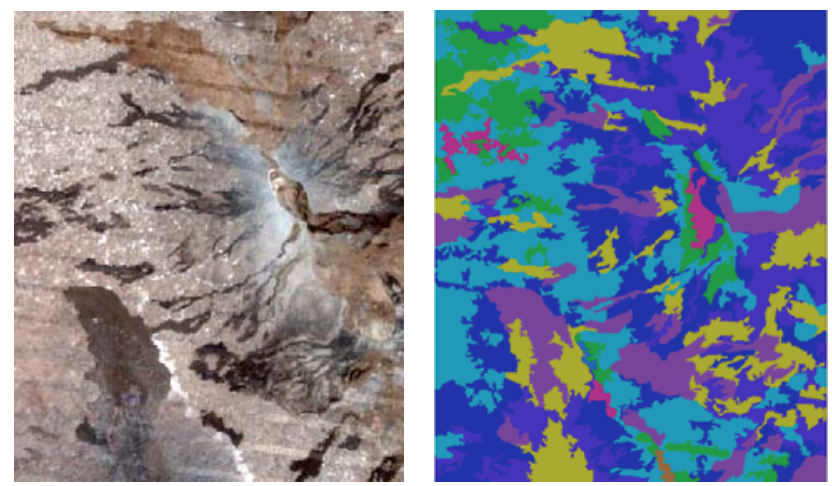

Figure 2. Left: Layered Landsat Imagery (4 layers, Bands 4,3,2 in RGB), Right: Results of segmentation using 10 classes.

\subsubsection{Adding of Attributes}

Following segmentation, the attributes for each polygon only relate to the polygon shape and k-means class. It is often required to view and use the values of initial raster layers. These can be aggregated for each polygon as an attribute value of the mean and standard deviation of the pixel values within the polygon for each layer. 


\subsection{4. $\quad$ Adding of Groundtruth Samples}

The attributes for each polygon can be extended if groundtruth point data is available. This is a very simple join of two datasets and effectively adds the attributes of a groundtruth data point to the relevant polygon. In this way the samples may be utilised to characterise the class type.

\subsubsection{Multibeam Echosounder (MBES) Wizard}

A specific wizard is available for multibeam echosounder bathymetry and backscatter imagery. It is designed to carry out a series of operations that are all available in the RSOBIA toolbar. The advantage of this is an easier and quicker method for the novice user. The wizard tool has been designed to have very few input parameters: the bathymetry and backscatter grids, the number of classes, the minimum size of the polygon and an output file. The wizard is programmed to work at any pixel resolution and will produce results at the resolution of the largest pixel of the two input files.

\subsection{Classification}

A manual classification tool is available for labelling polygons with a class name (e.g. for marine habitat mapping "Sandy Mud”). The user can choose pre-defined classification class names or type in their own class name.

The user can "paint" polygons with the appropriate colour and each polygon selected will be attributed the classification name (for example Sandy Mud).

\section{INSTALLATION}

The RSOBIA toolbar is an "addin” for ESRI's ArcMap 10.x. The Spatial Analyst extension is also required. Installation is generally a two stage setup. Two files are required: RSOBIA_setup.exe (about 138Mb) and RSOBIA.esriaddin (about 350Kb). These are both available from http://www.codemap.eu/Outputs together with full documentation.

Installation is designed to be quick and easy for the user. RSOBIA requires Python and specialised Windows libraries to be installed together in the same place. When installation is complete you should get the splash screen confirming success (Figure 5)

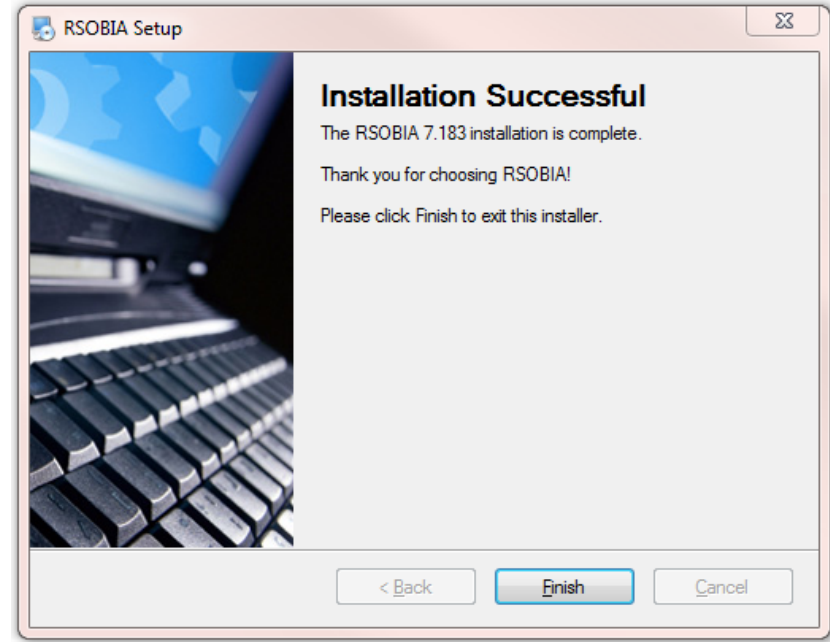

Figure 3: Splash screen after successful installation of the RSOBIA library functions

\section{USAGE}

The toolbar can be used on all types of data. Thematic maps from imagery data are often desired and the speed and reliability of objective mapping makes the RSOBIA toolbar a useful addition to ArcMap. Its ease of use is key. Testing has been done on Landsat imagery, Lidar data, Photogrammetric imagery and photographs and digital colour scans. Figure $\mathrm{X}$ shows versatility of the tool providing the segmentation from a very famous painting.
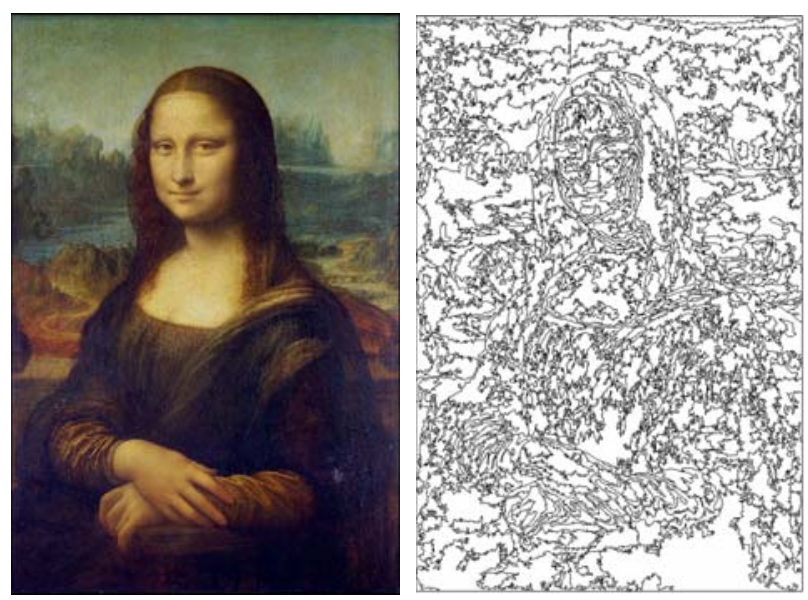

Figure 4: Example of the versatility of the segmentation process. Original imagery was scanned in 3 colours (1024 by 687 pixels) and the results of segmentation using 30 clusters (colours).

It has also very successfully been used on compilation datasets. Data from different data streams and sources have been combined by layering the data into a layered image. This layered image has then been segmented and has created a thematic map. The classes found were then classified using ground truth data and a final map produced.

If the segmentation results are not pleasing to the eye or seem to omit features, the parameters can be set differently and the process can be repeated. 


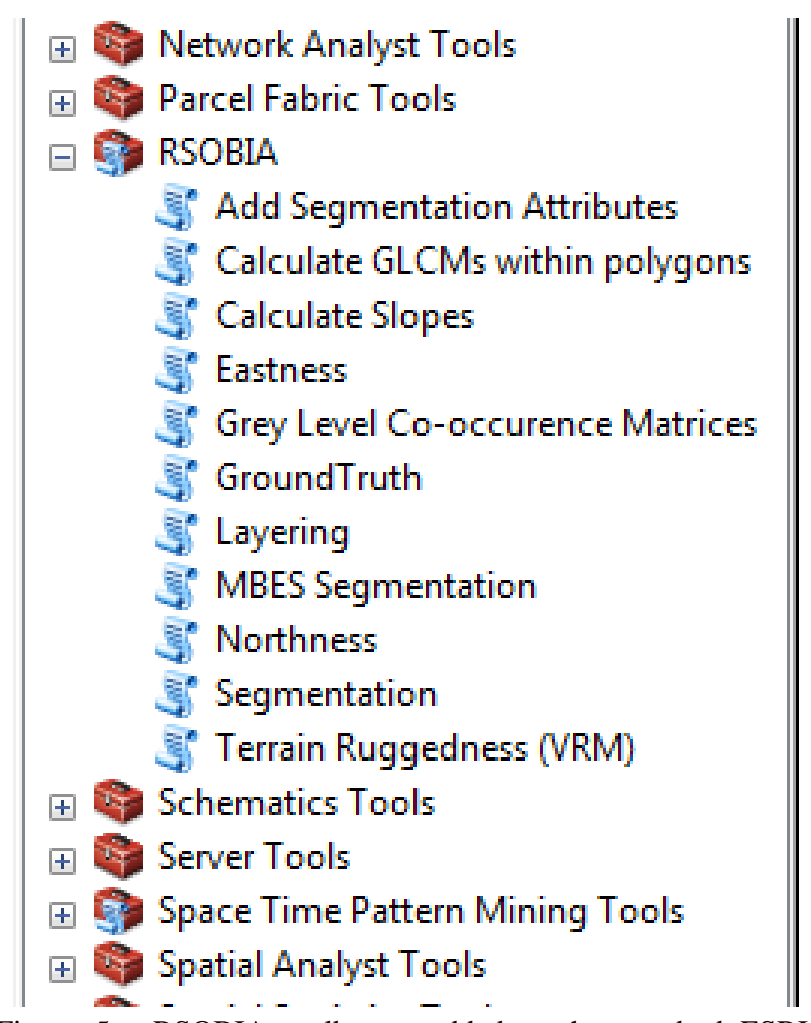

Figure 5: RSOBIA toolbox - added to the standard ESRI toolboxes.

A second way of using the tools is via the toolbox (see Figure 5 and 6). Users can create their own models using the RSOBIA tools together with standard ESRI tools. The model builder is very versatile and in this way the RSOBIA tools can be incorporated into user defined processes and data protocols.

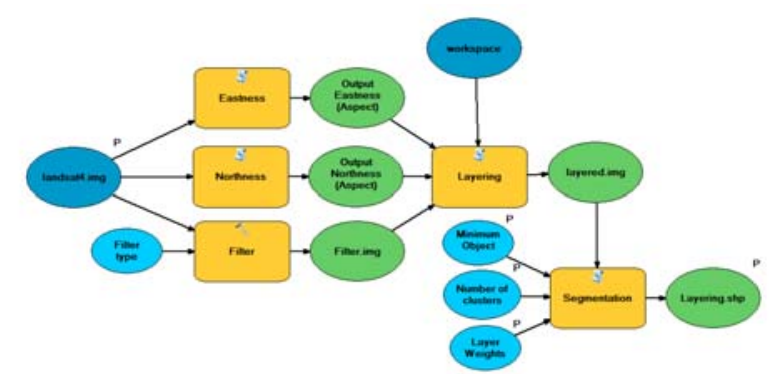

Figure 6: Example of the usage of the RSOBIA tools in a bespoke model using the model builder in ArcMap.

\section{CONCLUSIONS}

The RSOBIA toolbar is a new addition to the ArcMap set of tools. Object Based Image Analysis is a useful technique for analysis and interpretation of raster data and can be used on imagery and gridded data. Manual interpretation is slow, resource intensive and subjective. OBIA results are objective, repeatable and fast. The advantage of RSOBIA is that the process can be integrated into current workflows, and this improves efficiency of multiple processing results. The tools have been designed to be easy to learn and implement.

\section{REFERENCES}

Bunting, P.; Clewley, D.; Lucas, R.M.; Gillingham, S. The Remote Sensing and GIS Software Library (RSGISLib). Comput. Geosci. 2014, 62, 216-226.

Calinski, T., and J. Harabasz. "A dendrite method for cluster analysis." Communications in Statistics. Vol. 3, No. 1, 1974, pp. $1-27$.

Haralick, R.M., K. Shanmugam and I. Dinstein. Textural Features for Image Classification. IEEE Transactions on Systems, Man and Cybernetics. 1973 SMC vol. 3, no. 6, pp.610620.

Shepherd, J.D.; Bunting, P.; Dymond, J.R., Operational largescale segmentation of imagery based on iterative elimination. J. Appl. Remote Sens. 2014, in press.

Wright, D.J., Pendleton, M., Boulware, J., Walbridge, S., Gerlt, B., Eslinger, D., Sampson, D., and Huntley, E. 2012. ArcGIS Benthic Terrain Modeler (BTM), v. 3.0, Environmental Systems Research Institute, NOAA Coastal Services Center, Massachusetts Office of Coastal Zone Management. Available online at http://esriurl.com/5754. 


\title{
FUSION OF OPTICAL AND LIDAR IMAGES FOR URBAN OBJECTS RECOGNITION
}

\author{
W. Liao ${ }^{\text {a }}$, F. Van Coillie ${ }^{\text {b }}$, H. Zhang ${ }^{\text {c }}$, S. Gautama ${ }^{\text {a }}$, W. Philips ${ }^{\text {a }}$ \\ a iMinds-Telin-IPI, Faculty of Engineering, Ghent University, Belgium - (wliao, Sidharta.Gautama, Philips)@telin.ugent.be \\ ${ }^{\mathrm{b}}$ FORSIT Research Unit, Faculty of Bioscience Engineering, Ghent University, Belgium - Frieke.VanCoillie@ugent.be \\ c The State Key Laboratory of Information Engineering in Surveying, Mapping, and Remote Sensing, Wuhan University, \\ P. R. China - Hongyan.Zhang@telin.ugent.be
}

KEY WORDS: Data fusion, remote sensing, true orthophoto, LiDAR image, classification

\begin{abstract}
:
Nowadays, advanced sensor technology and image processing algorithms allow us to measure different aspects of the objects on the Earth's surface, from spectral characteristics in optical images, height information in LiDAR data, to spatial information generated by image processing technologies like commercial software eCognition ${ }^{\circledR}$. However, automatic recognition of objects in remote sensed scenes remains challenging. It is clear that single technology might not be sufficient to obtain reliable classification results (Debes, 2014). Multisensor data, once combined, can contribute to a more comprehensive interpretation of objects on the ground. For example, spectral reflections from optical image cannot recognize objects under shadows, while they can often be easily detected by LiDAR data. On the other hand, LiDAR data alone may fail to discriminate between objects that are quite similar in height.

Stacking multi-source data together is a widely applied data fusion technique for classification. These methods first apply feature extraction on each individual data source, after which all feature sources are concatenated into one stacked vector for classification. While such methods are appealing due to their simplicity, they do not always perform better than using a single data source. This is because the value of different components in the stacked feature vector can be significantly unbalanced. As a consequence, the information contained by different data sources is not equally represented or measured. Furthermore, the increase in the dimensionality of the stacked features, combined with the limited number of labelled samples, may together lead to the problem of the "curse of dimensionality" (Liao, 2015).

Therefore, we present a local graph fusion method to fuse true orthophoto and LiDAR image for urban object recognition. First, object-based spatial and height information are generated on true orthophoto and LiDAR image, respectively. Second, we build a local fusion graph within a sliding window where only the data points with similar spatial and height characteristics are connected. Finally, we solve the problem of multisensor data fusion by projecting multisensor data into a subspace, on which the advantages of different data sources are well exploited. Experimental results on fusion of true orthophoto and LiDAR image from 'ISPRS Test Project on Urban Classification and 3D Building Reconstruction' demonstrate the potential of the proposed method. Compared to the methods using only single data source or stacking them together, our approach has significant improvements in overall classification accuracy. Both the method's details and the results of a comprehensive test will be presented at GEOBIA 2016.
\end{abstract}

\section{ACKNOWLEDGEMENTS}

This work was supported by the FWO project G037115N: Data fusion for image analysis in remote sensing.

\section{REFERENCES}

Liao, W., Bellens, R., Pizurica, A., Gautama, S., Philips, W., 2015. Generalized Graph-Based Fusion of Hyperspectral and LiDAR Data Using Morphological Features. IEEE Geoscience and Remote Sensing Letters, vol. 12, no. 3, pp. 552-556.

Debes, C., Merentitis, A., Heremans, R., Hahn, J., Frangiadakis, N., Kasteren, T., Liao, W., Bellens, R., Pizurica, A., Gautama, S., Philips, W., Prasad, S., Du, Q., Pacifici, F., 2014. Hyperspectral and LiDAR Data Fusion: Outcome of the 2013 GRSS Data Fusion Contest. IEEE Journal of Selected Topics in

\footnotetext{
* Corresponding author
}

Applied Earth Observations and Remote Sensing, vol.7, no. 6, pp. 2405-2418. 


\title{
SEGMENTATION OPTIMIZATION VIA RECOGNITION OF THE PSE-NSR-ED2 PATTERNS ALONG WITH THE SCALE PARAMETER IN OBJECT-BASED IMAGE ANALYSIS
}

\author{
Y. Liu a, b, *, Y-D. Zhang a , Z. Huang a, M-M. Wang a , D. Yang a H-M. Ma a , Y-X. Zhang a , Y-F. Li a , H-W. Li a , X-G. Hu ${ }^{a}$ \\ ${ }^{a}$ College of Earth and Environmental Sciences, Lanzhou University, Lanzhou 730000, China -(liuy, zhangyd5)@1zu.edu.cn; \\ huangzh07@126.com; lystwmm@163.com; 764423127@qq.com; (mahm2014, zhangyongxu14, liyp13)@1zu.edu.cn; \\ lihongweibang@126.com; huxg13@1zu.edu.cn \\ ${ }^{\mathrm{b}}$ Key Laboratory of Western China's Environmental Systems (Ministry of Education), Lanzhou University
}

KEY WORDS: object based image analysis; discrepancy measure; image segmentation; optimization; data stack; scale parameter

\begin{abstract}
:
To create image objects for subsequent classification in object-based image analysis, an optimal segmentation threshold (OST) is a pre-requisite for image segmentation. However, an OST is practically acquired by assessing and ranking an exhaustive segment data stack constructed after foregoing image segmentation. In this paper, we propose an iterative exploration method via recognition of the Euclidean distance 2 (ED2)-scale parameter (SP) pattern with the least five tiles of segment data stack in each cycle among the Potential Segmentation Error (PSE)-Number-of-Segments Ratio (NSR)-ED2 patterns along with SP. We conducted two experiments. In the first experiment, we validated the general italic U-shaped ED2-SP pattern by constructing exhaustive segment data stacks and corresponding segment data stacks. In the second experiment, we adopted the proposed iterative exploration method for OST selection based on the ED2-SP pattern with respect to five equal-spacing SPs in each cycle. The bottom of the pattern was persistently approached by constructing updated segment data stacks and corresponding segment data stacks with five dynamically adjusted tiles. Our results showed that the PSE-NSR-ED2 discrepancy measure system is advantageous to OST selection.
\end{abstract}

\section{INTRODUCTION}

Image segmentation, as the first critical step in the object-based image analysis (OBIA) procedure, divides an image into nonoverlapping segment datasets and assigns each pixel within an image to an inner-homogeneous segment (Navulur, 2007; Tilton et al., 2016). In general, image segmentation is performed by predefining a threshold to assess the maximum allowable spectral or spatial heterogeneity within image objects (Baatz et al., 2008; Baatz and Schäpe, 2000; Blaschke, 2010; Blaschke et al., 2014; Ming et al., 2015). However, selecting an optimal threshold is often a tedious trial-and-error process (Castilla and Hay, 2008; Meinel and Neubert, 2004). In addition, the optimal threshold changes with respect to images and land cover types.

The exhaustive method is often employed for selecting an optimal segmentation threshold (OST) based on quantitative assessment and ranking with respect to an exhaustive segment data stack (Arvor et al., 2013; Im et al., 2014; Marpu et al., 2010; Myint et al., 2011). An exhaustive segment data stack consists of tiles of segment datasets, and each tile is related to a threshold. Reference datasets play an important role in quantitative assessment of image segmentation. A corresponding segment dataset is a subset of segments for a tile in an exhaustive segment data stack, which does not cover the entire image but corresponds simply to a reference dataset spatially based on areal-overlap-based criteria (Clinton et al., 2010; Yang et al., 2015a). In general, each tile of corresponding segment data stacks exhibits a complexity of overlapping, undersegmentation, and oversegmentation, as compared with reference datasets. An optimal segment dataset (OSD) associated with an OST is qualified for the subsequent segment classification because it has the least undersegmentation and less oversegmentation as compared with a reference dataset (Castilla and Hay, 2008; Liu et al., 2012; Marpu et al., 2010; Radoux and Defourny, 2008; Weidner, 2008).

Many approaches have focused on developing automatic or semiautomatic procedures to obtain the OST based on goodness or discrepancy measures for segmentation assessment (Anders et al., 2011; Drăguţ et al., 2014; Martha et al., 2011; Rasi et al., 2011; Schultz et al., 2015; Smith, 2010). They are generally based on tiles of exhaustive segment data stacks associated with a wide range of segmentation thresholds and dense threshold intervals. A critical problem for segmentation optimization is to pre-set the minimal, maximal threshold, and the increment between the minimum to maximum. If the interval between the minimal and the maximal threshold is pre-set closely around the OST, the obtained OST can be a small number of tiles and the time spent is short. However, if the interval is given apart from the OST. The exhaustive method would be unsuccessful to select the OST. On the other hand, the OST could be omitted between the coarse increments of the threshold. It takes extremely long times to construct an exhaustive segment data stack with a great number of tiles associated with a wide range of segmentation thresholds and a dense threshold increment.

Discrepancy measures assess segment datasets by comparing reference polygons with corresponding segments (Albrecht et al., 2008; Lang et al., 2010; Marpu et al., 2010; Tiede et al., 2010; Zhang, 1996). Many discrepancy measures have been proposed for ranking and selecting the OSD and OST (Liu et al., 2012; Weidner, 2008; Yang et al., 2015a; Yang et al., 2015b; Yang et al., 2014; Zhang et al., 2015). Our previous studies 
have shown that the Potential Segmentation Error (PSE)Number-of-Segments Ratio (NSR)-Euclidean distance 2 (ED2) discrepancy measure system is credible (Liu et al., in press).

In this study, we propose an iterative exploration method for selecting the OST via recognition of the distribution patterns between the PSE-NSR-ED2 discrepancy measure and the scale parameter (SP). To verify this method, image segmentation is conducted on the basis of the fractal net evolution algorithm (FNEA) with a combined segmentation threshold consisting of three parameters: scale, shape, and compactness (Baatz and Schäpe, 2000; Burnett and Blaschke, 2003; Trimble, 2014). In this combination, the SP plays an important role in controlling the segment size. The other two parameters are weight factors varying between zero and one, and are mainly involved in improving the geometric characteristics of image objects. Each segment data stack is constructed and is employed for the optimal scale parameter (OSP) selection with a set of SP and a fixed combination of shape and compactness parameters. Thus, the OST selection turns to rank all the OSPs for the entire combinations of the shape and compactness parameters.

The remainder of this paper is organized into as follows. Section 2 explains an italic U-shaped ED2-SP pattern and an iterative transformation method of five dynamically adjusted equal-spacing SP values for automatically exploring the OSP. Sections 3 and 4 describe two experiments for validating the ED2-SP pattern and compare the results from these two method. Finally, Section 5 concludes the paper.

\section{METHOD AND DATA PREPARATION}

\subsection{Italic U-shaped ED2-SP pattern}

In this section, an italic U-shaped ED2-SP pattern is proposed based on the analysis of the PSE-SP, NSR-SP, and ED2-SP curves. Firstly, a power function between the average area of segments and the SP is suggested, given a numerical combination of the shape and compactness parameters. It follows by regularly variations of PSE and NSR along with the SP. The italic U-shaped ED2-SP pattern is therefore deduced.

In the PSE-NSR-ED2 discrepancy measure system, PSE is the ratio between the total area of the under-segments and the total area of the reference polygons, NSR is the absolute number difference between the reference polygons and the corresponding segments divided by the number of reference polygons, and ED2 is a Euclidean distance associated with PSE and NSR (Liu et al., 2012).

Here, we denote a reference dataset with $m$ reference polygons as $R=\left\{r_{i}: i=1,2, \ldots, m\right\}$ and a corresponding segment dataset with $n$ segments as $S=\{s j: j=1,2, \ldots, n\}$ based on one-side areal-overlap-based criteria. Thus, PSE, NSR, and ED2 can be expressed as

$P S E=\frac{\left|S_{k}-R_{i}\right|}{\left|R_{i}\right|} ; \quad N S R=\frac{\mathrm{abs}(m-n)}{m} ; E D 2=\sqrt{P S E^{2}+N S R^{2}}$

where

$\left|S_{k}-R_{i}\right|=$ total areas of the under-segments

$\left|R_{i}\right|=$ total areas of reference polygons

abs ()$=$ absolute value function.

A given image having a total area TA is divided into $n$ segment polygons; the average area of the segment polygons is given by:

$$
M A=\frac{T A}{n}
$$

where $\quad \mathrm{TA}=$ total number of pixels in the image.

As discussed in Section 3.2, our study verifies that, given a numerical combination of the shape and compactness parameter the mean area of segments increases approximately in the form of a power function along with SP with positive proportional and power terms. Correspondingly, the number of segments decreases approximately in the form of a power function along with SP, but with a positive proportional term and a negative power term. Thus,

$$
M A=\alpha \cdot S P^{\beta} ; \quad n=\frac{T A}{\alpha} \cdot S P^{-\beta}
$$

where $\quad \mathrm{MA}=$ mean area of segments in a tile

$$
\alpha, \beta=\text { positive real number. }
$$

When the area and the number of reference polygons are constant, PSE and NSR vary approximately similar to MA and $\mathrm{N}$, respectively, along with $\mathrm{SP}$, with some bias due to differences of images and land cover types. When SP is small, the arithmetic relationship between the corresponding segments and the reference polygons is many-to-one, accordingly, PSE is close to zero and NSR is large. As shown in Equation (1), the maximal NSR is smaller than the ratio between $n$ and $m$. Along with the increased SP, MA increases and $\left|S_{k}-R_{i}\right|$ is gradually close to zero up to an interval where the arithmetic relationship becomes one-to-one and both PSE and NSR keep small (less than one at least). Beyond this interval, the area of the corresponding segments is larger than the area of the reference polygons and the difference tends to increase along with SP, and the increased undersegmentation and PSE follows the power function between MA and the SP. On the other hand, the arithmetic relationship between the corresponding segments and the reference polygons remains one-to-one, NSR tends to zero. Therefore, we establish an ED2-SP pattern showing the variation of ED2 in the form of the italic U-shaped curve along with SP (Figure 1).

It can be seen that ED2 varies regularly along with the SP axis. The lowest point for the minimal ED2 and OSP splits the italic $\mathrm{U}$-shaped pattern into the left and the right side. The italic Ushaped pattern is constructed because these two sides are asymmetrical. The right side generally has wider range of SP than the left side, provided by equal ED2 range. At the left side of this pattern, SP is smaller than the OSP, the italic U-shaped pattern is close to or overlaps with the decreasing NSR curve where PSE is very small. At the right side of the pattern, SP is larger than the OSP, the italic U-shaped curve is close or coincident to the PSE curve, where NSR is close to zero.

Owing to its regularity, it is possible to locate the OSP by recognition of this pattern. When ED2 is close to the minimum, the under-segment areas are very small comparing to the areas of reference polygons and the number of corresponding segments is close to that of reference polygons, therefore, PSE and NSR are less than one. In other word, ED2 associated with the OSP normally lies in a very limit interval and is small than one. 


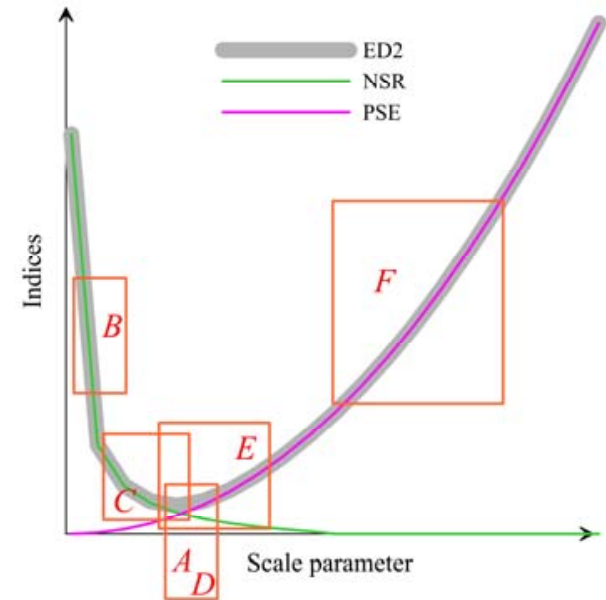

Figure 1. Italic U-shaped ED2-SP pattern under a numerical combination of the shape and compactness parameter. The ED2 curve coincides with the PSE and NSR curves at the two sides, respectively This italic U-shaped pattern is further divided into seven major cases, which are represented the ED2-SP pattern with five equal-spacing SPs in Figure 2 (from panel $A$ to $F$ ).

A critical aspect to consider in the OSP selection is that the pattern could vary with respect to the characteristics of remotely sensed imagery and land cover types. There is no universal coefficient setting for the ED2-SP pattern. The setting is unknown until a segment data stack and corresponding segment data stack are constructed. As indicated before, constructing an exhaustive segment data stack and corresponding segment data stack is bound to time-consuming. Alternatively, we proposed an iterative method for pattern recognition by constructing least five tiles of segment data stack and corresponding segment data stack for a combination of shape and compactness parameters. Provided by the entire OSP sets with respect to all the combinations of the shape and compactness parameters, the OST is selected by ranking the OSP set.

\subsection{Iterative exploration method for OSP selection via recognition of the ED2-SP pattern with five tiles of a segment data stack}

Graphically depicting a precise italic U-shaped pattern requires many ED2-SP dots. Reducing the dot number implies decreasing the tiles of segment data stack and the time spent for constructing the data stack. As performed in Section 4, five ED2-SP dots associated with five equal-spacing SPs for constructing a segment data stack and corresponding data stack are feasible to recognize the pattern in an iterative procedure.

The basic step is to construct a segment data stack with five tiles associated with five equal-spacing SP. Given a preliminary setting of the minimal and maximal SP, the other three SP can be interpolated equally between the ranges. Then, the PSENSR-ED2 measures are obtained and the ED2-SP pattern with five dots is constructed for each corresponding segment data stack.

Based on the pattern recognition with respect to the five ED2SP sets, a transformation to obtain updated five equal-spacing $\mathrm{SP}$ is conducted iteratively until the minimal ED2 at the bottom of the italic U-shaped pattern is found.
The ED2-SP pattern shown in Figure 1 represents only a general pattern. The actual pattern is offset owing to the differences with respect to images and reference datasets. Our study demonstrated that the variations of the five ED2 associated with the five equal-spacing SP can be classified into 17 cases. The pre-set minimal and maximal SP could be related to one of these cases. Six of them are the most important ones. They are shown as typical parts of the italic U-shaped ED2-SP pattern in Figure 1 and labelled also as panels $A-F$ in Figure 2. Case A could be prior to explore the OSP. However, it sometimes happens at the right side of the italic U-shaped pattern relevant to ED2s larger than 1. Case B is related to the left side of the pattern. Cases C-F happen at the bottom and the right side of the pattern. The other 11 Cases from $\mathrm{G}$ to $\mathrm{Q}$ can be meet mostly at the right side of the pattern relevant to ED2s larger than 1 .
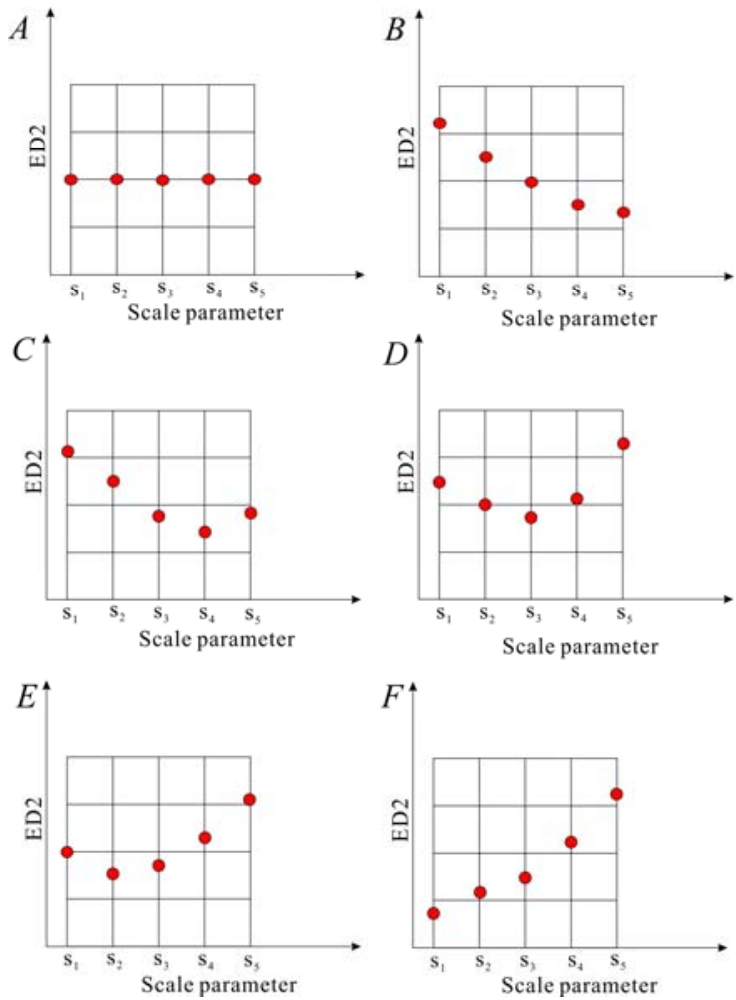

Figure 2. Major six cases of ED2 distribution associated with five equal-spacing SP.

To reach the target OSP interval as the case $A$ and $D$, a transformation is necessary to reset the SP range for the other cases based on their locations in the italic U-shape ED2-SP pattern. The basic transformations are one-dimensional zooming in, zooming out, left shifting, and right shifting along with the SP axis.

Initially, the SPs range $\left\{\mathrm{s}_{1}, \mathrm{~s}_{5}\right\}$ and the minimal interval between any two SPs, $d$ min, is predefined; $\mathrm{s}_{1}<\mathrm{s} 5$. To create a set of five SPs that are equally spaced from $\mathrm{s}_{1}$ to $\mathrm{s}_{5}$, we can sequentially interpolate the other three SPs, $\mathrm{s}_{2}, \mathrm{~s}_{3}$, and $\mathrm{s}_{4}$, as follows:

$d=\frac{1}{4}\left(s_{5}-s_{1}\right) ; \quad s_{3}=\frac{1}{2}\left(s_{1}+s_{5}\right) ; \quad s_{2}=\frac{1}{2}\left(s_{1}+s_{3}\right) ; \quad s_{4}=\frac{1}{2}\left(s_{3}+s_{5}\right)$ 
if the spacing between any pair of SPs is greater than the minimum, $d>d \min$. Otherwise, $\{\mathrm{s} 1, \mathrm{~s} 5\}$ need to be reset by increasing the spacing, $d$.

After setting these five SPs, s1-5, a segment data stack with five tiles is constructed. Further, a corresponding segment data stack is constructed with respect to a land cover type and the associated five ED2s are calculated.

The next step is to assess the pattern with the five ED2-SP dots in order to determine if one of the ED2s reaches the minimum and the OSP is coincident with the associated ED2. This assessment is performed case by case as follows.

In case $A$, the five ED2s associated with five equal-spacing SPs are equal or very close to each other. This case could occur in two situations. In the first situation, the five dots associated with the five ED2s really correspond to the bottom of the italic U-shaped pattern and indicate the minimal ED2. The OSP could be one of them. A further step is to confirm this situation by zooming out the five values by doubling the spacing, $d$, to update the five equal-spacing SPs:

$s_{1, \text { new }}=s_{1}-2 \cdot d ; \quad s_{2, \text { new }}=s_{1} ; \quad s_{3, \text { new }}=s_{3} ;$

$s_{4, \text { new }}=s_{5} ; \quad s_{5, \text { new }}=s_{5}+2 \cdot d$

where, S1-5, new represent the five updated SPs. If the updated pattern is coincident to the case $D$, as shown in Figure 2, the SP at $\mathrm{s}_{3, \text { new }}$ can be determined as the OSP.

In the second situation, these dots occur on the right side of the italic U-shaped pattern (Figure 1), associated with the ED2s larger than one. Then, a left-shifting operation can be conducted to reset these five SPs by decreasing the SP spacing, $d$, by four units.

$s_{1, \text { new }}=s_{1}-4 \cdot d ; \quad s_{2, \text { new }}=s_{1}+d ; \quad s_{3, \text { new }}=\frac{1}{2}\left(s_{1}+s_{5}\right) ;$

$s_{4, \text { new }}=s_{5}-d ; \quad s_{5, \text { new }}=s_{1}$

After this left-shifting operation, the routine returns to a new iterative cycle to construct a new segment data stack with the new five SPs given by Equation (6) in order to extract the corresponding segment data stack, recalculate the five ED2s for subsequent exploration, and recognize the new ED2-SP pattern.

In case $B$, the five ED2s decrease sequentially along with increasing SP. The minimal ED2 for this ED2-SP pattern could be at the right side of $\mathrm{s}_{5}$ or around it. A right-shifting operation can be conducted, where the SPs are obtained by doubling the spacing $d$.

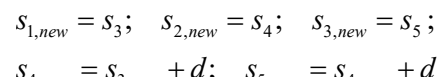

$s_{4, \text { new }}=s_{3, \text { new }}+d ; \quad s_{5, \text { new }}=s_{4, \text { new }}+d$

After this right-shifting operation, the routine returns to a new iterative cycle.

In case $C$, the minimal ED2 value occurs around S4. Furthermore, if $d \leq d \mathrm{~min}$ and the ED2 at $\mathrm{s} 4$ is smaller than one, then the SP can be determined as the OSP and the iterative operation terminates. If $d \geq d \mathrm{~min}$ and the ED2 at $\mathrm{s}_{4}$ is smaller than one, then the SP spacing is zoomed in to determine an improved estimation of the OSP. $d_{\text {new }}=\frac{1}{2} d ; \quad s_{1, \text { new }}=s_{2} ; \quad s_{5, \text { new }}=s_{4} ;$

$s_{2, \text { new }}=s_{1, \text { new }}+d ; \quad s_{3, \text { new }}=s_{2, \text { new }}+d ; \quad s_{4, \text { new }}=s_{5, \text { new }}-d ;$

Otherwise, if the ED2 at $\mathrm{s}_{4}$ is larger than one, then a rightshifting operation is conducted for decreasing the five SPs by one spacing $d$ :

$s_{1, \text { new }}=s_{2} ; \quad s_{2, \text { new }}=s_{3} ; \quad s_{3, \text { new }}=s_{4} ;$
$s_{4, \text { new }}=s_{5} ; \quad s_{5, \text { new }}=s_{5}+d$

In case $D$, the minimal ED2 occurs around $\mathrm{s}_{3}$. If $d \leq d \mathrm{~min}$ and the ED2 at $\mathrm{s}_{3}$ is smaller than one, then a zooming-in operation is conducted by halving the interval between $\mathrm{s} 1$ and $\mathrm{s} 5$, as shown in Equation (8). Otherwise, if the ED2 at $\mathrm{s}_{3}$ is greater than one, then a left-shifting operation is conducted to decrease these five SPs as shown in Equation (6).

In case $E$, the minimal ED2 occurs around $\mathrm{s}_{2}$. If $d \leq d \mathrm{~min}$ and the ED2 at s2 is smaller than one, then a zooming-in operation is conducted by halving the spacing between $\mathrm{s}_{1}$ and $\mathrm{s}_{5}$, as shown in Equation (8). Otherwise, if the ED2 at $\mathrm{s}_{2}$ is greater than one, then a left-shifting operation is conducted for decreasing the five SPs as shown in Equations (6).

In case $F$, these five ED2s increase sequentially along with the increasing SP. A left-shifting operation can be conducted for decreasing the SPs by doubling the spacing $d$.

$s_{5, \text { new }}=s_{3} ; \quad s_{3, \text { new }}=s_{1} ; \quad s_{4, \text { new }}=s_{2} ;$

$s_{5, \text { new }}=s_{3}-d ; \quad s_{1, \text { new }}=s_{2}-d$

For all the above cases with the left-shifting transformation, if the new $s_{1}$ is negative, then it must be reset to one and $s_{2-4}$ are recalculated, while s5 remains unchanged:

$s_{1, \text { new }}=1 ; \quad d=\frac{1}{4}\left(s_{5}-s_{1}\right) ; \quad s_{2, \text { new }}=\frac{1}{4} d ;$

$s_{3, \text { new }}=\frac{1}{2} d ; \quad s_{4, \text { new }}=\frac{3}{4} d ; \quad s_{5, \text { new }}=s_{5}$

\subsection{Data preparation}

Three types of images were principally employed in this study: QuickBird, WorldView II, and ALOS images. In addition, pansharpened multispectral data for the three images were constructed from all the multispectral bands and panchromatic band using the Gram-Schmidt algorithm. Therefore, we conducted our experiment using six sets of images, which, hereafter, are abbreviated for convenience as QB_MS, QB_sharpen, WV2_MS, WV2_ sharpen, ALOS_MS, and ALOS_sharpen.

Reference polygons were delineated for the six sets of images with regard to three types of land cover, namely farmland, rural housing, and water bodies, respectively. Each type of reference polygon was acquired using 30 samples for each image.

The computer employed in the following experiments had the following specification:

1) CPU: Intel ${ }^{\mathrm{TM}} \mathrm{Core}^{\mathrm{TM}} \mathrm{i} 7-3770,3.40 \mathrm{GHz}$;

2) Hard disk: Western Digital WD10 EALX-089BAO SATA $^{\mathrm{TM}}$, 1TB; 
3) Graphics adapter: NVIDIA ${ }^{\mathrm{TM}}, \mathrm{AMD}^{\mathrm{TM}}$ Radeon HD 7800 Series;

4) Operating System: Microsoft Windows 10 Professional, 64 bit.

\section{EXPERIMENT ONE: ED2-SP PATTERNS BASED ON EXHAUSTIVE METHOD}

The purpose of the experiment one is to verify (1) the power function between the average area of segments and the SP value; (2) the variations of PSE, NSR, and ED2 along with the SP based on the exhaustive segment data stacks from the six sets of images and corresponding segment data stacks associated with farmland, rural buildings, and water bodies.

\subsection{Construction of exhaustive segment data stacks and corresponding segment data stacks}

Image segmentation was conducted using the FNEA algorithm embedded in the eCognition ${ }^{\mathrm{TM}}$ software. To construct exhaustive segment data stacks, the minimal values, maximal values, and increments of SP were set to: 10, 100, and 5, respectively, for QB MS; 10, 190, and 20, respectively, for QB_sharpen; 5, 150, and 5, respectively, for WV2_MS; and 50, 350 , and 10, respectively, for WV2_sharpen. The minimal values, maximal values, and increments of both shape and compactness parameters were set to $0.1,0.9$, and 0.1 , respectively, for all the images. Therefore, there are 81 numerical combinations between the two parameter values for each SP, and the numbers of tiles for these six data stacks ranged from 810 to 2511 , as shown in Table 1 .

\begin{tabular}{|c|c|c|c|}
\hline Images & QB_MS & WV2_MS & ALOS_MS \\
\hline Image size & $1200 \times 1100$ & $1657 \times 1411$ & $290 \times 247$ \\
\hline $\begin{array}{c}\text { SP (min, max, } \\
\text { increment) }\end{array}$ & $10,100,5$ & $5,150,5$ & $10,150,10$ \\
\hline Number of tiles & 1539 & 2430 & 1215 \\
\hline$\alpha$ & $0.2918-1.1344$ & $0.1659-0.9975$ & $0.8487-6.0257$ \\
\hline$\beta$ & $1.7855-2.0144$ & $1.7745-2.1606$ & $1.8505-2.2414$ \\
\hline $\begin{array}{c}\text { Coefficient of } \\
\text { determinant }\end{array}$ & $0.9983-1.0000$ & $0.9793-0.9995$ & $0.9822-0.9991$ \\
\hline $\begin{array}{c}\text { Time spent } \\
\text { (hours) }\end{array}$ & 1.8407 & $\begin{array}{c}\text { S1: 6.0903; } \\
\text { S3: 7.3710; }\end{array}$ & 1.4805 \\
\hline Images & QB_sharpen & WV2_sharpen & $\begin{array}{c}\text { ALOS } \\
\text { sharpen }\end{array}$ \\
\hline Image size & $4800 \times 4400$ & $6630 \times 5644$ & $1067 \times 908$ \\
\hline $\begin{array}{c}\text { SP (min, max, } \\
\text { increment) }\end{array}$ & $10,190,20$ & $50,350,10$ & $50,650,50$ \\
\hline Number of tiles & 810 & 2511 & 1053 \\
\hline$\alpha$ & $0.2641-0.5544$ & $0.0778-1.0244$ & $0.7843-2.9019$ \\
\hline$\beta$ & $1.8218-2.1076$ & $1.6117-2.2615$ & $1.8175-2.0398$ \\
\hline $\begin{array}{c}\text { Coefficient of } \\
\text { determinant }\end{array}$ & $0.9961-0.9998$ & $0.9839-0.9997$ & $0.9932-0.9999$ \\
\hline $\begin{array}{c}\text { Time spent } \\
\text { (hours) }\end{array}$ & 17.6993 & $\begin{array}{c}\text { S1:108.6606; } \\
\text { S2:124.7088; } \\
\text { S3:123.7876 }\end{array}$ & 42.4262 \\
\hline Table 1. Statisics & for the prop & \\
\hline
\end{tabular}

Table 1. Statistics for the proportional and power coefficients, and the coefficient of determinant of the power function, and the time spent. The shape and compactness parameters are set from 0.1 to 0.9 at intervals of 0.1 with one accord.

Because of the large size of the WorldView II image $(6662 \times$ 5713 for eight bands and $26648 \times 22852$ for eight sharpened bands), it was divided into $(4 \times 4=) 16$ subsets. Only three subsets were selected in this experiment, based on the three dominant land cover types: farmland, rural housing, and water bodies. For convenience, they are abbreviated as FL, RH, and $\mathrm{WB}$, respectively.

\subsection{Results and discussion}

3.2.1 Power functions for the average area of segments and scale parameter

In this experiment, the power function for the average area of segments and SP was fitted with respect to each numerical combination of shape and compactness parameters. For this purpose, the average area of the segments in each data stack was calculated with respect to the SP.

The proportional and power coefficients $(\alpha$ and $\beta$ ) as well as the coefficients of determination of the power function, listed in Table 1, vary between 0.0778 and 6.0257 , and between 1.6117 and 2.2615, respectively, for the six types of images. Besides, all the coefficients of determinant take high values; the minimal coefficient is 0.9793 . It shows that the power function between the average area, number of segments, and SP is good for the given numerical combinations of shape and compactness parameter.

In addition, we conducted further data analysis using Chinese GF-1, Landsat 5 Thematic Mapper, and Landsat 8 Operational Land Imager data. Thus we confirmed that the power function is tenable with high coefficients of determination and various proportional and power coefficients.

\subsubsection{OST and time spent}

To extract corresponding segment data stacks, the reference datasets were employed for each land cover type with respect to the six sets of images. The overlapped, over-segmented, and under-segmented area were thus identified on the basis of the spatial overlap between the reference polygons and the corresponding segments. Furthermore, the PSE-NSR-ED2 discrepancy measure were calculated.

\begin{tabular}{|c|c|c|c|c|}
\hline $\begin{array}{l}\text { Image } \\
\text { sources }\end{array}$ & $\begin{array}{l}\text { Land } \\
\text { cover }\end{array}$ & $\begin{array}{c}\text { OST (scale, } \\
\text { shape, } \\
\text { compactness) }\end{array}$ & $\begin{array}{l}\text { Minimal } \\
\text { ED2 }\end{array}$ & $\begin{array}{l}\text { time spent } \\
\text { (hours) }\end{array}$ \\
\hline \multirow{3}{*}{ QB_MS } & FL & $15,0.3,0.9$ & 0.261 & 14.2382 \\
\hline & $\mathrm{RB}$ & $15,0.9,0.1$ & 0.368 & 15.0932 \\
\hline & WB & $50,0.5,0.7$ & 0.023 & 10.8182 \\
\hline \multirow{3}{*}{ WV2_MS } & $\mathrm{FL}$ & $25,0.6,0.4$ & 0.117 & 20.2653 \\
\hline & RB & $25,0.6,0.5$ & 0.292 & 21.5460 \\
\hline & WB & $150,0.3,0.6$ & 0.017 & 23.0166 \\
\hline \multirow{3}{*}{$\begin{array}{c}\text { ALOS_- } \\
\text { MS }\end{array}$} & FL & $30,0.2,0.3$ & 0.345 & 8.5680 \\
\hline & $\mathrm{RB}$ & $30,0.5,0.2$ & 0.264 & 7.8930 \\
\hline & WB & $20,0.4,0.7$ & 0.271 & 7.8930 \\
\hline \multirow{3}{*}{$\begin{array}{c}\mathrm{QB}- \\
\text { sharpen }\end{array}$} & FL & $70,0.1,0.8$ & 0.211 & 24.2243 \\
\hline & $\mathrm{RB}$ & $90,0.6,0.3$ & 0.324 & 23.9993 \\
\hline & WB & $190,0.6,0.7$ & 0.039 & 24.8993 \\
\hline \multirow{3}{*}{$\begin{array}{l}\text { WV2_- } \\
\text { sharpen }\end{array}$} & FL & $130,0.2,0.5$ & 0.107 & 124.0056 \\
\hline & RB & $90,0.3,0.4$ & 0.236 & 153.3053 \\
\hline & WB & $320,0.7,0.3$ & 0.076 & 157.9651 \\
\hline \multirow{3}{*}{$\begin{array}{l}\text { ALOS_- } \\
\text { sharpen }\end{array}$} & FL & $100,0.1,0.7$ & 0.246 & 47.6912 \\
\hline & $\mathrm{RB}$ & $100,0.3,0.9$ & 0.454 & 47.6912 \\
\hline & WB & $50,0.3,0.9$ & 0.123 & 52.9562 \\
\hline
\end{tabular}

Table 2. OST selection and time spent based on the exhaustive method.

The OSTs were selected by ranked ED2s for each corresponding segment data stack. Table 2 lists the OST, minimal ED2, tiles in each segment data stack, and time spent with respect to image and land cover. The OST apparently varies with land cover. Notably, the OST for water bodies is 
mainly characterized by larger SP and smaller ED2 than those for farmland and rural housing.

3.2.3 Assessment of the italic U-shaped ED2-SP pattern Based on the corresponding segment data stacks, the ED2-SP pattern was assessed with respect to PSE-SP and NSR-SP curves. In total, there were 1458 sets of curves for the six images of the three land cover types, associated with 81 combinations of shape and compactness parameters $(6 \times 3 \times 9 \times$ 9). Figure 3 shows that a set of PSE, NSR, and ED2 varies along with SP for farmland from the WV2_MS image, where shape and compactness parameters are set to 0.5 and 0.1 , respectively. It is characterized by a trend similar to the general italic U-shaped ED2-SP pattern with some modifications. PSE is smaller than 0.5 until SP is less than or equal to 25 ; then, it increases along with SP. In the interval of $130-135$ for SP, the curve becomes horizontal and PSE do not change. NSR decreases drastically and monotonically from a very large number associated with SP of 5 to zero associated with SP of 55 When SP is greater than 55, NSR is zero. Thus, the number of reference polygons equals the number of corresponding segments. The ED2-SP curve represents a combined form of the PSE-SP and NSR-SP with the italic U-shaped pattern. When SP is smaller than 25 , the curve coincides with the part for NSR; when SP is larger than 55, the curve coincides with the part for PSE. The minimal ED2 is very small and appears in the interval of $25-55$ for SP.

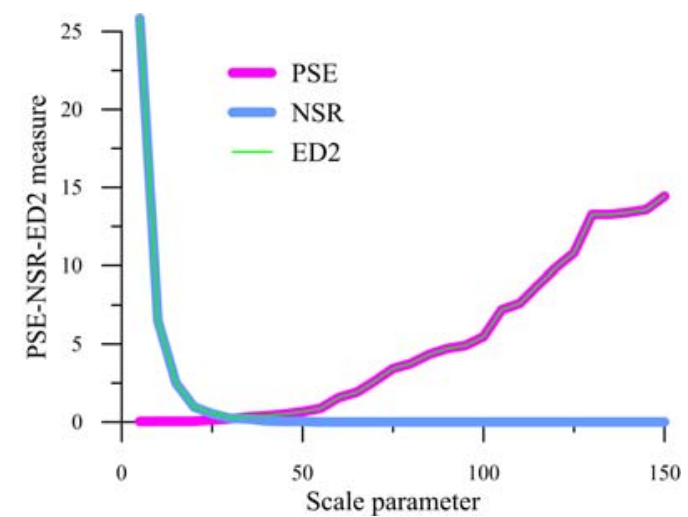

Figure 3. Variations in the PSE-NSR-ED2 along with SP for farmland from WV2 MS. The ED2 curve is shown by the thin green line superimposed on the other two curves (thick pink and blue lines) to indicate the local coincident relations among them.

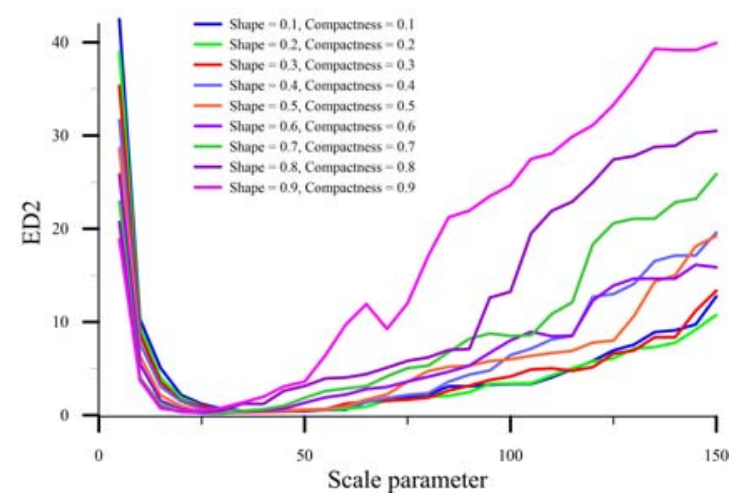

Figure 4. Italic U-shaped ED2-SP pattern obtained by some combinations of shape and compactness parameters.

Figure 4 shows the variations in ED2 along with SP with respect to nine sets of different shape and compactness parameters from the WV2_MS image for farmland. Some common trends for these nine curves are apparent. The ED2 variations along with SP present prominently diverse and is not well preserved as shown the general pattern in Section 2.1. At the left side of this curve, it decreases monotonically along with SP. However, the variations become complex in the right side, especially those associated with higher values of shape and compactness parameters. In some parts, ED2 are unchanged along with SP. When SP is larger than 0.6 , the monotonically increasing curve is reversed and local valleys appear sometimes. These abnormal changes occur when ED2 is larger than 1.0. All these variations are considered in the automatic exploration method, as discussed in Section 2.2.

\section{EXPERIMENT TWO: ITERATIVE OSP EXPLORATION METHOD BASED ON FIVE DOTS ED2-SP PATTERN}

The proposes of the experiment two are to verify the proposed iterative exploration method for OSP selection based on the five dots ED2-SP pattern and to compare the method with the exhaustive method.

\subsection{Construction of preliminary segment data stack with five tiles}

The second experiment was conducted using the same sets of image and reference datasets, as those employed in the first experiment, but with iterative exploration of the OSP with five equal-interval SPs. In this experiment, the preliminary minimal and maximal SPs were set to 10 and 50, respectively, for six images types and three land cover types, except for water bodies with respect to QB_sharpen (110 and 150, respectively), and for water bodies with respect to WV2_sharpen (410 and 450 , respectively). These values can be adjusted dynamically in an iterative routine to locate their position in the italic U-shaped ED2-SP patterns. Correspondingly, the minimal value, maximal value, and increment of both the shape and compactness parameters were set to $0.1,0.9$, and 0.1 , respectively. To recognize the ED2-SP pattern, both the minimum value and increment of SP were set to one, and two ED2s were regarded as equal if the difference between them is small than 0.0001 .

\subsection{Comparison between the exhaustive method and the iterative exploration method}

The results of the iterative exploration method are presented in Table 3. Comparing these results with those of the exhaustive method (shown in Table 2), it is clear that four sets of OSTs are consistent: the scale, shape, and compactness parameters are of $50,0.5$, and 0.7 for water bodies from QB MS; 25, 0.6, and 0.4 for farmland, and 25, 0.6, and 0.5 for rural housing from WV2_MS; and 70, 0.1, and 0.8 for farmland from QB_sharpen. More sets are close to the SP because the SP increment is set to one, which is smaller than that set in the exhaustive method, along with an improved ED2. Notably, the OST selected for water bodies based on WV2_MS and QB sharpen by the iterative exploration method lies outside of the pre-set intervals employed in the exhaustive method particularly for a larger SP (158 and 202, respectively).

The time spent changes from 7.425 hours for rural housing based on WV2_MS to 57.0375 hours for water bodies based on WV2_sharpen. Notably, the time spent does not decrease in all the cases, because the computational SP increment is set to one in order to track the italic U-shaped ED2 pattern. Provided by such a SP increment, the selection would take an extremely 
long time when employing the exhaustive method. In particular, the time spent is extremely long, e.g., for water bodies from WV2_MS, for farmland, rural housing, and water bodies from QB_sharp and WV2_sharpen, and for farmland and rural housing from ALOS_sharpen, because the initial setting of the minimum and maximum SP values exceed the results. Therefore, the initial setting of the SP for automatic exploration is critical for efficiently reducing the time spent.

\begin{tabular}{|c|c|c|c|c|c|}
\hline $\begin{array}{l}\text { Image } \\
\text { source }\end{array}$ & $\begin{array}{l}\text { Land } \\
\text { cover }\end{array}$ & $\begin{array}{l}\text { Number } \\
\text { of tiles }\end{array}$ & $\begin{array}{l}\text { OST (scale, } \\
\text { shape, } \\
\text { compactness) }\end{array}$ & ED2 & $\begin{array}{l}\text { Time } \\
\text { spent } \\
\text { (hours) }\end{array}$ \\
\hline \multirow{3}{*}{ QB_MS } & FL & 1296 & $17,0.1,0.1$ & 0.240 & 9.4500 \\
\hline & RH & 972 & $24,0.7,0.5$ & 0.353 & 8.1000 \\
\hline & WB & 1296 & $50,0.5,0.7$ & 0.023 & 10.8000 \\
\hline \multirow{3}{*}{$\begin{array}{c}\text { WV2 } \\
\text { MS }\end{array}$} & FL & 1053 & $25,0.6,0.4$ & 0.117 & 8.7750 \\
\hline & RH & 891 & $25,0.6,0.5$ & 0.292 & 7.4250 \\
\hline & WB & 2430 & $158,0.3,0.6$ & 0.017 & 20.2500 \\
\hline \multirow{3}{*}{$\begin{array}{c}\mathrm{ALOS}_{-} \\
\mathrm{MS}\end{array}$} & FL & 972 & $27,0.1,0.1$ & 0.327 & 8.1000 \\
\hline & $\mathrm{RH}$ & 1134 & $35,0.4,0.5$ & 0.225 & 9.4500 \\
\hline & WB & 1134 & $28,0.3,0.3$ & 0.207 & 9.4500 \\
\hline \multirow{3}{*}{$\begin{array}{c}\mathrm{QB}_{-} \\
\text {sharpen }\end{array}$} & FL & 1539 & $70,0.1,0.8$ & 0.211 & 41.6813 \\
\hline & RH & 1863 & $91,0.6,0.3$ & 0.308 & 50.4563 \\
\hline & WB & 1296 & $202,0.6,0.7$ & 0.030 & 35.1000 \\
\hline \multirow{3}{*}{$\begin{array}{l}\text { WV2_- } \\
\text { sharpen }\end{array}$} & FL & 1944 & $130,0.2,0.5$ & 0.107 & 52.6500 \\
\hline & $\mathrm{RH}$ & 1782 & $93,0.2,0.1$ & 0.228 & 48.2625 \\
\hline & WB & 2106 & $327,0.7,0.3$ & 0.076 & 57.0375 \\
\hline \multirow{3}{*}{$\begin{array}{l}\text { ALOS } \\
\text { sharpen }\end{array}$} & FL & 1782 & $96,0.1,0.7$ & 0.246 & 48.2625 \\
\hline & $\mathrm{RH}$ & 1863 & $82,0.3,0.7$ & 0.306 & 50.4563 \\
\hline & WB & 1215 & $41,0.3,0.7$ & 0.102 & 32.9063 \\
\hline
\end{tabular}

Table 3. OST selection and time spent for the automatic exploration method.

\section{CONCLUSIONS}

Based on the segment data stacks constructed with the fractal net evolution algorithm (FNEA) for image segmentation, this study verified that the mean area of segments increases approximately in the form of a power function along with SP, and the number of segments decreases approximately in the similar form of a power function. PSE and NSR vary approximately similar to the mean area and the number of segments, respectively, along with SP, with some bias due to differences of images and land cover types. Therefore, we establish an ED2-SP pattern showing the variation of ED2 in the form of the italic U-shaped curve along with SP. Owing to the regular variations of ED2 along with SP, the ED2-SP pattern is possible for recognition of the OSP. Furthermore, provided by the entire OSPs with respect to all the combinations of shape and compactness parameters, the OST is selected by ranking the OSPs.

In this study, we proposed and verified an iterative exploration method for OSP selection based on the italic U-shaped ED2-SP pattern. Given a preliminary range of SP, a segment data stack is constructed with five equal-spacing SPs, the PSE-NSR-ED2 measures are obtained and the five dots ED2-SP pattern with five pairs of ED2 and SP is constructed for the corresponding segment data stack associated with one land cover type. The variations of the five ED2 associated with the five equalspacing SP can be classified into 17 cases. Six of them are the most important. A linear transformation for the dynamical SP range is conducted iteratively until the minimal ED2 at the bottom of the italic U-shaped pattern is found. The bottom of the pattern was persistently approached by the transformation to obtain updated five equal-spacing SP and construction of updated segment data stacks and updated corresponding segment data stacks with five dynamically adjusted tiles.

The OSTs selected using the iterative exploration method are better than the exhaustive method. It is proved to be advantageous that an OSP is allowed for dense spacing SPs. Finally, an effective predefined SP range are advantageous for approaching the optimal SP value in the smallest time interval.

\section{ACKNOWLEDGEMENTS}

This research is supported by the National Science Foundation of China (NSFC) under grant 41271360.

\section{REFERENCES}

Albrecht, F., Lang, S., Hölbling, D., 2008. Spatial Accuracy Assessment of Object Boundaries for Object-Based Image Analysis, The International Archives of the Photogrammetry, Remote Sensing and Spatial Information Sciences, Vol. XXXVIII-4/C7.

Anders, N.S., Seijmonsbergen, A.C., Bouten, W., 2011. Segmentation optimization and stratified object-based analysis for semi-automated geomorphological mapping. Remote Sensing of Environment 115, 2976-2985.

Arvor, D., Durieux, L., Andrés, S., Laporte, M.-A., 2013. Advances in geographic object-based image analysis with ontologies: a review of main contributions and limitations from a remote sensing perspective. ISPRS Journal of Photogrammetry and Remote Sensing 82, 125-137.

Baatz, M., Hoffmann, C., Willhauck, G., 2008. Progressing from object-based to object-oriented image analysis, in: Blaschke, T., Lang, S. (Eds.), Object-based image analysis: spatial concepts for knowledge-driven remote sensing applications. Springer-Verlag, Berling.

Baatz, M., Schäpe, A., 2000. Multiresolution Segmentation: an optimization approach for high quality multi-scale image segmentation, in: Strobl, J., Blaschke, T., Griesebner, G. (Eds.), Angewandte Geographische Informations-Verarbeitung XIII, Wichmann, Heidelberg.

Blaschke, T., 2010. Object based image analysis for remote sensing. ISPRS Journal of Photogrammetry and Remote Sensing 65, 2-16.

Blaschke, T., Hay, G.J., Kelly, M., Lang, S., Hofmann, P., Addink, E., Feitosa, R.Q., van der Meer, F., van der Werff, H., van Coillie, F., 2014. Geographic object-based image analysis towards a new paradigm. ISPRS Journal of Photogrammetry and Remote Sensing 87, 180-191.

Burnett, C., Blaschke, T., 2003. A multi-scale segmentation/object relationship modelling methodology for landscape analysis. Ecological Modelling 168, 233-249.

Castilla, G., Hay, G.J., 2008. Image objects and geographic objects, in: Blaschke, T., Lang, S. (Eds.), Object-based image analysis: spatial concepts for knowledge-driven remote sensing applications. Springer-Verlag, Berling, Heidelberg, pp. 91-110.

Clinton, N., Holt, A., Scarborough, J., Yan, L., Gong, P., 2010. Accuracy Assessment Measures for Object-based Image 
Segmentation Goodness. Photogrammetric Engineering \& Remote Sensing 76, 289-299.

Drăguţ, L., Csillik, O., Eisank, C., Tiede, D., 2014. Automated parameterisation for multi-scale image segmentation on multiple layers. ISPRS Journal of Photogrammetry and Remote Sensing 88, 119-127.

Im, J., Quackenbush, L.J., Li, M., Fang, F., 2014. Optimum scale in object-based image analysis, in: Weng, Q. (Ed.), Scale Issues in Remote Sensing. John Wiley \& Sons, Inc, pp. 197-213.

Lang, S., Albrecht, F., Kienberger, S., Tiede, D., 2010. Object validity for operational tasks in a policy context. Journal of Spatial Science 55, 9-22.

Liu, Y., Bian, L., Meng, Y., Wang, H., Zhang, S., Yang, Y., Shao, X., Wang, B., 2012. Discrepancy measures for selecting optimal combination of parameter values in object-based image analysis. ISPRS Journal of Photogrammetry and Remote Sensing 68, 144-156.

Marpu, P.R., Neubert, M., Herold, H., Niemeyer, I., 2010. Enhanced evaluation of image segmentation results. Journal of Spatial Science 55, 55-68.

Martha, T.R., Kerle, N., van Westen, C.J., Jetten, V., Kumar, K.V., 2011. Segment Optimization and Data-Driven Thresholding for Knowledge-Based Landslide Detection by Object-Based Image Analysis. IEEE Transactions on Geoscience and Remote Sensing 49, 4928-4943.

Meinel, G., Neubert, M., 2004. A comparison of segmentation programs for high resolution remote sensing data, Commission VI on Proceeding to 20th ISPRS Congress, Istanbul.

Ming, D., Li, J., Wang, J., Zhang, M., 2015. Scale parameter selection by spatial statistics for GeOBIA: Using mean-shift based multi-scale segmentation as an example. ISPRS Journal of Photogrammetry and Remote Sensing 106, 28-41.

Myint, S.W., Gober, P., Brazel, A., Grossman-Clarke, S., Weng, Q., 2011. Per-pixel vs. object-based classification of urban land cover extraction using high spatial resolution imagery. Remote Sensing of Rnvironment 115, 1145-1161.

Navulur, K., 2007. Multispectral Image Analysis Using the Object-Oriented Paradigm. CRC Press, Taylor \& Grancis Group, Boca Raton.

Radoux, J., Defourny, P., 2008. Quality assessment of segmentation results devoted to object-based classification, in: Blaschke, T., Lang, S. (Eds.), Object-based image analysis: spatial concepts for knowledge-driven remote sensing applications. Springer-Verlag, Berling, Heidelberg, pp. 91-110.

Rasi, R., Bodart, C., Stibig, H.-J., Eva, H., Beuchle, R., Carboni, S., Simonetti, D., Achard, F., 2011. An automated approach for segmenting and classifying a large sample of multi-date Landsat imagery for pan-tropical forest monitoring. Remote Sensing of Environment 115, 3659-3669.

Schultz, B., Immitzer, M., Formaggio, A.R., Sanches, I.D.A., Luiz, A.J.B., Atzberger, C., 2015. Self-Guided Segmentation and Classification of Multi-Temporal Landsat 8 Images for Crop Type Mapping in Southeastern Brazil. Remote Sensing 7, 14482-14508.
Smith, A., 2010. Image segmentation scale parameter optimization and land cover classification using the Random Forest algorithm. Journal of Spatial Science 55, 69-79.

Tiede, D., Lang, S., Albrecht, F., Hölbling, D., 2010. Objectbased class modeling for cadastre-constrained delineation of geo-objects. Photogrammetric Engineering Remote Sensing 76, 193-202.

Tilton, J.C., Aksoy, S., Tarabalka, Y., 2016. Image segmentation algorithms for land categorization, in: Thenkabail, P.S. (Ed.). Taylor \& FRancis Group, LLC, Boca Raton, London, New York, pp. 317-342.

Trimble, 2014. eCognition Developer 9.0: User's Guide. Trimble Germany GmbH, Arnulfstrasse 126, D-80636 Munich, Germany.

Weidner, U., 2008. Contribution to the assessment of segmentation quality for remote sensing applications, The International Archives of the Photogrammetry, Remote Sensing and Spatial Information Sciences. ISPRS 2008, Beijing, pp. 479-484.

Yang, J., He, Y., Caspersen, J., Jones, T., 2015a. A discrepancy measure for segmentation evaluation from the perspective of object recognition. ISPRS Journal of Photogrammetry and Remote Sensing 101, 186-192.

Yang, J., He, Y., Weng, Q., 2015b. An Automated Method to Parameterize Segmentation Scale by Enhancing Intrasegment Homogeneity and Intersegment Heterogeneity. IEEE Geoscience and Remote Sensing.

Yang, J., Li, P., He, Y., 2014. A multi-band approach to unsupervised scale parameter selection for multi-scale image segmentation. ISPRS Journal of Photogrammetry and Remote Sensing 94, 13-24.

Zhang, X., Feng, X., Xiao, P., He, G., Zhu, L., 2015. Segmentation quality evaluation using region-based precision and recall measures for remote sensing images. ISPRS Journal of Photogrammetry and Remote Sensing 102, 73-84.

Zhang, Y.J., 1996. A survey on evaluation methods for image segmentation. Pattern Recognition 29, 1335-1346. 


\title{
A DEEP LEARNING APPROACH FOR URBAN LAND COVER CLASSIFICATION FROM HIGH-SPATIAL RESOLUTION IMAGERY AND GEOMORPHOMETRIC VARIABLES
}

\author{
I. Lizarazo ${ }^{\mathrm{a}, *}$, S. Ramirez \\ ${ }^{\text {a }}$ Facultad de Ciencias Agrarias, Universidad Nacional de Colombia, Bogota, D.C. - ializarazos@ unal.edu.co \\ ${ }^{\mathrm{b}}$ Facultad de Ingenieria, Universidad Distrital Francisco Jose de Caldas, Bogota, D.C. - sramirez@udistrital.edu.co
}

KEY WORDS: Urban Land Cover, Deep Learning, Geomorphometry, Digital Surface Models, Extreme Gradient Boosting, Random Forests

\begin{abstract}
:
This paper describes a deep learning approach for urban land cover classification in the context of the ISPRS 2D semantic labelling benchmark. A high spatial resolution digital surface model (DSM) and a true ortho-image over the city Potsdam (Germany) was used as input dataset for obtaining six target classes. The proposed approach focuses on augmenting the original input dataset with a combined set of geo-morphometric variables extracted from DSM -including slope/aspect transformation, second derivate of elevation, compound topographic index and hierarchical slope position-. Furthermore, it uses advanced deep learning architecture provided by $\mathrm{H} 2 \mathrm{O}$ framework which follows the model of multi-layer, feedforward neural networks for predictive modelling. Automatic hyperparameter tuning with random search was conducted for model selection. The method comprises five steps: (i) spectral segmentation of ortho-images; (ii) extraction of relevant geo-morphometric variables from DSM; (iii) multivariate land cover classification; and (iv) accuracy assessment. The proposed approach was used for classifying a selected ISPRS benchmark tile where a reference map is available. Thematic accuracy of the proposed approach was assessed using the traditional error matrix and compared with thematic accuracy of a deep learning classification based only on the original data set (i.e. DSM and multispectral imagery). In addition, the deep learning classification approach was compared with a random forest (RF) classification using both original and augmented input dataset. It is shown that: (i) thematic accuracy improves only slightly when geomorphological variables are used to enhance the input dataset; and (ii) deep neural nets provide a similar predictive power than random forests for urban remote sensing applications.
\end{abstract}

\section{INTRODUCTION}

\subsection{Machine Learning}

Machine learning (ML) is an effective empirical approach for supervised or unsupervised land cover classification (supervised or unsupervised). In ML, a comprehensive 'training dataset' of examples is constructed covering as much of the system parameter space as possible. Typically, a random subset of the data is put aside for a completely independent validation. ML is ideal for addressing those problems where our theoretical knowledge is still incomplete but for which we do have a significant number of observations and other data (Lary et al., 2016).

ML algorithms commonly used include artificial neural networks (ANN), support vector machines (SVM), self-organizing map (SOM), decision trees (DT), ensemble methods such as random forests (RF), case-based reasoning, neuro-fuzzy (NF), genetic algorithm (GA), multivariate adaptive regression splines (MARS). Application of these techniques in geosciences and remote sensing is relatively new but expanding (e.g. Atkinson and Tatnall, 1997; Brown et al., 2008; Lizarazo, 2008; Atzberger and Rembold, 2013; Hu et al., 2015; Langkvist et al., 2015; Liang and Li, 2016).

\subsection{Deep Learning}

Deep Learning (DL) techniques use neural networks with many hidden layers. Two options for applying DL are Deep Neural Networks (DNN) and Deep Belief Networks (DBN). A DBN has undirected connections between the top two layers (Hinton et al., 2012). This means that the topology of the DNN and DBN is

\footnotetext{
${ }^{*}$ Corresponding author
}

different by definition. The undirected layers in a DBN are called Restricted Boltzmann Machines. This layers can be trained using an unsupervised learning algorithm (i.e., contrastive divergence) that is very fast.

In contrast to DBNs, a DNN is a feed-forward, artificial neural network that has more than one layer of hidden units between its inputs and its outputs - and there are no undirected connections (Hinton et al., 2012). Each hidden unit, $j$, typically uses the logistic function (the closely related hyberbolic tangent is also often used and any function with a well-behaved derivative can be used) to map its total input from the layer below, $x_{j}$, to the scalar state, $y_{j}$ that it sends to the layer above (Hinton et al., 2012).

$$
y_{j}=\operatorname{logistic}\left(x_{j}\right)=\frac{1}{1+e^{-x_{j}}} ; x_{j}=b_{j}+\sum_{i=1} y_{i} w_{i j},
$$

where $b_{j}$ is bias of unit $\mathrm{j}, i$ is index over units in the layer below, and $w_{i j}$ is weight on a connection to unit $j$ from unit $i$ in the layer below.

For multiclass classification, output unit $j$ converts its total input, $x_{j}$, into a class probability, $p_{j}$, by using the 'softmax ' nonlinearity

$$
p_{j}=\frac{\exp \left(x_{j}\right)}{\sum \exp \left(x_{k}\right)},
$$

where $k$ is an index over all classes (Hinton et al., 2012).

A DNN can be discriminatively trained (DT) by back-propagating derivatives of a cost function that measures the discrepancy between the target outputs and the actual outputs produced for each training case. When using the soft-max output function, the nat- 
ural cost function $C$ is the cross entropy between the target probabilities $d$ and the outputs of the softmax, $p$ :

$$
C=-\sum_{j=1} d_{j} \log p_{j}
$$

where the target probabilities, typically taking values of one or zero, are the supervised information provided to train the DNN classifier (Hinton et al., 2012).

For large training sets, it is typically more efficient to compute the derivatives on a small, random 'minibatch' of training cases, rather than the whole training set, before updating the weights in proportion to the gradient. This stochastic gradient descent method can be further improved by using a 'momentum' coefficient, $0<\alpha<1$, that smooths the gradient computed for minibatch $t$, thereby damping oscillations across ravines and speeding progress down ravines:

$$
\Delta w_{i j}(t)=-\alpha \Delta w_{i j}(t-1)-\epsilon \frac{\partial C}{w_{i j}(t)} .
$$

The update rule for biases can be derived by treating them as weights on connections coming from units that always have a state of one (Hinton et al., 2012).

\subsection{Tree based Learning}

Tree based learning algorithms are considered to be one of the best and mostly used supervised learning methods. Tree based methods empower predictive models with high accuracy, stability and ease of interpretation. Unlike linear models, they map nonlinear relationships quite well. They are adaptable at solving any kind of problem at hand (classification or regression) (Breiman, 2001).

Methods like decision trees, random forest, gradient boosting are being popularly used in remote sensing problems (Belgiu and Dragut, 2016). Main advantages of such methods can be summarized as follows (Breiman, 2001): (i) Decision Trees (DTs) captures non-linear relationship and are robust to correlated features, feature distribution, and missing values. However, its performance is usually not very top-tiered. So there are two different categories of solutions for this problem, one is through bagging (RandomForest) and the second is through boosting (Gradient Boosting Machine); Random Forests (RF) are composed of many trees. Each tree is built on a sample of the features and on a sample of the observations (to increase variance of trees). Trees are independent with one another. It is easy to use since it has very few hyper-parameters to tune. And it runs pretty well with the default parameters. A main drawback is that it may be slow when it comes to scoring; and (iii) Gradient Boosting Machine (GBM) are also based on many trees. A GBM iteratively learns weak classifiers and adds them to a final strong classifier. After a weak learner is added, the data is re-weighted: examples that are misclassified gain weight and examples that are classified correctly lose weight. Thus, future weak learners focus more on the examples that previous weak learners misclassified. Trees are dependent with one another causing it to be not very robust to noisy data and outliers.

Results from several land cover classification studies (e.g. Pal, 2005; and Lizarazo, 2010) suggest that the RF classifier performs equally well to SVMs and other ML techniques in terms of classification accuracy and training time. Such studies also conclude that the number of user defined parameters required by RF classifiers is less than the number required for SVMs and easier to define. On the another hand, only a few number of studies have applied either DNN or DBN techniques for land cover classification (an exception being Lv et al, 2015 and Langkvist et al, 2016). Most studies focuses on 'scene classification', that is, giving each image an unique label according to a set of semantic categories (e..g. Castelluccio et al., 2015). Experimental results show that DNN and DBN based methods outperform other classification approaches and produce homogenous mapping results with preserved shape details.

\subsection{Objectives}

This article aims to establish whether urban land cover classification obtained from the ISPRS 2D semantic labelling benchmark (i.e a combination of a true color \& infrared orthophoto (TO) plus a digital surface (DSM)) using a DNN approach is thematically more accurate than the one obtained using a RF technique. In addition, potential contribution of terrain-related variables, derived from DSM, to improve thematic accuracy of land cover classification is evaluated.

\section{DATA AND METHODS}

\subsection{Data}

2.1.1 Original data set Performance of the proposed deep learning classification approach was evaluated using the ISPRS 2D semantic labeling benchmark. A high spatial resolution digital surface models (DSM) and a true ortho-image over the city Potsdam (Germany), namely the $6-11$ tile, were used as input dataset for obtaining six target classes. The ortho-image comprises 3 spectral bands in the visible range and 1 in the nearinfrared. Each band is $6000 \times 6000$ pixels with a spatial resolution of $0.05 \mathrm{~m}$. The DSM has similar spatial extent and pixel size. Figure 1 shows: (a) a true colour composition from orthophoto; (b) a pseudo color representation of DSM.

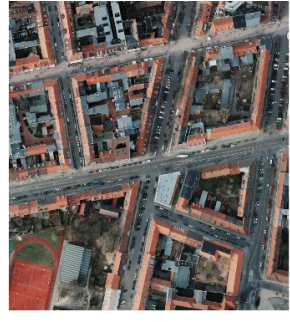

(a)

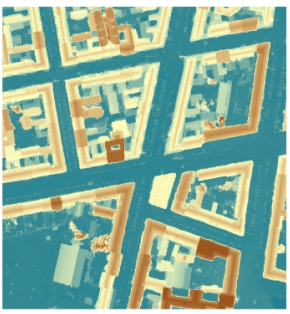

(b)
Figure 1. Input data set for this classification study: (a) Orthophoto's true colour composition; (b) DSM pseudo-color representation.

A Postdam reference map is available for the $6-11$ tile. It includes six different thematic classes:

1. Tree (green)

2. Low vegetation (cyan)

3. Clutter/background (red)

4. Building (blue)

5. Impervious surfaces (white)

6. Car (yellow) 
2.1.2 Training and validation data sets Two stratified random samples of approx. equal-sized number of points at each thematic class were selected from the reference map. For the training step, about 10000 sampling points were drawn (see Table 1). For validation of classification results, about 30000 samples were selected (see Table 1). At each sampling point a $3 \times 3$ window was considered for both establishing the response value (i.e. majority thematic class) and input data set values (i.e. mean of spectral and elevation bands).

\begin{tabular}{|c|c|c|}
\hline Class & Training & Testing \\
\hline 1 & 1763 & 3778 \\
2 & 1612 & 3639 \\
3 & 1654 & 3659 \\
4 & 1913 & 3912 \\
5 & 1791 & 3798 \\
6 & 1272 & 3302 \\
\hline
\end{tabular}

Table 1. Number of training and testing points at each thematic category.

2.1.3 Augmented data set The original input data set was augmented using a number of geo-morphometric variables extracted from DSM. Such variables include slope/aspect transformation (SAT), second derivate of elevation (CURV), compound topographic index (CTI), heat load index (HLI), and hierarchical slope position (HSI). Figure 2 depicts these terrain variables in a small window of $6-11$ tile.

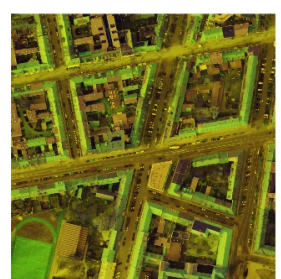

(a)

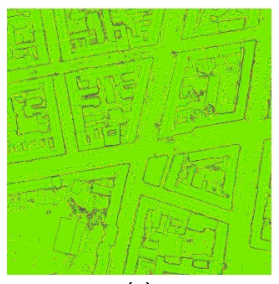

(c)

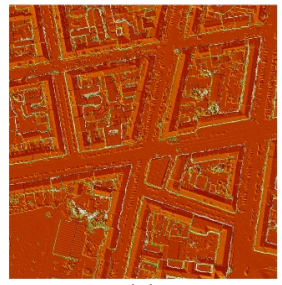

(e)

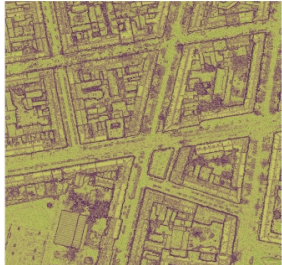

(b)

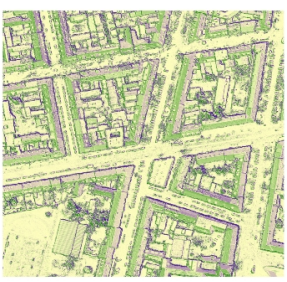

(d)

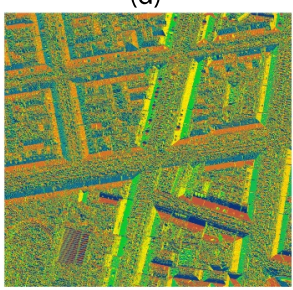

(f)
Figure 2. Geo-morphometric variables obtained from DSM: (a) RGB123 ortophoto colour composition ; (b) slope/aspect transformation (SAT), (c) second derivate of elevation (CURV), (d) compound topographic index (CTI), (e) heat load index (HLI), and (f) hierarchical slope position (HSI).

Slope/aspect transfomation was calculated using a simple ratio (Wilson, 2012):

$$
S A T=\frac{\text { slope }}{\text { aspect }}
$$

Curvature is second derivative of elevation and was calculated according to Shary (1995):

$$
C U R V=\frac{d^{2}(d z)}{d h^{2}}
$$

CTI is a steady state wetness index. CTI is a function of both the local slope $(\tan (B)$ in radians) and the upstream contributing area per unit width orthogonal to the flow direction $\left(A s\right.$ in $\left.m_{2}\right)$ (Hjerdt et al., 2004)

$$
C T I=\ln \frac{A_{s}}{\tan (B)},
$$

HLI is an aspect and slope based terrain metric. McCune and Keon (2002) 'fold' aspect and steepness so that 'heat' highest values are southwest and lowest values are northeast

$$
H L I=\frac{\text { aspect }- \text { slope }}{\text { aspect }+ \text { slope }},
$$

HSI is an index which captures terrain attributes in a hierarchical way by averaging slope over a number of windows at different scales (Florinsky, 2012):

$$
H S I=\frac{\sum_{j=1} d_{j} \text { slope }_{j}}{n}
$$

\subsection{Methods}

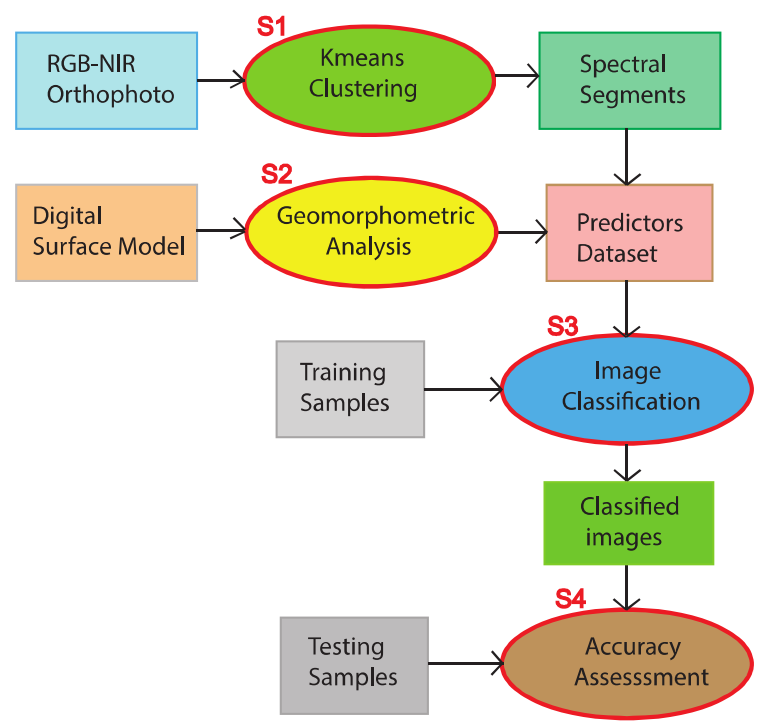

Figure 3. Flowchart of land cover mapping stages in this study.

Figure 3 depicts the four-stage method followed in this study: (S1) Image Segmentation; (S2) Terrain analysis; (S3) Supervised image classification; and (S4) Accuracy assessment. In the S1 stage, the Kmeans algorithm was applied to produce spectral segmentation into 48 clusters. In the $\mathrm{S} 2$ stage, five (5) geo-morphometric variables (i.e. SAT, CURV2, CTI, HLI and HSI) were obtained from the DSM. In the S3 stage, DNN and RF classification models were built and applied using as predictors features extracted from either the original input data set or the augmented data set. Original features include mean of Red (R), Green (G), Blue (B) 
and Infra-Red (IR) bands within spectral segments as well as pixel-based DSM values. Augmented features include original features plus pixel-based geo-morphometric values. After class prediction, a $13 \times 13$ majority filter was applied to smooth classified images. In the S4 stage, accuracy assessment of obtained thematic images was conducted using the traditional error matrix.

The $R$ system (R Core Team, 2015), a free software environment for statistical computing and graphics, was used to implement every stage of the proposed method. Raster and vector data sets reading, processing and analysis tasks were conducted using rgdal, sp, raster and rasterVis libraries. DNN and RF model building and prediction was accomplished using $h 2 o$ library. Tuning of DNN hyper-parameters was accomplished using a random search technique. Optimal values obtained include a deep neural net with five (5) hidden layers using 500, 1000, 2000, 1000 and 500 nodes per respective layer. RF application was conducted with no tuning technique, that is, using 500 trees, the default setting.

\section{RESULTS}

Figure 4 shows classification results for different methods corresponding to a small window near to the lower left zone of $6-11$ tile. This zoom in allows for a better visualization of results. However, interested readers may access the complete classified images at https://goo.gl/KogDXO. As reported in Tables 2, 3, 4, 5 the DNN approach using the augmented input data set obtained better results. Accuracy metrics show that most elusive categories to map are Tree (class 1) and Low vegetation (class 2) and that most reachable ones are Clutter / background (class 3) and Building (class 4).

A close look at Figure 4 and Tables 6, 7, 8, and 9 shows that DNN and RF techniques obtained similar results from both the original input dataset and the augmented input dataset. Results also show that inclusion of geo-morphometric variables in the input data set improves thematic accuracy only slightly.

Accuracy assessment was based on the computation of pixelbased error matrices per classification using the testing sample data set described in Section 2.1.2 and the reference data set with no boundary (i.e. testing points eventually falling on a boundary were ignored). From those matrices different measures are derived: per class we compute completeness (recall), correctness (precision) and $F 1$ score, and through the normalisation of the trace from error matrices overall accuracy $(\mathrm{OA})$ values were derived.

\begin{tabular}{|c|c|c|c|c|c|c|}
\hline Class & 1 & 2 & 3 & 4 & 5 & 6 \\
\hline 1 & $\mathbf{3 0 4 3}$ & 273 & 20 & 18 & 117 & 55 \\
2 & 475 & $\mathbf{2 7 1 2}$ & 7 & 25 & 96 & 18 \\
3 & 138 & 70 & $\mathbf{2 8 0 3}$ & 54 & 177 & 90 \\
4 & 40 & 39 & 12 & $\mathbf{3 7 2 1}$ & 32 & 15 \\
5 & 136 & 186 & 33 & 85 & $\mathbf{3 1 8 8}$ & 133 \\
6 & 18 & 5 & 44 & 0 & 57 & $\mathbf{2 4 3 5}$ \\
\hline
\end{tabular}

Table 2. Error matrix for DNN classification from original data set. Overall accuracy $(\mathrm{OA})$ is 0.878

In confusion matrices row direction gives reference values, while column direction shows the prediction. In precision tables cells show normalised values with respect to reference values. The True Positive $t_{p}$ pixels are derived from the main diagonal elements, while the False Positive $f_{p}$ is computed from the sum per column, excluding the main diagonal element. Likewise, the False Negative $f_{n}$ is the sum along the row, excluding the main

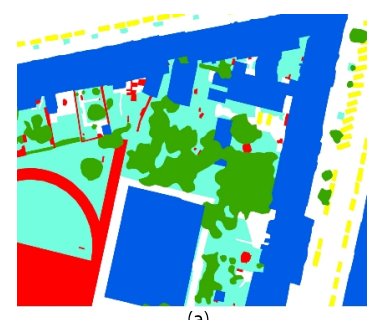

(a)
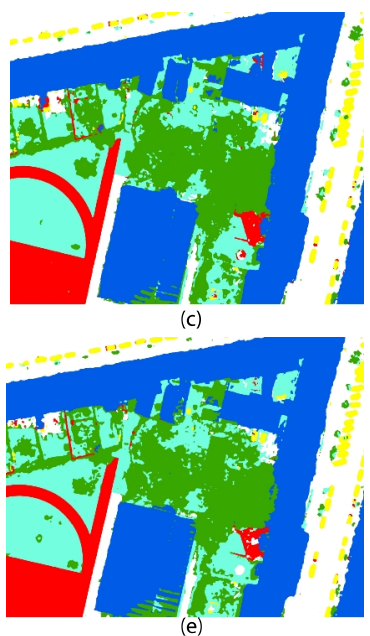

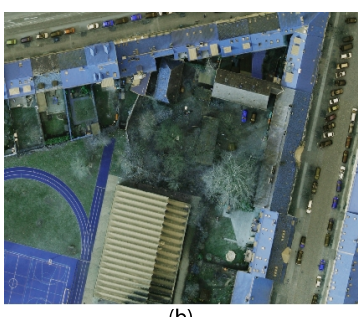

(b)

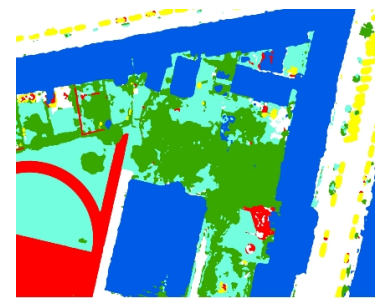

(d)

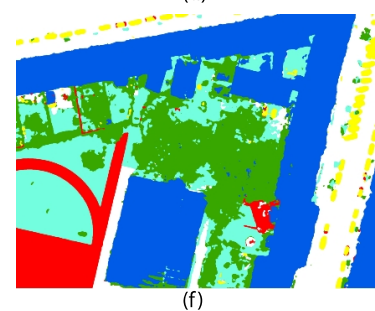

(f)
Figure 4. Classification results for a spatial subset of 611 tile of Potsdam data set: (a) reference; (b) orthophoto; (c) result for DNN on original data set; (d) result for DNN on augmented data set; (e) result for RF on original data set; (f) result for RF on augmented data set.

\begin{tabular}{|c|c|c|c|c|c|c|}
\hline Class & 1 & 2 & 3 & 4 & 5 & 6 \\
\hline 1 & $\mathbf{3 0 3 8}$ & 274 & 15 & 29 & 119 & 50 \\
2 & 448 & $\mathbf{2 7 3 5}$ & 9 & 30 & 92 & 19 \\
3 & 119 & 74 & $\mathbf{2 7 8 2}$ & 50 & 208 & 96 \\
4 & 34 & 29 & 10 & $\mathbf{3 7 2 6}$ & 42 & 18 \\
5 & 121 & 192 & 31 & 64 & $\mathbf{3 2 2 4}$ & 130 \\
6 & 16 & 7 & 50 & 1 & 57 & $\mathbf{2 4 2 9}$ \\
\hline
\end{tabular}

Table 3. Error matrix for DNN classification from augmented data set. Overall accuracy (OA) is 0.880 .

\begin{tabular}{|c|c|c|c|c|c|c|}
\hline Class & 1 & 2 & 3 & 4 & 5 & 6 \\
\hline 1 & $\mathbf{2 9 7 2}$ & 317 & 19 & 42 & 125 & 53 \\
2 & 635 & $\mathbf{2 5 6 3}$ & 1 & 46 & 74 & 13 \\
3 & 123 & 78 & $\mathbf{2 8 1 4}$ & 68 & 164 & 86 \\
4 & 67 & 41 & 10 & $\mathbf{3 7 0 7}$ & 25 & 8 \\
5 & 173 & 149 & 25 & 143 & $\mathbf{3 1 7 5}$ & 98 \\
6 & 11 & 13 & 28 & 2 & 76 & $\mathbf{2 4 2 8}$ \\
\hline
\end{tabular}

Table 4. Error matrix for RF classification from original data set. Overall accuracy $(\mathrm{OA})$ is 0.866 .

diagonal element. From those values following measures are derived:

$$
\text { Precision }=\frac{t_{p}}{t_{p}+f_{p}}
$$




\begin{tabular}{|c|c|c|c|c|c|c|}
\hline Class & 1 & 2 & 3 & 4 & 5 & 6 \\
\hline 1 & $\mathbf{2 9 9 3}$ & 262 & 13 & 34 & 165 & 54 \\
2 & 539 & $\mathbf{2 6 6 2}$ & 3 & 26 & 89 & 12 \\
3 & 155 & 82 & $\mathbf{2 7 8 6}$ & 54 & 181 & 74 \\
4 & 43 & 40 & 9 & $\mathbf{3 7 2 7}$ & 30 & 10 \\
5 & 162 & 199 & 28 & 98 & $\mathbf{3 1 6 4}$ & 115 \\
6 & 19 & 10 & 41 & 0 & 69 & $\mathbf{2 4 1 8}$ \\
\hline
\end{tabular}

Table 5. Error matrix for RF classification from augmented data set. Overall accuracy $(\mathrm{OA})$ is 0.872 .

$$
\text { Recall }=\frac{t_{p}}{t_{p}+f_{n}}
$$

The F1-score is defined as the harmonic mean of precision and recall:

$$
F_{1}=\frac{\text { Precision.Recall }}{\text { Precision }+ \text { Recall }}
$$

\begin{tabular}{|c|c|c|c|}
\hline Class & Precision & Recall & F1 \\
\hline 1 & 0.790 & 0.863 & 0.825 \\
2 & 0.825 & 0.814 & 0.819 \\
3 & 0.960 & 0.841 & 0.897 \\
4 & 0.953 & 0.964 & 0.959 \\
5 & 0.869 & 0.848 & 0.858 \\
6 & 0.887 & 0.951 & 0.918 \\
\hline
\end{tabular}

Table 6. Precision, Recall and F1 statistics for DNN classification from original data set $\left(\mathrm{DNN}_{o r}\right)$.

\begin{tabular}{|c|c|c|c|}
\hline Class & Precision & Recall & F1 \\
\hline 1 & 0.804 & 0.862 & 0.832 \\
2 & 0.826 & 0.820 & 0.823 \\
3 & 0.960 & 0.836 & 0.894 \\
4 & 0.955 & 0.965 & 0.960 \\
5 & 0.861 & 0.857 & 0.859 \\
6 & 0.886 & 0.949 & 0.916 \\
\hline
\end{tabular}

Table 7. Precision, Recall and F1 statistics for DNN classification from augmented data set $\left(\mathrm{DNN}_{a u}\right)$.

\begin{tabular}{|c|c|c|c|}
\hline Class & Precision & Recall & F1 \\
\hline 1 & 0.746 & 0.842 & 0.791 \\
2 & 0.811 & 0.769 & 0.789 \\
3 & 0.971 & 0.844 & 0.903 \\
4 & 0.924 & 0.961 & 0.942 \\
5 & 0.872 & 0.844 & 0.858 \\
6 & 0.904 & 0.949 & 0.926 \\
\hline
\end{tabular}

Table 8. Precision, Recall and F1 statistics for RF classification from original data set $\left(\mathrm{RF}_{o r}\right)$.

\begin{tabular}{|c|c|c|c|}
\hline Class & Precision & Recall & F1 \\
\hline 1 & 0.765 & 0.850 & 0.805 \\
2 & 0.818 & 0.799 & 0.808 \\
3 & 0.967 & 0.836 & 0.897 \\
4 & 0.946 & 0.966 & 0.956 \\
5 & 0.855 & 0.840 & 0.848 \\
6 & 0.901 & 0.946 & 0.923 \\
\hline
\end{tabular}

Table 9. Precision, Recall and F1 statistics for RF classification from augmented data set $\left(\mathrm{RF}_{a u}\right)$.

\section{DISCUSSION}

It is worth to have a closer look at classifications obtained in this study in order to understand why Tree (green) and Low vegetation (cyan) categories have the lowest accuracies. Figure 5 shows a small window near top right corner of $6-11$ tile. It shows: (a) reference map; (b) DNN classification from augmented input data set; (c) ortho-photo; (d) DSM. From the ortho-image, it can be seen that trees have dropped most of their leaves and confound easily with grass and low vegetation. In fact, by looking through tree branches it is possible to identify ground objects. Figure 5 also shows that cars (yellow) are well mapped and that small buildings (red) are poorly mapped.
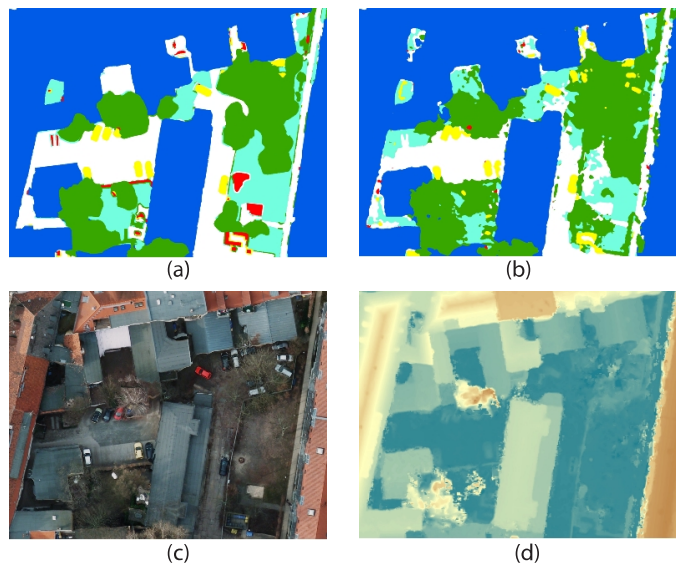

Figure 5. A closer look at the DNN land cover classification from the augmented data set: (a) reference map; (b) DNN classification from augmented input data set; (c) orthophoto; (d) DSM

\begin{tabular}{|c|c|c|c|c|}
\hline Method & OA & mPrecision & mRecall & mF1 \\
\hline DNN $_{o r}$ & 0.878 & 0.881 & 0.880 & 0.8794 \\
$\mathrm{DNN}_{a u}$ & 0.880 & 0.882 & 0.881 & 0.881 \\
$\mathrm{RF}_{o r}$ & 0.866 & 0.872 & 0.868 & 0.868 \\
$\mathrm{RF}_{a u}$ & 0.872 & 0.875 & 0.873 & 0.873 \\
\hline
\end{tabular}

Table 10. Overall Accuracy (OA), Precision and Recall macro statistics for all classified images

Table 10 summarizes thematic accuracy of results. From matrices presented in Section 3, it may be stated that, for practical applications, any of the DNN and RF techniques evaluated here allows for accurate classifications. However, a relevant discussion topic is to examine how expensive is each method regarding its use of computational resources and human effort. On such a matter, it should be said that this study confirms that DNN needs careful tuning of hyper-parameters as suggested from previous work (e.g. Hinton et al., 2012). Moreover, DNN seems to be a very unstable technique as minor variations in, for example, number of hidden layers or number of nodes, causes unexpected changes in model's behaviour and performance. While this happens to DNN, RF needs no significant work on parameterisation and works in a very stable way.

On the another hand, inclusion of geo-morphometric variables in the input data set did not improve accuracy of results significantly. This is confirmed by examination of variable importance ranking reported in Table 11. It should noted that elevation from DSM and Mean of IR band are the most important variables in both DNN and RF models. Geo-morphometric variables are the less important ones while their ranking is different in each 


\begin{tabular}{|c|c|c|}
\hline Variable & DNN $_{a u}$ & RF $_{a u}$ \\
\hline DSM & 1 & 1 \\
Mean of IR band & 2 & 2 \\
Mean of G band & 3 & 4 \\
Mean of R band & 4 & 3 \\
CURV & 5 & 8 \\
HSI & 6 & 5 \\
Mean of B band & 7 & 6 \\
CTI & 8 & 10 \\
HLI & 9 & 7 \\
SAT & 10 & 9 \\
\hline
\end{tabular}

Table 11. Overall Accuracy (OA), Precision and Recall macro statistics for all classified images

method. It should also noted that DSM is a pixel-wise variable -and this holds for every geo-morphometric variable in this study.

\section{CONCLUSIONS}

In this article, a new classification method that uses deep learning techniques for land cover classification from ortho-images and digital surface models (DSM) was investigated. The classification results indicate that deep neural networks (DNN) and random forest (RF) techniques can accurately classify target thematic categories. Furthermore, it is shown that neither DN nor RF outperforms each other method results in terms of thematic accuracy. It was also investigated the influence of geo-morphometric variables, obtained from DSM, on classification. It is shown that such variables increase thematic accuracy only slightly. Machine learning techniques examined in this study give very promising results, particularly the RF algorithm which is very robust, consistent and efficient. Future work will be focused on investigating causes of unstable performance of the deep learning implementation used in this study.

\section{REFERENCES}

Atkinson P. M. and Tatnall, A. R. L., 1997. Introduction: neural networks in remote sensing. International Journal of Remote Sensing, 18 (4), pp. 699-709.

Atzberger, C. and Rembold, F., 2013. Mapping the Spatial Distribution of Winter Crops at Sub-Pixel Level Using AVHRR NDVI Time Series and Neural Nets. Remote Sensing, 5(3), pp. 13351354.

Belgiu, M. and Dragut, L. 2016. Random forest in remote sensing: A review of applications and future directions ISPRS Journal of Photogrammetry and Remote Sensing, 114 (4), pp.24-31

Breiman, L., 2001. Random Forests. Machine Learning, 45(1), pp. 5-32.

Brown, M. E., Lary, D. J., Vrieling, A., Stathakis, D. and Mussa H., 2008. Neural networks as a tool for constructing continuous NDVI time series from AVHRR and MODIS. International Journal of Remote Sensing, 29 (24), pp. 7141-7158.

Castelluccio, M, Poggi, G., Sansone C. and Verdoliva, L., 2015. Land Use Classification in Remote Sensing Images by Convolutional Neural Networks. arXiv preprint, arXiv:1508.00092.

Florinsky I. 2012. Digital Terrain Analysis in Soil Science and Geology. Elsevier Academic Press: Oxford, UK.
Hinton, G., Deng, L., Yu, D., Dahl, G. E., Mohamed, A., Jaitly, N., Senior, A., Vanhoucke, V., Nguyen, P., Sainath, T. N. and Kingsbury, B., 2012. Deep Neural Networks for Acoustic Modeling in Speech Recognition. IEEE Signal Processing Magazine, 29(6), pp. 82-97.

Hjerdt, K. N.,McDonnell, J. J.,Seibert, J. and Rodhe, A., 2004. A new topographic index to quantify downslope controls on local drainage. Water Resources Research, 40(5), CiteID W05602.

Hu, F., Xia, G-S., Hu, J., and Zhang, L., 2015. Transferring Deep Convolutional Neural Networks for the Scene Classification of High-Resolution Remote Sensing Imagery. Remote Sensing, 7(11), pp. 14680-14707.

Langkvist, M., Kiselev, A., Alirezaie, M. and Loutfi, A., 2016. Classification and Segmentation of Satellite Orthoimagery Using Convolutional Neural Networks. Remote Sensing, 8(4), pp. 329350 .

Lary, D. J., Alavi, A. H., Gandomi, A. H., Walker, A. L., 2016. Machine learning in geosciences and remote sensing. Geoscience Frontiers,7(1), pp. 3-10.

Liang, H. and Li, Q., 2016. Hyperspectral Imagery Classification Using Sparse Representations of Convolutional Neural Network Features. Remote Sensing, 8(2), pp. 99-118.

Lizarazo, I., 2008. SVM based segmentation and classification of remotely sensed data. International Journal of Remote Sensing, 29 (24), pp. 7277-7283

Lizarazo, I., 2010. Fuzzy image regions for estimation of impervious surface areas. Remote Sensing Letters, 1(1), pp. 19-27.

Lv, Q., Dou, Y., Niu, X., Xu, J., Xu, J. and Xia, F., 2015. Urban Land Use and Land Cover Classification Using Remotely Sensed SAR Data through Deep Belief Networks. Journal of Sensors, 2015, 10 pages.

McCune, B. and Keon, D., 2002. Equations for potential annual direct incident radiation and heat load index. Journal of Vegetation Science, 13, pp. 603-606.

Pal, M., 2005. Random forest classifier for remote sensing classification. International Journal of Remote Sensing, 26 (1), pp. 217-222.

R Core Team, 2015. R: A Language and Environment for Statistical Computing. R Foundation for Statistical Computing, Viena, Austria.

Shary, P.A., 1991. The second derivative topographic method. In: Stepanov I. (Ed.) The Geometry of the Earth Surface Structures Pushchino Research Centre Press: Pushchino, USSR.

Wilson J. P. , 2012. Digital terrain modeling. Geomorphology, 137, pp. 107-121.

\section{ACKNOWLEDGEMENTS}

Authors thank ISPRS Working group III/4 for organizing benchmark on urban object detection. Acknowledgements also go for BSF Swissphoto for providing ISPRS with the Postdam input data set.

Article submitted on 10 July 2016 


\title{
OBJECT-BASED SYMMETRIC DIFFERENCE FOR LAND SURFACE SEGMENTATION SCALE PARAMETER OPTIMISATION
}

\author{
G. J. Louw ${ }^{\text {a }}$, A. Van Niekerk ${ }^{\mathrm{a}, \mathrm{c}}$, A. Rozanov ${ }^{\mathrm{b}}$ \\ ${ }^{\text {a }}$ Dept. of Geography and Environmental Science, Stellenbosch University - gerritlouw @ gmail.com, avn@sun.ac.za \\ ${ }^{\mathrm{b}}$ Dept. of Soil Science, Stellenbosch University - dar@sun.ac.za \\ ${ }^{c}$ School of Plant Biology, University of Western Australia, 35 Stirling Hwy, Crawley WA 6009, Perth, Australia
}

\begin{abstract}
KEY WORDS: GEOBIA, Geomorphometry, Land surface segmentation, Scale parameter optimisation
\end{abstract}

\begin{abstract}
Although multi-resolution segmentation (MRS) has been shown to delimit geomorphological boundaries more accurately than other available segmentation procedures, the selection of the appropriate parameters remains a challenge. The optimisation of scale has largely been focussed on deriving suitable scale parameters (SPs). Local variance (LV) has been shown to exploit spatial autocorrelation for identifying suitable SPs. However, further investigation into SP selection for land surface segmentation is necessary. This paper demonstrates how symmetric difference (SD) can be used to detect suitable SPs when segmenting continuous land surfaces. Comparisons with an established suitable SP selection technique (ESP 2) are made and, finally, it is concluded that SD provides a simple and effective way of selecting suitable SPs for land surface segmentation.
\end{abstract}

\section{INTRODUCTION}

The creation of fine scale geomorphological maps often involves the segmentation of continuous land surfaces into landform elements or landform components (Drăguţ and Eisank, 2011). Even though the pixel-based delimitation of landform components are well established (Evans, 2012), the segmentation of continuous terrain through object-orientated segmentation has received much attention in recently published literature (Drăguţ and Blaschke, 2008; Eisank et al., 2014, 2011; Van Niekerk, 2010). This can, at least partly, be ascribed to the presence of the salt-and-pepper effect in pixel-based approaches, which compromises landform component homogeneity (Blaschke, 2010; Gao et al., 2011).

To evaluate which segmentation algorithm consistently generates morphologically representative landform components, Van Niekerk (2010) evaluated three object-based segmentation algorithms - automated land component mapper (ALCoM), iterative self-organizing data analysis technique algorithm (ISODATA) and multi-resolution image segmentation (MRS) of which the MRS algorithm, implemented in eCognition (Trimble), performed the best.

MRS is a region-merging algorithm that creates homogenous objects based on a user defined scale parameter (SP) (Baatz and Schäpe, 2000). As the SP is primarily used to control the average size of the objects, it is the chief parameter through which the analyst can manipulate the output of MRS when segmenting continuous land surfaces (Drăguţ et al., 2011).

Drăguţ et al (2009) investigated the use of local variance (LV) to evaluate the relationship between object homogeneity and SP. This ultimately led to the development of the estimation of scale parameter (ESP) tool in eCognition (Drăguţ et al., 2010). The tool was later modified to allow for multi-layered approaches to SP optimisation, resulting in ESP 2 (Drăguţ et al., 2014).

LV-based SP selection is fundamentally based on the consideration of the indirect relationship between SP and object topology. As the SP is systematically increased, the object size will increase, and consequently object boundaries are affected. This, in turn, defines the interior object homogeneity and thus LV.

While LV-based SP selection has been shown to aid the delimitation of geomorphological features (Drăguţ et al., 2011), there is still a need for a topological investigation of the relationship between SP and object boundaries.

Accordingly, this study aims to evaluate the use of SD as a method for identifying suitable SPs in multi-resolution land surface segmentation. The authors demonstrate how SD can be used to identify suitable SPs using slope gradient as input to MRS, and conclusions are drawn within the context of finding a simple and effective technique for identifying SPs for objectbased geomorphometry applications.

\section{MATERIALS AND METHODS}

\subsection{Study Area}

A $420 \mathrm{~km}^{2}$ study area was chosen within the Letaba water catchment area (Limpopo province, South Africa), located in the Lowveld geomorphic province (Figure 1). Dominant tertiary sediments (manifested as river terraces) and planation surfaces occur throughout, whilst intermittently interrupted by inselbergs (Venter and Bristow, 1986).

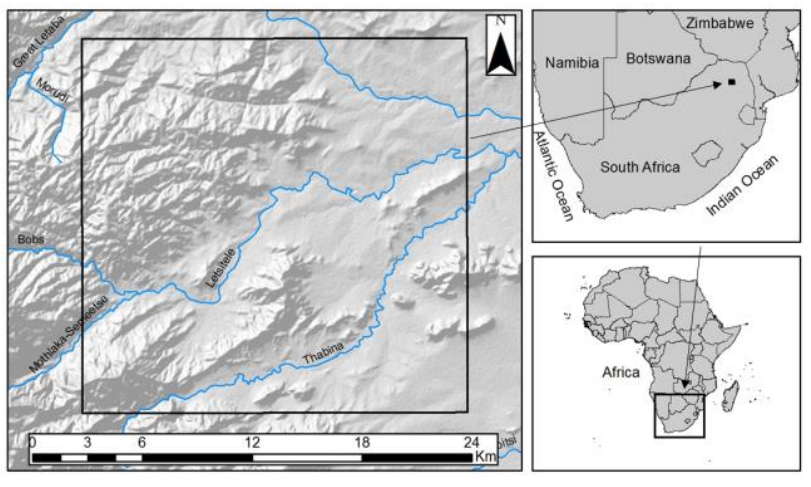

Figure 1 Map of the study area located in Limpopo, South Africa 


\subsection{DEM}

Fine scale land surface segmentation requires high resolution digital elevation models (DEMs). For this reason, level 2 of the $5 \mathrm{~m}$ Stellenbosch University DEM (SUDEM) was used. To construct the SUDEM, a combination of interpolators was used to create a continuous surface of elevation from both large scale contour and spot data. Finally, areas with low density contour and spot heights were supplemented with the $30 \mathrm{~m}$ void-filled SRTM (Van Niekerk, 2015).

\subsection{LSPs}

Since a multi-level segmentation is not necessary for illustrating SD-based SP selection, and in favour of simplicity, the only LSP chosen for the study was slope gradient. Using ArcGIS, a 10.3 slope gradient was calculated with a $3 \times 3$ kernel and the maximum averaging technique (ESRI, 2016).

\subsection{MRS Parameters}

MRS has three parameters to optimise for accurate land surface segmentation: shape, compactness and SP. First, shape and compactness were both set to zero as this permits the segmentation to only consider colour when merging pixels. SP, on the other hand, was iterated for every value between one and 100 .

\subsection{SP Optimisation Techniques}

\subsubsection{ESP 2}

ESP 2, an unsupervised SP optimisation technique, calculates $\mathrm{LV}$ at different SP intervals to estimate when objects approximate real-world features. Breaks in the LV graph are investigated to identify optimal SPs. However, the semivariogram-like LV trend shows too little variation to facilitate accurate optimal SP selection. Consequently, Drăguţ et al (2010) suggested the use of rate of change (ROC) (Bauer and Dahlquist, 1999) to evaluate the subtleties in LV from one SP to the next. In addition to LV-ROC, a non-linear cubic regression approach (with a very high $\mathrm{R}^{2}$ ) was also implemented to isolate LV residuals and to facilitate selection of LV peaks.

\subsubsection{SD}

The SD between object boundaries - derived from neighbouring SPs - is calculated to identify SPs where landform components are suitably delimited. As SP is systematically increased, the resultant objects' sizes will also increase until such a point where the objects match the real-world landform components. At this point, small systematic increases in SP will have little effect on SD. When SP is sufficiently increased, SD will show a distinct peak as mayor changes in object boundaries occur when the objects conform to a new landscape organisational level.

To calculate SD, SP is iterated for every value between one and 100. Each object set, derived from each SP, is then converted to polygons. The polygons are then exported as lines in the form of a shapefile. Lastly, the SD is calculated (using ArcGIS 10.3.1) between neighbouring SPs and the total SD distance is calculated in metres. Consequently, the length of the remaining object boundaries (SD) is quantified and can be visualised graphically for interpretation purposes.

\section{RESULTS}

The selection of optimal SPs from ESP 2 and SD necessitated visual inspection of both graph and map outputs. Only SPs between 1 and thirty were considered, as larger SPs resulted in non-geomorphologically representative objects.

\subsection{ESP 2}

Visual interpretations of peaks in LV-ROC resulted in seven suitable SPs (10, 13, 15, 18, 21, 23 and 30) being identified. Only five of these SPs corresponded with the ten SPs $(8,10,11$, $13,15,19,21,24,25$ and 30) obtained from peaks and knickpoints in the $\mathrm{LV}$ cubic regression $\left(\mathrm{R}^{2}=1, \mathrm{p}<0.0001\right)$ residuals graph (Figure 2). The process of selecting suitable SPs from the LV and LV-ROC graphs has been automated by Drăguţ et al (2014), but the SP as suggested by ESP 2 was 100, which resulted in objects that were too large to represent the geomorphology of the area.

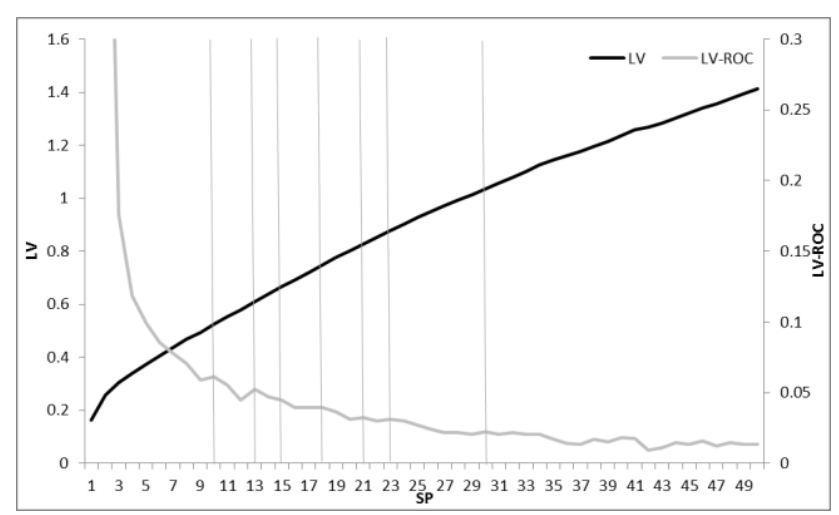

Figure $2 \mathrm{LV}$ and LV-ROC graphed across SP; derived using ESP 2. The grey vertical lines represent the position of suitable SPs based on LV-ROC peaks.

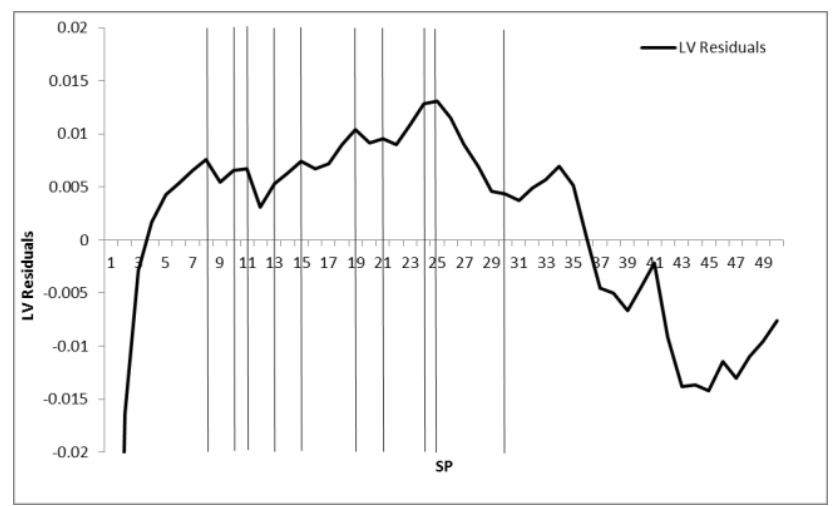

Figure $3 \mathrm{LV}$ cubic regression residuals $\left(\mathrm{R}^{2}=1, \mathrm{p}<0.0001\right)$. The black vertical lines represent the position of suitable SPs based on either knick-points or peaks.

\subsection{SD}

Four peaks, located at SP values 10, 12, 15 and 25 are clearly visible in the SD results (Figure 4). These peaks represent dramatic increases in SD as a result of the delayed responses in object boundary changes, ascribed to the systematic increase in SP. 


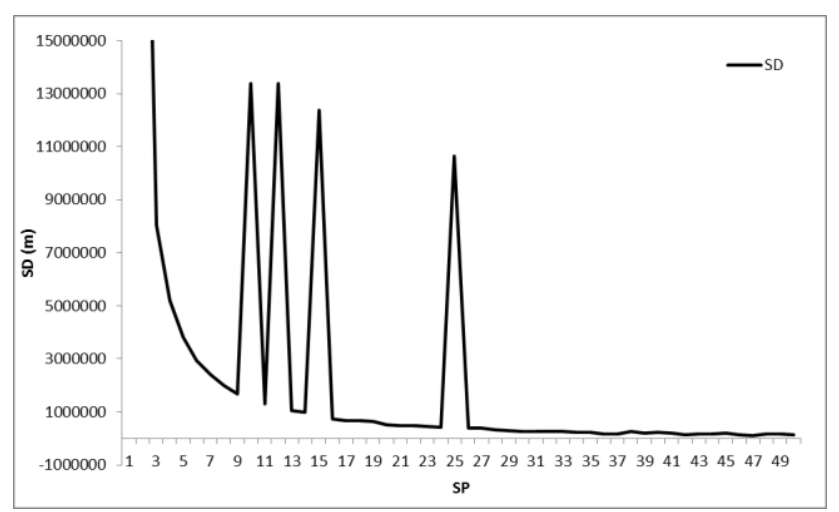

Figure 4 SD graphed across SP, showing four dramatic peaks indicative of large changes in object boundaries and, consequently, the position of suitable SPs.

Figure 5 maps the SD of SPs 8 to 12 for the study site. The four large peaks (dramatic increases in SD) in Figure 4 are clearly noticeable in Figures $8 \mathrm{~b}$ and $8 \mathrm{~d}$, while the remaining Figures $8 \mathrm{a}$ and $8 \mathrm{c}$ show comparatively fewer changes in object geometry.

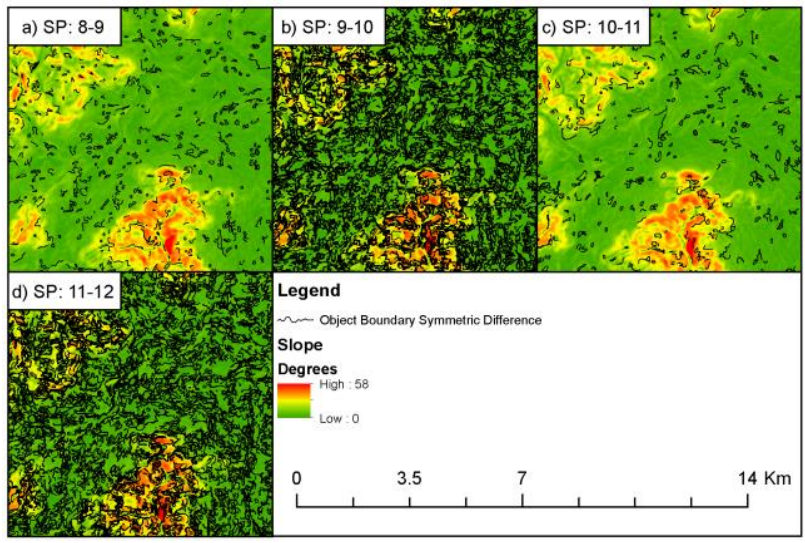

Figure 5 Spatial visualisation of SD across the study area, calculated as the difference of the object boundaries between neighbouring SPs 8 and 9, 9 and 10,10 and 11 , and finally, 11 and 12 .

\section{DISCUSSION AND CONCLUSION}

The homogeneity criterion, controlled by SP, serves as a common denominator between the LV and SD, and defines the causal relationship between object homogeneity and object topology. This relationship is further evident from the fact that three of the four SD selected SPs also registered in the LV response from object homogeneity.

The only SD-based selected SP not selected by ESP 2 was 12 , however, an unmistakable drop in LV at this particular position indicates that large changes in object topology between neighbouring SPs can result in either a peak or drop in LV. These results support observations by Gerçek et al. (2011) that drops in LV-ROC can also be indicative of suitable SPs.

We conclude that suitable SP selection using LV-ROC and regression residual graphs remains challenging as vital interpretations are not only subjective to analysts' interpretations, but are also greatly affected by the large number of possible suitable SPs represented as both peaks and drops in LV. In contrast, SD graphs are much easier to interpret and provide the added advantage that it can be visualised spatially (as demonstrated in Figure 5). SD clearly shows promise for guiding SP selection for geomorphometry applications, but more work is required to automate the process and to evaluate it in different areas and on different source DEMs.

\section{ACKNOWLEDGEMENTS}

This research is financially supported by the National Research Foundation (NRF). Additionally, the Centre of Geographical Analysis (CGA) from Stellenbosch University (SU) is thanked for suppling the $5 \mathrm{~m}$ SUDEM.

\section{REFERENCES}

Baatz, M., Schäpe, A., 2000. Multiresolution segmentation - An optimization approach for high quality multi-scale image segmentation. Angew. Geogr. Informationsverarbeitung XII 12-23. doi:Export Date 6 May 2013

Bauer, R.J., Dahlquist, J.R., 1999. Technical market indicators: Analysis \& performance. Wiley, New York.

Blaschke, T., 2010. Object based image analysis for remote sensing . ISPRS J. Photogramm. Remote Sens. 65, 2-16.

Drăguţ, L., Blaschke, T., 2008. Terrain segmentation and classification using SRTM data. Adv. Digit. terrain Anal. 141-158. doi:10.1007/978-3-540-77800-4_8

Drăguţ, L., Csillik, O., Eisank, C., Tiede, D., 2014. Automated parameterisation for multi-scale image segmentation on multiple layers. ISPRS J. Photogramm. Remote Sens. 88, 119-127. doi:10.1016/j.isprsjprs.2013.11.018

Drăguţ, L., Eisank, C., 2011. Object representations at multiple scales from digital elevation models. Geomorphology (Amst). 129, doi:10.1016/j.geomorph.2011.03.003

183-189.

Drăguț, L., Eisank, C., Strasser, T., 2011. Local variance for multi-scale analysis in geomorphometry. Geomorphology 130, 162-172. doi:10.1016/j.geomorph.2011.03.011

Drăguţ, L., Eisank, C., Strasser, T., Blaschke, T., 2009. A comparison of methods to incorporate scale in Geomorphometry. Proc. Geomorphometry 2009. Zurich, Switzerland, 31 August - 2 Sept. 2009 133-139.

Drăguţ, L., Tiede, D., Levick, S.R., 2010. ESP: a tool to estimate scale parameter for multiresolution image segmentation of remotely sensed data. Int. J. Geogr. Inf. Sci. 24, 859-871. doi:10.1080/13658810903174803

Eisank, C., Drăguț, L.D., Blaschke, T., 2011. A generic procedure for semantics-oriented landform classification using object-based image analysis. Geomorphometry 2011, 8 Sept. 2011, Redlands, USA 125-128.

Eisank, C., Smith, M., Hillier, J., 2014. Assessment of multiresolution segmentation for delimiting drumlins in digital elevation models. Geomorphology 214, 452-464. doi:10.1016/j.geomorph.2014.02.028

ESRI, 2016. ArcGIS - The Complete Geographic Information System [online] [WWW Document] URL http://www.esri.com/software/arcgis/index.html (accessed 6.2.16).

Evans, I.S., 2012. Geomorphometry and landform mapping: What is a landform? Geomorphology 137, 94-106. doi:10.1016/j.geomorph.2010.09.029

Gao, Y., Mas, J.F., Kerle, N., Navarrete Pacheco, J.A., 2011. Optimal region growing segmentation and its effect on classification accuracy. Int. J. Remote Sens. 32, 37473763. doi:10.1080/01431161003777189

Gerçek, D., Toprak, V., Strobl, J., 2011. Object-based classification of landforms based on their local geometry and geomorphometric context. Int. J. Geogr. Inf. Sci. 25, 
1011-1023. doi:10.1080/13658816.2011.558845

Van Niekerk, A., 2015. Stellenbosch University Digital Elevation Model ( SUDEM ) 2015 Edition. Stellenbosch.

Van Niekerk, A., 2010. A comparison of land unit delineation techniques for land evaluation in the Western Cape, South Africa. Land use policy 27, 937-945. doi:10.1016/j.landusepol.2009.12.007

Venter, F., Bristow, J., 1986. An account of the geopmorphology and drainage of the Kruger National Park. Keodoe. doi:10.4102/koedoe.v29i1.524 


\title{
An improved Bayesian nonparametric mixture model to fusing both panchromatic and multispectral images for classification
}

\author{
T. Mao ${ }^{\text {a, b }}$, H. Tang ${ }^{\text {a, b }}$, Y. Shu ${ }^{c}$, N. Yang ${ }^{\text {a,b }}$ \\ a State Key Laboratory of Earth Surface Processes and Resource Ecology, Beijing Normal University, 100875, China - (mting, \\ yns)@mail.bnu.edu.cn \\ ${ }^{\mathrm{b}}$ Key Laboratory of Environment Change and Natural Disaster, Beijing Normal University, 100875, China - tanghong@bnu.edu.cn \\ ${ }^{\mathrm{c}}$ The national disaster reduction center of China, 100875, China - sysun@ mail.bnu.edu.cn
}

KEY WORDS: Nonparametric Bayesian, Fusion, Classification, Object-Based Classification

\begin{abstract}
:
In this paper, we present an improved nonparametric Bayesian model based on a generalized metaphor of Chinese restaurant franchise (gCRF), which can take advantage of both panchromatic and multispectral images to obtain a classification map. There are two drawbacks in the gCRF when it is used to fuse panchromatic and multispectral image for classification, first, since superpixels which are obtained using other segmentation algorithm are considered as basic analysis units instead of pixels in the gCRF, the quality of final classification result depends on the calibre of over-segmentation map. Second, when classify PAN and MS image using the gCRF, semantic segments extracted from PAN image are sharing with MS image and then they are allocated clustering labels using MS image which is richer in spectral information. All the local semantic segments extracted from panchromatic image are supposed to be suitable for representing the local spatial structures in multispectral image, which is not objective in practice. In this paper we propose an improved gCRF, focusing on overcoming the two shortcoming of the gCRF. First of all, the formation of superpixels are integrated into the nonparametric Bayesian framework of the improved gCRF to obtain qualified superpixels. Second, the quality of the semantic segments is checked before sharing with MS image and corresponding measure is taken to dealing with the situation that the semantic segments are not suitable to represent the local structure of MS image. We evaluate the efficiency of the improved model and show it obtains the state-of-art results.
\end{abstract}

\section{INTRODUCTIONT}

With the development of improved sensors, many earth observation platforms can provide high-spatial-resolution remote sensing data, which often contain two types of images, i.e., panchromatic (PAN) and multispectral (MS) images, for example, QuickBird can provides us a PAN image with a resolution of $0.6 \mathrm{~m}$ and four-bands MS image with a resolution of $2.4 \mathrm{~m}$. Several methods are proposed previously in order to make full use of both images to obtain a better classification results. Image fusion is the most common method to integrate the two images which merges the high resolution PAN and lowresolution MS imagery to create and enhanced high-resolution MS image which can be used in subsequent classification (Wang,2013). These methods can be termed as sharpen-thenclassification. The shortcomings of these methods are twofold: firstly, classification results of these methods depend on the fusing algorithms which may have a negative effect on the classification due to the spectral distortion. Secondly, as pixelbased methods, the classification results may be unsatisfactory when dealing with high-spatial-resolution images due to the high degree of within-class spectral variability. Segmentation-thenclassification approaches are other ways widely used to classify high-resolution PAN and MS imageries, which uses a segmentation of an image with higher spatial resolution as an input for the classification of MS image. Mao (2016) propose a unsupervised segmentation-then-classification method, named a generalized metaphor of Chinese restaurant franchise ( $\mathrm{gCRF}$ ), to fuse both PAN and MS image for classification under a nonparametric framework. In the gCRF, two interactive random processes, i.e., table selection and dish selection are used to interpret the generalization of PAN and MS images respectively. In the process of table selection, the local spatial structures of PAN image are extracted, which use superpixels from an oversegmentation map obtained using eCognition as basic analysis units rather than pixels. In the following process of dish selection, MS image with richer spectral information is used to allocate the clustering label for the local spatial segments from PAN image. There are two deficiencies of the gCRF. Firstly, the quality of final classification result depends on the quality of oversegmentation map. Secondly, there is an assumption that all the local spatial segments of PAN image are supposed to be suitable for representing the spatial structure in MS image, which is not always in accordance with the fact. In this paper we propose an improved gCRF, focus on handling the two problems of the gCRF. The contribution of this paper are twofold: firstly, the formation of the superpixels is incorporated into the Bayesian framework; secondly, the examination that whether the local spatial segments of PAN image are suitable to represent the local spatial structures of MS images is taken into consideration and corresponding measures are taken to deal with the situation that some of the local spatial segments are not suitable to represent those in MS image. Experimental results show that the proposed method does improve the classification accuracy of the gCRF and has a state-of-art result than other comparative approaches, including sharpen-then-classification and segmentation-thenclassification methods.

\section{METHODOLOGY}

In this section, we first introduce the gCRF and its deficiencies. Then we present the proposed improved model concentrating on handling the problems of the gCRF.

\subsection{Generalized Chinese Restaurant Franchise}

To fuse PAN and MS images for classification, Mao (2016) proposed a unified framework to iteratively discover semantic segments from PAN image and allocate clustering label using MS image, where the probabilistic generalization process of PAN and MS images can be explained by the gCRF. The illustration of satellite classification using gCRF the is shown in Fig.1 As shown in Fig.1(a), during the pre-processing, PAN image $\mathbf{X}^{P}$ is over-segmented into a set of superpixels which are used as 
basic analysis units rather than pixels in the following processes and MS image $\mathbf{X}^{M}$ is up-sampled to the size of PAN image. Besides, both images are partitioned into L groups in an identical way, i.e., $\left\{\vec{x}_{1}^{P}, \vec{x}_{2}^{P}, \cdots, \vec{x}_{L}^{P}\right\}$ and $\left\{\vec{x}_{1}^{M}, \vec{x}_{2}^{M}, \cdots, \vec{x}_{L}^{M}\right\}$.

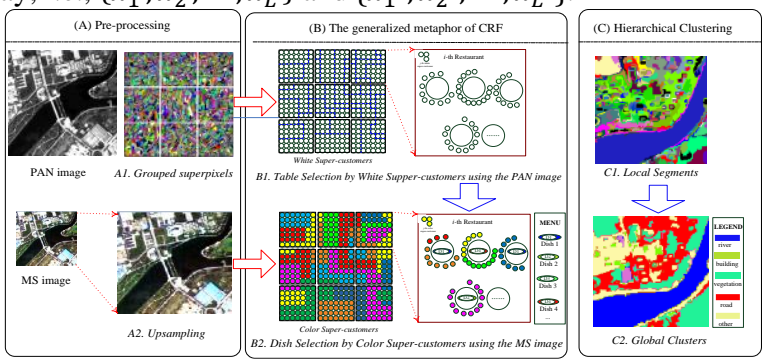

Fig.1 An illustration of satellite image classification using the $\mathrm{gCRF}$

[Mao, et al. 2016]

Then, superpixels from PAN and MS images can be explained by two types of people in $\mathrm{L}$ restaurants, i.e., white supercustomers $\boldsymbol{\theta}^{P}=\left\{\vec{\theta}_{1}^{P}, \vec{\theta}_{2}^{P}, \cdots, \vec{\theta}_{L}^{P}\right\}$ and colour supercustomers $\boldsymbol{\theta}^{M}=$ $\left\{\vec{\theta}_{1}^{M}, \vec{\theta}_{2}^{M}, \cdots, \vec{\theta}_{L}^{M}\right\}$ in the generalized metaphor of CRF shown in Fig.1(b). There are two random processes in the gCRF that can explain the formation of semantic segments from PAN image and allocating the clustering label using MS image, i.e., table selection and dish selection.

(1) Table selection

As shown in the first row of Fig.1(b), assuming that all the white supercustomers with exception of $\theta_{i j}^{P}$ have selected tables $\mathbf{T}_{\neg i j}$ and all of the tables with the exception of table $t$ in the $i$-th restaurant have been served with dishes $\mathbf{K}_{\neg i t}^{M}$ that were ordered by colour supercustomers. Supercustomer $\theta_{i j}^{P}$ choose a table t according to (1).

$$
\begin{aligned}
p\left(t_{i j}=t \mid \mathbf{T}_{\neg i j}, \mathbf{K}_{\neg i j}^{P}\right) & \propto\left\{\begin{array}{l}
n_{i t}^{\neg i j} f_{k_{i t}^{P}}^{P}\left(x_{i j}^{P}\right), \text { if table } t \text { has exist } \\
\gamma_{0} f_{k_{i t}^{P}}^{P}\left(x_{i j}^{P}\right), \text { if } t \text { is a new table }
\end{array}\right.
\end{aligned}
$$

Where $n_{i t}^{\neg i}$ is the number of customers with exception of $\theta_{i j}$ already chosen table $t, f_{k_{j t}}\left(x_{j i}\right)$ is the likelihood of observation $x_{j i}$ and $\gamma_{0}$ is the prior of a new table. If $t$ is a new table, a dish $\mathrm{k}_{\mathrm{it}}^{\mathrm{P}}$ would be selected with the probability

$\mathrm{p}\left(k_{i t^{\text {new }}}=k \mid T_{\neg i j}, K_{\neg i t}^{P}\right) \propto \gamma f_{k}^{P}\left(x_{i j}^{P}\right)$

Where $\mathrm{K}_{\neg \text { it }}^{\mathrm{P}}$,indicates the dish of all the tables selected by white supercustomers with exception of table $\mathrm{t}$ in $i$-th restaurant and $\gamma$ is the prior for a new dish

2) Dish selection

After all the white supercustomers have a meal, the table selected by them are shared with colour supercustomers from MS image which take in charge to select a dish for each table according to (3), which is shown in the second row of Fig.1(b).

$\mathrm{p}\left(k_{i t}^{M}=k \mid \mathbf{T}_{\neg i t}, \mathbf{K}_{\neg i t}^{M}\right) \propto$

$\left\{m_{\cdot k} f_{k_{i t}^{M}}^{M}\left(x_{i t}^{M}\right)\right.$, if $k$ is previously used

$\gamma f_{k}^{M}\left(x_{i t}^{M}\right), \quad$ if $k$ is a new dish

where $m_{\cdot k}$ indicates the number of tables already served dish $k$ chosen by colour supercustomers, and $\gamma$ is the prior for a new dish. For more detail please refer to (Mao, 2016).

There are two major deficiencies of the gCRF. Firstly, in the process of pre-treatment, an over-segmentation algorithm is applied to obtain the superpixels, for example, the multiresolution segmentation in eCognition. Thus the quality of classification result depends on the quality of the oversegmentation map. The inferior over-segmentation algorithm may result in poor classification result. Secondly, there is an assumption in the gCRF for sharing the tables between white supercustomers and colour supercustomers that all the semantic segments, i.e., tables, discovered from PAN image can represent the local spatial structure of MS image in the same area, which sometimes may not be accordance with the fact. For example, in the area of Miyun, Beijing, China, shown in Fig.3, the gray values of some buildings are quite similar with those of roads in PAN image. By contrast, the spectral values of the two in MS

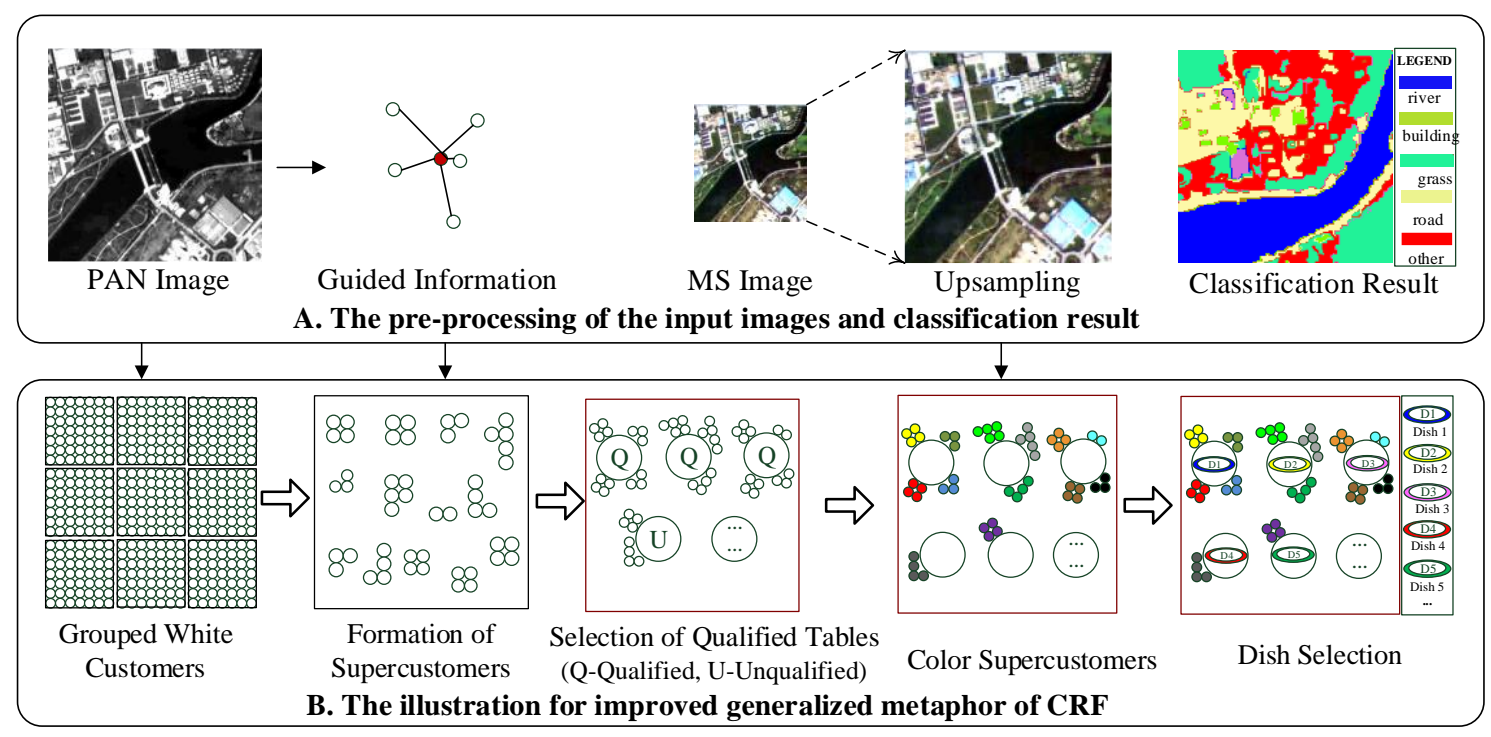

Fig. 2 Illustration of improved generalized metaphor of CRF 
image may be distinguishable. Thus those buildings and roads may be wrongly discovered in the same semantic segments in PAN image which is not suitable for sharing with MS image.

\subsection{The Improved Generalized Chinese Restaurant Franchise}

In this paper an improved gCRF are proposed concentrating on two aspects as shown in Fig.2: (1) the formation of supercustomers (i.e., superpixels) from raw PAN image; (2) the selection of qualified tables from PAN image which are suitable for sharing with MS image to allocate clustering labels.

2.2.1 Formation of supercustomers: The formation of superpixels from pixels of PAN image can be analogous to converging customers with similar characteristic to a supercustomer. To group customers to supercustomers, guided information which is the descriptor of combining spatial and spectral information among pixels is used (Shu et al., 2015). The guided information is defined as follows

$r_{m, n}=\exp \left(-\frac{\left\|x_{m}-x_{n}\right\|^{2}}{\sigma_{s p a}^{2}}-\frac{\left\|G_{m}-G_{n}\right\|^{2}}{\sigma_{s p e}^{2}}\right)$

where $x_{m}$ indicates the location of the $m$-th pixel, $G_{m}$ indicates the $m$-th pixel value in guided image, $\sigma_{s p a}^{2}$ and $\sigma_{s p e}^{2}$ are the spatial and spectral variance, respectively. $w$-th customer in $j$-th restaurant, indicated as $c_{j w}$, chooses a supercustomer $\theta_{j w}$ to join with proportional to

$p\left(\theta_{w j}=\theta \mid \boldsymbol{\theta}_{\neg w j}, \boldsymbol{K}_{\neg w j}\right)$

$\propto\left\{\begin{array}{c}\left(\sum_{\theta_{j l}=\theta} r_{j, l}\right) f_{k}\left(x_{j i}\right), \text { if supercustomer s has exist } \\ \alpha_{0}\left(x_{j i}\right) f_{k}\left(x_{j i}\right), \text { if } s \text { is a new supercustomer }\end{array}\right.$

where $\sum_{\theta_{j l}=\theta} r_{j, l}$ is the sum of guided information of all the customers in $j$-th restaurant who belong to supercustomer $\theta$ and $\alpha_{0}$ is the prior for a new supercustomer.

2.2.2 Selection of Qualified Tables: After we obtain the supercustomers, i.e., superpixels, each supercustomer selects a table (semantic segments) in a restaurant according to (1) and (2). In other words, superpixels in PAN image are grouped into semantic segments in the same way as that of gCRF. In the gCRF, the tables selected by white supercustomer are all shared with colour supercustomers, while in the improved model the quality of tables is checked before sharing with MS image. In other words, the validity of the semantic segments from PAN image are examined before transferred to MS image. During the examination, a semantic segment can be regarded as qualified to represent the local spatial structure of MS image if the spectral variability of the area in MS image with the same location of the semantic segments is lower than an experimental threshold, and it is regarded as unqualified if not. The qualified semantic segments of PAN image are shared with MS image and they are allocated a clustering label using MS image according to (3). While the unqualified semantic segments are split into superpixels contained in it. Then each superpixel in an unqualified semantic segments is regarded as a qualified table which is considered suitable to represent the local spatial structure of MS image and is allocated a clustering label of MS image according to (3), too.

The Gibbs-like sampling algorithm for the improved gCRF includes six steps.

1) Step 1: Initializing the model parameters and calculating the guided information.

2) Step 2: sampling a superpixel label for each pixel. Given the rest of the variables, sample a superpixel label $\theta_{w j}$ for w-th pixel in j-th sub-image according to (5).
3) Step 3: sampling a table label, i.e., semantic segments label for each superpixel using PAN image. Sampling table label $t_{i j}$ for $i$-th superpixel in $j$-th sub-image according to (1) and sampling a dish for new table according to (2).

4) Step 4: selecting qualified tables. Examine the quality of each semantic segments, semantic segments meet the requirement mentioned before are considered as qualified tables. Each unqualified semantic segment is split to superpixels contained in it and each superpixel is treated as a qualified table.

5) Step 5: allocating clustering labels using MS image. Allocate each qualified table a clustering label according to (3).

6) Step 6: checking the convergence of the clustering result. If not, repeat Step 2 to Step5.

\section{EXPERIMENT RESULT AND EVALUATION}

The experimental data we choose to evaluate the performance of the proposed method is shown in Fig. 3 including a PAN (2-m resolution) and MS image (6-m resolution; four bands: red, green, blue and near-infared) acquired by Mapping satellite-1 images with a size of $1200 * 1200$ pixels (PAN image), covering a suburban area (Miyun, Beijing, China) with five major geoobjects (water, grass/tree, building, road and bare soil), as shown in Fig.3(c).

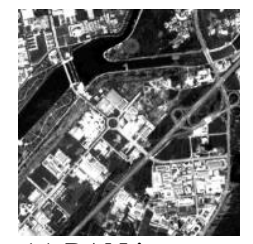

(a) PAN image

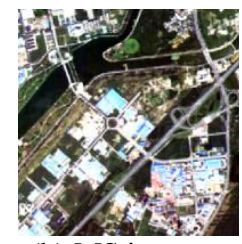

(b) MS image

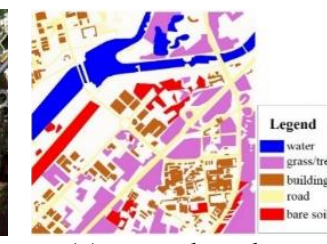

(c) ground-truth
Fig. 3 Panchromatic, multispectral satelliteimageries and ground-truth image

We compare the proposed method with gCRF and two types of approaches using both PAN and MS image to obtain classification results, (1) pixel-based methods: firstly, we use GS in ENVI to obtain the pan-sharpened image, then we use Kmeans and SVM to classify the pan-sharpened image, termed as Kmeans+GS+pix and SVM+GS+pix. (2) object-based methods: firstly, MS image is up-sampled to the size of PAN image and averaged according to a segment map obtained from PAN image. Then the pixels of averaged MS image in a segment is considered as basic analysis units instead of pixels and we perform Kmeans and SVM to obtain the classification results. Methods of this type are termed as Kmeans+MS+seg and SVM+MS+seg. The classification results and quantitative evaluation is shown in Fig.4 and TABLE I.

As shown in Fig.4, the proposed method performs similar with gCRF from visual inspection and better than Kmeans+GS+pix and Kmeans+MS+seg (Fig.4(c) and Fig.4 (e)). It also outperforms SVM+MS+seg, since the performance of the latter depends on the quality of segmentation map from PAN image. The proposed methods is comparable to SVM+GS+pix in Fig. 4 (b) by visual examination. 


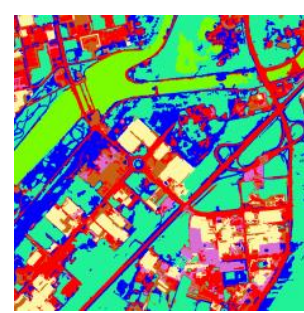

(a) improved gCRF
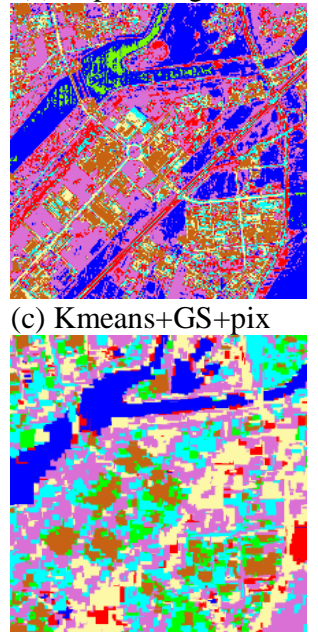

(e) Kmeans+MS+seg

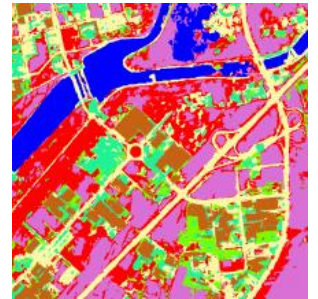

(b) $\mathrm{gCRF}$
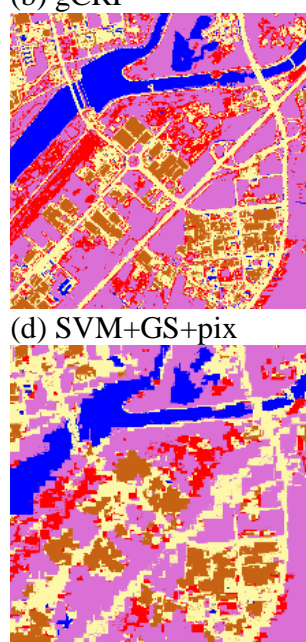

(f) $\mathrm{SVM}+\mathrm{MS}+\mathrm{seg}$
Fig.4 The classification results of all methods

As we can see from Table 1, the improved gCRF has the highest Kappa coefficient and lowest overall entropy except those of SVM + GS + pix. Since we know that the higher Kappa coefficient indicates higher classification accuracy, and the lower overall entropy indicates better homogeneity, the proposed method performs better than the gCRF, all the unsupervised methods which use Kmeans as the classifier, besides, it performs better than SVM+MS+seg which is a supervised method while it performs worse than $\mathrm{SVM}+\mathrm{MS}+$ seg according to Kappa and overall entropy.

\begin{tabular}{|l|l|l|l|l|l|l|}
\hline & $\begin{array}{l}\text { Impr } \\
\text { oved } \\
\text { gCRF }\end{array}$ & gCR & \multicolumn{2}{|l|}{ pixel-based methods } & \multicolumn{2}{|l|}{$\begin{array}{l}\text { object-based } \\
\text { methods }\end{array}$} \\
\cline { 4 - 7 } & & & $\begin{array}{l}\text { Kmeans }+G \\
\text { S+pix }\end{array}$ & $\begin{array}{l}\text { SVM } \\
+ \text { GS } \\
+ \text { pix }\end{array}$ & $\begin{array}{l}\text { Kmeans } \\
+ \text { +MS } \\
\text { seg }\end{array}$ & $\begin{array}{l}\text { SVM } \\
+ \text { se } \\
+ \text { seg }\end{array}$ \\
\hline Kappa & 0.8 & 0.79 & 0.39 & 0.80 & 0.35 & 0.72 \\
OE & 0.51 & 0.51 & 0.91 & 0.50 & 1.01 & 0.70 \\
\hline
\end{tabular}

Table 1 Evaluation of the kappa coefficient and overallentropies of different models

\section{CONCLUSION}

In this paper we proposed an improved nonparametric Bayesian model based on the gCRF, which focuses on overcoming the two defects of the gCRF when used to fuse PAN and MS image for classification, i.e. the dependence of other over-segmentation algorithm and lacking of examination of tables when sharing them between PAN image and MS image. In the future, more information such as edge, shape of the objects can be taken into consideration to extract the spatial information of images for a better classification.

\section{ACKNOWLEDGEMENTS}

This work is partly supported by the National Natural Science Foundation of China (no. 41571334) and the Fundamental Research Funds for the Central Universities.

\section{REFERENCES}

Shackelford, Aaron K., and Curt H. Davis, 2003. A hierarchical fuzzy classification approach for high-resolution multispectral data over urban areas. IEEE transactions on geoscience and remote sensing 41(9). pp. 1920-1932.

Bruzzone, L., \& Carlin, L., 2006. A Multilevel context-based system for classification of very high spatial resolution images. IEEE Transaction on Geosciences and Remote Sensing, 44(9), 2587-2600.

Johnson, B., Xie, Z., 2013. Classifying a high resolution image of an urban area using super-object information[J]. ISPRS journal of photogrammetry and remote sensing, 83, pp. 40-49.

Mao, T., Tang, H., He, S., Shu, Y. et al., 2015. Fusion of panchromatic and multispectral images for classification using the Chinese restaurant franchise with shaped tables. In: Joint Urban Remote Sensing Event (JURSE), Lausanne, Switzerland, pp. 1-4.

Shu, Y., Mao, T., Tang, H. et al., 2015. Unsupervised Classification of VHR Panchromatic Images Using Guided Chinese Restaurant Franchise Mixture Model. In: IEEE International Geoscience and Remote Sensing Symposium, Milan, Italy, pp. 2413-2416.

Teh, J. W., Jordan, M. I., Beal, M. J., et al. ,2006. Hierarchical dirichlet processes. Journal of the American statistical association, 101(476).

Wang, G., Liu, J. and He, G., 2013. A Method of Spatial Mapping and Reclassification for High-Spatial-Resolution Remote Sensing Image Classification. The Scientific World Journal, 2013. 


\title{
ASSESSING EDGE AND AREA METRICS FOR IMAGE SEGMENTATION PARAMETER TUNING AND EVALUATION
}

\author{
H. P. Meyer ${ }^{\mathrm{a}}$, A. Van Niekerk ${ }^{\mathrm{a}, *}$ \\ ${ }^{a}$ Dept. of Geography \& Environmental Studies, Stellenbosch University, South Africa Private Bag X1, Matieland, Stellenbosch, \\ 7602, South Africa
}

KEY WORDS: Segmentation, Edge metric, Area metric, Boundary delineation, Accuracy, Object-based image analysis

\begin{abstract}
:
Image segmentation algorithms allow the creation of variables that influence the attributes of the resulting objects. There is currently no set way of identifying the best segmentation parameters for a specific application. It would consequently be useful to calculate the accuracy of a particular segmentation for determining how well it can delineate an object of interest. This article assesses the use of both an area and edge metric to evaluate and quantify the ability of a segmentation algorithm to delineate objects of interest based on manually collected reference data. SPOT 5 imagery was used for the segmentation of two $2 \times 3 \mathrm{~km}$ study areas in the Eastern-Cape of South Africa. The aims of this study were to use the area metric to identify a scale parameter for which the ratio between oversegmentation (OS) and undersegmentation (US) yields the most favourable result and, use the edge metric to determine the accuracy to which the boundary of objects of interest is delineated. The usefulness of estimating scale parameter (ESP) and segmentation parameter tuner (SPT) are also investigated to identify the suitable scale parameter. The root mean square error (RMSE) between OS and US, was used in conjunction with the edge metric results to produce an overall accuracy value between 0 1. The ESP tool was found useful as a guide, but failed to identify the scale parameter yielding the most accurate results, whereas, the SPT tool succeeded in both cases to identify this scale parameter. It was concluded that the edge and area metric can be used in conjunction to, not only determine the ideal scale parameter, but also calculate the overall accuracy of the segmentation algorithm.
\end{abstract}

\section{INTRODUCTION}

The recent increase in availability of high resolution satellite imagery has led to a growing interest in object-based image analysis (OBIA). Fundamental to this relatively new paradigm in image analysis is the grouping of pixels with similar spectral or spatial attributes to form image objects (Blaschke, 2010; Blaschke et al., 2014; Lucieer \& Stein, 2002; Moller et al., 2007; Dragut et al., 2010). This process, called image segmentation, is not trivial as poor segmentations have a negative effect on the accuracy of any further classification or processing that follows (Baatz et al., 2008; Navulur, 2006). Image segmentation is a spatial clustering technique that divisions an image into non-overlapping regions or segments (Moller et al., 2007). Image segmentation can be categorized into edge detection methods and region-growing techniques. Edge detection segmentation regionalizes an image by locating feature boundary elements, while region-growing algorithms make use of seed pixels that are grown into objects, based on a region's homogeneity (Fortin et al., 2000; Munoz et al., 2003; Blaschke et al., 2014). Many factors affect the quality of segmentation results (Moller et al., 2007), and much research has been done on improving image object delineation by considering different segmentation algorithms (Meinel \& Neubert, 2004), tuning input parameters (Achanccaray et al., 2014; Achanccaray et al., 2015) and studying the effect that the spatial and spectral characteristics of the objects of interest have on the output (Lucieer \& Stein, 2002; Burnett \& Blaschke, 2003; Tian \& Chen, 2007; Blaschke, 2010; Petropoulos et al., 2012). Effective validation techniques that quantify a segmentation result's ability to delineate objects of interest remain elusive (Lucieer \& Stein, 2002; Benz et al., 2004; Clinton et al., 2010). The accuracy of image objects can be validated by studying their topological (area) and/or geometrical (edge) relationships (Molenaar, 1998; Zhan et al., 2005). Area metrics generally determine the extent of US and OS, where US is quantified by calculating the area of segments intersecting the boundaries of the reference object (Moller et al., 2007) and OS is determined by calculating the area of segments falling inside of the reference object. When using reference polygons for segmentation validation, the ideal result must adhere to two conditions, namely: 1) The number of segments within the reference polygon must be at its lowest; and 2) The area of intersecting segments falling outside of the reference polygon must be at a minimum (Benz et al., 2004). The first condition refers to OS, while the second refers to US.

In order for a segmentation to produce meaningful and accurate objects based on a reference dataset, it is important that the segmentation parameters are properly tuned. This can be a time consuming process of trials-and-errors. Segmentation parameter tuning (SPT) is a free software tool that automatically tests certain parameter values and compares the level of agreement between the segmented objects and a manually collected reference objects. The level of agreement between the segmentation output and reference objects are calculated for each parameter combination and referred to as segmentation goodness (Achanccaray et al., 2014; Achanccaray et al., 2015). The main purpose of the experiments was to determine to what extent edge and area metrics can be used to assess the output of segmentation algorithms and for selecting optimal input features and parameters. The estimated scale parameter (ESP) tool (Dragut et al., 2010) is also employed for comparison purposes.

\footnotetext{
* Corresponding author
} 


\section{STUDY AREA}

For the purpose of this study, two $2 \times 3 \mathrm{~km}$ study sites, representing a river and a dam respectively, were selected for carrying out the experiments. Study site 1 includes a section of the Kouga River and is situated in a mountainous region. This site was chosen as it includes a surface water body that is particularly challenging to delineate using remote sensing. The river course is relatively narrow due to the mountainous morphology, the intermediate channel gradient and stability, and the wandering channels associated with this type of landscape (Hogan \& Luzi, 2010).

Study site 2 covers the Glen Melville dam just north of Grahamstown which was built as part of the Orange-Great Fish Interbasin transfer scheme, and has a capacity of 6 million $\mathrm{m}^{3}$ (Lorraine \& Mullins, 2011). The dam is dominated by mountainous landscape to the north and relatively flat hills to the south.

The variation in reference object size and shape between the two study areas was taken into account and will consequently contribute to testing the robustness of the analysis.

\section{IMAGE DATA}

The data for the study consist of $10 \mathrm{~m}$ resolution multispectral SPOT5 images as well as $2.5 \mathrm{~m}$ resolution panchromatic images of the eastern-cape. The images used for study areas 1 and 2 were captured in March 2012 and March 2013 respectively. Images were ordered from SANSA (South African National Space Agency) Earth Observation. The $2.5 \mathrm{~m}$ panchromatic and $10 \mathrm{~m}$ multispectral SPOT5 imagery were orthorectified using $0.5 \mathrm{~m}$ ground sample distance orthorectified aerial photography, provided by the National Geo-spatial Information (NGI), as basis and geographical reference. The images were then atmospherically corrected (ATCOR 2) and pansharpened to $2.5 \mathrm{~m}$ using the PANSHARP tool in PCI Geomatica. The data used as reference were collected manually by digitizing the water bodies in both study sites to minimize error.

\section{METHODS}

\subsection{Area overlay}

The optimal segmentation for an intensive background feature (e.g. trees, buildings) would result in the reference polygon to be covered by a single object. This is however not expected to be the case for a spatially extensive feature (e.g. roads, rivers) which would likely contain multiple meaningful objects (Tian \& Chen, 2007). All the segmented objects intersecting the reference shape are divided into two classes namely, partially overlaying and overlaying. Therefore, a slightly modified equation from the one proposed by Clinton et al. (2010) is used for both the calculation of OS as well as US. With an increase in scale, OS drops due to the decrease in objects that fall completely within the borders of the reference object, and US increases. The area of intersecting objects falling outside of the reference object are used to calculate under segmentation, while all the resulting objects falling completely within the reference object are used to calculate over segmentation. The RMSE between US and OS is calculated and used as a measure of accuracy for the area metric.

\subsection{Distance between edge pixels}

In this study, the purpose of using the edge metric is, not to judge the goodness of fit between the objects, but the accuracy of boundary delineation which in order to obtain, we used the algorithm proposed by Delves et al. (1992) calculating the average distance from the reference shape to the nearest overlaying segment boundary.

The edge metric is used to calculate the average distance error between the boundaries of the resulting objects that intersect the reference object and the boundaries of the reference object. This is a measure of accuracy for the boundary delineation for the segmentation.

\subsection{Estimating scale parameter}

ESP is used to evaluate rate of change of local variation to identify suitable scales where the segmentation would have the greatest capacity to differentiate between spectral classes. ESP is a method presented by Dragut et al. (2010) that estimates scale parameter for the segmentation of remotely sensed data in eCognition. ESP is an iterative algorithm that builds on the principle of local variance (LV). It generates image objects on multiple scale levels to test the heterogeneity between objects. A measure called rate of change is used as a test for the LV variation between objects at certain scales

\subsection{Segmentation parameter tuner}

SPT is an automatic method that rely on optimization algorithms, to search the parameter space for an optimal set of values in order to produce the most accurate segmentation, and in that manner minimize human intervention (Achanccaray et al., 2015). The goodness of these segmentations are measured by a metric that expresses numerically the level of disagreement between the segmentation output and a set of reference polygons. In this study, differential evolution (Storn \& Price, 1997) is used as optimization algorithm for parameter tuning, while the multiresolution region growing algorithm (Happ et al., 2013 ) is used for the segmentation. The area-fit index is used to express segmentation quality, by comparing the segmentation results with the reference polygon and therefor helping to guide the optimization process (Achanccaray et al., 2015). The lower the value of the metric, the more similar the resulting segmentation is to the reference polygon. For this study, the updated version called SPT 3.1 has been used as a guide and verification to the scale, shape and compactness parameters chosen.

\section{RESULTS AND DISCUSSION}

The initial segmentation was run using the standard optical bands as input, and varying scale from 1-100. A range was identified, for both study areas, where the optimal scale parameter might lie. With this range restricted to 5-30, the processing time was reduced substantially and consequently the creation of redundant data prevented. In Table 1 below, are the parameters that produced the most accurate segmentation results according to both metrics, as well as the results obtained from ESP and SPT. Various shape and compactness parameters were tested, whereas only the most accurate is stated below.

\begin{tabular}{|l|c|c|}
\hline \multicolumn{3}{|c|}{ Results } \\
\hline & Study area 1 & Study area 2 \\
Shape & 1 & 0.5 \\
\hline
\end{tabular}




\begin{tabular}{|l|c|c|}
\hline Compactness & 0.5 & 0.5 \\
US & 0.366 & 0.390 \\
OS & 0.659 & 0.955 \\
RMS & 0.533 & 0.729 \\
Edge metric & 0.044 & 0.030 \\
Equilibrium (Scale) & 21 & - \\
Scale parameter & 12 & 25 \\
ESP output (Scale) & 8 & 14 \\
SPT output (Scale) & 12 & 24 \\
Overall accuracy & 0.024 & 0.022 \\
\hline
\end{tabular}

Table 1. Combined overall results

The segmentation results of the two study sites vary substantially. This is attributed to the size difference between the reference objects of both study areas as well as land cover characteristics. It therefore made it difficult to use one segmentation method for both study areas. The expected trend was that with a low starting scale, OS is high and US low. The RMSE is used in this case to observe the general accuracy of the area metric for the various scale parameters, as well as combined with the edge metric result, producing an overall accuracy value between $0-1$, with higher accuracy the closer the value is to 0 .

The trend for the edge metric however starts off with a relatively high error at a low scale, this error decreases as the scale increases until a lowest point (highest accuracy) and then increases again.

The smaller the scale, the higher the OS ratio, but because of each object being smaller, the error in boundary delineation is also lower.

\subsection{Study area 1}

In this segmentation based on optical bands, parameters shape and compactness were chosen as 1 and 0.5 respectively. OS and US was found to intersect at scale 19, at which the accuracy is 0.079 pixels. However, when the scale is lowered to 11 , a highest accuracy is reached of 0.042 pixels error. The expected nature of results obtained from the edge metric, would be accurate at a smaller scales and increase in error as scale increases. As the size of objects increase, so does the distance between the outline of the reference shape and the overlaying segmented objects. This is where the area metric helps to identify the scale parameter range where OS and US are equal. Although the results produced by the ESP tool aren't very accurate, the values suggested does fall within a small range from the value identified to produce the most accurate results. The SPT suggested the use of scale parameter as 12 and shape and compactness as 0.49 and 0.62 respectively. Although the scale parameter identified were correct, more accurate results were obtained from using different shape and compactness parameters.

At scale parameter 12 , the RMS error of 0.533 was multiplied by the edge metric result of 0.044 , which resulted in a lowest overall accuracy value of 0.024 .

\subsection{Study area 2}

Study area 2 contains a large object for which the assumption is not true, that the perfect segmentation will yield one object per reference object. Basing the accuracy on the OS/US ratio alone would therefore be problematic for smaller objects in the same study area, essentially resulting in the misclassification thereof. OS is based on the total area of objects falling inside the reference object, because this is expected to be high in the case of large objects, OS on its own is consequently neglected. The focus however shifts to US, which calculates the area of boundary objects falling outside of the reference object, and the RMSE between the two. Using the optical bands for the segmentation weighting with shape and compactness parameters both 0.5 , scale parameter 25 was identified as where the boundary delineation accuracy is at a high with an error of 0.03 pixels. The US ratio produced by the area metric at scale 25 is 0.389 , which is another example that would fit the norm of the previous study area and the use of optical bands to base segmentation on.

Again, the results suggested by ESP were not very useful. While SPT also yielded quite similar results with the shape and compactness parameters suggested not being accurate, but with scale parameter being out by one, at 24. Again the RMS error was calculated and multiplied with the edge metric result for scale 25 . The resulting value of 0.022 was found as the highest overall segmentation accuracy for study area 2 .

From the results obtained, it is possible for both instances to identify the parameters that yield the most accurate results. Determining the segmentation accuracy was achieved with both metrics, and a useful way of combining the results obtained to give an overall accuracy was proposed.

\section{CONCLUSION}

Both metrics have their advantages and weaknesses. Using them in conjunction with one another makes it possible to take into account the different facets of accuracy, whether it is area of overlap or boundary delineation. Favourable parameters changed from study area 1 being shape 1 and compactness 0.5 , to shape 0.5 and compactness 0.5 in the second area. This means that the importance of spectral and shape characteristics taken into account for region merging shifted due to the landscape change. ESP was found to be the least useful of the methods investigated, with only identifying scale parameters fairly close to the most accurate range. SPT in terms of scale parameter tuning was found quite useful, identifying the correct parameter in study area 1 and being out by 1 in study area 2 . With regard to parameters shape and compactness, SPT did not yield very accurate results. In both study sites, the edge metric succeeded in determining the accuracy and ability of a segmentation algorithm to delineate the boundary of a reference object. The area metric however can be divided into OS and US. US is useful to determine the percentage area of objects overlaying the reference object, which falls either within or outside the reference object. OS however is only useful for objects under which the assumption, that a perfect segmentation will yield one object for every reference object, is true. This is not expected for large reference objects and were therefore not of use in study area 2. The RMSE were calculated between US and OS, and used in conjunction with the edge metric result, to consequently yield a ratio value between the two metrics. The lower this number is, the more accurate the overall result of the segmentation. In both cases, the scale parameter for which this value was the smallest, were identified.

Being able to accurately map features of interest using an object oriented approach depend primarily on the segmentation algorithm's ability to delineate these features. It is confirmed that using three of the four investigated methods, that it is indeed possible, to ultimately quantify the accuracy to which this is achieved. 


\section{REFERENCES}

Achanccaray, P., Ayma, VA., Jimenex, LI., Bernabe, S., Garcia, S., Happ, PN., Feitosa, RQ. \& Plaza, A., 2014. A free software tool for automatic tuning of segmentation parameters. SouthEastern European Journal of Earth Observation and Geomatics 3(2):707-711

Achanccaray, P., Ayma, VA., Jimenex, LI., Bernabe, S., Happ, PN., Costa, GA., Feitosa, RQ. \& Plaza, A., 2015. SPT 3.1: A free software for automatic tuning of segmentation parameters in optical, hyperspectral and SAR images. IEEE International Geomatics and Remote Sensing Symposium. Milan, 26-31 July 2015. 4332-4335.

Baatz, M., Hoffmann, C. \& Willhauk, G., 2008. Progressing from object-based to object-oriented image analysis. In Blaschke T, Lang S \& Hay GJ (ed) Object-Based Image Analysis: Spatial Concepts for Knowledge-Driven Remote Sensing Applications. Springer, Berlin.

Benz, UC., Hofmann, P., Willhauck, G., Lingenfelder, I., \& Heynen M., 2004. Multi-resolution, object-oriented fuzzy analysis of remote sensing data for GIS-ready information. ISPRS Journal of Photogrammetry \& Remote Sensing 58, pp. 239-258.

Blaschke, T., 2010. Object based image analysis for remote sensing. ISPRS Journal of Photogrammetry and Remote Sensing 65, pp. 2-16.

Blaschke, T., Hay, GJ., Kelly, M., Lang, S., Hofmann, P., Addink, E., Feitosa, RQ., Van der Meer, F., Van der Werff, H., Van Coillie, F., and Tiede, D., 2014. Geographic Object-Based Image Analysis - Towards a new paradigm. ISPRS journal of Photogrammetry and Remote Sensing 87, pp. 180-191.

Burnett, C., \& Blaschke, T., 2003. A multi-scale segmentation/object relationship modelling methodology for landscape analysis. Ecological Modelling 168, pp. 233-249.

Clinton, N., Holt, A., Scarborough, J., Yan, L., \& Gong, P., 2010. Accuracy Assessment Measures for Object-based Image Segmentation Goodness. Photogrammetric Engineering \& Remote Sensing 76(3), pp. 289-299.

Delves, LM., Wilkinson, R., Oliver, CJ., \& White, RG., 1992. Comparing the performance of SAR image segmentation algorithms. International Journal of Remote Sensing 13(11), pp. 2121-2149.

Dragut, L., Tiede, D., \& Levick, S., 2010. ESP: a tool to estimate scale parameter for mutliresolution image segmentation of remotely sensed data. International Journal of Geographical Information Science 24(6), pp. 859-871.

Fortin, MJ., Olson, R., Ferson, S., Iverson, L., Hunsaker, C., Edwards, G., Levine, D., Butera, K., \& Klemas, V., 2000. Issues related to the detection of boundaries. Landscape Ecology 15, pp. 453-466.

Happ, P., Feitosa, R., Bentes, C., \& Farias, R., 2013. A region growing segmentation algorithm for GPUs. IEEE Geoscience and Remote Sensing Letters 10(6), pp. 1612-1616.

Hogan, DL., and Luzi, DS., 2010. Channel geomorphology: Fluvial forms, processes, and forest management effects. In:
Pike RG, Redding TE, Moore RD, Winkler RD, and Bladon KD (Eds.), Compendium of Forest Hydrology and Geomorphology in British Columbia. BC Ministry of Forests and Range, Forest Science Program, Victoria, BC, and FORREX Forum for Research and Extension in Natural Resources, Kamloops, BC Land Management Handbook No (Vol. 66, pp. 331-371).

Lorraine, R., \& Mullins, G., 2011. Grahamstown's Water Supply: a brief history from 1812 to 2008. Annals of the Eastern Cape Museums 7, pp. 1-64.

Lucieer, A., \& Stein, A., 2002. Existential uncertainty of spatial objects segmented from satellite sensor imagery. IEEE Transactions on Geoscience and Remote Sensing 40 (11), pp. 2518-2521.

Meinel, G., \& Neubert, M., 2004. A comparison of segmentation programs for high resolution remote sensing data. Leibniz Institute of Ecological and Regional Development (IOER). Dresden, Germany.

Molenaar, M., 1998. An Introduction to The Theory of Spatial Objecct Modelling. Research Monographs in Geographical Information Systems, Tayler \& Francis.

Moller, M., Lymburner, L., \& Volk, M., 2007. The comparison index: A tool for assessing the accuracy of image segmentation. International Journal of Applied Earth Observation and Geoinformation 9, pp. 311-321.

Munoz, X., Freixenet, J., Cufi, X., \& Marti, J., 2003. Strategies for image segmentation combining region and boundary information. Pattern Recognition Lett. 24, pp. 375-392.

Navulur, K., 2006. Multispectral Image Analysis Using the Object-Oriented Paradigm, CRC Press, New York.

Petropoulos, GP., Kalaitzidis, C., Prasad Vadrevu, K., 2012. Support vector machines and object-based classification for obtaining land-use/cover cartography from Hyperion hyperspectral imagery. Comput Geosci. 41, pp. 99-107.

Storn, R., \& Price, K., 1997. Differential Evolution - A simple and efficient heuristic for global optimization over continuous spaces. Journal of Global Optimization 11, pp. 341-359.

Tian, J., \& Chen, DM., 2007. Optimization in multi-scale segmentation of high resolution satellite images for artificial feature recognition. International Journal of Remote Sensing 28(20), pp. 4625-4644.

Zhan, Q., Molenaar, M., Tempfli, K., \& Shi, W., 2005. Quality assessment for geo-spatial objects derived from remotely sensed data. International Journal of Remote Sensing 26(14), pp. 29532974. 


\title{
MAPPING GREENHOUSE GAS EMISSIONS AND REMOVALS FROM THE LAND USE, LAND USE CHANGE, AND FORESTRY SECTOR AT THE LOCAL LEVEL
}

\author{
G. H. Mitri *, J. Karam \\ Land and Natural Resources Program, Institute of the Environment, University of Balamand, Kelhat, El Koura, Lebanon - \\ george.mitri@balamand.edu.lb
}

\begin{abstract}
KEY WORDS: Greenhouse gas emissions/removals, Land Use, Land Use Change, Forestry, Geographic Object-Based Image Analysis
\end{abstract}

\begin{abstract}
:
Greenhouse gas (GHG) emissions resulting from the Land Use, Land-Use Change, and Forestry sector (LULUCF) are estimated and reported in National Communications to the United Nations Framework Convention on Climate Change (UNFCCC). By definition, the LULUCF sector is a "greenhouse gas (GHG) inventory sector that covers emissions and removals of greenhouse gases resulting from direct human-induced land use, land-use change and forestry activities". In principle, the annual GHG national inventory should be transparent, consistent, comparable, complete, and accurate. Also, it should be able to systematically account for all changes in land use and forest cover over many years. In this context, it is essential to investigate the development of an automated approach for mapping local GHG emissions/removals from the LULUCF sector for integration at the national level. In view of that, the aim of this work was to develop a semi-automated model for estimating GHG emissions and removals form the LULUCF sector at the local level. The specific objectives were to 1) map changes in land use and forest cover between two consecutive years, and 2) assess GHG emissions and removals from the LULUCF sector. The methodology of work comprised the use of Geographic ObjectBased Image Analysis (GEOBIA) for modelling changes in the LULUCF sector and, subsequently, estimating GHG emissions/removals between two consecutive years. The combined use of Very High Resolution (VHR) SPOT imagery (2.5 m colour) and field data was involved in identifying and mapping land-use changes between 2014 and 2015. Subsequently, GHG emissions and removals were estimated using customized features in GEOBIA and following the 2003 Intergovernmental Panel on Climate Change "Good Practice Guidance for Land Use, Land-Use Change and Forestry", which adopts a land use category-based approach to estimate emissions/removals from all land categories and all relevant GHGs. An accuracy assessment of the initial classification was conducted with the use of reference data. The overall classification accuracy of the LULUCF mapping in 2014 was found to be 83\%, while the Kappa Index of Agreement (KIA) was 0.74. The developed GEOBIA model estimated for the year 2015 net annual GHG removals of $-1.613 \mathrm{Gg}$ of $\mathrm{CO} 2$ eq. (i.e., an approximate increase of $12.7 \%$ in removals between 2014 and 2015 ). Future work will involve further development of the model to account for all possible changes in the LULUCF sector and test the transferability of the model to other sites.
\end{abstract}

\section{INTRODUCTION}

Any process, activity, or mechanism, which removes a greenhouse gas from the atmosphere, is referred to as a sink under the United Nations Framework Convention on Climate Change (UNFCCC). Human activities can impact terrestrial sinks, through land use, land-use change and forestry (LULUCF) activities (Houghton et al., 2003; Watson et al., 2000). Accordingly, UNFCCC invited the Intergovernmental Panel on Climate Change (IPCC) to develop good practice guidance for LULUCF to assist countries in producing inventories for the land use, land-use change and forestry sector. As a result, the report on Good Practice Guidance for Land Use, Land-Use Change and Forestry (GPG-LULUCF) was developed to provide methods and good practice guidance for estimating, measuring, monitoring and reporting on carbon stock changes and greenhouse gas emissions from LULUCF activities under Article 3, paragraphs 3 and 4, and Articles 6 and 12 of the Kyoto Protocol (IPCC, 2003).

The annual GHG national inventory should be transparent, consistent, comparable, complete, and accurate. Also, it should be able to account all local changes in land use and forest cover. Accordingly, GPG-LULUCF describes three approaches for representing land areas (IPCC, 2003). Approach 1 identifies the total area of each individual land-use category, but does not provide detailed information on changes of area between categories and is not spatially explicit. Approach 2 introduces tracking of land-use changes between categories, while approach 3 extends approach 2 by allowing land-use changes to be tracked on a spatial basis. More specifically, the study area in approach 3 is subdivided into spatial units such as polygons appropriate to the scale of land-use variation. The spatial units must be utilized consistently over time and should be sampled using pre-existing map data with either a Geographic Information System (GIS) and/or in the field. Furthermore, observations may be acquired from satellite imagery, field visits, or questionnaires.

In this context, the use of objects (instead of pixels) that can facilitate the usage of, not only spectral, but also contextual and spatial information, considering the shape and texture of objects, as well as topological features (neighbour, superobjects, etc.) would be essential to be adopted in approach 3 .

\footnotetext{
* Corresponding author
} 
Geographic Object-Based Image Analysis (GEOBIA) has been devoted to developing automated methods of partitioning highresolution, remote-sensing imagery into meaningful imageobjects, and assessing their characteristics (Blaschke, 2010). GEOBIA of optical satellite data, ranging from low to very high spatial resolution, has been successfully used in several studies for mapping vegetation characteristics (Mitri et al., 2015; Mitri and Gitas, 2004) and landcover/land-use (Goodin et al., 2015). Based on the above, it would be worth exploring the capability of GEOBIA for the generation of time series of LULUCF area maps with the use of multi-temporal satellite imagery and field data acquisitions.

Previous national inventory reports (MoE/UNDP/GEF, 2011; $\mathrm{MoE} / \mathrm{UNDP}$, 1999) in Lebanon addressed GHG emissions/removals from the LULUCF sector mostly based on basic land-use data (i.e., tier 1 approach). Most recently, Lebanon's National GHG inventory for LULUCF involved the use of GEOBIA by employing multi-temporal satellite images for mapping specific changes in the landcover/land-use areas between consecutive years (MoE/UNDP/GEF, 2015). However, the automation of the process for mapping and assessing GHG emissions/removals was found to be essential in such analysis especially when the inventory covers an extensive multitemporal analysis of LULUCF. Accordingly, the aim of this work was to develop a semi-automated model for estimating GHG emissions and removals form the LULUCF sector at the local level. The specific objectives were to 1) map changes in land use and forest cover between two consecutive years, and 2) assess GHG emissions and removals from the LULUCF sector.

Data at fine scale, however, could directly account for units of land on which afforestation, reforestation or deforestation has occurred. Also, data available from satellite remote sensing can be combined with ancillary data to improve the accuracy of land-use classification.

\section{STUDY AREA AND DATASET DESCRIPTION}

\subsection{Study area}

The study area is the administrative area of Mounjiz (343' $\mathrm{N}$ and $36^{\circ} 14^{\prime}$ E) located in the north of Lebanon. The elevation ranges from $290 \mathrm{~m}$ to $350 \mathrm{~m}$ above sea level. Mounjiz has a population of 557 permanent inhabitants, counting 121 households. The surface of Mounjiz is 594 hectares, out of which 154 hectares are agricultural lands mainly consisting of vineyards and olive groves, and 39.2 hectares are occupied by a mixed forest of laurel trees (Laurus nobilis) and oaks (Quercus sp.). A total of ten hectares of grasslands were afforested in the year 2015 with stone pine (Pinus pinea), bay laurel (Laurus nobilis) and fruitful carob (Ceratonia silica) seedlings. The study area has a Mediterranean climate, which is characterized by mild rainy winters mainly between October and March, and hot dry summers between June and September. The registered mean annual precipitation is between $800-900 \mathrm{~mm}$ with an average number of 77 days of rain per year. The mean annual temperature is around $20^{\circ} \mathrm{C}(\mathrm{MOE} / \mathrm{UNDP}, 2011)$.

\subsection{Dataset description}

Satellite imagery namely SPOT ( $2.5 \mathrm{~m}$ colour) acquired on 15 9-2011 was collected. In addition, frequent field visits were conducted in 2014 and 2015 to survey the location and extent of afforested areas. A shapefile of afforested areas in 2015 was also employed. Field data observations of afforested areas comprised an exhaustive counting of planted seedlings, their survival rate, and their growth performance. Information about field wood gathering, estimated in 2014 at 80 tonnes, was collected from personal communications with the Municipality of Mounjiz.

\section{METHODOLOGY}

The methodology of work comprised the use of GEOBIA for mapping changes in the LULUCF sector and, subsequently, estimating GHG emissions/removals between two consecutive years, namely 2014 (i.e., reference year) and 2015. Ideally, changes in LULUCF between year 1 and year 2 are mapped using satellite images acquired on different dates, respectively. This approach was previously tested and adopted at the national level using spectral features from a series of Landsat Thematic Mapper images (MoE/UNDP/GEF, 2015).

In this work, Very High Resolution (VHR) SPOT imagery (2.5 $\mathrm{m}$ colour) was used in combination with field observations for mapping landcover/land-use in 2014. Changes in LULUCF between 2014 and 2015 were identified and mapped with the use of a shapefile produced from field data collection.

Consequently, GHG emissions and removals were estimated using customized features in GEOBIA and following the GPGLULUCF, which adopts a land-use category-based approach to estimate emissions/removals from all land categories and all relevant GHGs.

\subsection{Classification scheme}

The top-level land categories which were considered in the mapping were the following (IPCC, 2003): Forest land (F), Cropland (C), Grassland (G), Wetland (W), Settlement (S) and Other land (O). The abbreviations FF, CC, GG, WW, SS, OO denoted land-use categories undergoing no conversions; and the abbreviations LF, LG, LC, LW, LS, LO denoted land conversions to these land-use categories.

The inventory of year 2014 was considered a reference year and the areas extracted from the classification of the land cover/land use in 2014 were considered as reference values.

\subsection{GEOBIA}

The methodology of work involved 1) creating a classification scheme based on previously discussed land categories, 2) generating image objects or segments for subsequent classification, and 3) mapping the different land use and landuse change categories. The GEOBIA approach was adopted by employing the software "eCognition". More specifically, GEOBIA comprised two steps, namely, segmentation and classification of segmented images.

First, the segmentation of the VHR SPOT imagery at the pixels level was generated by using an average abstract scale of 10 and equal band weights. In general, the composition of homogeneity criterion included the following weights: $10 \%$ for shape, $90 \%$ for colour, and $50 \%$ for compactness. These same parameters were used to create two different levels of image segmentation, namely level 1 and level 2.

The land-use categories in 2014 were classified at level 1 using mainly layer mean values of the SPOT imagery (Figure 1). The 
classification was refined with the employment of land-use data collected in the field.

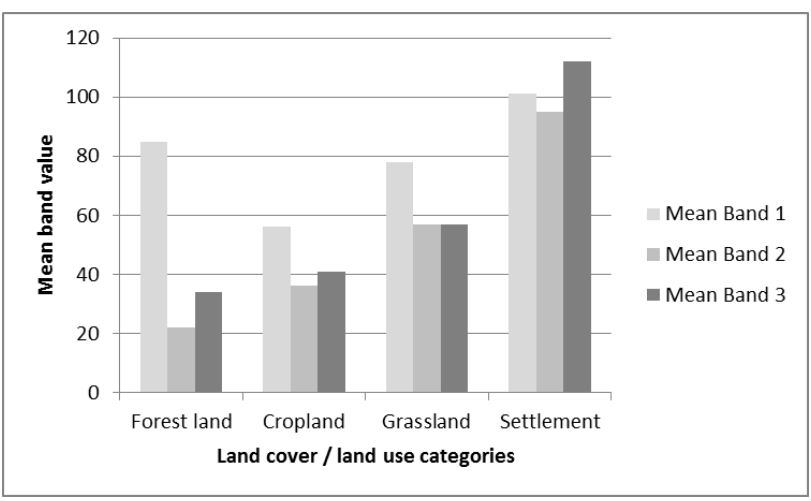

Figure 1. Mean band values per landcover/land-use categories

Changes in LULUCF in 2015 were classified at level 2. This incorporated mainly contextual features such as "existence of sub-objects" to classify conversion/non-conversion of lands. Field observations showed no significant changes (i.e., conversion of lands) other than the afforested areas. This was mainly attributed to the small size of the study area, the limited timeframe, and the location of the site in a rural area experiencing very little development. The feature "thematic object attribute" was used to classify afforested areas (i.e., objects) based on the employed shapefile of field-based data.

\subsection{Emission/removal factors}

Collection of the Emission/Removal (E/R) factors was done following two methodologies taking into account the availability and type of data (IPCC, 2003):

- Tier 1: IPCC GPG Default assumptions

- Tier 2: local data from surveys, and personal communications

Accordingly, customized features based on IPCC (2003) equations were built in "eCognition" for each of the following land-use categories: FF, CC, LF. Other land use categories are not reported as per GPG-LULUCF (IPCC, 2003). The summary equation, which estimates the annual emissions or removals from FF with respect to changes in carbon pools is given in Equation 1:

$\Delta \mathrm{C}_{\mathrm{FF}}=\left(\Delta \mathrm{C}_{\mathrm{FFLB}}+\Delta \mathrm{C}_{\mathrm{FFDOM}}+\Delta \mathrm{C}_{\mathrm{FFS} \text { oils }}\right)$

Equation 1

Where:

$\Delta \mathrm{CFF}_{\mathrm{FF}}=$ annual change in carbon stocks from forest land remaining forest land, tonnes ${\mathrm{C} \mathrm{yr}^{-1}}^{-1}$

$\Delta \mathrm{CFFLB}_{\mathrm{F}}=$ annual change in carbon stocks in living biomass (includes above- and belowground biomass) in forest land remaining forest land; tonnes $\mathrm{C} \mathrm{yr}^{-1}$

$\Delta$ CFFDOM $=$ annual change in carbon stocks in dead organic matter (includes dead wood and litter) in forest land remaining forest land; tonnes $\mathrm{C} \mathrm{yr}^{-1}$

$\Delta$ CFFSoils $=$ annual change in carbon stocks in soils in forest land remaining forest land; tonnes ${\mathrm{C} \mathrm{yr}^{-1}}^{-1}$

The summary equation, which estimates the annual change in carbon stocks in $\mathrm{CC}$ is given in Equation 2:
Where:

$\Delta \mathrm{C}_{\mathrm{CC}}=$ annual change in carbon stocks in cropland remaining cropland, tonnes $\mathrm{C} \mathrm{yr}^{-1}$

$\Delta \mathrm{C}_{\mathrm{CCLB}}=$ annual change in carbon stocks in living biomass, tonnes $\mathrm{C} \mathrm{yr}^{-1}$

$\Delta \mathrm{C}_{\mathrm{CCS} \text { oils }}=$ annual change in carbon stocks in soils, tonnes $\mathrm{Cyr}^{-1}$

Finally, the summary equation, which estimates the annual emissions or removals from LF with respect to changes in carbon pools is given in Equation 3:

\section{$\Delta \mathrm{C}_{\mathrm{LF}}=\Delta \mathrm{C}_{\mathrm{LFLB}}+\Delta \mathrm{C}_{\mathrm{LFDOM}}+\Delta \mathrm{C}_{\mathrm{LFS}}$ oils $\quad$ Equation 3}

\section{Where:}

$\Delta \mathrm{C}_{\mathrm{LF}}=$ annual change in carbon stocks in land converted to forest land, tonnes $\mathrm{C} \mathrm{yr}^{-1}$

$\Delta \mathrm{C}_{\mathrm{LFLB}}=$ annual change in carbon stocks in living biomass (includes above- and belowground biomass)

in land converted to forest land; tonnes $\mathrm{C} \mathrm{yr}^{-1}$

$\Delta \mathrm{C}_{\mathrm{LFDOM}}=$ annual change in carbon stocks in dead organic matter (includes dead wood and litter) in land converted to forest land; tonnes ${\mathrm{C} \mathrm{yr}^{-1}}^{-1}$

$\Delta \mathrm{C}_{\mathrm{LFSoil}} \mathrm{S}=$ annual change in carbon stocks in soils in land converted to forest land; tonnes ${\mathrm{C} \mathrm{yr}^{-1}}^{-1}$

\subsection{Accuracy assessment}

Mapping accuracy was assessed by means of ground-reference data. Accordingly, accuracy assessment of the initial classification was conducted with the combined use of field observations and VHR satellite data. Stratified random sampling was applied using the ERDAS Imagine software. A total of 30 points were randomly generated and checked for classification accuracy using field observations and visual interpretation of the VHR satellite imagery.

\section{RESULTS AND DISCUSSION}

The classification results at level 1 (Figure 2) showed the following coverage percentage: settlement/urban (3.37\%), grassland $(58.9 \%)$, forest land $(11.78 \%)$, and cropland $(25.92 \%)$.

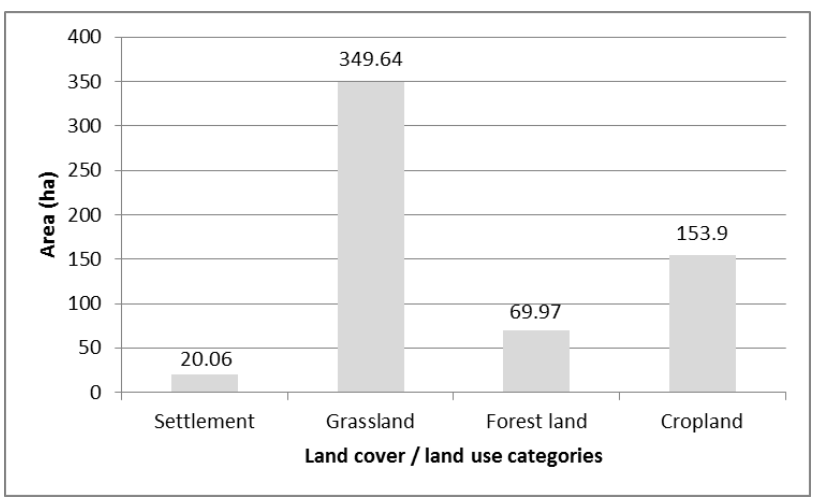

Figure 2. Areas of classified landcover/land-use categories at level 1 
The overall classification accuracy of the LULUCF mapping in 2014 was found to be $83 \%$, while the Kappa Index of Agreement (KIA) was 0.74. Main confusions were observed between the classes "Cropland" and "Grassland". This was mainly due to many agricultural lands being abandoned and vice versa.

The classification results at level 2 (Figure 3) indicated a total area of 10 ha classified as LF. More specifically, it was observed that these 10 ha of lands represented grassland areas, in the year 2014, converted to a forest land in 2015.

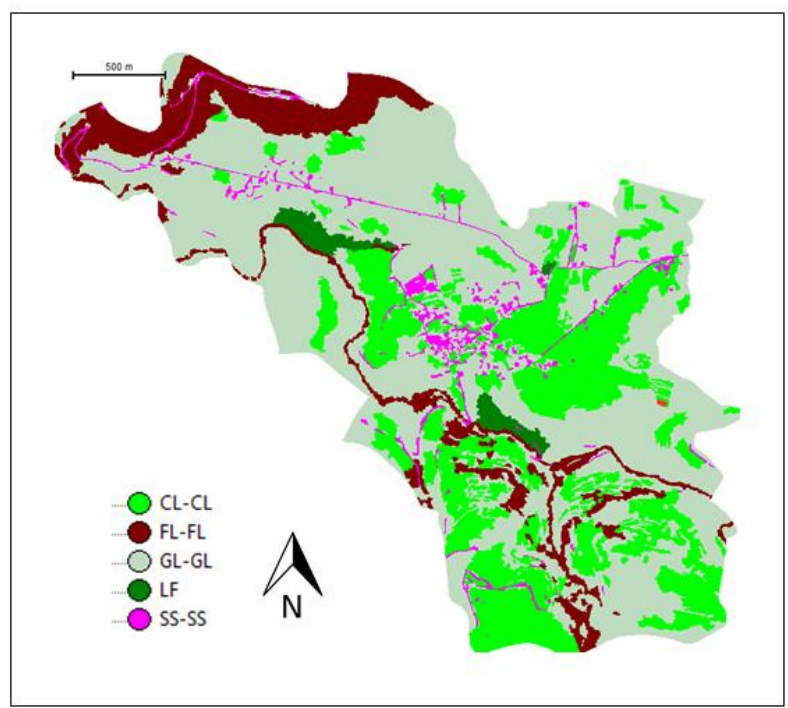

Figure 3. Classified map at level 2

Accordingly, the produced landcover/land-use statistics were used for assessing GHG emissions/removals in 2015 (Table 1).

\begin{tabular}{|l|l|}
\hline $\begin{array}{l}\text { Land-use categories undergoing either no } \\
\text { conversion or conversion between 2014 and 2015 }\end{array}$ & $\begin{array}{l}\text { Area } \\
\text { (ha) }\end{array}$ \\
\hline Forest land remaining forest land (FF) & 69.97 \\
\hline Grassland remaining grassland (GG) & 339.64 \\
\hline Cropland remaining cropland (CC) & 153.9 \\
\hline Settlement remaining settlement (SS) & 20.06 \\
\hline Grassland converted to forest land (LF) & 10 \\
\hline Total & 593.57 \\
\hline
\end{tabular}

Table 1. Changes in Land-use areas

The classified map was exported by integrating the previously build customized features for estimating GHG emissions/removals in each image object. As a result, the share of $\mathrm{CO}_{2}$ removals in 2014 was automatically calculated as follows: $-0.494956 \mathrm{Gg}$ of $\mathrm{CO}_{2}$ eq. (FF) and $-0.91245 \mathrm{Gg}$ of $\mathrm{CO}_{2}$ eq. (CC). The developed GEOBIA model estimated for the year 2015 net annual GHG removals of $-1.613 \mathrm{Gg}$ of $\mathrm{CO}_{2}$ eq. (i.e., an approximate increase of $12.7 \%$ in removals between 2014 and 2015).

\section{CONCLUSIONS}

In this work, changes in land use and forest cover between two consecutive years were mapped using the GEOBIA approach. Accordingly, GHG emissions/removals were automatically calculated with the use of emissions/removals equations that were integrated in the model. The semi-automated model is expected to provide a basic tool for potential use in the development of national inventory reports for estimating GHG emissions/removals from the LULUCF sector especially by allowing land-use changes to be tracked on a spatial basis. However, future work will include an extensive development of the model by 1) using multi-temporal satellite imagery of a larger area for mapping changes/no changes in landcover/land use, and 2) integrating additional customized features to address all possible land-use categories undergoing either conversion or non-conversion of lands.

\section{ACKNOWLEDGEMENTS}

This work was conducted within the framework of the ARDAC project (2014-2018), financed by the European Union, managed by the Lebanese Ministry of Agriculture and implemented by the University of Balamand in partnership with the Municipality of Mounjiz. The contents of this publication are the sole responsibility of the University of Balamand and can in no way be taken to reflect the views of the European Union.

\section{REFERENCES}

Blaschke, T., 2010. Object based image analysis for remote sensing, International Journal of Photogrammetry and Remote Sensing, 65, pp. 2-16.

Goodin, D., Anibas, K., and Bezymennyi, M. 2015. Mapping land cover and land use from object-based classification: an example from a comlex agricultural landscape. International journal of Remote Sensing, 36(18), pp. 4702-4723.

Houghton, R. A., and J. L. Hackler, 2003. Sources and sinks of carbon from land-use change in China, Global Biogeochemical Cycles, 17, 1034, doi:10.1029/2002GB001970.

IPCC, 2003. Good Practice Guidance for Land Use, Land-Use Change and Forestry (Edit. Penman et al.). Institute for Global Environmental Strategies (IGES) for the Intergovernmental Panel on Climate Change (IPCC), Kanagawa, Japan.

Mitri, G., and I. Gitas, 2004. A semi-automated object-oriented model for burned area mapping in the Mediterranean region using Landsat-TM imagery, International Journal of Wildland Fire. 13(3):367-376.

Mitri, G., Jazi, M., McWethy, D. 2015 Assessment of wildfire risk in Lebanon using Geographic Object-based image analysis. Photogrammetric Engineering \& Remote Sensing, 81(6), pp. 499-506.

MoE/UNDP, 2011. State and Trends of the Lebanese Environment (SOER) 2010, Beirut, Lebanon.

MoE/UNDP, 1999. Lebanon's Initial National Communication under the United Nations Framework Convention on Climate Change. Beirut, Lebanon.

MoE/UNDP/GEF, 2015. National Greenhouse Gas Inventory Report and Mitigation Analysis for the Land Use, Land-Use Change and Forestry Sector in Lebanon. Beirut, Lebanon.

MoE/UNDP/GEF, 2011. Lebanon's Second National Communication to the United Nations Framework Convention on Climate Change. Beirut. Lebanon.

Watson, R. T., Noble, I. R., Bolin, B. et al., 2000. Land use, land-use change, and forestry, Special Report of the IPCC, Cambridge University Press, 377, Cambridge, UK. 


\title{
MODELLING FOREST FIRE OCCURENCE IN LEBANON USING SOCIO-ECONOMIC AND BIOPHYSICAL VARIABLES IN OBJECT-BASED IMAGE ANALYSIS
}

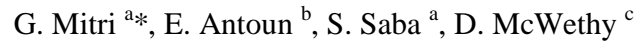 \\ ${ }^{a}$ Institute of the Environment, University of Balamand, Kelhat, El Koura, Lebanon - Email: george.mitri@balamand.edu.lb \\ ${ }^{\mathrm{b}}$ Directorate of Rural Development and Natural Resources, Ministry of Agriculture, Lebanon \\ ${ }^{\mathrm{c}}$ Department of Earth Sciences, Montana State University, Montana, USA
}

KEY WORDS: Mediterranean, fire, fire risk, wildland-urban interface, fire occurrence, socio-economic, biophysical, object-based

\begin{abstract}
:
Like many other countries in the Mediterranean, the occurrence and spread of forest fires in Lebanon are related to human activities. More specifically, landcover and land use changes (e.g., conversions of lands, abandonment of land and accumulation of fuel) driven by socio-economic changes occurring in the country have increased the probability of occurrence and spread especially in the Wildland-Urban Interface. The aim of this work was to model the influence of both socio-economic and biophysical variables on fire occurrence in Lebanon. The specific objectives were to 1) analyze socio-economic and biophysical drivers of forest fires, and 2) use object-based image analysis to derive a spatially explicit probability of fire occurrence across the country. Forward stepwise binary logistic regression analysis of 24 socio-economic and biophysical variables was used to predict wildfire occurrence. Spearman correlation analysis was conducted in order to eliminate multi-collinearity between selected variables. Eighty percent of the total number of administrative units was randomly selected for use in the development of the modelling, while the remaining $20 \%$ of units were used for testing and validating the final model. Object-based image analysis was used to map the spatial distribution of fire occurrence by modelling socio-economic and biophysical drivers including land-cover and land-use changes. The final map showed 5 different fire danger classes ranging from very low to very high. The quality of the classification results was evaluated and underand overestimations errors of fire occurrence were mapped. The accuracy of the fire occurrence mapping model was approximately $85 \%$ when tested on the validation data set. The probabilistic spatial output of the fire threat model was considered satisfactory given the challenges of using multi-source data in an object-based image analysis approach. Results suggest increasing the resolution of socio-economic data would improve modelling accuracy of fire occurrence in Lebanon.
\end{abstract}

\section{INTRODUCTION}

Many Mediterranean ecosystems are experiencing increases in fire frequency, intensity and severity (Vilar et al., 2014) resulting in negative ecological, social and economic consequences (Westerling et al., 2006; FAO, 2007). The increase in forest fires in the Mediterranean are mostly related to human activities as more than $90 \%$ of fires of known cause are related to deliberate or accidental human ignitions (Ganteaume et al., 2013).

Population expansion into the Wildland-Urban Interface (WUI) is increasing the risk of natural fire occurrence and spread (Koulelis et al., 2010). More specifically, socio-economic and demographic changes (i.e., rural exodus, abandonment of arable land, and increase in ex-urban populations) are leading to accumulation in forest fuels and the close proximity of communities to these fuels (Pausas et al., 2009; FAO, 2007).

In Lebanon, negligence was reported as the main cause of fires (i.e., $14 \%$ of reported fire causes), followed by human activities on natural landscapes (i.e., $9 \%$ of reported fire causes) although the causes of $\sim 67 \%$ of forest fires are not identified and reported (MOE/UOB, 2016). Landfill and agricultural practices represented $6 \%$ and $4 \%$, respectively, of the total fire causes.
In this context, it is important to identify the factors which contribute to wildfire occurrence and integrate these factors into a mathematical expression in order to evaluate and quantify fire threat level (Sebastian-Lopez et al., 2008). More specifically, knowledge of the impact of socio-economic factors and their contribution to wildfire occurrence and proximity to populations is essential for improved evaluation and management of wildfire risk. In recent years, many studies (De Torres Curth et al., 2012; Zumbrunnen et al., 2012; Martinez et al., 2009) have shown that socio-economic variables and human factors have a considerable impact on wildfire regime.

In order to accurately predict fire risk, new analytical methods need to be employed to evaluate multi-scale biophysical and socio-economic data (Mitri et al., 2014a). One powerful analysis approach that is useful for analysing multi-dimensional datasets is GEographic Object-Based Image Analysis (GEOBIA). This approach addresses the development of automated methods of generating meaningful image-objects and assessing their characteristics (Blaschke, 2010).

The aim of this work was to model the influence of both socioeconomic and biophysical factors on fire occurrence in Lebanon. The specific objectives were to 1) analyze socioeconomic and biophysical drivers of forest fires, and 2) use

\footnotetext{
* Corresponding author
} 
object-based image analysis to derive a spatially explicit probability of fire occurrence across the country.

\section{STUDY AREA AND DATASET DESCRIPTION}

\subsection{Study area}

The study area covers the country of Lebanon (with a tota surface of $10,452 \mathrm{~km}^{2}$ ) on the Eastern Mediterranean. It is divided into four distinct physiographic regions: the coastal plain, the Lebanon mountain range, the Beqaa Valley, and the Anti-Lebanon mountains (Mitri et al., 2015). Forests and other wooded land cover $24.5 \%$ of the Lebanese territory (MOE/UNDP/ECODIT, 2011).

The climate in Lebanon is characterized by dry summers extending from June to November (Salloum and Mitri, 2013) with average daytime temperatures above $30^{\circ} \mathrm{C}$, and little rain (i.e., around $90 \%$ of the total annual precipitation falling between November and March).

Mitri et al. (2014b) found that approximately $89 \%$ of the WUI in Lebanon occurs in areas classified between moderate to very high risk of fire spread and $37 \%$ of the roads occur in areas classified between moderate to very high risk of fire spread.

\section{Dataset description}

The administrative map of Lebanon used for this study comprises all administrative units at the level of cadastral unit representing the approximate extent of a municipality/village. Biophysical data included slope, aspect, and vegetation fuel type (Mitri et al., 2015). The Prometheus fuel type classification system (Lasaponara et al., 2006) was used for characterizing fuel type (Table 1).

\begin{tabular}{|c|c|c|}
\hline $\begin{array}{l}\text { Fuel } \\
\text { type }\end{array}$ & \% coverage & Description \\
\hline 1 & $\begin{array}{l}\text { Ground fuels } \\
(\text { cover }>50 \%)\end{array}$ & Grass \\
\hline 2 & $\begin{array}{l}\text { Surface fuels } \\
\text { (shrub cover > } \\
60 \% \text {; tree cover < } \\
50 \%)\end{array}$ & $\begin{array}{l}\text { Grassland, shrubland (smaller } \\
\text { than 0.3-0.6 m and with a high } \\
\text { percentage of grassland), and } \\
\text { clear-cuts, where slash was not } \\
\text { removed. }\end{array}$ \\
\hline 3 & $\begin{array}{l}\text { Medium-height } \\
\text { shrubs } \quad(\text { shrub } \\
\text { cover }>60 \% ; \text { tree } \\
\text { cover }<50 \%)\end{array}$ & Shrubs between 0.6 and $2.0 \mathrm{~m}$ \\
\hline 4 & $\begin{array}{l}\text { Tall shrubs }(\text { shrub } \\
\text { cover }>60 \% \text {; tree } \\
\text { cover }<50 \%)\end{array}$ & $\begin{array}{l}\text { High shrubs (between } 2.0 \text { and } \\
4.0 \mathrm{~m} \text { ) and young trees resulting } \\
\text { from natural regeneration or } \\
\text { forestation. }\end{array}$ \\
\hline 5 & $\begin{array}{l}\text { Tree stands }(>4 \\
\mathrm{m}) \text { with a clean } \\
\text { ground surface } \\
(\text { shrub cover < } \\
30 \%)\end{array}$ & $\begin{array}{l}\text { The ground fuel was removed } \\
\text { either by prescribed burning or } \\
\text { by mechanical means. This } \\
\text { situation may also occur in } \\
\text { closed canopies in which the } \\
\text { lack of sunlight inhibits the } \\
\text { growth of surface vegetation. }\end{array}$ \\
\hline 6 & $\begin{array}{l}\text { Tree } r \text { stands } \\
(>4 \mathrm{~m}) \quad \text { with } \\
\text { medium surface } \\
\text { fuels (shrub cover } \\
>30 \%)\end{array}$ & $\begin{array}{l}\text { The base of the canopies is well } \\
\text { above the surface fuel layer } \\
(>0.5 \mathrm{~m}) \text {. The fuel consists } \\
\text { essentially of small shrubs, } \\
\text { grass, litter, and duff. }\end{array}$ \\
\hline
\end{tabular}

\begin{tabular}{|c|c|c|}
\hline 7 & $\begin{array}{lr}\text { Tree } & \text { stands }(> \\
4 \mathrm{~m}) & \text { with heavy } \\
\text { surface } & \text { fuels } \\
(\text { shrub } & \text { cover }\end{array}$ & $\begin{array}{l}\text { Stands with a very dense surface } \\
\text { fuel layer and with a very small } \\
\text { vertical gap to the canopy base } \\
(<0.5 \mathrm{~m}) \text {. }\end{array}$ \\
\hline
\end{tabular}

Table 1. The Prometheus fuel type classes

Spatial distribution of fruit trees, mixed culture, field crop, olive trees, vineyard, and greenhouses were extracted from the landcover/land use map of Lebanon produced in 1998. Conversion of lands (i.e. cropland, forest, and grassland to settlement) between 1998 and 2005 were also acquired (MOE/UNDP/GEF, 2015). Animal production was obtained from the homogeneous agricultural zones data of the Ministry of Agriculture produced in 1998.

In addition, population density as acquired from the Central Administration of Statistics (CAS), distance to big cities (i.e., 1,000 to 10,000 inhabitants), distance to bigger cities (i.e., above 10,000 inhabitants), the agricultural-urban interface, and roads in agricultural lands were also acquired (Mitri et al., 2014b). The annual extent of burned areas from 1998 throughout 2012 was collected from MOE/UNDP/GEF (2015).

The list of datasets employed in this study, and their corresponding unit and sources were listed in Table 2.

\begin{tabular}{|c|c|c|}
\hline Description & Unit & Source/reference \\
\hline $\begin{array}{l}\text { Population } \\
\text { density }\end{array}$ & $\begin{array}{l}\text { Inhabitant } \\
/ \mathrm{km}^{2}\end{array}$ & $\begin{array}{lll}\text { Central Administration of } \\
\text { Statistics }\end{array}$ \\
\hline $\begin{array}{ll}\begin{array}{l}\text { Distance } \\
\text { big cities }\end{array} & \text { to } \\
(1,000 & \text { to } \\
10,000) & \end{array}$ & $\mathrm{Km}$ & Mitri et al, 2014b \\
\hline $\begin{array}{l}\text { Distance to } \\
\text { big cities } \\
(>10,000)\end{array}$ & $\mathrm{Km}$ & Mitri et al, 2014b \\
\hline $\begin{array}{l}\text { Roads } \\
\text { Density }\end{array}$ & $\mathrm{Km} / \mathrm{Km} 2$ & Mitri et al, 2014b \\
\hline $\begin{array}{l}\text { Agricultural } \\
\text { Urban } \\
\text { Interface } \\
\text { Length }\end{array}$ & $\mathrm{km}$ & Mitri et al, 2014b \\
\hline $\begin{array}{l}\text { Roads in } \\
\text { agricultural } \\
\text { land }\end{array}$ & $\mathrm{Km}$ & Mitri et al, 2014b \\
\hline Fruit trees & ha & $\begin{array}{l}1998 \text { landuse/landcover map- } \\
\text { MOE/UNDP/GEF, } 2015\end{array}$ \\
\hline Mixed culture & ha & $\begin{array}{l}1998 \text { landuse/landcover map- } \\
\text { MOE/UNDP/GEF, } 2015\end{array}$ \\
\hline Field Crop & ha & $\begin{array}{l}1998 \text { landuse/landcover map- } \\
\text { MOE/UNDP/GEF, } 2015\end{array}$ \\
\hline Olive trees & ha & $\begin{array}{l}1998 \text { landuse/landcover map- } \\
\text { MOE/UNDP/GEF, } 2015\end{array}$ \\
\hline Vineyard & ha & $\begin{array}{l}1998 \text { landuse/landcover map- } \\
\text { MOE/UNDP/GEF, } 2015\end{array}$ \\
\hline Greenhouses & ha & $\begin{array}{l}1998 \text { landuse/landcover map- } \\
\text { MOE/UNDP/GEF, } 2015\end{array}$ \\
\hline $\begin{array}{l}\text { Animal } \\
\text { production }\end{array}$ & $\begin{array}{l}\text { Big Cattle } \\
\text { Unit }\end{array}$ & Ministry of Agriculture \\
\hline $\begin{array}{l}\text { Conversion of } \\
\text { cropland to } \\
\text { settlement }\end{array}$ & ha & MOE/UNDP/GEF, 2015 \\
\hline Conversion of & ha & MOE/UNDP/GEF, 2015 \\
\hline
\end{tabular}




\begin{tabular}{|l|l|l|}
\hline $\begin{array}{l}\text { forest to } \\
\text { settlement }\end{array}$ & & \\
\hline $\begin{array}{l}\text { Conversion of } \\
\text { grassland to } \\
\text { settlement }\end{array}$ & ha & MOE/UNDP/GEF, 2015 \\
\hline Slope & $\%$ & Digital Elevation Model \\
\hline Aspect & degree & Digital Elevation Model \\
\hline $\begin{array}{l}\text { Fuel types (a } \\
\text { total of } 6\end{array}$ & $\begin{array}{l}\% \\
\text { coverage } \\
\text { variables) }\end{array}$ & Mitri et al. 2015 \\
\hline
\end{tabular}

Table 2. Datasets employed as independent variables

\section{METHODOLOGY}

\subsection{Logistic regression analysis}

A number of studies used logistic regression (Verdú et al., 2012; Catry et al., 2010; Vilar et al., 2010; Andrews et al., 2003) to model fire occurrence. This approach best describes binary variables (i.e., presence or absence of burned areas) and is considered a more flexible and statistically robust approach to use than multiple regression (Cohen et al., 2013). In this study, Forward Stepwise binary logistic regression was used in order to identify a group of predictors affecting wildfire occurrence in Lebanon.

The presence/absence of burned areas in each cadastral unit was considered the dependent variable and was coded in a binary format (i.e., 0 for no burned areas and 1 for burned areas). The analysis was carried out on randomly selected cadastral units representing $80 \%$ of the total number of units. The remaining $20 \%$ of cadastral units were employed for validation of the final model. Before running the regression analysis, Spearman correlation analysis was applied (Artusi et al., 2002) to eliminate highly collinear variables $(>0.60)$. Accordingly, the variable "distance to big cities $(1,000$ to 10,000 inhabitants" was removed. The final analysis was based on the following regression function:

$f(z)=1 /(1+e-z)$

Equation 1

Where $\mathrm{f}$ is the probability of occurrence of an event and $\mathrm{z}$ is the linear function of the independent variables:

$\mathrm{z}=\mathrm{b}_{0}+\mathrm{b}_{1} \mathrm{x}_{1}+\mathrm{b}_{2} \mathrm{x}_{2}+\ldots+\mathrm{b}_{\mathrm{n}} \mathrm{x}_{\mathrm{n}}$

Equation 2

Stepwise forward regression was used to identify the contribution of a group of predictors on wildfire occurrence. Eleven distinct regression models were generated and a final best fit model was selected (Martinez et al., 2008). More specifically, the introduction of variables in the model was based on Wald significance (i.e., $\mathrm{p}<0.05$ ) and their removal from the equation was applied when $\mathrm{p}>0.1$. The goodness of fit was estimated by the Nagelkerke's R2 test and the likelihood ratio $-2 \mathrm{LL}$ was used to test the predictive capability of the model.

In order to predict dichotomous fire occurrence $($ yes $=1$, no $=$ 0 ) cadastral units with a value exceeding 0.5 was considered equal to 1 (i.e., high risk of fire occurrence). Otherwise, cadastral units were considered low risk of fire occurrence (Hosmer et al., 2013).

\subsection{GEOBIA}

In previous study we used GEOBIA to map vegetation fuel type (Mitri et al., 2015) and conversion of lands (MOE/UNDP/GEF, 2015). In this work, GEOBIA was further developed to integrate additional socio-economic and biophysical datasets using the software ecognition.

Image segmentation was applied using the cadastral units thematic map. As a result, each of the generated polygon/segment corresponds to a cadastral unit. A customized feature was built to integrate the previously final best fit model in the mapping analysis. The final output of the logistic regression model for each image object was classified into one of the five classes of fire threat taking into account the equally divided ranges.

\subsection{Validation of the results}

The binary classification of image object (i.e., cadastral units) was compared with known fire occurrence values of the original dataset in order to assess over- or under-estimation of fire occurrence. Underestimations include those cadastral units where the model predicted low fire risk of occurrence and at least one known fire occurred, while overestimation include cadastral units where the model predicted high fire occurrence and no known fires occurred.

\section{RESULTS AND DISCUSSION}

The final logistic regression model included a total of 10 significant variables $(\mathrm{p}<0.05)$ for predicting fire occurrence:

$\mathrm{Z}=-2.078+0.045 \mathrm{x}_{1}+0.181 \mathrm{x}_{2}+0.347 \mathrm{x}_{3}+0.040 \mathrm{x}_{4}+0.014 \mathrm{x}_{5}+$ $0.094 \mathrm{x}_{6}+0.03 \mathrm{x}_{7}+0.042 \mathrm{x}_{8}+0.202 \mathrm{x}_{9}+0.004 \mathrm{x}_{10}$

Where

$\mathrm{x}_{1}$ : slope $-\mathrm{x}_{2}$ : area of grassland conversion to settlement (1998$2005)-x_{3}$ : area of forest conversion to settlement (1998-2005) $-\mathrm{x}_{4}$ : area of fuel type 6 and $7-\mathrm{x}_{5}$ : area of fuel type $4-\mathrm{x}_{6}$ : area of fuel type $3-\mathrm{x}_{7}$ : area of fuel type $2-\mathrm{x}_{8}$ : area of fuel type $1-$ $\mathrm{x}_{9}$ : length of Agricultural-Urban Interface $-\mathrm{x}_{10}$ : area of fruit trees cultivation.

The goodness of fit was estimated by the Nagelkerke's R2 test (i.e., 0.469) and the likelihood ratio -2LL (i.e., 943.815) to test the predictive capability of the model.

A $2 \times 2$ classification table (Table 3 ) of observed and predicted fire occurrence was developed.

\begin{tabular}{|c|c|c|c|c|c|c|c|}
\hline \multicolumn{2}{|c|}{ Observed } & \multicolumn{6}{|c|}{ Predicted } \\
\hline & & \multicolumn{3}{|c|}{ Model building data } & \multicolumn{3}{|c|}{ Validation data } \\
\hline & & \multicolumn{2}{|c|}{ Binary } & \multirow[t]{2}{*}{$\%$} & \multicolumn{2}{|c|}{ Binary } & \multirow[t]{2}{*}{$\%$} \\
\hline & & Low & High & & Low & High & \\
\hline \multirow[t]{2}{*}{ Binary } & 0 & 190 & 128 & 59.7 & 39 & 21 & 65 \\
\hline & 1 & 74 & 872 & 92.2 & 19 & 200 & 91.3 \\
\hline \multicolumn{4}{|c|}{ Overall \% } & 84.0 & & & 85.7 \\
\hline
\end{tabular}

Table 3. Comparison of predicted versus observed fire occurrence

Validation results showed that the model correctly predicted an average of $85.7 \%$ fire occurrences or non-occurrences. More 
specifically, $91.3 \%$ of fire occurrences were correctly predicted, while $65 \%$ of non-fire occurrences were correctly predicted.
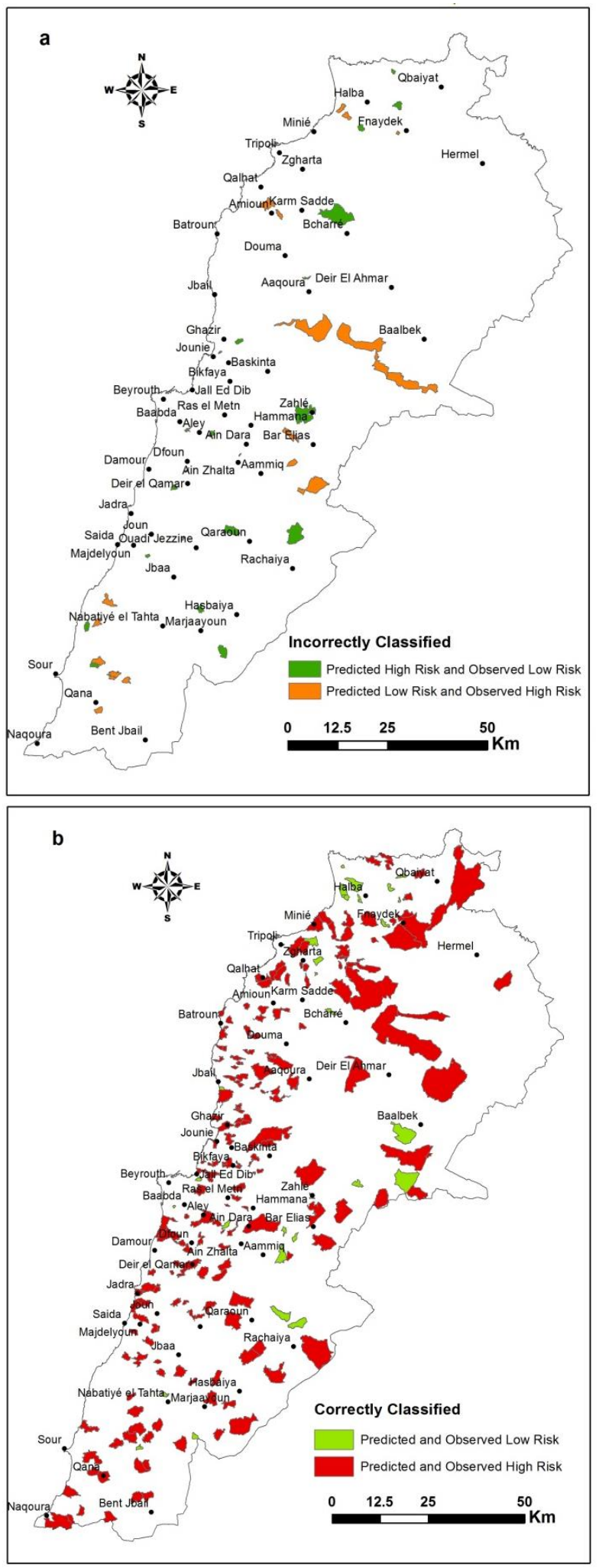

Figure 1. Cadastral units in which predicted fire occurrence did not match observed data (upper) and in which matched observed data (lower)

Figure 2 displays the spatial distribution of the model's final outcome at the level of the cadastral units. The range of estimated fire occurrence probabilities was divided into 5 equalsize classes. The majority of the cadastral units were characterized with a very high risk of fire occurrence. Cadastral units with very low to moderate risk of fire occurrence were mainly located in relatively highly urbanized or agricultural areas such as in the coastal zone and the central part of the Bekaa valley (i.e., inland).

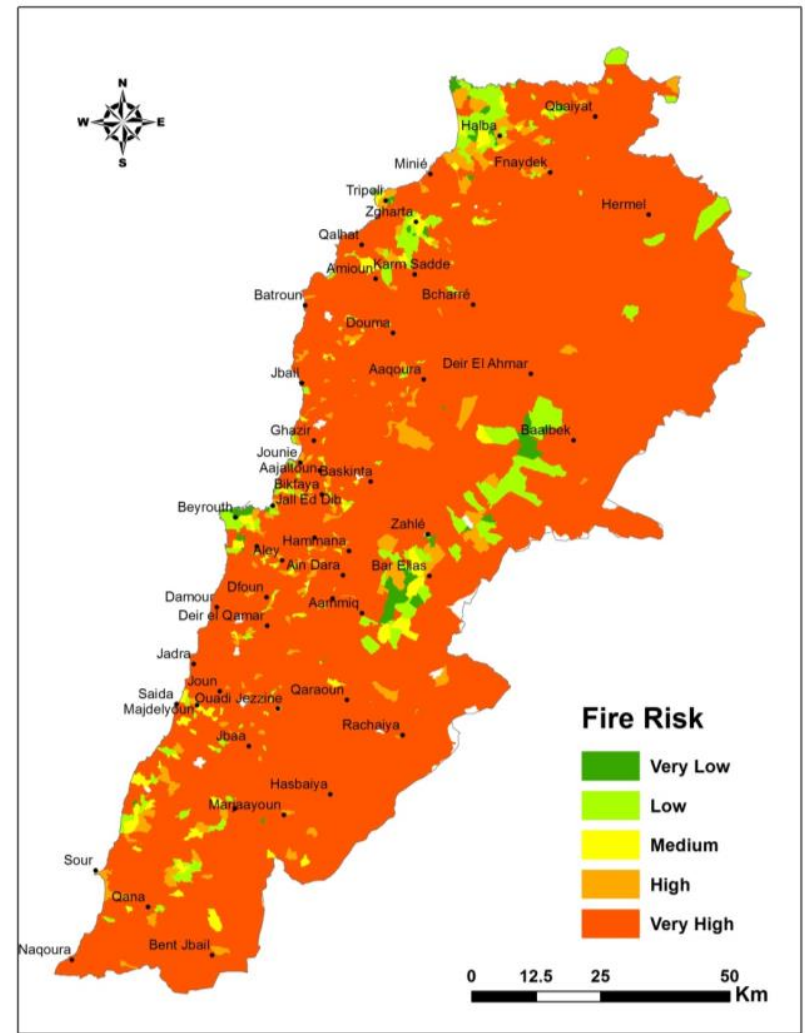

Figure 2. Spatial distribution of the logistic regression model outcome

The influence of the significant socio-economic variables in the model was identified through a sensitivity analysis. All the 10 variables were ranked according to a final score which was calculated by adding the ranks of the five evaluation criteria for significance (Wald test, standard coefficient (B), -2LL, Exp (B) and regression coefficient). The level of importance of the variables in the model increases with rank value (MartinezFernandez et al., 2013). Results showed that conversion of forest to settlement was considered as the most significant socio-economic variable increasing fire occurrence followed by the length $(\mathrm{km})$ of Agricultural-Urban Interface, conversion of grassland to settlement, and cultivation of fruit trees.

The final logistic regression model was evaluated in GEOBIA for analysis of multi-source socio-economic and biophysical spatial data. This allowed the assessment of fire occurrence at the level of individual polygons (i.e., cadastral units). GEOBIA was previously conducted to map landcover/landuse changes and vegetation fuel type with the use of satellite imagery (Mitri et al., 2015). It was used here to further integrate socioeconomic data to predict fire risk at the cadastral level. This approach showed that GEOBIA can serve as a tool for integrated analysis and assessment of fire occurrence for multiple purposes and scales. 


\section{CONCLUSIONS}

In this study, forward stepwise binary logistic regression analysis was conducted to assess fire occurrence using 24 socio economic and biophysical variables. A total of 10 socioeconomic and biophysical variables were finally used in the best performing predictive model. The resulting final logistic regression model was evaluated in GEOBIA to derive a spatially explicit probability of fire occurrence across the country.

Model validation showed an average accuracy of $85.7 \%$ in predicting fire occurrence. Socio-economic variables including conversion of forest to settlement, length of Agricultural-Urban Interface, conversion of grassland to settlement, and the cultivation of fruit trees were found to significantly contribute to increased probability of fire occurrence. The primary biophysical variables contributing to an increased probability of fire occurrence included fuel type and slope conditions.

Future work focusing on integrating higher-resolution socioeconomic datasets at the cadastral unit will help improve the accuracy of spatially-explicit mapping of fire occurrence across the Lebanon. GEOBIA serves as an essential tool for integrating multiple high-level datasets to predict the spatial probability of fire occurrence.

\section{ACKNOWLEDGEMENTS}

This work was published in association with the project "Towards a Better Assessment and Management of Wildfire Risk in the Wildland-Urban Interface in Lebanon: Gaining from the US experience" supported by the Partnerships for Enhanced Engagement in Research (PEER), sponsored by USAID. The contents do not necessarily reflect the views of USAID or the United States Government.

\section{REFERENCES}

Andrews, P. L., Loftsgaarden, D. O., and Bradshaw, L. S. 2003. Evaluation of fire danger rating indexes using logistic regression and percentile analysis. International Journal of Wildland Fire, 12(2), 213-226.

Artusi, R., Verderio, P., \& Marubini, E. 2002. Bravais-Pearson and Spearman correlation coefficients: meaning, test of hypothesis and confidence interval. International Journal of Biological Markers, 17(2), 148-151.

Blaschke, T., 2010. Object based image analysis for remote sensing. International Journal of Photogrammetry and Remote Sensing, 65, pp. 2-16.

Catry, F. X., Rego, F. C., Bação, F. L., \& Moreira, F. 2010. Modeling and mapping wildfire ignition risk in Portugal. International Journal of Wildland Fire, 18(8), 921931.

Cohen, J., Cohen, P., West, S. G., and Aiken, L. S. 2013. Applied multiple regression/correlation analysis for the behavioral sciences. Routledge.

De Torres Curth, M., Biscayart, C., Ghermandi, L., and Pfister, G. 2012. Wildland-Urban Interface fires and socioeconomic conditions: a case study of a Northwestern Patagonia City. Environmental Management 49(4): 876-891.

FAO 2007. Fire management global assessment. A thematic study prepared in the framework of the Global Forest Resources Assessment 2005. FAO Forestry Paper 151. Food and Agriculture Organization for the United Nations: Rome
Available at http://www.fao.org/forestry/fra2005/en [Verified on 24-5-2016].

Ganteaume, A., Camia, A., Jappiot, M., San-Miguel-Ayanz, J., Long-Fournel, M., Lampin, C. 2013. A Review of the Main Driving Factors of Forest Fire Ignition Over Europe. Environmental Management, 51(3), 651-662.

Hosmer Jr, D. W., Lemeshow, S., and Sturdivant, R. X. 2013. Applied logistic regression. John Wiley \& Sons.

Koulelis, P., and Mitsopoulos, I. 2010. A Study on the Socioeconomic Factors Influencing Wildfire Occurrence in Mediterranean Basin Countries. In. Proceedings of the Third International Symposium on Fire Economics, Planning, and Policy: Common Approaches and Problems (Vol. 227, p. 314). DIANE Publishing.

Lasaponara, R., A. Lanorte, and S. Pignatti, 2006. Characterization and Mapping of Fuel Types for the Mediterranean Ecosystems of Pollino National Park in Southern Italy by Using Hyperspectral MIVIS Data. Earth Interactions, 10(13): 1-11.

Martínez-Fernández, J., Chuvieco, E., \&Koutsias, N. 2013.Modelling long-term fire occurrence factors in Spain by accounting for local variations with geographically weighted regression. Natural Hazards and Earth System Science, 13(2), 311-327.

Martínez, J., Vega-Garcia, C., and Chuvieco, E. 2009. Humancaused wildfire risk rating for prevention planning in Spain. Journal of Environmental Management, 90(2), 12411252.

Martínez, J., Chuvieco, E., \& Martín, P. 2008. Estimation of risk factors of human ignition of fires in Spain by means of logistic regression. In Proceedings of the Second International Symposium on Fire Economics, Planning, and Policy: A Global View, Gen. Tech. Rep. PSW-GTR-208, pp. 265-278.

Mitri, G., Jazi, M., McWethy, D. 2015. Assessment of wildfire risk in Lebanon using Geographic Object-based image analysis. Photogrammetric Engineering \& Remote Sensing. Vol. 81, No. 6, June 2015, pp. 499-506.

Mitri, G., Nader, M., Van der Molen, I. and Lovett, J. 2014a. Evaluating exposure to land degradation in association with repetitive armed conflicts in North-Lebanon using multitemporal satellite data. Environmental Monitoring and Assessment. Volume 186, Issue 11, Page 7655-7672. http://dx.doi.org/10.1007/s10661-014-3957-5.

Mitri, G., Jazi, M., Antoun, E., and McWethy, D. 2014b. Managing wildfire risk in Lebanon. University of Balamand. Kelhat, El Koura, Lebanon.

MoE/UNDP/GEF (2015). National Greenhouse Gas Inventory Report and Mitigation Analysis for the Land Use, Land-Use Change and Forestry Sector in Lebanon. Beirut, Lebanon.

MOE/UNDP/ECODIT, 2011. State and Trends of the Lebanese Environment 2010, Ministry of Environment, Beirut, Lebanon. MOE/UOB. 2016. State of Lebanon's wildfire in 2015. A joint report of the Ministry of Environment and the University of Balamand, Beirut, Lebanon.

Pausas, J. G., Llovet, J., Rodrigo, A., \& Vallejo, R. (2009). Are wildfires a disaster in the Mediterranean basin? A review. International Journal of Wildland Fire, 17(6), 713-723. Salloum, L. and Mitri, G. 2014. Assessing the temporal pattern of fire activity and weather variability in Lebanon. International Journal of Wildland Fire. Volume 23(4): 503-509. http://dx.doi.org/10.1071/WF12101.

Sebastián-López, A., Salvador-Civil, R., Gonzalo-Jiménez, J., \& San Miguel-Ayanz, J. 2008. Integration of socio-economic and environmental variables for modelling long-term fire danger in Southern Europe. European Journal of Forest Research, 127(2), 149-163. 
Verdú, F., Salas, J., \& Vega-García, C. 2012. A multivariate analysis of biophysical factors and forest fires in Spain, 19912005. International Journal of Wildland Fire, 21(5), 498-509.

Vilar, L. Camia, A., San-Miguel-Ayanz, J. 2014. Modelling socio-economic drivers of forest fires in the Mediterranean Europe. Advances in forest fire research (Viegas, D.). Pp 18741882.

Vilar, L., Nieto, H., \& Martín, M. P. 2010. Integration of lightning-and human-caused wildfire occurrence models.Human and Ecological Risk Assessment: an International Journal, 16(2), 340-364.

Westerling A., Hidalgo H., Cayan D., Swetnam T. 2006. Warming and earlier spring increase western US forest wildfire activity. Science 313, 940-943.

Zumbrunnen, T., Menéndez, P., Bugmann, H., Conedera, M., Gimmi, U., and Bürgi, M. 2012. Human impacts on fire occurrence: a case study of hundred years of forest fires in a dry alpine valley in Switzerland. Regional Environmental Change, 12(4), 935-949. 


\title{
SYNERGY BETWEEN AERIAL IMAGERY AND LOW DENSITY POINT CLOUD FOR AUTOMATED IMAGE CLASSIFICATION AND POINT CLOUD DENSIFICATION
}

\author{
Hani Mohammed Badawy ${ }^{\mathrm{a},{ }^{*}}$, Adel Moussa ${ }^{\mathrm{a}, \mathrm{b}}$, Naser El-Sheimy ${ }^{\mathrm{a}}$ \\ ${ }^{a}$ Dept. of Geomatics Engineering, University of Calgary, Calgary, Canada (hmmohamm, amelsaye, elsheimy)@ucalgary.ca \\ ${ }^{\mathrm{b}}$ Dept. of Electrical Engineering, Port-Said University, Port-Said, Egypt
}

Commission VI, WG VI/4

KEY WORDS: Classification, Automated, LIDAR, Aerial, image, Point cloud

\begin{abstract}
:
In this paper a synergy scheme between aerial imagery and sparse LIDAR point clouds is proposed for an automated aerial image classification. In this scheme, a point cloud and an image are chosen for a certain urban area. The point cloud is automatically classified into buildings, vegetation and roads using PCA and intensity variation. Afterwards, a projection of the point cloud into an image is obtained, such that it is registered with the aerial image. The aerial image classifier is trained with the LIDAR classification result to generate an automated classifier for aerial images. The classifier is tested with another image to demonstrate its accuracy. Another benefit of the synergy proposed is to densify the planar patches of the low density point cloud using the segmented aerial image to help modelling applications achieve more precise boundaries.
\end{abstract}

\section{INTRODUCTION}

The vast number of available aerial images that cover most of the world to date is a great tool for object classification in different environments, such as urban areas, forests, etc. Most of the supervised classifiers require a training session with a large number of datasets to enhance the classification accuracy. Therefore, supervised training of datasets is a tedious task that requires human intervention. On the other hand, unsupervised classification could be achieved using 3D point clouds. However; these data are relatively expensive and are rarely updated on a regular basis, such that for a certain area, available high resolution point cloud data are fewer than their corresponding aerial imagery. Therefore; the problem of laborious supervised training of image classifier along with the problem of low density of rarely available point clouds, can be tackled simultaneously if both data are fused together.

In this paper a synergy scheme between aerial images and airborne laser data, of urban areas, is proposed to handle the limitations of both types of data. The proposed scheme comprises the following steps. The first step is to classify a low density point cloud while, at the same time, segmentation of an airborne image covering the same scene is being performed. Point cloud is automatically classified using Principal Component Analysis (PCA), Digital Terrain Model (DTM) generation and intensity (LIDAR reflectance) analysis. Image segmentation is achieved using region growing segmentation algorithm.

The second step is to register the airborne image with a projected image of the point cloud. Consequently, each segment in the image can be assigned the classification label of the corresponding point cloud. This process is equivalent to an automated training process of the image classifier. By repeating this process with different scenes, the image classifier is being enhanced.

The third step is the boundary regularized densification of the point cloud. Densification of the point cloud can be accomplished by adding 3D points, to the point cloud, that follow the geometrical constraints of the corresponding image segment boundary.
Ultimately, such synergy helps to classify image scenes that are not associated with point clouds using the gained information from the synergy. At the same time, the low density point cloud can be densified

The paper is organized as follows: the rest of section (1) summarizes that related work and the paper contribution. Section (2) describes the detailed synergy scheme. Section (3) demonstrates the data set and the result obtained. The final section is a conclusion followed by the proposed future work.

\subsection{Related work and paper contribution}

The fusion of LIDAR and aerial images has been discussed in several literatures for different purposes. For example, 3D modelling as in (Nakagawa \& Shibasaki, 2003), Building extraction as (Mao, Liu, \& Zeng, 2009) and (Zhou \& Zhou, 2014). (Mao et al., 2009) integrated LIDAR with CCD images on the same board and used LIDAR return to filter vegetation, and then the remaining points were clustered to determine the buildings' roofs and walls. (Zhou \& Zhou, 2014) utilized geometrical features and structures of houses to extract the building and create Digital Building Models (DBM).

In (Li, Li, Wang, Wang, \& Li, 2014) the aerial images with the normalized Digital Surface Model (nDSM) were used to filter the different objects in the urban areas.

Most of the authors targeting the integration of LIDAR with aerial images require both devices to be on the same board and to be utilized together for all images used in the classification process.

The main contribution of this paper is the synergy of LIDAR data with any aerial image that does not have to be on the same board, and generate a classifier that is trained by the classified LIDAR data. Consequently, the classifier can classify other aerial images without the need of further LIDAR data for the new image.

\section{SYNERGY SCHEME}

The Synergy scheme is illustrated in figure (1) below. 


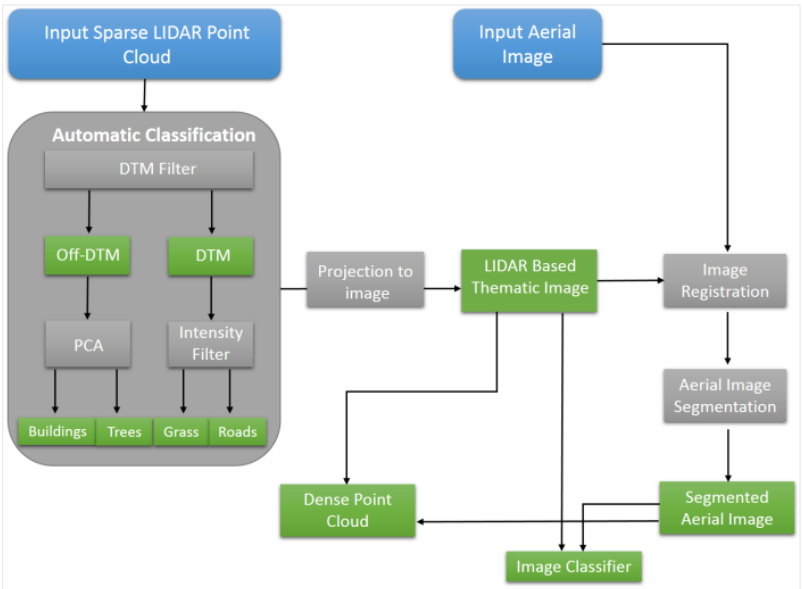

Figure (1): Synergy scheme for building unsupervised image classifier and densifying point cloud.

In the stage of generating an image classifier the input comprises an aerial image and airborne LIDAR point cloud. The point cloud is being classified (automatically) using the Digital Terrain Model (DTM) filter which discriminates the ground (DTM data) from the trees and buildings (off-DTM data). Principal Component Analysis (PCA) is then applied to the offDTM data to discriminate between the trees and buildings. Grass and roads are extracted from the land surface based on the LIDAR return intensity (reflectance).

After classification, the point cloud is projected to an image by eliminating the z-coordinate. A LIDAR based thematic map is obtained from this projection.

By default, the aerial image and the airborne point cloud are georeferenced. That simplifies the image registration between the thematic map and the aerial image.

The aerial image is segmented and information about each segment is fed from the LIDAR based thematic map to the generated image classifier. Simultaneously, the segmented image constrains the point cloud densification to certain shape boundaries.

In the following subsection each of these processes is discussed in detail.

\subsection{LIDAR data classification}

As discussed in the previous subsection, LIDAR data is classified via DTM filter, PCA and the intensity filter, in this paper we follow the work done in (Badawy, Moussa, \& ElSheimy, 2014) with a slight modification to the DTM filter.

\subsubsection{DTM filter}

DTM generation was discussed in many literature, such as (Badawy et al., 2014), (Sithole, 2001) and (Vosselman, 2000). The main idea in those papers was that the height gradient from one point to another is computed and tested against a threshold. In this paper, the same process is done but with a different approach. First, the data are divided by a plane passing through the z-axis. The point with minimum height $h_{0}$ is selected and the plane, with z-component equals to $h_{0}+\epsilon$, is used to divide the data. $\epsilon$ is a parameter that equals to few meters and is chosen according to the data characteristics.

If the height gradient is used in a local area of few meters it usually identifies points with sudden change in height as offDTM points. In case of a roof of a building with an area of a few meters; the height on such roof will have a zero gradient. Consequently; a point on the roof of a building could be falsely identified as DTM point while it actually belongs to the set of off-DTM points. Hence, the plane cut will group all the buildings points and their roofs in the off-DTM set of points except for few points below the cutting plane.

Now, these off-DTM points that are embedded in the DTM points can be filter with the following DTM filter. First the area is divided into grids of an equal size. This allows for the identification of local minima of the height in each grid as shown in figure (2).

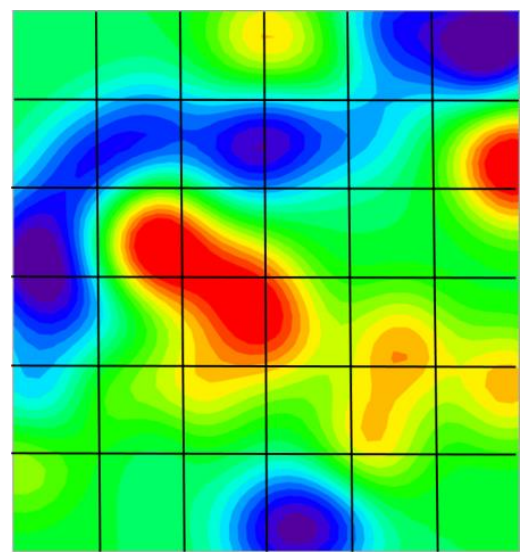

Figure (2): Equally divided grids with different height minima.

The gradient is computed for each point in the grid as compared to the grid's local minima. This can be written mathematically as (Badawy et al., 2014):

$$
\begin{aligned}
& p_{\text {min }}=\left\{p_{i} \in N_{i} \subset C: \forall p_{j} \in N_{i}: h_{p_{i}} \leq h_{p_{j}}\right\} \\
& D T M=\left\{p_{j} \in N_{i} \subset C: h_{p_{j}}-h_{p_{m}} \leq \Delta h\left(d\left(p_{j}, p_{\text {min }}\right)\right)\right\}
\end{aligned}
$$

where $p_{\min }$ are the local minima (point with minimum height value) in the neighbourhood which is a subset of the point cloud $C$. The height function $\Delta h$ is a function of distance from the minima point $d\left(p_{j}, p_{\min }\right)$ and is defined to be $0.25 d$.

The DTM filter results in two data sets, the DTM data defining the ground of the test area, and the off-DTM. Off-DTM data are further classified using the PCA and DTM data are classified via the LIDAR intensity.

\subsubsection{PCA}

The principal component analysis (PCA) is well documented in the literature. In this paper, it is used to classify the off-DTM data into trees and buildings.

The data are organized via the $\mathrm{kD}$-tree data structure. The covariance matrix is computed for each subset of points in the $\mathrm{kD}$-tree search radius $R$. Covariance matrix determines the geometry at which the LIDAR points are spread. This can be inferred from the eigenvalues of the covariance matrix.

If the covariance matrix is denoted $C_{v}$ the eigenvalue problem is expressed as:

$$
\left(C_{v}-I \lambda\right) X=0
$$

where $I$ is the identity matrix, $\lambda$ is the eigenvalue corresponding to the eigenvector $X$.

Since the covariance matrix is $3 \times 3$, there will be three eigenvalues corresponding to three eigenvectors. If the largest eigenvalue is much larger than the two other eigenvalues, then the points are spread along a line (representing edges). Similarly, if the points are spread over a plane (roofs and walls), two eigenvalues will be close in value and larger than the third. 
If the three eigenvalues are close in values, the points are scattered in the 3D space (representing trees). These three conditions are summarized here:

1) Planar points, if $\lambda_{1} \approx \lambda_{2} \gg \lambda_{3}$.

2) Scattered points if $\lambda_{1} \approx \lambda_{2} \approx \lambda_{3}$.

3) Linear points, if $\lambda_{1} \gg \lambda_{2} \approx \lambda_{3}$.

Further details about PCA can be found in (Shi \& Zakhor, 2011) and (Carlberg, Gao, Chen, \& Zakhor, 2009).

\subsubsection{Intensity Filter}

LIDAR data are usually combined with the intensity (reflectance) of the reflected laser beam. The reflectance is dependent on the object's material. Typically, vegetation has larger reflectance than roads. Therefore, based on the reflectance, one can classify the DTM data into roads and grass. Since the DTM is either roads or grass and their return will be relatively constant, the histogram of the reflectance will normally show two peaks. The peak at larger reflectance corresponds to the vegetation and the other corresponds to the roads. There might be other small peaks which will be ignored and classified as ambiguous objects. These objects do not contribute to the training process of the classifier.

\subsection{Projection of the LIDAR data to an image}

The LIDAR data are projected to an image by eliminating the $\mathrm{z}$ coordinate and using the $(x, y)$ coordinates of the LIDAR data and projected to an image, where a transformation is performed to map from the $(x, y)$ ground coordinate to $\left(x_{p}, y_{p}\right)$ image coordinates. The equations of projection used here are discussed in (Mohammed, 2015) and are listed as:

$$
\begin{array}{r}
\left(x_{k}, y_{k}\right)=P_{k}(X, Y) * S \\
\left(x^{\prime}, y^{\prime}\right)=\left(x_{k}-x_{m}, y_{k}-y_{m}\right) \\
i=-x_{k}+\frac{r}{2} \\
j=y_{k}+\frac{c}{2} \\
f(i, j)=R G B_{P_{k}}
\end{array}
$$

The point cloud is first scaled when multiplied by the scale factor. Then the points are shifted to the centre by subtracting the centre of mass of the point cloud from each point. Afterwards, the indices of the pixel representing the point in the point cloud are obtained from equations (6) and (7) where $c$ is the number of columns and $r$ is the number of rows in the generated image. The RGB values associated with the point cloud are assigned to the image at the corresponding pixels as in equation (8).

The registration of the two images (The projected LIDAR based image and the Aerial image) is based on their georeferencing information.

\subsection{Migrating the LiDAR based classification into spectral attributes of aerial images}

For training the classifier, spectral attributes of the image segments are fed as input features while the corresponding classes are obtained using the previous LiDAR based classified image. To build such input features/corresponding classes, the aerial image of the training scene is segmented using a region growing segmentation approach conducted over the $L * a * b *$ representation of the image. Since the spectral components of each segment are used directly as input features, the employed segmentation has been intentionally forced to have high number of small segments (over segmentation) to increase the spectral purity of the segments and to avoid generating segments of more than one dominant spectral tone.

After the segmentation of the aerial image, the class that has the highest count of pixels in each segment is assigned to this segment. The segments that has significantly mixed classes (percentage of pixels belongs to dominant class $<75 \%$ ) are excluded from the training data set. This exclusion helps to avoid the mixed areas typically found between trees and other classes of the scene. The mean values of the RGB and the $\mathrm{L} * \mathrm{a} * \mathrm{~b} *$ components of the accepted segments are computed to serve as input features.

\subsection{Aerial image classification algorithm}

The training features and classes obtained in the previous step are employed for classification of aerial images of nearby scenes in an object based image analysis fashion.

For a new aerial image to be classified, the image has to be segmented first and the spectral attributes are computed for each segment (mean RGB and L*a*b* components). These attributes are used as an input to $\mathrm{K}$ Nearest Neighbour (KNN) classifier using the training features and classes obtained in the previous step. Over segmentation of test scene has also been emphasized to avoid generating segments of many different spectral tones.

\subsection{Point cloud densification}

Densification of the point cloud is another advantage of the synergy between point clouds and images. As the aerial image is segmented, each segment can be used to fill the gaps in the projected LIDAR based image. Those gaps are a result of the projection of LIDAR points without interpolation. In fact filling the gaps from the information from the segmented image can be considered as an interpolation process. The interpolated points in the image space need to be transformed to 3D space to obtain a densified point cloud. Equations (4) through (8) are used to derive the inverse mapping equation to obtain the $\left(x_{k}, y_{k}\right)$ from the indices $(i, j)$. Obtaining the $\mathrm{z}$-coordinate can be done via fitting a plane using the neighbourhood points. The segmented image is used to regularize the densified point cloud.

\section{RESULT ANALYSIS AND DISCUSSION}

\subsection{Dataset}

The data used for the test are obtained for parts of the state of Indiana, USA. The aerial images are obtained from Google Earth. The Airborne LIDAR data (IndianaMap Framework Data) are obtained from OpenTopography source with links : http://www.indianamap.org http://dx.doi.org/10.5069/G9959FHZ

The aerial image which is used for the training process of the classifier is shown in figure (3) below. 


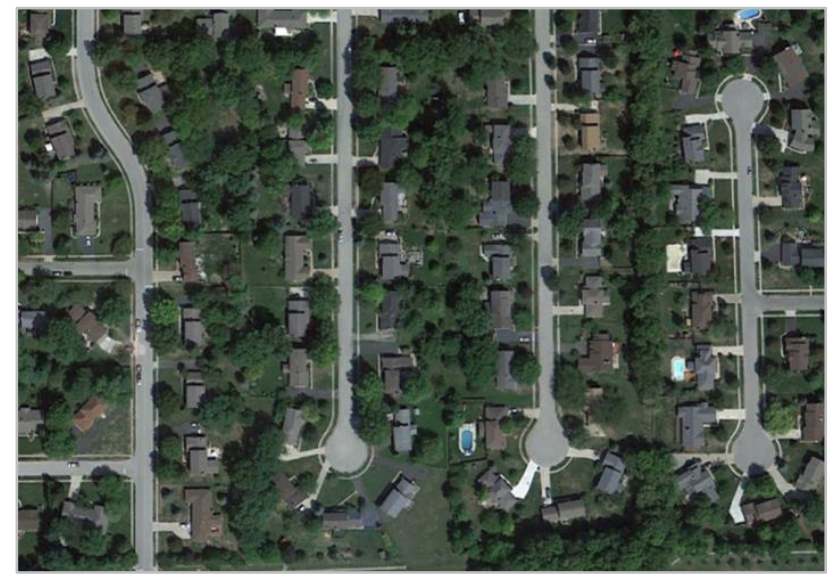

Figure (3): Aerial image of the original scene used for training the classifier.

\subsection{Result analysis}

\subsubsection{Lidar DTM extraction}

Figure (4) shows the result obtained for the DTM (ground) and off-DTM extraction. The points in red represent the off-DTM data, while the grey points represent the DTM data.

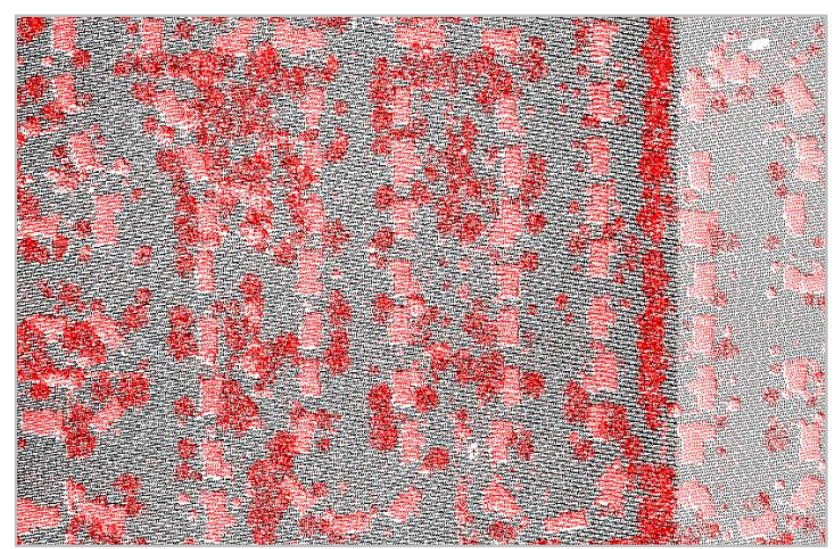

Figure (4): DTM and off-DTM data extracted using the DTM

\subsubsection{Intensity filter}

filter.

The DTM data are classified into roads, grass and ambiguous data based on the histogram of the LIDAR reflectance. In figure (5) the histogram is plotted with two main peaks one around the reflectance value of 20 (representing roads) and the other around the reflectance value of 222 (representing grass). A window is taken around both values as a threshold for the ground data classification. Between the two peaks there are few other peaks corresponding to data that are left unclassified. The reason for excluding those data points from the classification and training process is that they will be most probably misclassified. Therefore, ignoring those points is preferable.

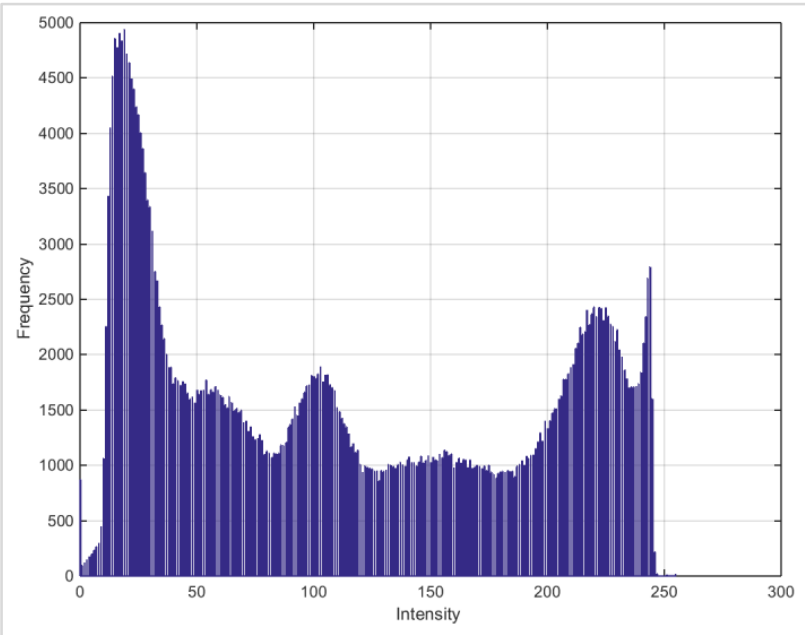

Figure (5): Histogram of the reflectance (intensity) of the LIDAR data.

\subsubsection{PCA}

PCA was used to determine the geometry of the objects in the point cloud. Then they are given the brown label if they are planar (representing buildings) or given a green label to represent the trees.

Figure (6) is a projection of the completely classified LIDAR data where the green colour represents trees, the brown colour represents buildings, the roads are represented with black colour, and the grass is represented with the yellow colour. The blue colour represents unclassified areas. It can be clearly noticed that the classification result agrees with the aerial image in figure (3).

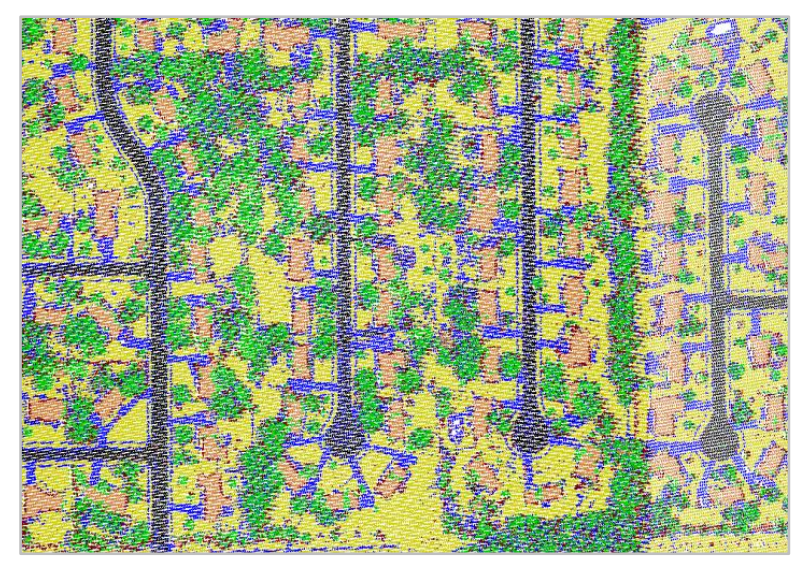

Figure (6): Projection of the LIDAR data after complete classification.

\subsubsection{Aerial image segmentation}

Figure (7) shows the segmented aerial image. The image is over-segmented so that when it is registered with the LIDAR based thematic map each segment lies inside the classified object in the point cloud. This ensures that segmented regions will contain spectral attributes from only one object. 


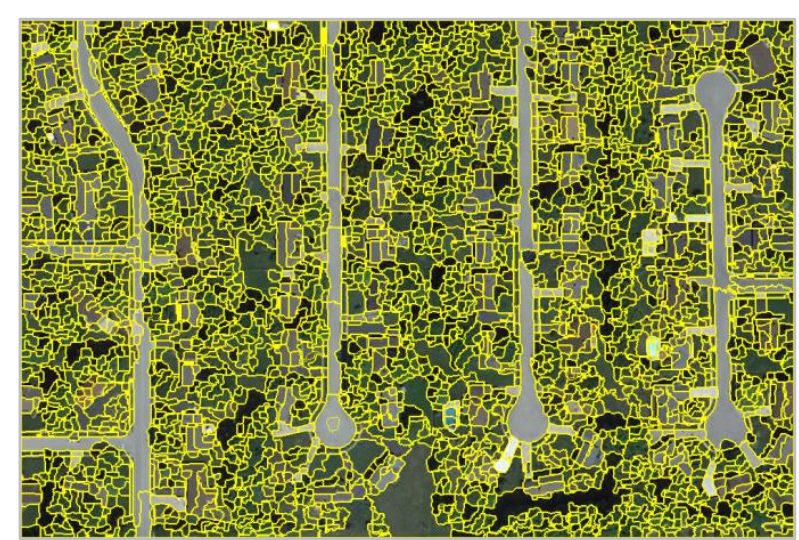

Figure (7): Aerial image over-segmented to avoid generating segments of many different spectral tones.

Each segment is recognized automatically using the LIDAR data classification. Those segments are then used to train the classifier and the classifier is tested with a test image.

\subsubsection{Test scene}

The test image is acquired for the same state of Indiana in USA to ensure that the spectral characteristics of the original and the test images are similar. Otherwise; there will be misclassification especially between roads and buildings.

Figure (9) shows the segmented aerial image for the test scene. The result of classification is shown in figures (10), (11) and (12) for the buildings, the vegetation and the roads.

The results exhibit very good classification accuracy especially in the classification of the vegetation area. Those results are also supported with the confusion matrix in table (1).

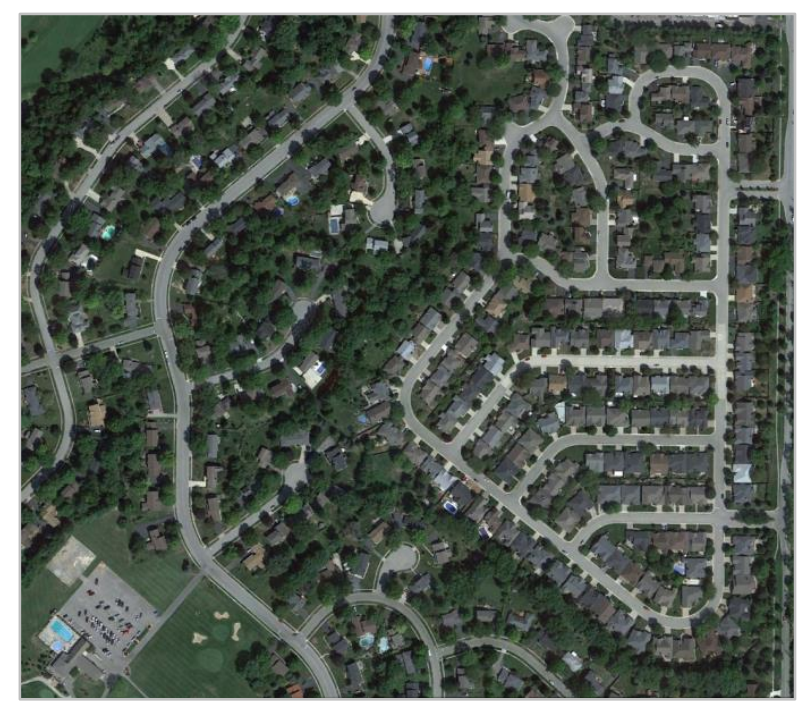

Figure (8): Test image at the same state of Indiana, USA.

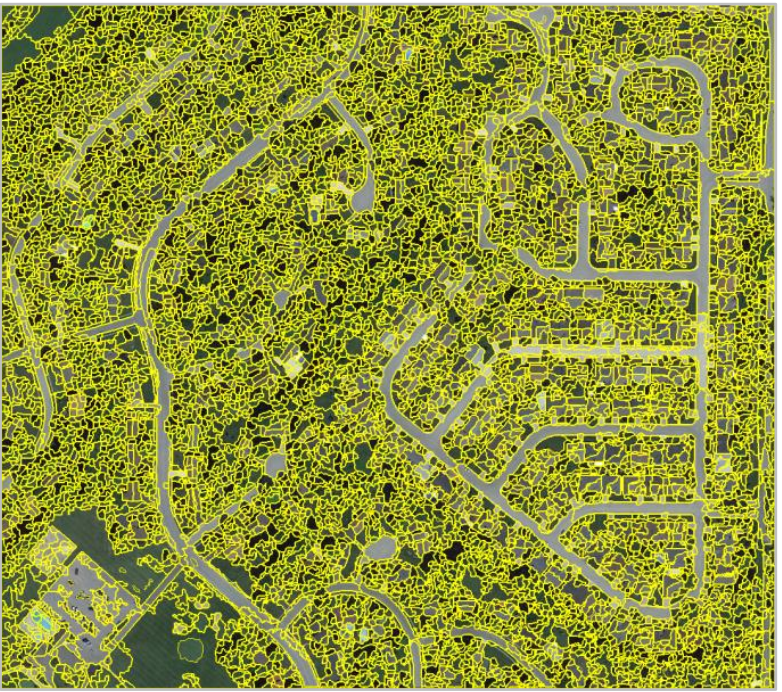

Figure (9): segmentation of the test scene that is used with the trained classifier.

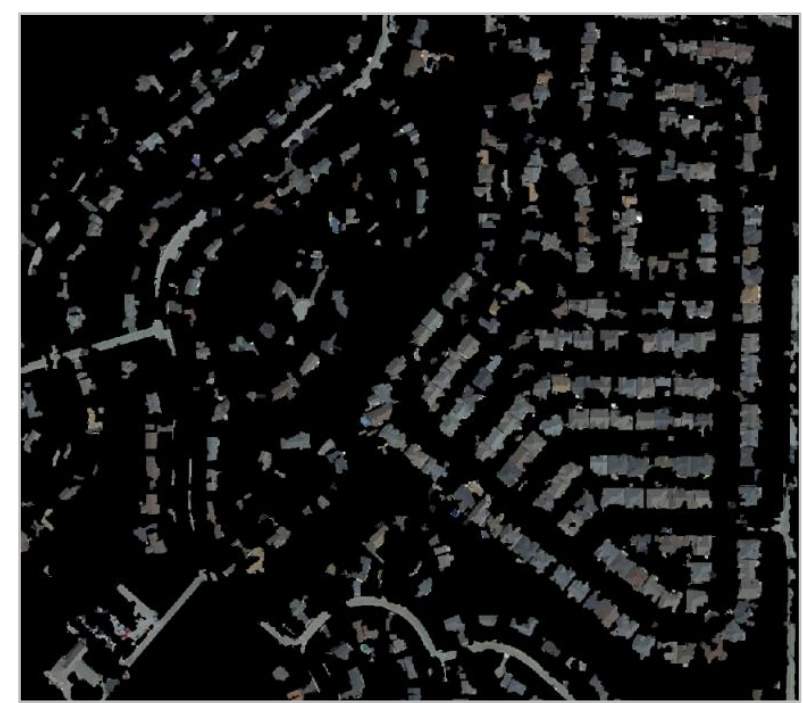

Figure (10): Buildings extracted from the test image using the trained classifier.

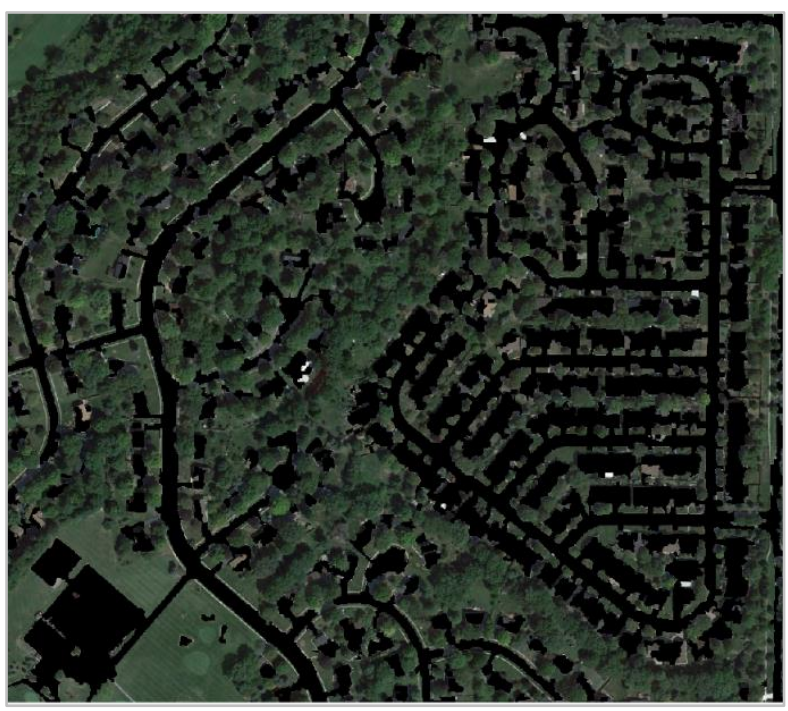

Figure (11): Vegetation extracted from the test image using the trained classifier. 


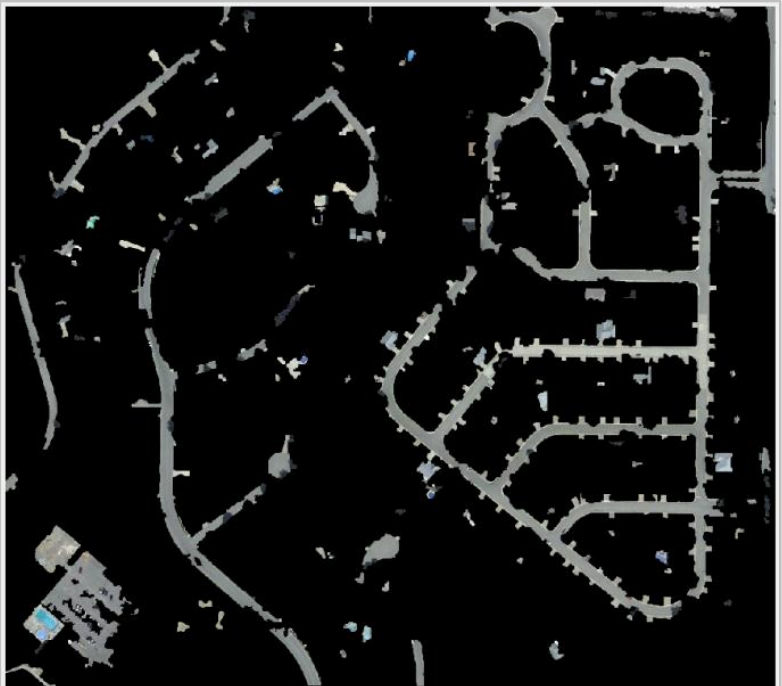

Figure 12: Roads extracted from the test image using the trained classifier.

Parts of the roads are misclassified as buildings due to the similarity of their spectral attributes in parts of the image.

\subsection{Classification quality assessment}

To assess the classification result, a manual classification was performed and used as a ground truth. The confusion matrix is listed in table (1). This matrix describes the amount of agreement between the classified objects in the test image and the ground truth.

Associated with the confusion matrix is the Cohen kappa coefficient $\kappa$ (Cohen, 1960) and (Monserud \& Leemans, 1992) given by:

$$
\kappa=\frac{P_{0}-P_{e}}{1-P_{e}}
$$

where

$$
\begin{gathered}
P_{0}=\sum p_{i i} \\
P_{e}=\sum_{i \neq j} p_{i} p_{j}
\end{gathered}
$$

And $p_{i}$ are the elements of the confusion matrix.

Table (1): Confusion matrix of the classification result.

\begin{tabular}{|c|c|c|c|c|}
\hline \multicolumn{2}{|c|}{ Actual (\%) } & \multicolumn{3}{c|}{ Classified } \\
\cline { 3 - 5 } & Buildings & vegetation & roads \\
\hline Buildings & 100 & 72.7977 & 26.5766 & 0.6257 \\
\hline Vegetation & 100 & 0 & 100 & 0 \\
\hline Roads & 100 & 19.7390 & 11.5642 & 68.6968 \\
\hline
\end{tabular}

The computed value of $\kappa$ for this test is $\kappa=0.7809$ which indicates a very good to excellent agreement.

Both of the confusion matrix and the value of $\kappa$ are in agreement with the results of figures (10) to (11). For example; it can be clearly seen from figure (11) that the vegetation is perfectly classified without misclassifications with roads or buildings due to sharp difference in spectral attributes between vegetation and the other objects. When looking at the confusion matrix, it is seen that $100 \%$ of the vegetation are classified as vegetation.

\subsection{Point cloud densification result}

The densification of the sparse point cloud was performed based on the segmented aerial image. As discussed earlier, segmented image offer a smoother boundaries for the densified point cloud. Moreover; the point cloud obtained can be correctly labelled using the aerial image.

Figure (13) demonstrates part of the point cloud for a building roof before and after densification. Distortion in the image can be seen clearly in the (a). While in the right image (b) the rood of the building is denser and more regular.

In figure (14) the result of densification is demonstrated with the surrounding environment of the building. The geometry of the building is more accurate with increased data points. The increase of regularized data points is useful if a surface mesh is to be computed, since gaps in the data points lead to inaccurate surface reconstruction. It can be seen also that the ambiguous points can be removed using the segmented image.

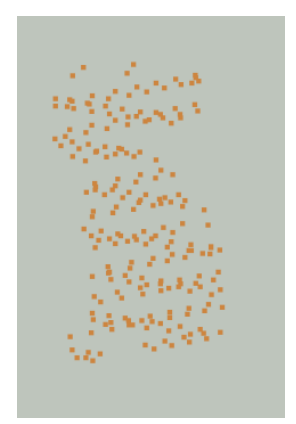

(a)

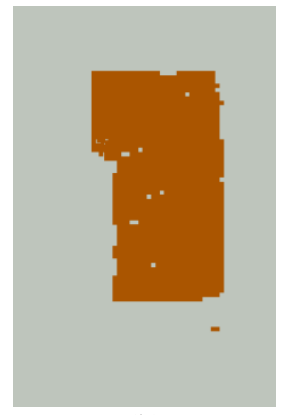

(b)
Figure (13): Part of the sparse point cloud of a building roof before densification in (a) and after densification (b).

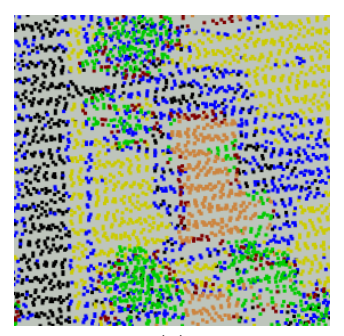

(a)

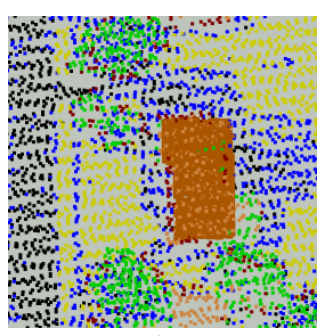

(b)
Figure (14): Part of the building with its surroundings before densification in (a) and after densification (b).

\section{CONCLUSION}

This paper investigates the possibility of using LIDAR point cloud with aerial images, mainly, to obtain unsupervised (automated) classification of aerial images and to densify the point cloud associated with the original image. Different classification steps have been employed on the LIDAR data, such as DTM filter, intensity filter and PCA to accurately classify the point cloud. This classification is used to train the image classifier. The classification result was shown to be very good. The densification algorithm proved to provide a regularized dense point cloud from a sparse point cloud. The limitation of the classification algorithm appears in the misclassification between buildings and roads due to the similarity of the spectral attributes between them. Shadow is another problem that faces the classification.

\section{ACKNOWLEDGEMENTS}

This work was supported by Dr. Naser El-Sheimy research funds from NSERC and Canada Research Chairs programs. This material is based on [data, processing] services provided by the OpenTopography Facility with support from the National Science Foundation under NSF Award Numbers 1226353 \& 1225810 . 
The 'IndianaMap Framework Data are found at: http://www.indianamap.org

http://dx.doi.org/10.5069/G9959FHZ

\section{REFERENCES}

Badawy, H. M., Moussa, A., \& El-Sheimy, N. (2014). Automatic Classification of Coarse Density Lidar Data in Urban Area, $\quad X L$ (June), 2500. http://doi.org/10.5194/isprsarchives-XL-5-77-2014

Carlberg, M., Gao, P., Chen, G., \& Zakhor, A. (2009). Classifying urban landscape in aerial lidar using 3D shape analysis. In Proceedings - International Conference on Image Processing, ICIP (pp. 1701-1704). http://doi.org/10.1109/ICIP.2009.5413385

Cohen, J. (1960). A coefficient of agreement of nominal scales. Educational and Psychological Measurement, 20(1), 3746. http://doi.org/10.1177/001316446002000104

Li, S., Li, Z., Wang, H., Wang, J., \& Li, L. (2014). Urban land cover classification using aerial LiDAR and CCD images. In 2014 IEEE Geoscience and Remote Sensing Symposium (pp. 1967-1970).

Mao, J., Liu, X., \& Zeng, Q. (2009). Building extraction by fusion of LIDAR data and aerial images. In 2009 Joint Urban Remote Sensing Event. http://doi.org/10.1109/URS.2009.5137631

Mohammed, H. (2015). Fusion of Terrestrial and Airborne Laser Data for 3D Modeling Applications. University of Calgary.

Monserud, R. A., \& Leemans, R. (1992). Comparing global vegetation maps with the Kappa statistic. Ecological Modelling, 62(4), 275-293. http://doi.org/10.1016/03043800(92)90003-W

Nakagawa, M., \& Shibasaki, R. (2003). Integrating high resolution air-borne linear CCD (TLS) imagery and LIDAR data. In 2nd GRSS/ISPRS Joint Workshop on Remote Sensing and Data Fusion over Urban Areas, $\begin{array}{llll}\text { URBAN } & 2003 & \text { 236-240). }\end{array}$ http://doi.org/10.1109/DFUA.2003.1219994

Shi, X., \& Zakhor, A. (2011). Fast approximation for geometric classification of LiDAR returns. Proceedings International Conference on Image Processing, ICIP, 2925-2928. http://doi.org/10.1109/ICIP.2011.6116272

Sithole, G. (2001). Filtering of laser altimetry data using a slope adaptive filter. In ... Archives of Photogrammetry Remote Sensing and ... (Vol. XXXIV, pp. 22-24). Retrieved from

http://lr.tudelft.nl/fileadmin/Faculteit/LR/Organisatie/Afd elingen_en_Leerstoelen/Afdeling_RS/Optical_and_Laser _Remote_Sensing/Publications/Papers/0182001/doc/sithole_annapolis.pdf

Vosselman, G. (2000). Slope based filtering of laser altimetry data. International Archives of Photogrammetry and Remote Sensing, Vol. 33, Part B3/2, 33(Part B3/2), 678684. http://doi.org/10.1016/S0924-2716(98)00009-4

Zhou, G., \& Zhou, X. (2014). Seamless fusion of LiDAR and aerial imagery for building extraction. IEEE Transactions on Geoscience and Remote Sensing, 52(11), 7393-7407. http://doi.org/10.1109/TGRS.2014.2311991 


\title{
ROBUSTNESS OF RULE SETS USING VHR IMAGERY TO DETECT INFORMAL SETTLEMENTS - A CASE OF MUMBAI, INDIA
}

\author{
V. Naorem ${ }^{\text {a }}$, M. Kuffer ${ }^{\text {a* }}$, J. Verplanke ${ }^{\mathrm{a}}$ and D. Kohli ${ }^{\mathrm{a}}$ \\ ${ }^{\text {a }}$ Faculty of Geo-Information Science and Earth Observation (ITC), University of Twente, PO Box 217, 7500AE Enschede, The \\ Netherlands - (v.naorem, m.kuffer, j.verplanke, d.kohli)@utwente.nl
}

KEY WORDS: informal settlements, slum ontology, robustness, rule set, OBIA, Mumbai, India

\begin{abstract}
:
Robust monitoring approaches for informal settlements using very high-resolution (VHR) satellite imagery can deliver essential information for supporting the formulation of pro-poor policies. Such information can complement census methods or participatory approaches. With the increasing availability of VHR satellite imagery, detection of the informal settlements benefits from the conceptualization of location-specific knowledge in the form of a locally-adapted generic slum ontology (GSO). In this study, we developed the local slum ontology for Mumbai, India, by incorporating local knowledge with image-based proxies. Then, we translated the local ontology into a rule set using Object Based Image Analysis (OBIA) to identify informal settlements by using spectral, spatial, geometric and texture measures. The method was applied to three subsets of a Worldview- 2 imagery. The robustness of the initial rule set was analysed with the help of membership functions. The results showed that the normalized difference ratio of near infrared (NIR) and blue band and grey level co-occurrence matrix (GLCM) features are most effective in all three subsets in the identification of informal settlements. The results suggest that the rule sets developed in this study can potentially be applied to other study areas of Worldview-2 imagery for informal settlements identification.
\end{abstract}

\section{INTRODUCTION}

\subsection{General Instructions}

The rapid growth of informal settlements in cities of the Global South is a cause of concern to local governments and a challenging task for the overall development of countries. On one hand, rapidly growing cities have high demand for workers in constructing infrastructures, retail and many other sectors. On the other hand, inhabitants of rural areas are attracted to cities because of employment and education opportunities and the general hope for improved living conditions. However, the high cost of living in formal areas and other social factors makes them to settle in informal areas commonly characterized by overcrowded, dilapidated housing and inadequate access to basic services (UN-Habitat, 2010). Moreover, locations of informal settlements are often at polluted and hazardous spots, e.g. areas close to rivers and places of unhygienic environment (Kit, Lüdeke, \& Reckien, 2012; Kohli, Sliuzas, Kerle, \& Stein, 2012; Kuffer, Pfeffer, \& Sliuzas, 2016). Eventually, all these factors severely affect the health conditions of the inhabitants of informal settlements(Subbaraman et al., 2014). As an initial step to tackle these problems in a decisive and effective manner there is a need to detect the location, boundaries and growth dynamics of informal settlements. This may provide important base data for planning and decision making to the concerned authorities. Allowing monitoring of development dynamics can assist in pro-poor policy formulation, as stepping stone towards inclusive development.

\subsection{Remote sensing for mapping informal areas}

Several methods like census methods, participatory mapping and advanced image analysis have been used to identify informal settlements. However, the utility of the census method is limited by the long temporal gap between two census surveys (Ebert, Kerle, \& Stein, 2009). Also, many settlements are not included in a census as they are not officially recognised (Nolan, 2015). The degree of the data aggregation can also be a problem (Kohli et al., 2012). Participatory mapping methods provide very rich ground information allowing incorporation of spatial and non-spatial data but are very time-consuming in large areas (Kohli et al., 2012). VHR images can help to outline informal settlements even in very heterogeneous areas and allow frequent mapping due to a high temporal resolution, which is of particular relevance for fast growing cities (Netzband \& Rahman, 2009 ).

OBIA has been successfully used in several studies to identify informal settlements in a fast and efficient way (e.g. Hofmann, Strobl, Blaschke, \& Kux, 2008; Kohli, Stein, Sliuzas, \& Kerle, 2015). Kohli et al. (2012) introduced the generic slum ontology (GSO) which defines the general characteristics of informal settlements and can be adapted and extended for operational tasks. The GSO supports the conceptualization of informal settlement characteristics for the creation of OBIA rule sets. However, one of the main limitations of OBIA is the robustness and transferability of rule sets across space and time. Rule sets commonly require extensive manual adaptation to obtain comparable results when applied to other images (even of the same city) (Hofmann, Blaschke, \& Strobl, 2011; Kohli, Warwadekar, Kerle, Sliuzas, \& Stein, 2013). Hofmann et al. (2011) defined robustness as the ability to generate comparable results on similar images with minimum adaptations. According to Hofmann (2014), robustness can be quantified by two factors, i.e., classification quality and deviation of the rule set, while five main deviations of the rule set exists:

- $\quad$ addition or removal of classes

- addition or removal of rules

\footnotetext{
* Corresponding author
} 
- changing logical operators

- changing relational operators

- changing the thresholds

Very few studies exist that focus on measuring the robustness of rule sets for mapping informal settlements. Thus there is a need to find the most robust features for mapping informal settlements. This study contributes in this direction by presenting a case of Mumbai, India.

\section{METHODOLOGY}

\subsection{Study area}

The study area in Mumbai, India has an urban population of 18.4 million according to the Indian Census 2011 (Government of India, 2015). The acquired WorldView-2 images (Pan: $0.5 \mathrm{~m}$ and Multispectral: $2 \mathrm{~m}$ ) covers the area surrounding the airport which contains large settlements (Figure 1) which have the typical features of informal settlements: small building size, high building density, organic pattern and diverse roof materials (Kuffer, Pfeffer, Sliuzas, \& Baud, 2016). Three subsets were chosen based on local knowledge to cover different morphologies and patterns, for instance, variability of shape and configuration.

- Subset 1 covers vegetation, river, formal buildings and Dharavi, which is known to be a large informal settlement.

- Subset 2 covers a mix of several informal and formal settlements.

- Subset 3 contains a mixture of vegetation, informal settlements located mainly close to old factories.

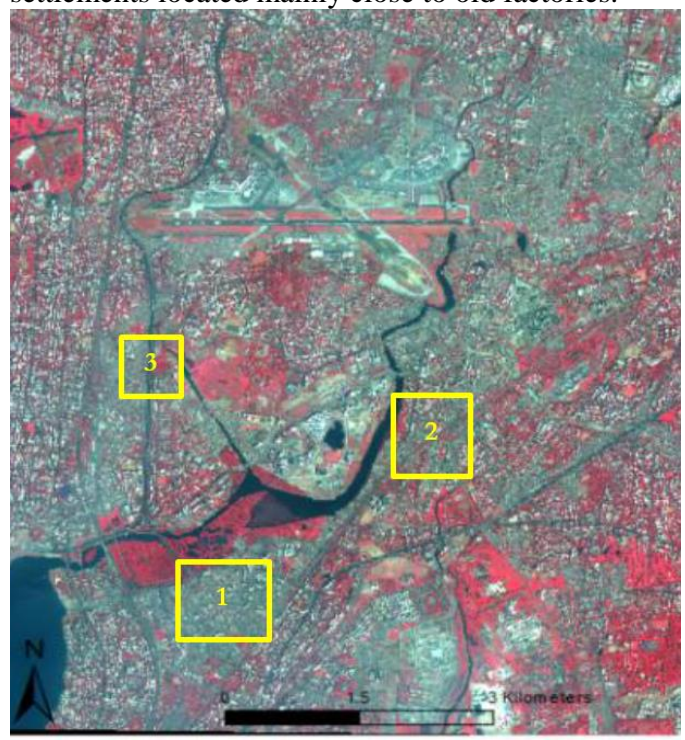

Figure1. Location of subsets (WorldView-2 image 2009)

Fieldwork was conducted in 2015. 150 ground control points were collected within the subsets using quota sampling.

\subsection{General Methodology}

Before performing OBIA, radiometric correction was conducted to ensure that the analysis was not much influenced by difference in atmospheric conditions using the FLAASH tool (Jinguo \& Zheng, 2008). For the translation of ontology and the development of rule sets, image analysis was done in two steps: segmentation and OBIA. The general overview of the methodology is given in Figure 2, details are explained in the following subsections.

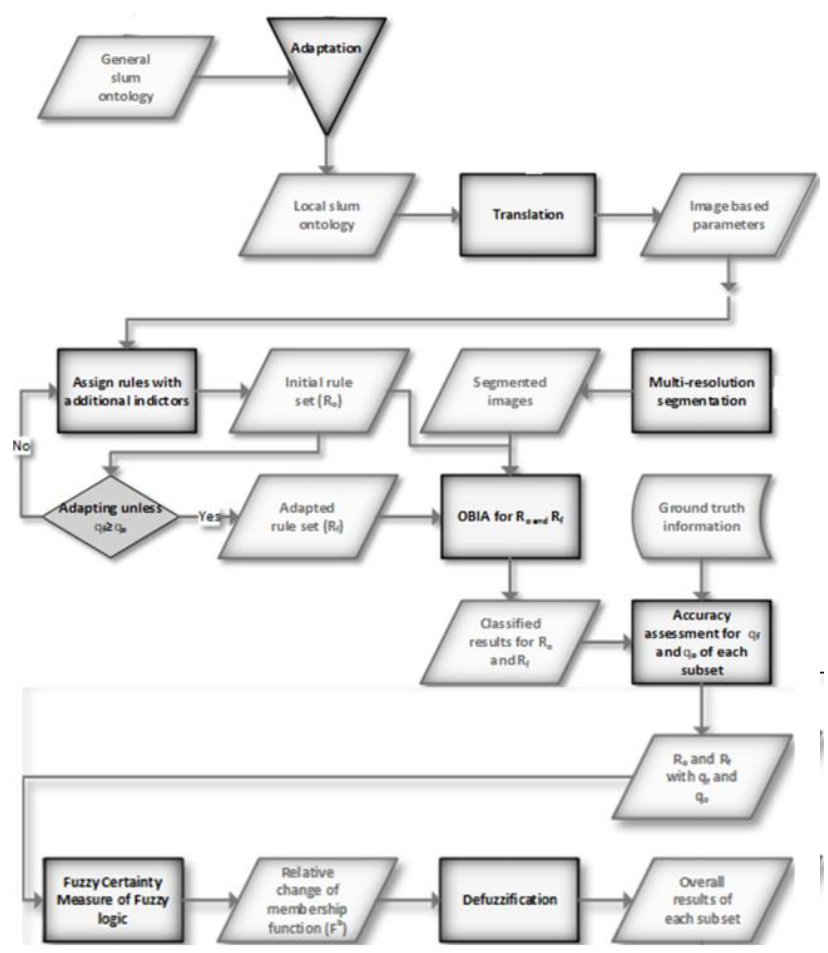

Figure 2. Overview of the methodology

\subsection{Development of the Local Ontology}

The local ontology describes the local features of informal settlements mainly based on morphological features (Kohli et al., 2012). A local ontology always needs to be developed since the GSO cannot be used for translating the image based parameters for classification directly. For this purpose, commonly expert knowledge is required. After obtaining local knowledge via a questionnaire from local experts, the main morphological indicators were conceptualized. These main indicators contributed to translate the local slum ontology into image based parameters. The local characteristics of informal settlements from the experts were organized according to the six general indicators given in GSO that are grouped at three spatial levels (i.e. object level, settlement and environs level).

\subsection{Image segmentation}

Before segmentation, the estimated scale parameter (ESP) tool for multiresolution segmentation was used to calculate the optimal scale parameter (Drăguț, Tiede, \& Levick, 2010). The tool measures the changing aspects of local variance (LV) from an object level to another so that the suitable scale parameter can be generated using rate of change (ROC):

$R O C=\left[\frac{L-(L-1)}{L-1}\right] * 100$

where $\quad \mathrm{L}=\mathrm{LV}$ at target level and

$$
\mathrm{L}-1=\mathrm{LV} \text { at next lower level. }
$$

In order to measure the segmentation quality of the study, we used the location discrepancy of a segmented object to a referenced object (Montaghi, Larsen, \& Greve, 2013), using forty manually delineated image objects for the assessment: 
$D_{s r}=\frac{1}{n} \sum_{i=0}^{n} \sqrt{\left(X_{s(i)}-X_{r}\right)^{2}+\left(Y_{s(i)}-Y_{r}\right)^{2}}$

where $D_{\text {sr }}$ is the average of the distance in the Euclidian plane between the centroid coordinates of the $\mathrm{i}^{\text {th }}$ segmented object $(\mathrm{Xs}(\mathrm{i})$ and $\mathrm{Ys}(\mathrm{i}))$ and the centroid coordinates of the reference object $\left(\mathrm{X}_{\mathrm{r}}\right.$ and $\left.\mathrm{Y}_{\mathrm{r}}\right)$.

When $\mathrm{D}_{\text {sr }}$ tends to be zero, the quality of the segmentation increases whereas increase of the $D_{\text {sr }}$ values represents underand over-segmentation.

\subsection{Classification}

The image based parameters were used for the development of the initial rule set to perform the classification of subset 1 . Each parameter was assigned with threshold values in the membership function of the class description. The class description was used for the classification of the image objects. Using the rule sets, we executed OBIA by employing the initial rule set for subset 1 as well as for subset 2 and subset 3 . Rule sets for subset 2 and subset 3 were adapted until a similar classification quality was achieved. The classification comprised of several hierarchical steps to extract the class features, i.e. vegetation, bare soil, roads, formal settlements, water, shadow and informal settlements in all 3 subsets. First, the class features vegetation, roads, water and shadow were classified. Second, formal settlements were classified followed by bare soil classification. Once all other classes were classified, the remaining objects were assigned to the class "informal settlements". The following parameters were used in all the three subsets for classification:

- $\quad$ Normalized difference green and red edge (NDGR) (used to separate bare soil and formal settlement areas) $\frac{\text { Green-Red edge }}{\text { Green+Red edge }}$ (Hamedianfar \& Shafri, 2015)

- $\quad$ Normalized difference NIR1 and blue (NDNB) (used to extract shadows) $\frac{\text { Blue-Near InfraRed }}{\text { Blue+Near InfraRed }}$ (Hamedianfar \& Shafri, 2015)

- Brightness (used to distinguish the formal areas)

- $\mathrm{GLCM}_{\mathrm{red}}$ entropy (used to distinguish formal and informal areas)

- $\quad$ NDVI (used to classify vegetation)

- GIS layers from open street maps (OSM) (used to classify roads and water).

Fuzzy rule sets were used to give membership values to each object of a class. To quantify the value of the relative deviation of feature parameters, a curve, the membership function, has been assigned to each parameter.

\subsection{Accuracy Assessment}

For this purpose ground truth information as well as manually generated reference data through visual interpretation were used. A sample of 35 points for informal settlements and 15 points for formal settlements were collected during the fieldwork. 25 additional points for water class features were used. The manual points were cross-checked with Google Earth imagery also referring to the ground points which have similar ground truth information. As per Lee, Shan, \& Bethel (2003), the mathematical equation of the quality percentage is the following:

$$
\begin{aligned}
& \text { Completeness }=\frac{T P}{(T P+F N)} \\
& \text { Correctness }=\frac{T P}{(T P+F P)} \\
& \text { Quality Percentage }=100 \times \frac{T P}{(T P+F P+F N)}
\end{aligned}
$$

where TP; FP and $F N$ are True Positive, False Positive and False Negative respectively.

\subsubsection{Robustness Measurements}

The measurement of the robustness of a rule set was adopted from Hofmann et al. (2011). The assessment of robustness uses two steps. First, the classification quality without adaption is assessed between the subsets. Achieving a similar quality without adaption is considered robust. Second, when adaption is required, the rule set is adapted until a quality comparable to subset 1 is achieved. Here a deviation the membership function $\delta \mathrm{F}$ was considered as the measurement of robustness. Hence the classification qualities for subset 2 and subset 3 were first compared with the quality of subset 1 . The quality for all three subset was measured on the basis of manually delineated settlements by local experts during the questionnaire.

The equation by Hofmann et al., (2011) gives the relative change of the adapted rule set:

$$
\delta \mathrm{F}_{i}=\delta \mathrm{a}_{i}+\delta \mathrm{v}_{i}
$$

where $\delta F_{i}$ is the relative change of a fuzzy membership function in the $\mathrm{i}^{\text {th }}$ subset

$\delta \mathrm{a}_{\mathrm{i}}$ is the relative change for shifts in the $\mathrm{i}^{\text {th }}$ subset $\delta v_{i}$ is the relative change for stretch or compression in the $\mathrm{i}^{\text {th }}$ subset

The following equation by Hofmann et al., (2011) measures the robustness of a particular rule set:

$$
r_{i}=\frac{q_{i} / q_{o}}{d+1}
$$

where $r_{i}$ is the robustness of the rule set in the $i^{\text {th }}$ subset, $\mathrm{d}$ is the sum of all rule set deviation,

$q_{i}$ is the quality of classification of test imagery ( $\left.\mathrm{I}_{\mathrm{f}}\right)$ using the rule set $\left(\mathrm{R}_{\mathrm{f}}\right)$; $\mathrm{q}_{\mathrm{o}}$ is the quality of classification of referenced imagery (Io) using the rule set $\left(\mathrm{R}_{\mathrm{o}}\right)$.

\section{RESULTS}

\subsection{Local Ontology}

The feature characteristics of informal settlements are summarised in the table 1 . This information was used for conceptualising the local knowledge of informal settlements and completing the set of indicators for the image based parameters. The overall feature types (quantitative indicators) for classification were spectral values, geometry and textural association. 
Table 1: Local ontology of informal areas in Mumbai

\begin{tabular}{|c|c|c|c|c|}
\hline Level & $\begin{array}{l}\text { General } \\
\text { indicators }\end{array}$ & $\begin{array}{l}\text { Image } \\
\text { domain }\end{array}$ & $\begin{array}{l}\text { Real world domain } \\
\text { (experts) }\end{array}$ & $\begin{array}{l}\text { Image based } \\
\text { parameters for } \\
\text { rule set }\end{array}$ \\
\hline \multirow[t]{2}{*}{ Environs } & $\begin{array}{l}\text { Site location } \\
\text { (hazards) }\end{array}$ & $\begin{array}{l}\text { Slope, } \\
\text { pattern, } \\
\text { secondary } \\
\text { data }\end{array}$ & $\begin{array}{l}\text { Waterlogging, along } \\
\text { railway lines, } \\
\text { proximity to high } \\
\text { voltage power lines }\end{array}$ & $\begin{array}{l}\text { Association - } \\
\text { distance to features }\end{array}$ \\
\hline & $\begin{array}{l}\text { Neighbourhood } \\
\text { characteristics }\end{array}$ & $\begin{array}{l}\text { Pattern, } \\
\text { secondary } \\
\text { data }\end{array}$ & $\begin{array}{l}\text { Often located in } \\
\text { between well- } \\
\text { developed areas, } \\
\text { close to employment }\end{array}$ & $\begin{array}{l}\text { Association - } \\
\text { distance to planned } \\
\text { areas and } \\
\text { employment } \\
\text { opportunities }\end{array}$ \\
\hline \multirow[t]{3}{*}{$\begin{array}{l}\text { Settlement } \\
\text { level }\end{array}$} & $\begin{array}{l}\text { Density and } \\
\text { compactness }\end{array}$ & Pattern & $\begin{array}{l}\text { Highly compact } \\
\text { compared to planned } \\
\text { areas }\end{array}$ & $\begin{array}{l}\text { Association - } \\
\text { distance to less } \\
\text { compact areas }\end{array}$ \\
\hline & \multirow[t]{2}{*}{$\begin{array}{l}\text { Shape of the } \\
\text { settlement }\end{array}$} & \multirow[t]{2}{*}{ Texture } & $\begin{array}{l}\text { Planned areas with } \\
\text { medium density and } \\
\text { very dense irregular } \\
\text { layouts }\end{array}$ & Texture - entropy \\
\hline & & & $\begin{array}{l}\text { Lack of visibility of } \\
\text { road network and low } \\
\text { vegetation }\end{array}$ & $\begin{array}{l}\text { Geometry - area of } \\
\text { vegetation, service } \\
\text { area of road } \\
\text { network }\end{array}$ \\
\hline \multirow[t]{6}{*}{$\begin{array}{l}\text { Object } \\
\text { level }\end{array}$} & \multirow{3}{*}{\begin{tabular}{|l|}
$\begin{array}{l}\text { Characteristics } \\
\text { of building } \\
\text { structure }\end{array}$ \\
\end{tabular}} & Shape & Rectangular & $\begin{array}{l}\text { Geometry - } \\
\text { rectangular }\end{array}$ \\
\hline & & Size & $10-30 \mathrm{~m}^{2}$ & Geometry - area \\
\hline & & Material & $\begin{array}{l}\begin{array}{l}\text { Iron, abestos, plastics, } \\
\text { concrete }\end{array} \\
\end{array}$ & $\begin{array}{l}\begin{array}{l}\text { Spectral - layer } \\
\text { mean values }\end{array} \\
\end{array}$ \\
\hline & \multirow{3}{*}{\begin{tabular}{|l|} 
Road network \\
(type and nature \\
of roads)
\end{tabular}} & Shape & Irregular & $\begin{array}{l}\text { Geometry - } \\
\text { rectangular }\end{array}$ \\
\hline & & Type & $\begin{array}{l}\text { Unpaved, concrete, } \\
\text { tarmac (metalled), } \\
\text { Paved }\end{array}$ & $\begin{array}{l}\text { Spectral - } \\
\text { length/width ratio, } \\
\text { layer mean value }\end{array}$ \\
\hline & & Widths & $1-2 \mathrm{~m}^{2}$ & Geometry - area \\
\hline
\end{tabular}

\subsection{Image segmentation quality}

Multiresolution segmentation in eCognition 9.0 was employed. A value of 0.5 was used for shape and compactness. Calculating $\mathrm{D}_{\text {sr }}$ (equation 2) we obtained the following results:

$$
\begin{array}{ll}
\text { - } & \text { subset } 1 \text { is } 4.97 \text { metres } \\
\text { - } & \text { subset } 2 \text { is } 3.81 \text { metres } \\
& \text { subset } 3 \text { is } 3.99 \text { metres }
\end{array}
$$

The results of $D_{\text {sr }}$ indicate an acceptable segmentation quality. Problems are particularly caused by informal settlements forming groups of buildings in many segmented objects because of high densities, while in formal settlements, individual buildings and trees can be extracted. Integration of thematic layers of roads helped in segmentation.

\subsection{Classification and Accuracy Assessment}

After preforming the classification of subset 1 (Figure 3), the classification accuracy was assessed by calculating the overall accuracy using the standard error matrix. The classification accuracy for subset 1 was $87 \%$ (Kappa: 0.85). Best performing classes in subset 1 were vegetation, water and roads, with above $90 \%$ accuracies. Formal settlements were classified by using the combination of three parameters, namely GLCM, NDNB and brightness. Formal settlements were classified with lower accuracy (76\%), showing some mix-up with the classes bare soil and informal settlements. However, working with fuzzy membership function improved the performance of the classification of formal settlements. Bare soil was also classified with a relatively low accuracy of $72 \%$, due to similar spectral values of old roof materials and soil. Lastly, the remaining unclassified features were assigned as informal settlements and were classified with an accuracy of $72 \%$. We found that the parameters area and height, derived from digital elevation model (DEM), refined the classification because these parameters could be used to reduce the false-positives of formal and informal settlements. The accuracy result of subset 1 was used as a benchmark for the comparison with the accuracies of subset 2 and 3.

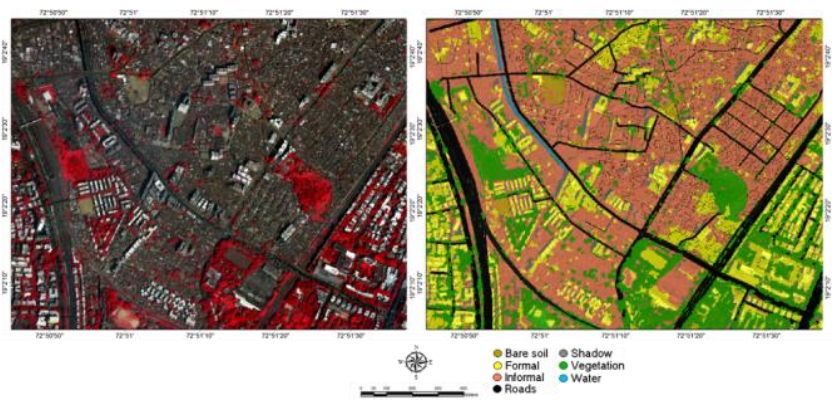

Figure 3: Image Subset 1 (left) and classification result (right)

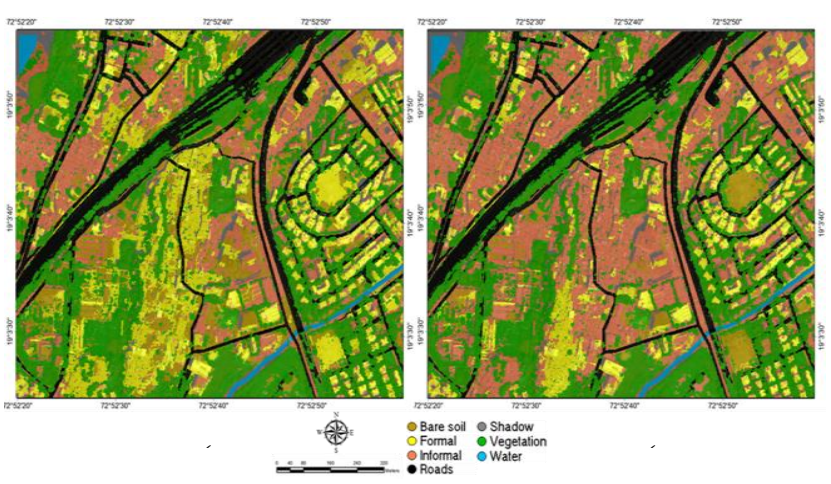

Figure 4. Subset 2 before (left) and after adaption (right)
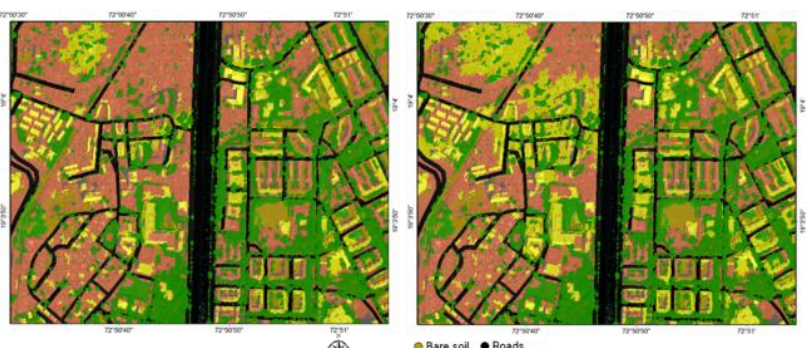

.

Figure 5. Subset 3 before (left) and after adaption (right)

Next, the same rule set was applied on subset 2 and 3 (Figure 4 and 5) and accuracies were measured. The overall accuracies before adaptation for subset 2 and 3 were $79 \%$ and $82 \%$ respectively. Thus for both subsets the accuracies were lower than that of subset 1 . This required adapting the parameters to improve the classification accuracies to be similar or better than for subset 1. After adapting the rule sets, the overall classification accuracies for subset 2 and subset 3 were $85 \%$ and $86 \%$, while for the informal settlements class, the accuracies were $82 \%$ and $86 \%$ respectively. Henceforth, we considered not only qualities but also the deviation of relative change of membership functions in evaluating the robustness of rule sets.

\subsection{Robustness Measurement of Rule Set}

The classification quality was analysed via, completeness, correctness and a quality percentage (equation 3-5). The 
classification quality of subset 1 was $52.4 \%$ and for subset 2 it was $5.6 \%$ which was very low in comparison (Table 2). The classification quality of subset 3 , however, was $58 \%$. Therefore, deviation of relative change of the membership function was considered for subset 2 but not for subset 3 .

Table 2: Classification qualities

\begin{tabular}{|c|c|c|c|c|}
\hline & Subsets & $\begin{array}{c}\text { Completeness } \\
\left(\mathbf{C}_{1}\right)\end{array}$ & $\begin{array}{c}\text { Correctness } \\
\left(\mathrm{C}_{2}\right)\end{array}$ & $\begin{array}{l}\text { Quality percentage } \\
\text { (QP) }\end{array}$ \\
\hline 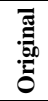 & Subset 1 & $61.29 \%$ & $78.28 \%$ & $52.38 \%$ \\
\hline \multirow{2}{*}{ 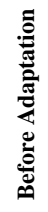 } & Subset 2 & $58.04 \%$ & $5.88 \%$ & $5.64 \%$ \\
\hline & Subset 3 & $61.02 \%$ & $91.78 \%$ & $57.86 \%$ \\
\hline \multirow{2}{*}{ 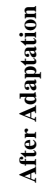 } & Subset 2 & $65.86 \%$ & $70.90 \%$ & $51.85 \%$ \\
\hline & Subset 3 & $63.61 \%$ & $80.69 \%$ & $55.21 \%$ \\
\hline
\end{tabular}

After measuring the quality criterion the deviation of relative change of the membership function was calculated. Equation 6 provided the results of deviation for each parameter used in the class features. We found that the parameters of thematic attributes roads, GLCM red entropy and NDGR were found to be stable throughout the classification by achieving deviations below 0.5 in all subsets. The overall deviation $\left(\mathrm{d}_{1}\right)$ for subset 2 was 6.04 whereas deviation $\left(\mathrm{d}_{2}\right)$ for subset 3 after adaptation was 6.89. Using equation 7 , the robustness of the developed rule set is found to be 0.14 out of 1 .

\section{DISCUSSION}

The results of this research show that the employed spectral indices (NDNB and NDGR) have an important role in detecting informal settlements using VHR satellite images. In the study of Hamedianfar and Shafri (2015),these indices were employed to distinguish between roof and non-roof surfaces, while in this research they are used to distinguish between formal settlements, bare soil and informal settlements.

A limitation of this study is the use of non-random sampling frame. This was done because of the limited accessibility of informal settlements. In the adaption process, we observed that the performance of parameters dropped when the first parameter was optimized. For instance in the case of detecting formal settlements three OBIA parameters were used. The first parameter was the GLCMred entropy. After optimizing this parameter for classification, the remaining parameters showed problems. However, the parameter NDNB did not require much adaptation. For example, while trying to detect shadows using the parameter NDNB, it showed good potential for detecting formal settlements as well.

Estimation of stable parameters in different subsets was done by selecting image based parameters for which membership values below 0.50 were found in the different subsets. Using the membership function samples for the classification must be taken for each rule in order to generate the fuzzy membership function automatically in the class description rather than manually inserting the membership function type. Moreover, the fuzzy membership function plays an important role in incorporating local knowledge while developing the rule set.
The deviation of the rule set is also highly influenced by the selected scales. If all other parameters, such as changing classes, operators, connectors, rules and thresholds were included as additional conditions in measuring the deviations of the rule sets, then it might contribute to strengthen the identification of the stable parameters. For example, the deviation of relative change of membership function because of absence of the water class in subset 3 negatively impacted the robustness. The robustness of the rule set could be increased up to 0.62 when considering cases in which classification quality is close to the quality of the subset 1 and ignoring the deviation of relative change of the membership function.

\section{CONCLUSIONS}

In this study, local expert knowledge was used to produce a local adaptation of GSO for Mumbai, resulting in spatial, geometry and texture based indicators. Furthermore, we used spectral indices (NDRR and NDNB) as an important feature for extracting informal settlements. These spectral indices are specific to WV-2 satellite imagery with its very unique 8 bands. In our study, we have tested these indices in three subsets showing their relative robustness. However, we cannot generalise this beyond our study area. Using spatial, geometry, spectral and texture indices we found the classification accuracy was less in subset 2 and 3 as compared to that of subset 1 . The addition of thematic layers (e.g. roads) did help in improving the segmentation quality as well as the classification accuracy.

To evaluate the robustness of the initial rule set, we used two criteria i.e. the classification quality and the deviation of relative change of the membership functions. We found that the degree of robustness is 0.14 (out of 1 ) which is considerably similar to earlier studies (e.g. Hofmann et al., 2011). For future research, other criteria in addition to deviation could allow more insights on robustness. In this study, parameters such as NDGR of spectral index and GLCM texture are found to be most stable in all subsets and therefore these parameters can be used to strengthen the robustness in future research.

\section{ACKNOWLEDGEMENTS (OPTIONAL)}

We would also like to thank Mr. Ronald Soubam and Mr. Kakchingtabam Lenin Sharma for their support during the fieldwork.

\section{REFERENCES}

Drăguţ, L., Tiede, D., \& Levick, S. R. (2010). ESP: a tool to estimate scale parameter for multiresolution image segmentation of remotely sensed data. International Journal of Geographical Information Science, 24(6), 859-871. doi: 10.1080/13658810903174803

Ebert, A., Kerle, N., \& Stein, A. (2009). Urban social vulnerability assessment with physical proxies and spatial metrics derived from air- and spaceborne imagery and GIS data. Nat. Hazards, 48(2), 275-294. doi: 10.1007/s11069-008-9264-0

Government of India. (2015). Population Census 2011. Retrieved 07-06-2016, from http://www.census2011.co.in/

Hamedianfar, A., \& Shafri, H. Z. M. (2015). Detailed intraurban mapping through transferable OBIA rule sets using WorldView-2 very-high-resolution satellite images. Int. J. Remote Sens., 36(13), 3380-3396. doi: 10.1080/01431161.2015.1060645 
Hofmann, P. (2014). Defining robustness measures for OBIA framework: A case study for detecting informal settlements. In Q. Weng (Ed.), Global Urban Monitoring and Assessment through Earth Observation (pp. 303-324). Boca Raton, FL, USA: CRC Press.

Hofmann, P., Blaschke, T., \& Strobl, J. (2011). Quantifying the robustness of fuzzy rule sets in object-based image analysis. Int. J. Remote Sens., 32(22), 7359-7381. doi: 10.1080/01431161.2010.523727

Hofmann, P., Strobl, J., Blaschke, T., \& Kux, H. (2008). Detecting informal settlements from QuickBird data in Rio de Janeiro using an object based approach. In T. Blaschke, S. Lang \& G. Hay (Eds.), Object-Based Image Analysis (pp. 531-553). Berlin|Heidelberg, Germany: Springer.

Jinguo, Y., \& Zheng, N. (2008, 30 June -2 July 2008). Evaluation of atmospheric correction using FLAASH. Paper presented at the International Workshop on Earth Observation and Remote Sensing Applications, 2008. EORSA 2008, Beijing (China).

Kit, O., Lüdeke, M., \& Reckien, D. (2012). Texture-based identification of urban slums in Hyderabad, India using remote sensing data. App. Geogr., 32(2), 660667. http://dx.doi.org/10.1016/j.apgeog.2011.07.016

Kohli, D., Sliuzas, R. V., Kerle, N., \& Stein, A. (2012). An ontology of slums for image-based classification. Computers Environment and Urban Systems, 36(2), 154-163.

Kohli, D., Stein, A., Sliuzas, R. V., \& Kerle, N. (2015). Identifying and Classifying Slum Areas Using Remote Sensing. (PhD), University of Twente Faculty of GeoInformation and Earth Observation (ITC), Enschede, The Netherlands. Retrieved from http://www.itc.nl/library/papers_2015/phd/kohli.pdf

Kohli, D., Warwadekar, P., Kerle, N., Sliuzas, R., \& Stein, A. (2013). Transferability of object-oriented image analysis methods for slum identification. Remote Sens., 5(9), 4209-4228.

Kuffer, M., Pfeffer, K., \& Sliuzas, R. (2016). Slums from Space-15 Years of Slum Mapping Using Remote Sensing. Remote Sens., 8(6), 455.

Kuffer, M., Pfeffer, K., Sliuzas, R., \& Baud, I. (2016). Extraction of slum areas from VHR imagery using GLCM variance. IEEE J. Sel. Top. Appl. Earth Observ. and Remote Sens., 9(5), 1830-1840. doi: 10.1109/JSTARS.2016.2538563

Montaghi, A., Larsen, R., \& Greve, M. H. (2013). Accuracy assessment measures for image segmentation goodness of the Land Parcel Identification System (LPIS) in Denmark. Remote Sens. Lett., 4(10), 946955. doi: 10.1080/2150704X.2013.817709

Netzband, M., \& Rahman, A. (2009 ). Physical characterisation of deprivation in cities. How can remote sensing help to profile poverty (slum dwellers) in the megacity of Delhi/India? Paper presented at the Proceedings of the IEEE Joint Urban Remote Sensing Event, Shanghai, China. <Go to ISI > //WOS:000270972300186

Nolan, L. B. (2015). Slum Definitions in Urban India: Implications for the Measurement of Health Inequalities. Popul. Dev. Rev., 41(1), 59-84. doi: 10.1111/j.1728-4457.2015.00026.x

Subbaraman, R., Nolan, L., Shitole, T., Sawant, K., Shitole, S., Sood, K., . . . Patil-Deshmukh, A. (2014). The psychological toll of slum living in Mumbai, India: A mixed methods study. Soc. Sci. Med., 119, 155-169. doi:

http://dx.doi.org/10.1016/j.socscimed.2014.08.021

UN-Habitat. (2010). State of the world's cities 2010- 2011: Bridging the urban divide. Nairobi. 


\title{
SCENE CLASSIFICATION OF URBAN AREAS EXPLOITING MULTI-VIEW HIGH RESOLUTION AERIAL IMAGES
}

\author{
F. Nex ${ }^{a^{*}}$, M. Dalla Mura ${ }^{b}$ \\ ${ }^{a}$ University of Twente, ITC Faculty, Department of Earth Observation Sciences, Enschede, The Netherlands - f.nex@utwente.nl \\ ${ }^{\mathrm{b}}$ GIPSA-lab, Grenoble Institute of Technology, Grenoble, France - mauro.dalla-mura@ gipsa-lab.grenoble-inp.fr
}

KEY WORDS: Scene classification, multi-view aerial images, DSM, photogrammetry, urban remote sensing

\begin{abstract}
:
Many supervised and unsupervised algorithms for the automated and reliable classification of large regions using high resolution data have been presented in the remote sensing community in the last decades. Most of these approaches exploit a single input data: high resolution orthophotos or 3D point clouds. An increasing number of contributions has more recently exploited the combined use of orthophotos and LiDAR DSM taking advantage from the complementarity of these inputs. Nevertheless, very few applications have considered the use of overlapping multi-view images on the same area for classification. In this paper the first tests to investigate this classification architecture are presented. Different typologies of DSM and orthophoto as well as a variable number of images on the same area have been considered in the experiments. The preliminary results on the two available test areas will be shown, in order to draw the first conclusions on this approach and discuss the further developments of this research.
\end{abstract}

\section{INTRODUCTION AND MOTIVATIONS}

In the last decades, the improvements in the technical development of airborne image sensors and the mass production of UAVs have boosted the acquisition of extremely high resolution images for the generation of reliable and automated Digital Surface Models (DSMs) and orthoimages. On the other hand, the need to monitor, map and model urban and rural areas has led to the improvement of several algorithms for the automated and reliable classification of large regions using high resolution data (Mallet et al., 2011).

Some of the approaches presented in the literature exploit orthoimages or DSMs for performing a scene classification. More recently an increasing number of contributions combines the complementary nature of both spectral and depth information to overcome the limits of each input separately. A large variety of features from both DSMs and orthoimages have been therefore developed to make the classification process more reliable and accurate (Rottensteiner et al., 2014; Gerke and Xiao, 2014).

In all these implementations, the spectral information is only taken from the orthophoto, while the full information provided by the overlapping input images is neglected in the classification process. However, each pixel of the DSM could be easily mapped on the corresponding images thanks to the projective geometry equations, allowing the exploitation of the information from different points of view.

This paper aims at investigating the benefits for the classification process given by the use of multi-view images acquired on the same area. The conventional classification generated using (i) DSM, (ii) orthophoto and (iii) their combination has been compared to the classification provided combining together (iv) the DSM and the several images captured on the same area.

The first experiments and the first promising results will be discussed in the following sections. In the performed tests, different set of features have been adopted in each experiment and the same training sample has been used for each data configuration to compare the different results using the same input. Two different test areas have been considered in order to make the investigation independent from the input data.

The paper is organized as follow: in the following section the background on the use of photogrammetric techniques for the generation of point clouds and orthophotos will be shortly presented. Then, the adopted testing methodology will be introduced in Section 3, while the first results will be presented in Section 4. The conclusions and the future developments of our tests will be finally discussed in the last section.

\section{BACKGROUND}

Photogrammetry is the science of using image measurements to extract tridimensional information. Each image is ideally modelled as a central projection according to the pinhole camera model (Hartley and Zisserman, 2004): the projection center, the image point and the corresponding point in the space lie on the same line. The projective lines are then used to retrieve the position of points in the $3 \mathrm{D}$ space, given their $2 \mathrm{D}$ position on two or more images and vice-versa, to determine the position of a point on the images from its $3 \mathrm{D}$ coordinates in the space.

The photogrammetric process can be divided in three main steps: (i) image orientation, (ii) DSM generation and (iii) orthophoto generation.

The position (geo-referencing) and the attitude (rotation towards the coordinates system) of each acquisition is obtained by estimating the image orientation. In the dense point cloud generation, 3D point clouds are generated from a set of images, while the orthophoto are generated in the last step combining the oriented images projected on the generated point cloud, which leads to orthorectified images. The outputs from the last two steps (point clouds and orthophotos) can be directly used as input in the classification process. As a consequence, the way these products are generated and their final quality can have a direct effect on the classification of the area.

In the following sections, the dense point cloud and the orthophoto generation are reported, focusing on the possible 
problems these products can have and the consequences for the final classification quality.

\subsection{DSM generation and orthophoto drawbacks}

Image matching represents the simultaneous establishment of correspondences between primitives (i.e. points, lines) extracted from two or more images and the estimation of the corresponding position in the 3D space using the collinearity or projective models (Remondino et al., 2014). Image matching algorithms are the bedrock for the automated generation of Digital Surface Models (DSM).

All of the proposed matching methods are based on similarity or photo-consistency measures, i.e. they compare pixel values between the images. In image space this process produce a depth map (that assigns relative depths to each pixel of an image) while in object space it is called point cloud. Anyway, the establishment of dense and accurate image correspondences is still a challenging task, and very different solutions have been conceived in the last three decades. Even if image matching algorithms have been greatly improved, they are not still able to generate error-free point clouds. Outliers are still visible in the most occluded parts of the scene. Regions with low textures or shadows can greatly increase the level of noise of the point clouds too. These problems afflict all the available solutions (Haala, 2013), producing unwanted spikes and locally wrong surface models. Of course, these problems affect in a negative way the quality of meshes and orthophotos generated from photogrammetric DSM.

Airborne (GSD $<20 \mathrm{~cm}$ ) and UAV images, with high overlaps $(>70 \%)$ are more often used in the point cloud generation. The high image resolution and the high overlaps increase the quality of the achieved DSM, improving the quality in the surface reconstruction and reducing the number of outliers and mismatches in correspondence of occlusions. On the other hand, the geometric resolution (up to $40 \mathrm{~cm}$ ) and the limited stereoscopic coverage of the satellite stereo images is nowadays still insufficient to provide DSM comparable to the aerial cases (Arefi et al., 2011)

The orthophoto is the orthogonal parallel projection of the input images where the projective distortions of the image are geometrically corrected using a 3D surface model. A Digital Terrain Model (DTM) was traditionally used for this kind of process but the extensive use of high resolution images in the last two decades has pointed out the limits of this kind of surface model on urban areas. Large artefacts or double mapping in correspondence of complex structures were often generated and the relief displacement was unacceptable for mapping purposes in correspondence of the high buildings (i.e. the position of building roofs is displaced from the correct position).

For this reason, the Digital Terrain Model is usually used in the orthogonal projection (Ahmar et al., 1998) and their output is therefore called true-orthophoto. Reliable true-orthophotos can be usually generated thanks to the use of high overlaps and high resolution images and the generally good performances of image matching algorithms.

As already mentioned, inaccurate DSMs can negatively influence the quality of the true-orthophoto, generating double mapping or wrong point projections. Then, many images with significant scene-to-scene radiometric variations can be often fused together to generate an orthophoto. This problem can be only partially mitigated using radiometric balancing and blending algorithms (such as min-cut, seamless stitching, graphcut and watershed) to prevent/reduce patchy appearance in the final result.

\section{METHODOLOGY}

All the above mentioned problems usually reflect in the classification process as both DSMs and orthophotos are directly used in input. These issues become more relevant when high resolution images are used, giving misclassification in correspondence of wrong reconstructed regions of the DSM or abrupt and erroneous radiometric changes in neighbouring and homogenous regions of the orthophoto (i.e. on the same roof).

From this perspective, the use of the original images could reduce the negative impact given by these artefacts. The wrong DSMs reconstructions would be mitigated by the combined use of images from different perspectives. The different radiometric content of the images would be averaged reducing the misclassification due to inaccurate radiometric blending between images.

The developed methodology is based on the use of the original images in the attempt to make the classification more robust and less error prone in correspondence of critical areas.

For this purpose, a set of overlapping images, the generated photogrammetric DSM and the corresponding true-orthophoto are considered as input. Then, different configurations of input and different training sets are considered: the results achieved using only the DSM and only true-orthophoto as well as they combined used are initially considered. Then, the additional information provided by a variable number of images (from 1 to 4) on the same region are compared in order to assess the possible benefits given by the use of overlapping images in the classification. Each image is initially orthorectified using the available DSM: the information from different images on the same point can be therefore overlaid in the same reference system. The information provided by the images and the orthophoto is finally merged using a majority voting decision rule.

From each input data and each performed test, a minimum number of features has been considered in this stage of the research: the three bands from the orthophoto, the normalized height information and the spectral information from a variable number of images have been considered. The same training sample has been used for each data configuration to compare different results using the same input. The Random Forest algorithm has been adopted to generate the classification results. DSMs generated using different image matching algorithms has been considered in order to make our investigation software independent.

The classification process has been performed on two different areas, using different camera and different image bands in order to make our investigation independent from the considered scenario.

\section{TESTS}

\subsection{Data description}

Two different datasets have been adopted in the performed experiments. The first area is on Transacqua, a small town in Italy, while the second dataset is on the city of Vaihingen, Germany. The Transacqua dataset was acquired using an amateur D3X camera installed on a helicopter. The flight was performed at $800 \mathrm{~m}$ height with $80 \%$ along track and $60 \%$ across track overlaps: an average Ground Sample Distance (GSD) of $9 \mathrm{~cm}$ was achieved on the test area. The Vaihingen data is a subset of the data used for the test of digital aerial cameras (Cramer et al., 2010) carried out by the German Association of Photogrammetry and Remote Sensing (DGPF) 
and currently available thanks to the ISPRS benchmark on the 2D semantic labelling contest (http://www2.isprs.org/commissions/comm3/wg4/semanticlabeling.html). The GSD is $8 \mathrm{~cm}$ and Red, Green and NearInfrared bands have been stored in 11 bits radiometric resolution image. Both 8 bit and 16bit images are available for participants. In the performed tests only the 8bit images have been used.

The DSM generation of the two datasets have been performed using two different algorithms. The first dataset has been processed using the open-source MicMac software (PierrotDeseilligny and Paparoditis, 2006), while an implementation of the semi-global algorithm (Trimble Match-T software) has been adopted in the second image block processing (Hirshmuller, 2008).

The generated point clouds have been used to ortho-rectify the images and produce a high resolution image. The first orthophoto was generated using the tool Porto of the MicMac library. The orthophoto on Vaihingen area has been processed with the commercial software Trimble INPHO OrthoVista.

A labelled ground truth has been manually generated on the first dataset. Five different classes (building, road, ground, vegetation and shadows) have been considered on this area. On the other hand, the ground truth provided by the ISPRS benchmark has been adopted on the second dataset.

\subsection{First results and discussion}

In the following, the first performed tests are reported. The results on the Transacqua area are reported in Table 1, while the results achieved on Vaihingen are shown in Table 2.

\begin{tabular}{lcccc} 
& $\mathbf{5}$ & $\mathbf{1 0}$ & $\mathbf{5 0}$ & $\mathbf{1 0 0}$ \\
\hline ortho+ndsm & $76.7(3.3)$ & $81.9(3.1)$ & $89.6(1.6)$ & $92.1(0.8)$ \\
\hline ortho & $57.0(4.5)$ & $60.4(5.7)$ & $75.2(1.9)$ & $79.9(1.0)$ \\
\hline allviews+ndsm & $75.3(6.2)$ & $81.3(6.1)$ & $90.1(1.5)$ & $92.2(1.2)$ \\
\hline view1+ndsm & $77.6(6.3)$ & $83.2(2.8)$ & $90.2(1.3)$ & $92.2(1.0)$ \\
\hline view2+ndsm & $78.5(4.8)$ & $83.1(4.2)$ & $90.6(1.1)$ & $92.8(1.1)$ \\
\hline view3+ndsm & $78.8(5.2)$ & $83.3(4.1)$ & $90.4(0.9)$ & $92.0(1.0)$ \\
\hline majvot_3views & $\mathbf{8 1 . 8 ( 4 . 2 )}$ & $\mathbf{8 5 . 8 ( 3 . 2 )}$ & $\mathbf{9 1 . 8}(1.2)$ & $\mathbf{9 3 . 5}(0.9)$ \\
\hline
\end{tabular}

Table 1. Classification results on the Transacqua test area using different input data configurations. Best results are in bold.

\begin{tabular}{lcccc} 
& $\mathbf{5}$ & $\mathbf{1 0}$ & $\mathbf{5 0}$ & $\mathbf{1 0 0}$ \\
\hline ortho+ndsm & $59.4(6.3)$ & $58.0(5.7)$ & $56.7(1.7)$ & $59.6(3.1)$ \\
\hline ortho & $41.7(12.1)$ & $46.9(8.3)$ & $46.7(0.0)$ & $47.1(0.9)$ \\
\hline allviews+ndsm & $55.4(2.5)$ & $\mathbf{5 9 . 2 ( 3 . 7 )}$ & $\mathbf{6 0 . 0}(1.9)$ & $\mathbf{6 2 . 0}(2.4)$ \\
\hline view1+ndsm & $61.0(6.0)$ & $58.3(4.4)$ & $58.5(2.5)$ & $59.1(2.2)$ \\
\hline view2+ndsm & $60.3(9.0)$ & $55.9(5.5)$ & $58.0(3.4)$ & $59.1(3.8)$ \\
\hline view3+ndsm & $57.9(5.7)$ & $56.4(6.6)$ & $56.8(5.8)$ & $55.2(1.8)$ \\
\hline view4+ndsm & $61.0(7.0)$ & $53.3(4.7)$ & $56.6(1.4)$ & $56.5(1.0)$ \\
\hline majvot_4views & $\mathbf{6 1 . 7 ( 5 . 0 )}$ & $58.2(3.2)$ & $58.7(1.8)$ & $59.0(1.2)$ \\
\hline
\end{tabular}

Table 2. Classification results on the Vaihingen test area using different input data configurations. Best results are in bold.

Different combinations are reported in these tables: on the rows the input data are reported, while in the column the number of samples used to train the algorithm.
The contribution of the multiview images has been tested considering one image per time (viewX, with $\mathrm{X}$ the id of the image) or all the images together (allviews) and combining the results of the classifications of the single images via majority voting (majvot option). The results look quite similar when a single image or the true-orthophoto are considered in the classification: in these cases, no substantial improvement can be detected. On the other hand, the use of multiple images on the same area (either in the allview or majvot configurations) systematically overcomes the standard configuration $($ ortho $+n d s m)$ in both the test areas. By using a high number of images in the classification process, the classification accuracy always improves. This positive contribution is more relevant when a reduced number of samples is used to train the data.

Analysing the classification maps, it can be noticed that the use of multiple images reduces the noise in the results, decreasing the number of ambiguous and wrong classifications in most of the analysed cases. As an example, in Figure 1 the noise on the roofs is less when the number of images increases.

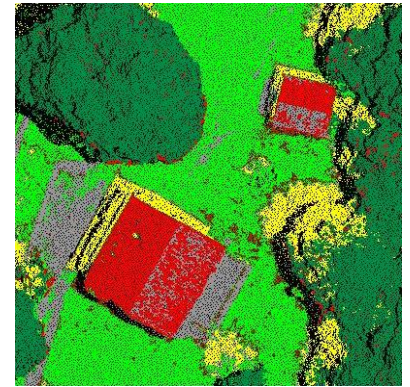

(a)

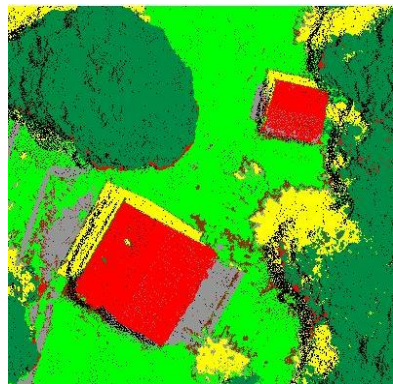

(b)
Figure 1. Example of classification in the Transacqua dataset using the true-orthophoto and the DSM (a) and adding to this configuration three images.

\section{CONCLUSIONS AND FUTURE DEVELOPMENTS}

The use of overlapping images in the classification of very high resolution data has been discussed in this paper. By means of photogrammetric techniques, the corresponding points on different images are jointly exploited to classify the scene in a more robust and efficient way.

The presented investigation is just the first step of more thorough investigations that will be completed in the near future. However, the presented results have both confirmed that the use of multi-image can improve the classification results. The classification improvement is more relevant when a reduced number of training samples is used.

New and more extensive investigations will be performed in the future with more exhaustive investigations. The use of different DSM and different set of features will be first considered. The adoption of different DSM on the same area will allow to estimate the robustness of the presented approach in relation to the quality of the 3D data, observing different behaviours in presence of noisy or wrongly reconstructed regions. Different sets of features will help to understand if the use of the overlapping images is still relevant when more complete sets of features are adopted.

\section{ACKNOWLEDGEMENTS}

The authors would like to acknowledge the Project "Automated scene information extraction from a joint analysis of aerial 
remote sensing images and their photogrammetric DSM" (in the Galileo 2013 Project framework) that supported the initial stage of this investigation.

\section{REFERENCES}

Ahmar, f., Jansa, J., Riess, C., 1998. The generation of true orthophotos using a 3D building model in conjunction with a conventional dtm, IAPRS, vol. 32, pp. 16-22.

Arefi, H., d'Angelo, P., Mayer , H., Reinartz , P., 2011. Iterative approach for efficient digital terrain model production from CARTOSAT-1 stereo images. Journal of Applied Remote Sensing, 2011 (5), 19 p. DOI: 10.1117/1.3595265.

Gerke, M., Xiao, J., 2014. Fusion of airborne laserscanning point clouds and images for supervised and unsupervised scene classification. ISPRS Journal of Photogrammetry and Remote Sensing, 87, 78-92.

Hartley, R., Zisserman, A., 2004. Multiple view geometry in Computer Vision. Cambridge University press ISBN 0521540518 .

H. Hirschmüller, 2008. Stereo processing by semiglobal matching and mutual information. IEEE Trans. Pattern Anal. Mach. Intell., vol. 30, no. 2, pp. 328-341.

Mallet, C., Bretar, F., Roux, M., Soergel, U., Heipke, C., 2011. Relevance assessment of full-waveform lidar data for urban area classification. ISPRS Journal of Photogrammetry and Remote Sensing, vol.66 (6), pp.S71-S84.

Pierrot-Deseilligny, M., Paparoditis, N., 2006. A multiresolution and optimization-based image matching approach: An application to surface reconstruction from SPOT5-HRS stereo imagery. In: IAPRS, vol. XXXVI, pp. 1-5.

Remondino, F., Spera, M.G., Nocerino, E., Menna, F., Nex, F., 2014. State of the art in high density image matching, Photogramm. Rec., vol. 29, no. 146, pp. 144-166. 


\title{
GEOBIA SYSTEMS FOR MASSIVE DATA PROCESSING
}

\author{
N. Ahles ${ }^{\text {a }}$, S. MacFaden ${ }^{\text {a }}$, J. O’Neil-Dunne ${ }^{\text {a }}$, A. Royar ${ }^{\text {a }}$, T. Engel ${ }^{\text {a }}$ \\ ${ }^{\text {a }}$ University of Vermont, Spatial Analysis Laboratory, Burlington, Vermont USA - (nahles, smacfade, jonieldu, aroyar, \\ tengel)@uvm.edu
}

KEY WORDS: geographic object-based image analysis (GEOBIA); eCognition; LiDAR; multispectral imagery; massive data processing; ISPRS

\begin{abstract}
:
Large portions of the Earth's landscape are now captured by high-resolution remotely-sensed datasets and turned into corresponding thematic data. Despite these advancements the number of comprehensive, high-resolution land-cover maps is surprisingly low. The value of high-resolution land-cover data in landscapes that are increasingly fragmented and heterogeneous is great, but so are the challenges associated with turning these disparate datasets into information. We argue that effective geographic object-based image analysis (GEOBIA) system design, while rarely discussed in the literature, is perhaps the most important factor in determining the success of projects whose focus is on broad-area mapping. Human resources, data, hardware, and software must be tightly integrated to make the system efficient and effective. At the same time, the object-based approaches used by such systems for land-cover mapping must try to replicate the human cognitive process as much as possible, using stable, context-based approaches to feature extraction that leverage the strengths of the various input datasets while minimizing their weaknesses. Drawing on our experience deriving 12 terabytes of high-resolution land cover for more than $232,000 \mathrm{~km}^{2}$ in the United States, we describe the design considerations for GEOBIA systems that are capable of processing huge volumes of data. In addition, we provide examples of the techniques and approaches deployed within these systems that overcome the challenges associated with mapping land cover from massive, disparate datasets.
\end{abstract}

\section{INTRODUCTION}

The limiting factor of spatial analytics is no longer the lack of upto-date remote-sensing data but our ability to rapidly turn those data into information. High-resolution multispectral satellite and aerial imagery has greatly proliferated in recent years, and some acquisition programs (e.g., Planet Labs) are now approaching single-day temporal resolution for the entire globe. LiDAR, while not as universally accessible as imagery, has also grown dramatically in availability and quality, and linear LiDAR systems continue to provide the market with datasets covering much of the developed world. LiDAR coverage and revisit rates will improve further with Single Photon and Geiger-Mode LiDAR systems, which are capable of dwarfing linear LiDAR acquisition rates. Much of the world has already turned these imagery and LiDAR datasets into thematic information in some capacity, most often in the form of vector GIS layers. Developed countries in particular have highly detailed datasets funded through governmental initiatives. Many developing countries have not yet invested in such GIS datasets but nonetheless have access to road networks, building footprints, and other vector features through crowd-sourced initiatives such as OpenStreetMap. However, very few areas of the globe have comprehensive, high-resolution land-cover maps. Such maps are crucial for the effective management and understanding of gray and green infrastructure (Benz, 2004).

A considerable amount of work in the GEOBIA field has focused on the development of individual algorithms, comparative analysis, and case studies. Relatively little has been published on what makes an effective GEOBIA system, particularly one capable of capitalizing on this modern area of vast quantities of geospatial data.
In this paper, we discuss the framework we have developed to integrate data preparation, GEOBIA, and manual editing into an iterative system that streamlines the production of land-cover datasets covering large geographic extents. High-resolution image analysis at county, state and regional scales necessitates efficient system design and processing at multiple steps, and personnel, hardware, software, and input datasets all must be coordinated in this effort. Specialized analysts and technicians are trained to manage isolated aspects of production, relying on multi-core workstations to maximize processing efficiency, standard operating procedures that codify workflows, videos and other training materials that expedite information transfer, extensive script templates and libraries that encapsulate preexisting modeling routines, and data servers that allow crossnetwork processing and editing.

This framework moves GEOBIA from the realm of localized feature extraction to regional mapping efforts covering entire drainage basins and political administrative units (e.g., individual American states). We are currently mapping some of the largest sub-meter resolution land-cover projects ever attempted in the United States, including the Chicago Region $\left(14,530 \mathrm{~km}^{2}\right)$, the Chesapeake Bay Watershed $\left(165,760 \mathrm{~km}^{2}\right)$, and the Delaware River Basin $\left(35,066 \mathrm{~km}^{2}\right)$. Overall, our workflows have mapped land cover for more than 1 trillion pixels of data across North America. And size is not the only factor requiring efficiency; many of our large-extent mapping projects must be completed on very short timelines, often less than a year. The maps produced for these regions will document baseline conditions and serve as inputs for future land-cover and change-detection analyses.

\footnotetext{
* Corresponding author
} 
Many projects also focus on improving or updating existing datasets rather than mapping landscapes from scratch. GEOBIA is uniquely positioned to perform this type of mapping; it can ingest existing land-cover datasets and vector GIS layers (e.g., building footprints, road polygons), extract new features from updated imagery or LiDAR, and detect changes using contextual information. This process mimics human cognition (Blaschke, 2010). When a high density of roads and buildings is observed, it can be assumed with a high degree of probability that parking lots and other impervious surfaces are nearby. Similarly, extensive concentrations of large, tall buildings are much more likely to occur in an urban zone than an agricultural landscape. This understanding of contextual relationships, and the ability to incorporate them into GEOBIA rule sets, is essential to effective land-cover mapping across large, heterogeneous geographic areas (O’Neil-Dunne, 2011).

\section{METHODOLOGY}

\subsection{Framework}

A GEOBIA system is ultimately a collection of data, hardware, software and people. Development of accurate high-resolution, comprehensive land cover maps requires the effective synergy of these technological and human resources.

\subsubsection{Data}

We have found that the most efficient and effective approach to high-resolution land cover mapping is to leverage all existing remotely-sensed and thematic datasets. There is no point in extracting features that have already been mapped. Moreover, any land cover mapping should insure consistency, to the extent possible, with existing mapped information. Flawed datasets are not excluded in our workflow, rather they are evaluated and understood so that they can be harnessed to minimize their limitations and maximize their valuable information. We draw from datasets that exist in raster, vector, and point cloud formats.

\subsubsection{Software}

Although the term "image" is still part of the GEOBIA definition we are of the opinion that the true power of GEOBIA lies in its ability to serve as a data fusion platform in which the object breaks down the barriers that exist between traditional geospatial formats. As such, one of our key requirements is that the software be capable of handling point cloud, raster, and vector datasets in their native format without the need for conversion. Furthermore, the system must be capable of applying vector, raster, and point cloud algorithms. eCognition (Trimble) serves as the foundation of our GEOBIA system. eCognition fulfils our key requirements in terms of data fusion while at the same time supporting enterprise-level, distributed processing of massive datasets. In addition, we make use of a number of domain-specific software packages for data preparation and manual corrections. Key data preparation tasks include LiDAR point cloud classification, generating raster mosaics, and editing vector data layers.

\subsubsection{Hardware}

A given regional high-resolution land cover project may entail working with hundreds of gigabytes or even terabytes of input datasets. In a GEOBIA processing object information is stored in computer memory, making large amounts of RAM a key requirement of our hardware systems. Multiple, highperformance CPUs also form a key foundation of our GEOBIA- specific hardware. Much less stringent hardware requirements exist for our computer workstation used for manually editing. Online storage with fast networking and regular backups are also a key component of our GEOBIA system.

\subsubsection{People}

The human resource component of the GEOBIA system is the one we consider to be the most important. There exist two facets of the people component of our GEOBIA system, the first is the individual skills that each person possess, the second is the ability to function as a team. Personnel on our team are assigned to one of three sections: data preparation, GEOBIA feature extraction, and manual corrections. The data preparation section is responsible for assembling input datasets, including such tasks as mosaicking raster data, classifying LiDAR point clouds, and checking the quality and consistency of vector data. The GEOBIA section develops automated approaches to feature extraction using GEOBIA software. The manual corrections section has the responsibility of reviewing the output from the automated process, determining if it meets the standard, and making manual corrections to the data.

The ability to recognize features from a broad array of types of remotely sensed data is the foundational skill that we consider to be paramount to all team members regardless of their role. Image interpretation is a qualitative skill, one that is developed over time through the application of the elements of image interpretation. Our GEOBIA feature extraction analysts, who are the most seasoned members of our team spent many thousands of hours manually extracting information from remotely sensed data. We see this development as a crucial preparatory phase that enables them to harness the elements of image interpretation for automated feature extraction.

Communication is essential. We use a cloud-based team collaboration software to insure that all project personnel must understand project goals, the prescribed workflow, and their specific responsibilities in the workflow. By developing standard but adaptable protocols, it is possible to build a team of technicians and analysts (sometimes including 40 or more contributors) that works time- and cost-efficiently toward final products that satisfy end-user needs. A second but no less important goal is to document and store each final product for historical use: what were its inputs, how was it processed, and where is it located?

\subsection{Approach}

Our approach to broad-area, wall-to-wall land cover mapping with GEOBIA includes three primary phases: data preparation, GEOBIA rule-set development and processing, and manual editing (Figure 1). These tasks require different levels of technical expertise, ranging from undergraduate students who have completed GIS and remote-sensing coursework to seasoned research analysts with considerable experience in GEOBIA theory and practice. They also require unique considerations with respect to the geospatial hardware and software employed.

\subsubsection{Data Preparation}

Data preparation includes acquisition and evaluation of all existing imagery, LiDAR, and vector datasets that could be useful in the feature extraction process. It also includes initial data processing and creation of derivative products that aid object segmentation and classification (e.g., rasterized LiDAR surface models). Our typical projects will incorporate anywhere from a 
few to over twenty existing vector layers. These vector layers, representing individual features (e.g. buildings) or geographies (e.g. property parcel boundaries) are valuable, but most likely outdated when compared to the source imagery and LiDAR. A crucial part of the data preparation phase is the evaluation of these datasets, to understand their strengths, weaknesses, and their value in the GEOBIA feature extraction process. The review of these datasets is also crucial for determining whether or not

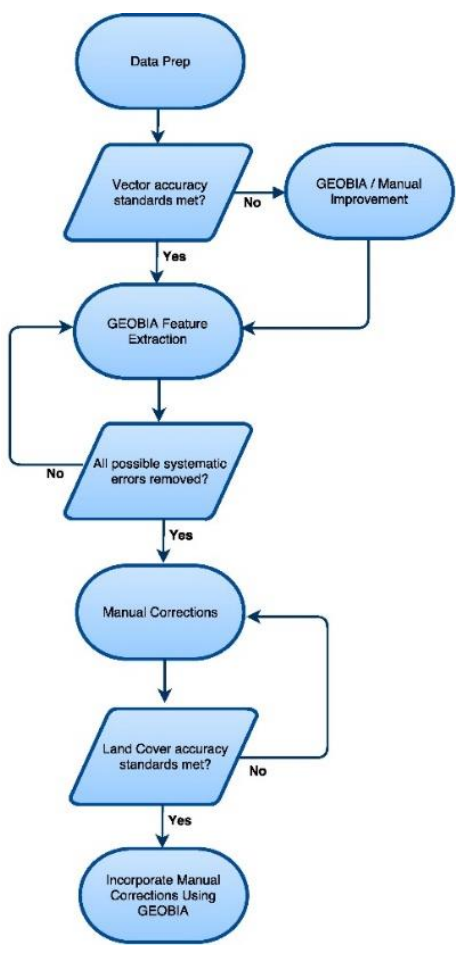

Figure 1. Flowchart depicting the GEOBIA process.

manual corrections should be made to the data prior to incorporating them into the GEOBIA system. An example of this is an incomplete building dataset that is serving as an input to a high-resolution land cover mapping project. If the dataset contains a relatively low number of missing or changed buildings manual editing would be a more efficient approach than an automated GEOBIA workflow. A larger number would make a manual editing process too costly. Determinations such as this are judgement calls, requiring input from both the GEOBIA feature extraction analysts and manual editing technicians.

\subsubsection{GEOBIA Rule Set Development and Processing}

In the GEOBIA step, rule-set development is the primary task, requiring iterative modification and testing in eCognition. Individual rule sets are generally produced for separate LiDAR collections, which in the United States is often by county but can sometimes include larger portions of states. This focus on LiDAR collections helps maximize data quality and temporal consistency, especially in counties or states that have coordinated programs for acquiring or updating imagery, LiDAR, and planimetric vector layers. However, the rule sets are usually structured with a generic modeling flow (e.g., preliminary identification of tall features followed by discrimination of tree canopy from buildings) that can be readily modified for use with other LiDAR collections. By building a library of rule sets, GEOBIA analysts can adapt modeling routines from project to project, re-using well-tested approaches while adjusting specific segmentation and classification parameters as necessary; we do not start from scratch unless project goals or input datasets necessitate it. It is also possible to apply templates to specific modeling tasks depending on data availability. For example, if no planimetric building footprints exist for an individual study area, we can implement a previously-created routine that uses a combination of imagery and LiDAR to model footprints. This routine would replace a step that simply incorporates existing buildings into the draft classification. Similarly, if no roads polygons exist for an area of interest but road centerlines are available, the centerlines can be used to estimate road surfaces with a preexisting routine designed for this purpose.

\subsubsection{Manual Review}

Once a rule set has been sufficiently honed by iterative testing, a point typically reached when all systematic errors have been addressed, a draft land-cover dataset is produced for manual review and editing. The team responsible for this effort usually consists of well-trained undergraduate students led by one or more experienced research technicians. Each member reviews individual tiles of the draft map, comparing it to the imagery and LiDAR used in initial modelling and identifying obvious errors of omission and commission (MacFaden, 2004). Polygons are then drawn around the misclassified features and labelled according to the specific changes required (e.g., a powerline erroneously classified as tree canopy must be re-assigned to the unclassified category). These polygons are then incorporated into a final classification in a second eCognition run (Figure 2). The manual corrections team follows a project-specific image interpretation key, and the lead research technicians review edits and provide feedback on the quality and volume of edits. The tiling scheme used to distribute sections for editing provides an effective system for gauging and documenting progress.

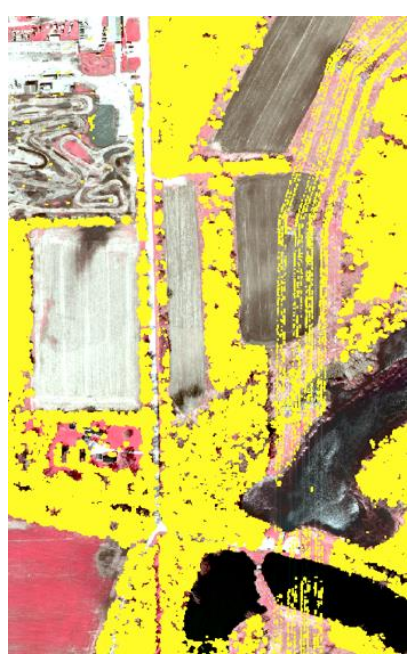

(a)

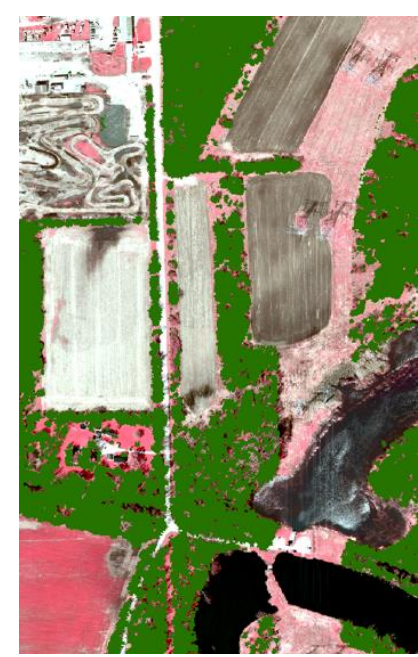

(b)
Figure 2. Tree canopy after automated feature extraction (a).

Powerlines and other false tree canopy removed during manual review (b).

\subsubsection{Data Management}

Data management and organization are the pillars on which the GEOBIA workflow is built. Projects often require dozens of input layers possessing heterogeneous acquisition dates, data types, projections, resolutions, and quality (O’Neil-Dunne, 
2013). As such, it is essential to know when and how each was acquired or developed.

\subsubsection{Workflow Examples}

GEOBIA is an iterative process, not only within eCognitionbased modeling but also between automation and manual corrections. One way of mimicking human cognition in a rule set is by applying contextual metrics using distance and density maps derived from building footprints and road centerlines. By using these maps in tandem, it is possible to depict approximate areas of urbanization (O'Neil-Dunne, 2011). However, these contextual analyses are only as good as their input datasets. For example, when classifying buildings using a combination of current imagery and an older LiDAR dataset, new buildings constructed after LiDAR acquisition will inevitably be classified as non-building impervious surfaces due to their low Normalized Difference Vegetation Index (NDVI) value, used to identify healthy vegetation, and ground level elevation. This is an error that is not easily remedied using an automated approach, but a round of manual corrections focused on reclassifying misidentified buildings quickly fixes the problem. Once these errors are fixed, the new building dataset will improve the building-distance and density maps, which in turn improves the contextual analysis of agricultural fields, impervious surfaces, and sidewalks.

Similarly, existing planimetric vector datasets depicting buildings, roads, and other impervious surfaces often need to be improved before they can be incorporated into a landcover rule set. If available imagery and LiDAR datasets are more recent than an existing vector layer, improvements can be performed using an automated approach. For example, an old set of building footprints can be augmented to remove non-building features such as ground level porches by segmenting with new LiDAR and then evaluating new aboveground features with a combination of simple height thresholds and the size of the resulting image objects (Figure 3 ). This approach maximizes the resources already devoted to the buildings layer and minimizes the errors of commission in unwanted features. A similar combination of LiDAR metrics and spectral values can be used to identify newly built buildings since the creation of the original dataset.

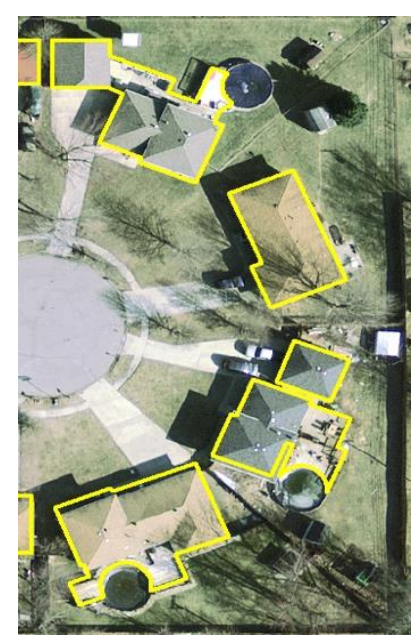

(a)

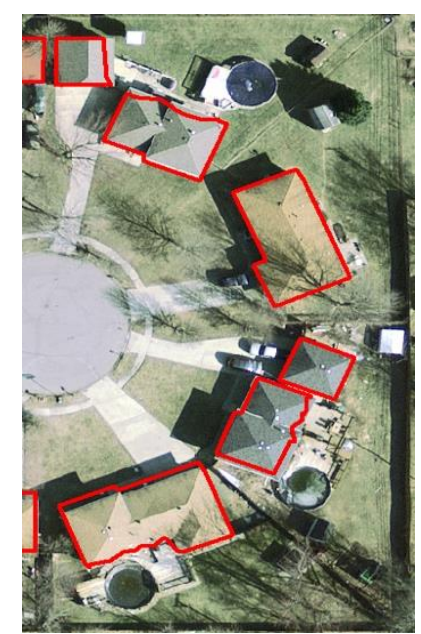

(b)
Figure 3. Old buildings vector dataset with porches (a). Porches automatically removed using OBIA (b).

\section{CONCLUSIONS}

GEOBIA is perhaps the only tool for converting the abundance of available remote-sensing datasets into timely, GIS-ready information. Much thought has been given to individual GEOBIA algorithms and applications, but little has been written on building effective GEOBIA systems, and the hardware, software, data, and human resources that are required to successfully apply this technology. Our experiences show that by implementing a framework that combines GEOBIA with standardized data processing and thorough manual review, it is possible to leverage previous investments in imagery and GIS data while providing essential land-cover data to policy makers and urban planners who need reliable, comprehensive maps for establishing baseline conditions and formulating tangible greeninfrastructure goals. Additionally, this information is equally important to researchers who are working toward a better understanding of complex landscape phenomena and their social and environmental dependencies, including tree-canopy change, global carbon stocks, urban land use, water and air pollution, and human health. The time is now for GEOBIA to inform and shape landscape analysis and planning at broad regional scales.

\section{REFERENCES}

Benz, U.C.; Hofmann, P.; Willhauck, G.; Lingenfelder, I.; Heynen, M. Multi-resolution, object-oriented fuzzy analysis of remote sensing data for GIS-ready information. ISPRS J. Photogramm. Remote Sens. 2004, 58, 239-258.

Blaschke, T. Object based image analysis for remote sensing. ISPRS J. Photogramm. Remote Sens. 2010, 65, 2-16.

MacFaden, S.W.; O’Neil-Dunne, J.P.M.; Royar, A.R.; Lu, J.W. T.; Rundle, A.G. High-resolution tree canopy mapping for New York City using LiDAR and object-based image analysis. J. Appl. Remote Sens. 2012, 6, doi:10.1117/1.JRS.6.063567.

O’Neil-Dunne, J.P.M.; MacFaden, S.W.; Pelletier, K.C. Incorporating contextual information into object-based image analysis workflows. In Proceedings of ASPRS 2011 Annual Conference, Milwaukee, WI, USA, 1-5 May 2011.

O’Neil-Dunne, J.P.M.; MacFaden, S.W.; Royar, A.R.; Pelletier, K.C. An object-based system for LiDAR data fusion and feature extraction. Geocarto. Int. 2013, 28, 227-242. 


\title{
SUSCEPTIBILITY MAPPING OF LINEAR EROSION PROCESSES USING OBJECT- BASED ANALYSIS OF VHR IMAGES
}

\author{
D. P. Passo ${ }^{\text {a }}$, E. S. Bias ${ }^{\text {a }}$, R. S. Brites ${ }^{\text {a }}$, G. A. O. P. Costa ${ }^{\text {b }, ~ R . ~ R . ~ A n t u n e s ~}{ }^{\text {a }}$ \\ a Federal University of Brasilia, Brazil - geodenilson@gmail.com, edbias@gmail.com, brites.ricardo@gmail.com and \\ rodrigorantunes@hotmail.com \\ ${ }^{\mathrm{b}}$ Rio de Janeiro State University - gilson.costa@ime.uerj.br
}

\begin{abstract}
:
Linear erosion is a natural phenomenon. However, inadequate occupation of the environment or the implementation of engineering works, without the due care, accelerates this process, which has been acknowledged as the main cause of land degradation worldwide. The use of high-resolution satellite imaging to map risk areas for this process, may contribute to devising prevention strategies. Linear erosion is a process dependent on thresholds controlled by many variables. This study has used only topographic variables (altimetry, slope, curvature profile, curvature plan, slope orientation, accumulation flow, humidity index, sediment transport capacity, potential flow and drainage network) and a vegetation index, which were selected due to their influence on linear erosion processes. The study was developed in two 6,000 x 4,500 meter areas, located in the eastern part of the Federal District - Brazil. The classification model building was done using open source software packages, namely InterIMAGE and WEKA. The aim of this study was to develop a routine for automatic mapping of areas susceptible to linear erosion. The accuracy rate achieved by the model was $87.5 \%$, as 21 of 24 linear erosion processes were identified. The percentage of the mapped area in relation to the total study area also showed that the classification was not overestimated.
\end{abstract}

KEYWORDS: RapidEye, Segmentation, Linear Erosion, Digital Elevation Model.

\section{INTRODUCTION}

The erosion process represents a major environmental impact caused by inappropriate occupation of rural and urban areas. This process results from the disintegration and natural movement of the particles of the soil; and it can be accelerated by anthropic action. It may occur when, for example, the original vegetation is removed and the uncovered soil becomes more vulnerable. It also happens when the appropriate conservation techniques are not used to manage the soil, causing imbalances in the interaction between soil, climate, relief and vegetation, and also instability in the system as a whole.

Studies regarding erosion processes have been developed by many researchers for a long time, usually based on empirical equations regarding the loss of soil layers. According to Lanza (2011), there are many approaches to this analysis, such as: the creation of cartography of areas at risk, field observation and experiment activities, spatial incidence of centers of erosion features, and also estimation of soil loss rates.

There is, however, a gap in erosion studies concerning automatic mapping of linear erosion processes. Measurements need to be taken on site to assess and monitor these processes. For the measurements to be taken on site, a great number of people and a large amount of equipment is necessary on the edges and in the interior of the process, which may worsen it, and puts the researchers on the field at risk. When it comes to locating, assessing and monitoring these processes, a viable alternative is to use remote sensing based methods, such as automatic classification of highresolution spatial imagery.

Some of the features that make linear erosion a different process with respect to other classes of erosion are: spectral heterogeneity (mixed types of soil, vegetation, and water), complexity of the target, caused by its size and varied shapes (irregular shapes, variable dimensions, asymmetry, and variable width/length proportion), and altimetry variation (which can range from centimeters to meters, with steep declivities on the edges). So it is necessary to devise an approach that take these specific features into account.

Among high-resolution remote sensing imagery classification approaches, Geographic Object-Based Image Analysis GEOBIA (Hay and Castilla, 2008) has gained relevance in the last years. This approach represents an improvement in respect to conventional automatic remote sensing analysis approaches, as it makes it possible to model human knowledge regarding specific characteristics of the target classes of objects, with the aid of features like brightness, texture, shape, proximity relations, among others, as well as being able to be integrate with other techniques, such as mining, Fuzzy logic, and fractal geometry. GEOBIA focuses on the classification of image segments, which is different from traditional classification approaches, which perform pixel-wise classification.

Information obtained with the object-based method proposed in this work provide an important support to environmental and territorial planning and managing. Such information helps in the efforts of locating the distribution and expansion of linear erosion processes, as well as making it possible to monitor changes in short intervals of time.

The objective of this study is to employ object-based classification on high-resolution images, using topographic variables to automatically map areas susceptible to linear erosion.

\section{MATERIALS AND METHODS}

The area under study is in Distrito Federal, one of 27 Brazilian states, located in the Midwestern region of the country, on the Central Plateau, with an area of 5,814 square kilometers. 


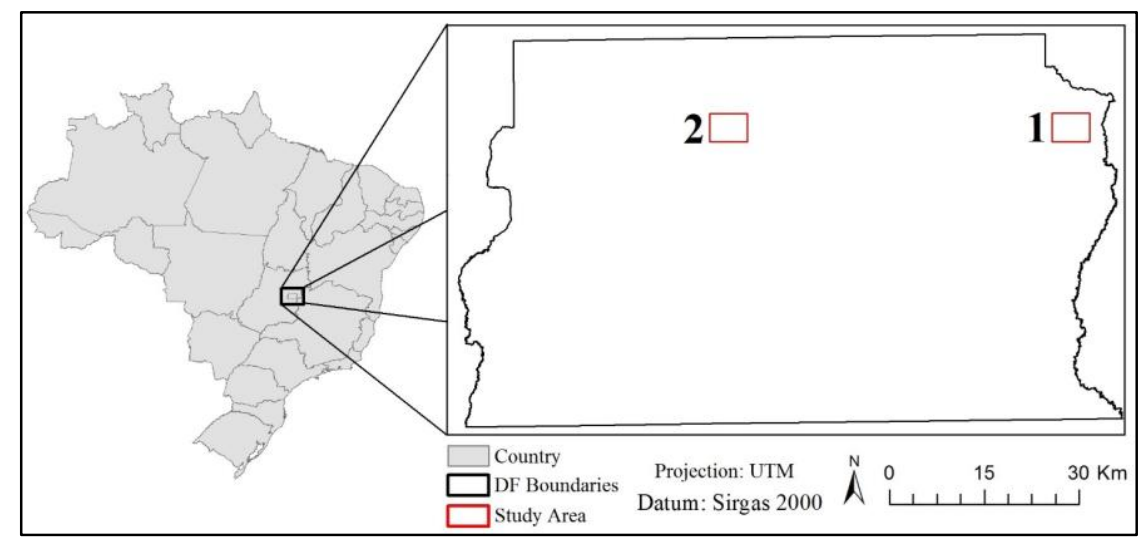

Picture 1 - Location of test-areas.

The proposed methodology was applied in two test-areas (Picture 1). Each test-area is 6,000 X 4,500 meters in size: that is, $27,000 \mathrm{~m}^{2}$. The delimitation of these areas took into account the existence of several ongoing erosion processes in the areas under study.

Test-area 1 was used for selecting samples to train the data mining procedure, so as to build a decision tree that aids mapping of ravines and gullies. Test-area 2 was chosen to test if the decision tree could be applied to an environment that was not influenced by the presence of training samples.

The following products were used to execute this study:

a) RapidEye image, with 5 meters' spatial resolution, with a spectral range between 440 and $850 \mathrm{~nm}$, and a 12-bit radiometric resolution;

b) vector data: hydrography, height level curves, and critical points;

c) Digital elevation model (DEM) generated by interpolation of topographic height level curves and critical points;

d) DEM-generated data: altimetry, declivity, profile curvature, plan curvature, aspect, flow accumulation, humidity index, sediment transport volume, and drainage potential.

The following software packages were used to execute this study:

a) ArcGIS v10.3 (ESRI, 2008);

b) ENVI v5.3 (ITT, 2009);

c) InterIMAGE v1.42 (Costa et al. 2010);

d) WEKA v3.7 (Witten and Frank, 2005).

The proposed methodology starts by selecting and acquiring a high-resolution remote sensing system. The RapidEye imaging system was chosen because of its spatial resolution and because MMA - Ministry of the Environment of Brazil provides it at no cost for research projects.

TERRACAP - Land Development Company of Brasília provided vector data (level curves, critical points, and drainage network) that made possible the construction of the DEM. This data was gathered during a cartographic mapping of the Federal District, at 1:10,000 scale, undertaken by TOPOCART in 2010.
After generating the following classification image planes from the DEM: altimetry, declivity, profile curvature, plan curvature, aspect, flow accumulation, humidity index, sediment transport volume and drainage potential; those planes were cropped so they would fit the limits of the testareas and could, then, be inserted on InterIMAGE as bands of a single, synthetic image, which was subjected to segmentation using the multi resolution segmentation algorithm proposed in (Baatz and Shäpe 2000).

Once the image segments were produced, segments representing linear erosion processes were selected as training samples for the data mining procedure. This selection was based on a mapping produced by Oliveira (2011) through visual interpretation of aerial and satellite imagery, that aimed to identify and analyze factors related to linear erosion processes in the Federal District.

After creating a single synthetic image by stacking the input image planes, segmentation and feature extraction was carried out with InterIMAGE. Then, a table was created, each line corresponding to a segment and each column, to a computed feature. The data thus obtained was transferred to the data mining software, through which the features were analyzed, and the most relevant features were selected to take part of in the classification model.

The J48 decision tree algorithm in the WEKA data mining software package was used to define a decision tree based on the grouping of specific segment features. The generated decision tree was then translated into decision rules in InterIMAGE, which was responsible for the final classification.

In order to assess classification quality, a verification based on a generic accuracy metric (non-site specific accuracy) was conducted. In this verification only the total area of the mapped category is analyzed, without taking the location of the category into account. In other words, it is a comparison (ratio) between the area of the category on the map (linear erosion susceptible areas in this case), which is generated by comparing the classification and the reference data (ground truth) (Congalton and Green, 1999). 


\section{RESULTS AND DISCUSSION}

The segmentation process preceded that of classification. Segmentation parameter values were input on the operator TA_Baatz_Segmenter (Baatz and Shäpe 2000), which is capable of multi-resolution segmentation, by accepting values for the relative weights of the synthetic image bands, and for compacity, color, and scale. The "weight" parameter defines each image band's relevance in the segmentation. In the experiments the same weight was defined for all bands. The "compacity" parameter represents the way the pixels in each segment are grouped, with a higher value meaning more compact objects. The "color" parameter refers to the relative importance of spectral information, i.e., pixel intensity values, in relation to the morphological characteristics of the segments, i.e., shape information. The "scale" parameter value roughly defines the size of the final image segments.

The J48 decision tree algorithm in the WEKA package can be tuned to constrain the model to be generated. Therefore, the parameter "MinNumObj" was set. This parameter controls the size and the complexity of the tree to be generated, and was set so that a minimum of 4 objects (segments) per leaf of the decision tree should be selected. This means that decisions that did not classify at least 4 segments were automatically eliminated from the model.

To build the decision tree (Table 1), the J48 algorithm selected eight features as the most relevant for the mapping of areas susceptible to linear erosion process. These features were altimetry, NDVI, profile curvature, plan curvature, topographic humidity index, sediment transport capacity index, proximity to drainage network, and aspect.

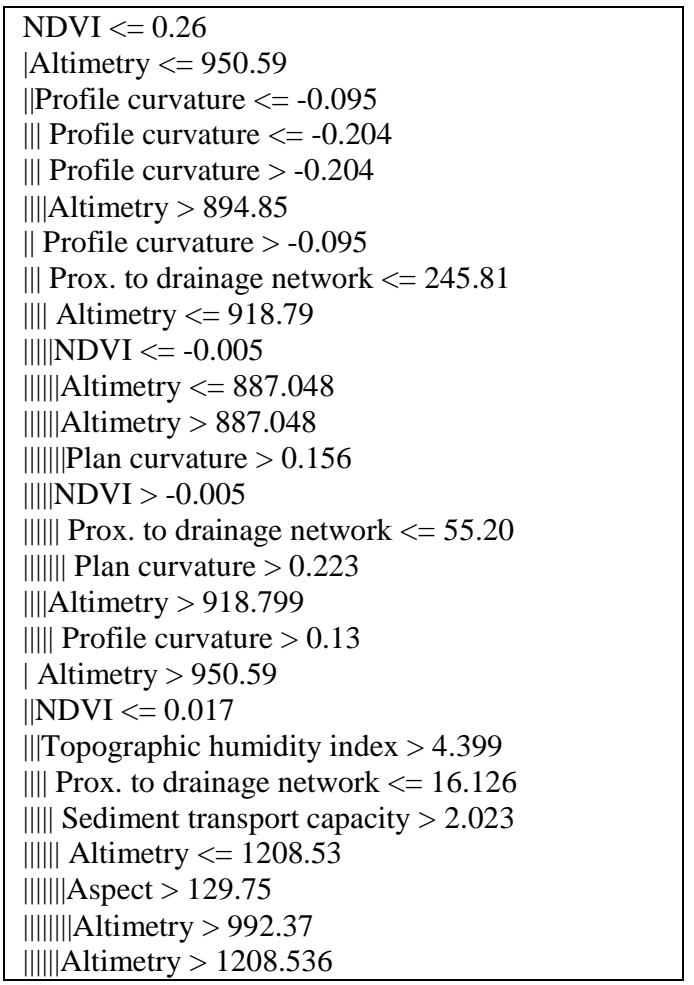

Table 1 . Tree in textual form, generated by the J48 algorithm.
The altimetry feature was then used as an indirect measurement of spatial variations in temperature and/or precipitation, as well as to estimate spatial distribution of climatic variables, such as average seasonal and annual precipitation, maximum daily precipitation, monthly average and temperature (Moore et al. 1991). This justifies the feature being chosen by the data mining algorithm, because, according to Pruski (2006), rain is the agent responsible for providing the necessary energy for hydric erosion to occur, because of the direct impact of water drops on the surface of the soil and also because of it is capability of producing superficial drainage.

The data mining algorithm selected NDVI as its first criterion to divide the areas (segments) susceptible to linear erosion processes, which confirms Lima (2003), who states that the presence of vegetation is a very important characteristic in the study of erosion, because it is a factor related to the direct protection of the soil against the impact of water drops. Vegetation also stops erosive action originated from superficial drainage.

The profile curvature and plan curvature are present because both are a characteristic of the terrain associated with hydrological, pedological, and transport of solid matter, as well as many other aspects, indirectly. The combination of both curvatures is represented by a concave-convergent form (maximum drainage concentration and accumulation) and by a convex-convergent form (maximum drainage dispersion), as described by Araújo (2006).

Even though the declivity feature is defined as inclination of slopes, which causes locations with a higher declivity to have more frequent linear erosion processes, the data mining algorithm did not select it directly. It was used indirectly, nevertheless, because it was part of the calculation to obtain other variables taken into account, such as: topographic humidity index and sediment transport capacity index.

The aspect variable was also selected by the data mining algorithm, which shows the importance of this feature in the mapping. Proof of this importance is the fact that this feature is used as local attribute in geological investigations, and may indirectly influence linear erosion processes by controlling exposure to different climatic conditions (time of exposure to sunlight, precipitation intensity, humidity retention, etc.) and vegetation (Dai et al., 2001; Çevik and Topal, 2003; Pulice et al., 2009; Conforti et al., 2010).

The values of the flow accumulation and declivity features were used to calculate other features taken into account, such as the humidity topographic index and the sediment transport capacity index.

The fact that the data mining algorithm selected the humidity index feature may be explained by the relationship between this index and saturated areas, as well as the generation of superficial drainage.

The feature concerning proximity to drainage network was also chosen because a relationship between this variable and linear erosion processes was inferred, which confirms Werlang (2004), who states that the relation between headwaters and linear erosion processes may be observed through the similarity between the concepts of incised river channels in valley headwaters and linear erosion processes. Incised river channels are the upper limit of flow and 
sediment transport. Linear erosion processes are natural incised channels that originate from natural or anthropic imbalances.

For test-area 1 (Figure 1), where training samples were selected, the classification model achieved a $95.83 \%$ accuracy value for the mapping in relation to the amount of linear erosion processes identified. This means the mapping was able to find 46 of 48 existing linear erosion processes.
Test-area 2 was used for confirmation, and it was analyzed using the decision tree defined by the training samples obtained in test-area 1 . The accuracy rate achieved by the model in Test-area 2 was $87.5 \%$, that is, 21 of 24 linear erosion processes were identified (Figure 2). The percentage of the mapped erosion susceptible area in relation to the total area of the test-area was $17.5 \%$ which shows that the classification was not overestimated.

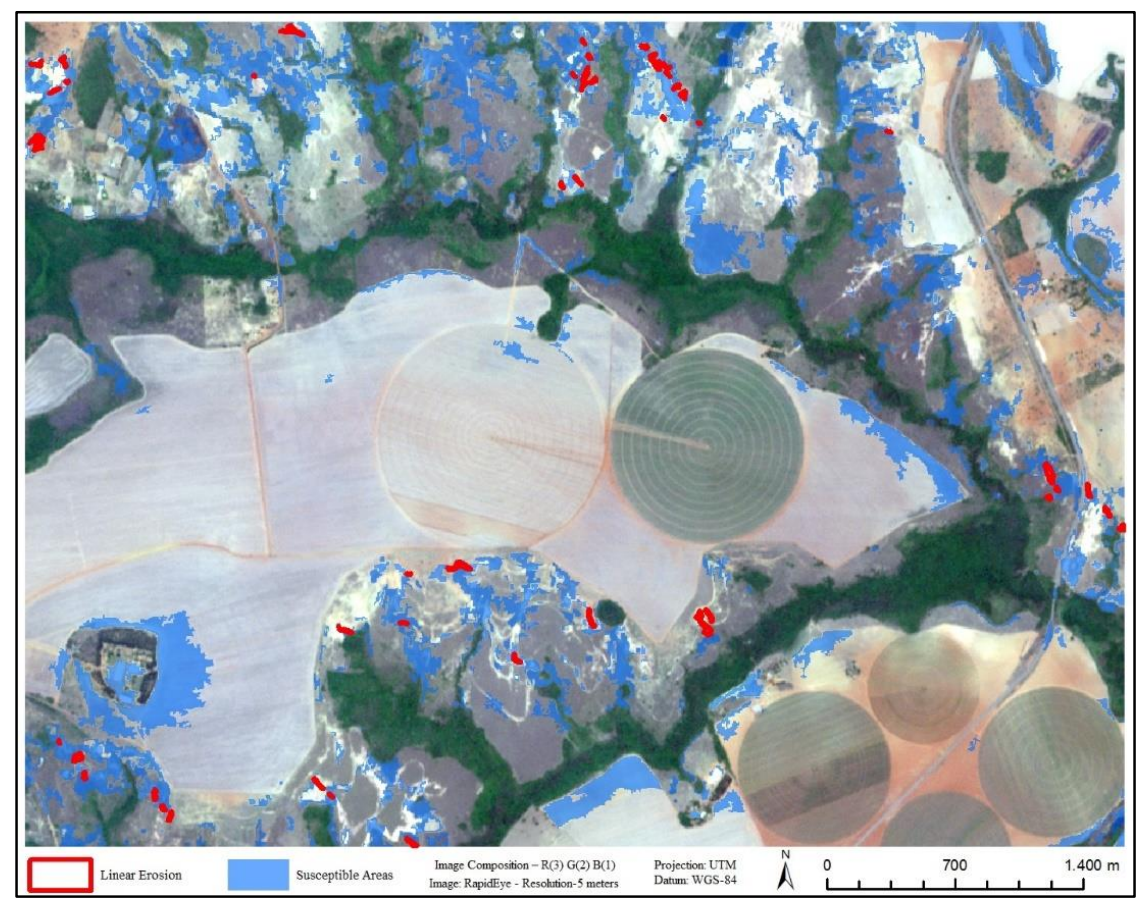

Figure 1. Map of susceptibility to linear erosion processes in test-area 1.

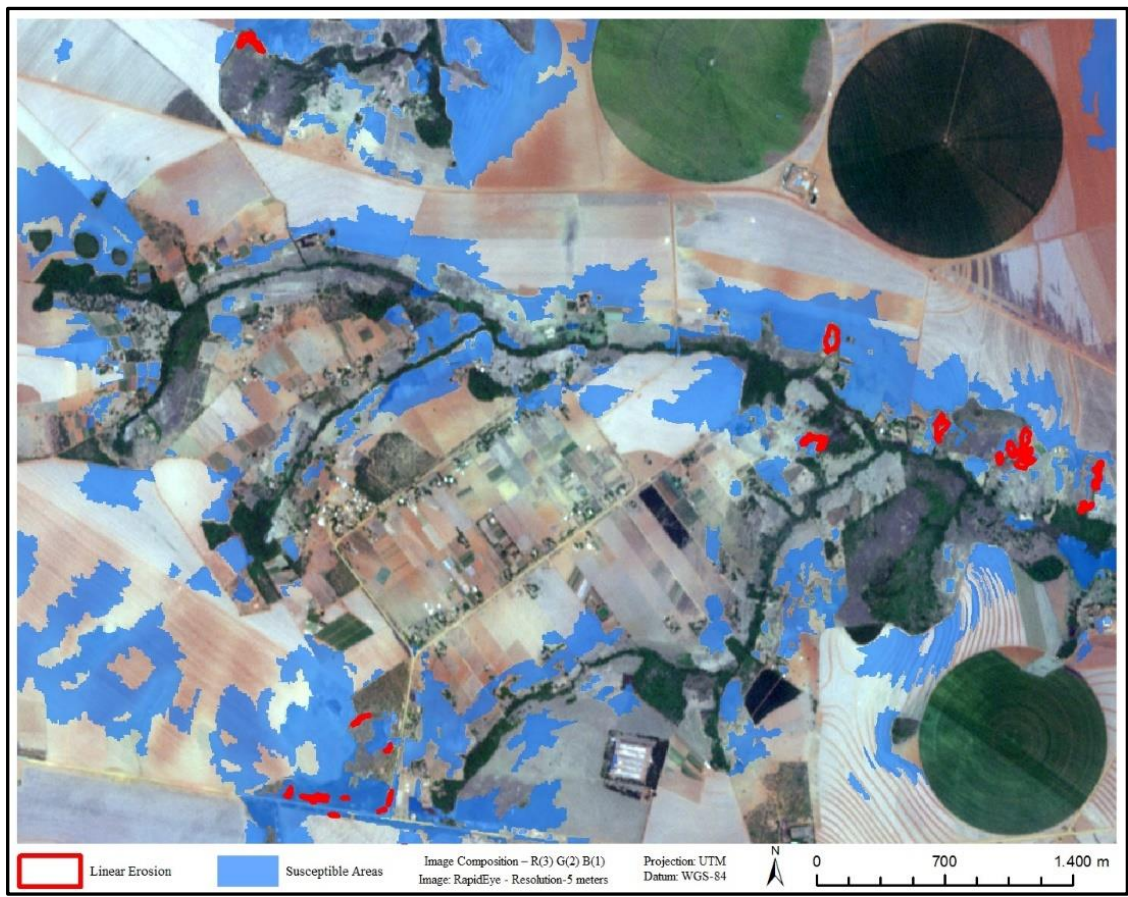

Figure 2. Map of susceptibility to linear erosion processes in test-area 2. 


\section{FINAL CONSIDERATIONS}

The association of object-based analysis and data mining has proven to be very effective for identifying areas susceptible to linear erosion processes.

The areas that were identified by the model coincided with a significant number of existing linear erosion processes, which led to high accuracy rates. As a result, using topographic attributes to map areas susceptible to linear erosion processes can be considered a coherent and effective method, and represents a useful tool for studies of this nature.

The study of topographic aspects has shown the strong relationship between physical elements of the terrain, hydrological processes, and, consequently, linear erosion processes. This relationship made it possible to devise an object-based methodology that delivered high accuracy rates. Due to the high accuracy obtained, the methodology has been found recommendable for the mapping of areas susceptible to linear erosion processes.

\section{REFERENCES}

Araújo, E. P., 2006. Aplicação de dados SRTM à modelagem da erosão em microbacias por geoprocessamento. Master's Thesis, São José dos Campos: Instituto Nacional de Pesquisas Espaciais (INPE).

Baatz, M., Schäpe, A., 2000. Multiresolution segmentation: an optimization approach for high quality multi-scale image segmentation. In: XII Angewandte Geographische Informations verarbeitung, AGIT Symposium. Proceedings. Karlsruhe, Germany: Herbert Wichmann Verlag, Salzburg - Austria, pp. 12-23.

Çevik, E., Topal, T., 2003. GIS-based landslide susceptibility mapping for a problematic segment of the natural gas pipeline, Hendek, Turkey. Environ Geol, n. 44, pp. 949-962.

CODEPLAN. Companhia de Desenvolvimento do Planalto Central. (1971). Diagnóstico do Espaço Natural do Distrito Federal. Brasília: Edição CODEPLAN.

Congalton, R. G., Green, K., 1999. Assessing the accuracy of remotely sensed data: principles and practices. New York: Lewis Publisher.

Costa, G.A.O.P., Feitosa, R.Q., Fonseca, L.M., Oliveira, D.A.B., Ferreira, R.S., Castejon, E., 2010KnowledgeBased Interpretation of Remote Sensing Data with the Interimage System: Major Characteristics and Recent Developments. In: Proceedings of the 3rd International Conference on Geographic Object-Based Image Analysis - GEOBIA 2010. The International Archives of the Photogrammetry, Remote Sensing and Spatial Information Sciences XXXVII. ITC, Enshede. 2010.

Dai, F. C. et al. 2001. Assessment of landslide susceptibility on the natural terrain of Lantau Island, Hong Kong. Environ Geol, n. 40, pp. 381-391.

Environmental Systems Research Institute (ESRI), 2015. ArcGIS Professional GIS for the desktop.
Hay, G. J., Castilla, G., 2008. Geographic Object-Based Image Analysis (GEOBIA): A new name for a new discipline? In: Blaschke, T., Lang, S., Hay, G. J. (Eds.) Object-based image analysis spatial concepts for knowledge-driven remote sensing applications. Berlin: Springer, pp. 75-89.

ITT Visual Information Solutions, 2009. ENVI 4.7, version 4.7. Boulder, Colorado, EUA.

Lanza, D. S., 2011. Diagnóstico da erosão laminar na alta e média bacia do rio Paraopeba. Master's Thesis, Belo Horizonte: Universidade Federal de Minas Gerais.

Lima, E. R. V., 2003. Erosão do solo: fatores condicionantes e modelagem matemática. Cadernos do LOGEPA. Série pesquisa. João Pessoa: Universidade Federal da Paraíba.

Moore, I. D., Grayson, R. B., Ladson, A. R., 1991. Digital terrain modeling: A review of hydrological, geomorphological and biological applications. Hydrological Processes, v. 5, pp. 3-30.

Oliveira, B. E. N., 2011. Mapeamento, identificação e análise dos fatores relacionados aos processos erosivos no Distrito Federal (DF) - Ênfase nas Voçorocas. Master's Thesis, Brasilia: Universidade de Brasília.

Pruski, F. F., 2006. Conservação do solo e da água: práticas mecânicas para o controle da erosão hídrica. Viçosa: UFV, pp. 131-171.

Pulice, I. et al. 2009. Studio multidisciplinare di forme e processi denudazionali nell'area di Vrica (Calabria orientale). Bollettino della Societa Geografica Italiana 87(I-II), pp. 399-414.

Werlang, M. K., 2004. Configuração da rede de drenagem e modelado do relevo: conformação da paisagem na zona de transição da bacia do Paraná na Depressão Central do Rio Grande do Sul. PhD Thesis, Santa Maria-RS: Universidade Federal de Santa Maria.

Witten, I., Frank, E., 2005. Data Mining: Practical Machine Learning Tools and Techniques. 2. ed. San Francisco, CA: Morgam Kaufmann Publishers. 


\title{
APLICATION OF OBJECT-BASED ACCURACY ASSESSMENT FOR LAND COVER CLASSIFICATION USING RAPIDEYE IMAGES IN SOUTHEASTERN BRAZIL
}

\author{
D. F. C. Prado ${ }^{\text {a }}$, L. M. T. Carvalho ${ }^{\text {a } *}$ \\ ${ }^{\text {a }}$ Federal University of Lavras - UFLA, Lavras, Brazil-danfernando010@gmail.com, passarinho@dcf.ufla.br.
}

KEY WORDS : Validation, Sampling, Thematic accuracy, Object geometry, Similarity measurements

\begin{abstract}
:
Maps of land cover generated by Geographic Object-Based Image Analysis (GEOBIA) approach provide several advantages in relation to the pixel-based methodology, thus leading to significant gains for the accuracy of the final map. The accuracy indices that frequently express such gains are obtained from the traditional pixel-based validation. Then, given the absence of a validation representing the geographical object, new object-based methodologies to assess the accuracy have been developed with the similarity matrix STEP (Shape, Thematic, Edge and Position). In this sense, from the STEP methodology, this study aims to evaluate the thematic and geometric accuracy of land cover mapping generated by GEOBIA, as well as identify and analyse the main sources of errors. The study area is located in the municipalities of São José do Barreiro (São Paulo state) and Resende (Rio de Janeiro state), Southeastern Brazil. The map of land cover was generated from RapidEye Images acquired in year of 2011, with $5 \mathrm{~m}$ of spatial resolution. The global accuracy of the classification in relation to the traditional matrix was of $92 \%$. The methodology integrates four similarity measurements (Shape, Thematic, Edge and Position), each one generating two error matrices (individual and aggregate thematic class). A global thematic accuracy of $76.6 \%$ was obtained, and for the measurements position (81.8\%), edge (99.1\%) and shape $(93.3 \%)$. The methodology may be considered applicable and effective to validate thematic maps generated by GEOBIA. However, there are some limitations to be considered in relation to the size and extent of the objects.
\end{abstract}

\section{INTRODUCTION}

Remote sensing consists in a wide and attractive source for making maps, which enable the description of information on land cover through representations of the land surface (Foody, 2002). Furthermore, remote sensing has been showed to be useful in agriculture ( $\mathrm{Li}$ et al, 2016), urban analy sis (Zang \& Du, 2016), in change detections ( $\mathrm{Yu}$ et $\mathrm{al}, 2016$ ) and in the monitoring the vegetation at local, regional (Kempneers et al, 2013) and global scales (Homer et al, 2007, Xian et al, 2011)

Such information may be used for several purposes, like supporting public policies, decision making and scientific researches. In this sense, the accuracy of this information is determinant when using these products, what requires the measurement of the accuracy level, thus establishing a reliability parameter of the product.

Problems related to the inaccuracy of thematic maps may be influenced by several factors from the moment of data acquisition to the processing and generation of the final product. Characteristics like heterogeneity, shape and extension of targets or lack of experience of the interpreter may cause errors during the image analysis, thus decreasing its accuracy level.
Some constraints regarding the accuracy of cartographic products are also related to the source data, it means, the images. The design of targets and their allocation may be influenced by distortions caused during their acquisition. Nowadays it is possible to work with high-resolution and highly geometrically accurate images in function of advances on orbital remote sensors and pre-processing of images, for example the launch of a constellation with five RapidEye satellites in 2008 (BLACKBRIDGE, 2013). High-quality data provide more detailed information without distortions.

Additionally, new methodologies have been developed to improve techniques of aut omated classification. The Geographic Object-Based Image Analysis (GEOBIA) is a recent approach used to classify images supported by the object-based paradigm, which includes the location and spatial context as keycomponents of the analy sis (Hay and Castilla, 2008).

GEOBIA is a useful approach for mapping the land cover, since it allows a more significant classification of the landscape when compared to traditional pixel-based methods of image processing, especially using high-resolution spatial data (BLASCHKE and STROBL, 2001; ZHOU and TROY, 2008).

* Corresponding author 
In this sense, high-resolution data allied to new methodologies of analysis, such as those based in objects, have an enormous potential for generating information. On the other hand, there is a great challenge to evaluate such information, since the traditional pixel-based assessment is still used for this type of product. The assessment of thematic and geometric accuracy is one of the main challenges when using the object-based approach, but some methodologies have been developed in order to substitute the traditional ones (PERSELLO and BRUZONE, 2010; HERNANDO et al, 2012; MÖLLER et al, 2013, MÖLLER, BIRGER, GLÄSSER, 2014; LIZARAZO, 2014).

Identifying the source of errors of data classification is fundamental to measure the reliability of thematic maps. In this sense the present work aimed to assess the thematic and geometric accuracy of land cover mapping generated from RapidEye images, as well as identify and analyzed the main sources of errors in the generated product.

\section{STUDY AREA}

The study area is situated in Southeastern Brazil in the frontier between the municipalities of São José do Barreiro and Resende, São Paulo and Rio de Janeiro states, respectively. The land cover is compounded by fragments of Atlantic Forest, pasture, urban areas, small eucalypt crops and water bodies, with a total area of 2991.47 hectares, according to Figure 1.

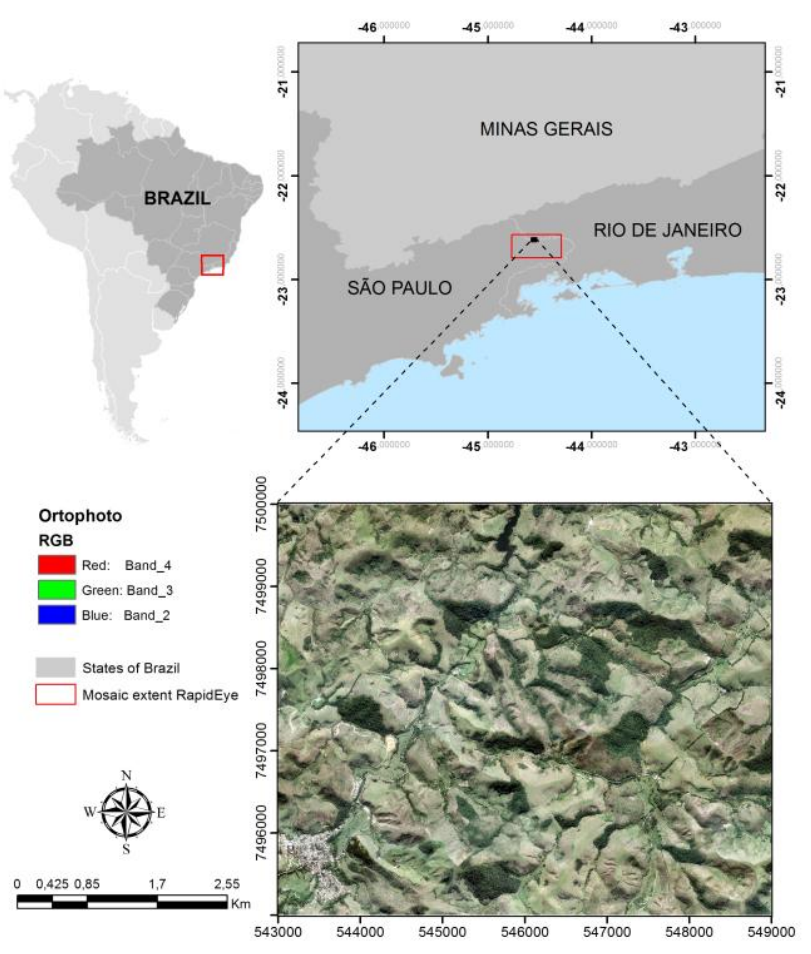

Figure 1. Localization of the study area.

\section{MATERIAL AND METHODS}

\subsection{Satellite images}

RapidEye images corresponding to the tiles 2328617 and 2328616 with acquisition dates in 09/27/2011 and 08/16/2011, respectively, were used to classify the land cover in the study area. The images have spatial resolution of $5 \mathrm{~m}$ and 5 spectral bands: Blue (B), Green (G), Red (R), Red-Edge(R-edge) and Infra-red (IR). The images were provided by Ministério do Meio Ambiente (MMA) and were mosaicked, since the study area is situated in the frontier between images. An ortophoto from 2012 with $0.4 \mathrm{~m}$ of spatial resolution and 4 spectral bands (B, G, R, NIR) was used for the collection of reference samples.

\subsection{Image classification}

The thematic classification of RapidEye images was made through GEOBIA. The included classes were vegetation (fragments of forests and areas of natural vegetation), anthropic area (pasture, crops, exposed soil and areas of human intervention), urban area (conurbations, cities and districts) and water bodies. The class anthropic area represented $81.39 \%$ of all the studied area, followed by the classes vegetation (17.36\%), urban area $(1.14 \%)$ and water bodies $(0.11 \%)$. The area of analysis is limited to the ortophoto extension, what led to the reduction of thematic classification for comparisons among objects. Overall the classification resulted in 184 classified objects that comprised 152 objects of vegetation, 22 of anthropic area, 9 objects of water bodies and 3 of urban area. The thematic classification attained a global accuracy of $92 \%$ and 0.74 for the Kappa coefficient.

\subsection{Reference data}

In order to obtain the global accuracy based on the traditional error matrix (Congalton and Green, 1999), 200 random sampling points were distributed proportionally to the area of each class. For each point one thematic class corresponding to the ground truth was attributed. The targets were manually vectorized from the sampling points to represent the reference objects based on the ortophoto. Overall, 13 reference objects were digitized, which comprised 10 objects of vegetation and one for each of the other classes. The class anthropic area presented the largest area and more sampling points. However, since this class comprises almost all the extension of the area continuously, it resulted in only one reference object.

\subsection{Accuracy analysis}

The method proposed by Lizarazo (2014) makes a thematic and geometric validation for classification data generated by GEOBIA. The analysis consists on comparing corresponding classified objects and reference objects, it means, those with intersection. The reference object is the analysis parameter, in 
which all corresponding objects are analyzed. Based on the correspondence, the indexes of thematic and geometric similarity among objects are calculated: Shape, Theme, Edge and Position. Each index is calculated individually for each object, the value for each cell results in an interval ranging from 0 to 1 in which 0 corresponds to any similarity and 1 to total similarity.

The index of shape similarity (S) is calculated in relation to the normalized perimeter index (NPI) of each object (classified and reference) (Angel, Parent and Civco, 2010). The index is the results of the ratio between the circular perimeter with equal area $\left(P_{e a c}\right)$ and the perimeter of the analyzed object $\left(P_{o b j}\right)$ according to Equation 1. The ratio among NPIs of objects expresses the shape similarity, in which the $k$ value is 1 when the ratio $\mathrm{r}_{n p i}$ is equal to or lower than 1.0, otherwise the $k$ value is -1 according to Equation 2.

$$
\mathrm{NPI}=P_{e a c} / P_{o b j} \quad \text { (1) } \quad S=\mathrm{r}_{n p i}{ }^{k}
$$

The index of theme similarity $(\mathrm{T})$ is calculated by the percentage of intersection area between the reference object and classified object divided by the area of the reference object $\left(A_{\text {ref }}\right)$ according to Equation 3.

$$
T=A_{\text {int }} / A_{\text {ref }}
$$

The index of edge similarity (E) is calculated through the percentage of the edge length coincident between the classified object and the reference object $\left(l_{\text {int }}\right)$ in relation to the total edge length of the reference object $\left(p_{r e f}\right)$. The index of edge similarity is expressed by Equation 4. The $k$ value is attributed to 1 when $l_{\text {int }}$ is lower than or equal to $p_{\text {ref }}$, otherwise $k$ is equal to -1 .

$$
E=\left(l_{i n t} / p_{r e f}\right)^{\mathrm{k}}
$$

A "width" is considered in relation to the edge of reference data, namely the Epsilon Band, which is defined as a zone of uncertainty around an encoded line within which there is a certain probability of observing the 'actual' line (Lizarazo, 2014). This distance is based in the spatial accuracy of reference data that in this case was $1.25 \mathrm{~m}$ with $95 \%$ of confidence level.

Finally, the index of position similarity is calculated based on the position of the objects centroid. The index consists on dividing the Euclidian distance between corresponding centroids $\left(d_{\text {cent }}\right)$ by the diameter of the combined circular area of the reference object and the classified object $\left(d_{\text {cac }}\right)$ according to Equation 5. $d_{\text {cac }}$ corresponds to the diameter of the circle with area equal to the sum of the corresponding objects.

$$
P=1-d_{\text {cent }} / d_{\text {cac }}
$$

The results expressed by the indexes of similarity are presented in a matrix in which rows correspond to the reference objects and columns to the classified objects. The values contained in each cell consist in the similarity values of the corresponding objects. Objects without correspondence are expressed with value 0.00 in the matrix.
Resultant values of the individual similarity matrix are summarized in a similarity matrix aggregated by thematic classes. The matrix is constructed by aggregating lines and columns of the individual matrix in which the resultant number of rows and columns correspond to the number of thematic classes. The aggregation of line and columns is conducted separately. The aggregation of columns is made in relation to the reference objects according to Equation 6. For the aggregation of theme index, $\mathrm{T}_{j}$ is the aggregated value of the theme index $(\mathrm{T})$ of the reference object $j, \mathrm{a}_{\mathrm{i}}$ is the percentage of the reference area occupied by the object $i$ and $\mathrm{T}_{i}$ is the theme value of object $i$.

$$
T_{j}=\sum_{i=1}^{n}\left(a_{i} T_{i}\right)
$$

The aggregation of lines is made according to the weight of each reference object. The weight (Equation 7) is calculated from the probability of one reference object be selected within its thematic category (Equation 8). The aggregation of lines is conducted according to Equation 9, in which $\mathrm{T}_{a g}$ is the aggregated value of the theme index for each thematic category; $\mathrm{w}_{\mathrm{j}}$ is the weight given to the reference object $j$, and $\mathrm{T}_{j}$ is the resultant value of the theme index for the object $j$.

$$
w_{j}=\frac{1}{p_{j}} \quad \text { (7) } \quad P_{j}=\frac{a_{j}}{A_{c}} \quad \text { (8) } \quad T_{a g}=\frac{\sum_{j=1}^{n}\left(w_{j} T_{j}\right)}{\sum_{j=1}^{n}\left(w_{j}\right)}
$$

Furthermore, in order to compensate the differences of areas sampled by thematic category, the method still proposes an analy sis of aggregated matrix by thematic class weighted by area, in which values of area superimposed on the reference object of each cell of the aggregated matrix is weighted according to the normalized weight for its thematic category.

The normalized weight is obtained from the ratio between the simple weight of one category and the sum of weights of all categories. The simple weight of each class $\left(\mathrm{W}_{\mathrm{x}}\right)$ is calculated by the Equation 10 as the ratio between the total sampled area $\left(A_{t}\right)$ and the sampled area of each category $\left(a_{x}\right)$.

$$
\mathrm{W}_{\mathrm{x}}=\mathrm{A}_{\mathrm{t}} / \mathrm{a}_{\mathrm{x}}
$$

From the weighted matrix the producer, user and global accuracy corresponding to each index (Shape, Theme, Edge and Position) was calculated.

\section{RESULTS AND DISCUSSION}

Similarity matrices for each index (Shape, Theme, Edge and Position) were constructed from the comparison of individual objects. Only similarity matrices aggregated by thematic classes and matrices weighted by area were used for a general analysis of classes. Table 1 presents the results of similarity matrices aggregated by thematic class. In the aggregated similarity matrix of theme the class anthropic area presented the better results, with 0.96 , followed by urban area with 0.91 . The classes 
vegetation and water bodies presented the lowest values, 0.79 and 0.75 , respectively.

\begin{tabular}{|c|c|c|c|c|}
\hline & Theme & & & \\
\hline True / Class & Anthropic & Vegetation & Water & Urban \\
\hline Antropic & $\begin{array}{l}0.96 \\
\end{array}$ & $\begin{array}{l}0.04 \\
\end{array}$ & 0.00 & $\begin{array}{l}0.00 \\
\end{array}$ \\
\hline \begin{tabular}{|l|} 
Vegetation \\
\end{tabular} & 0.21 & 0.79 & 0.00 & 0.00 \\
\hline \begin{tabular}{|l|} 
Water \\
\end{tabular} & 0.02 & 0.22 & 0.75 & 0.00 \\
\hline \multirow[t]{2}{*}{ Urban } & 0.08 & 0.01 & 0.00 & 0.91 \\
\hline & Edge & & & \\
\hline True / Class & Anthropic & Vegetation & Water & Urban \\
\hline Antropic & $\begin{array}{l}0.55 \\
\end{array}$ & \begin{tabular}{|l|}
0.00 \\
\end{tabular} & 0.00 & \begin{tabular}{|r|}
0.00 \\
\end{tabular} \\
\hline \begin{tabular}{|l|} 
Vegetation \\
\end{tabular} & 0.12 & 0.64 & 0.00 & 0.00 \\
\hline \begin{tabular}{|l|} 
Water \\
\end{tabular} & 0.02 & 0.21 & 0.69 & 0.00 \\
\hline \multirow[t]{2}{*}{$\begin{array}{l}\text { Urban } \\
\end{array}$} & 0.03 & 0.00 & 0.00 & 0.70 \\
\hline & Shape & & & \\
\hline True / Class & Anthropic & Vegetation & Water & Urban \\
\hline Antropic & \begin{tabular}{|r|}
0.87 \\
\end{tabular} & \begin{tabular}{|l|}
0.01 \\
\end{tabular} & 0.00 & \begin{tabular}{|r|}
0.00 \\
\end{tabular} \\
\hline \begin{tabular}{|l|} 
Vegetation \\
\end{tabular} & 0.05 & 0.69 & 0.00 & 0.00 \\
\hline \begin{tabular}{|l|} 
Water \\
\end{tabular} & 0.02 & 0.04 & 0.60 & 0.00 \\
\hline \multirow[t]{2}{*}{ Urban } & $\begin{array}{l}0.04 \\
\end{array}$ & 0.01 & 0.00 & 0.83 \\
\hline & Position & & & \\
\hline True / Class & Anthropic & Vegetation & Water & Urban \\
\hline Antropic & $\begin{array}{l}0.95 \\
\end{array}$ & $\begin{array}{l}0.03 \\
\end{array}$ & 0.00 & 0.00 \\
\hline \begin{tabular}{|l|} 
Vegetation \\
\end{tabular} & 0.00 & 0.68 & 0.00 & 0.00 \\
\hline \begin{tabular}{|l|} 
Water \\
\end{tabular} & 0.00 & 0.00 & 0.73 & 0.00 \\
\hline \begin{tabular}{|l} 
Urban \\
\end{tabular} & 0.01 & 0.00 & 0.00 & 0.89 \\
\hline
\end{tabular}

Table 1. Matrix aggregated by thematic class of Theme, Shape, Edge and Position similarities.

The vegetation of the studied area consists in forest fragments, most of them linked by corridors. Differences between canopy size, textural variations in the image or shaded areas were also common in the area, which also have a more rugged relief. Such factors may cause errors of classification in function of the design of vegetation objects.

The class vegetation presented the lowest value in the aggregated matrix of position in relation to the other classes (0.68). According to Figure 2-A, errors in classification like disjoint classified objects, cause great difference between the position of objects centroids. In this case, both the number of centroids analyzed and the distance among them increases, what decreases the level of positional accuracy.

During the construction of the individual similarity matrix of position, negative values were observed in some cells, which correspond to the intersection between objects of different classes. Negative values occur when the distance of centroid $\left(d_{\text {cent }}\right)$ among objects is greater than the value of circular diameter with the same area $\left(d_{c e a}\right)$ (equation 5). In this case, the analysis of several different classes of objects presented a large distance between the centroids (Figure 3), thus generating negative values. These negative values were assigned to objects without any positional correspondence and were considered the value 0.00 in the individual similarity matrix.
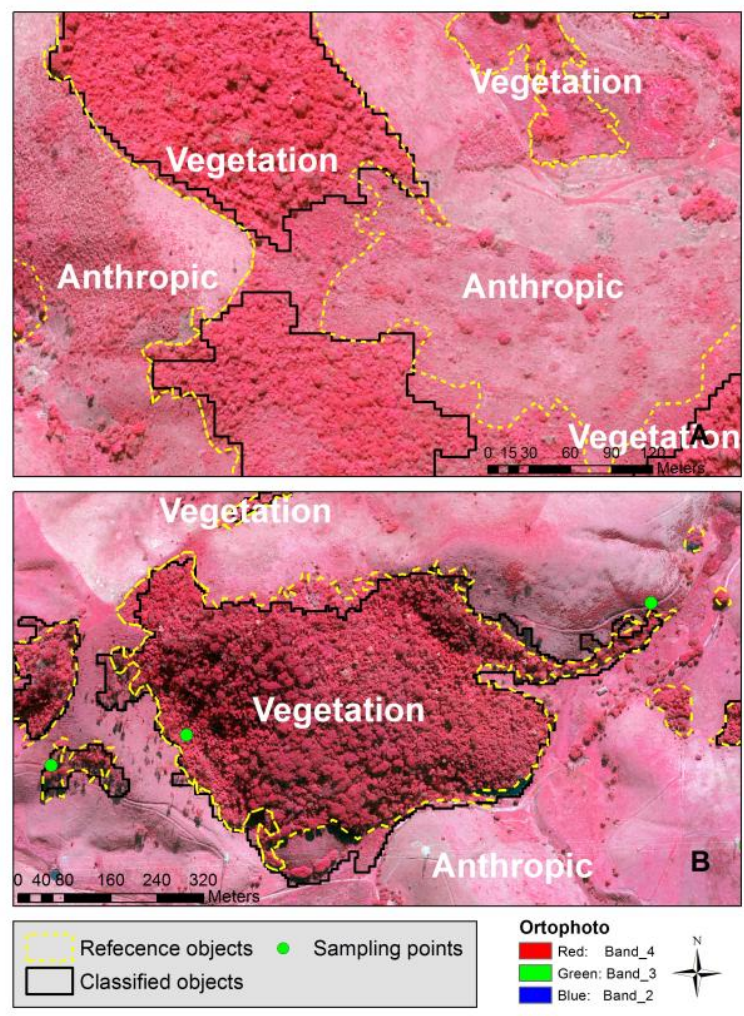

Figure 2A - Error in the discontinuity of the classified object. B - Differences between the edge of objects.

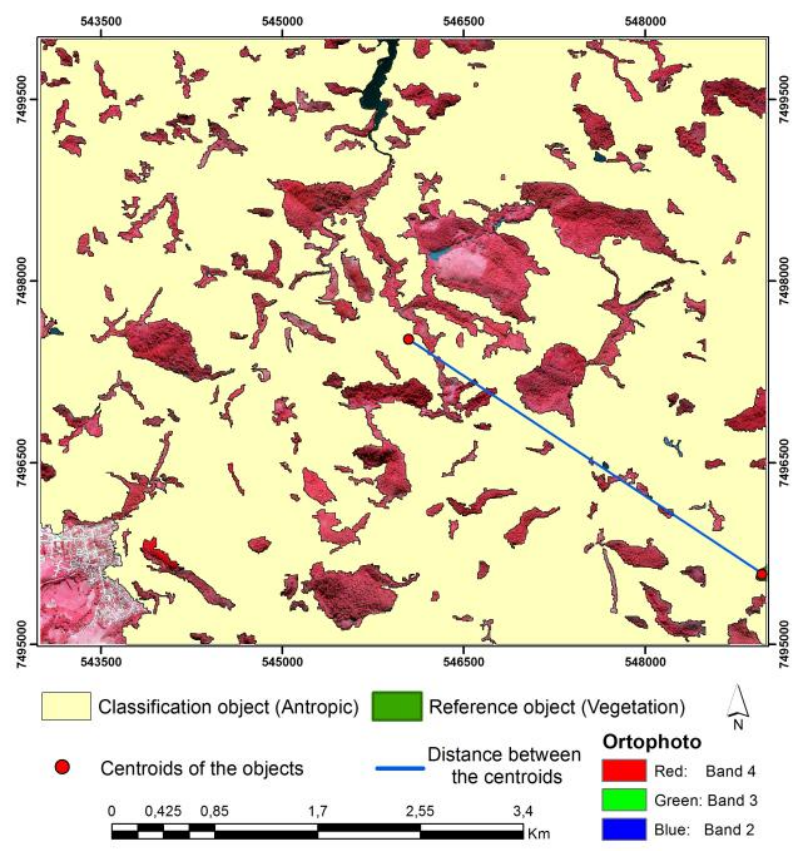

Figure 3. An example of the distance between objects with low correlation and different classes.

The similarity matrix of edge presented the lowest results in all the classes. In this matrix the anthropic area presented the lowest accuracy level (0.55). Problems related to the non- 
correspondence of edge often occur as in Figure 2B, for example, which are caused by classification errors and differences between the design of objects.

The weighted error matrix aims to compensate the differences of area in relation to the size of reference objects. Thus, classes with large areas receive low weight, while classes with small areas receive higher weights (Table 2).

\begin{tabular}{l|r|l|c} 
Class & $\begin{array}{c}\text { Reference object } \\
\text { area }\end{array}$ & $\begin{array}{l}\text { Simple } \\
\text { weigth }\end{array}$ & $\begin{array}{c}\text { Normalized } \\
\text { weigth }\end{array}$ \\
\hline Anthropic & 23438514 & 1.113327 & 0.000413 \\
\hline Vegetation & 2298875 & 11.35109 & 0.004209 \\
\hline Water & 10000.32 & 2609.391 & 0.967523 \\
\hline Urban & 347348.8 & 75.12547 & 0.027855
\end{tabular}

Table 2. Weight values for each thematic class

It is possible to observe in the weighted error matrix of theme (Table 3) that the class anthropic area presented the lowest producer accuracy $(50.33 \%)$. On the other hand, the class urban area obtained the highest producer $(99.77 \%)$ and user accuracy $(99.68 \%)$. The lowest values of user accuracy were obtained by the classes vegetation and water bodies, with $65.9 \%$ and $56.58 \%$, respectively. The global accuracy of the thematic similarity index was $76.67 \%$.

The highest percentage of error was observed for the classes vegetation and water bodies, which have the highest percentage of area within other reference object, therefore it is the anthropic class that receives the greater amount of area. This also reflects in a high omission error for the class anthropic area.

The weighted matrix of edge presented the highest results, with global accuracy of $98.73 \%$ and producer and user accuracy between $96.11 \%$ and $100 \%$. The values presented in the weighted error matrix for this index were more satisfactory than those presented by the aggregated matrix, what occurs because the area of edge overlap of different classes is smaller when compared to the correspondence area of objects. Thus, the omission and commission errors are low in relation to the weighted area of objects.

Results of shape and position weighted matrices presented global accuracy of $92.21 \%$ and $82.10 \%$, respectively. The classes vegetation and water bodies also presented the lowest values of user accuracy $(79.55 \%$ and $85.88 \%$ for shape and $62.68 \%$ and $66.08 \%$ for position, respectively), while the class anthropic area presented the lowest values of producer accuracy ( $81.04 \%$ for shape and $61.07 \%$ for position).

In relation to results by thematic class, it is possible to observe that the higher omission and commission errors repeated among the classes anthropic area, vegetation and water bodies. Therefore, there is an evident direct relationship between the errors of thematic classification and errors regarding the geometry of objects (shape, edge and position). In this study, only the edge similarity did not present the same cases of error in relation to the weighted area.

\begin{tabular}{|c|c|c|c|c|c|c|}
\hline & & Theme & & & & \\
\hline True / Class & Anthropic & Vegetation & Water & Urban & Total & Prod. Acc \\
\hline Antropic & 9247.22 & 4335.39 & 4763.48 & 26.37 & 18372.5 & $50.33 \%$ \\
\hline \begin{tabular}{|l} 
Vegetation \\
\end{tabular} & 123.34 & 8414.37 & 829.14 & 0.00 & 9366.85 & $89.83 \%$ \\
\hline Water & 0.10 & 9.35 & 7288.71 & 0.00 & 7298.17 & $99.87 \%$ \\
\hline Urban & 11.67 & 9.10 & 0.00 & 8827.82 & 8848.59 & $99.77 \%$ \\
\hline Total & 9382.33 & 12768.22 & 12881.33 & 8854.19 & 43886.1 & \\
\hline \multirow[t]{2}{*}{ User Acc. } & $98.56 \%$ & \begin{tabular}{|l|}
$65.90 \%$ \\
\end{tabular} & $56.58 \%$ & $99.70 \%$ & & $76.97 \%$ \\
\hline & & Edge & & & & \\
\hline True / Class & Anthropic & Vegetation & Water & Urban & \begin{tabular}{|l|} 
Total \\
\end{tabular} & Prod. Acc \\
\hline Antropic & 5337.66 & 208.14 & 7.75 & 0.06 & 5553.61 & $96.11 \%$ \\
\hline \begin{tabular}{|l|} 
Vegetation \\
\end{tabular} & 75.38 & 5809.07 & 10.93 & 0.00 & 5895.38 & $98.54 \%$ \\
\hline \begin{tabular}{|l} 
Water \\
\end{tabular} & 0.07 & 8.70 & 6632.73 & 0.00 & 6641.5 & $99.87 \%$ \\
\hline $\begin{array}{l}\text { Urban } \\
\end{array}$ & 3.94 & 0.71 & 0.00 & 6709.14 & 6713.79 & $99.93 \%$ \\
\hline Total & 5417.04 & 6026.62 & 6651.41 & 6709.20 & 24804.3 & \\
\hline \multirow[t]{2}{*}{ User Acc. } & $98.53 \%$ & $96.39 \%$ & $99.72 \%$ & $100.00 \%$ & & $98.73 \%$ \\
\hline & & Shape & & & & \\
\hline True / Class & Anthropic & Vegetation & Water & Urban & Total & Prod. Acc \\
\hline \begin{tabular}{|l|} 
Antropic \\
\end{tabular} & 8472.09 & 1289.57 & 685.49 & 6.59 & 10453.7 & $81.04 \%$ \\
\hline \begin{tabular}{|l} 
Vegetation \\
\end{tabular} & 43.37 & 5053.39 & 273.42 & 0.00 & 5370.18 & $94.10 \%$ \\
\hline \begin{tabular}{|l|} 
Water \\
\end{tabular} & 0.08 & 1.50 & 5830.97 & 0.00 & 5832.54 & $99.97 \%$ \\
\hline \begin{tabular}{|l} 
Urban \\
\end{tabular} & 5.32 & 7.65 & 0.00 & 8033.31 & 8046.29 & $99.84 \%$ \\
\hline \begin{tabular}{|l} 
Total \\
\end{tabular} & 8520.87 & 6352.12 & 6789.87 & 8039.91 & 29702.8 & \\
\hline \multirow[t]{2}{*}{ User Acc. } & $99.43 \%$ & \begin{tabular}{|r|}
$79.55 \%$ \\
\end{tabular} & $85.88 \%$ & $99.92 \%$ & & $92.21 \%$ \\
\hline & & Position & & & & \\
\hline True / Class & Anthropic & Vegetation & Water & Urban & Total & Prod. Acc \\
\hline \begin{tabular}{|l|} 
Antropic \\
\end{tabular} & 9240.78 & 2866.92 & 3013.30 & 10.81 & 15131.8 & $61.07 \%$ \\
\hline \begin{tabular}{|l|} 
Vegetation \\
\end{tabular} & 2.48 & 4819.91 & 578.22 & 0.00 & 5400.6 & $89.25 \%$ \\
\hline \begin{tabular}{|l} 
Water \\
\end{tabular} & 0.00 & 0.00 & 6997.17 & 0.00 & 6997.17 & $100.00 \%$ \\
\hline \begin{tabular}{|l|} 
Urban \\
\end{tabular} & 1.92 & 2.28 & 0.00 & 8651.26 & 8655.47 & $99.95 \%$ \\
\hline Total & 9245.18 & 7689.11 & 10588.68 & 8662.07 & 36185.1 & \\
\hline User Acc. & $99.95 \%$ & $62.68 \%$ & $66.08 \%$ & $99.88 \%$ & & $82.10 \%$ \\
\hline
\end{tabular}

Table 3. Weighted error matrix of theme, edge, shape and position.

Regarding global results, it is possible to observe that similarities of shape and edge obtained the highest success (more than 90\%). On the other hand, the similarity of position obtained a global accuracy of $82.1 \%$ and theme similarity of $76.97 \%$.

In an object-based accuracy analysis we may consider that geometric and thematic characteristics affect directly results of both of them. However, in general the theme and position similarities are the responsible by classification errors detected in the thematic map.

\section{CONCLUSION}

The method is able to assess jointly the thematic and positional accuracy of a thematic map with efficiency. The object-based assessment provided a more detailed analysis of the thematic product, thus allowing to observe how the inconsistencies between objects may reflect misclassification. Classification errors were well identified and measured by the method both in relation to the thematic and geometric information, what represents a great advantage in relation to traditional pixel-based analy ses. 


\section{ACKNOWLEDGMENTS}

I thank Fundação de Amparo à Pesquisa de Minas Gerais FAPEMIG for financial support and Coordenação de Aperfeiçoamento de Pessoal de Nível Superior - CAPES - for the scholarship provided.

\section{REFERENCES}

Angel, S., Parent, J., Civco, D. L., 2010. Ten compactness properties of circles: measuring shape in geography. The Canadian Geographer, 4 (54).

Blackbridge. Satellite Imagery Product Specifications. 2015.

Blaschke, T., Strobl, J., 2001. What's wrong with pixels? Some recent developments interfacing remote sensing and GIS. GISZeitschrift für Geoinformationssy steme 14 (6), 12-17.

Congalton, R. G., \& Green, K. ,1999. Assessing the accuracy of remotely sensed data: principles and practices. Boca Raton, FL: Lewis Publishers, pp.137.

Foody, G. M., 2002.Status of land cover classification accuracy assessment. Remote Sensing of Environment 80, pp. 185-201.

Hay, G.J., Castilla, G., 2008. Geographic object based image analysis: a new name for a new discipline.In: T. Blaschke, S. Lang, and G. Hay, (eds.), Object-based image analysis. Berlin:Springer, pp.91-111.

Hernando, G. M., D. Tiede, F. Albrecht, and S. Lang, 2012. Spatial and Thematic Assessment of Object-based Forest Stand Delineation Using an OFA-matrix. International Journal of Applied Earth Observation and Geoinformation 19, pp. 214225.

Homer, C. G., Dewitz, J., Coan, M., Hossain, N., Larson, C., Herold, N., et al., 2007. Completion of the 2001 National Land Cover Database for the conterminous United States. Photogrammetric Engineering and Remote Sensing, 73, pp. 337 341 .

Kempeneers, P., McInerney, D., Sedano, F., Gallego, J., Strobl, P, Kay, S., Korhonen, K. T., San-Miguel-Ayanz, J., 2013. Accuracy Assessment of a Remote Sensing-Based, PanEuropean Forest Cover Map Using Multi-Country National Forest Inventory Data. IEEE Journal of selected topics in applied earth observations and remote sensing, 6 (1).

Li, M., Ma, L., Blaschke, T., Chenga, L., Tiede, D., 2016. A systematic comparision of different object-based classification techniques using high spatial resolution imagery in agriculture enviroments. International journal of applied earth observation and geoinformation, 49, pp. 87-98.
Lizarazo, I., 2014. Accuracy assessment of object-based image classification: another STEP. International journal of remote sensing., 16 (35), pp. 6135-6156.

Möller, M., Birger, J., Gidudu, A., Glässer, C., 2013. A Framework for the Geometric Accuracy Assessment of Classified Objects." International Journal of Remote Sensing 34 , pp. 8685-8698.

Möller, M., Birger, J., Glässer, C., 2014. Geometric Accuracy Assessment of Classified Land Use/Land Cover Changes. Photogrammetrie - Fernerkundung - Geoinformation, 2, pp. 91100.

Persello, C., Bruzzone, L., 2010. A Novel Protocol for Accuracy Assessment in Classification of Very High Resolution Images. IEEE Transactions on geoscience and remote sensing, 48 (3), pp. 1232-1244.

Xian, G., Homer, C., Dewitz, J., Fry, J., Hossain, N., \&Wickham, J. (2011). Change of impervious urface area between 2001 and 2006 in the conterminous United States. Photogrammetric Engineering and Remote Sensing, 77,758-762.

Yu, W., Zhou, W., Qian, Y., Yan, J., 2016. A new approach for land cover classification and change analysis: Integrating backdating and an object-based method. Remote Sensing of Environment, 177, pp. 37-47.

Zhang, X., Du, S., 2016. Learning selfhood scales for urban land cover mapping with very-high-resolution satellite images. Remote Sensing of Environment 178, pp. 172-190.

Zhou,W. Q., \& Troy, A. (2008). An object-oriented approach for analysing and character- izing urban landscape at the parcel level. International Journal of Remote Sensing, 29, 3119-3135. 


\title{
UNCERTAINTIES IN ANALYSING THE TRANSFERABILITY OF THE GENERIC SLUM ONTOLOGY
}

\author{
J. Pratomo ${ }^{\text {a }}$, M. Kuffer ${ }^{\mathrm{a},}{ }^{*}$, J. Martinez $^{\mathrm{a}}$, D. Kohli ${ }^{\mathrm{a}}$ \\ ${ }^{a}$ Faculty of Geo-Information Science and Earth Observation (ITC), University of Twente, PO Box 217, 7500 AE Enschede, The \\ Netherlands - (j.pratomo, m.kuffer, j.martinez, d.kohli)@utwente.nl
}

KEYWORDS: GSO, Uncertainties, Transferability, Qualitative, Quantitative

\begin{abstract}
:
The Generic Slum Ontology (GSO) was developed to assist the detection of slums using Geographic Object-Based Image Analysis (GEOBIA). When applying the GSO locally, uncertainties exist in slum detection and transferability. Slums often have fuzzy boundaries and different ways to conceptualise. This study focuses on inherent uncertainties when analysing the transferability of the GSO across space, time and conceptualizations in the city of Jakarta, Indonesia. To measure the transferability of the GSO, we developed quantitative and qualitative indicators in multi-temporal Pleiades imagery (2012-2015) of two purposely-selected subsets. This framework allows assessing whether the developed ruleset is transferable across different spatial and temporal images. We applied two classification stages: background removal with a low scale parameter (SP) followed by slum extraction with a coarser SP. Both quantitative and qualitative indicators showed limited spatial and temporal transferability. Three sources of uncertainties can explain this result. First, the static concept of the employed ruleset and dynamic changes of slums. Real-world objects evolve over time, but their description remains static. Second, the gap between the real world (subjective conceptualization of objects) and image domain (quantitative values). For instance, the roof materials of slums (i.e. asbestos) have a similar spectral property with parking lot (from concrete), which resulted in misclassification. Third, the use of references data from local experts and municipal data introduce uncertainties that related to local ground knowledge and politics of slum declarations. Thus, this research contributes to the development of transferability measurements for the GSO and the understanding of underlying uncertainties.
\end{abstract}

\section{INTRODUCTION}

\subsection{Generic Slum Ontology (GSO)}

The Generic Slum Ontology was developed by Kohli, Sliuzas, Kerle, and Stein (2012) to assist the detection of slums in the GEOBIA domain. The GSO consist of three spatial levels, i.e., object, settlement, and environs (Kohli et al., 2012). At the environs level, Kohli et al. (2012) characterise slums by their location and neighbourhood characteristics; slums are often located in hazardous areas that may be close to employment opportunities. Meanwhile, on the settlement level, slums are characterised by their shape and density, where they often follow physical features, e.g. elongated settlements along rivers (Kohli et al., 2012). Lastly, at the object level, slums are characterised by building characteristics and access networks. However, not all slum characteristics can be determined by satellite imagery. Thus, for indicators that are not directly detectable from imageries, Kohli et al. (2012) recommended combining various other attributes that could act as proxy variables, i.e. roof type and building footprint area.

To implement the GSO in slum detection has three challenges (Pratomo, 2016). First, slums have unique spatial and temporal characteristics compared to formal areas, but can show considerable diversity within a city and even within a settlement (Kuffer, Pfeffer, \& Sliuzas, 2016). Second, slums have different definitions depending on the context. For this challenge, Kohli et al. (2012) recommended adopting the GSO to the local context. Third, due to the dynamics of slums, the GSO needs to be adapted to perform in multi-temporal images.

\subsection{Transferability}

Transferability, as mentioned by Kohli, Warwadekar, Kerle, Sliuzas and Stein (2013) refers to the capability of a method to result in comparable outputs with minimum adaptations, when applied in different spatiotemporal conditions. Selecting a single evaluation method to measure transferability is difficult (Bamberger, 2012). The usage of mixed-methods can combine the strength of quantitative and qualitative methods, which can overcome their respective weaknesses (Bamberger, 2012).

For quantitative methods to measure the transferability, Tuanmu et al. (2011) suggested three indicators to assess the transferability of a model. First, the model should result in a similar performance under different temporal conditions. Second, the model should result in a similar performance in various places. Third, the models should lead to similar spatial patterns (Tuanmu et al., 2011). Meanwhile, the usage of qualitative methods in measuring transferability aims to enhance, complement and follow up unexpected results from quantitative methods (Harwell, 2011). A study from Sori (2012) proposed several qualitative indicators, such as reliability, efficiency, utility and generality, and validity. 


\section{MATERIALS AND METHOD}

\subsection{Study Area}

Jakarta, the capital city of Indonesia, is inhabited by 30 million people within its metropolitan area (Demographia, 2015). The inability of local authorities to provide affordable housing has forced many low-income citizens to live in slums. To increase the quality of settlements, the national government of Indonesia developed policies to achieve the $100-0-100^{1}$ target by 2019 (UN-Habitat, 2014). Therefore, to monitor the achievement of this target, reliable data regarding slums is crucial.

Monitoring the dynamics of slums in the study area faces three main sources of uncertainties. First, the different definitions used to characterise slums (Pratomo, 2016). For instance, the Indonesian Central Board of Statistics (BPS) (2013) characterise slums by four indicators, i.e., lacking access to clean water, poor building materials, poor sanitation, and inadequate living space. Meanwhile, a different definition is used by the Ministry of Public Works and Public Housing (2014), using six characteristics, i.e., poor disposal, low coverage and poor quality of access network, an area inundated during rain, poor quality of water sources, high density and poor quality of settlements. The second uncertainty in monitoring slums refers to different levels to measure slums (Pratomo, 2016), commonly either on settlement level or household level. According to the official data obtained from the Department of Building and Settlements (DKI, 2014), the extent of slum settlements in Jakarta shows a decreasing trend between 2004 to 2013 (Figure 1). Meanwhile, the number of slum inhabitants increased between 1997 and 2013 (Department of Building and Settlements DKI, 2014). This fluctuation is partially due to the different levels used to measure slum extent and population.

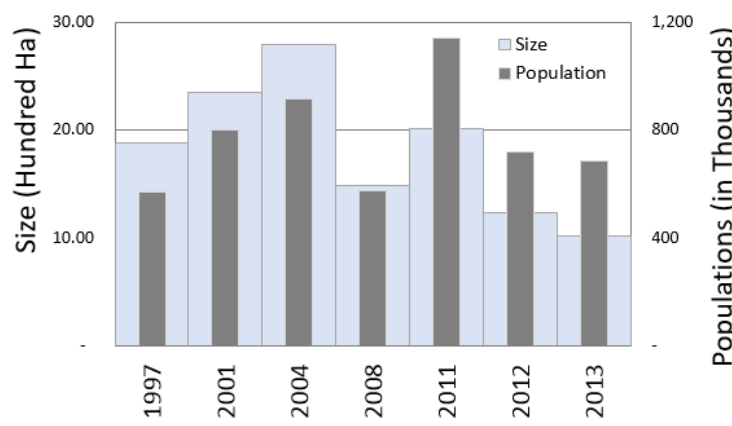

Figure 1. Trend of Slums in Jakarta (Pratomo, 2016)

The third source of uncertainties in monitoring slums is due to the existence of kampungs. This housing typology appeared in Jakarta more than half a century ago when the formal planning institutions were not established (Rukmana, 2008). To some extents, Kampungs are informal settlements, which share similar characteristics with slums, e.g., high density and unorganised layout. However, we cannot categorise all kampungs as slums, since many kampungs' residents have a formal ownership on land.

\footnotetext{
1 This programs aimed to achieve $100 \%$ access of clean water, $0 \%$ slums and $100 \%$ access to sanitation.
}

\subsection{Data Sources}

2.2.1 Image Selection. Multitemporal Pleiades imageries from the year 2012 to 2015 were used in our research. The spatial resolution of these images is 0.5 meters (after pansharping). We selected one image for each year with cloud coverage of less than $10 \%$. All images were radiometrically corrected. To analyse the transferability across different spatial conditions, we selected a subset that has variations in its spatial characteristics. The chosen area was the Tebet District in Jakarta (Figure 2). The subset size was 21 square kilometres. This district was selected due to three factors. Firstly, various urban features, from high-class residential, CBD to slums are located in this district. Secondly, a river passes through the district; regular floods occur here, and slums are often found near rivers. Lastly, various typologies of slums exist in this district, e.g., slums located on the riverbank and near the railroad. For the accuracy assessment, we employed groundtruth data collected in 2015 combined with slum delineations by the local experts.

2.2.2 The usage of ancillary data. To assist slum detection in GEOBIA, we employed several ancillary data (Table 1). The utility of combining VHR imagery with ancillary data for slum detection was demonstrated by Netzband (2009), Sori (2012), and Kohli et al. (2013).

\begin{tabular}{|l|c|l|}
\hline \multicolumn{1}{|c|}{ Data } & Year & \multicolumn{1}{c|}{ Source } \\
\hline Google Earth Image & 2013 & Google Earth \\
Google Street Map & 2013,2015 & Google Streetmap \\
Land use planning & 2014 & Govt of Jakarta \\
River, waterbody & 2015 & Openstreet Map \\
Road/rail network & 2014 & Openstreet Map \\
Socio-Economic & 2010,2015 & Statistical Bureau \\
District Boundary & 2014 & Govt of Jakarta \\
Slums neighbourhood & $2007-2013$ & Govt of Jakarta \\
\hline
\end{tabular}

Table 1. Ancillary Data

\subsection{Slum Detection in GEOBIA}

2.3.1 Formulation of the Local Ontology. To develop the local slums ontology (LSO), we conducted topic-focused interviews (Groenendijk \& Dopheide, 2003) with five local experts with experience on slum issues in Jakarta having different backgrounds. These were two experts from the government (central and local), one expert from an NGO, and two experts from consultants. During the interviews, experts indicated the local characteristics of slums and delineated the slum locations in the subset image.

2.3.2 Development of the ruleset. In our study, we developed two rulesets. The first ruleset was developed via a local adaptation of the GSO. The second ruleset was, however, developed based on the local slum characteristics listed by the experts, here called local slum ontology (LSO). The different characteristics of slums were then linked to image characteristics of slums, e.g., tone, shape, size, association and texture (Table 2).

\begin{tabular}{|l|l|c|c|}
\hline \multicolumn{1}{|c|}{ Real-world } & \multicolumn{1}{|c|}{ Image Domain } & GSO & LSO \\
\hline $\begin{array}{l}\text { Located on } \\
\text { riverbank/railroad }\end{array}$ & $\begin{array}{l}\text { Association: Distance } \\
\text { to river/railroad }\end{array}$ & $\sqrt{ }$ & $\sqrt{ }$ \\
\hline $\begin{array}{l}\text { Close to economic } \\
\text { hotspot }\end{array}$ & $\begin{array}{l}\text { Association: Distance } \\
\text { to CBD/industry }\end{array}$ & $\sqrt{ }$ & $\sqrt{ }$ \\
\hline Built in illegal land & Ancillary: land use & - & $\sqrt{ }$ \\
\hline
\end{tabular}




\begin{tabular}{|l|l|c|c|}
\hline \multicolumn{1}{|c|}{ Real-world } & \multicolumn{1}{c|}{ Image Domain } & GSO & LSO \\
\hline Irregular pattern & Shape: Irregular & $\sqrt{ }$ & $\sqrt{ }$ \\
\hline High density & Texture: GLCM & $\sqrt{ }$ & - \\
\hline $\begin{array}{l}\text { Absence of green } \\
\text { space/ vegetation }\end{array}$ & $\begin{array}{l}\text { Association: vegeta- } \\
\text { tion/green space }\end{array}$ & $\sqrt{ }$ & - \\
\hline Irregular roads & Shape: Irregular & $\sqrt{ }$ & $\sqrt{ }$ \\
\hline Small building size & Size: small & $\sqrt{ }$ & $\sqrt{ }$ \\
\hline Poor roof materials & Tone: Asbestos, iron & $\sqrt{ }$ & $\sqrt{ }$ \\
\hline Poor sanitation & Ancillary: Sanitation & - & $\sqrt{ }$ \\
\hline
\end{tabular}

Table 2. Application of Real-world into Image domain

2.3.3 Selection of the test area. To test the ruleset that was developed from the GSO and LSO, we purposively selected two test areas in Tebet District (Figure 2). For the first area, we selected an area where local experts had a relatively similar agreement on the slums extent. For the second sample, we selected an area where local experts had very different slum delineations. To reduce the computation time, we selected relatively small test areas, of 1 square kilometre.

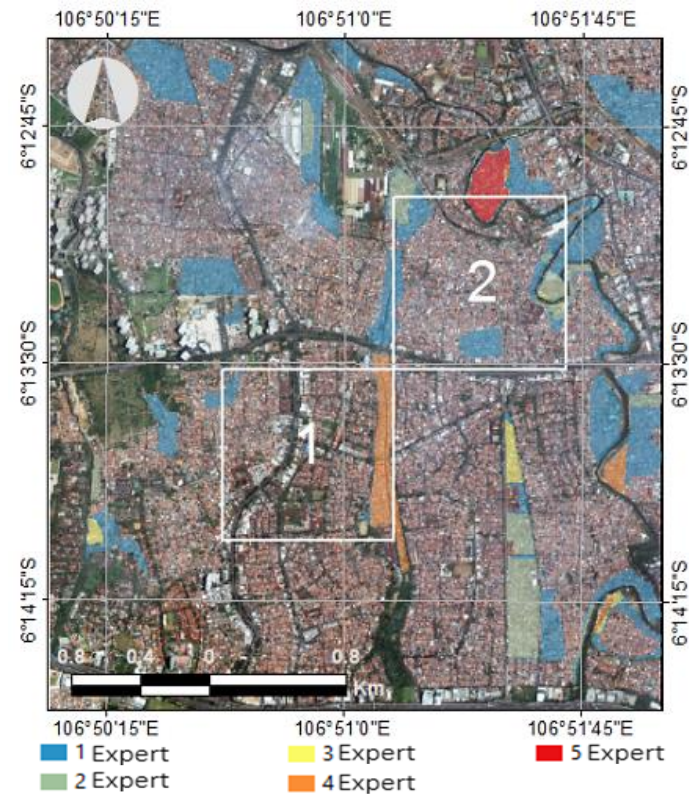

Figure 2. Selection of the test area

2.3.4 Segmentation. Among various segmentation algorithms, we selected the multi-resolution segmentation, being widely used due to its capability to create homogeneous objects based on user-defined thresholds (Baatz \& Schäpe, 2000). Some studies demonstrated the usage of multi-resolution segmentation in the slums domain, e.g., Kuffer, Barros, and Sliuzas (2014), and Kohli, Kerle, and Sliuzas (2012).

Within multi-resolution segmentation the setting of an appropriate scale parameter (SP) is necessary (Drăguț, Tiede, \& Levick, 2010). The SP controls the heterogeneity of an object, and its value correlates with the object size (Baatz \& Schäpe, 2000). The major drawback of the usage of multi-resolution segmentation is the commonly employed trial-and-error approach in selecting an appropriate SP (Whiteside, Boggs, \& Maier, 2011). To facilitate the estimation of an appropriate SP, Drăguţ et al. (2010) proposed a tool for Estimation of Scale Parameter (ESP), based on the idea of the Local Variance from Woodcook and Strahler (1987). In our study, we employed two levels of segmentation. At the first level, we employed a low $\mathrm{SP}$, with equal value for the smoothness and compactness parameter. The image objects resulting from the first level of segmentation were used as an input for background extraction. For the second level segmentation, we employed coarser SP, where the value was obtained from ESP.

2.3.5 Classification. Similar to the segmentation process, also for classification we employed a two-level approach. For the level 1 classification, we focused on extracting background classes, which are vegetation, road, railroad and river. For vegetation, we used the NDVI. The features roads, railroads and river, were classified using vector layers obtained from Openstreet Map (OSM). After we had extracted the background classes, the remaining unclassified image objects were segmented again with a coarser SP (see 2.3.4). Next, we employed two rulesets, i.e., from GSO and LSO.

\subsection{Transferability Measurements}

To measure transferability, we employed both quantitative and qualitative measurements. For quantitative measurements, we adopt the transferability framework of Tuanmu et al. (2011). For the qualitative measurement, we adopted indicators from Sori (2012).

2.4.1 Quantitative measurements. We measured the spatial and temporal transferability according to the resulted accuracy from the classification process. The ruleset is transferable, regarding spatial transferability, if it produces a similar accuracy with minimum adaptation when it is applied to a different location (image of the same date) (Figure 3). Meanwhile, for temporal transferability, similar accuracies should be obtained when implementing the ruleset to an image of a different date (at the same place) (Figure 4). Regarding spatiotemporal patterns, rulesets are transferable if they result in similar spatiotemporal patterns (Figure 5). For analysing the spatiotemporal patterns, we compared the spatial metrics of the extracted slums (from the GSO and LSO). We used the Unplanned Settlement Index (USI) developed by Kuffer et al. (2014), as indices.

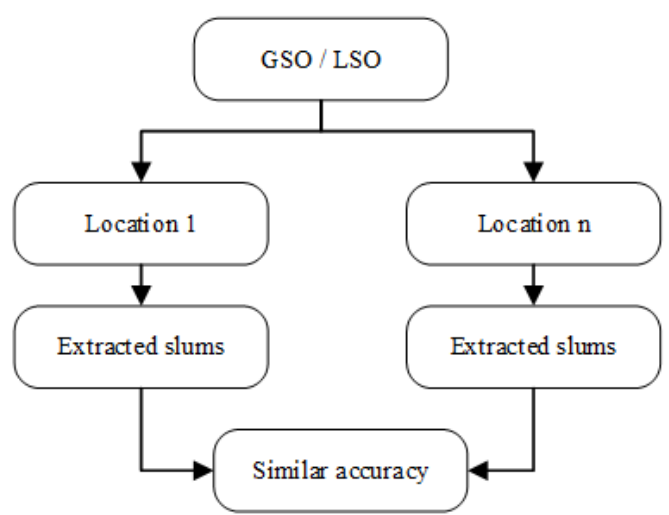

Figure 3. Spatial Transferability Framework 


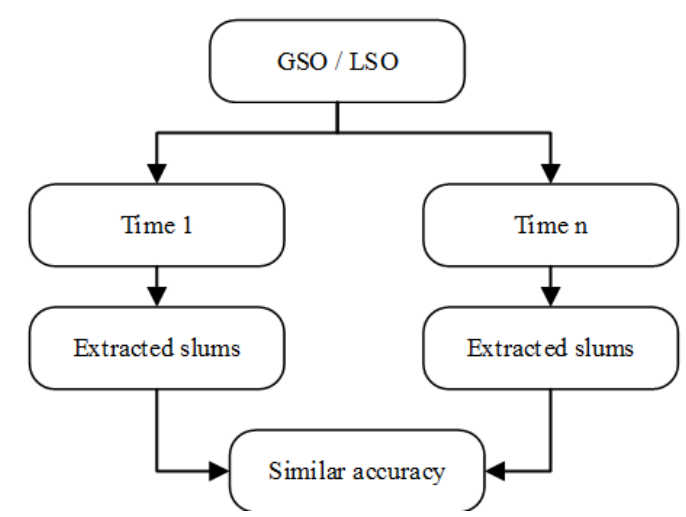

Figure 4. Temporal Transferability Framework

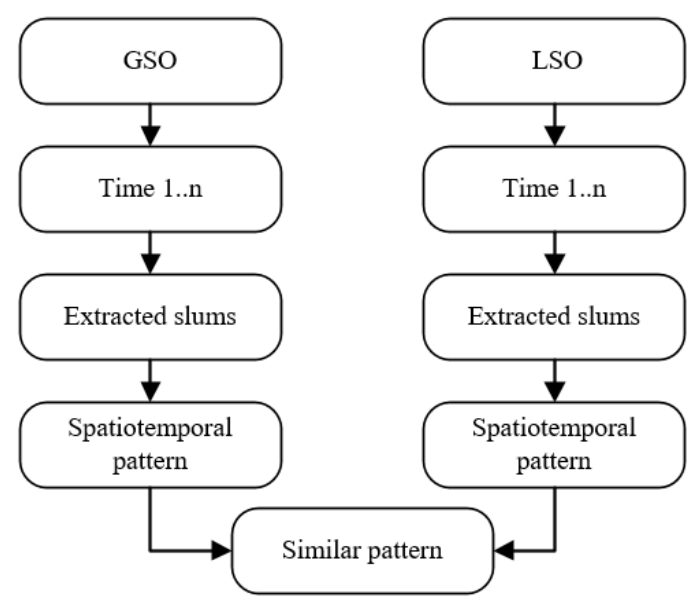

Figure 5. Spatiotemporal Transferability Framework

Three accuracy measurements were used to measure the spatial and temporal transferability, which are Overall Accuracy (OA), Kappa Index and quality of classification. For the quality of classification, the number of True Positives is compared with every possible result (True Positive, False Positive, and False Negative) (equation 2), as suggested by McKeown and Cochran (1999). The TP, FP and FN are defined by comparing the classification results from automated (GEOBIA) and manual delineations (from local experts).

$$
\text { Quality }=100 * \frac{T P}{(T P+F P+F N)}
$$

$$
\begin{aligned}
& \text { where } \quad \text { TP }=\text { True Positive } \\
& \text { FP }=\text { False Positive } \\
& \text { FN }=\text { False Negative }
\end{aligned}
$$

For the spatiotemporal comparison, we used spatial metrics to compare the classification results. Among various spatial metrics that may be utilised, we used Landscape Division Index, Patch Density, Aggregation Index, Shannon's Evenness Index, and Contagion Index.
2.4.2 Qualitative measurements. We employed two transferability indicators suggested by Sori (2012). For the first indicator, we measured the transferability according to the utility. A high utility is obtained when a large number of criteria (from the GSO and LSO), can be transformed into the image domain. For the second indicator, we used generality. The ruleset is transferable if the number of required adaptation for extracting slums is limited.

\section{RESULTS AND DISCUSSION}

\subsection{Slums Detection}

We detected the slums according to the GSO and LSO in the first and second area (Figure 6). For the 2013 data, we employed two temporal imageries. The 2013-2 data is applicable in both area, while the 2013-1 is only applicable in the first area due to high cloud cover in the second area.

3.1.1 GSO in the first area. Slums are reducing between 2012 and 2015 (first column, first to the fifth row). The slums can be divided into two patches, an eastern and western patch. Both patches show different slum extents. Some misclassification occurred in the west patch, which is a car park with similar spectral properties. This shows the uncertainties that resulted from transforming the real world characteristics of slums (i.e. poor roof materials), into image domain characteristics (i.e. tone of the roof) (see Table 2).

3.1.2 LSO in the first area. Slums that were detected resulted in a smaller area (second column, first to the fifth row) compared to the slums delineated by the experts. In the western part, only small areas of slums were detected. Although we can notice irregular patterns in the eastern parts, this area is not detected as slums, because of using the land tenure as an indicator. The use of references data from local experts (see Figure 2) and municipal data (see Figure 1) introduce uncertainties that relate to local ground knowledge and politics of slum declarations.

3.1.3 GSO in the second area. For the second area, we excluded the image obtained from May 2013 (2013-1) due to high cloud cover (Figure 6). Detecting slums in this area is more challenging since slums and non-slums are more difficult to distinguish. In general, slums that are located near the railroad stretching from south to north. They were changing between 2012 to 2015. A similar result is obtained for the slums that are located on the riverbank in the eastern parts. Meanwhile, slum areas along the railroad from east to west are relatively stable.

3.1.4 LSO in the second area. We can notice relatively stable slum locations in the western part (fourth column, row 1, 3-5). Only small changes occurred in this location during 2012 and 2015. A similar location, which is more stable, is found nearby the railroad in east-west direction. This pattern apparently is consistent with the results from the GSO. Outside this location, we also found slum areas that are relatively stable on the riverbank in the north side of the area. 

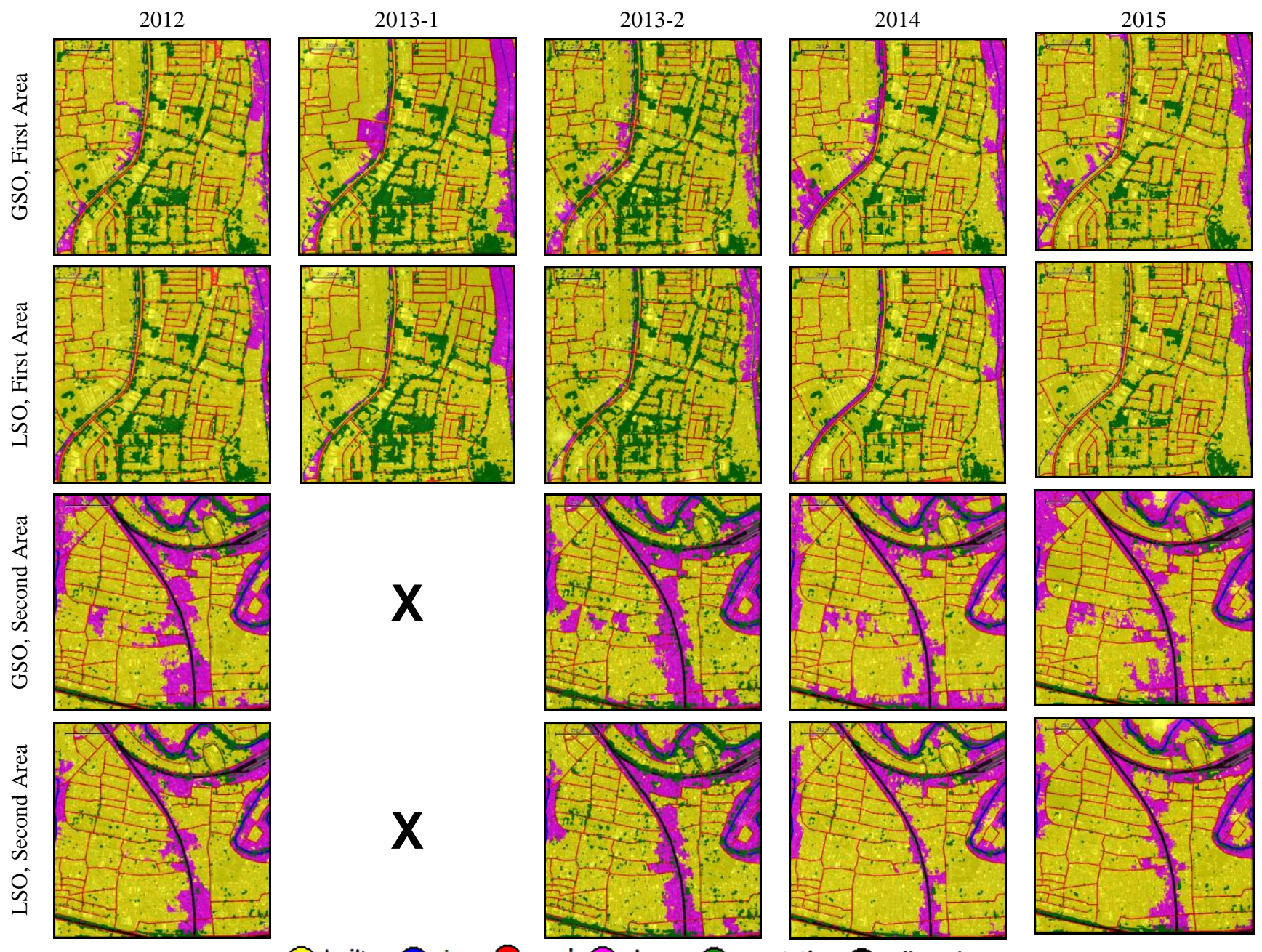

Figure 6. Result of Slum Detections

\subsection{Quantitative Measurements of Transferability}

3.2.1 Spatial Transferability. The ruleset should result into similar accuracies when applied in different areas. Hence, the difference of accuracies should be as low as possible. Accuracy results can be seen in Figure 7.

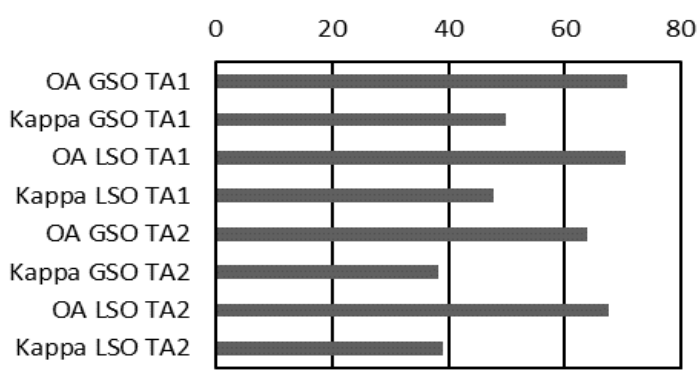

Figure 7. Confusion matrix-based accuracy (average)

For OA, the GSO resulted in a higher value compared with the LSO in the first area (TA1). For the second area (TA2), both rulesets led to the same result. We also noticed that the OA and kappa for this area are higher, compared with the first area. For the quality of classification (equation 2), the different concepts and delineations of slums (Table 2 and Figure 2) made it difficult to decide on the actual slum boundary (Figure 8). As a result, we found that the quality obtained from GSO and LSO is low (Table 3).

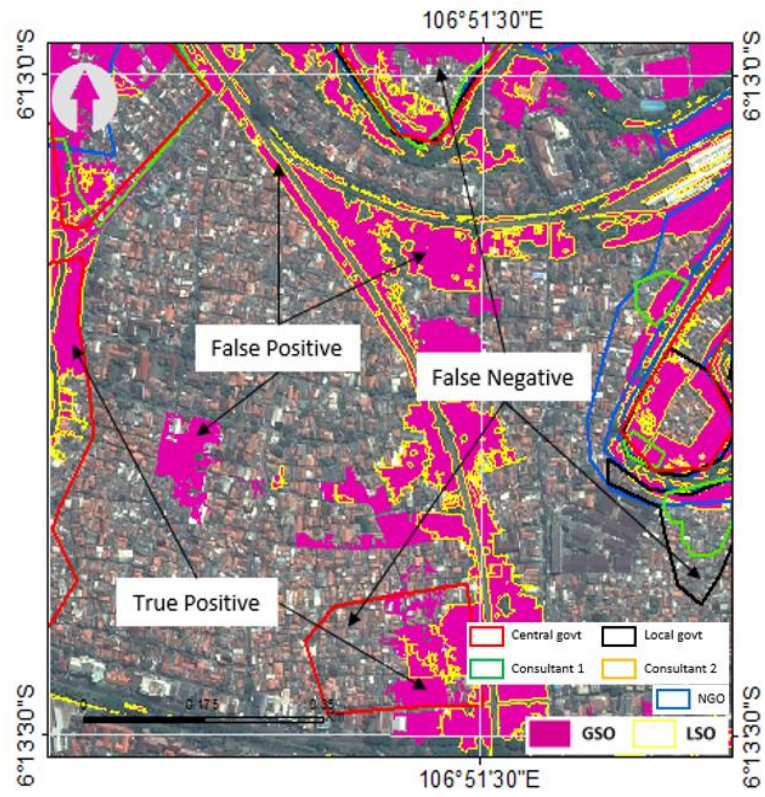

Figure 8. Automated and Manual Slum Extraction (area 2) 


\begin{tabular}{|c|c|r|r|r|}
\hline $\begin{array}{c}\text { Area, } \\
\text { ruleset }\end{array}$ & $\mathrm{TP}\left(\mathrm{m}^{2}\right)$ & \multicolumn{1}{|c|}{$\mathrm{FP}\left(\mathrm{m}^{2}\right)$} & $\mathrm{FN}\left(\mathrm{m}^{2}\right)$ & $\mathrm{Q}(\%)$ \\
\hline 1, GSO & $40,776.5$ & $14,508.1$ & $74,634.2$ & 31.39 \\
1, LSO & $34,809.8$ & $4,696.3$ & $80,600.9$ & 28.98 \\
2, GSO & $85,331.7$ & $145,663.6$ & $106,208.9$ & 25.31 \\
2, LSO & $52,969.3$ & $99,405.9$ & $138,571.3$ & 18.21 \\
\hline \multicolumn{5}{|c}{ Table 3. Quality of Classification Results }
\end{tabular}

The best result (Table 3) is obtained by the GSO in the first area, whereas the lowest is by the LSO in the second area. The GSO led to a higher TP in both test areas compared to the LSO, which indicates that the GSO led to a larger overlap area between automated and manual delineation. However, the GSO also resulted in a higher FP and lower FN compared to the LSO. Although the quality of the classification is low, the first area resulted in a higher quality compared to the second area. This result is as we expected since the difference between slum and non-slum areas in the first area is clearer than in the second area. However, the lower accuracy achieved by the LSO is not as we expected. We expect that the LSO should have higher accuracies since the LSO represents the local ontology based on the local characteristics stressed by experts. We argue that the low accuracy values of the LSO be due to the smaller slum area extracted by the automated detection. The tenure status ${ }^{2}$ was added as a slum characteristic for the LSO (Table 2). We combined the results from confusion matrix-based accuracy and quality of classification. As shown in Figure 3, a transferable ruleset should lead to similar accuracies when applied to a different area. We calculate the difference of accuracy result between the first and the second area for each ontology. To make the graph easier to interpret, we inversed the values. Thus, the higher the value, the less different the accuracy between the first and the second area; the more transferable the ruleset. We visualised the result in Figure 9.

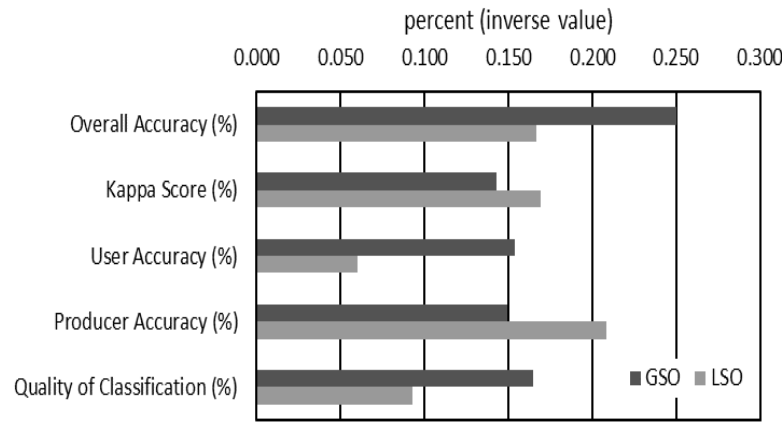

Figure 9. Spatial Transferability

According to Figure 9, the GSO resulted in higher transferability (lower difference of accuracies) for the three measurements, whereas the LSO shows a better Kappa and producer accuracy. Hence, the GSO is slightly more transferable than LSO. This result is unexpected since we assumed the local adaptation (LSO) would increase the accuracy of extracting slums.

\footnotetext{
2 Tenure status is obtained from the land use map. Developments are prohibited in green areas or buffer zones. Thus, by overlay the settlement with the land use data, we can indicate illegal settlements, which did not have a tenure status.
}

3.2.2 Temporal Transferability. For the first area, the GSO resulted in a slightly higher average of accuracy than the LSO, which is $70.8 \%$ and $70.4 \%$ respectively. Meanwhile for the second area, the LSO led to a higher average OA, which is $67.5 \%$. The average of the user accuracy obtained by the LSO in the first area is much greater compared to the GSO. For temporal transferability, the ruleset should result in a similar performance when applied to a different temporal image. Hence, we calculated the average of the accuracy changes. To make the comparison easier, we inversed the values (Figure 10). Thus, the higher the value, the less different the accuracy across the years; the more transferable the ruleset is.

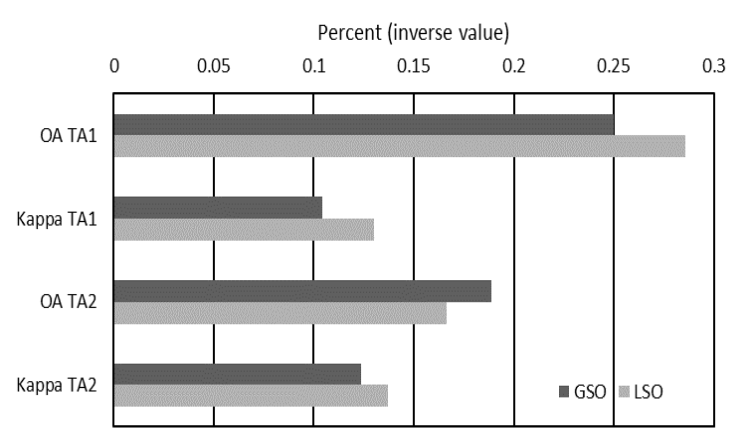

Figure 10. Temporal Transferability

For the first area, we can notice that the LSO produced a higher score (lower difference of accuracy across years) in three of four indicators. Meanwhile, in the second area, the GSO resulted in a higher score. Therefore, regarding temporal transferability, the LSO is more transferable to be applied in the first area, while the GSO is more transferable in the second area.

3.2.3 Spatiotemporal Transferability. The low values (Figure 11) indicate that the spatiotemporal results for the first and second area are similar; thus the ruleset is more transferable. Regarding Landscape Division, between 2012 and 2013 both rulesets are transferable. Meanwhile for 2014, both rulesets are more transferable to the first area, where in 2015 they are more transferable to the second area. For the Patch Density, both rulesets are transferable in both areas in 2012 and 2015. In addition, both rulesets are transferable in 2014 only for the first area. Regarding the Aggregation Index, GSO and LSO show good transferability for the second area. The Shannon's Evenness and Contagion Index resulted in similar patterns. Both of them indicated that both rulesets are not transferable. Firstly, the difference between rulesets is large in both test areas. A slightly smaller difference appeared in 2013 for the second test area. Secondly, the difference between GSO and LSO in both areas shows an increasing trend. 


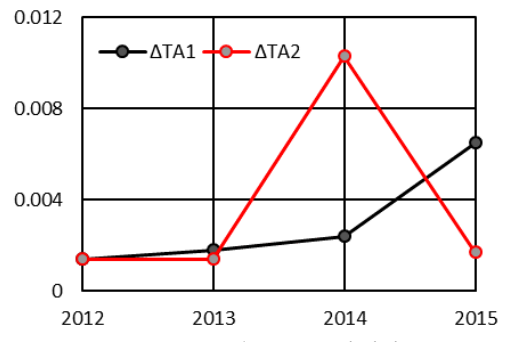

a. Landscape Division

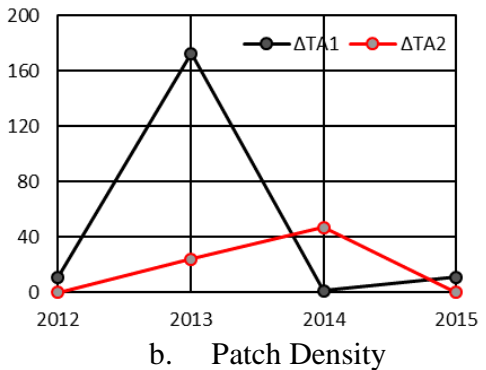

b. Patch Density

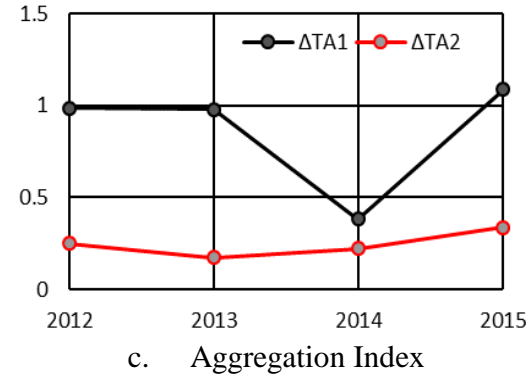

c. Aggregation Index

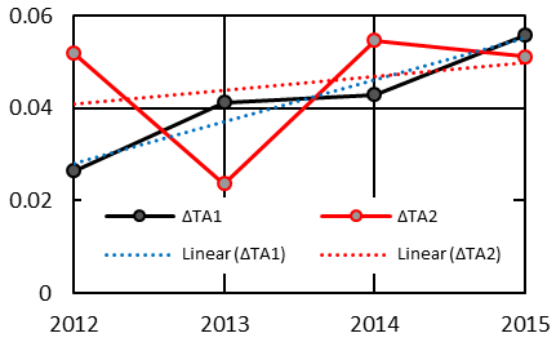

d. Shannon's Evenness Index

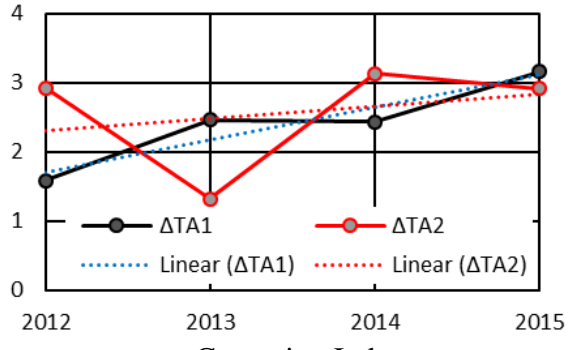

e. Contagion Index

Figure 11. Spatiotemporal Pattern

\subsection{Qualitative Measurement of Transferability}

3.3.1 Utility. Among eight real-world domain indicators from the GSO (Table 2), five of them can be used to detect slums in the study area. Meanwhile, three of them cannot be used due to different levels of aggregation, (e.g. distance to the economic hotspot) and unavailability of data (e.g. irregular road and small building size). Therefore, $62.5 \%$ of the GSO indicators can be used to detect slums. Meanwhile, from eight real-world domain indicators from the LSO (Table 2), only four of them $(50 \%)$ can be used to detect the slums. The reasons were the different levels of aggregation and unavailability of data. Therefore, the GSO is more transferable than LSO, in term of utility.

3.3.2 Generality. For the GSO, two adaptations are needed to detect the slums, which are the adaptation of NDVI values (for detecting vegetation), and the mean red/green ratio (for detecting the roof materials of slums). Meanwhile, since the LSO did not use the association to vegetation, the adaptation needed is only for roof materials. Therefore, in terms of generality, the LSO is more transferable compared to the GSO.

\section{CONCLUSIONS}

In measuring the spatial transferability, we conclude that the GSO resulted in a higher transferability compared to the LSO. This result is unexpected since we assumed that the local adaptation would increase the accuracy. We argue that the different perceptions on slum boundaries by the local experts that were used as reference caused this unexpected result (Figure 9). Furthermore, the usage of the tenure status as an indicator, which is not observable in an image, has affected the accuracy of the LSO since the reference data for the accuracy assessment are based on visual image interpretation by local experts. Therefore, the LSO resulted in a lower accuracy. Similar to the research of Kohli (2015), the reference from visual image interpretation introduces uncertainties since slums often have fuzzy boundaries and different ways to conceptualise. Similar with the temporal transferability, we expected that the LSO would show a higher transferability than the GSO in every area. However, the result shows the GSO had a higher temporal transferability in the first area. This result is unexpected. Three sources of uncertainties might explain this unexpected result. Firstly, the static concept and dynamic changes. As mentioned by Arvor et al. (2013), a real-world object changes over time, but the description of an object remains static in the ontology. For instance, we assumed the tenure status of a particular area did not change between 2012 and $2015^{3}$, but in the real world, this changed. Secondly, real world characteristics need to be translated into the image domain. Our knowledge of particular characteristics is often qualitative and subjective, but the information contained in an image is quantitative (Arvor et al., 2013). For instance, we argue that the lack of open green space be one characteristic of slums. This real world definition needs to be translated into the image domain. Although we applied different NDVI thresholds for different images, it is also possible that these thresholds do not capture the phenomenon sufficiently. For instance, during the dry season, leafs of trees are brown coloured. Lastly, similar due to the uncertainties in spatial transferability, the usage of tenure status also resulted in a low accuracy of the LSO. The result shows that assessing the transferability of slum detection methods across space, time and conceptualizations helps in detecting and understanding sources of uncertainties, which ultimately will support the development of more transferable and robust methods. A thorough understanding of uncertainties in slum mapping is essential for the development of policyrelevant information, such as the monitoring of large-scale slum improvement initiatives.

\section{ACKNOWLEDGEMENTS}

Multitemporal Pleiades imagery that was used in this research was obtained from the European Space Agency (ESA).

\footnotetext{
${ }^{3}$ Tenure status was obtained from the Land use Planning documents, which have a period of 20 years and are reviewed every 5 years
} 


\section{REFERENCES}

Arvor, D., Durieux, L., Andrés, S., \& Laporte, M. A. (2013). Advances in Geographic Object-Based Image Analysis with ontologies: A review of main contributions and limitations from a remote sensing perspective. ISPRS Journal of Photogrammetry and Remote Sensing, 82, 125-137. http://doi.org/10.1016/j.isprsjprs.2013.05.003

Baatz, M., \& Schäpe, A. (2000). Multiresolution Segmentation: an optimization approach for high quality multi-scale image segmentation. In Angewandte Geographische Informationsverarbeitung XII. Beiträge zum AGIT-Symposium Salzburg 2000, Karlsruhe, Herbert Wichmann Verlag (pp. 12 23). Retrieved

http://www.ecognition.cc/download/baatz_schaepe.pdf

Bamberger, M. (2012). Introduction To Mixed Methods in Impact Evaluation. Impact Evaluation Notes. Retrieved from http://www.interaction.org/sites/default/files/Mixed Methods in Impact Evaluation (English).pdf

Demographia. (2015). Demographia World Urban Areas (11th ed.). Demographia. Belleville. Retrieved from http://www.demographia.com/db-worldua.pdf

Department of Building and Settlements DKI. (2014). Pemetaan Direktori Kumuh [Directory of Slum Settlements]. Unpublished Manuscript, Jakarta.

Drăguţ, L., Tiede, D., \& Levick, S. R. (2010). ESP: a tool to estimate scale parameter for multiresolution image segmentation of remotely sensed data. International Journal of Geographical Information Science, 24(6), 859-871. http://doi.org/10.1080/13658810903174803

Groenendijk, E. M. C., \& Dopheide, E. J. M. (2003). Planning and management tools. ITC Special Lecture Notes Series. Enschede: The International Institute for Geo-Information Science and Earth Observation (ITC). Retrieved from http://www.itc.nl/library/Papers_2003/tech_rep/groenendijk.pdf

Harwell, M. R. (2011). Research Design in Qualitative/Quantitative/ Mixed Methods. In C. F. Conrad \& R. C. Serlin (Eds.), The SAGE Handbook for Research in Education (2nd ed., pp. 147-163). SAGE Publications Ltd.

Indonesian Central Board of Statistics (BPS). (2013). Indikator Rumah Tangga Kumuh [Slum Household Indicator]. Retrieved January 20, 2016, from http://sirusa.bps.go.id/index.php?r=indikator/view\&id=453

Kohli, D. (2015). Identifying and classifying slum areas using remote sensing. University of Twente. Retrieved from http://purl.org/utwente/doi/10.3990/1.9789036540087

Kohli, D., Kerle, N., \& Sliuzas, R. (2012). Local ontologies for object-based slum identification and classification. In Proceedings of the 4th GEOBIA (p. 201). Rio de Janeiro. Retrieved from https://www.researchgate.net/publication/230667153_Local_on tologies_for_object-

based_slum_identification_and_classification

Kohli, D., Sliuzas, R., Kerle, N., \& Stein, A. (2012). An ontology of slums for image-based classification. Computers, Environment and Urban Systems, 36(2), 154-163. http://doi.org/10.1016/j.compenvurbsys.2011.11.001

Kohli, D., Warwadekar, P., Kerle, N., Sliuzas, R., \& Stein, A. (2013). Transferability of object-oriented image analysis methods for slum identification. Remote Sensing, 5(9), 4209-
4228. http://doi.org/10.3390/rs5094209

Kuffer, M., Barros, J., \& Sliuzas, R. V. (2014). The development of a morphological unplanned settlement index using very-high-resolution (VHR) imagery. Computers, Environment and Urban Systems, 48, 138-152. http://doi.org/10.1016/j.compenvurbsys.2014.07.012

Kuffer, M., Pfeffer, K., \& Sliuzas, R. (2016). Slums from Space - 15 Years of Slum Mapping Using Remote Sensing. Remote Sensing, 8(6), 1-29. http://doi.org/10.3390/rs8060455

McKeown, D. M., \& Cochran, S. D. (1999). Fusion of HYDICE hyperspectral data with panchromatic imagery for cartographic feature extraction. IEEE Transactions on Geoscience and Remote Sensing, 37(3 I), 1261-1277. http://doi.org/10.1109/36.763286

Ministry of Public Works and Public Housing. (2014). Panduan Penyusunan SPPPIP dan RPKPP [Guideline for Urban Settlement and Infrastructure Development]. (Directorate of Settlement Development, Ed.) (5th ed.). Jakarta. Retrieved from http://www.scribd.com/doc/147120807/Buku-PanduanPenyusunan-SPPIP-dan-RPKPP\#scribd

Netzband, M. (2009). Physical Characterisation of Deprivation in Cities. In Urban Remote Sensing Event, 2009 Joint (pp. 1-5). Shanghai: IEEE. http://doi.org/10.1109/URS.2009.5137652

Pratomo, J. (2016). Transferability of The Generic and Local Ontology of Slum in Multi-temporal Imagery, Case Study: Jakarta. University of Twente. Retrieved from http://www.itc.nl/library/papers_2016/msc/upm/pratomo.pdf

Rukmana, D. (2008). Planning the Megacity: Jakarta in the Twentieth Century. Journal of the American Planning Association, 74(2), 263-264. http://doi.org/10.1080/01944360801940995

Sori, N. D. (2012). Identifying and Classifying Slum Development Stages from Spatial Data. University of Twente. Retrieved from https://www.itc.n1/library/papers_2012/msc/upm/dinsasori.pdf

Tuanmu, M.-N., Viña, A., Roloff, G. J., Liu, W., Ouyang, Z., Zhang, H., \& Liu, J. (2011). Temporal transferability of wildlife habitat models: implications for habitat monitoring. Journal of Biogeography, 38(8), $1510-1523$ http://doi.org/10.1111/j.1365-2699.2011.02479.x

UN-Habitat. (2014). Indonesia prepares National Report for Habitat III. Retrieved February 11, 2016, from $\mathrm{http}$ //unhabitat.org/indonesia-prepares-national-report-forhabitat-iii/

Whiteside, T. G., Boggs, G. S., \& Maier, S. W. (2011). Comparing object-based and pixel-based classifications for mapping savannas. International Journal of Applied Earth Observation and Geoinformation, 13(6), 884-893. http://doi.org/10.1016/j.jag.2011.06.008

Woodcook, C., \& Strahler, A. (1987). The factor of scale in remote sensing. Remote Sensing of Environment, 21, 311-332. http://doi.org/10.1016/0034-4257(87)90015-0 


\title{
LAND COVER AND LAND USE CHARACTERIZATION WITH GEOBIA IN THE PITANGUI RIVER BASIN AREA, PARANÁ-BRAZIL.
}

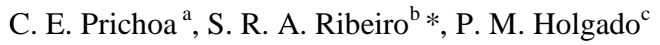 \\ ${ }^{a}$ Department of Geography, Autonomous University of Madrid, Spain, Street Francisco Tomas y Valiente 1 Ciudad Universitaria \\ Cantoblanco, 28049, Madrid - pedro.molina@uam.es; prichoa.carla@gmail.com \\ ${ }^{\mathrm{b}}$ Department of Geography, State University of Ponta Grossa, Avenida General Carlos Cavalcanti, 4748 - Paraná, 84030-900, Brazil - \\ selmar.aranha@gmail.com
}

KEY WORDS: GEOBIA, Land Cover and Land Use, Compact and Solidity.

\begin{abstract}
:
GEOBIA - Geographic Object-Based Image Analysis - is considered to be a technique that takes advantage of several Remote Sensing dimensions - like spectral, spacial, morphologic and contextual information - together with functionalities of Geographic Information Systems (GIS) as well. GEOBIA unit of analysis is an image object that obtains complete information. It includes texture, shape and spatial relations to neighboring objects and ancillary spatial data to different resolutions. In this study, it was sought to identify - in the spatial, spectral and texture descriptor group generated with GEOBIA - those that most represent the study unity. The technique was applied in part of the scene 221/77 of Landsat 5 TM satellite from year 2010 in color composition R5G4B3. This area belongs to the Pitangui river basin located at the center-east portion of the State of Paraná - Brazil, between coordinates $600.987 \mathrm{~m} \mathrm{E}$ and $7.240 .620 \mathrm{~m} \mathrm{~N}$ to $614.973 \mathrm{~m} \mathrm{E}$ and $7.231 .568 \mathrm{~m} \mathrm{~N}$ and zone $22 \mathrm{~S}$. Segmentation tests were done adjusting scale and merging levels according to the proposed objective (analysis of land use). Using a group of 35 descriptors with spatial, spectral and texture characteristics, Principal Component Analysis (PCA) and clustering were respectively applied with the aim of decreasing dimensionality of descriptors and grouping similar ones according to the shortest euclidean distance. From 14 resulting descriptors analyzed in the dendrogram generated in the clustering procedure, only compactness and solidity were grouped in the shortest euclidean distance. In the descriptors graph, maximum and minimum values for compactness and solidity have presented linear increase enabling the previous analysis.
\end{abstract}

\section{INTRODUCTION}

Remote sensing orbital images are considered to be one of the most traditional sources to acquire information about the earth's surface. This information is the basis for a great deal of practical applications including local, regional and global resource monitoring, land cover and its changes, as well as basis for other environmental studies.

The increasing ability to collect and manage high-resolution data has become a challenge to the traditional methods of image analysis, specially to the ones based on spectral characteristics. Advanced techniques such as GEOBIA - Geographic ObjectBased Image Analysis - are alternatives to the traditional pixelbased paradigm, specially when dealing with the data originating from high-resolution images. Thus, besides spectral properties, objects have numerous descriptors associated with shape, texture, contextual and semantic relationships which can be used for image analysis; it approaches this way human cognitive processes of photo interpretation (Navulur, 2006; Marpu, 2009; Camargo et al, 2009a) and therefore it can be applied to environmental studies for various purposes, and it serves as basis for land management.

The unit of analysis GEOBIA is an image-object that obtains complete information. It includes texture, shape and spatial relations with neighboring objects and ancillary spatial data for different spatial resolutions (Gutiérrez-Aguirre et al, 2012; Bock et al, 2005). This allows for an exploration of spatial context which is similar to a human analyst, who intuitively identifies objects in an image rather than individual pixels. It considers different properties (size, texture, shape) and spatial arrangements of these objects in order to understand its semantics (Addink et al, 2012. Marceau, 1999).

Compared to pixel-based approaches, GEOBIA facilitates multiscale analysis allowing to outline the landscape at different levels, reducing small spurious changes and provides a significant increase in the number of features that can be examined in a given analysis (HayandCastilla, 2008).

Previous studies have shown that improved results and higher accuracy in image classification is achieved when GEOBIA is applied (Desclée et al, 2006; Im et al, 2008b; Johansen et al, 2010; Lingcao et al, 2010; McDermid et al, 2008; Robertson and King, 2011; Zhou et al, 2008a).

The image is divided into sub-objects where scale and merging may be adjusted according to a proposed objective. The choice of the scale parameter and the similarity criteria are related to the spatial and spectral resolutions of the image. The term object is characterized as the main element in this type of analysis. This demonstrates that the necessary semantic information to interpret an image is not inserted into the pixel, but in the object produced in the image and the relationships between them (Definiens 2008). This fact increases the amount of features used for identifying different targets from the image;

\footnotetext{
* Corresponding author
} 
it is possible, however, to reduce dimensionality through statistical techniques like Principal Component Analysis (PCA). PCA is an alternative to describe heterogeneous areas such as the one in this study.

Based on these assumptions, the objective of this study is to use the GEOBIA technique in part of the scene 221/77, colorful makeup R5G4B3 from Landsat $5 \mathrm{TM}$, to identify those descriptors among the set of spatial, spectral and textural descriptors resulting from segmentation, that better represent its physical aspects, land cover and land use in 2010.

\section{STUDY AREA AND METHODOLOGY}

The study area (Figure 1) is part of the Devonian Escarpment ${ }^{1}$ in the Pitangui river basin located in the central eastern part of the State of Paraná - Brazil, between coordinates: $600.987 \mathrm{~m} \mathrm{E}$ and $7.240 .620 \mathrm{~m} \mathrm{~N}$ to $614.973 \mathrm{~m} \mathrm{E}$ and $7.231 .568 \mathrm{~m} \mathrm{~N}$ and zone $22 \mathrm{~S}$.

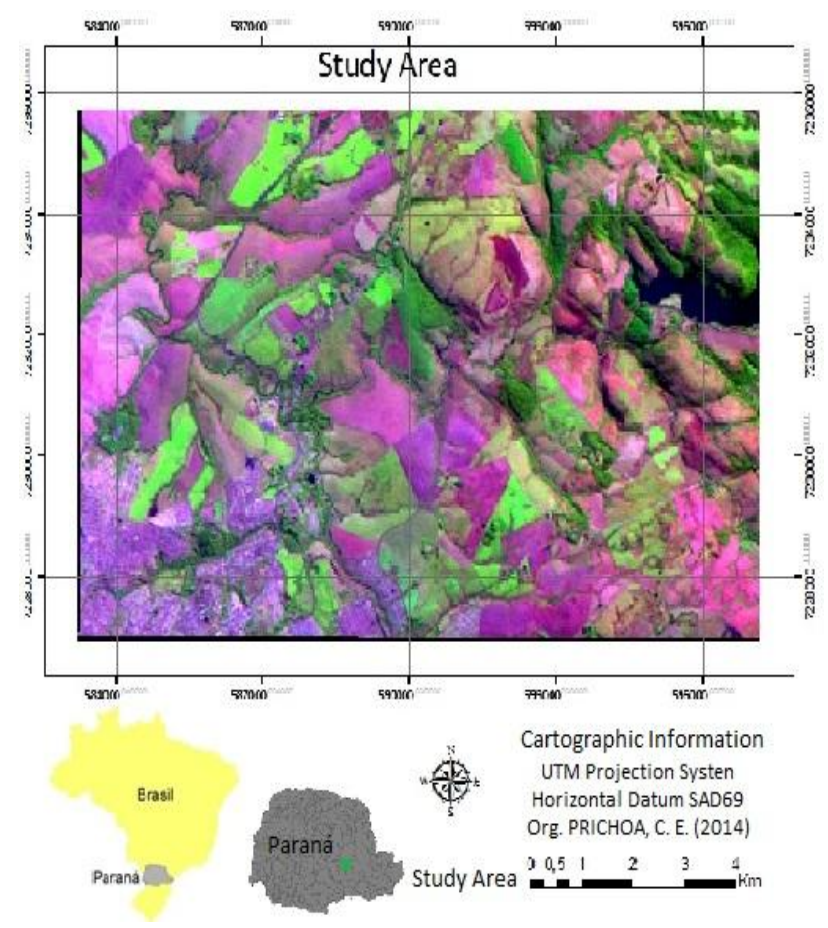

Figure 1. Location of the study area

In the performed segmentation tests, pixels were grouped according to the proposed objective: to analyze the use, utilizing Merge Level 91.7 and Scale Level 45.6. Departing from the set of 35 descriptors with spatial, spectral and textural features obtained through GEOBIA; Principal Component Analysis (PCA) and Cluster Analysis (CA) were applied in order to, respectively, reduce the dimensionality of the descriptors and group similar ones in shorter Euclidean distance. This resulted in 14 descriptors and two of them, Compact and Solidity that were analyzed in the dendrogram generated by CA, grouped themselves in the shortest Euclidean distance. Thus, compact and solidity are descriptors that have

${ }^{1}$ The origin of the name is due to the rock that supports it (Furnas Formation) from Devonian age (about 400 million years). best represented the study area and the ones through which the description of physical features of land cover and use can be conducted.

The calculation of PCA consists in a matrix whose rows are the eigenvectors of the covariance matrix estimated from data. The covariance matrix $\Sigma$ is a positive and symmetric matrix that contains information about the variances in all axes where data is distributed. The calculation is estimated as follows:

$$
S=\frac{1}{n} \sum_{i=1}^{n}\left(x_{1}-\bar{x}\right)\left(x_{1}-\bar{x}\right)^{n}
$$

Where: $\mathrm{n}$ is the number of data samples, $\mathrm{xl}$ is the mean of the set.

CA consists in grouping the closest pairs of variables, using the Euclidean distance for such and then replacing them with a new variable located at the half-distance between them. Euclidean distance (xjh) refers to the distance between two variables $\mathrm{j} e \mathrm{~h}$ in n-dimensional space and is given by:

$$
x_{j h}^{2}=\sum_{i=1}^{n}\left(x_{I j}-x_{i n}\right)^{2}
$$

\section{RESULTS AND DISCUSSION}

The study area presents heterogeneous characteristics as to the physical aspects and to land cover and use. It is predominantly an agricultural area, formed by valleys of the Pitangui river and tributaries, and an urban portion in the southwest as well. The descriptors compact and solidity (Figures 2 and 3) were those which displayed greater representativeness in the ACP and $\mathrm{CA}$ and thus best characterize the area.

The compact descriptor can vary from 0 to 1 . Nevertheless, in Figure 2, compact shapes are distributed within limits ranging from 0.072 (lower compactness, less rounded) to 0.294 (greater compactness, the more rounded shapes). Although values of compactness of the area are low, the descriptor provides pertinent representativeness attributes of land cover and use. Once that presenting the shortest Euclidean distance it hence represents forms originated by land use, mainly agriculture which are characterized in their great majority by plots displayed as solid and rectangular of various perimeters, just as highlighted in the image (circles, orange and green plots). 


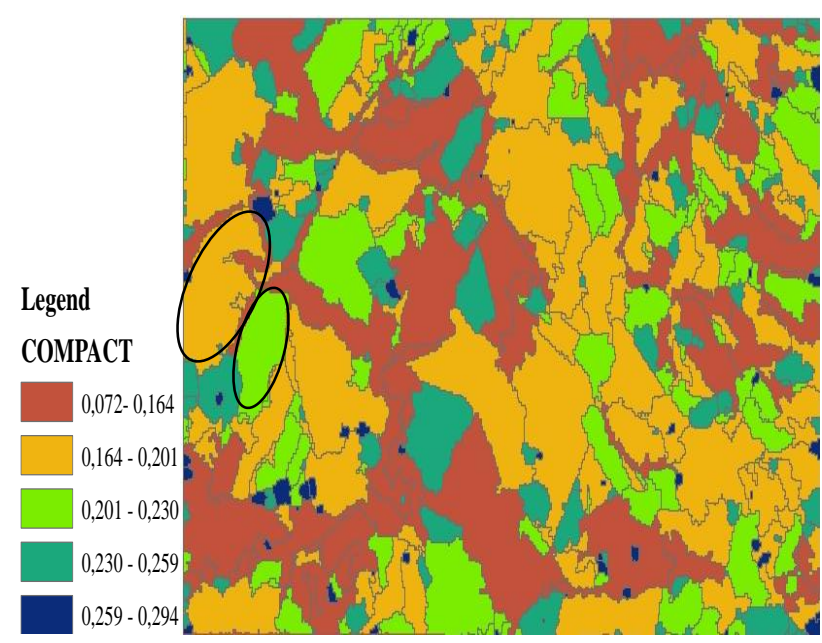

Figure 2: Compact Descriptor

Solidity varies from 0 to 1 and also offers high representativeness to the study area according to PCA and CA. In Figure 3, minimum and maximum values of descriptor are respectively distributed between 0.370 and 1 . The most solid objects refer to those of lower compactness (circles); they represent delimited agricultural areas of various geometry and for the most part rectangles without divisions or spurious elements. Intermediate values of solidity and low compactness in most of the area are associated with large scale agricultural land use, considering that this region has appropriate geomorphological conditions for such: from mild relief to wavy with flat tops and fertile soils.

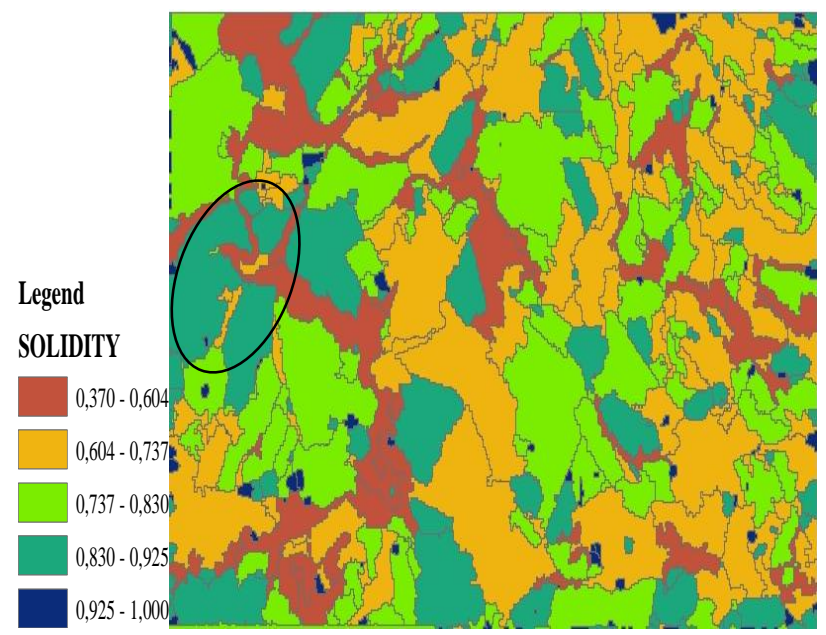

Figure 3: Descriptor Solidity

Each descriptor resulting from the GEOBIA technique possesses numerical properties that represent real-world features. In the graph (Figure 4) characteristics of descriptors compact and solidity are represented by the variation of the maximum and minimum values in each point. The graph shows that the values of descriptors compact and solidity increase coming close to a straight line, i.e. they display a linear relationship.
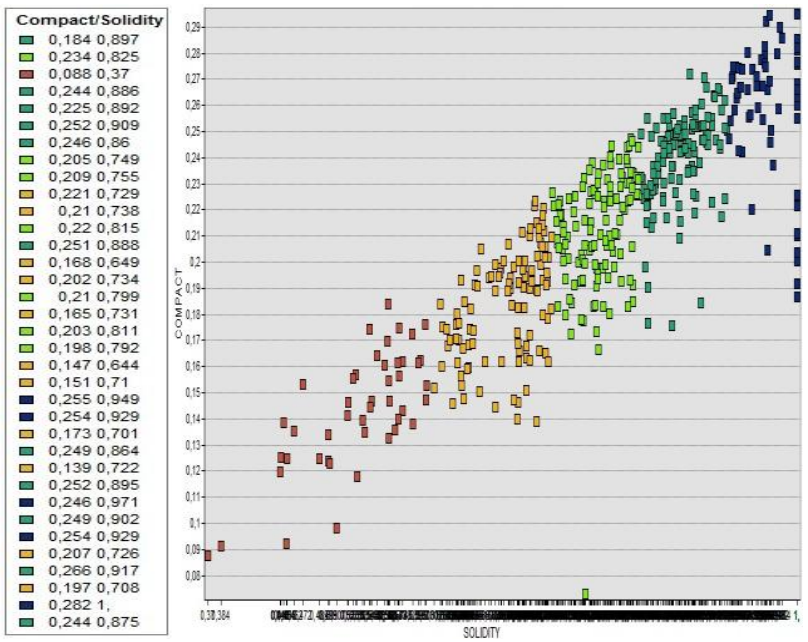

Figure 4: Maximum and minimum values of descriptors compact and solidity.

\section{CONCLUSIONS}

Land cover and land use can be identified and characterized by means of descriptors (compact and solidity) arising out of the GEOBIA technique when applied to an area that presents heterogeneous uses. The GEOBIA technique enabled the extraction of information from spatial context, incorporating to the spectral information characteristics of shape, size and texture of objects as well as other spatial phenomena of interest. GEOBIA applied in combination with the analysis of data dimension reduction and clustering made possible both the identification of homogeneous objects and their association with the study area. The descriptors compact and solidity presented the highest correlation when PCA and CA tests were performed, as well as the distribution of pairs in the graph (Figure 4) that are linearly positioned to one another.

The result proved to be suitable for this type of study, which emphasizes geometric shapes of objects of intermediate solidity and low compactness, associating them to land use and then to polygons with large perimeters. It is hereafter suggested to apply this method in monoculture areas in order to relate them to the type of cultivation.

\section{REFERENCES}

Addink, E. A., Van Coillie, F. M. B., De Jong, S. M., 2012. Introduction to the GEOBIA 2010 special issue: from pixels to geographic objects in remote sensing image analysis. International Journal of Applied Earth Observation and Geoinformation 15, 1-6.

Aguirre-Gutiérrez, J., Seijmonsbergen, A. C., Duivenvoorden, J. F., 2012. Optimizing land cover classification accuracy for change detection, a combined pixel-based and object-based approach in a mountainous area in Mexico. Applied Geography 34, 29-37. 
Bock,M., Xofis, P., Mitchley, J., Rossner, G., Wissen, M., 2005. Object-oriented methods for habitat mapping at multiple scales - case studies from Northern Germany and Wye Downs, UK. Journal for Nature Conservation $13,75-89$.

Definiens. Ecognition: user guide 3. Germany. http://www.definiens-imaging.com/down/ecognition.

Report.html (January 2014).

Desclée, B., Bogaert, P., Defourny, P., 2006.Forestchange detection by statistical object-based method. Remote Sensing of Environment 102, 1-11.

Hay, G. J. and Castilla, G., 2008. Geographic ObjectBased Image Analysis (GEOBIA). In: Blaschke, T., Lang, S., Hay, G. J. (eds) Object-Based Image Analysis Spatial concepts for knowledge-driven remote sensing applications. Chapter 1.4, pp. 81- 92 Springer. 700 p.

Im, J., Jensen, J.R., Tullis, J.A., 2008b. Object-based change detection using correlation image analysis and image segmentation. International Journal of Remote Sensing 29, 399-423.

Johansen, K., Arroyo, L.A., Phinn, S., Witte, C., 2010.Comparison of geo-object based and pixel-based change detection of riparian environments using high spatial resolution multi-spectral imagery. Photogrammetric Engineering \& Remote Sensing 76, 123-136.

Lingcao, H., Guo, Z., Yang, L., 2010. An object-based change detection approach by integrating intensity and texture differences.In: 2010 2nd International Asia 104 M. Hussain et al. / ISPRS Journal of Photogrammetry and Remote Sensing 80 (2013) 91- 106 Conference on Informatics in Control, Automation and Robotics (CAR), pp. 258-261.

Marceau, D.J., 1999. The scale issue in social and natural sciences. Canadian Journal of Remote Sensing 25, $347-$ 356.

MARPU, P. R. Geographic Object-based Image Analysis. Freiberg, 2009. 121f. Thesis (Doctorate in Engineering) Faculty of Geosciences, Geo-Engineering and Mining Technische Universität Bergakademie Freiberg, 2009.

McDermid, G.J., Linke, J. Pape, A. D., Laskin, D. N., McLane, A. J., Franklin, S.E.,2008. Object-based approaches to change analysis and thematic map update: challenges and limitations. Canadian Journal of Remote Sensing 34, 462-466.

Navulur, K. Multispectral image analysis using the object-oriented paradigm. Boca Raton: Taylor \& Francis Group, 2006. 165p.
Robertson, L.D., King, D.J., 2011. Comparison of pixeland object-based classification in land cover change mapping. International Journal of Remote Sensing 32, 1505-1529.

Zhou, W., Troy, A., Grove, M., 2008a. A Comparison of Object-Based with Pixel-Based Land Cover Change Detection in the Baltimore Metropolitan Area using Multitemporal High Resolution Remote Sensing Data, Geoscience and Remote Sensing Symposium, 2008. IGARSS 2008. IEEE International, pp.IV-683-IV-686. 


\title{
DETECTION OF PHOTOVOLTAIC INSTALLATIONS IN RGB AERIAL IMAGING: A COMPARATIVE STUDY.
}

\author{
Steven Puttemans*, Wiebe Van Ranst and Toon Goedemé
}

EAVISE, KU Leuven - Campus De Nayer, Sint-Katelijne-Waver, Belgium

(steven.puttemans, wiebe.vanranst, toon.goedeme)@kuleuven.be

KEY WORDS: Photovoltaic Installations, Automated Object Detection, Fraud Detection

\begin{abstract}
:
In this work, we compare four different approaches for detecting photovoltaic installations from RGB aerial images. Our client, an electricity grid administrator, wants to hunt down fraud with unregistered illegal solar panel installations by detecting installations in aerial imagery and checking these against their database of registered installations. The detection of solar panels in these RGB images is a difficult task. Reasons are the relatively low resolution (at $25 \mathrm{~cm} /$ pixel an individual solar panel only measures about $9 \times 7$ pixels), the undiscriminating colour properties of the object (due to in-class variance and specular effects) and the apparent shape variability (rotation and skew due to the different roofs slant angles). Therefore, straightforward object segmentation techniques do not yield a satisfying solution, as proven in this paper. We compared four state-of-the-art object detection approaches for this task. First we experimented with a machine learning object detection technique based on pixel-based support vector machine classification. Secondly we developed an approach using MSER based colour segmentation and shape analysis. Finally a dual approach based on object categorization using the boosted cascade classifier technique of Viola \& Jones and the aggregate channel features technique of Dollár et al., is introduced, learning a combination of colour and gradient feature based classifiers from a given training set. We successfully evaluate these four different approaches on a fully labelled test set of a $8000 \times 8000$ pixel, 4 square km zone containing 315 solar panel installations with in total more than 10.000 individual panels.
\end{abstract}

\section{INTRODUCTION}

Solar panels provide a solution in generating energy from nonpolluting resources and therefore placing a solar panel installation is subsidized and encouraged by governments and electricity grid administrators. However due to the given funding and tax reduction, these 'greener' energy generating alternatives also give raise to malicious fraud. Our client, an electricity grid administrator wants to use the power of computer vision to track down these fraud cases to ensure that no financial benefits are given to people that are not correctly registered at the grid administrator with their solar panel installation.

Fully automated solar panel detection in RGB images yields some major challenges, as seen in Figure 1 First of all a solar panel is an object shape with only a few distinct visual features like shape and colour, due to its simple shape and colour distribution. Secondly the images that are freely available on the FGIA portal have a very limited resolution $(25 \mathrm{~cm} / \mathrm{pixel})$ resulting in a solar panel size of only $9 \times 7$ pixels, which is very little considering the real dimensions are around $1 \times 1.5 \mathrm{~m}$. This is clearly visible in Figure 1 (a). Due to the material properties of solar panels, specular reflections tend to change the visual properties of the panels, depending on the position of the sun. This could lead to solar pan-

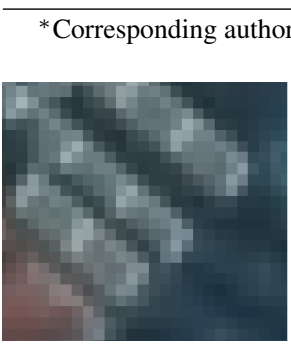

(a)

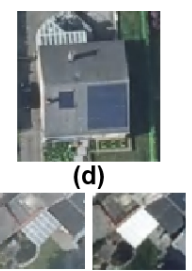

(b)

(b')

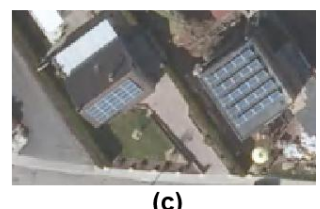

(c)
Figure 1. Challenges when dealing with solar panel detection. els occurring as a flat white overexposed part of the image. This difference can be seen in Figure 1 (b) and $\left(b^{\prime}\right)$. The orientation in which the solar panels are placed also varies (south, southeast, south-west), raising the need of a full $360^{\circ}$ rotational search of the image for object instances. Combined with the different roofs' slants $\left(30^{\circ}, 45^{\circ}, 60^{\circ}\right.$, etc.), panels get optically deformed and thus appear shorter or longer in 2D images, despite the fixed physical size, as seen in Figure 1 (c). Finally solar panels come in different materials (mono- and polycrystalline, full black, etc.) introducing even more intra class variance into the problem. For example, due to the small resolution per panel, full black installations appear as a black square, yielding no visual features to train computer vision techniques, as seen in Figure 1(d).

Our goal is to develop a computer vision based approach that is able to automatically detect solar panels in aerial imagery and return the location of these installations with a high certainty. This in turn can avoid putting in huge amounts of manual labour to manually locate solar panel installations for fraud detection.

\section{RELATED WORK}

The automated analysis of solar panel installations from aerial images using techniques of the computer vision fields, limits itself to the analysis of solar panel efficiency and defects, using RGB and thermal cameras, as seen in (Sánchez-Friera et al. 2011. Li and Tsai. 2012; Tsai et al., 2013), while automated solar panel detection and localisation seem to be unexplored. While object detection is a well studied problem in the field of computer vision, many other fields still have not discovered the power of these state-of-the-art techniques in autonomous object detection and localisation. The community of computer vision has however already performed object detection research in the field of aerial imagery, focussing on roads (Hinz and Baumgartner 2003), buildings (Mayer 1999) or vehicles (Gleason et al. 2011), always using state-of-the-art computer vision algorithms and thus giving 

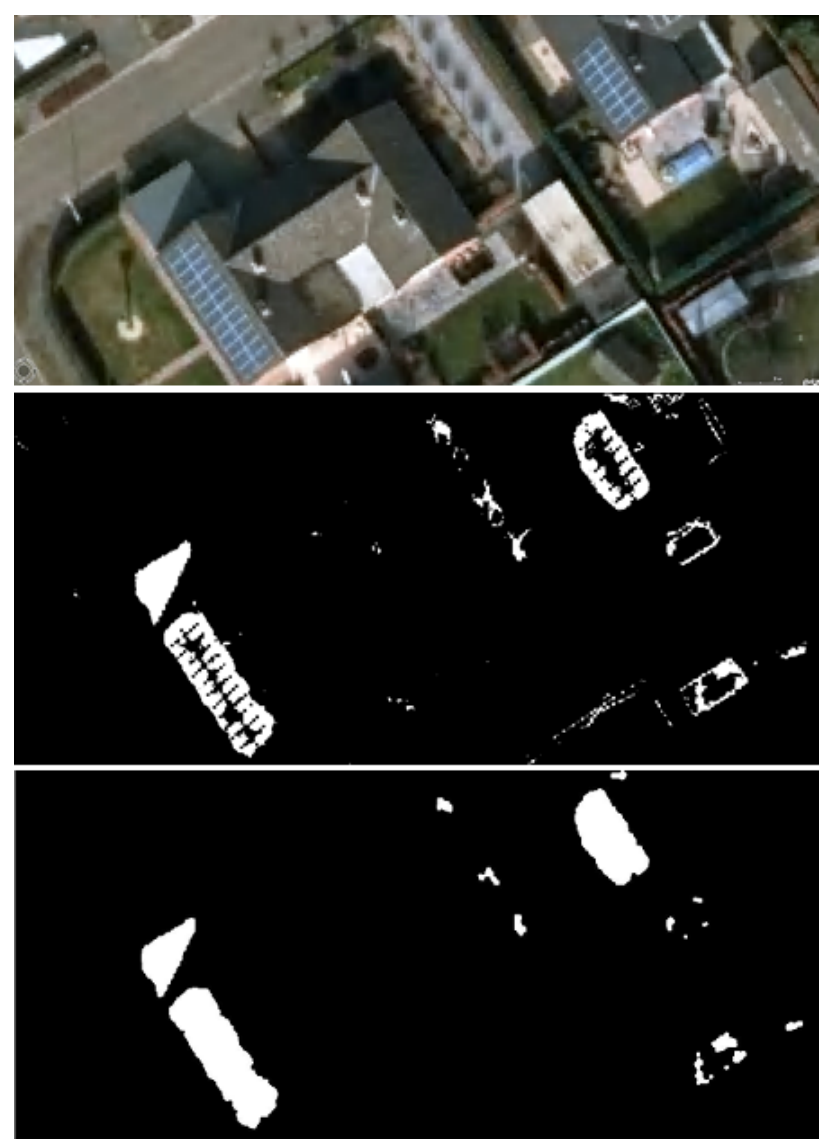

Figure 2. Example of pixel based colour classification using neural networks as learning tool. (top) original image (middle) pixel classification result (bottom) cleaned up segmentation.

perfect example cases that can be expanded to solar panel detection and localisation.

While the intuitive way would be to use segmentation based approaches, where the RGB input image is transformed to a colour space where separating object pixels from background pixels using strict (learned) thresholds is easier, they tend to fail when a wide range of other objects in the images have similar colour ranges. Furthermore these techniques only take into account object colour information. Object categorisation techniques like (Viola and Jones, 2001, Felzenszwalb et al., 2010, Dollár et al. 2009. Dollár et al. 2014) tend to go one step further and use object properties like colour, shape, texture, etc. to uniquely define features that describe the object class, which are then used to successfully separate objects from the background.

\section{DATASETS}

For developing and testing our suggested approaches, we used the freely available medium-scale $(25 \mathrm{~cm} /$ pixel resolution) aerial footage from FGIA (Flanders Geographical Information Agency) covering the grid area of Flanders where our electricity grid adminstrator is active. From this publicly available dataset, a set of 2500 individual solar panels where manually annotated and a set of more than 150000 random negative samples (containing everything except solar panels) were collected and used to learn the models used by our state-of-the-art object detection algorithms.

To test the four suggested approaches an aerial image of $2 \times 2 \mathrm{~km}$ of the city centre of Sint-Truiden was obtained. At a resolution of $25 \mathrm{~cm} /$ pixel this results in a $8000 \times 8000$ pixel image, which is up-scaled using a bi-cubic operator to $16000 \times 16000$ pixels to ensure the solar panels are covered by enough pixels per panel. Inside this test image 313 solar panel installations where manually annotated, by drawing polygons around the installations, to use as ground truth when validating the fully automated object detection techniques suggested in this work.

The complete dataset, including training data, test data and annotations can be found for research purposes at http://www . eavise.be/SolarPanelDataset/

\section{SUGGESTED APPROACH}

To find an optimal solution for fully automated solar panel detection in aerial images, a comparative study was performed. In the following subsections each of the four state-of-the-art approaches, will be explained and discussed in detail. Section 5 will then take a closer look at the accuracy and time complexity achieved by each individual technique.

\subsection{Pixel based colour classification using support vector ma- chines}

Our pixel based colour classification, as seen in Figure 2 uses the internal colour area of each solar panel (blue-grey colour range) without looking at the bright edges of the panel, in order to ensure that the pixel colour distribution of the training pixels are separable in the HSV colour space. We manually collected 1000 internal solar panel pixels and 2000 randomly selected non-solar panel background pixels. Both solar panel and non-solar panel pixels are transformed to the HSV colour space, a space where separating colours is easier then inside the RGB colour space. Based on this training data, a support vector machine classifier with linear kernel is trained, able to autonomously separate solar panel from non-solar panel pixels.

When a test image is presented to the support vector machine classifier, each pixel is processed and is given a certainty score, indicating how certain we are that the pixel is actually part of a solar panel installation. This certainty score allows us to set a minimal certainty threshold, generating a binary image as seen in Figure 2 On top of that, binary image opening and closing operators can be applied to remove noise, followed by contour detection and contour filling to achieve a cleaner result.

\subsection{MSER based colour segmentation and shape analysis}

Maximally stable extremal regions (Matas et al. 2004) is a technique used to detect blobs inside a given image. By systematically increasing the threshold on a given greyscale input image, from very sloppy to very strict, we create a set of sequential binary images. Inside those images the algorithm looks for regions that stay stable over the different thresholds and then approximates those regions by their fitted ellipse. Due to the higher response of solar panels in the blue channel compared to the red and green channels, we only process the blue channel data, removing the need to explicitly convert the RGB image to a greyscale image. Applying the MSER algorithm generates a selection of blob candidates, as seen in Figure 3(a) To further filter the obtained regions, we start by discarding blobs of an incorrect size (see Figure 3(b) , then look for blobs with a axis ratio that deviates maximally $30 \%$ of the ideal $1.5: 1$ ratio (see Figure $3(\mathrm{c})$ ) and finally we apply HSV colour segmentation on the remaining blobs, using the technique discussed in subsection 4.1 The known size range of the blobs can be explained by the fact that aerial imagery is taken on a constant hight, while the limited ratio deviation is explained by the fixed physical size of the solar panels. Finally 


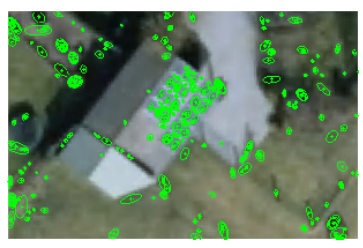

(a)

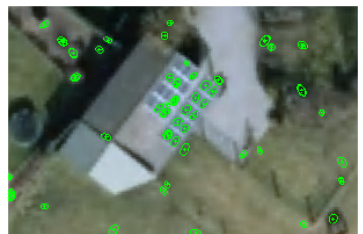

(c)

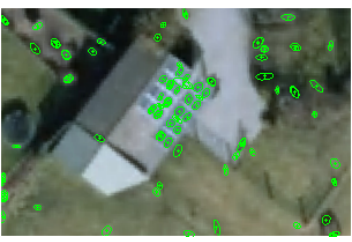

(b)

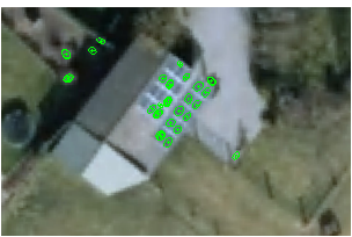

(d)
Figure 3. MSER based colour segmentation (a) MSER detected blobs (b) area restriction (c) ratio restriction (d) colour restriction.

since our solar panel installation training set contains solar panels with a known and shared colour range in the HSV colour space, we can allow colour based segmentation for further filtering.

\subsection{Boosted cascade of weak classifiers}

The previous techniques have only a very limited training time, since most processing is done on the fly when providing new test samples. This is different for object categorization techniques, where a model is learned from a set of positive object samples and a large set of random background samples. From each training sample a set of specific features is learned that is smartly combined into a model, able to separate objects from non-object patches inside the image.

For our first object categorization approach, we use the framework by Viola and Jones (2001), based on a boosted, using AdaBoost (Freund et al. 1999), cascade of weak classifiers (simple decision trees). This technique was originally developed for efficient face detection, but recent advances in computer vision (Puttemans et al. 2015: Puttemans and Goedemé 2015) have proven that this technique still achieves top notch results in other application fields, focussing on more general object detection test cases.

The boosted cascade framework used does not incorporate colour information, but rather looks at the structure and texture of objects a greyscale image. It describes local binary pattern features (Liao et al. 2007), features that look at local gradient information in training samples. Since solar panels have a colour range that

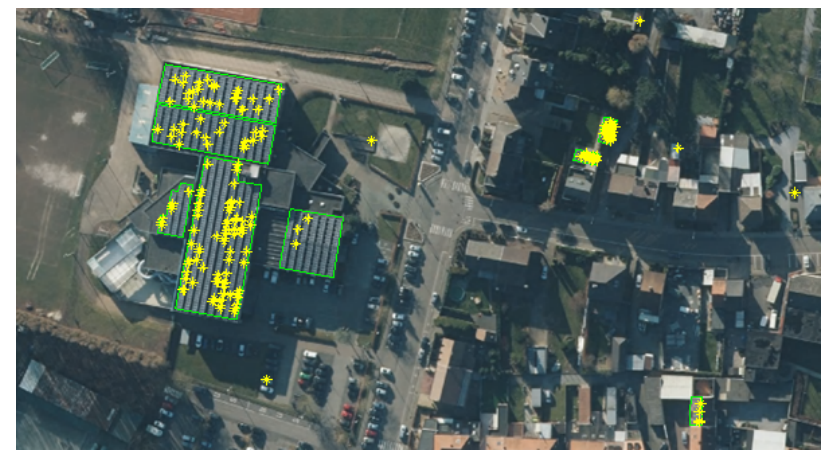

Figure 4. Detection of solar panels using a boosted cascade of weak classifiers.

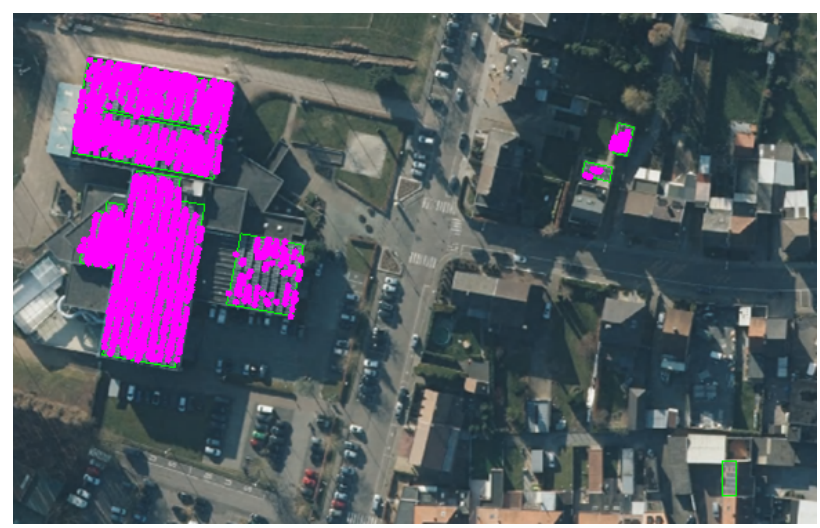

Figure 5. Detection of solar panels using the aggregate channel features technique.

has a higher response in the blue channel, we decided not to use a greyscale image, but the blue channel instead, explicitly forcing the framework to use colour information.

A downside of the boosted cascade classifier framework, is that it trains a model for a fixed orientation. For this we explicitly rotated all solar panel examples to a horizontal position, resulting in a model able to detect horizontal solar panels. However solar panels occur in different orientations. To cope with that, we simply rotate the input image over different angles, with a predefined angle step, and then warp back the retrieved detections. This allows us to build a full 360 degree capable solar panel detector using a single orientation model.

When running the detector on a test image, the detector will apply a fully rotational sliding window based evaluation of the image, triggering a detection at each position that gets classified as an object by the trained model. An example of such a detection output can be seen in Figure 4

\subsection{Aggregate channel features}

The technique suggested by Dollár et al. (2009)(Dollár et al. 2014) is in fact an extension to the latter technique. Where the boosted cascade framework ignores colour information from the start, this technique proved that using colour information for certain object detection tasks can yield serious advantages and yield a higher detection accuracy. This is one of the main reasons why we decided to test this framework for our solar panel detection case. Besides that, we also have an internally developed C++ implementation of this framework (De Smedt and Goedemé, 2015) available, which allows us to easily run extra tests.

The technique does not take a single feature representation of the input image, like the previously used framework, but rather combines several feature channels, including colour, gradients, gradient histograms, etc. From this larger feature pool, the technique decides on its own which feature is good enough to efficiently separate positive and negative training data.

Running this more recent object categorization detector on top of a given test image generates similar output as the previous technique, as seen in Figure 5 However keep in mind that both Figure 4 and 5 are a sample detection output at specific detection thresholds of the algorithms. Deciding which algorithm performs better will be done thoroughly in section 5

\section{RESULTS}

We started out with comparing our four state-of-the-art algorithms in processing time, as seen in Table 1 . We can see a clear differ- 
Table 1. Comparison of training (given the training sets described in section 3 and detection times (based on the upscaled $16000 \times$ 16000 pixel image) combined with the complete system configurations used.

\begin{tabular}{|c|c|c|c|}
\hline Technique used & Training time & Detection time & System configuration used \\
\hline HSV + SVM & $10 \mathrm{sec}$ & $10 \mathrm{sec}$ & Intel(R) Core(TM) CPU i7-4500U @ 2.4GHZ \\
\hline MSER & $0 \mathrm{sec}$ & $100 \mathrm{sec}$ & Intel(R) Core(TM) CPU i7-4500U @ 2.4GHZ \\
\hline Boosted Cascade & $3.5 \mathrm{hour}$ & $10 \mathrm{~min}$ & Intel(R) Xeon(R) CPU E5-2630 v2 @ 2.60GHz \\
\hline Aggregate Channel Features & $36 \mathrm{~min}$ & $6 \mathrm{hour}$ & Intel(R) Xeon(R) CPU E5-2630 v3 @ 2.40GHz \\
\hline
\end{tabular}

ence in training time between the more basic HSV pixel segmentation and the MSER approach on the one hand, and the object categorization techniques on the other hand. While the boosted cascade and the aggregate channel features approach take quite a bit longer to train on the given dataset, this task should only be done once, because a trained model can be reused as many times as we desire. However when looking at detection time, we notice a steady increase in processing time when computational complexity of the algorithm increases. Where the standard pixel based segmentation takes only 10 seconds for a $16000 \times 16000$ pixel image, the basic object categorization framework already takes 600 times that long.

However these timings should be interpreted with caution because they highly depend on the available infrastructure, which is also specified in Table 1 Furthermore the implementation of the aggregate channel features technique was developed in-house and was not yet optimized for parallel processing, thus needing to process everything in a sequential order.

One of the main reasons why object categorization techniques take a lot more time, is because these techniques are trained for a specific orientation. In order to be able to detect objects in every possible orientation, we rotated the original image for a full 360 degrees, with a single degree step. This again can be heavily optimized when desired, by for example rotating the model instead of rotating the image.

To evaluate the accuracy of the developed techniques, we suggest to use precision recall curves, used to compare the actual detection output with the manually obtained ground truth polygons. To generate these curves, seen in Figure 6 , the generated detection maps are first downscaled to a $4000 \times 4000$ pixels, combining scores of the detections obtained on the same locations. On top of the resulting score map, a threshold is applied (which is the varying parameter used to generate the different precision-recall values for each algorithm). This is followed by a dilation (make detection centres as large as solar panel dimensions) and erosion

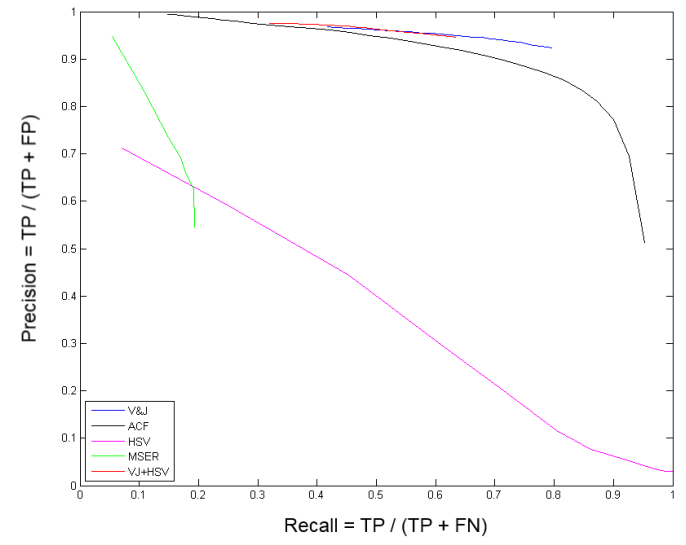

Figure 6. Precision - Recall curves for all techniques tested and validated on the $16000 \times 16000$ test image of the Sint-Truiden city centre. (remove detections that are lonely and not grouped) operations resulting in a clear binary image. This binary image is then compared to calculate the amount of true positives, false positives and false negatives, which are in turn used to calculate correct precision and recall values.

Figure 7 shows the comparison of that binary output which is in turn compared to the manual annotations. By using the three channels of an RGB image, knowledge of both results can be visually combined. Detection centres are visualised as dots on the red channel, the ground truth is visualized on the green channel and finally the detection output regions are visualised on the blue channel. Combining those three colour channels yields a set of pixel based classification labels. Cyan labels indicate true positive detections, black labels indicate true negative detections, blue labels indicate false positive detections and green labels indicate false negative detections. The visualisation is done for both the boosted cascade and the aggregate channel technique.
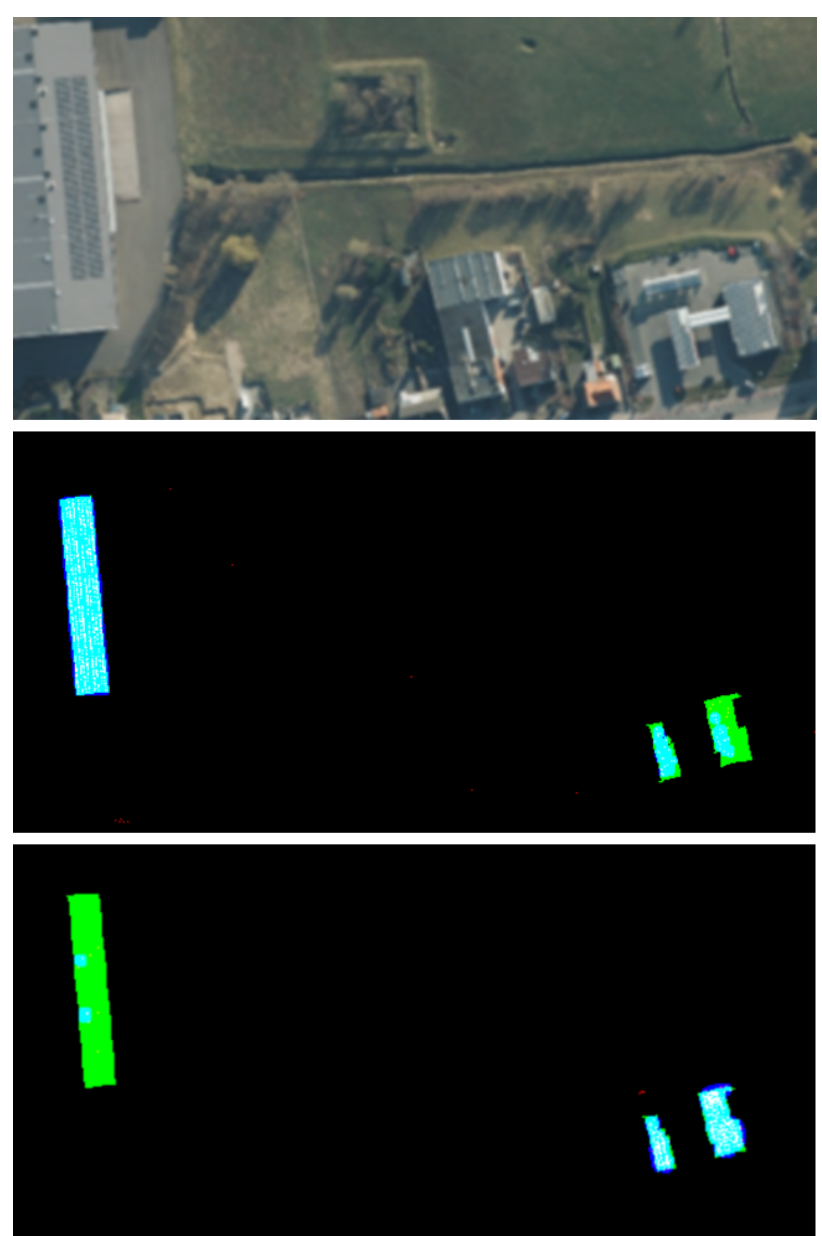

Figure 7. Score processing map for both boosted cascade and aggregate channel features technique. (top) original input image (middle) boosted cascade result (bottom) aggregate channel features result 
The precision-recall curves clearly show that object categorization techniques outperform the other, more basic computer vision techniques. We notice that the boosted cascade technique performs still a bit better than the aggregate channel technique, which indicates that adding extra feature channels like colour and other gradient filters is overkill for solar panel detection. To test this theory we added HSV pixel based segmentation as a postprocessing step to our boosted cascade detector, displayed as the red curve in Figure 6 We see almost no increase in efficiency which made us to decide to drop this extra processing step, in order to obtain a smaller computational complexity and thus a faster running time.

\section{DISCUSSION AND CONCLUSION}

Our goal is to compare techniques for automatically detecting photovoltaic solar panel installations in RGB aerial images, considering the very challenging conditions due to the limited resolution, the limited amount of visual features, the existance of specular reflections and the different orientations of the solar panel object class.

Using pixel based segmentation for solar panel detection yields only moderate results. In any given test image, it is impossible to only separate blobs that are pure solar panels, since the colour distribution of the panels, also tends to return in many background patches. Furthermore there are several solar panel types that tend to deviate from the average colour distribution, like the full black type, generating even more missed detections.

Applying smarter feature based techniques, like the MSER based approach, seems promising and even yields decent results in specific areas, but still has quite a fast drop in accuracy when considering larger regions where solar panels need to be detected, as seen from the resulting precision-recall curve based on the $2 \times 2$ $\mathrm{km}$ area of Sint-Truiden.

However, our tests clearly showed that object categorization techniques like boosted cascades and aggregate channel features can outperform more basic techniques with the only downside that training data needs to be collected and that the single training setup for building the model takes a bit longer.

We proved that computer vision is a working solution for efficient and fully automated solar panel detection in RGB based aerial imagery for fraud detection. For a fully automated system, achieving a precision of $93 \%$ at a recall of $80 \%$ is not bad at all. Certainly if you take into account some considerations. Due to the heavy deformations a roofs' slant angle can introduce to the solar panel object, training multiple models to cope with the missed detections could be a first improvement. Furthermore, we performed the research on mid resolution images, at $25 \mathrm{~cm} / \mathrm{pixel}$. However there are already measurements of the same area at a resolution of $8 \mathrm{~cm} /$ pixel available, which would again yield an accuracy increase. Since our training set consists mainly of industrial solar panel arrays, it is also quite understandable that the model is not able to cope with the larger deformations found on solar panels placed on domestic housing.

Finally one could argue that the fully automated system should not be able to detect $100 \%$ of the solar panels with $100 \%$ precision. In most set-ups, an operator is still available for slight semi-automated input. We could provide that percentage of object that we did not found, or that yield a lower certainty, to the operator and ask him to define these cases of doubt by manual inspection. This suggested semi-automated approach would still reduce the manual labour drastically and reduce the time needed to process larger datasets.
As future research based on this paper we suggest to take a closer look at CNN based detectors Girshick et al. (2014). In many object detection tasks they achieve top notch performance and could thus improve the obtained results.

\section{ACKNOWLEDGEMENTS}

This work is supported by the KU Leuven, Campus De Nayer, through a scholarship. We would like to thank Infrax, for providing the solar panel datasets and manual annotations.

\section{References}

De Smedt, F. and Goedemé, T., 2015. Open framework for combined pedestrian detection. In: VISAPP, Vol. 2, pp. 551-559.

Dollár, P., Appel, R. et al., 2014. Fast feature pyramids for object detection. TPAMI 36(8), pp. 1532-1545.

Dollár, P., Tu, Z. et al., 2009. Integral channel features. In: $B M V C$.

Felzenszwalb, P. F., Girshick, R. B. et al., 2010. Cascade object detection with deformable part models. In: $C V P R$, pp. 22412248.

Freund, Y., Schapire, R. et al., 1999. A short introduction to boosting. JSAI Journal 14(771-780), pp. 1612.

Girshick, R., Donahue, J. et al., 2014. Rich feature hierarchies for accurate object detection and semantic segmentation. In: CVPR, pp. 580-587.

Gleason, J., Nefian, A. et al., 2011. Vehicle detection from aerial imagery. In: ICRA, IEEE, pp. 2065-2070.

Hinz, S. and Baumgartner, A., 2003. Automatic extraction of urban road networks from multi-view aerial imagery. Journal of Photogrammetry and Remote Sensing 58(1), pp. 83-98.

Li, W.-C. and Tsai, D.-M., 2012. Wavelet-based defect detection in solar wafer images with inhomogeneous texture. Pattern Recognition 45(2), pp. 742-756.

Liao, S., Zhu, X. et al., 2007. Learning multi-scale block local binary patterns for face recognition. In: Advances in Biometrics, Springer, pp. 828-837.

Matas, J., Chum, O. et al., 2004. Robust wide-baseline stereo from maximally stable extremal regions. Image and vision computing 22(10), pp. 761-767.

Mayer, H., 1999. Automatic object extraction from aerial imagerya survey focusing on buildings. CVIU 74(2), pp. 138149.

Puttemans, S. and Goedemé, T., 2015. Visual detection and species classification of orchid flowers. In: $M V A$, IEEE, pp. 505-509.

Puttemans, S., Goedemé, T. et al., 2015. Automated walking aid detector based on indoor video recordings. In: $E M B C$, IEEE, pp. 5040-5045.

Sánchez-Friera, P., Piliougine, M. et al., 2011. Analysis of degradation mechanisms of crystalline silicon pv modules after 12 years of operation in southern europe. Progress in photovoltaics: Research and Applications 19(6), pp. 658-666.

Tsai, D.-M., Wu, S.-C. et al., 2013. Defect detection in solar modules using ica basis images. IEEE Transactions on Industrial Informatics 9(1), pp. 122-131.

Viola, P. and Jones, M., 2001. Rapid object detection using a boosted cascade of simple features. In: $C V P R$, Vol. 1, IEEE, pp. I-511. 


\title{
COMPARING MACHINE LEARNING CLASSIFIERS FOR OBJECT-BASED LAND COVER CLASSFICATION USING VERY HIGH RESOLUTION IMAGERY
}

\author{
Yuguo Qian a, Weiqi Zhou ${ }^{\text {a }}{ }^{*}$, Jingli Yan ${ }^{\text {a }}$, Weifeng Li ${ }^{\text {a }}$ Lijian Han ${ }^{\text {a }}$, \\ a State Key Laboratory of Urban and Region Ecology, Research Centre for Eco-Environmental Sciences, Chinese Academy of \\ Sciences - (ygqian, wzhou, li.wf, ljhan)@ rcees.ac.cn, jlyan24@163.com
}

KEY WORDS: Object-based classification, Machine learning classifiers, Very high resolution image, Urban area, Tuning parameters

\begin{abstract}
:
This study evaluates and compares the performance of four machine learning classifiers - support vector machine (SVM), normal Bayes (NB), classification and regression tree (CART) and K nearest neighbour (KNN) - to classify very high resolution images, using an object-based classification procedure. In particular, we investigated how tuning parameters affect the classification accuracy with different training sample sizes. We found that: (1) SVM and NB were superior to CART and KNN, and both could achieve high classification accuracy (>90\%); (2) the setting of tuning parameters greatly affected classification accuracy, particularly for the most commonly-used SVM classifier; the optimal values of tuning parameters might vary slightly with the size of training samples; (3) the size of training sample also greatly affected the classification accuracy, when the size of training sample was less than 125. Increasing the size of training samples generally led to the increase of classification accuracies for all four classifiers. In addition, NB and KNN were more sensitive to the sample sizes. This research provides insights into the selection of classifiers and the size of training samples. It also highlights the importance of the appropriate setting of tuning parameters for different machine learning classifiers and provides useful information for optimizing these parameters.
\end{abstract}

\section{INTRODUCTION}

Urban landscapes are extremely complex and heterogeneous. To adequately quantify the heterogeneity of urban land cover, high spatial resolution images are needed. A considerable amount of research has shown that object-based approaches are superior to traditional pixel-based methods in the classification of high spatial resolution data. Consequently, object-based approaches have been increasingly used for urban land cover classification (Duro, Franklin et al. 2012).

With object-based classification approaches, objects generated from image segmentation can be typically classified using a rule-based procedure (a set of rules) or using machine learning algorithms (MLA) based on training samples. While rule-based procedures, which use expert knowledge, have been increasingly used for classification, the majority of the studies have used supervised classifications (Laliberte, Rango et al. 2004; Mathieu and Aryal 2007). Many different kinds of MLA have been applied for supervised classifications. These algorithms are commonly categorized as parametric and nonparametric classifiers. The two widely-used types of parametric algorithms are the maximum likelihood classifier (MLC) and Bayes classifiers, and the frequently-used non-parametric classifiers include $\mathrm{K}$ nearest neighbour $(\mathrm{KNN})$, decision tree (DT) and support vector machine (SVM).

Previous studies have shown that the use of different classifiers may lead to different classification results. Therefore, many studies have been conducted to investigate the effectiveness and efficiency of different classifiers (McInerney and Nieuwenhuis 2009; Song, Duan et al. 2012). However, these studies have been mostly conducted using pixel-based approaches. With the wide use of object-based approaches, there has been an increasing interest in comparing different machine learning classifiers using object-based methods. When using these machine learning classifiers, we should consider at least four key factors that can dramatically affect the classification accuracy and efficiency. Specifically, these are image segmentation, training sample selection, feature selection and tuning parameter setting. While the first three factors have been investigated in many previous studies (Wieland and Pittore 2014), few studies have investigated the effects of the setting of tuning parameters. However, setting tuning parameters is the very first step, as well as one of the most important steps to appropriately use these machine learning classifiers. In addition, previous comparison studies of machine learning classifiers have been mostly focused on non-urban areas, such as grasslands, farmlands and coal mine area.

The overall objective of this study is to evaluate the four most frequently used MLAs for urban land cover classification, with an object-based approach, using very high spatial resolution imagery.

In particular, we aim to investigate how tuning parameters affect the classification results, especially with different training sample sizes. The four classifiers are: (1) normal Bayes (NB), a parametric algorithm; (2) SVM, a statistical learning algorithm; (3) $\mathrm{KNN}$, an instance-based learning algorithm; and (4) the classification and regression tree (CART) classifier, a commonly-used DT algorithm. The results from this study can provide insights into classifier selection and parameter setting for high resolution urban land cover classification.

\section{MAIN BODY}

\footnotetext{
* Corresponding author
} 


\subsection{Study Area and Data}

The study site is an urban area located in the Haidian District of Beijing, China, between latitudes $39^{\circ} 58^{\prime} 30^{\prime \prime}$ and $40^{\circ} 0^{\prime}$ $47^{\prime \prime}$ and longitudes $116^{\circ} 17^{\prime} 5^{\prime \prime}$ and $116^{\circ} 20^{\prime} 12^{\prime \prime}$. The study area is a complex urban area with many land use types, including parks, universities, construction sites and residential areas. Land cover types are mainly impervious surface, vegetation cover, bare soil and water, which are typical in urban areas. The dominant land cover in parks is vegetation and water, while in the universities and the residential areas, the primary land cover is impervious surfaces, mixed with dispersed small patches of greenspace. Bare soil is the dominant land cover type in construction sites (Figure 1)

We used WorldView-2 satellite imagery, acquired on 14 September 2012, for land cover classification. WorldView-2, launched in October 2009, is the first high resolution 8-band multispectral commercial satellite. Four land cover types were identified for the study area: (1) impervious surfaces; (2) vegetation; (3) water; and (4) bare soil. Impervious surfaces were mainly roads and building roofs. Vegetation included trees and grass. Water mostly occurred in parks and bare soil in construction sites. Shadows from buildings and trees are common in very high resolution images of urban areas. Therefore, we included the shadow class and separated shadows from unshaded land cover types.

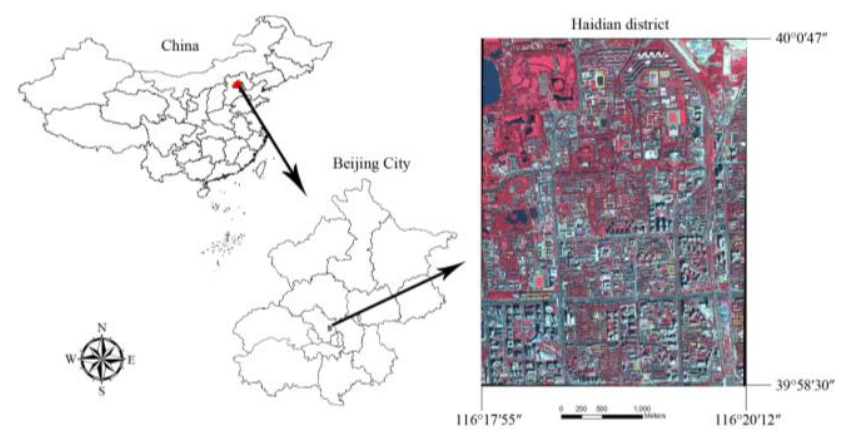

Figure 1. The study area, an urban area located in the Haidian District of Beijing, China.

\subsection{Methods}

The object-based classification procedure includes image segmentation, training sample selection, classification feature selection, tuning parameter setting and, finally, algorithm execution. We first segmented the image into land cover segments and then chose a certain amount of segments of different land cover types as training samples. After comparing the training sample characteristics of different land cover types, we selected certain object features for classification. Finally, we adjusted the tuning parameters of different classifiers to generate high classification accuracy. For all four classifiers, we used the same procedure for image segmentation and the selection of training samples and classification features. The optimal setting of tuning parameters, however, were determined separately for each classifier. Following the classifications, object-based accuracy assessment was applied to evaluate different classifiers.

Using Google Earth, we randomly chose 1500 object samples, 300 for each class, for the classifications and accuracy assessment. We then randomly divided the 1500 object samples into two sets: 1000 as training samples and 500 as testing samples. To investigate the sensitivities of classifiers to the size of training samples, 8 training sample subsets were generated by randomly sampling from the total training sample set. The sizes of the training samples of those subsets were 125, 250, 375, 500, $625,750,875$ and 1000 , respectively. Within a training subset, the numbers of samples for each of the five classes were equal, and thus, the sample numbers of each class within the 8 training sample subsets were 25, 50, 75, 100, 125, 150, 175 and 200, respectively. Likewise, in the testing sample set, there were 100 samples per class.

For DT, we tested the value of "maximum depth" from 1 to 20. For SVM, we systematically tested 10 values for both $\mathrm{C}$ and gamma. Specifically, we tested the 10 values of $\mathrm{C}-10^{-1}$, $10^{\circ}, 10^{1}, 10^{2}, 10^{3}, 10^{4}, 10^{5}, 10^{6}, 10^{7}$ and $10^{8}$ - and 10 values of gamma- $10^{-5}, 10^{-4}, 10^{-3}, 10^{-2}, 10^{-1}, 10^{\circ}, 10^{1}, 10^{2}, 10^{3}$ and $10^{4}$. For $\mathrm{KNN}$, we examined $\mathrm{K}$ values from 1 to 20 . All 8 training sample subsets have tested by these four classifier with different parameter settings.

For each of the 4 classifiers, we chose one thematic map with the highest overall classification accuracy for each of the 8 training sample subsets and then compared these 32 thematic maps based on overall accuracy, the kappa coefficient and the user's and producer's accuracy. In addition, we repeated the random selection of training and testing samples within the 1,500 samples 10 times and then compared average overall accuracy for all classifiers with the optimal parameter values.

\subsection{Results and Discussion}

The results showed that SVM generally had the best performance among the four classifiers (Table 3). With the optimal parameter setting, the minimum overall accuracy and kappa coefficient of SVM was $92.6 \%$ and 0.9075 , which was higher than the maximum overall accuracy and kappa coefficient of DT $(88.4 \%$ and 0.855$)$ and $\mathrm{KNN}(86.8 \%$ and $0.835)$. Figure 2 shows the classification results with the highest overall accuracy for each classifier. Using the Z-statistics, we found that the overall accuracies of SVM were significantly greater than those of DT and KNN, regardless of the size of training samples. In addition, SVM had significantly higher overall accuracy than NB, when the size of training samples was relatively small. However, when training samples were greater than or equal to 100 per class, the classification accuracies of NB and SVM were similar and significantly higher than the accuracies of the other two classifiers.

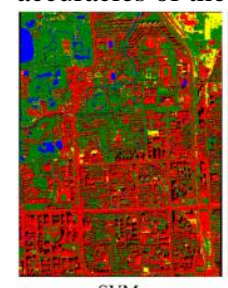

SVM

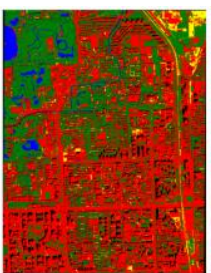

NB

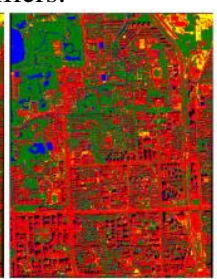

DT

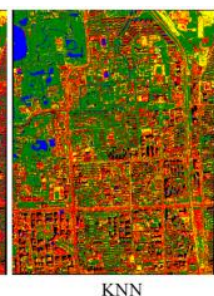

Figure 2. The classification results with the highest overall

accuracy for each classifier.

In general, when the size of samples per class was less than 125 , the accuracies of the four classifiers increased with increasing size of the training samples, and $\mathrm{NB}$ and $\mathrm{KNN}$ were more sensitive to sample sizes than SVM and DT. When the size of training samples increased from 25 to 125 per class, the classification accuracies of NB and $\mathrm{KNN}$ increased by $17 \%$ and 
$8.2 \%$, while SVM and DT increased by $3.6 \%$ and $4.6 \%$, respectively (Table 1; Figure 3 ). NB was the most sensitive to sample size. This may be because this parametric classifier used training samples to estimate parameter values for the data distribution, and thus, more training samples can lead to more accurate parameter estimation. In contrast, SVM is the least sensitive to sample sizes, because SVM only uses the support vectors instead of all training samples to build the separating hyperplane. Thus, adding more training samples may not significantly affect the classification accuracy. However, when the size of the samples is more than 125 per class, all four classifiers become insensitive to the increase of sample sizes. The classification accuracies of NB, KNN, SVM and DT fluctuated from $95 \%$ to $96.4 \%, 85.2 \%$ to $86.8 \%, 96.2 \%$ to $97.6 \%$ and $87.2 \%$ to $87.6 \%$, respectively, with the sample size increasing from 125 to 200 per class. This result indicated that the training sample size of 125 per class might be a turning point, beyond which the increase of sample size does not necessarily lead to a significant increase in classification accuracies. These results have important implications for determining the appropriate sample size.

Table 1. The highest overall accuracy for the four classifiers using eight training sample sets and the corresponding parameter values. NB, normal Bayes; DT, decision tree.

\begin{tabular}{lllll|llll}
\hline $\begin{array}{l}\text { Sample } \\
\text { Size }\end{array}$ & C & Gamma & Accuracies & Kappa & $\begin{array}{l}\text { Sample } \\
\text { Size }\end{array}$ & $\begin{array}{l}\text { Max } \\
\text { Depth }\end{array}$ & Accuracies & Kappa \\
\hline SVM & & & & & DT & & & \\
\hline 25 & 10,000 & 0.001 & 0.926 & 0.9075 & 25 & 3 & 0.838 & 0.7975 \\
50 & $1,000,000$ & 0.00001 & 0.94 & 0.925 & 50 & 5 & 0.866 & 0.8325 \\
75 & $1,000,000$ & 0.00001 & 0.958 & 0.9475 & 75 & 12 & 0.884 & 0.855 \\
100 & $1,000,000$ & 0.00001 & 0.944 & 0.93 & 100 & 6 & 0.884 & 0.855 \\
125 & 10,000 & 0.0001 & 0.962 & 0.9525 & 125 & 8 & 0.874 & 0.8425 \\
150 & 10,000 & 0.0001 & 0.964 & 0.955 & 150 & 5 & 0.876 & 0.845 \\
175 & $1,000,000$ & 0.00001 & 0.976 & 0.97 & 175 & 5 & 0.876 & 0.845 \\
200 & $1,000,000$ & 0.00001 & 0.964 & 0.955 & 200 & 6 & 0.872 & 0.84 \\
\hline NB & & & & & KNN & & & \\
\hline 25 & & & 0.794 & 0.7425 & 25 & 1 & 0.77 & 0.7125 \\
50 & & & 0.836 & 0.795 & 50 & 1 & 0.796 & 0.745 \\
75 & & & 0.912 & 0.89 & 75 & 3 & 0.81 & 0.7625 \\
100 & & & 0.936 & 0.92 & 100 & 3 & 0.828 & 0.785 \\
125 & & & 0.964 & 0.955 & 125 & 1 & 0.852 & 0.815 \\
150 & & & 0.95 & 0.9375 & 150 & 1 & 0.852 & 0.815 \\
175 & & & 0.958 & 0.9475 & 175 & 3 & 0.856 & 0.82 \\
200 & & & 0.964 & 0.955 & 200 & 3 & 0.868 & 0.835 \\
\hline
\end{tabular}

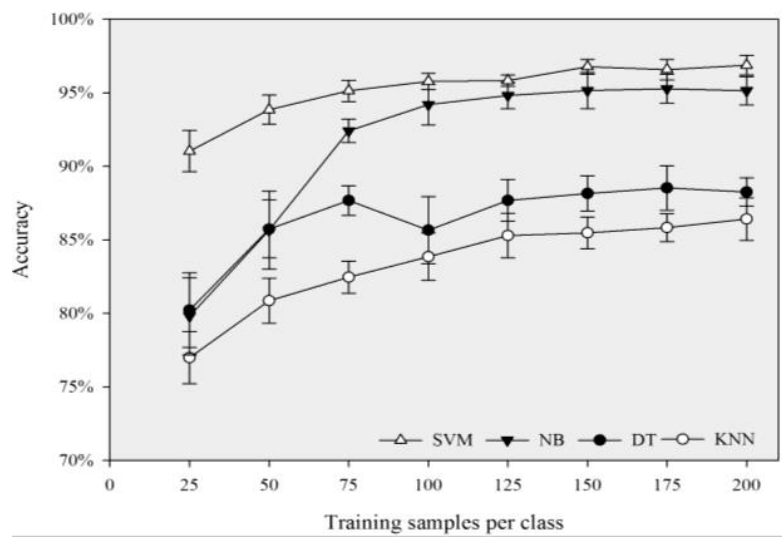

Figure 3. Overall accuracies of the four classifiers with increasing size of training samples.

\section{CONCLUSION}

SVM and NB were superior to CART and KNN in urban land classification. Both SVM and NB could achieve very high classification accuracy, with appropriate setting of the tuning parameters and/or enough training samples. However, each of the two classifiers has its advantages and disadvantages, and thus, the choice of the appropriate one may be case dependent. SVM could achieve relatively high accuracy with a relatively small amount of training samples, but the setting of tuning parameters could be subjective and time-consuming. In contrast, NB does not need the setting of any tuning parameter, but generally requires a large amount of training samples to achieve relatively high accuracy.

Both the size of training samples and the setting of tuning parameters have great impacts on the performance of classifiers. When the size of training samples is less than 125 per class, increasing the size of training samples generally leads to the increase of classification accuracies for all four classifiers, but $\mathrm{NB}$ and KNN were more sensitive to the sample size. Increasing the size of training samples does not seem to significantly improve the classification once the size of training samples reaches 125 per class. The tuning parameters of the classifier had a great impact on the classification accuracy. While the optimal settings of tuning parameters varied with the size of training samples, some general patterns occurred. For SVM, setting $\mathrm{C}$ between 1,000,000 and 100,000,000, and gamma between 0.00001 and 0.001 usually achieved the best overall accuracy. With DT, the best classification accuracy was generally achieved when the max depth of classification tree was between five and eight. For KNN, the optimal $\mathrm{K}$ value was either one or three. These findings provide insights into the selection of classifiers and the size of training samples when implementing an object-based approach for urban land classification using high resolution images.

\section{REFERENCES}

Duro, D. C., S. E. Franklin and M. G. Dube (2012). "A comparison of pixel-based and object-based image analysis with selected machine learning algorithms for the classification of agricultural landscapes using SPOT-5 HRG imagery." Remote Sensing of Environment 118: 259-272.

Laliberte, A. S., A. Rango, K. M. Havstad, J. F. Paris, R. F. Beck, R. McNeely and A. L. Gonzalez (2004). "Object-oriented image analysis for mapping shrub encroachment from 1937 to 2003 in southern New Mexico." Remote Sensing of Environment 93(1-2): 198-210.

Mathieu, R. and J. Aryal (2007). "Object-based classification of Ikonos imagery for mapping large-scale vegetation communities in urban areas." Sensors 7(11): 2860-2880.

McInerney, D. O. and M. Nieuwenhuis (2009). "A comparative analysis of $\mathrm{kNN}$ and decision tree methods for the Irish National Forest Inventory." International Journal of Remote Sensing 30(19): 4937-4955.

Song, X., Z. Duan and X. Jiang (2012). "Comparison of artificial neural networks and support vector machine classifiers for land cover classification in Northern China using a SPOT-5 HRG image." International Journal of Remote Sensing 33(10): 3301-3320.

Wieland, M. and M. Pittore (2014). "Performance Evaluation of Machine Learning Algorithms for Urban Pattern Recognition from Multi-spectral Satellite Images." Remote Sensing 6(4): 2912-2939. 


\title{
MAP LEGEND AND RESPONSE DESIGN: HOW DO THEY AFFECT ACCURACY OF GEOBIA RESULTS
}

\author{
J. Radoux ${ }^{\mathrm{a} *}$, P. Bogaert ${ }^{\mathrm{a}}$ \\ ${ }^{a}$ Earth and Life Institute, Université catholique de Louvain, 1348 Louvain-la-Neuve, Belgium - (julien.radoux,patrick.bogaert)@uclouvain.be
}

KEY WORDS: Accuracy assessment, quality, segmentation, legend, overall accuracy

\begin{abstract}
:
The legend used to map a given region is most of the time the first characteristic assessed by end users. In GEOBIA, the choice of a classification system is primarily guided by the scale of analysis and the underlying data model. This study focuses on the classification systems related to spatial regions. Depending on the purpose of the GEOBIA analysis, three types of classification systems have been identified: majority-based, LCCS like and feature-based. The validation of the classification results then relies on an appropriate choice of response design, which in turn influences the accuracy results. The impact of the different response designs is highlighted with a real case study. A high accuracy pixel-based classification of the segmented image is used as synthetic reference and classification errors are simulated on the object database. The results shows that all methods are equivalent if all polygons are pure, but that the uncertainty conferred by under-segmented or heterogeneous polygons affects the accuracy estimates in different ways: some methods tolerate delineation or under-segmentation while others are directly affected. This experiment also shows some differences and similarities between the point-based and the object-based validation frameworks, which are both valid depending on the circumstances but could be misleading when they are not applied with the right purpose. The thematic precision of the legend is also discussed: the LCCS authorize mixtures of different spatial entities in the class definition, but the information content differs depending of the purity of the majority of the classes.
\end{abstract}

\section{INTRODUCTION}

GEOBIA is now considered as a new image classification paradigm. It is therefore important to identify good practices for the quality assessment of GEOBIA results. As central part of the fitness to purpose assessment, the legend used to map a given region is most of the time the first characteristic considered by end users. In GEOBIA, the choice of a classification system is primarily guided by the scale of analysis and the underlying data model. Image-segments are indeed groups of pixels that may be used to represent spatial objects (which can be unambiguously delimited) or spatial regions (which do not have precise boundaries at the scale of the analysis and could be defined as a mixture of different entities). Classification systems for spatial objects usually imply a majority-based class assignment guided by pure reference objects. The spatial delineation of the image-segments is very important in this case. On the other hand, spatial regions are more often used to describe a continuous landscape with softer boundaries: in this case, partitioning classification systems like the LCCS should prevail while the positional errors along the boundary are usually not the primary quality issue.

This study focuses on the classification systems related to spatial regions. Depending on the purpose of the GEOBIA analysis, three types of classification systems have been identified: majority-based, LCCS (Di Gregorio and Jansen, 2000) like and feature-based. The validation of the classification results then relies on an appropriate choice of response design, which in turn influences the accuracy results. The impact of the different response designs is highlighted with a real case study. A high accuracy pixel-based classification of the segmented image is used as synthetic reference and classification errors are simulated on the object database. The results shows that all methods are equivalent if all polygons are pure, but that the uncertainty conferred by

\footnotetext{
${ }^{*}$ Corresponding author
}

under-segmented or heterogeneous polygons affects the accuracy estimates in different ways: some methods tolerate delineation or under-segmentation while others are directly affected. This experiment also shows some differences and similarities between the point-based and the object-based validation frameworks, which are both valid depending on the circumstances but could be misleading when they are not applied with the right purpose. The thematic precision of the legend is also discussed: the LCCS authorize mixtures of different spatial entities in the class definition, but the information content differs depending of the purity of the majority of the classes. Finally, it is shown that the accuracy of the LCCS-like classification system can be optimized for a given number of classes by selecting thresholds that minimize the confusion errors.

\section{METHOD}

A framework based on realistic synthetic data was set up in order to compare the different validation approaches. This is achieved by assuming that the delineation corresponds to the end user needs and using an existing classification as if it was the true land cover. Thematic classification errors are then simulated based on transposed confusion matrix of the classified map, but the delineation is not modified in order to avoid interactions between thematic and spatial errors. The study area could have been completely synthetic, but the use of a real world example allows us to better illustrate the discussion. This real world example uses a pixelbased classification and image segmentation based on the same data set. It covers the Walloon region at a resolution of 2 meters and the mean polygon size is around 6700 pixels with a minimum mapping unit of 1000 square meters.

\subsection{Classification systems}

The choice of a legend affects both the accuracy of a map and its fitness to purpose. Three classifications are used in this study: 
- Majority rule assigns a label based on the dominant land cover inside each polygon. This type of legend includes the minimum number of classes necessary to represent each of the basic classifiers. It is most suited when GEOBIA aims at identifying spatial object, which are pure with respect to the classifiers.

- FAO LCCS rules are built upon a set of thresholds on the proportion of each land cover within the spatial unit. When correctly applied, those rules generate a comprehensive partition of the feature space. They are well suited to coarse resolution (due to mixed pixels) or to the characterization of heterogeneous land cover classes based on spatial region. In this study, the LCCS rules used in the global land cover CCI project (Bontemps et al., 2013) have been applied. Considering the region, possible classes are 10, 30, 40, 60, 70, 90, 100, 110, 120, 190, 200 and 210. Those classes are characterized with an unique code that guarantees a good interoperability.

- In addition to those two expert-based rules, a third objectbased legend was driven by the data itself based on k-mean clustering. This legend is more difficult to interpret but the aim is to test its robustness relatively to expert-based legends.

Those labels were first assigned based on their reference values. In a second stage, random errors have been introduced into the pixel-based classification and labels were assigned on the altered data.

\section{RESPONSE DESIGNS AND SAMPLING UNITS}

Obviously, the ideal response design would consist in assigning a class to each object by measuring the proportion of each classifier and applying the legend rules. This method is however the most time consuming, which reduces the advantage of using polygonbased instead of point-based sampling (Radoux et al., 2011). The bias on the overall accuracy due to the sampling unit was therefore estimated for different alternative that could be applied on the field:

- Centroid-based sampling : similar sampling scheme to the standard probabilistic sampling, but polygons are selected from a list of all polygons then the point samples are defined as the centroids of each polygon.

- Parcel-based sampling: polygons centroids are first selected then the pixels in a circular plot around those centroids are used. In this study a 5 pixels radius $(10 \mathrm{~m})$ was arbitrarily selected. The different classification rules are then applied on the proportions of pixels belonging to each circular plot in order to determine their final label, which is compared with the polygon label.

In order to estimate the errors, ten random samples of 600 points were created using the centroids of randomly selected polygons. The values were then extracted from the unaltered pixel-based classification. Both classification accuracy (proportion of correctly classified polygons) and overall accuracy (proportion of the map area that is correctly classified) are then derived based on (Radoux and Bogaert, 2014).

\section{RESULTS}

The results show that the difference between a centroid-based interpretation and an object-based legend can be substantial. The bias of a single point sampling and the maximum or the LCCS object-based legends are $17.7 \%$ and $28.3 \%$, respectively. This bias is smaller with the parcel-based sampling, but it is still large: it reaches $11.5 \%$ for the majority label and $21.1 \%$ with the LCCS. As expected, the differences are linked with the heterogeneity of the spatial regions. Indeed, polygons that are close to purity (more than $90 \%$ of a single class) are matched with a simple point-based sampling in $98.3 \%$ of the cases. With parcelbased sampling, this value is even larger with $98.8 \%$. This suggest that centroid-based or parcel-based sampling unit could be used as a pragmatic alternative for the validation of spatial objects, but should be avoided with heterogeneous spatial regions. On the other hand, centroid-based sampling is not equiprobable because points along the object boundaries are less likely to be selected. Because object delineation errors are more likely around the edges, centroid-based classification overestimate the quality of spatial object classification. This issue can be solved by using standard probabilistic sampling or by assessing the delineation quality independently of the thematic accuracy.

\section{CONCLUSION}

This study demonstrates that the choice of sampling unit and response design have a major impact on the accuracy assessment results. Detailed results and discussion will be presented at the GEOBIA conference.

\section{ACKNOWLEDGEMENTS}

This study was supported by the Fédération Wallonie-Bruxelles in the frame of the Lifewatch-WB project.

\section{REFERENCES}

Bontemps, S., Defourny, P.and Radoux, J., Van Bogaert, E., Lamarche, C., Achard, F., Mayaux, P., Boettcher, M., Brockmann, C., Kirches, G., Zulkhe, M., Kalogirou, V., Seifert, F. M. and Arino, O., 2013. Consistent global land cover maps for climate modelling communities: Current achievements of the esa' land cover cci. ESA Living Planet Symposium, ESA-ESRIN, Frascati.

Di Gregorio, A. and Jansen, L., 2000. Land cover classification system (lccs): Classification concepts and user manual. GCP/RAF/287/ITA Africover-East Africa Project and Soil Resources, Management and Conservation Service, Food and Agriculture Organization.

Radoux, J. and Bogaert, P., 2014. Accounting for the area of polygon sampling units for the prediction of primary accuracy assessment indices. Remote Sensing of Environment 142, pp. 919.

Radoux, J., Bogaert, P., Fasbender, D. and Defourny, P., 2011. Thematic accuracy assessment of geographic object-based image classification. International Journal of Geographical Information Science 25(1), pp. 1365-8816. 


\title{
USING LIDAR AND AERIAL PHOTOGRAPHY TO BUILD A GEOGRAPHIC OBJECT DATABASE TUNED FOR ECOLOGICAL MODEL
}

\author{
J. Radoux ${ }^{\text {a }}$, P. Defourny ${ }^{\mathrm{a}}$ \\ a Earth and Life Institute, Université catholique de Louvain, 1348 Louvain-la-Neuve, Belgium - (julien.radoux,pierre.defourny)@uclouvain.be
}

Commission VI, WG VI/4

KEY WORDS: Spatial regions, LIDAR, biodiversity, segmentation, topography

\begin{abstract}
:
Ecological models require a large variety of variables in order to describe the biotic and abiotic conditions that define wildlife habitats and biotope distribution. Instead of providing this information using regular grids or categorical maps, geographic object-based image analysis allows to describe the land cover based on meaningful spatial regions which are closer to the natural habitats than regular grids and more flexible to the specific needs of models than categorical data. In the frame of the Lifewatch/Wallonia-Brussels project, we use GEOBIA to create a geographic object database closely related to the ecological concept of ecotope. Through an iterative process with ecological modellers, this database has been designed in order to define its key characteristics : it had to provide a quantitative description of the land cover within topographically relevant spatial regions and it integrates contextual information from different scales. The proof of concept of this new type of geographic database was done over the Wallon region in Belgium. This study area covers approximately 16800 square kilometers with a very fragmented landscape. A dataset including aerial photographs at $0.25 \mathrm{~cm}$ resolution and LIDAR at $0.8 \mathrm{pts} / \mathrm{m}$ has been provided by the Walloon region for the study. The data were resampled at $2 \mathrm{~m}$ resolution for the purpose of the analysis. The data processing workflow includes three steps : pixel-based image classification, image segmentation and object-based integration. Pixel-based image classification consists in a supervised classification with the spectral values from the aerial photographs (NIR/Red/green/blue), the Digital Height Model extracted from the LIDAR and the intensity of the first LIDAR return. This yielded a classification into broadleaved trees, needleleaved trees, grass, bare soil, crop, pavement, building, water and shadows with more than $80 \%$ overal accuracy. The image segmentation approach is the main novelty of this research. In order to fit with the biotopes, image segments indeed had to take the type of slope into account. This was achieved by computing pseudo-hillshades for North-South and West-East orientation and including those two files together with the spectral information from the aerial photographs. The result of this analysis is a set of topographically relevant ecotope delineation. The last step applied contextual decision rules to consistently aggregate the land cover information at the ecotope level and add more information from ancillary datasets.
\end{abstract}

\section{INTRODUCTION}

Habitat suitability mapping and biotope prediction models are necessary to fill the gaps of field observation for biodiversity monitoring. Remotely sensed data are of paramount importance in providing some spatially comprehensive information that is necessary to the prediction over large regions. In this context, models are historically based on regular grids linked with permanent structured inventories. However, with the democratization of geopositioning devices and the raise of citizen science, the precision of the observation has tremendously increased. An alternative approach to grid based habitat and biotope prediction could therefore emerge with a partitioning of landscape based on GEOBIA.

In this study, we demonstrate the feasibility of automated land units characterization under the concept of ecotopes (Ellis et al., 2006). Ecotope is not a new concept in ecology but it was rarely used in practice due to the combined absence of automated method and spatially precise positioning of biodiversity observations. Contrary to ecological land unit, which are created by the intersection of multiple categorical data, ecotopes are defined as the smallest ecologically homogenous landscape units. A method was therefore designed to delineate and characterize those units at large scale.

\footnotetext{
${ }^{*}$ Corresponding author
}

\section{DATA AND STUDY AREA}

The study area is the Walloon region in the Southern part of Belgium. This is a very fragmented landscape including coniferous forests (mainly spruce), broadleaved forests (mainly oaks and beeches), crops, natural and managed grasslands, peatlands, small water bodies, extraction areas and urban areas.

Two types of input data were available. First, a mosaic of orthorectified aerial photographs upscaled to $2 \mathrm{~m}$ resolution and including four 8-bit bands; Second, a LIDAR dataset rasterized at 2 $m$ resolution. The DEM and the height of the vegetation were derived from this LIDAR dataset. This dataset was heterogeneous and required specific mathematical morphology analysis: an closing was applied on the West part (captured at $1550 \mathrm{~nm}$ ) in order to compensate for the lack of multiple returns and an opening was applied the East part in order to remove power lines.

\section{METHOD}

The delineation and the characterization are two independent steps of the processing chain. The ecotopes are delineated and quantitatively characterized with a set of ecologically meaningful variables obtained by remote sensing or from other ancillary variables.

\subsection{Delineation}

The three variables of interest to discriminate ecological function at the scale of the analysis are the land cover, the topography 
and the soil type. However, soil type information was not precise enough and could be partly inferred by the topography. We therefore focused on topography and land cover. A commonly used trial and error segmentation with the multiresolution segmentation algorithm (Baatz and Schäpe, 2000) was applied for the ecotope delineation. The efficiency of a segmentation combining LIDAR height and multispectral image had already been proven (Geerling et al., 2009). However, the main originality of our method was to combine the spectral information from the orthophotos with the DEM in addition to the height information from the LIDAR. Including topography in a segmentation however require a transformation of the DEM data to highlight the different slope types and identify breaks. Slope aspect could however not be used the the segmentation algorithm because it is undefined when the slope in null and because it is a circular metric that jumps from 360 to 0 . We therefore created two synthetic hillshade maps along the North-South and the East-West transects. The seven layer were then combined in a single image that was segmented with band weights providing equal contribution to spectral values, height and hillshade.

\subsection{Characterisation}

A large set of attributes have been derived from existing database and GIS analysis. This set includes bioclimatic variables, soil variables, topographic variables and land cover variables. Those variables have been selected based on expert knowledge and their contribution to habitat suitability models.

For the land cover variables, a pixel-based classification was first performed using the 4 spectral bands of the orthophoto and the LIDAR information. LIDAR data was used to mask out most water bodies based on the absence of returns and extreme intensities linked with the specular reflection on the waves. A maximum likelihood classifier was trained (Radoux et al., 2014) based on a reference dataset combining an existing land cover maps and a model to predict shadow position based on the LIDAR DSM. The a priori probability was computed based on the frequency of each land cover type within two height classes (below and above $50 \mathrm{~cm}$ ). This step was particularly useful to discriminate forests, shrubs and buildings from the other land covers.

\section{RESULTS AND DISCUSSION}

Approximately 1.2 million image-segments were automatically created. Figure 1 shows a subset of the segmentation result highlighting the impact of the topography on the segments created inside the otherwise homogeneous forest. Indeed, homogeneous slope types are delineated in addition to the land cover induced delineation. As a result, the heterogeneity of the topographic attributes decreases and the separability of ecotopes increases. On the other hand, relevant continuous patches are delineated without the artefacts that risk to occur when two (or more) categorical maps are overlaid in a GIS (Hong et al., 2004).

From a modelling perspective, habitat suitability mapping was improved by using the ecotopes versus categorical maps. Furthermore, it was shown that the biotope mapping of Aceron pseudoplatani (ravine maple forests) was improved when using a topographic based segmentation.

\section{CONCLUSION}

This study highlighted how GEOBIA could provide appropriate input data for habitat suitability and biotope mapping. Detailed results and discussion will be presented at the GEOBIA conference.

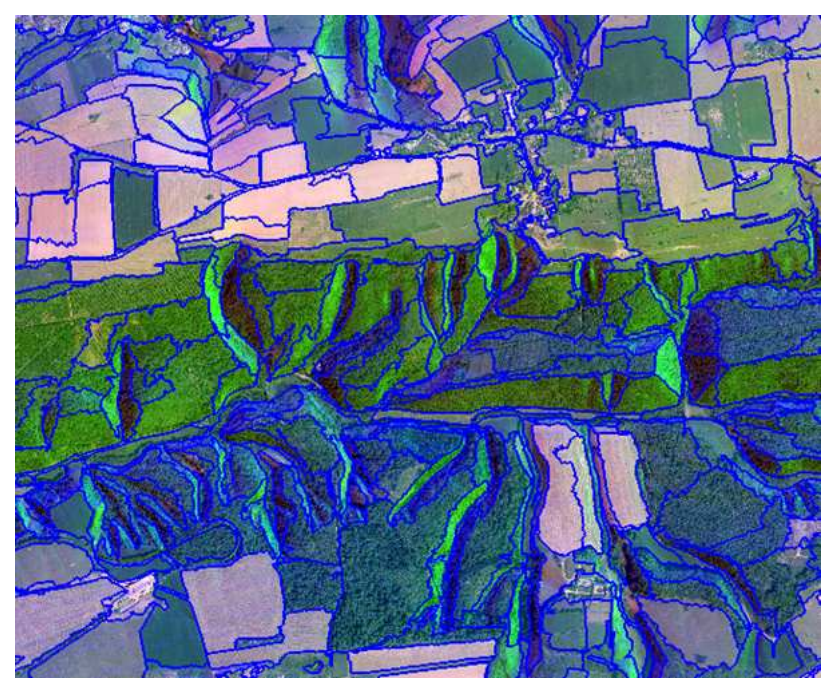

Figure 1. Image-segment overlaid on the input data. The color composite used the red, green and blue bands of the orthophoto in the RGB channels as well as the NS and EW hillshades with the red and green channels.

\section{ACKNOWLEDGEMENTS}

This study was supported by the Fédération Wallonie-Bruxelles in the frame of the Lifewatch-WB project.

\section{REFERENCES}

Baatz, M. and Schäpe, A., 2000. Multiresolution segmentation - an optimization approach for high quality multi-scale image segmentation. In: J. Strobl, T. Blaschke and G. Griesebner (eds), Angewandte Geographische Informationsverarbeitung XII, Wichmann-Verlag, Heidelberg, pp. 12-23.

Ellis, E. C., Wang, H., Xiao, H. S., Peng, K., Liu, X. P., Li, S. C., Ouyang, H., Cheng, X. and Yang, L. Z., 2006. Measuring longterm ecological changes in densely populated landscapes using current and historical high resolution imagery. Remote Sensing of Environment 100(4), pp. 457 - 473.

Geerling, G. W., Vreeken-Buijs, M. J., Jesse, P., Ragas, A. M. J. and Smits, A. J. M., 2009. Mapping river floodplain ecotopes by segmentation of spectral (casi) and structural (lidar) remote sensing data. River Research and Applications 25(7), pp. 795813.

Hong, S.-K., Kim, S., Cho, K.-H., Kim, J.-E., Kang, S. and Lee, D., 2004. Ecotope mapping for landscape ecological assessment of habitat and ecosystem. Ecological Research 19(1), pp. 131139.

Radoux, J., Lamarche, C., Van Bogaert, E., Bontemps, S., Brockmann, C. and Defourny, P., 2014. Automated training sample extraction for global land cover mapping. Remote Sensing 6(5), pp. 3965. 


\title{
DECISION TREE CLASSIFICATION MODEL FOR DETECTING AND TRACKING PRECIPITATING OBJECTS FROM SERIES OF METEOROLOGICAL IMAGES
}

\author{
S. Ramirez ${ }^{\mathrm{a} *}$ I. Lizarazo $^{\mathrm{b}}$ \\ ${ }^{a}$ Facultad de ingenería, Universidad Distrital Francisco Jose de Caldas, Bogota DC., Colombia - seramirezf@correo.udistrital.edu.co \\ ${ }^{\mathrm{b}}$ Facultad de Ciencias Agrarias, Universidad Nacional, Bogota, DC., Colombia - ializarazos@ unal.edu.co
}

KEY WORDS: Mesoscale Convective Systems, Precipitating Objects, Classification Model, Decision Trees, Meteorological Images

\begin{abstract}
:
Accurate detection and identification of convective (cumulonimbus) clouds, which are potentially precipitating objects, as well as tracking cloud movement, are important tasks to locate and predict precipitation. In the present work, a Decision Tree classification model was used to locate and track precipitating objects from series of GOES-13 meteorological image sub-scenes covering the territory of Colombia, located to the northwest corner of South America. Results show that it is possible to infer a classification model that can be used repeatedly for accurately locating and tracking precipitating objects from multispectral meteorological images.
\end{abstract}

\section{INTRODUCTION}

Knowledge of convective systems' cloud processes and their evolution is important for understanding weather and climate, particularly over the tropics (Vila et al., 2008). In both equatorial and mid tropical latitudes, cumulonimbus $(\mathrm{Cb})$ clouds are responsible for causing extreme weather conditions; these clouds intense convection causes the formation of Mesoscale Convective Systems (MCS) (Liu et al., 2014). A MCS is a convective system' $C b$ cloud that produces a contiguous precipitation area $\geq 100 \mathrm{~km}$ in at least one direction (Houze, 2004).

SCM pose a serious risk to aviation and may impact crops and urban populations because rapidly changing weather on various spatial and temporal scales may occur within and near $C b$ clouds (Liu et al., 2014). A large number of MCS is located in Colombia, which according to data from Tropical Rainfall Measuring Mission (TRMM) during the period 1998-2002, contribute approximately $70 \%$ of annual precipitation (Sakamoto et al., 2011). In this country disasters caused by heavy precipitation produced by SCM, hit much of the country between the second half of 2010 and the first half of 2011, causing devastating effects on the population and on local, regional and national economy (Colombia Humanitaria, 2010).

Observations of SCM are an important source of data for assimilation in weather forecasting and for monitoring climate trends (Liu et al., 2014). In this regard, images of the Geostationary Operational Environmental Satellites (GOES) have shown a high potential for detection and monitoring of SCM (Goyens et al., 2012).

A recent study investigated the possibility to improve precipitating objects detection over the Colombian continental territory from five meteorological channels rather than a single IR channel (Ramírez and Lizarazo, 2014). That study was conducted to detect and extract precipitating objects from a series of GOES13 meteorological image sub-scene. Such study applied three machine learning algorithms including support vector machine (SVM), random forest (RF) and decision tree (DT) and compared them with the infrared brightness temperature threshold (IR-BT)

\footnotetext{
${ }^{*}$ Corresponding author
}

method. Accuracy assessment was conducted using STEP (Lizarazo, 2014) using a set of NASA Tropical Rainfall Measuring Mission (TRMM) images as reference.

The results of that study suggested that the machine learning DT is more accurate for detection of precipitating objects, compared to the conventional method IR-TB, while that SVM and RF results may not be acceptable for practical applications. The DT algorithm yielded a classification model tree which exhibits a very simple structure, with only two partitions, selecting thermalinfrared (TIR1) and water vapour (WV) as the most important channels.

In contrast to such study, this article aims to: establish whether it is possible to have a classification model that can be used repeatedly, and not requiring visual interpretation of imagery for every cloudy day. The evaluation was performed for the driest day and the wettest day of 2014 and 2015. In this case, a set of Global Precipitation Measurement (GPM), a well-established satellite weather radar system, with higher resolution than TRMM was used as reference.

\section{DECISION TREE CLASSIFICATION MODEL}

DT decomposes the space's attributes in P disjoint sets, $K_{r}$ with $n \in\{1, \ldots, P\}$, using decision rules or cuts that are orthogonal to the axes' attributes. Therefore, a decision rule can be described as a simple expression of the form $X_{i} \geq T_{i}$, o $X_{i}<T_{i}$ where $T_{i}$ is some threshold, which makes this operation easy to understand by the user (Lizarazo, 2008).

In previous study, the DT algorithm yielded a tree which exhibits a very simple structure, from a meteorological image taken by the Geostationary Operational Environmental Satellite (GOES13) for twenty-tow half-hour periods for one day in 2013. Date and time for this study were selected to ensure a similar number of class representatives at the instant on April 03, 2013 at 21:15 hours Coordinated Universal Time (UTC) (201304032115, this is the date format used in the data set) taken as reference.

This tree was obtained using the Gini impurity measure, selecting thermal-infrared (thermal-IR) and water vapour (WV) as the most 
important bands. The Gini impurity measure, is a measure of the distribution of the class' label on the node, taking values in $[0,1]$, where 0 is obtained when all the elements in a node fall into the same class. DT uses the Gini impurity measure to select the cut decision with the lowest impurity in each node (Tan et al., 2005). Formally, the impurity measure Gini given by Equation 2, for $x_{i}$ attribute with $i \in\{1, \ldots, n\}$ in the $t$ node (Khalilia et al., 2011).

$$
I\left(t_{x_{i}}\right)=1-\sum_{c=0}^{c}\left(\frac{n_{c i}}{a_{i}}\right)^{2}
$$

where $\quad n$ is the number of branches in the node $t$

$N$ is the samples' number

$n_{c i}$ is the samples' number with value $x_{i}$ belonging to class $c$

$a_{i}$ is the samples' number with value $x_{i}$ in the node $t$

The Gini index of a partition is the weighted average of the Gini impurity measure the different values of $X$ attribute, given by equation 2 (Khalilia et al., 2011).

$$
\operatorname{Gini}(t, X)=\sum_{j=1}^{j} \frac{a_{i}}{N} I\left(t_{x_{i}}\right)
$$

Figure 1 shows DT algorithm classification model obtained previously, which exhibits a very simple structure, with only two partitions. Thermal-infrared (TIR1) and water vapour (WV) channel were selected as the most important channels, and the model accuracy was $98 \%$.

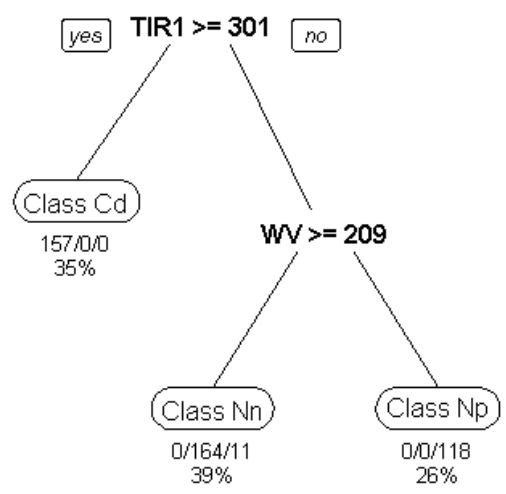

Figure 1. Decision tree obtained by the DT algorithm

The tree in Figure 1 defines the following classification rules in terms of brightness temperatures (Tb): (i) If channel TIR1 is $<$ $301 \mathrm{~K}$ then the object is Cs (Clear Sky); (ii) If channel TIR1 is $\geq 301 \mathrm{~K}$ and the channel WV is $<209 \mathrm{~K}$ then the object is $\mathrm{Nr}$ (Non-Rainfall Cloud); and (iii) If channel TIR 1 is $\geq 301 \mathrm{~K}$ and the channel WV is $\geq 209 \mathrm{~K}$, then the object is Rc (Rainfall Cloud). The $35 \%$ of training pixels fulfil the first rule, $39 \%$ the second rule and $26 \%$ the last rule. This result is similar to obtained in a previous work (Feidas et al., 2000), where the cloud classification scheme developed for the thick opaque convective clouds correspond to classes with the lower $\mathrm{Tb}$ in both the IR channel (below $223 \mathrm{~K}$ ) and WV channel (below $223 \mathrm{~K}$ ).

\section{DATA AND METHODS}

\subsection{Study area}

This study was conducted over a tropical region comprising 5062400 $\mathrm{km}^{2}$, with predominance of Colombian territory, which is located in the northwest of South America as shown in the dashed window of Figure 2a. s

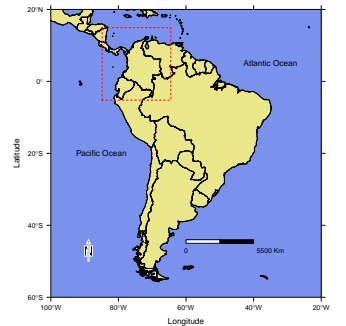

(a)

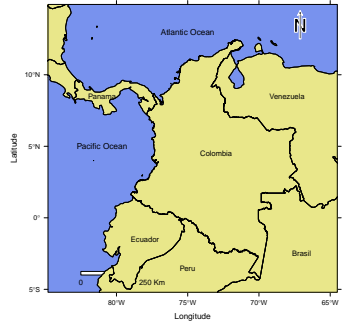

(b)
Figure 2. The study area:(a) South America. (b) Colombia.

\subsection{Data}

Meteorological images comprise a series of 88 sub-scenes in mode A (8-bits), taken by the Geostationary Operational Environmental Satellite (GOES-13) every half hour from 11:45 to 22:15 hours Coordinated Universal Time (UTC) for four different dates: (i) May 08, 2014, the rainiest day of 2014 with a precipitation of $19307.4 \mathrm{~mm}$; (ii) December 22, 2014, the least rainy day of 2014 with a precipitation of $225.9 \mathrm{~mm}$; (iii) January 06, 2015, the least rainy day of 2015 with a precipitation of $81 \mathrm{~mm}$; and (iv) October 13,2015 , the rainiest day of 2015 with a precipitation of 11900 $\mathrm{mm}$. The GOES Imager covers from visible (VIS) to thermal infrared (TIR) spectral regions with 5 spectral channels at spatial resolutions ranging from $1 \mathrm{~km}$ to $8 \mathrm{~km}$ (Table 1 ).

\begin{tabular}{|c|c|c|l|}
\hline Channel number & Central wavelength & Spatial Resolution & Channel description \\
\hline 1 & $0.52-0.72 \mu \mathrm{m}$ & $1 \mathrm{~km}$ & Visible (VIS) \\
2 & $3.78-4.03 \mu \mathrm{m}$ & $4 \mathrm{~km}$ & Mid Infrared (MIR) \\
3 & $6.47-7.02 \mu \mathrm{m}$ & $8 \mathrm{~km}$ & Water Vapor (WV) \\
4 & $10.2-11.2 \mu \mathrm{m}$ & $4 \mathrm{~km}$ & Thermal Infrared (TIR1) \\
5 & $12.9-13.8 \mu \mathrm{m}$ & $4 \mathrm{~km}$ & Thermal Infrared (TIR2) \\
\hline
\end{tabular}

Table 1. Characteristics of GOES Imager

Reference data for accuracy assessment was selected from a NASA Global Precipitation Measurement (GPM) data set, well-established satellite weather radar system, merged with high quality (IR) precipitation and monthly surface precipitation gauge data that provides half hourly consistently calibrated, uniformly gridded with spatial resolution about $10 \mathrm{~km}$ at the Equator, global precipitation data product GPM_3IMERGHH with appropriate error and metadata information (Hou et al., 2014).

\subsection{Methods}

The method proposed here comprises (Figure 3): (i) data preprocessing to convert image counts to physical quantities using the Equations 3 and 5 and match pixel size, spatial extent and spatial reference systems; (ii) pixel-based supervised cloud classification using the DT algorithm classification model obtained previously; (iii) creation of precipitating objects by binarisation and vectorization of the classified image; and (iv) accuracy assessment. The GPM precipitation amounts were also binarised, thus obtaining a set of reference precipitating objects to perform validation using STEP method (Lizarazo, 2014). STEP provides 


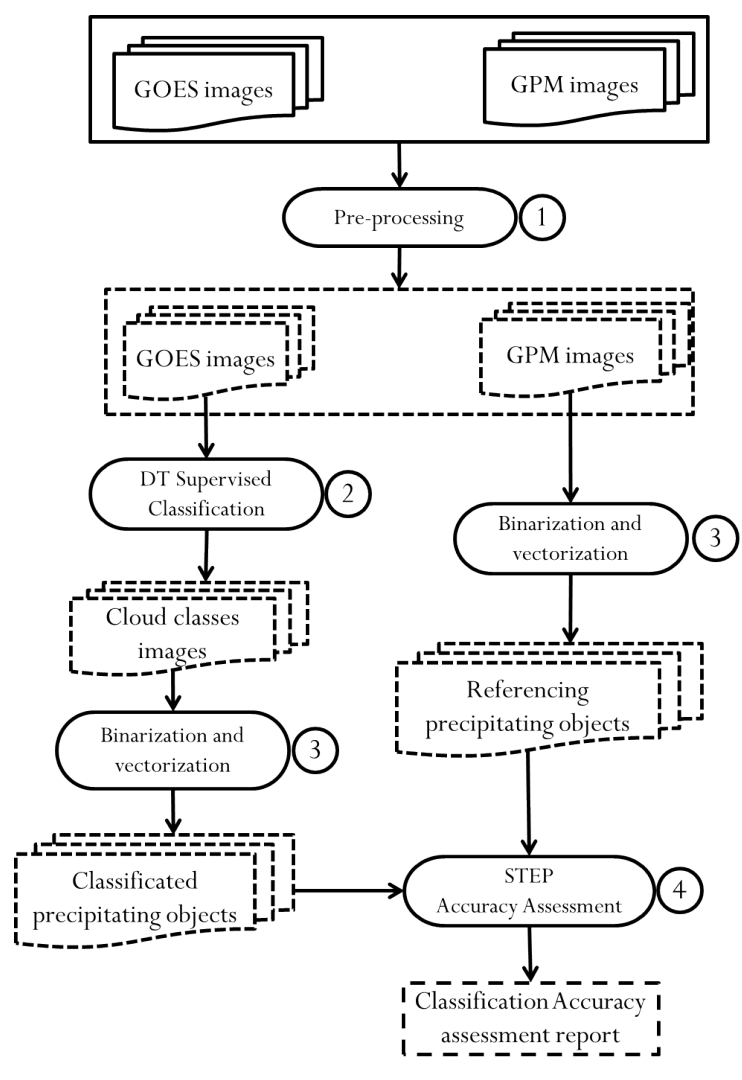

Figure 3. Methodology

four (4) similarity metrics for characterization of classified objects compared with reference objects: (i) shape similarity; (ii) theme similarity; (iii) edge similarity; and (iv) position similarity. The accuracy assessment focuses on convectives system' clouds having an area larger than $2400 \mathrm{~km}^{2}$. This threshold was chosen following a previous work (Sakamoto et al., 2011) on life cycle convective systems over Western Colombia.

Albedo (A) is calculated as:

$$
A=\left(\frac{N D_{V I S}}{255}\right)^{2}
$$

where $\quad N D_{V I S}$ are counts values in Mode-A visible channel

Brightness temperature $(\mathrm{Tb})$ is calculated as:

$$
\begin{aligned}
& T b=330 K-\frac{N D_{I R}}{2} ; \text { if } N D_{I R} \leq 176 \\
& T b=418 K-N D_{I R} ; \text { if } N D_{I R}>176
\end{aligned}
$$

where $\quad N D_{I R}$ are counts values in Mode-A infrared channel

\section{RESULTS AND DISCUSSION}

Overall accuracy was computed, similar to how overall accuracy is computed in the traditional error matrix, from shape, theme, edge and position similarity OBIA indices calculated by comparing thematic and geometric properties of classified precipitating objects with their corresponding reference precipitation objects, using a weighted area instead of the actual area for compensation of the unequal probability sampling, assigning to each classified precipitation object that matches (intersects) a given reference precipitation object a similarity value in the $[0,1]$ interval (Lizarazo, 2014).

Table 2 summarizes overall STEP similarity indices for each twentytwo date under evaluation. For the rainiest day of 2014 (20140508) the shape index presents variations in validation interval between $92 \%$ and $95 \%$, the theme index between $87 \%$ and $94 \%$, the edge index between $97 \%$ and $99 \%$ and the position index was $100 \%$ for all instants. In contrast for the rainiest day of 2015 (20151013) the shape index presents variations in validation interval between $95 \%$ and $97 \%$, the theme index between $91 \%$ and $93 \%$, the edge index between $98 \%$ and $100 \%$ and the position index was $100 \%$ between $98 \%$ and $100 \%$ for all instants.

\begin{tabular}{|c|c|c|c|c|}
\hline Day & Shape & Theme & Edge & Position \\
\hline 20140508 & {$[0.92,0.95]$} & {$[0.87,0.94]$} & {$[0.97,0.99]$} & {$[1.00,1.00]$} \\
20141222 & {$[0.94,1.00]$} & {$[0.93,1.00]$} & {$[0.99,1.00]$} & {$[0.98,1.00]$} \\
20150106 & {$[0.98,0.99]$} & {$[0.98,0.99]$} & {$[1.00,1.00]$} & {$[1.00,1.00]$} \\
20151013 & {$[0.95,0.97]$} & {$[0.91,0.93]$} & {$[0.98,1.00]$} & {$[1.00,1.00]$} \\
\hline
\end{tabular}

Table 2. Overall thematic and geometric accuracy predictors for the MCS

While for the least rainy day of 2014 day (20141222) the shape index presents variations in validation interval between $94 \%$ and $100 \%$, the theme index between $93 \%$ and $100 \%$, the edge index between $99 \%$ and $100 \%$ and the position index between $98 \%$ and $100 \%$. In contrast for the least rainy day of 2015 (20150106) the shape index presents variations in validation interval between $98 \%$ and $99 \%$, the theme index between $98 \%$ and $99 \%$, the edge index and the position index were $100 \%$ for all instants.

The results suggests that the best aspect is position, followed in their respective order by edge, shape and theme. There are no significant differences between the two dates rainiest and the two dates less rain, shown be suitable for practical applications.

A visual comparison among the time's instants under evaluation also was performed for detected cloud objects. Figure 4 shows an instant by each date under evaluation.

For the instant 201405081545 UTC (Figure 4a) of rainiest day of 2014 the overall STEP similarity was shape $95 \%$, theme $93 \%$, edge $99 \%$ and position $100 \%$, for this instant there are 21 precipitating objects which are located mainly on the Pacific, central and eastern regions of Colombia, on west region of Venezuela, on Amazon region of Brazil and on Ecuador and Peru, with areas between $1922 \mathrm{~km}^{2}$ and $117164 \mathrm{~km}^{2}$.

For the instant 201510131945 UTC (Figure 4d) of rainiest day of 2015 the overall STEP similarity was shape $96 \%$, theme $92 \%$, edge $98 \%$ and position $100 \%$, for this instant there are 55 precipitating objects which are located mainly on the Pacific, the Caribbean, central and west regions of Colombia and on Venezuela, with areas between $1005 \mathrm{~km}^{2}$ and $170715 \mathrm{~km}^{2}$.

For the instant 201412221715 UTC (Figure 4b) of least rainy day of 2014 the overall STEP similarity was shape $96 \%$, theme $97 \%$, edge $100 \%$ and position $100 \%$, for this instant there are 2 precipitating objects which are located mainly on the Colombian and Brazilian Amazon region, with areas of $3544 \mathrm{~km}^{2}$ and 11475 $\mathrm{km}^{2}$. 


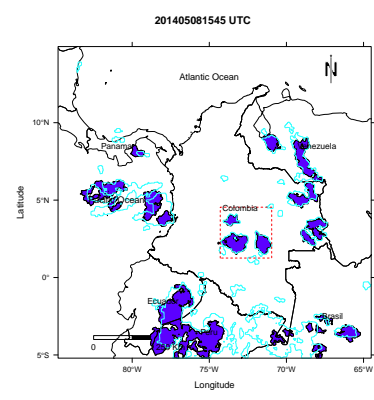

(a) 201405081545 UTC

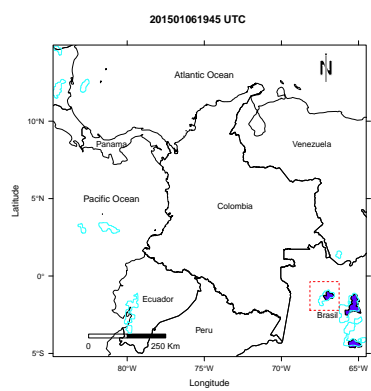

(c) 201501061945 UTC

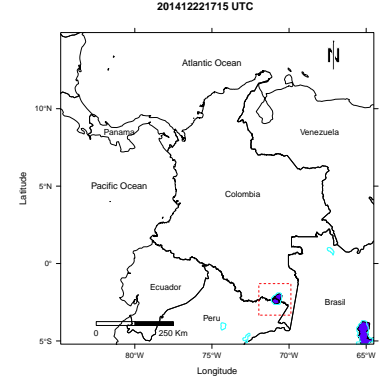

(b) 201412221715 UTC

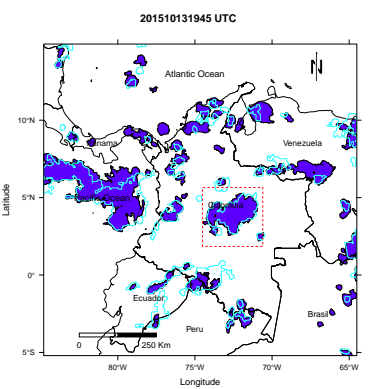

(d) 201510131945 UTC
Figure 4. Precipitating cloud objects detected at four dates. Blue colour fill represent DT classified precipitation objects, cyan colour border represent GPM reference precipitation objects and dashed red colour represent a window for analysis.

For the instant 201501061945 UTC (Figure 4b) of least rainy day of 2015 the overall STEP similarity was shape $98 \%$, theme $98 \%$, edge $100 \%$ and position $100 \%$, for this instant there are 3 precipitating objects which are located mainly on the Brazilian Amazon region, with areas between $2873 \mathrm{~km}^{2}$ and $7690 \mathrm{~km}^{2}$.

Figure 5 shows views of precipitating objects that correspond to the dashed windows in the Figure 4. In this Figure it is possible to observe that there is a strong locational match between predicted precipitation objects and reference prediction objects. However there are slight differences regarding shape and edge.

Results demonstrate how well a decision tree (DT) based classification model, inferred from a single-date GOES imagery, can be repeatedly used on different dates. This eventual transferability of empirical obtained models needs to be confirmed in more extensive experiments. However, these results suggest that machine learning techniques may be useful for better understanding meteorological phenomena as well as for improving manual methods currently used for cloud detection and tracking.

\section{CONCLUSIONS}

The main objectives of the study were accomplished: (i) evaluating the potential of using the classification model yielded by DT on many dates; and (ii) establishing that it is possible to have a classification model that can be used repeatedly for locating and tracking precipitating objects from multispectral meteorological images.

According to this study's results the best geometry aspect in the detection of precipitating objects from DT model is the position, that is, DT is able to locate accurately precipitating objects.

From the practical point of view, this work suggest a transferable decision classification model that can be used repeatedly for

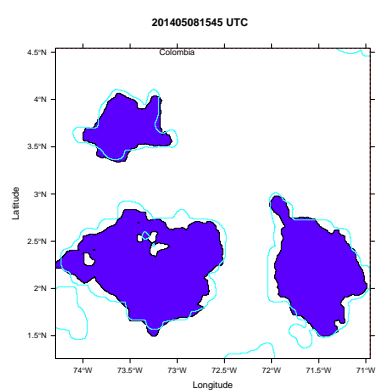

(a) 201405081545 UTC

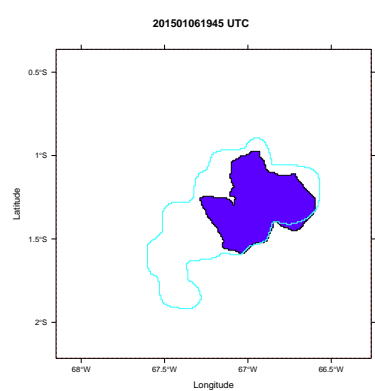

(c) 201501061945 UTC

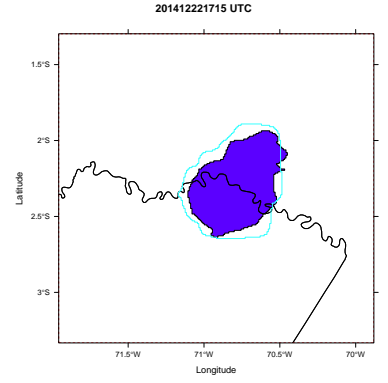

(b) 201412221715 UTC

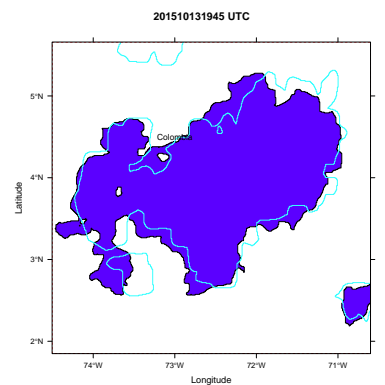

(d) 201510131945 UTC
Figure 5. Views of precipitating objects. Blue polygons represent DT classified precipitation objects and cyan edge polygons represent GPM reference precipitation objects.

locating and tracking precipitating objects from multi-temporal meteorological images, defined explicit classification rules useful for operational applications, with no visual interpretation of imagery for every cloudy day.

\section{ACKNOWLEDGEMENTS}

The authors are grateful to Instituto de Hidrología, Meteorología y Estudios Ambientales (IDEAM), the Colombian national weather service, for providing the GOES-13 meteorological images ModeA and the technical resources used in this study. The Global Precipitation Measurement (GPM) product GPM_3IMERGHH images was obtained from the National Aeronautics and Space Administration (NASA), available at http://giovanni.gsfc.nasa.gov/.

\section{REFERENCES}

Colombia Humanitaria, 2010. Reporte diario ola invernal 20102011.

Feidas, H. N., Cartalis, C. and Cracknell, A. P., 2000. Use of Meteosat imagery to define clouds linked with floods in Greece. International Journal of Remote Sensing 21(5), pp. 1047-1072.

Goyens, C., Lauwaet, D., Schröder, M., Demuzere, M. and Van Lipzig, N., 2012. Tracking mesoscale convective systems in the Sahel: relation between cloud parameters and precipitation. International Journal of Climatology 32(12), pp. 1921-1934.

Hou, A. Y., Kakar, R. K., Neeck, S., Azarbarzin, A. A., Kummerow, C. D., Kojima, M., Oki, R., Nakamura, K. and Iguchi, T., 2014. The global precipitation measurement mission. Bulletin of the American Meteorological Society 95(5), pp. 701-722.

Houze, R., 2004. Mesoscale convective systems. Reviews of Geophysics 42, pp. 1-43. 
Khalilia, M., Chakraborty, S. and Popescu, M., 2011. Predicting disease risks from highly imbalanced data using random forest. BMC medical informatics and decision making 11(1), pp. 51.

Liu, Y., Xi, D.-g., Li, Z.-1. and Shi, C.-x., 2014. Automatic Tracking and Characterization of Cumulonimbus Clouds from FY-2C Geostationary Meteorological Satellite Images. Advances in $\mathrm{Me}$ teorology.

Lizarazo, I., 2008. Clasificación de la cobertura y del uso del suelo urbano usando imágenes de satélite y algoritmos supervisados de inteligencia. UD y la GEOMÁTICA 0(2), pp. 4-18.

Lizarazo, I., 2014. Accuracy assessment of object-based image classification: another STEP. International Journal of Remote Sensing 35(16), pp. 6135-6156.

Ramírez, S. and Lizarazo, I., 2014. Digital classification of cloud masses from weather imagery using machine learning algorithms. Revista Facultad de Ingeniería Universidad de Antioquia (73), pp. 43-57.

Sakamoto, M., Ambrizzi, T. and Poveda, G., 2011. Moisture sources and life cycle of convective systems over Western Colombia. Advances in Meteorology 2011, pp. 1-11.

Tan, P., Steinbach, M. and Kumar, V., 2005. Classification: Basic Concepts, Decision Trees, and Model Evaluation. In: Introduction to Data Mining, 1st edn, Addison-Wesley, chapter Chapter 4, pp. 145-205.

Vila, D., Machado, L., Laurent, H. and Velasco, I., 2008. Forecast and Tracking the Evolution of Cloud Clusters (ForTraCC) Using Satellite Infrared Imagery: Methodology and Validation. Weather and Forecasting 23(2), pp. 233-245.

Article submitted on 10 July 2016 


\title{
ASSESSMENT OF POINT CLOUD ANALYSIS IN IMPROVING OBJECT-BASED AGRICULTURAL LAND COVER CLASSIFICATION USING DISCRETE LIDAR DATA IN CABADBARAN, AGUSAN DEL NORTE, PHILIPPINES
}

\author{
T. A. M. Rollan ${ }^{1}$, A. C. Blanco ${ }^{1,2}$ \\ ${ }^{1}$ Phil-LiDAR 2, UP Training Center for Applied Geodesy and Photogrammetry \\ ${ }^{2}$ Department of Geodetic Engineering, College of Engineering \\ University of the Philippines - Diliman, Quezon City, Philippines - therese.rollan@gmail.com, ariel.blanco@coe.upd.edu.ph
}

KEY WORDS: agriculture, land cover, LiDAR, point cloud analysis, object-based image analysis, OBIA, eCognition

\begin{abstract}
:
Cabadbaran City is the capital of Agusan del Norte which is located at the north eastern portion of Mindanao, Philippines. Almost $30 \%$ of its land area is devoted to agriculture (mainly rice, corn, coconut, banana, vegetables and abaca). Currently, the city government and agriculture office are implementing programs focusing on improving coconut and vegetable productivity, controlling banana disease and infestation, and enhancing abaca production industry. In support of decision making, the current situation must first be assessed by answering the basic questions what and where through detailed and accurate resource mapping. In this study, only discrete LiDAR datasets were utilized. Corresponding orthophotos were used only for training and validation. Land cover classification was done using two workflows using Support Vector Machines (SVM) as the classifier. In the first workflow, land cover classes were classified using rasterized point cloud metrics such as minimum, maximum, standard deviation, skewness, kurtosis, quartile average, mode and median. In the second workflow, point cloud analysis was used to derive additional features for classification which was integrated and executed in the same object-based software through Cognition Network Language $(\mathrm{CNL})$. The derivations of the additional features were conducted after running an initial segmentation which means that the distribution of points was analysed within the delineated objects. Classes that benefited to point cloud-based metrics are mostly non-ground agricultural classes namely coconut, mango and palm trees. These classes have obtained increase in accuracies by an average of $11.2 \%$ using validation sample set 1 and an average of $18.2 \%$ using validation sample set 2 . Ground classes, particularly barren land and rice, appeared to be incompatible to these point cloud metrics as shown by the decrease in accuracies for Methods 2 and 3 by about $18.1 \%$ using validation sample set 1 and about $16.4 \%$ using validation sample set 2 . Exploring other useful point cloud-based metrics and testing on sites with other land cover classes are highly recommended.
\end{abstract}

\section{INTRODUCTION}

Agriculture is vital in every country's food security. It supports the people's daily needs not only in terms of food but also in providing raw materials essential for the production of medicines, clothing, and many more. In the Philippines, the agricultural sector feeds more than a hundred million citizens (Philippine Statistics Authority, 2016) which most of the time is said to be unable to provide sufficient supply of goods particularly rice. Some of the reasons are the lack of allotted area for plantation, scarcity of water during the dry season that leads to lower yields and higher production cost caused by a number of typhoons that hit the country (Dy, 2015). Because of these, there is a need to import goods to fill up these gaps. A way to deal this is to monitor and plan the yields and the allotment of area for the sector such that the volume of yields will fit the intensity of demand. There should also be a way to monitor and regulate the conversion of land use from agricultural to residential or commercial. In order to do these, the sector must know the current status of the crop areas starting from the basic questions what and where. What crops are being cultivated and where are they situated? These can be answered by resource mapping. Resource maps are maps showing specific type/s of target land cover/s. A variety of methods can be conducted to produce a resource map. In this study, two methodologies will be presented using LiDAR technology and object-based image analysis (OBIA).

\section{RELATED LITERATURE}

\subsection{Point Cloud Analysis and Applications}

LiDAR (Light Detection and Ranging) is a form of a remote sensing system of acquiring data by radiating a pulsed laser to obtain measurements to the earth (National Oceanic and Atmospheric Administration, 2015). At a given target, distance from the platform can be calculated knowing the speed of the laser pulse and the time it takes for the pulse to return to the sensor (LiDAR-UK). A GPS (Global Positioning System) and Inertial Measurement Unit provide position and orientation information to the system respectively (Bao, et al., 2008). The output point cloud data consist of 3D coordinates $\mathrm{x}, \mathrm{y}$ and $\mathrm{z}$ and Intensity i. Based on these four attributes, various rasters can be derived such as Intensity, Number of Returns, and Digital Elevation Model (DEM) images (Carranza, et al., 2014). Moreover, from these images, we can further derive height textures and metrics. In the study conducted by Qiao and Zhang (2009), height and intensity textures were used to classify a LiDAR dataset into five classes: cropland, bare land, water body, man-made constructions, and sparse tree land. These textures include mean, entropy, variance, second moment and homogeneity that served as criteria for the Artificial Neural Network (ANN) classifier. Aside from land cover mapping, LiDAR point cloud can also be used for environmental management. One example is how Peterson et al. (2005) predicted canopy bulk density (CBD) and canopy base height $(\mathrm{CBH})$ for a portion of the Sierra National Forest, Nevada. Moreover, they also used LiDAR data for canopy fuel mapping 
which served as input for a fire behaviour model (FARSITE). Kandrot (2013) tackled a new approach and its advantages on coastal monitoring particularly the dynamics of sand dunes morphology. More uses of point cloud analysis include urban (Harrap \& Lato, 2006), disaster mitigation (Disaster Risk and Exposure Assessment for Mitigation (DREAM) Program, 2016), and medical applications (Schultz \& Kindlmann, 2013).

\subsection{Support Vector Machines (SVM)}

Support Vector Machines (SVM) is a type of supervised classification in which a hypothesis space of linear functions in a high dimensional feature space (Cristianini \& Shawe-Taylor, 2000). According to Cristianini and Shawe-Taylor (2000), SVM algorithm finds a way on obtaining efficient separating hyperplanes such that the generalization bounds are optimized.

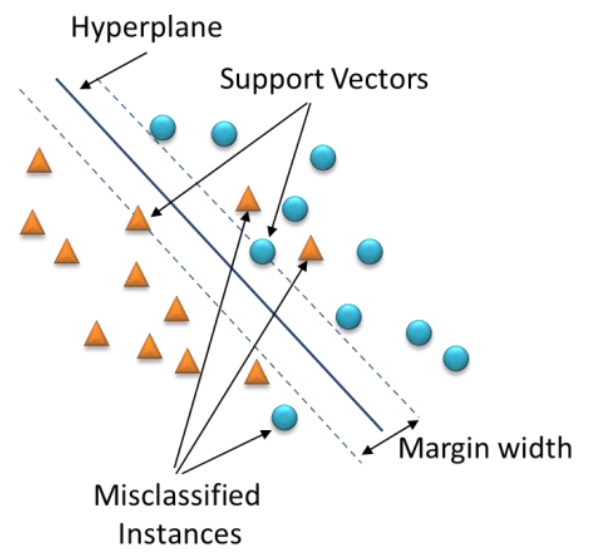

Figure 1. Simple Illustration of How SVM works as adapted from Burges (1998)

Despite its promising and very strong theoretical background, it remained unnoticed in its early publications (Kecman, 2005). The user may tweak on its parameters upon using it to certain application. These include $\mathrm{C}$ or the penalty factor (Alpaydin, 2014), Epsilon or the value that indicates the percentage or ratio of the support vectors to the number of samples (Mattera \& Haykin, 1999), Gamma which defines the extent of influence of a training sample (scikit-learn.org) and Kernel functions which are used to project non-linearly separable samples into linear separable feature space (Hofmann, 2006). There are four types of kernel functions namely Linear, Polynomial, Radial Basis Function (RBF) and Sigmoid (McCue, 2009). Kecman mentioned in his book section that it is only when practical applications yielded excellent results that SVM became known and accepted. These applications include digit recognition, computer vision and text categorization. For land cover classification, Zhang, Lin and Ning (2013) utilized SVM for airborne LiDAR point clouds in urban areas. Roli and Fumera (2001) introduced and applied SVM with varying parameters to multisensory images and compared its performance to Multilayer Perceptrons Neural Network and k-NN classifiers. Their output classes include sugar beets, stubble, bare soil, potatoes and carrots. A data fusion was done by Waske and Benediktsson (2007) using multitemporal synthetic aperture data and optical imagery. Their proposed SVM-based fusion methodology had significantly increased the accuracy results of a single run of SVM consisting classes namely arable crops, cereals, canola, root crops, grassland, orchard, forest, and urban areas.

\section{MATERIALS AND METHODOLOGY}

\subsection{Study Area}

Agusan del Norte is situated at the south-eastern part of the Philippines. The province's major crops include rice, corn, coconut, abaca, banana and mango (Provincial Agricultural Office of Agusan del Norte). A portion of the municipality of Cabadbaran approximately located at $9^{\circ} 06^{\prime} \mathrm{N}, 125^{\circ} 34^{\prime} \mathrm{E}$ was selected as the study site which covers an area of about 6 sq. $\mathrm{km}$. It is comprised of land covers built up areas, water body, grassland, bare land, rice, corn, non-agricultural trees, coconut, mango, banana and oil palm trees. A banana plantation is located at the leftmost portion while mango plantations are distributed within the study area. Coconut and oil palm trees are often located in the same area. Corn parcels contain sparse plants but can still be identified.
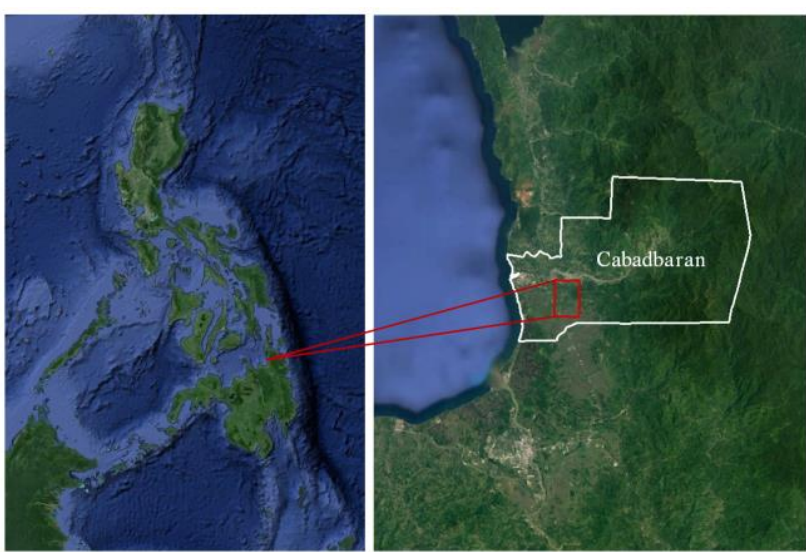

Figure 2. Study Area (Google, 2012)

\subsection{Datasets}

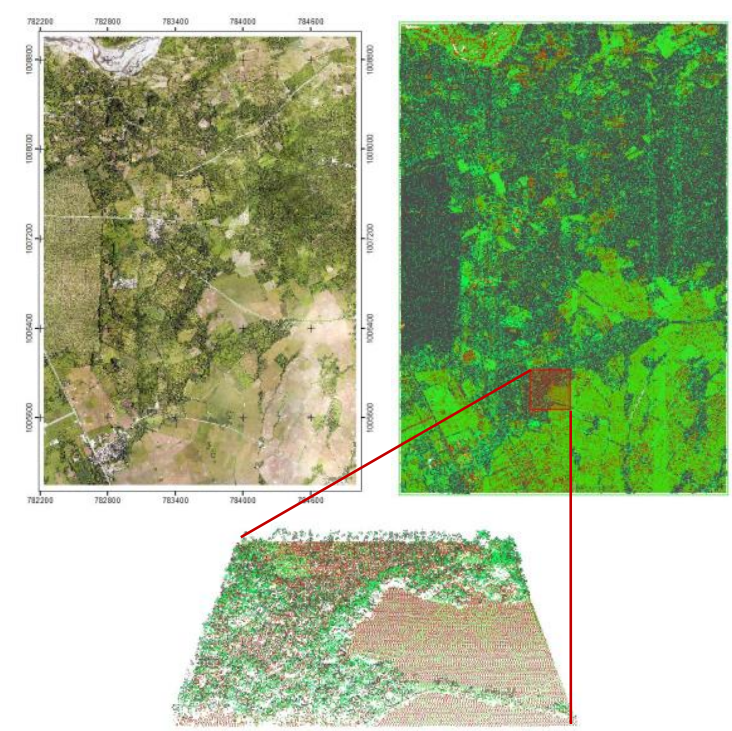

Figure 3. Datasets: Orthophoto (left), Point Cloud (right) and Point Cloud Perspective View (bottom)

The data used include an orthophoto with $0.5 \times 0.5 \mathrm{~m}$ resolution and a pre-classified LiDAR point cloud (.las) with 2 points per sq. km. resolution. The orthophoto contains three bands: Red, Green and Blue. The point cloud was pre-classified into general classes: ground, vegetation (low, mid and high), and buildings. 


\subsection{Methodologies}

Three classification methodologies were applied and corresponding results were compared. The first method utilized raster-based derivatives (see Figure 4), the second used point cloud-based derivative layers, and the third is a combination of the two sets of derivatives. Orthophotos are available but were only utilized for the selection of training and validation points as this research is on the classification of agricultural cover using LiDAR data only.
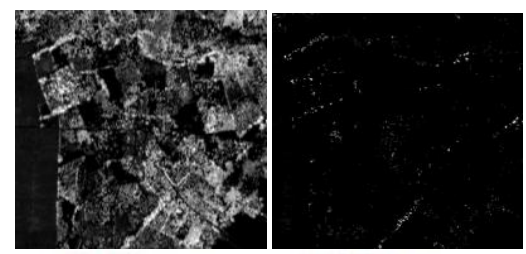

Height Average Value

High : 31.6

Low: 0

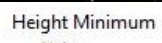

Value

High : 20.96

ow : 0

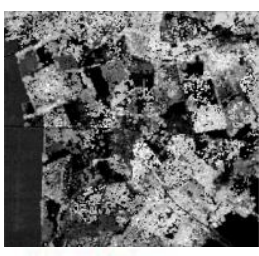

Height Maximum

Value

High: 37.8

ow: 0
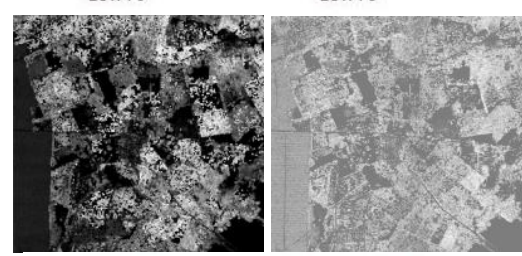

Height Std. Dev.

Number of Returns

Value

High: 15.74

Low : 0

High : 4

Low: 1
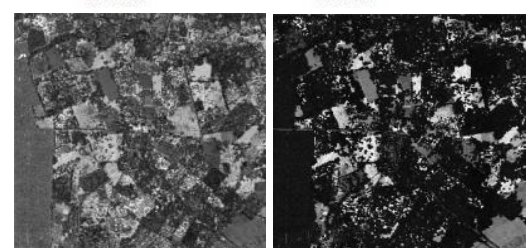

Intensity Average

Intensity Minimum

Value

Value
High: 179

High : 17

Low: 3

Low: 0

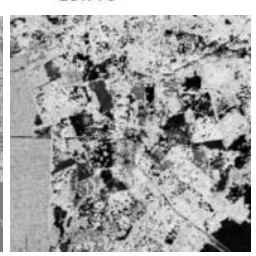

Slope 2x Exag

Value

High: 89.0411

Low:0

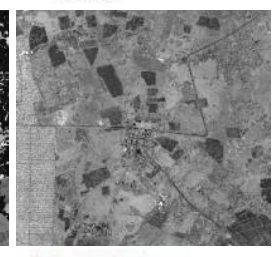

Intensity Maximum

Value

High : 255

Low : 3
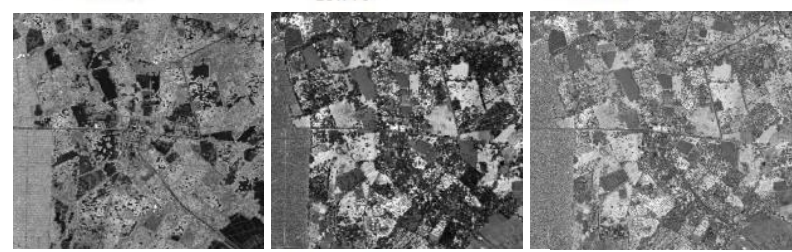

Intensity Std. Dev.

Intensity Median

High: 86

,

Low: 0
Intensity Highest 1st Returr Value

How

Low: 1

Figure 4. Examples of LiDAR Derivatives used

Figure 5 illustrates the classification workflow for method 1 in which height and intensity metrics were calculated per specified pixel size. A relatively large pixel size of 5 x 5 meters was used to be able to feed enough number of points for the computation of the metrics (Jerez, et al., 2015) using Lastools. LiDAR derivatives include normalized Digital Surface Model (nDSM) or the height image, intensity, slope, number of returns, height metrics (average, minimum, maximum, standard deviation, mode and median or the $50^{\text {th }}$ percentile), and intensity metrics

(average, minimum, maximum, first returns maximum, last returns minimum, standard deviation, mode and median) (see Figure 4). These derivatives were then imported in eCognition where initial segmentation was done using nDSM to separate non-ground from ground features. Non-ground and ground objects were further segmented using nDSM and intensity respectively until meaningful objects were obtained. Meaningful objects indicates no multiple type of feature are in the same object. Moreover, minimal over-segmentation (i.e., single type of feature were broken into smaller pieces) was committed.

Training points were selected using the orthophoto. Separate SVM classifications for the two general classes were conducted in order to avoid confusion between low and high vegetation (Jerez, et al., 2015). The parameters that were used include $1 /$ (number of dimensions or the derivatives used) for the Gamma parameter which is the default value, 200 for the C parameter, and RBF kernel function (Carranza, et al. 2014). Output final classes include water body, grassland, bare land, rice and corn for ground and built up areas, non-agricultural trees, coconut, mango, banana and oil palm trees for nonground.

Validation samples for the accuracy assessment were then selected such that they do not overlap with the training samples and the number of samples follows the ratio $70 \%$ training - 30\% validation (Jerez, et al., 2015). From the same reference, individual accuracies especially for the agricultural classes were examined and necessary improvement was done in order to meet the minimum required accuracy of $85 \%$ set for classification using LiDAR dataset only (no orthophoto).

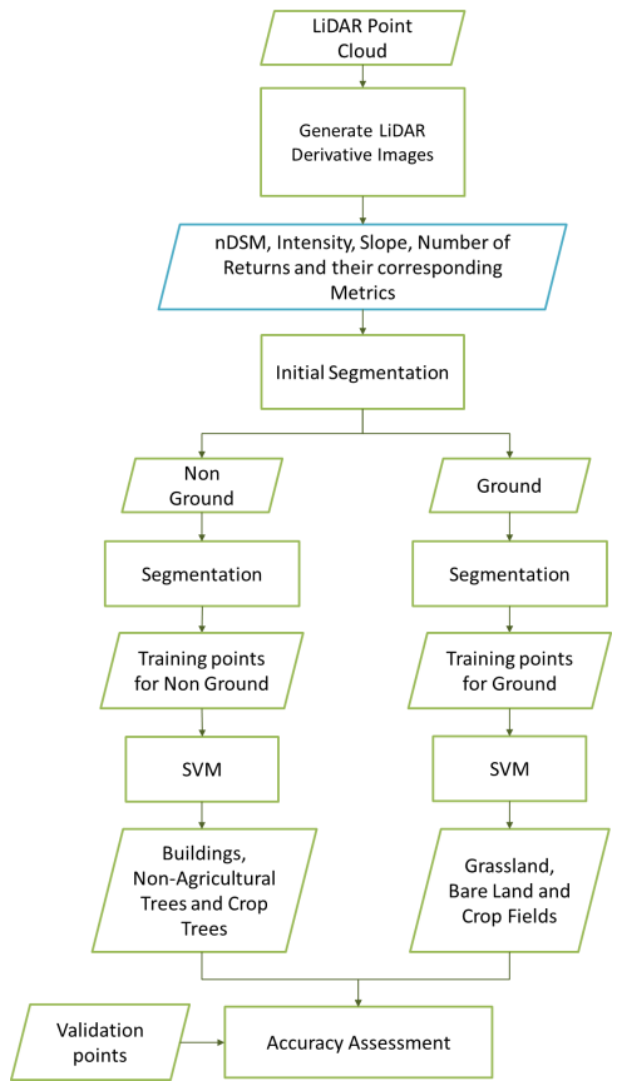

Figure 5. Method 1: Using Raster-Based Metrics for Land Cover Classification 
In the second methodology, height and intensity metrics were computed directly from the point clouds per object. Figure 6 shows the workflow in which deriving the metrics (in blue box) were not done prior to the whole classification procedure. The same set of ground and non-ground objects were used in the first two methodologies to ensure that the differences in the output will only be caused by the difference in the computation of the metrics. In the third methodology, derivatives from Methods 1 and 2 were combined.

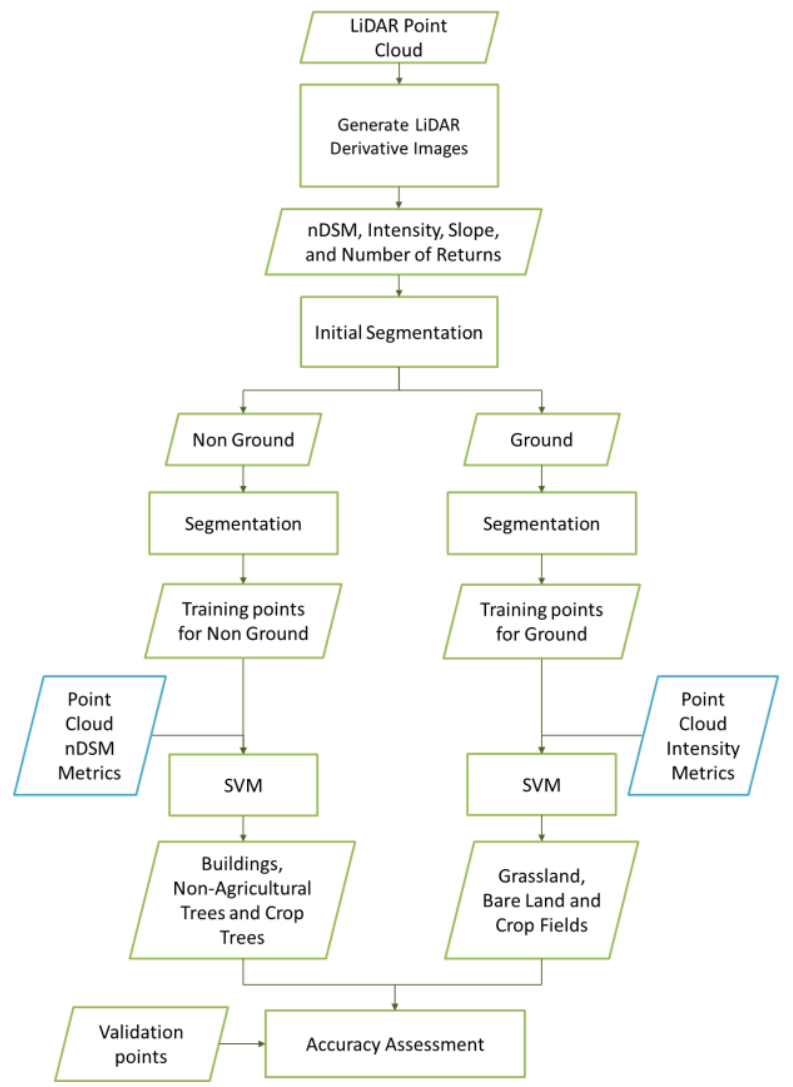

Figure 6. Method 2: Using Point Cloud-Based Metrics for Land Cover Classification

Lastly, the output accuracies from the three methodologies were compared per class. Two sets of validation samples were obtained in order to check the consistency of the results.

\section{RESULTS AND DISCUSSION}

Final classes include barren, corn, grassland, rice, banana, coconut, palm, mango, non-agricultural trees and buildings. The classification results for the three methodologies were visually examined. To quantify the differences among results, accuracy assessment was conducted using two sets of validation samples. Trends of the accuracy values were also observed per class from method 1 to 3 . Figure 7 shows the classification outputs from the 3 methodologies. Some portions of the classified area are presented in Figure 8.

By visual inspection, differences are not too apparent except for rice and barren. Methodologies 2 and 3 have similar classification outputs as compared to that of Methodology 1 . The banana plantation located at the left side was captured by the three methodologies and differs at some parts only. At the lower right of the classified images, the differences are most apparent where barren and rice parcels are mostly located. Method 1 had detected the least area of rice parcels and, consequently, the largest barren areas.

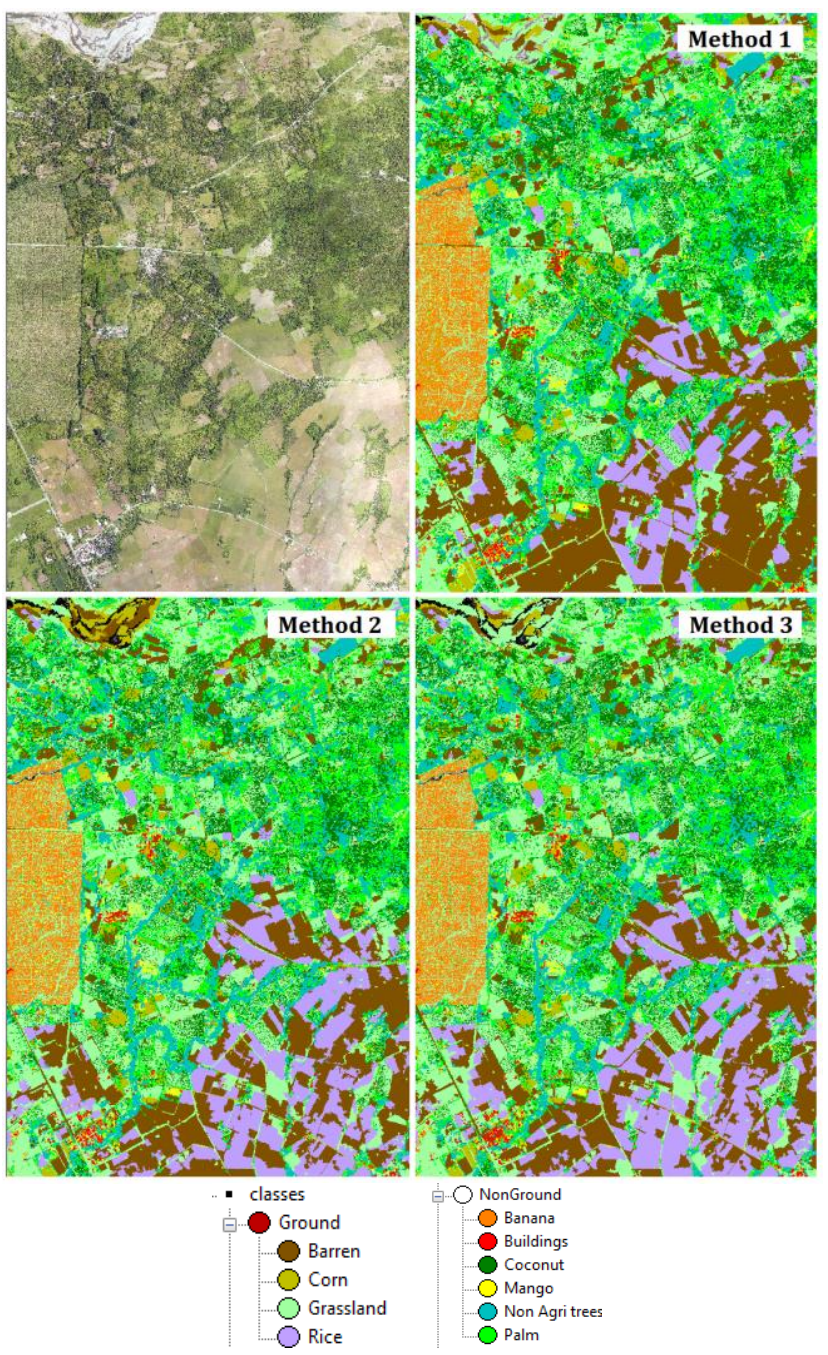

Figure 7. Orthophoto (upper left) and Classification Results for Methods 1, 2 and 3

The top three highest accuracies for validations sample set 1 are from corn (1.000 from all methods), banana (1.000 from all methods), and grassland (1.000 from Methods 1 and 3) while for validation sample set 2 are grassland, corn, coconut, banana, and buildings ( 1.000 from all methods). On the other hand, the top 3 lowest accuracies from validation sample set 1 are from mango (0.588 from Method), barren (0.661 from Method 2), and rice (0.705 from Method). For validation sample set 2, top 3 lowest accuracies are from rice ( 0.451 from Method 2), barren (0.540 from Method 2), and mango (0.651 from Method 1). Method 1 yielded the highest overall accuracy of 0.944 and Method 2 yielded the lowest overall accuracy of 0.771 . 

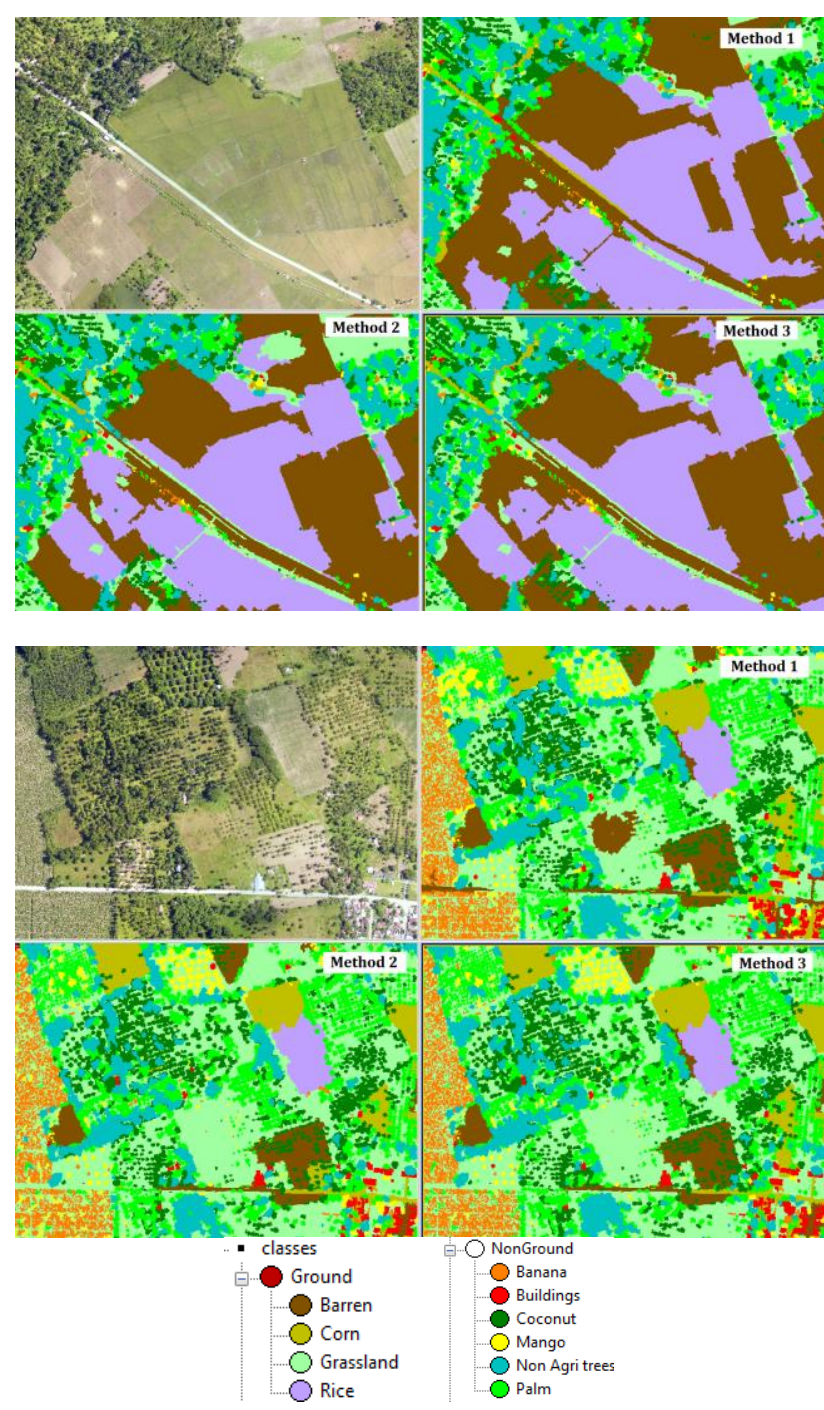

Figure 8. Portion of the Classification Result for Methods 1, 2 and 3

Observing the trend of accuracies for validation samples set 1 from Method 1(raster-based) to 2(point cloud-based) (Table 1 and Figure 9), accuracies of three (Coconut, Mango and Palm trees) out of ten classes have increased values, four (Barren, Grassland, Rice and Non-Agricultural trees) classes and the Overall Accuracy obtained lower values and the remaining (Corn, Banana and Buildings) have retained their accuracy level. On the other hand, observing the accuracies using validation sample set 2 from Method 1 to 2 (Table 2 and Figure 10), two classes (Mango and Palm Trees) obtained increase in accuracy values, three classes (Barren, Rice and NonAgricultural trees) and the Overall Accuracy have obtained decrease in accuracies, and five (Grassland, Corn, Coconut, Banana and Buildings) have retained their accuracy level.

Comparing the results of processing the two sets of derivatives (Method 1: raster- and Method 2: point cloud-based) separately to the combined derivatives (Method 3) using validation set 1 (Table 1 and Figure 9), the classification of the following classes improved with Method 3: Grassland, Mango, Palm and Non-Agricultural trees. However, Barren land, Rice, Corn, Coconut, Banana, Building and the Overall Accuracy did not obtain any increase in accuracy. For validation sample set 2
(Table 2 and Figure 10), accuracy improvement was observed for the Barren land, Rice, Mango, Non-Agricultural trees classes, as well as in the Overall Accuracy. For the remaining classes namely Grassland, Corn, Coconut, Banana, Palm, and Buildings, no increase in accuracies were obtained.

Table 1. Accuracy Results of Validation Sample Set 1

\begin{tabular}{|l|c|c|c|}
\hline \multirow{4}{*}{ Class } & \multicolumn{3}{c|}{ Method } \\
\cline { 2 - 4 } & $\mathbf{1}$ & $\mathbf{2}$ & $\mathbf{3}$ \\
\hline Barren land & 0.872 & 0.661 & 0.620 \\
\hline Grassland & 1.000 & 0.985 & 1.000 \\
\hline Rice & 0.935 & 0.785 & 0.705 \\
\hline Corn & 1.000 & 1.000 & 1.000 \\
\hline Coconut & 0.976 & 0.979 & 0.930 \\
\hline Banana & 1.000 & 1.000 & 1.000 \\
\hline Mango & 0.588 & 0.831 & 0.957 \\
\hline Palm & 0.774 & 0.864 & 0.812 \\
\hline Non-Agricultural Trees & 1.000 & 0.966 & 0.981 \\
\hline Buildings & 0.851 & 0.851 & 0.851 \\
\hline Overall Accuracy & 0.944 & 0.854 & 0.829 \\
\hline
\end{tabular}

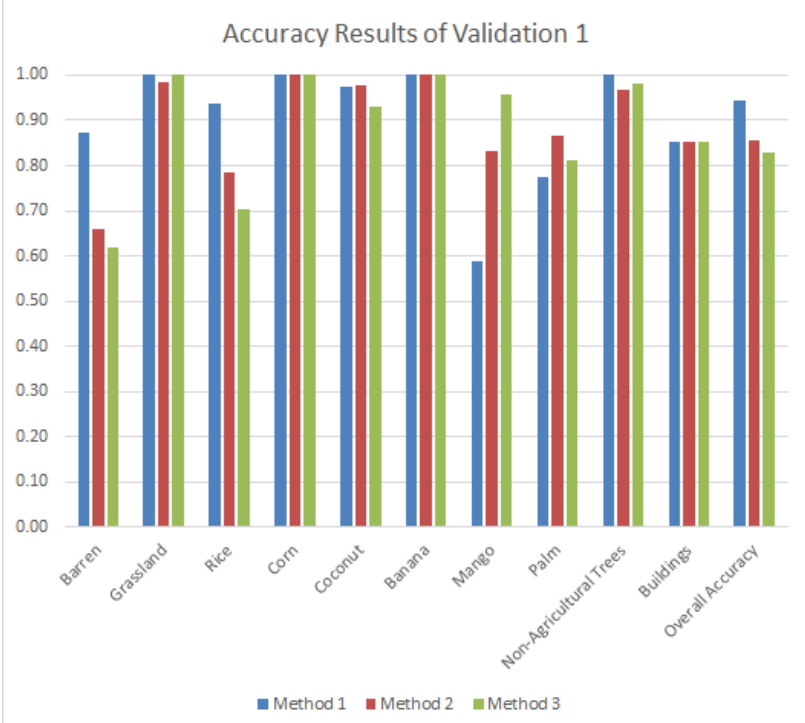

Figure 9. Accuracy Results using Validation Sample Set 1

Table 2. Accuracy Results using Validation Sample Set 2

\begin{tabular}{|l|c|c|c|}
\hline \multirow{4}{*}{ Class } & \multicolumn{3}{c|}{ Method } \\
\cline { 2 - 4 } & $\mathbf{1}$ & $\mathbf{2}$ & $\mathbf{3}$ \\
\hline Barren land & 0.590 & 0.540 & 0.571 \\
\hline Grassland & 1.000 & 1.000 & 1.000 \\
\hline Rice & 0.728 & 0.451 & 0.583 \\
\hline Corn & 1.000 & 1.000 & 1.000 \\
\hline Coconut & 1.000 & 1.000 & 1.000 \\
\hline Banana & 1.000 & 1.000 & 1.000 \\
\hline Mango & 0.651 & 0.941 & 0.891 \\
\hline Palm & 0.877 & 0.951 & 0.865 \\
\hline Non-Agricultural Trees & 1.000 & 0.953 & 0.968 \\
\hline Buildings & 1.000 & 1.000 & 1.000 \\
\hline Overall Accuracy & 0.810 & 0.771 & 0.791 \\
\hline
\end{tabular}




\section{REFERENCES}

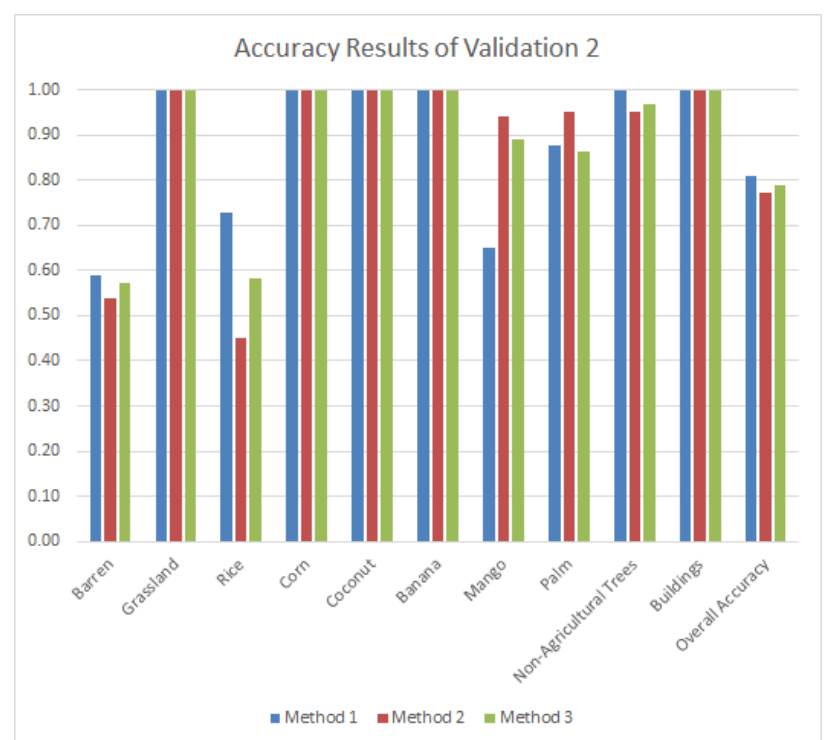

Figure 10. Accuracy Results using Validation Samples Set 2

\section{CONCLUSION AND RECOMMENDATIONS}

Majority of the classes with improved accuracies after applying point cloud-based derivatives belong to Non-Ground classes, specifically, coconut, mango and palm trees. The use of the derivatives on Ground classes in this dataset did not yield satisfactory results. Since there are more classes with decreased accuracy values upon utilizing point cloud-based metrics, the overall accuracy had also shown a decreasing trend in general. Although Method 2 and 3 did not perform well on all classes, we can still utilize them for Non-Ground classes for higher classification accuracy.

For further research, other study sites with varying classes and distribution are highly advisable in order to test the methodology and see if other factors affect the resulting accuracies. Also, try using tree-dominated areas and classify them accordingly. Moreover, other metrics can be computed to determine which point cloud-based derivatives are much more useful in improving the classification results.

\section{ACKNOWLEDGEMENTS}

This study is made possible by the Phil-LiDAR 2 Project funded by the Department of Science and Technology (DOST) and monitored by the Philippine Council for Industry, Energy and Emerging Technology Research and Development (PCIEERD). The Project is also supported by the Department of Agriculture through the Information Technology Center for Agriculture and Fisheries (ITCAF).

The methodologies were developed using the aerial and point cloud data from DREAM/Phil-LiDAR 1 Program.
Alpaydin, E. (2014). Introduction to Machine Learning (Third Edition). Massachusetts: The Massachusetts Institute of Technology Press.

Application, U. o. (2005). Peterson, Birgit; Dubayah, Ralph; Hyde, Peter; Hofton, Michelle; Blair, J. Bryan; Fites-Kaufman, JoAnn. Proceedings of the Seventh Annual Forest Inventory and Analysis Symposium (pp. 193-200). Portland: United States Department of Agriculture, Forest Service, 2007.

Bao, Y., Li, G., Cao, C., Li, X., Zhang, H., He, Q., et al. (2008). Classification of lidar point cloud and generation of $\mathrm{dtm}$ from lidar height and intensity data in forested area. International Archives of the Photogrammetry, Remote Sensing and Spatial Information Sciences, 37 (Part 3B), 313-318.

Burges, C. J. (1998). A Tutorial on Support Vector Machines for Pattern Recognition. Data Mining and Knowledge Discovery 2, 121-167.

Carranza, C., Rollan, T., Tañada, E., Guerrero, J., Jerez, M., \& Blanco, A. (2014). Phil-LIDAR 2 OBIA Training Session Exercises Manual. Nationwide Detailed Resource Assessment using LIDAR (Phil-LIDAR 2) Program Project 1 Agricultural Resources Extraction from LIDAR Surveys (PARMAP). Quezon City, Metro Manila, Philippines.

Cristianini, N., \& Shawe-Taylor, J. (2000). Introduction to Support Vector Machines and Other Kernel-Based Learning Methods. Cambridge: The Press Syndicate of the University of Cambridge.

Disaster Risk and Exposure Assessment for Mitigation (DREAM) Program. (2016). The UP DREAM Program. Retrieved June 29, 2016, from Disaster Risk and Exposure Assessment for Mitigation (DREAM) Program: https://dream.upd.edu.ph/about/

Dy, R. T. (2015, April 20). Why we have to import rice. Retrieved June 23, 2016, from Business World Online: http://www.bworldonline.com/content.php?section=Opinion\&ti tle=why-we-have-to-import-rice $\&$ id $=106455$

Google. (2012, October 16). Cabadbaran, Agusan del Norte, Philippines $9^{\circ} 06^{\prime} 03^{\prime \prime} \mathrm{N}, 1^{\circ} 5^{\circ} 32^{\prime} 26^{\prime \prime} \mathrm{E}$, Eye Alt $23683 \mathrm{ft}$. Retrieved June 22, 2016, from Google Earth Pro v7.1.2.2041: http://www.google.com/earth/index.html

Harrap, R., \& Lato, M. (2006). An Overview of LIDAR for Urban Applications. Kingston, Ontario, Canada.

Hofmann, M. (2006, June 26). Support Vector Machines Kernels and the Kernel Trick. Bamberg, Germany.

Jerez, M. V., Carranza, C. U., Gatdula, N. B., Rollan, T. M., Tañada, E. M., Pagkalinawan, H. M., et al. (2015, December 31). Phil-LiDAR 2 Project 1: Agricultural Resources Extraction from LiDAR Surveys (PARMap) Technical Narrative Report. Quezon City, Metro Manila, Philippines.

Kandrot, S. M. (2013). Coastal Monitoring: A New Approach. Chimera 26: Geographical Journal, University College Cork, 75-89. 
Kecman, V. (2005). Support Vector Machines - An Introduction. In L. Wang, Support Vector Machines: Theory and Applications (pp. 1-47). Heidelberg: Springer-Verlag Berlin Heidelberg.

LiDAR-UK. (n.d.). LiDAR-UK. Retrieved June 2, 2015, from The uses of LiDAR: What applications are there for LiDAR systems?: http://www.lidar-uk.com/usage-of-lidar/

Mattera, D., \& Haykin, S. (1999). Support vector machines for dynamic reconstruction of a chaotic system. In B. Schölkopf, C. J. Burges, \& A. J. Smola, Advances in Kernel Methods: Support Vector Learning (pp. 211-241). Massachusetts: The Massachusetts Institute of Technology Press.

McCue, R. (2009, November 29). A Comparison of the Accuracy of Support Vector Machine and Naive Bayes Algorithms in Spam Classification. Santa Cruz, California, United States of America. Retrieved June 30, 2015, from University of California Santa Cruz: https://classes.soe.ucsc.edu/cmps242/Fall09/proj/RitaMcCueRe port.pdf

National Oceanic and Atmospheric Administration. (2015, May 29). National Oceanic and Atmospheric Administration. Retrieved June 1, 2016, from What is LiDAR?: http://oceanservice.noaa.gov/facts/lidar.html

Philippine Statistics Authority. (2016, May 19). Highlights of the Philippine Population 2015 Census of Population. Retrieved June 23, 2016, from Philippine Statistics Authority: https://www.psa.gov.ph/content/highlights-philippinepopulation-2015-census-population

Provincial Agricultural Office of Agusan del Norte. (n.d.). Agricultural Crops. Retrieved June 20, 2016, from Official Website of Agusan del Norte: http://www.agusandelnorte.gov.ph/index.php/socio-ecoprofile/economic-profile/agricultural-crops

Qiao, J.-G., \& Zhang, Y.-H. (2009). Texture-Based LiDAR Gray Image Segmentation using Artificial Neural Network. Guangzhou, China.

Roli, F., \& Fumera, G. (2001). Support vector machines for remote-sensing. Proceedings of the International Society for Optics and Photonics, Vol. 4170, 160-166.

Schultz, T., \& Kindlmann, G. L. (2013). Open-Box Spectral Clustering: Applications to Medical Image Analysis. IEEE Computer Society.

scikit-learn.org. (n.d.). RBF SVM parameters. Retrieved 2014, from scikit-learn.org: http://scikitlearn.org/stable/auto_examples/svm/plot_rbf_parameters.html

Waske, B., \& Benediktsson, J. A. (2007). Fusion of Support Vector Machines for Classification of Multisensor Data. IEEE Transactions on GeoScience and Remote Sensing, Volume 45, 3858-3866.

Zhang, J., Lin, X., \& Ning, X. (2013). SVM-Based Classification of Segmented Airborne LiDAR Point Clouds in Urban Areas. Remote Sensing (ISSN 2072-4292): Advances in Mobile Laser Scanning and Mobile Mapping, 3749-3775. 


\title{
SEMANTIC SEGMENTATION OF SETTLEMENT PATTERNS IN GRAY-SCALE MAP IMAGES USING RF AND CRF WITHIN AN HPC ENVIRONMENT
}

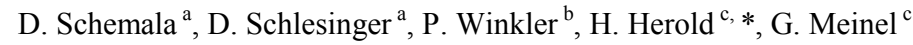 \\ ${ }^{\text {a }}$ Chair of Computer Vision, Institute of Artificial Intelligence, TU Dresden, Germany - \\ dmytro.shlezinger@tu-dresden.de \\ ${ }^{\mathrm{b}} \mathrm{ZIH}$ - Center for Information Services and High Performance Computing TU Dresden, Germany - \\ peter.winkler1@tu-dresden.de \\ ${ }^{c}$ Leibniz Institute of Ecological Urban and Regional Development, Dresden, Germany - \\ h.herold@ioer.de
}

KEY WORDS: Segmentation, Maps, Machine Learning, Random Forest, Conditional Random Field, High Performance Computing

\begin{abstract}
:
The segmentation of complex and compound geographical objects such as settlement areas in images is a challenging task. This is particularly true if the images are scanned historical maps, which are only available as gray-scale images. On the other hand, their vast availability and spatio-temporal coverage make these maps a valuable source of information for historical land use and land cover (LULC) research and urban geography. To facilitate the laborious information extraction from these maps, we present a twostage machine learning-based approach for segmenting settlement and non-settlement areas in the map scans. We employ a Conditional Random Field (CRF) which obtains its unary potentials from a Random Forest (RF). The method is tested using two inference algorithms. To evaluate the performance and the scalability of the approach over large amounts of data sets, we conduct parallel computing experiments within a High Performance Computing (HPC) environment at the Center for Information Services and High Performance Computing at TU Dresden. Experimental results indicate the suitability of both the methodological approach as well as its parallel implementation.
\end{abstract}

\section{INTRODUCTION}

Semantic segmentation of complex geographical objects such as settlements in historical map images is a challenging task. However, the historical information is of great interest, e.g. for land use and land cover change (LULCC) research, urban geography or archaeology. In order to facilitate the laborious manual information extraction from historical maps, research on automated map interpretation and cartographic pattern recognition has a long-standing tradition (cf. Herold et al., 2013). While existing approaches to map interpretation are mostly based on extraction of single object types from colour map images, here we follow a different approach. We develop and present a methodology to segment settlement areas as a whole from gray-scale map scans using only a small set of training samples. We evaluated the methodology on a specific map type (in German so-called Messtischblaetter) of large spatial and temporal coverage, which makes them particularly valuable for land change research and historical geoinformation systems (HGIS).

\section{METHODOLOGY}

The supervised segmentation approach comprises two stages combining a pixel-wise classification and a graphical model, which are described in the following section. Subsequently, in section 2.2 an approach and the technical environment to scale the method to large amounts of input images is described.

\subsection{Semantic Segmentation}

For pixel-wise segmentation we use a Random Forest (Breiman, 2001). A Random Forest consists of a number of Decision Trees (DT) which, when applied to a pixel, yield the estimated probability that this pixel belongs to settlement area. The outcome of all Decision Trees is added up and normalized to obtain the total probability estimation of the RF. A DT makes its decisions based on the gray levels of the input pixel and of its surrounding pixels. Every test function checks whether or not the difference between two neighbouring pixels is smaller than a threshold. The offsets of these pixels and the threshold are learned during the training phase. Every DT in the Forest is trained individually using an equal number of settlement and non-settlement pixels which are sampled from the training images. The depth of a DT is restricted to a maximum depth to reduce overfitting. We used the information gain as splitting criterion. Since we know a-priori that settlements usually cover largish, connected areas in the image, we smooth the outcome of the RF using a CRF (Lafferty et al., 2001).

The unary potentials for each output variable are simply the probability assigned to by the RF. The pairwise potentials between neighbouring output variables are determined by a constant function which yields a cost e for adjacent variables with different labelling and zero otherwise. A good value of the constant $e$ as well as the various parameters for building the RF are learned using cross validation. As inference algorithm we used the Min-Cut/Max-Flow implementation in (Boykov et al., 2004) for Maximum A-Posteriori Inference. We also applied Maximum-Marginal Inference using Gibbs Sampling.

\footnotetext{
* Corresponding author
} 


\subsection{Parallelization}

To evaluate the scalability of the approach for the large and ever increasing amount of available map scans, we perform parallelization experiments on the high performance computing (HPC) cluster TAURUS cluster located at the Center for Information Services and High Performance Computing at TU Dresden. The Taurus architecture has 7,184 CPU cores and a peak performance of 137 TFLOPS.

As the input data comprise a large number of individual images, the appropriate way of parallelization is to perform several hundred independent parallel segmentation jobs.

\section{RESULTS}

For the evaluation of both the segmentation performance as well as the scalability various experiments have been conducted. Section 3.1 describes the experimental setup and Section 3.2 and 3.3 the results for both the segmentation quality and the parallelization performance.

\subsection{Experiments}

We divided the data set into training data, test data and validation data. The following experiments have been conducted: We measured the influence of the parameters $t$ (number of trees in the RF), $d$ (the maximum depth of a tree), $w$ (width of the window around each pixel), $p$ (number of samples while building a decision function), $e$ and $N$ (the number of steps when doing Gibbs Sampling) on the segmentation performance. Then, we measured the performance when using different amounts of training data. Furthermore, we tested the generalization of the approach, since the used maps come in several types, each with a slightly different mark-up of settlement area and other elements. We measured the outcome when using test data of the same type as the training data and of a different type. The performance measures used was Precision and Recall, combined into the F-measure.

\subsection{Segmentation}

Figure 1 depicts how the F-measure depends on the number of training pixels (cf. Schemala 2016). For the same map image type, the number of necessary training samples is low. Figure 2 shows the F-measure against the pairwise cost $e$ (cf. Schemala 2016). Thus, $e$ is a very crucial parameter to tune.

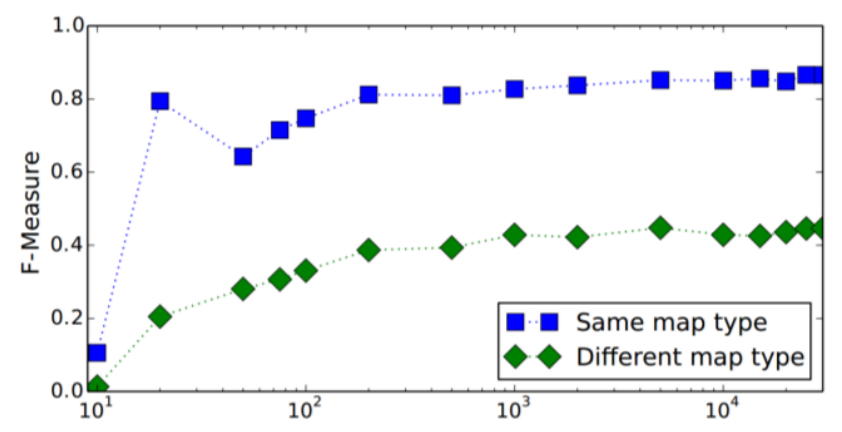

Figure 1. The F-measure plotted against the number of pixels used for the training.

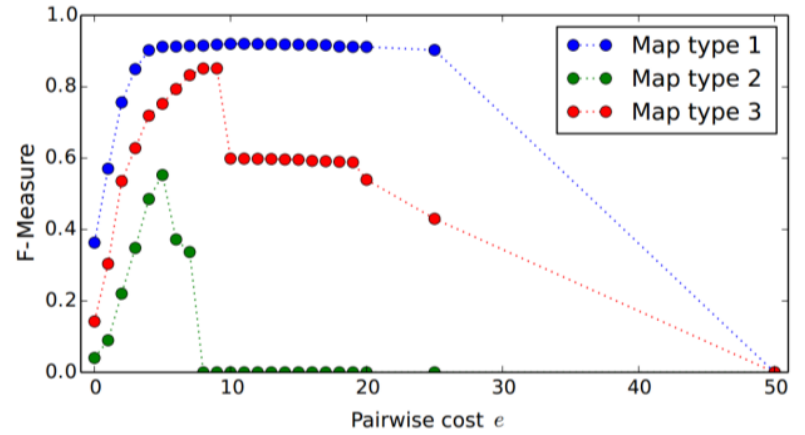

Figure 2. F-measure plotted against the pairwise cost $e$

\subsection{Parallelization}

Segmentation of 391 maps with a total storage volume of 45 GB has been performed on the TAURUS cluster in 245 seconds (approximately 4 minutes) using 391 cores with $10 \mathrm{~GB}$ main memory each. Such a rapid segmentation process facilitates a thorough validation procedure and extensive parameter testing concerning the intrinsic model parameters of the RF and CRF.

\section{ACKNOWLEDGEMENTS}

Parts of this research are funded by the Federal Ministry of Education and Research (BMBF) under grant number: 01IS14014A, Project: Competence Center for Scalable Data Services and Solutions (ScaDS). The authors greatly appreciate this funding. The authors also thank the Saxon State and University Library Dresden (SLUB) and its Virtual Map Forum for providing the georeferenced map images.

\section{REFERENCES}

Breiman, L., 2001. Random forests. Machine learning, 45(1), pp. 5-32.

Boykov, Y., Kolmogorov, V., 2004. An experimental comparison of min-cut/max-flow algorithms for energy minimization in vision. Pattern Analysis and Machine Intelligence, IEEE Transactions on, 26(9), pp. 1124-1137.

Herold, H. 2013. Advances and Recent Challenges in Cartographic Pattern Recognition. In: Proceedings of the $26^{\text {th }}$ ICA International Cartographic Conference, 2013.

Lafferty, J., McCallum, A., Pereira, F. (2001). Conditional random fields: Probabilistic models for segmenting and labeling sequence data. Proc. 18th International Conference on Machine Learning. Morgan Kaufmann. pp. 282-289.

Schemala, D., 2016. Semantische Segmentierung historischer topographischer Karten. Diploma thesis, TU Dresden, 57 p. 


\title{
SATELLITE BASED MULTI-SCALE METHODS TO SUPPORT THE GOVERNANCE OF BRAZILIAN LOW-CARBON AGRICULTURE (ABC PLAN)
}

\author{
M. Simões ${ }^{\mathrm{a}, *}$, R. P. D. Ferraz ${ }^{1 \mathrm{~b}}$, A. Béguéc, B. Bellón ${ }^{\mathrm{c}}$, P. L. Freitas ${ }^{\mathrm{b}}$, P. L. O. A. Machado ${ }^{\mathrm{d}}$, M. L. Neves ${ }^{\mathrm{b}}$, L. Skorupa ${ }^{\mathrm{e}}$, C.Manzatto $^{\mathrm{e}}$. \\ ${ }^{\text {a}}$ Embrapa Solos and UERJ/FEN/DESC/PPGMA, Rio de Janeiro, Brazil margareth.simoes@ embrapa.br; \\ ${ }^{\text {b} E m b r a p a ~ S o l o s, ~ R i o ~ d e ~ J a n e i r o, ~ B r a z i l ~(r o d r i g o . d e m o n t e, ~ p e d r o . f r e i t a s, ~ m a t h e u s . n e v e s) @ e m b r a p a . b r ~}$ \\ ${ }^{\mathrm{c}}$ Cirad, UMR Tetis, Montpellier, France (agnes.begue, beatriz.bellon)@ teledetection.fr \\ ${ }^{\mathrm{d}}$ Embrapa Headquarters, Brasilia, Brazil - pedro.machado@embrapa.br \\ ${ }^{e}$ Embrapa Meio Ambiente, Jaguariúna, Brazil - (ladislau.skorupa, celso.manzatto)@embrapa.br
}

KEY WORDS: Land Management Changes, Integrated Crop Livestock System, Remote Sensing, Temporal Time Series, Segmentation

\begin{abstract}
:
Brazilian Low Carbon Agriculture Plan (ABC Plan) is one the initiatives that places the climate in the agricultural agenda towards a more sustainable and adapted agriculture under global changes. Among the several practices listed and supported by the ABC Plan, zero tillage and integrated crop-livestock-forestry systems including the recovery of degraded pasture are the most relevant ones. Those practices introduced a complexity to agriculture landscape image analyse. The objective of this paper is to present the GeoABC Project and preliminary procedures using OBIA and satellite based multi-scale methods to monitor the introduced scenario of a new agriculture land use dynamics.
\end{abstract}

\section{INTRODUCTION}

There is no humanitarian goal more crucial than feeding a world population projected to expand beyond nine billion by 2050 and agriculture lies at the heart of many fundamental global challenges faced by humanity including food security, environmental degradation and climate change (Dickie et al., 2014). Several initiatives such as the Climate Smart Agriculture supported by the World Bank and FAO, the 4 per 1000 Initiative by France and supported by CGIAR, and Brazil's $\mathrm{ABC}$ Plan are examples of the key role played by agriculture in a more sustainable world. These developments indicate the special role to multifunctional landscapes in the process to establish a more sustainable agriculture. The purpose of this work is to present the Geo-ABC Project, an innovative project to monitor the implementation of the $\mathrm{ABC}$ Plan and aiming at the development of remote sensing methods to monitor agricultural systems listed in the ABC Plan and adopted at local scale. These are mixed farming systems such as integrated croplivestock-forestry system at various combinations and at a regional scale, to monitor sustainable farming systems through landscape patterns, in order to provide spatial indicators to improve governance of the ABC Plan.

\subsection{Project context}

The increased demand for food is projected to be satisfied through productivity gains, with modest changes in crop area and livestock herds. Yield improvements are projected to account for $80 \%$ of the increase in crop output (OECD/FAO, 2016). There is some scope to increase agricultural area sustainably, mainly in parts of Latin America and Sub-Saharan Africa. This includes increased multiple cropping and shortening of fallow periods. Climate change with its severe and erratic events increases the risk of the agricultural activity and not only mitigation, but also adaptation is essential to avoid severe depletions. Adaptation includes good management practices with low carbon footprint.

Conservation agriculture is a key component of the ABC Plan and low carbon agriculture such as zero tillage, recovery of degraded pasture, and planted forests give a special role to multifunctional landscapes in the process towards a more sustainable agriculture. Indeed, landscapes must be considered as a whole system at the heart of human-nature relationships that need to be efficiently managed in order to preserve and restore ecosystem services.

Consequently, there is an urgent need to better characterize agricultural systems at global, regional and local scales, with a particular emphasis on the various pathways towards agricultural intensification, since those systems are the key for understanding land use sustainability in agricultural territories.

\subsection{International context}

G20 launched in June 2011 the Group on Earth Observations Global Agricultural Monitoring (GEOGLAM) initiative and the Agricultural Market Information System (AMIS) initiatives. G20 has consulted the GEO Agriculture Community of Practice to implement GEOGLAM with the main objective to improve crop yield forecasts as an input to the Agricultural Market Information System (AMIS) to foster stabilization of markets and increase transparency on agricultural production. The objectives of GEOGLAM are to (i) Enhance national agricultural reporting systems (ii) Establish a "global" network of experts in agricultural monitoring (iii) Create an operational global agricultural monitoring system of systems based on Earth Observation and in situ data. This initiative was further supported by the G20 meeting of agricultural vice ministers and deputies, including Brazilian governmental representatives in Mexico City in May 2012. All representatives and delegates recognized Earth Observation as an important tool to provide information on agriculture, at the present time and in the future. 
The Group on Earth Observations (GEO) started in 2002 Summit on Sustainable Development in South Africa, which is a voluntary partnership of governments and international organizations with the main goal to support sustainable management of the earth's resources making use of remote sensing. Its main vision is to build a Global Earth Observation System of Systems (GEOSS) through a coordination of remote sensing activities around the globe. As one of its strategic targets GEOSS aims to expand application capabilities to advance sustainable agriculture.

Geo-ABC project is aligned, with the goals of the EC Framework Program (FP7) SIGMA Project (Stimulating Innovation for Global Monitoring of Agriculture and its Impact on the Environment in support of GEOGLAM) which intends to contribute to the establishment of a global observation system for the assessment of the impact of cropland areas and change on the environment and as such strengthen global agricultural monitoring by improving the use of Earth Observation for crop production projections. Both intensification and expansion of agricultural land are therefore the primary focus of SIGMA and the Brazilian equivalent GeoABC Project which supports the ABC Plan. SIGMA uses the JECAM (Joint Experiment for Crop Assessment and Monitoring) sites (local agricultural sites of 50x50 km located on different countries) as defined by the GEOGLAM community to reach a convergence of approaches, develop monitoring and reporting protocols and best practices for a variety of global agricultural systems (see www.jecam.org). The Brazilian JECAM sites are currently being used as study sites in this proposed project, taking advantage of the JECAM facility to access satellite data (different sensors and high periodicity).

\subsection{National context}

Geo-ABC is aligned with the $\mathrm{ABC}$ Plan presented as a voluntary initiative by the Brazilian Government at the UNCCC-COP15 in 2009 in Copenhagen, Denmark.

Since 2011, the ABC Plan provides an initial credit line of $\mathrm{R} \$ 2$ billion (US\$ $1.1 \mathrm{bn}$ ) over ten years to finance different low carbon agricultural practices that use technologies to reduce greenhouse gas emissions. The national goal is to reduce carbon equivalent emissions from Brazilian agriculture by up to 176 million tons by the year 2020 .

Zero tillage system is one of the practices promoted by the $\mathrm{ABC}$ Plan, which includes crop rotation with cover crops for mulching and avoidance of soil ploughing and harrowing. Brazil is one of the largest producers of soybean worldwide and totally in zero tillage system and vast use of biological nitrogen fixation, that is no mineral nitrogen fertilizer. This procedure preserves nutrients in the soil, thus increasing crop yields and protects the field from external erosion factors. Through the ABC program, the Ministry of Agriculture plans to expand the use of this technique to cover an area of 33 million hectares from the 25 million hectares on which it is currently implemented. The adoption of zero tillage systems may reduce emissions by $16-20$ million tons of $\mathrm{CO}_{2}$ over a ten-year period.

Integrated crop-livestock-forestry (ICLF) system based on zero tillage is a more complex farming system supported by the federal government. Farmers or ranchers alternate from pasture to agriculture to forestry on the same paddock promoting continuous soil cover and carbon accumulation throughout the year. The ABC Plan aims to increase the use of Integrated CropLivestock-Forest system (ICLF) by 4 million hectares over the next decade, while reducing $\mathrm{CO}_{2}$ equivalent emissions by $18-22$ million tons.

The measurement of the impact of the $\mathrm{ABC}$ Plan is envisaged by the federal government as presented during the UNCCCCOP15. Considering this scenario, the use of satellite based multi-scale methods is important in order to create metrics to support the governance of the Low Carbon Agriculture Plan.

\section{METHODS AND PRELIMINARY RESULTS}

Different methodological approaches are being used in the GeoAbc Project to evaluate how local variables can be scaledup to be monitored at a regional scale (Bégué et al., 2015). In particular, the developed methodologies focus on the use of spatial, temporal and textural indicators derived from coarseresolution satellite images to represent crop systems at a landscape level. Crop distribution modelling, up-scaling methods based on satellite-derived variables (temporal, spectral and spatial indicators) and Object Based Image Analysis (OBIA) techniques are being applied and tested in the study area of the State of Tocantins and at ICLF system sites.

At the state scale (regional scale), a landscape stratification was carried out through segmentation of coarse-resolution satellite derived variables (Bellón et al., 2016). A principal component analysis transformation was used on a MODIS NDVI annual time-series (250m spatial resolution MOD13Q1 product) to obtain the principal vegetation physiognomic and phenologic characteristics on the first three principal component images. In addition, a textural index (GLCM homogeneity index) was calculated for MODIS NDVI images of three different dates within the annual series, i.e. before the growing season, middle of main plant cycle and middle of following plant cycle (Haralick \& Shanmuga, 1973). The derived radiometric (principal component) and textural indices was segmented using the multiresolution segmentation algorithm of the Definiens Developer software and the optimal segmentation parameters were evaluated by a statistical unsupervised evaluation method (Johnson \& Xie, 2011) (Figure 1).

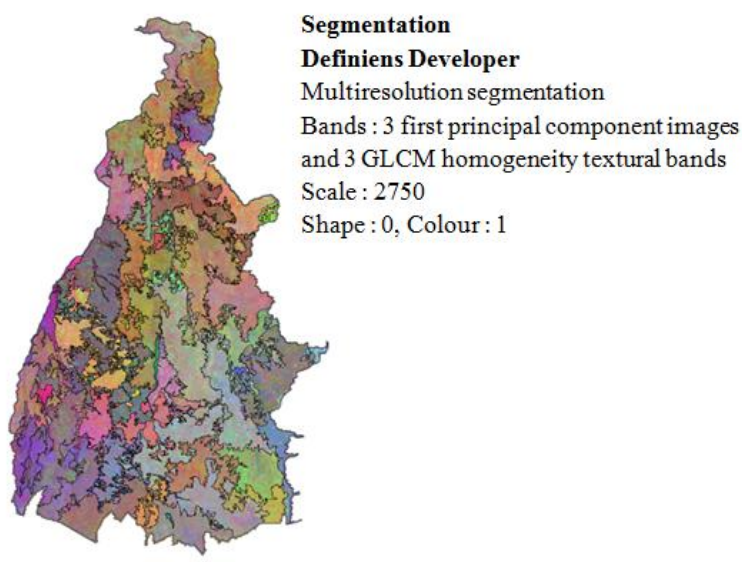

Figure 1 - Regional scale landscape stratification of MODIS NDVI derived radiometric and textural indices (Bellón et al., 2016).

At local scale, temporal profiles were analyzed by experts in order to detect metrics and different behaviour of vegetation index on different Low Carbon Agricultural systems (ABC), such as Integrated Crop-Livestock-Forest systems (Figure 2). 


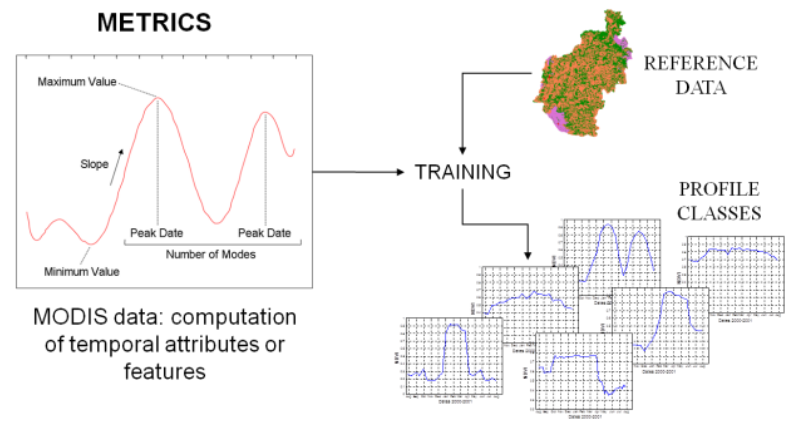

Figure 2 - Computation of MODIS temporal attributes

ICLF systems commonly presents a mixed growth of both crop and pasture grass (Figure 3). This procedure, adopted by farmers, enables both plants to take advantage of the residual rains in the humid season and pasture formation in the dry season due to the deep rooting of the forage grass.

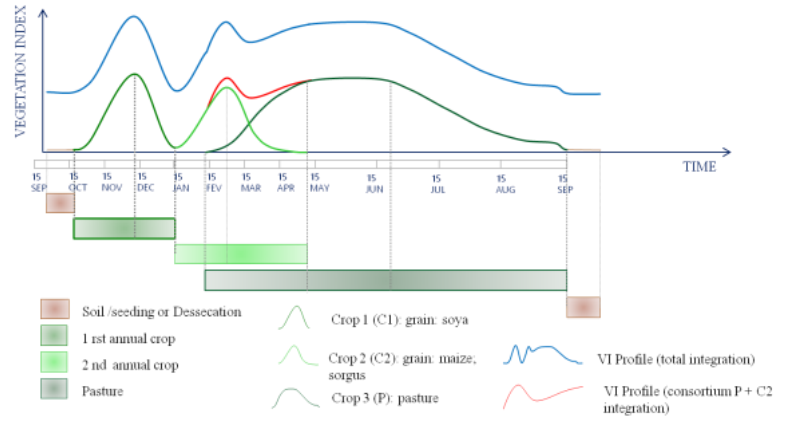

Figure 3 - Integrated Crop-Livestock Hypothetical temporal profile

At local scale, the study area was a 190-ha farm paddock. The soil type is a clayey and acidic Ferralsol under degraded pasture grass, mostly Urochloa spp. Pasture was recovered using grain crop after liming and fertilizer application. By the end of October 2006, the soil was prepared using a heavy disc harrow and soybean was sown by mid November. After harvest of soybean by mid March 2007, maize together with pasture grass (Urochloa ssp) was sown and after harvest of maize in JuneJuly, the area was kept under pasture grass for beef cattle. Between October-November 2007, soybean under zero-tillage was sown in the area followed by maize or sorghum between March and June. Regrowth of pasture grass allowed enabled grazing by beef cattle. This double cropping followed by grazing pasture was kept until 2012 (Figure 4).

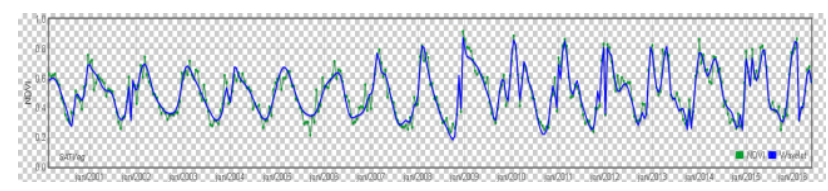

Figure 4 Integrated-Crop-Livestock System VI temporal profile

\section{CONCLUSION}

This work presented preliminary studies for the assessment of complexes agriculture landscapes, induced by the implementation of low carbon agriculture systems. By the end of the project a complete set of methodological approach will establish protocols to obtain a systematic monitoring of $\mathrm{ABC}$ Plan. GEOBIA integrated with multi-scale Remote Sensing techniques can provide metrics and spatial-temporal indicators that can be used as inputs for monitoring the goals of the ABC Plan. At the scientific context, those methods will provide inputs for studies: on the dynamics of land use related with the adoption of low carbon agriculture, for assessment of trends and establishment of future scenarios (land use dynamics); on the dynamics of land use, based on the expansion of low carbon agriculture related to the mitigation of environmental impacts (environmental impacts); on the dynamics of land use, based on the expansion of low-carbon agriculture production related to the mitigation of the greenhouse effect: (a) carbon stocks in soil and biomass; (b) reduction of GHGS; (c) water balanceecosystem services; (iv) on the dynamics of land use, based on the expansion of low-carbon agriculture production and the relation with Climate Change (climate change).

\section{ACKNOWLEDGEMENTS}

The authors would like to acknowledge the financial support of Capes/Cofecub Programme for the GeoABC Project $\left(\mathrm{N}^{\circ}\right.$ 845/15), as well as CIRAD and the SIGMA European Collaborative Project (FP7-ENV-2013 SIGMA), the sponsors of Beatriz Bellon PhD studies.

\section{REFERENCES}

Bellón, B., Bégué, A., Lo Seen, D., Simões, M., Ferraz, R., Lebourgeois, V., Gaetano, R. 2016. A remote sensing multiscale approach for agricultural systems zoning. In: Proceedings of the Global Land Project 3rd Open Science Meeting, Beijing, October 24-27, 2016 (to be published).

Bégué, A., Arvor, D., Lelong, C., Vintrou, E., Simões, M., 2015. Agricultural system studies using remote sensing. In: Tenkabail, P.S. (ed.) Land resources monitoring, modeling, and mapping with remote sensing. Boca Raton: CRC Press, 2015. cap. 5, p. 113-130.

Haralick, R. M., Shanmugam, K.K. 1973. Textural features for image classification. IEEE Transactions on systems, man, and cybernetics, (6), 610-621.

Dickie, A., Streck, C., Roe, S., Zurek, M., Haupt, F., Dolginow, A., 2014. Strategies for mitigating climate change in agriculture: abridged report. Climate Focus and California Environmental Associates and The Climate and Land Use Alliance. Available at www.agriculturalmitigation.org

Johnson, B., Xie, Z. 2011. Unsupervised image segmentation evaluation and refinement using a multi-scale approach. ISPRS Journal of Photogrammetry and Remote Sensing, 66(4), 473483.

OECD/FAO, 2016. OECD-FAO Agricultural Outlook 20162025, OECD Publishing, Paris. http://dx.doi.org/10.1787/agr_outlook-2016-en

Simões, M., Bégué, A., Ferraz, R. 2015. Methodologies and technological innovations for low-carbon agriculture monitoring and planning for the ABC Plan governance support - Geo ABC. In: World Congress on Integrated Crop Livesctock Forest Systems; International Symposium on Integrated CropLivestock System, 3, Brasília, DF. Towards sustainable intensification: proceedings. Brasília, DF: Embrapa http://ainfo.cnptia.embrapa.br/digital/bitstream/item/137498/1/2 015-140.pdf 


\title{
FIRST EXPERIMENTS USING THE IMAGE FORESTING TRANSFORM (IFT) ALGORITHM FOR SEGMENTATION OF REMOTE SENSING IMAGERY
}

\author{
A. R. Soares ${ }^{\text {a }}$,T. S. Körting ${ }^{\text {a }}$, L. M. G. Fonseca ${ }^{a}$ \\ ${ }^{a}$ Image Processing Division, National Institute of Space Research, INPE, São José dos Campos, Brazil \\ (anderson.soares,thales.korting,leila.fonseca)@inpe.br
}

KEY WORDS: Image Segmentation, Image Foresting Transform, Multiresolution Segmentation, Segmentation Comparison

\begin{abstract}
:
Image segmentation is a traditional method in Remote Sensing and a fundamental problem in image processing applications. It has been widely used, especially with the emergence of the Geographic Object-Based Image Analysis (GEOBIA). The results of segmentation must create uniform areas, which must allow a simpler interpretation by the users and simpler representation for classification algorithms. Several algorithms were proposed through the years, using different approaches. One that is widely used in Remote Sensing applications is the Multiresolution algorithm, that is based on the region growing method. Other, which has great potential and is applied in other research areas, is available on the Image Foresting Transform (IFT) framework, which has several image operators developed primarily for medical images. The Watershed from Grayscale Marker operator uses an edge image to perform the segmentation, however, we propose an extension of the edge detection algorithm, by summing normalized gradients of each band. This work aims to evaluate and compare these two segmentation algorithms, by comparing their results through supervised segmentation from reference regions, that were defined manually by an expert user. Quality measures were evaluated by four metrics, that represent the positional adjustment based the center of gravity, intensities, size, and the amount of overlap between the segment created by the algorithms and the reference segment. We selected 21 objects of a WorldView-2 multispectral image that were used to compute the metrics. Both methods reached similar results, by comparing the aforementioned 4 metrics applied to the 21 reference regions, IFT achieved better results for majority of regions. The IFT generated segments with similar shape when compared with the references, and the multiresolution generated results with similar sizes and positional adjustments. It may be explained by the fact that IFT uses an edge image to perform the segmentation. Both algorithms obtained similar agreement for intensity.
\end{abstract}

\section{INTRODUCTION}

Image segmentation is a fundamental problem in image processing, (Soille, 1999) defined segmentation as a process to split an image grouping the pixels by a similar attribute, such as the grey level, so the line which splits the areas, ideally, must be an edge. According to (Körting, 2012) the segmentation is one of the most challenging tasks in digital image processing.

Geographic Object-Based Image Analysis (GEOBIA) is devoted to developing automated methods to partition Remote Sensing imagery into meaningful image-objects, and assessing their characteristics through spatial, spectral and temporal scales (Hay and Castilla, 2008). It became widely used because it offers the potential to exploit geographical information.

The development of GEOBIA required a search for new methods for image segmentation. During the last decades, numerous techniques have been developed and applied in Remote Sensing analysis (Bins et al., 1996, Hay et al., 2005). The image segmentation algorithm that generates appropriate results has been the Multiresolution Segmentation (MRS) (Baatz and Schäpe, 2000), which probably is the most popular algorithm applied in GEOBIA.

Besides MRS good results, the search for new algorithms for image segmentation is still a necessity, and can not be limited by the MRS popularity. Different approaches may, also, produce similar results or even better results.

The Image Foresting Transform is a general tool for the design, implementation, and evaluation of image processing operators

\footnotetext{
${ }^{*}$ Corresponding author
}

based on connectivity (Falcão et al., 2004), it defines a minimumcost path forest in a graph, whose nodes are the image pixels and whose arcs are defined by an adjacency relation between pixels. The cost of a path is determined by a path-cost function $f$, which usually depends on local image properties (color, gradient, or pixel position) along the path between the nodes and the root.

The IFT Watershed from Grayscale Marker (called Watergray from this point), is one IFT operator in which the segmentation is computed from a gray scale image. According (Lotufo et al., 2002) it puts together several steps used in classical watershed morphological segmentation strategies in a single algorithm.

This paper evaluates and compares the Watergray operator, adapted to the Remote Sensing context, with the MRS algorithm.

\section{METHODOLOGY}

We compared the algorithms using 4 quality measures applied in 21 selected regions (Figure 1), on a WorldView-2 Multispectral image.

As the Watergray uses an edge image to perform the segmentation we implemented on MATLAB an adaptation of the traditional way to compute the gradient of the image. As described in Equation 1 we computed the traditional gradient for each band and normalized each one. With this preprocessing we expect that each band has the same importance when all gradients are summed.

$$
\nabla_{\text {image }}=\sum_{i}^{N} w_{N} * \nabla_{N}
$$




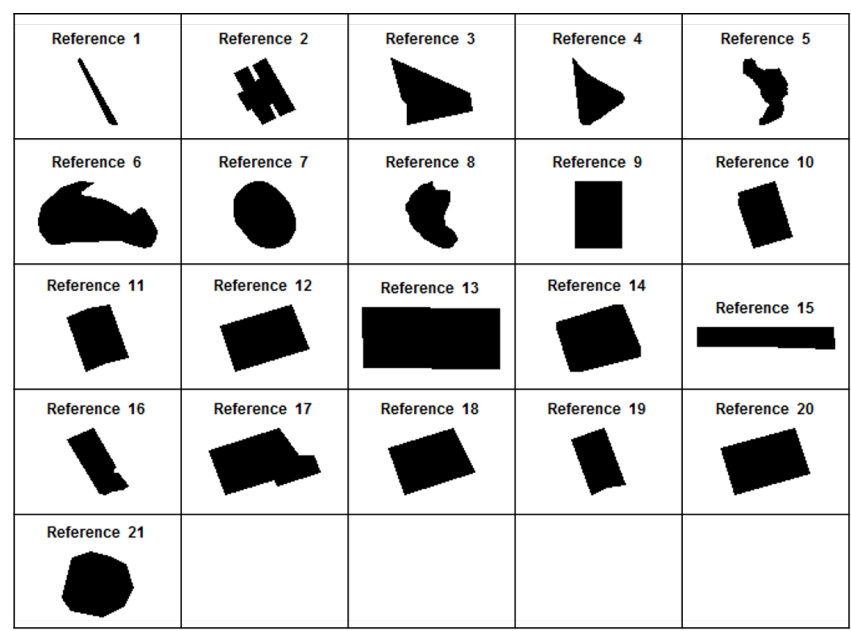

Figure 1. Rerefence regions.

In comparison with MRS algorithm, by using our approach it is possible to create a gradient image with different weights for each band. This way, we can enhance some feature or object, considering its spectral characteristics.

Since Watergray is not able to handle georeferenced images, we developed an algorithm in C++ using the TerraLib library (Câmara et al., 2008), to project the labeled image provided by Watergray to the same reference system of the WorldView-2 image.

To perform the experiment we used the following tuning parameters for MRS algorithm: 45 scale, 0.3 shape e 0.7 compactness, and weight 2 for infrared bands and 1 for other bands. For the Watergray the cost applied was 12 and weight 2 for infrared bands and 1 for the other bands.

For evaluation of the segmentation algorithms we applied a supervised approach, as proposed by (Delves et al., 1992). Each region of the reference segmentation is selected to be evaluated in the comparison. Consider a reference segmentation with $K$ segments and the evaluated segmentation with $L$ segments, both with $W$ columns and $H$ rows, a given region $i$ in the reference segmentation, and a given object in the segmentation being evaluated, $f$. The $\left\langle g_{i}>\right.$ notation represents the average of the measure $g$ in a given region $i, N(i)$ represents the number of pixels of $i$ and $x_{i}$ and $y_{i}$ indicate the location of some pixel inside $i$ region, respectively the column and row (Reis et al., 2015). Two matrices, $G f$ and Fit, both with $H$ rows and $W$ columns, are constructed using the following equations:

$$
\begin{gathered}
G f(i, f)=\frac{N(i \bigcap f)}{N(i \bigcup f)} \\
F i t=\frac{x d+y d+\frac{p d+i d}{2}}{G f(i, f)}
\end{gathered}
$$

in which:

$$
\begin{aligned}
& x d=\frac{\left|<x_{i}>-<x_{f}\right\rangle \mid}{H} \\
& y d=\frac{\left|<y_{i}>-<y_{f}>\right|}{W}
\end{aligned}
$$

Table 1. Average and Standard Deviation of Metrics

\begin{tabular}{c|c|c|c|c} 
& \multicolumn{2}{|c|}{ MRS } & \multicolumn{2}{c}{ Watergray } \\
\hline Metric & Average & Std. & Average & Std. \\
\hline FIT XY & 0.8993 & 0.1170 & 0.8821 & 0.1376 \\
FITI & 0.6086 & 0.1966 & 0.6146 & 0.1840 \\
Gshape & 0.6491 & 0.1804 & 0.6632 & 0.1836 \\
FIT N & 0.5555 & 0.2105 & 0.5390 & 0.2210 \\
FIT M & 0.6781 & 0.0750 & 0.6738 & 0.0843
\end{tabular}

$$
\begin{gathered}
p d=\frac{|N(i)-N(f)|}{|N(i)+N(f)|} \\
i d=\frac{|<V(i)>-<V(f)>|}{|<V(i)>+<V(f)>|}
\end{gathered}
$$

With those matrices, this approach computes 4 metrics: FIT XY, FITI, FITN, and Gshape. Each measure is calculated for each region considering a reference, that were defined manually by an expert user.

The metric FITXY, Equation 8, evaluates the displacement between the center of gravity of polygon generated by segmentation algorithms and the reference polygon. FITI, Equation 9, checks the grey level intensity of the segments, using for that, the original image.

The FITN, Equation 10, assesses the similarity between the size of the reference polygon and the size of the segment generated by the analyzed algorithms. Gshape, Equation 11, evaluates the shape similarity between the segments generated by algorithms and the reference.

$$
\begin{gathered}
F I T X Y=1-\frac{x d+y d}{2} \\
F I T I=1-\frac{|<V(i)>-<V(f)>|}{<V(i)>+<V(f)>} \\
F I T N=1-\frac{|N(i)-N(f)|}{N(i)+N(f)} \\
\text { Gshape }(i, f)=\frac{N(i \bigcap f)}{N(i \bigcup f)}
\end{gathered}
$$

All metrics produce values in the interval $[0,1]$, with 1 being the best. In order to merge all metrics in a single value, we computed also the average value of the aforementioned metrics, and it was denoted by FITM.

\section{PRELIMINARY RESULTS}

Table 1 shows the average obtained by each metric analyzed for both algorithms in all 21 reference regions. By analyzing this table we can observe that Watergray achieved better results for Gshape metric, while the MRS obtained better results for FITN and FITXY. For the other metrics, the results were similar.

\subsection{Positional adjustment - FITXY}

As can be observed on Figure 2 the MRS and Watergray generated similar segments, however, some segments generated by Watergray were slightly shifted, the difference was subtle. 


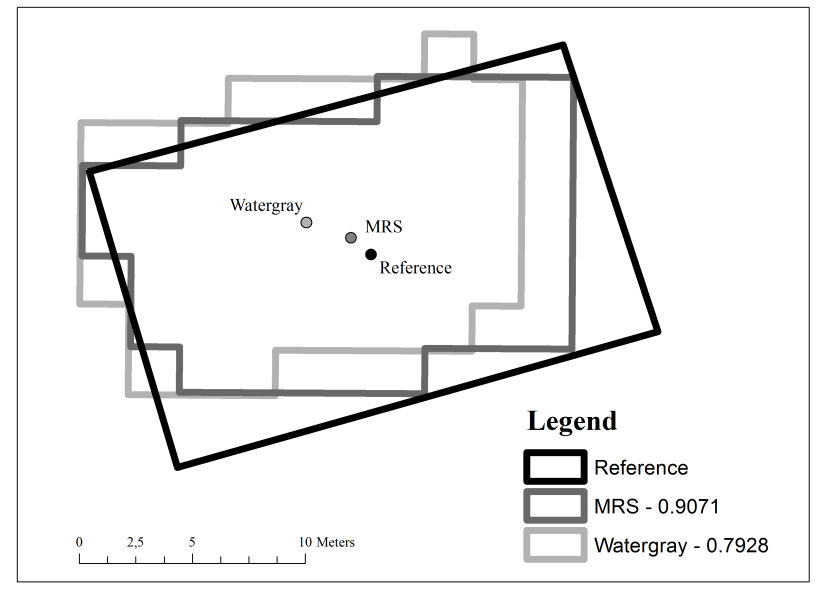

Figure 2. Center of gravity of segments - The FITXY metric.

\subsection{Intensities - FITI}

Again both algorithms produced similar responses, the difference of averages was below 0.001 . However, it is important to address that the standard deviation for this metric was high for both algorithms (around 0.19).

\subsection{Overlap - Gshape}

For this metric the Watergray had slightly better results, around 0.02 , this can be explained by the edge image used by Watergray, that allowed a better definition of the objects. Both algorithms had a high standard deviation, around 0.18 .

\subsection{Size - FITN}

For this metric the MRS naturally has an advantage, since it has a specific parameter that influences on the average size of resultant polygons, while the Watergray does not have such capability. In some cases the Watergray generated segments smaller than the references, or created some holes on the segment. Also, it is important to call attention to the fact that this metric had the worst results for both algorithms, and also the highest standard deviation, around 0.21 for MRS and 0.22 for Watergray.

\subsection{Average - FITM}

As aforementioned, the FITM is obtained by the average of the 4 metrics, therefore, it provides a global understanding of the similarity between the reference and the segment generated by algorithms. For most of the polygons the differences were small, around 0.004 . Therefore, the results indicate a good agreement between the algorithms and the analyzed polygons.

\subsection{Regularity}

We also evaluated if the regularity of the objects influences on the performance of the algorithms. From the 21 analyzed polygons, 13 were regular, while 8 had irregular shapes. Both algorithms obtained better results for the irregular polygons in almost all metrics, only the Gshape metric had poor results, around 0.59 . The FITI metric had the major gain, almost 0.4 for both algorithms. The small advantage obtained by MRS on the FITN and FITXY metrics, when all polygons were analyzed together, persisted only for the regular objects.

\section{CONCLUSION}

In this paper we evaluated and compared the Watergray and the MRS algorithms for Remote Sensing image segmentation. The Watergray had better results for the Gshape metric, however, it did not obtain as good results for FITN as MRS. Both algorithms obtained similar agreement for FITXY and FITI.

Those results indicate that both algorithms produced equivalent segmentations, however, new tests must be conducted to evaluate the Watergray on images of different domains and spatial resolutions. We also conclude from the results that Watergray has a great potential to segmentation of Remote Sensing imagery. For further studies, we will adapt the Watergray to handle small polygons and implement it on the TerraLib library (Câmara et al., 2008).

\section{REFERENCES}

Baatz, M. and Schäpe, A., 2000. Multiresolution segmentation: an optimization approach for high quality multi-scale image segmentation. In: Wichmann-Verlag (ed.), XII Angewandte Geographische Informationsverarbeitung, Vol. pp, Herbert Wichmann Verlag, Heidelberg, pp. 12-23.

Bins, L., Fonseca, L., Erthal, G. and Ii, F., 1996. Satellite imagery segmentation: a region growing approach. Simpósio Brasileiro de Sensoriamento Remoto 8, pp. 677-680.

Câmara, G., Vinhas, L., Ferreira, K., Queiroz, G., Souza, R., Monteiro, A., Carvalho, M., Casanova, M. and Freitas, U., 2008. TerraLib: An open source GIS library for large-scale environmental and socio-economic applications. Open Source Approaches in Spatial Data Handling 2, pp. 247-270.

Delves, L., Wilkinson, R., Oliver, C. and White, R., 1992. Comparing the performance of sar image segmentation algorithms. International Journal of Remote Sensing 13(11), pp. 2121-2149.

Falcão, A., Stolfi, J. and de Alencar Lotufo, R., 2004. The image foresting transform: theory, algorithms, and applications. IEEE Transactions on Pattern Analysis and Machine Intelligence 26(1), pp. 19-29.

Hay, G. and Castilla, G., 2008. Geographic Object-Based Image Analysis (GEOBIA): A new name for a new discipline. In: T. Blaschke, S. Lang and G. Hay (eds), Object-Based Image Analysis: Spatial Concepts for Knowledge-Driven Remote Sensing Applications, Springer-Verlag, Berlin, Heidelberg, chapter 1.4 , pp. $75-89$.

Hay, G. J., Castilla, G., Wulder, M. A. and Ruiz, J. R., 2005. An automated object-based approach for the multiscale image segmentation of forest scenes. International Journal of Applied Earth Observation and Geoinformation 7(4), pp. 339-359.

Körting, T., 2012. GeoDMA: A toolbox for data mining, object-based and multi-temporal analysis of remote sensing imagery. PhD thesis, Brazil's National Institute for Space Research (INPE).

Lotufo, R. A., O, A. X. F. and Zampirolli, F. A., 2002. IFTWatershed From Gray-Scale Marker. In: SIBGRAPI'02, pp. 146-152.

Reis, M. S., de Oliveira, M. A. F., Korting, T. S., Pantaleo, E., Sant'Anna, S. J. S., Dutra, L. V. and Lu, D., 2015. Image segmentation algorithms comparison. In: 2015 IEEE International Geoscience and Remote Sensing Symposium (IGARSS), pp. 43404343.

Soille, P., 1999. Morphological image processing: Principles and Applications. Cambridge University Press. 


\title{
THREE-YEAR ASSESSMENT OF THE SPACE-TIME DYNAMICS OF BURNED FOREST IN THE BRAZILIAN AMAZON, STATE OF MATO GROSSO
}

\author{
E. de Souza ${ }^{\mathrm{a}, *}$, R. Beuchle ${ }^{\mathrm{a}}$, R. C. Grecchi ${ }^{\mathrm{a}}, \mathrm{F}$. Achard ${ }^{\mathrm{a}}$ \\ a Joint Research Centre - Ispra, Institute for Environment and Sustainability, Forest Resource and Climate Unit. Via E. Fermi 2749 \\ TP261VA 21027 - ISPRA, - Italy, eliana.de-souza@jrc.ec.europa.eu
}

KEY WORDS: Amazon rainforest, forest degradation, forest fire, image classification, segmentation

\begin{abstract}
:
The use of fire in tropics for land management and for forest clearing contributes to forest degradation and to carbon emissions in the atmosphere. Yet, a comprehensive understanding of the spatial and temporal dynamics of forest fires is needed to mitigate such impacts. The primary aim of this study is to map burned forest in Brazilian Amazon by means of an object-based classification applied on Landsat imagery. Our secondary aim is to understand the spatial and temporal dynamics of forest fires. For this, we compare the forest cover maps with the map of burned forest generated during the dry season. In our study we consider three consecutive bi-annual periods (2013-2014, 2014-2015, 2015-2016) over four sites, in Mato Grosso State. From Landsat-8 imagery acquired during May or June of each year we derived NDVI and fraction images (soil, vegetation and shade) in order to map the following land cover classes: intact forest, burned forest, forest regrowth and non-forest. In addition, we map the burned areas during the fire season for each year. Intact forest, initially covering $55 \%$ of the area has lost $3.4 \%$ of its area over the course of three years. The annual burning increased from 2.6\% to 5.9\%, from 2013 to 2015 . Within the annually burned areas, non-forest areas showed a lower percentage of burning, compared to forest areas, varying from $3.8 \%$ to $7.9 \%$, while amongst the forest classes, burned forest showed the highest percentage. Re-burning on a biannual period is more than double of re-burning on a thrice-year.
\end{abstract}

\section{INTRODUCTION}

Although a decrease in deforestation in the Brazilian Amazon has been observed in the last ten years (INPE, 2015), studies on the Amazon Forest have pointed out a constant high intensity of forest fires in the states of Pará, Rondônia and Mato Grosso (Miettinen et al. 2015; Morton et al., 2013; Sousa et al., 2013; Lima et al., 2012) which contribute to forest degradation.

Degraded and intensely burned areas result in a decrease to the tree crown cover and consequently in an increased exposure of the soil, as well as in a loss of biodiversity (Cochrane and Schulze 1993; Silva Monteiro et al, 2004).

Selective logging and forest fire are the main drivers of forest degradation in the Brazilian Amazon (Souza et al., 2013; Matricardi et al., 2010). Forest fires have been associated with land-use change processes, in which fire escapes from pasture management (Fanin and van der Werf, 2015; Cano-Crespo et al., 2014, Lima et al., 2012), with changes in the weather conditions due to severe droughts (Aragão et al., 2008). Forest fires have however been identified as non-correlated to unmanaged land (Morton et al., 2013).

The relationship between burning and land cover is not yet fully understood. The distinct spectral characteristics of burned scars allow the mapping of burned forest areas through the use of satellite imagery, hence, the primary aim of this study is to map burned forest for three consecutive years in the State of Mato Grosso, Brazilian Amazon (2013-2014-2015) by means of an object-based classification applied to recent Landsat imagery. Our secondary aim is to understand the spatial and temporal dynamics of the fire related to land cover.

\section{MATHERIAL AND METHODS}

\subsection{Data sets and classification}

The study was carried out for an area of $40,616 \mathrm{~km}^{2}$, located in an active deforestation frontier in south-eastern Mato Grosso State in the Brazilian Amazon (Figure 1). The area is composed of subsets of four neighbouring Landsat scenes, image reference (path-row) and respective areas of the sub-scenes are as follows: 1 - 225/69 (3,450 km²), 2 - 226/68 (12,368 km²), 3 - 226/69 $\left(12,031 \mathrm{~km}^{2}\right), 4$ - 227/68 $\left(12,776.4 \mathrm{~km}^{2}\right)$. We consider three consecutive bi-annual periods (2013-2014, 2014-2015, 20152016) in the land cover change for the study.

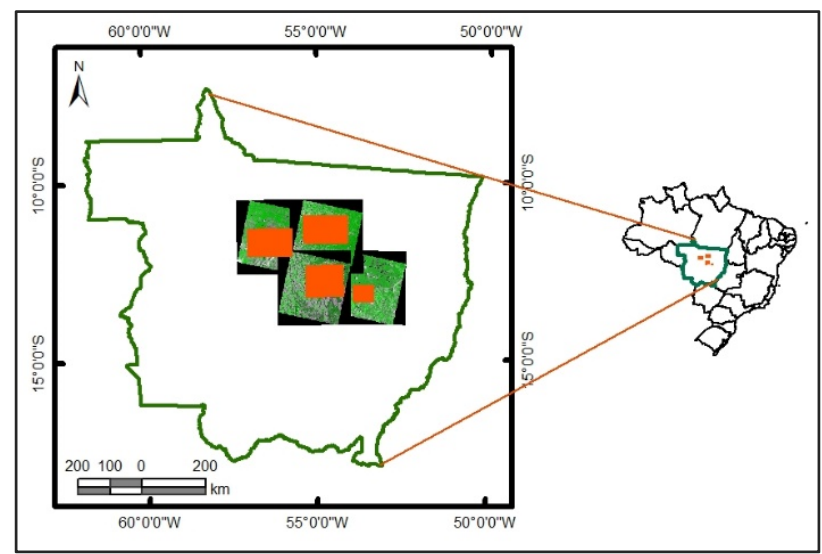

Figure 1. Study area in the State of Mato Grosso, Brazil.

A total of 64 scenes of Landsat-8 imagery, downloaded from the USGS archive, were processed. The visible (1, 2, and, 3), near infrared (4) and short-wave infrared (5 and 7) bands of each 
Landsat scene were used. Image processing was carried out using IMPACT software (Simonetti et al., 2016), which performed the image layer-stacking, clipping and, generated the NDVI (Normalized Difference Vegetation Index) and the soil, vegetation and shade fraction images, based on the linear spectral mixture model (Shimabukuro and Smith, 1991).

From images acquired on May or June of each year (beginning of the burning season), forest cover maps were produced with the following land cover classes: intact forest, burned forest, forest regrowth and non-forest. Burned areas were mapped during the early, middle and end of the dry season (i.e. July to November) of each year and the maps summed up to calculate the cumulative annual burning.

The segmentation was based on single date images with the same parameters applied for all dates. eCognition Developer 9.0 software (Benz et al., 2004) was used for segmenting and classifying the images. The classification of burned areas, as well as the land cover classification were threshold-based, using customized thresholds for each class and date. For classifying burned areas, the NDVI and the shade fraction images were used, while for the land cover classification, all fraction images (soil vegetation, shade) were used together with NDVI. Subsequently to the classification step, manual editing of the result was applied to ensure a high mapping accuracy.

The cumulative annual burning and the frequency of re-burning for bi-annual and thrice-year periods were calculated after overlaying the cumulative annual burning maps. Annual burned area maps were cross tabulated with respective maps of land cover of each year to relate burned areas to land cover. Changes in land cover over a three-year period were analysed by cross tabulating land cover information of 2013 and 2016. We analysed land cover changes of the entire study area and burned areas for the first year.

\section{RESULTS AND DISCUSSION}

The land cover maps generated for the years 2013, 2014, 2015 and 2016 show the majority of the area being covered by intact forest (Table 1). The forest area decreased from 54.9\% in 2013 to $51.5 \%$ on 2016 . This $3.4 \%$ of tree-cover loss $\left(758 \mathrm{~km}^{2}\right)$ has contributed to a $6.3 \%$ increase of non-forest areas, in addition to $2.6 \%$ of areas from regrowth and $0.3 \%$ from burned forest.

The annual burned areas increased from $2.6 \%$ on 2013 to $5.9 \%$ on 2015. The increase was higher from the first to second year (2.2\%) compared to the second to the third year (1.1\%). The total area burned $\left(3,940 \mathrm{~km}^{2}\right)$ represent $9.7 \%$ of the entire of this study. Miettinen et al. (2015) used a sampling approach to estimate the burning forest for the entire Amazon Biome within the Mato Grosso State for three dates in a five-year interval (2000-20052010). Similarly to what was found in the present study, these authors report a lower increase of burning for the second period (8.5\%) 2005 to 2010, compared to the first period (44.4\%) (20002005).

The present study mapped a total $1,087 \mathrm{~km}^{2}$ of intact forest disturbed by burning, in the course of three years (Table 2). This disturbed area, representing $4.9 \%$ of the total forest, which was much higher compared to what was found in the study of Matricardi et al. (2010), that conducted a detailed analysis of the relationship between selective logging and forest fire in an area on the Mato Grosso State during the period of 1992 to 2004. The findings of Matricardi et al. (2010) showed that fire alone was responsible for $2.9 \%$ of the forest disturbance, while combined effect of logging and forest fire resulted in a contribution of 5.5\% to forest disturbance.

The three years re-burning areas account for a total area of 5,381 $\mathrm{km}^{2}$ of land under fire at least once during the three years of analysis (Table 2). The analysis of burned areas by land cover class shows a predominance of fire in areas previously burned, burned forest and regrowth areas, with $39.8 \%$ and $23.4 \%$ of burned areas, respectively, in 2015. Non-forest areas showed relatively lower percentages of burning compared to intact forest.

\begin{tabular}{|c|c|c|c|c|}
\hline & 2013 & 2014 & 2015 & 2016 \\
\hline & \multicolumn{4}{|c|}{$\mathrm{km}^{2}$} \\
\hline Non-forest & 15,966 & 17,034 & 17,898 & 18,525 \\
\hline Forest burned & 601 & 204 & 551 & 506 \\
\hline Forest & 22,285 & 22,005 & 21,211 & 20,897 \\
\hline \multirow[t]{2}{*}{ Regrowth } & 1,765 & 1,374 & 957 & 688 \\
\hline & \multicolumn{4}{|c|}{$\%$} \\
\hline Non-forest & 39.3 & 41.9 & 44.1 & 45.6 \\
\hline Forest burned & 1.5 & 0.5 & 1.4 & 1.2 \\
\hline Forest & 54.9 & 54.2 & 52.2 & 51.5 \\
\hline Regrowth & 4.3 & 3.4 & 2.4 & 1.7 \\
\hline
\end{tabular}

Table 1 - Area of land cover classes and percentage of occurrence for the years 2013-2016.

Similar results were observed by Fanin and van der Werf (2015), comparing burning occurrence in the areas of forest and pasture during a ten-year period (2002-2012) on selected areas on Mato Grosso. These authors observed higher percentage of burning occurring in forest areas compared to non-forest (pasture) areas, with $7 \%$ and $8 \%$ for forest areas against 86 and $87 \%$ for pastures and forest during the years 2007 and 2010. This trend of higher burning on forest areas is an indication of fires being used for clearing areas more than for land management.

Lima et al. (2012) also observe a direct relation between fire and the process of land cover change, in a study for an area on the boarder of Rondônia and Mato Grosso `s States, with fire widely affecting previously degraded vegetation. Lima et al. (2012) reported 31\% of area burned in 2005 had been deforested in one of three preceding years.

\begin{tabular}{|lrrrr|}
\hline & 2013 & 2014 & 2015 & 3-years \\
\cline { 2 - 5 } Non-forest & \multicolumn{4}{c}{$\mathrm{km}^{2}$} \\
Forest burned & 71 & 1,151 & 1,410 & 2,042 \\
Forest & 176 & 383 & 219 & 246 \\
Regrowth & 187 & 324 & 224 & 1,087 \\
Total & 1,040 & 1,944 & 2,397 & 365 \\
& \multicolumn{4}{c}{$\%, 940$} \\
\cline { 2 - 5 } Non-forest & 3.8 & 6.8 & 7.9 & \\
Forest burned & 11.7 & 42.2 & 39.8 & 42.8 \\
Forest & 0.8 & 1.7 & 2.6 & 4.0 \\
Regrowth & 10.6 & 23.6 & 23.4 & 32.0 \\
Total & 2.6 & 4.8 & 5.9 & 9.7 \\
\hline
\end{tabular}

Table 2 - Area burned, by land cover class, and its percentage for the years 2013, 2014 and 2015 and, 3-years cumulative.

The frequency of re-burning over the three-year period accounts for $2.6 \%$ of the total area $\left(1,040 \mathrm{~km}^{2}\right)$. The percentage of reburning on biannual periods (2013-2014, 2013-2015 and 20142015) varied from $7.3 \%$ to $10.7 \%$ (Table 3 ). Over the three-year period, a total of $5,381 \mathrm{~km}^{2}$ were burned. From this cumulative burned area re-burned re-burning occurred at least once in the biannual periods. Twice re-burning accounts for $80.7 \%$ of the accumulated burned area, while a thrice re-burning was detected in $19.3 \%$ of the burned area. 
The analysis of non-forest areas show that approximately 50\% have been re-burned in a biannual or three-year period. This high percentage of re-burning on non-forest areas, compared with reburning in forest areas, can be related to fire been used for managing pastures. Regrowth areas show re-burning as varying from $15 \%$ to $19 \%$ while forest areas vary from $7 \%$ to $27 \%$ over the years examined. The percentage of burning recurrence on burned forest areas is $5 \%$ to $7 \%$, and thus lower if compared to regrowth and burned forest areas, reflecting the land cover class in which burning is less needed for clearing areas previously under fire (Table 3).

\begin{tabular}{|lcccc|}
\hline & 2013 & 2014 & 2013 & 3-years \\
\cline { 2 - 5 } & -2014 & -2015 & -2015 & \multicolumn{4}{c|}{$\mathrm{km}^{2}$} \\
Non-forest & 1,640 & 2,193 & 1,766 & 606 \\
Forest burned & 177 & 266 & 230 & 71 \\
Forest & 604 & 1,166 & 913 & 176 \\
Regrowth & 563 & 716 & 527 & 187 \\
Total & 2,984 & 4,341 & 3,437 & 1,040 \\
\cline { 2 - 5 } & & $\%$ & 51 & 58 \\
Non-forest & 55 & 51 & 6.7 & 6.8 \\
Forest burned & 5.9 & 6.1 & 6.7 \\
Forest & 20.2 & 26.9 & 26.6 & 16.9 \\
Regrowth & 18.9 & 16.5 & 15.3 & 18 \\
Total & 7.3 & 10.7 & 8.5 & 2.6 \\
\hline
\end{tabular}

Table 3. Frequency of re-burning for biannual period and for the 3-years period (2013, 2014 and, 2015) based on 2013 land cover map.

Although an annual increase of burned areas is observed, with 2015 burned areas $\left(2,397 \mathrm{~km}^{2}\right)$ being is more than double of the areas in $2013\left(1,040 \mathrm{~km}^{2}\right)$, a high variability is observed regarding the spatial distribution of re-burning in forest areas, as shown on the re-burning frequency (Table 3) with $16.9 \%$ for a three-year period and an average of $25 \%$ for a twice re-burning. The maps of annual burning and burning frequency (Figure 2) shows no spatial tendency on areas that have been re-burned twice or thrice.
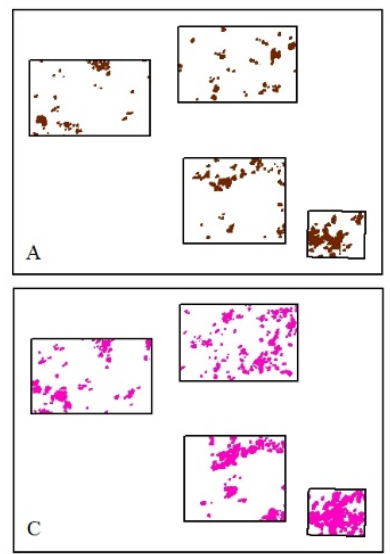

Figure 2 - Burning occurrence on: A) 2013, B) 2014, C) 2015 and, D) burning frequency (blue $=$ twice, red $=$ thrice).

The land cover change over the three-year period (Table 4) shows intact forest with $88.8 \%$ of its area in 2013 maintained as forest in 2016 , with $1.8 \%$ changing to burned forest and $8.5 \%$ to nonforest. The classes, regrowth and burned forest, are the classes most prone to land cover change. $50.6 \%$ of the regrowth areas of 2013 regenerated into forest, $28.9 \%$ turned into non-forest and $18 \%$ continued as a regrowth in 2016.

The burned forest class showed $35.5 \%$ of its total area recovering as forest, while around $26 \%$ of the area changed into regrowth or non-forest.

\begin{tabular}{|l|c|c|c|c|}
\hline \multirow{2}{*}{2013} & $\begin{array}{l}\text { Non- } \\
\text { forest }\end{array}$ & $\begin{array}{l}\text { Forest } \\
\text { burned }\end{array}$ & Forest & Regrowth \\
\cline { 2 - 5 } Non-forest & \multicolumn{4}{|c|}{$\%$} \\
Forest burned & 100 & 29.3 & 8.5 & 28.9 \\
Forest & & 9.6 & 1.8 & 2.5 \\
Regrowth & & 35.4 & 88.8 & 50.6 \\
\hline
\end{tabular}

Table 4. Area of land cover conversion from 2013 to 2016.

Table 5 shows areas that were burned in 2013, and the conversion of the class from 2013 to 2016. A restoration process is observed for burned forest and regrowth converting into forest (21.9\% and $16.8 \%$ respectively). Nevertheless, the percentage of areas that are converted from forest areas to non-forest is higher: to regrowth (55\%), followed by burned forest (39\%), and intact forest $(35.4 \%)$. These figures indicate the feasibility of clearing forest under different stage of preservation.

\begin{tabular}{|l|c|c|c|c|}
\hline $2013 \quad 2016$ & $\begin{array}{l}\text { Non- } \\
\text { forest }\end{array}$ & $\begin{array}{l}\text { Forest } \\
\text { burned }\end{array}$ & Forest & Regrowth \\
\cline { 2 - 5 } Non-forest & 100 & 39 & 35.4 & 55.2 \\
Forest burned & & 9.8 & 7.4 & 2 \\
Forest & & 21.9 & 44.8 & 16.8 \\
Regrowth & & 29.3 & 12.4 & 26 \\
\hline
\end{tabular}

Table 5. Area of land cover conversion from 2013 to 2016 over areas burned in 2013.

Further steps towards understanding the spatial dynamic of burning forest will consider the use of Sentinel-2 imagery to increase the temporal and spatial accuracy of mapping, as well as classify the burned forest in level of severity to estimation carbon emission.

\section{ACKNOWLEDGEMENTS}

The Brazilian Foundation Conselho Nacional de Desenvolvimento Científico e Tecnológico, of the Ministry of Science, Technology and Innovation of Brazil (CNPq), is gratefully thanked for granting a scholarship for the first author to develop this study.

\section{REFERENCES}

Aragão, L.E.O.C.; Malhi, Y.; Barbier, N.; Lima, A.; Shimabukuro, Y.; Anderson, L.; Saatchi, S. 2008. Interactions between rainfall, deforestation and fires during recent years in the Brazilian Amazonia, Philos. T. R. Soc. B, 363, 1779-1785,

Benz, U.; Hofmann, P.; Willhauck, G.; Lingenfelder, I.; Heynen M. 2004. Multi-resolution, object-oriented fuzzy analysis of remote sensing data for GIS-ready information. ISPRS Journal of Photogrammetry and Remote Sensing, 58: 239-258. 
Cano-Crespo, A.; Oliveira, P.J.C.; Cardoso, M.; Thonicke, K. 2014. Tropical forest degradation in the Brazilian Amazon relation to fire Forest fires contribute to greenhouse-gas emissions. In: VIEGAS, D. X. (ED). Advances in forest fire research. Imprensa da Universidade de Coimbra.

Cochrane, M.A.; Schulze, M.D. 1993. Fire as a recurrent event in tropical forests of the eastern Amazon: effects on forest structure, biomass, and species composition. Biotropica 31: 2-16.

Fanin, T.; van der Werf, G. R. 2015. Relationships between burned area, forest cover loss, and land cover change in the Brazilian Amazon based on satellite data. Biogeosciences, 12: 6033-6043.

INPE. 2015. Amazon Program - Monitoring the Brazilian Amazon by satellite: The PRODES, DETER, DEGRAD and TerraClass Systems. Available at www.inpe.br (accessed 10 of May 2015).

Lima, A.; Silva, T.S.F.; Aragão, L.E.O.C.; Feitas, R.M.; Adami, M.; Formaggio, Antônio Roberto; Shimabukuro, Y.E. 2012. Land use and land cover changes determine the spatial relationship between fire and deforestation in the Brazilian Amazon. Appl. Geogr., 34:239-246.

Matricardi, E.A.T.; Skole, D.L.; Pedlowski, M.A.; Chomentowski, W.; Fernandes, L.C. 2010. Assessment of tropical forest degradation by selective logging and fire using Landsat imagery. Remote Sens. Environ., 114:1117-1129.

Miettinen, J.; Shimabukuro, Y.E.; Beuchle, R.; Grecchi, R.C.; Gomez, M.V.; Simonetti, D.; F., Achard. 2016. On the extent of fire-induced forest degradation in Mato Grosso, Brazilian Amazon, in 2000, 2005 and 2010. Int. J. Wildland Fire, 25:129136.

Morton, D.C.; Le Page, Y.; DeFries, R.; Collatz, G.J.; Hurtt, G.C. 2013. Understorey fire frequency and the fate of burned forests in Southern Amazonia,” Philos. Trans. Roy. Soc. B Biol. Sci., 368: 20120163.

Simonetti, D.; Marelli, A.; Eva H.D. IMPACT Toolbox, a Portable Open Source GIS Toolbox for Image Processing and Land Cover Mapping, Publications Office of the European Union, doi: 10.2788/143497. Last accessed: jun. 2016.

Shimabukuro, Y.E.; Smith, J.A. 1991. The least squares mixing models to generate fraction images derived from remote sensing multispectral data. IEEE Trans. Geosci. Remote Sens., 29:16-20.

Silva Monteiro, A.L.; Barreto, P.G.; Pantoja, F.L.D.S.; Gerwing, J.J. 2004. Impactos da exploração madeireira e do fogo em florestas de transição da Amazônia Legal. Scientia Forestalis, 65:11-21.

Souza, C.; Siqueira, J.; Sales, M.; Fonseca, A.; Ribeiro, J.; Numata, I.; Cochrane, M.; Barber, C.; Roberts, D.; Barlow, J. 2013. Ten-Year Landsat Classification of Deforestation and Forest Degradation in the Brazilian Amazon. Remote Sens., 511:5493-5513. 


\title{
USING SPATIAL POINT PATTERN ANALYSIS AS SUPPLEMENT FOR OBJECT-BASED IMAGE CLASSIFICATION OF TREE CLUSTERS
}

\author{
E. L. M. Tañada ${ }^{1}$, A. C. Blanco ${ }^{1,2}$ \\ ${ }^{1}$ Phil-LiDAR 2, Training Center for Applied Geodesy and Photogrammetry \\ ${ }^{2}$ Department of Geodetic Engineering, College of Engineering \\ University of the Philippines, Diliman, Quezon City, Philippines - elmtanada@gmail.com, ariel.blanco@coe.upd.edu.ph
}

KEY WORDS: trees, LiDAR, point pattern analysis, nearest neighbour, object-based image analysis, eCognition

\begin{abstract}
:
Single tree detection is important for monitoring tree plantations in order to estimate crop yield, which can be used for assessing food and biofuels. In the field of remote sensing, there have been studies regarding single tree extraction using Light Detection and Ranging (LiDAR) point cloud data. Canopy Height Model (CHM) can be simply derived from these LiDAR data where trees can be analysed using different physical characteristics such as tree crown width, and height. However, using these physical characteristics can't be very useful for identifying which trees are within a plantation, a forest, or a single tree. This is the motivation for developing algorithms of automated differentiation of random trees from plantation trees. Object Based Image Analysis (OBIA) was used to acquire the locations of individual trees. Trees were separated from other land cover in the image using a CHM derived from LiDAR point cloud data. Using watershed algorithm in eCognition, trees with good canopy spacing was easily distinguished. A spatial point pattern analysis was done from the extracted locations of trees to differentiate regularly patterned points from randomly distributed points. Nearest neighbour statistics was done to provide measurement of the distribution of the extracted points. The ZScore parameter from nearest neighbor was then added to the layers in eCognition as supplement to the available height derivatives such as mean, standard deviation, and different textural parameters. Supervised type of classification was used in order to classify the trees on the image. Training samples of each tree from the image were entered in Support Vector Machine (SVM) to classify the different trees. Two sets of validation were done to compare the results of using the object parameters only, and the object parameters and Z-Score. Addition of the Z-Score from the object parameters increased the obtained accuracy on both validation sets. Classification accuracy based on validation set 1 and set 2 increased by about $20 \%$ and $6 \%$, respectively. Additional spatial pattern analysis methods and validation can further prove the use of such layers in improving classification of trees.
\end{abstract}

\section{INTRODUCTION}

Based on different studies, classification of objects using object-based image analysis is dependent on the characteristics of each object. Mean, standard deviation and other textural parameters such as homogeneity and entropy are some of the information used. The methodology for classifying treedominant images used by Rollan, et al. (2015) utilized different LiDAR derivatives such as Normalized Digital Surface Model (NDSM), intensity, number of returns, and other textural data. The study achieved an overall accuracy of $87 \%$. It was also stated that it is important to follow an iterative process in classifying using Support Vector Machine (SVM) while not getting the expected output, and it is important to create sufficient samples for different variations of a specific class.

This study aims to extend the defining characteristic of each tree outside its crown footprint by adding spatial pattern analysis on top of the individual object characteristics in order to classify clusters of trees accurately. Observing the distribution of the trees near the target tree might provide additional information to lessen the confusion on classifying a randomly distributed tree to a spatially patterned tree.

\section{RELATED LITERATURE}

\subsection{Point Pattern Analysis}

Point pattern analysis is the evaluation of pattern, or distribution, of a set of points on a surface. The analysis of spatial point patterns came to prominence in geography during the late 1950s and early 1960s (Gatrell, et al., 1995). Spatial point patterns can be characterized as ranging from dispersed to clustered, with random point patterns having elements of both dispersion and clustering (Robeson, et al., 2014). It has been proven that spatial pattern analysis can be used on different problems. Ek (1969) identified three forest patterns, clustered, random and regular, using spatial point pattern analysis. Ek obtained indices of nonrandomness (coefficients of randomization) for the spatial distribution of stems using a method described by Pielou (1959). A study by Li and Zhang (2007) compared different point pattern analysis methods for classifying the spatial distributions of spruce-fir stands. The author presented four methods of spatial point pattern analysis (nearest neigbor, refined nearest neighbour, ripley's K function, and pair correlation function analysis), and performed them on 50 separate plots which were patterned as complete spatial random (CSR), cluster, or regular. Though $\mathrm{Li}$ and Zhang successfully compared the four methods, they pointed out that they did not use height and crown measurements of each tree.

\subsection{Support Vector Machine}

A Support Vector Machine (SVM) is a discriminative classifier formally defined by a separating hyperplane. In other words, given labelled training data (supervised learning), the algorithm outputs an optimal hyperplane which categorizes new examples (Ervin, D., n.d.).

SVM has become widely used in image classification. Also, the performance of SVM is comparable to other supervised classifiers like Multilayer Perceptrons neural networks and k- 
nearest neighbour statistical classifier (Roli, et al., n.d.). Mountrakis et al. (2010) stated in his review on SVM in remote sensing that SVMs outperformed even best neural networks when it comes to data with no normal or seen distribution, which is highly possible in remotely sensed images.

\section{MATERIALS AND METHODOLOGY}

\subsection{Study Area}

Study area is located in Nasipit, Agusan del Norte, Philippines. Selected area was based primarily on the available data, and the presence of different variations of trees and tree distribution. The study site includes coconut, mango, and banana plantations, and a few other trees (Figure 1).
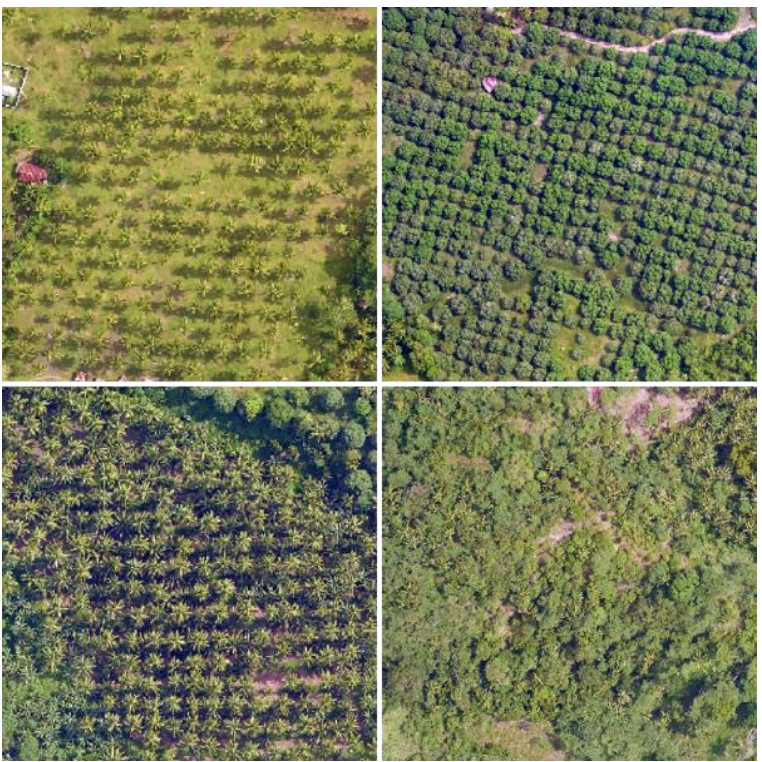

Figure 1. Orthophotos showing widely spaced coconut trees (upper left), coconut trees (lower left), mango trees (upper right), randomly distributed trees (lower right)

\subsection{Datasets}

Light Detection and Ranging (LiDAR) point cloud, generated by the DREAM Phil-LiDAR 1 Program, was used for the classification of trees. The point cloud has a density of 2 points per sq. m.. The data was pre-classified into ground and nonground. Orthophotos, which includes red, green and blue bands, were available but was only used for the selection of training and validation points. This is because the area contains four adjacent orthophotos which are uncalibrated, and using these images will result in confusion in the classification.

\subsection{Methodology}

\subsubsection{LiDAR Derivatives}

LiDAR point cloud was converted to a raster image using Lastools. Normalized Digitized Surface Model (NDSM) with a 0.5 meter resolution was derived from the point cloud.

\subsubsection{Object Based Image Analysis (OBIA)}

NDSM was imported in eCognition and it was used as the main image for OBIA. Roads, water, buildings, and power lines were initially segmented and classified. The following procedure was then focused on the remaining unclassified objects, ground and trees. Unclassified objects were segmented using multiresolution segmentation with a large scale to extract cluster of trees (Figure 2). The scale parameter used for the segmentation was 100; it was tweaked until the observed objects are homogeneous. Watershed algorithm and local maxima filter in eCognition was used to determine location of trees (Wulder, et al., 2000, Rollan, et al., 2015). The locations of trees produced in eCognition are 1 pixel size each; these points were exported as a point shapefile.

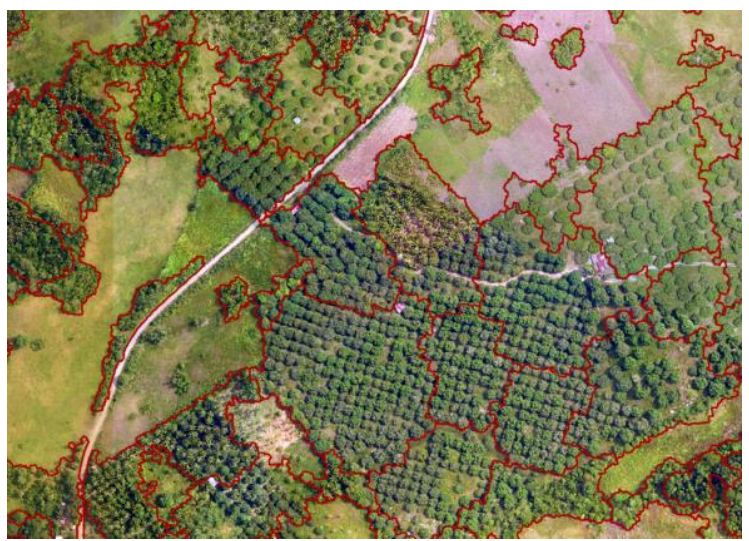

Figure 2. Initial segmentation to extract clusters of trees.

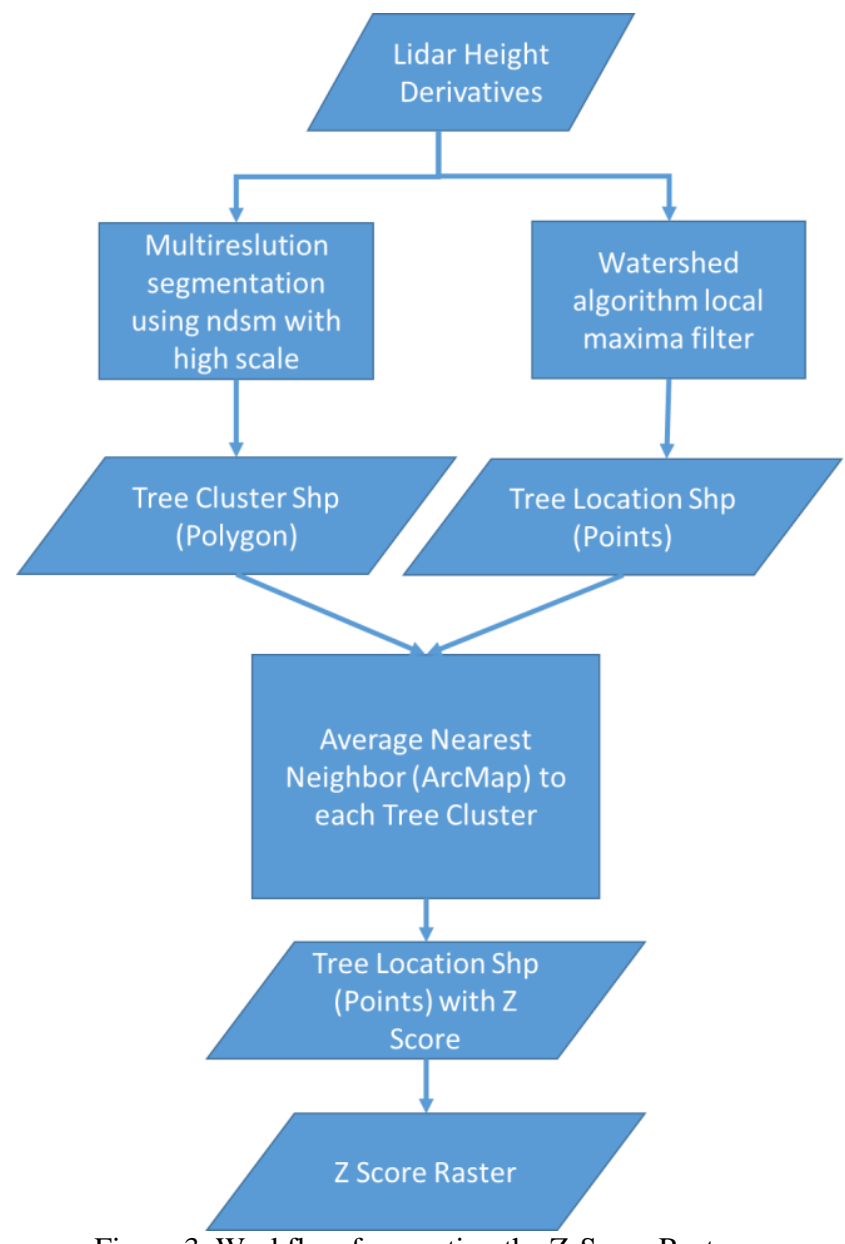

Figure 3. Workflow for creating the Z-Score Raster. 


\subsubsection{Point Pattern Analysis}

Tree cluster and tree location shapefiles were then imported in ArcMap. A tool was created using the ArcMap Model Builder to automatically iterate the Average Nearest Neighbor tool. This tool adds attributes in the cluster shapefile which shows the calculated nearest neighbour for each set of tree points inside each cluster. A raster image of Z-Scores of each cluster was created using the calculated attributes in the cluster shapefile (Figure 4), which was then imported in eCognition.

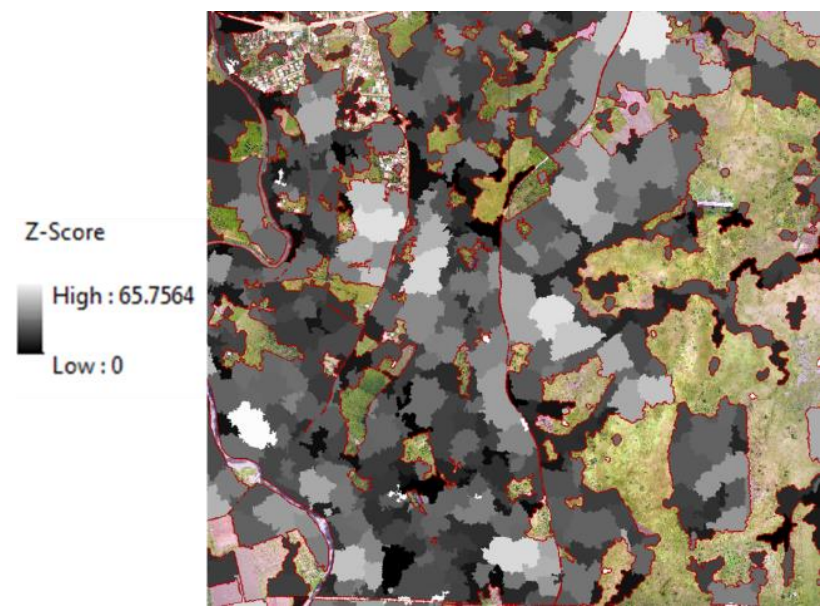

Figure 4. Z-Scores of each tree cluster.

\subsubsection{Tree Classification}

SVM was used for the classification of trees. The parameters that were used include Radial Basis Function kernel function, 200 for the $\mathrm{C}$ parameter, 1/(number of dimensions used) for the Gamma parameter (Carranza, et al., 2014). Training points were selected using the orthophoto. Around 50 samples per tree cluster type (coconut plantation, mango plantation, and randomly distributed) were selected for the classifier. Different combinations between NDSM (mean, standard deviation, homogeneity, and entropy) and Z-Score were tested to see if the addition of Z-Score is beneficial for the classification.

Combination A uses height statistics and height textures. And then, for combination B, Z-Score was added to combination A. For combination $\mathrm{C}$, height texture layers were removed from combination B. Lastly, Z-Score was tested by itself.

Table 1. Combination of layers used in SVM.

\begin{tabular}{|c|l|}
\hline \multicolumn{2}{|c|}{ Combination of Layers used in SVM } \\
\hline Combination & \multicolumn{1}{|c|}{ Layers } \\
\hline A & $\begin{array}{l}\text { NDSM (Mean, Standard Deviation, } \\
\text { Homogeneity, Entropy) }\end{array}$ \\
\hline B & $\begin{array}{l}\text { NDSM (Mean, Standard Deviation, } \\
\text { Homogeneity, Entropy), Z-Score }\end{array}$ \\
\hline C & $\begin{array}{l}\text { NDSM (Mean, Standard Deviation), Z- } \\
\text { Score }\end{array}$ \\
\hline D & Z-Score \\
\hline
\end{tabular}

There were two sets of validation samples created. Both sets were done similarly, but made sure the samples were not overlapping each other, and with the training samples. 20 samples for each tree cluster (coconut, mango, and random tree), for a total of 60 samples per set, were randomly selected for validation based on the orthophoto. Difference in accuracy was observed on the different combinations to analyse the addition of Z-Score on the SVM classifier. Also, the results from the two validation sets were compared to each other.

\section{RESULTS AND DISCUSSION}

Based on initial observation, Z-Score displayed a clear trend. Clusters with low Z-Score are those trees which were randomly distributed, and clusters with high Z-Score are trees arranged in patterns (Figure 5 and 6).
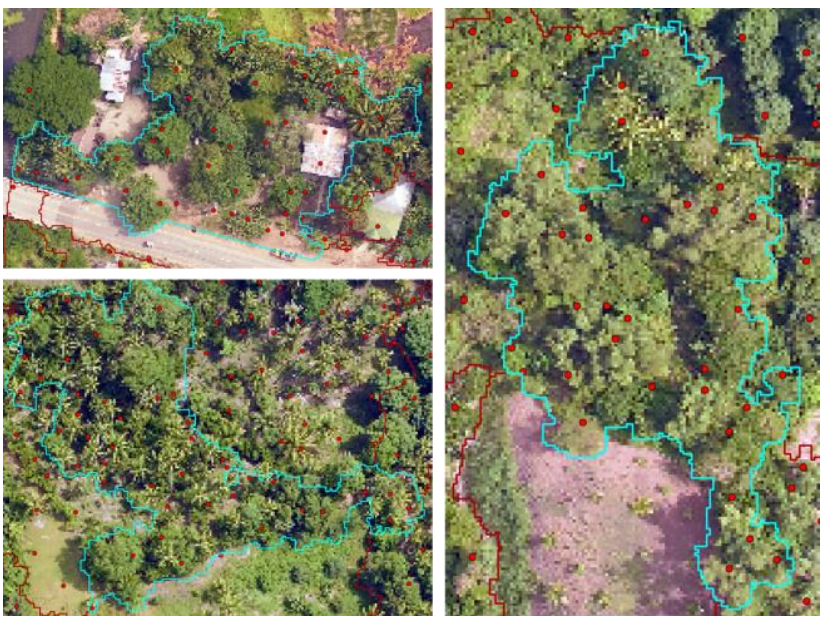

Figure 5. Tree clusters with low Z-Score
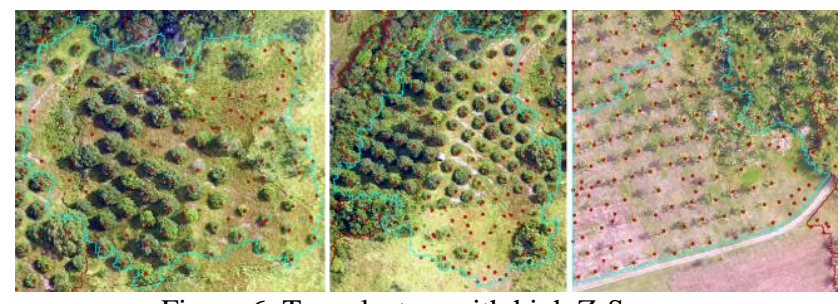

Figure 6. Tree clusters with high Z-Score

Table 2 shows the result of the accuracy of the classification using the two validation sets.

For validation set 1 , accuracy of combination A reached $73.21 \%$. Accuracy was raised to $93.76 \%$ when the Z-Score was added. And then, the accuracy went back down when the height textures was removed from combination B. Lastly, Z-Score alone scored the least accuracy of $74.96 \%$.

Table 2. Overall accuracy of tree cluster classification.

\begin{tabular}{|c|c|c|}
\hline \multirow{3}{|c|}{ Accuracy Assessment } \\
\hline \multirow{2}{*}{ Combination } & \multicolumn{2}{|c|}{ Validation Set } \\
\cline { 2 - 3 } & 1 & 2 \\
\hline A & $73.21 \%$ & $81.08 \%$ \\
\hline B & $93.76 \%$ & $87.45 \%$ \\
\hline C & $77.21 \%$ & $79.14 \%$ \\
\hline D & $74.96 \%$ & $55.81 \%$ \\
\hline
\end{tabular}

For validation set 2, accuracy of combination A resulted to $81.08 \%$. Adding the Z-Score raised the accuracy to $87.45 \%$ which is similar to validation set 1 . And then, it went down upon removal of the height textures. Z-Score by itself acquired the lowest accuracy.

Comparing the two validation sets, it was observed that in both of them, combination B reached the highest accuracy. But 
using Z-Score alone attained the lowest accuracy. Removing either the height textural layers or the Z-Score from combination $\mathrm{B}$ resulted to a decrease in accuracy.

\section{CONCLUSION AND RECOMMENDATIONS}

The use of information on the distribution of a tree with respect to its neighboring trees, together with the statistical and textural parameters of the object, increased the accuracy of classification using SVM. Aside from the parameters derived from the point cloud data, extending the analysis outside the object can help in identifying and classifying trees by using the Z-Score of the tree clusters. Other point statistics layers may further improve the classification. Additional spatial pattern analysis methods and validation can further prove the use of such layers in improving classification of trees.

\section{ACKNOWLEDGEMENTS}

This study is made possible by Phil-LiDAR 2 Program funded by the Department of Science and Technology (DOST) and monitored by the Philippine Council for Industry, Energy and Emerging Technology Research and Development (PCIEERD). The Project is also supported by the Department of Agriculture Information Technology Center for Agriculture and Fisheries (DA-ITCAF).

The methodologies were developed using the aerial and point cloud data from DREAM/Phil-LiDAR 1 Program implemented by the University of the Philippines Training Center for Applied Geodesy and Photogrammtery.

\section{REFERENCES}

Disaster Risk and Exposure Assessment for Mitigation (DREAM) Program. (2016). The UP DREAM Program. Retrieved June 29, 2016, from Disaster Risk and Exposure Assessment for Mitigation (DREAM) Program: https://dream.upd.edu.ph/about/

Ek, A. R., (1969). Stem Map Data for Three Forest Stands in Northern Ontario. Information Report 0-X-113, Canadian Forest Service, Department of Fisheries and Forestry.

Ervin, D. (n.d.). Point Pattern Analysis. Retrieved June 2016, from http://gispopsci.org/point-pattern-analysis/

Gatrell, A. C., Bailey, T. C., Diggle, P. J., Rowlingson, B. S., (1995). Spatial Point Pattern Analysis and its Application in Geographical Epidemiology. Transactions of the Institute of British Geographers, New Series 21 (1): 256-274

Jerez, M. V., Carranza, C. U., Gatdula, N. B., Rollan, T. M., Tañada, E. M., Pagkalinawan, H. M., et al. (2015). Phil-LiDAR 2 Project 1: Agricultural Resources Extraction from LiDAR Surveys (PARMap) Technical Narrative Report. Quezon City, Metro Manila, Philippines.

Li, F., Zhang, L., (2007). Comparison of Point Pattern Analysis Methods for Classifying the Spatial Distributions of Spruce-Fir Stands in the North-East USA. Forestry 80 (3): 337-349

Mountrakis, G., Ogole, C., \& Im, J. (2011). Support vector machines in remote sensing: A review. ISPRS Journal of Photogrammetry and Remote Sensing, 247-259.
Pielou, E. C., (1959). The Use of Point-to-Plant Distances in the Study of the Pattern of Plant Populations. The Journal of Ecology 47(3): 607-613

Robeson, S. M., Li, A., \& Huang, C. (2014). Point Pattern Analysis on the Sphere. Spatial Statistics 10: 76-86

Roli, F., Fumera, G., (n.d.). Support Vector Machines for Remote-Sensing Image Classification. Retrieved June 2016 from

http://citeseerx.ist.psu.edu/viewdoc/download?doi=10.1.1.11.58 $30 \&$ rep $=$ rep $1 \&$ type $=$ pdf.

Rollan, T. A., Carranza, C., Jerez M., Blanco A.C., (2015). Combining Watershed Transformation and Local Maxima Approach in Developing a Tree Detection and Counting. PhilLiDAR 2 Project 1: Agricultural Resources Extraction from LiDAR Surveys (PARMap) Technical Narrative Report. Quezon City, Metro Manila, Philippines.

Wulder, M., Niemann, K. O., Goodenough, D. G., (2000). Local Maximum Filtering for the Extraction of Tree Locations and Based Area from High Spatial Resolution Imagery. Retrieved June 2016 from http://www.sciencedirect.com/science/article/pii/S00344257000 01012 


\title{
GULLY EROSION MAPPING WITH HIGH RESOLUTION IMAGERY AND ALS DATA BY USING TREE DECISION, HIERARCHICAL CLASSIFICATION AND OBIA
}

\author{
A. Tedesco ${ }^{\text {a }, \text { A. F. B. Antunes }}{ }^{\text {b }}$, S. R. A. Ribeiro ${ }^{a}$ \\ a Dept. of Geosciences, Ponta Grossa State University, Paraná, Brasil - (atedesco@uepg.br, selmar.aranha@gmail.com) \\ ${ }^{\mathrm{b}}$ Dept. of Geomatic, Federal University of Paraná, Paraná, Brasil - felipe@ufpr.br
}

KEY WORDS: Gully Erosion, ALS Data, Multiespectral Data, High Resolution Imagery, OBIA, Multirresolution Segmentation, Hierarchical Classification, Decision Tree.

\begin{abstract}
:
The gully erosion presents spectral and spatial heterogeneity and altimetry variation. It is not a land use class, but an object and it can be mapped as a subclass, using OBIA. This study presents a methodology for delimitation of gullies in rural environments, based on image classification procedures. For such, two study areas were selected: one located in Minas Gerais, Brazil and another one located in Queensland, Australia. There were used high resolution images and ALS data. The objects were generated by multiresolution segmentation method. The most important attributes in the definition of gullies were selected using decision tree induction algorithms, being these attributes spectral, altimetry and texture. Classifications hierarchical and by decision trees were carried out. Using decision tree the classification is performed only by a factor of scale, not allowing the identification of all the constituent features of the gully system. In hierarchical classification, the procedure is performed at different scales and allowing to use of fuzzy logic. The classification obtained with hierarchical classification showed results more reliable with the field of reality, by allowing the use of different scales, fuzzy logic and integration of knowledge (the established rule base) compared to the automatic classification by decision tree. As different gullies erosion are similar when presents the same evolution stage and soil type, it is not possible to select attributes to classify all gully systems, being necessary to investigate attributes for each gully erosion, based on available data and existing land use classes in the area.
\end{abstract}

\section{INTRODUCTION}

The gullies are the biggest erosive processes and, consequentely, responsible for ambiental, social and financial damages. Corrective and preventive measures need mapping and monitoring, which can be made by local measurements or by remote sensing.

Local measurements can be done by staking (Hessel e Van Asch, 2003; Morgan, 2005), by topographic surveys, by GNSS receivers, or using TLS (Terrestrial LASER Scanning) (Perroy et al., 2010). However, these methods needs traversal and equipment installation on edges and inside the gullies, which can aggravate erosive processes and it can be a risk for surveyours.

Remote sensing monitoring has been carried out by using aerophotos (Marzolff; Poesen, 2009), or multiespectral images (King et al., 2005; Vrieling; Rodrigues; Sterk, 2005), or DTM (Digital Terrain Model) (Martínez-Casasnovas; Ramos; Poesen, 2004), or ALS (Airborne LASER Scanning) data (James; Watson; Hanse, 2007; Eustace; Pringle; Witte, 2009). Recently, researches have used OBIA for detection, mapping, monitoring, volume calculation and predictive models of erosion risk.

In relation to the remote sensing, the gully erosion presents spectral heterogeneity (soil, vegetation, shade and water mix), spatial heterogeneity (existence of features as head, canals and digits with irregular forms and variable dimensions) and altimetry variation (with high declivity on the edges). Due to spectral heterogeneity, it is not enough use only spectral data, being necessary auxiliary data, as altimetry and texture data. Using auxiliary data is recommended to use data mining.

In this context, this study proposed a methodology for delimitation of gullies on image classification procedures based on OBIA (Object Based Image Analysis), identifying attributes to establish a decision rule base. For such, there were used an Ikonos image, an orthophoto and ALS altimetry and intensity data of an area located in Uberlandia - Minas Gerais - Brasil and of an area located in Queensland - Australia. The objects were generated by multiresolution segmentation (FNEA-Fractal Net Evolution Approach method). The most important attributes in the gullies mapping were selected by decision tree, being these attributes spectral, altimetry and texture, and a classification by tree decision was carried out. The hierarchical classification was carried out and presented satisfactory results, by allowing the use of different scale factors, uncertainty insert (by fuzzy logic) and integration of knowledge (the established rule base) compared to the automatic classification by decision tree.

\section{METHODS}

\subsection{Data}

For the Brazilian study area, there were used a 1 meter spatial resolution and 11 bits radiometric resolution Ikonos image, illustrated by Figure 1 (presented in a coloured composition $\mathrm{R}=3, \mathrm{G}=4, \mathrm{~B}=1$, with coordinates related to WGS84 - UTM zone $51^{\circ} \mathrm{W}$ ), and ALS data from ALTM 2025 Optech (1 meter spatial resolution rasterized).

For the Australian study area, there were used a 0,5 meter spatial resolution and 8 bits radiometric resolution orthophoto, illustrated by Figure 2 (with coordinates related to GDA94 MGA 1994 zone 55), and ALS data from Riegl LMS-Q560 (0,5 meter spatial resolution rasterized).

The procedures were carried out by using ENVI (The Environment for Visualizing Images) 4.7, ALDPAT (Airborne LiDAR Data Processing and Analysis Tools) and eCognition Developer 8.8. 


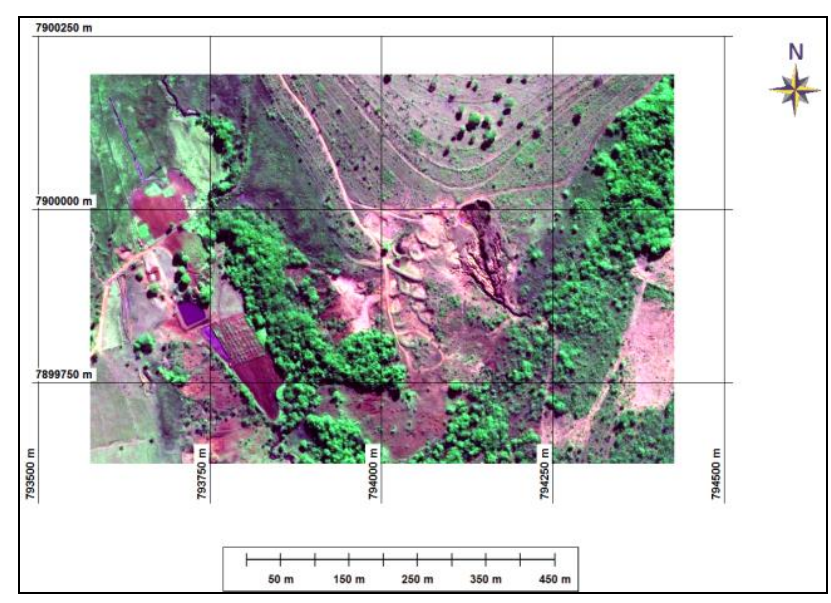

Figure 1. Study area in Minas Gerais, Brazil

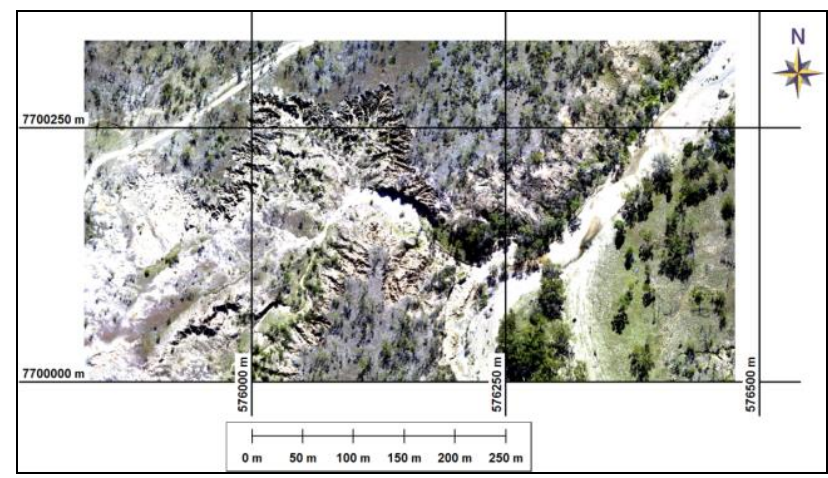

Figure 2. Study area in Queensland, Australia

\subsection{Methods}

\subsubsection{Spectral and altimetry data processing}

From the ALS data, there were generated the DSM (Digital Surface Model) and intensity image for both study areas, by linear interpolation. From the DSM, there were generated the DTM, the nDSM (Normalised Digital Surface Model) and the slope map.

The NDVI (Normalised Difference Vegetation Index) was generated by Ikonos Image. For the Australian study area, as the infrared image is not available, we propose a new index based on intensity image. The Intensity Based Contrast Index - IBCI, can be obtained from:

$$
\mathrm{IBCI}=(\operatorname{Red}-\text { Intensity }) /(\operatorname{Red}+\text { Intensity })
$$

Unlike NDVI, the IBCI highlights the ground and not the vegetation. There is obviously a need for spectral and altimetry data and it has been acquired at the same time and therefore are georeferenced and correspond to the same date.

\subsubsection{Generating objects}

The objects were obtained by using multiresolution segmentation applied to spectral data (because there are not a perfect coincidence between edges in the image and in the ALS data), ranging the scale factor between 5 and 100, with multiple range of 5 . The composition of homogeneity criterion was shape $=0.1$ and compactness $=0.5$.

\subsubsection{Classification}

For the Brazilian study area, there were identified these land use classes: tree vegetation, ground vegetation, shadow, water, bare soil and gully erosion. For the Australian study area, there were identified these land use classes: vegetation, shadow, bare soil and gully erosion.

The spectral, geometric, texture and context attributes available in eCognition software were processed using CART (Classification And Regression Trees) algorithm, and a decision tree was obtained for each study area, for objects generated with a scale factor $(\mathrm{SF})=50$. A classification was carried out applying this decision tree.

Refining the attributes and ranges obtained from the decision tree and inserting the expertise knowledge, a hierarchical classification was carried out.

A hierarchical classification evaluation, for both study areas, was performed by confusion matrix, selecting verifying samples.

\section{RESULTS}

\subsection{Spectral and altimetry data processing}

The Figure 3 shows the DTM and the Figure 4 shows the slope map obtained for Brazilian study area. Figures 5 and 6 showed the same for the Australian study area. It is possible to verify that altimetry data is an important auxiliary data in gully erosion mapping. In the slope map the gully system edge is evidenced because of its high declivity.

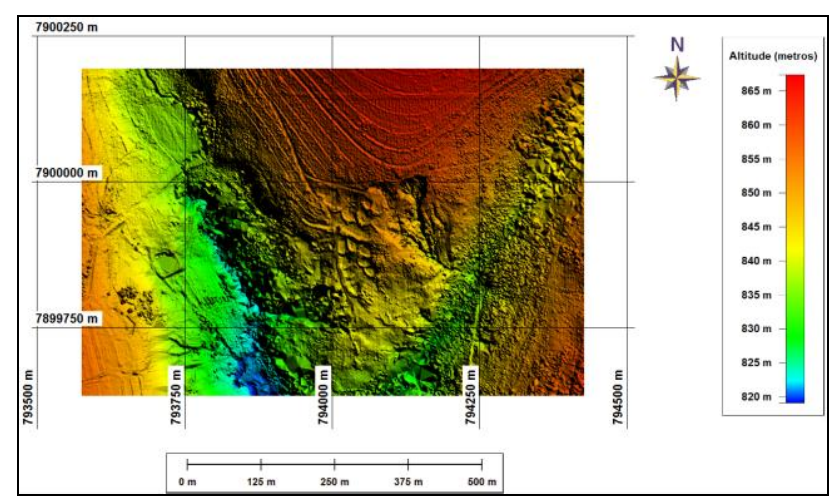

Figure 3. DTM - Brazilian study area

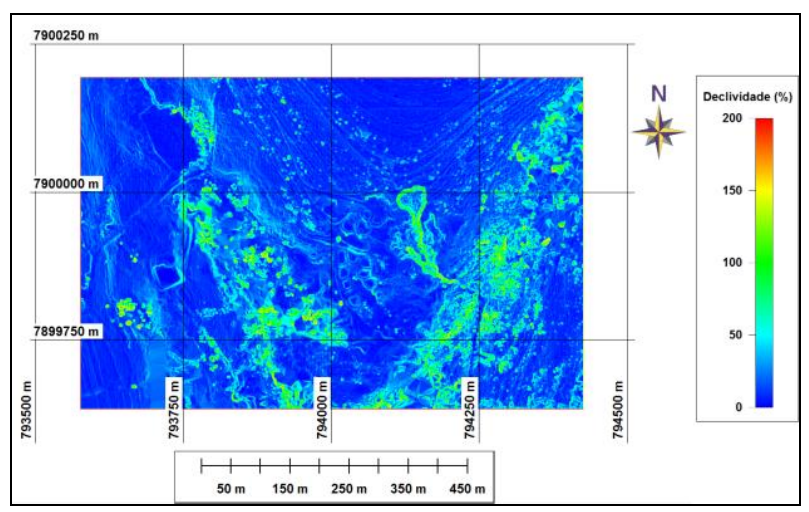

Figure 4. Slope map - Brazilian study area 


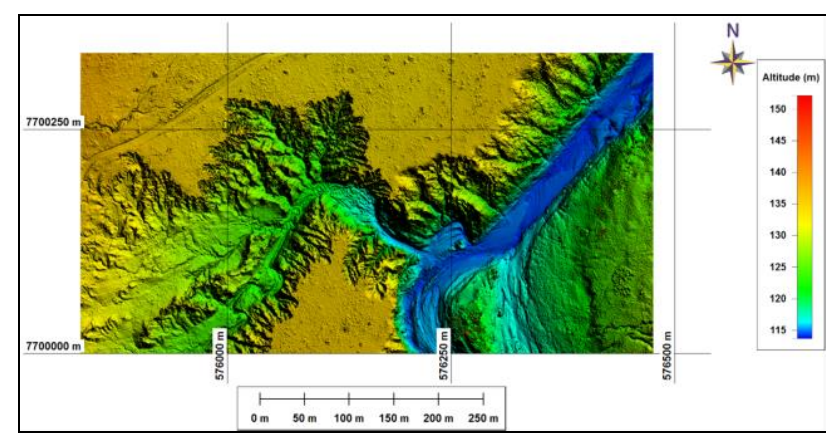

Figure 5. DTM - Australian study area

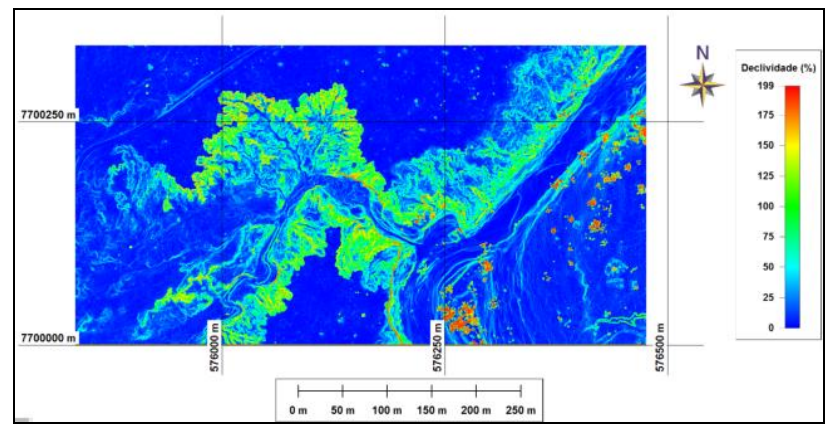

Figure 6. Slope map - Australian study area

The Figure 7 shows NDVI image for the Brazilian study area. The vegetated and not vegetated areas can be discriminated, as well tree vegetation and ground vegetation areas. The IBCI image was generated for the Australian study area, as shown in the Figure 8. The areas with soil are evidenced. The Figure 9 shows the IBCI image for the Brazilian study area. Comparing to Figure 7, it is evident the featured in the soil areas.

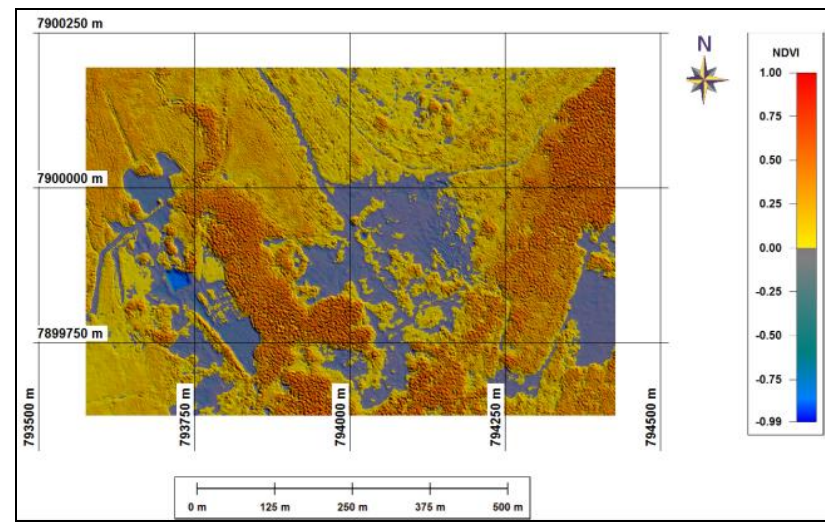

Figure 7. NDVI image - Brazilian study area

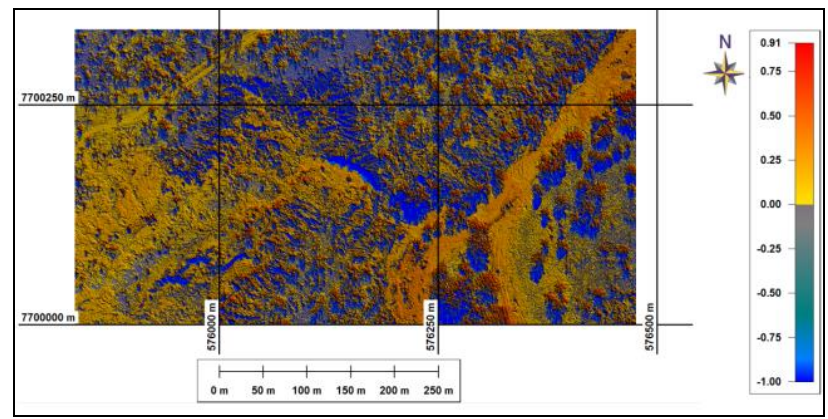

Figure 8. IBCI image - Australian study area

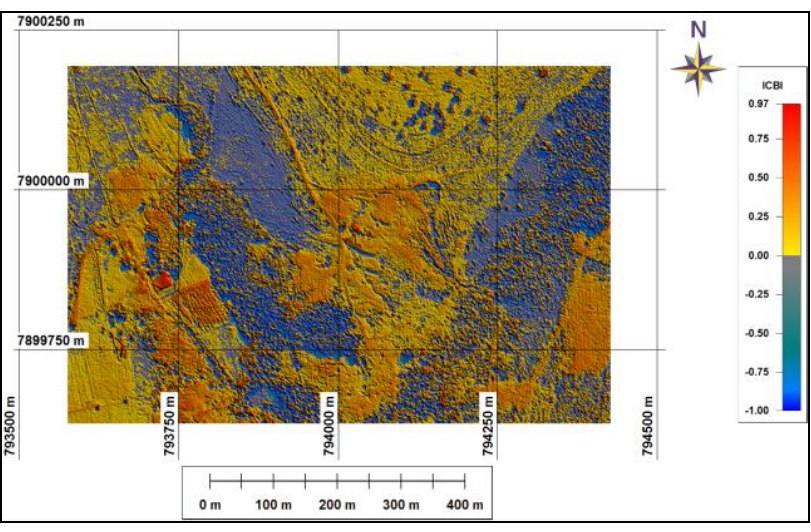

Figure 9. IBCI image - Brazilian study area

\subsection{Classification}

\subsubsection{Classification using tree decision}

The data mining carried out by CART algorithm resulted in the decision trees showed in the Figures 10 and 11 (Brazilian and Australian areas, respectively). For the Brazilian study area, in a first node, using circular mean applied to nDSM, the samples were divided in an intermediate node 1 and in a leaf of the Tree Vegetation class. In the node 1, using circular mean applied to NDVI, the samples were divided in a leaf of the Water class and in the intermediate node 2 . In the node 2 , using NDVI, the samples were divided in intermediate nodes 3 and 4 . In node 3, using circular mean applied to slope map, the samples were classified in Soil class or in Gully erosion class. In node 4, using Brightness, the samples were classified in Shadow or Ground Vegetation classes. For this data set, the major attribute for gully classification was the slope.

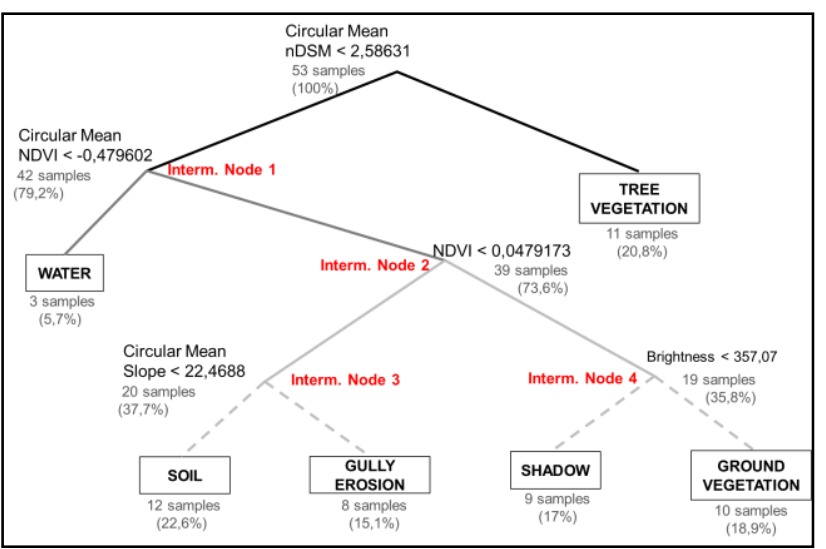

Figure 10. Decision tree - Brazilian study area

For the Australian study area, in a first node, using Texture after Haralick (GLDV entropy), applied to slope map, the samples were divided in an intermediate node 1 and in a leaf of the Gully Erosion class. In the node 1, using Green band, the samples were divided in a leaf of the Shadow class and in the intermediate node 2 . In the node 2 , using Blue band, the samples were divided in a leaf of the Vegetation class and in the intermediate node 3 . In the node 3, using Texture after Haralick (GLDV entropy) applied to slope map, the samples were classified in Soil class or in Vegetation class. 


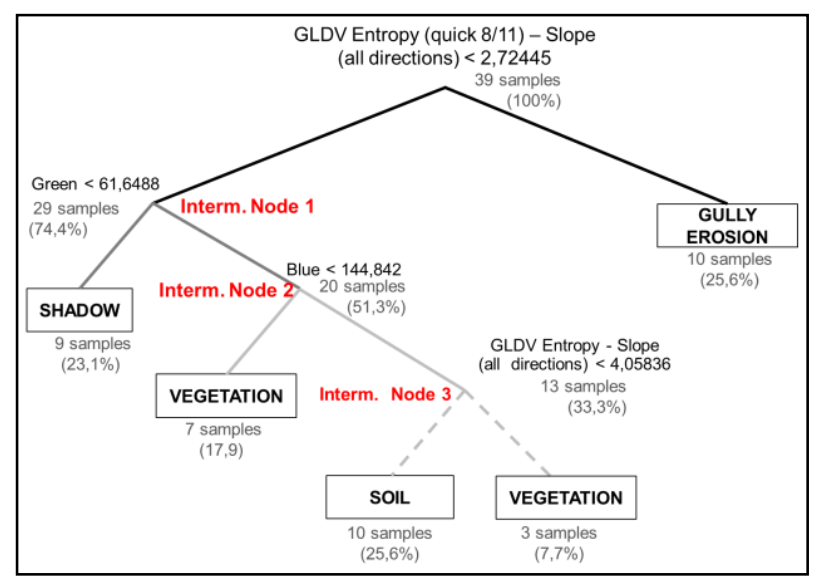

Figure 11. Decision tree - Australian study area

The Figure 12 shows the Brazilian's study area classification by decision tree. It is possible verify that occurred confusion between gully erosion and vegetation classes, in the edges of the tree vegetation due to the high declivity in these areas (height difference between ground level and tree tops). Even with the similarity between soil and gully erosion classes there was few confusion areas due to the use of the declivity attribute to separate these classes. The gully system neighbourhood was incorporated to the gully class because it was used the circular mean of the slope map attribute which expanded gully erosion area to the soil area. There was confusion between tree and ground vegetation and the shadows areas were evidenced.

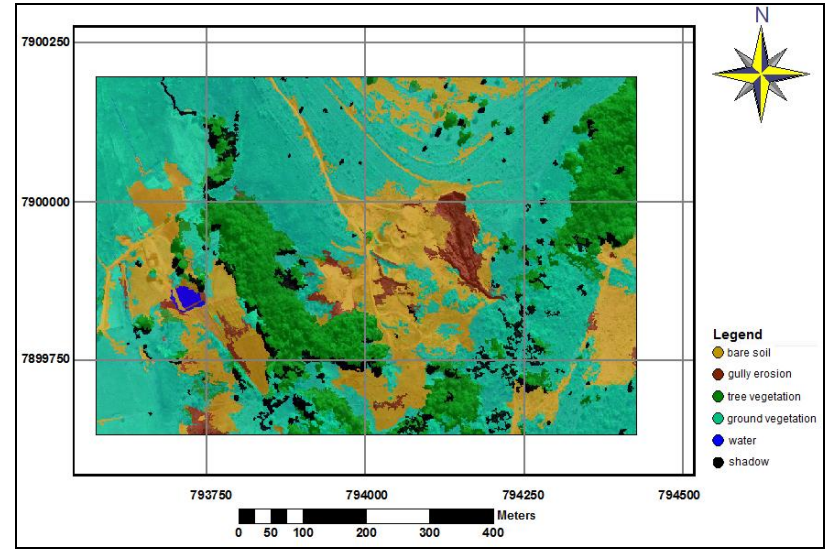

Figure 12. Classification by decision tree - Brazilian study area

The Figure 13 shows the Australian's study area classification by decision tree. There was confusion between gully erosion and vegetation classes, due to the high declivity between ground level and tree tops. The shadows areas were evidenced.

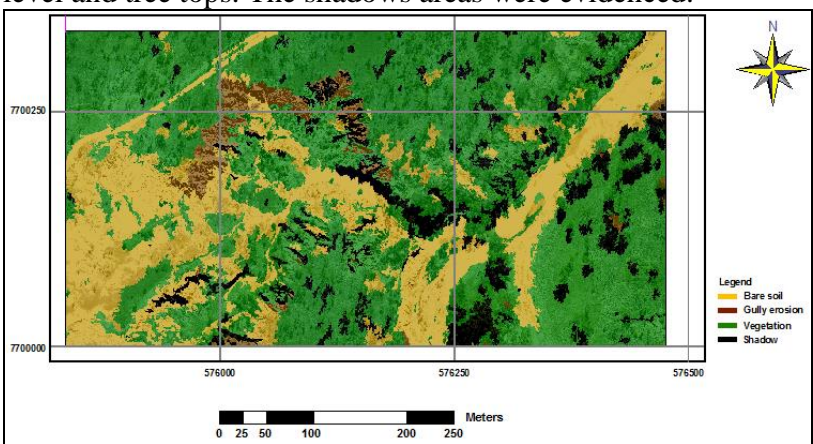

Figure 13. Classification by decision tree - Australian study area

\subsubsection{Hierarchical Classification}

Based on the attributes selected by CART algorithm and in the expertise knowledge, were selected attributes, fuzzy membership functions and scale factors (SF) to discriminate each class. The Figure 14 and 15 shows the hierarchical rule bases for Brazilian and Australian study areas respectively.

* note: $\mathrm{NN}=$ nearest neighbour algorithm; $\mathrm{SF}=$ scale factor

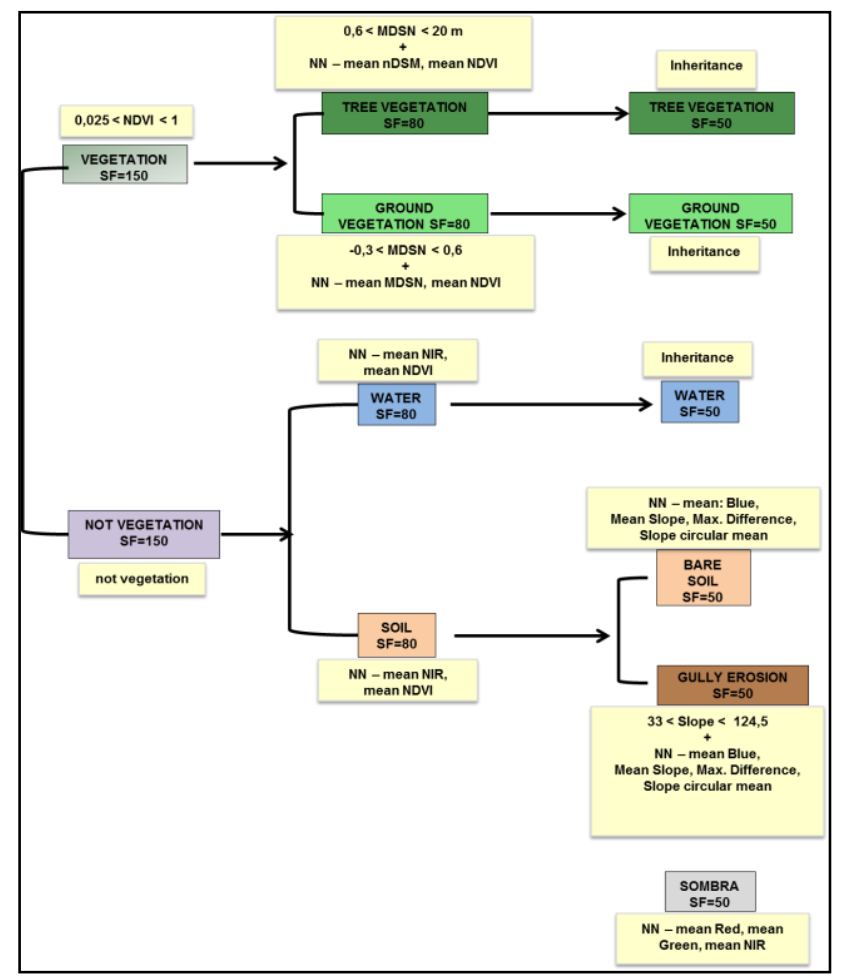

Figure 14. Hierarchical rule base - Brazilian study area

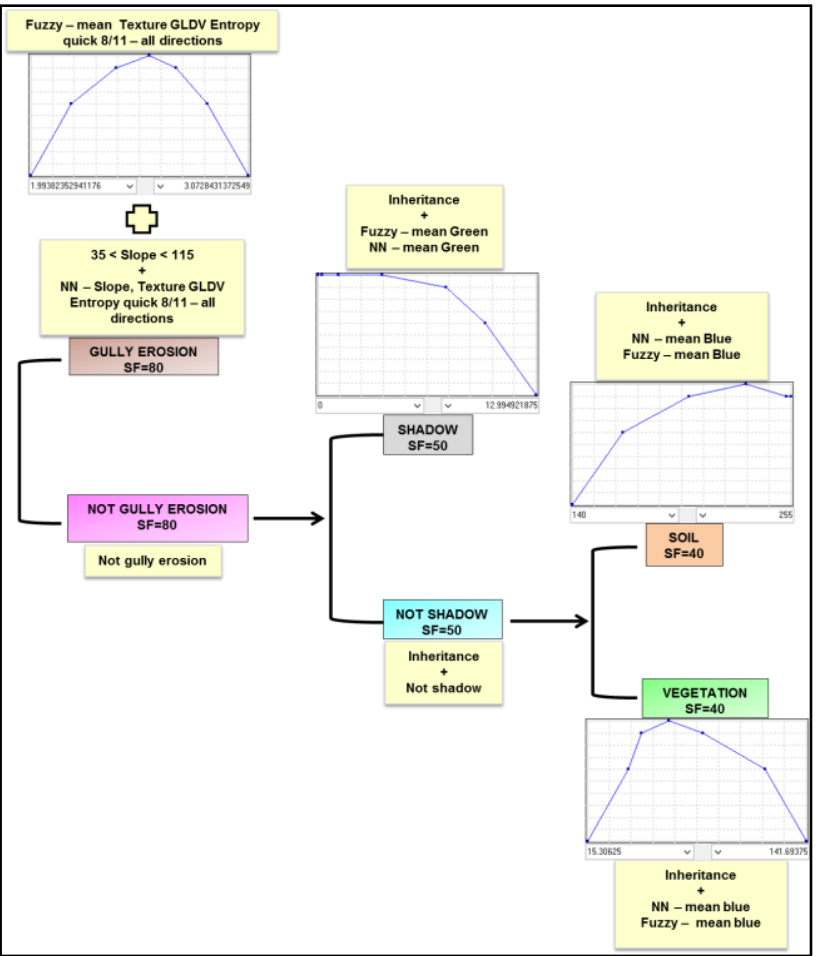

Figure 15. Hierarchical rule base - Australian study area 
The Figure 16 shows the Brazilian's study area hierarchical classification. The gully system edge was better mapped than by tree decision classification. The inside areas of the gully system were classified in Soil class due to the use of slope attribute. (the declivity is low inside the gully). The water body and vegetated areas were better mapped in this classification.

In the Brazilian study area image there are 2394 image objects and 217 samples were selected, with $95 \%$ confidence interval (error $=6.35 \%$ ), yielding kappa index $=0.75$ and overall accuracy $=82 \% .3$ of 14 gully samples were classified as bare soil and 1 as ground vegetation, 3 of 42 bare soil samples were classified as gully.

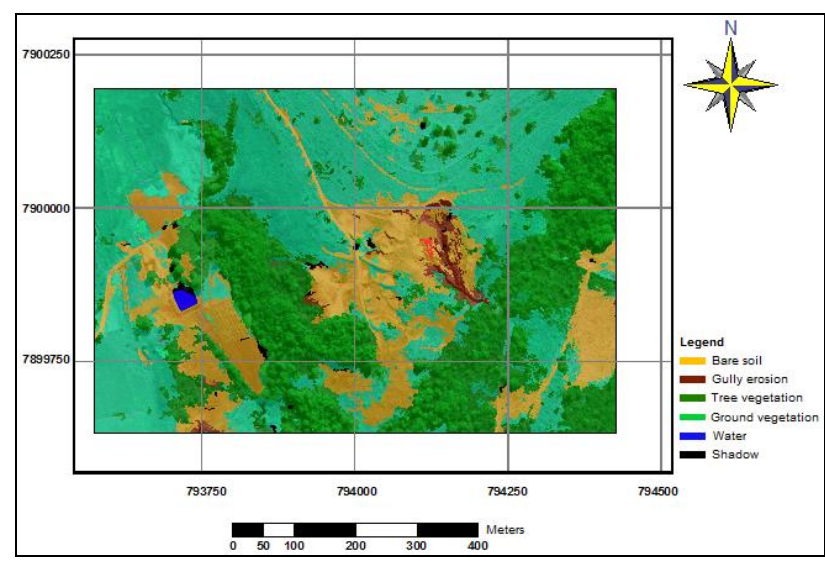

Figure 16. Hierarchical classification - Brazilian study area

The Figure 17 shows the Australian's study area hierarchical classification. The gully system was better classified than in the classification by tree decision. The mainly attributes selected for gully erosion classification were slope and texture. As vegetated areas have high declivity and texture too, there was confusion between gully an vegetation classes.

In the Australian study area image there are 2265 image objects and 865 samples were selected, with $99 \%$ confidence interval (error $=5 \%$ ), yielding kappa index $=0.46$ and overall accuracy $=64.05 \% .3$ of 102 gully samples were classified as vegetation, 29 as bare soil and 1 as shadow. 61 of 127 vegetation samples were classified as gully, 27 of 309 of bare soil samples were classified as gully and 11 of 16 shadow samples were classified as gully.

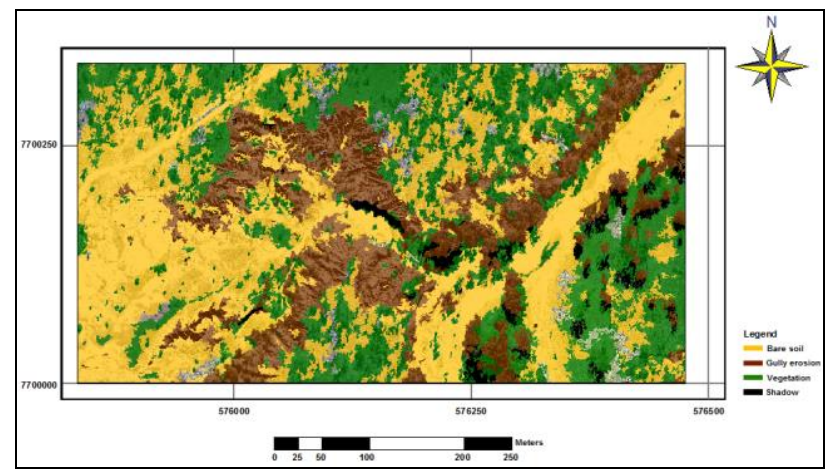

Figure 17. Hierarchical classification - Australian study area

\section{CONCLUSIONS}

As the gully systems are composed of features with highly variable shapes and sizes, the gullies could be mapped from high resolution imagery, with auxiliary altimetry data and by OBIA. Using hierarchical classification, it is possible to select different scale factors appropriated for gully features with variable sizes. For both data sets the scale factor equals to 50 was enough to map gullies.

As the gully systems have higher similarities when they correspond to the same stage of evolution and soil type, for example, there is no way to select attributes that are appropriate to the classification of all systems, requiring the investigation of discriminant attributes for each gully system, including on the basis of available data and existing land use classes in the scene.

Data mining by decision tree allowed rapid analysis and the best attributes selection, subsidizing the decision making process, replacing empiricism, providing a preliminary decision rule base, which can be adjusted according to the expert knowledge to realization of hierarchical classification. A disadvantage of the classification by decision trees is that you can only do it at one level of segmentation.

For data sets available and the specificities of the two study areas, the attributes that were more relevant to the discrimination of the gully class were the slope and texture. Regarding the data set, it is emphasized that the use of spectral data, high spatial resolution, coupled with the use of altimetry data allows the classification of gullies.

As the gully is an object and not a land use class, only using object-oriented classification procedures is that it can be defined.

Note also that the index proposed IBCI allowed the enhancement of the soil, being an alternative in case of unavailability of the infrared band, but the availability of ALS intensity band.

\section{ACKNOWLEDGEMENTS}

The authors wish to thank the Remote Sensing Centre, Queensland Department of Environment and Resource Management (Mr. Dan Tindall) for giving ALS data and orthophoto of the Queensland study area. Also thank the Engesat Imagens de Satélite Ltda (Mrs. Jaquelin Cavalli) for giving Ikonos imagery and Esteio Engenharia $\mathrm{e}$ Aerolevantamentos S.A. (Mrs. Elizabete B. A. Peixoto) for giving ALS data of the Uberlandia study area.

\section{REFERENCES}

Eustace, A.; Pringle, M.; Witte, C., 2009. Give me the dirt: Detection of Gully Extent and Volume Using High-Resolution LIDAR. In: Innovations in Remote Sensing and Photogrammetry: Lecture Notes in Geoinformation and Cartography, Berlin, Springer publisher, pp. 255-269.

Hessel, R.; Van Asch, T., 2003. Modelling gully erosion for a small catchment on the Chinese Loess Plateau. In: Catena 54, pp. 131-146.

James, L. A.; Watson, D. G.; Hanse, W. F., 2007. Using LiDAR data to map gullies and headwater streams under forest canopy: South Carolina, USA. In: Catena. 71, pp. 132-144. 
Johansen, K.; Taihei, S.; Tindall, D.; Phinn, S., 2012. Objectbased Monitoring of Gully Extent and Volume in North Australia using LiDAR Data. In: Proceedings of the 4th GEOBIA, Rio de Janeiro, pp. 168-173.

King, C.; Baghdadi, N.; Lecomte, V.; Cerdan, O., 2005. The application of remote-sensing data to monitoring and modelling of soil erosion. In: Catena 62, pp. 79-93.

Martínez-Casanovas, J. A.; Ramos, M. C.; Poesen, J., 2004. Assessment of sidewall erosion in large gullies using multitemporal DEMs and logistic regression analysis. In: Geomorphology 58, pp. 305-321.

Marzolff, I.; Poesen, J., 2009. The potential of 3D gully monitoring with GIS using high-resolution aerial photography and a digital photogrammetry system. In: Geomorphology 111, pp. $48-60$.

Morgan, R. P. C., 2005. Soil Erosion and Conservation, Oxford: Blackwell Publishing.

Perroy, R. L.; Bookhagen, B.; Asner, G. P.; Chadwick, O. A., 2010. Comparison of gully erosion estimates using airborne and ground-based LiDAR on Santa Cruz Island, California. In: Geomorphology 118, pp. 288-300.

Shruthi, R. B. V.; Kerle, N.; Jetten, V., 2011. Object based gully feature extraction using high spatial resolution imagery. In: Geomorphology 134, pp. 260-268.

Shruthi, R. B. V.; Kerle, N.; Jetten, V., 2012. Extracting gully features and its dynamics from high spatial resolution imagery using object based image analysis. In: Proceedings of the 4th GEOBIA, pp. 7-12.

Vrieling, A.; Rodrigues, S. C.; Sterk, G., 2005. Evaluating erosion from space: a case study near Uberlândia. In: Revista Sociedade \& Natureza, pp. 683-696.

Wang, T.; He, F.; Zhang, A.; Gu, L.; Wen, Y.; Jiang, W.; Shao, H., 2014. Quantitative Study of Gully Erosion Based on ObjectOriented Analysis Techniques: A Case Study in Beiyanzikou Catchment of Qixia, Shandong, China. In: The Scientific World Journal, pp. 1-11. 


\title{
NATIONAL FUEL TYPE MAPPING METHODOLOGY USING GEOGRAPHIC OBJECT BASED IMAGE ANALYSIS AND LANDSAT 8 OLI IMAGERY.
}

\author{
M. Tompoulidou ${ }^{\text {a, } *}$, A. Stefanidou ${ }^{\text {a }}$, D. Grigoriadis ${ }^{\text {a }}$, E. Dragozi ${ }^{\text {a }}$, D. Stavrakoudis ${ }^{\text {a }}$, I.Z. Gitas ${ }^{\text {a }}$ \\ ${ }^{a}$ Forest Management and Remote Sensing Laboratory, Aristotle University of Thessaloniki 55124 Thessaloniki, P.O. Box 248, GR- \\ 54124, Greece
}

KEY WORDS: Fuel type mapping, Landsat-8 OLI, Object based image analysis, LUCAS points

\begin{abstract}
:
A key issue in modern fire management planning is the accurate fuel type mapping, required at many different spatial and temporal scales. Fuel type classification is critical for improving fire prevention schemes, developing accurate fire dispersion models and designing effective measures for mitigating the impacts of a potential wildfire event on the ecosystem. Remote sensing offers the potential to provide spatially distributed information on fuel types and it has been widely used both at regional and local scale. The aim of this study is to develop and evaluate a fuel type mapping methodology on a national level, based on the geographic objectoriented classification approach and Landsat-8 OLI imagery. The proposed methodology was developed in the case study of Chalkidiki in the northern part of Greece and it was further tested for its transferability in the regional unit of Preveza and the whole administrative region of Attica. The classification scheme was determined taking under consideration the existing fuel models (Prometheus, FBP etc) and fuel type products (JRC FUELMAP, LIFE10 ArcFUEL) so as the final fuel type map could be easily adjusted and/or compared. Fifty-four Landsat-8 OLI images for summer and for winter season, covering the national territory, were acquired and several features (vegetation indices, textural and spectral features) were calculated for both seasons. The optimized feature selection for the discrimination of each fuel type category was empirically obtained. Results showcase the effectiveness of the employed object-oriented classification approach in obtaining highly accurate fuel type maps. Specifically, the generated fuel map of Chalkidiki exhibited an overall accuracy of $89.47 \%$, with a Kappa Index of Agreement (KIA) equal to 0.844 . The application of the model to the two other regions resulted in overall accuracies of $80.30 \%$ (KIA=0.706) for Attica and 91.74\% (KIA=0.867) for Preveza. Summarizing, the results proved the good transferability properties of the proposed methodology enabling the implementation of the model across the country.
\end{abstract}

\section{INTRODUCTION}

Efficient forest fire management is the key element for alleviating the catastrophic impacts of wildfires, which involves both adequate planning and preparedness before the start of the fire season as well as a quantification of environmental impacts in case of wildfires (Martín-Gómez et al. 2015). Landscapelevel fuel maps are essential for local fire management, as they describe fire potential for planning and prioritizing specific burn incidents (Chuvieco et al. 1989). The design and consequent success of such measures is highly dependent on the availability and reliability of up-to-date fire-related data. The quality, completeness and reliability of such data need to be further and constantly improved, offering operational and procedural standardization and data interoperability (SanMiguel-Ayanz et al. 2012). Remote sensing offers the potential to provide spatially distributed information regarding the state of the managed areas and it has been widely used both at regional and local scale (Koetz et al. 2008). Traditionally, such generation of fuel maps has been based in the analysis of medium - to high- resolution sensors, such as Landsat MSS and TM data (Riaño et al. 2002). As a recent example of such effort is the fuel type methodology developed within the LIFE 2010 ArcFUEL project. Under the scope of the specific project, a fuel type map for four Mediterranean regions was generated based on Landsat TM imagery and pixel- based approach (Toukiloglou et al. 2013). Nevertheless, advanced classification methodologies such as object- oriented method, have been employed in several studies for local fuel type mapping in order to achieve more accurate results (Mallinis et al. 2014), (Arroyo et al. 2006), (Gitas et al. 2006). The aim of this study is to develop a methodology for fuel type mapping on a national level, using advanced classification techniques (Object Based Image Analysis-OBIA) and the newly Landsat 8 OLI imagery.

\section{STUDY AREA AND DATASET}

The fuel type mapping methodology was initially developed and tested in the case study of the Chalkidiki Regional Unit. The region is characterized as a typical Mediterranean area covered primarily with coniferous and beech forests, but also includes a variety of other habitats such as pastures, cultivations, streams and stony hills. In addition, the understory vegetation comprises maquis species and low herbaceous vegetation. The developed classification model was further applied to two other study areas, namely Attiki and Preveza, in order to test its transferability before implementing it on a national level.

The dataset employed for the analysis was the recently provided Landsat-8 OLI imagery. Particularly, fifty-four Landsat-8 OLI images - both during the summer and the winter seasons-were acquired, covering the whole country. To assist the classification, ancillary datasets were used such as the Land Identification Parcel System (LPIS) thematic layer, which is a geo-database that provides the accurate boundaries mainly of the agricultural areas as well as land parcels defined by natural breaks, provided by the Hellenic Agricultural Payments Organization (OPEKEPE) in accordance to the European Union's (EU) Common Agricultural Policy (CAP). The official 
vegetation layer provided by the Ministry of Environment and Energy was also used in this study. In addition, points from the Land Use and Cover Area frame Survey (LUCAS) provided by Eurostat on 2013, were employed for the accuracy assessment process.

\section{METHODOLOGY}

\subsection{Dataset pre-processing}

Pre-processing included a radiometric correction of the images converting the raw digital values into Top Of the Atmosphere (ToA) reflectance values (Mishra et al. 2014). Atmospheric correction was applied in order to enhance the classification outputs by employing the Dark Object Subtraction (DOS) technique (Gilmore et al. 2015). Finally, the two images per area, one for summer and one for winter season, were stacked together and a final image of 16 layers was generated. The dataset derived from that process will be henceforth referred as Landsat ${ }_{16 \text { bands. }}$

\subsection{Object-based image analysis}

A subset of the Landsat ${ }_{16 \text { bands }}$ covering the area of Chalkidiki was segmented using the multiresolution segmentation algorithm embedded in the commercial software eCognition Developer 8.7. Following the Fractal Net Evolution Approach (FNEA) concept (Baatz et al. 2000), three levels of segmentations were applied. In each level the segmentation parameters were determined empirically in order to produce highly homogenous objects in specific resolutions and for specific purposes. The classification scheme was determined taking under consideration the existing fuel models (Prometheus, FBP etc.) and fuel type products (JRC FUELMAP, LIFE10 ArcFUEL) so as the final fuel type map could be easily adjusted and/or compared. The fuel type categories classified per level were:

- $\quad$ Level 1: Agricultural areas, Urban areas

- $\quad$ Level 2: Non-vegetated areas, Waterbodies

- Level 3: Deciduous Broadleaves (three density classes: $0-40 \%, 41-70 \%$, and $71-100 \%$ ), Coniferous (three density classes as before), Evergreen Broadleaves (three density classes as before), Grasslands and Shrublands.

Classification was based on fuzzy logic rules, defined by means of fuzzy sets. A fuzzy set describes the degree of certainty to which an input pattern (object in this case) belongs to the class of the rule, with respect to the specific feature that it is defined for. The certainty degree for each feature value is derived through an appropriately defined membership function. Fuzzy rules are constructed by selecting a subset of features for each class and the final classification is derived as the class of the rule that exhibits the highest adaptation degree. In this study, a total number of 63 features was identified as a superset of features that can be potentially used to discriminate the categories of fuels (Table 1). Several vegetation indices were calculated from the images of both seasons (winter and summer), along with textural and spectral features embedded in the eCognition software. The subset of features involved in each fuzzy rule and the definition of the respective membership functions were determined empirically, as well as through expert knowledge.

\begin{tabular}{|c|c|c|}
\hline $\begin{array}{c}\text { Feature } \\
\text { Categories }\end{array}$ & Object Features & $\begin{array}{c}\text { Number of } \\
\text { Features }\end{array}$ \\
\hline $\begin{array}{l}\text { Customi- } \\
\text { zed } \\
\text { (Indices) }\end{array}$ & $\begin{array}{l}\text { DVI (s), DVI (w), MSAVI2 } \\
\text { (s), MSI (s), NDVI (s), NDVI } \\
\text { (w), NDWI (s), RVI (s), RVI } \\
\text { (w), SAVI (s), SAVI (w), } \\
\text { SUM, VIN (s), VIN (w), } \\
\text { Minus NDVI }\end{array}$ & 15 \\
\hline $\begin{array}{l}\text { Layer } \\
\text { Values }\end{array}$ & $\begin{array}{l}\text { Brightness, Max. Difference, } \\
\text { Mean (for all image layers), } \\
\text { Standard Deviation (for all } \\
\text { image layers), Ratio (for the } \\
\text { image layers Blue (s), Green } \\
\text { (s), Red (s), NIR (s), SWIR1 } \\
\text { (s), SWIR2 (s)), Min pixel } \\
\text { value (for the image layers } \\
\text { Green (s), NIR (s)), Border } \\
\text { Contrast (for the image layers } \\
\text { Blue (s), NIR (s)), Circular } \\
\text { Mean (for the image layers } \\
\text { Blue (s), NIR (s), NIR (w), } \\
\text { Red (s)) }\end{array}$ & 44 \\
\hline Geometry & $\begin{array}{l}\text { Border length, Length/Width, } \\
\text { Compactness, Roundness }\end{array}$ & 4 \\
\hline $\begin{array}{c}\text { Total } \\
\text { amount }\end{array}$ & & 63 \\
\hline
\end{tabular}

(s) and (w) denote features derived from summer and winter season images, respectively.

Table 1. List of object features divided into three categories according to eCognition's categorization.

To test the transferability of the developed methodology we applied the object-based classification model in other two study areas, namely Attiki and Preveza. Those areas were selected since they present differences in the fuel type's distribution. Apart from some adjustments in the parameters' values of some fuzzy sets, the classification model's structure (e.g., number rules, features selected for each rule, etc.) was maintained. Subsequently, the model was applied to the rest of the country, in order to obtain the whole fuel type map.

\subsection{Accuracy Assessment}

The final products were evaluated for their accuracy, using the confusion matrix method (Foody 2002). A total set of 7626 LUCAS points (2013) covering the Greek territory was used as control points in the accuracy assessment process. At this point it should be mentioned that the agricultural and urban areas that were classified using the LPIS thematic layer, were excluded from the accuracy assessment process.

\section{RESULTS}

The derived fuel type maps of Chalkidiki, Attiki and Preveza are depicted in Figure 1 and the national fuel type map in Figure 2. The Overall Accuracy (OA) of the final map showed that the classification exhibits very satisfactory results in terms of overall performance and kappa coefficient $(\mathrm{OA}=87.80 \%$ and kappa $=0.837)$. Particularly, in Chalkidiki, where the classification model was developed, the overall accuracy was equal to $89.47 \%(\mathrm{KIA}=0.844)($ Fig1a). Results showed that the transferability of the model in the next two study areas seemed to be well adjustable, with OA equal to $89.28 \%(\mathrm{KIA}=0.839)$ 
(Fig $1 \mathrm{~b}$ ) in the study area of Attiki and OA equal to $91.74 \%$ $(\mathrm{KIA}=0.868)$ in the study area of Preveza (Fig1c) (Table 2).
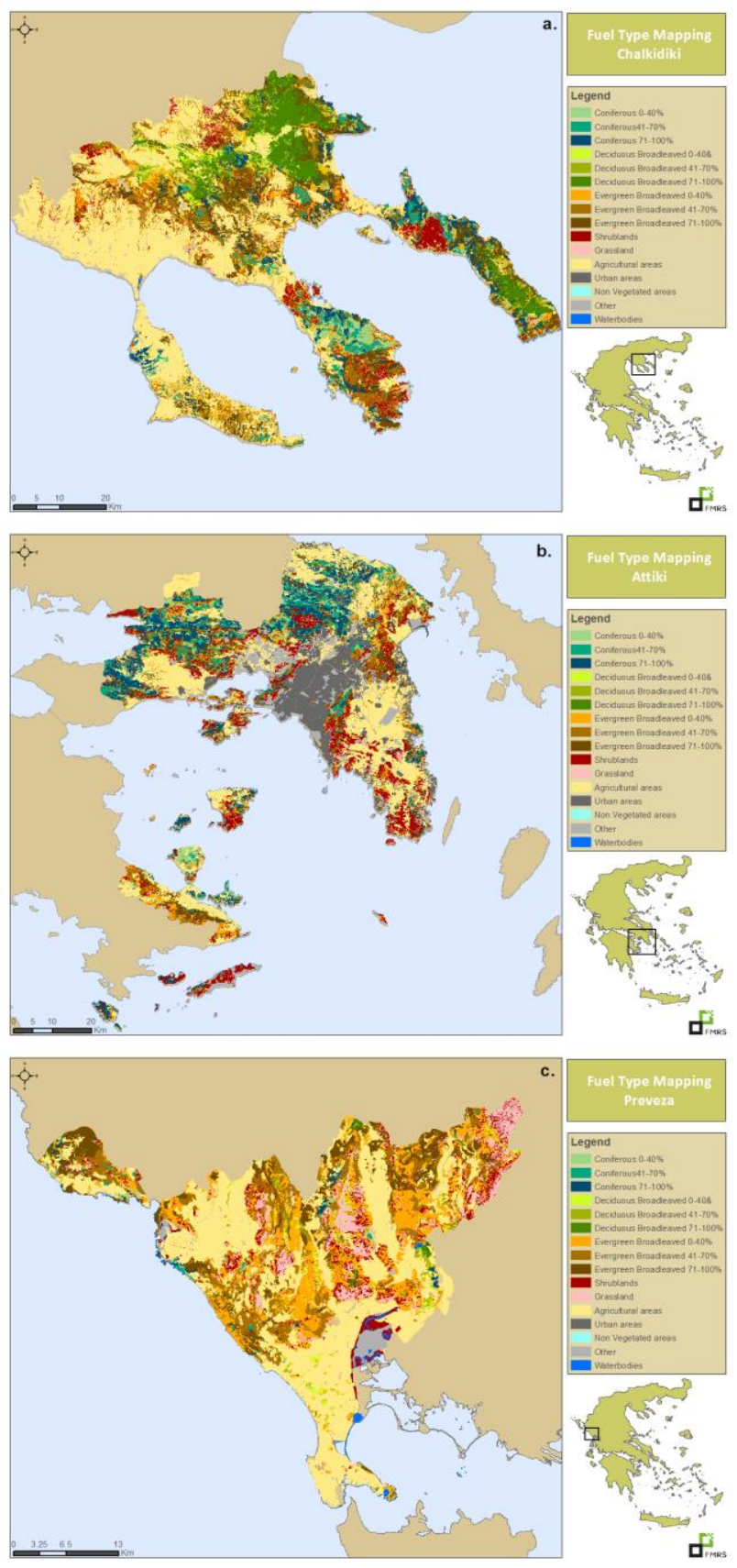

Figure 1. Fuel type classifications for the three study areas. The object-based classification model was developed in the first area of Chalkidiki (a) and it was further tested for its transferability in the other areas, Attiki (b) and Preveza (c) in accordance.

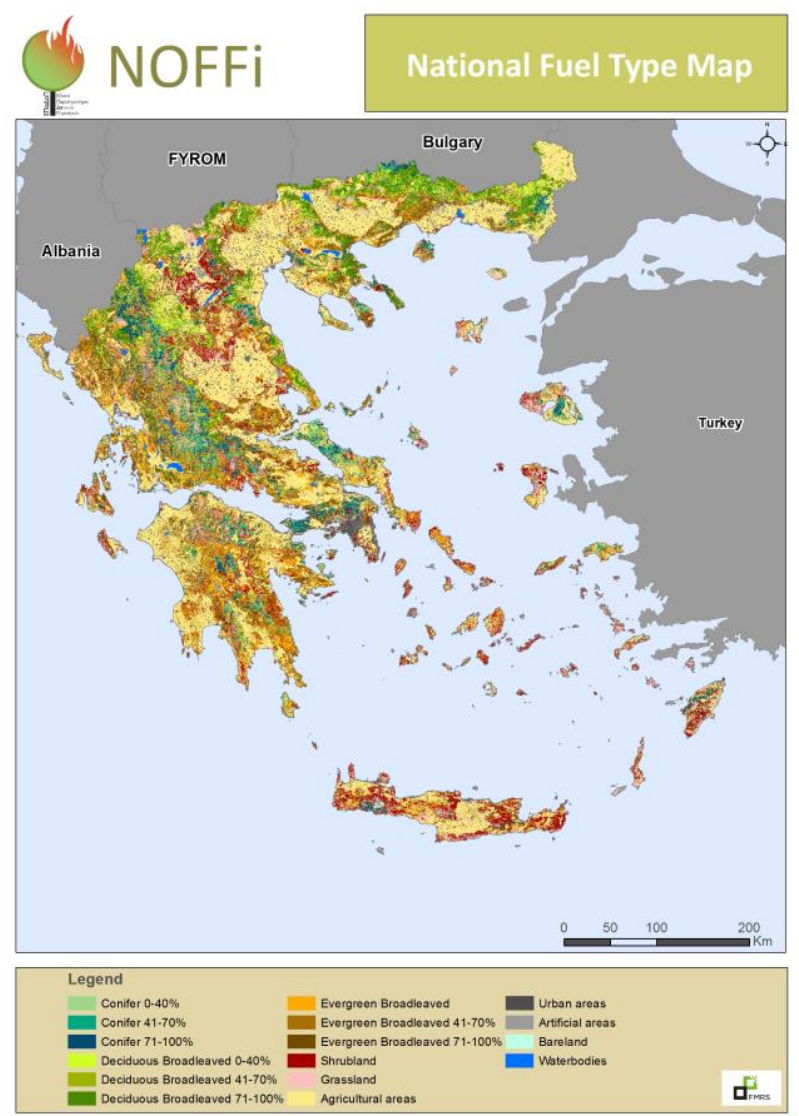

Figure 2. National fuel type map produced by object-based image analysis and Landsat 8 OLI imagery.

\begin{tabular}{|c|c|c|c|c|}
\cline { 2 - 5 } \multicolumn{2}{c|}{} & Chalkidiki & Attiki & Preveza \\
\hline \multirow{2}{*}{$\begin{array}{c}\text { Deciduous } \\
\text { Broadleaved }\end{array}$} & $U A$ & 97.53 & N/A & 83.33 \\
\cline { 2 - 5 } & $P A$ & 96.34 & N/A & 74.07 \\
\hline \multirow{2}{*}{ Coniferous } & $U A$ & 86.11 & 86.79 & 76.47 \\
\cline { 2 - 5 } & $P A$ & 74.7 & 97.18 & 100 \\
\hline \multirow{2}{*}{$\begin{array}{c}\text { Evergreen } \\
\text { Broadleaved }\end{array}$} & $U A$ & 88.06 & 91.28 & 94.39 \\
\cline { 2 - 5 } & $P A$ & 92.19 & 82.93 & 92.96 \\
\hline \multirow{2}{*}{ Grassland } & $U A$ & 85.71 & 80.00 & 92.86 \\
\cline { 2 - 5 } & $P A$ & 85.71 & 100 & 88.64 \\
\hline \multirow{2}{*}{ Shrubland } & $U A$ & 83.87 & 90.12 & 84.62 \\
\cline { 2 - 5 } & $P A$ & 92.86 & 86.90 & 97.06 \\
\hline \multirow{2}{*}{ Unvegetated } & $U A$ & 100 & 100 & 100 \\
\cline { 2 - 5 } & $P A$ & 100 & 100 & 90.91 \\
\hline \multirow{2}{*}{\begin{tabular}{c} 
Waterbodies \\
\cline { 2 - 5 }
\end{tabular}} & UA & 100 & 100 & 100 \\
\hline $\begin{array}{c}\text { Kappa } \\
\text { Coefficient }\end{array}$ & 100 & 100 & 100 \\
\hline $\begin{array}{c}\text { Overall } \\
\text { Accuracy } \%)\end{array}$ & & $\mathbf{8 9 . 4 7}$ & $\mathbf{8 9 . 2 8}$ & $\mathbf{9 1 . 7 4}$ \\
\hline
\end{tabular}

Table 2. User's accuracies (UA) and Producer's accuracies (PA) of the three classifications.

In the case of Chalkidiki, the results in general, show high values of PA and UA in all classes. This is expected since the model was developed based on the specific area. Apart from 
"Unvegetated" and "Waterbodies", that were accurately classified, the "Deciduous Broadleaves" present the highest PA and UA with minor of $2 \%$ deviation. However, in the case of Preveza, the specific class is underestimated. A closer examination of the map revealed that the misclassified objects were mainly located in shadowed areas (mainly shadows from the rough terrain). On the other hand, "Coniferous" seem to be overestimated since their spatial distribution is rather sparse resulting to spectral heterogeneity. What is commonly observed in all three cases is the underestimation and overestimation of "Grassland" and "Shrubland" classes, which can be attributed to two conditions. First, many small areas (even comparable to the pixel size) are characterized by complex patterns comprising both types of vegetation. For example, in typical grassland several individual shrubs are scattered, whereas areas of low vegetation are frequently observed within shrublands. Secondly, such intermixing often occurs in areas smaller than the pixel size, resulting in the existence of mixed pixels. This in turn leads to the creation of rather spectral heterogeneous objects during the segmentation process.

\section{CONCLUSIONS}

In this study, we develop an object oriented methodology for accurate fuel type mapping on a national level. The classification procedure was developed based on Landsat 8 OLI imagery for summer and winter season. Initially, the classification model was developed in the study area of Chalkidiki. In further, it was applied in other two areas, namely Attiki and Preveza in order to test and evaluate its transferability. Summarizing, the results showcase the effectiveness of the employed object-oriented classification approach in obtaining highly accurate fuel type maps for operational use on a national level.

\section{ACKNOWLEDGEMENTS}

This research work was conducted as part of the project "National Observatory of Forest Fires (NOFFi)", which is implemented in collaboration with the Directorate General for the Development and Protection of Forests and Rural Environment of the Hellenic Ministry of Environment and Energy and financially supported by Greece's Green Fund.

\section{REFERENCES}

Arroyo, L. A., S. P. Healey, et al. (2006). "Using object-oriented classification and high-resolution imagery to map fuel types in a Mediterranean region." Journal of Geophysical Research: Biogeosciences (2005-2012) 111(G4).

Baatz, M. and A. Schäpe (2000). "Multiresolution segmentation: an optimization approach for high quality multiscale image segmentation." Angewandte Geographische Informationsverarbeitung XII: 12-23.

Chuvieco, E. and R. G. Congalton (1989). "Application of remote sensing and geographic information systems to forest fire hazard mapping." Remote sensing of Environment 29(2): 147-159.

Foody, G. M. (2002). "Status of land cover classification accuracy assessment." Remote Sensing of Environment 80(1): 185-201.
Gilmore, S., A. Saleem, et al. (2015). "Effectiveness of DOS (Dark-Object Subtraction) method and water index techniques to map wetlands in a rapidly urbanising megacity with Landsat 8."

Gitas, I., G. Mitri, et al. (2006). "Fuel type mapping in Anopolis, Crete by employing QuickBird imagery and objectbased classification." Forest Ecology and Management 234(1): S228.

Koetz, B., F. Morsdorf, et al. (2008). "Multi-source land cover classification for forest fire management based on imaging spectrometry and LiDAR data." Forest Ecology and Management 256(3): 263-271.

Mallinis, G., G. Galidaki, et al. (2014). "A comparative analysis of EO-1 Hyperion, Quickbird and Landsat TM imagery for fuel type mapping of a typical Mediterranean landscape." Remote Sensing 6(2): 1684-1704.

Martín-Gómez, C., J. Vergara-Falces, et al. (2015). "Geographic information system software application developed by a regional emergency agency." Case Studies in Fire Safety 4: 1927.

Mishra, N., M. O. Haque, et al. (2014). "Radiometric Cross Calibration of Landsat 8 Operational Land Imager (OLI) and Landsat 7 Enhanced Thematic Mapper Plus (ETM+)." Remote Sensing 6(12): 12619-12638.

Riaño, D., E. Chuvieco, et al. (2002). "Generation of fuel type maps from Landsat TM images and ancillary data in Mediterranean ecosystems." Canadian Journal of Forest Research 32(8): 1301-1315.

San-Miguel-Ayanz, J., E. Schulte, et al. (2012). "Comprehensive monitoring of wildfires in Europe: the European Forest Fire Information System (EFFIS)."

Toukiloglou, P., G. Eftychidis, et al. (2013). ArcFuel methodology for mapping forest fuels in Europe. First International Conference on Remote Sensing and Geoinformation of Environment, International Society for Optics and Photonics. 


\title{
MULTISPECTRAL IMAGE SEGMENTATION BASED ON CARTESIAN COMPLEXES AND THEIR ASSOCIATED ORIENTED MATROIDS
}

\author{
J. A. Valero ${ }^{\text {a }}$, I. Lizarazo $^{\text {b }}$, P. A. Arbeláez ${ }^{\text {c }}$ \\ ${ }^{a}$ Facultad de Ingeniería, Universidad Distrital, Bogotá, Colombia - jvalero@ udistrital.edu.co \\ ${ }^{\mathrm{b}}$ Facultad de Ciencias Agrarias, Universidad Nacional de Colombia, Bogotá, Colombia - ializarazos@ unal.edu.co \\ ${ }^{\mathrm{c}}$ Universidad de los Andes, Bogotá, Colombia - pa.arbelaez@uniandes.edu.co
}

KEY WORDS: Image Segmentation, Locally Finite Spaces, Matroids, Cartesian Complexes

\begin{abstract}
:
A number of strategies have been used to include spatial and topological properties in the image segmentation stage. It is generally accepted that grouping of nearby pixels by modelling neighbourhood relationships as $(a, b)$ connected graphs may lead to meaningful image objects. In such approach, however, topological concepts may suffer from ambiguity since image elements (pixels) are two dimensional entities. This paper evaluates whether an alternative representation of digital images based both on Cartesian complexes and oriented matroids may improve multispectral image segmentation by enforcing topological and geometric properties and then be used in the classification stage. A conceptual model is defined, using Cartesian complexes, in order to link combinatorial properties of axiomatic locally finite spaces and their associated oriented matroids for involving topological properties. The proposed approach uses a layered architecture going from a physical level, going next through logical geospatial abstraction level and then through the Cartesian complex logical level. Additionally, there is a layer of oriented matroids composed by conceptual elements in terms of combinatorics for encoding relevant features to multispectral image segmentation. First, it is conducted an edge detection task, next an probability contour map using a Cartesian complex space rather than the conventional image space and finally, an image classification using random forest method. A computational solution including several components was developed using a framework for parallel computing. The performance of this solution was assessed using a small subset of GEOBIA2016 benchmark dataset. It is shown that the usage of a partial implementation of Cartesian complexes and associated oriented matroids is computationally but does not increase classification accuracy.
\end{abstract}

\section{INTRODUCTION}

A number of strategies have been used to include spatial and topological properties in the image segmentation stage. The most common practice for grouping nearby pixels is to model neighbourhood relationships as (a, b) connected graphs as it has been accepted that it may lead to meaningful image objects. In this approach, however, topological concepts may suffer from ambiguity since image elements (pixels) are two dimensional entities composing a spatially uniform grid cell in which there are not uni-dimensional nor zero-dimensional elements to build boundaries. This paper at establishing to stablish whether an alternative representation of digital images based both on Cartesian complexes (Kovalevsky, 2005) and oriented matroids (Whitney, 1935)(Oxley, 2006) may improve multispectral image segmentation by enforcing topological and geometric properties. A conceptual model is defined, using axiomatic locally finite spaces (ALFS) provided by Cartesian complexes, in order to link ALFS combinatorial properties and their associated oriented matroids in order to take into account topological properties.

The proposed approach uses a Cartesian complex layered architecture (CCLA) (Figure 1) going from a physical level represented by the multispectral image data set, and then passing through the logical geospatial abstraction level (GDAL) that allows manipulation of the underlying physical level. This abstraction level supports Cartesian complex logical level which provides required topological and geometric elements. Those elements allow for conducting first an edge detection task and then a hierarchical watershed using a Cartesian complex space rather than the conventional uniform grid cell. Additionally, there is a layer of oriented matroids composed by conceptual elements in terms of combinatorics for encoding relevant features to calculate oriented deriva- tive filters and oriented texture gradients that builds on (Arbelaez et al., 2011) but this time using a Cartesian complex space.

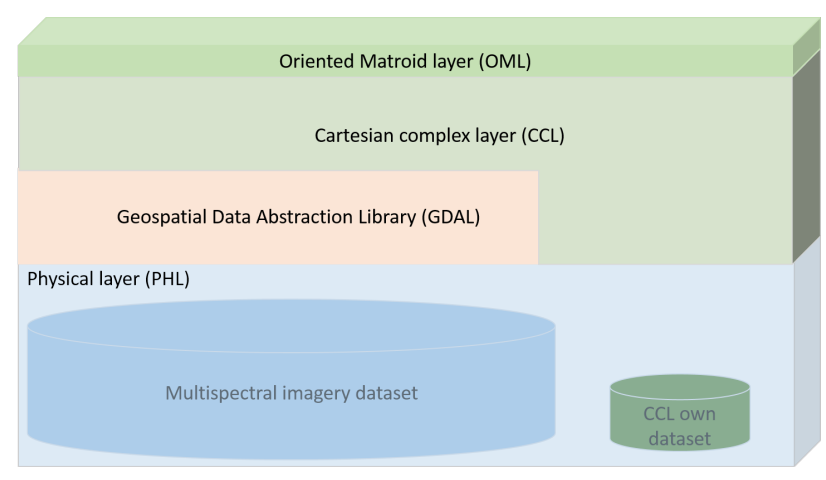

Figure 1. Multispectral image segmentation conceptual model

The complete implementation includes several components: (i) Cartesian complex core (CCC), on top of the GDAL implements a topological and geometrical space: (ii) Region boundary encoding component (RBE) provides a block complexes (BC) boundary encoding: (iii) Directional filtering (DF) supports texture detection based on filter banks. (iv) Orient gradient (OG) implements the calculation, based on oriented matroids, required to produce the maximum gradient image: (iv) Boundary cell affinity (BCA) supports the affinity analysis between cracks on detected digital straight line segments: (v) Ultrametric contour map (UCM), using mathematical morphology on a point and crack graph, provides means to produce the final Cartesian complex segmentation. 
For this study, a CCLA computational implementation (CCLAI) using only CCC, GDAL, DF and OG components was developed applying the Fork/Join framework for parallel computing. The performance of this partial solution was assessed using a small subset of GEOBIA2016 benchmark dataset. The segmented image was classified using random forest (RF) method (Tso and Mather, 2009).

This article is organized as follows. Section 2 discusses first the two most common topological ambiguities found in a conventional pixel space representation and and describes the dataset used. Descriptions of boundary strength, watershed transform and classification methods used in the experiments are also included. Results and discussion are presented in Sections 3 and 4 respectively and finally, main conclusions are drawn in Section ??.

\section{DATA AND METHODS}

\subsection{Conventional space representation ambiguities}

In the conventional representation of images, i.e. a tessellated space, topological concepts may suffer from ambiguity since image elements (pixels) are two dimensional entities. This inaccuracy is present in representations of images such as 4-connected, 8-connected and (a,b)-connected graphs (Rosenfeld, 1970) (Kong and Rosenfeld, 1991).

Figure 2(a) shows on the left, in a continuous space, how withdrawing points from the boundary between the interior and exterior sets - previously disconnected by a Jordan curve- these sets become connected and on the right how boundaries of the interior and exterior sets, are one-dimensional and coincide perfectly (red line).
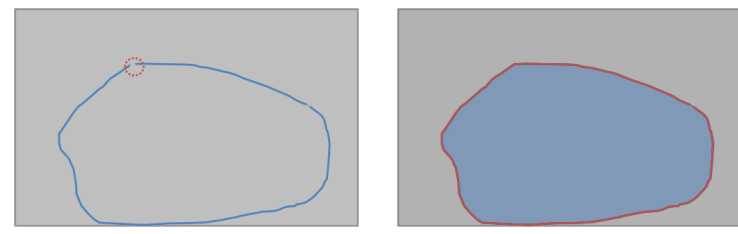

(a) Continuous space

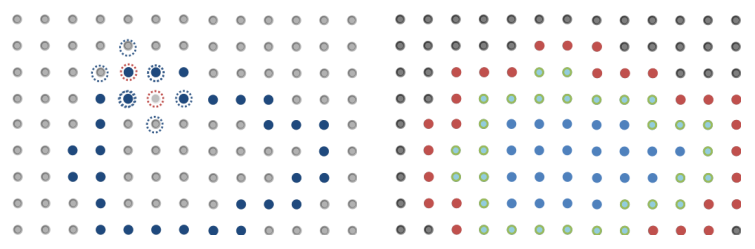

(b) Conventional digital space

Figure 2. Connectedness and Boundary definition

Figure 2(b) shows on the left,in a conventional digital space and using the 4-connected criterion, how by removing an element (encircled in red) from the boundary (depicted using blue points), the corresponding interior and exterior sets do not become connected. Actually, in the conventional digital space, it is not possible either to establish unambiguously such a connectivity using the 8-connected criterion. In this case, the two sets remain always connected even without removing the element under consideration from the boundary. The right of this figure shows how each of the two sets have different boundary (exterior's boundary is red and interior's boundary is green), and that both boundaries are two-dimensional (all the elements comprising this space are two-dimensional). These paradoxes may be particularly significant for image segmentation and classification when considering topological relationships such as neighbourhood, contiguity, connectivity, and boundary.

Digital image processing based on representations of space that do not meet the topological axiomatic postulates (Munkres, 1999) may cause geometric algorithms to be affected by paradoxes that lead to ambiguous or erroneous decisions. While it is commonly accepted that a raster image matches human perception to some degree, it seems that several important concepts for image analysis, such as the connectivity of regions, their boundaries, and the adjacency between them, are not explicitly present. This lack of topological awareness of grid-based image representation is clearly an important limitation for image analysis in computer environments (Kovalevsky, 1989).

On the other hand, it is well known that algorithms based on geometric characteristics have high complexity since the number of possible situations to be considered increases significantly with the number of dimensions of the objects under evaluation (Worboys and Duckham, 2004). Additionally, another problem that arises during the implementation phase of geometric algorithms is the impossibility of having an accurate arithmetic in terms of real numbers. This leads to resort to an accurate arithmetic in terms of integer or rational numbers that decreases performance (de Berg et al., 2008).

To sum up, conventional methods for multispectral image segmentation are based on digital structures that violate well-established topological axioms and geometric algorithms that assume a continuous space computing model. This study explores an alternative approach to address such a problem.

\subsection{Data}

For the boundary reliability assessment Berkeley Segmentation Data Set and Benchmarks 500 (BSDS500) (Martin et al., 2001) was used. A training dataset with two hundred natural images along their ground truth segmentations were used for producing the precision and recall curves. The data set used in the segmentation and classification experiments are further described.

The International Society for Photogrammetry and Remote Sensing (ISPRS) has launched the "semantic labeling contest" and provided airborne image datasets consisting of very high resolution true ortho photo (TOP) tiles and corresponding digital surface models (DSMs) of Vaihingen, a small village area in Germany, derived from dense image matching techniques.

The TOP are 8 bit TIFF files with three bands: near infrared, red and green bands delivered by the camera. The DSM are TIFF files with one band; the grey levels (corresponding to the DSM heights) are encoded as 32 bit float values. Images cover urban scenes that have been classified manually into five land cover classes: Building, Low vegetation, Tree, Car and Impervious surfaces.

In this study, it was selected tile 11 of Vaihingen data set. Two non-overlapping 1000 X 1000 pixel windows, one from $(400,400)$ to $(1400,1400)$ for the training stage and the other one from $(893,1400)$ to $(1893,2400)$ were subset from tile 11 for the final classification and assessment stages. A whole tile was not used because available computations resources were not good enough to work with images of that size in all conducted in the experiments. 


\subsection{Boundary strength and watershed transform}

Previous work on topology and geometry preserving image segmentation includes definition of a predicate for measuring evidence for a boundary between two regions using a graph-based representation (Felzenszwalb and Huttenlocher, 2004); abstract cell complexes for implementing topological relations needed to perform unambiguously segmentation of digital images in computer vision (Kovalevsky, 2005); image objects generation using the fractal network evolution (FNEA) (Yu et al., 2006); multiscale image segmentation approach in which urban land use is divided into different levels forming a hierarchical network structure (Kong et al., 2006); hybrid split-merge method for image segmentation based on computational geometry and topology using persistent homology (Letscher and Fritts, 2007); objectoriented spectral and textural classification algorithm for producing a map of structural classes after a textural analysis (Johansen et al., 2007).

Previous work also includes an active image segmentation method that distorts the image using B-Spline free-form deformations in order to match initial outlines and segments multiple objects simultaneously (Li and Sun, 2010), unified approach to contour detection and image segmentation using first an Oriented Watershed Transform (OWT) to build a hierarchical segmentation and then, from an initial RAG, a hierarchy formed based on the links weights as a measure of dissimilarity between regions (Arbelaez et al., 2011), a method for integrating topological image properties within a random field image segmentation model, which does not pose topological restrictions in the energy minimization stage (Chen et al., 2011) and a bottom-up strategy improving the agglomeration using more information besides boundary. Using supervised machine learning techniques it is possible to predict when two super pixels should be merged (Nunez-Iglesias et al., 2013).

In this study the first experiment includes a preliminary boundary reliability assessment in order to ensure CCLAI is good enough for producing boundary definitions. The assessment consisted in a gradient magnitude stage followed by a watershed production stage in both CCLAI and Matlab software. In the first stage, a gray scale image was computed from the original datasets (using Equation 1) by a three band lineal combination which was $x$ and $y$ sobel (Szeliski, 2010) filtered for finally calculating the gradient magnitude (using Equation 2).

$$
\text { grayimage }=\frac{1}{3}\left(b_{1}+b_{2}+b_{3}\right)
$$

where

$$
b_{i}=i \text { image band }
$$

$$
\text { gradientmagnitud }=\sqrt{\text { sobel }_{x}^{2}+\text { sobel }_{y}{ }^{2}}
$$

where $\quad$ sobel $_{x}=$ horizontal sobel filter

$$
\begin{aligned}
& \text { sobel }_{x}=\text { horizontal sobel filter } \\
& \text { sobel }_{y}=\text { vertical sobel filter }
\end{aligned}
$$

As CCLAI has available interpixel elements, it was used a $2 \times 3$ sobel fiter for horizontal edgels ( 1 -cells) and a $3 \times 2$ for verticals in contrast with the two $3 \times 3$ sobel filters used in Matlab. Raw gradient magnitude images where thinned using non-maximum suppression (Canny, 1986). The assessment methodology defined in (Arbelaez et al., 2011) was applied on the BSD500 images.

In the other experiment previously computed gradient magnitude images were introduced as watershed hierarchy production inputs. In this case the non maximum suppression procedure was not applied as the watershed transform should obtain boundary definitions. The watershed production implementation was based on the algorithm proposed in (Vincent and Soille, 1991) modified to use edgels (1-cells) available in the Cartesian complex space.

For the watershed hierarchy production a hierarchical grouping based on watershed ids with two to seven number of clusters was done. This ids were used as a watershed id produced by CCLAI framework is a poset in which the partial order relationship is given by the flooding process implicit in (Vincent and Soille, 1991) algorithm. It was necessary to match Matlab watershed ids to this order by a relabelling procedure.

On the architecture mentioned in section 1 the watershed production process shown on Figure 3 was implemented and used for obtaining classification superpixel datasets.

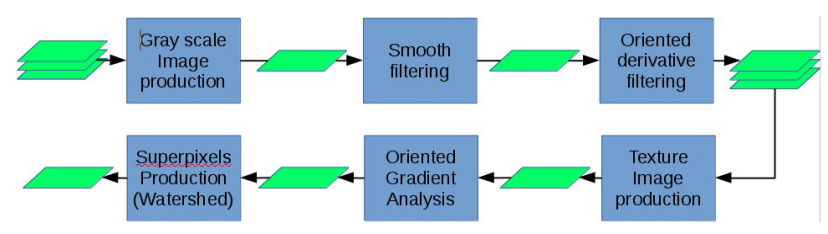

Figure 3. Superpixel production pipe line

First, a gray scale image was computed from the original datasets and then it was Gaussian filtered. Next an oriented derivative filter bank with seven orientations was applied from which a texture image was produced by a k-means grouping (Kanungo et al., 2002). Then, an oriented gradient image was calculated from the so produced texture (Leung and Malik, 1996) image taking the maximum for each of seven orientations. Finally, the watershed algorithm CCLAI implementation was applied to obtain the superpixel images.

Also, training and testing texture images were produced for the classification stage. For that, as can be seen on Figure 4 first a gray scale image was computed from the original datasets over which an oriented Gaussian filter bank with three orientations and three scales was applied in order to generate the edgel (1cell) texton response image. The number of filters in the bank was 21 - 3 even-symmetric (Gaussian second derivative), 3 oddsymmetric (Gaussian second derivative with Hilbert transform) and one center-surround all of them at each one of three scales but as each pixel has two own edgels (Kovalevsky, 2005) the final number of response texton channels were 42. Finally, "texture" images were obtained by k-means grouping with 64 clusters applying training texture image cluster centres to texton test image to obtain the testing texture image. Each texture image has two channels one for vertical edgels and one for horizontal ones.

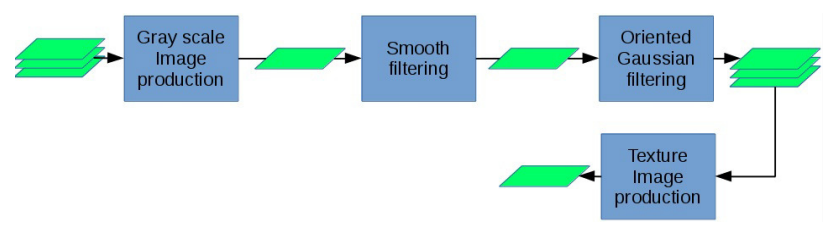

Figure 4. Superpixel production pipe line

All algorithms were implemented on the underlying Cartesian complex 2-dimensional space using specifically edgels (1-cells) interpixel space elements instead of the pixels available in a conventional digital space. Hyperplane central arrangements from 
the Oriented Matroid Layer (OML) were used to compute oriented derivative filtering responses. Fourteen histogram sectors used in oriented gradients calculation were encoded by using OML layer to compute combinatorially the final gradient image. The edgel gradient value was calculate as in (Arbelaez et al., 2011). The resulting over segmented image was used for classification describing each superpixel with multispectral, "texture" (k-means grouping), normalized difference vegetation index (NDVI) (calculated using Equation 3) and Digital Surface Model (DSM) mean responses inside it. The classification stage was conducted as described in Section 2.4.

$$
N V D I=\frac{b_{1}-b_{2}}{b_{1}+b_{2}}
$$

where

$$
\begin{aligned}
& b_{1}=\text { near infrared band } \\
& b_{2}=\text { red band of the image }
\end{aligned}
$$

\subsection{Classification procedure and assessment of classifications}

Three classification scenarios were defined. In the first, shown on Figure 5, the selected window was classified taking into account only the three spectral bands available for both the original pixel image and super pixel image produced by watershed transform previously computed.

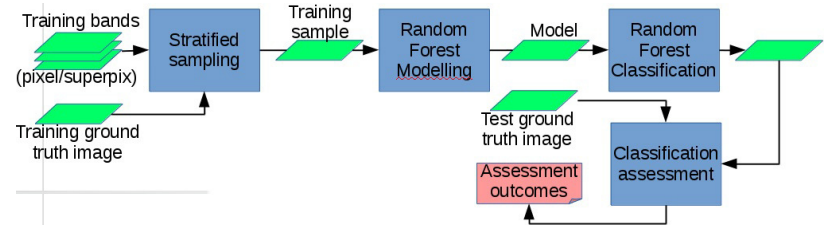

Figure 5. Spectral bands scenario

In the second scenario shown on Figure 6 the selected window was classified taking into account besides three spectral bands those corresponding to texture channels for both original pixels and super pixels.

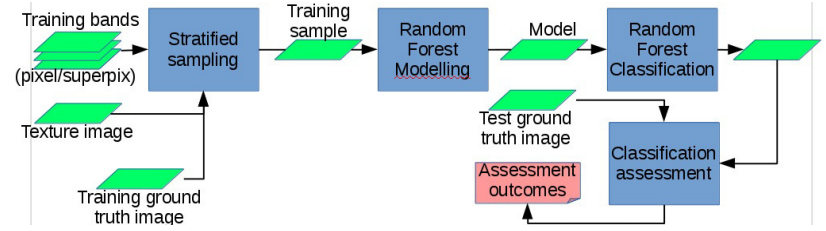

Figure 6. Spectral bands and texture scenario

In the third scenario shown on Figure 7 the selected window was classified taking into account besides three spectral bands and those corresponding to the texture channels, channels corresponding to NDVI and DSM for both original pixels as super pixels. At the end six random forest classification were run.

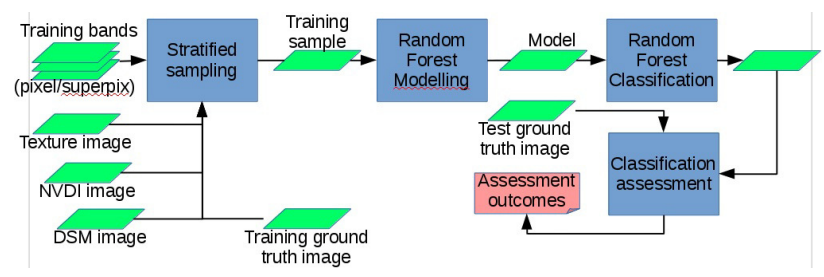

Figure 7. Spectral bands, texture, NDVI and DSM scenario
An overview of general steps of image classifications and its assessment is described in the following.

1. Training: A set of 3000 observation points was extracted from training window. A stratified sampling procedure was used in order to guarantee the same category rate in the sample as in the training window. The same set of observation points was used in all six classifications.

2. Extracting spectral signatures: the next step computed statistics from radiometric values for each class based on training sample feature space for each one of the classification scenarios previously described. Then, the model with the characteristic patterns of each class was created training each random forest classification model with 50 trees. The same model was used for both pixel and superpixel images.

3. Image classification: the image classification was carried out by random forest technique applying the respective mode generated in the previous step to the selected window. All three scenarios for both pixel and superpixel were considered to producing six random forest classifications.

4. Thematic accuracy evaluation: this step used the whole selected window reference classification (one million pixels) as validation data set. A normalized confusion matrix, the classification overall accuracy and the precision and recall were calculated for each classification outcome.

Reference classification images are shown on Figure 8. Blue areas correspond to building category, cyan to Low vegetation, green to Tree, yellow to Car and white to Impervious surfaces.

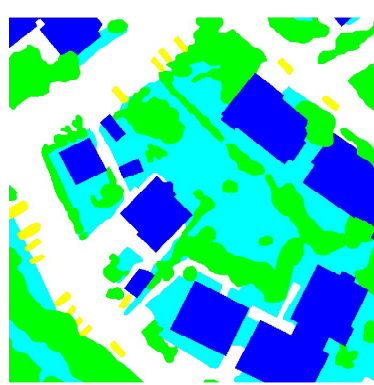

(a) Training

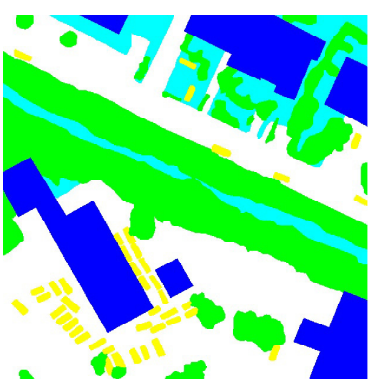

(b) Test
Figure 8. Reference classification images

\section{RESULTS}

\subsection{Segmentation}

Figure 9(a) shows a comparative boundary strength precision and recall assessment between this work outcome (green line) and conventional Matlab watershed implementation (red line) against BSDS500. The boundary strength was produced by computing the gradient magnitude from $x$ and $y$ sobel filtering. 


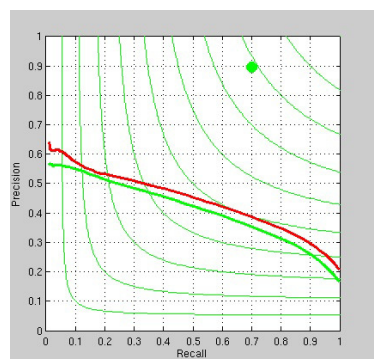

(a) Boundary strength

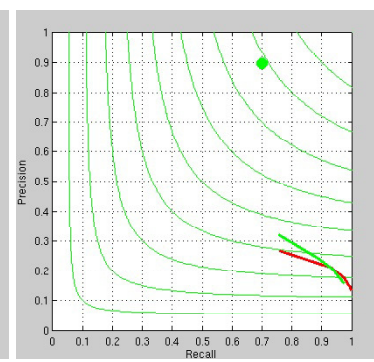

(b) Watershed
Figure 9. Precision and recall assessment

Figure 9 (b) shows a watershed precision and recall comparison between this work outcome (green line) and the conventional Matlab watershed implementation (red line) using BSDS500 assessment methodology against the train dataset.

The over segmentation image procedure allow to group 1000000 pixels into 201143 super pixels.

\subsection{Classification}

Obtained classification images for defined scenarios and their assessment outcomes are described in the following. Table 1 to Table 6 rows correspond to ground truth and columns (in the same order) to prediction.

Figure 10 shows classified images obtained only based on three spectral bands (scenario 1).

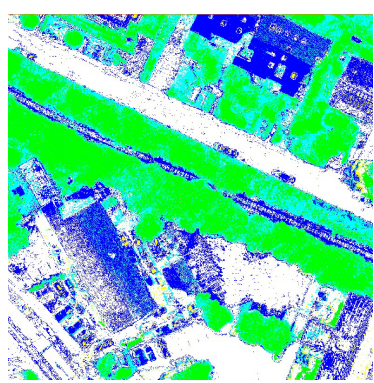

(a) Normal pixels

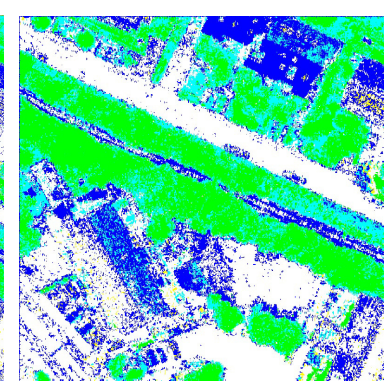

(b) Superpixels
Figure 10. Only spectral band classification images

Table 1 and Table 2 show confusion matrices for random forest pixel and superpixel (respectively) classification for the multispectral feature space.

\begin{tabular}{|l|r|r|r|r|r|}
\hline Category & \multicolumn{5}{|c|}{ Prediction(\%) } \\
\hline Building & 55.3 & 31.8 & 0.7 & 11.0 & 1.3 \\
Impervious surfaces & 14.4 & 72.8 & 3.2 & 8.6 & 1.1 \\
Tree & 1.4 & 1.6 & 72.1 & 24.9 & 0.2 \\
Low vegetation & 19.6 & 15.1 & 25.3 & 39.9 & 0.2 \\
Car & 42.7 & 38.5 & 2.2 & 9.6 & 7.1 \\
\hline
\end{tabular}

Table 1. Pixel based spectral feature space classification confusion matrix

\begin{tabular}{|l|r|r|r|r|r|}
\hline Category & \multicolumn{5}{|c|}{ Prediction(\%) } \\
\hline Building & 55.3 & 34.1 & 0.6 & 11.4 & 1.6 \\
Impervious surfaces & 15.0 & 72.8 & 3.0 & 8.2 & 1.0 \\
Tree & 1.8 & 1.6 & 71.7 & 24.8 & 0.1 \\
Low vegetation & 20.8 & 15.8 & 24.8 & 38.6 & 0.1 \\
Car & 42.7 & 41.3 & 1.8 & 8.6 & 5.6 \\
\hline
\end{tabular}

Table 2. Superpixel based spectral feature space classification confusion matrix

Figure 11 shows classified images based on a feature space composed by three spectral bands and the texture image channels (scenario 2).

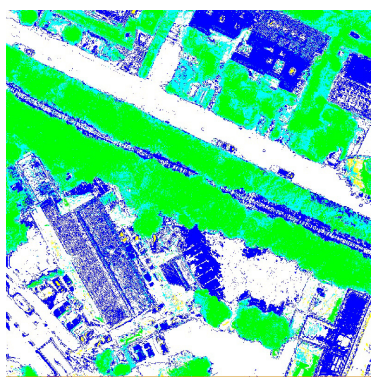

(a) Normal pixels

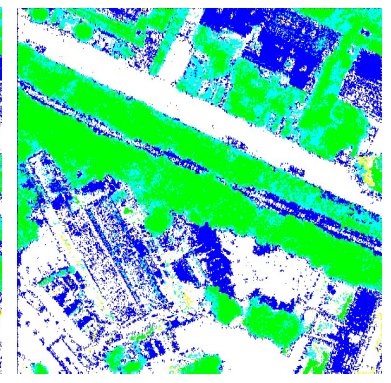

(b) Superpixels
Figure 11. Extended by texture feature space classification images

Table 3 and Table 4 show confusion matrices for random forest pixel and superpixel (respectively) classification for the feature space extended with channels for texture feature.

\begin{tabular}{|l|r|r|r|r|r|}
\hline Category & \multicolumn{5}{|c|}{ Prediction(\%) } \\
\hline Building & 57.3 & 34.7 & 0.8 & 6.5 & 0.7 \\
Impervious surfaces & 14.6 & 74.1 & 3.3 & 7.3 & 0.8 \\
Tree & 1.8 & 1.2 & 77.3 & 19.3 & 0.0 \\
Low vegetation & 19.5 & 15.1 & 26.6 & 38.7 & 0.1 \\
Car & 40.2 & 46.1 .6 & 2.4 & 6.6 & 4.7 \\
\hline
\end{tabular}

Table 3. Pixel based texture extended feature space classification confusion matrix

\begin{tabular}{|l|r|r|r|r|r|}
\hline Category & \multicolumn{5}{|c|}{ Prediction(\%) } \\
\hline Building & 53.8 & 38.5 & 0.8 & 6.2 & 0.8 \\
Impervious surfaces & 14.6 & 74.5 & 3.1 & 7.1 & 0.7 \\
Tree & 2.1 & 1.2 & 76.9 & 19.8 & 0.0 \\
Low vegetation & 19.8 & 15.9 & 25.1 & 39.0 & 0.1 \\
Car & 40.1 & 48.3 & 1.8 & 5.9 & 3.8 \\
\hline
\end{tabular}

Table 4. Superpixel based texture extended feature space classification confusion matrix

Figure 12 shows classified images based on a feature space composed by three spectral bands, texture image channels, NDVI channel and DSM channel (scenario 3). 


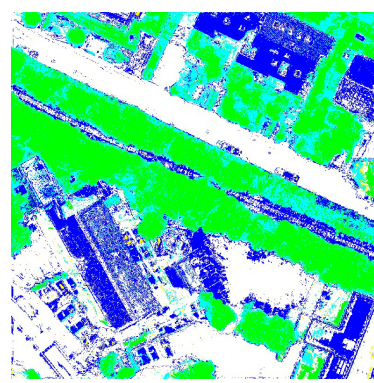

(a) Normal pixels

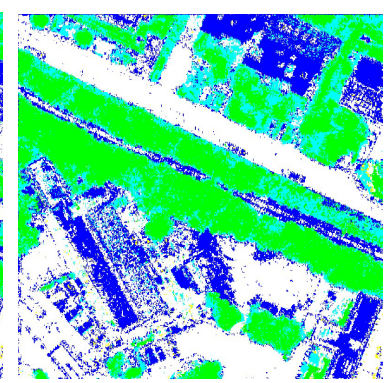

(b) Superpixels
Figure 12. Extended by texture, NDVI and DSM feature space classification images

Table 5 and Table 6 show confusion matrices for random forest pixel and superpixel (respectively) classification for the feature space extended with channels for texture, NDVI and DSM features.

\begin{tabular}{|l|r|r|r|r|r|}
\hline Category & \multicolumn{5}{|c|}{ Prediction(\%) } \\
\hline Building & 61.3 & 30.7 & 0.9 & 6.6 & 0.4 \\
Impervious surfaces & 13.3 & 74.6 & 3.3 & 8.2 & 0.5 \\
Tree & 2.0 & 1.0 & 76.3 & 20.7 & 0.0 \\
Low vegetation & 19.5 & 15.6 & 24.6 & 40.2 & 0.1 \\
Car & 39.6 & 46.6 & 2.1 & 7.7 & 4.1 \\
\hline
\end{tabular}

Table 5. Pixel based texture, NDVI and DSM extended feature space classification confusion matrix

\begin{tabular}{|l|r|r|r|r|r|}
\hline Category & \multicolumn{5}{|c|}{ Prediction(\%) } \\
\hline Building & 59.8 & 32.0 & 0.9 & 6.7 & 0.6 \\
Impervious surfaces & 13.9 & 74.6 & 3.0 & 8.1 & 0.5 \\
Tree & 2.1 & 1.0 & 75.6 & 21.4 & 0.0 \\
Low vegetation & 20.2 & 15.6 & 23.8 & 40.3 & 0.1 \\
Car & 39.2 & 48.7 & 1.7 & 7.1 & 3.3 \\
\hline
\end{tabular}

Table 6. Superpixel based texture, NDVI and DSM extended feature space classification confusion matrix

From confusion matrices the average classification accuracy (ACA) shown in Table 7 was calculated.

\begin{tabular}{|r|r|r|r|r|r|}
\hline \multicolumn{2}{|c|}{ Scenario1 } & \multicolumn{2}{c|}{ Scenario 2 } & \multicolumn{2}{c|}{ Scenario 3 } \\
\hline Pixel & Superpix & Pixel & Superpix & Pixel & Superpix \\
\hline 64.1 & 63.3 & 63.0 & 66.4 & 65.8 & 67.0 \\
\hline
\end{tabular}

Table 7. Overall accuracy

Precision and recall were also calculated for defined scenarios and respective outcomes are shown in Tables 8 and 9

\begin{tabular}{|l|r|r|r|r|r|r|}
\hline Category & \multicolumn{2}{|c|}{ Scenario1 } & \multicolumn{2}{c|}{ Scenario 2 } & \multicolumn{2}{c|}{ Scenario 3 } \\
\hline & Pixel & Super & Pixel & Super & Pixel & Super \\
Building & 50.3 & 47.7 & 50.9 & 49.0 & 53.9 & 52.4 \\
Impervious & 76.5 & 75.3 & 75.5 & 74.1 & 77.0 & 76.4 \\
surfaces & & & & & & \\
Tree & 84.4 & 84.8 & 84.8 & 85.4 & 85.1 & 85.8 \\
Low veg- & 23.8 & 23.3 & 28.3 & 28.5 & 27.4 & 27.2 \\
etation & & & & & & \\
Car & 25.3 & 21.5 & 25.8 & 22.6 & 30.2 & 27.3 \\
\hline
\end{tabular}

Table 8. Precision

\begin{tabular}{|l|r|r|r|r|r|r|}
\hline Category & \multicolumn{2}{|c|}{ Scenario1 } & \multicolumn{2}{c|}{ Scenario 2 } & \multicolumn{2}{c|}{ Scenario 3 } \\
\hline & Pixel & Super & Pixel & Super & Pixel & Super \\
Building & 55.3 & 52.3 & 57.3 & 53.8 & 61.3 & 59.8 \\
Impervious & 72.8 & 72.8 & 74.1 & 74.5 & 74.6 & 74.6 \\
surfaces & & & & & & \\
Tree & 72.1 & 71.8 & 77.7 & 76.9 & 76.3 & 75.6 \\
Low veg- & 39.9 & 38.6 & 38.7 & 39.0 & 40.2 & 40.3 \\
etation & & & & & & \\
Car & 7.1 & 5.6 & 4.7 & 3.8 & 4.0 & 3.3 \\
\hline
\end{tabular}

Table 9. Recall

\section{DISCUSSION}

As shown in Figure 9(a), $\frac{1}{3}$ of data used by a conventional convolution algorithm, it was possible to obtain similar sobel filter outcome. Figure 9(b) shows that Cartesian complex watershed implementation was as good as Matlab so it was appropriate for this study. Both implementations follow the same procedure described in section 2.3; the only difference is that CCLAI implementation uses edgels available in the underlying cartesian complex space.

It is possible to notice that when additional criteria are included in the feature space, the texture image first (Figure 11) and then the normalized difference vegetation index (NDVI) and SDM (Figure 12), the classified images get improved with respect to the classification obtained based only on the three spectral bands (Figure 11).

When comparing the respective classification images for pixels and superpixels in a same scenario, it is possible to notice how boundary superpixels have a rough finish and how extending the inside out and the outside in. This effect is due to the current implementation of the watershed transform and prevents the results for superpixels from being better. Despite the above, confusion matrices Table 1 / Table 2, Table 3 / Table 4 and Table 5 / Table 6 pairwise comparatives show that although creating the super pixels based on the watershed transform significantly decreased the number of elements of the space to be classified, the overall accuracy remained, so the performance gain by having to classify fewer elements did not mean a sacrifice of accuracy. It was better to have to classify 201143 superpixels than 1000000 pixels as the overall accuracy was kept.

Table 3 shows how including texture feature in the classification raised the correctly classified pixel percentage except for low vegetation and car categories in the case of pixel classification and 4 and Building and Car in the case of superpixels. This effect was stronger when NDVI and DSM features were included as shown in Figure 12 and Table 5 except for Car category. 
The overall accuracy always was below 70 percent but it should be considered that the assessment stage was conducted against the whole ground truth window (10000000 pixels).

As shown in Table 7 the use of a extended feature space with texture, NDVI and DSM data did not produce a significant effect in overall accuracy. However, Scenario 1 column, which did not included texture feature, shows how including superpixels and texture feature the overall accuracy decreases while Scenario 2 and Scenario 3 show how by including it increases overall accuracy.

Table 8 allows observing how, except for Impervious surfaces, the texture feature inclusion (scenario 2) slightly improves the precision regarding scenario 1.This tendency is kept with NDVI and SDM features inclusion which can be evidenced in scenario 3 with precision values higher for all categories.

Recall shows a similar effect in the case of scenario 3 (see Table 9) except for Car category, and except for Low vegetaton and Car categories in the case of scenario 2.

The inclusion of texture features taking into account geometric features in three different scales, starting at the most detailed possible, was key to improve the final classification results. This supports the argument that texture analysis is essential in the process of classification because it can provide an additional criterion to avoid erroneously mixing or separating adjacent space elements.

The use of watershed transform based on gradient texture favoured the proper definition of super pixels which, although it is true that it did not improve overall accuracy classification it did manage to preserve, with the added benefit of having a number of elements significantly reduced in the geometric space. This means that consequently the processing is significantly improved regardless of the classification method used.

It was possible to establish that the potential of superpixels in the classification is conditional on the simultaneous use of texture analysis. This suggests that processes not considering classifications texture analysis could reconsider the use of superpixels. Now, this study shows that it is necessary to improve the watershed transform in order to get better outcomes.

\section{CONCLUSIONS}

Sobel implementation based on edgels (1-cells) allows improving the processing independently of the algorithm used as it uses filter sizes of $2 \times 3$ and $3 \times 2$. This means that it uses a third less of the data than a conventional space based implementation using two $3 \times 3$ sized filters. In addition, the result is associated with a one-dimensional element as should happen from the topological point of view.

The availability of a underlying cartesian complex space provides a topologically correct oriented gradient calculation. In a conventional space when a disk is defined it is recommended not using the pixel linear arrangement which meets the diameter along the orientation as it does not lay in any of two sides but in the orientation. However, in a complex Cartesian space, there are interpixel elements that allow representing this situation properly by modelling that dividing line based on edgels and not on pixels.

The use of oriented matroids and their hyperplane central arrangement allows to improve the oriented gradient computation as the respective histograms are calculated once by sector and, at the final calculation, combined in agreement with each one of the specific orientations. The histogram calculation does not have to be done as many times as the number of orientations but it is conducted once and combined as many times as the number of orientations.

The benefits in processing and guaranteeing the topological model expressed in the previous paragraphs and although the results of the experiments show that the benefits of the inclusion of an underlying space based on Cartesian complex at the moment are very small, are evidence that it is worth continuing with the research.

As this study did not demonstrate improvement of thematic accuracy when using the proposed alternative space for land cover classification, in near future it will be investigated whether a complete implementation of a computational framework based on Cartesian complexes is able to accomplish such a challenge.

\section{ACKNOWLEDGEMENTS}

The Vaihingen data set was provided by the German Society for Photogrammetry, Remote Sensing and Geoinformation (DGPF) (Cramer, 2010).

Cristian Dallos wrote derivative filters, watershed and k-means first version for CCLAI.

Centro de Computo de Alto Desempeo of Universidad Distrital (CECAD) provided the matlab license and a Open SUSE Linux virtual machine for deployment.

\section{REFERENCES}

Arbelaez, P., Maire, M., Fowlkes, C. and Malik, J., 2011. Contour detection and hierarchical image segmentation. IEEE Trans. Pattern Anal. Mach. Intell. 33(5), pp. 898-916.

Canny, J., 1986. A computational approach to edge detection. IEEE Transactions on Pattern Analysis and Machine Intelligence PAMI-8(6), pp. 679-698.

Chen, C., Freedman, D. and Lampert, C. H., 2011. Enforcing topological constraints in random field image segmentation. In: Computer Vision and Pattern Recognition (CVPR), 2011 IEEE Conference on, pp. 2089-2096.

Cramer, M., 2010. The dgpf-test on digital airborne camera evaluation overview and test design. http://www.ifp.unistuttgart.de/dgpf/DKEP-Allg.html.

de Berg, M., Cheong, O., van Kreveld, M. and Overmars, M., 2008. Computational Geometry. Third edn, Springer Berlin Heidelberg.

Felzenszwalb, P. F. and Huttenlocher, D. P., 2004. Efficient graph-based image segmentation. Int. J. Comput. Vision 59(2), pp. 167-181.

Johansen, K., Coops, N. C., Gergel, S. E. and Stange, Y., 2007. Application of high spatial resolution satellite imagery for riparian and forest ecosystem classification. Remote Sensing of Environment $110(1)$, pp. $29-44$

Kanungo, T., Mount, D. M., Netanyahu, N. S., Piatko, C. D., Silverman, R. and Wu, A. Y., 2002. An efficient k-means clustering algorithm: analysis and implementation. IEEE Transactions on Pattern Analysis and Machine Intelligence 24(7), pp. 881-892.

Kong, C., Xu, K. and Wu, C., 2006. Classification and extraction of urban land-use information from high-resolution image based on object multi-features. Journal of China University of Geosciences 17(2), pp. $151-157$. 
Kong, T. and Rosenfeld, A., 1991. Digital topology: a comparison of the graph-based and topological approaches. g.m. reed, a.w. roscoe, r.f. wachter (eds.), topology and category theory in computer science, clarendon press, oxford, great britain. pp. 273287.

Kovalevsky, V., 2005. Algorithms in Digital Geometry Based on Cellular Topology. Springer Berlin Heidelberg, Berlin, Heidelberg, pp. 366-393.

Kovalevsky, V. A., 1989. Finite topology as applied to image analysis. In: Computer Vision, Graphics, and Image Processing, pp. 141-161.

Letscher, D. and Fritts, J., 2007. Image Segmentation Using Topological Persistence. Springer Berlin Heidelberg, Berlin, Heidelberg, pp. 587-595.

Leung, T. K. and Malik, J., 1996. Detecting, localizing and grouping repeated scene elements from an image. In: Proceedings of the 4th European Conference on Computer Vision-Volume I - Volume I, ECCV '96, Springer-Verlag, London, UK, UK, pp. 546-555.

Li, C. and Sun, Y., 2010. Active image: A shape and topology preserving segmentation method using b-spline free form deformations. In: 2010 IEEE International Conference on Image Processing, pp. 2221-2224.

Martin, D., Fowlkes, C., Tal, D. and Malik, J., 2001. A database of human segmented natural images and its application to evaluating segmentation algorithms and measuring ecological statistics. In: Proc. 8th Int'l Conf. Computer Vision, Vol. 2, pp. 416-423.

Munkres, J., 1999. Topology; a First Course. 2nd edition edn, Prentice-Hall.

Nunez-Iglesias, J., Kennedy, R., Parag, T., Shi, J. and Chklovskii, D. B., 2013. Machine learning of hierarchical clustering to segment $2 \mathrm{~d}$ and $3 \mathrm{~d}$ images. PLoS ONE 8(8), pp. 1-11.

Oxley, J. G., 2006. Matroid Theory (Oxford Graduate Texts in Mathematics). Oxford University Press, Inc., New York, NY, USA.

Rosenfeld, A., 1970. Connectivity in digital pictures. J. ACM 17(1), pp. 146-160.

Szeliski, R., 2010. Computer Vision: Algorithms and Applications. 1st edn, Springer-Verlag New York, Inc., New York, NY, USA.

Tso, B. and Mather, P., 2009. Classification Methods for Remotely Sensed Data. Environmental engineering, CRC Press, Taylor \& Francis Group.

Vincent, L. and Soille, P., 1991. Watersheds in digital spaces: an efficient algorithm based on immersion simulations. IEEE Transactions on Pattern Analysis and Machine Intelligence 13(6), pp. 583-598.

Whitney, H., 1935. On the abstract properties of linear dependence. American Journal of Mathematics 57, pp. 509-533.

Worboys, M. and Duckham, M., 2004. GIS: A Computing Perspective, 2Nd Edition. CRC Press, Inc., Boca Raton, FL, USA.

Yu, Q., Gong, P., Clinton, N., Biging, G., Kelly, M. and Schirokauer, D., 2006. Object-based detailed vegetation classification with airborne high spatial resolution remote sensing imagery. Photogrammetric Engineering \& Remote Sensing 72(7), pp. 799 811. 


\title{
GETTING THE ACT TOGETHER: SEGMENTATION-BASED LAND COVER CLASSIFICATION USING RAPIDEYE IMAGERY AND OPEN STREET MAP ANCILLARY DATA
}

\author{
L. Valozic ${ }^{\mathrm{a}}$ \\ ${ }^{a}$ University of Zagreb, Faculty of Science, Department of Geography, Marulicev trg 19, 10000 Zagreb, Croatia - \\ lvalozic@geog.pmf.hr
}

KEY WORDS: Land Cover Classification, OBIA, Rule-Based, GIS, RapidEye, Open Street Map

\begin{abstract}
:
The research deals with land cover classification by means of segmentation-based image analysis and geoprocessing in GIS software. The goal of the research is the overall improvement of classification results obtained from rule-based land cover classification, by utilising ancillary vector data. The area of interest is the City of Zagreb in Croatia. This research is primarily based on 2015 RapidEye satellite imagery. The assumption that the mixture of urban, peri-urban, rural areas, that makes the territory of the City of Zagreb, is sufficiently interwoven with OpenStreetMap data was confirmed after examination of acquired shapefiles. Intent of the research was to find a solution for land cover classification of geographically heterogeneous region by reconciling the need for convenient and affordable spatial data. Preprocessing and postprocessing of spatial data, as well as the principle component analysis of the imagery, were performed in ESRI ArcGIS. The classification process was performed using Trimble eCognition Developer. Rule-based classification was employed on image segments that were created by the multiresolution segmentation algorithm. Threshold values for statistical features, vegetation indices, and values derived from PCA were set after careful examination of all input datasets. Overall classification accuracy of $91.5 \%$ was assessed by error matrix. Imagery of higher spatial resolution were used as reference data for the error matrix.
\end{abstract}

\section{INTRODUCTION}

\subsection{Aim and Objective}

The aim of this research is the successful land cover classification of urban and peri-urban areas by means of objectbased image analysis of remotely sensed imagery. The evaluation criteria for the success of the land cover classification have been set as the overall accuracy of the classification, as well as the classification accuracies for the individual land cover classes, to be equal or more than 85 percent (Anderson, 1971).

The purpose of such classification tasks is the improvement of the spatial awareness of apparent geographic dichotomies of the urban versus rural, or artificial versus natural (Besussi et al, 2010).

The objective of this research work was the production of a land cover dataset by means of segmentation-based image analysis of multispectral satellite images with the support of ancillary GIS vector data layers.

Final land cover dataset created by classification consists of four classes: vegetation, impervious or built-up surfaces, bare soil, and water. Classification scheme is based on vegetation impervious surface - soil (V-I-S) model for urban ecosystem analysis (Ridd, 1995).

\subsection{Study Area}

The study area is the administrative area of the City of Zagreb in the Republic of Croatia. This county-level administrative unit is the country's capital and the most populous city with almost 800000 inhabitants. The City of Zagreb has an area of $641 \mathrm{sq}$ $\mathrm{km}$ and is comprised of 70 urban, peri-urban, or even rural settlements. Even though this area has been characterised by a considerable growth in population and built-up surfaces during the last several decades, significant parts of this administrative unit are either agricultural areas or inside the protected area of Medvednica Nature Park and are covered with forests. Most of the water surfaces in the City of Zagreb are highly regulated rivers and streams, or artificial lakes and ponds.

\subsection{Data}

The choice of suitable remote sensing data requires the settling of multiple criteria such as the quality and the quantity of data, as well as the financial costs. Two main data sources were used in the research: archived RapidEye's multispectral satellite imagery from 2015, and shapefiles of OpenStreetMap data. Former dataset comes from a commercial remote sensing vendor. RapidEye images have been chosen because of relatively convenient combination of scene tile size $(25 \times 25$ $\mathrm{km}$ ), spatial resolution $(5 \mathrm{~m})$ and spectral resolution (blue, green, red, red edge, near infrared). Five image scenes acquired for this work (3A) have already been geometrically and radiometrically corrected. Latter dataset, the free and easily obtainable OpenStreetMap data shapefiles, have been chosen because they have become a rich data source, and represent the dynamic crowdsourced knowledge of the area of interest (Rosina et al, 2016). More precisely, point, polyline, and polygon features that represent various urban objects, building footprints, and pedestrian, road, or rail networks, have been used as datasets that should help to classify the land cover of City of Zagreb. OpenStreetMap shapefiles have been acquired online from Geofabrik GmbH (Geofabrik, 2015).

DigitalGlobe satellite imagery found in GoogleEarth application and digital colour orthophoto of City of Zagreb available in WMS Service (CDOF2012, 2016) were used as reference data for the accuracy assessment. 


\section{METHODOLOGY}

\subsection{Approach}

This research was based on processing of raster images and vector datasets, by both remote sensing and GIS software in order to produce the final land cover dataset.

The preprocessing and postprocessing tasks of the datasets were performed in ESRI ArcGIS 10.3. and they include: geometry checks and geometry repairs of OSM polygon shapefiles, selection of features by attributes, overlays of data layers that originate from different sources, and principle component analysis of multispectral imagery. Additionally, GIS software was used for spatial and attribute data management operations, and visualizations, from initial previews to the final representations of the results of the research.

After the geoprocessing in GIS software, spatial data was imported in Trimble's eCognition Developer 9, software that offers a myriad of tools for image segmentation and classification. Imported raster datasets included original RapidEye five-band images and five raster image layers created by PCA. They were joined by OpenStreetMap shapefiles as thematic layers.

Image segmentation was performed by using multiresolution segmentation algorithm (Benz et al, 2004; Trimble, 2014). Original image bands and thematic layers for OSM points, roads, railways, and buildings, were used for the segmentation process. Additional weight (2) was set for the NIR and red edge bands. Scale parameter was set to 75 , shape criterion weight to 0.1 , and compactness criterion to 0.5 .

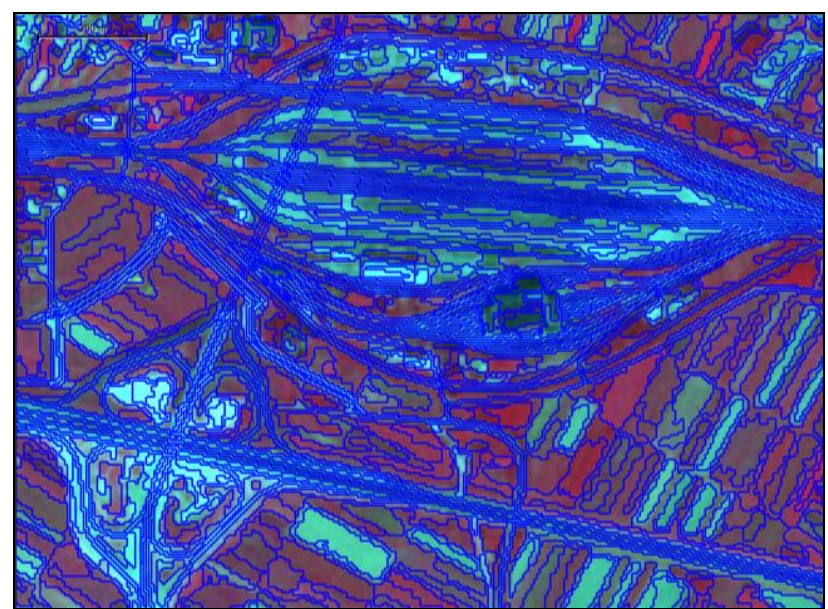

Figure 1. Example of image segments produced by segmentation algorithm executed on RapidEye satellite image and OpenStreetMap data, (C) 2016 Planet Labs Netherlands BV, (C) 2016 Geofabrik GmbH, (c) 2016 Trimble Germany GmbH

The rule-based classification (Zhu, 2009) of image segments in the eCognition environment is the foundational part of this research. A step-wise process tree that assigns image segments to different classes according to determined threshold values has been developed. Careful examination of features and a priori knowledge of the area of interest were crucial for defining of the threshold settings.

Thematic layers imported from OSM shapefiles were used immediately for the classification of impervious surfaces (builtup areas) because they already represented buildings and transport networks. Points and polylines that did not represent artificial surfaces were removed from the dataset in advance by actions in GIS software. Decisions about removal of certain features were made based on inspection of attribute data and a priori knowledge of the City of Zagreb area.

Most of the other object features that were used for classification are statistical data (mean, SD) on image segments for individual image layers (original image bands and PCA bands), arithmetic features such se ratios between couple or more image bands, HSI transformation, and vegetation or water indices such as SAVI (Huete, 1988), NDWI (McFeeters, 1996), BNDVI (Yang et al, 2004).

Executed process tree resulted with a crisp classification of image segments into land cover classes with binary membership values assigned to them.

\subsection{Results and Discussion}

The final results were vector datasets of four land cover classes for the area of City of Zagreb. Accuracy assessment was performed by error matrix (Story, Congalton, 1986). Reference and classification data samples were extracted on locations that were obtained by stratified random sampling. Colour digital orthophotos of Zagreb area and imagery from Google Earth were used as reference data sources for the purpose of accuracy assessment. As indicated in the Table 1., most of the classification errors occurred between classes of impervious surfaces and bare soil.

\begin{tabular}{|l|c|c|c|c|}
\hline \multicolumn{1}{|c|}{$\%$} & $\begin{array}{c}\text { Impervious } \\
\text { Surface }\end{array}$ & Bare Soil & Vegetation & Water \\
\hline $\begin{array}{l}\text { Producer's } \\
\text { Accuracy }\end{array}$ & 88.5 & 83.3 & 95 & 100 \\
\hline $\begin{array}{l}\text { User's } \\
\text { Accuracy }\end{array}$ & 90 & 85.7 & 95 & 92 \\
\hline $\begin{array}{l}\text { Overall } \\
\text { Accuracy }\end{array}$ & \multicolumn{4}{|c|}{91.5} \\
\hline
\end{tabular}

Table 1. Accuracy assessment results

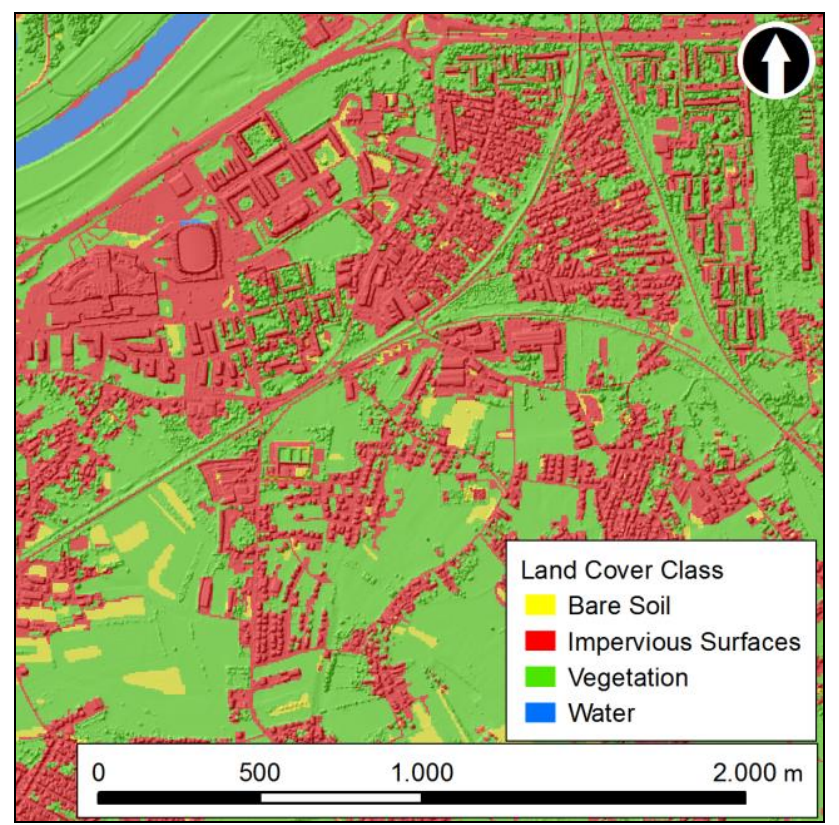

Figure 2. Part of a land cover thematic map of the City of Zagreb produced in the research

Visual inspection of the classification results (Figure 2.) has revealed the main advantage of OpenStreetMap shapefiles' usage as ancillary data for land cover classification. Relatively small or narrow anthropogenic objects that do not come to light 
because of spatial resolution or are secluded by e.g. tree canopy, are clearly mapped because of vector datasets and methods used for their processing.

\section{CONCLUSIONS}

On the basis of the accuracy assessment, the land cover classification performed for this research work can be regarded quite successful. The imperfections of both remote sensing raster data and GIS vector data have been settled by their combined use.

Obtained land cover data can used for several purposes. Primarily, it is used in spatial analyses and thematic mapping in the discipline of urban geography. Such data can help the study of relationships between built-up or artificial surfaces, and vegetation or natural (quasi-natural) land covers. In an environment where naturally occurring bare surfaces are rare, data on bare soil land cover can indicate environmental transition on the observed locations. In combination with population data, even land cover data based on simple classification schemes as one employed in this research, improve the mapping of population distribution and density.

Even though development of rule-based classification requires relatively large amount of time and a certain a priori knowledge of the area of interest, once created rulesets can be executed rapidly and used on similar datasets (similar environments or geographical areas) with just some fine-tuning of threshold values.

\section{ACKNOWLEDGEMENTS}

This research is part of CRORURIS - interdisciplinary project that is focused on the study of changes in the rural areas of Croatia and aims to develop framework for identification and geographical differentiation of predominant trends and key uncertainties as well as their projection by using statistical modelling and Delphi method, and to construct alternative future scenarios and relate them to the context of rural Europe.

\section{REFERENCES}

Anderson, J. R., 1971. Land-Use Classification Schemes, Photogrammetric Engineering 37(4), pp. 379-387.

Benz, U. C., Hofmann, P., Willhauck, G., Lingenfelder, I., Heynen, M., 2004. Multi-resolution, object-oriented fuzzy analysis of remote sensing data for GIS-ready information, ISPRS Journal of Photogrammetry \& Remote Sensing, 58, pp. 239- 258 .

Besussi, E., Chin, N., Batty, M., Longley, P. A., 2010. The Structure and Form of Urban Settlements. In: Remote Sensing of Urban and Suburban Areas, Remote Sensing and Digital Image Processing 10 (ed. Rashed, T., Jürgens, C.), Springer, Dordrecht, Heidelberg, London, New York, pp. 13-31.

Huete, A. R., 1988. A soil-adjusted vegetation index (SAVI), Remote sensing of environment, 25 (3), pp. 295-309.

McFeeters, S. K., 1996. The use of the Normalized Difference Water Index (NDWI) in the delineation of open water features, International journal of remote sensing, 17 (7), pp. 1425-1432.

Ridd, M. K., 1995. Exploring a VIS (vegetation-impervious surface-soil) model for urban ecosystem analysis through remote sensing: comparative anatomy for cities, International Journal of Remote Sensing, 16 (12), pp. 2165-2185.

Rosina, K., Hurbánek, P., Cebecauer, M., 2016. Using OpenStreetMap to improve population grids in Europe, Cartography and Geographic Information Science, 43 (5).

Story, M., Congalton, R. G., 1986. Accuracy assessment - A user's perspective, Photogrammetric Engineering and remote sensing, 52 (3), pp. 397-399.

Trimble Documentation, 2014. Trimble eCognition Developer 9 User Guide, Trimble Germany GmbH.

Yang, C., Everitt, J. H., Bradford, J. M., \& Murden, D., 2004. Airborne hyperspectral imagery and yield monitor data for mapping cotton yield variability, Precision Agriculture, 5 (5), pp. 445-461.

Zagrebačka infrastruktura prostornih podataka, Gradski ured za strategijsko planiranje i razvoj grada, "Digitalni ortofoto 2012 (CDOF2012)", Zagreb, Croatia https://geoportal.zagreb.hr/ Public/Ortofoto2012_Public/MapServer/WMSServer (13 Jun. 2016).

Zhu, A. X., 2008. Rule based mapping. In: The handbook of geographic information science (ed. Fotheringham, A. S., Wilson, J. P.), Blackwell, Malden, Oxford, Carlton, pp. 273291. 


\title{
RIVER FLOODPLAIN VEGETATION CLASSIFICATION USING MULTI-TEMPORAL HIGH-RESOLUTION COLOUR INFRARED UAV IMAGERY
}

\author{
W.K. van Iersel ${ }^{\text {a* }}$ E.A. Addink ${ }^{\mathrm{a}}$, M.W. Straatsma ${ }^{\mathrm{a}}$, H. Middelkoop $^{\mathrm{a}}$ \\ ${ }^{a}$ Utrecht University, Department of Physical Geography, Faculty of Geosciences, Heidelberglaan 2, 3584 CS Utrecht, \\ the Netherlands - (w.k.vaniersel@uu.nl)
}

KEY WORDS: river floodplains, land cover classification, multi-temporal data, aerial photography, UAV, DSM

\begin{abstract}
:
To evaluate floodplain functioning, monitoring of its vegetation is essential. Although airborne imagery is widely applied for this purpose, classification accuracy $(\mathrm{CA})$ remains low for grassland $(<88 \%)$ and herbaceous vegetation $(<57 \%)$ due to the spectral and structural similarity of these vegetation types. Increased availability of Unmanned Aerial Vehicles (UAV) allows low-cost production of high-resolution orthophotos and digital surface models (DSMs). Multi-temporal DSMs and orthophotos may be used as input for an improved classification methodology, using differences in phenological changes between vegetation types. The aim of this study was (1) to evaluate the improvement of the CA when using multi-temporal UAV-derived imagery and (2) to determine which layers of a multi-temporal imagery and derived DSMs yield an optimal balance between CA and acquisition effort. During six field surveys with six to ten weeks intervals over one year, a floodplain section along the lower Rhine, the Netherlands, was recorded with true-colour and false-colour imagery with a UAV. In several segmentation-classification-evaluation loops we determined the most important set of variables and the data layers providing them. Our main conclusions are (1) Multi-temporal data input greatly improve CAs of grassland and herbaceous vegetation classes in floodplains: user's accuracies exceed $90 \%$, and (2) the input data contributing most to these high CAs are NDVI layers from winter, spring and summer, and nDSM layers from winter and end of summer.
\end{abstract}

\section{INTRODUCTION}

The different user functions of river floodplains might compete with each other, such as water storage during peak discharge, ecological value, agricultural practices and recreation. They also result in a different land cover of the floodplain, or may have demands concerning land cover characteristics, such as a low hydraulic roughness for flood protection. Ecological restoration in floodplains has generally resulted in more heterogeneous floodplain vegetation (Göthe et al., 2016). Due to vegetation phenology and succession, both ecological and hydraulic characteristics of the floodplain change over time. To evaluate floodplain functioning, monitoring of its vegetation is essential. However, using the current classification methods of airborne surveys classification accuracy (CA) remained low for grassland and herbaceous vegetation (Knotters and Brus, 2013) due to the spectral and structural similarity of these vegetation types (Geerling et al., 2007). For floodplains along the lower Rhine river in the Netherlands, Knotters and Brus (2008) reported a user's accuracy (UA) of $22-57 \%$ for herbaceous vegetation and $74-88 \%$ for grassland. Moreover, current vegetation mapping of these floodplains is based on manual identification and classification of vegetation objects on airborne imagery. Until now vegetation classification has mainly been performed using single-moment datasets. The increased availability of Unmanned Aerial Vehicles (UAV) allows low-cost production of high-resolution orthophotos and digital surface models (DSMs). Multi-temporal DSMs and orthophotos may be used as input for an improved classification methodology, using the differences in development over time of object variables as vegetation height, colour and photosynthetic activity (Xie et al., 2008; Yan et al., 2008). The aims of this study were (1) to evaluate the improvement of the CA with multi-temporal data and (2) to determine which layers of a multi-temporal imagery and derived DSMs yield an optimal balance between CA and acquisition effort.

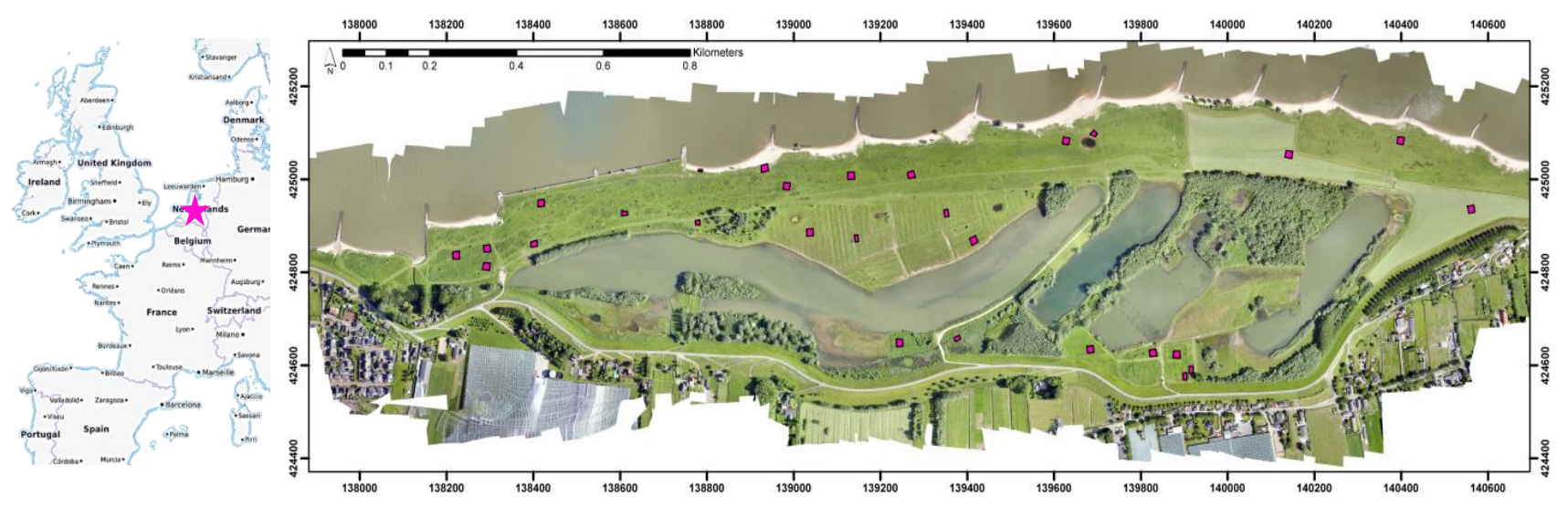

Figure 1. Location of study area in the Netherlands (left). True colour orthophoto of Breemwaard in June with reference plots (magenta). 


\section{STUDY AREA}

We studied the Breemwaard floodplain, sized 116 ha, in the Netherlands. Approximately $30 \%$ of the area is used as hayfield and is cut several times per year. Several parts of the floodplain are managed by private owners and used as willow fields, reed fields or for grazing ponies. The remaining part is a nature area, which is managed by the state forestry and grazed by cows and ponies. Certain part of the nature area, which are fenced off for cattle, developed into riparian woodland. This variability in management of the area has resulted in heterogeneous vegetation types and structure, which made it well suited for classification experiments of riparian vegetation.

\section{METHODS}

\subsection{Imagery and DSMs data layers}

During six field surveys with six to ten weeks intervals over one year, the entire study area was recorded with true-colour and false-colour imagery at $5 \mathrm{~cm}$ resolution from a UAV. Surveys were done in February, April, June, September, November 2015 and January 2016. The UAV images were converted to true and false colour orthophotos and point cloud DSMs of the study area for each survey using the Structure-from-Motion (SfM) method (Lucieer et al., 2014). The false colour orthophotos were used to derive the Normalized Difference Vegetation Index (NDVI) with the near-infrared and blue band. The true and false colour imagery was used to establish respectively RGB DSMs and NIR DSMs. To determine the height of the vegetation above the terrain from the imagery, the DSM of each survey was normalized by abstracting the DSM of February 2015 from it. Thereby, it was implicitly assumed that the February DSM represents the Digital Terrain Model (DTM), which is reasonable for herbaceous vegetation in leaf-off conditions. The DTM of February was denoised by gridding the initial point cloud obtained from the SfM preprocessing to a 0.2 $\mathrm{m}$ resolution grid. Average spacing of the initial dense point cloud was $0.08 \mathrm{~m}$. Large water areas were masked in the normalized DSMs (nDSMs), because of unreliably high values in the dense point clouds.

A layer is a raster-based derivative of a UAV campaign, with nine layers per campaign: R-G-B from the true color orthophoto NIR-R-G from the false colour orthophoto, RGB-nDSM, NIRnDSM, and NDVI. The six campaigs provided a total of 54 layers.

Field reference data for the vegetation classes was collect during the survey in September 2015. Vegetation classes pioneer, grassland, herbaceous and reed vegetation were assigned to field plots of $15 \mathrm{~m} \times 15 \mathrm{~m}$ based on dominant species and vegetation height in the field.

\subsection{Segmentation-classification-evaluation loop}

The layers (section 3.1) were used as input for the segmentation, leading to segment attributes: the mean and standard deviation of the 54 layers, plus the length-with ratio, giving a total of 109 attributes. These segment attributes were used as explanatory variables in the RF classifier. An overview of the segmentationclassification-evaluation loop is shown in figure 2 . To find the most important variables the segmentation was performed with Definiens Developer. Initially all 54 data layers were used as input for segmentation. A scale parameter of 50 showed the best fit in object size with previous results based on multi-temporal NIR imagery only. Shape and compactness were both kept at 0.1 during every loop. The segments overlapping with field plots were classified using the Random Forest classification
(Breiman, 2001). The object variables available for the classification were the length/width ratio of the objects and their mean and standard deviation from all orthophotos, NDVI and nDSM layers for each time step. The classification was trained with $50 \%$ of the reference data from the field and validated with the remaining 50\%. The 10 most important variables for the RF classification, based on the decreased mean accuracy and gini index, were used to determine which layers were to be used in a new segmentation-classification-evaluation. Only those layers with the 10 most important variables were used to perform the segmentation again. For each segmentation, the scale parameters were adjusted to keep the number of objects constant (deviation max. \pm 10.000 ) over the segmentation loops. The new segmentation would again be classified by taking all the same segment features as in the first segmentation into account.

This segmentation-classification-evaluation loop was repeated three times until convergence to a static set of variables was obtained (Figure 2). The final set of variables was each time one of the 10 most important layers in all of the three loops. During the final classification of the study area only the final set of variables was used to segment and classify the study area.

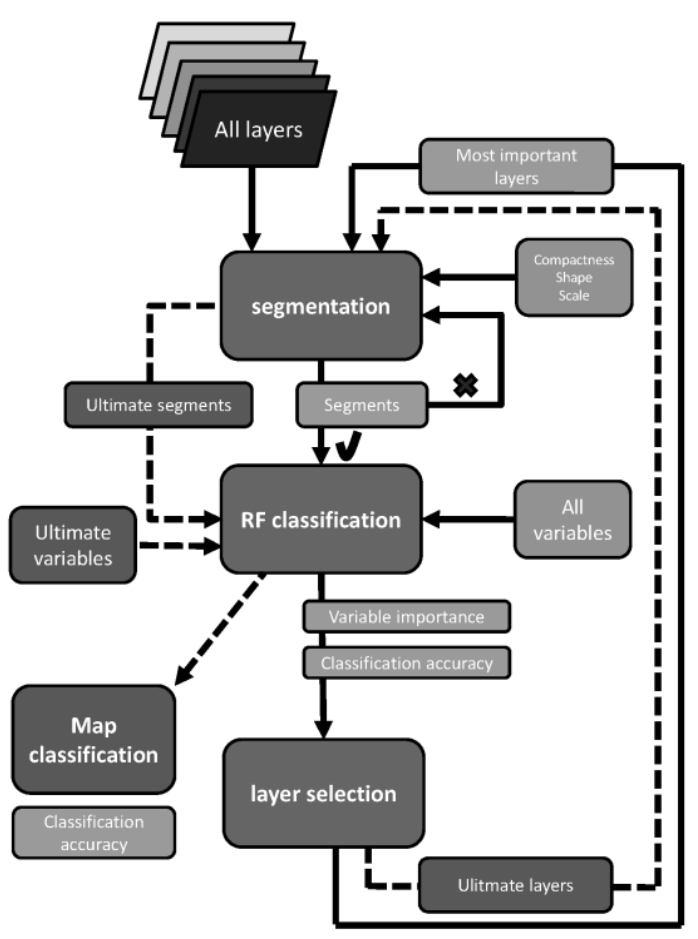

Figure 2. Segmentation-classification-evaluation loops, which were performed three times, plus an additional segmentation and classification with only the ultimate selection of layers.

\section{RESULTS}

\subsection{Multi-temporal segmentation-classification-evaluation}

For the segmentation of the first loop with all data layers a scale parameter of 50 was used. In the second loop only nine data layers were used, in the third loop ten data layers and in the fourth loop also nine (table 1). To keep objects of approximately the same size over the loops, the scale parameters could be kept as 50 in the second and third loop, but was adjusted to 45 in the fourth loop. 


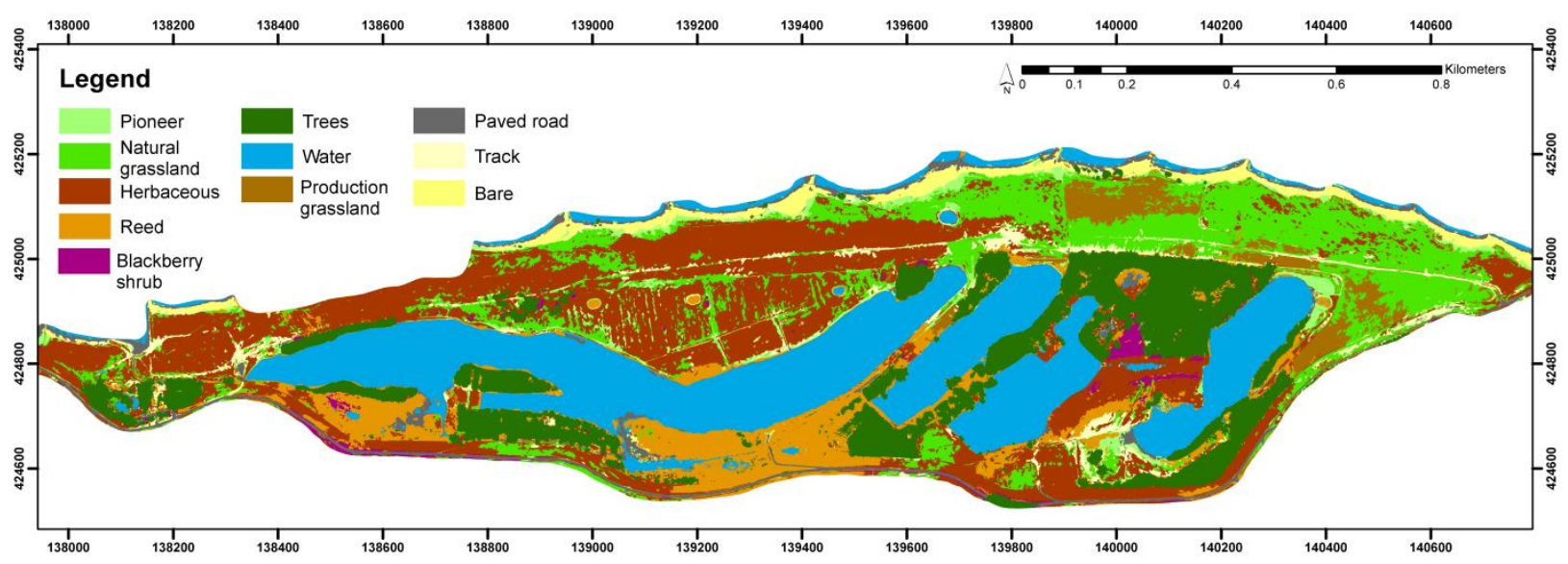

Figure 3. Map of classification of the Breemwaard study area.

Table 1. Most important variables for the four iterations. The position of the final variables is highlighted in grey shading in the first three loops. $M=$ mean, first 4 numbers = year, last two number = month, last part of code is the data layer.

\begin{tabular}{c|l|l|l|l}
\hline \multirow{2}{*}{ Top 10} & $\begin{array}{l}\text { Explanatory variables of } \\
\text { loop1 }\end{array}$ & $\begin{array}{l}\text { Explanatory variables of } \\
\text { loop2 }\end{array}$ & $\begin{array}{l}\text { Explanatory variables of } \\
\text { loop3 }\end{array}$ & \multicolumn{2}{l}{ Final selection of variables } \\
\hline 1 & M_201502_nDSM_NIR & M_201601_NDVI & M_201504_NDVI & M_201504_NDVI \\
2 & M_201504_NDVI & M_201506_NDVI & M_201601_NDVI & M_201502_nDSM_NIR \\
3 & M_201506_NDVI & M_201511_RGB_B & M_201509_NIR_G & M_201506_NDVI \\
4 & M_201506_NIR_NIR & M_201504_NDVI & M_201506_NDVI & M_201509_nDSM_NIR \\
5 & M_201509_nDSM_NIR & M_201509_nDSM_NIR & M_201509_nDSM_NIR & M_201509_nDSM_RGB \\
6 & M_201509_NIR_G & M_201509_nDSM_RGB & M_201509_nDSM_RGB & M_201601_NDVI \\
7 & M_201509_NIR_B & M_201502_nDSM_NIR & M_201509_NIR_B & \\
8 & M_201509_nDSM_RGB & M_201601_NIR_NIR & Length/width & \\
9 & M_201601_NDVI & M_201601_RGB_B & M_201502_nDSM_NIR & \\
10 & Length/Width & M_201502_NIR_B & SD_NIR_R & \\
\hline
\end{tabular}

Each segmentation-classification-evaluation loop had a different set of 10 most important variables (table 1). However, all loops had six variables present top 10 of most important variables for their RF classification, which are highlighted in table 1 . The ultimate set of layers is composed of NDVI and nDSM mean values from different time steps. Moreover, the important mean nDSM of February is obtained from false colour imagery and the mean nDSM from November from true and false colour imagery. The important mean NDVI variables are obtained from two moments in spring (April and June) and one in winter (January).

\subsection{Ultimate multi-temporal segmentation and classification}

For the segmentation with the ultimate data layers (table 1) the scale parameters was adjusted to 37 to maintain comparable object sizes with the other loops. The RF classification using only the ultimate variables layers was applied on all objects resulting from the segmentation with only the ultimate data layers. Even with this data reduction of $94.5 \%$ user's accuracies for grassland and herbaceous vegetation are still $92.6 \%$ and $92.0 \%$ respectively. The map of all classified object in general represent the spatial arrangement of the different vegetation classes well (figure 3). Some classes still get mixed up in the classification, like paved road and water surfaces.

\section{DISCUSSION AND CONCLUSIONS}

Even though the data reduction is $94.5 \%$, the ultimate variables are still from 5 different time steps. This means that still data acquisition needs to take place at five moments in time to get the desired classification accuracies. On the other hand, five out of six ultimate variables are derived from false colour imagery. Moreover, these ultimate layers cover the extremes of the phenology of the vegetation, since (1) the NDVI changes most from winter to spring and summer, and (2) vegetation height difference is largest between winter and the end of summer.

It also needs to be noted that high correlation can be expected between nDSM_NIR and nDSM_RGB, resulting in an even further data reduction. The contribution of the individual layers has not been checked yet.

For practical reasons it will also be interesting to see the CA when the procedure is repeated with fewer time steps. For this purpose the approach needs to be changed from keeping layers for the next segmentation-classification loop to dropping all layers of non-important variables during the evaluation. Each loop a time step can be removed, until the classification accuracy becomes unacceptably low.

The confusion of the classes water and paved road in the ultimate mapped classification can be explained by their low mean NDVI and low mean height values which also do not change over time. 
Our main conclusions are:

- Multi-temporal data input greatly improved CAs of grassland an herbaceous vegetation classes in floodplains: user's accuracies exceed $90 \%$.

- The input data contributing most to this high CAs are multi-temporal NDVI layers from both winter, spring and summer and nDSM layers from winter and end of summer. These layers cover the extremes of the phenology of the vegetation.

- Non-vegetation classes behaving similarly in NDVI and height over time, like water and paved roads, still get confused in the classification.

\section{ACKNOWLEDGEMENTS}

This research is part of the STW-funded RiverCare project.

\section{REFERENCES}

Breiman, L. (2001), Random Forests, Machine Learning 45(1), 5-32.

Geerling, G. W., Labrador-Garcia, M., Clevers, J. G. P. W., Ragas, A. M. J., \& Smits, A. J. M. (2007). Classification of floodplain vegetation by data fusion of spectral (CASI) and LiDAR data. International Journal of Remote Sensing, 28(19), pp. 4263-4284.

Göthe, E., Timmermann, A., Januschke, K., \& BaattrupPedersen, A. (2016). Structural and functional responses of floodplain vegetation to stream ecosystem restoration. Hydrobiologia, 769(1), pp. 1-14

Knotters, M., \& Brus, D. J. (2013). Purposive versus random sampling for map validation: a case study on ecotope maps of floodplains in the Netherlands. Ecohydrology, 6(3), 425-434.

Lucieer, A., De Jong, S. M., \& Turner, D. (2014). Mapping landslide displacements using Structure from Motion (SfM) and image correlation of multi-temporal UAV photography. Progress in Physical Geography, 38, pp. 97-116

Xie, Y., Sha, Z., \& Yu, M. (2008). Remote sensing imagery in vegetation mapping: a review. Journal of plant ecology, 1(1), pp. 9-23.

Yan, E., Wang, G., Lin, H., Xia, C., \& Sun, H. (2015). Phenology-based classification of vegetation cover types in Northeast China using MODIS NDVI and EVI time series. International Journal of Remote Sensing, 36(2), 489-512. 


\title{
Local Climate Zone Mapping: A Case Study In Belgium
}

\author{
M.L. Verdonck ${ }^{\mathrm{a}, *}$ and F. Van Coillie ${ }^{\mathrm{a}}$ \\ ${ }^{a}$ FORSIT, Faculty of Bioscience Engineering, University Ghent, Belgium- marieleen.verdonck@ ugent.be
}

KEY WORDS: Local climate zones, Landsat-8, WUDAPT, Belgium, urban, characteristic properties, OBIA

\begin{abstract}
:
Classifying a city according to the local climate zone (LCZ) scheme can provide insights into the thermal issue of urban planning. The different zones of the LCZ scheme feature particular morphological properties in terms of terrain roughness class, aspect ratio, sky view factor, surface admittance, etc. Our goal is to define the values for remote sensing and/or GIS based properties that can be used to extract local climate zones in Brussels, Belgium. The results showed similar values for LCZ 2, LCZ 3, LCZ 6, LCZ 8, LCZ 9, LCZ A, LCZ B and LCZ D compared to the zone property threshold set in the LCZ datasheets. We were not able to delineated characteristic values for aspect ratio, terrain roughness, surface admittance, albedo and Anthropogenic heat flux. Overall we can conclude that this preliminary study shows good results for the city of Brussels. More research should be done to confirm these results and to explore the OBIA approach based on these results.
\end{abstract}

\section{INTRODUCTION}

Urban areas are one of the most important human habitats (Mills, 2007). Nowadays, already 50\% of the world's population is living there and this percentage is expected to rise to $70 \%$ by 2050 (Zhou et al. 2011; UN 2012). Global warming and the increasing world population will only put more pressure on the living conditions in urban habitats (Aertsens et al. 2012; WMO 2013). From a thermal comfort point of view, it is clear that there is a need for sustainable urban planning that integrates the impact of new developments on the urban temperature regime. It is therefore key to delineate zones with a uniform thermal behaviour. Such zones were developed by Stewart and Oke (2012): local climate zones (Appendix 1, table $1)$.

\begin{tabular}{|l|l|}
\hline \multicolumn{1}{|c|}{ Built zones } & \multicolumn{1}{c|}{ Natural zones } \\
\hline \hline LCZ 1: Compact high-rise & LCZ A: Dense trees \\
\hline LCZ 2: Compact mid-rise & LCZ B: Scattered trees \\
\hline LCZ 3: Compact low-rise & LCZ C: Bush, Scrub \\
\hline LCZ 4: Open high-rise & LCZ D: Low plants \\
\cline { 1 - 1 } LCZ 5: Open mid-rise & LCZ E: Bare rock or paved \\
\hline LCZ 6: Open low-rise & LCZ F: Bare soil or sand \\
\cline { 1 - 1 } LCZ 7: Large low-rise & LCZ G: Water \\
\cline { 1 - 1 } LCZ 8: Large low-rise & \\
\cline { 1 - 1 } LCZ 9: Sparsely built & \multicolumn{1}{|c}{ Table 1. LCZ list }
\end{tabular}

Since 2015 a remote sensing based method is developed (Bechtel et al. 2015; Bechtel and Daneke 2015) to delineated LCZs in a spatial explicit manner. Verdonck et al. (in review) showed that this method might not be optimal for small heterogeneous cities. They developed a method which is based on the original one, using contextual information. One of the remaining issues of using a spatially explicit method to delineate LCZ maps is the transferability of the properties assigned to the different zones by Stewart and Oke (2012). Both methods mentioned above use visually selected training data to classify Landsat images using a random forest classifier. This selection stage is subjective and therefore we need to examine whether the zone properties derived from a spatially explicit mapping approach are conform to the thresholds set by Stewart and Oke (2012)(Appendix 2).

The objective of this preliminary study is to examine the values of the properties in the spatially explicit LCZ maps and the LCZ conceptual framework from Stewart and Oke (2012).

\section{MATERIALS AND METHODS}

\subsection{Data}

For this preliminary study we used a LCZ map of Brussels based on the method described in Verdonck et al. (in review). The map contains 10 local climate zones: LCZ 1 (Compact high-rise), LCZ 2 (Compact mid-rise), LCZ 3 (Compact lowrise), LCZ 6 (Open low-rise), LCZ 8 (Large low-rise), LCZ 9 (Sparsely built), LCZ A (Dense trees), LCZ B (Scattered trees), LCZ D (Low plants), LCZ G (Water). The remaining zones cover too small a surface to be classified correctly (LCZ 4: Open high-rise and LCZ 5: Open mid-rise) or are not present at all (LCZ 7: Large low-rise, LCZ 10: Heavy industry, LCZ C: Bush, Scrub, LCZ E: Bare rock or paved, LCZ F: Bare soil or sand)

To evaluate building height and sky view factor (SVF), high resolution Digital Surface and Digital Terrain models were used. For the region of Flanders this data is freely distributed by AGIV (AGIV, 2015). SVF maps were generated using the sky view factor tool in SAGA GIS.

Fraction maps for impervious, pervious and building surface were used to determine percentage land cover. These maps are generated using hyperspectral images for Brussels. The study area covered is visualised in figure 1 .

\footnotetext{
* Corresponding author
} 


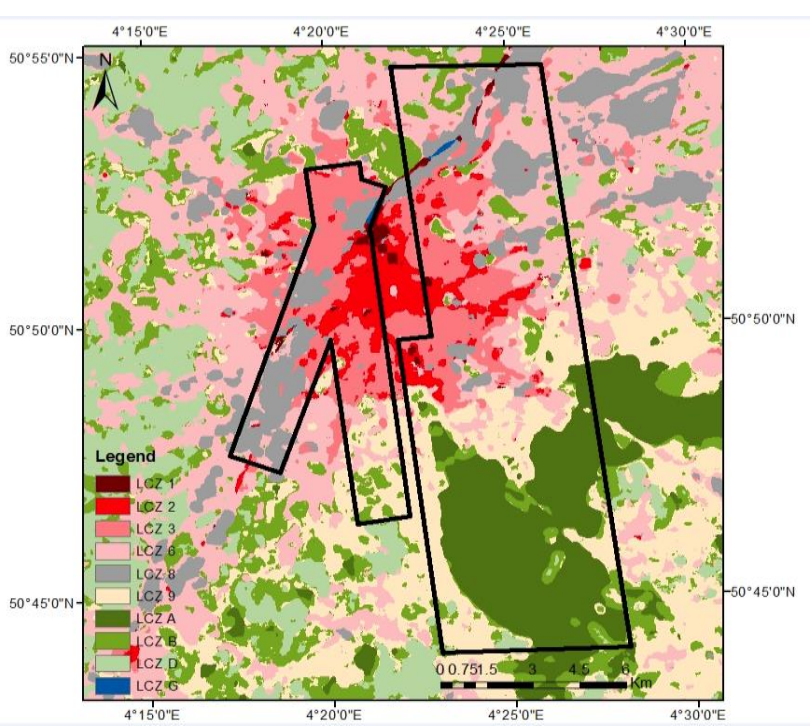

Figure 1. Visualised study area on spatially explicit LCZ map

\subsection{Method}

Seeing as this is a preliminary study we only evaluated 5 properties (SVF, building/tree height, Building surface fraction, Impervious surface fraction, pervious surface fraction) for a small portion of Brussels. For each LCZ the mean value and the standard deviation of each property is calculated.

\section{RESULTS AND DISCUSSION}

The values for the properties based on the spatially explicit LCZ map are shown in table 2 .

\begin{tabular}{|c|c|c|c|c|c|c|c|c|c|c|}
\cline { 2 - 12 } \multicolumn{1}{c|}{} & \multicolumn{2}{|c|}{ SVF } & \multicolumn{2}{c|}{$\begin{array}{c}\text { Building } \\
\text { Height }\end{array}$} & \multicolumn{2}{c|}{$\begin{array}{c}\text { Building } \\
\text { Fraction }\end{array}$} & \multicolumn{2}{c|}{$\begin{array}{c}\text { Impervious } \\
\text { Fraction }\end{array}$} & \multicolumn{2}{c|}{$\begin{array}{c}\text { Pervious } \\
\text { Fraction }\end{array}$} \\
\cline { 2 - 12 } \multicolumn{1}{c|}{} & M & S & M & S & M & S & M & S & M & S \\
\hline LCZ 1 & 0.70 & 0.20 & 18.41 & 11.37 & 0.27 & 0.31 & 0.27 & 0.28 & 0.45 & 0.38 \\
\hline LCZ 2 & 0.59 & 0.21 & 16.03 & 9.80 & 0.46 & 0.30 & 0.29 & 0.25 & 0.24 & 0.27 \\
\hline LCZ 3 & 0.61 & 0.20 & 11.10 & 5.77 & 0.49 & 0.27 & 0.25 & 0.23 & 0.25 & 0.26 \\
\hline LCZ 6 & 0.76 & 0.18 & 10.06 & 6.33 & 0.22 & 0.23 & 0.22 & 0.23 & 0.56 & 0.31 \\
\hline LCZ 8 & 0.82 & 0.17 & 10.27 & 5.99 & 0.34 & 0.34 & 0.37 & 0.30 & 0.29 & 0.30 \\
\hline LCZ 9 & 0.67 & 0.19 & 9.89 & 6.26 & 0.14 & 0.18 & 0.10 & 0.16 & 0.75 & 0.25 \\
\hline LCZ A & 0.29 & 0.22 & 15.91 & 10.06 & 0.00 & 0.04 & 0.06 & 0.09 & 0.93 & 0.10 \\
\hline LCZ B & 0.73 & 0.22 & 10.40 & 7.19 & 0.04 & 0.11 & 0.08 & 0.16 & 0.87 & 0.22 \\
\hline LCZ D & 0.90 & 0.13 & 5.25 & 3.37 & 0.01 & 0.05 & 0.03 & 0.09 & 0.95 & 0.13 \\
\hline LCZ G & 0.87 & 0.12 & 9.17 & 4.38 & 0.09 & 0.19 & 0.15 & 0.22 & 0.75 & 0.33 \\
\hline
\end{tabular}

Table 2. Properties based on the spatially explicit LCZ map for Brussels $(\mathrm{M}=$ mean, $\mathrm{S}=\mathrm{STDEV})$

The results show that most properties fit between the thresholds set by Stewart and Oke (2012) (Appendix 2). Some of the zones such as LCZ 1 and LCZ G do not fit the scheme. Both zones only cover a small surface area in the study area, it was also shown by Verdonck et al. (in review) that these zones were not wel classified. Next to that we see high standard deviations for building height. This is due to the heterogeneous nature of the study area.

\section{CONCLUSION}

The results of this preliminary study show that a visual selection of training data does result in a spatial explicit LCZ map with property values ranging between the thresholds set by Stewart and Oke (2012). Further research should be conducted to analyse whether valuable maps can be produced using an Object based method.

\section{ACKNOWLEDGEMENTS}

We thank USGS, the NASA and AGIV for the freely available Landsat and Lidar data. We also thank the Belgian Federal Science Policy Office for providing research funds to make this research, as part of the UrbanEARS project, possible.

\section{REFERENCES}

Aertsens J., De Nocker L., Lauwers H., Norga K., Simoens I., Meiresanne L., Turkelboom F. \& Broekx S., 2012. Daarom Groen! Waarom u wint bij groen in u stad of gemeente. Studie uitgevoerd in opdracht van: ANB-Afdeling Natuur en Bos

Agentschap voor Geografische informatie Vlaanderen, 2015. Standaardproducten Digitaal Hoogtemodel II. https://download.agiv.be/Producten/Detail?id=966\&title=Stand aardproducten_Digitaal_Hoogtemodel_Vlaanderen_II (1 July 2016)

Bechtel, B.; Alexander, P.; Böhner, J.; Ching, J.; Conrad, O.; Feddema, J.; Mills, G.; See, L.; Stewart, I., 2015. Mapping Local Climate Zones for a Worldwide Database of the Form and Function of Cities. ISPRS Int. J. Geo-Information, 4, pp. 199 219.

Bechtel, B.; Daneke, C., 2012. Classification of local climate zones based on multiple earth observation data. IEEE J. Sel. Top. Appl. Earth Obs. Remote Sens., 5, pp. 1191-1202.

Mills, G., 2007. Cities as agents of global change. International journal of climatology, 27, pp. 1847-1857

Stewart, I. D., \& Oke, T. R. , 2012. Local climate zones for urban temperature studies. Bulletin of the American Meteorological Society, 93(12), pp. 1879-1900.

UN, United Nations, Department of Economic and Social Affairs, 2012. World Urbanization Prospects, the 2011 Revision. Highlights. New York

Verdonck M.L., Okujeni A., van der Linden S., Demuzere M., De Wulf R. and Van Coillie F., (in review). Influence of neighbourhood on 'Local Climate Zone' mapping in heterogeneous cities. Remote Sensing

World Meteorological Organization, 2013. The global climate 2001- 2010 a decade of climate extremes summary report

Zhou W., Huang G. \& Cadenasso M.L., 2011. Does spatial configuration matter? Understanding the effects of land cover pattern on land surface temperature in urban landscapes. Landscape and urban planning ,102, pp. 54-63 


\section{APPENDIX}

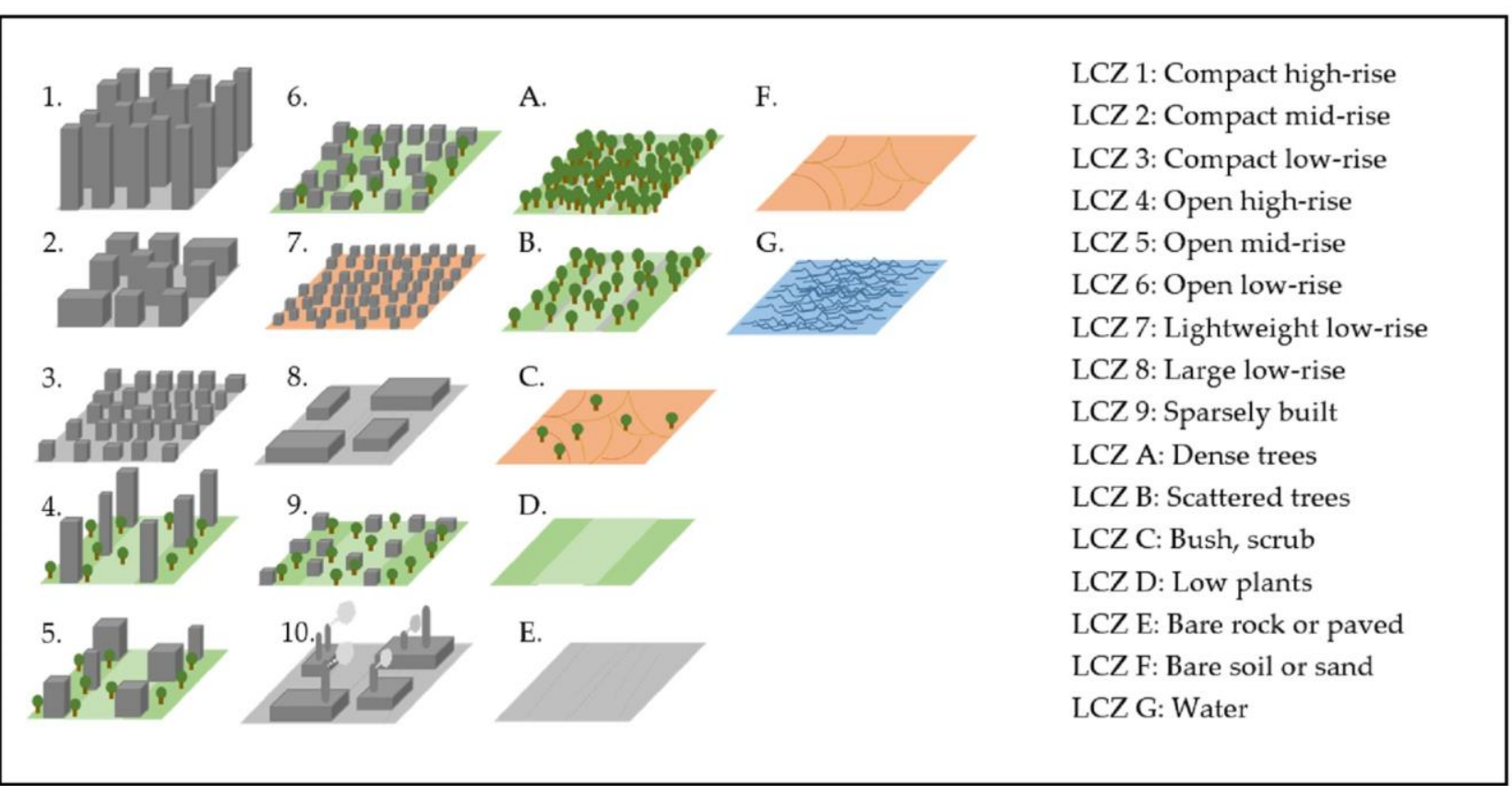

Appendix 1. LCZ scheme

\begin{tabular}{|c|c|c|c|c|c|c|c|c|c|c|c|c|c|c|c|c|c|}
\hline & LCZ 1 & LCZ 2 & LCZ 3 & LCZ 4 & LCZ 5 & LCZ 6 & LCZ 7 & LCZ 8 & LCZ 9 & LCZ 10 & LCZ A & LCZ B & LCZ C & LCZ D & LCZ E & LCZ F & LCZ G \\
\hline SVF & $0.2-0.4$ & $0.3-0.6$ & $0.2-0.6$ & $0.5-0.7$ & $0.5-0.8$ & $0.6-0.9$ & $0.2-0.5$ & $0.7-1$ & $0.8-1$ & $0.6-0.9$ & $0-0.4$ & $0.5-0.8$ & $0.9-1$ & $0.9-1$ & $0.9-1$ & $0.9-1$ & $0.9-1$ \\
\hline Aspect ratio & $2-3$ & $0.75-2$ & $\begin{array}{c}0.75- \\
1.5 \\
\end{array}$ & $\begin{array}{l}0.75- \\
1.25 \\
\end{array}$ & $0.3-0.75$ & $0.3-0.75$ & $1-2$ & $0.1-0.3$ & $0.1-0.25$ & $0.2-0.5$ & $1-3$ & $\begin{array}{l}0.25- \\
0.75 \\
\end{array}$ & $0.25-0.3$ & $0-0.1$ & $0-0.1$ & $0-0.1$ & $0-0.1$ \\
\hline Building/Tree height (m) & $25-50$ & $10-25$ & $3-10$ & $25-50$ & $10-25$ & $3-10$ & $2-4$ & $3-10$ & $3-10$ & $5-15$ & $3-30$ & $3-15$ & $0-2$ & $0-1$ & $0-0.25$ & $0-0.25$ & $0-0.25$ \\
\hline Terrain roughness class & 8 & $6-7$ & 6 & $7-8$ & $5-6$ & $5-6$ & $4-5$ & 5 & $5-6$ & $5-6$ & 8 & $5-6$ & $4-5$ & $3-4$ & $1-2$ & $1-2$ & 1 \\
\hline Building surface fraction $(\%)$ & $40-60$ & $40-70$ & $40-70$ & $20-40$ & $20-40$ & $20-40$ & $60-90$ & $30-50$ & $10-20$ & $20-30$ & $0-10$ & $0-10$ & $0-10$ & $0-10$ & $0-10$ & $0-10$ & $0-10$ \\
\hline Impervious surface fraction $(\%)$ & $40-60$ & $30-50$ & $20-50$ & $30-40$ & $30-50$ & $20-50$ & $0-20$ & $40-50$ & $0-20$ & $20-40$ & $0-10$ & $0-10$ & $0-10$ & $0-10$ & $90-100$ & $0-10$ & $0-10$ \\
\hline Pervious surface fraction $(\%)$ & $0-10$ & $0-20$ & $0-30$ & $30-40$ & $20-40$ & $30-60$ & $0-30$ & $0-20$ & $60-80$ & $40-50$ & $90-100$ & $90-100$ & $90-100$ & $90-100$ & $0-10$ & $90-100$ & $90-100$ \\
\hline Surface admittance $\left(\mathrm{Jm}^{-2} \mathbf{S}^{1 / 2} \mathbf{K}^{-1)}\right.$ & $\begin{array}{l}1100- \\
2200\end{array}$ & $\begin{array}{l}1100- \\
2200 \\
\end{array}$ & $\begin{array}{l}1000- \\
2200 \\
\end{array}$ & $\begin{array}{l}1100- \\
2200\end{array}$ & $\begin{array}{l}1000- \\
2200\end{array}$ & $\begin{array}{l}1000- \\
2200\end{array}$ & $\begin{array}{l}400- \\
1800 \\
\end{array}$ & $\begin{array}{l}1000- \\
2000\end{array}$ & $\begin{array}{l}1000- \\
2200 \\
\end{array}$ & $\begin{array}{l}1000- \\
2500 \\
\end{array}$ & $\begin{array}{l}1000- \\
1800 \\
\end{array}$ & $\begin{array}{l}1000- \\
1800 \\
\end{array}$ & $\begin{array}{l}1000- \\
1800\end{array}$ & $\begin{array}{c}1000- \\
1800\end{array}$ & $\begin{array}{l}1300- \\
3000\end{array}$ & $\begin{array}{l}1000- \\
1800\end{array}$ & 1500 \\
\hline Albedo & $0.1-0.2$ & $0.1-0.2$ & $0.1-0.2$ & $\begin{array}{l}0.12- \\
0.25 \\
\end{array}$ & $\begin{array}{l}0.12- \\
0.25 \\
\end{array}$ & $\begin{array}{l}0.12- \\
0.25 \\
\end{array}$ & $\begin{array}{l}0.15- \\
0.35 \\
\end{array}$ & $\begin{array}{l}0.15- \\
0.25 \\
\end{array}$ & $\begin{array}{l}0.12- \\
0.25 \\
\end{array}$ & $0.12-0.2$ & $0.1-0.2$ & $\begin{array}{l}0.15- \\
0.25 \\
\end{array}$ & $0.15-0.3$ & $\begin{array}{l}0.15- \\
0.25 \\
\end{array}$ & $\begin{array}{l}0.15- \\
0.25 \\
\end{array}$ & $\begin{array}{l}0.20- \\
0.35 \\
\end{array}$ & $0.02-0.1$ \\
\hline Anthropogenic heat flux $\left(\mathrm{Wm}^{-2}\right)$ & $50-300$ & $50-300$ & $0-75$ & $0-50$ & $0-25$ & $0-25$ & $0-35$ & $0-50$ & $0-10$ & $0-300$ & 0 & 0 & 0 & 0 & 0 & 0 & 0 \\
\hline
\end{tabular}




\title{
TOWARDS AUTOMATED SATELLITE IMAGE SEGMENTATION AND CLASSIFICATION FOR ASSESSING DISASTER DAMAGE USING DATA- SPECIFIC FEATURES WITH INCREMENTAL LEARNING
}

\author{
A. Vetrivel*, N. Kerle, M. Gerke, F. Nex, G. Vosselman
}

\author{
University of Twente, Faculty of Geo-Information Science and Earth Observation (ITC), P.O. Box 217, 7500 AE, Enschede, \\ The Netherlands \\ \{a.vetrivel, n.kerle, m.gerke, f.nex, george.vosselman\}@utwente.nl
}

KEY WORDS: Online learning, CNN features, building damage, satellite imagery, streaming training samples

\begin{abstract}
:
Automated damage assessment based on satellite imagery is crucial for initiating fast response actions. Several methods based on supervised learning approaches have been reported as effective for automated mapping of damages using remote sensing images. However, adopting these methods for practical use is still challenging, as they typically demand large amounts of training samples to build a supervised classifier, which are usually not readily available. With the advancement in technologies local and detailed damage assessment for individual buildings is being made available, for example through analysis of images captured by unmanned aerial vehicles, monitoring systems installed in buildings, and through crowdsourcing. Often such assessments are being done in parallel, with results becoming available progressively. In this paper, an online classification strategy is adopted where a classifier is built incrementally using the streaming damage labels from various sources as training samples, i.e. without retraining it from the scratch when new samples stream in. The Passive-Aggressive online classifier is used for the classification process. Apart from the classifier, the choice of image features plays a crucial role in the performance of the classification. The features extracted using recently reported deep learning approaches such as Convolutional Neural Networks (CNN), which learns features directly from images, have been reported to be more effective than conventional handcrafted features such as gray level co-occurrence matrix and Gabor wavelets. Thus in this study, the potential of CNN features is explored for online classification of satellite image to detect structural damage, and is compared against handcrafted features. The feature extraction and classification process is carried out at an object level, where the objects are obtained by over-segmentation of the satellite image. The proposed online framework for damage classification achieves a maximum overall accuracy of about $73 \%$, which is comparable to that of batch classifier accuracy (74\%) obtained for the same training and testing samples, however at a significantly lesser time and memory requirements. Moreover, the CNN features always significantly outperform handcrafted features.
\end{abstract}

\section{INTRODUCTION \& RELATED WORKS}

Very high resolution satellite images, which are usually made available within a few hours after a disaster event such as an earthquake, serve as an ideal data source for rapid damage assessment over large areas for fast response actions (Kerle and Hoffman, 2013). Though many automated methods have been proposed for damage mapping using satellite imagery, in practice operational damage mapping continues to be based on manual interpretation of satellite images, which is time- and labourintensive. There are many reasons for automated methods not yet being adopted for automatic processing, including the limited spatial resolution of satellite imagery compared to increasingly available aerial alternatives, and the complexity of the scene. However, the primary reason is that most of the automated methods are based on supervised learning approaches that require a large volume of training samples to build accurate models which are often not readily available (Dong and Shan, 2013). Also adopting a pre-trained supervised model that was previously developed for a different geographic area or image data type typically has limited transferability. In such cases at least a small number of training samples reflecting the study area is required to fine-tune the pre-trained model. Either for constructing a new model or for calibrating the existing model, a significant number of study-area-specific training samples is required. However, training samples representing the distribution of damage characteristics of the study area, are usually not readily available. Moreover, manual construction of a new training dataset with a large number of samples is not feasible at the time of emergency. With the advancement in technologies, local and detailed damage assessments for individual buildings are made available from various sources. For example, it is becoming increasingly common to assess damages locally after an earthquake. UAV images substantially exceed satellite images in terms of spatial resolution and multi-perspective coverage of individual buildings (Vetrivel et al., 2015). Therefore, automated damage estimation based on UAV images can be more accurate and reliable. Furthermore, damage information for specific locations is made available on-line from many other sources such as buildings equipped with automated monitoring systems (Sdongos et al., 2014) and crowdsourcing (Ghosh et al., 2011; Sdongos et al., 2014). Local damage estimates from such sources could be used to construct the study-area-specific training data for building a required supervised classifier. However, two major challenges need to be addressed when constructing a supervised classifier with such training data:

1) Handling streaming training samples: In the considered scenario damage assessment results from the aforementioned sources arrive at different points in time, also depending on whether data processing takes place locally or remotely. Therefore, a classifier is needed that can be trained dynamically, i.e., when new samples arrive the classifier should learn without retraining from scratch, and reclassify the image if required. This kind of learning is referred to as incremental or on-line learning (i.e. learning without having access to all the samples at once) (Crammer

\footnotetext{
* Corresponding author.
} 
et al., 2006). Conventional batch-learning methods such as Random Forests or Support Vector Machines (SVM) are not suitable for this kind of learning (Narayanan et al., 2016). Many on-line learning algorithms have been developed, and have been shown to perform similarly to batch-learning methods (Hoi et al., 2014; Wang et al., 2014).

2) Data-specific feature for damage classification: Even when a large number of training samples is available, it is critical to choose the features and a representation strategy that is suitable for the specific data, study area and application. For example, in earlier work (cf. Vetrivel et al. (2016)) we examined various image features for identifying damage using images from various geographic locations. It was observed that specific features are performing well for specific study areas. Hence, selection of appropriate features specific to the study area is crucial for improved assessment. Recent research revealed that supervised feature learning methods such as Convolutional Neural Networks (CNN) could learn the data-specific features and their representation directly from the image pixel values (Bengio et al., 2013). These features are found to be far superior to conventional handcrafted features, which are described in the later section (Bengio et al., 2013).

Generally, the regions corresponding to heavy damage are determined through the identification of damage patterns corresponding to rubbles piles, debris and spalling in an image (Kerle and Hoffman, 2013). The recognition process of those damage patterns can be performed by analysing features extracted either at pixel or region level (Dong and Shan, 2013; Kaya et al., 2010; Miura et al., 2013). However, the pixel level analysis is not meaningful for very high spatial resolution images, particularly in the context of damage assessment, as the evidences are identified based on the characteristics of their radiometric distribution pattern, which can be captured more precisely at a region- or object-level. Therefore, super-pixels or segments derived from object-based image analysis approaches are considered as the primary entity for performing feature extraction and classification. Moreover, this kind of segmentbased (super-pixels) approach has been demonstrated as a more efficient approach in several studies compared to pixel-based methods, particularly in applications dealing with very high resolution images (Blaschke, 2010; Blaschke et al., 2014).

Another challenging task is how to compile information from various sources to label each super-pixel as damaged or undamaged. However, this is not the focus of this paper. Instead we center on how to build an incremental classifier if the labels for super-pixels are made available from streaming data sources. Hence, in this study the labels for super-pixels are manually annotated and they are synthetically framed as a stream of training labels obtained from various sources to carry out the online-classification.

To the best of our knowledge online classification with CNN features has not yet been tested for remote sensing applications, particularly for damage assessment. Thus the objective is to develop a framework to use online-learning and $\mathrm{CNN}$ together to build an incremental classifier with data-specific features for automated satellite image-based damage classification from streaming samples.

\section{METHODOLOGY}

The proposed methodology comprises three pipeline processes. As a first step, the satellite image is split into super-pixels using an over-segmentation approach. In the second step, feature extraction is carried for the super-pixels, and as a step 3 an online classifier is constructed based on the extracted features, by considering them as streaming training samples.

\section{Step 1: Over-segmentation of image}

Super pixel construction is a mandatory pre-processing step in many image processing applications. For that a range of methods has been reported (Achanta et al., 2012; Aksoy and Akcay, 2005; Salem et al., 2013). Among them Simple Linear Iterative Clustering (SLIC) (cf. Achanta et al. (2012)) is widely used and reported to be effective for obtaining objects in uniform size, which is suitable to create rectangular image patches, the input format required by common $\mathrm{CNN}$ implementation for extracting features.

\section{Step 2: Feature extraction}

Many kinds of feature extraction techniques, such as statistical, filtering and morphological operations, have been reported for image processing applications (Zhang and Tan, 2002). Among them filtering is recognized as the most effective approach (Arivazhagan et al., 2006; Tian, 2013). For example, many popular features such as Gabor-, Sobel-, Gaussian- and waveletfeatures are based on filtering techniques. Such filtering-based features have proved to be effective for many image processing applications, particularly for image classification (Arivazhagan et al., 2006; Tian, 2013). These filters are designed based on standard mathematical functions and they are referred to as handcrafted features. However, these features are not especially designed for specific data types or applications. It is challenging to choose appropriate features, i.e. designing filters with appropriate weights that give the best image representation for a specific application (Vetrivel et al., 2016). CNN is one of the deep learning approaches where the filters' weights are learned directly from the images chosen for a specific application, instead of using a mathematical function (Krizhevsky et al., 2012). It has been reported that filters that are directly learned from images outperform conventional handcrafted features (Antipov et al., 2015; Chen et al., 2015; Krizhevsky et al., 2012) .

In this study, both hand-crafted- and CNN features are examined and compared for the damage classification capacity.

a) Hand-crafted features: Two kinds of hand-crafted texture features that have been widely reported as effective features for damage classification are considered. They are 1) features based on gray-level co-occurrence matrix (GLCM) and 2) Gabor wavelet features. Details about the extraction of GLCM- and Gabor wavelet- features can be found in Preethi and Sornagopal (2014) and Arivazhagan et al. (2006), respectively.

b) Deep learning features based on $\mathrm{CNN}$ : $\mathrm{CNN}$ can be used for classification in three different scenarios:

1) Training from scratch: Designing and training of new $\mathrm{CNN}$ requires a large amount of training data to avoid overfitting. This method can be adopted if large number of training sample is available.

2) Tuning a pre-trained model: Another common approach is to adopt a pre-trained CNN model that is trained for a related domain (e.g., general image classification) using a large amount of training samples: the network weights of a pre-trained model are fine-tuned using a domain specific 
training samples. This approach also requires relatively many training samples.

3) Pre-trained model without tuning: If only a small amount of training sample is available for the designated application (here damage classification), then a common approach is to extract the features using the pre-trained model and use them to perform classification based on any supervised classifier such as SVM. The activations of one of the fully connected layers in $\mathrm{CNN}$ architecture are considered as the $\mathrm{CNN}$ features for a given image patch (super-pixels). For more details about the layers in $\mathrm{CNN}$ architecture refer to Zeiler and Fergus (2014) and Krizhevsky et al. (2012).

The third approach is most suitable for our application where it is usually common to obtain only small amount of training samples from the aforementioned sources i.e., few hundreds to thousands of samples depending on the nature of the disaster event. Moreover, this approach has been reported to be effective for image classification in various domains, including remote sensing applications such as land cover classification using very high resolution images (Hu et al., 2015). Hence, this approach is adopted in this study where the features for super-pixels are obtained using the pre-trained model and using these features an independent supervised classifier is built for performing the final classification.

\section{Step 3: online classification}

Many online-learning algorithms have been proposed and among them the widely used Passive-Aggressive (PA) algorithm is adopted here for building the online classifier (Crammer et al., 2006). The classifier is built incrementally by providing one sample at a time, where it predicts the label and confidence rate of the provided unseen sample. Further, the classifiers gets updated for each wrong and low confidence prediction. The details of the PA algorithm can be found in Crammer et al. (2006).

The overall work flow is depicted in Figure 1.

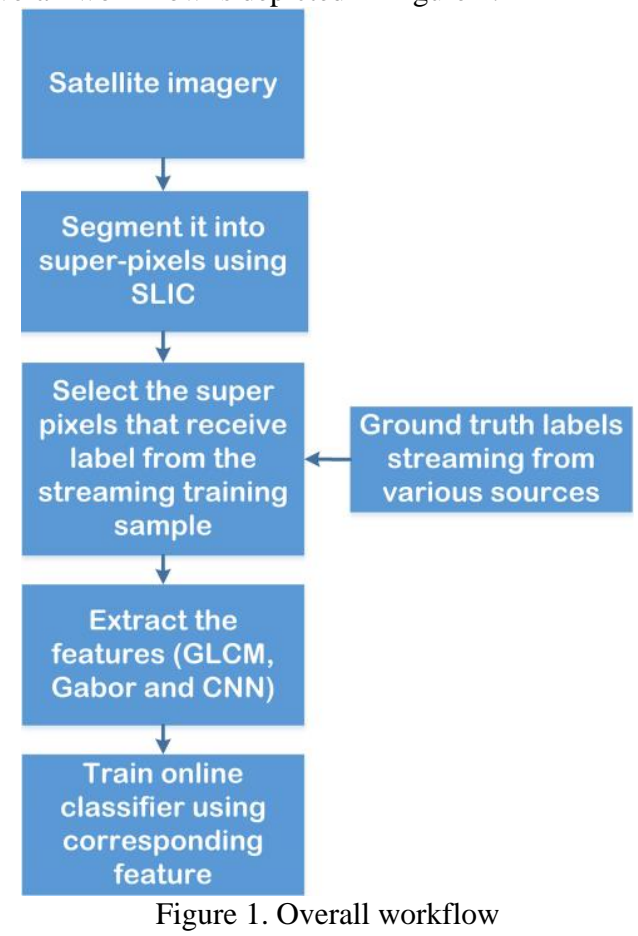

\section{EXPERIMENTS}

\subsection{Data used:}

A subset of a Geoeye satellite image with $50 \mathrm{~cm}$ nominal ground resolution of Port-au-Prince captured after the 2010 Haiti earthquake was considered for this experiment. The damaged and undamaged regions in the image were manually delineated using a polygon and annotated as damaged and undamaged, respectively, to generate the training samples for building the classifier. These polygons are considered as the streaming ground truth information for analyzing the proposed online classifier.

\subsection{Experimental steps and implementation details:}

Step 1: The super-pixels were generated for the selected image subset using SLIC method. As stated earlier, we need superpixels with more uniform size and shape. To achieve this, the parameter ' $m$ ' in SLIC that controls the compactness of a superpixel was empirically determined as 40 .

Step 2: The manually delineated polygons annotated with damage labels were overlaid on the super-pixels of the image. The super-pixels having at least 50\% of their area overlapping with a polygon were assigned with corresponding polygon's label, and considered for training and testing the classifier. In total, 2553 super-pixels were labelled, of which $70 \%$ of the samples were considered for building the classifier, while the remaining $30 \%$ samples were used for testing.

Step 3: Three kinds of features (GLCM, Gabor and CNN features) as described in the methodology section were extracted for the selected super-pixels. The CNN features were extracted using a pre-trained CNN model 'imagenet-caffe-alex' developed byKrizhevsky et al. (2012). This model demands the input of image patch with size $227 \times 227 \times 3$. Hence the super-pixels were converted into rectangular patches and then scaled to above said dimension. In general the images are scaled to larger size using an interpolation technique that significantly degrades the quality of the image. This might have an impact on the quality of the $\mathrm{CNN}$ features. Hence, in this study the image patches based on super-pixels were resized to aforementioned dimensions using two approaches: 1) images resized based on interpolation techniques and 2) images resized based on zero padding. CNN features from image patches obtained based on these two approaches are compared as well.

In total four different features - GLCM, Gabor, CNN_pad (image resized by zero padding) and CNN_nopad (image resized by interpolation technique) are analyzed independently by fitting the PA online classifier.

\subsection{Results}

The results of the online classification are shown in Figure 2, which depicts the cumulative error rate for each feature. Also this figure implicitly shows the number of times the classifier gets updated. The overall accuracy estimated based on their error rates for GLCM, Gabor, CNN_pad and CNN_nopad are 66.5\%, $70.0 \%, 71.0 \%$ and $74.5 \%$, respectively.

It is evident that the online learning algorithm does not need to be re-trained from scratch every time a sample streams in, leading to significantly superior time and memory efficiency. However, this might adversely affect the accuracy of the model. Hence, we intend to determine whether the accuracy diminishes in the online learning setting, and if so, how significant would that be. To this end, we compare the online classifier with canonical batch classifier SVM, using all aforementioned features. The SVM classifier was trained using the same $70 \%$ of the samples. 
Subsequently, both SVM and PA classifiers were evaluated using the remaining $30 \%$ of samples and the accuracies are reported in Table 1 .

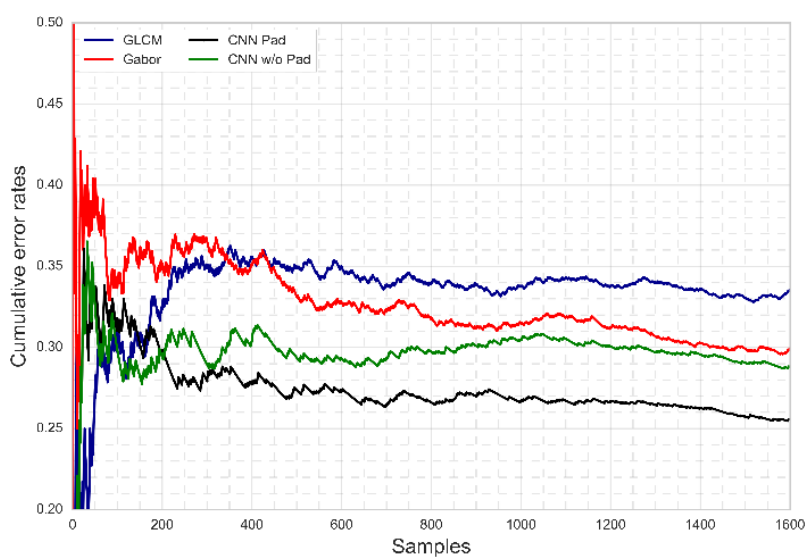

Figure 2. The cumulative error plot of PA online classifiers when associated with GLCM, Gabor and CNN features

\begin{tabular}{|l|l|l|l|l|}
\hline \multirow{2}{*}{ Classifier } & \multicolumn{4}{|c|}{ Overall accuracy in \% } \\
\cline { 2 - 5 } & GLCM & Gabor & CNN_nopad & CNN_pad \\
\hline PA & 57.3 & 59.5 & 68.1 & 73.2 \\
\hline SVM & 63.4 & 63.7 & 70.9 & 74.7 \\
\hline
\end{tabular}

Table 1. Overall accuracy of online- and batch- classifiers when associated with different features.

\section{DISCUSSIONS, CONCLUSIONS AND FUTURE WORK}

A framework was developed for automated mapping of building damage from satellite imagery, using an online classifier that dynamically learns from streaming training samples based on local damage assessments from different sources. In this study, the potential of $\mathrm{CNN}$ features from pre-trained model, in combination with online PA classifier was explored for automated damage classification of satellite imagery. Further, the accuracy retrieved from $\mathrm{CNN}$ features was compared against the standard hand-crafted features GLCM and Gabor by fitting them using the PA classifier. The results show that CNN features are performing better than both handcrafted features. Moreover, the cumulative error graph (cf. Figure 2) shows that CNN features generalize better with fewer training samples than the handcrafted features. For example, the error curve (cf. Figure 2) of CNN tends to be smoother (shows better generalization) after being trained with 1000 samples, while the error curves of handcrafted features fluctuates, indicating that it could not learn fast and generalize well compared to CNN features. Also the error graph shows that the choice of image resizing approach used to resize the super-pixel based image patch in a dimension required for $\mathrm{CNN}$ feature extraction have a significant impact on the performance of the final classification accuracy. For example, $\mathrm{CNN}$ features extracted from the image patch resized using an interpolation technique were found to be significantly inferior to the $\mathrm{CNN}$ features extracted from image patch resized using zero padding (cf. Figure 2).

The features evaluated in online learning settings were also evaluated using the canonical batch mode classifier SVM. The results show that the selected online classifier performs slightly inferior to the batch classifier, irrespective of the features (cf. Table 1). However, the difference in accuracy is only around $1 \%$ concerning $\mathrm{CNN}$ features. There is significant difference between the accuracies of online and batch classifiers when employing the handcrafted features. This is because the online classifiers based on the handcrafted features could not learn and generalize well. The overall conclusion is that the choice of features has significant impact on the performance of the online classifier. Also it is observed that compared to handcrafted features the $\mathrm{CNN}$ features are more effective and consistent for online classification, as they yield similar accuracy when used in the batch learning setting (cf. Table 1).

Though CNN features were reported to be effective for many remote sensing application such as land cover classification, the maximum classification accuracy obtained for damage classification using satellite imagery was around $73 \%$ (cf. Table 1), signalling a continuing limitation of automated damage classification with satellite imagery (Kerle, 2010). This inferior accuracy might be due to many reasons such as insufficient number of training samples used for building the classifier, and complexity of the scene. However, the major reason would be the limitation of the satellite imagery where the considered image resolution is around $50 \mathrm{~cm}$. With this level of spatial resolution even manual interpretation is difficult, and similar problems have previously been reported (Kerle, 2010). The available pre-event satellite imagery could be of help and is usually available. In such a case, the classifier can be trained with $\mathrm{CNN}$ features from both epochs, which could provide better results and we intend to explore this in the future. On the other hand, the training samples considered in this study are from single satellite imagery of a single geographic location, where the characteristics of the streaming samples are not highly variable. However, the usefulness and potential of online learning can be realized when there is high variability in the streaming samples in terms of characteristics of the study area, weather condition and spatial resolution of the images. Hence, as a future work we intend to explore the potential of the proposed framework by applying it to much better spatial resolution satellite or aerial imagery obtained for different geographic locations varying in aforementioned characteristics.

\section{ACKNOWLEDGEMENTS}

The work was funded by FP7 project: RECONASS (Reconstruction and Recovery Planning: Rapid and Continuously Updated Construction Damage and Related Needs Assessment; grant no 312718).

\section{REFERENCES}

Achanta, R., Shaji, A., Smith, K., Lucchi, A., Fua, P., Susstrunk, S., 2012. SLIC superpixels compared to state-of-the-art superpixel methods. IEEE Transactions on Pattern Analysis and Machine Intelligence, 34 (11), pp. 2274-2282.

Aksoy, S., Akcay, H.G., 2005. Multi-resolution segmentation and shape analysis for remote sensing image classification, Proceedings of 2nd International Conference on Recent Advances in Space Technologies, RAST 2005. , pp. 599-604.

Antipov, G., Berrani, S.-A., Ruchaud, N., Dugelay, J.-L., 2015. Learned vs. hand-crafted features for pedestrian gender recognition. Proceedings of the 23rd ACM international conference on Multimedia, Brisbane, Australia, pp. 1263-1266.

Arivazhagan, S., Ganesan, L., Priyal, S.P., 2006. Texture classification using Gabor wavelets based rotation invariant features. Pattern Recognition Letters, 27 (16), pp. 1976-1982.

Bengio, Y., Courville, A., Vincent, P., 2013. Representation learning: A review and new perspectives. IEEE Transactions on Pattern Analysis and Machine Intelligence, 35 (8), pp. 17981828. 
Blaschke, T., 2010. Object based image analysis for remote sensing. ISPRS Journal of Photogrammetry and Remote Sensing, 65 (1), pp. 2-16.

Blaschke, T., Hay, G.J., Kelly, M., Lang, S., Hofmann, P., Addink, E., Queiroz Feitosa, R., van der Meer, F., van der Werff, H., van Coillie, F.,Tiede, D., 2014. Geographic object-based image analysis - towards a new paradigm. ISPRS Journal of Photogrammetry and Remote Sensing, 87, pp. 180-191.

Chen, M., Zhang, L., Allebach, J.P., 2015. Learning deep features for image emotion classification, IEEE International Conference on Image Processing (ICIP) pp. 4491-4495.

Crammer, K., Dekel, O., Keshet, J., Shalev-Shwartz, S., Singer, Y., 2006. Online Passive-Aggressive algorithms. The Journal of Machine Learning Research, 7, pp. 551-585.

Dong, L., Shan, J., 2013. A comprehensive review of earthquakeinduced building damage detection with remote sensing techniques. ISPRS Journal of Photogrammetry and Remote Sensing, 84, pp. 85-99.

Ghosh, S., Huyck, C.K., Greene, M., Gill, S.P., Bevington, J., Svekla, W., DesRoches, R., Eguchi, R.T., 2011. Crowdsourcing for rapid damage assessment: The global earth observation catastrophe assessment network (GEO-CAN). Earthquake Spectra, 27 (S1), pp. S179-S198.

Hoi, S.C., Wang, J., Zhao, P., 2014. Libol: A library for online learning algorithms. The Journal of Machine Learning Research, 15 (1), pp. 495-499.

Hu, F., Xia, G.-S., Hu, J., Zhang, L., 2015. Transferring deep convolutional neural networks for the scene classification of high-resolution remote sensing imagery. Remote Sensing, 7 (11), pp. 14680.

Kaya, G.T., Ersoy, O.K.,Kamasak, M.E., 2010. Spectral and spatial classification of earthquake images by support vector selection and adaptation. International Conference of Soft Computing and Pattern Recognition (SoCPaR), pp. 194-197.

Kerle, N., 2010. Satellite-based damage mapping following the 2006 Indonesia earthquake- - How accurate was it? International Journal of Applied Earth Observation and Geoinformation, 12 (6), pp. 466-476.

Kerle, N., Hoffman, R.R., 2013. Collaborative damage mapping for emergency response: the role of Cognitive Systems Engineering. Natural hazards and earth system sciences (NHESS), 13 (1), pp. 97-113.

Krizhevsky, A., Sutskever, I., Hinton, G.E., 2012. Imagenet classification with deep convolutional neural networks, Advances in neural information processing systems, pp. 1097-1105.

Miura, H., Midorikawa, S., Kerle, N., 2013. Detection of building damage areas of the 2006 Central Java, Indonesia, earthquake through digital analysis of optical satellite images. Earthquake Spectra, 29 (2), pp. 453-473.

Narayanan, A., Liu, Y., Chen, L., Liu, J., 2016. Adaptive and scalable android malware detection through online learning. arXiv preprint arXiv:1606.07150.

Preethi, G., Sornagopal, V., 2014. MRI image classification using GLCM texture features, International Conference on Green Computing Communication and Electrical Engineering (ICGCCEE), pp. 1-6.

Salem, M., Ibrahim, A.F., Ali, H.A., 2013. Automatic quick-shift method for color image segmentation, 8th International Conference on Computer Engineering \& Systems (ICCES), pp. 245-251.
Sdongos, E., Tsertou, A., Georgakopoulos, N., Loupos, K., Amditis, A., Joram, N., Ellinger, F., Lindner, B., Edwan, E., Ehlerding, A., Camarinopoulos, S., Kallidromitis, V., Bairaktaris, D., Bairaktaris, E., Naundrap, J., Kerle, N., Gerke, M., Vierhuß-Schloms, K., Markus, M., 2014. A novel \& practical approach to structural health monitoring -The RECONASS vision: Local positioning, sensor networks, secure communications and remote sensing at the service of structural monitoring to assess construction damage and related needs, IEEE Workshop on Environmental Energy and Structural Monitoring Systems (EESMS) pp. 1-6.

Tian, D.P., 2013. A review on image feature extraction and representation techniques. International Journal of Multimedia and Ubiquitous Engineering, 8 (4), pp. 385-396.

Vetrivel, A., Gerke, M., Kerle, N.,Vosselman, G., 2015. Identification of damage in buildings based on gaps in 3D point clouds from very high resolution oblique airborne images. ISPRS Journal of Photogrammetry and Remote Sensing, 105, pp. 61-78.

Vetrivel, A., Gerke, M., Kerle, N.,Vosselman, G., 2016. Identification of Structurally Damaged Areas in Airborne Oblique Images Using a Visual-Bag-of-Words Approach. Remote Sensing, 8 (3), pp. 231.

Wang, J., Zhao, P.,Hoi, S.C.H., 2014. Cost-sensitive online classification. IEEE Transactions on Knowledge and Data Engineering, 26 (10), pp. 2425-2438.

Zeiler, M.D.,Fergus, R., 2014. Visualizing and Understanding Convolutional Networks. European Conference on Computer Vision (ECCV), pp. 818-833.

Zhang, J., Tan, T., 2002. Brief review of invariant texture analysis methods. Pattern Recognition, 35 (3), pp. 735-747. 


\title{
DEVELOPMENT OF A KNOWLEDGE DRIVEN RULE SET FOR CLASSIFICATION OF SUBMERGED AQUATIC VEGETATION (SAV) IN A CLEAR WATER STREAM: WHERE DO YOU DRAW THE BOUNDARIES...?
}

\author{
Fleur Visser $^{\mathrm{a}}{ }^{*}$, Kerst Buis $^{\mathrm{b}}$, Veerle Verschoren ${ }^{\mathrm{b}}$, Jonas Schoelynck ${ }^{\mathrm{b}}$ \\ ${ }^{\text {a }}$ Institute of Science and the Environment, University of Worcester, Henwick Grove, Worcester WR2 6AJ, UK - \\ f.visser@worc.ac.uk \\ ${ }^{\mathrm{b}}$ Department of Biology, Ecosystem Management Research Group, University of Antwerp, Universiteitsplein 1C, B-2610 Wilrijk, \\ Belgium - (kerst.buis, veerle.verschoren, jonas.schoelynck)@uantwerpen.be
}

KEY WORDS: Macrophytes, OBIA, Remote Sensing, VHR image data, knowledge-based

\begin{abstract}
SUMMARY
A recent attempt at mapping submerged aquatic vegetation (SAV) species composition of a clear water stream in Belgium from ultrahigh resolution, multispectral photographs, using object based image analysis (OBIA), resulted in a low, but consistent overall classification accuracy (53-61\%). Since the results were obtained with a single rule set they show promise for the development of an automated tool to map SAV despite the challenges of its submerged environment. This extended abstract investigates to what extent difficulties with species delineation in the validation data may have influenced the results. We compare class boundaries, as drawn by experts along image segmentation outlines, with the results from the expert knowledge driven classification rules. A comparison for 'pure' objects, where the expert is certain about the assigned object class, resulted in a moderately good overall similarity (68\%), while inclusion of ambiguous objects reduces the results to 59\%. Under ideal circumstances the rule set seems capable of $74 \%$ similarity with expert validation data.
\end{abstract}

\section{INTRODUCTION}

\subsection{Introduction}

The mapping of submerged aquatic vegetation (SAV) using remote sensing techniques has not been attempted much until quite recently. The detailed scale required to study SAV and the absorption of light in water have been major barriers to the effective use of remote sensing techniques. Very High or Ultra High Resolution (VHR) image data in combination with Object Based Image Analysis (OBIA) has brought some change to this situation, as it reduces the reliance on spectral information for vegetation species detection.

Although species can show very subtle differences that even experts find difficult to detect when observing the plants up close, they also display considerable differences in their morphology that allow for broad mapping of SAV species cover by eye from the bank side of a river. For this reason we expected that expert driven OBIA could be a useful tool to provide some form of automation to this mapping process.

An initial attempt at developing a ruleset for the mapping of $\mathrm{SAV}$ in the Desselse Nete, a clearwater stream in Flanders, Belgium has been presented in Visser et al. (accepted). The classification accuracies that we achieved in this study were however not as good as we hoped. We are currently in the process of improving our methods in a number of ways. Adjustments of the ruleset for effects of water depth and variable illumination conditions are two things that we expect may improve the work. Another issue, which may have influenced the results is the complexity of delineating the species class boundaries.

The canopy morphology of the species in our target river varies from very dense with sometimes fuzzy edges (e.g. Blunt-fruited Water Starwort: Callitriche obtusangula Le Gall) to very open groups of narrow, but well defined leaves (e.g. European Burreed: Sparganium emersum L.). Other species included in this study were Water Crowfoot (Ranunculus aquatilis L.), Curlyleaf Pondweed (Potamogeton crispus L.) and Broad-Leaved Pondweed (Potamogeton natans L.). In many cases it is difficult, if not impossible to identify where one species cover starts and another ends. However, the only means to check the classifications we produced was by manually delineating class boundaries in images of our study area. We soon realized that hand-drawn boundaries could not achieve the same detail as automated classification and would therefore not necessarily provide a fair assessment of mapping accuracy. To overcome this problem we decided on a slightly different approach to obtain validation data. Instead we asked our experts to use the Level 1 OBIA image segmentation as basis for their validation map and assign each object to what they think would be its appropriate class.

This still resulted in a number of notable issues. Firstly, although the segmentation applied to the image was to our knowledge the most appropriate, it certainly was not perfect and the experts noted various under-segmented objects, where an additional boundary would have been beneficial in order to assign the object a distinct class. Similarly for the more open canopies where only one or two leaves fall within an object, covering a relatively small part of the object surface area, decisions on how to classify that object are debatable. Where the vegetation of the more open canopies varied in submergence depth another difficulty was caused by the visibility of the target. At some point it becomes difficult to judge from the image whether slight spectral variation is part of the bottom surface texture or presence of vegetation a greater depth. 
This extended abstract further describes and discusses how the validation data was created and how effective it was at assessing the performance of the knowledge-driven classification ruleset.

\section{METHODS}

\subsection{Image data acquisition}

Images used for this project were collected from the Desselse Nete, a lowland River in Flanders, Belgium, during the spring/summer of 2012. The Fujifilm IS-Pro NIR sensitive DSLR camera was used in combination with a radio controlled shutter to produce $3024 \times 2016$ pixels photos in 8-bit GEOTIFF format from a telescopic pole fixed in position with guy ropes at approximately $4.5 \mathrm{~m}$ at nadir over the centre line of the river. Multi-spectral image composites were created by adding different filters to the Tamron AF Aspherical 28-80 mm f/3.55.6 lens. Red, Green and Blue image bands were obtained by adding a NIR blocking filter (model XNite CC1, LDP LLC, Carlstadt, USA, formerly 'maxmax.com', here referred to as ' $\mathrm{CC} 1$ '). A single band covering most of the NIR spectrum (NIR(R72)) was obtained by adding a Hoya R72 VIS blocking filter and a further two bandpass filters (XNite Bandpass IR Filters, LDP LLC, Carlstadt, USA), were used to obtain one narrow NIR wavelength band around $710 \mathrm{~nm}$ (model XNite $\mathrm{BPB}$, here referred to as 'NIR(BP1)') and one around $828 \mathrm{~nm}$ (model XNite BPG, here referred to as 'NIR(BP2')).

\subsection{Image pre-processing and mage segmentation}

The use of a low-cost image data collection approach meant that image data layers could not be collected simultaneously and needed co-registration before they could be combined in an image composite. A significant error is introduced at this stage, more so because the submerged vegetation target is highly dynamic. This meant that plant elements such as leaves were not located in exactly the same position in each image data layer and would not show up as distinct objects during a segmentation based on multiple image layers.

To minimize the effect of this on the further classification we decided to use a segmentation based on a single data layer. For this purpose the NIR(BP1) band was used. This relatively narrow band of the NIR spectrum was noted by the experts involved to show SAV species most clearly, allowing for the most relevant object delineation.

Several issues affected the image quality, for example sun and sky glint at the river water surface. However, no radiometric pre-processing of the data was undertaken, as it was not expected to significantly improve further analysis for reasons described in Visser et al. 2015.

\subsection{Image classification}

The result from this project are based on a ruleset, which was created based on two 'development' images and a validation image, results of which have been published in Visser et al. (accepted). The ruleset has however been modified to enable classifications of one species at a time. We also checked that each included rule related to an identification step undertaken by the experts involved. Examples of this are given in the Results section.

The classification rule set makes use of two segmentation levels. The first level (Level 1) outlines patches of different vegetation species, while objects at the second level (Level 2) delineated distinct plant morphological elements (e.g. individual leaves and stem segments). The parameter settings used for each segmentation level were based on a trial and error approach.

\subsection{Classification validation}

In pixel-based classification studies a sample of cells is generally used to assess the accuracy of a classification. For OBIA classifications the sampling units should consist of polygons rather than pixels (Radoux et al., 2011). However, a universal method that can deal with objects varying both in size and class definition has not yet been devised. Due to the difference in the way automatic classifications and manual delineation of polygons work, they can produce maps with significantly differ ranges in shape and size of polygons. It actually makes the selection of a random sample of polygons to estimate the accuracy of the full classification impossible.

Rather than manually outlining each patch we used the Level 1 segmentation polygons and manually classified all those into one of the available classes, using all six data layers and a field sketch, for confirmation. The validation dataset then consisted of polygons with the same shape and size as the automatic classification. This approach would allow an accuracy assessment based on probability sampling. However, this study is a bit exceptional since there is full validation cover for the classified image, so a direct comparison can be made between the maps without the need to rely on statistical inference.

In the original study of Visser et al. (accepted) similarity values were still relatively low despite the use of Level 1 polygons to create the validation data, with $61 \%$ the best overall accuracy levels obtained. These poor results could be due to inadequate classification rules. However the 'accuracy' of the validation data may also be questioned. The submerged environment is notoriously difficult to map even when done by hand from the bank of a river. Overlap between vegetation species and variation in visibility make it difficult to draw boundaries between SAV species as as well as determine vegetation and substrate boundaries.

Issues with image object composition, as those mentioned in the Introduction made it impossible to assign all objects to an appropriate SAV class. In order to assess the effect of these ambiguous objects, a number of additional combination classes were defined. Where an object would for example consist of mostly river bottom with a few $S$. emersum elements, it could be assigned to $S$. emersum/bottom combination class.

Running rulesets separately for each class resulted in overlap where individual objects fitted the rules of more than one class. All possible classes were noted for each object and compared with the expert classification result to assess how well each class was represented by the set of rules.

\section{RESULTS}

Figure 1 shows the classification of SAV for section of the Desselse Nete river based on a knowledge-driven ruleset.

The following are examples of plant spectral and morphological characteristics for C. Obtusangula, as used by an expert to identify the species:

- Bright overall, but especially in NIR and green with little spectral variation $=>$ Mean NIR(BP1) $>70$ 
Mean NIR(BP2) $>25$

- 'Fluffy' appearance due to rosette shaped leaves/canopy $=>$ Relative area of sub-object 'Rosettes' $>0.23$

- Rosettes shaped leaves or 'Rosettes' => Level 2 objects with: Length/Width $<2$

Similarly rules for S. emersum were as follows:

- Bright narrow and elongated leaves $=>$ Level 2 objects with: Length/Width $>4$

Mean NIR(BP1) > 60

Relative border with brighter objects BP $1<0.5$ Width (18 pxl)

- A certain density of $S$. emersum leaves and absence of $P$. natans leaves $=>$

Number of sub-objects $S$. emersum $>=1$

Relative area of sub-objects. S emersum $>0.1$

Number of sub-objects $P$. natans $<=1$

The chosen parameter values and settings for each of the two segmentation levels are as follows:

Level 1: Multiresolution segmentation: Scale parameter 100; Shape 0.2 Compactness 0.2: NIR(BP1) data layer only.

Level 2: Multiresolution segmentation: Scale parameter 20; Shape 0.2 Compactness 0.2 : NIR(BP1) data layer only.

Figure 1 shows the full classification of the river section.

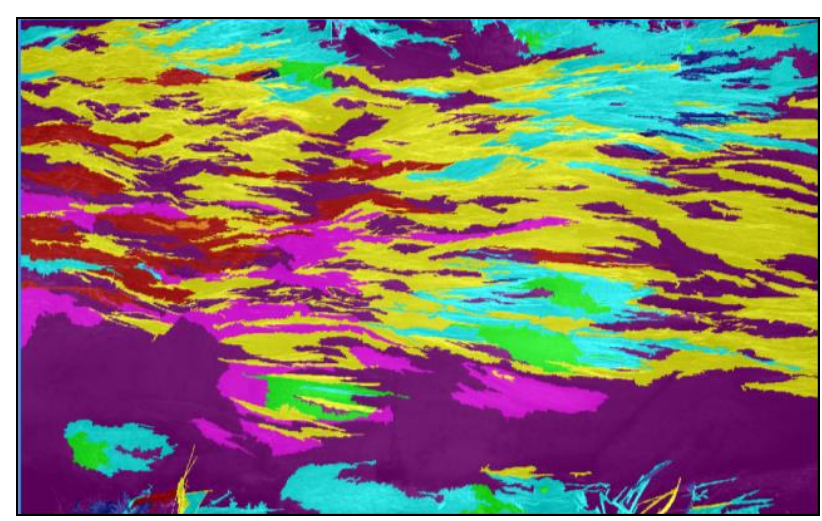

Bank/emergent vegetation
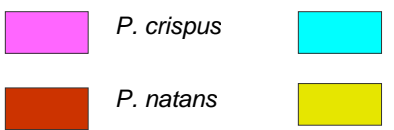

R. aquatilis

Bottom

P. natans

S. emersum

C. obtusangula

Figure 1. Original classification of the river section.

Figures 3a-d provide examples of the classification vs. validation results for four of the 8 classes defined for this project. The figures show areas where the classifications are identical and where they differ for both the automatic classification and the manual validation data.

Table 1 shows percentage similarities of the comparisons of the classification result and the manual validation data.
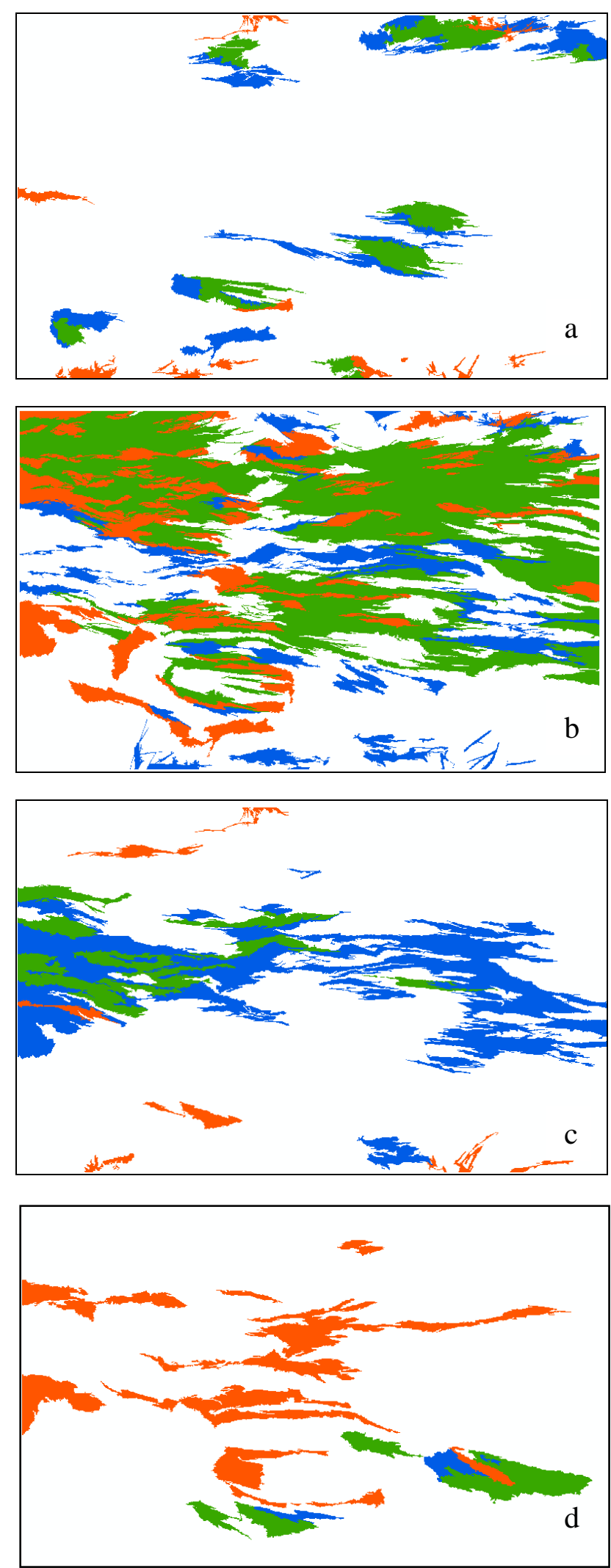

Classification

Validation

Both

Figure 3. Overview of objects that are part of the classification, validation and of both validation and classification, for $C$. obtusangula, (a) S. emersum, (b) P. natans (c) and P. Crispus (d). 


\begin{tabular}{|c|c|c|c|c|c|c|}
\hline & \multicolumn{4}{|c|}{ Per class } & \multicolumn{2}{|c|}{ All classes } \\
\hline & $\begin{array}{c}\text { Correctly } \\
\text { classified / } \\
\text { total in pure } \\
\text { class }\end{array}$ & $\%$ & $\begin{array}{l}\text { Correctly } \\
\text { classified / } \\
\text { total in } \\
\text { class+ } \\
\text { boundary } \\
\text { cases }\end{array}$ & $\%$ & $\begin{array}{l}\text { Correctly } \\
\text { classified / } \\
\text { total }\end{array}$ & $\%$ \\
\hline $\begin{array}{l}\text { Bank/emergent } \\
\text { vegetation }\end{array}$ & $11 / 45$ & 24 & $11 / 45$ & 24 & $11 / 45$ & 24 \\
\hline C. obtusangula & $22 / 39$ & 56 & $24 / 53$ & 45 & $32 / 53$ & 60 \\
\hline P. crispus & $8 / 10$ & & $8 / 11$ & 73 & $8 / 10$ & 80 \\
\hline S. emersum & $259 / 299$ & 87 & $301 / 381$ & 79 & 343 / 375 & 91 \\
\hline R. aquatilis & $10 / 16$ & 63 & $10 / 16$ & 63 & $10 / 16$ & 63 \\
\hline P. natans & $13 / 69$ & 19 & $16 / 118$ & 14 & $51 / 113$ & 45 \\
\hline Total & & 68 & & 59 & & 74 \\
\hline
\end{tabular}

Table 1. Percentages of objects that have the correct class assigned out of 1 or more 'fitting' classes

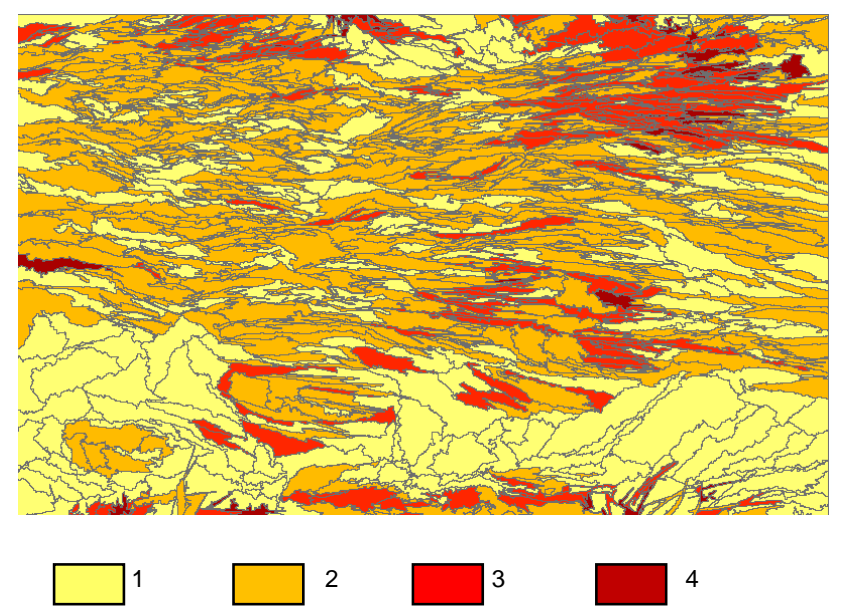

Figure 4. Class membership numbers for each object polygon

When only objects are considered that were manually assigned to one of the six cover classes the classification rules correctly classified $68 \%$ of the objects. The best performing class was $S$. emersum with $87 \%$ of the object correctly classified. P. natans preformed least well with only $19 \%$ of objects correctly classified. When also objects were considered that could not be classed unambiguously by the expert the percentage correctly classified objects is reduced to $59 \%$, with the same best and worst performing classes. However, because there is overlap between the rules of the different classes, this means some objects are part of more than one class (membership numbers are shown in Figure 4). This means in a best case scenario when the best fitting class is chosen for an object a $74 \%$ accuracy could be achieved with the current ruleset.

\section{DISCUSSION}

Table 1 shows that when pure class objects are considered our rules perform moderately well to classify certain plant species with a $68 \%$ overall 'accuracy'. However, the results deteriorate when objects are included that could not be classified unambiguously by the expert, often because they clearly included elements of more than one class. This raises the question exactly how/where the validation class boundaries should be drawn or whether rules should be rewritten.

To some extent problems were created by basing the validation data on image objects, as the object boundaries often did not follow the boundaries the expert would have liked to draw. However, it would be an impossible task to draw boundaries around for example individual $S$. emersum leaves to create the perfect validation dataset.

Alternatively the expert could be requested to assign class membership values to each object, however memberships are difficult to estimate by the expert in the validation data set and also difficult to quantify in an object based environment, where the membership of a polygon to a particular class may be dependent on a combination object features such as the number of leave-shaped sub-object present.

An important consideration is that where an expert will make mistakes and inconsistent decisions on class definitions/content, a digitally applied OBIA ruleset will be able to provide much more objective classification results. This is great strength of the remote sensing approach and should be developed further. However, one does need to bear in mind that the expert-driven ruleset development is also a result of human understanding and perception of class definitions and boundaries, which is a weakness of the approach that requires further work (Arvor et al., 2013).

\section{ACKNOWLEDGEMENTS}

FWO Flanders (Belgium) partly funded this project via the multidisciplinary research project 'Linking optical imaging techniques and 2D-modelling for studying spatial heterogeneity in vegetated streams and rivers' (G.0290.10, Antwerp University, Ghent University, 2010-2013). V.V. obtained research funding from the Institute for the Promotion of Innovation through Science and Technology in Flanders (IWTVlaanderen) and J.S. is a postdoctoral fellow of FWO (project no. $12 \mathrm{H} 8616 \mathrm{~N})$.

\section{REFERENCES}

Arvor, D., L. Durieux, S. Andrés \& M-A. Laporte, 2013. Advances in Geographic Object-Based Image Analysis with ontologies: A review of main contributions and limitations from a remote sensing perspective. ISPRS Journal of Photogrammetry and Remote Sensing, 82, pp. 125-137.

Radoux, J., P. Bogaert, D. Fasbender, \& P. Defourny, 2011. Thematic accuracy assessment of geographic object-based image classification. International Journal of Geographical Information Science, 25, pp. 895-911.

Visser, F., Buis, K., Verschoren, V. \& Schoelynck, J., accepted. Mapping of submerged aquatic vegetation in rivers from very high resolution image data, using Object Based Image Analysis combined with expert knowledge. Hydrobiologia.

Visser, F., K. Buis, V. Verschoren \& P. Meire, 2015. Depth Estimation of Submerged Aquatic Vegetation in clear water streams using low-altitude optical remote sensing. Sensors, 15, pp. 25287-25312. 


\title{
A SEMI-AUTOMATIC CROPLAND MAPPING APPROACH USING GEOBIA AND RANDOM FORESTS ON BLACK-AND-WHITE AERIAL PHOTOGRAPHY
}

\author{
M. F. A. Vogels ${ }^{a}, *$, S. M. de Jong ${ }^{\text {a }}$, G. Sterk ${ }^{\text {a }}$, E. A. Addink ${ }^{\text {a }}$ \\ ${ }^{\text {a }}$ Utrecht University, Department of Physical Geography, PO box 80115, 3508 TC Utrecht, The Netherlands - (M.F.A.Vogels, \\ S.M.deJong, G.Sterk, E.A.Addink)@uu.nl
}

KEY WORDS: Agricultural cropland expansion, land-use change, black-and-white (historical) aerial photography, GEOBIA, Random Forests

\begin{abstract}
:
For decades land-use and land-cover (LULC) conversions have had an important impact on land- and ecosystem degradation, accordingly (historical) LULC information is important for the assessment of such impacts. This information can be derived from black-and-white (B\&W) aerial photography. Such photography is often visually interpreted, which is a very time-consuming approach. This study shows that machine learning can be applied on only brightness to derive LULC information. Cropland acreage is semi-automatically mapped by means of Geographic Object-Based Image Analysis (GEOBIA) and Random Forest classification in two study sites in Ethiopia and in The Netherlands. The result is a thematic map with two classes: 1) agricultural cropland and 2) other types of land cover. Overall mapping accuracies attained are $90 \%$ and $96 \%$ for the two study areas respectively. This mapping method increases the timeline at which historical cropland expansion can be mapped purely from brightness information in B\&W photography up to the 1930s.
\end{abstract}

\section{INTRODUCTION}

During the past decades major LULC conversions occurred in Africa, Southeast Asia and South America as a consequence of a growing population and economy. Most dominant is the conversion of natural vegetation into agriculture. Unsustainable management practices in the agricultural sector are seen as the major driver of land degradation (Foley et al., 2005), which poses a threat for food security, biodiversity, biomass productivity and environmental sustainability (Millennium Ecosystem Assessment 2005; Mueller et al., 2014).

Historical land-use (change) maps are important information in the assessment of LULC change on land- and ecosystem degradation. This can be derived from $\mathrm{B} \& \mathrm{~W}$ aerial photography. The use of such photography is limited due to its panchromatic spectral information. An image analyst can visually identify cropland plots in B\&W aerial photography by the cropland plot's characteristic rectangular shape, and smooth, regular texture, but such imagery holds little information for machine learning.

This study has developed a semi-automated procedure to map cropland acreage in B\&W photography using GEOBIA and Random Forest classification. To assess the universal applicability of this mapping approach, the method is tested and validated in two contrasting study areas with respect to LULC types: 1) the Awassa Lake region in the Central Rift Valley in Ethiopia and 2) the Bladel Kempen region in the Netherlands.

\section{METHODOLOGY}

\subsection{Data description}

B\&W aerial photography was simulated from the Web Map Service layer named World Imagery (ESRI, 2015). The methodology was evaluated on 15 sectors for each region comprising a variety of LULC. For the Awassa Lake region panchromatic imagery from WorldView-1 (0.5 m spatial resolution) was used. For the Bladel Kempen region aerial data (average of three bands: $400-700 \mathrm{~nm}$ ) from the UltraCam-G camera ( $0.3 \mathrm{~m}$ spatial resolution) was used. The slope is added as a covariate for the classification of cropland.

\subsection{Workflow}

Four stages were involved in this mapping procedure: 1) the original $\mathrm{B} \& \mathrm{~W}$ photograph was segmented into coherent landscape elements (objects), e.g. cropland plots, 2) a training and validation set were generated by means of interpretation of B\&W photography and ancillary data sources by randomly selecting a number of objects, 3) these landscape objects were then classified by a Random-Forest algorithm into either 'cropland' or 'other land cover', on the basis of object attributes including textural, shape, slope, neighbour and spectral variables (the 'other land cover' class incorporates all other LULC), and 4) accuracy statistics of this classification procedure were calculated to validate the method.

\section{RESULTS AND DISCUSSION}

\subsection{Classification performance}

Cropland classification in the Awassa Lake region and the Bladel Kempen region have an overall accuracy and kappa coefficient of $90 \%$ and 0.77 and $96 \%$ and 0.91 respectively. Two sectors in the Awassa Lake region show significant swamp land cover in the B\&W photography, but this is not well depicted in the LULC maps where it is miss-classified as cropland. Also in the Bladel Kempen region classification proves to be more difficult in large natural vegetated area's.

\footnotetext{
* Corresponding author
} 
Here a small number of objects; $0.8 \%$ and $6.8 \%$ of the objects in two sectors, are wrongly classified as cropland.

\subsection{Classification uncertainty}

Historical aerial photography is subject to common issues such as light falloff, distortion and image noise (Aber et al., 2010; Morgan et al., 2010). Differences in Field of View and time of day will not be consistent over time and space and information to correct for those absent. This is a source of uncertainty that cannot be quantified and may influence the segmentation results, the training- and validation set generation and, hence classification performance. The high performance of this methodology on imagery from Web Map Service layers in this study highlights the even higher potential when applied on actual aerial photography, which generally have a higher radiometric resolution and therefore hold more information for the distinction of landscape elements.

\section{CONCLUSIONS}

The classification procedure shows good results throughout the two study areas. It proved more difficult to classify objects in dense homogeneous natural vegetated areas, where some objects were confused with cropland. A solution was found by adding slope as an additional variable. The application of the method in the two study area's is assumed to cover a wide range of current landscape patterns and LULC globally, making the method portable to other regions. It provides valuable information on LULC change, which is important to assess the impacts of land-cover change on land- and ecosystem degradation processes such as runoff, erosion, and water availability. The method is fast and efficient in mapping the expansion or shrinking of cropland acreage at the regional scale wherever historical B\&W aerial photography is available, which is especially valuable for regions where historical land-use statistics are mostly absent.

\section{ACKNOWLEDGEMENTS (OPTIONAL)}

This study is funded by Climate-KIC (Task ID: ARED0004_2013-1.1-008_P001-06).

\section{REFERENCES}

Aber, J. S., Marzolff, I., Ries, J., 2010. Small-format aerial photography: principles, techniques and geoscience applications, 1st Edition. Elsevier, Amserdam, Oxford, p. 76.

ESRI, 2015. ArcGIS Online Standard Service: World Imagery Collection, Map Server. Maps throughout this book were created using $\operatorname{ArcGIS}^{\circledR}$ software by ESRI. ArcGIS ${ }^{\circledR}$ and ArcMap $^{\mathrm{TM}}$ are the intellectual property of ESRI and are used herein under license. Copyright ${ }^{\odot}$ ESRI. For more information about ESRI ${ }^{\circledR}$ software: www.esri.com, Accessed 25 February 2015.

Foley, J. A., Defries, R., Asner, G. P., Barford, C., Bonan, G., Carpenter, S. R., Chapin, F. S., Coe, M. T., Daily, G. C., Gibbs, H. K., Helkowski, J. H., Holloway, T., Howard, E. A., Kucharik, C. J., Monfreda, C., Patz, J. A., Prentice, I. C., Ramankutty, N., Snyder, P. K., 2005. Global consequences of land use. Science, 309, pp. 570-574.

Millennium Ecosystem Assessment, 2005. Ecosystems and human well-being: biodiversity synthesis. Tech. rep., World Resources Institute, Washington D.C., p. 30.
Morgan, J. L., Gergel, S. E., Coops, N. C., 2010. Aerial photography: a rapidly evolving tool for ecological management. BioScience, 60, pp. 47-59.

Mueller, E. N., Wainwright, J., Parsons, A. J., Turnbull, L., 2014. Land degradation in drylands: an ecogeomorphological approach. In: Patterns of land degradation in drylands: understanding self-organised ecogeomorphic systems. Springer, Dordrecht, Heidelberg, New York, London, p. 3. 


\title{
MAP BASED SEGMENTATION OF AIRBORNE LASER SCANNER DATA
}

\author{
Y. Wang ${ }^{\text {a }}$, S.J. Oude Elberink ${ }^{\mathrm{a}, *}$ \\ ${ }^{\text {a }}$ Faculty of Geo-Information Science and Earth Observation, University of Twente, Netherlands - yanchengwang1990@163.com, \\ s.j.oudeelberink@utwente.nl
}

KEY WORDS: Segmentation, Data Fusion, Airborne Laser Scanning, point cloud processing, lidar

\begin{abstract}
:
The task of segmenting point clouds is to group points that belong to the same (part of an) object. In this project we make use of an existing topographic map as a kind of background layer to segment point clouds acquired by airborne laser scanning systems. This map is an object based representation into polygons which are labelled into topographic classes like buildings, roads, terrain, water and vegetation.

We implemented a point-in-polygon operation which is succeeded by a relabeling step at locations of roof overhangs. Next, the topographic class of the object is used to correctly process the point cloud into meaningful segments. The type of segmentation, e.g. planar or smooth segmentation or connect component analysis, depends on the corresponding topographic class of the object. The segmentation step is extended with a classification step where points are labelled as actually belonging to the corresponding map class or not. This is helpful when dealing with point clouds of cars on roads, or powerlines above the terrain. We segment for example the points on the individual cars into one segment each, but we label those points as not belonging to the class 'road'. This is useful information to filter points from the point cloud when used to generate a 3D landscape model. The result of the map based segmentation algorithm is a point cloud enriched with information on the corresponding topographic class, corresponding map object, a segment number, and an indication whether this point actually belongs the map class or not.

Results on two national datasets show that the use of map information is beneficial compared to a standard segmentation approach. Improvements are shown at situations where in one class a smooth segmentation is more suitable, whereas in the other class a planar segmentation is better, which can only be achieved if the class is known before the segmentation.
\end{abstract}

\section{INTRODUCTION}

Lidar is probably the most important technology introduced in the mainstream topographic mapping in the last decades (Shan and Toth, 2008). As the data collected by Lidar is a set of unstructured point clouds, it does not directly show objects, so a series of processes is needed for the point clouds to extract the information. An important step in using Lidar for mapping is segmentation of the point cloud. Segmentation is a process to label segment ID for laser points, so the points which belong to a surface or object are given the same segment ID. The problem is that many segmentation algorithms are fixed in terms of ways of grouping points together. Planar segmentations are very useful when dealing with planar surfaces such as roof planes (Vosselman, 1999; Verma et al 2006; Dorninger and Pfeifer (2008) and Xiong et al (2014), but fail when the scene contain curved shapes.

The main problem is that for a good segmentation, it is needed to know which class the object belong to. For a correct classification of a point cloud however, we need to have some context information to see which groups belong to a certain object. To overcome this chicken-and-egg problem we make use of a large scale topographic map to have an initial guess about the class of the object.

The topographic map gives clear hints on what to expect in the point cloud and how to segment those points into meaningful pieces. For example, if the map indicates that there is a building in a certain area, it is logical to find planar segments in the point cloud. First task is to correctly match point clouds with the corresponding map object, which is challenging not only because of potential small registration errors but also because of differences in object appearances. For example a building in a map may represent the wall outlines whereas the point clouds captures the complete roof, including a roof overhang.

After the fusion process we segment the laser data into logical object wise segments, based on the class knowledge. After the segmentation, it is possible to indicate which points are likely belonging to that class and which do not. At the end of the process the point cloud is enriched by four point attributes, namely the polygonID, the classID, segmentation number and an attribute which indicates the likeliness that this point actually belongs to the class.

The objective of this paper, which is based on the MSc research from Wang (2016), is to present an accurate segmentation model for ALS point clouds of multiple-land-cover landscapes, with the help of maps. Next, the goal is to indicate which segments within each polygon actually belong to corresponding map class. This is helpful for map updating purposes as this is the first indication that a newly acquired point cloud data set may not fit will to the expected object class from an outdated map. After the explanation of the segmentation rules in section 2 , results are shown in section 3 and discussed in the conclusions.

\section{METHODOLOGY}

\subsection{Introduction}

The general order of the algorithms is:

\footnotetext{
* Corresponding author
} 
- $\quad$ Filtering of point clouds into ground and nonground

- $\quad$ Point-in-polygon operation

- $\quad$ Reassignment of points on overhanging roofs and walls

- $\quad$ Per class per object segmentation

- $\quad$ Grouping of all segmentation results

Although the first two processes are not part of the innovation of the approach, we shortly discuss them here to explain the assumptions of how the data appears when starting the map based segmentation.

The Lidar data is assumed to be filtered into ground and nonground data, e.g. using filtering techniques as designed by Axelsson (1999). Next, the Lidar data is expected to be georeferenced in the same coordinate system as the map with a planimetric accuracy in the order of $10-20 \mathrm{~cm}$. In the datasets we used, the above described assumptions are realistic as these are standard properties of the national height model (AHN) of the Netherlands. A point-in-polygon operation is used to link the points to polygons based on the planimetric positions.

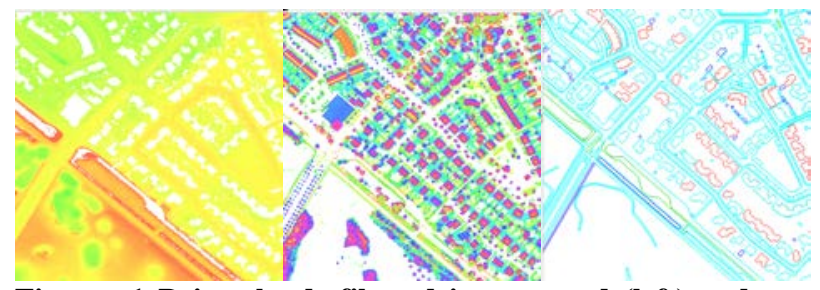

Figure 1 Point clouds filtered into ground (left) and nonground points (middle). Right: a corresponding map.

We implemented an addition to a normal point-in-polygon operation which corrects for unavoidable small registration errors between map and laser scanner data. Specially points on walls are by definition close to a polygon boundary. A smart assigning procedure is needed to connect walls points to building polygons. So an addition is needed to ensure a correct assignment of laser points on overhanging roofs and walls to the corresponding building polygon.

The procedure is that any non-ground laser point within the vicinity of a building polygon is checked whether it is likely belonging to a building than another class. This is done by calculating its local normal direction for detecting vertical planes, and checking whether its fits to a planar segment, e.g. a roof plane or a wall plane.

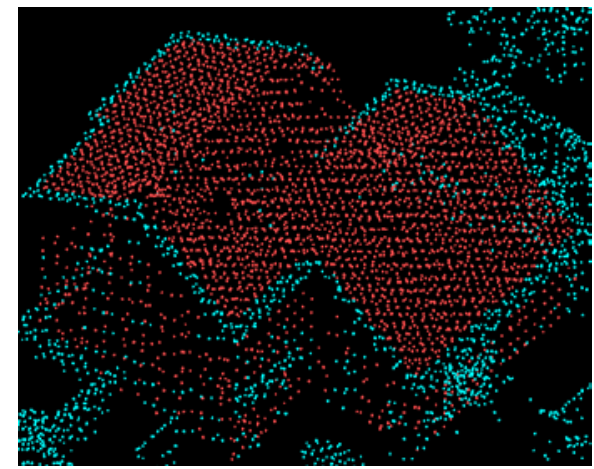

Figure 2 Points within (red) a building polygon. Points of roof overhang and some points on walls (cyan points) are outside the building polygon.

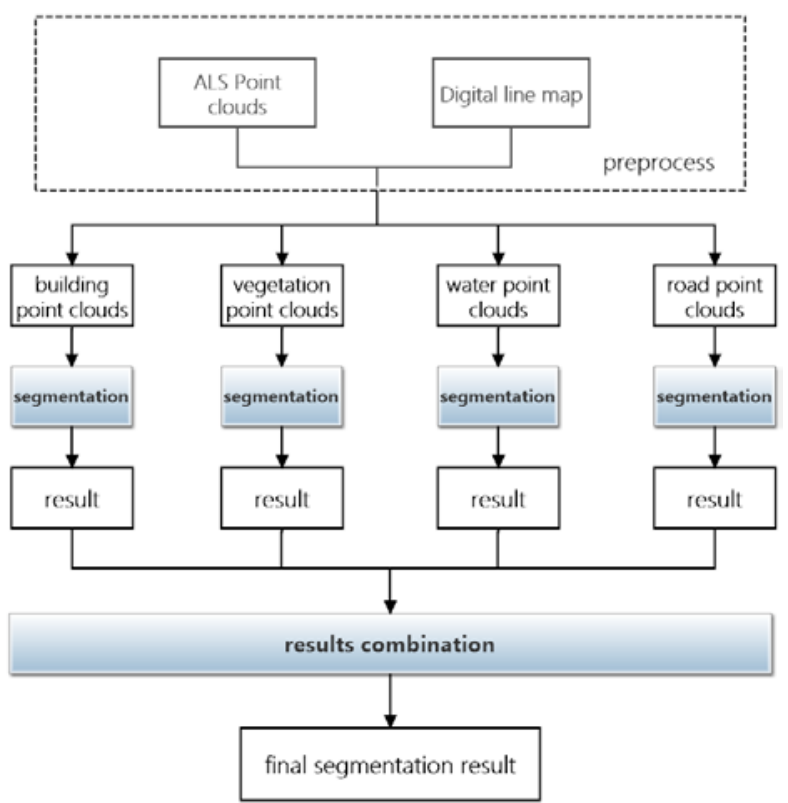

Figure 3 Overall workflow of our map based segmentation approach.

\subsection{Point attribute features}

To guide the segmentation process, point attributes are calculated to be able to group points based on proximity in combination with constraints on local geometric features. Features like flatness, normal, segment size, max height difference are used in this segmentation model. Flatness is used to differentiate regular manmade surfaces from irregular plant surfaces. Normal of the planes is used to identify wall surface and water surface which are vertical and horizontal respectively. The height difference between the lowest and highest part of a segment is used in re-segmentation of multi-tree components and to differentiate the low vegetation components from tree components.

\subsection{Per class segmentation design}

After the correct assignment of points to polygons by the adjusted point-in-polygon operation, points within polygons are selected and segmented according to the rules of the corresponding topographic class of that polygon. The rules are explained for the four main classes: buildings, terrain (including vegetated areas), roads and water. The rules are summarized in table 1 , and further explained in the following text.

\begin{tabular}{|l|l|l|l|}
\hline Building & Terrain & Roads & Water \\
\hline $\begin{array}{l}\text { Non-ground } \\
\text { points }\end{array}$ & $\begin{array}{l}\text { Ground and } \\
\text { non-ground } \\
\text { (for trees) }\end{array}$ & Ground & Ground \\
\hline $\begin{array}{l}\text { Planar } \\
\text { segmentation }\end{array}$ & $\begin{array}{l}\text { Connected } \\
\text { components } \\
\text { per filter type }\end{array}$ & $\begin{array}{l}\text { Smooth } \\
\text { surface } \\
\text { segmentation }\end{array}$ & $\begin{array}{l}\text { Planar, } \\
\text { horizontal }\end{array}$ \\
\hline $\begin{array}{l}\text { Connected } \\
\text { components } \\
\text { for smaller } \\
\text { details }\end{array}$ & $\begin{array}{l}\text { Individual } \\
\text { tree detection }\end{array}$ & $\begin{array}{l}\text { Large } \\
\text { growing } \\
\text { radius }\end{array}$ \\
\hline
\end{tabular}

Table 1 Segmentation rules and processes per map class. 


\subsubsection{Buildings}

Non-ground laser points assigned to building polygons are first segmented into planar segments. The larger planar segments are very likely belonging to the building class. The remaining points are filtered for outliers, followed by a connected component algorithm to group points on larger structures. Structures with many locally planar points, such as dormers and chimneys are considered to be moderate likely to be part of buildings. Other connected components with little planar points, such as vegetation above buildings, will get a label of less likely belonging to buildings.

\subsubsection{Terrain}

Laser points that fall within polygons with the class terrain contains information on the ground, but also above the ground such as power lines, trees and other vegetation. As such the name "terrain" is confusing but it contains all land cover objects other than buildings, roads and water. Segmentation of the points is done on both types of point clouds: the ground dataset, and the non-ground dataset. For the ground a connected component algorithm is used to group points on the earth surface into large segments. For the non-ground data first the planar segments are found and labelled as unlikely to be part of the terrain class. Next connected component algorithm is used to group points on above ground objects. It is checked whether points belong to linear objects, such as power lines. Multi-tree components are split into smaller segments based on local maxima information, as shown in Figure 4.

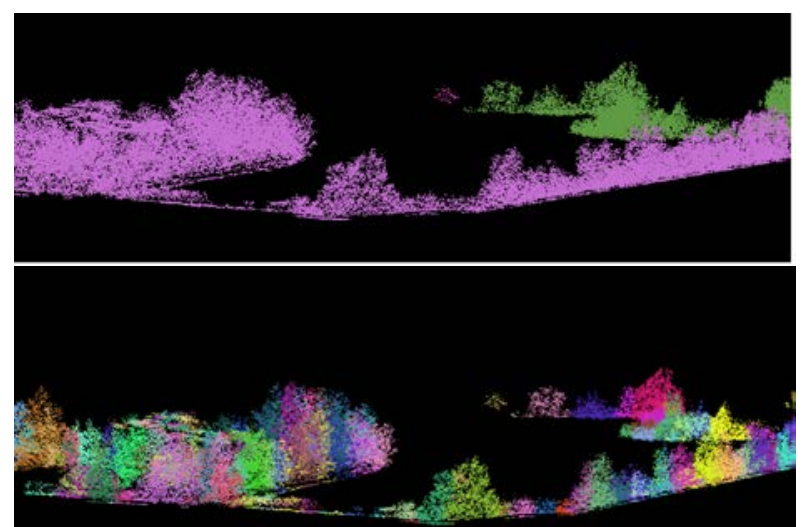

Figure 4 Multi tree components (top) are split into single tree segments.

\subsubsection{Roads}

Per road polygon ground points are considered to be belonging to the class, in case they fit to large and smooth segments. All non-ground points get a label of not belonging to the road class, and are segmented via a connected component algorithm to group points on objects like cars, and street furniture.

\subsubsection{Water}

Ground points within water polygons are segmented using a planar segmentation algorithm, where the largest horizontal segment is used as a reference for the main water body. All other horizontal segments at the same height are added to the largest segment in order to bridge possible gaps due to pulse absorption by the water.

\section{RESULTS}

Results of the map based segmentation are shown and discussed per class. Our algorithms have been tested on area of 1 by $1 \mathrm{~km}$ near Den Bosch in The Netherlands. We have made use of two national datasets: point clouds come from the national height model AHN-2, and the map information is from the large scale topographic map BGT. Building points have been segmented in planar segments and small connected components (Figure 5). Points which are within building polygons but unlikely belonging to the class buildings are coloured green in Figure $6 x$. These are mainly points on vegetation hanging over roof planes.

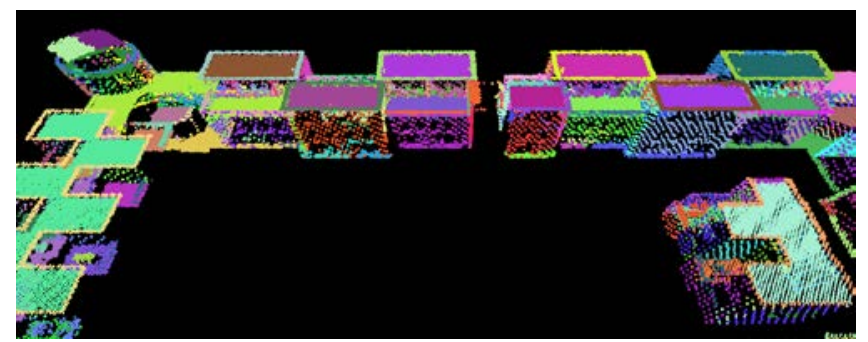

Figure 5 Map based segmentation results on buildings, including points on walls.

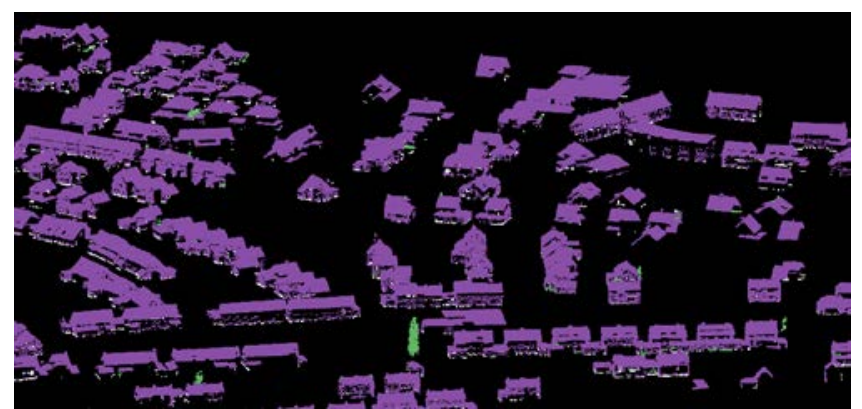

Figure 6 Segmented points likely belonging to building class (purple) and not likely (green).

Segmented terrain points from the points in the 'ground' dataset are shown in Figure 7. Non-ground points on vegetation and powerlines are shown in Figure 8. Based on linearity measures points on linear objects such as power lines are labelled unlikely belong to the class, as shown in Figure 9.

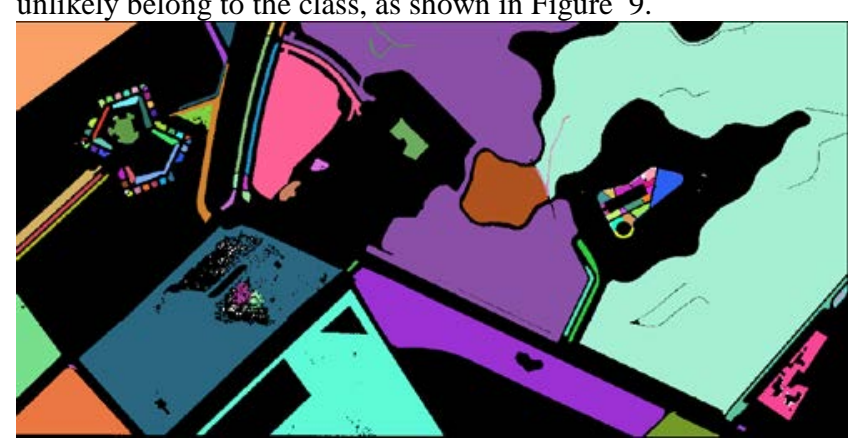

Figure 7 Terrain segments of ground points.

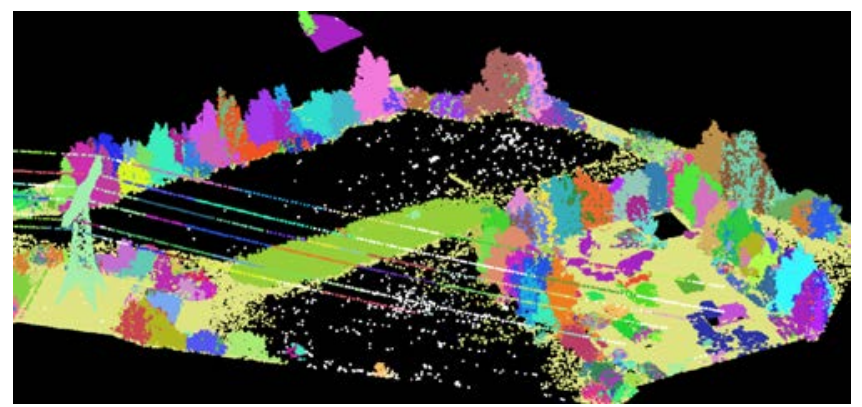

Figure 8 Terrain segments after multi-tree component analysis of non-ground points. 


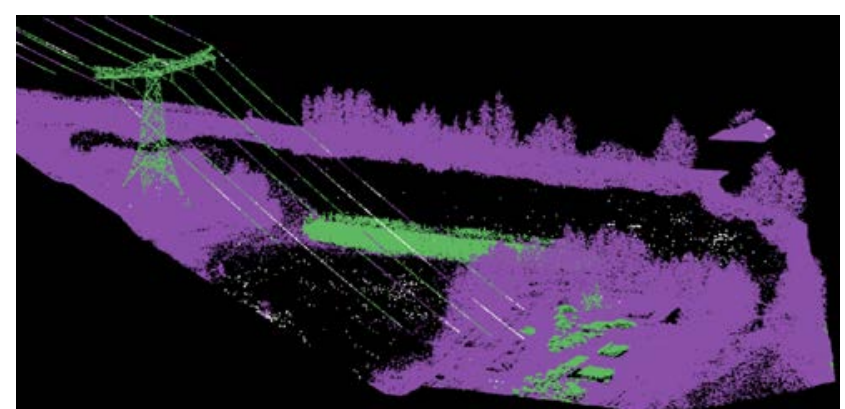

Figure 9 Segmented points likely belonging to terrain class (purple) and not likely (green).

Per road polygon points have been segmented using a smooth segmentation algorithm on the ground points (Figure 10 and Figure 11). A connected component algorithm is applied on the non-ground points to group points on cars, trees and street furniture, see Figure 12.

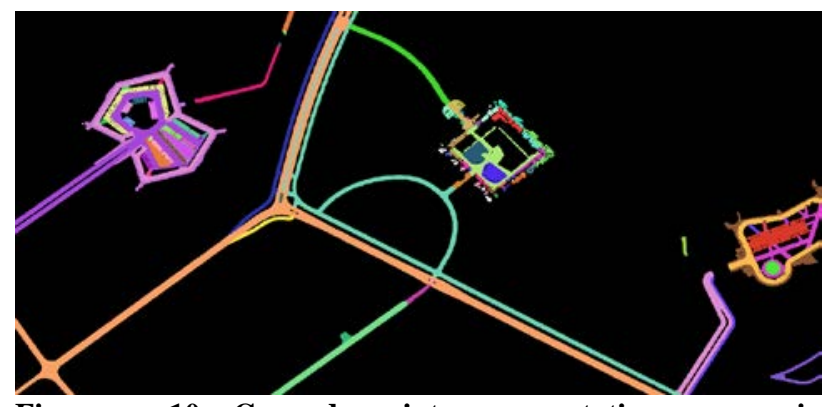

Figure 10 Ground points segmentation on main infrastructural objects.

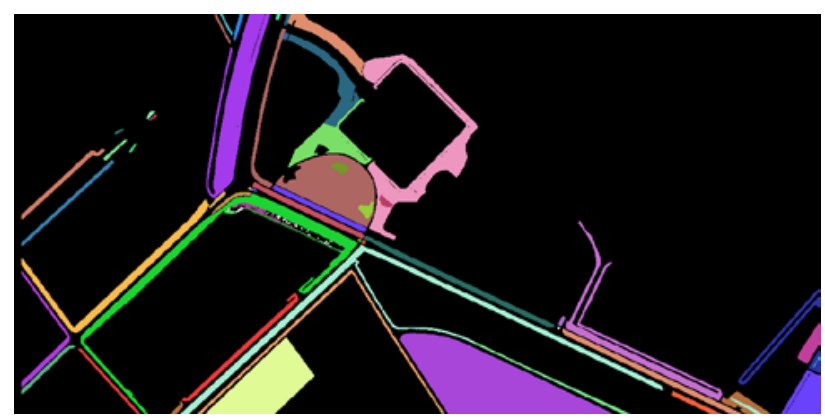

Figure 11 Ground points segmentation on secondary infrastructural objects.

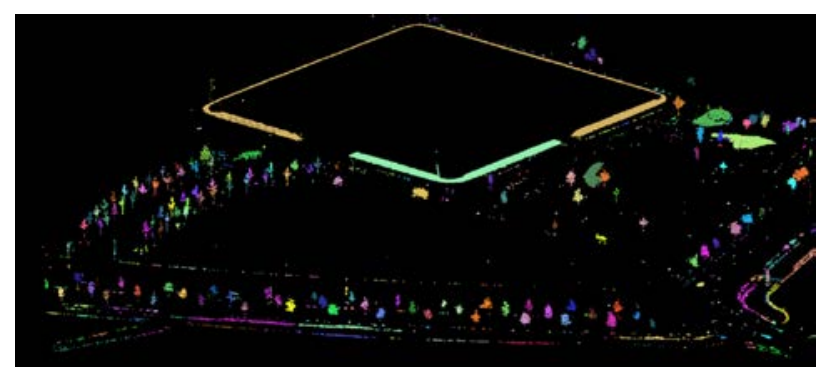

Figure 12 Segmentation on infrastructural objects of nonground points.

Segments on water surfaces are shown in Figure 13. The area contains a few lakes and many ditches.

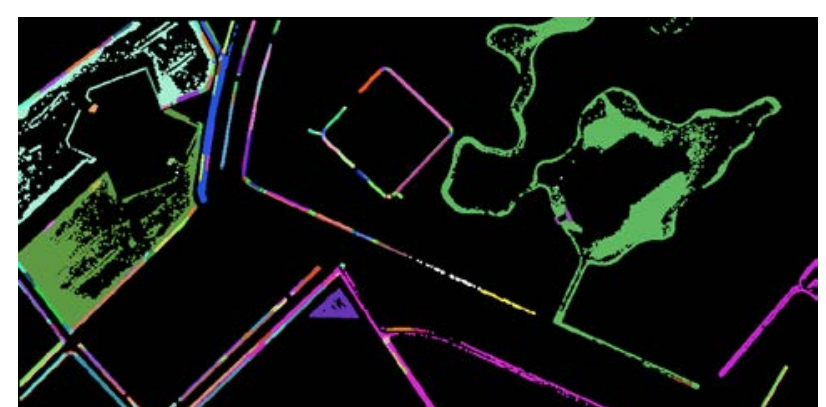

Figure 13 Ground points segmentation of water objects.

All segmentation results are grouped by renumbering of the segment numbers when combining all map based segments. Compared to a normal planar segmentation or connected component approach the map based approach is capable of segmenting both a hilly surface and planar roof faces, see Figure 14. Another reason why the map based segmentation approach appears to be more object based is that it shows boundaries of objects which do not have a height difference, just because there is an object boundary in the map.

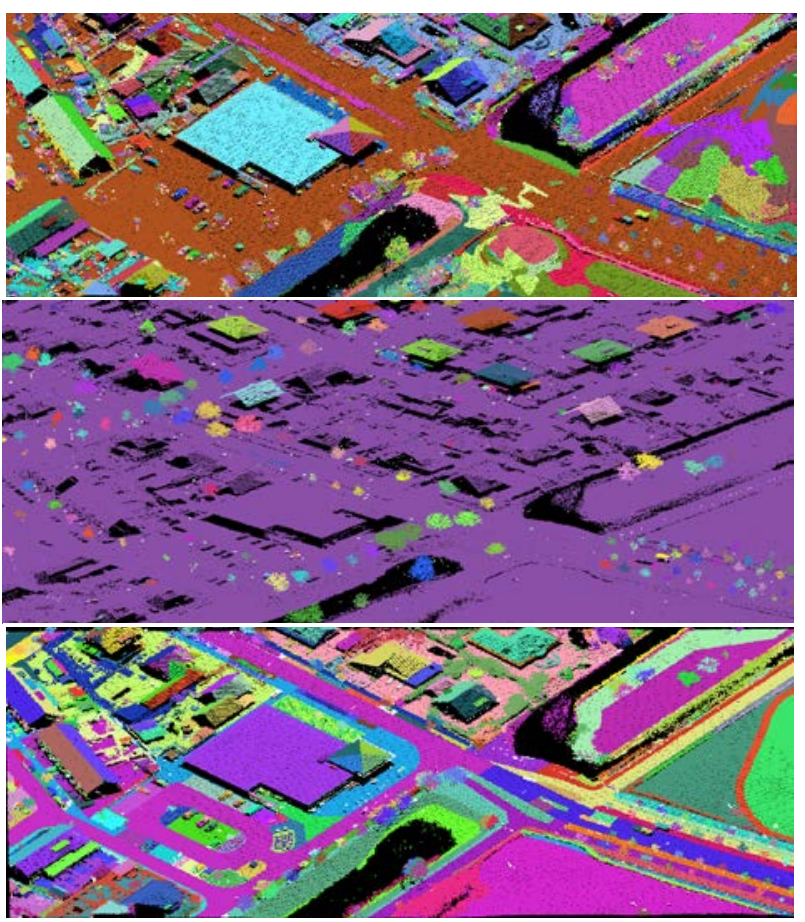

Figure 14 Planar segmentation (top), traditional connected components (middle) and map based segmentation (bottom) results.

At this stage there has not been a quantitative accuracy assessment on the correctness of the segmentation due to absence of reliable and independent ground truth data. In the future we aim to conduct a thorough accuracy check to optimize the segmentation parameters, and to show the potential of this segmentation in 3D modelling and map updating.

\section{CONCLUSION}

We have presented a segmentation approach for airborne laser scanner point clouds which is adaptive to its corresponding map object and its class. Our approach is able to deal with small registration errors between map and laser data at locations of buildings, as we can reassign wall points and points on 
overhang roof faces. At other situations we did not explicitly check whether points actually belong to the neighbouring polygon although we do assign a likelihood that a segment actually belongs to that class.

Our approach ensures a more logical grouping of points towards an object based processing of point clouds. It is shown that by performing a map guided segmentation the point cloud is cleaner in the sense that it is clear which points are useful for further processing. That is beneficial for researchers who use the segmentation result to continue processing for 3D modeling for buildings. They can select the points which are likely belonging to the corresponding map class.

This paper is only the start of using this object based approach in applications. In the future a quantitative accuracy is needed to optimize the segmentation parameters, and to use the cleaned dataset in further mapping steps.

\section{REFERENCES}

Axelsson, P., 2000. DEM generation from laser scanner data using adaptive TIN models. International Archives of Photogrammetry and Remote Sensing, 33(B4/1; PART 4): 111118.

Dorninger, P. and Pfeifer, N., 2008. A Comprehensive Automated 3D Approach for Building Extraction, Reconstruction, and Regularization from Airborne Laser Scanning Point Clouds. Sensors, 8(11): 7323-7343.

Shan, J. and Toth, C.K., 2008. Topographic laser ranging and scanning: principles and processing. CRC press.

Verma, V., Kumar, R. and Hsu, S., 2006. 3D Building Detection and Modeling from Aerial LIDAR Data, IEEE Computer Society Conference on Computer Vision and Pattern Recognition (CVPR'06). IEEE Computer Society, Washington, DC, USA, pp. 2213-2220.

Vosselman, G., 1999. Building Reconstruction Using Planar Faces in Very High Density Height Data. International Archives of Photogrammetry, Remote Sensing and Spatial Information Sciences, XXXII(part 3/2W5), 87-92.

Wang, Y., 2016. Map based segmentation of airborne point clouds, University of Twente, Enschede, The Netherlands.

Xiong, B., Elberink, S.O. and Vosselman, G., 2014. A graph edit dictionary for correcting errors in roof topology graphs reconstructed from point clouds. ISPRS Journal of Photogrammetry and Remote Sensing, 93: 227-242. 


\title{
Object-Based Image Analysis based on A Region-Line Primitive Association Framework
}

\author{
M. Wang and J. Wang \\ Key Laboratory of Virtual Geographic Environment (Nanjing Normal University), Ministry of Education, Nanjing, Jiangsu, P.R. \\ China, 210023 -sysj0918@126.com; wangjieyl@sina.com
}

KEY WORDS: Region, Straight Line, Primitive, Image Segmentation, Image Classification, Road Extraction

\section{INTRODUCTION}

Common object-based image analysis (OBIA) applications generally follow the "segment and then classify" framework. However, information extraction based only on regions has many limitations. For example, image edges are not considered during common OBIAs. Image edge lines generally denote object boundaries or borders, which exhibit strong semantic connotations. However, region boundaries obtained by image segmentation do not necessarily match image edge lines in quantity and location. Thus, image edges should be additionally used in OBIA. In this study, straight lines are used in our OBIA framework for extracting man-made objects, because the latter generally have straight line-shaped boundaries.

The current study introduces a region-line primitive association framework (RLPAF) for OBIA. Regions and lines are used collaboratively from low-level image processing (segmentation) and feature extraction to high-level image analysis (classification and recognition). Several region-line associating techniques for analysing object shapes and relationships were designed. Our techniques have been validated by impervious surface and road network extraction from high spatial resolution (HSR) images.

\section{METHODS}

\subsection{Technical Framework of RLPAF}

In Fig. 1 which illustrates RLPAF, HBC-SEG and Burn's phase-grouping method are used to obtain the region primitives and straight line primitives. Region and line features, including the spectra and shapes of the regions as well the lengths and directions of the lines are calculated. The association model RLPAF is built based on region and line topologies and orientation relationships. Thematic information extraction, image classification, and change detection can then be conducted by both considering the two kinds of primitives.

In RLPAF, direction relationships of a region to a line are classified as "above" "bilateral" and "below". The topology of a region to a line includes separation, intersection, tangent, and inclusion. The combination of different direction and topology relationships creates several region-line relationships, which can be used as new features for OBIAs.

In this study, a set of line-based concepts is proposed and used in subsequent OBIAs, which include:

-Unilateral and Tangent Relationship

- Ipsilateral Neighbors
- Ipsilateral and Homogeneous Chain

- Line-Based Length-to-Width Ratio

- Region and Line Mutual Conversion Operators

Figure 3 illustrates the concept of line-based length-to-Width Ratio (LBLW). The width of the region is defined by its tangent straight line by moving the line along its perpendicular direction and record the span where the touching length declines dramatically. A region can have multiple LBLW measures based on its multiple tangent straight lines, which thus describes irregular shapes objectively and precisely.

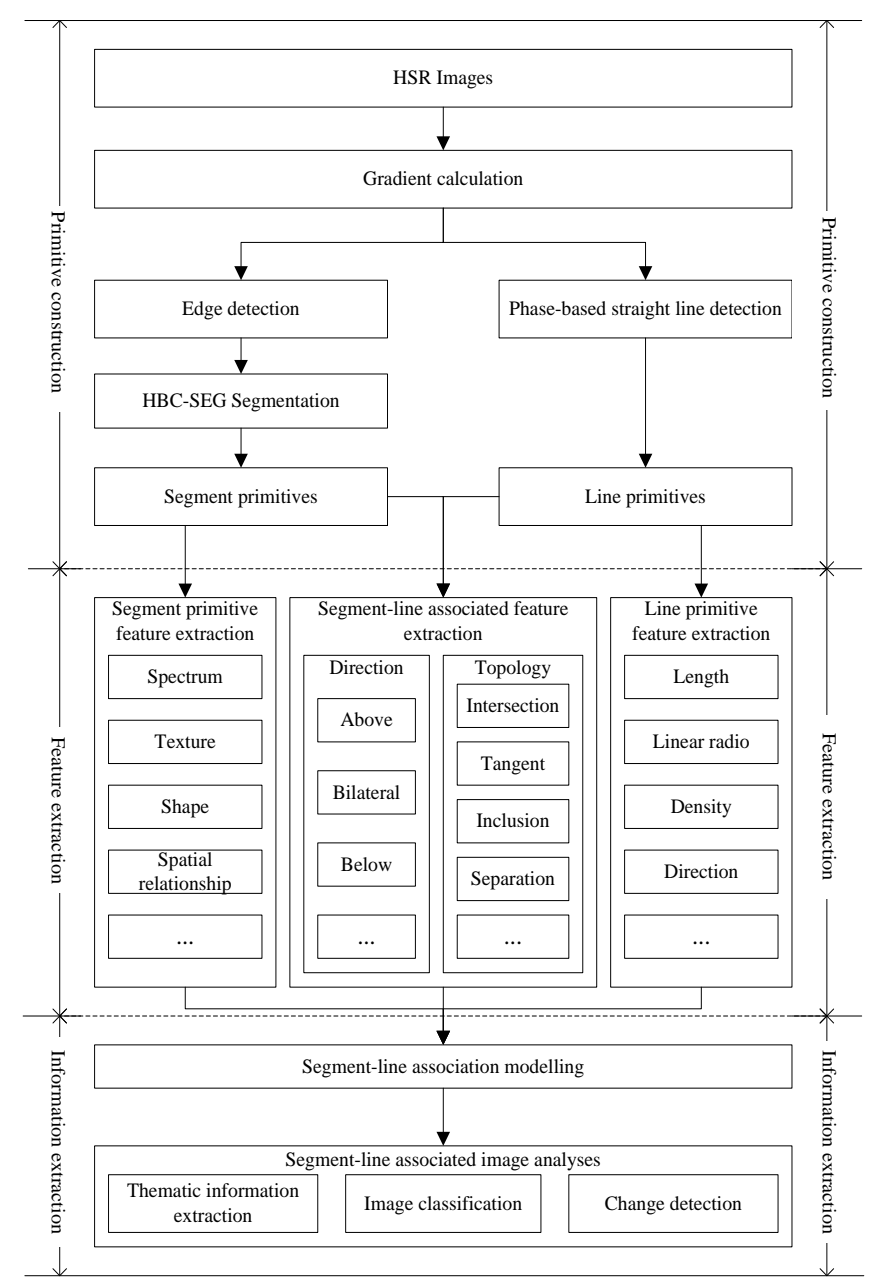

Figure 1. Technical framework. 


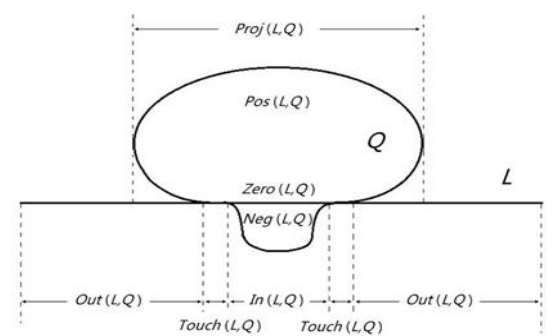

(a)
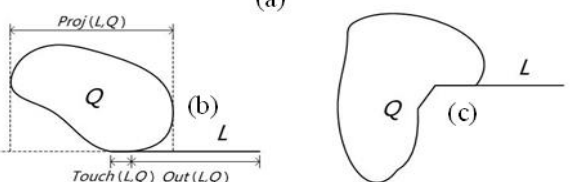

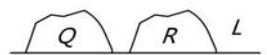

(d)
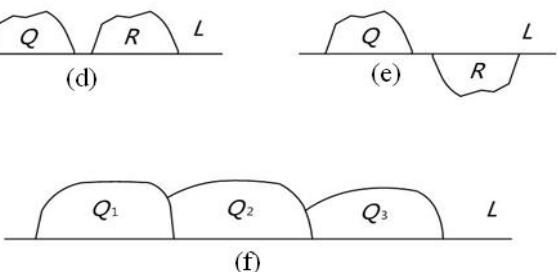

Figure 2. Region-line relationship modeling. (a) A pair of touching region and line. (b) A unilateral case. (c) A tangent relationship that is not unilateral. (d) An IPSL-neighborhood relationship. (e) Regions that are not IPSL-neighbors because they are located bilaterally to line $L$. (f) An IPSL chain.

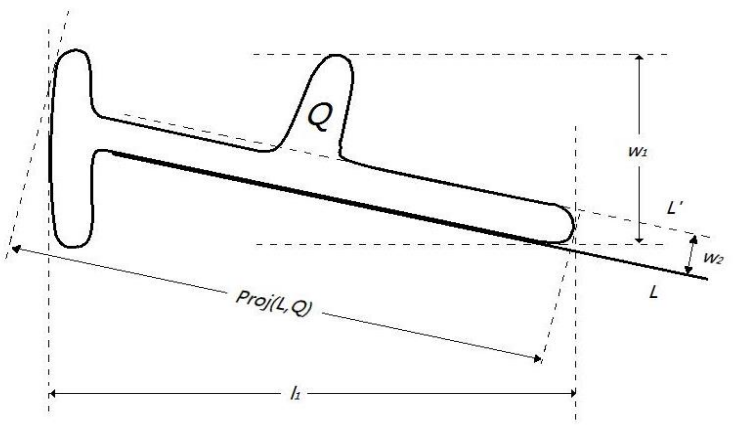

Figure 3. Line-based length-to-width ratio.

\subsection{RLPAF-based road-extraction}

An RLPAF-based road-extraction method was designed to exemplify and validate PRLAF (Figure 4). First-level, coarsegrained supervised classification based on region spectral signatures was initially performed to separate impervious surfaces. After that, second-level rule-based classification extracts high $L B L W$ segments within an impervious surface as roads. After that, a straight line-guided depth-searching step was designed to extend initial, broken road regions into a road network. This searching step involves several region-to-line, line-to-line, and line-to-region conversions. Road regions with low $L B L W \mathrm{~s}$ and sufficient line-based widths $(L B W \mathrm{~s})$ are thus appended into the initial road segments and formed the final road network.

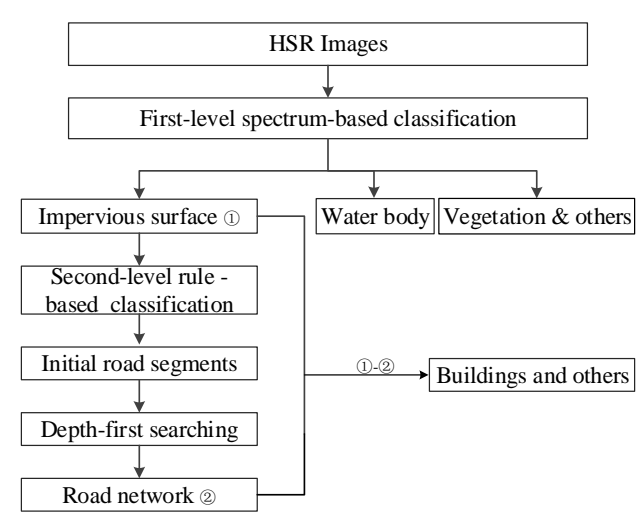

Figure 4. Impervious surface and road extraction scheme.

\section{EXPERIMENTS}

The methods were applied in different HSR images for validation. In the experiments, impervious surfaces were first extracted from HSR images by first-level supervised classification using region spectral features. Then, three road extraction schema, i.e., using common $L W$ measure (the $L W$ method), $L B L W$ measure (the LBLW method), and $L B L W$ measure combined with depth searching (the LBLW\&DS method), were applied on the impervious surface to extract road regions with high $L W \mathrm{~s}$ or $L B L W \mathrm{~s}$. Accuracy measures, including Recall, Precision, and F-measure, were employed to evaluate road-extraction accuracy.

In the experiments, given a length-to-width threshold $T$, the recall ratios of the three methods were LBLW\&DS > LBLW > LW, whereas their precision measures were reversed as LW $>$ LBLW > LBLW\&DS. However, the LBLW and LBLW\&DS methods obviously performed better than the LW method on the $F$-measure, given the same threshold $T$. The compensation on recall ratio far exceeded that on precision loss when the LBLW and LBLW\&DS methods were compared with the LW method. In addition, the LBLW\&DS method had flat $F$-measure curves in all the experiments.

In Figure 5, by visual interpretation, the LW method retrieved the minimum road regions and formed a broken road network. The LBLW and LBLW\&DS methods obtained significantly denser road regions and formed continuous road networks while inducing additional errors. As illustrated in Fig. 5(g), in a zoomed-in section of the experimental area, broken roads are connected by the depth-searching process of the LBLW\&DS method.

In all the experiments, straight lines played at least two important roles. 1) Region shape analysis became more accurate than that in common region only-based OBIA. 2) The linebased depth-searching process offered precise directions and the capability to override gaps. The LBLW and LBLW\&DS methods obviously performed better than the LW method on both the quantitative and qualitative analyses.

In conclusion, the proposed framework and methods refine primitive shape and spatial relationship analyses, as well as obtain higher method accuracy, than OBIAs based on only regions. However, the extracted road network exhibited many defects and might only serve as an initial extracted result for subsequent refinement. Possible segmentation and classification errors, as well as the simplicity of current road-extraction rules 
are the main causes of these defects. Sophisticated OBIA rules or processing are necessary to improve the accuracy of road extraction particularly in urban areas. Nevertheless, the proposed scheme can serve as an initial step for road extraction because of its simplicity and effectiveness.
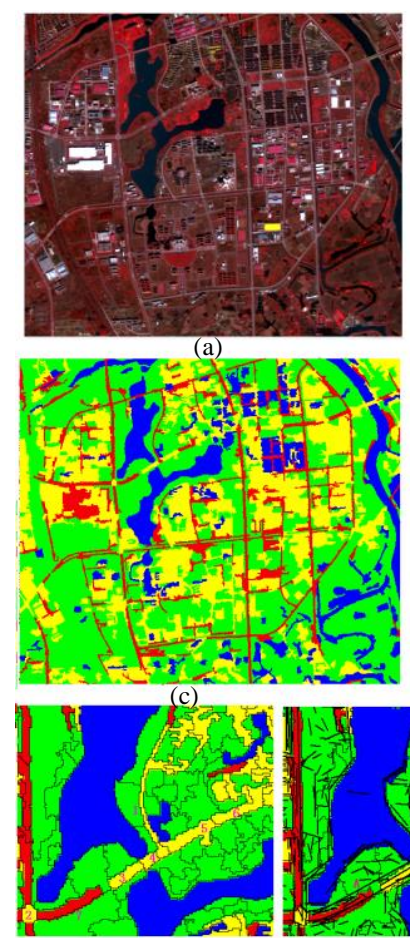

(e)
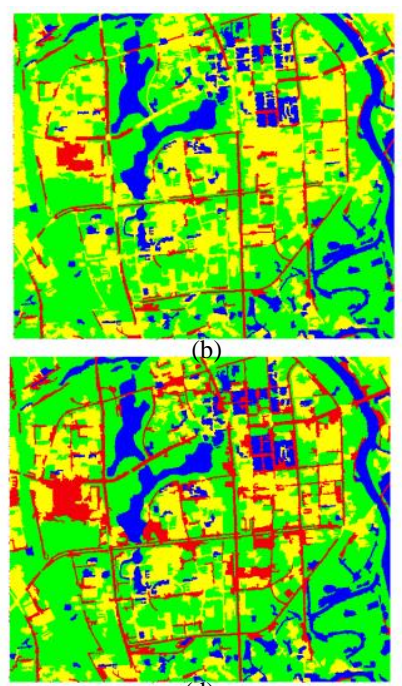

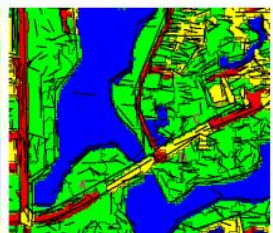

(f)

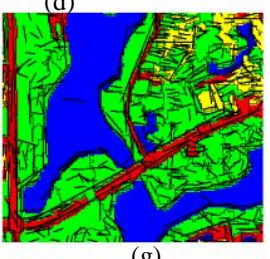

(g)

Figure 5. Experimental area. (a) Original data. (b), (c), and (d) The red regions are roads extracted by the LW, LBLW, and LBLW\&DS methods, respectively. (e), (f), and (g) The zoomedin images of (b), (c), and (d), respectively.

\section{CONCLUSTIONS}

RLPAF comprehensively utilizes line and region primitives in OBIA by image segmentation, straight line detection, and region-line relationship modelling. The proposed framework is then applied and validated in extracting road networks from HSR images. In the road extraction task, region shape analysis and spatial relationship reasoning are facilitated by considering both region and line primitives. In future work, we will investigate to extend the proposed framework and methods, as well as optimizing their performance in extensive application scenarios.

\section{ACKNOWLEDGMENT}

This work is jointly supported by the Natural Science Foundation of Jiangsu Province, China (BK20140042), the Qing Lan Project, and the Priority Academic Program Development of Jiangsu Higher Education Institutions.

P.S. A paper version of this abstraction can be found in "M. Wang and J. Wang. A Region-Line Primitive Association Framework for Object-Based Remote Sensing Image Analysis. Photogrammetric engineering \& Remote sensing, 2016, 82(2):149-159." Some of these figures used in the current abstract are from or modified from the aforementioned paper. 


\title{
DETECTION, SEGMENTATION AND LOCALIZATION OF INDIVIDUAL TREES FROM MMS POINT CLOUD DATA
}

\author{
M. Weinmann ${ }^{\mathrm{a}, \mathrm{b}}$, C. Mallet ${ }^{\mathrm{a}}$, M. Brédif ${ }^{\mathrm{a}}$ \\ ${ }^{a}$ Université Paris-Est, IGN, LaSTIG, MATIS, \\ 73 avenue de Paris, 94160 Saint-Mandé, France - (martin.weinmann, clement.mallet, mathieu.bredif)@ign.fr \\ ${ }^{\mathrm{b}}$ Institute of Photogrammetry and Remote Sensing, Karlsruhe Institute of Technology (KIT), \\ Englerstr. 7, 76131 Karlsruhe, Germany - martin.weinmann@kit.edu
}

KEY WORDS: Mobile Mapping Systems, Point Cloud, Feature Extraction, Classification, Segmentation

\begin{abstract}
:
In this paper, we address the extraction of objects from 3D point clouds acquired with mobile mapping systems. More specifically, we focus on the detection of tree-like objects, a subsequent segmentation of individual trees and a localization of the respective trees. Thereby, the detection of tree-like objects is achieved via a binary point-wise classification based on geometric features, which categorizes each point of the $3 \mathrm{D}$ point cloud into either tree-like objects or non-tree-like objects. The subsequent segmentation and localization of individual trees is carried out by applying a 2D projection and a mean shift segmentation on a downsampled version of that part of the original 3D point cloud which represents all tree-like objects, and it also involves a segment-based shape analysis to only retain plausible tree segments. We demonstrate the performance of our framework on a benchmark dataset which contains 10.13M 3D points and has been acquired with a mobile mapping system in the city of Delft in the Netherlands.
\end{abstract}

\section{INTRODUCTION}

Due to the technological advancements in 3D data acquisition, there has been an increasing interest for the acquisition and automated analysis of large scenes in recent years. Particularly mobile mapping systems are meanwhile widely used since they allow to capture data in the form of $3 \mathrm{D}$ point clouds representing densely sampled object surfaces. The automated analysis of such $3 \mathrm{D}$ point cloud data has been addressed with respect to different avenues of research such as 3D point cloud classification, the segmentation of pole-like objects, the detection of roads and/or road inventory, or the identification of individual trees.

In this paper, we present a novel two-stage framework for tree extraction from 3D point clouds acquired with mobile mapping systems. The first stage of this framework focuses on the detection of tree-like objects via a binary point-wise classification based on geometric features, which categorizes each point of the 3D point cloud into either tree-like objects or non-tree-like objects. The second stage exploits the derived classification results and performs a segmentation and localization of individual trees, whereby a $2 \mathrm{D}$ projection and a mean shift segmentation are applied on a downsampled version of that part of the original $3 \mathrm{D}$ point cloud which represents all tree-like objects. To only retain plausible tree segments, this stage also involves a segment-based shape analysis.

We demonstrate the performance of our framework on a benchmark dataset acquired with a mobile mapping system in the city of Delft in the Netherlands. For our experiments, we consider the provided subset comprising 26 tiles with a total number of about 10.13M points for which reference labels with respect to a binary classification are available and for which the results of a segmentation and localization of individual trees may easily be verified by visual inspection. This evaluation strategy allows a comparison to those results achieved in the scope of the recent IQmulus Processing Contest IQPC'15, where the first step consists in a classification of $3 \mathrm{D}$ points corresponding to trees and other 3D points and the second step consists in segmenting the $3 \mathrm{D}$ points corresponding to the tree class into clusters referring to the respective individual trees. Based on the derived results, we discuss the strengths and limitations of both the framework and the involved methods in detail.

For our framework, appropriate classification results represent an important prerequisite for the segmentation and localization of individual trees. In the evaluation, particular attention is therefore paid to the impact of using different feature sets on the classification results. With a detailed evaluation in this regard, we draw conclusions about which features to use, about the computational efficiency of feature extraction and classification, and about how to further increase the quality of the derived results.

After briefly discussing related work in Section 2, we explain our novel framework and its components in detail in Section 3. Subsequently, we demonstrate the performance of this framework on a publicly available benchmark dataset in Section 4 and discuss the derived results. Finally, in Section 5, we provide concluding remarks and suggestions for future work.

\section{RELATED WORK}

To describe related work relying on the use of mobile laser scanning data, we focus on recent progress in two research directions represented by (i) a semantic classification which aims to assign a semantic class label to each 3D point (Weinmann et al., 2015a; Weinmann, 2016) and (ii) a semantic segmentation which aims to provide a meaningful partitioning of a set of 3D points into smaller, connected subsets which correspond to objects of interest or to parts of these (Melzer, 2007; Vosselman, 2013).

\subsection{Point Cloud Classification}

An automatic point-wise semantic labeling of 3D point clouds typically relies on the use of meaningful features. In this regard, a variety of geometric features describing the spatial arrangement of 3D points within the local neighborhood of a considered 3D point has been proposed (West et al., 2004; Munoz et al., 2008; Weinmann et al., 2013; Hackel et al., 2016). Since an appropriate neighborhood is a crucial prerequisite for the extraction of 
distinctive features, further investigations focus on an automatic selection of an appropriate neighborhood size for each individual 3D point (Lalonde et al., 2005; Demantké et al., 2011; Weinmann et al., 2015a; Weinmann, 2016) and on the use of multi-scale neighborhoods relying on one neighborhood type with varying scale parameter (Brodu and Lague, 2012) or on different entities such as voxels, blocks and pillars (Hu et al., 2013).

The derived features are typically concatenated to a feature vector serving as input for classification, whereby a standard classification based on the derived feature vectors may be used (Weinmann et al., 2015a; Weinmann, 2016) as well as a contextual classification also taking into account relationships among 3D points within the local neighborhood in order to assign the class label (Munoz et al., 2008; Munoz et al., 2009a; Munoz et al., 2009b; Xiong et al., 2011).

Among the classes which are considered in the task of multi-class classification based on MLS data, several benchmark datasets contain at least one class referring to vegetation (Munoz et al., 2009a; Serna et al., 2014; Brédif et al., 2014). However, a particular focus on the binary classification of MLS point clouds with respect to tree and non-tree classes has been set in recent investigations presented in (Sirmacek and Lindenbergh, 2015), where a $2 \mathrm{D}$ probability matrix is defined on a horizontal plane and each entry of the matrix represents a probability value calculated by checking the respective point density. Assuming that tree trunks correspond to high values in the derived $2 \mathrm{D}$ probability matrix, local maxima are selected as tree trunks and further points are assigned to these tree trunks if they appear in the close proximity.

\subsection{Point Cloud Segmentation}

There are different approaches which may be applied for 3D point cloud segmentation (Vosselman, 2013). While the segmentation may generally address a variety of objects, we focus on detecting single trees from 3D point cloud data, i.e. the derived segments should correspond to individual trees. In order to achieve a respective segmentation, many approaches rely on a voxelization of 3D space. In (Yao and Fan, 2013), an approach is presented which derives a 2D accumulation map on a horizontally oriented plane and - based on respective features - allows to separate natural objects such as trees from man-made objects. Those $3 \mathrm{D}$ points corresponding to natural objects are transferred to a voxel space and, subsequently, a normalized cut segmentation based on the voxel structure is carried out (Reitberger et al., 2009). A different strategy consists in performing a voxelization of 3D space, deriving connected components and separating the components further if they contain multiple clusters (Gorte et al., 2015). Alternatively, tree individualization may be achieved by a downsampling and retiling of the original 3D point cloud data via voxelization, where a subsequent $2 \mathrm{D}$ gridding allows to find local maxima in point density and thus potential tree locations (Lindenbergh et al., 2015). Based on these tree locations, individual trees are finally segmented via octree-based region growing and thresholding techniques.

Besides approaches involving a voxelization of 3D space, there are also approaches which perform tree individualization by considering the original data on point-level. An exemplary approach focuses on the calculation of geometric descriptors for each $3 \mathrm{D}$ point, the projection of these descriptors onto a horizontally oriented 2D accumulation map and the consideration of a spatial filtering to obtain individual tree clusters (Monnier et al., 2012). Furthermore, an approach for tree individualization on point-level has been proposed which relies on deriving connected components for those $3 \mathrm{D}$ points categorized into a tree class, and the connected components are further split via an upward and downward growing algorithm if there are multiple seeds at a height between $0.5 \mathrm{~m}$ and $1 \mathrm{~m}$ (Gorte et al., 2015; Oude Elberink and Kemboi, 2014). Alternatively, a direct consideration of the original data on point-level may be achieved by applying a standard clustering technique such as $k$-means clustering or hierarchical clustering (Gupta et al., 2010), or the mean shift algorithm presented in (Fukunaga and Hostetler, 1975). The latter has for instance been applied on 3D point cloud data in (Ferraz et al., 2012; Schmitt et al., 2013; Yao et al., 2013; Shahzad et al., 2015). However, such an approach can be computationally demanding, particularly for a large number of considered $3 \mathrm{D}$ points. To improve computational efficiency, it seems desirable to apply the mean shift algorithm on a $2 \mathrm{D}$ projection of a $3 \mathrm{D}$ point cloud as e.g. described in (Schmitt et al., 2015) for 3D point cloud data acquired via tomographic SAR processing. However, the point density will be significantly higher for a ground-based acquisition of 3D point cloud data as e.g. given when using mobile mapping systems.

\section{METHODOLOGY}

In this paper, we present a novel framework for detecting, segmenting and localizing individual trees from MMS point cloud data. Our framework first addresses the detection of tree-like structures in a considered 3D point cloud via classification (Section 3.1), which is followed by a segmentation and localization of individual trees (Section 3.2).

\subsection{Detection of Tree-Like Structures via Classification}

In the scope of this work, we mainly focus on the use of geometric features to obtain a point-wise semantic labeling of a considered $3 \mathrm{D}$ point cloud. Accordingly, a respective local neighborhood has to be recovered for each $3 \mathrm{D}$ point $\mathbf{X}$ in order to appropriately describe the local 3D structure at $\mathbf{X}$ (Section 3.1.1). Subsequently, a variety of geometric features may be extracted for $\mathbf{X}$ based on those $3 \mathrm{D}$ points within its local neighborhood (Section 3.1.2). If available, intensity and color information may also be considered to define features. Depending on the features of interest, we may define different feature sets (Section 3.1.3) which allows us to conclude about their absolute and relative performance with respect to the classification task (Section 3.1.4).

3.1.1 Neighborhood Selection: To appropriately describe the local 3D structure at a considered 3D point $\mathbf{X}$, we may generally consider different neighborhood types (Weinmann et al., 2015a). Since we focus on a processing of MMS point cloud data for which the point density is rather high, we favor the use of a spherical neighborhood. Furthermore, we intend to avoid including prior knowledge about the scene and/or the data for specifying the neighborhood. Accordingly, we involve a generic solution which automatically selects a suitable neighborhood size for each individual 3D point $\mathbf{X}$. Respective approaches typically rely on a neighborhood formed by the $k$ nearest neighbors of $\mathbf{X}$, and we determine the optimal scale parameter $k_{\text {opt }}$ for each individual 3D point via eigenentropy-based scale selection (Weinmann et al., 2015a; Weinmann, 2016) which has proven to be favorable in comparison to other approaches.

3.1.2 Feature Extraction: Based on the defined neighborhood, we extract a set of 14 geometric 3D features (Weinmann et al., 2013; Weinmann et al., 2015a). These features comprise eight local 3D shape features (West et al., 2004; Pauly et al., 2003):

$$
\text { - Linearity: } L_{\lambda}=\frac{\lambda_{1}-\lambda_{2}}{\lambda_{1}}
$$$$
\text { - Planarity: } P_{\lambda}=\frac{\lambda_{2}-\lambda_{3}}{\lambda_{1}}
$$ 
- Sphericity: $S_{\lambda}=\frac{\lambda_{3}}{\lambda_{1}}$

- Omnivariance: $O_{\lambda}=\sqrt[3]{\lambda_{1} \lambda_{2} \lambda_{3}}$

- Anisotropy: $A_{\lambda}=\frac{\lambda_{1}-\lambda_{3}}{\lambda_{1}}$

- Eigenentropy: $E_{\lambda}=-\lambda_{1} \ln \left(\lambda_{1}\right)-\lambda_{2} \ln \left(\lambda_{2}\right)-\lambda_{3} \ln \left(\lambda_{3}\right)$

- Sum of eigenvalues: $\Sigma_{\lambda}=\lambda_{1}+\lambda_{2}+\lambda_{3}$

- Local surface variation: $C_{\lambda}=\frac{\lambda_{3}}{\lambda_{1}+\lambda_{2}+\lambda_{3}}$

Here, the $\lambda_{i}$ with $i \in\{1,2,3\}, \lambda_{1} \geq \lambda_{2} \geq \lambda_{3} \geq 0$ and $\lambda_{1}+\lambda_{2}+$ $\lambda_{3}=1$ represent the normalized eigenvalues of the 3D structure tensor calculated based on all 3D points within the neighborhood of $\mathbf{X}$. Furthermore, the set of geometric 3D features comprises six basic geometric $3 \mathrm{D}$ properties of the considered $3 \mathrm{D}$ point $\mathbf{X}$ and its neighborhood:

- Height $H=Z$ of the considered 3D point

- Radius $R_{k \text {-NN }}$ of the neighborhood

- Local point density: $D=\frac{\# 3 \mathrm{D} \text { points within the local neighborhood }}{\text { volume of the local neighborhood }}$

- Verticality: $V=1-\mathbf{n}_{Z}$ where $\mathbf{n}$ is the normal vector

- Maximum height difference $\Delta H_{k \text {-NN }}$ within the neighborhood

- Standard deviation of height values $\sigma_{H, k \text {-NN }}$ within the neighborhood

Since urban environments contain many man-made objects with almost perfectly vertical structures (e.g. building façades, walls, poles or traffic signs), we also consider features relying on a projection of $\mathbf{X}$ and its nearest neighbors onto a horizontally oriented plane. For the resulting 2D space, we define local 2D shape features in analogy to the $3 \mathrm{D}$ case:

- Sum of eigenvalues: $\Sigma_{\lambda, 2 \mathrm{D}}=\lambda_{1,2 \mathrm{D}}+\lambda_{2,2 \mathrm{D}}$

- Ratio of eigenvalues: $R_{\lambda, 2 \mathrm{D}}=\lambda_{2,2 \mathrm{D}} / \lambda_{1,2 \mathrm{D}}$

where $\lambda_{1,2 \mathrm{D}}$ and $\lambda_{2,2 \mathrm{D}}$ are the eigenvalues of the $2 \mathrm{D}$ structure tensor. Furthermore, we use two geometric 2D properties:

- Radius $R_{k \text {-NN,2D }}$

- Local point density $D_{2 \mathrm{D}}$

To also account for the vertical behavior of 3D scene points around $\mathbf{X}$, we introduce a further neighborhood definition in the form of a spatial binning resulting from the discretization of the horizontally oriented plane into quadratic bins with a side length of $0.25 \mathrm{~m}$ and, for each bin, we derive features for $\mathbf{X}$ from the statistics of those $3 \mathrm{D}$ points assigned to the respective bin:

- Number $N_{\text {bin }}$ of 3D points falling into the respective bin

- Maximum height difference $\Delta H_{\text {bin }}$ within the bin

- Standard deviation of height values $\sigma_{H \text {,bin }}$ within the bin

If available, reflectance and color information may be considered to define radiometric features which can be used in addition to all these geometric features.
3.1.3 Feature Selection: For our experiments, we test different feature sets which are defined as follows:

- The feature set $\mathcal{S}_{\text {dim }}$ contains the dimensionality features:

$$
\mathcal{S}_{\operatorname{dim}}=\left\{L_{\lambda}, P_{\lambda}, S_{\lambda}\right\}
$$

- The feature set $\mathcal{S}_{\mathrm{EV}, 3 \mathrm{D}}$ contains eight local 3D shape features:

$$
\mathcal{S}_{\mathrm{EV}, 3 \mathrm{D}}=\left\{L_{\lambda}, P_{\lambda}, S_{\lambda}, O_{\lambda}, A_{\lambda}, E_{\lambda}, \Sigma_{\lambda}, C_{\lambda}\right\}
$$

- The feature set $\mathcal{S}_{3 \mathrm{D}}$ contains all defined 3D features, i.e. the local 3D shape features and the geometric 3D properties:

$$
\begin{aligned}
\mathcal{S}_{3 \mathrm{D}}= & \left\{L_{\lambda}, P_{\lambda}, S_{\lambda}, O_{\lambda}, A_{\lambda}, E_{\lambda}, \Sigma_{\lambda}, C_{\lambda},\right. \\
& \left.H, R_{k-\mathrm{NN}}, D, V, \Delta H_{k-\mathrm{NN}}, \sigma_{H, k-\mathrm{NN}}\right\}
\end{aligned}
$$

- The feature set $\mathcal{S}_{3 \mathrm{D}+2 \mathrm{D}^{*}}$ contains all 3D and 2D features relying on the $k$-NN neighborhood, i.e. the local 3D shape features, the geometric $3 \mathrm{D}$ properties, the local $2 \mathrm{D}$ shape features and the geometric 2D properties:

$$
\begin{gathered}
\mathcal{S}_{3 \mathrm{D}+2 \mathrm{D}^{*}=}=\left\{L_{\lambda}, P_{\lambda}, S_{\lambda}, O_{\lambda}, A_{\lambda}, E_{\lambda}, \Sigma_{\lambda}, C_{\lambda},\right. \\
H, R_{k-\mathrm{NN}}, D, V, \Delta H_{k-\mathrm{NN}}, \sigma_{H, k-\mathrm{NN}}, \\
\left.\Sigma_{\lambda, 2 \mathrm{D}}, R_{\lambda, 2 \mathrm{D}}, R_{k-\mathrm{NN}, 2 \mathrm{D}}, D_{2 \mathrm{D}}\right\}
\end{gathered}
$$

- The feature set $\mathcal{S}_{3 \mathrm{D}+2 \mathrm{D}}$ contains all 3D and 2D features, i.e. the local 3D shape features, the geometric 3D properties, the local 2D shape features, the geometric 2D properties and the features based on the $2 \mathrm{D}$ accumulation map:

$$
\begin{aligned}
\mathcal{S}_{3 \mathrm{D}+2 \mathrm{D}}=\{ & L_{\lambda}, P_{\lambda}, S_{\lambda}, O_{\lambda}, A_{\lambda}, E_{\lambda}, \Sigma_{\lambda}, C_{\lambda}, \\
& H, R_{k-\mathrm{NN}}, D, V, \Delta H_{k-\mathrm{NN}}, \sigma_{H, k-\mathrm{NN}}, \\
& \Sigma_{\lambda, 2 \mathrm{D}}, R_{\lambda, 2 \mathrm{D}}, R_{k-\mathrm{NN}, 2 \mathrm{D}}, D_{2 \mathrm{D}}, \\
& \left.N_{\text {bin }}, \Delta H_{\text {bin }}, \sigma_{H, \mathrm{bin}}\right\}
\end{aligned}
$$

- The feature set $\mathcal{S}_{3 \mathrm{D}+2 \mathrm{D}+\mathrm{I}}$ contains all 3D and 2D features as well as the given reflectance information:

$$
\begin{aligned}
\mathcal{S}_{3 \mathrm{D}+2 \mathrm{D}+\mathrm{I}}=\left\{L_{\lambda}, P_{\lambda}, S_{\lambda}, O_{\lambda}, A_{\lambda}, E_{\lambda}, \Sigma_{\lambda}, C_{\lambda},\right. & \\
& H, R_{k-\mathrm{NN}}, D, V, \Delta H_{k-\mathrm{NN}}, \sigma_{H, k-\mathrm{NN}}, \\
& \Sigma_{\lambda, 2 \mathrm{D}}, R_{\lambda, 2 \mathrm{D}}, R_{k-\mathrm{NN}, 2 \mathrm{D}}, D_{2 \mathrm{D}}, \\
& N_{\text {bin }}, \Delta H_{\text {bin }}, \sigma_{H, \text { bin }}, \\
& I\}
\end{aligned}
$$

- The feature set $\mathcal{S}_{3 \mathrm{D}+2 \mathrm{D}+\mathrm{I}+\mathrm{RGB}}$ contains all defined 3D and 2D features as well as reflectance and color information:

$$
\begin{gathered}
\mathcal{S}_{3 \mathrm{D}+2 \mathrm{D}+\mathrm{I}+\mathrm{RGB}}=\left\{L_{\lambda}, P_{\lambda}, S_{\lambda}, O_{\lambda}, A_{\lambda}, E_{\lambda}, \Sigma_{\lambda}, C_{\lambda},\right. \\
H, R_{k-\mathrm{NN}}, D, V, \Delta H_{k-\mathrm{NN}}, \sigma_{H, k-\mathrm{NN}}, \\
\Sigma_{\lambda, 2 \mathrm{D}}, R_{\lambda, 2 \mathrm{D}}, R_{k-\mathrm{NN}, 2 \mathrm{D}}, D_{2 \mathrm{D}}, \\
\quad N_{\text {bin }}, \Delta H_{\text {bin }}, \sigma_{H, \text { bin }}, \\
\\
I, R, G, B\}
\end{gathered}
$$

For each feature subset, the respective features are concatenated to a feature vector and a subsequent normalization is carried out so that the values of each dimension are mapped to the interval $[0,1]$. Thereby, the normalization is defined based on the minimum and maximum values of each dimension for the training examples. The test data is mapped accordingly and values outside of $[0,1]$ are mapped to the closest border of the interval. 
3.1.4 Classification: The normalized feature vectors serve as input for classification for which we use a Random Forest (RF) classifier (Breiman, 2001) as representative of modern discriminative methods. Generally, the RF classifier consists of an ensemble of decision trees as weak learners, where each decision tree is trained for a subset of the training data which is randomly drawn with replacement. Due to this random selection, it may be expected that the weak learners are all randomly different from each other, so that taking the majority vote across the hypotheses of all weak learners results in a generalized and robust hypothesis of a single strong learner.

\subsection{Segmentation and Localization of Individual Trees}

The procedure to get from $3 \mathrm{D}$ points categorized into the tree class to 3D segments corresponding to trees mainly relies on our previous work (Weinmann et al., 2016) which involves a downsampling of the original data, a projection of the downsampled data onto a horizontally oriented plane, a mean-shift-based segmentation of the projected points, a transfer of the segmentation results to the original data, a refinement of the segmentation results via segment-based shape analysis, and a localization of respective tree trunks. However, we also take into account that misclassifications might occur for 3D points corresponding to flat surfaces (Gorte et al., 2015), and we therefore introduce an initial filtering based on the feature of verticality. Normalized to the interval $[0,1]$, this feature characterizes horizontal surfaces in case of low values (e.g. in $\left[0, T_{1}\right]$ ) and high values (e.g. in $\left[0,1-T_{1}\right]$ ), while a vertical structure is indicated by a value of $\approx 0.5$ (e.g. in $\left[0.5-T_{2}, 0.5+T_{2}\right]$ ). Accordingly, we apply an initial thresholding whereby the thresholds are selected based on the histogram of values for the feature of verticality $\left(T_{1}=0.1, T_{2}=0.2\right)$.

3.2.1 Downsampling: To improve efficiency with respect to processing time and memory consumption, we take into account that MMS point cloud data provides a dense representation of object surfaces near the acquisition system and that the point density may significantly be decreased while still being able to detect individual trees in the respective $3 \mathrm{D}$ point cloud data. The reduced $3 \mathrm{D}$ point cloud in turn might facilitate time-consuming tasks such as a generic segmentation. For this reason, we introduce a downsampling of the 3D points classified as tree by only keeping every $k$-th point, whereby we heuristically select a parameter of $k=10$ as done in (Weinmann et al., 2016). To avoid such a manual selection, a pruning of this parameter could be conducted based on the local point density (Caraffa et al., 2015).

3.2.2 2D Projection: Since - due to human intervention in nature and due to planning processes - urban areas typically provide a larger spacing and less overlap between individual trees in comparison to forested areas, we neglect the occurrence of dominant, co-dominant or dominated trees in urban environments and assume that individual trees may still sufficiently be delineated when only considering a $2 \mathrm{D}$ projection of the downsampled $3 \mathrm{D}$ point cloud data corresponding to the tree class onto a horizontally oriented plane.

3.2.3 Mean Shift Segmentation: To derive a meaningful partitioning, the $2 \mathrm{D}$ projections of the downsampled $3 \mathrm{D}$ point cloud data corresponding to the tree class onto a horizontally oriented plane are provided as input for the mean shift algorithm (Fukunaga and Hostetler, 1975; Cheng, 1995; Comaniciu and Meer, 2002) which represents an iterative technique for locating the maxima / modes of a probability density function by only considering discrete data sampled from that probability density function. Thereby, the probability density function does not have to be determined explicitly, and there is no need to make assumptions on a specific geometric model or the number of modes.
Considering the derived 2D projections as discrete data points sampled from an empirical 2D probability density function, the mean shift algorithm takes each data point and iteratively $(i)$ calculates the weighted mean of data points within a window defined by a kernel $\mathcal{K}$ (typically an isotropic kernel such as a Gaussian kernel or an Epanechnikov kernel (Comaniciu and Meer, 2002)), (ii) defines the mean shift vector $\mathbf{m}$ as the difference between the data point and the weighted mean of data points within the considered window, and (iii) moves the data point along the mean shift vector. Thereby, the magnitude of the mean shift vector will be large in areas of low point density, whereas it will be low in areas of high point density. Accordingly, the mean shift algorithm iteratively performs an adaptive gradient ascent until convergence (up to numerical accuracy). The stationary points correspond to regions of high point density and represent the modes of the underlying distribution of data points. Finally, all data points leading to the same mode are considered as cluster or segment. In the scope of our work, the single clusters / segments are expected to represent the individual trees in the considered scene.

The number of detected modes depends on the specification of the involved kernel for which we select an isotropic Gaussian kernel with the same bandwidth $h$ in all directions. Such a choice is intuitively justified since we want to detect individual trees and it may rely on prior knowledge about the shape and size of the trees in the considered scene. Accordingly, we carried out different tests and heuristically selected a value of $h=3.8 \mathrm{~m}$ for our experiments (Weinmann et al., 2016).

3.2.4 Data Transfer: Since the segmentation results are derived for the downsampled 3D point cloud data corresponding to the tree class, a transfer of these results to the original 3D point cloud data is required. For this purpose, we focus on an intuitive, simple and straightforward approach which assigns each 3D point of the respective part of the original 3D point cloud data the segment label of the closest 3D point in the downsampled version of the original data. Thereby, we conduct a nearest neighbor search based on Euclidean distances.

3.2.5 Shape Analysis: In contrast to our previous work (Weinmann et al., 2016), we focus on a segment-based shape analysis which relies on a feature extraction on the basis of a segment as respective neigborhood, i.e. those geometric features presented in Section 3.1.2 may also be derived for each segment. Defining the derived segments as neighborhood, we first discard small segments which are not likely to correspond to the objects of interest. Accordingly, those segments comprising less than 500 points are removed since, for 3D point clouds acquired with mobile mapping systems, a much larger number of 3D points may be expected for meaningful segments corresponding to trees. For the remaining segments, we take into account that misclassifications may occur for 3D points corresponding to building façades which e.g. becomes visible in the results for one of the approaches presented in (Gorte et al., 2015). To address this issue, we take into account that the ratio $R_{\lambda, 2 \mathrm{D}}$ of the eigenvalues of the $2 \mathrm{D}$ structure tensor reveals line-like structures as e.g. given for the $2 \mathrm{D}$ projection of a building façade onto the horizontally oriented plane. Since such line-like structures in the $2 \mathrm{D}$ projection are rather elongated, we may simply discard all segments for which $R_{\lambda, 2 \mathrm{D}}$ is below a certain threshold $t_{R}$. Thereby, we heuristically select a value of $t_{R}=0.3$ indicating that the smaller eigenvalue has to be equal to or even above a value of $30 \%$ of the larger eigenvalue for a segment corresponding to a tree.

3.2.6 Localization: For all plausible tree segments, we define their location via the respective mode determined during the mean shift segmentation based on the $2 \mathrm{D}$ projections of the downsampled 3D point cloud data. 

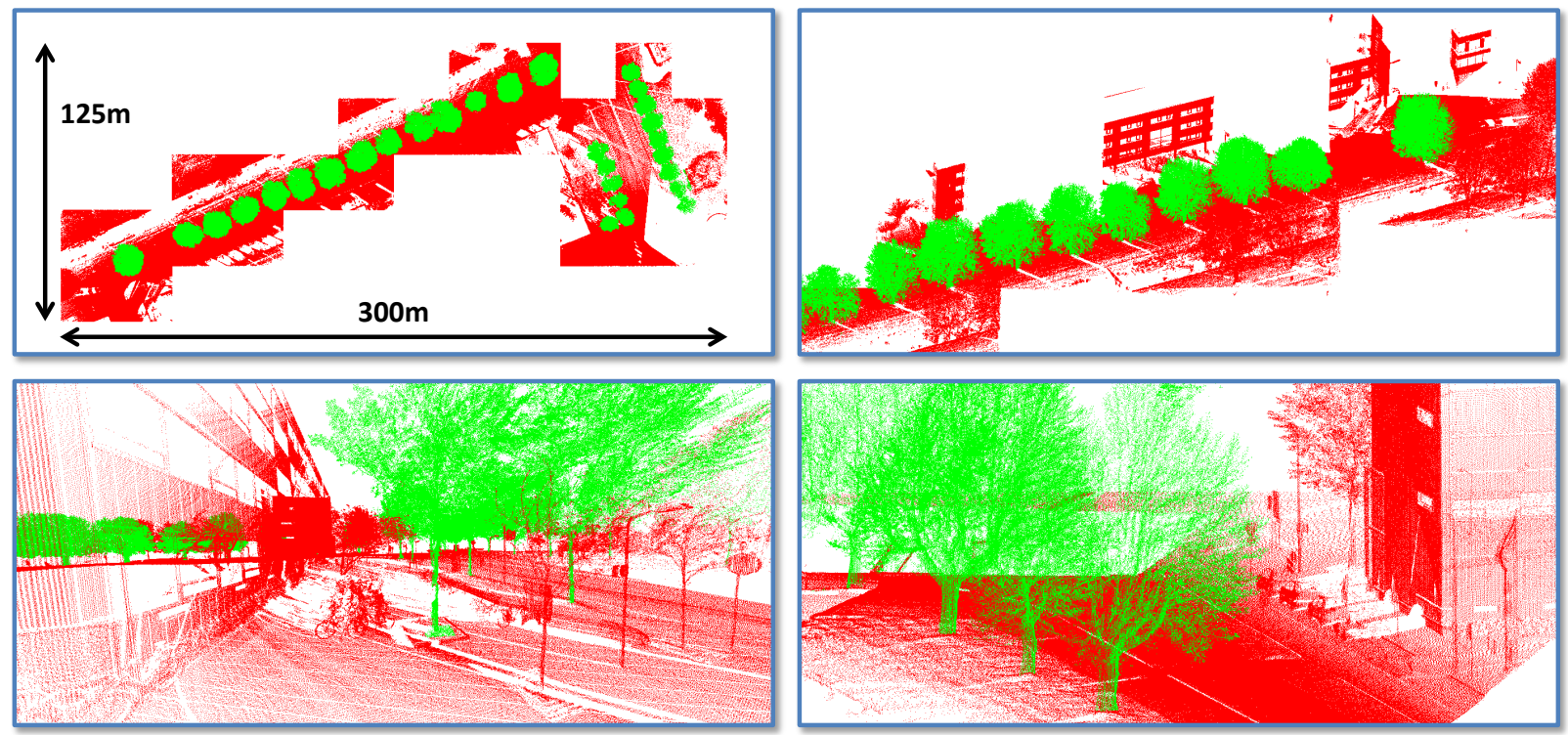

Figure 1. Visualization of the used benchmark dataset with about 10.13M labeled 3D points (top row: nadir view and side view; bottom row: more detailed views): $3 \mathrm{D}$ points categorized into the tree class are colored in green and all other 3D points are colored in red.

\section{EXPERIMENTAL RESULTS}

In the following, we provide details on the involved dataset (Section 4.1) and, subsequently, we present results obtained for the task of tree classification (Section 4.2) as well as results obtained for the task of tree segmentation and localization (Section 4.3).

\subsection{Dataset}

The considered benchmark dataset has been acquired in the vicinity of the campus of TU Delft in the Netherlands with the Fugro DRIVE-MAP system (Gorte et al., 2015). For our experiments, we use the provided subset consisting of 26 tiles with a total number of 10,126,500 labeled 3D points (see Figure 1), where the class labels refer to a binary classification to distinguish between (i) 3D points corresponding to trees and (ii) other 3D points. Thereby, the tree class comprises about $1.78 \mathrm{M}$ points $(17.6 \%)$ and the non-tree class comprises the remaining $3 \mathrm{D}$ points.

\subsection{Task 1: Tree Classification}

To evaluate the performance of our framework with respect to tree classification, we consider the results after the binary classification categorizing $3 \mathrm{D}$ points with respect to the tree class and the non-tree class. Accordingly, the local neighborhood is first derived for each 3D point via eigenentropy-based scale selection. This neighborhood serves as the basis for deriving point-wise feature vectors which serve as input for the involved RF classifier. For training this classifier, we take into account that an unbalanced distribution of training examples per class might have a detrimental effect on the training process (Chen et al., 2004; Criminisi and Shotton, 2013). For this reason, we randomly select 1,000 training examples per class for training the classifier, i.e. we use 2,000 points as training set and the remaining $10,124,500$ points as test set. The number $N_{T}$ of decision trees used for the RF classifier has been selected heuristically via grid search and is given with $N_{T}=100$ for all the feature sets introduced in Section 3.1.3. The respectively derived RF-based classification results (averaged across 10 runs) are provided in Table 1, where the following evaluation metrics are provided: overall accuracy $(\mathrm{OA})$, Cohen's kappa coefficient $(\kappa)$, precision for the tree class $(P($ tree $))$ and for the non-tree class $(P($ non-tree $))$, and recall for the tree class ( $R$ (tree) $)$ and for the non-tree class ( $R$ (non-tree $)$ ). A visualization of the classification results derived for the feature set $\mathcal{S}_{3 \mathrm{D}+2 \mathrm{D}^{*}}$ is provided in Figure 2.

These results clearly indicate that only using the three dimensionality features for classification does not lead to accurate results. The respective values for $\mathrm{OA}$ and $\kappa$ are relatively low, while the respective standard deviation across the 10 runs is relatively high. By adding more features, the results are significantly improved and the standard deviation is reduced in most of the cases. When for instance extending the feature set $\mathcal{S}_{\mathrm{dim}}$ comprising the three dimensionality features to the feature set $\mathcal{S}_{3 \mathrm{D}+2 \mathrm{D}}$ comprising 21 low-level geometric 3D and 2D features (Weinmann et al., 2013; Weinmann et al., 2015a), a gain of about $17.43 \%$ in OA and about $39.99 \%$ in $\kappa$ may be observed, while the standard deviation $\sigma_{\mathrm{OA}}$ of the overall accuracy $\mathrm{OA}$ is reduced by $0.99 \%$. When only using the feature set $\mathcal{S}_{3 \mathrm{D}+2 \mathrm{D}^{*}}$ comprising all $3 \mathrm{D}$ and $2 \mathrm{D}$ features relying on the $k$-NN neighborhood, the gain is still about $15.69 \%$ in OA and about $35.83 \%$ in $\kappa$, while $\sigma_{\mathrm{OA}}$ is reduced by $0.26 \%$. The use of $\mathcal{S}_{3 \mathrm{D}+2 \mathrm{D} *}$ can be motivated by the fact that the calculation of features relying on a $2 \mathrm{D}$ accumulation map is not required. This is meaningful since the computational effort for calculating respective features reveals a non-linear behavior, whereas the computational effort for calculating the remaining 18 geometric features - all depending on characteristics of the same 3D points within the local neighborhood determined via eigenentropy-based scale selection - reveals a linear behavior (Weinmann et al., 2015b). For both $\mathcal{S}_{3 \mathrm{D}+2 \mathrm{D}}$ and $\mathcal{S}_{3 \mathrm{D}+2 \mathrm{D}^{*} \text {, how- }}$ ever, the improvement in comparison to $\mathcal{S}_{\mathrm{dim}}$ can be considered as significant. Yet, additionally considering radiometric information (i.e. reflectance or color information) does not seem to lead to an improvement of the classification results for our application.

A more detailed consideration of failure cases (see Figure 3) reveals the following insights:

- Incorrect labeling: As shown in Figure 3, some trees are completely labeled as non-tree-like objects. If training examples corresponding to respective 3D points are selected, the generalization capability of the classifier might be reduced. Furthermore, the incorrect labeling causes that the evaluation on the test set considers a significant number of correctly classified 3D points as classification errors. 


\begin{tabular}{|c|c|c|c|c|c|c|c|}
\hline Feature Set & \# Features & OA [\%] & $\kappa[\%]$ & $P($ tree $)[\%]$ & $P($ non-tree $)[\%]$ & $R($ tree $)[\%]$ & $R($ non-tree $)[\%]$ \\
\hline $\mathcal{S}_{\mathrm{dim}}$ & 3 & $74.34 \pm 1.47$ & $35.62 \pm 1.36$ & $38.32 \pm 1.35$ & $93.20 \pm 0.33$ & $74.59 \pm 2.07$ & $74.29 \pm 2.22$ \\
\hline $\mathcal{S}_{\mathrm{EV}, 3 \mathrm{D}}$ & 8 & $84.42 \pm 0.70$ & $57.06 \pm 1.33$ & $53.52 \pm 1.32$ & $96.95 \pm 0.21$ & $87.65 \pm 0.93$ & $83.73 \pm 0.94$ \\
\hline $\mathcal{S}_{3 \mathrm{D}}$ & 14 & $90.12 \pm 1.07$ & $71.67 \pm 2.42$ & $64.60 \pm 2.64$ & $99.40 \pm 0.13$ & $97.49 \pm 0.57$ & $88.54 \pm 1.38$ \\
\hline $\mathcal{S}_{3 \mathrm{D}+2 \mathrm{D}} *$ & 18 & $90.03 \pm 1.21$ & $71.45 \pm 2.75$ & $64.44 \pm 2.96$ & $99.37 \pm 0.12$ & $97.36 \pm 0.53$ & $88.46 \pm 1.55$ \\
\hline $\mathcal{S}_{3 \mathrm{D}+2 \mathrm{D}}$ & 21 & $91.77 \pm 0.48$ & $75.62 \pm 1.18$ & $68.80 \pm 1.36$ & $99.40 \pm 0.11$ & $97.46 \pm 0.47$ & $90.55 \pm 0.61$ \\
\hline $\mathcal{S}_{3 \mathrm{D}+2 \mathrm{D}+\mathrm{I}}$ & 22 & $91.74 \pm 0.60$ & $75.61 \pm 1.44$ & $68.68 \pm 1.71$ & $99.47 \pm 0.10$ & $97.73 \pm 0.45$ & $90.46 \pm 0.79$ \\
\hline $\mathcal{S}_{3 \mathrm{D}+2 \mathrm{D}+\mathrm{I}+\mathrm{RGB}}$ & 25 & $91.34 \pm 0.50$ & $74.53 \pm 1.19$ & $67.68 \pm 1.39$ & $99.36 \pm 0.09$ & $97.28 \pm 0.41$ & $90.07 \pm 0.65$ \\
\hline
\end{tabular}

Table 1. Mean values and standard deviation for the averaged classification results across 10 runs (2D*: only those 2D features relying on the $k$-NN neighborhood).
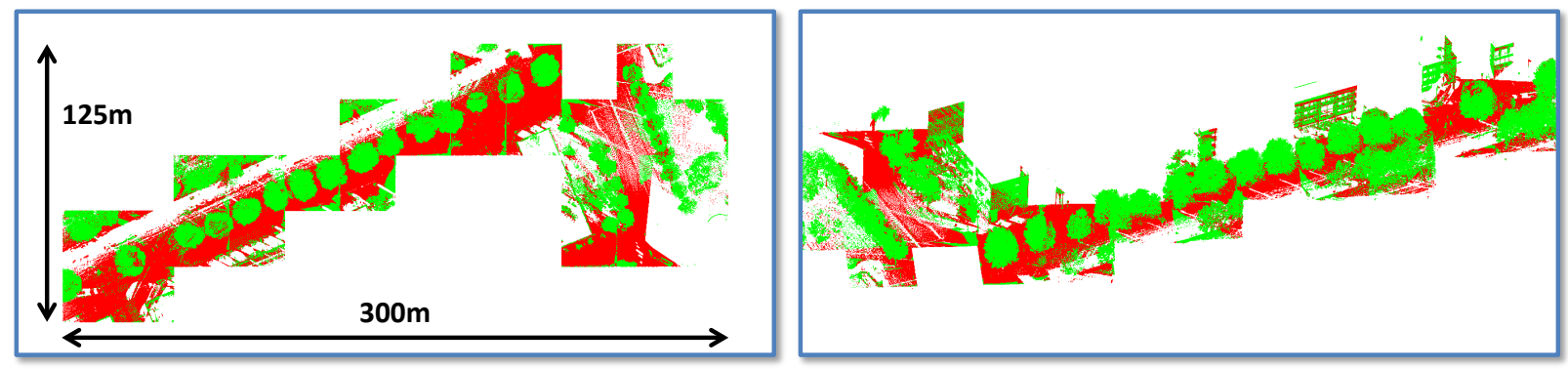

Figure 2. Visualization of exemplary classification results derived for the feature set $\mathcal{S}_{3 \mathrm{D}+2 \mathrm{D}^{*}}$ (left: nadir view; right: side view): $3 \mathrm{D}$ points classified as tree are colored in green and all other 3D points are colored in red.

- Registration errors: A closer look on the classified point cloud also reveals that there seems to be a slight misalignment of different MLS point clouds, resulting in the fact that 3D points on some building façades are characterized by a volumetric behavior when considering local neighborhoods derived via eigenentropy-based scale selection.

- Significant variations in point density: Some regions are not appropriately classified since the point density is extremely high (which results in an extremely small neighborhood tending to be rather meaningless) or extremely low (which results in an extremely large neighborhood tending to smooth details of the local 3D structure).

- Edge effects: There are some misclassifications which occur at the boundary of tiles. This might be solved by considering small padding regions at the borders of each tile, so that those $3 \mathrm{D}$ points within the small padding around each tile are also used if they are within the neighborhood of any 3D point within the considered tile (Weinmann et al., 2015b).

As a consequence, misclassifications might mainly depend on the considered dataset and less on the proposed methodology.

\subsection{Task 2: Tree Segmentation and Localization}

To evaluate the performance of our framework with respect to tree segmentation and localization, we use the results of Task 1 focusing on tree classification and delivering a classified 3D point cloud, where the single $3 \mathrm{D}$ points are categorized either into the tree class or into the non-tree class. All those 3D points belonging to the non-tree class are removed, and the remaining 3D points serve as input for the segmentation pipeline.

In the following, we consider the classification results derived when considering the feature set $\mathcal{S}_{3 \mathrm{D}+2 \mathrm{D}^{*}}$ containing all $3 \mathrm{D}$ and 2D features relying on the $k$-NN neighborhood. After a filtering based on the feature of verticality, the segmentation pipeline involves a mean shift segmentation on a suitable subspace of the data for reasons of efficiency. A visualization of the segmentation results derived from the classification results depicted in Figure 2 is provided in Figure 4 and also shows intermediate results after different subtasks.

A closer look on these segmentation results reveals that the derived segmentation results are sufficiently accurate for the benchmark dataset (i.e. almost all derived segments correspond to individual trees) and that only minor segmentation errors occur at segment borders if adjacent trees are relatively close to each other. However, the latter also becomes visible in the results presented in (Gorte et al., 2015). Besides, the proposed approach for individual tree segmentation and localization is rather simple and easy-to-use. It directly works on the given data without relying on a voxelization as e.g. presented in (Gorte et al., 2015; Lindenbergh et al., 2015), where the voxel size as well as the voxel orientation might strongly influence the respective segmentation results. The consideration on point-level remains efficient since time-consuming tasks such as the mean shift algorithm are applied on a subspace of the considered $3 \mathrm{D}$ point cloud and respective results are subsequently transferred back to the input data, whereby the subspace is defined via a downsampling and a $2 \mathrm{D}$ projection. The downsampling increases efficiency while still allowing to detect individual trees in the respective 3D point cloud data (Weinmann et al., 2016), and the 2D projection further reduces the computational effort since a mean shift segmentation in 2D can be conducted much faster than a mean shift segmentation in 3D (Ferraz et al., 2012; Schmitt et al., 2013).

The prototype of our framework has been implemented in Matlab and tested on a high-performance computer (Intel Core i7-3820, $3.6 \mathrm{GHz}, 64 \mathrm{~GB}$ RAM). For the first task focusing on classification, the processing times are significant $(8.34 \mathrm{~h}$ for neighborhood selection, $10.84 \mathrm{~h}$ for feature extraction, $0.34 \mathrm{~s}$ for training, $23.81 \mathrm{~s}$ for testing), while the second task focusing on a segmentation and localization of individual trees requires less than $1 \mathrm{~min}$ in total. Yet, our implementation is not fully optimized and a significant speed-up of the first task may be achieved via parallelization.

\section{CONCLUSIONS}

In this paper, we have presented a framework for detecting, segmenting and localizing individual trees from MMS point cloud 

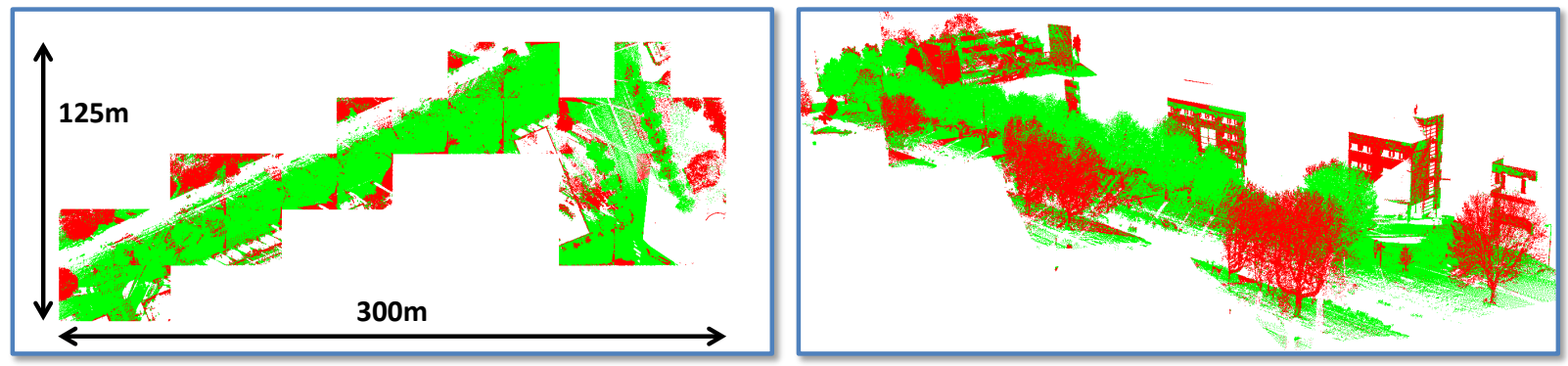

Figure 3. Visualization of the main failure cases in the form of misclassifications for trees and building façades (left: nadir view; right: side view): correctly classified 3D points are colored in green and all other 3D points are colored in red.

data. The main novelty of this framework consists in an endto-end processing workflow from an acquired $3 \mathrm{D}$ point cloud to individual trees, whereby all steps are performed on point level. The derived results indicate that $(i)$ classification results of highquality may be achieved by only involving geometric features and (ii) appropriate segmentation results may be derived based on the classified 3D point cloud and the derived point-wise features.

For future work, we plan to integrate parts of the proposed framework into the IQmulus platform (Böhm et al., 2016) focusing on large-scale scene analysis, where one goal consists in the extraction of individual trees for a dataset which represents about $10 \mathrm{~km}$ of streets and has been acquired in the city of Toulouse, France.

\section{ACKNOWLEDGEMENTS}

This work was partially supported by the European Commission's Seventh Framework Programme under the grant agreement FP7-ICT-2011-318787 (IQmulus: A High-Volume Fusion and Analysis Platform for Geospatial Point Clouds, Coverages and Volumetric Data Sets).

\section{REFERENCES}

Böhm, J., Brédif, M., Gierlinger, T., Krämer, M., Lindenbergh, R., Liu, K., Michel, F. and Sirmacek, B., 2016. The IQmulus urban showcase: automatic tree classification and identification in huge mobile mapping point clouds. In: The International Archives of the Photogrammetry, Remote Sensing and Spatial Information Sciences, Prague, Czech Republic, Vol. XLI-B3, pp. 301-307.

Brédif, M., Vallet, B., Serna, A., Marcotegui, B. and Paparoditis, N., 2014. TerraMobilita/IQmulus urban point cloud classification benchmark. In: Proceedings of the IQmulus Workshop on Processing Large Geospatial Data, Cardiff, UK, pp. 1-6.

Breiman, L., 2001. Random forests. Machine Learning 45(1), pp. 5-32.

Brodu, N. and Lague, D., 2012. 3D terrestrial lidar data classification of complex natural scenes using a multi-scale dimensionality criterion: applications in geomorphology. ISPRS Journal of Photogrammetry and Remote Sensing 68, pp. 121-134.

Caraffa, L., Brédif, M. and Vallet, B., 2015. 3D octree based watertight mesh generation from ubiquitous data. In: The International Archives of the Photogrammetry, Remote Sensing and Spatial Information Sciences, La Grande Motte, France, Vol. XL-3/W3, pp. 613-617.

Chen, C., Liaw, A. and Breiman, L., 2004. Using random forest to learn imbalanced data. Technical Report, University of California, Berkeley, USA.

Cheng, Y., 1995. Mean shift, mode seeking, and clustering. IEEE Transactions on Pattern Analysis and Machine Intelligence 17(8), pp. 790-799.

Comaniciu, D. and Meer, P., 2002. Mean shift: a robust approach toward feature space analysis. IEEE Transactions on Pattern Analysis and Machine Intelligence 24(5), pp. 603-619.

Criminisi, A. and Shotton, J., 2013. Decision forests for computer vision and medical image analysis. Advances in Computer Vision and Pattern Recognition, Springer, London, UK.
Demantké, J., Mallet, C., David, N. and Vallet, B., 2011. Dimensionality based scale selection in 3D lidar point clouds. In: The International Archives of the Photogrammetry, Remote Sensing and Spatial Information Sciences, Calgary, Canada, Vol. XXXVIII-5/W12, pp. 97-102.

Ferraz, A., Bretar, F., Jacquemoud, S., Gonçalves, G., Pereira, L., Tomé, M. and Soares, P., 2012. 3-D mapping of a multi-layered Mediterranean forest using ALS data. Remote Sensing of Environment 121, pp. 210-223. Fukunaga, K. and Hostetler, L., 1975. The estimation of the gradient of a density function, with applications in pattern recognition. IEEE Transactions on Information Theory 21(1), pp. 32-40.

Gorte, B., Oude Elberink, S., Sirmacek, B. and Wang, J., 2015. IQPC 2015 Track: Tree separation and classification in mobile mapping lidar data. In: The International Archives of the Photogrammetry, Remote Sensing and Spatial Information Sciences, La Grande Motte, France, Vol. XL-3/W3, pp. 607-612.

Gupta, S., Weinacker, H. and Koch, B., 2010. Comparative analysis of clustering-based approaches for 3-D single tree detection using airborne fullwave lidar data. Remote Sensing 2(4), pp. 968-989.

Hackel, T., Wegner, J. D. and Schindler, K., 2016. Fast semantic segmentation of 3D point clouds with strongly varying density. In: ISPRS Annals of the Photogrammetry, Remote Sensing and Spatial Information Sciences, Prague, Czech Republic, Vol. III-3, pp. 177-184.

Hu, H., Munoz, D., Bagnell, J. A. and Hebert, M., 2013. Efficient 3-D scene analysis from streaming data. In: Proceedings of the IEEE International Conference on Robotics and Automation, Karlsruhe, Germany, pp. 2297-2304.

Lalonde, J.-F., Unnikrishnan, R., Vandapel, N. and Hebert, M., 2005. Scale selection for classification of point-sampled 3D surfaces. In: Proceedings of the International Conference on 3-D Digital Imaging and Modeling, Ottawa, Canada, pp. 285-292.

Lindenbergh, R. C., Berthold, D., Sirmacek, B., Herrero-Huerta, M., Wang, J. and Ebersbach, D., 2015. Automated large scale parameter extraction of road-side trees sampled by a laser mobile mapping system. In: The International Archives of the Photogrammetry, Remote Sensing and Spatial Information Sciences, La Grande Motte, France, Vol. XL-3/W3, pp. 589-594.

Melzer, T., 2007. Non-parametric segmentation of ALS point clouds using mean shift. Journal of Applied Geodesy 1(3), pp. 159-170.

Monnier, F., Vallet, B. and Soheilian, B., 2012. Trees detection from laser point clouds acquired in dense urban areas by a mobile mapping system. In: ISPRS Annals of the Photogrammetry, Remote Sensing and Spatial Information Sciences, Melbourne, Australia, Vol. I-3, pp. 245-250.

Munoz, D., Bagnell, J. A., Vandapel, N. and Hebert, M., 2009a. Contextual classification with functional max-margin Markov networks. In: Proceedings of the IEEE Conference on Computer Vision and Pattern Recognition, Miami, USA, pp. 975-982.

Munoz, D., Vandapel, N. and Hebert, M., 2008. Directional associative Markov network for 3-D point cloud classification. In: Proceedings of the International Symposium on 3D Data Processing, Visualization and Transmission, Atlanta, USA, pp. 63-70.

Munoz, D., Vandapel, N. and Hebert, M., 2009b. Onboard contextual classification of 3-D point clouds with learned high-order Markov random fields. In: Proceedings of the IEEE International Conference on Robotics and Automation, Kobe, Japan, pp. 2009-2016.

Oude Elberink, S. and Kemboi, B., 2014. User-assisted object detection by segment based similarity measures in mobile laser scanner data. In: The International Archives of the Photogrammetry, Remote Sensing and Spatial Information Sciences, Zurich, Switzerland, Vol. XL-3, pp. 239246. 

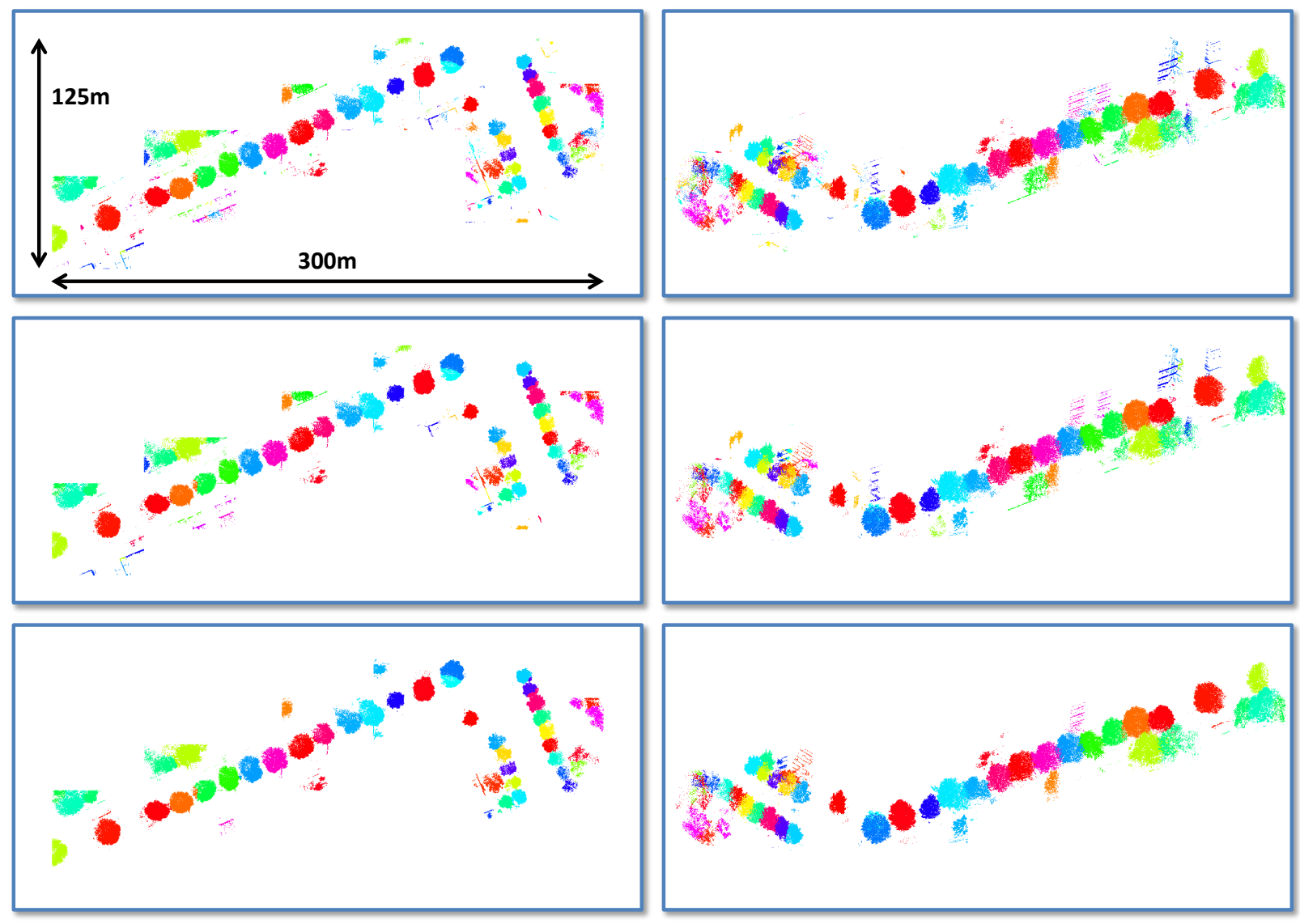

Figure 4. Visualization of the segmentation results derived from the classification results depicted in Figure 2 (left: nadir view; right: side view): single segments are indicated in different color. The figure illustrates the results after the transfer of the mean shift segmentation results to 3D space (top), after removing segments with less than 500 points (center) and after the refinement based on shape analysis (bottom).

Pauly, M., Keiser, R. and Gross, M., 2003. Multi-scale feature extraction on point-sampled surfaces. Computer Graphics Forum 22(3), pp. 81-89.

Reitberger, J., Schnörr, C., Krzystek, P. and Stilla, U., 2009. 3D segmentation of single trees exploiting full waveform lidar data. ISPRS Journal of Photogrammetry and Remote Sensing 64(6), pp. 561-574.

Schmitt, M., Brück, A., Schönberger, J. and Stilla, U., 2013. Potential of airborne single-pass millimeterwave InSAR data for individual tree recognition. In: Tagungsband der Dreiländertagung der DGPF, der OVG und der SGPF, Freiburg, Germany, Vol. 22, pp. 427-436.

Schmitt, M., Shahzad, M. and Zhu, X. X., 2015. Reconstruction of individual trees from multi-aspect TomoSAR data. Remote Sensing of Environment 165 , pp. 175-185.

Serna, A., Marcotegui, B., Goulette, F. and Deschaud, J.-E., 2014. Parisrue-Madame database: a 3D mobile laser scanner dataset for benchmarking urban detection, segmentation and classification methods. In: Proceedings of the International Conference on Pattern Recognition Applications and Methods, Angers, France, pp. 819-824.

Shahzad, M., Schmitt, M. and Zhu, X. X., 2015. Segmentation and crown parameter extraction of individual trees in an airborne TomoSAR point cloud. In: The International Archives of the Photogrammetry, Remote Sensing and Spatial Information Sciences, Munich, Germany, Vol. XL3/W2, pp. 205-209.

Sirmacek, B. and Lindenbergh, R., 2015. Automatic classification of trees from laser scanning point clouds. In: ISPRS Annals of the Photogrammetry, Remote Sensing and Spatial Information Sciences, La Grande Motte, France, Vol. II-3/W5, pp. 137-144.

Vosselman, G., 2013. Point cloud segmentation for urban scene classification. In: The International Archives of the Photogrammetry, Remote Sensing and Spatial Information Sciences, Antalya, Turkey, Vol. XL-7/W2, pp. 257-262.

Weinmann, M., 2016. Reconstruction and analysis of 3D scenes - From irregularly distributed $3 D$ points to object classes. Springer, Cham, Switzerland.
Weinmann, M., Jutzi, B. and Mallet, C., 2013. Feature relevance assessment for the semantic interpretation of 3D point cloud data. In: ISPRS Annals of the Photogrammetry, Remote Sensing and Spatial Information Sciences, Antalya, Turkey, Vol. II-5/W2, pp. 313-318.

Weinmann, M., Jutzi, B., Hinz, S. and Mallet, C., 2015a. Semantic point cloud interpretation based on optimal neighborhoods, relevant features and efficient classifiers. ISPRS Journal of Photogrammetry and Remote Sensing 105, pp. 286-304.

Weinmann, M., Mallet, C. and Brédif, M., 2016. Segmentation and localization of individual trees from MMS point cloud data acquired in urban areas. In: Tagungsband der Dreiländertagung der DGPF, der OVG und der SGPF, Bern, Switzerland, Vol. 25, pp. 351-360.

Weinmann, M., Urban, S., Hinz, S., Jutzi, B. and Mallet, C., 2015b. Distinctive $2 \mathrm{D}$ and $3 \mathrm{D}$ features for automated large-scale scene analysis in urban areas. Computers \& Graphics 49, pp. 47-57.

West, K. F., Webb, B. N., Lersch, J. R., Pothier, S., Triscari, J. M. and Iverson, A. E., 2004. Context-driven automated target detection in 3-D data. Proceedings of SPIE 5426, pp. 133-143.

Xiong, X., Munoz, D., Bagnell, J. A. and Hebert, M., 2011. 3-D scene analysis via sequenced predictions over points and regions. In: Proceedings of the IEEE International Conference on Robotics and Automation, Shanghai, China, pp. 2609-2616.

Yao, W. and Fan, H., 2013. Automated detection of 3D individual trees along urban road corridors by mobile laser scanning systems. In: Proceedings of the International Symposium on Mobile Mapping Technology, Tainan, Taiwan, pp. 1-6.

Yao, W., Krzystek, P. and Heurich, M., 2013. Enhanced detection of 3D individual trees in forested areas using airborne full-waveform lidar data by combining normalized cuts with spatial density clustering. In: ISPRS Annals of the Photogrammetry, Remote Sensing and Spatial Information Sciences, Antalya, Turkey, Vol. II-5/W2, pp. 349-354. 


\title{
ROBUST AND REPEATABLE RULESET DEVELOPMENT FOR HIERARCHICAL OBJECT-BASED MONITORING OF REVEGETATION USING HIGH SPATIAL AND TEMPORAL RESOLUTION UAS DATA
}

\author{
T. G. Whiteside ${ }^{\text {a }}$ R. E. Bartolo ${ }^{\text {a }}$ \\ ${ }^{a}$ Supervising Scientist, Department of the Environment, PO Box 461, Darwin NT, 0801, (tim.whiteside, \\ renee.bartolo)@environment.gov.au
}

\begin{abstract}
KEY WORDS: UAV, Time series analysis, GEOBIA, NDVI, Ultra-high spatial resolution, Minesite rehabilitation, Monitoring
\end{abstract}

\begin{abstract}
:
The monitoring of mine site rehabilitation, particularly revegetation, involves assessing biophysical parameters over time to determine success or otherwise. Resource and logistical constraints limit the spatial and temporal scale of standard field-based monitoring methods. Unmanned aerial systems (UAS) can provide the capability to acquire optical data with coverage of an entire site at the spatial and temporal scales, suitable for the monitoring of relevant biophysical parameters. UAS data are not without challenges, such as radiometric variation between flights and sensors, different sun angles and spatial variation between dates. Therefore, a ruleset (a stepwise set of analysis algorithms) is needed that is robust and repeatable across each image regardless of spatial and radiometric variability. This study reports on the development and implementation of such a ruleset. Using a fixed wing unmanned aircraft, colour and near infrared imagery was captured over the Jabiluka mine site, located in the Northern Territory, Australia on 7 dates between April 2014 and October 2015. The imagery was radiometrically and geometrically corrected and 4 band mosaics for each date were created using photogrammetric techniques. Image analysis involved the creation of vegetation indices relevant to the data available, the segmentation of the image to delineate plants from the background and the creation of a hierarchy of objects for analysis that was consistent across all dates. Accuracy assessment showed that the GEOBIA-derived measures compared well with visual assessments of the imagery. From the analysis, the proportional cover of green plants and number of plant objects per unit area could be calculated. The time series analysis showed that proportional cover across the site varied between dates but gradually increased over time, although there was a high level of plant mortality. In addition, there was natural recruitment of volunteer plants. The same ruleset was applied to the imagery from each date demonstrating that it is robust enough to be used for further monitoring on the site. In addition, the ruleset in a modified form is currently being used for change analysis using scanned historical aerial photography.
\end{abstract}

\section{INTRODUCTION}

The monitoring of mine site rehabilitation, particularly revegetation, involves assessing biophysical parameters over time to determine success or otherwise. Resource and logistical constraints limit the spatial and temporal scale of standard fieldbased monitoring methods. One useful parameter that can be used to monitor the progress of mine site rehabilitation is the amount of vegetation foliage cover on the site. An analysis of changes in vegetation cover over time can indicate, whether a mine site is heading on a trajectory toward meeting rehabilitation goals.

Unmanned aerial systems (UAS) can provide the capability to acquire optical data with coverage of an entire site at the spatial and temporal scales, suitable for the monitoring of relevant biophysical parameters (Fletcher and Erskine 2013). UAS data are not without challenges, such as radiometric variation between flights and sensors, different sun angles and spatial variation between dates.

Geographic object-based image analysis (GEOBIA) provides a suitable framework for the analysis of ultra-high spatial resolution imagery where the above issues exist as well as for data where there exists increased within-field variability resulting from the pixel size being much smaller than the features under investigation. Time series data of a high temporal resolution are useful for monitoring the progress of rehabilitation particularly in the early stages where growth rate is rapid and tree mortality high. The project reported here describes a GEOBIA approach to monitor change in vegetation cover using a UAS data time series.

\section{METHODS}

\subsection{Study site}

Jabiluka mine site $\left(12^{\circ} 29.9^{\prime} \mathrm{S}, 132^{\circ} 54.8^{\prime} \mathrm{E}\right)$ with an area of 12 ha is located within the Jabiluka mineral lease belonging to Energy Resources of Australia (ERA) (Figure 1). The mineral lease is surrounded by the World Heritage listed Kakadu National Park approximately $300 \mathrm{~km}$ east of Darwin, Northern Territory, Australia. The local climate is monsoonal wet/dry tropics with a short intense wet season (Dec-Mar) and a longer slightly cooler dry season (April-Nov). The study site is situated on the western edge of the Arnhem Land escarpment. Although the mine was never operational, a retention pond was constructed and associated preparatory earthworks were undertaken. During 2013, the mining company conducted rehabilitation works including the removal of the retention pond and shaping of the surface. Plantings of seedlings (tubestock) occurred in two events: (1) over 3600 tubestock were planted across the site during November and December 2013; and (2) a further 4000 tubestock were planted in November 2014. 


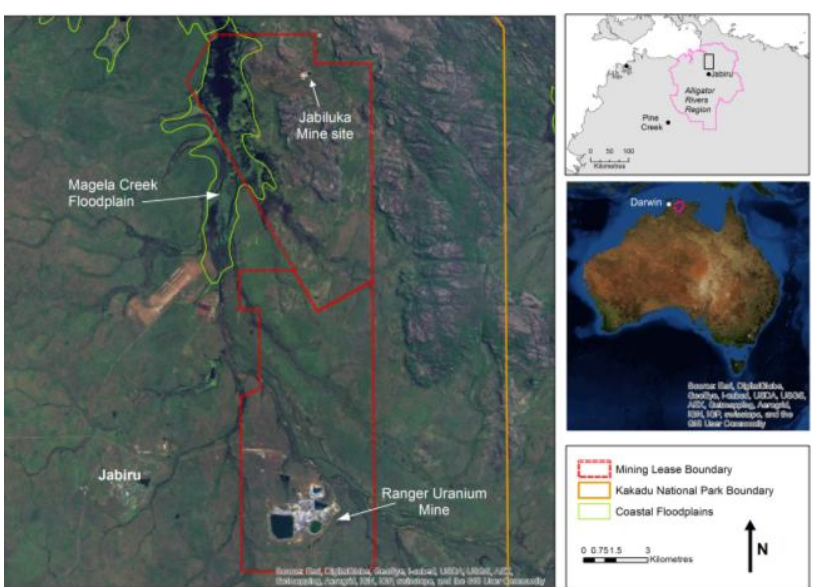

(a)

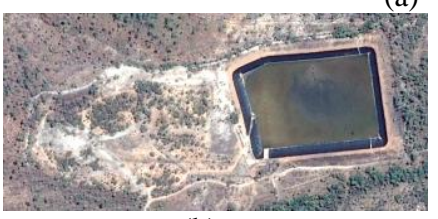

(b)

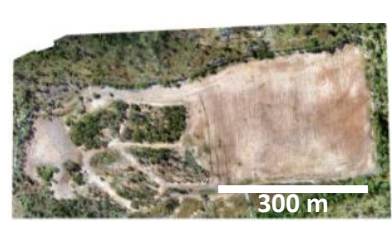

(c)
Figure 1. (a) Location of the study site. Jabiluka mine site February 2013 and after earthworks April 2014; (b) Jabiluka mine site prior to rehabilitation works (February 2013) and (c) post works (April 2014).

\subsection{Platform and sensors}

The UAS platform used for image acquisition was the Swampfox X5 fixed wing (Figure 2a) manufactured by Skycam UAV (www.kahunet.co.nz). The aircraft has a maximum all up weight of $4.7 \mathrm{~kg}$ and a payload capacity of $1.1 \mathrm{~kg}$. It is battery powered with flight duration of approximately 45 minutes maintaining a cruising airspeed of $60 \mathrm{kph}$. The sensor system (Figure 2b) used for most flights consisted of two Sony NEX5 cameras: i) red, green, blue (RGB) and ii) modified near infrared (NIR). The payload for the last flight was the Sony Nex5 RGB and a MicaSense RedEdge multispectral camera. Data was captured at a frame rate of 1 photo per 1.5 seconds along with GPS (time, heading, location and altitude) and attitude information (pitch, roll and yaw). Flights of the study site were conducted on 7 dates between 28 April 2014 and 9 October 2015. Flight patterns ensured at least $75 \%$ forward and $50 \%$ side overlap (75\% for the MicaSense flight) between images. Unfortunately, no NIR data were captured during the 23 April 2015 mission, due to sensor malfunction.

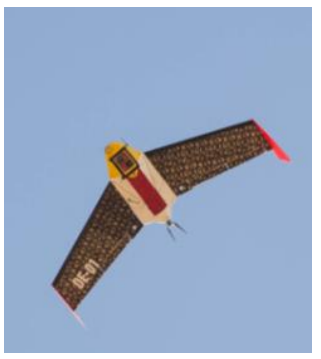

(a)

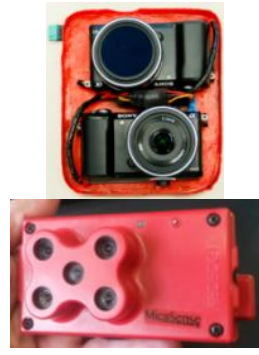

(b)
Figure 2. (a) The Skycam Swampfox aircraft and (b) the sensors used in this project: Sony NEX-5 RGB and NIR (top) and MicaSense RedEdge (bottom).
A minimum of 6 ground control points were marked on the site for each flight and their location recorded with a differential GNSS receiver (Figure 3). Also placed upon the surface were pseudo-invariant calibration panels for the radiometric correction of the imagery.

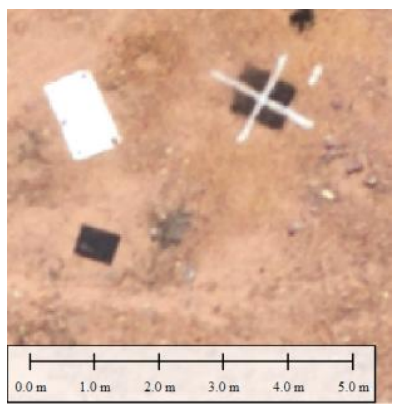

Figure 3. Ground control points and calibration panels.

\subsubsection{Data}

Table 1 shows the data captured for each flight with all fights capturing a minimum of 350 photos.

\begin{tabular}{llccc}
\hline Year & Date & RGB & NIR & RE \\
\hline 2014 & 28 April & $\checkmark$ & $\checkmark$ & \\
& 13 June & $\checkmark$ & $\checkmark$ & \\
& 23 September & $\checkmark$ & $\checkmark$ & \\
& 23 December & $\checkmark$ & $\checkmark$ & \\
\hline 2015 & 23 April & $\checkmark$ & & \\
& 10 July & $\checkmark$ & $\checkmark$ & \\
& 9 October & $\checkmark$ & $\checkmark$ & $\checkmark$
\end{tabular}

Table 1. Data captured for each date. RGB $=$ red, green, blue bands; NIR = near infrared band; RE = red edge band.

\subsubsection{Pre-processing}

Prior to photogrammetric processing, images were corrected for vignetting lens distortion and sensor variability. All 2014 imagery was then processed into orthomosaics by aerial survey and photogrammetric company, Aerometrex PL. All 2015 imagery was processed by Supervising Scientist staff using Pix4DMapper. Processing of UAS data typically involves several steps (Strecha et al. 2012). Firstly, the geometric alignment of photos using a feature matching algorithm and the image position and orientation based on the autopilots photolog are used to conduct a bundle block adjustment. Based on this reconstruction, a 3D point cloud is created which is interpolated forming a mesh which provides a digital surface model (DSM). The DSM is then used to project every image pixel and a georeferenced orthomosaic is calculated. RGB and NIR data were stacked to create 4 band images. Finally, the data were radiometrically calibrated to surface reflectance using the known reflectance of the panels and an empirical line method (Smith and Milton 1999).

\subsubsection{Analysis}

The analysis workflow applied to the imagery from each date is shown in Figure 4. The first step consisted of the calculation of a vegetation index. The normalised difference vegetation index (NDVI) was created for all dates with NIR data. For the one date with no NIR data a surrogate, the Triangular Greenness 
Index (Hunt et al. 2013), was used. Each index layer was then subjected to a $5 \times 5$ Gaussian low pass convolution filter to remove noise. The filtered index was then segmented based on a threshold to create a layer of objects (L1) representative of plant cover on the site. The value of the vegetation index threshold variable was set at 0 . Objects that contained pixels with values greater than the threshold were classified vegetation cover, while those less than the threshold were classified as background.

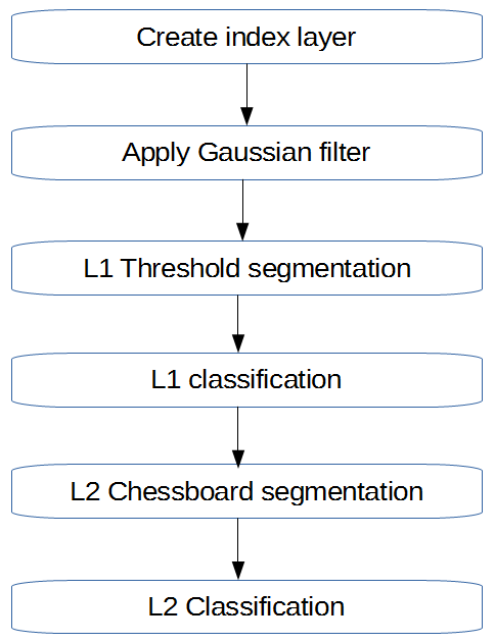

Figure 4. Image analysis workflow

A second layer (L2) of super-objects was created using a chessboard segmentation to create a grid of $10 \times 10 \mathrm{~m}$ objects (Figure 5). Proportional cover of L1 plant objects was then calculated per L2 object. Each L2 object was then assigned to a class based on the proportion of L1 plants sub objects: Less than $1 \%, 1-10 \%, 11-20 \%, 21-30 \%, 31-40 \%, 41-50 \%$, and greater than $50 \%$. Along with proportional cover, the number of plant objects and the average size of plant objects per L2 object were calculated. Change analysis was then conducted between dates on the L2 objects.

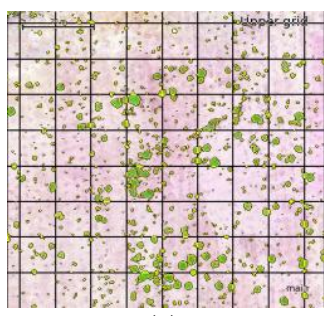

(a)

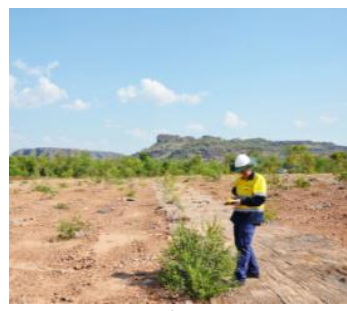

(b)
Figure 5. (a) A sample of the study area showing L1 objects (plants) and L2 objects (10 x $10 \mathrm{~m}$ grid). (b) Collecting field data.

\subsubsection{Validation}

Validation of accuracy was conducted using two methods. Field data were collected during the September 2014 capture, plant location (differential GNSS) and condition recorded for a transect across the site (Figure 5b). This data were compared to the L1 vegetation cover layer for that date. Further validation for other dates was conducted comparing the proportional cover for a random sample of $\mathrm{L} 2$ objects derived from this study to proportional cover estimated from manual aerial photointerpretation.

\section{RESULTS AND DISCUSSION}

All trees that were recorded in the field along the transect were detected in the L1 woody cover layer. There was also some commission error $(<5 \%)$ as a number of non tree objects were detected. These tended to be felt mats that were used as a mulch layer for the tubestock. The comparison between this objectbased proportional cover and the manually derived cover estimates also showed strong correlation $\left(r^{2}>0.8\right)$. Figure 6 shows the proportional cover maps for the mine site for each of the seven dates. The time series shows the change in cover over time highlighting an increase in relative cover within the eastern portion of the site. The maps also show a seasonal influence on the cover, with green growth during the wet season and some senescence in mature plants and mortality in juveniles during the drier months. A snapshot of 30 randomly selected L2 objects shows a trend of increasing relative cover from June 2014 onwards (Figure 7). The mean relative cover increased from less than 5\% in June 2014 to over 20\% in October 2015. Over the same time period however, there has been a decrease in the number of plant objects per L2 object (Figure 8). The mean number of L1 objects per L2 object was 44 in June 2014 decreasing to just over 16 in October 2015. This suggests that while there is plant mortality, the plants that are surviving are growing. It may also suggest that plant crowns are starting to overlap. The peak (April 2015) and trough (August 2015) in Figure 7 may be associated with the second planting (November 2014) and subsequent mortality through the dry season. Alternatively, the spike might be an artefact of the use of a different index for the April 2015 imagery. This will require further analysis. In addition, there was an observed natural recruitment of volunteer plants on site.
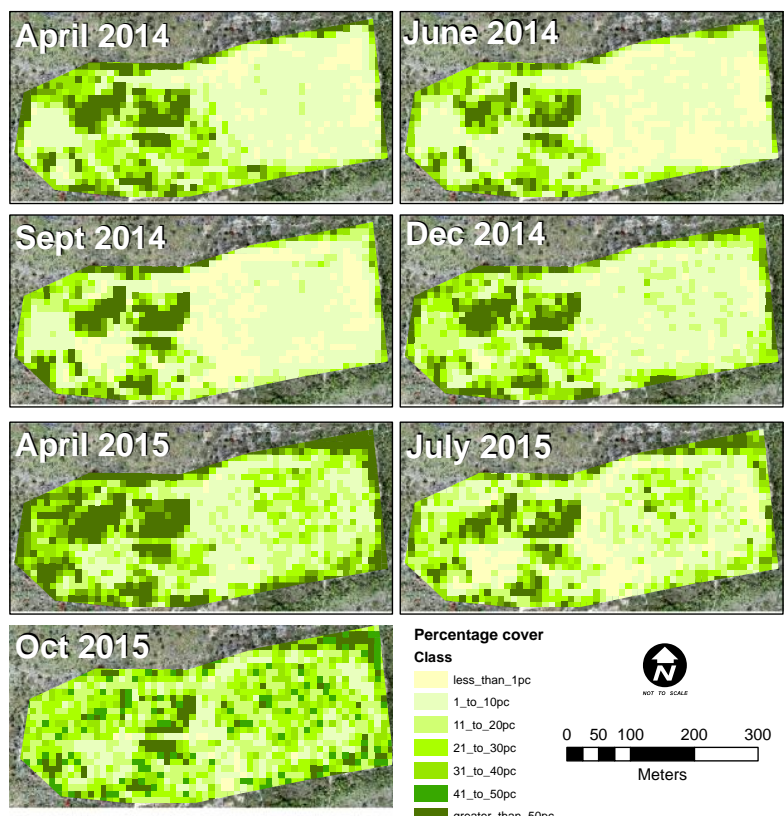

Figure 6. Proportional cover maps for the seven dates of image capture.

\section{CONCLUSION}

The study undertaken here has shown that multispectral UAS data of ultra-high spatial and temporal resolution can be used to provide information relevant to monitoring of mine site rehabilitation progress. Relevant bio-physical parameters, particularly the proportion of vegetation cover for the whole of site can be measured and assessed over time. Information such 
as this will be useful in determining whether a rehabilitation project is capable of achieving its desired outcomes. Further analysis will include a rigorous accuracy assessment of the time series analysis, the addition of more recent image captures, and whether it is possible to use the data to discriminate species. In addition, an assessment of the how much of the regrowth is from planted tubestock versus natural recruitment might also be useful.

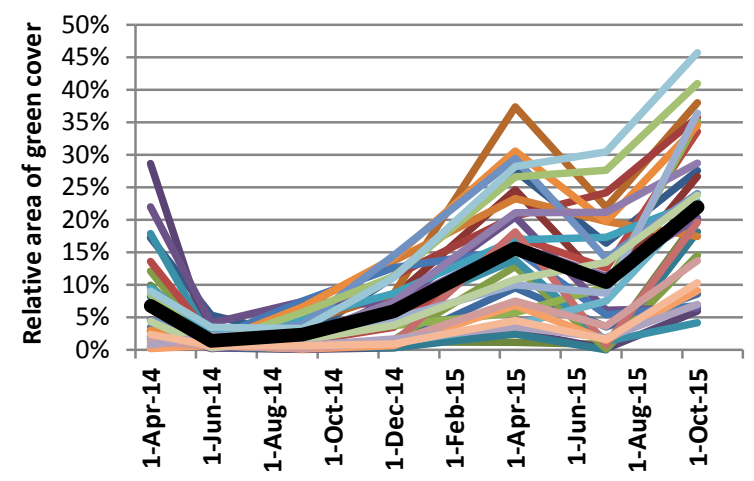

Figure 7. Change over time in the relative cover per the 30 randomly selected L2 objects. Thick black line is the mean relative cover for the objects selected.

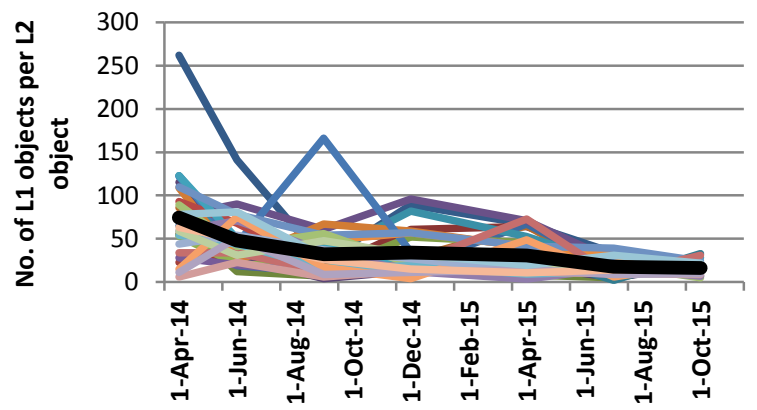

Figure 8. Change over time in the number of L1 objects per the 30 randomly selected L2 objects. Thick black line is the mean value for the objects selected.

\section{ACKNOWLEDGEMENTS}

Sandra Grant, Kirrilly Pfitzner and Andrew Esparon from the Supervising Scientist for field assistance.

\section{REFERENCES}

Fletcher, A.T., \& Erskine, P.D. 2013. Rehabilitation closure criteria assessment using high resolution photogrammetrically derived surface models. International Archives of Photogrammetry, Remote Sensing and Spatial Information Sciences, $X L-1 / W 2$, pp. 137-140.

Hunt, E.R., Doraiswamy, P.C., McMurtrey, J.E., Daughtry, C.S., Perry, E.M., \& Akhmedov, B. 2013. A visible band index for remote sensing leaf chlorophyll content at the canopy scale. International Journal of Applied Earth Observation and Geoinformation, 21, pp. 103-112.
Smith, G.M., \& Milton, E.J. 1999. The use of the empirical line method to calibrate remotely sensed data to reflectance. International Journal of Remote Sensing, 20, pp. 2653-2662.

Strecha, C., Bronstein, A.M., Bronstein, M.M., \& Fua, P. 2012. LDAHash: Improved matching with smaller descriptors. IEEE Transactions on Pattern Analysis and Machine Intelligence, 34, pp. 66-78. 


\title{
DROPBAND: A CONVOLUTIONAL NEURAL NETWORK WITH DATA AUGMENTATION FOR SCENE CLASSIFICATION OF VHR SATELLITE IMAGES
}

\author{
Naisen Yang ${ }^{\mathrm{a}, \mathrm{b}}$, Hong Tang ${ }^{\mathrm{a}, \mathrm{b}}$, Hongquan Sun ${ }^{\mathrm{c}}$, Xin Yang ${ }^{\mathrm{b}}$ \\ ${ }^{a}$ The Key Laboratory of Environmental Change and Natural Disaster,Beijing Normal University, China - \\ yns@mail.bnu.edu.cn \\ b The State Key Laboratory of Earth Surface Processes and Resource Ecology,Beijing Normal University, China- \\ (tanghong, yangxin)@bnu.edu.cn \\ ${ }^{c}$ China Institute of Water Resources and Hydropower Research, China - sunhq@iwhr.com
}

KEY WORDS: dropband, dropout, data augmentation, neural networks, model combination

\begin{abstract}
:
Data augmentation is a common method that can prevent the overfitting of classification tasks in deep neural networks. This paper presents another kind of data augmentation method called DropBand that is useful for remote sensing image classification. Data augmentation is usually used along two dimensions of the image plane. This method executes this operation in the third dimension formed by all the spectral bands of an input image. With dropping a band of images out, the error rate of deep neural networks can be reduced. This method can also be viewed as a peculiar version of deterministic Dropout. The normal Dropout does not work well when it is applied to input channels of neural networks. To release this issue, dropping a band of input by schedule is employed. Moreover, model synthesis plays a key role in this procedure. To exclude the influence of increasing parameters, extra comparison groups are set up. The final experimental result shows that deep neural networks indeed benefit from the method of DropBand. This method improves the state-of-the-art on the latest SAT-4 and SAT-6 benchmarks.
\end{abstract}

\section{INTRODUCTION}

Data augmentation is widely used for preventing overfitting in a diverse range of machine learning technologies. It is also employed for training Deep Neural Networks (DNN) (Krizhevsky et al., 2012, Simonyan and Zisserman, 2014, Szegedy et al., 2015). Data augmentation enlarges the training datasets without touching the architectures of neural networks. By using label-preserving transformations, training dataset covers more regions of the input space.

In many previous works, the main forms of data augmentation, such as cropping and flipping, are performed along two dimensions of the image plane (Howard, 2013, Szegedy et al., 2015, Ciresan et al., 2012). Other forms of data augmentation include color casting (Wu et al., 2015) and intensity altering (Krizhevsky et al., 2012). But it is not thorough enough for remote sensing images. This paper proposes a new form of data augmentation that executes cropping along the third dimension formed by all the spectral bands of an input image. Below this method is referred as DropBand. We focus on convolutional neural networks (CNN) (Fukushima, 1980, LeCun et al., 1998) and show that deep neural networks trained with a subset of input channels can also achieve comparable accuracy.

Unsurprisingly, combining all these individual models trained on different subset of input bands can reduce error rate of models. Furthermore, we set up extra comparison groups which consist of models trained on all available bands with different random initialization. We observed that DropBand groups always surpass the corresponding comparison groups.

This paper is organized as follows. Section 2 describes the architecture of our method. The experimental setup and results are presented in section 3 and 4 respectively. In the end, section 5 gives a conclusion.
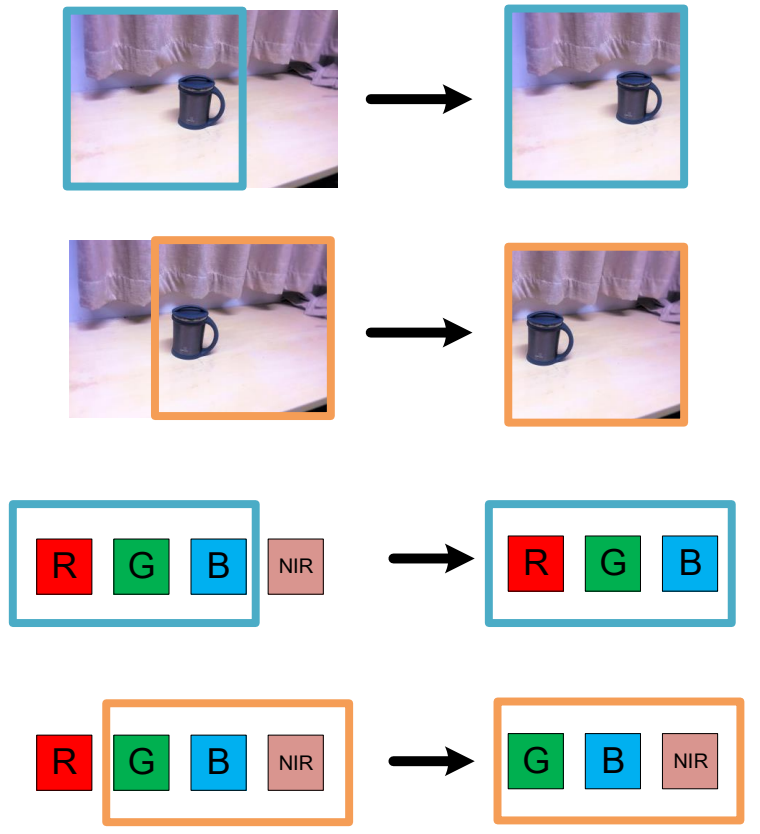

Figure 1. The concept of DropBand is an extension of cropping out a patch of the original input image for data augmentation. This method selects a subset of the input bands - four bands in this figure: red (R), green (G), blue (B) and Near Infrared (NIR).

\section{METHODS}

In this section, we give a concise definition of DropBand , and discuss the connection between this method and other related methods. 


\subsection{DropBand}

The DropBand method simply selects the subsets of input bands as training data. As shown on the top of Figure 2, DNN is trained on the dataset enlarged by DropBand. At test time, the scores of final predictions are a combination of predictions of each subset of input bands. Actually, this form of combination can be viewed as a combination of a number of networks that share same weights (on the bottom of Figure 2). For the reason that the model trained by DropBand can only capture the relevance among the subsets of bands, a base net trained with whole bands is added to the final model.
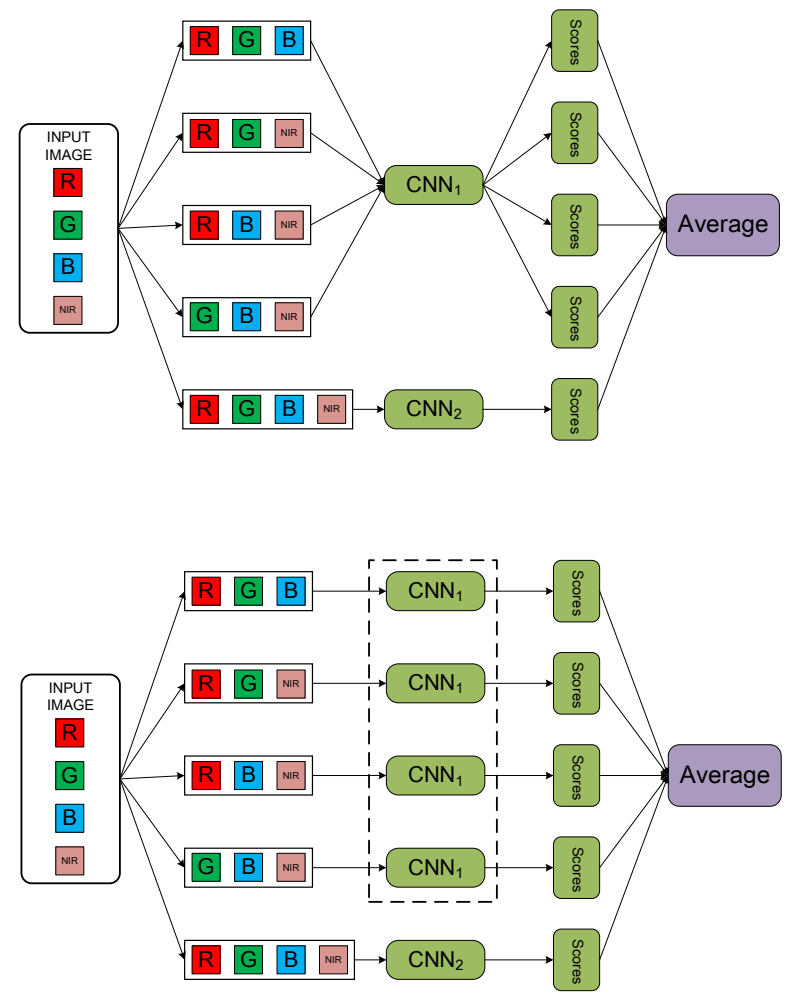

Figure 2. Architecture of the shared DropBand. The model on the bottom is an equivalence of the model on the top.

Unfortunately, by comparing with the base net, feeding the enlarged datasets to the input directly would lead to the changing of hyperparameters of neural networks. For the consideration of parameter tuning, we also train individual models on each maximum proper subset of whole input bands. This version of DropBand is illustrated in Figure 3. To distinguish the two forms of the DropBand method, we refer the model with weight sharing as the shared DropBand (on the top of Figure 2), and name the model in Figure 3 DropBand.

\subsection{From A Data Augmentation Perspective}

Augmenting data by domain knowledge improves the generalization of models (Krizhevsky et al., 2012, Szegedy et al., 2015). Transformations of data augmentation applied to samples preserve their original labels. This is the most common method to enlarge the dataset with little extra effort. In image classification tasks, the most common forms of data augmentation are flipping, cropping, and rotation. These operations are performed along two dimensions of the image plane.

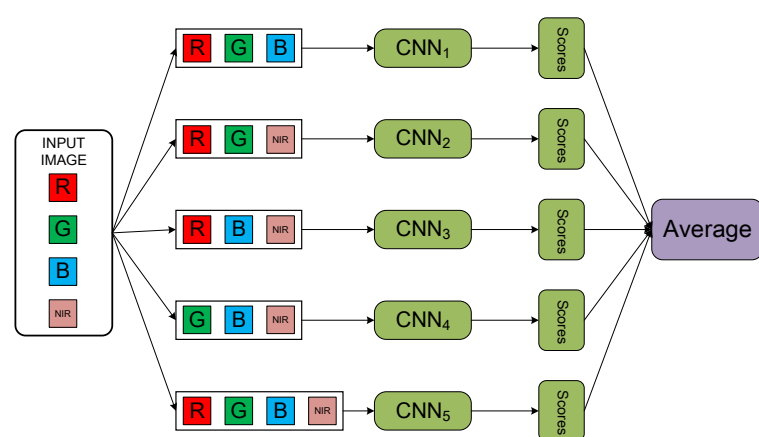

Figure 3. Architecture of the DropBand.

Altering intensities of RGB channels is also adopted widely (Krizhevsky et al., 2012). But for remote sensing images, it is not thorough enough. The most significant difference between the images of everyday objects and remote sensing images is that the latter have a wide range of available bands. These bands form the third dimension, so we can crop part of them along this dimension to augment datasets. Cropping along the dimension of bands amounts to set intensities of some bands to zero. In other words, this means that we have to abandon other bands at the same time. Therefore we call this form of data augmentation DropBand, and it has a deep relationship with Dropout (Srivastava et al., 2014) (this will be explained in section 2.3).

\subsection{From A Dropout Perspective}

By far, the most simple regularization method for training deep neural networks is Dropout (Srivastava et al., 2014). Dropout is an efficient method of model combination with weight sharing. It randomly drops a portion of the hidden units out of the neural network during training time. This amounts to training a single thinned network. All the hidden units work together during testing. Dropout significantly reduces overfitting and prevents the hidden units from co-adapting.

The original Dropout is a stochastic technique that brings noises to the units. DropBand can be regarded as a deterministic version of the original Dropout. It drops some of the input channels consistently instead of at random. This deterministic manner can ameliorate the drawback of misconvergence for the case of dropping the input channels out. When applying Dropout to the input channels with some probabilities of retaining an unit, the validation accuracy of neural network does not follow the increasing training accuracy and oscillates dramatically. This is shown in Figure 4. After using the DropBand, this phenomenon does not appear any more.

\subsection{From A Feature Bagging Perspective}

Feature bagging (Sutton et al., 2006) is to ameliorate the weight undertraining of conditional random fields (CRFs) (Lafferty et al., 2001). Weight undertraining is caused by which a few strong features dominate the result of classifiers. They build a collection of feature bags. Each feature bag consists of overlapping subset of input features. Different models are trained on the corresponding feature bags. Finally, the synthetic model is obtained by averaging the individual CRFs.

DropBand can also be interpreted as a kind of feature bagging if the input bands are seen as a kind of features. The proposed paper (Sutton et al., 2006) have shown the validity of feature bagging 


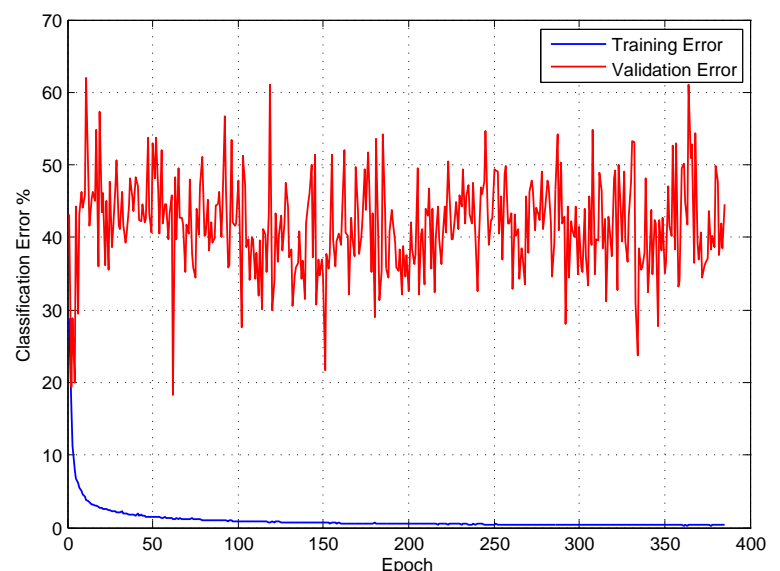

Figure 4. Effect of applying Dropout to the input channels directly. It is obtained by adding Dropout to the input channels with $p=0.75$ on the SAT-6 dataset. Details of this model is described in section 3 .

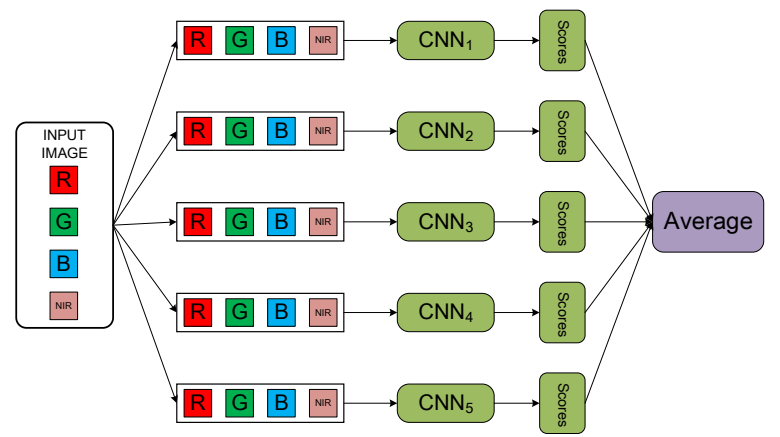

Figure 5. Architecture of comparison group. It consists of five base networks.

by comparing the synthetic model with a single CRF. Furthermore, we set the comparison groups. These are also the synthetic models consisting of the individual models. But these individual models of comparison groups are trained on all the input bands with different initialization (as shown in Figure 5).

\section{EXPERIMENTS}

This section presents the datasets and the configuration of the CNNs used in the experiments.

\subsection{Datasets}

3.1.1 SAT-6 The SAT-6 dataset, released in 2015 (Basu et al., 2015 ), consists of 405,000 image patches of size $28 \times 28$ selected from the National Agriculture Imagery Program (NAIP). It has 6 landcover classes : barren land, trees, grassland, roads, buidings and water bodies. One fifth of them are testing dataset $(81,000$ images) and the remains are training dataset (324,000 images). Each image contains 4 bands - red (R), green $(G)$, blue (B) and Near Infrared (NIR).
3.1.2 SAT-4 The SAT-4 dataset (Basu et al., 2015) is similar to SAT-6. It has 500,000 image patches in total and contains four classes - barren land, trees, grassland and a class composed of all the other landcover classes. One fifth of the SAT-4 dataset are testing dataset $(100,000$ images) and the remains are training dataset (400,000 images). Each image also contains 4 bands red (R), green (G), blue (B) and Near Infrared (NIR).

\subsection{Experimental Setup}

The DNN architecture used in this paper is VGG-like (Simonyan and Zisserman, 2014) (as shown in Figure 6). The convolutional kernel is $3 \times 3$ without zero-padding. The nonlinear activation function uses rectified linear unit (ReLU). Dropout is used for the purpose of preventing the overfitting (Srivastava et al., 2014).

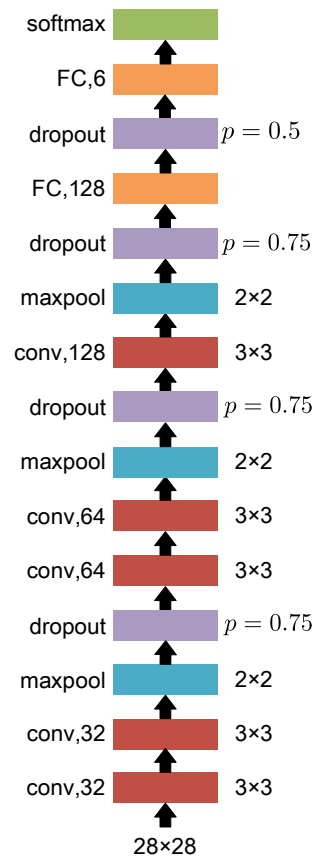

Figure 6. Basic CNN architecture for the SAT-6 dataset. It is also used for the SAT-4 dataset. For the reason that the SAT-4 dataset contains four classes, the last fully connected (FC) layer of the network for SAT-4 has four output channels.

All the network weights follow the Glorot initialization (Glorot and Bengio, 2010). We use the Adadelta optimization method (Zeiler, 2012) for training. Configuration of the Adadelta's parameters follows the recommendation in the proposed paper (Zeiler, 2012). Mini-batch size is 512 in both SAT-6 and SAT-4.

We split out one fifth of training dataset for validation in each experiment. After selecting hyperparameters, the validation dataset is not incorporated to the training dataset.

\section{RESULTS AND DISCUSSION}

SAT-6: Experimental results of DropBand and the shared DropBand on SAT-6 are summarized in Table 1. Due to the large number of training samples, the single base $\mathrm{CNN}$, which achieves a good enough performance, has a testing error rate of $0.038 \%$. The shared DropBand (shown on the bottom of Figure 2) yields 
a very low $0.010 \%$ error rate. It is identical with the error rate of the comparison group consisting of five base networks with different initializations (Figure 5). The DropBand achieves the best result of $0.006 \%$. By comparing the DropBand with the comparison group, we can observe that they have nearly equal number of parameters. This means that the improvement of accuracy comes from DropBand rather than increasing number of models.

\begin{tabular}{lc}
\hline Method & Test error \% \\
\hline DeepSat (Basu et al., 2015) & 6.084 \\
GoogLeNet (Ma et al., 2016) & 3.963 \\
One Base CNN & 0.032 \\
Five Base CNNs (comparison group) & 0.010 \\
Shared DropBand & 0.010 \\
DropBand & $\mathbf{0 . 0 0 6}$ \\
\hline
\end{tabular}

Table 1. Comparison of different methods on SAT-6.

SAT-4: Table 2 shows results on the SAT- 4 dataset. Comparing to the SAT-6 dataset, the SAT-4 dataset contains more samples but less classes. Therefore, the DropBand method obtains almost perfect result of $0.003 \%$. It means that only 3 images are misclassified in testing dataset (100,000 images). Comparison group also reach an error rate of $0.004 \%$, which means that 4 images are misclassified. For the model of the shared DropBand, underfitting occurs.

Taken together, our method, DropBand, improves the state-ofthe-art on SAT- 6 and SAT- 4 .

\begin{tabular}{lc}
\hline Method & Test error \% \\
\hline DeepSat (Basu et al., 2015) & 2.054 \\
GoogLeNet (Ma et al., 2016) & 1.592 \\
One Base CNN & 0.018 \\
Five Base CNNs (comparison group) & 0.004 \\
Shared DropBand & 0.017 \\
DropBand & $\mathbf{0 . 0 0 3}$ \\
\hline
\end{tabular}

Table 2. Comparison of different methods on SAT-4.

\section{CONCLUSION}

In this work, a new form of data augmentation, DropBand, is proposed. It has shown that this method can improve the performance of deep neural network significantly. Besides, results of the comparison groups also demonstrated that the effectiveness of DropBand does not come from the increasing number of models.

Abundant information of colors, which is underutilized by the deep neural networks, is contained in remote sensing images. It is the most notable characteristic different from other datasets. Therefore, an interesting area for future work is creating more refined structures of neural networks to make full use of multispectral information.

\section{ACKNOWLEDGEMENTS}

This work was supported in part by the National Natural Science Foundation of China under Grant 41571334, in part by the Fundamental Research Funds for the Central Universities, and in part by IWHR Research\&Development Support Program (JZ0145B592016).

\section{REFERENCES}

Basu, S., Ganguly, S., Mukhopadhyay, S., DiBiano, R., Karki, M. and Nemani, R., 2015. Deepsat: A learning framework for satellite imagery. In: Proceedings of the 23rd SIGSPATIAL International Conference on Advances in Geographic Information Systems, GIS '15, ACM, New York, NY, USA, pp. 37:1-37:10.

Ciresan, D., Meier, U. and Schmidhuber, J., 2012. Multi-column deep neural networks for image classification. In: Computer Vision and Pattern Recognition (CVPR), 2012 IEEE Conference on, IEEE, pp. 3642-3649.

Fukushima, K., 1980. Neocognitron: A self-organizing neural network model for a mechanism of pattern recognition unaffected by shift in position. Biological cybernetics 36(4), pp. 193-202.

Glorot, X. and Bengio, Y., 2010. Understanding the difficulty of training deep feedforward neural networks. In: International conference on artificial intelligence and statistics, pp. 249-256.

Howard, A. G., 2013. Some improvements on deep convolutional neural network based image classification. arXiv preprint arXiv:1312.5402.

Krizhevsky, A., Sutskever, I. and Hinton, G. E., 2012. Imagenet classification with deep convolutional neural networks. In: F. Pereira, C. Burges, L. Bottou and K. Weinberger (eds), Advances in Neural Information Processing Systems 25, Curran Associates, Inc., pp. 1097-1105.

Lafferty, J., McCallum, A. and Pereira, F., 2001. Conditional random fields: Probabilistic models for segmenting and labeling sequence data. In: Proceedings of the eighteenth international conference on machine learning, ICML, Vol. 1, pp. 282-289.

LeCun, Y., Bottou, L., Bengio, Y. and Haffner, P., 1998. Gradientbased learning applied to document recognition. Proceedings of the IEEE 86(11), pp. 2278-2324.

Ma, Z., Wang, Z., Liu, C. and Liu, X., 2016. Satellite imagery classification based on deep convolution network. World Academy of Science, Engineering and Technology, International Journal of Computer, Electrical, Automation, Control and Information Engineering 10(6), pp. 1031-1035.

Simonyan, K. and Zisserman, A., 2014. Very deep convolutional networks for large-scale image recognition. CoRR.

Srivastava, N., Hinton, G., Krizhevsky, A., Sutskever, I. and Salakhutdinov, R., 2014. Dropout: A simple way to prevent neural networks from overfitting. The Journal of Machine Learning Research 15(1), pp. 1929-1958.

Sutton, C., Sindelar, M. and McCallum, A., 2006. Reducing weight undertraining in structured discriminative learning. In: Proceedings of the main conference on Human Language Technology Conference of the North American Chapter of the Association of Computational Linguistics, Association for Computational Linguistics, pp. 89-95.

Szegedy, C., Liu, W., Jia, Y., Sermanet, P., Reed, S., Anguelov, D., Erhan, D., Vanhoucke, V. and Rabinovich, A., 2015. Going deeper with convolutions. In: Proceedings of the IEEE Conference on Computer Vision and Pattern Recognition, pp. 1-9.

Wu, R., Yan, S., Shan, Y., Dang, Q. and Sun, G., 2015. Deep image: Scaling up image recognition. arXiv preprint arXiv:1501.02876.

Zeiler, M. D., 2012. Adadelta: An adaptive learning rate method. arXiv preprint arXiv:1212.5701. 


\title{
Object-oriented Land Cover Mapping in China National Geographical Conditions Census
}

\author{
Liang Zhai, Huiyong Sang, Qinghua Qiao \\ Chinese Academy of Surveying and Mapping, Lianhuachi West Road 28, Haidian District, Beijing, 100830, China-(zhailiang, \\ huiyong.sang, qiaoqh)@casm.ca.cn
}

KEY WORDS: Nationwide General Survey of Geographic Conditions, Object-oriented Decision Tree Algorithm, Land Cover Classification, Image Segmentation, GLC Classifier, WorldView-2 images

\begin{abstract}
:
Remote sensing image classification is one of the important methods of obtaining land cover/use information for nationwide general survey of geographical conditions in China. In this study, an object-oriented decision tree algorithm is utilized for land cover and land use mapping from high resolution WorldView-2 satellite remote sensing images. The major steps of this approach include image segmentation to get image objects and pixel-based classification based on the image objects. Multi-resolution segmentation approach was adopted in this study, which utilizes spatial and spectral information of land covers in the image to segment into different small objects with particular structural, spectral and texture attributes. A new decision tree classifier called AdaTree. Weight Leaf (AdaTree. WL) was applied to conduct classification process based on the segmented objects, which is modeled by combining the algorithms C4.5 and AdaBoost. The decision algorithm is integrated in the software--GLC (Global Land Cover Classification) classifier. Several study sites are selected from northwest and southeast in China to test GLC classifier, and compared to SVM (Support Vector Machine), the GLC classifier could reaches better results (mean kappa coefficient is $84.61 \%$ ) in different scenarios with WorldView-2 images.
\end{abstract}

\section{INTRODUCTION}

In order to investigate national geographical conditions for the purpose of economic society development and ecological civilization construction, Chinese government initiated the program of the first national geographical conditions census. Land cover mapping is one of the main objectives in this program (NGCC, 2013). Moreover, to better understand the basic conditions of natural ecology and human activity, the timely mapping land-use/land-cover distribution and detecting its change is required (Zhang, J, 2013). Remote-sensing technologies are useful tools in providing these services. Current remote sensing image classification models commonly used pixel-based and object-oriented method according to different studying objects and purposes. Pixel-based classifiers, such as maximum likelihood, decision tree, SVM and neural networks, can be extensive applied for land use classification with moderate or low resolution remote sensing images (Lackner M, 2014, Dengsheng Lu, 2013, Vieira, I. C. G, 2003). With the increase of spatial resolution of satellite image, the traditional per-pixel classification results in the well-known salt and pepper effect, while the object-oriented method has provided a new method for this situation (Lackner M, 2014, Sun Z P, 2010, Chaudhary R C F N, 2008). Meanwhile, decision tree algorithm becomes more mature (Zhai L, 2015, Wang P, 2015, Chasmer L, 2014). GLC tree classifier integrated the improved C4.5 and AdaBoost algorithm (Zhai L, 2015, Dou P, 2013). In the global surface coverage classification of Oceania, GLC tree has been successfully applied to Landsat satellite images (2000 and 2010, 2,796 scenes) category, and the average classification accuracy reached more than $85 \%$. Therefore, the objectives of this research are (1) to develop a new method to improve land use classification accuracy through the integrated use of object-oriented method and GLC tree algorithm with high resolution WorldView-2 satellite remote sensing images and (2) to propose the automatic classification technology for the national geographical conditions census.

\section{METHOD}

\subsection{Data Source}

WorldView-2 remote sensing images are the main data source of nationwide general survey of geographic conditions. It provides eight bands (coastal zone, blue, green, red, yellow, red edge band, and two near infrared bands) with $2 \mathrm{~m}$ pixel size, and one panchromatic band with $0.46 \mathrm{~m}$ pixel size. While in the census, there are four bands (blue, green, red and near infrared) mainly used in extracting the land-cover information. Therefore, these four bands are adopted in this study.

\subsection{Method}

The major steps of this approach include image segmentation to get image objects and pixel-based classification based on the image objects. The strategy of object-oriented decision tree algorithm for land cover classification is illustrated in Figure 1. The major steps include (1) taking image segmentation on WorldView-2 image to get homogeneous polygons; (2) selecting and extracting features for classification, taking crossvalidation on sample quality after collecting specimens; (3) achieving automatic classification by using GLC tree classifier; (4) mapping land cover with manual editing. 


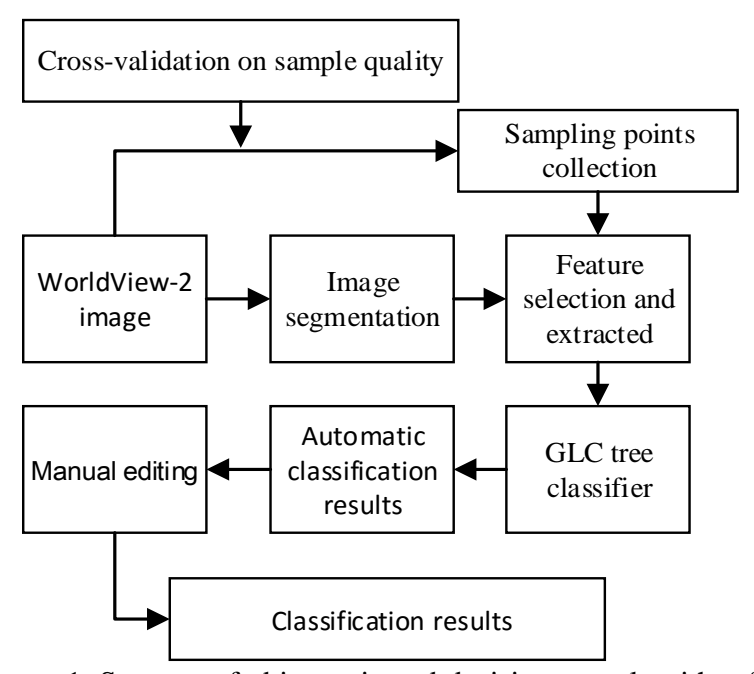

Figure 1. Strategy of object-oriented decision tree algorithm for land cover classification

\subsubsection{Image segmentation and feature selection}

Multi-resolution segmentation approach is adopted in this study, which utilizes spatial and spectral information of land covers in the image to segment into different small objects with particular structural, spectral and texture attributes. Table 1 shows some of the most common features among the classification based on object-oriented method.

Table 1. Features of remote sensing images

\begin{tabular}{|c|c|}
\hline Type & Characteristic \\
Spectrum & $\begin{array}{c}\text { Brightness, Mean, Ratio, Standard Deviation, } \\
\text { Normalized Difference Vegetation Index (NDVI), } \\
\text { etc } \\
\text { Structure } \\
\text { Aspect Ratio, Perimeter, rectangle fit, Density, } \\
\text { Area, Compactness, etc } \\
\text { Texture }\end{array} \begin{array}{c}\text { Homogeneity (HOM), Entropy (ENT), Contrast } \\
(\mathrm{COM}), \text { Angular Second Moment (ANG), etc }\end{array}$ \\
\hline
\end{tabular}

\subsubsection{Sampling points collection}

The classifications of land cover are defined in Contents and indexes of national geographical conditions census, and classified into three levels (the first level has 12 second classes, and the second level are divided into 49 third classes, and then reclassified into 86 subclasses). It's suggested to flexibly define the land coverage in this research. User-defined land cover types are also utilized in the sample collection. Croplands and fallow lands with evident spectral difference are further differentiated from the land cover class "agricultural lands". There are several suggestions that may help extracting land cover samples:

(1)The classification accuracy is determined by the quality of training samples. It is better to collect samples by the technicians with rich experience.

(2)Training samples should generally be no less than five in each image.

(3)The classification accuracy of land cover classes is related with the number of training samples.

(4)The samples are selected from the centres of land cover types. (5)Usually the collected samples have a uniform spatial distribution.

\subsubsection{GLC tree classifier}

A new decision tree classifier called AdaTree. Weight Leaf (AdaTree. WL) was applied to conduct classification process based on the segmented objects. AdaTree. WL is modeled by combining the algorithms C4.5 and AdaBoost. Unlike C4.5, this algorithm develops a single decision tree by conducting a binary recursive partitioning process and predefining a depth threshold for this tree. AdaTree. WL also modifies the final hypothesis of AdaBoost by setting a prediction weight for each leaf. The prediction weight is given by its prediction accuracy and the weight of the decision tree it belongs to. The decision algorithm is integrated in the software--GLC (Global Land Cover Classification) classifier. There are three steps to achieve automatic classification results (Figure 2). Based on this method, it takes a set of rules in process of training samples, and the single one can be expressed as follows:

count $=$ " $2 "$ cover $=" 6 "$ correct $=" 6 "$ pre $=" 1.0518 "$ class $=$ "8";

att $=$ " $4 "$ cut $=$ " $1170.5 "$ result $="<="$;

att $=$ " $4 "$ cut $=" 601 "$ result $=">"$

where $\quad$ count $=$ number of judgment condition

cover $=$ sample size of the single rule

correct $=$ correct number of predicted value pre $=$ weighted prediction of the single rule

class $=$ class code

att $=$ attributive judgment

cut $=$ threshold

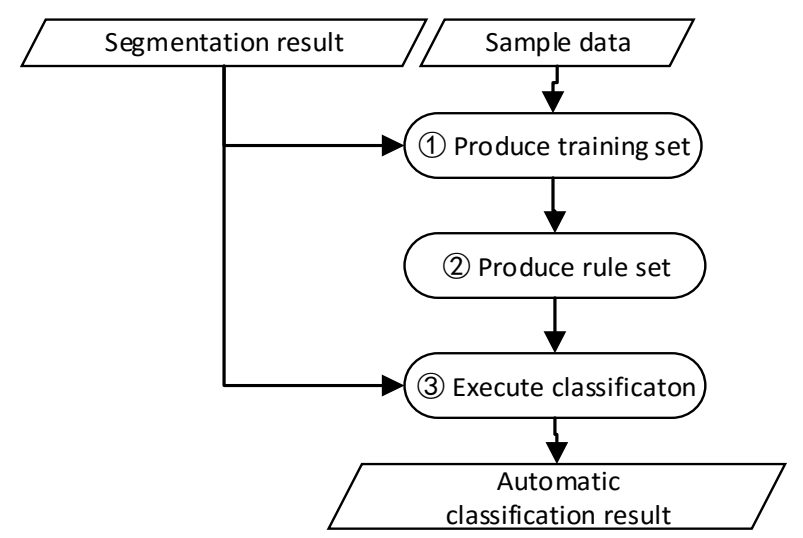

Figure 2. Workflow of GLC tree classifier for land cover classification

\section{RESULT AND DISCUSSION}

In order to test the object-oriented decision tree algorithm, several study sites are selected from northwest northeast and south east in China. Meanwhile, a comparison of the results with SVM-based method shows that whether the GLC classifier is creditable.

\section{(1) Experiment 1 - Lingtong Shanxi}

In this experiment, segmentation scale is set to 100 , and training samples and test samples are 368 and 201, respectively. 


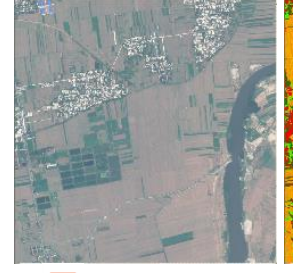

impervious suface

A

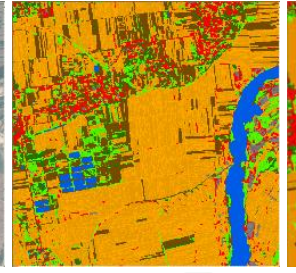

grassland $\square$ bare land

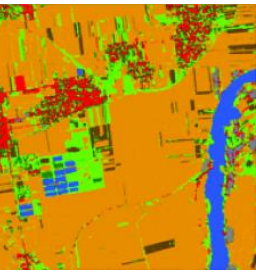

forest
Figure 3. A comparison of classification results from different classifiers. $\mathrm{A}$ is the WorldView-2 image somewhere in Lingtong. B, C are classified images based on GLC and SVM classifier, respectively.

\section{(2) Experiment 2 - Northeast China}

For the second experiment, parameter settings are 200, 285 and 236.

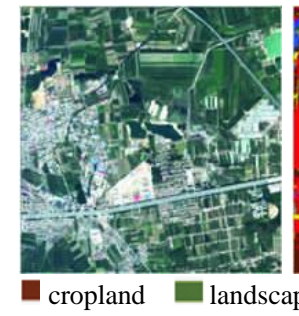

A

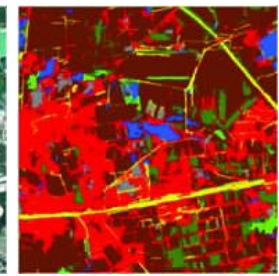

bare land

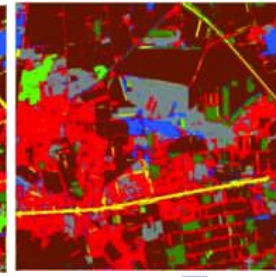

tion $\square$ road $\square$ water C
Figure 4. A comparison of classification results from different classifiers. $\mathrm{A}$ is the WorldView-2 image somewhere in Northeast China. B, C are classified images based on GLC and SVM classifier, respectively.

\section{(3) Experiment 3 - Hangzhou Zhejiang}

The segmentation scale is 150 , training samples and test samples are set in 406, 206.

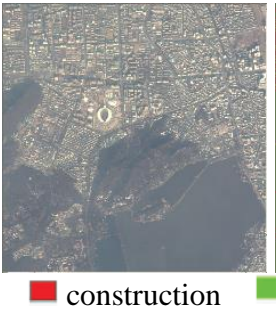

A

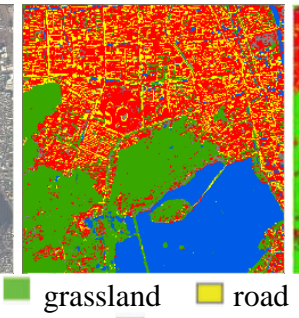
water $\square$ bare land B

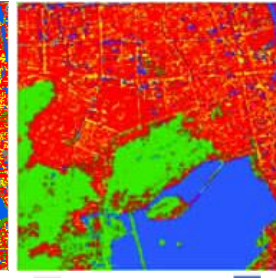

$\mathrm{C}$
Figure 5. A comparison of classification results from different classifiers. A is the WorldView-2 image somewhere in Hangzhou. B, C are classified images based on GLC and SVM classifier, respectively.

Compared to the distinction of land cover from original images among different study areas, classification difficulty increases gradually. Comparative analysis of the land cover classification results (Figure 3,4 and 5) shows that GLC tree algorithm is helpful in improving classification accuracies. The land cover classification results (Table 2) based on image segmentation indicate that, compared to SVM method, GLC increased OA (Overall Accuracy) by $1.06 \%-4.65 \%$. In particular, the precision are willing to decline as the difficulty of classification. Meanwhile, based on AdaBoost lifting scheme, GLC classifier can improve classification accuracy, but there existed some differences each experiment for that algorithm uses random function to reselect different samples based on a new weight. While there is no remarkable difference between each classification, so this approach remains suitable for national geographical conditions census.

Table 2. Comparison of classification results from different classifiers based on WorldView-2 data

\begin{tabular}{|c|c|c|c|c|}
\hline \multirow{2}{*}{$\begin{array}{c}\text { Evaluation } \\
\text { Index }\end{array}$} & \multicolumn{2}{|c|}{ GLC-Tree } & \multicolumn{2}{c|}{ SVM } \\
\cline { 2 - 5 } & $\begin{array}{c}\text { Kappa } \\
\text { Coefficient }\end{array}$ & $\begin{array}{c}\text { Overall } \\
\text { Accuracy }\end{array}$ & $\begin{array}{c}\text { Kappa } \\
\text { Coefficient }\end{array}$ & $\begin{array}{c}\text { Overall } \\
\text { Accuracy }\end{array}$ \\
\hline $\begin{array}{c}\text { Experiment } \\
1\end{array}$ & $93.53 \%$ & $92.42 \%$ & $89.55 \%$ & $87.77 \%$ \\
$\begin{array}{c}\text { Experiment } \\
2\end{array}$ & $87.29 \%$ & $85.19 \%$ & $84.65 \%$ & $82.70 \%$ \\
$\begin{array}{c}\text { Experiment } \\
3\end{array}$ & $81.30 \%$ & $79.23 \%$ & $80.19 \%$ & $78.17 \%$ \\
Mean & $87.37 \%$ & $85.61 \%$ & $84.80 \%$ & $82.88 \%$ \\
\hline
\end{tabular}

\section{CONCLUTIONS}

The method developed in this paper, which is based on the object-oriented decision tree algorithm that combine image segmentation and GLC-based algorithm, can effectively extract land cover with WorldView-2 images. Meanwhile, three images from different regions in China were choose and processed with GLC classifier, and the mean kappa coefficient of result reaches $84.61 \%$. In particular, the automatic classification technology is recommended in geographical conditions census for detailed land cover classification. In view of improving work efficiency in the project, we will concentrate on setting up a rule database for land cover classification based on GLC tree algorithm in the future.

\section{ACNOWLEDGEMENTS}

This work was supported by the National Geographical Conditions Monitoring Project (grant number B1605); Program for the Young Academic and Technological Leaders of NASG, funded by Key Laboratory of Geo-informatics of NASG (grant number E1604); Beijing Key Laboratory of Urban Spatial Information Engineering. We would like to thank the reviewers for their valuable comments and suggestions. We also wish to thank Siwei Worldview Technology Co., Ltd for the WorldView-2 data used in this research.

\section{REFERENCES}

NGCC, 2013. The notification of first national geographical conditions census.

http://ngcc.sbsm.gov.cn/article/kjcj/zdxmcg/201307/201307000 02626.shtml.

Zhang, J., 2013. Understanding geographical conditions monitoring: a perspective from China. International Journal of Digital Earth, 8:38-57.

Zhang J., 2014. A comparison of pixel-based and object-based land cover classification methods in an arid/semi-arid environment of Northwestern China[C]// Earth Observation and Remote Sensing Applications (EORSA), 2014 3rd International Workshop on. IEEE, 2014:403-407.

Lackner M., 2014. Determining land-use information from land cover through an object-oriented classification of IKONOS imagery. Canadian Journal of Remote Sensing, 34(2):77-92. 
Dengsheng Lu., 2013. A comparison of multisensor integration methods for land cover classification in the brazilian amazon. Giscience \& Remote Sensing, 48(3):345-370.

Vieira, I. C. G., 2003. Classifying successional forests using landsat spectral properties and ecological characteristics in eastern amazônia. Remote Sensing of Environment, 87(4):470481.

Sun Z P., 2010. Object-oriented land cover classification using HJ-1 remote sensing imagery. Science China, 53(S1):34-44.

Chaudhary R C F N., 2008. Multi-scale Image Segmentation and Object-Oriented Processing for Land Cover Classification. Giscience \& Remote Sensing, 45(4):377-391.

Zhai L., 2015. A new approach for mapping regional land cover and the application of this approach in Australia. Remote Sensing Letters, 6(4):267-275.

Dou P., 2013. Research and application of object-oriented remote sensing image classification based on GLC. Geomatics \& Spatial Information Technology, 36(11):68-71.

Wang P., 2015. A Land Cover Classification Method for Antarctica Using Support Vector Machine and Decision Tree. Open Cybernetics \& Systemics Journal, 9(1):2920-2928.

Chasmer L., 2014. A decision-tree classification for low-lying complex land cover types within the zone of discontinuous permafrost. Remote Sensing of Environment, 143(10):73-84. 